\title{
FY 2019 DOE-EERE Materials Vehicle Technologies Office Consolidated Report
}

Sarah Kleinbaum, Gordon E Holt, Gurpreet Singh, Jerry L. Gibbs, H. Felix Wu, Ph. D

April 2020

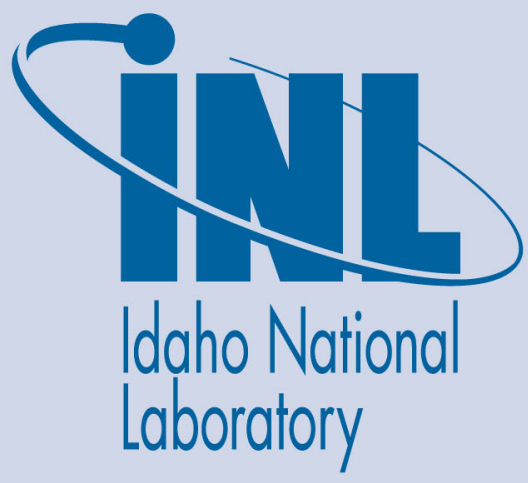

The INL is a U.S. Department of Energy National Laboratory operated by Battelle Energy Alliance 


\section{FY 2019 DOE-EERE Materials Vehicle Technologies Office Consolidated Report}

Sarah Kleinbaum, Gordon E Holt, Gurpreet Singh, Jerry L. Gibbs, H. Felix Wu, Ph. D

April 2020

Idaho National Laboratory Idaho Falls, Idaho 83415

http://www.inl.gov

Prepared for the U.S. Department of Energy

Office of Energy Efficiency and Renewable Energy

Under DOE Idaho Operations Office

Contract DE-AC07-05ID14517 


\section{Office of}

ENERGY EFFICIENCY \& RENEWABLE ENERGY

\section{Materials}

2019 Annual Progress Report

Vehicle Technologies Office 
(This page intentionally left blank) 


\section{Disclaimer}

This report was prepared as an account of work sponsored by an agency of the United States government. Neither the United States government nor any agency thereof, nor any of their employees, makes any warranty, express or implied, or assumes any legal liability or responsibility for the accuracy, completeness, or usefulness of any information, apparatus, product, or process disclosed or represents that its use would not infringe privately owned rights. Reference herein to any specific commercial product, process, or service by trade name, trademark, manufacturer, or otherwise does not necessarily constitute or imply its endorsement, recommendation, or favoring by the United States government or any agency thereof. The views and opinions of authors expressed herein do not necessarily state or reflect those of the United States government or any agency thereof. 


\section{Acknowledgements}

First and foremost, the Principal Investigators from industry, academia, and the National Laboratories who supplied the content of these reports are to be acknowledged and commended. It is their work that moves our nation forward to improved and more affordable transportation, as well as increased energy security.

Thank you to the project managers at the National Energy Technology Laboratory for continued support administering these projects.

We would also like to acknowledge Energetics for their help in preparing and publishing this report.

\section{Gurpreet Singh}

Acting Program Manager

Materials Technology Program

Vehicle Technologies Office

\section{Sarah Kleinbaum}

Technology Development Manager

Multi-Material Joining

Lightweight Materials

Vehicle Technologies Office
Jerry L. Gibbs

Technology Development Manager

Propulsion Materials

Vehicle Technologies Office

\section{H. Felix Wu, Ph. D}

Technology Development Manager

Carbon Fiber and Polymer Composites

Lightweight Materials

Vehicle Technologies Office 


\title{
Acronyms and Abbreviations
}

\author{
symbols \\ $\alpha_{\text {def }}$ \\ deformed ferrite \\ $\alpha_{\text {rx }}$ \\ ferrite recrystallization \\ $\Delta \mathrm{a}_{\mathrm{e}}$ \\ crack extension \\ $\varepsilon$ \\ strain \\ $\gamma$ \\ austenite formation \\ $\gamma^{\prime}$ \\ major strengthening phase \\ $\mu \mathrm{m}$ \\ micrometer \\ $\mu \mathrm{s}$ \\ microsecond \\ $\rho$ \\ density \\ $\sigma$ \\ stress (or strength, when referring to physical properties) \\ $\sigma$ \\ electrical conductivity (when referring to electricity) \\ $\sigma_{\text {UTS }}$ \\ ultimate tensile strength \\ $\sigma_{\mathrm{ys}}$ \\ yield strength \\ $\tau_{10}$ \\ a compositional phase of a ternary intermetallic, such as aluminum ( $\mathrm{Al})-$ silicon ( $\mathrm{Si}$ ) \\ light alloy reinforced with a cast-iron insert (e.g., Al-iron [Fe]-Si) \\ $\Psi$ \\ loss coefficient defined as $\psi=\Delta U / U$ \\ $\Psi_{\mathrm{m}}$ \\ energy of morphed networks \\ $\Psi_{\mathrm{d}}$ \\ energy of deactivated networks \\ $2 \mathrm{D}$ \\ two-dimensional \\ $3 \mathrm{D}$ \\ three-dimensional \\ $3 \mathrm{M}$ \\ Minnesota Mining and Manufacturing Company (company's former name) \\ $21-2 \mathrm{~N}$ \\ an austenitic stainless steel with 21 percent chromium $(\mathrm{Cr})$ and 2 percent nickel (Ni) \\ used in stock engine exhaust valve applications \\ a nitrogen-alloyed austenitic stainless steel with 23 percent $\mathrm{Cr}, 8$ percent $\mathrm{Ni}$, and \\ 2.5 percent manganese $(\mathrm{Mn})$ high-temperature $\left(450 \sim 900^{\circ} \mathrm{C}\right)$ valve applications \\ $316 \mathrm{~L}$ \\ $5 \times x x$ \\ the low carbon version of 316 stainless steel commonly used in chemical and \\ petrochemical industry, in food processing, pharmaceutical equipment, medical \\ devices, in potable water, wastewater treatment, in marine applications and \\ architectural applications near the seashore or in urban areas \\ $6 \mathrm{xxx}$ \\ series designation for $\mathrm{Al}$ alloyed with magnesium $(\mathrm{Mg})$ \\ $7 \mathrm{xxx}$ \\ series designation for $\mathrm{Al}$ alloyed with $\mathrm{Mg}$ and $\mathrm{Si}$ \\ series designation for $\mathrm{Al}$ alloyed with zirconium $(\mathrm{Zr})$
}


A

A356

A365

AA

AA3003

AA5083

AA5182

AA5754

AA6022

AA6061

AA6111

AA7055

AA7075

ABAQUS

ABL

AC

ACEC

ACMZ

ACN

AC-STEM

AET

AFA

AFM-1

AFM-D
$\mathrm{Al}$ alloy with greater elongation, higher strength, and considerably higher ductility than Alloy 356 because of lower Fe content that is typically used for airframe castings, machine parts, truck chassis parts, aircraft and missile components, and structural parts requiring high-strength

a primary die-casting alloy ( $\left.\mathrm{Al}-\mathrm{Si}_{9}-\mathrm{Mg}-\mathrm{Mn}\right)$ widely used to manufacture automotive parts

Aluminum Association

$\mathrm{Al}$ alloy with moderate strength, which can be increased by cold working, and good corrosion resistance

$\mathrm{Al}$ alloy with $\mathrm{Mg}$ and traces of $\mathrm{Mn}$ and $\mathrm{Cr}$ that is highly resistant to attack by seawater and industrial chemicals

wrought alloy with good corrosion resistance and weldability containing $4.5 \% \mathrm{Mg}$, $0.35 \% \mathrm{Mn}$, and the balance $\mathrm{Al}$

wrought $\mathrm{Al}$ alloy containing $94.2 \%$ to $97.4 \% \mathrm{Al}, 0.3 \%$ max $\mathrm{Cr}, 0.1 \%$ max copper $(\mathrm{Cu}), 0.4 \% \max \mathrm{Fe}, 2.6 \%$ to $3.6 \% \mathrm{Mg}, 0.5 \% \max \mathrm{Mn}, 0.4 \% \max \mathrm{Si}, 0.15 \% \max$ titanium (Ti), $0.2 \%$ max $\mathrm{Zn}$, and $0.15 \%$ max residuals formed by rolling, extrusion, and forging, but not casting that can be cold worked to produce tempers with a higher strength but a lower ductility

heat treatable low $\mathrm{Cu}$ precipitation hardenable $\mathrm{Al}$ sheet alloy containing $0.8 \%$ to $1.5 \% \mathrm{Si}, 0.45 \%$ to $0.70 \% \mathrm{Mg}$, and $0.25 \%$ zinc $(\mathrm{Zn})$

precipitation hardening $\mathrm{Al}$ alloy containing $0.8 \%$ to $1.2 \% \mathrm{Mg}$ and $0.4 \%$ to $0.8 \% \mathrm{Si}$ as its major alloying elements

wrought $\mathrm{Al}$ alloy that is a heat treatable and possesses high-strength and excellent stretch-forming characteristics containing $0.6 \%$ to $1.1 \% \mathrm{Si}, 0.5 \%$ to $1.0 \% \mathrm{Mg}, 0.1 \%$ to $0.45 \% \mathrm{Mn}, 0.5 \%$ to $0.9 \% \mathrm{Cu}$, and $0.15 \% \mathrm{Zn}$

$\mathrm{Al}$ alloy used in the aerospace sector and other high-strength requirement areas

$\mathrm{Al}$ alloy with strength comparable to many steels, good fatigue strength, and average machinability

software suite for finite element analysis and computer-aided engineering

acrylonitrile-butadiene-lignin

Allen-Cahn

Advance Combustion Engine and Emission Control

designation for $\mathrm{Al}$ alloys containing $\mathrm{Al}, \mathrm{Cu}, \mathrm{Mg}$, and $\mathrm{Zr}$

acrylonitrile

aberration-corrected scanning transmission electron microscope (or microscopy)

Applied Engineering \& Technology Integration, Inc.

$\mathrm{Al}_{2}$-oxygen $(\mathrm{O})_{3}$-forming austenitic alloys (or alumina-forming alloys)

antiferromagnetic - single layer

antiferromagnetic - double layer 
AHSS

$\mathrm{Al}$

$\mathrm{Al}_{2} \mathrm{O}_{3}$

$\mathrm{A} / \mathrm{m}^{2}$

AM

AMC

AMIPC

ANL

AP

APS

APS

APS-U

APT

AR

Arconic

ASCENDS

ASME

ASTM

at. $\%$

Aural 2

AZ

AZ21

AZ31B

B

B117

bar

BCC or bec

BF

BIW

BJ

BM

BMW advanced high-strength steel

aluminum

Al oxide

amps per square meter

additive manufacturing or additive manufactured (when referring to processes)

Al matrix composites

additively manufactured interpenetrating phase composite

Argonne National Laboratory

atmospheric plasma

Atmospheric Plasma Solutions (when referring to treatment of $\mathrm{Mg}$ )

Advanced Photon Source (when referring to materials characterization)

Advanced Photon Source Upgrade

atom probe tomography

as-received

company specializing in lightweight metals engineering and manufacturing

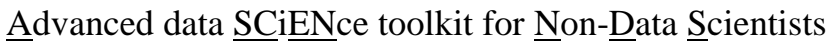

American Society of Mechanical Engineers

American Society for Testing and Materials

atomic percent

$\mathrm{Al}$ alloy used for thin wall structural components

$\mathrm{Mg}$ alloy designation with $\mathrm{Al}$ and $\mathrm{Zn}$ as the principal alloying metals

$\mathrm{Mg}$ alloy with $2 \% \mathrm{Al}$ and $1 \% \mathrm{Zn}$

most widely available $\mathrm{Mg}$ grade alloy, high-strength-to-weight ratio with a composition of $2.5 \%$ to $3.5 \% \mathrm{Al}$ and $0.7 \%$ to $1.3 \% \mathrm{Zn}$

ASTM standard salt spray test used to produce relative corrosion resistance information on metals and coated metals

measurement of pressure equal to one atmosphere

body-centered cubic

bright field

body-in-white

binder-jetting

base metal

Bayerische Motoren Werke AG; a German luxury automobile, motorcycle, and engine manufacturing company 


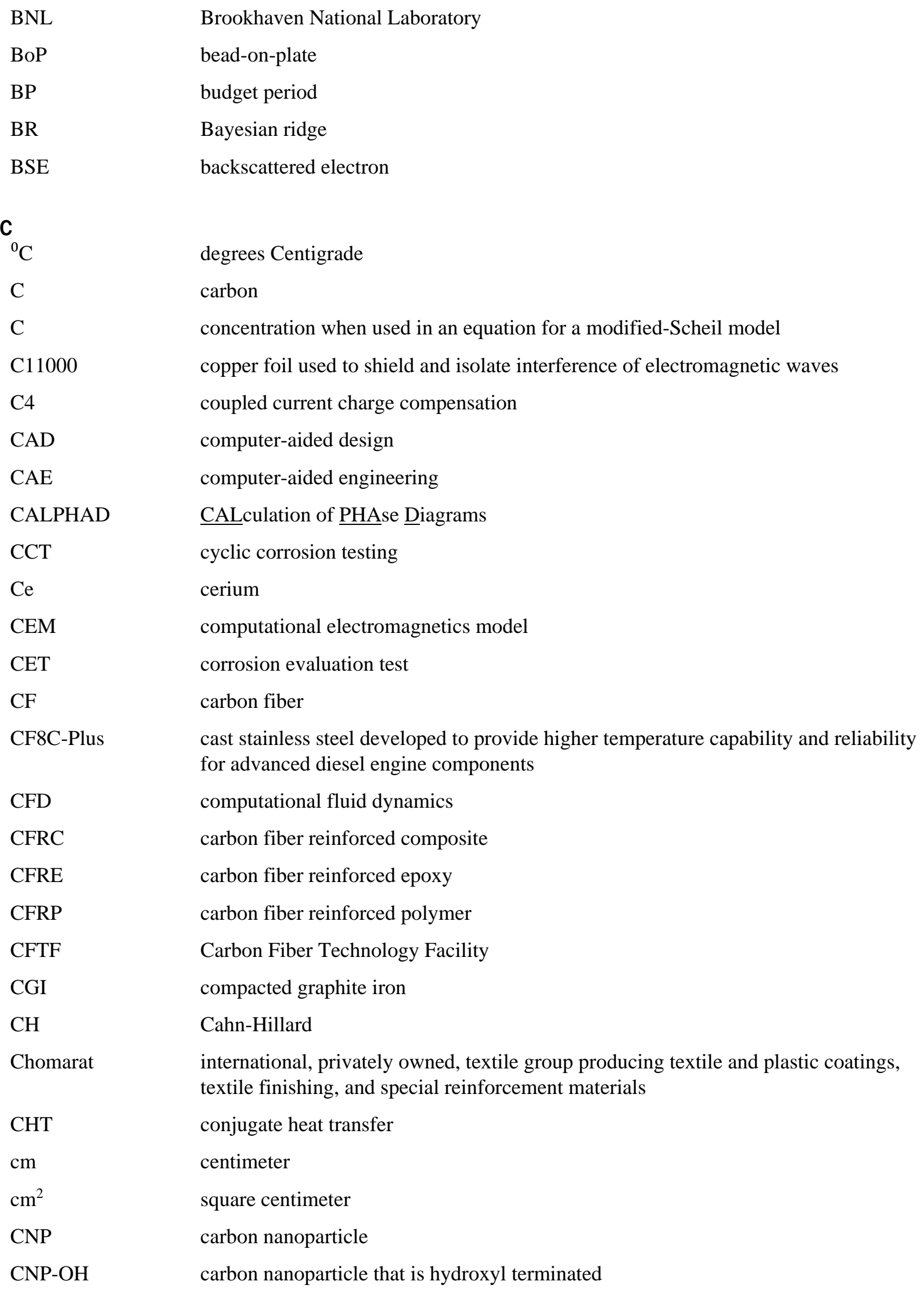




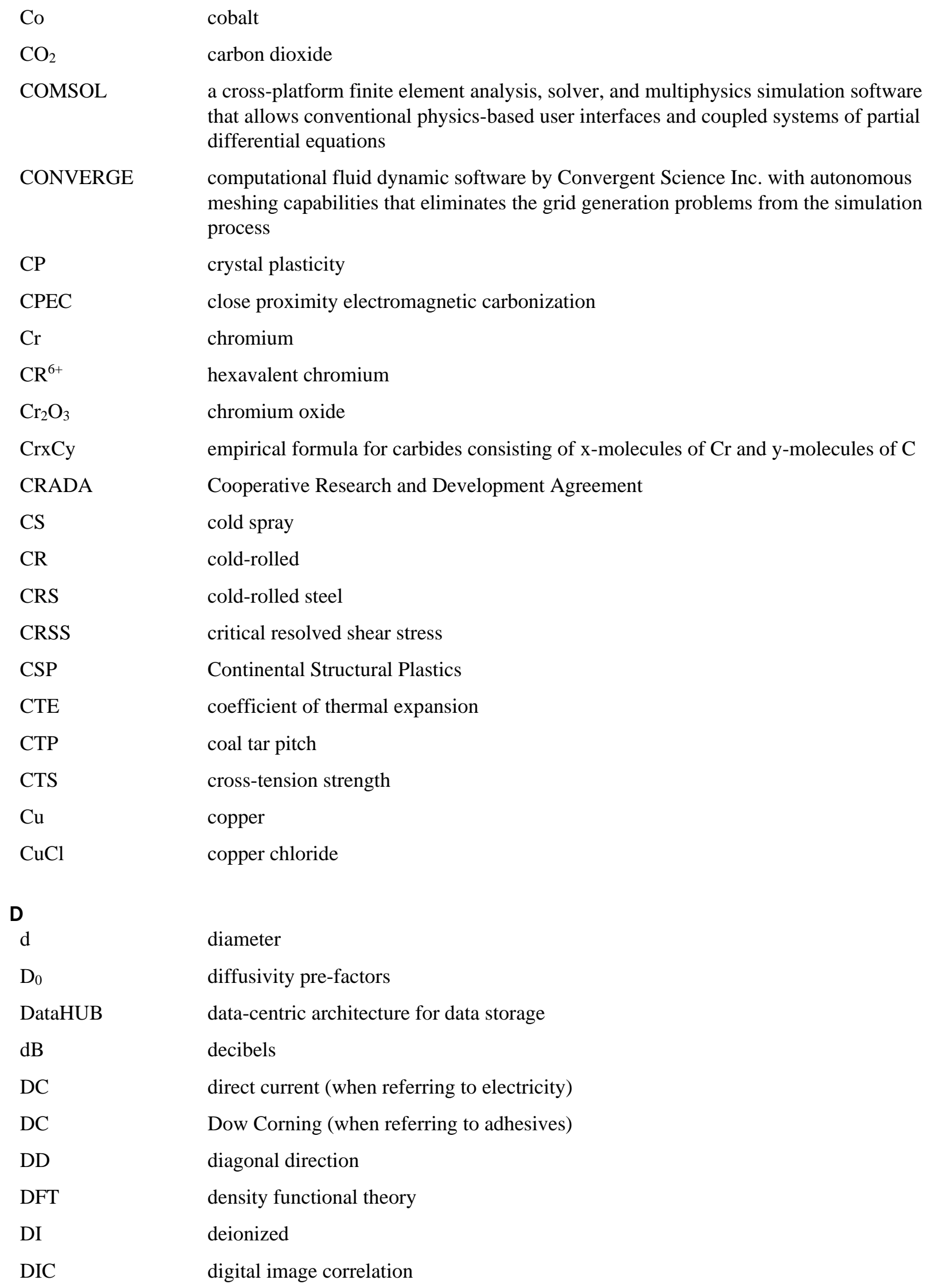




\section{DICTRA}

DL

DMA

DOE

DoE

DP

DP980

DSA

DSC

\section{E}

e.g.

E

$\mathrm{E} 1 / 3 \tan \delta / \rho$

EBSD

E-coat

ECorr

EDAG

EDS

EDXS

EERE

EFP

EIS

EPIKOTETM

EPMA

$\operatorname{ESE}(\mathrm{T})$

ESI

et al.

EV

EV

EWI

ex situ
DIffusion-Controlled TRAnsformations in multicomponent systems, a software diffusion module within Thermo-Calc for accurate simulation of diffusion-controlled reactions in multicomponent alloy systems

difunctional linker

dynamic mechanical analysis

U.S. Department of Energy (when referring to the agency)

design of experiments (when referring to experimental procedures)

dual-phase

dual-phase steel consisting of a ferrite matrix containing a hard second-phase

dynamic strain agency

differential scanning calorimeter (or calorimetry)

abbreviation meaning "for example"

stiffness or Young's modulus

definition for a damping figure of merit

electron backscatter diffraction

electrophoretic coating

corrosion potential

EDAG, Inc., is a company that provides integrated development and optimization of vehicles, production facilities, derivatives and modules

energy dispersive spectroscopy

energy dispersive $\mathrm{x}$-ray spectroscope

Office of Energy Efficiency and Renewable Energy

E-Form Plus

electrochemical impedance spectroscopy

EPIKOTE $^{\text {TM }}$ Resin 05475 with EPIKURE Curing Agent 05443: a system with lowviscosity, a relatively long injection window, excellent wetting and adhesion to CFs, and superior thermal and mechanical performance

electron probe microanalysis or electron probe micro analyzer

designation for an extended compact specimen for testing using ASTM E647 standard

Engineered Solutions, Inc. Group

abbreviation meaning "and others"

exhaust valve (when referring to engine parts)

electric vehicle (when referring to types of vehicles)

Edison Welding Institute

offsite or not in place 
F

F

FADI-AMT LLC a limited liability company that does testing of automotive materials; the name is a composite of the first name of the company's president (Fadi Abu-Farha) and the abbreviation for automotive materials testing

FBJ

FCA US LLC

FCC or fcc

$\mathrm{Fe}$

FE

FEA

FEM

FE-SEM

FI

FIB

FLCA

FLTM

FOM

FSE

FSI

FSLW

FSP

FSPR

FSS

FSSW

FSW

$\mathrm{ft}$

FTIR

ft-lbf

FY

\section{G}

$\mathrm{g}$

$\mathrm{Ga}$

gal

$\mathrm{g} / \mathrm{sec}$ friction bit joining

Fiat Chrysler Automobiles U.S. LLC

face-centered cubic

iron

finite element

finite element analysis

finite element method (or model)

field emission scanning electron microscopy

fuel injector

focused ion beam

front lower control arm

Ford Laboratory Test Method

figure of merit

friction stir extrusion

friction stir interlocking

friction stir lap welding

friction stir processing

friction self-piercing riveting

friction stir scribe

friction stir spot welding (or weld)

friction stir welding (or weld)

foot or feet

Fourier Transform Infrared

foot-pound force

fiscal year

$\operatorname{gram}(\mathrm{s})$

gallium

gallon

grams per second 


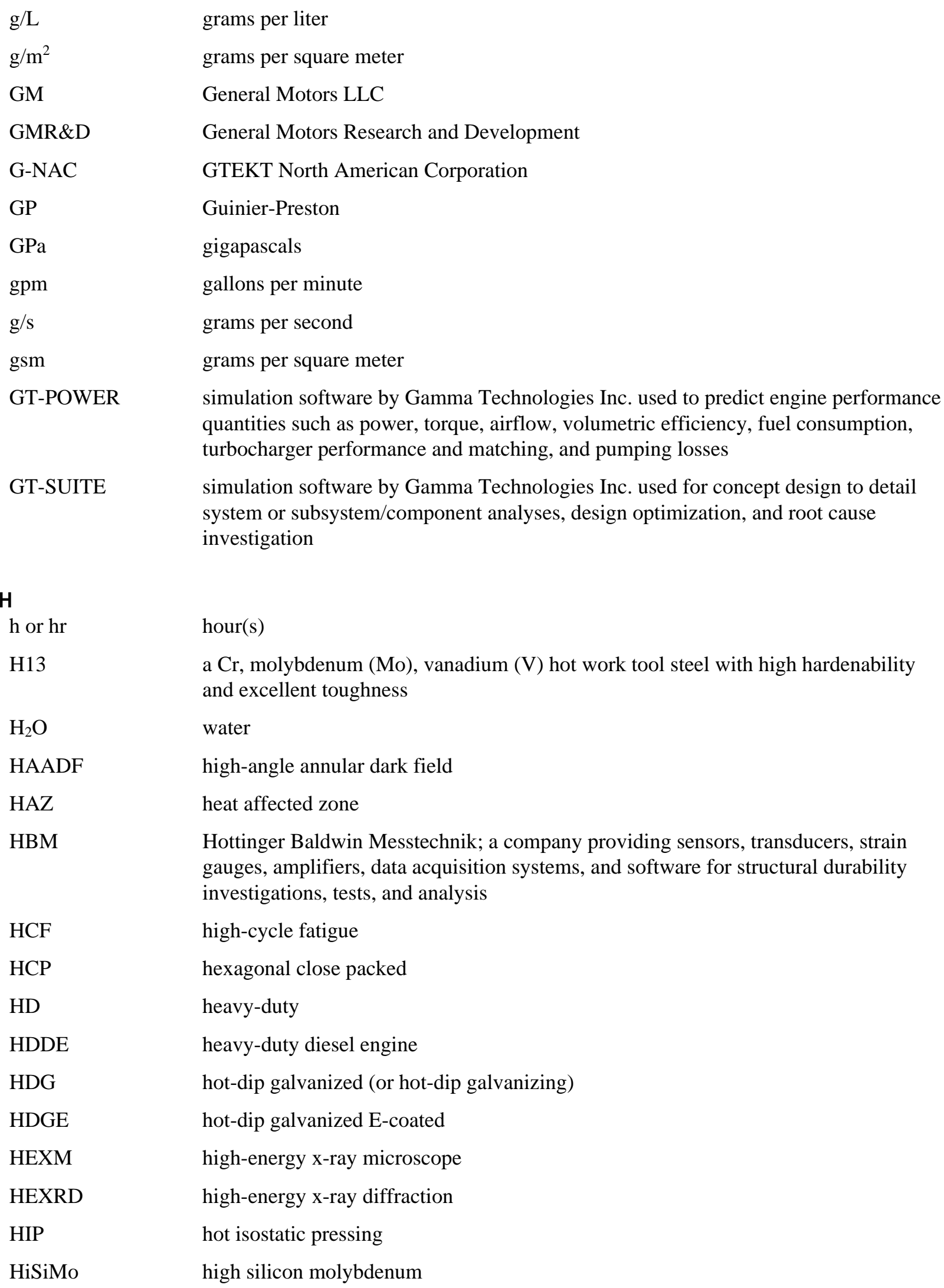




\begin{tabular}{|c|c|}
\hline $\mathrm{HK} 30 \mathrm{Nb}$ & $\begin{array}{l}\text { a grade of cast stainless steel with a composition of } 25 \% \mathrm{Cr}, 21 \% \mathrm{Ni}, 1.75 \% \mathrm{Si} \text {, } \\
1.5 \% \mathrm{Mn} \text {, and trace amounts of } \mathrm{C}, \mathrm{P}, \mathrm{S} \text {, and Mo }\end{array}$ \\
\hline $\mathrm{HOF}$ & Herman's Orientation Factor \\
\hline HP-RTM & high-pressure resin transfer molding \\
\hline HRA & Honda R\&D Americas, Inc. \\
\hline HR-STEM & high-resolution scanning transmission electron microscope (or microscopy) \\
\hline HRTEM & high-resolution transmission electron microscope (or microscopy) \\
\hline HSLA & high-strength low alloy \\
\hline HSLA 340 & steel intended for general presswork, bending, and forming \\
\hline HSS & high-strength steel \\
\hline HTC & heat transfer coefficient \\
\hline Hy & Vickers pyramid number (measurement of hardness) \\
\hline $\mathrm{Hz}$ & hertz \\
\hline i.e. & abbreviation for "id est," a Latin phrase meaning "that is" \\
\hline i3 & five-door urban electric vehicle \\
\hline i8 & coupe with an advanced plugin hybrid drivetrain \\
\hline$i_{\text {galv }}$ & galvanic current \\
\hline$i_{\text {corr }}$ & corrosion current density \\
\hline I & x-ray intensity used in SAXS and WAXS analyses \\
\hline I & electrical current (when referring to mathematical equations) \\
\hline IA & intercritical annealing \\
\hline IACMI & Institute for Advanced Composites Manufacturing Innovation \\
\hline IACS & International Annealed Copper Standard \\
\hline IBS & inner belt stiffener \\
\hline ICME & integrated computational materials engineering \\
\hline ID & identification \\
\hline IDZ & interdiffusion zone \\
\hline IIHS & Insurance Institute for Highway Safety \\
\hline ImageJ & $\begin{array}{l}\text { a Java-based software program by the National Institutes of Health used for image } \\
\text { analysis and processing }\end{array}$ \\
\hline $\mathrm{IM}$ & intermetallic \\
\hline IMC & intermetallic compound \\
\hline in. & inch \\
\hline in $/ \mathrm{sec}$ & inch per second \\
\hline
\end{tabular}




$\begin{array}{ll}\text { in situ } & \text { onsite or in place } \\ \text { INL } & \text { Idaho National Laboratory } \\ \text { IPF } & \text { inverse pole figure } \\ \text { IQ } & \text { image quality } \\ \text { IR } & \text { infrared } \\ \text { IV } & \text { intake valve }\end{array}$

\section{J} JAVA script high-level, interpreted programming language which is characterized as dynamic, weakly typed, prototype-based and multi-paradigm

K

$\mathrm{K}$

k

k

$k_{B}$

$\mathrm{K}_{\mathrm{R}}$

$\mathrm{K}_{\mathrm{s}}$

$\mathrm{K}_{\mathrm{s} 0}$

$\mathrm{K}_{\mathrm{TH}}$

$\mathrm{k}_{\mathrm{f}}$

$\mathrm{kg}$

$\mathrm{kgf}$

$\mathrm{kHz}$

kJ

$\mathrm{kN}$

$\mathrm{kN} / \mathrm{mm}$

Kovar

$\mathrm{k}_{\mathrm{p}}$

$\mathrm{K} / \mathrm{ps}$

$\mathrm{Ksi}, \mathrm{ksi}$, and kpsi

$\mathrm{kWh} / \mathrm{kg}$

L

$l$

$\mathrm{Ll}_{2}$

\section{degree Kelvin}

thermal conductivity (when used in heat transfer equations)

abbreviation for 1000

the Boltzmann constant

effective stress intensity

rate of chain scission

the Arrhenius rate factor

threshold stress intensity

the constant value that represents the constant force required to extrude $\mathrm{Mg}$

kilogram

kilograms force

kilohertz

kilojoules

kilonewton

kilonewton per millimeter

a low thermal expansion alloy with the composition of $\mathrm{Ni}_{29}-\mathrm{Co}_{17}-\mathrm{Fe}_{53}$-trace 1

parabolic rate constant which describes the isothermal oxidation kinetics during hightemperature dwells

degree Kelvin per picosecond

kilopound per square inch

kilowatt-hour per kilogram

length

designation for the major strengthening phase precipitates of $\mathrm{Al}$ alloys 


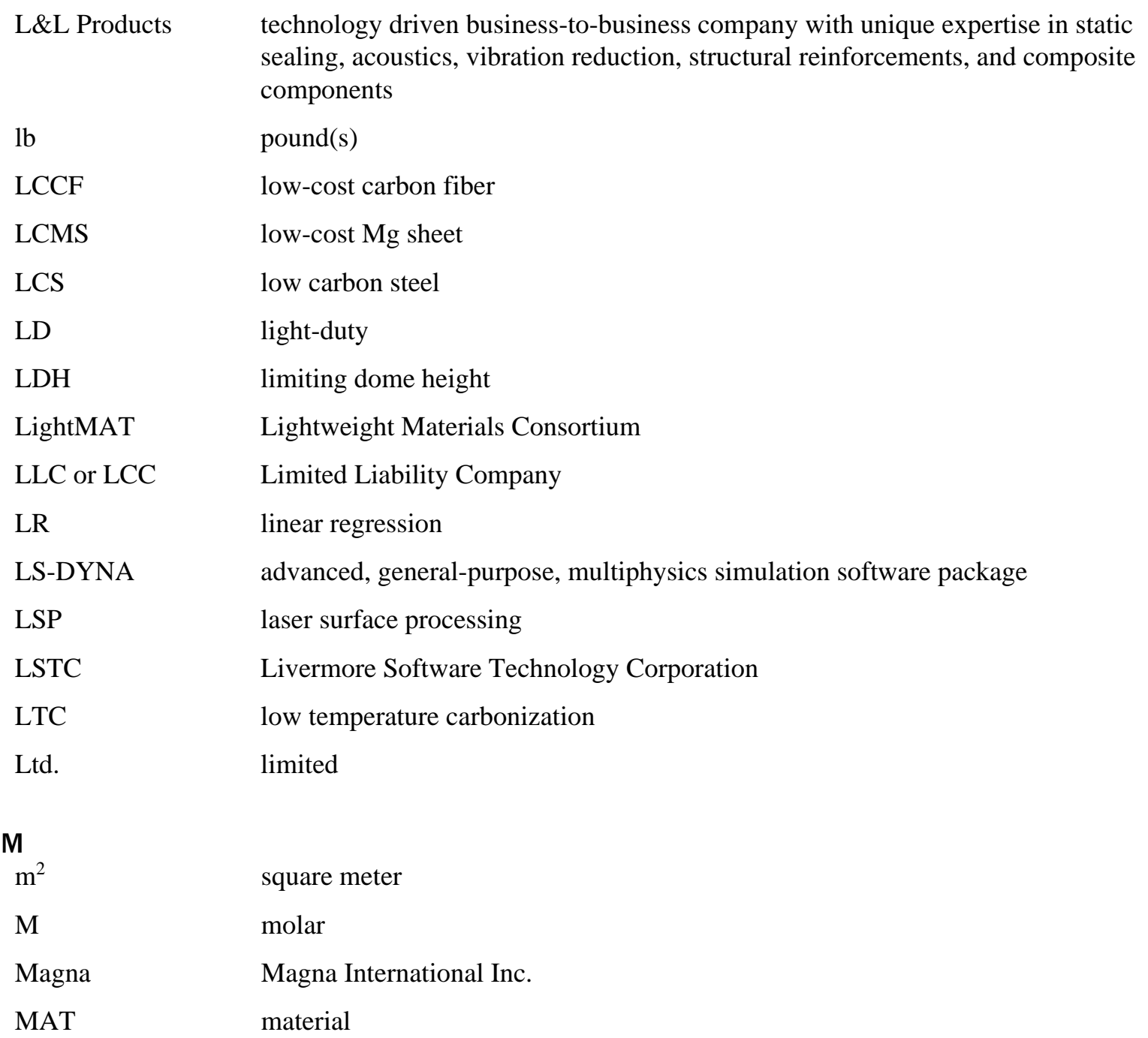

*MAT233 a LS-DYNA material model for hexagonal closed packet metals capable of describing the yielding asymmetry between tension and compression for such materials

$\begin{array}{ll}\text { MATLAB } & \text { MATrix LABoratory, a multi-paradigm numerical computing environment and } \\ \text { programming language } & \text { major ampullate spidroin } \\ \text { MaSp } & \text { minor ampullate spidroin } \\ \mathrm{MD} & \text { molecular dynamic } \\ \mathrm{MDB} & \text { moving deformable barrier } \\ \mathrm{MEMS} & \text { micro-electro-mechanical systems } \\ \mathrm{MEUMAPPS} & \text { microstructure evolution using massive parallel phase-field simulations } \\ \mathrm{Mg} & \text { magnesium } \\ \mathrm{MgCO} & \text { magnesium carbonate } \\ \mathrm{MgO} & \text { magnesium oxide } \\ \mathrm{Mg}_{2} \mathrm{Si} & \text { magnesium silicide }\end{array}$




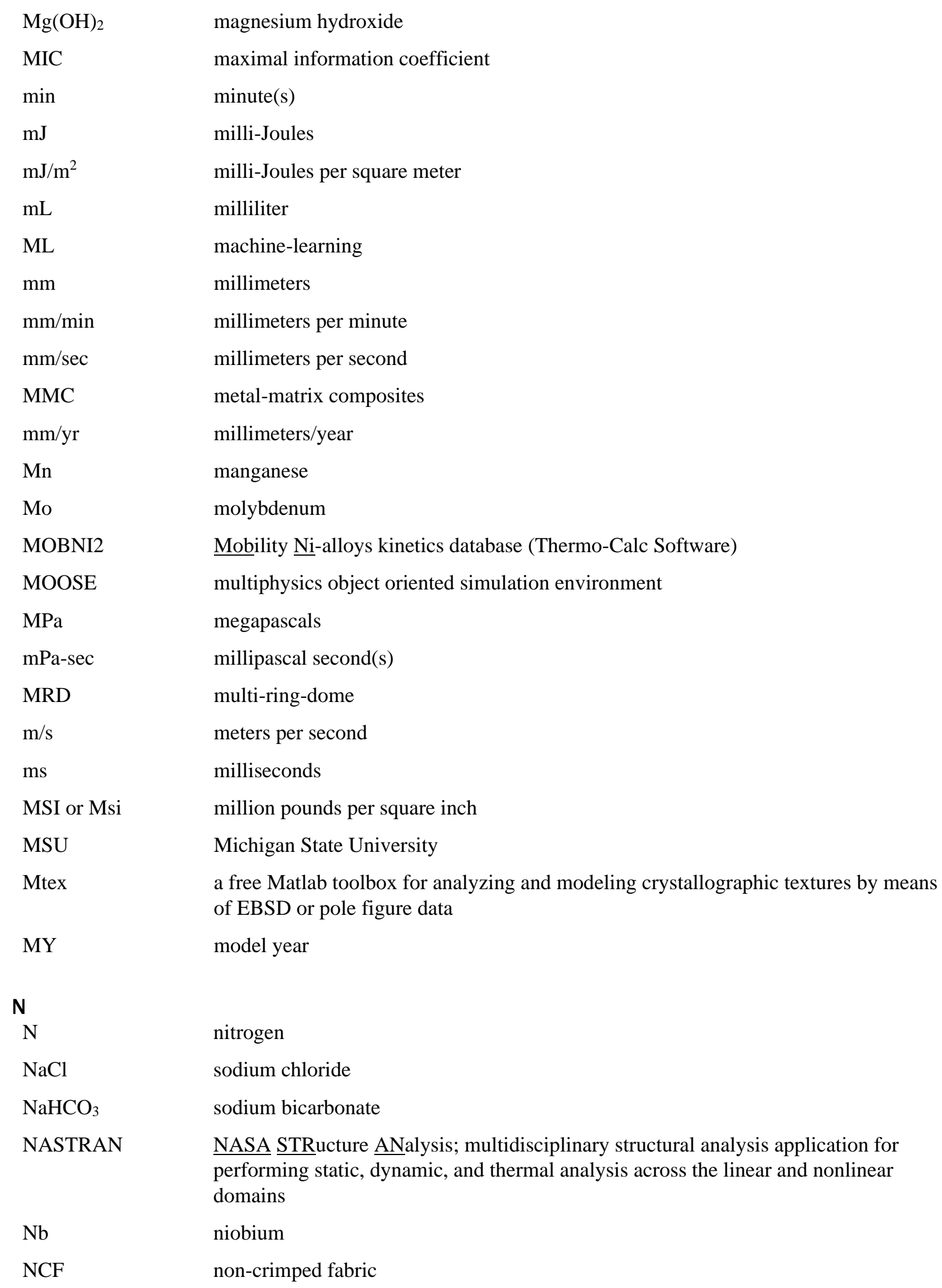




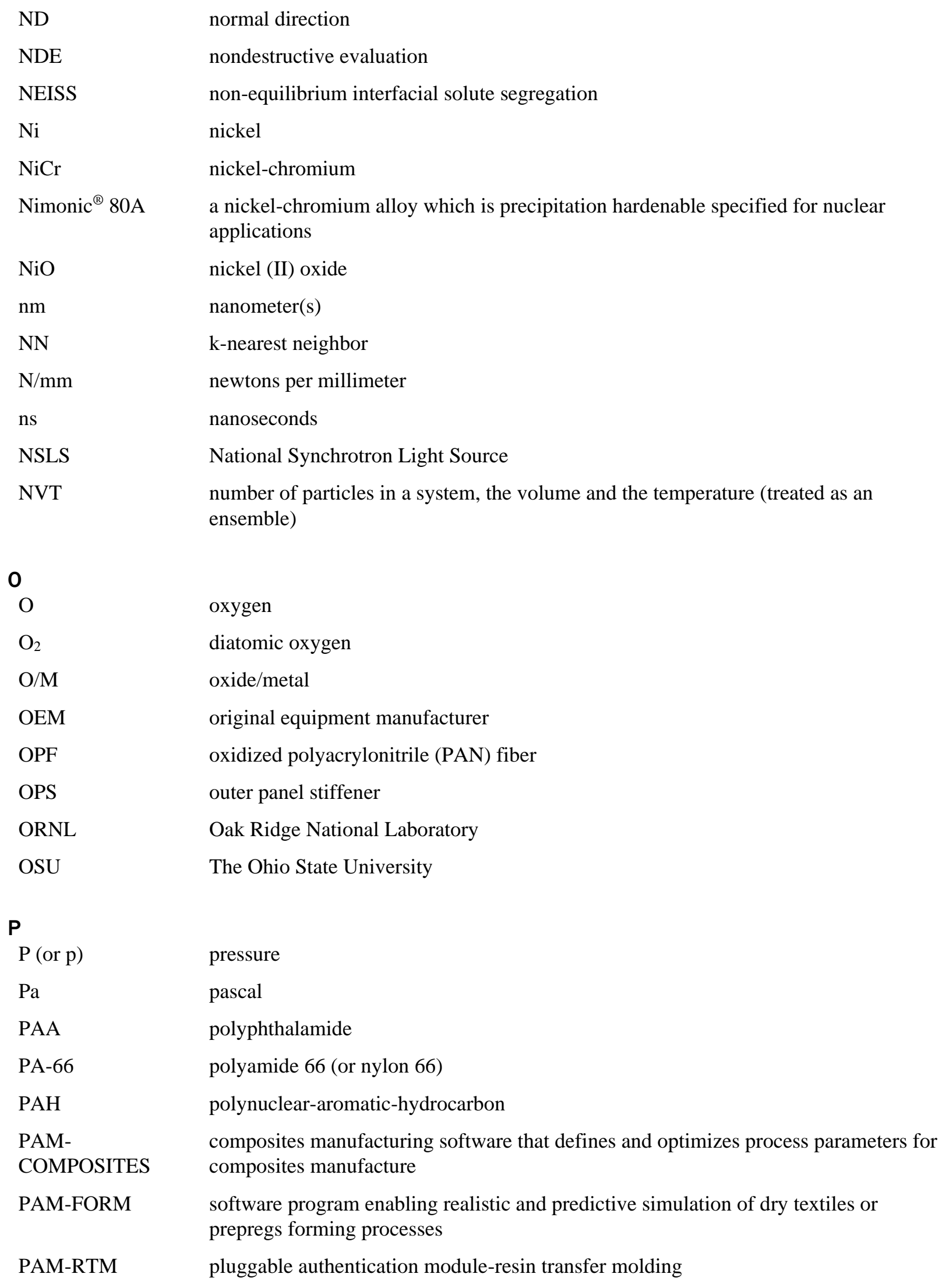




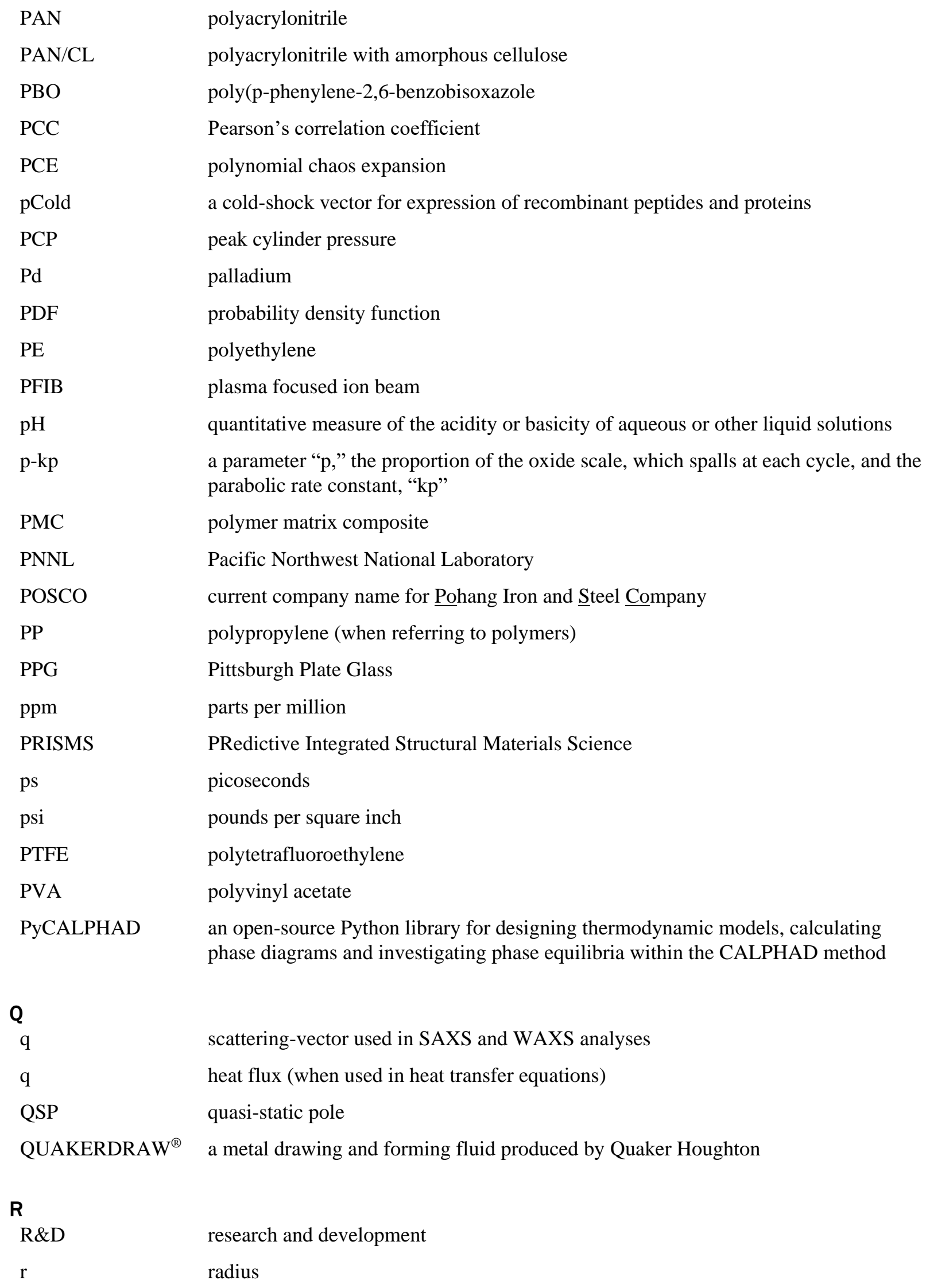




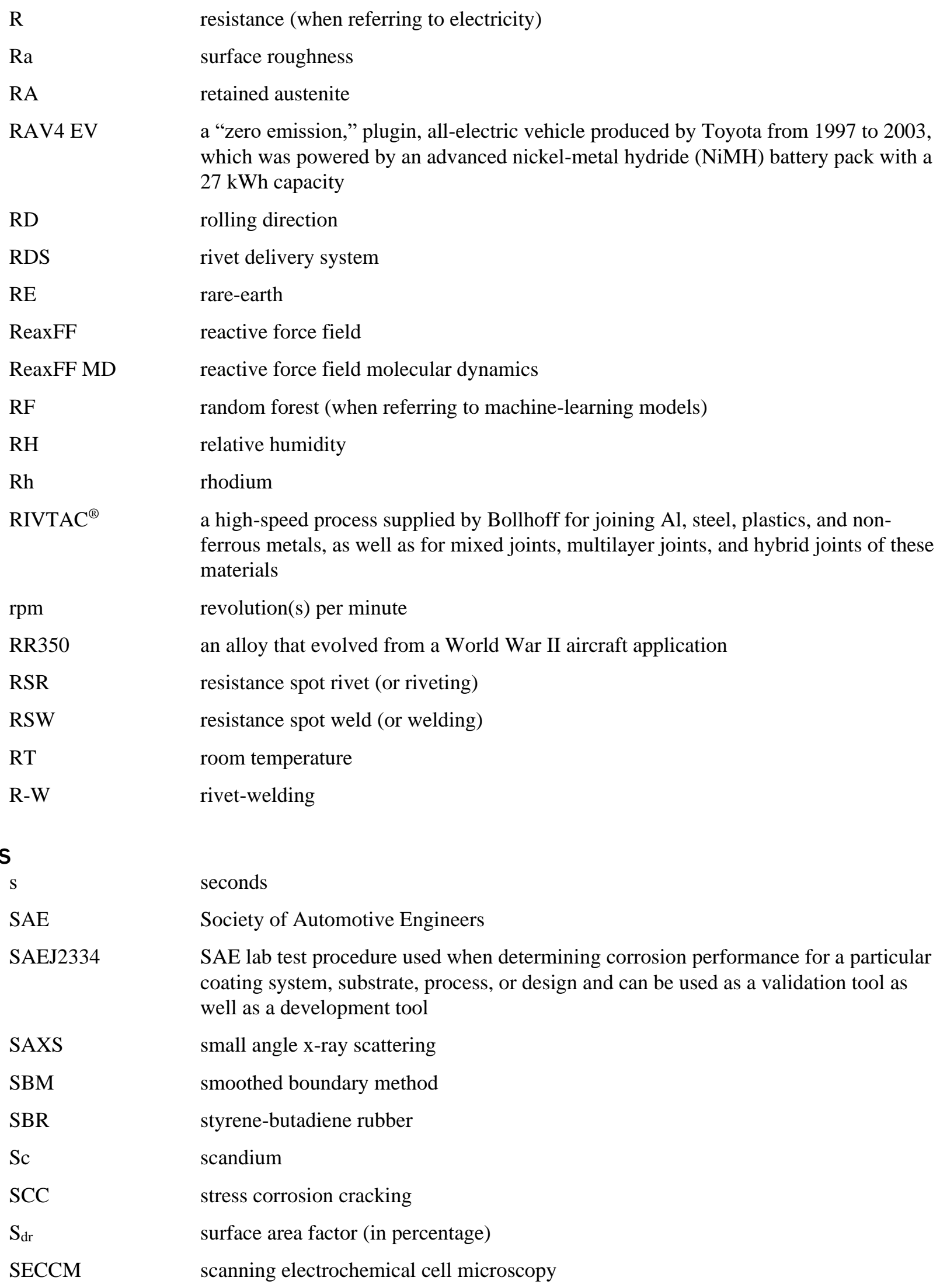

R

$\mathrm{Ra}$

RA

RAV4 EV

RD

RDS

RE

ReaxFF

ReaxFF MD

RF

$\mathrm{RH}$

$\mathrm{Rh}$

RIVTAC $^{\circledR}$

rpm

RR350

RSR

RSW

RT

R-W

S

SAE

SAEJ2334

SAXS

SBM

SBR

$\mathrm{Sc}$

SCC

$\mathrm{S}_{\mathrm{dr}}$

SECCM

resistance (when referring to electricity)

surface roughness

retained austenite

a "zero emission," plugin, all-electric vehicle produced by Toyota from 1997 to 2003, which was powered by an advanced nickel-metal hydride (NiMH) battery pack with a $27 \mathrm{kWh}$ capacity

rolling direction

rivet delivery system

rare-earth

reactive force field

reactive force field molecular dynamics

random forest (when referring to machine-learning models)

relative humidity

rhodium

a high-speed process supplied by Bollhoff for joining Al, steel, plastics, and nonferrous metals, as well as for mixed joints, multilayer joints, and hybrid joints of these materials

revolution(s) per minute

an alloy that evolved from a World War II aircraft application

resistance spot rivet (or riveting)

resistance spot weld (or welding)

room temperature

rivet-welding

seconds

Society of Automotive Engineers

SAE lab test procedure used when determining corrosion performance for a particular coating system, substrate, process, or design and can be used as a validation tool as well as a development tool

small angle x-ray scattering

smoothed boundary method

styrene-butadiene rubber

scandium

stress corrosion cracking

surface area factor (in percentage)

scanning electrochemical cell microscopy

$\mathbf{S}$ 
SEM

ShAPETM

SI

$\mathrm{Si}$

$\mathrm{SiC}$

SL

SLM

SLPS

SMART

SMC

SP

SPR

sq. ft.

$\mathrm{Sr}$

SRS

SS

SSE

SSS

SStAC

STEM

SVM

T

$\mathrm{t}$

$\mathrm{T}$

$\mathrm{T}_{\mathrm{g}}$

T6

T7

$\tan \delta$

TCAL5 scanning electron microscope (or microscopy)

shear-assisted processing and extrusion

side-impact

silicon

silicon carbide

solid lubricants

selective laser melting

supersolidus liquid-phase sintering

partnership between Swatch and Mercedes (s+m) and their design of an "artful" little car

sheet molding compound

softening point

self-pierce riveting or self-piercing rivet

square foot

strontium

strain-rate sensitivity

stainless steel

stored strain energy

solid-state sintering

Stainless Steel Alloy Corrosion (the name of a simulation tool)

scanning transmission electron microscope (or microscopy)

support vector machine regression

time

temperature

glass transition temperature

temper designation for a material heat-treated at a temperature between 325 and 400 degrees $\mathrm{F}$ to strengthen the $\mathrm{Al}$

temper designation for solution heat-treated and then artificially overaged

value used in small-strain dynamic material analysis and quasi-static large-strain experiments

a thermodynamic database for Al-based alloys for use with Thermo-Calc and the addon Diffusion Module (DICTRA) and/or Precipitation Module (TC-PRISMA) that is based on the critical evaluation of binary, ternary and important higher order systems which enables predictions to be made for multicomponent systems and alloys of industrial importance 


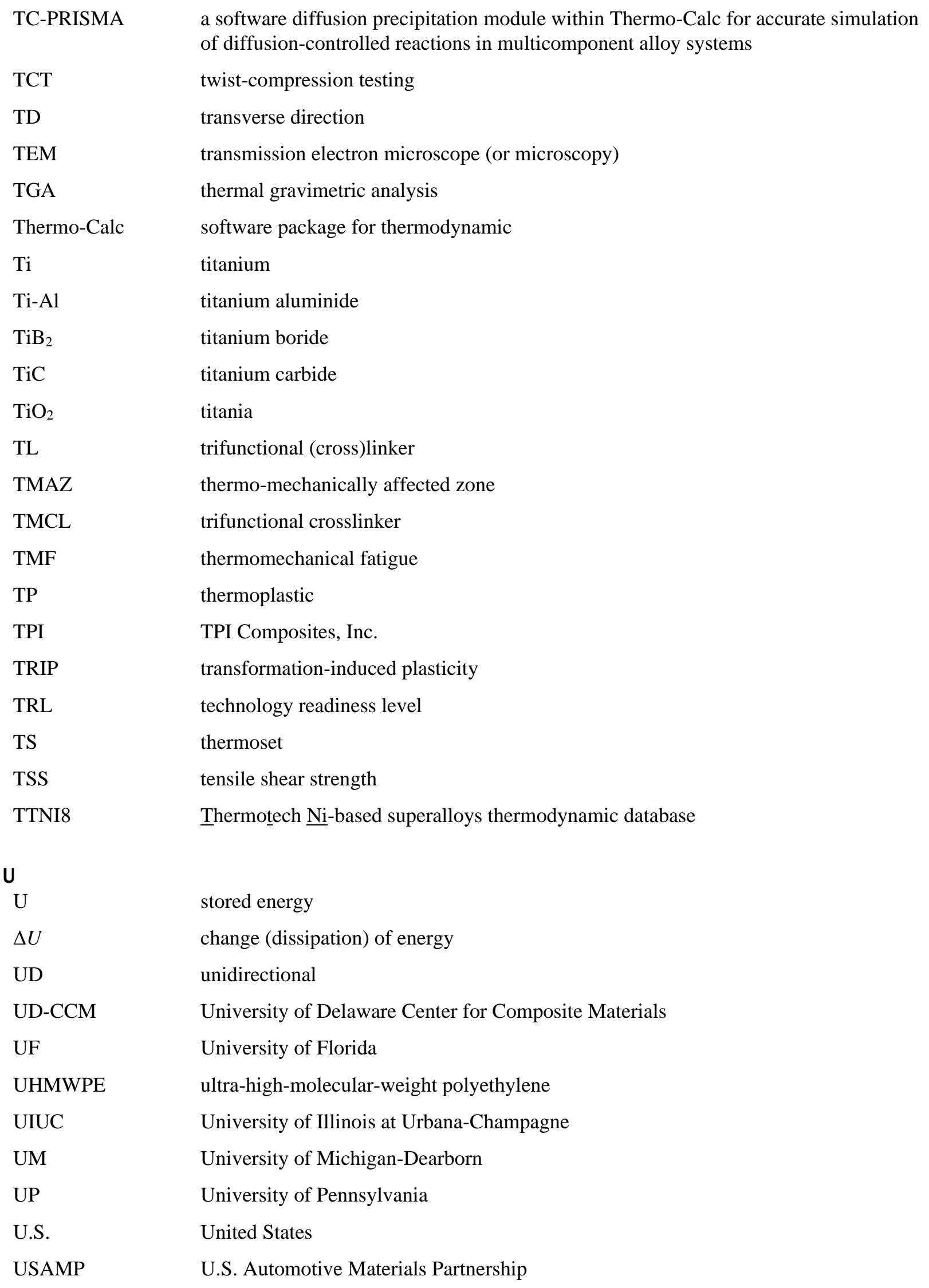



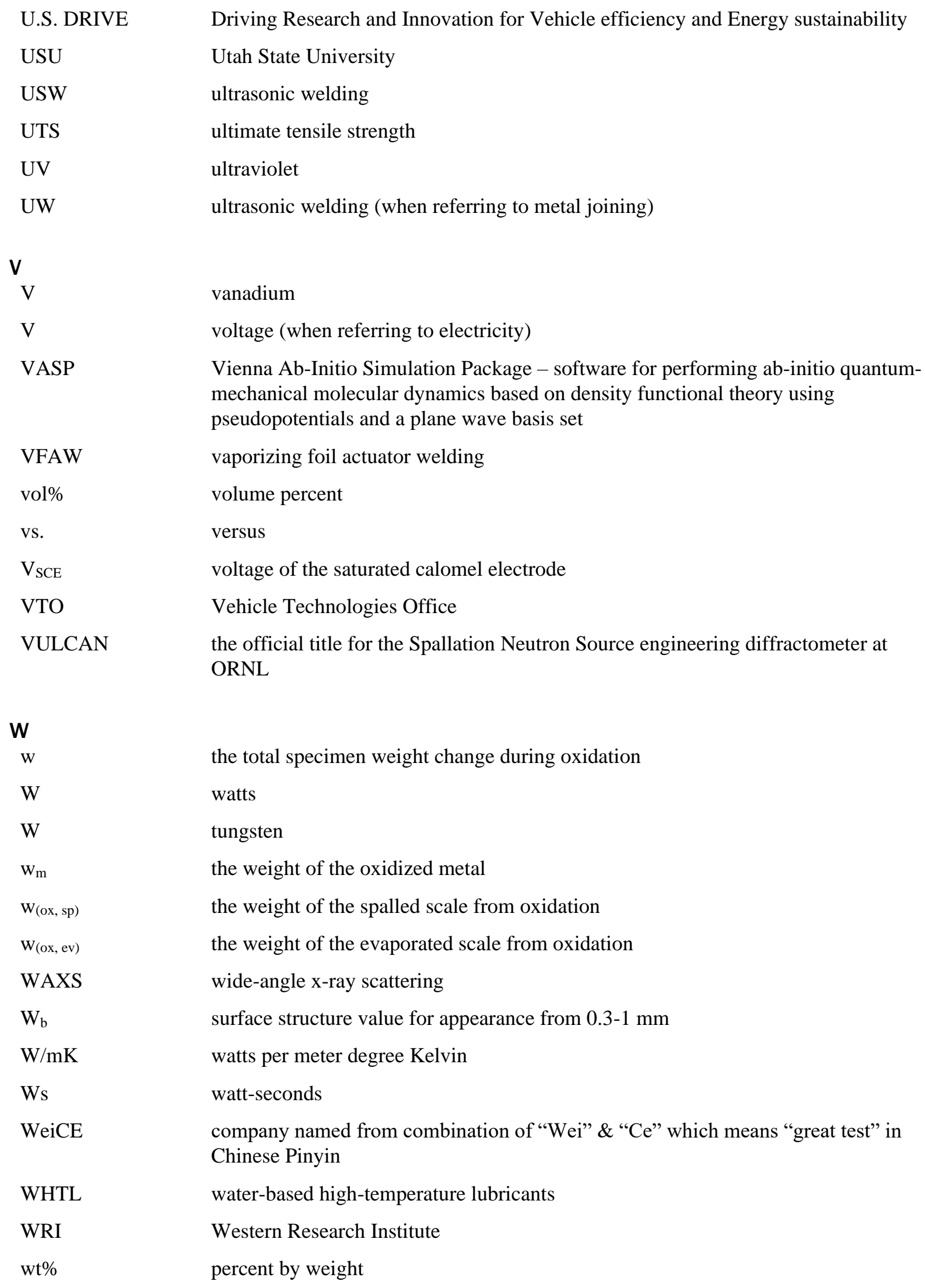


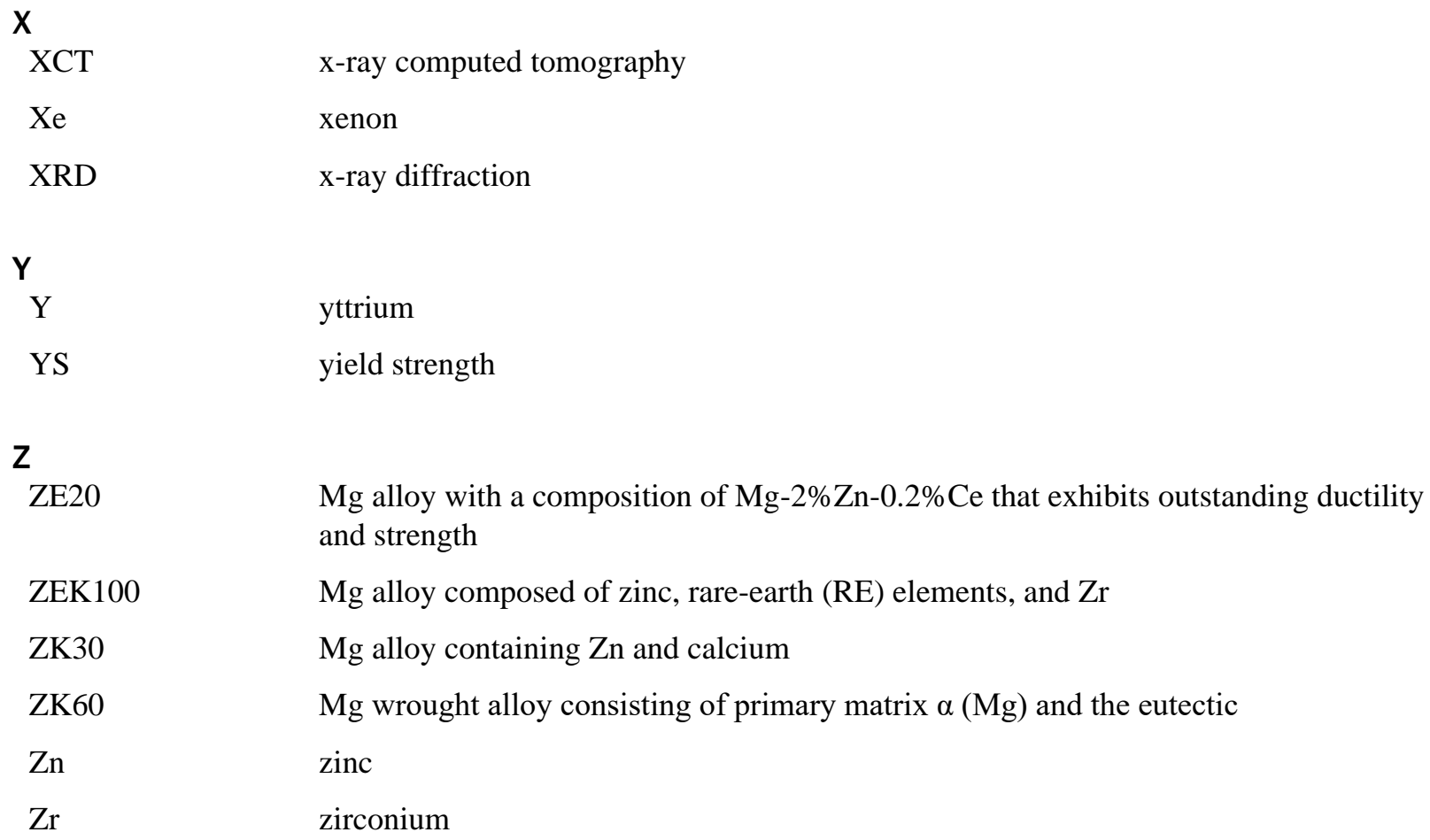




\section{Executive Summary}

The Materials Technology Program supports the Vehicle Technologies Office's (VTO's) mission to help consumers and businesses reduce their transportation energy costs while meeting or exceeding vehicle performance expectations. The propulsion materials research portfolio seeks to develop higher performance materials that can withstand increasingly extreme environments and address the future properties needed for a variety of high-efficiency powertrain types, sizes, fueling concepts, and combustion modes. The lightweight materials research portfolio enables improvements in fuel economy by providing properties that are equal to or better than traditional materials at a lower weight. Because it takes less energy to accelerate a lighter object, replacing cast-iron and traditional steel components with lightweight materials such as advanced high-strength steels, magnesium ( $\mathrm{Mg}$ ) alloys, aluminum ( $\mathrm{Al}$ ) alloys, and fiber-reinforced polymer composites can directly reduce a vehicle's fuel consumption. By 2025, Materials Technology research activities seek to enable a 25\% weight reduction of the glider for light-duty (LD) vehicles including body, chassis, and interior as compared to a 2015 baseline at no more than a $\$ 5 / \mathrm{lb}$-saved increase in cost.

\section{Propulsion Materials}

In fiscal year (FY) 2019, the propulsion materials portfolio included research in three main areas: (1) use of multiscale modeling to predict corrosion and oxidation of engine components and the material properties needed for future engines; (2) development of new lightweight alloys for high-temperature engine components; and (3) additive manufacturing (AM) of powertrain alloys. In addition to these three main thrusts, work was completed on several exploratory projects in novel materials, coatings, processing techniques, and characterization methods relevant to propulsion materials.

The environment within an engine and exhaust system during operation is extremely harsh, with high temperatures and pressures, combustion, and corrosive exhaust gases. This environment corrodes components, including engine intake and exhaust valves, valve seats, and exhaust manifolds. Three projects aim to develop practical and accurate models that can rapidly evaluate corrosion/oxidation of materials such as stainless steel, nickel (Ni)-chromium ( $\mathrm{Cr}$ )-based alloys, and cast-iron ( $\mathrm{Fe})$, and predict their performance. These new models will help decrease the time and cost of development as materials are selected for new engines that operate at higher temperatures.

Automotive applications need low-cost, lightweight, high-temperature alloys to enhance efficiencies in systems such as internal combustion engines. One project focuses on increasing the compositional range of the $\mathrm{Al}-\mathrm{Fe}$-silicon ( $\mathrm{Si}$ ) system via alloy design and non-equilibrium processing, such as AM. Another effort seeks to develop a fundamental understanding of the features that impart thermal stability to cast Al precipitate microstructures, such as those found in Al-copper $(\mathrm{Cu})$ and ACMZ alloys. Some components, such as exhaust valves, are exposed to even higher temperatures and the development of lower-cost, improved wroughtprocessed Ni-based alloys is needed to achieve the combination of yield and fatigue strengths, oxidation resistance, and cost specific to the operating characteristics and lifetime expectations. Pistons in heavy-duty (HD) diesel engines also have unique requirements, so researchers are investigating existing commercial alloys, new developmental alloys, and alloys with thermal barrier coatings to enable increased operating temperature, but require only moderate cost increases. Lastly, corrosion-resistant coatings are also being evaluated to determine whether the operating temperatures of alloys can be increased by improving cyclic oxidation resistance without impacting the alloy high-cycle fatigue behavior.

AM offers unprecedented possibilities to fabricate unique and complex near net-shape components leading to significant savings by decreasing tooling and materials cost, accelerating prototype development, offering unique properties, and increasing system efficiency through advanced designs not achievable via conventional fabrication processes. In-depth understanding of the relationship between microstructure and processing parameters could result in unique microstructures and enhanced or geometrically tailored properties for a wide 
range of powertrain materials and components. Work in this area addresses the specific challenges and opportunities associated with AM for future LD and HD engine components. Key factors - such as cost, influence of feedstock characteristics, volatilization of species, cooling rates, non-equilibrium phases, materials properties, non-uniform geometry-dependent thermal history, thermal cycling, etc.- are assessed for existing and new materials via both modeling and experimental studies.

\section{Lightweight Materials}

In FY 2019, the lightweight materials portfolio included research in the following three areas: (1) improving the properties and manufacturability of light metals such as advanced high-strength steels (AHSSs), Al alloys, and $\mathrm{Mg}$ alloys; (2) reducing the cost of carbon fibers (CFs) and developing Integrated Computational Materials Engineering (ICME) frameworks for the manufacturing of CF composites; and (3) developing novel joining methods to enable multi-material systems.

Substitution of light metals for mild steel can result in weight-savings of $25 \%$ to $60 \%$ per component, which increases fuel efficiency. However, there are several challenges to the increased use of light metals including material cost, room temperature formability, and corrosion mitigation. One project is working to lower the cost of $\mathrm{Al}$ castings to be more competitive with cast-iron by using novel processing methods to break up the detrimental iron-intermetallic phases that can form in less-expensive secondary $\mathrm{Al}$ alloys. Similarly, the use of high-performance $\mathrm{Mg}$ alloys in the automotive industry is currently limited due to the addition of costly rare-earth (RE) elements, the need for high-temperature forming, and the difficulties in corrosion mitigation. Two projects in this report aim to eliminate the need for RE additives: (1) through alloy development; and (2) through a novel solid-phase processing method. Two additional projects seek to validate methods of preventing $\mathrm{Mg}$ corrosion.

Carbon fiber reinforced polymer (CFRP) composites also have the potential to reduce component weight by more than $60 \%$. One of the main barriers to widespread implementation is the high cost of CF, which is due in large part to the cost of the input material (precursor) and the CF conversion process. In addition to a successfully commercialized plasma oxidation technology, one project is addressing this challenge by developing higher throughput, low temperature carbonization of $\mathrm{CF}$, thus further lowering manufacturing costs and increasing production rates. Two projects are investigating potential low-cost CF precursors utilizing ICME models to guide their research to meet the U.S. Department of Energy's (DOE's) targets. Another challenge that the lightweight materials subprogram is addressing is the lack of predictive modeling available, which is necessary to accelerate the development and deployment of CFRP automotive components. One project is creating ICME models in order to predict the manufacturing and structural performance of CF composites. Materials design and manufacturing process information, such as geometric dimension, fiber layout, and fiber orientation, are inputs for multiscale models to generate local material constitutive relationships used in addressing noise, vibration, and harshness $(\mathrm{NVH})$; crash worthiness and safety; and durability challenges. The integrated tools are being applied to component design and optimization, demonstrating that the ICME approach generates powerful tools for the most efficient usage of CFRP composites, leading to optimized weight-savings with a minimal cost increase.

The most effective way to reduce the overall weight of a vehicle is to tailor the material selection to each component's needs. However, joining dissimilar materials to create a multi-material structure is a significant challenge. The Joining Core Program continues its efforts to address specific challenging material pairsincluding Mg-CFRP, CFRP-Steel, and Mg-Steel - as well as beginning a new effort focused on galvanic corrosion in joints. The methods being developed in the Joining Core Program include ultrasonic welding (UW), novel methods for mechanical fastening, over molding, adhesives, and friction stir welding (FSW). Two projects investigate the use of solid-state welding processes to join $\mathrm{Al}$ to steel, as well as dissimilar $\mathrm{Al}$ alloy combinations. Solid-state welding allows for joining materials with vastly different melting temperatures, which is not possible with fusion welding. Two additional projects incorporate fasteners into welding 
processes - both solid-state and fusion — in order to provide a strong spot joint comparable to resistance spot welds (RSWs) in steel to steel joints. Research in this portfolio also includes adhesives that address concerns with the coefficient of thermal expansion (CTE) mismatch seen in dissimilar material joints. Three new projects model the effect of galvanic corrosion on a wide variety of joining methods and material combinations to predict the effect on joint strength and fatigue life. 


\section{Table of Contents}

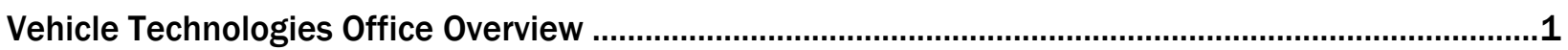

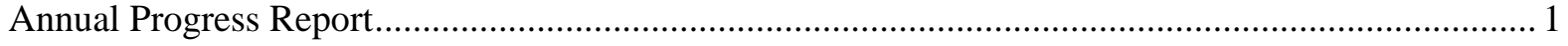

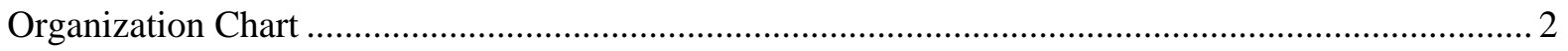

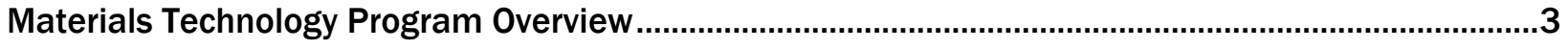

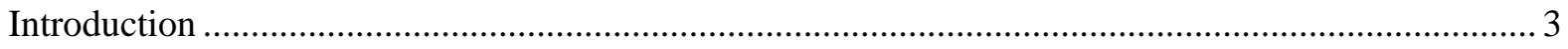

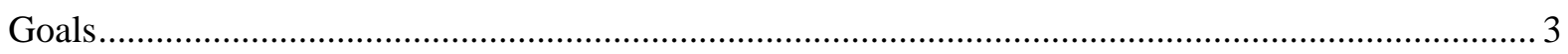

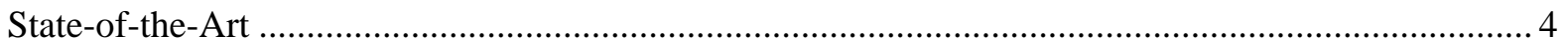

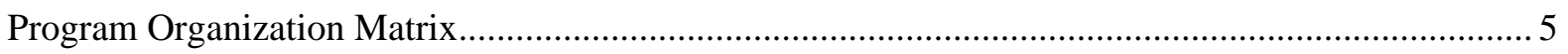

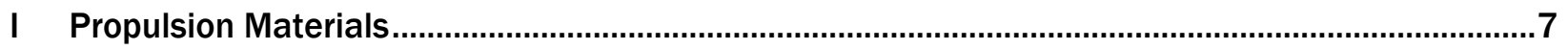

I.1 Modeling of Powertrain Materials ......................................................................................... 7

I.1.1 Future Engine Requirements - Modeling Advanced Engine Materials (Oak Ridge

National Laboratory) ................................................................................................ 7

I.1.2 Lightweight High-Temperature Alloys Based on the Al-Fe-Si System (University of

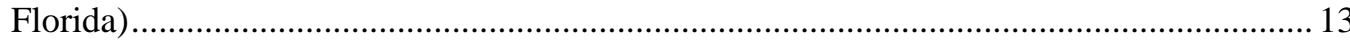

I.1.3 Multiscale Modeling of Corrosion and Oxidation Performance and Their Impact on High-Temperature Fatigue of Automotive Exhaust Manifold Components (Ford Motor

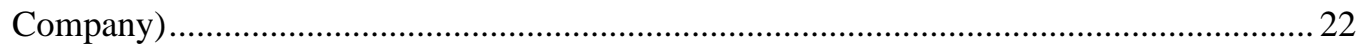

I.1.4 Machine-Learning and Supercomputing to Predict Corrosion/Oxidation of HighPerformance Valve Alloys (Oak Ridge National Laboratory) ........................................... 31

I.1.5 Multiscale Development and Validation of the Stainless Steel Alloy Corrosion Tool for High-Temperature Engine Materials (University of Florida) ............................................... 38

I.2 Solid-State Processing and Metal-Matrix Composites ............................................................. 47

I.2.1 Direct Extruded High Conductivity Copper for Electric Machines (Pacific Northwest

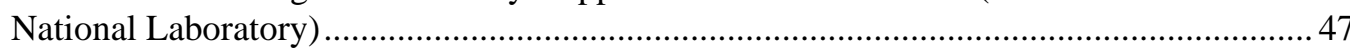

I.2.2 Metal-Matrix-Composite Brakes Using Titanium Diboride - LightMAT DFA1 (Pacific

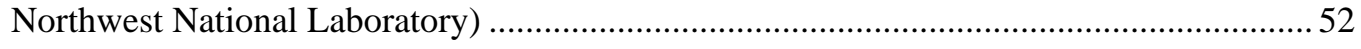

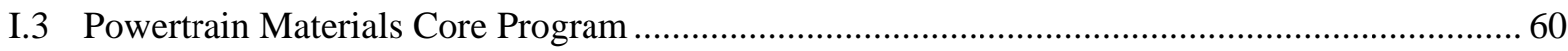

I.3.1 High-Temperature Lightweight Engine Materials - Thrust 1 (Oak Ridge National

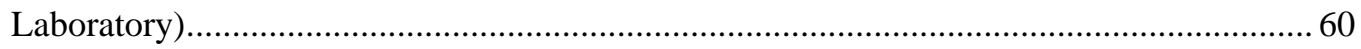

I.3.1.1 New Aluminum Alloys with Improved High-Temperature Performance (1A).............60

I.3.1.1.1 Fundamental Studies of Complex Precipitation Pathways (1A1) ...............60

I.3.1.1.2 New Higher Performance Aluminum Alloys (1A2)...................................66

I.3.1.1.3 Intermediate Temperature Variants of Cast ACMZ Alloys (1B1) ...............71

I.3.2 Cost-Effective Higher Temperature Engine Alloys - Thrust 2 (Oak Ridge National

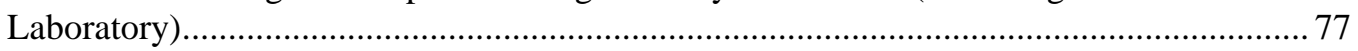

I.3.2.1 Advanced Affordable Wrought Engine Alloys (2A and 2B) ...................................... 77

I.3.2.1.1 Oxidation-Resistant Valve Alloys for $900^{\circ} \mathrm{C}-950^{\circ} \mathrm{C}$ Operation (2A1) .....78

I.3.2.1.2 Higher Temperature Heavy-Duty Piston Alloys (2A2).............................85

I.3.2.1.3 Advanced, Affordable Wrought Engine Alloys, High-Temperature

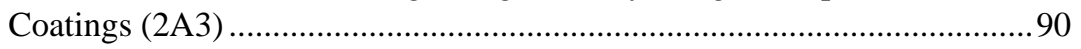

I.3.2.1.4 Development of Cast, Higher Temperature Alloys (2B1) ..........................96

I.3.2.1.5 Selective Material Processing to Improve Local Properties (2B2)............ 103

I.3.3 Additive Manufacturing - Thrust 3 (Oak Ridge National Laboratory)............................. 109 
I.3.3.1 Additive Manufacturing of Powertrain Alloys (3A) .....

I.3.3.1.1 Fundamental Development of Lightweight Alloys for Additive Manufacturing (3A1)

I.3.3.1.2 Hybrid Manufacturing of Additive Manufactured Interpenetrating Phase Composites (3A2).....

I.3.3.1.3 Fundamentals of Austenitic Alloys Processing by Additive Manufacturing (3B1).

I.3.3.1.4 Development of Ferritic Alloys for Heavy-Duty Pistons by Additive Manufacturing (3B2).

I.3.4 Advanced Characterization and Computational Methods - Thrust 4 (Oak Ridge National Laboratory).

I.3.5 Exploratory Research - Thrust 5 (Oak Ridge National Laboratory)......

I.3.5.1 Novel Materials for Polymer Composite Engine Blocks (Oak Ridge National Laboratory)

I.3.5.2 Advanced Anticorrosion Coatings on Lightweight Magnesium Alloys by Atmospheric $\mathrm{CO}_{2}$ Plasma Treatment (Oak Ridge National Laboratory) . 160

I.3.5.3 Novel Aluminum Matrix Composite for Powertrain Applications (Oak Ridge National Laboratory).

I.3.5.4 High-Strength Aluminum-Graphene Composite for Powertrain System (Pacific Northwest National Laboratory)

I.3.5.5 Development of High-Temperature Sample Environment for Advanced Alloy Characterization Utilizing High-Speed, Micron Resolution X-ray Imaging Techniques (Argonne National Laboratory) .

\section{Lightweight Materials}

II.1 Automotive Metals

II.1.1 Non-Rare Earth Magnesium Bumper Beam (Pacific Northwest National Laboratory/Magna International CRADA)

II.1.2 Low-Cost Mg Sheet Component Development and Demonstration Project (Fiat Chrysler Automobile, U.S., LLC).

II.1.3 Mitigating Corrosion in Mg Sheet in Conjunction with a Sheet-Joining Method that Satisfies Structural Requirements within Subassemblies (Pacific Northwest National Laboratory).

II.1.4 Low-Cost Corrosion Protection Techniques for Magnesium Alloys (Pacific Northwest National Laboratory)

II.1.5 Reducing Mass of Steel Auto Bodies Using Thin Advanced High-Strength Steels with Carbon-Reinforced Epoxy Coating (Idaho National Laboratory).

II.1.6 Optimizing Heat-Treatment Parameters for Third-Generation Advanced High-Strength Steels Using an Integrated Experimental-Computational Framework (Pacific Northwest National Laboratory).

II.1.7 Overcoming the Barriers to Lightweighting by Enabling Low-cost and Highperformance Structural Automotive Aluminum Castings (Pacific Northwest National Laboratory).

II.1.8 Room Temperature Stamping of High-Strength Aluminum Alloys (Pacific Northwest

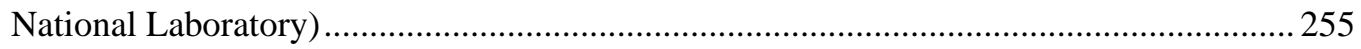

II.1.9 Graphene-Based Solid Lubricants for Automotive Applications (Argonne National Laboratory). 
II.2.1 Integrated Computational Materials Engineering Predictive Tools for Low-Cost Carbon

Fiber for Lightweight Vehicles (University of Virginia) ...

II.2.2 Consortium for the Production of Affordable Carbon Fibers in the United States (Western Research Institute)

II.2.3 Ultralight Hybrid Composite Door Design and Rapid Manufacture

(TPI Composites Inc.)

II.2.4 Functionally Designed Ultra-Lightweight Carbon Fiber Reinforced Thermoplastic

Composites Door Assembly (Clemson University)

II.2.5 Development and Integration of Predictive Models for Manufacturing and Structural Performance of CF Composites in Automotive Applications (General Motors) ................ 344

II.2.6 Integrated Computational Materials Engineering Development of CF Composites for Lightweight Vehicles (Ford Motor Company).

II.2.7 Continuous Fiber Malleable Thermoset Composites with Sub-1-minute Dwell Times;

Validation of Impact Performance and Evaluation of the Efficacy of the Compression-

Forming Process (Mallinda LLC)

II.2.8 Ultra-Lightweight, Ductile Carbon Fiber Reinforced Polymers (Oak Ridge National

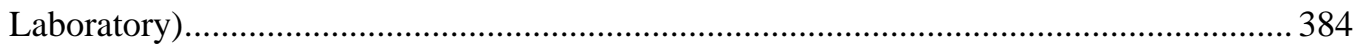

II.2.9 Spider Silk as Carbon Fiber Precursor (Oak Ridge National Laboratory) .......................... 393

II.2.10 Carbon Fiber Technology Facility (Oak Ridge National Laboratory) ............................... 399

II.2.11 Proximity Electromagnetic Carbonization (Oak Ridge National Laboratory) ..................... 407

II.3 Multi-Material Joining.

II.3.1 A Multiscale Computational Platform for Predictive Modeling of Corrosion in AIuminum-Steel Joints (University of Michigan).

II.3.2 Phase-Field Modeling of Corrosion for Next-Generation Aluminum-Magnesium Vehicle Joints (Worcester Polytechnic Institute)

II.3.3 A Hybrid Physics-based Data-Driven Approach to Model Damage Accumulation in Corrosion of Polymeric Adhesives (Michigan State University)...

II.3.4 High-Strength Steel-Aluminum Components by Vaporizing Foil Actuator Welding (The Ohio State University).

II.3.5 Corrosion Control in Carbon Fiber Reinforced Polymer Composite-Aluminum Closure Panel Hem Joints (PPG Industries, Inc.).

II.3.6 Assembly of Dissimilar Aluminum Alloys for Automotive Applications (Pacific Northwest National Laboratory)

II.3.7 Corrosion Protection of Dissimilar Material and Joining for Next-Generation Lightweight Vehicles (Arconic, Inc.).

II.3.8 Adhesive-Bonding of CF Reinforced Polymer to Advanced High-Strength Steels (Oak Ridge National Laboratory) .

II.3.9 Solid-Phase Joining of Magnesium Sheet to High-Strength Steel (Pacific Northwest National Laboratory).

II.3.10 Joining Magnesium Alloys to Carbon Fiber Reinforced Polymers (Pacific Northwest National Laboratory).

II.3.11 High-Performance Computing and High-throughput Characterizations towards Interfaces-by-Design for Dissimilar Materials Joining (Oak Ridge National Laboratory) 523

II.3.12 Solid-State BIW Spot Joining of Al to AHSS at a Prototype-Scale (Oak Ridge National Laboratory).

II.3.13 Nondestructive Inspection of Aluminum-Steel Weld Bond (Oak Ridge National Laboratory). 
II.3.14 Corrosion Mechanisms in Magnesium-Steel Dissimilar Joints (Pacific Northwest National Laboratory and Oak Ridge National Laboratory). 


\section{List of Figures}

Figure I.1.1.1. Elastic stresses [psi] from head bolt preload and (engine-cold) combustion pressure (a) compared with preload and (ambient-pressure) combustion temperature loading (b) for CGI-450. Source: ORNL

Figure I.1.1.2. Stresses [psi] at the baseline (190 bar) condition for CGI-450, with an elastic-only model (a) compared with a model including plasticity (b). Source: ORNL.

Figure I.1.1.3. Materials surface temperature $\left[{ }^{\circ} \mathrm{C}\right]$ maps, with maxima labeled, for 190 bar and 225 bar operation with three cooling scenarios. The red contour $\left(>380^{\circ} \mathrm{C}\right)$ is the same on all frames. Source: ORNL.

Figure I.1.1.4. Strains in CGI-450 at the baseline (a) and higher-flux (b) conditions. Plasticity is accounted for, but the effects of creep are not. Source: ORNL.

Figure I.1.2.1. Experimentally measured isothermal sections of the Al-Co-Fe ternary system:

(a) $1000^{\circ} \mathrm{C}$; (b) $900^{\circ} \mathrm{C}$; and (c) $800^{\circ} \mathrm{C}[1]$.

Figure I.1.2.2. Experimentally measured isothermal sections of the Al-Cu-Fe ternary system:

(a) $1000^{\circ} \mathrm{C}$; (b) $800^{\circ} \mathrm{C}$; and (c) $700^{\circ} \mathrm{C}[2]$.

Figure I.1.2.3. Al-Fe-Si-Mn quaternary alloys with 3.5 to 5.5 at.\% $\mathrm{Mn}$ that were annealed at $800^{\circ} \mathrm{C}$ for 350 to $400 \mathrm{~h}$ : (a)-(e) SEM-BSE images showing the microstructure of the heat-treated alloys and (f)-(j) XRD patterns confirming the existence of the $\tau_{10}$ single phase.

Source: University of Florida......

Figure I.1.2.4. SEM-BSE images showing the microstructure of the Al-Fe-Si-Mn quaternary alloys with 4.5 at.\% $\mathrm{Mn}$ and 8.0 to 14.0 at.\% Si that were annealed at $800^{\circ} \mathrm{C}$ for 350 to $400 \mathrm{~h}$ :

(a) Al-20.0Fe-8.0Si-4.5Mn; (b) Al-20.0Fe-9.0Si-4.5Mn; (c) Al-20.0Fe-12.0Si-4.5Mn; and

(d) Al-20.0Fe-14.0Si-4.5Mn. All the compositions are in at.\% Source: University of Florida.

Figure I.1.2.5. Microstructure of the laser-melted areas of the Al-Fe-Si-Mn quaternary alloy with 4.5 at.\% Mn: (a) Schematic diagram showing the laser test locations and the melted areas;

(b) Typical SEM-BSE image taken from the melted area using a laser power of $750 \mathrm{~W}$ and a scan speed of $20 \mathrm{~mm} / \mathrm{s}$; and (c) High magnification image of the red box location in (b) clearly showing the HAZ boundary. Source: University of Florida.

Figure I.1.2.6. Plot showing the measured dendrite arm spacing and depth of the HAZs in the laser-melted areas for two laser powers and two laser scan speeds. Source: University of Florida... 18

Figure I.1.2.7. (a) SEM secondary electron image taken from the powders with a size distribution of 15-53 $\mu \mathrm{m}$. (b) Plot showing the measured dendrite arm spacing and depth of the HAZs in the laser-melted areas. Source: University of Florida.

Figure I.1.2.8. Photograph of the eighteen laser printed samples. The numbers correspond to the run order listed in Table I.1.2.2. Source: University of Florida......

Figure I.1.3.1. Graphical representation of initial ReaxFF MD simulation setting for: (a) bcc alloys; and (b) fcc alloys showing system sizes, gas environment, and temperatures.

Source: The Ohio State University. 
Figure I.1.3.2. Graphical representation of the configurations and stress conditions after $300 \mathrm{ps}$ ReaxFF MD simulation for (a) bcc-Fe-20at\%X-5at\%Y at different temperatures; (b) bcc- and fcc-Fe-25at\%X-25at\%Y at 800K; and (c) pure bcc-Fe and bcc-Fe-25at\%X-25at\%Y at 1200K showing the influence of alloying elements, $X$ and $Y$, on corrosion and oxidation performance for Iron with the bcc structure in exhaust gas environment. Source: The Ohio State University.

Figure I.1.3.3. Graphical representation of the transformation of the oxidation scales from crystalline structures to amorphous 1 ns ReaxFF MD simulation showing that the employed force field needed to be improved. Source: The Ohio State University.

Figure I.1.3.4. (a) The total weight changes; (b) the weight loss due to de-carbonization; and (c) the mass changes associated with oxidation for base HiSiMo-type alloys showing that the oxidation behavior at different temperatures. Source: Missouri Science and Technology.

Figure I.1.3.5. Graphical representation of: (a) surface topology; and (b) cross-section of base HiSiMo-type alloys at in a combustion atmosphere at $700^{\circ} \mathrm{C}$ and $800^{\circ} \mathrm{C}$ showing the oxidation behavior at different temperatures. Source: Missouri Science and Technology.

Figure I.1.3.6. FIB/TEM imaging showing the oxidization layer structure for HiSiMo-type alloys in a combustion atmosphere at $750^{\circ} \mathrm{C}$ for $100 \mathrm{~h}$. Source: Missouri Science and Technology.

Figure I.1.4.1. The top 15 features determined by correlation analysis between all input features (i.e., oxidation-testing temperature, $\mathrm{T}$, and the mass fraction of each element) and pkp_kp using (a) database A; and (b) database B. Source: ORNL

Figure I.1.4.2. Accuracy of five trained machine-learning models with: (a) database A; and (b) database B. The lines connecting the data points are only for visual purposes. Source: ORNL. .. 34

Figure I.1.4.3. Comparison between measured and calculated mass change for Nimonic ${ }^{\circledR} 80 \mathrm{~A}$ in 1h cycles in dry air and air $+10 \% \mathrm{H}_{2} \mathrm{O}$ at: (a) $900^{\circ} \mathrm{C}$; and (b) $950^{\circ} \mathrm{C}$. Source: ORNL 34

Figure I.1.4.4. Comparison between the measured and calculated mass change for Nimonic ${ }^{\circledR} 90$, HAYNES ${ }^{\circledR} 282^{\circledR}$, and Udimet 520 in $1 \mathrm{~h}$ cycles under air $+10 \% \mathrm{H}_{2} \mathrm{O}$ at $900^{\circ} \mathrm{C}$. Source: ORNL.

Figure I.1.4.5. Calculated $\mathrm{Cr}$ concentration at the scale/alloy interface for Nimonic ${ }^{\circledR} 80 \mathrm{~A}$ in $1 \mathrm{~h}$ cycles in dry air and air $+10 \% \mathrm{H}_{2} \mathrm{O}$ at $900^{\circ} \mathrm{C}$ and $950^{\circ} \mathrm{C}$. (a) Symbol represents measured (electron probe microanalysis) $\mathrm{Cr}$ concentration. (b) Measured mass change/area for Nimonic ${ }^{\circledR}$ $80 \mathrm{~A}$ in $1 \mathrm{~h}$ cycles in air $+10 \% \mathrm{H}_{2} \mathrm{O}$ at $950^{\circ} \mathrm{C}$. Source: ORNL.

Figure I.1.5.1. Extreme environmental degradation to an alloy 21-4-3 valve head due to local temperature spikes resulting from improper seating. Source: Tenneco......

Figure I.1.5.2. Summary of the multiscale approach to be used to develop the SStAC tool. Note both the macro- and meso-scale tools will be built using the MOOSE framework. Source: University of Florida...... 39

Figure I.1.5.3. High-temperature corrosion experiment. Source: University of Wisconsin-Madison... 40 Figure I.1.5.4. (a) Low magnification SEM image with carbides particles and the corresponding EDS analysis; and (b) image quality maps on two regions of the 21-2N valve.

Source: University of Wisconsin-Madison.

Figure 1.1.5.5. Simulation domain of Fe corrosion in oxygen where the phases from left to right are metal, oxide, and oxygen. Source: University of Florida. 
Figure I.1.5.6. Gibbs free energy plot of Fe-FeO-O system. The two tangent lines (colored orange and light blue, not specified in the legend) define five equilibrium regions from left to right: $\mathrm{Fe}$, $\mathrm{Fe}+\mathrm{FeO}, \mathrm{FeO}, \mathrm{FeO}+\mathrm{O}$, and O. Source: University of Florida.

Figure I.1.5.7. Surface energy of each alloy constituent for FCC-Fe and BCC-Fe.

Source: University of Florida..

Figure I.1.5.8. Summary of the $\mathrm{n}$-type $\mathrm{C}_{2} \mathrm{O}_{3}$ formation mechanism.

Source: University of Wisconsin-Madison.

Figure I.1.5.9. An example of fitting existing data of high chromium SS with the C4 n-type model.

Source: University of Wisconsin-Madison.

Figure I.2.1.1. Induction motor rotor and an example of a shorting bar. Source: PNNL

Figure I.2.1.2. Four-point probe method for measuring electrical conductivity and current density. Source: PNNL.

Figure I.2.1.3. Electrical resistivity of copper-composite with 'B' measured from $20-250^{\circ} \mathrm{C}$ using the four-point probe method in a furnace. Source: Thermophysical Properties Research Laboratory, Inc.

Figure I.2.1.4. Microstructure of: (a) sample 'A' (control) made with consolidated C110000 foils;

(b) ShAPETM extrudate manufactured using pure copper billet; and (c) sample 'C' containing 0.0006 wt.\% graphene. Source: PNNL.

Figure I.2.2.1. Conventional Brake design utilizing an Al caliper and a cast-iron brake disc. Source: PNNL.

Figure 1.2.2.2. PNNL tilt-pour stir-casting equipment used for the casting trials. Flat-bladed

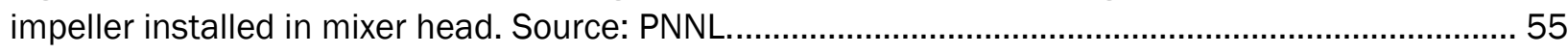

Figure I.2.2.3. Impeller designs for mixing trials. Source: PNNL. ..................................................... 55

Figure I.2.2.4. Brake friction pair test stand at PNNL. Source: PNNL. .............................................. 56

Figure I.2.2.5. Brake pad holder and tested subscale brake rotor. Source: PNNL. .......................... 56

Figure I.2.2.6. (a) Wear track profilometry; and (b) transfer layer chemistry characterization. Source: Rockwell Science Center.

Figure I.2.2.7. (a) Fixture for stir-casting trials and (b) the gravity cast plates for 5, 10 , and 15 vol. \% TiB2 reinforced A356 alloy. Source: PNNL. .................................................................... 58

Figure I.2.2.8. Subscale rotors ready for friction/wear-testing. Source: PNNL................................. 58

Figure I.3.1.1.1.1. Illustration of the approach for high-temperature alloy design in this effort.

See text for details. Source: ORNL

Figure I.3.1.1.1.2. An example of advanced characterization (STEM) of key $\theta^{\prime} /$ matrix Al interface (left) guiding atomistic calculations by DFT (right). Source: ORNL.

Figure I.3.1.1.1.3. Solute segregation profiles predicted by phase-field simulations compared to empirical segregation profile. (a) 2D contour plot of Mn. (b) The simulated solute segregation profile with only the lattice strain mechanism activated. (c) 2D contour plot of Zr. (d) The simulated segregation profile with both the lattice strain and interfacial energy mechanisms activated. Source: ORNL 
Figure I.3.1.1.1.4. (a) In situ HR-STEM results showing transformations from $\theta^{\prime}$ to $\theta$. (b) Phasefield simulations show that for small aspect ratios, small precipitates can rapidly transform the entire precipitate, but larger aspect ratios favor the $\theta^{\prime}$ phase. (c) Analytic theory demonstrates the temperature-dependent map of behaviors. Source: ORNL.

Figure I.3.1.1.1.5. (a) HR-STEM results showing a single layer of $\mathrm{Al}_{3} \mathrm{Zr}$ at the interface between the $\mathrm{Al}$ matrix and the $\mathrm{Al}_{2} \mathrm{Cu} \theta^{\prime}$ precipitate. (b) Full nucleation of $\mathrm{Al}_{3} \mathrm{Zr} \mathrm{L} 1_{2}$ on $\theta^{\prime}$ precipitate.

(c) "Ghost" layers of $\mathrm{Al}_{3} \mathrm{Zr}$ ( $\left(1_{2}\right.$ structure) near the $\theta^{\prime}$ precipitates after higher temperature aging. Source: ORNL.

Figure I.3.1.1.2.1. The NEISS mechanism for stabilization of precipitation-hardened Al-Cu alloy microstructures using: (a) atom probe calculations of interfacial $\mathrm{Zr}$ concentration; and (b) an $\mathrm{L}_{2}$ phase on the coherent interface and the solute concentration corresponding to this $\mathrm{Al}_{3} \mathrm{Zr}$ phase. Source: ORNL.

Figure I.3.1.1.2.2. DFT calculated values of interfacial energy for the semi-coherent, coherent

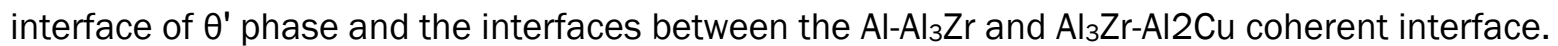
Source: ORNL.

Figure I.3.1.1.2.3. In situ neutron diffraction during room temperature tensile testing of an Al-Cu alloy under various testing conditions. (a) Solution-treated (or naturally aged); (b) $200^{\circ} \mathrm{C}$ preconditioned; and (c) $300^{\circ} \mathrm{C}$ preconditioned. Source: ORNL.

Figure I.3.1.1.2.4. Schematic displaying the stages of deformation-as well as their grain-and precipitate-scale deformation mechanisms for each aging condition. Source: ORNL.

Figure I.3.1.1.3.1. Low magnification scanning electron micrographs of the as-aged alloys showing particles in (a) $6.0 \mathrm{Cu}$; (b) $6.6 \mathrm{Cu}$; (c) $7.3 \mathrm{Cu}$; (d) $8.0 \mathrm{Cu}$; and (e) $9.0 \mathrm{Cu}$ alloys. (f) Higher magnification scanning electron micrograph of $8.0 \mathrm{Cu}$ alloy showing $\theta^{\prime}$ precipitates.

Source: ORNL.

Figure I.3.1.1.3.2. (a) Room temperature engineering stress-strain curves of as-aged alloys; and (b) variation of yield strength, ultimate tensile strength, and elongation as a function of copper content. Source: ORNL.

Figure I.3.1.1.3.3. (a) Representative micrograph of an ACMZ alloy after tensile test showing fractured grain boundary $\theta$ particles. (b) Fractograph of an ACMZ alloy illustrating extensive coverage of fracture surface by grain boundary $\theta$ particles. Comparison between: (c) mean particle sizes; and (d) circularity of overall particle distribution before tensile test (all particles) and unfractured particle distribution after tensile test. Source: ORNL.

Figure I.3.1.1.3.4. (a) A finite element model of the alloy; and (b) a comparison between simulated and experimental values of elongation. Source: ORNL.

Figure I.3.1.1.3.5. Low-cycle fatigue lives of: (a) as-aged alloys tested at $150^{\circ} \mathrm{C}$; and (b) as-aged alloys exposed to $250^{\circ} \mathrm{C}$ for $100 \mathrm{~h}$ and tested at $250^{\circ} \mathrm{C}$. Source: ORNL.

Figure I.3.2.1.1.1. Initial yield strengths and yield strengths after $250 \mathrm{~h}$ of aging of IN ${ }^{\circledR} 751$ and new ORNL Alloys 163 and 200. Source: ORNL 80

Figure I.3.2.1.1.2. SEM image showing the presence of $\gamma^{\prime}$ precipitates in Alloy 163 after $250 \mathrm{~h}$ aging at $900^{\circ} \mathrm{C}$. Source: ORNL. 
Figure I.3.2.1.1.3. High-cycle fatigue lives at $900^{\circ} \mathrm{C}$ and $200 \mathrm{MPa}$ of laboratory-scale heats for several ORNL alloys compared to the Alloy 751 baseline. Source: ORNL.

Figure I.3.2.1.1.4. Newly designed alumina-forming alloys showed oxidation behavior comparable to commercial alumina-forming Ni-based alloys at $950^{\circ} \mathrm{C}$ in air $+10 \%$ water vapor. The high rate of mass loss of the chromia-forming Udimet 520 alloy indicates poor oxidation resistance compared to the three alumina-forming alloys. Source: ORNL.

Figure I.3.2.1.1.5. Cross-sectional images of alumina-forming ORNL alloy $4 \mathrm{~K}$ shows the presence of an Al-rich oxide scale after $600 \mathrm{~h}$ at $950^{\circ} \mathrm{C}$, in air $+10 \%$ water vapor. Source: ORNL 82

Figure I.3.2.1.1.6. Yield strengths of new alumina-forming alloys satisfied FY 2019 targets.

Source: ORNL.

Figure I.3.2.1.2.1. (a) Yield strength of 418 and 422 steel at test temperatures of $600^{\circ} \mathrm{C}$, $650^{\circ} \mathrm{C}$, and $700^{\circ} \mathrm{C}$, and at $600^{\circ} \mathrm{C}$ and $650^{\circ} \mathrm{C}$ after aging for $500 \mathrm{~h}$ at each temperature.

(b) Oxidation mass gain of T91, 422, and 418 during cyclic oxidation testing ( $1 \mathrm{~h}$ cycles) at $600^{\circ} \mathrm{C}$ in air plus $10 \%$ water vapor. Source: ORNL.

Figure I.3.2.1.2.2. Oxidation mass changes of two developmental alloys along with commercial alloys T91, 422, and 418 during cyclic oxidation testing ( $1 \mathrm{~h}$ cycles) at $600^{\circ} \mathrm{C}$ in air plus $10 \%$ water vapor. Source: ORNL.

Figure I.3.2.1.2.3. Yield strength of developmental alloy HTC-OR and 422 steel at test temperatures of $550^{\circ} \mathrm{C}, 600^{\circ} \mathrm{C}, 650^{\circ} \mathrm{C}$, and $700^{\circ} \mathrm{C}$. Source: ORNL.

Figure I.3.2.1.2.4. Ultimate tensile strengths of commercial alloys 418,422 , as well as developmental alloys $\mathrm{HTCR}$-Plus, $\mathrm{HTC}-\mathrm{OR}$, and $\mathrm{BCO}$ at test temperatures of $550^{\circ} \mathrm{C}, 600^{\circ} \mathrm{C}$, $650^{\circ} \mathrm{C}$, and $700^{\circ} \mathrm{C}$. Source: ORNL.

Figure I.3.2.1.3.1. Cross-section optical micrographs of aluminide slurry coated specimens annealed for $4 \mathrm{~h}$ at $1121^{\circ} \mathrm{C}$ in vacuum showing a $\sim 20-25 \mu \mathrm{m}$ coating formed at the surface of the (a) 2686, (b) 2687, and a $\sim 40$ um coating at the surface of (c) 31V. (d) Mass changes at $900^{\circ} \mathrm{C}$ in air $+10 \% \mathrm{H}_{2} \mathrm{O}$ with $1 \mathrm{~h}$ cycle for bare 2687 , bare $31 \mathrm{~V}$, and coated 2687 coupons showing a significant improvement of the oxidation behavior for the coated 2687 coupon compared to the bare coupon. Source: ORNL.

Figure I.3.2.1.3.2. Top view of 2687 coupons dipped in Al Ceral slurries showing that only the Ceral 10A green coating effectively wet the 2687 coupon. Source: ORNL

Figure I.3.2.1.3.3. (a) Calculated phase distribution for the slurry aluminized $31 \mathrm{~V}$ specimen after aluminizing at $1100^{\circ} \mathrm{C}$ for $3 \mathrm{~h}$ followed by a heat-treatment at $850^{\circ} \mathrm{C}$ for $4 \mathrm{~h}$ and at $730^{\circ} \mathrm{C}$ for $4 \mathrm{~h}$. (b) Elemental mappings of the cross-section of the corresponding aluminized $31 \mathrm{~V}$ specimen and (c) EBSD spectroscopy phase map showing that the model predicted well the microstructure evolution in the coating and the substrate during the coating heat-treatment. Source: ORNL.

Figure I.3.2.1.3.4. (a) Backscattered electron image of the cross-section of the as-aluminized $31 \mathrm{~V}$ specimen after a multi-step heat-treatment for $3 \mathrm{~h}$ at $1100^{\circ} \mathrm{C}, 4 \mathrm{~h}$ at $850^{\circ} \mathrm{C}$, and $4 \mathrm{~h}$ at $730^{\circ} \mathrm{C}$ and after (b) a single-step heat-treatment for $16 \mathrm{~h}$ at $760^{\circ} \mathrm{C}$ highlighting the impact of the heat-treatment on the coating microstructure. Source: ORNL. 
Figure I.3.2.1.3.5. Predictions of the influence of varying heat-treatments on the coating microstructure: (a) after the single-step heat-treatment; and (b) after the multi-step heattreatment showing that the impact of the heat-treatment on the coating/substrate microstructure is well captured by the model. Source: ORNL

Figure I.3.2.1.4.1. Specific mass changes in various cast austenitic steels after cyclic oxidation with $1 \mathrm{~h}$ cycles at: (a) $900^{\circ} \mathrm{C}$ and (b) $950^{\circ} \mathrm{C}$ in air with $10 \% \mathrm{H}_{2} \mathrm{O}$ Specimens exhibiting rapid mass gains or losses indicate poor oxidation resistance. Source: ORNL.

Figure I.3.2.1.4.2. Cross-sectional SEM-BSE images and STEM-dark field images, together with element distribution maps acquired by STEM-EDS, of as-cast AFA2 and AFA 5 after a total of $1000 \mathrm{~h}$ exposure time in $1 \mathrm{~h}$ cycles at $950^{\circ} \mathrm{C}$ in Air $+10 \% \mathrm{H}_{2} \mathrm{O}$. Source: ORNL.

Figure I.3.2.1.4.3. Comparison of creep-rupture lives of various cast austenitic steels tested at $900^{\circ} \mathrm{C}$ and $50 \mathrm{MPa}$ including lab-scale heats based on AFA2 and AFA5 with a variation of low Ni contents. Source: ORNL

Figure I.3.2.1.4.4. Specific mass changes in cast AFA2 and AFA5 base alloys with a variation of $\mathrm{Ni}$ contents after cyclic oxidation with $1 \mathrm{~h}$ cycles at $950^{\circ} \mathrm{C}$ in air with $10 \% \mathrm{H}_{2} \mathrm{O}$, together with the results from the commercially produced cast AFA heats. Source: ORNL

Figure I.3.2.1.4.5. Preliminary TEM characterization results of: (a) cast AFA2; and (b) AFA5, tested at $900^{\circ} \mathrm{C}$ and $50 \mathrm{MPa}$. Source: PNNL.

Figure I.3.2.1.4.6. Images of the first commercial heats of AFA2 and AFA5, together with a cross-sectional macrostructure of AFA5 (heat \#190201) showing no visible solidification defects.

Source: ORNL.

Figure I.3.2.1.4.7. Tensile properties of the cast austenitic stainless steels at $700^{\circ} \mathrm{C}, 900^{\circ} \mathrm{C}$ (yield strength), or RT (ductility), comparing the commercial and lab-scale heats of AFA2 and AFA5 together with the benchmark cast materials. Source: ORNL.

Figure I.3.2.1.5.1. Previous results of fatigue testing showing significant improvements in fatigue performance can be achieved when applying FSP to cast Al alloys. Source: PNNL.

Figure I.3.2.1.5.2. FSP can be used to mitigate stress concentrations around: (a) oil holes in crankshaft steels; and (b) a rotating beam fatigue (RBF) test specimen by improving local fatigue properties. (c) Fatigue test results showing improved performance using FSP. Source: PNNL. ....... 105

Figure I.3.2.1.5.3. Torque-tension measuring rig used to quantify the thread pullout performance of a FSP region. Source: PNNL.

Figure I.3.2.1.5.4. Ten cranks were received from GMR\&D for processing trials. The top crank is as-forged and bottom crank is as-machined. Source: PNNL. 107

Figure I.3.3.1.1.1. (a) RT hardness of two Al-Ce-Cu-based alloys as a function of prior thermal exposure time at $350^{\circ} \mathrm{C}$ showing that the addition of small amounts of $\mathrm{Mn}$ has a significant increase for the hardness of the AICeCuZrMn alloy due to additional solid-solution strengthening. (b) Micrographs of the alloy structures. Source: ORNL. 
Figure I.3.3.1.1.2. (a) UTS for the AICeMn and AICeNi AM alloys following 30 min. exposure time as compared to similar exposure times for other Al alloys. (b) Yield strength of the AICeMn alloy as a function of time compared to 2618 showing much greater thermal stability for the AICeMn alloy. (c) Micrographs used for advanced characterization to identify the phase structure and strengthening mechanisms that enable the excellent high-temperature performance of the AICeMn alloy. Source: ORNL

Figure I.3.3.1.2.1. Process for synthesizing interpenetrating phase composites using the PrintCasting technique. Source: ORNL.

Figure I.3.3.1.2.2. Build plate of six different lattice geometries of PrintCast preforms fabricated by SLM using 316L steel prior to infiltration. Source: ORNL

Figure I.3.3.1.2.3. (a) Experimental and simulated engineering stress-strain curves. (b) A lowmagnitude optical image of an as-tested 20 vol.\% 316L composite with an insert showing stress distribution simulation and (c) A low-magnitude optical image of an as-tested 50 vol.\% 316L composite. Source: ORNL.

Figure I.3.3.1.2.4. (a) Averaged lattice-strains measured via in situ neutron diffraction as for $316 \mathrm{~L}$ reinforcement and A356 matrix in the axial and radial direction to the loading axis used to estimate stress during tensile testing. (b) Experimental and simulated averaged hydrostatic stress for the $316 \mathrm{~L}$ reinforcement and the A356 matrix showing tensile stresses for $316 \mathrm{~L}$ but mainly compressive stresses for A356. Source: ORNL.

Figure I.3.3.1.2.5. Second generation of PrintCast for (a) octahedral and (b) fcc reinforcement designs. Source: ORNL.

Figure I.3.3.1.3.1. As-fabricated SLM 316L: (a) build configuration; (b) optical cross-section micrograph of the rod showing very few large defects; (c) EBSD map highlighting elongated grains along the build direction with no preferential orientation; (d) EBSD map transverse to the build direction; (e) etched BSE-SEM image for the as-fabricated SLM316L and (f) etched BSESEM image of SLM316L after annealing for $5 \mathrm{~h}$ at $800^{\circ} \mathrm{C}$. Source: ORNL.

Figure I.3.3.1.3.2. (a) Comparison of the RT tensile properties of SLM 316L with literature data showing superior ductility but low yield strength for ORNL's SLM316L [1-11]. (b) Plastic elongation at rupture showing significant decrease of ductility at $T>200^{\circ} \mathrm{C}$. (c) Yield strength results. (d) Ultimate tensile strength results. Source: ORNL.

Figure I.3.3.1.3.3. (a) Cross-section BSE-SEM micrograph and (b) further magnification of the single-track laser-melted area for the CF8C+ steel highlighting the disappearance of coarse carbides and the presence of a cellular structure in the melted area. (c) Phase fraction in the CF8C+ steel based on the Sheil's equation to assess the impact of very fast cooling on the alloy microstructure. Source: ORNL

Figure I.3.3.1.4.1. (a) Powder size distribution; (b) micrograph of the Carpenter powder; (c) micrograph of the Sandvik powder; (d) flexural strength showing the impact of powder size and binder saturation on green part strength; and (e) dimensional tolerance with respect to the specified dimensions for Carpenter and Sandvik powders highlighting good dimension tolerance.

Source: ORNL. 
Figure I.3.3.1.4.2. (a) Optical images showing a lack of shape retention at $1400^{\circ} \mathrm{C}$ sintering temperature. (b) Densification in the phase fractions reaching a maximum at $\mathrm{T}>1380^{\circ} \mathrm{C}$.

(c) X-ray diffraction analysis of sintered samples used to calculate phase fractions.

Source: ORNL.

Figure I.3.3.1.4.3. (a) Optical micrograph showing embedded spherical powder particles, (b) SEM micrograph highlighting grain boundary carbides and (c) Scheil simulation for solidification of the liquid post-SLPS for the $105 \%$ sample sintered at $1380^{\circ} \mathrm{C}$ used to better understand microstructure evolution during solidification. Source: ORNL.

Figure I.3.3.1.4.4. Tensile behavior of the samples in the as-sintered and HIP plus heattreatment conditions with the inset showing the representative micrographs for each condition along with porosity reconstructions for the HIP+HT samples Source: ORNL.

Figure I.3.4.1. Cross-sectional $\theta^{\prime}$ 2D composition plots for: (a) ACMZ; and (b) RR350 exposed to $300^{\circ} \mathrm{C}$ for $200 \mathrm{~h}$ reveal the interfacial segregation profiles for $\mathrm{Si}, \mathrm{Zr}$, and $\mathrm{Mn}$. Si shows segregation to regions of the coherent and semi-coherent interfaces, Zr segregates only to the coherent interfaces with additional segregation to the coherent/semi-coherent intersection, and Mn segregates to the coherent and semi-coherent interfaces with additional segregation to the semi-coherent interface [1]. Source: ORNL.

Figure I.3.4.2. APT data for a commercial 422 martensitic stainless steel double tempered at $650^{\circ} \mathrm{C}$. (a) APT atom map with $\mathrm{Fe}, \mathrm{Cr}, \mathrm{N}$, and $\mathrm{V}$ atoms displayed as magenta, blue, green, and purple dots, respectively. A 3 at.\%, the $\mathrm{V}+\mathrm{N}$ iso-concentration surface is displayed to highlight $\mathrm{V}-\mathrm{N}$ particles. A 1D concentration profile was taken across a V-N particle as marked by the black arrow. (b) The $\mathrm{V}$ and $\mathrm{N}$ profile across the $\mathrm{V}-\mathrm{N}$ particle. (c) The Fe and Cr profile across the same $\mathrm{V}-\mathrm{N}$ particle. Source: ORNL

Figure I.3.4.3. A Ni/NiO interface for oxidized $\mathrm{Ni200}$ at $1000^{\circ} \mathrm{C}$ for $24 \mathrm{~h}$. (a) APT atom map of the $\mathrm{NiO} / \mathrm{Ni}$ interface with $\mathrm{Ni}$ and $\mathrm{O}$ ions shown as green and blue spheres, respectively. (b) An $\mathrm{O}$ concentration profile across the $\mathrm{NiO} / \mathrm{Ni}$ interface in the direction shown by the black arrow with a sigmoidal fit overlaid. (c) A magnified view of the 0 concentration profile highlighted by the blue rectangle in (b) showing $\mathrm{O}$ infiltration into the $\mathrm{Ni}$ metal. Control experiments are being conducted to determine the accuracy of the APT measurements of $\mathrm{O}$ solubility in Ni. Source: ORNL

Figure I.3.4.4. (a) Tip of the double-tilt heating holder showing the Micro-Electro-Mechanical Systems (MEMS) heater at the red arrow installed with an inset image showing the atomic structure at the surface of an $\mathrm{Al}_{2} \mathrm{Cu}\left(\theta^{\prime}\right)$ precipitate from (d) and (b) through (c) the progression of the $\theta$ coalescence with heating at $200^{\circ} \mathrm{C}$. Source: ORNL.

Figure I.3.4.5. (a) Tested alloy scattering variation caused by different microstructures as a function of q. (b) The scattering curves change as a function of time at $900^{\circ} \mathrm{C}$. Source: ANL 138

Figure I.3.4.6. (a) The environmental reactor chamber attached to APT at PNNL. (b) The APT run of as-received ACMZ alloy. (c) 2D compositional analysis across precipitate and matrix. (d) Compositional analysis after $300^{\circ} \mathrm{C}$ for $1 \mathrm{~h}$. (e) 2D compositional mapping. (f) Compositional analysis after an additional $3 \mathrm{~h}$ of annealing. (g) 2D compositional map. Source: PNNL. 
Figure I.3.4.7. Microstructural characterization results of HCC1163-2018-3 Aged+250h at $900^{\circ} \mathrm{C}$. (a) SEM image of the overall microstructure. (b) STEM image of the alloy showing matrix and L12 precipitates from which selected area diffraction patterns given in (c) and (d) where obtained. (e) STEM-EDS maps showing element portioning. (f) APT results showing primary and secondary L12 precipitates. Source: PNNL.

Figure I.3.4.8. (a) SEM image of the AFA5 alloy showing the different precipitates. (b) SEM-EDS image showing solute partitioning. (c) TEM weak beam dark field imaging identifying dislocation structures. (d) STEM image of dislocation structures. (e) Area diffraction from different phases in the alloy corresponding to the numbers in (d). (f) STEM-EDS mapping showing the compositional partitioning between phases. The different phases are numbered 1, 2, 3, and 4, respectively. Source: PNNL.

Figure I.3.4.9. Three-dimensional simulations of the precipitation of $\theta^{\prime}$ phase in Al-Cu binary alloy performed using MEUMAPPS in ORNL Summitdev [4]. Source: ORNL.

Figure I.3.4.10. (a) Calculated phase distribution for the aluminized $31 \mathrm{~V}$ specimen after aluminizing at $1100^{\circ} \mathrm{C}$ for $3 \mathrm{~h}$ followed by a heat-treatment at $850^{\circ} \mathrm{C}$ for $4 \mathrm{~h}$ and at $730^{\circ} \mathrm{C}$ for $4 \mathrm{~h}$ and (b) elemental and phase mappings of the cross-section of the corresponding aluminized 31V. Source: ORNL.

Figure I.3.4.11. (a) The stitched SEM images of a 9\% Cu AZMZ alloy. (b) The corresponding microstructure-based FE model. (c) The comparison of the simulation results of engineering strain at failure with those of the experiments. Source: ORNL.

Figure I.3.4.12. Comparison of the FE analysis and experimental results for body-centered lattice structure AMIPC at (a) 20 vol\%; and (b) 50 vol\%. (c) Stress-strain curves for simulated and experimental results. Source: ORNL.

Figure I.3.4.13. Pearson Correlation Coefficient plots for: (a) bulk alloy composition; and (b) the top five best and worst computational features. Source: ORNL.

Figure I.3.4.14. Solidification path simulation of: (a) Al-Fe-Ce; (b) Al-Ni-Ce; and (c) Al-Fe-Ni-Ce showing $\sim 25,50$, and 75 mol\% eutectic in as-solidified microstructure. Source: ORNL.

Figure I.3.4.15. The Scheil-solidified microstructure and equilibrium microstructure of a CF8C-Plus alloy for (a) the equilibrium phases; (b) the solidification path; and (c) the solidification phases. Source: ORNL.

Figure I.3.4.16. (a) Predicted part shrinkage for the cube shape. (b) The part shrinks about $3 \mathrm{~mm}$ in $\mathrm{x}-, \mathrm{y}-$, and $\mathrm{z}$-direction. (c) The phenomenological model predicts the shrinkage within minor error. Source: ORNL.

Figure I.3.5.1.1. (a) Temperature-resistant phenolics are preferred material for engine block applications. However, these compositions are very brittle (magenta colored data). (b) Use of crosslinker (both matrix and fiber surface modifier) changes in failure mode from fiber/matrix debonding to fiber breakage. (c) With 12 wt.\% CF, the toughened composition exhibits 1.6\% strain to failure. Source: ORNL

Figure I.3.5.1.2. Mechanical properties for (a) tensile strength and (b) modulus of a renewable variant of toughened phenolic resin. Source: ORNL

Figure I.3.5.1.3. Tensile strength of unidirectional renewable composite containing $~ 12 \% \mathrm{CF}$. Source: ORNL. 
Figure I.3.5.1.4. Thermogravimetric analysis of the neat resin and its two composites.

Source: ORNL.

Figure I.3.5.1.5. Two views of the single cylinder 2-stroke GE35 engine purchased for parametric studies. Source: ORNL.

Figure I.3.5.1.6. Computer-aided design geometry of the Pegasus GE-35 engine that was imported into a preprocessor for the FEA. Source: ORNL.

Figure I.3.5.2.1. (a) System for the inline $\mathrm{CO}_{2} \mathrm{AP}$ treatment on a $\mathrm{Mg}$ specimen inside a $\mathrm{CO}_{2}$ enriched chamber. (b) The proposed approach where the $\mathrm{CO}_{2}$ plasma converts the surface to an $\mathrm{MgCO}_{3}$ protective layer. (c) SEM and cross-sectional TEM images showing a $\mathrm{CO}_{2}$ plasma-driven protective layer that consisted of particle deposition on a dense layer with $\sim 1.5 \mu \mathrm{m}$ thickness. Source: (a) Atmospheric Plasma Solution (APS); (b) and (c) ORNL.

Figure I.3.5.2.2. Formation of protective layers on various $\mathrm{Mg}$ specimens via $\mathrm{CO}_{2}-\mathrm{AP}$ treatment (i.e., 5 sweeps, $0.15 \mathrm{in}$. height, $300 \mathrm{in./min}, 0.025 \mathrm{in}$. spacing) showing microscopic surface morphology changes of (a) AP-treated Mg and (b) DI water pretreated Mg (DI-Mg) specimens. Source: ORNL.

Figure I.3.5.2.3. Anticorrosion effects of $\mathrm{CO}_{2} \mathrm{AP}$-driven protective layer on $\mathrm{Mg}$ specimen: (a) AP-treated $\mathrm{Mg}$ specimens after a $20 \mathrm{~h}$ immersion corrosion test in $3.5 \mathrm{wt} . \% \mathrm{NaCl}$ solution; and (b) the electrochemical corrosion rate measurement on the AP-driven protective layer on the $\mathrm{Mg}$ surface where the potentiodynamic polarization measurement was in $3.5 \mathrm{wt} . \% \mathrm{NaCl}$ solution. Source: ORNL.

Figure I.3.5.2.4. (a) Hydrogen collection test on various Mg surfaces in $3.5 \mathrm{wt} \% \mathrm{NaCl}$. Inset pictures are corroded Mg and AP-Mg surfaces after $24 \mathrm{~h}$ immersion. (b) The potentiodynamic polarization measurements of various $\mathrm{CO}_{2} \mathrm{AP}$-treated $\mathrm{Mg}$ specimens with different sweeping numbers and pretreatment. Source: ORNL

Figure I.3.5.2.5. (a) Photo-image of the $\mathrm{CO}_{2}$-AP-treated $\mathrm{Mg}$ surface showing superhydrophobicity and (b) photo-images of immersed $\mathrm{Mg}$ samples under $3.5 \mathrm{wt} . \% \mathrm{NaCl}$ solution. Source: ORNL. ....... 166

Figure I.3.5.2.6. (a) High magnification cross-sectional HAADF STEM images of $\mathrm{CO}_{2}$-AP-treated $\mathrm{Mg}$ along with EDS elemental mapping showing the distribution of $\mathrm{C}, \mathrm{O}$, and $\mathrm{Mg}$ in the treated layer. (b) EDS pattern for the $\mathrm{CO}_{2}$-AP-treated $\mathrm{Mg}$ (five sweeps). Source: ORNL.

Figure I.3.5.2.7. (a) Hydrogen collection test of the $\mathrm{CO}_{2}-\mathrm{AP}-5$ sweep on AZ31B, compared with an untreated sample. The reduced $\mathrm{H}_{2}$ evolution indicates lower rate of corrosion in the $3.5 \mathrm{wt} . \%$ $\mathrm{NaCl}$ immersion test. Insets are surface features of $\mathrm{CO}_{2}$-AP-treated AZ31B after $160 \mathrm{~h}$ immersion test. (b) The potentiodynamic polarization measurement on various AP-treated AZ31B surfaces in

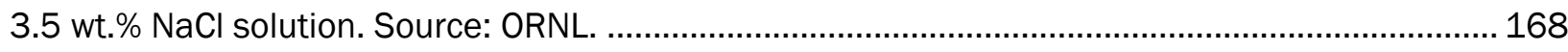

Figure I.3.5.3.1. Friction stir-processed AA6061 block with nano additives in the processed region. Source: ORNL

Figure I.3.5.3.2. AA7075 machined chips as feedstock material. Source: ORNL.

Figure I.3.5.3.3. Progressive process refinement to achieve fully dense consolidated material blocks for property testing. Source: ORNL. 173

Figure I.3.5.3.4. Microhardness results by adding IMs and SiC to AA6061 matrix. Source: ORNL. . 174 
Figure I.3.5.4.1. Al connecting rod for a 4-stroke engine failed by fatigue, which subsequently impacted the crankshaft and caused catastrophic engine damage. Source: Wikipedia. 176

Figure I.3.5.4.2. Schematic diagram of the ShAPETM process. Source: PNNL. 178

Figure I.3.5.4.3. Al billet loaded with graphene powders as a precursor of ShAPETM. Source: PNNL. 178

Figure I.3.5.4.4. Experimental setup of ShAPETM process. Source: PNNL 178 Figure I.3.5.4.5. (a) Al-graphene composite wires produced by ShAPETM . (b) A cross-section of 7075-graphene ShAPETM wire. Source: PNNL.

Figure I.3.5.4.6. (a) Backscatter electron image of Al-graphene ShAPETM wire. (b) EDS image shows uniform carbon dispersion. Source: PNNL 180

Figure I.3.5.4.7. ShAPE-processing temperature of making 7075-G composite, as compared to other processes. Source: PNNL.

Figure I.3.5.5.1. Advanced in situ material characterization helps the accelerating alloy development process for automotive applications. Source: ANL.

Figure I.3.5.5.2. Schematic diagram of zoom-in-out imaging setup for the future HEXM beamline (APS-U project). The project will develop a high-temperature environment for the imaging setup.

Source: [1].

Figure I.3.5.5.3. Test of heating characteristic of SiC under various dimensions and coil geometry. Source: ANL.

Figure I.3.5.5.4. (a) 3D model of high-temperature cell. (b) The setup consists of a SiC susceptor and ceramic holder. Source: ANL.

Figure I.3.5.5.5. (a) Overall temperature profile and (b) heating and cooling rates that are characteristic of the high-temperature cell. Source: ANL

Figure II.1.1.1. (a) ShAPE ${ }^{\mathrm{TM}}$ machine installed in PNNL's Solid-Phase Processing Laboratory and (b) thin-walled ZK60 circular tubing extruded by ShAPETM. Source: PNNL.

Figure II.1.1.2. (a) ZK60 billet mounted in rotating spindle of ShAPETM machine and (b) with the clamshell container assembly. Source: PNNL

Figure II.1.1.3. (a) Two-piece portal bridge die design showing: (b) scrolled-face (front side); and (c) mandrel (back side). Source: PNNL.

Figure II.1.1.4. (a) Back side of die head after an initial extrusion trial showing re-combination of four material streams in the weld chamber and (b) bare mandrel before extrusion. Source: PNNL.190

Figure II.1.2.1. Illustration of the 2013 Ford Fusion door showing the progression from baseline steel to Multi-Material Lightweight Vehicle Al to an Al-intensive door with $\mathrm{Mg}$ inner and outer panels selected for demonstration in warm-forming. Source: Ford Motor Company.

Figure II.1.2.2. (a) Tensile test results of USAMP Alloy 2 Plus at annealing condition; and (b) comparison of Alloy 2 Plus with other Mg sheet alloys reported in literature. Source: OSU. 194

Figure II.1.2.3. Mechanical properties of USAMP Alloy 2 Plus sheet and summary of formability and strength of the existing Mg sheets. Source: OSU. 
Figure II.1.2.4. ORNL-rolled Alloy 2 Plus sheet (size is $\sim 1.65-\mathrm{mm}$-thick and $\sim 11-\mathrm{cm}$ wide).

Source: ORNL.

Figure II.1.2.5. Shear roll arrangement for asymmetric rolling. Source: ORNL

Figure II.1.2.6. Sections of symmetric + asymmetric rolled Mg sheet (left) and symmetric rolled Mg sheet (right) sent to OSU for analyses. Source: ORNL.

Figure II.1.2.7. Effect of solutes on the yield stress required to move (a) basal and $c+a$ or (b) and (c) twinning edge dislocations at RT. Source: UIUC.

Figure II.1.2.8. Grain size distributions and optical micrographs (inset) showing the rolling direction (RD) and transverse direction (TD) of (a) Mg-0.2Ca and (b) Mg-1.5Sn sheets with 20\% thickness reduction annealed at $400^{\circ} \mathrm{C}$ for 120 hours. Source: UF.

Figure II.1.2.9. (a) X-ray pattern obtained from the homogenized alloys. (b) Spot EDS spectrum of a $\mathrm{Mg}_{2} \mathrm{Ca}$ particle from homogenized $\mathrm{Mg}-0.35 \mathrm{Ca}-0.5 \mathrm{Zn}$ alloy with the inset showing the particle and location diffraction from which the spectrum was obtained. Source: UF.

Figure II.1.2.10. (a) Representative stress-strain curves of Mg-0.35Ca-0.5Zn compressed at $250^{\circ} \mathrm{C}$ showing serrations at $10^{-3} \mathrm{~S}^{-1}$. " $\mathrm{X}$ " represents the fracture point of the sample. (b) SRS changes as a function of temperature from $10^{-3}$ to $10^{-2} \mathrm{~s}^{-1}$ for $\mathrm{Mg}$-Ca binary and $\mathrm{Mg}$-Ca-Zn ternary alloys showing the DSA regime. Source: UF.

Figure II.1.2.11. Photograph of a ZX30 sample subjected to plane-strain compression. The top and bottom of the sample was unconstrained, and the middle of the specimen is the deformed region with a refined microstructure. Source: UM.

Figure II.1.2.12. (a) EBSD inverse pole figure map and (b) pole figures of a ZX30 Gleeble sample subjected to ten passes of 0.2 true strain with a strain-rate of $0.5 \mathrm{~s}^{-1}$. Source: UM

Figure II.1.2.13. The Mg alloy sheets and tensile specimens. Source: PNNL

Figure II.1.2.14. EBSD results from as-rolled Mg-0.2Ca (a-c) and recrystallized Mg-0.2Ca (d-f). $(a, d)$ The IQ map for the IPF map (b, e). The inserted color scale is projected along the RD. The nonindexed phase in the IPF map is black. (c, f) The pole figures. Three plots in each figure are set to equal color scale. The $X$ direction is along the RD, while the pole axis is along the ND of the rolling sheet. The pole figures were calculated by choosing the EBSD area in (b, e); however, the largest grain in (e) was not considered. Source: PNNL.

Figure II.1.2.15. EBSD results from as-rolled Mg-1.5Sn (a-c) and recrystallized Mg-1.5Sn (d-f). $(a, d)$ The IQ map for the IPF map (b, e). The inserted color scale is projected along the RD. The nonindexed phase in the IPF map is black. (c, f) The pole figures. Three plots in each figure are set to equal color scale. The $X$ direction is along the RD, while the pole axis is along the ND of the rolling sheet. The pole figures were calculated by choosing the EBSD area in (b, e); however, the largest grain in (e) was not considered. Source: PNNL.

Figure II.1.2.16. (a) Uniaxial stress-strain responses; (b) initial EBSD measurement; and (c) the pole figure measurement for E-Form Plus Mg sheet. Source: Inal Tech Inc.

Figure II.1.2.17. Taylor-type Mg CP formulation [14]. 208

Figure II.1.2.18. Experimental data shows a distinctive difference in the hardening rates in the different directions. Source: Inal Tech Inc. 
Figure II.1.2.19. Different strain paths are simulated to produce anisotropic measurements to calibrate a desired yield function. Source: Inal Tech Inc.

Figure II.1.2.20. Example of LS-DYNA formatted material card with the populated values.

Source: LSTC.

Figure II.1.2.21. Cross-section micrograph showing good adhesion in a $2-\mu \mathrm{m}$ thick Zn-coating on E-Form Plus. Source: Xtalic.

Figure II.1.2.22. Larger 19 in. x 26 in. Zn-plated panels with a superimposed dicing pattern. Anode connections can be seen at the top of the image. Source: Xtalic.

Figure II.1.2.23. Bend test after humidity exposure showing no apparent increase in oxidation.

Source: Henkel.

Figure II.1.2.24. FE-SEM of coated E-Form Plus Mg showing (a) the control and (b) bent at 5000x magnification showing moderate cracking on the surface. Source: Henkel.

Figure II.1.2.25. A typical automotive pretreatment sequence before applying the electrocoat. Source: PPG.

Figure II.1.2.26. X-ray photoelectron spectroscopic depth profiles of E-Form Plus immersed in an alkaline cleaner, then in Cleaner 2 for various lengths of time. The concentration of each element is shown changing in both time (along the x-axis), as well as at sample depth (light pink $\rightarrow$ dark pink). The decrease in oxygen content is clearly observed over time, which is attributed to the conversion of $\mathrm{Mg}(\mathrm{OH})_{2}$. Source: $\mathrm{PPG}$.

Figure II.1.2.27. E-Form Plus (EFP) joined to AA6111, HDGE, CRS, and itself using two selfpiercing rivets and subjected to different cleaning and pretreating combinations after three weeks of filiform corrosion testing. Source: PPG.

Figure II.1.3.1. (a) Corrosion test chamber for the ASTM B117 test where (b) the samples are vertically hung. Source: PNNL.

Figure II.1.3.2. The condition of joint coupons before and after $1500 \mathrm{~h}$ of ASTM B117 test:

(a) Arplas RSW joint front side; (b) Arplas RSW joint back side; (c) Al rivet joint front side; and

(d) Al rivet joint back side. Source: PNNL.

Figure II.1.3.3. Relative change in weight of corrosion coupons as a function test duration, ASTM B117 method, Arplas RSW goes through least amount of corrosion attack. Source: PNNL.

Figure II.1.3.4. RT lap-shear strength of $\mathrm{Mg} / \mathrm{Mg}$ similar joints before and after exposure to corrosion test chamber (a) Arplas RSW; (b) rivet joint, deburred hole; and (c) rivet joint, nondeburred hole. Source: PNNL.

Figure II.1.3.5. Stereo micrograph of joint interface of corrosion coupons following a $1500 \mathrm{~h}$ exposure and subsequent lap-shear test. The occurrence of corrosion is noted by the white arrows for: (a) the Arplas RSW; (b) the non-deburred hole rivet; and (c) the deburred hole rivet. Source: PNNL.

Figure II.1.4.1. (a) LSP and BM coupons hanging within the environmental test chamber for ASTM B117 test; (b) \% change in weight of LSP vs BM coupons as a function of test duration and (c) physical appearance of BM and three different LSP coupons after 56 days of corrosion testing with the BM showing heavy corrosion and deposition of white colored corrosion products.

Source: PNNL. 230 
Figure II.1.4.2. SEM images showing the plan view of (a) the LSP surface and (b) the non-LSP BM surface. Through-thickness backscattered electron images of (c) the LSP sample showing the presence of a continuous layer indicated by red arrows and (d) the non-LSP sample showing the absence of any distinct surface layer. Source: PNNL.

Figure II.1.4.3. (a) Through-thickness FIB image of LSP-treated AZ31 sample. (b) BF image from LSP-treated sample showing the top surface layer at black arrows and second-phase particles indicated by red arrows. (c) HAADF image from LSP sample, bright particles are an AI-Mn rich IM phase. Source: PNNL.

Figure II.1.4.4. (a) HAADF image of LSP-treated AZ31 sample showing the surface film and subsurface AZ31 matrix. (b) Elemental concertation profile from the yellow arrow region shown in (a). (c) Higher magnification HAADF image of top surface film and adjoining AZ31 matrix. (d) Elemental concentration profile from the yellow arrow region shown in (c). Source: PNNL.

Figure II.1.5.1. Test coupons used for corrosion testing of CFRE-coated AHSS: (a) CFRE bonded lap-shear tensile test; (b) CFRE-coated flexural strength test coupons; and (c) a CFRE-coated CET coupon scribed in the middle to evaluate delamination. Source: INL

Figure II.1.5.2. (a) Coating tensile stress at yield point as a function of corrosion cycles after the SAE J2334 test. (b) Flexural strength at yield point as a function of corrosion cycles. (c) Flexural strength at yield point as a function of humidity cycles. (d) Flexural strength after environmental cycling at maximum loads. Each data point is an average of at least 10 values. All error bars are standard deviations. CFRE coating thickness on all specimens was $0.5 \mathrm{~mm}$. Source: INL.

Figure II.1.5.3. Coupon J116 (a) immediately after 30 cycles of testing; and (b) after the corrosion has been cleaned out of the scribe line area. Source: INL

Figure II.1.6.1. Temporal HEXRD assessments for a portion of the $2 \theta$ range collected in situ during IA with a heating rate of $0.7625^{\circ} \mathrm{C} / \mathrm{s}$ and an isothermal holding at $650^{\circ} \mathrm{C}$ for $1800 \mathrm{~s}$ showing cementite $(\theta)$ and austenite $(\mathrm{Y})$ diffraction peaks. The full $\mathrm{y}\{200\}$ intensity is shown in (a), while the truncated intensity in (b) shows the $\theta$ peaks. Source: PNNL.

Figure II.1.6.2. Field emission SEM images of the microstructure produced from IA with a heating rate of $0.7625^{\circ} \mathrm{C} / \mathrm{s}$ and an isothermal hold at $650^{\circ} \mathrm{C}$ for (a) $1000 \mathrm{~s}$ and (b) $1800 \mathrm{~s}$. Image (a) exhibits the elongated austenite and fine cementite, while image (b) exhibits a higher magnification of intra-lath rod-like cementite. Source: Colorado School of Mines.

Figure II.1.6.3. Austenite volume fraction predictions from Simulations 1 and 2 for isothermal holding at $650^{\circ} \mathrm{C}$, as well as the austenite volume fraction assessments from the in situ HEXRD during isothermal holding at $650^{\circ} \mathrm{C}$. Source: Colorado School of Mines.

Figure II.1.6.4. Mn distribution predictions from (a) DICTRA Simulation 1 and (b) Simulation 2 for isothermal holding at $650^{\circ} \mathrm{C}$ and at isothermal hold times ranging from $100-50,000 \mathrm{~s}$.

Source: Colorado School of Mines.

Figure II.1.6.5. Dilatometry assessments of austenite volume fraction from the three conditions of the $0.0005 \mathrm{C}-7.19 \mathrm{Mn}$ steel during IA with a $1000 \mathrm{~s}$ isothermal hold at $650^{\circ} \mathrm{C}$ and heating rates of: (a) $0.05^{\circ} \mathrm{C} / \mathrm{s}$; and (b) $96^{\circ} \mathrm{C} / \mathrm{s}$. Source: Colorado School of Mines.

Figure II.1.6.6. Field emission SEM images of the microstructure produced from heating the CR50 condition of the $0.0005 \mathrm{C}-7.19 \mathrm{Mn}$ steel with a heating rate of $0.7625^{\circ} \mathrm{C} / \mathrm{s}$ to $650^{\circ} \mathrm{C}$ and quenching. Austenite is observed to form at elongated, deformed ferrite boundaries, labeled $\mathrm{A}$, as well as at recrystallized ferrite boundaries, labeled B. Source: Colorado School of Mines. 
Figure II.1.6.7. Exemplary MICRESS $®$ simulation results of IA with an isothermal holding temperature of $650^{\circ} \mathrm{C}$ and a heating rate of $96^{\circ} \mathrm{C} / \mathrm{s}$ for: (a) phase maps; (b) SSE maps; and (c) Mn distribution maps. Source: Colorado School of Mines.

Figure II.1.6.8. Austenite volume fraction predictions during isothermal holding from phase-field simulations incorporating: (a) different amounts of austenite nucleation sites; and (b) diffusivity pre-factors for $\mathrm{Mn}$ in ferrite. Source: Colorado School of Mines.

Figure II.1.6.9. Predictions for Mn distribution across the $\alpha-y$ interface after a $500 \mathrm{~s}$ isothermal hold at $650{ }^{\circ} \mathrm{C}$ for simulations incorporating different diffusivity pre-factors $\left(D_{0}\right)$ for $\mathrm{Mn}$ diffusivity in ferrite. Source: Colorado School of Mines.

Figure II.1.7.1. Benchtop setup constructed at PNNL for ultrasonic melt-processing experiments. Source: PNNL.

Figure II.1.7.2. (a) As-cast A206 Al alloy tensile bars; and (b) modified solutionization heattreatment procedure implemented in the experiments. Source: PNNL.

Figure II.1.7.3. As-cast SEM microstructure of A356 Al alloy: (a) without ultrasonication; and (b) with ultrasonication. Source: PNNL.

Figure II.1.7.4. (a) Phase identification in the A356 cast microstructure and (b) EDS mapping showing the evidence of the identified phases. No impurity elements, such as Ti from the ultrasonic probe, were detected. Source: PNNL.

Figure II.1.7.6. SEM micrographs showing (a) as-cast microstructure and further effects of solutionization and T7 treatments for solutionization times of (b) $0 \mathrm{~h}$ and (c) $12 \mathrm{~h}$ in A206 Al alloy. Source: PNNL

Figure II.1.7.7. Graphs showing the effect of different solutionization times (i.e., $0 \mathrm{~h}$ and $12 \mathrm{~h}$ ) followed by natural aging/ $\mathrm{T} 7$ treatments on: (a) volume fraction of $\mathrm{Al}_{2} \mathrm{Cu}$; and (b) size of $\mathrm{Al}_{2} \mathrm{Cu}$. Source: PNNL.

Figure II.1.8.1. Images of the steel side-impact beam: (a) top view, (b) bottom view. Source: PNNL.

Figure II.1.8.2. (a) Dark field TEM image showing a GP-I zone. (b) Natural aging behavior of AA 7075 (flow stress vs time) after solutionization and quenching heat-treatment. (c) Modeling results obtained from microstructure evolution models and strength governing Equations 1-5, assuming strengthening only from GP-I zones (red curve) or only from GP-II zones (blue curve). Source: PNNL.

Figure II.1.8.3. Physical meaning of the constants used in Equations 1-6. Source: PNNL.

Figure II.1.8.4. Strength prediction in deformed AA 7075 during aging using the coupled deformation-precipitation model. Source: PNNL.

Figure II.1.8.5. Intensity (I) versus scattering-vector (q) plots from in situ SAXS/WAXS studies at NSLS-II, BNL, to study the precipitation kinetics during natural aging in AA 7075. Scattering at low angles (small q) is clearly visible owing to precipitate size and volume fraction evolution. Time is varied from $0.56 \mathrm{hr}$ to $14.5 \mathrm{hr}$ after solutionization and quench procedures.

Source: PNNL/BNL. 259

Figure II.1.9.1. Approach in the development of lubricants. Source: ANL. 262 
Figure II.1.9.2. (top) WHTL1 coating on the steel sample and Raman spectroscopy of the coating deposited on to the substrate with a wear volume on the bare disc of $0.613 \mathrm{~mm}^{3}$. (bottom) The graph of friction at a high-temperature with the lubricant down to 0.1 which was minimal and a wear volume after the coating was removed of $0.465 \mathrm{~mm}^{3}$. Source: ANL.

Figure II.1.9.3. (top) WHTL2 coating on the Al substrate and the Raman spectra of the lubricant showing characteristic peaks of the $2 \mathrm{D}$ materials present and a wear volume on the bare disc of $1.115 \mathrm{~mm}^{3}$. (bottom) A graph of the friction at a high-temperature showing $66 \%$ reduction in friction when compared to the lubricant-free substrate after wear-testing with the coating removed showing no calculable wear loss. Source: ANL.

Figure II.1.9.4. Friction coefficient recorded on the twist-compression-testing machine with the developed lubricants during warm-forming in a temperature range of $275-480^{\circ} \mathrm{C}$. (a) The bare substrate was observed to have a friction of about 0.35-0.4. A commercial lubricant lowered the friction to 0.1 and WHTL1 produced a friction of 0.03-0.05. (b) WHTL2 produced a friction of 0.02-0.03. Source: ANL

Figure II.1.9.5. Friction recorded on the twist-compression testing machine with lubricants for high-temperature $\left(790^{\circ} \mathrm{C}\right)$. The hot-forming lubricants (SL1) was observed to have a friction of 0.1-0.15 with whereas the commercial lubricant had shown a friction of 0.05. The lubricant is seen to show consistent performance over three turns as shown in the graph. Source: ANL.

Figure II.2.1.1. Cross-sectional SEM micrographs of carbonized (a) neat nylon, (b) nylon/PVA and (c) nylon/PVA/0.1 wt.\% graphene. XRD patterns of neat nylon, nylon/PVA, and nylon/PVA/graphene (d) without any treatment, (e) after a 25-hour oxidation and (f) after carbonization. Source: University of Virginia.

Figure II.2.1.2. (a) Raman spectra of carbonized nylon, nylon/PVA, and nylon/PVA/0.1 wt.\% graphene fibers. (b) TGA results of oxidized nylon, nylon/PVA, and nylon/PVA/graphene.

(c) Stress-strain curves of carbonized nylon, nylon/PVA, and nylon/PVA/graphene.

Source: University of Virginia.

Figure II.2.1.3. (a) Graphic of the stepwise microwave heating time. (b) Infrared image of the nylon/PVA/graphene during microwave irradiation. (c) Surface temperature, measured with the thermal camera, of the microwave-treated fibers with increasing treatment duration.

Source: University of Virginia.

Figure II.2.1.4. Simulation snapshots representing carbon monoxide production for samples with (a) $2 \mathrm{~K} / \mathrm{ps}$ and (b) $700 \mathrm{~K} / \mathrm{ps}$ heating rate. Source: Pennsylvania State University.

Figure II.2.1.5. Total error during parameterization and comparisons between ReaxFF and the quantum reference data after the force field training: (a) Total error versus iteration (220 times of the initial parameters) during parameterization; (b) Comparisons of S-S, $\mathrm{H}-\mathrm{S}-\mathrm{S}-\mathrm{H}$, and $\mathrm{H}-\mathrm{S}-\mathrm{H}$ bond dissociation energies between ReaxFF and the quantum data; Comparisons of (c) S-S, (d) S-H, (e) S-S, (f) C=S, and (g) C-C bond dissociation energies between ReaxFF and the quantum data; Comparisons of (h) C-S-C, (i) C-S-H, and (j) H-S-H angle distortion energies between ReaxFF and the quantum data. Source: Pennsylvania State University.

Figure II.2.1.6. (a) The nylon 6 with copper chloride ions in water solution simulations; and (b) densities for each solution. Source: Pennsylvania State University.....

Figure II.2.1.7. (a) Six-membered, all carbon ring formation and (b) carbon content of 5-layered graphene/PAN for various carbonization temperatures. Source: Pennsylvania State University. .... 277 
Figure II.2.1.8. (a) A comparison of the data from carbonization simulation for the PBO (b) with and (c) without box deformation on the x-direction during the carbonization simulation at $2800 \mathrm{~K}$. Source: Pennsylvania State University.

Figure II.2.1.9. A 6-membered ring production from the carbonization simulation at a temperature of $2800 \mathrm{~K}$ for the polymers with four monomers illustrated in the color boxes. Source: Pennsylvania State University.

Figure II.2.1.10. A possible UV treatment simulation with ReaxFF. A system of 6 aliphatic dicycloepoxide molecules was consider without (a) and with (b) bond-boost applied to carbonepoxy oxygen bond. Source: Pennsylvania State University.

Figure II.2.1.11. (top) Visual representations of large-scale MD simulations of the effect of graphene sheet and (bottom) the accompanying distribution of ring normals. (a) An isolated graphene sheet represents maximum degree of alignment. (b) A system with graphene sheet obtains a high degree of alignment. (c) A system without graphene sheet maintains random alignment of polymers. Source: University of Virginia.

Figure II.2.1.12. Computational approach for identifying structure/mechanical properties relationships: (a) 3D grid of rectangular cuboids for a simulated microstructure; (b) local stress distribution; (c) local strain distribution; and (d) correlation between local strain and degree of graphitization at different global strain levels marked on the panel. The contour plots shown in (b) and (c) corresponds to the global strain level of 0.04. Source: University of Virginia.

Figure II.2.1.13. Results of the atomistic simulations performed for a large (e.g., 3.8 million atoms) system representing CF microstructure. $(a, b)$ Snapshots of the 3D microstructure, where all carbon atoms are colored green in (a), while the graphitic phase is shown by blue in (b). (c) Void size distribution. (d) Stress-strain curves from ReaxFF tensile testing simulations performed for systems of two sizes. Source: University of Virginia.

Figure II.2.1.14. (a) The preparation procedure for nylon CF developed at ORNL. (b, c) Micrographs of produced CF. Source: ORNL.

Figure II.2.1.15. Experimental testing with nylon fibers demonstrate the effect of: (a) graphene content; and (b) spinning nozzle diameter. (c) Raman spectra of fibers after spinning, stabilization, and carbonization. Source: ORNL.

Figure II.2.2.1. Organization of the Consortium for Production of Affordable CFs in the United States. Source: WRI.

Figure II.2.2.2. Cross-polarized optical-microscopy micrographs for CTP6 under different reaction conditions (above the micrograph) to produce increasing mesophase content and SP (below the micrograph in green font). Source: WRI.

Figure II.2.2.3. Scanning electron micrographs of the well-stabilized CTPC mesophase CF at: (a) $10.2 \mathrm{~mm} \times$ 4.50k magnification; and (b) $8.7 \mathrm{~mm} \times 3.00 \mathrm{~K}$ magnification. Source: ORNL.

Figure II.2.2.4. Scanning electron micrographs of various fiber defects from an interfilament fusing: (a) at $10.0 \mu \mathrm{m}$; and (b) at $30.0 \mu \mathrm{m}$. (c) Poor stabilization is seen at $20.0 \mu \mathrm{m}$.

Source: ORNL. 296

Figure II.2.2.5. Testing protocol developed by UW for tow-level resin composites and feedback loops that will be used for FEA and other modeling efforts. Source: UW. 
Figure II.2.2.6. Heat map showing positive and negative correlations according to the Pearson method for CF mechanical properties and chemical, compositional, and physical characterization of the corresponding isotropic and mesophase pitch. Source: UW.

Figure II.2.2.7. The targeted approach that is being applied to data to generate ML predictive models using chemical and physical insights through correlation analysis. Source: MIT.

Figure II.2.2.8. Correlations between various chemical and physical properties of isotropic and mesophase pitch. Source: WRI.

Figure II.2.2.9. First principles energetic calculations based on hydrogen abstraction acetylene addition mechanism. (a) A hypothesized pathway for indeno[1,2,3-cd]pyrene [1] is assessed regarding both (b) energetics and minimum energy path. (c) The automatic enumeration of all possible pathways for indeno[1,2,3-cd]pyrene and (d) polynucleus condensation mechanism for pyrene dimerization and trimerization. (e-f) HOMO and total charge density of pyrene dimer. Source: MIT.

Figure II.2.2.10. Coarse-grained approach used to model the chemical and structural evolution of bio-ACN into bio-PAN-based fibers. Source: MIT.

Figure II.2.3.1. Systems approach for reducing weight in complex automotive structures including the use of FE tools for detailed design. Source: University of Delaware ............................................305

Figure II.2.3.2. Integrated predictive engineering environment. Source: University of Delaware..... 306 Figure II.2.3.3. Composites allow part consolidation, further reduces cost and weight. Source: Composites World [1]. 307

Figure II.2.3.4. Preform materials types: (a) chain stitch; (b) tricot stitch; and (c) braided broadgood fabric. Source: TPI Composites.

Figure II.2.3.5. (a) Bending stiffness measurement apparatus. (b) An example image used for measuring curvature and the extent of bending. Source: TPI Composites.

Figure II.2.3.6. Nonlinear shear behavior used in draping modeling. Source: OEM Partner.

Figure II.2.3.7. Comparison of draping experiment and simulation for the preform 1.

Source: OEM Partner.

Figure II.2.3.8. Comparison of draping experiment and simulation for preform 2.

Source: OEM Partner.

Figure II.2.3.9. Single hat section beam used for the study. Source: OEM Partner.........................313

Figure II.2.3.10. Selected design points for conducting flexural tests. Source: OEM Partner.....

Figure II.2.3.11. Experimental three-point bend test results for the two beam types: (a) average force-displacement trends; and (b) damage pattern representatives. Source: OEM Partner.

Figure II.2.3.12. Comparison of the flexural test and the simulation result for Beam D: (a) loaddisplacement trend; and (b) damage pattern. Source: OEM Partner.

Figure II.2.3.13. Comparison of the flexural test and the simulation result for the Beam $\mathrm{H}$ :

(a) load-displacement trend; and (b) damage pattern. Source: OEM Partner.

Figure II.2.3.14. Al preform tool with the silicone vacuum bag in (a) the open position and (b) the closed position. Source: TPI Composites. 
Figure II.2.3.15. Preform fabrication (a) on the mold and (b) after consolidation and trimming. Source: TPI Composites.

Figure II.2.3.16. Preform breakup (a) for the door inner (b). Source: TPI Composites.

Figure II.2.3.17. The Fraunhofer facility. Source: Fraunhofer Project Center.

Figure II.2.3.18. HP-RTM molded intrusion beam hats. Source: TPI Composites.

Figure II.2.3.19. The mold cavity for the HP-RTM intrusion beam hat. Source: TPI Composites....... 321

Figure II.2.3.20. Dry area. Source: TPI Composites.

Figure II.2.3.21. Sliding preform at the mirror mount showing: (a) the resin-rich area; and (b) the preform out of position. Source: TPI Composites.

Figure II.2.3.22. Preform joint designs for: (a) the lap-joint for the new preforms; and (b) the tabjoint for the old preforms. Source: TPI Composites.

Figure II.2.3.23. Part wrinkling in the NCF preform. Source: TPI Composites.

Figure II.2.3.24. Door mass breakdown. Source: TPI Composites.

Figure II.2.3.25. Breakdown for: (a) the baseline door cost; and (b) the baseline door mass. Source: TPI Composites.

Figure II.2.4.1. Door concept comparison: (a) composite door with older OPS design; (b) newer composite door design with OPS version 1; (c) newer composite door design with OPS version 2; and (d) final composite door design with simplified inner panel geometry and OPS version 2.

Source: Clemson University.

Figure II.2.4.2. (a) Latest door design; (b) new ply layup with mixed woven and UD plies; and (c) shape of bottom four woven plies. Source: Clemson University.

Figure II.2.4.3. Thickness distribution obtained after static optimization under linear load cases for the door components: (a) inner panel and (b) IBS. Source: Clemson University.

Figure II.2.4.4. Thickness distribution of the door components: (a) inner panel; and (b) IBS, which satisfies both linear and nonlinear load cases. Source: Clemson University.

Figure II.2.4.5. Reaction force plot for the QSP test comparing the baseline door with the composite door. Source: Clemson University......

Figure II.2.4.6. Subcomponent tool for hat production. Source: University of Delaware.

Figure II.2.4.7. Process conditions used to fabricate subcomponent.

Source: University of Delaware.

Figure II.2.4.8. (a) Fabricated spine; (b) hat geometry tool; and (c) finished hat section.

Source: University of Delaware.

Figure II.2.4.9. Measurement points of: (a) hat; and (b) spine for thickness.

Source: University of Delaware.

Figure II.2.4.10. Completed subcomponent assembly after adhesive-bonding: (a) top view; and

(b) end view. Source: University of Delaware.

Figure II.2.4.11. Subcomponent configuration, boundary conditions, and simulation summary. 
Figure II.2.4.12. Predicted contact force over time for the simulation conditions in Figure II.2.4.13. Source: University of Delaware. 336

Figure II.2.4.13. Location of the bonded strain gauges on (a) the side and (b) bottom in order to record strain during impact. Source: University of Delaware.

Figure II.2.4.14. Subcomponent test progression showing the impactor and the subcomponent before the impact, at impact, and after the impact. Source: University of Delaware.

Figure II.2.4.15. (a) Comparison between the simulated and experimental results. (b) If loaded, the composite, as a whole, is able to absorb and withstand far higher loads.

Source: University of Delaware.

Figure II.2.4.16. Composite door manufacturing process. Source: Clemson University

Figure II.2.4.17. Fully assembled door frame on the existing vehicle. Source: Clemson University. 340 Figure II.2.4.18. Cost distribution of the individual parts of the door. Source: Clemson University.. 341 Figure II.2.4.19. Normal distribution of the total cost of the door. Source: Clemson University.

Figure II.2.5.1. CF composite rocker floor assembly built for the demonstration of ICME tools. Source: GM.

Figure II.2.5.2. Tool design of the reinforcement ( $P$ denotes pressure sensor location; $C$ denotes dielectric sensors). Source: GM.

Figure II.2.5.3. Determining the 2D-flat blank for the reinforcement component. Source: GM 348

Figure II.2.5.4. Reinforcement preforms (Pattern 1 in blue, Pattern 2 in green, and the flange patches in brown and dark blue). Source: GM.

Figure II.2.5.5. The six preform 2D blank patterns used to produce the reinforcement. Source: GM.

Figure II.2.5.6. Adjustable preforming frame with clamps. Source: GM.

Figure II.2.5.7. Preform patterns after forming with measurement lines: (a) middle pattern 1;

(b) middle pattern 2; (c) side pattern 1; and (d) side pattern 2. Source: GM.

Figure II.2.5.8. Draw-in results for the middle patterns: (a) middle pattern 1; and (b) middle pattern 2. Source: GM.

Figure II.2.5.9. Press and metering cycle timesteps control. Source: GM.

Figure II.2.5.10. HP-RTM metering system installed at GM R\&D for the project. Source: GM.

Figure II.2.5.11. (a) Molded reinforcement panel. (b) Process parameters including injection pressures and flow rates where in-mold pressures were measured from the pressure sensors. Source: GM.

Figure II.2.5.12. In-mold temperature and log ion viscosity at C1, C2, and C3. Source: GM.

Figure II.2.5.13. Comparison of pressure profile between the experiment and the numerical prediction. Source: GM.

Figure II.2.5.14. (a) Critical areas during single pole-side impact crash (red). (b) Locations of slits (green and blue) in non-critical areas. Source: GM.

Figure II.2.5.15. Coats preforming patterns. Source: GM. 358 
Figure II.2.5.16. Coats 3D preform. Source: GM. 358

Figure II.2.5.17. Lattice ${ }^{\mathrm{TM}}$ preform with carbon and glass fibers selectively placed: (a) CAD generated and (b) finished product. Source: GM. 359

Figure II.2.5.18. Reinforcement panel molded with carbon and glass eliminating the scrap. Source: GM. 359

Figure II.2.5.19. Floor upper tool port and sensor locations. Source: GM. 360

Figure II.2.5.20. Floor preforming patterns for the main blank (top) and large patch (bottom). Source: GM. 360

Figure II.2.5.21. Floor preforming pattern for the main blank, draped on the tool. Source: GM....... 361

Figure II.2.5.22. Example of an untrimmed floor. Source: GM. 361

Figure II.2.5.23. Viewgraph of pole-side impact simulation at different timesteps (in milliseconds). Source: GM...

Figure II.2.5.24. Prediction for the force-displacement results. Source: GM. 362

Figure II.2.5.25. Experimental stress-strain curves from (a) bending and (b) tensile tests. Source: GM 364

Figure II.2.5.26. Eigenvalues of diffusion. Source: GM. 365

Figure II.2.5.27. Training sets (a) and augmented sets (b) for bending (top) and tensile (bottom) experiments. Source: GM.

Figure II.2.5.28. PDF of the (a) flexural and (b) tensile stresses at different strain levels; leftmost curves are in the linear elastic regime while the rightmost curves are in the inelastic regime.

Source: GM.

Figure II.2.5.29. PDF of stress at specified strain levels when conditioned on specific values of fiber properties or tow geometry. The legend on the right side of each graphic shows the conditioning state, while the legend at the top of each graphic indicates the quantity of interest being plotted. Source: GM.

Figure II.2.5.30. PDF of fiber modulus (a) when conditioned on values of stress at strain levels or (b) when conditioned on the full experimental stress-strain curve. Source: GM.

Figure II.2.6.1. Proposed initial subframe design at 30\% weight save and additional cost of $\$ 4.01$ per pound saved material distribution. Source: Ford Motor Company.

Figure II.2.7.1. (a) Successful impregnation of CF/UHMWPE hybrid-fiber. (b) Successful demonstration of compression-forming precured vitrimer-matrix parts with complex curvature. Source: Mallinda.

Figure II.2.7.2. Application of C-scan ultrasonic analysis to vitrimer-matrix composites. (a) Though visibly similar, the difference in consolidation conditions can be clearly seen in (b) the C-scan images. Source: PNNL.

Figure II.2.7.3. Application of split-Hopkinson bar (Kolsky bar) testing to vitrimer-matrix composites for (a) tension configuration and (b) compression configuration. Source: SNL. 381

Figure II.2.7.4. Dynamic tensile stress-strain curves at three test rates. Source: SNL 382 
Figure II.2.7.5. Dynamic compressive stress-strain curves in (a) the out-of-plane and (b) the inplane directions. Source: SNL

Figure II.2.8.1. Customized multi-material AM system and fabricated samples. (a) Schematic of multi-material projection micro-stereolithography process integrated with the tape-casting method. (b) Schematic of the recoating process, depicting the behavior of the viscous resin during the process. (c)-(d) Complex 3D structures fabricated by the system. (e) Multi-material octet-truss unit cell complying with CFRP and polyethylene glycol diacrylate resin. (f) Closed cell made of CFRP in a large-scale. (g-h) Arbitrary gyroid 3D structure with a minimum feature of 150 $\mu \mathrm{m}$. Source: Virginia Tech.

Figure II.2.8.2. The effect of soft phase on the bulk CFRP stiffness-damping properties.

(a) Stiffness $(E)$ versus loss tangent $(\tan \delta)$ for various two-phase configurations incorporating CFRP and soft phase of bulk material. The percentage shown here corresponds to the volume fraction of the stiff phase. Graphical legends are provided on the bottom of this figure.

(b) $E^{1 / 3} \tan \delta / \rho$ versus the volume fraction of the soft phase $\left(V_{2}\right)$. This index reaches its maximum at $V_{2}=0.9 \%$. Source: Virginia Tech.

Figure II.2.8.3. Multi-material architecture. (a) Design of lightweight, stiff, high-damping microlattice with two-phase materials incorporating CFRP and soft phase. (b) Fabricated lightweight cellular CFRP microlattice having $\rho=7 \%$ with $V_{\text {soft }}=9 \%$. (c) SEM image showing the interface between the two phases. Source: Virginia Tech.

Figure II.2.8.4. Intrinsic damping properties of the lightweight cellular CFRP microlattice having a relative density of $7 \%$ with different soft phase ratios. (a) The effective modulus $(E)$ as a function of the soft phase ratio $V_{\text {soft. }}(b)$ Loss tangent (tan $\delta$ ) as a function of $V_{\text {soft. }}$ (c) Damping FOM as a function of $V_{\text {soft. }}$ Solid lines in these figures represent the curve fit. Source: Virginia Tech.

Figure II.2.8.5. Structural damping properties of the lightweight cellular CFRP microlattice obtained from large-strain quasi-static cyclic compression tests. (a)-(b) Hysteresis loops from multicyclic compression tests for 30 cycles and evolution of the loss coefficient with the cycle number. (a) Sample with $\rho=4 \%$ having $V_{\text {soft }}=20 \%$. (b) Sample with $\rho=12 \%$ having $V_{\text {soft }}=20 \%$. (c) Normalized stress-strain hysteresis loops of samples having $V_{\text {soft }}=20 \%$ with various $\rho$. Elastic buckling behavior was observed for $\rho=4 \%$ and diminished with an increase in $\rho$. (d) The effective modulus $(E)$ as a function of $V_{\text {soft. }}$ The modulus was inversely proportional to an increase in $V_{\text {soft, }}$ showing its asymptotic value. (e) Loss tangent (tan $\delta$ ) as a function of $V_{\text {soft. }}$ An increase in $V_{\text {soft }}$ led to an improvement in loss tangent. (f) Damping FOM as a function of $V_{\text {soft. }}$ FOM can be tuned to have a peak when $V_{\text {soft }}$ is approximately $10 \%$. Source: Virginia Tech.

Figure II.2.8.6. Tunability maps for: (a) intrinsic; and (b) structural damping performance in terms of the damping FOM obtained from the experimental measurements. Source: Virginia Tech. 390

Figure II.2.8.7. Assessment of the lightweight cellular CFRP microlattice developed in this work. The orange ellipse represents the envelope of experimental results for an overall damping property. The CFRP microlattice exhibits similar stiffness as commercially available CFRP composites while providing high-damping as like that of elastomers. (Image is created using CES Granta [7]) The property of the lightweight microlattice (in orange color) is from our experimental results. All the other material properties are from CES Granta library. Source: Virginia Tech. 390

Figure II.2.9.1. (a) Spooled samples of $100 \%$ bacterially produced spider silk fiber; and (b) comparative photos of the natural (white) and transgenic silkworm silk (green). Source: USU. . 395

Figure II.2.9.2. Spinning equipment used at USU for the filament extrusion. Source: USU. 395 
Figure II.2.9.3. (a) Two examples of isolated MaSp1 filament by optical-microscopy. These two pictures highlight the irregularity of the filament cross-sections. (b) Observation of the crosssection of a MaSp1 non-degummed strand before conversion. The filaments were fused during the spinning phase. Source: ORNL.

Figure II.2.9.4. The typical failure mode observed during the conversion process is shown in the red dotted frame with the failure in the processed material on the right and the carrier material on the left. Source: ORNL.

Figure II.2.10.1. Tensile Strength of Textile CF. Source: ORNL...................................................... 401

Figure II.2.10.2. Tensile Modulus of Textile CF. Source: ORNL....................................................... 401

Figure II.2.10.3. (a) Old packaging method that was bulky and time-consuming; and (b) new simplified packing configuration. Source: ORNL.............................................................................. 402

Figure II.2.10.4. Paperless packaging system for downstream processing. Source: ORNL. ............ 403

Figure II.2.10.5. (a) Textile CF on cardboard cores. (b) Textile CF on plastic cores. Source: ORNL. . 403

Figure II.2.10.6. Flexural strength of TCF in various resin systems. Source: IACMI. ....................... 404

Figure II.2.10.7. Flexural modulus of TCF in various resin systems. Source: IACMI......................... 404

Figure II.2.10.8. Strongwell pultrusion equipment at the CFTF. Source: ORNL............................... 405

Figure II.2.10.9. (a) Textile CF wet out resin being pulled through the pultruded process.

(b) Pultruded textile CF being pulled through the pultruded process to remove excess resin.

Source: ORNL. .....

Figure II.2.11.1. An example of a virtual network analyzer measurement of one port of the vessel. Two bands are showing a low reflection $(<30 \mathrm{~dB})$, ensuring an effective power transfer at these frequencies. Data generated at 4XTechnologies.

Figure II.2.11.2. A bundle of several hundred of grey filaments between $10 \mathrm{~mm}$ and $120 \mathrm{~mm}$ was found among the 8 tows of the static batch. Its total length was measured to ca. 8 in.

Source: 4XTechnologies.

Figure II.2.11.3. By using a regular multimeter, the fiber can be measured to be conductive in the area of interest, which confirms that the material has been carbonized. Source: 4XTechnologies. 412

Figure II.2.11.4. TGA characterization for the OPF conversion. Sample analyzed at ORNL.

Figure II.3.1.1. Joint samples: (a) RSW, uncoated samples and E-coated samples; (b) SPR; and (c) R-W. Source: University of Michigan.

Figure II.3.1.2. Cross-sections of an: (a) uncoated RSW joint; and (b) uncoated SPR joint.

Source: Penn State University.

Figure II.3.1.3. Corroded samples (a) RSW; (b) SPR joints uncoated after lap-shear tests; and (c) lap-shear results. Source: University of Michigan.

Figure II.3.1.4. SPR joints uncoated with corrosion after (a) 1 cycle and (b) 26 cycles. Source: University of Michigan.

Figure II.3.1.5. DFT calculated phase stability of Al-Fe IMCs at O K. Red lines indicate convex hull. Source: Penn State University. 419 
Figure II.3.1.6. Equilibrium AI-Fe phase diagram by CALPHAD modeling and the metastable phase region (Liquid+BCC/B2, the dotted \& dashed lines). Source: Penn State University.....

Figure II.3.1.7. Scheil simulations for $(\mathrm{a}) \mathrm{X}_{\mathrm{Fe}}=0.3$ and 0.6 and $(\mathrm{b})$ non-equilibrium Al-Fe phase diagram based on Scheil simulations. Source: Penn State University.

Figure II.3.1.8. (a) Crevice localized at the interface between the rivet and top sheet in a SPR joint. (b) 2D FE model of galvanic/crevice corrosion. (c) Electrolyte potential change contours and structural deformation during the corrosion evolution process.

Source: University of Illinois Urbana-Champaign.

Figure II.3.1.9. (a) The 3D visualization of crevice corrosion schematic in the FE model.

(b) Electrolyte potential change contours along the anode (Al) surface.

Source: University of Illinois Urbana-Champaign.

Figure II.3.1.10. Scheme of the user-defined material interface in LS-DYNA.

Source: Livermore Software Technology Corporation.

Figure II.3.2.1. Al-Mg sheet diffusion-bonding photos: (a) argon tube furnace; (b) manually debonded sample; (c) screw-driven fixture providing compression force; and (d) diffusion-bonded piece. Source: PNNL.

Figure II.3.2.2. (a) Polished samples submerged in mineral oil ready for diffusion-bonding. (b) $\mathrm{Mg}$ and Al samples loaded in the fixture for diffusion-bonding. Source: PNNL.

Figure II.3.2.3. FSW weld development approach. Source: PNNL.

Figure II.3.2.4. (a) Weld geometry (top) and preliminary optical characterization with the length direction from left to right, and (b) lap-shear test results. Source: PNNL.

Figure II.3.2.5. Optical micrograph of $\mathrm{Mg} / \mathrm{Al}$ joint cross-section: (a) in the middle of the weld line; and (b) at the exit hole. Source: PNNL.

Figure II.3.2.6. Load as a function of extension for Mg-Al joints showing progress in load-bearing capacity. Note the variation in joint strength from 115-180N/mm. Source: PNNL.

Figure II.3.2.7. (a) Gamry paracell kit; and (b) Reference 600 potentiostat. Source: Gamry Instruments.

Figure II.3.2.8. Linear polarization test graphs for Solution 1: (a) Al and (b) Mg; and Solution 2: (c) Al and (d) Mg. Source: WPI.

Figure II.3.2.9. (a) WeiCe CCT equipment; and (b) corrosion chamber with samples. Source: WPI.. 429

Figure II.3.2.10. (a) Al sheet EBSD inverse pole figure map showing the [001] texturing. (b) The pole figure of the Al sheet is typical of textured materials. Source: ORNL

Figure II.3.2.11. SEM-BSE micrograph of as-received Mg sheet. Source: ORNL

Figure II.3.2.12. (a) SEM-BSE micrograph of as-received Al sheet; and (b) phase map of asreceived Al sheet. Source: ORNL.

Figure II.3.2.13. SEM images of pure Al and Mg sheet diffusion-bonded samples, from left to right: $430^{\circ} \mathrm{C}$ for two hours; $400-425^{\circ} \mathrm{C}$ for two hours; $400-430^{\circ} \mathrm{C}$ for two hours; and $390-$ $410^{\circ} \mathrm{C}$. Note that none of the interfaces were adherent. Source: ORNL.

Figure II.3.2.14. X-ray maps of pure $\mathrm{Mg}$ and Al sheet diffusion-bonded samples.

Source: ORNL/WPI. 
Figure II.3.2.15. The evolution of pit morphology is shown for: (a) a single grain metal; and (b) for a polycrystalline metal. Source: [1]

Figure II.3.2.16. PF model of IMC layer growth in diffusion-bonding for (a) the initial condition and (b) after significant growth. The scale bar on the right shows Mg mole fraction. Note the faster growth of $\mathrm{Al}_{3} \mathrm{Mg}_{2}$ (grey) than $\mathrm{Al}_{43} \mathrm{Mg}_{57}$ (pink) as seen in the experiments. Source: WPI.

Figure II.3.2.17. Al-Mg binary free energy function used in the PF model of diffusion-bonding. Source: WPI.

Figure II.3.3.1. Inelastic effects in silicon adhesives.

Source: MSU High Performance Materials Group.....

Figure II.3.3.2. Comparison of model against (a) UT tests after 1, 1000, and 2000 cycles and (b) stress-relaxation over the number of loading cycles [1].

Source: MSU High Performance Materials Group.

Figure II.3.3.3. Comparison of model to measured constitutive behavior for: (a) failure of DC Dowsil 7091 silicone adhesive aged for 30 days at $95^{\circ} \mathrm{C}$; (b) cyclic behavior of DC Dowsil 7091 silicone adhesive aged for 10 days at $80^{\circ} \mathrm{C}$; (c) failure for $3 \mathrm{M}-590$ polyurethane aged for various times at $95^{\circ} \mathrm{C}$; and (d) cyclic behavior of $3 \mathrm{M}-590$ polyurethane aged for 30 days at $95^{\circ} \mathrm{C}$. Source: MSU High Performance Materials Group...

Figure II.3.3.4. Constitutive behavior of UV test samples for (a) Dow Corning adhesive, (b) black polyurethane adhesive, (c) acrylic, and green polyurethane.

Source: MSU High Performance Materials Group.

Figure II.3.3.5. SBR sample constitutive behavior at various aging times shown in: (a) cyclic tests performed at $60^{\circ} \mathrm{C}$ and (b) at failure of the adhesive.

Source: MSU High Performance Materials Group.

Figure II.3.3.6. (a) Shape functions $(\mathrm{N})$ as a function of time and temperature; and (b) the reconstructed surface of $N(t, T)$. Source: MSU High Performance Materials Group.

Figure II.3.3.7. Model validation for aging of control compound SBR in multiple conditions. Constitutive behavior for samples aged for 6 days at: (a) $60^{\circ} \mathrm{C}$ and (b) $80^{\circ} \mathrm{C}$ and those aged for 10 days at (c) $60^{\circ} \mathrm{C}$ and (d) $80^{\circ} \mathrm{C}$. Source: MSU High Performance Materials Group.

Figure II.3.4.1. Illustrations of: (a) the lap-joint showing the dimple length (L) and width (W), which varied; and (b) the VFAW layup with the interlayer. Source: OSU.

Figure II.3.4.2. Illustrations of the two-shot VFAW: (a) the first shot welds the flat interlayer to the deformed target; and (b) the second shot welds the flyer to the welded flyer-target stack-up. Source: OSU.

Figure II.3.4.3. Welds of two directions: (a) the length of the weld is perpendicular to the length of the sample; and (b) the length of the weld is parallel to the length of the sample. Source: OSU..... 448

Figure II.3.4.4. (a) Pre-strained samples and (b) pre-strained and deformedsamples. The prestrain increases from left to right as labeled. Source: OSU.

Figure II.3.4.5. Fatigue matrix of 4-mm-thick AA5754-0 to 2.2-mm-thick HSLA 340 with the single-shot method. Source: OSU. 
Figure II.3.4.6. Welds of $4 \mathrm{~mm}$ AA5052-HSLA 340 using the two-shot method created with the pre-prototype welding head \#1: (a) results of lap-shear tests; (b) a weld of interlayer to target after first shot; and (c) a weld created using the two-shot method. Source: OSU.

Figure II.3.4.7. Lap-shear tests of welds of $4 \mathrm{~mm}$ AA5052-HSLA 340 using: (a) the two-shot method; and (b) the single-shot method created with pre-prototype welding head \#2.

Source: OSU.

Figure II.3.4.8. Lap-shear tests of (a) a weld of with $4.5 \mathrm{~mm}$ AA5182-HSLA 340 in the perpendicular direction; (b) welds of $5.1 \mathrm{~mm}$ hot-rolled AA5052-HSLA 340 in both directions; and (c) a photo of the front and back sides of an AA5182-HSLA 340 weld in the perpendicular direction. Source: OSU.

Figure II.3.4.9. Upgrade design of subframe: (a) frontside; and (b) backside. Source: Magna 452

Figure II.3.4.10. (a) FLCA locations and (b) results of modeling of extreme loading condition. Source: Magna.

Figure II.3.4.11. (a) A pre-prototype system and (b) a closer view of the pre-prototype welding head \#1. Source: OSU.

Figure II.3.4.12. Pre-prototype welding head \#2 (a) top and (b) front. Source: OSU

Figure II.3.4.13. (a) Weld head mounted on a 6-axis robot. (b) VFAW head in configuration with lower initial gap between anvil and breech for welding on the outside flange of the prototype component. (c) Close-up view of the weld head shown in closed position with the foil in place. Source: Coldwater Machine.

Figure II.3.4.14. Fully automated work cell with all equipment installed. Source: OSU.

Figure II.3.5.1. Potentiodynamic polarization of primed (100, 50), unprimed CFRP (Twill, Random), and $\mathrm{AA}(6111,6022)$ in 5 wt.\% aerated $\mathrm{NaCl}$ at $27^{\circ} \mathrm{C}$ (May 2018 results for comparison). Source: The Ohio State University.

Figure II.3.5.2. Potentiodynamic polarization of (a) unprimed, primed $(0,50,100)$ CFRP-Twill and (b) CFRP- Random and AA $(6111,6022)$ in $3.5 \mathrm{wt} . \%$ aerated $\mathrm{NaCl}$ at $27^{\circ} \mathrm{C}$.

Source: The Ohio State University....

Figure II.3.5.3. Effective stress intensity as a function of effective crack extension for slow strainrate testing at crosshead displacement rate of $4.50 \times 10-8 \mathrm{in} / \mathrm{sec}$. A PS plastic zone size correction was applied to calculate effective values, and full immersion SCC tests are plotted with an in-air test for comparison to "no-environment" cracking behavior.

Source: The Ohio State University......

Figure II.3.5.4. Plot of threshold stress intensity $\left(\mathrm{K}_{T H}\right)$ as a function of applied polarization potential for rising displacement SCC testing of AA6111-T8 in $3.5 \mathrm{wt} . \% \mathrm{NaCl}$ using ASTM E1820 and ASTM \#561. (a) $\mathrm{K}_{T H}$ results at displacement rates of $4.50 \times 10^{-8} \mathrm{in} / \mathrm{sec}$ and $1.05 \times 10^{-6}$ in/sec as a function of polarization potential. (b) $\mathrm{K}_{\mathrm{TH}}$ results at displacement rate of $4.50 \times 10^{-8}$ in/sec as a function of polarization potential. Source: The Ohio State University.

Figure II.3.5.5. Storage modulus (representative of $\mathrm{Tg}$ ) and $\tan \delta$ as a function of temperature for two developmental adhesives. Source: PPG Industries, Inc. 464 
Figure II.3.5.6. (a) Lap-shear (b) and t-peel results comparing the control formula baked at $175^{\circ} \mathrm{C}$ with a few prototype formulations baked at $150^{\circ} \mathrm{C}$ and showing progress in optimizing anticorrosion properties for low cure adhesive systems. Source: PPG Industries, Inc.

Figure II.3.5.7. Long-beam CFRP-Al adhesive specimens made from high (top sample) and low temperature (bottom sample) bake adhesives with a comparison of the deflection for structural vs. prototype adhesives. Source: Ford Motor Company.

Figure II.3.5.8. Accelerated corrosion coupon geometry and adhesive placement conditions. Source: Ford Motor Company.

Figure II.3.5.9. (a) \& (b) Prototype PPG adhesive showing excellent adhesion and toughness between Al and CFRP at (a) a bond gap of $0.25 \mathrm{~mm}$ and (b) a bond gap of $1.25 \mathrm{~mm}$.

Source: Ford Motor Company...

Figure II.3.5.10. Laser scanning output of liftgates (a) manufactured with a low-temperature cure E-coat and flexible adhesive, and (b) control process and materials. Maximum deflection is approximately 50\% less with a low-temperature process. Source: Ford Motor Company.

Figure II.3.6.1. Examples of sample placement used for exposure orientation assessment. Source: OSU.

Figure II.3.6.2. 7055 Load-bearing capacity comparison. (a) Load per unit weld width plotted against measured extension during lap-shear testing of two [7055 (2 mm)-7055 (2 mm] and three sheets [7055 (2 mm)-7055 (2 mm)-6022 (1 mm)] FSLW joints. Tensile test results for base 7055 sheet and bead-on-plate welds are also shown for reference. The dotted line indicates the minimum load-bearing capacity required for RSW for $15 \mathrm{~mm}$ spacing as per AWS D17.2. (b) A representative joint cross-section for a 3 sheet joint. Full-field hardness color map showing HAZ minimum hardness regions around the weld nugget. Source: PNNL.

Figure II.3.6.3. Bar chart representing the average load-bearing capacity observed in three sheet FSLW for three types of alloys considered. The corresponding welding speeds and $Z$ forces are also shown. Standard deviations with three samples in each case are also shown. In all cases, the target set by HRA was met. Source: PNNL.

Figure II.3.6.4. Several types of fracture modes observed in three sheet FSLW. Results are shown for whole welds samples for each case with a 30-mm weld length. Source: PNNL.

Figure II.3.6.5. Load per unit weld length plotted against displacement for T-peel FSLW samples for the three alloys layup. An example of a fractured sample is shown for each fracture mode for the 6111-6111-6022 set. Note that the start and stop of the weld is within the tested joint. The total weld length in each case was $30 \mathrm{~mm}$. Source: PNNL

Figure II.3.6.6. Clockwise from bottom left: (a) KS2 sample testing fixture holding a KS2 sample; (b) KS2 welding fixture with KS2 sample clamped; (c) a top view of KS2 sample; (d) a side view of tested fractured KS2 (7055-7055-6022); (e) a top view of fractured KS2 (7055-7055-6022). Source: PNNL.

Figure II.3.6.7. (a) A CAD of demonstration hat section atop of fixture with desired clamping scheme. Source: HRA. (b) A side view of axial crush sample loaded on the hat section fixture. Source: PNNL. (c) Hat sections clamped down on fixture ready to be welded. Source: PNNL. (d) FSLW setup sketch illustrating welding configuration for axial crush sample. (e) An example of a welded axial crush sample with three weld lines on each side. Source: PNNL. (f) FSLW setup sketch illustrating welding configuration for the 3-point bend sample. Source: HRA. 
Figure II.3.7.1. Examples of sample placement used for exposure orientation assessment.

Source: OSU. 480

Figure II.3.7.2. Surface corrosion location definitions. Source: OSU.

Figure II.3.7.3. Maximum sustained load of samples joined by SPR, RSR, or FDS after ASTM G85A2 exposure. Source: OSU.

Figure II.3.7.4. Cross-section data of AA7055-T76 joined to 980 steel via RSR orientation after 21 days of ASTM G85-A2 exposure: (a) RSR exposed up and (b) RSR exposed down.

Source: OSU. 482

Figure II.3.7.5. Cross-section data of AA7055-T76 joined to 980 steel via FDS orientation after 21 days of ASTM G85-A2 exposure: (a) FDS exposed up and (b) FDS exposed down.

Source: OSU.

Figure II.3.7.6. Cross-section data of AA6013-T4 joined to 590 steel via RSR orientation after 21 days of ASTM G85-A2 exposure: (a) RSR exposed up and (b) RSR exposed down. Source: OSU..... 483 Figure II.3.7.7. Cross-section data of AA6013-T4 joined to 590 steel via SPR orientation after 21 days of ASTM G85-A2 exposure: (a) SPR exposed up and (b) SPR exposed down. Source: OSU. .... 483 Figure II.3.7.8. Cumulative probability of corrosion depths of AA7055 joined to 980 steel via RSR or FDS after 21 days ASTM G85-A2 exposure. Note: the data was fit to a line and symbols were added for legibility. The symbols do not represent a single data point collected. Source: OSU.

Figure II.3.7.9. Cross-section data of AA5754-0 joined to 590 steel via RSR after 32.4 days exposure: (a) ASTM B117; (b) ASTM G85-A2; and (c) CCT-1. Source: OSU. 485

Figure II.3.7.10. Cross-section data of AA6013-T4 joined to 980 steel via RSR after 32.4 days exposure: (a) ASTM B117; (b) ASTM G85-A2; and (c) CCT-1. Source: OSU.

Figure II.3.7.11. Cross-section data of Aural2 joined to 980 steel via RSR after 32.4 days exposure: (a) ASTM B117; (b) ASTM G85-A2; and (c) CCT-1. Source: OSU.

Figure II.3.7.12. Honda CCT results - TSS coupons (with Pilot Holes). Source: Honda.

Figure II.3.7.13. Honda CCT test results - CTS coupons (with Pilot Holes). Source: Honda. 487

Figure II.3.7.14. Honda CCT TSS Coupons (with pilot holes). Source: Honda.

Figure II.3.7.15. Production condition specimen examples (e.g., rivet offset, angularity, gap).

Source: Arconic.

Figure II.3.7.16. Effect of angularity for self-piloted (no hole) joints between 1.0-mm MMHF-T4 Al and two sheets of 1.2-mm 980-MPa steel. Source: Arconic.

Figure II.3.7.17. Effect of angularity for self-piloted (no hole) joints between 3-mm Aural2-T7 Al and two sheets of 1.2-mm 980-MPa steel. Source: Arconic.

Figure II.3.7.18. Effect of gap testing for self-piloted (no hole) joints between 1.0-mm MMHF-T4 $\mathrm{Al}$ and two sheets of 1.2-mm 980-MPa steel. Source: Arconic.

Figure II.3.7.19. Effect of gap testing for self-piloted (no hole) joints between 3-mm Aural2-T7 Al and two sheets of 1.2-mm 980-MPa steel. Source: Arconic.

Figure II.3.7.20. Double hat assembly used to demonstrate multi-axis RSR robotic capability. Source: Arconic. 
Figure II.3.7.21. Demonstration assembly process with the first step to design maturation.

Source: Honda. 493

Figure II.3.7.22. Demonstration assembly manufacturing sequences. Source: Honda. 493

Figure II.3.8.1. Overall research plan. Source: ORNL. 496

Figure II.3.8.2. Summary of adhesive-bonding strength for DP980-DP980 (no-zinc) and DP980 (no-zinc)-TS-CFRP with different modulus of adhesives. Source: ORNL.

Figure II.3.8.3. (a) Shear-strain map from the micro-DIC image with the strain field during lapshear tensile testing and (b) the fractograph of the sample. Source: ORNL.

Figure II.3.8.4. Laser profilomerty in micrometers for (a) laser-surface-textured TP-CFRP-PPA and (b) AR TP-CFRP-PPA. Source: ORNL.

Figure II.3.8.5. Wetting envelopes for surface polished and laser-surface-textured PPA resin only, TP-CFRP-PPA, TS-CFRP, and DP980. Source: PNNL. 500

Figure II.3.8.6. Optical profilometry for: (a) AR-CFRP-PPA; (b) mechnically polished with $1 \mu \mathrm{m}$ alumina; (c) mechanically abraded with 320 grit; (d) example of tensile pull sample; and (e) tensile pull strength test values for surface roughened PPA resin only and TP-CFRP-PPA. Source: PNNL. 501

Figure II.3.8.7. Adhesive-bonding strength for DP980 (no-zinc)-TP-CFRP-PPA before and after laser-surface-textured on TP-CFRP-PPA. Source: PNNL 502

Figure II.3.9.1. (a) Representative load versus displacement curves obtained during nano indentation of the weld interface. (b) Snapshot of joint cross-section at various magnification shows nano indents at AZ31 weld nugget near the interface, IM layer at the interface, and steel near the interface. Source: PNNL.

Figure II.3.9.2. Comparison of (a) hardness and (b) elastic modulus calculated from nano indentation tests near the weld interface and base material. For IMC, the base represents the IMC layer found away from the weld region. Source: PNNL.

Figure II.3.9.3. SEM micrographs of a fractured Mg/steel joing. Images 1 and 3 are the two hook locations while 2 is the area in between the hooks. The $\mathrm{Zn} / \mathrm{Mg}$ eutectic is distinctly observed in elemental color mapping in the upper left micrograph. Source: PNNL.

Figure II.3.9.4. SEM fractography of the $\mathrm{Mg}$ and steel side on scribe joints made with bare steel. Source: PNNL.

Figure II.3.9.5. Progressively decreasing hook features $(350 \mu \mathrm{m}-60 \mu \mathrm{m})$ at the interface of $\mathrm{Mg} / \mathrm{steel}$ joints. Source: PNNL.

Figure II.3.9.6. Fracture load plotted against fracture extension for various Mg/steel joints made at 2 scribe swept radius. Pictures and sketch on the left shown two swept radius scribe tooling. Source: PNNL.

Figure II.3.9.7. SEM and EDX mapping of a USW sample made of AZ31B Mg alloy to Zn-coated DP590 steel. Source: ORNL.

Figure II.3.9.8. (a) Lap-shear USW samples of AZ31B and bare DP590 (top) and pure Mg and bare DP590 (bottom) and (b) results for the lap-shear strength. Source: ORNL 
Figure II.3.9.9. SEM and EDX mapping of a USW sample made of AZ31B Mg alloy to bare DP590 steel. Source: ORNL.

Figure II.3.9.10. SEM images of the joint interface of pure Mg-bare DP590 USW samples.

Source: ORNL.

Figure II.3.9.11. STEM image (left) and EDX mapping (right) of the joint interface of pure Mg-bare DP590 USW samples. Source: ORNL.

Figure II.3.10.1. Schematic of Mg (AZ31) and CFRC plates joined via (a) BoP configurations, and (b) FSI. (c) Top view of the Mg-CFRC BoP joint showing a defect-free surface. (d) Microstructure of the Mg-CFRC BoP joint showing minimal CFRC deflection and no defects. Source: PNNL.

Figure II.3.10.2. Top view (a) and cross-section view (b) of Mg-CFRC joint made using FSI; SEM image (c) of the Mg-CFRC interface. Source: PNNL.

Figure II.3.10.3. (a) Top and (b) bottom view of Mg-CFRC joint made using FSI; (c) fractures MgCFRC sample post lap-shear testing and (d) load per sample width as a function of sample extension depicting the lap-shear behavior of the multiple Mg-CFRC FSI joints manufactured with

4 degrees tool tilt angle (legend labels indicate sample names). Source: PNNL.

Figure II.3.10.4. Schematic of immersion corrosion testing of bolted thermoset CFRC-AZ31B joint. Source: ORNL.

Figure II.3.10.5. Optical images of post-corroded lap-shear samples for: (a) the baseline control; (b) isolated for the three-step process; and (c) isolated plus the coating with beeswax.

Source: ORNL.

Figure II.3.10.6. Optical images of post-corroded lap-shear samples with different conditions. (a) Side view of selective exposure areas at the FSPR joint, and (b) and (c) backside view of the FSPR joint displaying progressive corrosion. Source: ORNL.

Figure II.3.10.7. Load versus displacement curves from lap-shear testing for five weld-bonded samples. Source: ORNL.

Figure II.3.11.1. Prediction of heat generation for UW: (a) predicted temperature distribution and (b) comparison of predicted and measured temperature histories under different welding conditions. Source: ORNL.

Figure II.3.11.2. SEM and TEM micrograph showing the AZ31/DP590 impact weld result by VFAW. (a) SEM micrograph of the AZ31/DP590 impact weld sample cross-section. (b) Higher magnification SEM image of the corner region, which shows an interlayer between AZ31 and DP590. (c) HRTEM images of the interlayer and the interface between interlayer and DP590, abundant Fe particles are observed. (d) STEM-EDS image of chemical components of the interlayer. Source: ORNL.

Figure II.3.11.3. Eulerian FEM simulation results of the AZ31-DP590 impact welding process. (a) Model setup with applied initial boundary conditions. (b) AZ31 material flow ejected from the impact front, known as jetting, is formed as a result of the impact angle. (c, d) A simulation showing the corresponding temperature and velocity distributions, respectively. (e) the velocity distribution after the jet flow propagates to the flat region of impact interface. Source: ORNL. 
Figure II.3.11.4. MD simulation prediction of impact interface nanostructure evolution: (a) setup of MD simulation with applied initial boundary conditions; (b) at the begining (10 ps after) of the impact; (c) before the unloading; (d) after cooling for 0.6 ns to $2500 \mathrm{~K}$, segregation of $\mathrm{Mg} / \mathrm{Fe}$ atoms at the interface is observed; and (e) after cooling to RT, Fe atoms segreagated into particles in Mg matrix and lattice structure formed at interface. Source: ORNL.

Figure II.3.11.5. Roughness measurements of filed surfaces. (a) AZ31 and (b) DP590. Source: ORNL.

Figure II.3.11.6. Temperature by infrared measurement for USW with different surface conditions: (a) Peak temperature and (b) at center. Source: ORNL.

Figure II.3.11.7. Horn-anvil welding setup and results of Mg-Steel (uncoated) USW. (a) Sheet stack-up and dimension; (b) predicted temperature distribution in two-sided filed sheets; and (c) the effect of surface engineering on the temperature mark on the steel surface.

Source: ORNL. 531

Figure II.3.11.8. Influence of horn tip diameter on the heat generation for three different diameters. Source: ORNL.

Figure II.3.11.9. Numerical simulation of horn diameter effect with: (a) temperature distribution; and (b) pressure distribution. Source: ORNL.

Figure II.3.11.10. Comparison of USW using sharp-tooth sonotrode and flat-tooth sonotrode: (a) temperature; and (b) contact pressure. Source: ORNL 533

Figure II.3.11.11. (a) SEM image of a representative Mg-steel FSS joint cross-section with an overlay of interfacial geometry. FEM for the Mg-Steel lap-joint: (b) and (c) different material regions considered in the $\mathrm{Mg}$ and steel plates respectively. (d) Dimensions and FE mesh for the model. (e) Close-up of the FEM close to the interface where red line indicates region with cohesive interaction and yellow line indicates penalty contact region to avoid any penetration. Source: PNNL.

Figure II.3.11.12. (a) Deformed configuration of the Mg-steel joint tested in lap configuration showing the contour plot for von Mises stress. Highlighted regions indicate the most likely locations for failure. (b) Load-displacement curves obtained for different hook shapes. Source: PNNL.

Figure II.3.11.13. Schematics of the mechanical tests in: (a) cross-tension; (b) T-peel; and (c) U-peel configurations to determine the interfacial parameters for a dissimilar friction stir welded joint. Source: PNNL.

Figure II.3.11.14. (a) Contour plot showing the maximum principal stress (MPa) in the welded plates loaded in the T-peel configuration. (b) Load-displacement behavior obtained through the experiments and FE simulation of the T-peel test. (c) Load-displacement behavior obtained through the experiments and FE simulation of the U-peel test. Source: PNNL.

Figure II.3.12.1. Comparison of gap measured by DIC and predicted numerical model at peakbake temperature. Source: ORNL.

Figure II.3.12.2. Predicted distortion with different weld pitch from $900 \mathrm{~mm}$ to $55 \mathrm{~mm}$ at peak paint-bake tempeature. Source: ORNL.

Figure II.3.12.3. (a) Predicted gap between Al and steel panel with different weld pitch from 900 $\mathrm{mm}$ to $55 \mathrm{~mm}$ at peak paint-bake tempeature. (b) After cool down to RT. Source: ORNL. 542 
Figure II.3.12.4. Finalized new FBJ machine design. Source: ORNL 543

Figure II.3.12.5. Finalized new FBJ machine and joining/assembly cell design. Source: ORNL. 544

Figure II.3.13.1. IR NDE methods used were (a) inline imaging, (b) off-line imaging using flash lamp pulsed auxiliary heating and (c) post-weld imaging using auxiliary induction heating.

Source: ORNL.

Figure II.3.13.2. Examples of steel, Al, and Al-steel weld cross-sections with nugget diameters highlighted by red arrows. Source: [1].

Figure II.3.13.3. Predicted diameters versus real measured diameters for the entire sample set, where measurements are denoted by the black dots and the expected trend is represented by the diagonal grey line, for (a) inline IR NDE, (b) off-line pulsed induction method and (c) off-line pulsed flash lamp. Source: ORNL.

Figure II.3.14.1. (a) Simplification of the geometry to produce (b) one domain for COMSOL. Source: PNNL. 553

Figure II.3.14.2. Selectively masked FSSW and USW joint specimens: (a) plan and (b) side view. The lower side of joints were remained unmasked with $1 \mathrm{~cm}$ length as shown in (b). (c) The schematic of exposed and corroding surfaces in a selectively masked joint and the resulting corrosion depth on Mg. Source: ORNL.

Figure II.3.14.3. Electrolyte potential profile (color surface) and current density distribution (arrows) using COMSOL Multiphysics ${ }^{\circledR}$ software for $\mathrm{Mg}$ on top, $1 \mathrm{~cm}$ sheet thickness, and $6 \mathrm{~cm}$ top and bottom lengths. Source: PNNL.

Figure II.3.14.4. Mg current distribution for selected cases where $I_{\text {top }}=$ l $_{\text {bottom. }}$ Source: PNNL. ........ 555

Figure II.3.14.5. Mg loss rate for selected cases where Itop $=$ I $_{\text {bottom. }}$ Source: PNNL......................... 555

Figure II.3.14.6. Peak Mg current density at selected steel lengths. Source: PNNL........................556

Figure II.3.14.7. Peak Mg current density for Mg on top and Steel on top at various thicknesses as a function of the ratio of bar lengths. Source: PNNL................................................................. 556

Figure II.3.14.8. (a) Optical microstructure taken after measurements showing the different locations where data was generated to produce the (b) Tafel and (c) Nyquist plots for the corresponding points marked. The starting measurement point was relatively large. Yellow circles represent individual grains and red circles are grain boundaries. Source: PNNL.

Figure II.3.14.9. Plan view of corroded (a) USW joint of Mg and bare steel; (b) USW joint of $\mathrm{Mg}$ and Zn-coated steel; and (c) FSSW joint of Mg and Zn-coated steel after $208 \mathrm{~h}$ immersion in 0.1 $\mathrm{M} \mathrm{NaCl}$. The related corrosion morphologies reconstructed by an image analysis software are shown in (d)-(f), respectively. Notable difference of $\mathrm{Mg}$ corrosion volume can be observed between the joints. Source: ORNL.

Figure II.3.14.10. (a) The maximum corrosion depths measured for the two sides of Mg (left- and right-half filled symbols for the left and right sides, respectively) and their average trends (dotted lines) as a function of time; and (b) the corrosion potentials of the joints measured during $0.1 \mathrm{M}$ $\mathrm{NaCl}$ immersion using saturated calomel reference electrode also as a function of time. Arbitrary trend lines are added for corrosion potential data in (b). Source: ORNL. 560 
Figure II.3.14.11. Multi-length scale imaging of post-corrosion Mg to Zn-coated steel USW joint. $\mathrm{OM}$ and 3D imaging were used to survey the fracture surface of the USW weld. SEM and X-ray maps determined two types of corrosion product present post-corrosion. Mg and O-rich corrosion product and a Mg, ,Zn and O-rich feature were present on the Zn side of the fractured USW joint. Source: ORNL.

Figure II.3.14.12. Post-corrosion USW sample, Mg side of sample in Figure II.3.14.9: (a) The SEM micrograph shows that un-corroded Mg existed near the USW joint, in addition to the Mg oxide corrosion product; (b) X-ray maps show the distribution of Mg oxide scale near the joint, the Zn which diffused from the steel coating and $\mathrm{Cl}$ ions left over from the immersion. Source: ORNL...... 561

Figure II.3.14.13. Post-corrosion analysis of PNNL FSSW Mg/Zn-coated steel joint cross-section.

Source: ORNL. 562 


\section{List of Tables}

Table I.1.2.1. Laser Parameters Tested on the Al-Fe-Si-Mn Bulk Alloy............................................ 17

Table I.1.2.2. Laser Parameters Tested on the Al-Fe-Si-Mn Alloy Powders........................................ 19

Table I.1.4.1. Grouping of Different NiCr Alloys Based on Their Composition in wt.\%. ..................... 34

Table I.1.5.1. Chemical Composition of the Two Different Studied Alloys 21-2N and 23-8N in wt.\%

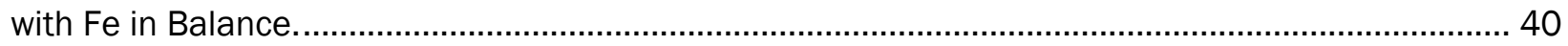

Table I.2.1.1 Composition of the Copper-Carbon Composite Samples Manufactured in FY 2019.... 48

Table I.2.1.2 Electrical Conductivity of the Copper-Carbon Composite Wires Measured by the

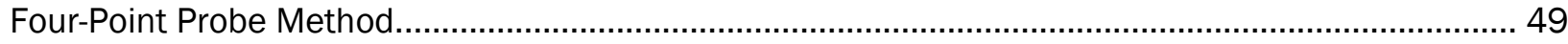

Table I.2.2.1. List of Tasks to be Accomplished by this Project...................................................... 54

Table I.3.1.1.3.1. Mean Values of Al Grain Size as Well as Area and Volume Fraction of Grain Boundary $\Theta$ Particles in the As-Aged ACMZ Alloys. ........................................................................ 72

Table I.3.2.1.1.1. Target Properties and Metrics Suggested by a Piston Manufacturer.................... 79

Table I.3.2.1.3.1. Alloy Composition in wt\%............................................................................... 91

Table I.3.2.1.4.1. Alloy Compositions of Cast Austenitic Stainless Steel in the Present Study.......... 97

Table I.3.2.1.5.1. Targeted Applications of Selective Processing.................................................... 108

Table I.3.3.1.3.1. Chemical Composition of the Cast Alloys Exposed to Laser Beam Single-Track

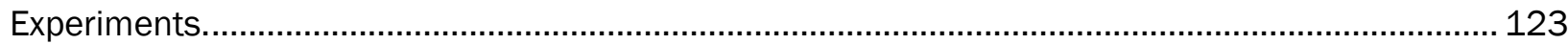

Table I.3.4.1. Phase Fraction of Metastable $\theta^{\prime}$ and Equilibrium $\theta$ Phases of Al5CuNi (RR350) after Different Heat-Treated Conditions. ......................................................................................... 137

Table I.3.4.2. Chemical Compositions of Alloys for Solidification and Phase Equilibrium Simulation.

Table I.3.4.3. Comparison of the Predicted Relative Density to the Experiment after Sintering....... 149

Table I.3.5.2.1. The Results of Potentiodynamic Corrosion Tests in 3.5 wt.\% NaCl Solution and Comparisons with State-of-the Art Mg Anticorrosion Coating Methods. .......................................... 165

Table II.1.2.1. Status of Project Tasks and Milestones at the End of FY 2019............................... 193

Table II.1.2.2. Summary of Processing Parameters of USAMP Alloy 2 and Alloy 2 Plus................... 196

Table II.1.2.3. DSA Regime for Mg-Ca Binary and Mg-Ca-Zn Ternary Alloys Compressed at $200^{\circ} \mathrm{C}$

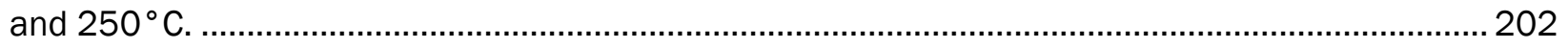

Table II.1.2.4. Loading Conditions for the CP Formulation Virtual Experiments...............................210

Table II.1.2.5. Summary of Surface Roughness Measurements on Control and Bent Coated E-

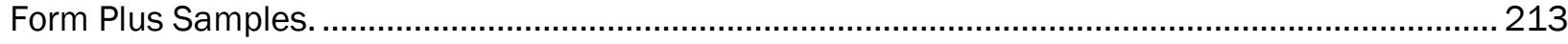

Table II.1.2.6. Processes used to Clean and Pretreat Panels Treated with Each Task 3 TeamMember's Coating. 218

Table II.1.5.1. Flexural Load in Newtons (N) of DP500 with the CFRE Coating Applied with Varying Thicknesses of CFRE and Steel.. 235 
Table II.1.5.2. Full Coverage of DP490 Steel with $0.50 \mathrm{~mm}$-thick CFRE with Mass and the Cost of Steel and CFRE Compared with the Baseline Materials of $0.70 \mathrm{~mm}$ Uncoated Steel. 236

Table II.1.7.1. Standardized Compositions of the A356 Al and A206 Al Alloy. 249

Table II.2.1.1. Project Parameters and Requirements. 268

Table II.2.1.2. Summary of Key Lab-Scale Results (Upper Limits Only). 270

Table II.2.1.3. Summary of Key Pilot-Scale Results (Upper Limits Only). 287

Table II.2.2.1. Project Parameters and Requirements. 292

Table II.2.2.2. Milestones for BP2. 294

Table II.2.2.3. Mechanical Properties of Successful CF Candidates Obtained During BP1 for Use during BP2. 296

Table II.2.3.1. Optimization Results. 314

Table II.2.3.2 Status to Target. 326

Table II.2.4.1. Project Participants. 328

Table II.2.4.2. Static Performance of Composite Door. 331

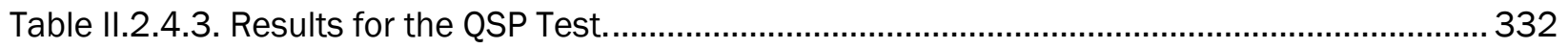

Table II.2.4.4. Results For Full Pole and Deformable Barrier Dynamic Load Cases.......................... 332

Table II.2.4.5 Statistical Distribution of Individual Parameters and Total Cost. ............................... 342

Table II.2.5.1. Input Parameters for Numerical Simulations of Microscale Simulations. ...................363

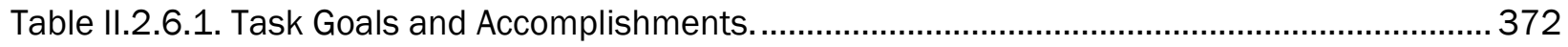

Table II.2.9.1. Detail of the Major Calendar Steps of the Spider Silk Conversion Project. ................ 394

Table II.2.10.1. Task 3 Milestones, Task Descriptions and Status. .................................................. 400

Table II.2.10.2. Characterization of the Top Three Montefibre Carbon Materials-Based on

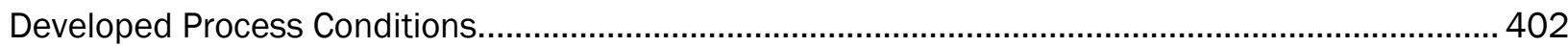

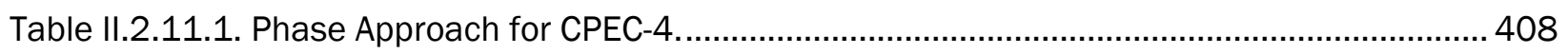

Table II.2.11.2. Measurements of the Crosstalk between the Antennas and Their Lines with Two Configurations at Two Power Levels. 410

Table II.3.5.1. Formulation Details, Key Properties, and Throwpower for the Selected Prototype

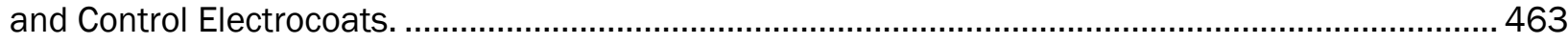

Table II.3.5.2. Full Test Matrix for Coupons Used in Accelerated Corrosion Testing........................ 467

Table II.3.5.3. Test Matrix of Liftgates Manufactured to Prove Out Dimensional Stability and 468

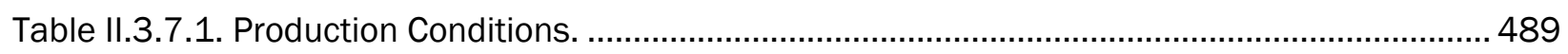

Table II.3.8.1. Summary of Various Adhesives with Different Modulus. ......................................... 497

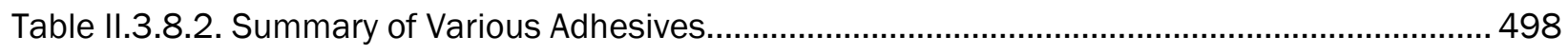

Table II.3.10.1. Summary of Immersion Corrosion Testing Condition.............................................. 518 
Table II.3.10.2. Summary of Corrosion Potential Measurement for Individual Material. 520

Table II.3.11.1. Surface Roughness (Ra) of Specimen under Different Engineering Conditions. ..... 529

Table II.3.11.2. Bonding Strength of Mg-Steel USW Joint under Different Process Conditions. 531

Table II.3.12.1. Summary of Lap-Shear Tensile Testing for FBJ Samples by the New FBJ Machine. 543

Table II.3.13.1. Spot Welding Experiments with Destructive Evaluation. 546

Table II.3.14.1. Resistance Calculated From DC and AC Methods. 558 


\section{Vehicle Technologies Office Overview}

Vehicles move our national economy. Annually, vehicles transport 11 billion tons of freight-about $\$ 35$ billion worth of goods each day ${ }^{1}$-and move people more than 3 trillion vehicle-miles. ${ }^{2}$ Growing our economy requires transportation and transportation requires energy. The transportation sector accounts for approximately $30 \%$ of total U.S. energy needs ${ }^{3}$ and $70 \%$ of U.S. petroleum consumption. ${ }^{4}$ The average U.S. household spends over $15 \%$ of its total family expenditures on transportation, making it the most expensive spending category after housing. ${ }^{5}$

The Vehicle Technologies Office (VTO) has a comprehensive portfolio of early-stage research to enable industry to accelerate the development and widespread use of a variety of promising sustainable transportation technologies. The research pathways focus on fuel diversification, vehicle efficiency, energy storage, and mobility energy productivity that can improve the overall energy efficiency and efficacy of the transportation or mobility system. VTO leverages the unique capabilities and world-class expertise of the National Laboratory system to develop innovations in electrification, including advanced battery technologies; advanced combustion engines and fuels, including co-optimized systems; advanced materials for lighterweight vehicle structures; and energy efficient mobility systems.

VTO is uniquely positioned to address early-stage challenges due to strategic public-private research partnerships with industry (e.g., U.S. DRIVE, $21^{\text {st }}$ Century Truck Partnership) that leverage relevant expertise. These partnerships prevent duplication of effort, focus DOE research on critical R\&D barriers, and accelerate progress. VTO focuses on research that industry does not have the technical capability to undertake on its own, usually due to a high degree of scientific or technical uncertainty, or that is too far from market realization to merit industry resources.

\section{Annual Progress Report}

As shown in the organization chart (below), VTO is organized by technology area: Batteries \& Electrification R\&D, Materials Technologies, Advanced Engine \& Fuel R\&D, Energy Efficient Mobility Systems,

Technology Integration, and Analysis. Each year, VTO's technology areas prepare an Annual Progress Report (APR) that details progress and accomplishments during the fiscal year. VTO is pleased to submit this APR for Fiscal Year (FY) 2019. In this APR, each project active during FY 2019 describes work conducted in support of VTO's mission. Individual project descriptions in this APR detail funding, objectives, approach, results, and conclusions during FY 2019.

\footnotetext{
${ }^{1}$ Bureau of Transportation Statistics, Department of Transportation, Transportation Statistics Annual Report 2018, Table 4-1. https://www.bts.gov/tsar. 2 Transportation Energy Data Book 37th Edition, Oak Ridge National Laboratory (ORNL), 2019. Table 3.8 Shares of Highway Vehicle-Miles Traveled by Vehicle Type, 1970-2017.

3 Ibid. Table 2.1. U.S. Consumption of Total Energy by End-use Sector, 1950-2018.

4 Ibid. Table 1.12. U.S. Transportation Petroleum Use as a Percent of U.S. Petroleum Production, 2018.

5 Ibid. Table 10.1, Average Annual Expenditures of Households by Income, 2016.
} 


\section{Organization Chart}

\section{Vehicle Technologies Office}

February 2020

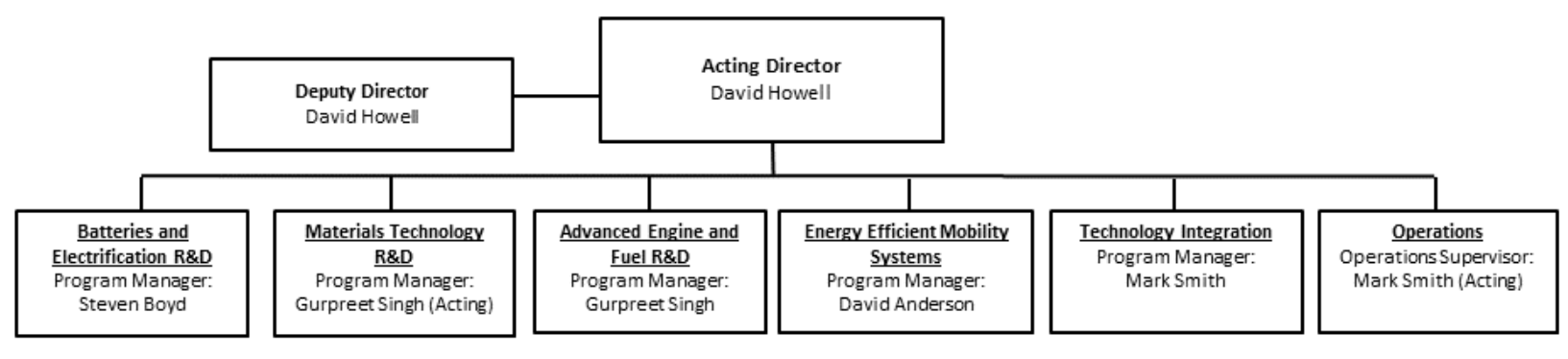




\section{Materials Technology Program Overview}

\section{Introduction}

The Materials Technology Program supports vehicle lightweighting and improved propulsion (powertrain) efficiency through early stage research and development (R\&D) to increase understanding of novel materials and to enable industry to develop and deploy more fuel efficient LD and HD vehicles. The research focus is on activities that have a high degree of scientific or technical uncertainty, or that are too far from market realization to merit sufficient industry focus and critical mass. The Materials Technology Program accomplishes its technical objectives through research programs with academia, National Laboratories, and industry.

The Propulsion Materials portfolio is closely aligned with other VTO subprograms to identify critical materials needs for next-generation high-efficiency powertrains for both LD and HD vehicles. Strategies for achieving high-efficiency powertrains include improved powertrain thermal efficiency, increased power density resulting from high-strength and lightweight materials, and petroleum displacement through fuel substitution strategies. In FY 2019, the Propulsion Materials portfolio launched a new National Laboratory consortium to target critical engine components based on thermal loading and structural requirements and utilize an ICME approach linking advanced characterization to high-performance computing methods to accelerate the development of new material families.

The Lightweight Materials team works closely with industry through the U.S. DRIVE (Driving Research and Innovation for Vehicle efficiency and Energy sustainability) partnership to understand HD and LD vehicle structural weight reduction goals and to identify technical challenges that prevent the deployment of lightweight materials. The most promising and likely approach for lightweighting is a multi-material approach that focuses on the use of the right material for the application. The Lightweight Materials research portfolio addresses significant technology gaps for each family of structural materials: $\mathrm{Mg}$, AHSS, Al, and CF composites. In addition, there is a growing focus on dissimilar material joining in order to enable the reliable assembly of these multi-material systems.

\section{Goals}

The Materials Technology Program supports the VTO's mission to help consumers and businesses reduce their transportation energy costs while meeting or exceeding vehicle performance expectations. Lighter vehicles with more efficient engines reduce fuel use and save consumers money. The structural and powertrain systems that we target to improve are limited by materials performance. By improving the properties of powertrain and structural materials, we can enable a significant improvement in fuel economy for future vehicles. Increasing the strength and oxidation resistance of high-temperature materials will enable new combustion strategies while improving properties in structural materials such as stiffness, strength, joinability, and crash energy absorption - all with lower-cost - will accelerate the deployment of lightweight materials in the automotive market. The specific performance and cost target for the Materials Technology Program is to:

- $\quad$ Enable a 25\% weight reduction for LD vehicle gliders including body, chassis, and interior as compared to a 2015 baseline at no more than a $\$ 5 / 1 \mathrm{lb}$.-saved increase in cost by 2025 .

- Validate a $25 \%$ improvement in high-temperature $\left(300^{\circ} \mathrm{C}\right)$ component strength relative to components made with a 2010 baseline cast Al alloys (A319 or A356) for improved efficiency LD engines by 2025. 


\section{State-of-the-Art}

Automakers are seeking to improve fuel economy while maintaining or improving vehicle performance and safety. Reducing the weight of the structure of the vehicle improves fuel economy and reduces the strain on the powertrain of the vehicle, thereby allowing for further fuel savings. For structural components, the market is shifting from traditional steel components to lighter-weight materials, such as AHSSs, Al alloys, $\mathrm{Mg}$ alloys, and CF composites. Lighter structures allow for downsized engines and increased power density, which requires lightweight materials with low density, high-strength, and high-stiffness at elevated temperatures.

In order to meet material property requirements in increasingly severe combustion environments, new cast $\mathrm{Al}$ alloys are needed. This is due, in part, to inadequate thermal performance, as well as very high peak cylinder pressures (PCPs) in advanced engine applications. As engine thermal efficiency improves, the temperature experienced by downstream exhaust components is decreased. This leads to a need for low temperature catalyst development in order to maintain effective reduction of tailpipe emissions. Moreover, inadequate databases, modeling, and design tools are significant barriers for further development of new materials. By evaluating existing computational tools, identifying gaps that must be overcome to achieve seamless integration across multiple-length scales, and increasing understanding of the basic behavior of the material (i.e., effects of solute at the atomistic level, microstructural development, microstructure/property relationships, fracture and failure mechanisms, durability, temperature-dependent behavior, etc.), more accurate design tools and predictive models can be established. High quality, consistent, and accessible databases are needed to support this development.

AHSS is the most mature lightweight material in terms of widespread use in industry due to its compatibility with existing manufacturing infrastructure and vehicle materials. The application of third-generation highstrength steel has the potential to reduce component weight by up to $25 \%$, particularly in strength-limited designs. However, technical challenges remain to achieve room temperature formability, improved weldability, and reliable and accurate predictive performance models. Al continues to see steady growth in market share in the automotive industry ${ }^{6}$ despite issues of material cost, room temperature formability, and limitations within the existing manufacturing infrastructure. This is due to the $40 \%$ weight-savings that can be achieved with $\mathrm{Al}$ along with the well-established domestic supply chain. $\mathrm{Mg}$ has the potential to significantly reduce vehicle component weight by $55 \%$ or greater; however, there are several significant technical barriers preventing increased use of this material in vehicle designs. $\mathrm{Mg}$ has high raw material costs and price volatility, relatively low specific stiffness, difficulty in forming sheet at low temperatures, low ductility of finished components, and a limited alloy set, among other challenges. CF composites have the potential to reduce component weight by more than $60 \%$. The main barriers to widespread CF use are the high cost to manufacture the CF, lack of high-volume composite manufacturing methods, and a need for reliable predictive tools for both part design and performance prediction.

When combinations of the above lightweight materials are used, the resulting multi-material structures have challenges of their own. Traditional joining methods used in automotive assembly, such as RSW and riveting, are inefficient for the joining of dissimilar metals and for some combinations, infeasible. In the near-term, friction stir scribe welding and resistance spot riveting (RSR) are showing promising advances for the joining of AHSS and $\mathrm{Al}$ (the more mature lightweight metals). An additional challenge posed by multi-material structures is the increased risk of corrosion due to galvanic coupling. As the barriers to the introduction of $\mathrm{Mg}$ and $\mathrm{CF}$ are overcome, breakthroughs in joining technology will also be necessary.

6 Ducker Worldwide, Drive Aluminum, 2017. Al content in North American light vehicles 2016 to 2028. http://www.drivealuminum.org/wpcontent/uploads/2017/10/Ducker-Public FINAL.pdf. 


\section{Program Organization Matrix}

The Materials Technology Program is led by Acting Program Manager, Gurpreet Singh.

1. Propulsion Materials consists of one primary research portfolio:

- High-Temperature Materials (Jerry L. Gibbs) - which includes research on the material characteristics that influence temperature-dependent behavior, as well as alloy development.

2. Lightweight Materials consists of three research portfolios:

- Light Metals (Sarah Kleinbaum/Jerry L. Gibbs) - which includes research on property improvement and processing advances for AHSS, $\mathrm{Al}$, and Mg.

- Composites (Felix Wu) - which includes research on low-cost production of $\mathrm{CF}$ and predictive performance models of $\mathrm{CF}$ composites.

- Joining of Dissimilar Materials (Sarah Kleinbaum) - which includes research on solid-state and mechanical joining methods for dissimilar materials, as well as galvanic corrosion mitigation.

All of the activities within the Materials Technology Program utilize computational methods for material discovery, prediction of structure, and understanding failure mechanisms, including corrosion and the effects of processing on properties. 
(This page intentionally left blank) 


\section{Propulsion Materials}

\section{I.1 Modeling of Powertrain Materials}

\section{I.1.1 Future Engine Requirements - Modeling Advanced Engine Materials (Oak Ridge National Laboratory)}

\section{Charles E.A. Finney, Principal Investigator}

Oak Ridge National Laboratory

National Transportation Research Center

2360 Cherahala Blvd

Knoxville, TN 37932

E-mail: finneyc@ornl.gov

\section{Jerry L. Gibbs, DOE Technology Development Manager}

U.S. Department of Energy

E-mail: jerry.gibbs@ee.doe.gov

Start Date: October 1, 2016

Project Funding (FY19): \$210,000

End Date: September 30, 2019

DOE share: $\$ 210,000 \quad$ Non-DOE share: $\$ 0$

\section{Project Introduction}

HD internal combustion engines for the transportation sector operate at high PCPs to achieve required performance and brake thermal efficiency. Current PCP operation for on-road HD vehicle engines peaks at about 190 bars $\left[1 \mathrm{bar}=10^{5} \mathrm{~Pa}\right.$ ], where it has been maintained for almost two decades following nearly four decades of steady increase. A general trend in both HD and LD engines over the past few decades has been an increase in the specific engine output, meaning the amount of torque or power output per displacement volume of the engine. Generally, higher specific output requires more intense combustion with a higher combustion thermal loading per displacement volume, and this usually translates to higher temperatures in the cylinder materials containing the combustion charge.

This pause in increased PCP and specific output operation has a variety of causes, with a prominent reason being engine materials limitations. The current generation of materials, such as the family of grey cast-irons, is widely considered inadequate for the projected elevated-pressure and temperature environments of future higher efficiency engines. Although candidate replacement materials, such as compacted graphite iron (CGI), are being developed and adopted for some applications, a robust understanding of engine cylinder materials stresses and the materials properties necessary to enable desired engine efficiency and specific output of future HD engines is lacking. Internal combustion engines, in both LD and HD applications, have been trending toward higher specific output to enable downsizing for fuel economy benefits.

Numerical simulations offer insight into the cylinder environment at such elevated operating cylinder pressures and temperatures and can help identify design needs and changes more comprehensively than can be identified experimentally, particularly with materials temperatures and heat fluxes. The current practice of simulation can achieve low- or high-dimensional modeling of the combustion environment or the cylinder/engine structure, but a fully coupled, high-dimensional computational fluid dynamics (CFD)/finite element analysis (FEA) simulation of combustion, heat transfer, and consequential material temperatures and stresses is a fundamental development need.

Part of this limitation is the time and level of detail required to set up simulations. For FEA particularly, a proper meshing of complex geometries for high-fidelity calculation of materials stresses with conjugate heat 
transfer can be very labor-intensive. However, with simplifications, a sufficient first order estimate of future required materials properties can be gauged with simulations. This project is employing an incremental process for this problem, starting with the use of CFD results, guided by low-dimensional modeling, as inputs to FEA simulations and fatigue and creep analysis. A concurrent experimental project measured the properties of a relevant grade of CGI, which has provided the needed data for the combustion, materials modeling, and analysis.

\section{Objectives}

An important objective of this project is to study the effects of operating HD internal combustion engines at increased levels of specific output, which will require higher PCPs and temperatures, with a goal to increase engine and system efficiency. This objective will require evaluation of the performance of currently available materials at current and projected future specific output. The principal objective of this project is to identify properties requirements of materials suitable to withstand a lifetime of operation at the elevated temperatures and pressures in future higher efficiency HD engines. This objective will account for fatigue and creep properties of candidate materials.

\section{Approach}

The project approach utilizes several computational tools informed by selective experimental materials measurements. The first stage is estimation of the thermal environment in the cylinder materials boundaries as a function of varying degrees of combustion intensification. The second stage is to use the thermomechanical boundary conditions (e.g., heat fluxes and pressures) to evaluate stresses and strains in the cylinder materials boundaries using FEA. The third stage is to utilize a constitutive model of the materials to estimate fatigue life, or the inverse problem of defining the materials properties based on a fatigue life required by a lifetime of commercial operation.

Combustion modeling is performed using two approaches: (1) a high-level ( $0-1$ dimensional) modeling suite; and (2) a high-dimensional (3D with fine spatial resolution) CFD package. The low-dimensional modeling is implemented using GT-Suite and GT-Power (Gamma Technologies, Inc.) [1], which is an industry-standard simulation package. A unique feature of GT-Suite is the ability to integrate a 3D thermal solution with the lowdimensional engine model. The solution speed allows for rapid evaluation of large parameter spaces and makes it suitable for defining boundary conditions for and verifying trends in the higher-fidelity CFD simulations. The high-dimensional modeling is implemented using CONVERGE CFD (Convergent Science, Inc.) [2], an industry-standard software package. This code employs state-of-the-art models and numerical techniques to achieve a high degree of fidelity, but with higher computational expense.

Both combustion models utilize design data for a 2013 model year 15-liter diesel engine provided by a HD engine manufacturer. Both models implement schemes for the co-solution of combustion with materials heat transfer in a technique called conjugate heat transfer (CHT). The use of CHT provides more accurate simulations, especially in defining wall heat fluxes, than using a fixed estimate of wall temperatures, which was standard practice until recently. The CHT solutions span the entire engine block and head and utilize materials data from standard references for conventional materials and from an ongoing experimental program at ORNL for an engine-grade compacted graphite iron, CGI-450.

The overall approach is to model combustion at the baseline condition (190 bar) and at various levels of intensified combustion to achieve high specific output using the same engine geometry, which results in higher pressures (exceeding 250 bar) and temperatures. The heat fluxes across the cylinder boundaries - the head, valves, liner, and piston - are then used in the FEA simulations, which employ ANSYS (ANSYS, Inc.) [3], and utilize materials properties from standard references or from ORNL CGI-450 measurement data. At each combustion condition, stresses and strains in the cylinder components of interest are evaluated using a nonlinear stress model. The principal area of interest is in the cylinder head, specifically in the bridge between 
the exhaust valves, where the highest materials temperatures are anticipated, and which could be an area of concern in actual operation.

The results of the FEA simulations are used in a fatigue and creep model to estimate the service life at each combustion condition. The required service life is estimated from the established HD Federal certification cycle where the highest or most stressed load conditions are identified.

\section{Results}

Work in FY 2019 included the refinement of the combustion simulations, but primarily focused on refinement and evaluation of the FEA model to investigate areas of greatest concern in the head.

An important aspect of the combustion modeling is the effect of materials properties on combustion, as reflected in thermal diffusivity, as well as the heat flux from the cylinder through the engine material to the environment. As engine-specific output is increased from the baseline condition of the engine design, the peak pressures and temperatures rise because of the intensified combustion. One benefit of the FEA modeling is the ability to apply loading in stages so that the relative contributions of the constituent loadings may be evaluated. Here, a "loading" refers to a force placed onto the structure of the material, where combinations of the following were evaluated: (1) the preload, largely imposed by the tightening of the head bolts; (2) mechanical loading, caused by PCP during combustion; and (3) thermal loading, caused by the thermal expansion of the material related to the steady-state engine temperature during the target engine operating condition.

With such a loading decomposition, the FEA model predicts that the preload with thermal loading causes stresses at an order-of-magnitude greater than the preloading with mechanical loading. A spatial distribution of stresses in the head on the fire-deck surfaces is seen in Figure I.1.1.1. Ports for the fuel injector (FI), intake valves (IV), and exhaust valves (EV) are labeled. The simulation was for the baseline (190 bar) operation for CGI-450, and the solids-stress model was elastic-only. It is seen here that the predicted baseline stresses are greater than the yield stresses for this material and the temperatures, so material plasticity must be accounted for. Using data measured at ORNL in this project, plasticity for CGI-450 was incorporated into the FEA model. The effects of the addition to this model are seen in Figure I.1.1.2, which shows stress maps on the firedeck surface in the head with elastic-only and plastic models. The areas within the plastic regime could be subject to long-term creep leading to materials structural failure.

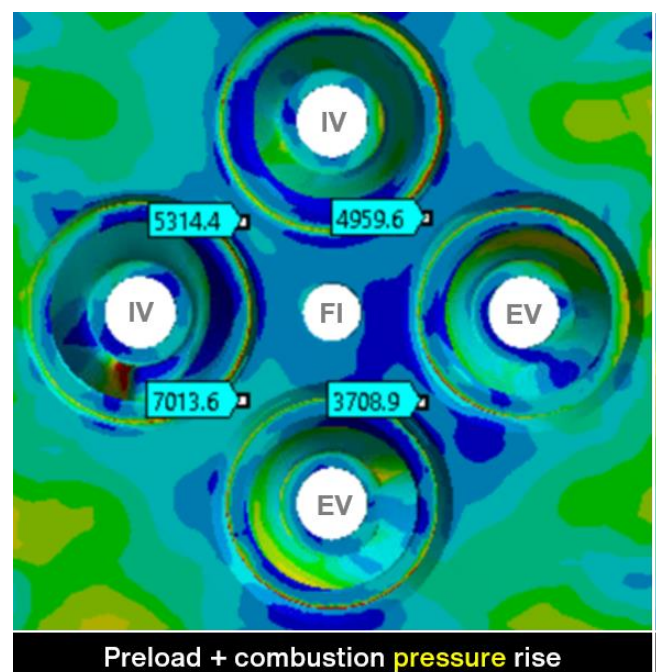

(a)

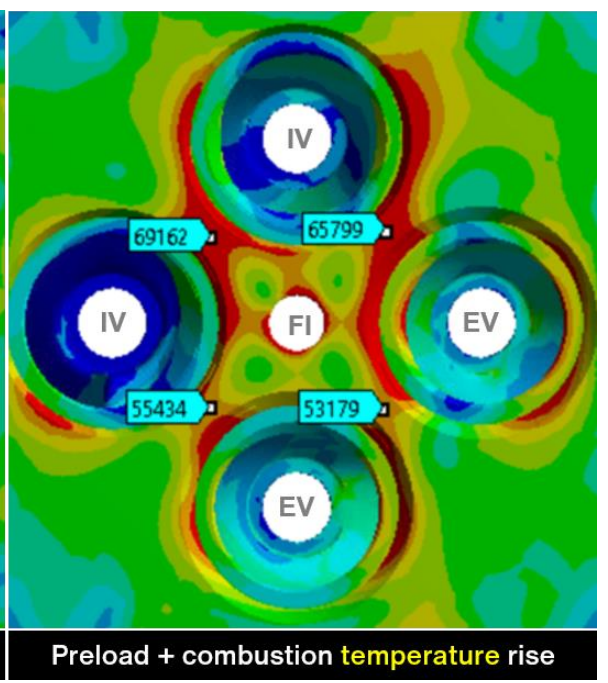

(b)

Figure I.1.1.1. Elastic stresses [psi] from head bolt preload and (engine-cold) combustion pressure (a) compared with preload and (ambient-pressure) combustion temperature loading (b) for CGI-450. Source: ORNL. 


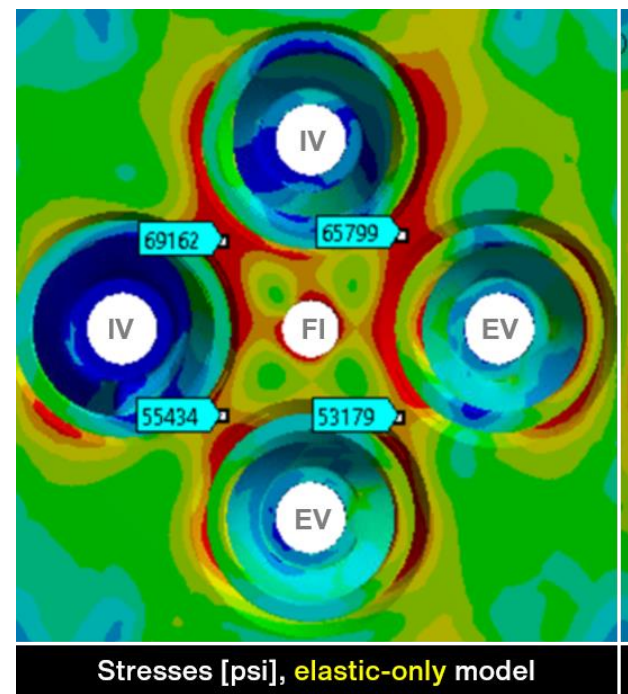

(a)

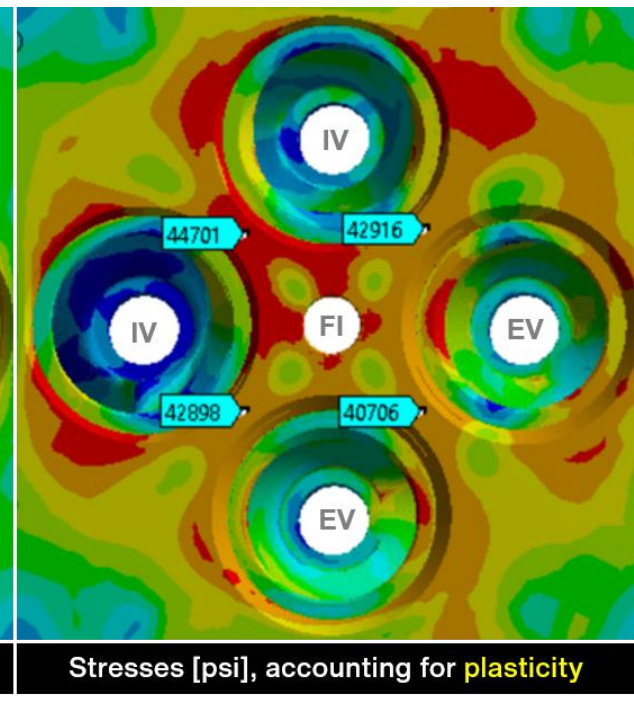

(b)

Figure I.1.1.2. Stresses [psi] at the baseline (190 bar) condition for CGI-450, with an elastic-only model (a) compared with a model including plasticity (b). Source: ORNL.

The effects of increasing specific output by $\sim 25 \%$, resulting in a PCP of $\sim 225$ bar, on CGI-450 head temperatures are seen in Figure I.1.1.3. As can be seen in Figure I.1.1.3, for the higher-flux conditions, three cooling scenarios are treated: (1) cooling that is the same as the baseline (i.e., coolant temperature and heat transfer coefficient [HTC]); (2) 25\% higher HTC cooling, representing a larger coolant flow rate at the same temperature; and (3) 50\% higher HTC cooling.

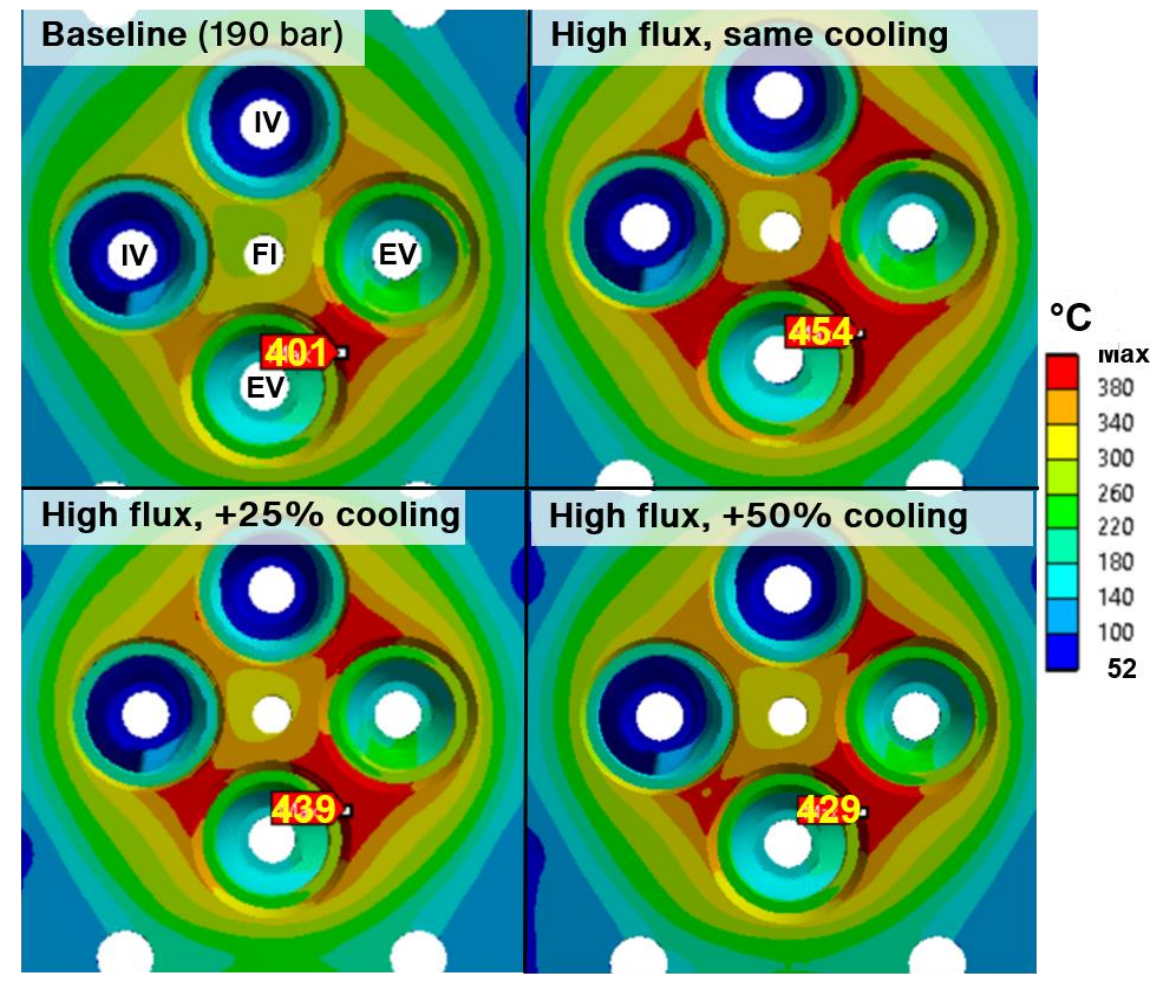

Figure I.1.1.3. Materials surface temperature $\left[{ }^{\circ} \mathrm{C}\right]$ maps, with maxima labeled, for 190 bar and 225 bar operation with three cooling scenarios. The red contour $\left(>380^{\circ} \mathrm{C}\right)$ is the same on all frames. Source: ORNL. 
Higher specific output will raise materials temperatures significantly, where even aggressive cooling cannot compensate. This observation is important because the $400-450^{\circ} \mathrm{C}$ range is where most cast-iron metals lose strength precipitously with small increases in temperature. Future engine designs must account for cooling in the design or employ materials that can tolerate the thermal stresses with increased specific output.

The thermal expansion of the materials causes stresses and strains. These strains, as a relative material deformation, can be manifest in creep and crack initiation. As seen with stresses and temperatures, higher engine-specific output with more intense heat-loading per volume can also be expected to increase strains. This effect is seen in Figure I.1.1.4, which depicts a strain map in the head materials in the baseline (190 bar) and higher-flux ( 225 bar) engine operating conditions for CGI-450. Both frames are displayed with the same color map, while labels show strains on the centerlines between adjoining valve centers on the fire-deck surface.

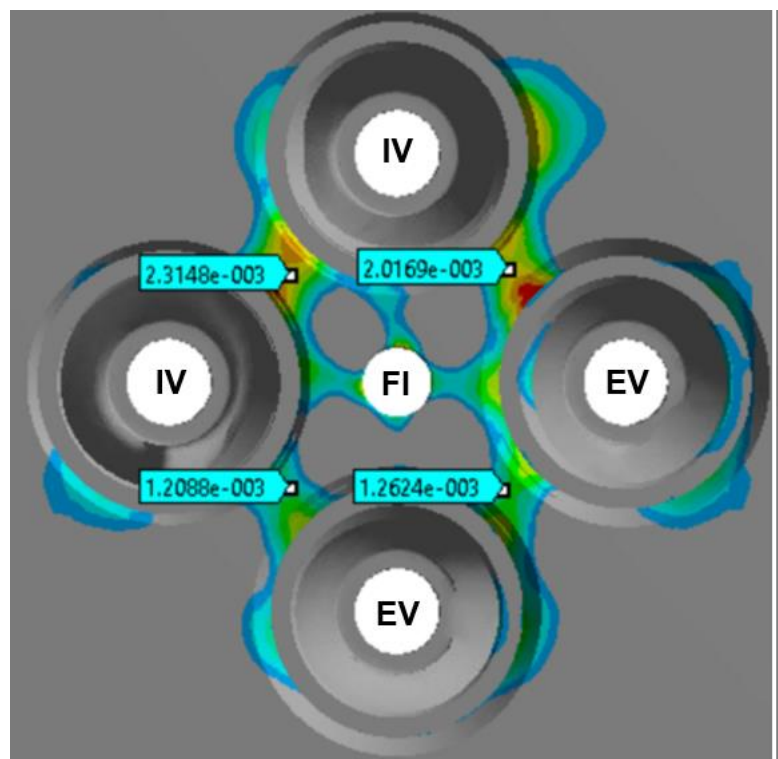

Stains [-], baseline (190 bar) condition

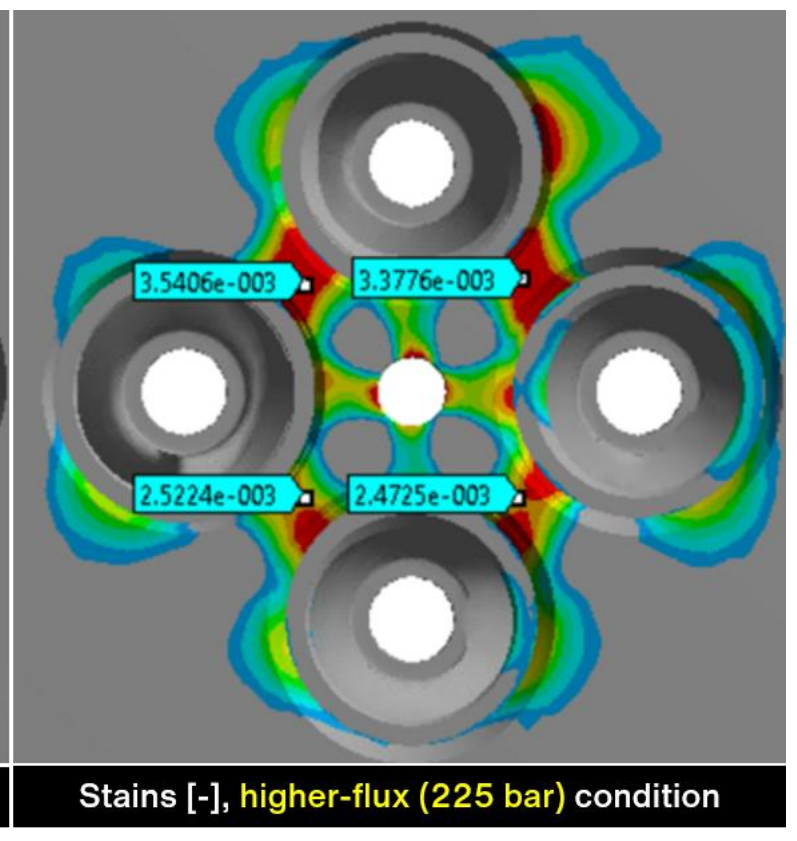

(b)

Figure I.1.1.4. Strains in CGI-450 at the baseline (a) and higher-flux (b) conditions. Plasticity is accounted for, but the effects of creep are not. Source: ORNL.

Work done in this project continues to consider creep, which can affect material integrity throughout the volume; follow-on work should continue to do this. The techniques developed in this project are anticipated to be used to consider materials needs in LD applications, where there are greater materials and cost constraints. A scoping study was completed this year on LD engines [4].

\section{Conclusions}

An integrated experimental and computational approach shows promise for the virtual evaluation of materials properties in the absence of an extensive experimental program; this approach can also provide guidance for future materials research. The computational strategies employed in the project are evolving to reflect best practices to provide the most confidence in simulated results. From the simulations and experimental mechanical properties measurement data, it is apparent that current materials will be highly stressed with increased combustion intensification. 
The simulations used in this project are used for scoping relative trends for several reasons. First, detailed temperature and heat flux measurements in operating engines can be a costly and time-consuming effort, while validation data to calibrate these simulations are not readily available without a large experimental effort. Second, over a range of engine model years and designs among different manufacturers, the relative effects of different materials properties is of more interest to provide materials design guidance.

Ongoing activities in this project should include the following:

- An evaluation of the thermal and mechanical stresses and strains in selected engine components at increased specific output conditions using FEA and estimated fatigue life

- The identification of properties needed for candidate materials to be suitable for future production environments

- A continuation on the integration of the constitutive model for CGI-450 to assist in engine analysis and design.

\section{Key Publications}

1. Mills, Z. G., C. E. A. Finney, K. D. Edwards, and J. A. Haynes, 2019, "Benefits of higher-temperature operation in boosted SI engines enabled by advanced materials," Proceedings of the 2018 Fall Technical Conference of the ASME Internal Combustion Engine Division, Paper ICEF2018-9739.

2. Finney, C. E. A., G. Muralidharan, Z. G. Mills, and J. A. Haynes, 2018, "Future engine requirements," 2018 U.S. DOE Vehicle Technologies Office Annual Merit Review, MAT057.

\section{References}

1. Gamma Technologies, LLC. GT-Suite software. https://www.gtisoft.com.

2. Convergent Science, Inc. CONVERGE CFD software. https://convergecfd.com.

3. ANSYS, Inc. ANSYS Mechanical Enterprise software. https://www.ansys.com/products/structures.

4. Mills, Z. G., C. E. A. Finney, K. D. Edwards, and J. A. Haynes, 2019, "Benefits of higher-temperature operation in boosted SI engines enabled by advanced materials," Proceedings of the 2018 Fall Technical Conference of the ASME Internal Combustion Engine Division, Paper ICEF2018-9739.

\section{Acknowledgements}

Co-investigators in this project include Govindarajan Muralidharan and Zachary Mills of ORNL and Andrew Miles of the SimuTech Group of Atlanta, Georgia. 


\section{I.1.2 Lightweight High-Temperature Alloys Based on the Al-Fe-Si System (University of Florida)}

\section{Michele V. Manuel, Principal Investigator}

University of Florida

549 Gale Lemerand Dr.

Gainesville, FL 32611

E-mail:mmanuel@mse.ufl.edu

\section{Richard G. Hennig, Co-Principal Investigator}

University of Florida

549 Gale Lemerand Dr.

Gainesville, FL 32611

E-mail: rhennig@ufl.edu

\section{Jerry L. Gibbs, DOE Technology Development Manager}

U.S. Department of Energy

E-mail: jerry.gibbs@ee.doe.gov

Start Date: October 1, 2016

Total Project Funding: \$1,102,081
End Date: December 31, 2019

DOE share: $\$ 991,873$
Non-DOE share: $\$ 110,209$

\section{Project Introduction}

Automotive applications need low-cost, lightweight, high-temperature alloys to enhance efficiencies in systems such as internal combustion engines. The Al-Fe-Si system provides an opportunity to develop such a material, as it comprises three of the lowest cost elements abundant in nature. Specifically, the $\tau_{10}-\mathrm{Al}-\mathrm{Fe}-\mathrm{Si}$ $\left(\mathrm{Al}_{4} \mathrm{Fe}_{1.7} \mathrm{Si}\right)$ ternary intermetallic phase is a lightweight and low-cost phase that exhibits excellent mechanical properties at high temperatures. However, the $\tau_{10}-\mathrm{Al}_{4} \mathrm{Fe}_{1.7} \mathrm{Si}$ phase has an extremely small compositional range, which should be expanded in order to use this alloy at commercial scale. To increase this compositional range, the equilibrium phase boundaries can be manipulated and expanded via alloy design and non-equilibrium processing and the parts can potentially be fabricated by AM.

\section{Objectives}

The objective of this project is to design alloy chemistries to create phases with an excellent combination of mechanical properties and to 3D laser print them to exploit the nearly non-equilibrium nature of the total melting and solidification process. Additions of a quaternary solute $\mathrm{X}$ to the Al-Fe-Si ternary system will be examined. It is expected that the alloys produced will have three times the tensile strength of competing intermetallic systems and have a density comparable to Ti-Al alloys.

\section{Approach}

An integrated computational approach was used that spans electronic structure calculations to thermodynamic models that are paired with strategic experiments for alloy characterization and thereby validation to produce revolutionary automotive components that will supersede current commercial and research-grade hightemperature alloys. 
The project will be conducted in three budget periods:

Budget Period 1: Candidate solute elements were identified that have the potential to increase the compositional range over which the $\tau_{10}-\mathrm{Al}_{4} \mathrm{Fe}_{1.7} \mathrm{Si}$ phase is stable. Ternary and quaternary alloys were cast via arc melting. The Go/No Go milestone decision was to deliver alloys that have the potential to display a stable $\tau_{10} \mathrm{Al}-\mathrm{Fe}-\mathrm{Si}-\mathrm{X}$ phase. This deliverable was achieved through the experimental demonstration of the desired $\tau_{10}$ phase in the Al-Fe-Si-X alloy system (where $\mathrm{X}=\mathrm{Ni}$ ).

Budget Period 2: For the candidate solute elements, the compositional range of the $\tau_{10}$ phase was expanded by computationally evaluating its energetic and thermodynamic stability. The Go/No Go milestone decision was to create a list of chemistries suitable for powder processing via 3D printing. This deliverable was achieved through computational prediction and experimental demonstration of the desired $\tau_{10}$ phase in the Al-Fe-Si-X alloy system (where $\mathrm{X}=$ copper $(\mathrm{Cu})$, manganese $(\mathrm{Mn})$, nickel $(\mathrm{Ni})$ and zinc $(\mathrm{Zn})$ ).

Budget Period 3: Specimens for mechanical and microstructural characterization are being produced using 3D powder-bed processing for component manufacturing. The Go/No Go milestone decision will be based on the production of mechanical test samples using AM. Argon-atomized Al-Fe-Si-Mn alloy powders were fabricated and the components for mechanical testing are being produced by 3D laser printing.

\section{Results}

In Budget Period 2, quaternary alloying elements were explored to expand the composition range of the $\tau_{10}-\mathrm{Al}_{4} \mathrm{Fe}_{1.7} \mathrm{Si}$ phase by combination of thermodynamic modeling, density functional theory calculations, and diffusion couple experiments. Thermodynamic modeling via Thermo-Calc software and the TCAL5 thermodynamic database was performed to predict the solubility of quaternary elements in the competing $\mathrm{Al}_{13} \mathrm{Fe}_{4}$ phase and the phase stability of $\tau_{10}$. However, we found the current $\mathrm{Al}$ thermodynamic databases cannot provide a good prediction for the alloy systems containing cobalt $(\mathrm{Co})$ and $\mathrm{Cu}$, which is due to the lack of key experimental data for several constituent ternary systems, specifically $\mathrm{Al}-\mathrm{Co}-\mathrm{Fe}$ and $\mathrm{Al}-\mathrm{Cu}-\mathrm{Fe}$. Therefore, we collected additional experimental data for these two ternary systems for future thermodynamic assessments to improve the current $\mathrm{Al}$ thermodynamic databases for designing Al-Fe-Si-based alloys.

Both diffusion triples and equilibrated alloys were used to determine the $\mathrm{Al}-\mathrm{Co}-\mathrm{Fe}$ and $\mathrm{Al}-\mathrm{Cu}-\mathrm{Fe}$ phase diagrams. $\mathrm{Co} / \mathrm{Fe} / \mathrm{Al}_{5} \mathrm{Fe}_{2}$ and $\mathrm{Cu} / \mathrm{Fe} / \mathrm{Al}_{5} \mathrm{Fe}_{2}$ diffusion triples were fabricated using custom-built Kovar diffusion couple jigs. Forty-three Al-Co-Fe and forty-seven $\mathrm{Al}-\mathrm{Cu}-\mathrm{Fe}$ ternary alloys were prepared by arc melting in a water-cooled copper hearth under an argon atmosphere. Following the heat-treatments ranging from 700 $1000^{\circ} \mathrm{C}$, the diffusion triples and alloy samples were analyzed using scanning electron microscopy (SEM), especially backscattered electron (BSE) imaging, energy dispersive spectroscopy (EDS), electron probe microanalysis, and X-ray diffraction (XRD). Based on the experimental results obtained, three $\mathrm{Al}-\mathrm{Co}-\mathrm{Fe}$ isothermal sections at $800^{\circ} \mathrm{C}, 900^{\circ} \mathrm{C}$, and $1000^{\circ} \mathrm{C}$; and three $\mathrm{Al}-\mathrm{Cu}-\mathrm{Fe}$ isothermal sections at $700^{\circ} \mathrm{C}, 800^{\circ} \mathrm{C}$, and $1000^{\circ} \mathrm{C}$ were established over the whole composition range, as shown in Figure I.1.2.1 and Figure I.1.2.2 $[1,2]$, respectively. The measured isothermal sections provide a more detailed description of the overall Al$\mathrm{Co}-\mathrm{Fe}$ and $\mathrm{Al}-\mathrm{Cu}-\mathrm{Fe}$ phase diagrams than what had been reported in the literature previously. The large amount of new phase equilibrium data is essential for future thermodynamic reassessments of the two systems with the CALPHAD (CALculation of PHAse Diagrams) approach to improve the fidelity of the current Al thermodynamic databases. 

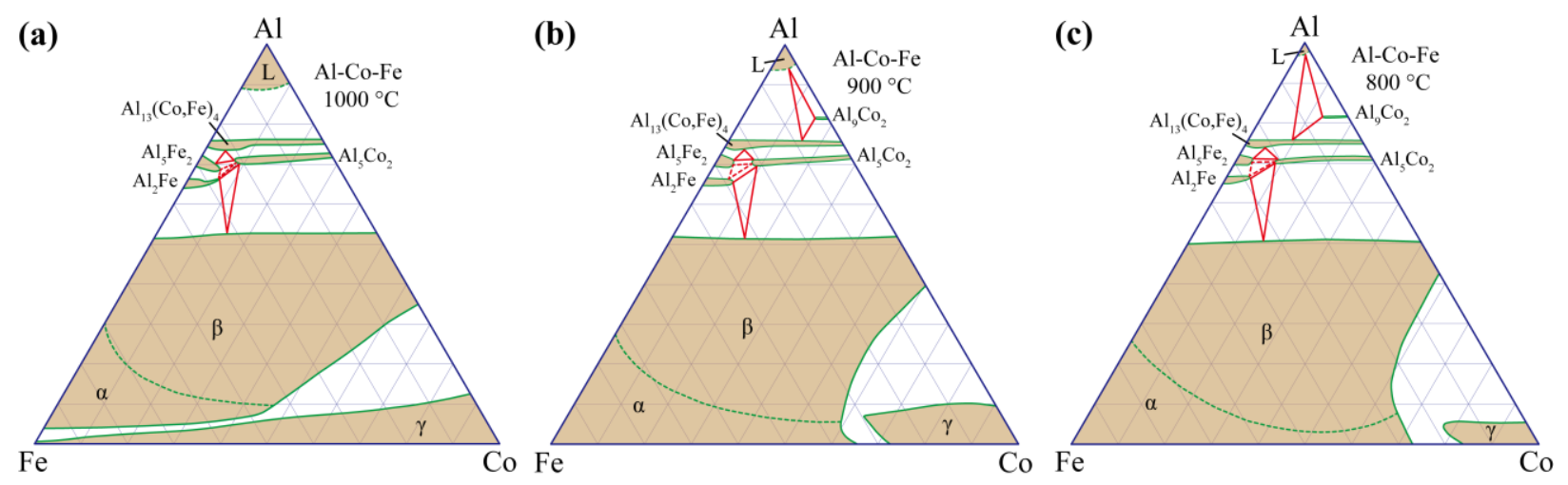

Figure I.1.2.1. Experimentally measured isothermal sections of the Al-Co-Fe ternary system: (a) $1000^{\circ} \mathrm{C}$; (b) $900^{\circ} \mathrm{C}$; and (c) $800^{\circ} \mathrm{C}[1]$.
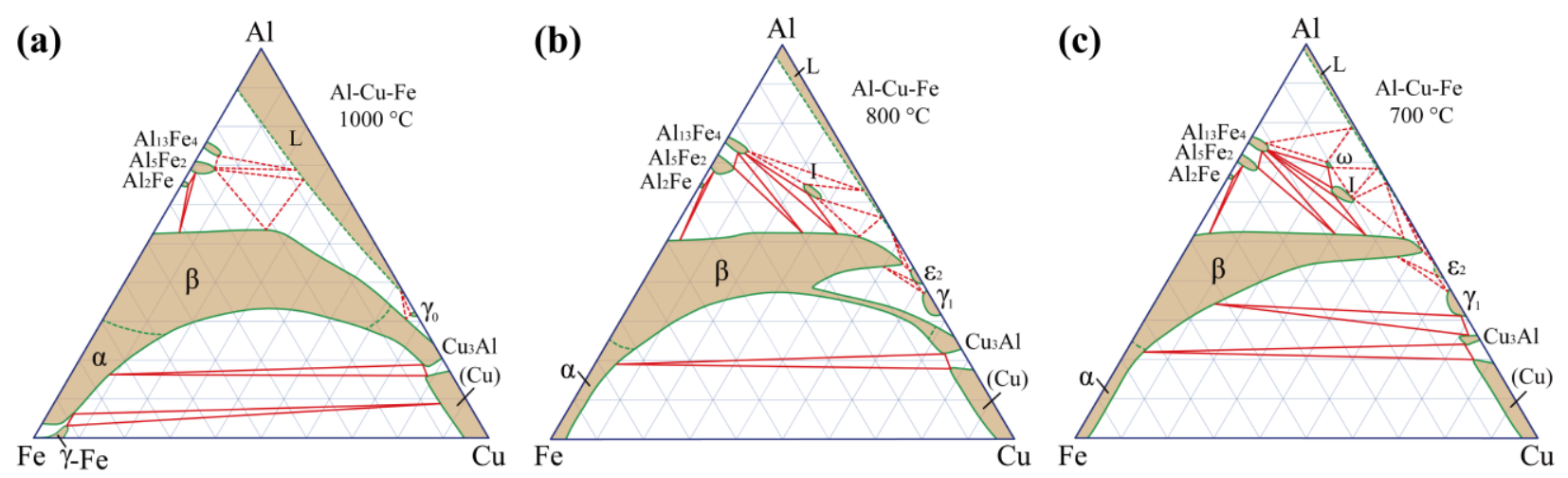

Figure I.1.2.2. Experimentally measured isothermal sections of the Al-Cu-Fe ternary system: (a) $1000^{\circ} \mathrm{C}$; (b) $800^{\circ} \mathrm{C}$; and (c) $700^{\circ} \mathrm{C}[2]$.

From the main findings from Budget Period 2, five candidate elements of $\mathrm{Co}, \mathrm{Cu}, \mathrm{Mn}, \mathrm{Ni}$, and $\mathrm{Zn}$ were demonstrated to have the potential to stabilize the $\tau_{10}$ phase and expand its composition range. In Budget Period 3, more detailed investigations were carried out to study the stability of the Al-Fe-Si-Mn alloys. $\tau_{10}$-Al-Fe-Si-Mn alloys with 0.5 to 5.5 at. $\% \mathrm{Mn}$ were fabricated using arc melting and then annealed at $800^{\circ} \mathrm{C}$ for 350-400 h. Following the heat-treatment, the samples were analyzed using XRD, SEM, and EDS. As can be seen in Figure I.1.2.3, the $\tau_{10}$ single phase was confirmed in the alloys with nominal compositions of 3.5 to 5.5 at. $\% \mathrm{Mn}$. In order to determine the effect of $\mathrm{Mn}$ on the $\mathrm{Al}$ and Si concentrations in the $\tau_{10}$ phase, a series of $\mathrm{Al}-\mathrm{Fe}-\mathrm{Si}-\mathrm{Mn}$ alloys with 4.5 at.\% $\mathrm{Mn}$ and 8.0 to 14.0 at.\% Si were prepared and heat-treated at $800^{\circ} \mathrm{C}$ for $350-400 \mathrm{~h}$. The microstructure of these alloys is shown in Figure I.1.2.4, thereby suggesting a single $\tau_{10}-\mathrm{Al}-\mathrm{Fe}-\mathrm{Si}-\mathrm{Mn}$ phase for the Si concentration ranges from 11 to 14 at.\%

Based on these results, the quaternary alloy of $\mathrm{Al}-20.0 \mathrm{Fe}-11.0 \mathrm{Si}-4.5 \mathrm{Mn}$ (in at.\%) was selected for 3D printing processing, specifically in a selective laser melt powder-bed system at University of Nebraska-Lincoln. Printing parameters were first tested on the $\mathrm{Al}-20.0 \mathrm{Fe}-11.0 \mathrm{Si}-4.5 \mathrm{Mn}$ bulk alloy, which was casted by arc melting and annealed at $800^{\circ} \mathrm{C}$ for $400 \mathrm{~h}$. Table I.1.2.1 lists the laser parameters used for this preliminary testing. Figure I.1.2.5 (a) is a schematic diagram showing locations of the four laser tests and the melted areas. Figure I.1.2.5 (b) and (c) show the typical microstructures taken from the corresponding melted area using a laser power of $750 \mathrm{~W}$ and a scan speed of $20 \mathrm{~mm} / \mathrm{s}$. The melt pool area, the heat affected zone (HAZ), and the boundary between them can be clearly identified. Figure I.1.2.6 plots the measured dendrite arm spacing and the depth of the HAZs with different laser parameters. As can be observed, there is not a significant difference in the dendrite arm spacing, indicating there is no significant change in the cooling rate of the melt pool. Similarly, no significant difference was found in the measured depth of the HAZs. 

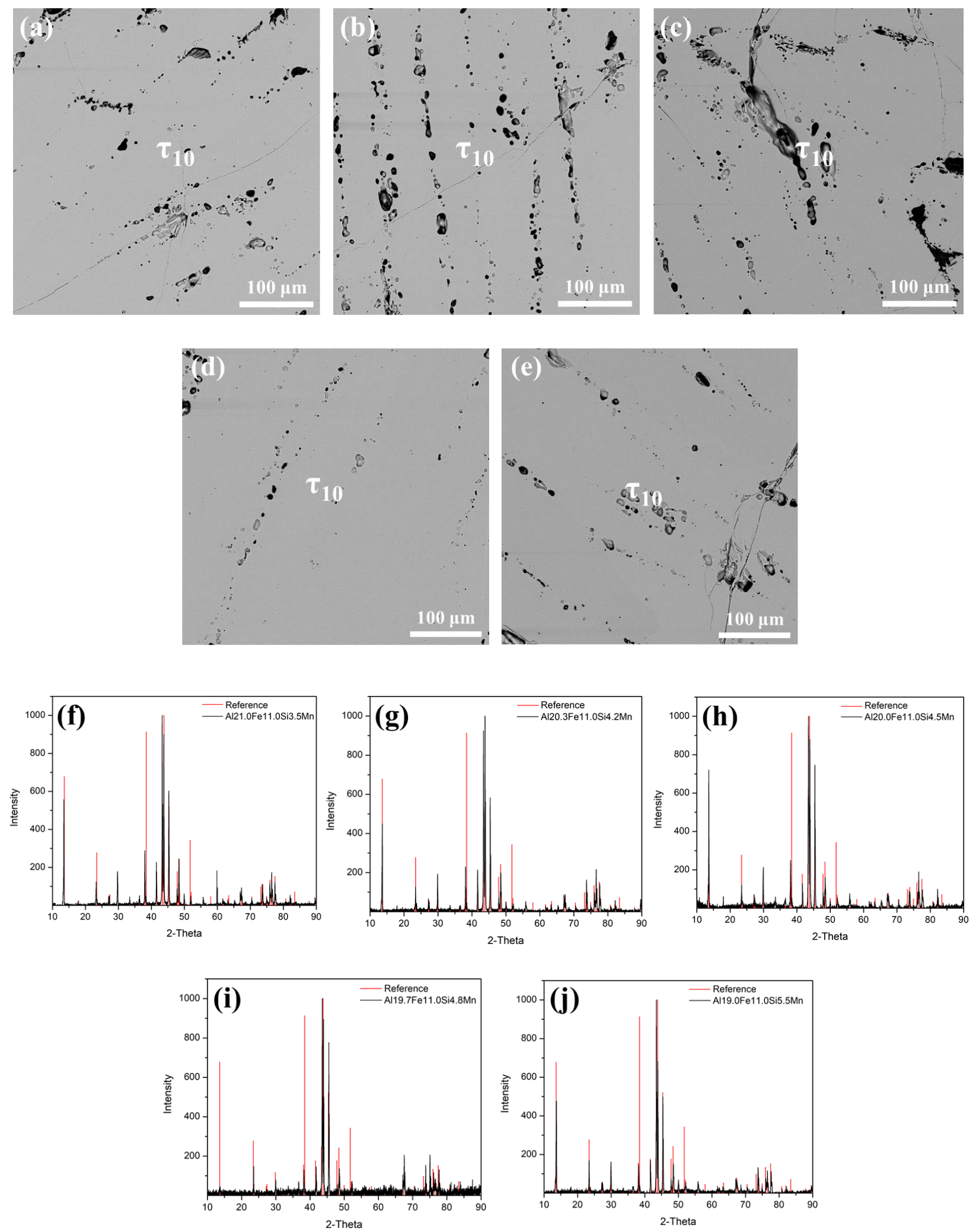

Figure I.1.2.3. Al-Fe-Si-Mn quaternary alloys with 3.5 to 5.5 at. $\% \mathrm{Mn}$ that were annealed at $800^{\circ} \mathrm{C}$ for 350 to $400 \mathrm{~h}$ : (a)-(e) SEM-BSE images showing the microstructure of the heat-treated alloys and (f)-(j) XRD patterns confirming the existence of the $\tau_{10}$ single phase. Source: University of Florida. 

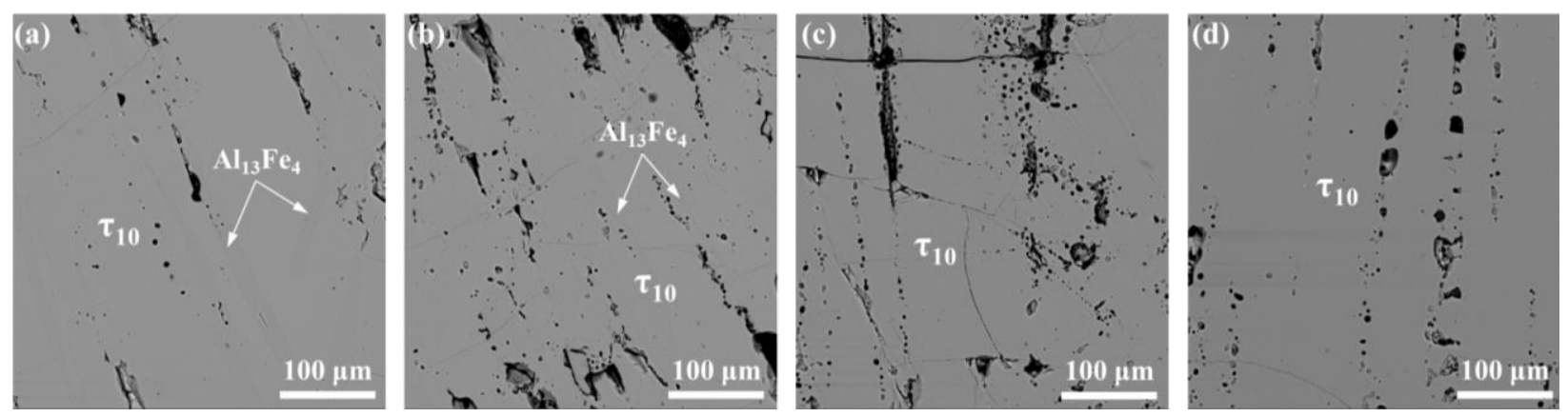

Figure I.1.2.4. SEM-BSE images showing the microstructure of the Al-Fe-Si-Mn quaternary alloys with 4.5 at.\% Mn and 8.0 to 14.0 at.\% Si that were annealed at $800^{\circ} \mathrm{C}$ for 350 to $400 \mathrm{~h}$ : (a) Al-20.0Fe-8.0Si-4.5Mn; (b) Al-20.0Fe-9.0Si-4.5Mn;

(c) Al-20.0Fe-12.0Si-4.5Mn; and (d) Al-20.0Fe-14.0Si-4.5Mn. All the compositions are in at.\%

Source: University of Florida.

Table I.1.2.1. Laser Parameters Tested on the AI-Fe-Si-Mn Bulk Alloy.

\begin{tabular}{|c|c|}
\hline Laser Power (W) & Scan Speed $(\mathbf{m m} / \mathbf{s})$ \\
\hline 550 & 20 \\
\hline 750 & 20 \\
\hline 550 & 10 \\
\hline 650 & 15 \\
\hline
\end{tabular}
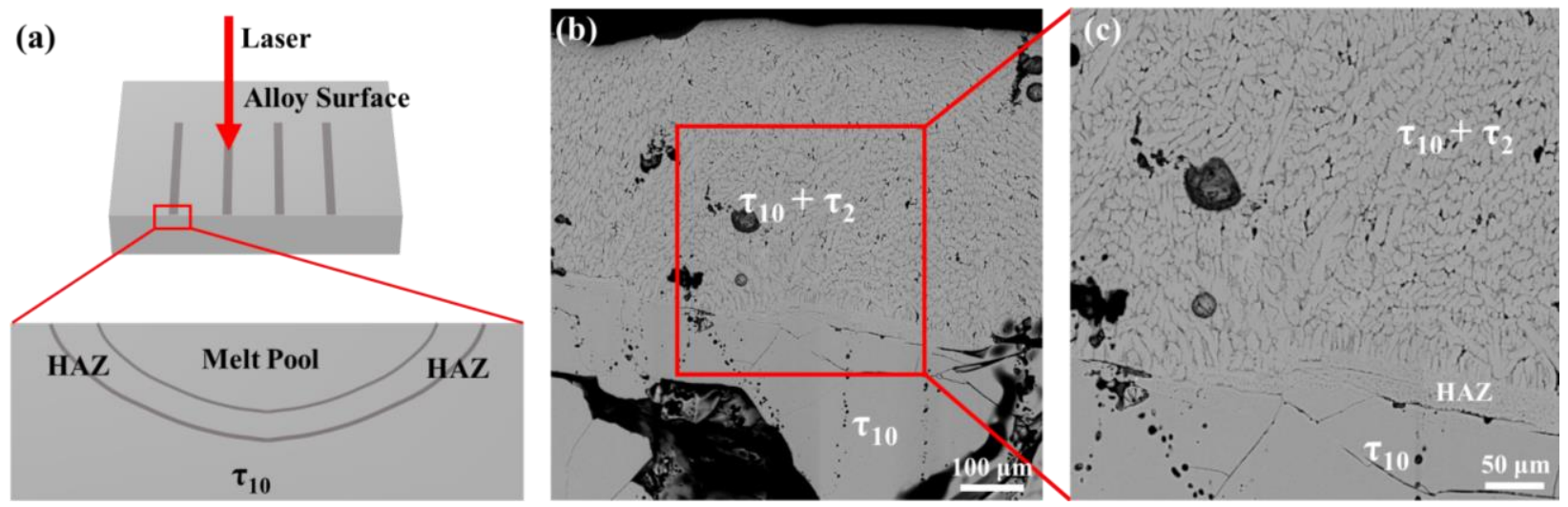

Figure I.1.2.5. Microstructure of the laser-melted areas of the Al-Fe-Si-Mn quaternary alloy with 4.5 at.\% Mn: (a) Schematic

diagram showing the laser test locations and the melted areas; (b) Typical SEM-BSE image taken from the melted area using a laser power of $750 \mathrm{~W}$ and a scan speed of $20 \mathrm{~mm} / \mathrm{s}$; and (c) High magnification image of the red box location in (b) clearly showing the HAZ boundary. Source: University of Florida.

Argon-atomized powders with a nominal composition of Al-20.0Fe-11.0Si-4.5Mn (in at.\%) were purchased from Jiangsu Vilory Advanced Materials Technology Co., Ltd. The powders are spherical particles with two different size distributions of 15-53 $\mu \mathrm{m}$ and $45-150 \mu \mathrm{m}$. The concentration of oxygen impurity was measured to be less than 0.2 at.\%. Figure I.1.2.7 (a) is an SEM secondary electron image taken from the powders with a size distribution of 15-53 $\mu \mathrm{m}$. The obtained XRD patterns are shown in Figure I.1.2.7 (b), confirming the presence of the $\tau_{10}$ single phase in the powders. 

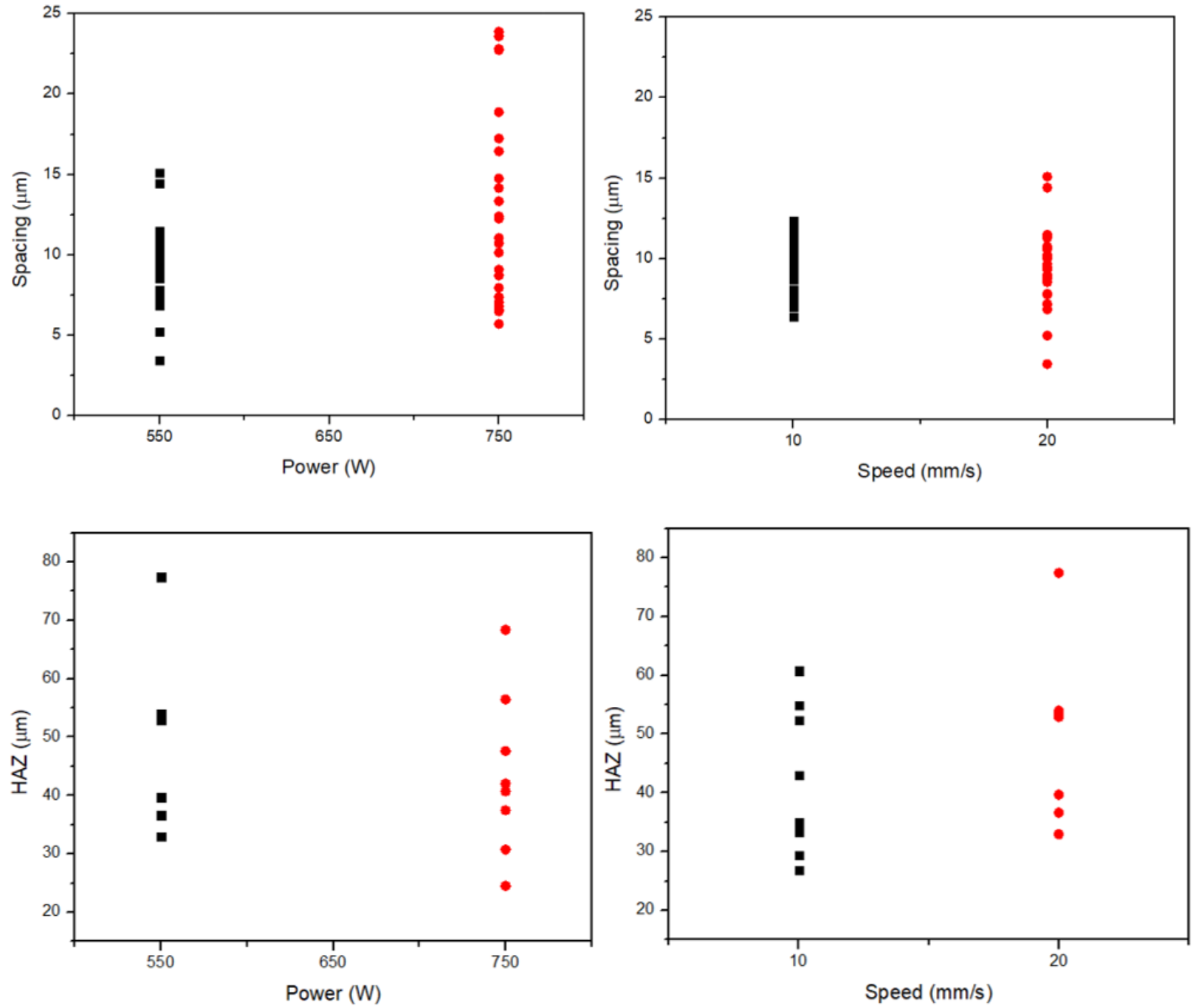

Figure I.1.2.6. Plot showing the measured dendrite arm spacing and depth of the HAZs in the laser-melted areas for two laser powers and two laser scan speeds. Source: University of Florida. 

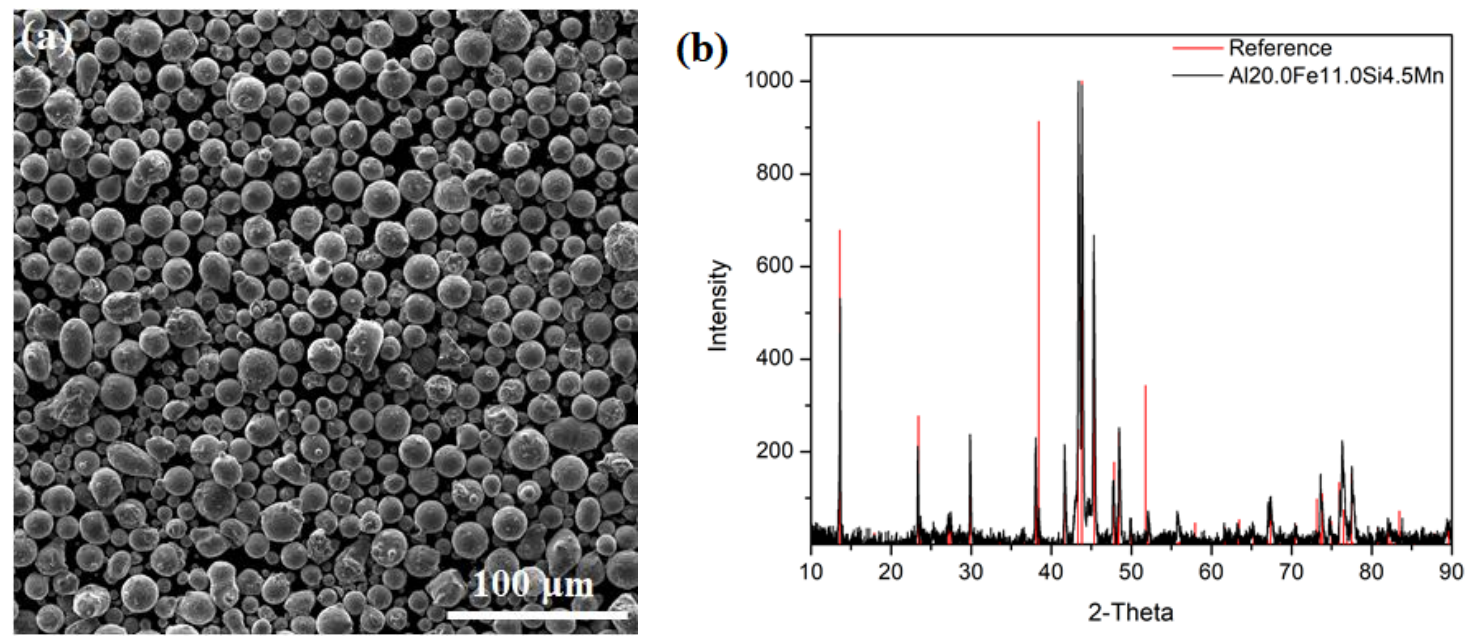

Figure I.1.2.7. (a) SEM secondary electron image taken from the powders with a size distribution of 15-53 $\mu \mathrm{m}$. (b) Plot showing the measured dendrite arm spacing and depth of the HAZs in the laser-melted areas. Source: University of Florida.

The powders with a size distribution of $45-150 \mu \mathrm{m}$ were first used for 3D printing to fabricate specimens for mechanical testing and microstructural analysis. Before starting the fabrication of the specimens, optimum printing parameters for this specific alloy need to be explored. Therefore, a design of experiment approach with different laser parameters was created, as summarized in Table I.1.2.2, to determine the optimal printing parameter combinations. These parameters were used to print plates with dimensions of $5 \mathrm{~mm} \times 5 \mathrm{~mm} \times$ $1 \mathrm{~mm}$. The eighteen printed samples are shown in Figure I.1.2.8. As can be seen, low laser power and high scan speed were not enough to sinter the powders. On the other hand, high laser power and low scan speed could melt the powders and form some large spheres (i.e., Run Orders 1 and 8). However, these spheres (i.e., Run Orders 3 and 15) were still not completely sintered into dense bulk samples. In the next laser printing experiments, higher laser power $(\geq 750 \mathrm{~W})$ and lower scan speed $(\leq 10 \mathrm{~mm} / \mathrm{s})$ will be tested.

Table I.1.2.2. Laser Parameters Tested on the Al-Fe-Si-Mn Alloy Powders.

\begin{tabular}{|c|c|c|c|c|c|}
\hline Run Order & Laser Power $(\mathbf{W})$ & Scan Speed $\mathbf{( m m} / \mathbf{s})$ & Run Order & Laser Power $\mathbf{( W )}$ & Scan Speed $\mathbf{( m m} \mathbf{s})$ \\
\hline $\mathbf{1}$ & 250 & 30 & 10 & 450 & 20 \\
\hline 2 & 250 & 10 & 11 & 550 & 30 \\
\hline 3 & 550 & 10 & 12 & 450 & 30 \\
\hline 4 & 650 & 20 & 13 & 650 & 10 \\
\hline 5 & 350 & 20 & 14 & 450 & 10 \\
\hline 6 & 350 & 30 & 15 & 750 & 10 \\
\hline $\mathbf{7}$ & 750 & 20 & 16 & 650 & 30 \\
\hline 9 & 250 & 20 & 17 & 550 & 20 \\
\hline
\end{tabular}




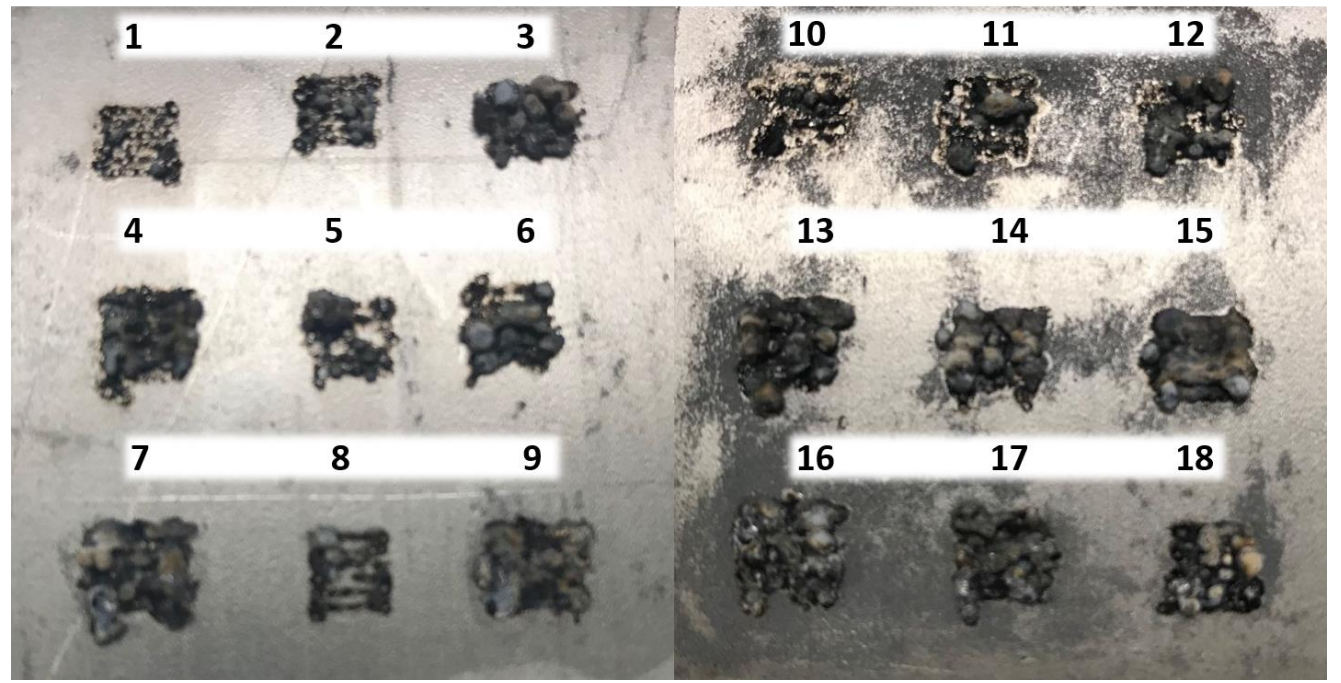

Figure I.1.2.8. Photograph of the eighteen laser printed samples. The numbers correspond to the run order listed in Table I.1.2.2. Source: University of Florida.

\section{Conclusions}

In Budget Period 3, we carried out more detailed investigations to study the stability of Al-Fe-Si-Mn alloys and explore the optimal laser printing parameters to fabricate the components for mechanical testing and microstructural characterization. The main conclusions drawn from this budget period are as follows:

- $\quad \mathrm{Al}-\mathrm{Co}-\mathrm{Fe}$ and $\mathrm{Al}-\mathrm{Cu}-\mathrm{Fe}$ phase diagrams were experimentally determined over the whole composition range. The newly obtained phase equilibrium data are essential for future thermodynamic reassessments of the two systems to improve the current $\mathrm{Al}$ thermodynamic databases for designing Al-Fe-Si-based alloys.

- The stable compositional range of the $\tau_{10}-\mathrm{Al}-\mathrm{Fe}-\mathrm{Si}-\mathrm{Mn}$ single phase was determined to be Al-(18.022.3)Fe-(10.5-14)Si-(3.5-5.5)Mn, at.\%.

- $\quad$ Argon-atomized powders with a nominal composition of $\mathrm{Al}-20.0 \mathrm{Fe}-11.0 \mathrm{Si}-4.5 \mathrm{Mn}$ (in at.\%) were produced for 3D laser printing process.

- Laser power and scan speed were explored to provide a basis for the optimal laser printing parameters for the $\tau_{10}-\mathrm{Al}-\mathrm{Fe}-\mathrm{Si}-\mathrm{Mn}$ intermetallic phase.

\section{Key Publications}

1. Manuel, M. V., S. Soto-Medina, L. L. Zhu, B. Rijal, and R. G. Hennig, 2019, "High-temperature lightweight Al-Fe-Si-based alloys," Invention disclosure, July 2019.

2. Zhu, L. L., S. Soto-Medina, R. G. Hennig, and M. V. Manuel, 2020, "Experimental investigation of the Al-Co-Fe phase diagram over the whole composition range," J. Alloy. Compd., Vol. 815, Art. \#152110.

3. Zhu, L. L., S. Soto-Medina, W. Cuadrado-Castillo, R. G. Hennig, and M. V. Manuel, 2020, "New experimental studies on the phase diagram of the $\mathrm{Al}-\mathrm{Cu}-\mathrm{Fe}$ quasicrystal-forming system," Mater. Design, Vol. 185, Art. \#108186.

4. Zhu, L. L., S. Honrao, B. Rijal, R. G. Hennig, and M. V. Manuel, 2019, "Phase equilibria and diffusion coefficients in the Fe-Zn binary system," Mater. Design, in review.

5. Soto-Medina, S., L. L. Zhu, B. Rijal, R. G. Hennig, and M. V. Manuel, 2019, "Experimental measurement of the phase boundary of the $\tau_{10}-\mathrm{Al}_{4} \mathrm{Fe}_{1.7} \mathrm{Si}$ intermetallic phase," to be submitted. 


\section{References}

1. Zhu, L. L., S. Soto-Medina, R. G. Hennig, and M. V. Manuel, 2020, "Experimental investigation of the Al-Co-Fe phase diagram over the whole composition range," J. Alloy. Compd., Vol. 815, Art. \#152110.

2. Zhu, L. L., S. Soto-Medina, W. Cuadro-Castillo, R. G. Hennig, and M. V. Manuel, 2020, "New experimental studies on the phase diagram of the $\mathrm{Al}-\mathrm{Cu}-\mathrm{Fe}$ quasicrystal-forming system," Mater. Design, Vol. 185, Art. \#108186.

\section{Acknowledgements}

Dr. Daniel Shoemaker at the University of Illinois at Urbana-Champaign is acknowledged for providing feedback on the study of site-lattice occupancy of elements within the $\tau_{10}$ phase. Dr. Bai Cui from the University of Nebraska-Lincoln is acknowledged for exploring AM process to fabricate Al-Fe-Si-X components. The authors also acknowledge Drs. Anil Sachdev and Andrew Bobel at General Motors (GM) for their valuable knowledge gleaned from many discussions. 


\title{
I.1.3 Multiscale Modeling of Corrosion and Oxidation Performance and Their Impact on High-Temperature Fatigue of Automotive Exhaust Manifold Components (Ford Motor Company)
}

\author{
Dr. Mei Li, Principal Investigator \\ Ford Motor Company \\ 2101 Village Rd. RM 2014 \\ Dearborn, MI 18124 \\ E-mail: $\underline{\text { mli9@ford.com }}$
}

\section{Jerry L. Gibbs, DOE Technology Development Manager}

U.S. Department of Energy

E-mail: jerry.gibbs@ee.doe.gov

Start Date: October 1, 2018

Project Funding (FY19): \$2,660,000
End Date: September 30, 2021

DOE share: $\$ 2,080,000 \quad$ Non-DOE share: $\$ 580,000$

\section{Project Introduction}

Corrosion and oxidation of exhaust manifold components made from cast-iron or steel under typical service conditions can be very severe and are a chief concern within the automotive industry, yet an understanding of what causes this remains elusive [1]. This is partly due to the lack of knowledge of failure in structural components having been built upon either corrosion/oxidation or mechanical loads independently. In real applications, however, materials are susceptible to experiencing corrosion/oxidation in synergy with mechanical loads, which is more severe than the effects produced by these two mechanisms independently.

In this project, it is proposed to develop a multiscale computational model to predict:

1. The oxidation/corrosion performance of two different categories of iron-based materials used for exhaust manifold in Advance Combustion Engine and Emission Control (ACEC) gas compositions at two different temperatures (e.g., $805^{\circ} \mathrm{C}$ and $1050^{\circ} \mathrm{C}$ ). This model, spanning from the atom level to the microstructure level, aims to study the oxidation/corrosion susceptibility of materials of interest to ACEC gas, and the diffusion-controlled growth of oxidation/corrosion production.

2. The lives of exhaust manifold components, based on a comprehensive database of high-temperature corrosion/oxidation and fatigue durability data, and a thorough and detailed investigation of the physical mechanisms that cause damage in high-temperature fatigue, with special consideration of the synergy effects of corrosion/oxidation.

\section{Objectives}

- To develop multiscale computational models capable of predicting the location and extent of hightemperature corrosion/oxidation of automotive exhaust manifold materials (e.g., cast steels, Ni-resist, and cast-iron) in ACEC gas compositions for temperature up to $1050^{\circ} \mathrm{C}$.

- To conduct static and thermal cycling corrosion/oxidation trials in controlled atmosphere and demonstrate the model capable of predicting the corrosion/oxidation performance of the exhaust manifold materials exposed to high temperatures and realistic combustion gases to within $10 \%$ of experimental measurements.

- To develop a robust computational model to predict the high-temperature fatigue life of the material under corrosive/oxidizing conditions, and demonstrate the model is capable of predicting the corrosion/oxidation-fatigue performance of exhaust manifold materials exposed to high temperatures and realistic environment to within $10 \%$ of experimental measurements. 
- To demonstrate the predictive utility of the developed corrosion/oxidation-fatigue model in accelerating the development of new exhaust manifold alloys.

\section{Approach}

- Develop atomistic and mechanistic models of the influence of composition, microstructure, and environment on corrosion and oxidation performance of exhaust manifold materials in high-temperature combustion gases.

- Validate the corrosion/oxidation performance models using static and thermal cycling corrosion/oxidation trials in controlled, realistic combustion gases atmosphere.

- Develop and validate the corrosion/oxidation-fatigue interaction model for exhaust manifold components.

- Demonstrate success of the project by quantifying the acceleration of developing new exhaust manifold alloys with computational tools.

\section{Results}

In FY 2019, the corrosion/oxidation performance of high silicon molybdenum (HiSiMo)-type cast-ironincluding based alloys and alloys with alloying elements - in exhaust gas were evaluated from both simulations and experiments.

For the simulation effort, preliminary reactive force field molecular dynamics (ReaxFF MD) [2-5] simulations were performed for $\mathrm{Fe} / \mathrm{X} / \mathrm{Y}$ alloys, where $\mathrm{X}$ and $\mathrm{Y}$ are the promising alloying elements to improve corrosion/oxidation performance, with body-centered cubic (bcc) structures in an ACEC gas environment, using potentials from the literature [6]. ReaxFF MD enables the simulation of breaking and forming bonds during chemical reactions, which describes the case for corrosion/oxidation of Fe-based alloys in an ACEC gas environment. The key features of ReaxFF MD include: (1) smooth transitions from non-bonded to single-, double-, and triple-bonded systems are obtained via a bond length/bond-order relationship, where bond orders are updated every iteration; (2) all connectivity-dependent interactions (i.e., valence and torsion angles, $\mathrm{H}$ bonds) are made bond-order dependent, ensuring that their energy contributions disappear upon bond dissociation; (3) non-bonded interactions (e.g., van der Waals, Coulomb) are calculated between every atom pair, irrespective of connectivity, where excessive close-range non-bonded interactions are avoided by shielding and (4) ReaxFF uses an Electronegativity Equalization Method, a geometry-dependent charge calculation scheme that accounts for polarization effects.

The first ReaxFF MD simulation set was performed for three bcc Fe-based alloys with compositions of pure $\mathrm{Fe}, \mathrm{Fe}-20 \mathrm{at} \% \mathrm{X}-5 \mathrm{at} \% \mathrm{Y}$, and Fe-25at $\% \mathrm{X}-25 \mathrm{at} \% \mathrm{Y}$ under conditions of a constant number of atoms in a constant volume where the temperature is controlled using a thermostat. The total number of atoms for bcc alloys is 2000. The gas environment compositions were obtained from Milella et al. [7] and Gersten et al. [8], which consisted of $300 \mathrm{CO}_{2}$ species, $600 \mathrm{H}_{2} \mathrm{O}$ Species, and $300 \mathrm{O}_{2}$ species. The simulation volume was $30 \AA \times 30 \AA \times$ $100 \AA$, as described as Figure I.1.3.1 (a). The alloys were arranged in the middle of the simulation domain while the gas species were placed at the two ends. Three temperatures $-800 \mathrm{~K}, 1200 \mathrm{~K}$, and $1500 \mathrm{~K}$ - were studied. Based on the number of gas species, 1200, and the size simulation domain, the gas pressure was around 5000 times atmosphere pressure, which is much higher than the actual pressure in an exhaust manifold. Such high-pressure is used to accelerate ReaxFF MD simulation for preliminary testing and will be modified to approach the actual pressure in an exhaust manifold later. To study the influence of crystal structure on the corrosive/oxidation performance, the ReaxFF MD simulation was carried out for face-centered cubic (fcc) Febased alloy with composition of Fe-25at $\%$ X-25at\% Y as well. The total number for the fcc alloy is 4000 . The simulation setting for the fcc alloy is similar to that for the bcc alloys, as shown in Figure I.1.3.1 (b). The appearance if greater density for the fcc structure is expected because the fcc structure has denser packing than bcc structure. This was the initial setting for the simulation. 


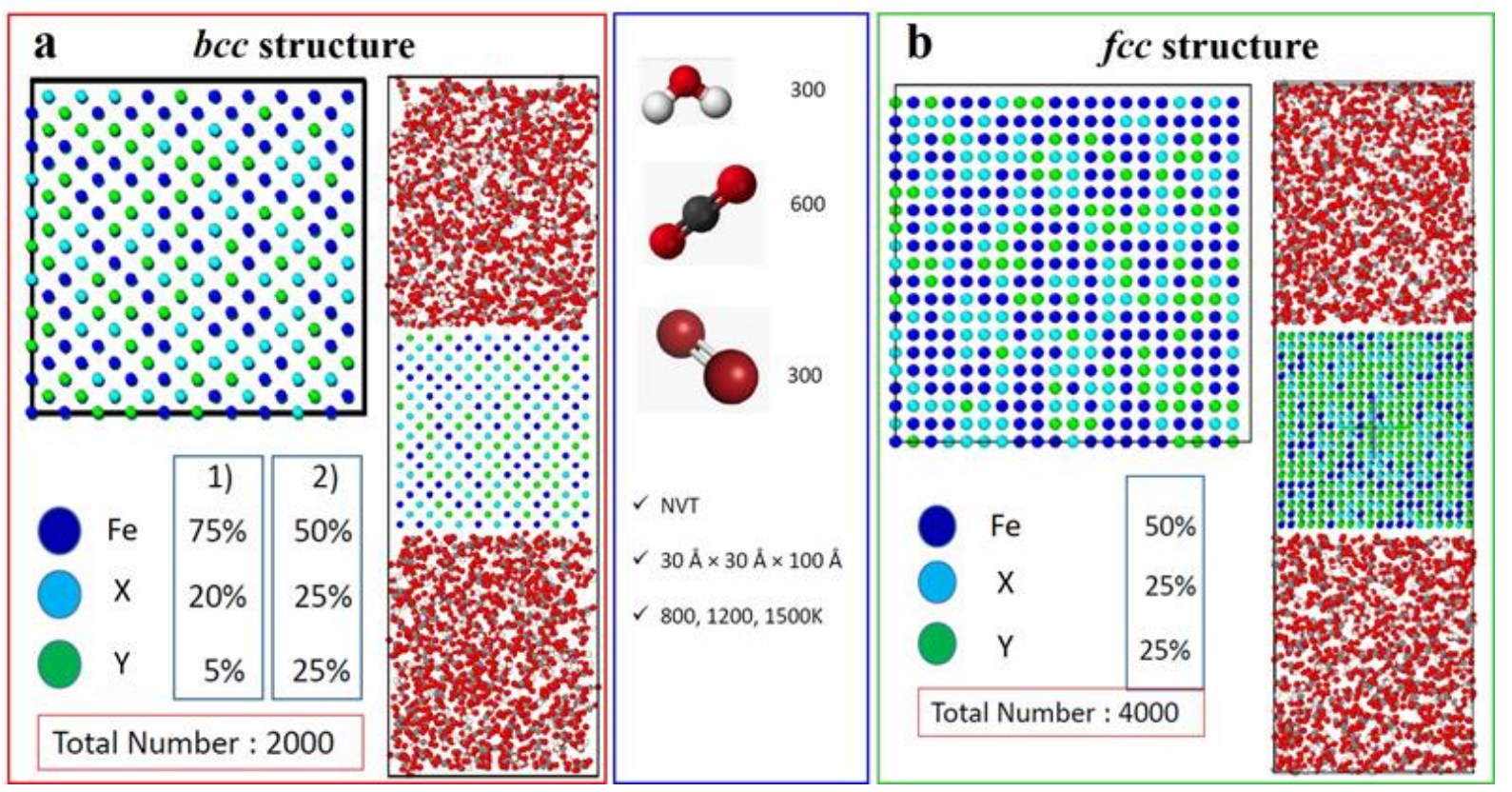

Figure I.1.3.1. Graphical representation of initial ReaxFF MD simulation setting for: (a) bcc alloys; and (b) fcc alloys showing system sizes, gas environment, and temperatures. Source: The Ohio State University.

Figure I.1.3.2 (a) illustrates the scale formation with temperatures for bcc alloys with composition of Fe$20 \mathrm{at} \% \mathrm{X}-5 \mathrm{at} \% \mathrm{Y}$ in the gas environment described previously. The snapshots for configuration and stress at three different temperatures were taken from ReaxFF MD simulation at a time of $300 \mathrm{ps}$. Indications are that thicker oxidation layers and higher stresses are built up for higher temperatures at the same time. In addition, for the same composition (e.g., Fe-25at\%X-25at\%Y), the fcc structure has a higher stress level than compared to the bcc structure on the oxide scale based on the ReaxFF MD simulation at the same temperature and simulated time - 300 ps — as indicated in Figure I.1.3.2 (b). Finally, ReaxFF MD simulation at 300 ps indicates that additions of $\mathrm{X}$ and $\mathrm{Y}$ are able to improve the corrosion/oxidation performance of the bec structure since thinner oxide scale and lower stress were observed for Fe-25at\%X-25at\%y alloys than pure Fe, as shown in Figure I.1.3.2 (c). 

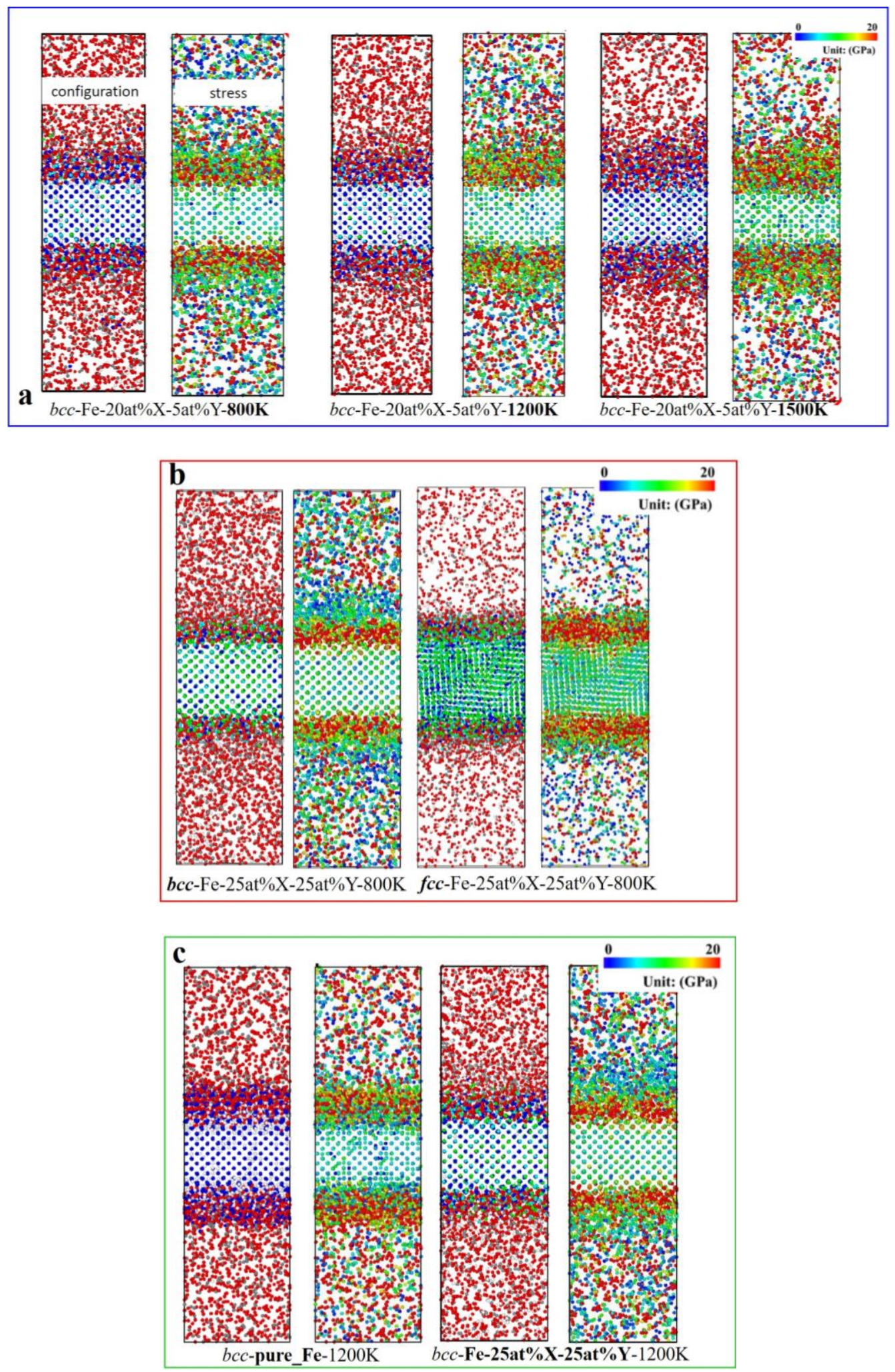

Figure I.1.3.2. Graphical representation of the configurations and stress conditions after 300 ps ReaxFF MD simulation for (a) bcc-Fe-20at\%X-5at\%Y at different temperatures; (b) bcc- and fcc-Fe-25at\%X-25at\%Y at 800K; and (c) pure bcc-Fe and bcc-Fe-25at\%X-25at\% $Y$ at $1200 \mathrm{~K}$ showing the influence of alloying elements, $X$ and $Y$, on corrosion and oxidation performance for Iron with the bcc structure in exhaust gas environment. Source: The Ohio State University. 
Based on the ReaxFF MD simulation results, it is realized that the oxidation scales-including $\mathrm{Fe}_{2} \mathrm{O}_{3}, \mathrm{X}_{2} \mathrm{O}_{3}$, and $\mathrm{Y}_{2} \mathrm{O}_{3}$ - exhibit non-order structures. This is inconsistent with experimental observations in which the oxidation scale always has periodic crystal structures. The stability of the oxidation scales in an ACEC gas environment need to be studied by ReaxFF MD simulation, which will provide critical validation for force field used in simulation. The three oxidation scales with corresponding crystal structures were used as an initial condition. After $1 \mathrm{~ns}$ of MD simulation, however, all three oxidation scales lost their periodic structure and transformed to amorphous structures as shown in Figure I.1.3.3. Therefore, more robust potentials to describe the force field of the oxidation scale are seriously needed. The next step is to develop such potentials using density function theory. In addition, another simulation method, the potential energy landscape, will be utilized as well to access the developed potential and provide insights for corrosion/oxidation along grain boundaries.

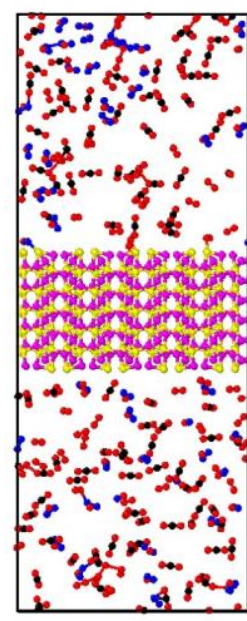

initial

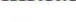

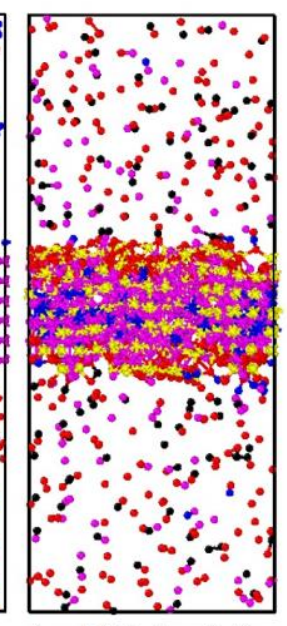

1ns MD simulation

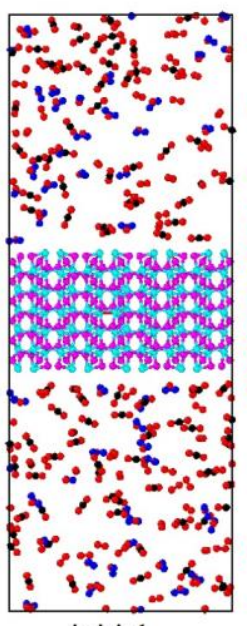

initial

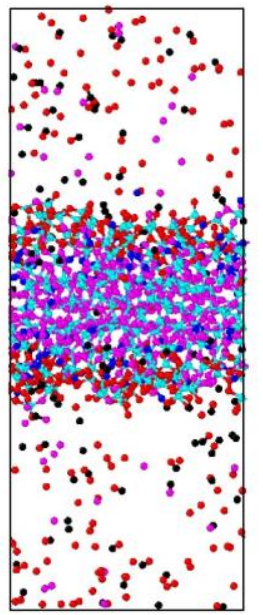

lns MD simulation

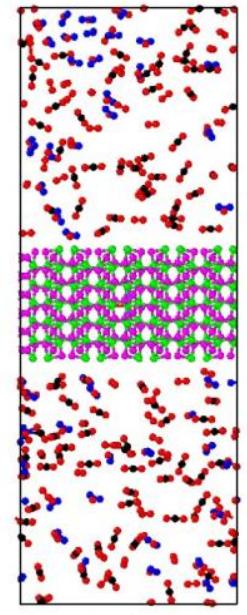

initial

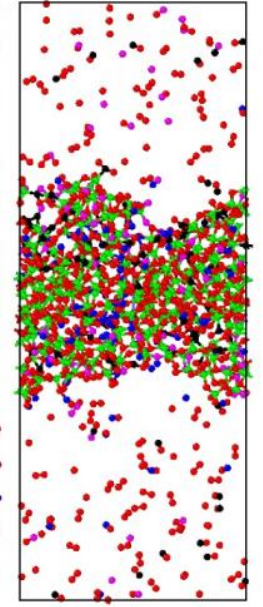

1ns MD simulation

$\mathrm{O}$ (oxidation products)

$\mathrm{Fe} \bigcirc \mathrm{X}$

Y

Figure I.1.3.3. Graphical representation of the transformation of the oxidation scales from crystalline structures to amorphous 1 ns ReaxFF MD simulation showing that the employed force field needed to be improved.

Source: The Ohio State University.

Progress was made on experimental evaluation of the corrosion/oxidation behavior of base HiSiMo-type castiron in combustion gas. The static oxidation experiments for base HiSiMo-type alloys were conducted in a combustion atmosphere similar to the exhaust gas of diesel engine. A sealed horizontal tube furnace with a two-zone temperature control was employed. Temperature stability was verified by thermocouples inserted inside into tube next to samples within $+/-2^{\circ} \mathrm{C}$. High-precision four-channel mass flow meters $(0-500 \mathrm{ml} / \mathrm{min})$ were used to premix $\mathrm{CO}_{2}, \mathrm{~N}_{2}$, and $\mathrm{O}_{2}$. An oxygen meter was used to verify the $\mathrm{O}_{2}$ level. Water vapor percentage was controlled by temperature in a bubbling vaporizer. The gas humidity was determined from the time at which the gas passed through the water vaporizer. Also, the total water vaporized was measured by change water weight during the experiment. Samples with a dimension of $36 \mathrm{~mm} \times 12 \mathrm{~mm} \times 5 \mathrm{~mm}$ were placed in several open alumina crucibles, and the system was purged for $30 \mathrm{~min}$ before the start of the heating cycle. Applied gas atmosphere was $68 \mathrm{vol} \% \mathrm{~N}_{2}, 15 \mathrm{vol} \% \mathrm{CO}_{2}, 2$ vol\% $\mathrm{O}_{2}$, and 15 vol\% $\mathrm{H}_{2} \mathrm{O}$. The samples were oxidized in a two-zone tube furnace at $700^{\circ} \mathrm{C}, 750^{\circ} \mathrm{C}$, and $800^{\circ} \mathrm{C}$ where a synthetic combustion atmosphere similar to exhaust gas of diesel engine, consisting of $68 \mathrm{vol} \% \mathrm{~N}_{2}, 15 \mathrm{vol} \% \mathrm{CO}_{2}, 2 \mathrm{vol} \% \mathrm{O}_{2}$, and $15 \mathrm{vol} \% \mathrm{H}_{2} \mathrm{O}$, was applied. 
First, the total weight changes, $\Delta \mathrm{G}_{\text {total }}$, were measured, as shown in Figure I.1.3.4 (a). The mass changes associated with oxidation, $\Delta \mathrm{G}_{\mathrm{o}}$, shown in Figure I.1.3.4 (c), were then evaluated by subtracting the weight loss due to de-carbonization, $\Delta \mathrm{G}_{\text {De-C}}$, shown in Figure I.1.3.4 (b), from the total weight change. The inserted SEM imaging in Figure I.1.3.4 (b) illustrates an example of de-carbonization at the surface. It is indicated that the total weight changes for HiSiMo-type alloys at $700^{\circ} \mathrm{C}, 750^{\circ} \mathrm{C}$ shows a monotonic increase with time, and at $800^{\circ} \mathrm{C}$, exhibits a parabolic behavior. The de-carbonization measured as a total decrease of $\mathrm{C} \%$ is very significant at $800^{\circ} \mathrm{C}$, compared with $700^{\circ} \mathrm{C}$ and $750^{\circ} \mathrm{C} . \Delta \mathrm{G}_{\mathrm{o}}$ was calculated and presented as grams $(\mathrm{g}) /$ area of the sample surface. Oxidation increased with time, but it was not linear with temperature. This could be related to $\mathrm{O}$ and $\mathrm{C}$ diffusion in fcc and bcc iron or other causes, such as the stability of oxide (spalling) or developing silica protection sublayer. This will be studied in the future.
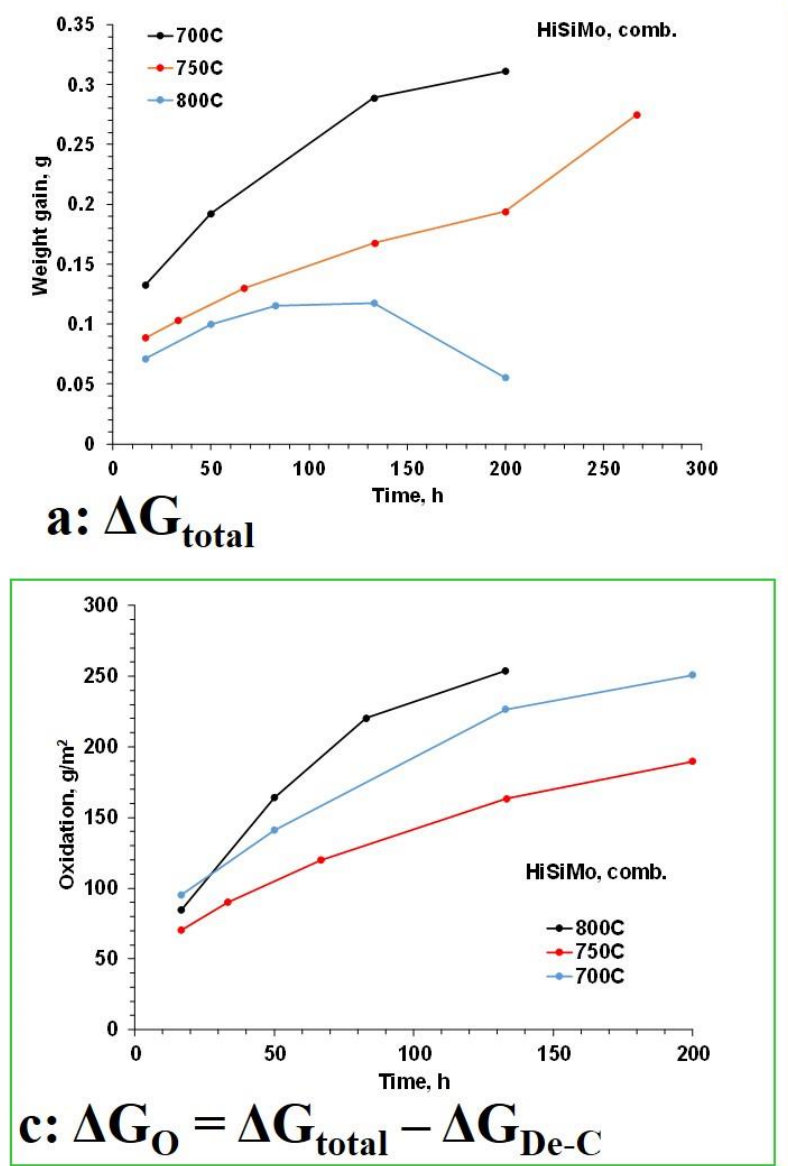

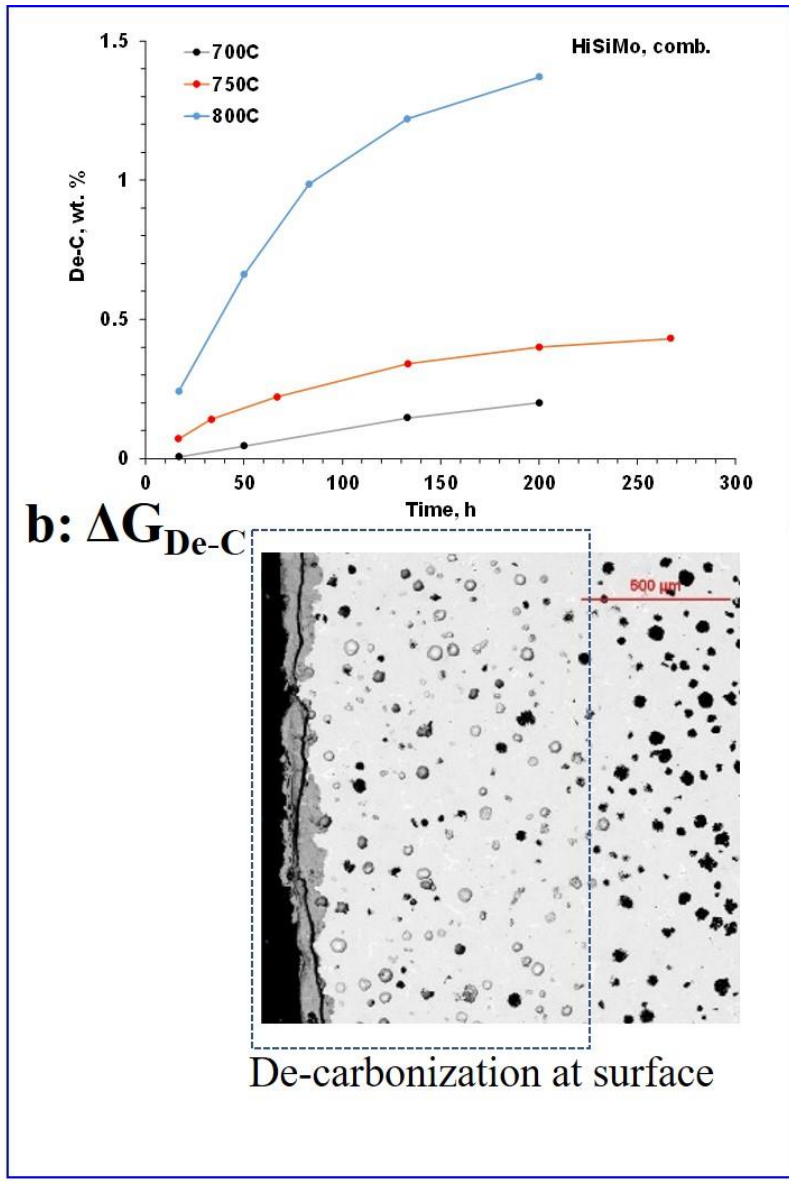

Figure I.1.3.4. (a) The total weight changes; (b) the weight loss due to de-carbonization; and (c) the mass changes associated with oxidation for base HiSiMo-type alloys showing that the oxidation behavior at different temperatures. Source: Missouri Science and Technology.

Second, the surface topology of the HiSiMo-type cast-iron after $16 \mathrm{~h}$ in a combustion atmosphere at $700^{\circ} \mathrm{C}$ and $800^{\circ} \mathrm{C}$ was characterized by 3D optical profiler and SEM imaging as shown in Figure I.1.3.5 (a). Indications were that roughness at $800^{\circ} \mathrm{C}$ increased 5 times when compared to $700^{\circ} \mathrm{C}$. In addition, the oxidized surfaces were also characterized by SEM imaging. The scale cross-section at $700^{\circ} \mathrm{C}$ and $800^{\circ} \mathrm{C}$ for 16 and $100 \mathrm{~h}$ are shown in Figure I.1.3.5 (b). With an increase in exposure time, more complicated layer structures that consist of several sublayers were developed for samples at $700^{\circ} \mathrm{C}$. The de-carbonization layer thickness increases with exposure time for $800^{\circ} \mathrm{C}$ as well. 

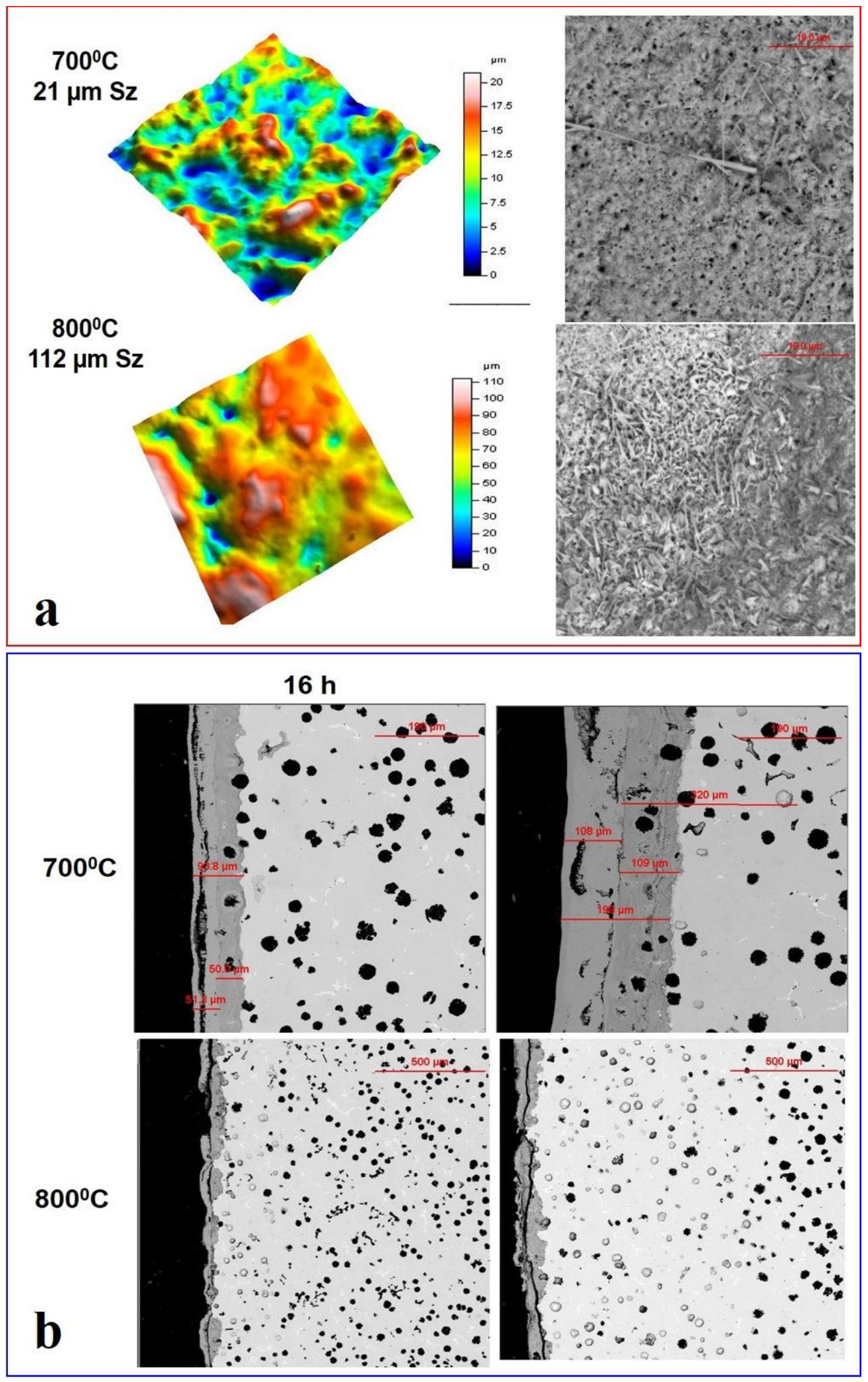

Figure I.1.3.5. Graphical representation of: (a) surface topology; and (b) cross-section of base HiSiMo-type alloys at in a combustion atmosphere at $700^{\circ} \mathrm{C}$ and $800^{\circ} \mathrm{C}$ showing the oxidation behavior at different temperatures.

Source: Missouri Science and Technology. 
Finally, focused ion beam (FIB)/transmission electron microscopy (TEM) was employed to study the structures of oxidation layers. Figure I.1.3.6 shows a multilayer structure consisting of metal-matrix, silica, and Fe oxide in the samples that were oxidized in a combustion atmosphere at $750^{\circ} \mathrm{C}$ for $100 \mathrm{~h}$. In the metalmatrix, a MnS boundary precipitated, which was identified by the selected area diffraction pattern in Figure I.1.3.6, and formed a nearly oxidized surface indicating Mg diffusion out to the boundary, leaving free $\mathrm{S}$ to react with less strong sulfide in the former MnS. This indicated a possibility of different exchange reactions near the oxidized surface. Si formed an amorphous sublayer with a thickness of $0.5-0.7$ micron. In addition, a visible gap was developed between metal-matrix and silica amorphous layer, which indicates an irregular coverage of the surface in a combustion atmosphere.

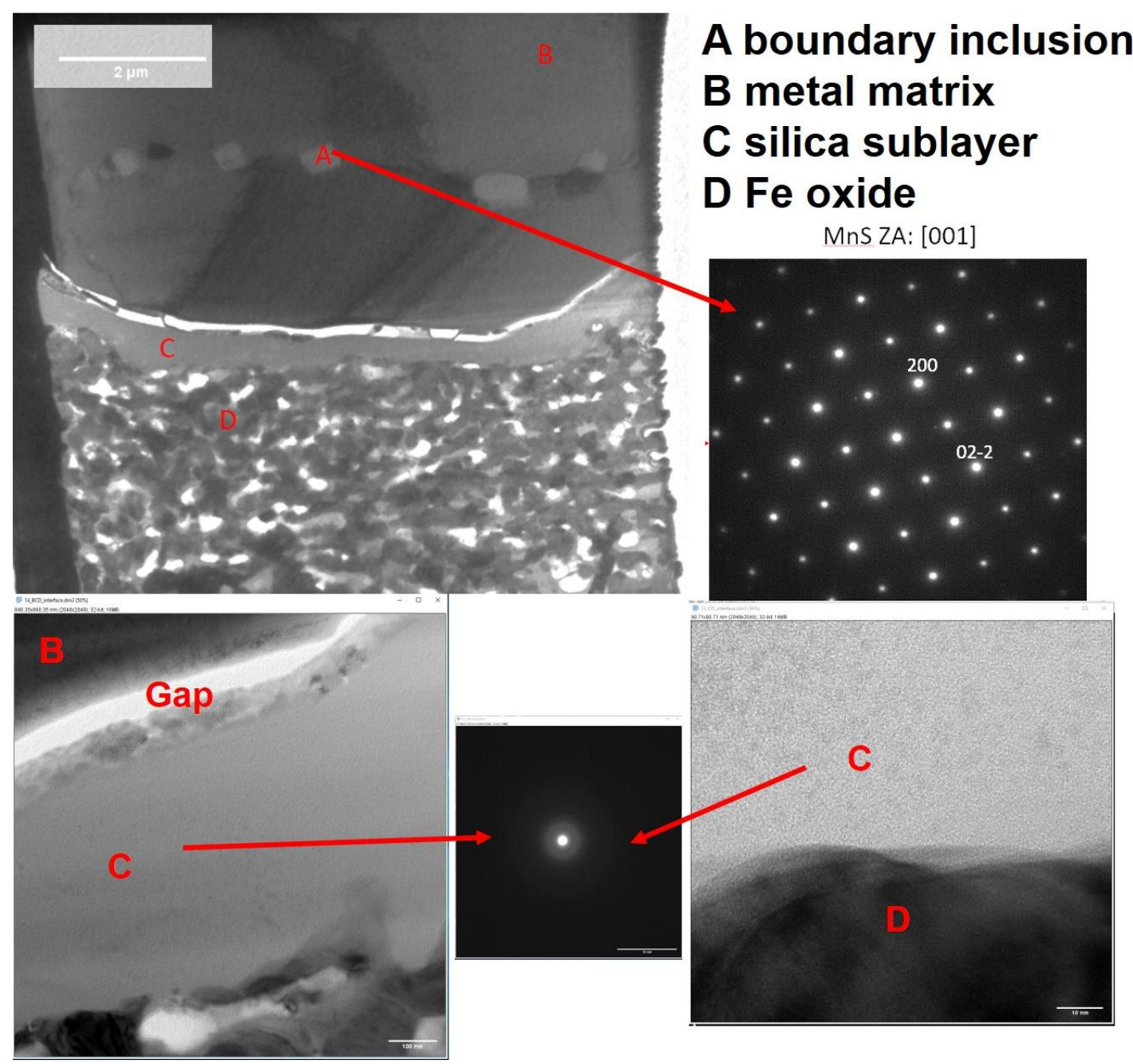

Figure I.1.3.6. FIB/TEM imaging showing the oxidization layer structure for HiSiMo-type alloys in a combustion atmosphere at $750^{\circ} \mathrm{C}$ for $100 \mathrm{~h}$. Source: Missouri Science and Technology.

The experimental investigation of the corrosion/oxidation performance of based HiSiMo-type cast-iron in exhaust gas was completed. For future research, alloying elements will be added to assess if they can improve the corrosion/oxidation performance of HiSiMo-type cast-iron. In addition to the static oxidation experiments, thermal cycling experiments with a temperature range from $500-800^{\circ} \mathrm{C}$ using $1 \mathrm{~h}$ cycle time will be conducted for both base and improved HiSiMo-type cast-iron. The testing environment will include dry air, air $+10 \%$ water vapor and inert gas. Meanwhile, thermomechanical fatigue (TMF) rigs will be equipped with ACEC gas chambers to conduct in situ corrosion/oxidation TMF experiments. 


\section{Conclusions}

The following conclusions are drawn based on both the simulation and experimental results:

The capability of ReaxFF MD to study the corrosion/oxidation performance of cast-iron in exhaust gas were evaluated using potentials from the literature. Some preliminary insights were obtained:

1. The thickness of the oxide layers is around $2 \mathrm{~nm}$ for all the alloys and pure Fe cases. The thickness of the oxidation scales increases with temperature for bcc alloy structures, and the thickness of the oxide scale for the fcc is bigger than for the bcc alloy and pure Fe.

2. The stress of the oxide layer increases with temperature for all the alloys and pure Fe cases.

3. Interestingly, especially for the higher alloying concentration bcc alloy, the stress inside the oxide layer decreased at some locations. This decrease of stress is associated with mole fraction of oxygen among the other gas species. However, the oxidation scales exhibit non-order crystal structures, showing that the force field employed in ReaxFF MD simulation needs to be improved. Therefore, density functional theory will be used to develop a more robust force field.

The corrosion/oxidation performance of based HiSiMo-type cast-iron was studied using several experimental methods, including weight, optical profiler, SEM/EDS, and TEM. In the future efforts, the corrosion/oxidation performance of improved alloys will be evaluated by static and cyclic corrosion/oxidation. In addition, the in situ TMF experiment will be conducted.

\section{References}

1. Ekström, M., 2015, "Oxidation and corrosion fatigue aspects of cast exhaust manifolds," Doctoral dissertation, Stockholm: KTH Royal Institute of Technology.

2. Senftle, T. P., S. Hong, M. M. Islam, S. B. Kylasa, Y. Zheng, Y. K. Shin, C. Junkermeier, R. EngelHerbert, M. J. Janik, H. M. Aktulga, T. Verstraelen, A. Grama, and A. C. T. van Duin, 2016, "The ReaxFF reactive force field: development, applications and future directions," NPJ Computational Materials, Vol. 2, No. 1, Art. \#15011.

3. Jeon, B., Q. van Overmeere, A. C. T. van Duin, and S. Ramanathan, 2013, "Nanoscale oxidation and complex oxide growth on single crystal iron surfaces and external electric field effects," Phys. Chem. Chem. Phys., Vol. 15, pp. 1821-1830.

4. Zou, C., Y. K. Shin, A. C. T. van Duin, H. Fang, and Z.-K. Liu, 2015, "Molecular dynamics simulations of the effects of vacancies on nickel self-diffusion, oxygen diffusion and oxidation initiation in nickel, using the ReaxFF reactive force field," Acta Mater., Vol. 83, pp. 102-112.

5. DorMohammadi, H., Q. Pang, L. Árnadóttir, and O. Burkan Isgor, 2018, "Atomistic simulation of initial stages of iron corrosion in pure water using reactive molecular dynamics," Comp. Mater. Sci., Vol. 145, pp. 126-133.

6. Shin, Y. K., H. Kwak, A. V. Vasenkov, D. Sengupta, and A. C. T. van Duin, 2015, "Development of a ReaxFF reactive force field for $\mathrm{Fe} / \mathrm{Cr} / \mathrm{O} / \mathrm{S}$ and application to oxidation of butane over a pyrite-covered $\mathrm{Cr}_{2} \mathrm{O}_{3}$ catalyst," ACS Catal., Vol. 5, No. 12, pp. 7226-7236.

7. Milella, P. P., 2013, Fatigue and Corrosion in Metals, Springer Verlag, Italy, pp. 771-806.

8. Gersten, J. I., and F. W. Smith, 2001, The Physics and Chemistry of Materials, John Wiley \& Sons, Inc., New York, NY, USA, pp. 344-347. 


\title{
I.1.4 Machine-Learning and Supercomputing to Predict Corrosion/Oxidation of High- Performance Valve Alloys (Oak Ridge National Laboratory)
}

\author{
Scott Whalen, Principal Investigator \\ Oak Ridge National Laboratory \\ 1 Bethel Valley Rd. \\ Oak Ridge, TN 37831 \\ E-mail: shind@ornl.gov
}

\section{Jerry L. Gibbs, DOE Technology Development Manager}

U.S. Department of Energy

E-mail: jerry.gibbs@ee.doe.gov

Start Date: October 1, 2018

Project Funding (FY19): \$500,000
End Date: September 30, 2021

DOE share: $\$ 500,000 \quad$ Non-DOE share: $\$ 0$

\section{Project Introduction}

This project aims to develop practical and highly accurate machine-learning models that can rapidly evaluate corrosion/oxidation of complex, multicomponent high-performance valve alloys for turbocharged engines. We will leverage high quality experimental nickel-chromium $(\mathrm{NiCr})$-based alloy oxidation data that has been systematically obtained over decades at Oak Ridge National Laboratory (ORNL). Trained machine-learning models will provide predictive capability in evaluating corrosion/oxidation of high-temperature alloys, without performing computationally extensive simulations, with an agreement with experimental results within $10 \%$ accuracy. Available supercomputing resources at ORNL will enable the rapid population of a large volume of scientific alloy features and fundamental data from physics-based simulations. A generated synthetic alloy feature dataset (e.g., depletion of critical alloying elements, oxygen permeabilities within the alloy matrix, etc.) will augment the 'raw' experimental dataset and help us to better understand underlying mechanisms, as well as to improve the accuracy of machine-learning models. Predicted corrosion/oxidation behavior of NiCr-based alloys will be experimentally validated via both lab-scale and real engine/rig testing.

\section{Objectives}

1. Data Analytics. The objective of this study is to use data analytics (i.e., correlation analysis and machinelearning) to predict the cyclic oxidation resistance of $\mathrm{NiCr}$ alloys.

2. Physics-based Alloy Oxidation Modeling. The objective of this study is to model oxidation kinetics and oxidation-induced compositional changes in the alloy to provide the machine-learning procedures with physics-based parameters/descriptors.

\section{Approach}

\section{Data Analytics}

The experimental data analyzed in this work were consistently collected from cyclic oxidation experiments performed at ORNL. The selected dataset covers $29 \mathrm{NiCr}$-based alloys with up to 23 elements at temperatures of $800,850,900,950$, and $1000^{\circ} \mathrm{C}$. These experimental data are divided into two databases based on the experimental atmosphere (i.e., air with water vapor [database A] and dry air [database B]). The parabolic rate constant, $\mathrm{k}_{\mathrm{p}}$, was calculated using the $\mathrm{p}-\mathrm{k}_{\mathrm{p}}$ model [1] (denoted as pkp_ $\mathrm{k}_{\mathrm{p}}$ hereafter). The complete experimental data were used to calculate the pkp_k $\mathrm{k}_{\mathrm{p}}$ and the influence of $\mathrm{pkp} \_\mathrm{k}_{\mathrm{p}}$ on the performance of machine-learning models was compared. 


\section{Physics-based Alloy Oxidation Modeling}

The following approach was chosen to achieve the project objective:

- Assimilation of existing oxidation data for relevant alloys in a single database

- Development of an oxidation model to describe oxide growth and oxide loss kinetics (oxide spallation due to cycling and oxide evaporation due to moisture)

- Performance of coupled thermodynamic-kinetic simulations to predict the compositional evolution in the alloys.

\section{Oxidation Model}

Several models have been proposed in the literature to describe the oxidation kinetics of high-temperature materials [2-4]. However, the complexity of considering oxide growth and oxide loss due to spallation during thermal cycling and oxide evaporation of the chromia scale in wet atmospheres in a single model has not yet been dealt with in the literature. An initial attempt was made during the first year of this project to describe oxidation kinetics in the following manner:

$$
\frac{\boldsymbol{d} \boldsymbol{w}}{\boldsymbol{d} \boldsymbol{t}}=\frac{\boldsymbol{d}}{\boldsymbol{d} \boldsymbol{t}}\left(\left(\frac{1}{a}\right) w_{m}\right)-\frac{\boldsymbol{d}}{\boldsymbol{d} t}\left(w_{o x, s p}\right)-\frac{\boldsymbol{d}}{\boldsymbol{d} \boldsymbol{t}}\left(w_{o x, e v}\right)
$$

where $\boldsymbol{w}$ is the total specimen weight change, $w_{m}$ is the weight of the oxidized metal, $w_{o x, s p}$ is the weight of the spalled scale, and $w_{o x, e v}$ is the weight of the evaporated scale. Parabolic growth kinetics were assumed and the parabolic growth constant, $k_{p}$, was estimated from the measured mass change data for the initial 100h. It was assumed based on experimental observations that the effect of evaporation and spallation on the oxide growth was minimal up to $100 \mathrm{~h}$ for most alloys. The evaporation kinetics were estimated based on the thermodynamic data for chromia evaporation in the literature and assuming the boundary layer transport theory [5]. The spallation kinetics were fitted to the observed oxide loss kinetics in dry air (negligible of chromia evaporation).

\section{Coupled Thermodynamic-Kinetic Simulations}

The oxidation-induced compositional changes in alloys were predicted by a coupled thermodynamic-kinetic model [6]. The modeling procedure utilizes commercially available data to calculate thermodynamic phase equilibria as a function of temperature, composition, and kinetic data to determine the evolution in the alloy. All chemical interactions between alloying elements are considered. The recession of the oxide/metal $(\mathrm{O} / \mathrm{M})$ interface due to metal consumption to oxidation is calculated and the computational mesh is accordingly regenerated, thus enabling a more realistic calculation of $\mathrm{Cr}$ depletion profiles. The thermodynamic (Thermotech Ni-based Superalloys Database [TTNI8]) and kinetic databases (Thermo-Calc Software Ni-alloys Mobility Database [MOBNI2]) were employed in the calculations performed for the candidate alloys.

\section{Results}

\section{Data Analytics}

The correlation analysis and machine-learning process were performed using the open-source Advanced data SCiENce toolkit for Non-Data Scientists (ASCENDS), which was recently developed by the ORNL members of this research team. The correlation between the input features and $\log \left(\mathrm{k}_{\mathrm{p}}\right)$ was analyzed using both Pearson's Correlation Coefficient (PCC) and the Maximal Information Coefficient (MIC) [7] analyses. PCC can evaluate the strength of the linear relationship, while MIC can identify the strength of both the linear and nonlinear relationships between the two variables. In this work, both databases were used to train five machine-learning models: (1) linear regression (LR) [8]; Bayesian ridge (BR) [9]; k-nearest neighbor (NN) [10]; random forest (RF) [11]; and support vector machine (SVM) regression. These models were trained with different numbers of top-ranking features based on the MIC or PCC analyses ranking to explore the effects of the input features on the performance of these models. The hyperparameters for each model were tuned up to 
150 iterations to find the optimum parameters. Each model was trained five times for a given number of features to determine an averaged accuracy and its standard deviation.

Figure I.1.4.1 summarizes the results from the correlation analysis between all input features and parabolic rate constant, pkp_kp, from both MIC and PCC analyses using databases A and B. Both of the MIC and PCC analyses identified oxidation-testing temperature, $T$, as the strongest feature influencing the parabolic rate constant, pkp_k $\mathrm{k}_{\mathrm{p}}$, which is in good agreement with the fact that as the oxidation temperature increases, the growth rate of oxides increases as well, consequently resulting in a larger parabolic rate constant. The formation of a continuous chromia $\left(\mathrm{Cr}_{2} \mathrm{O}_{3}\right)$ layer on the alloy surface can significantly reduce the oxidation rate of an alloy, leading to better oxidation resistance; thus, it is reasonable that the present PCC analysis identified $\mathrm{Cr}$ content as the third and second strongest features in databases A (see Figure I.1.4.1 (a)) and B (see Figure I.1.4.1 (b)), respectively. The present results suggest that correlation analysis is an effective approach to evaluate the strength of the correlation between the input features and the parabolic rate constant.
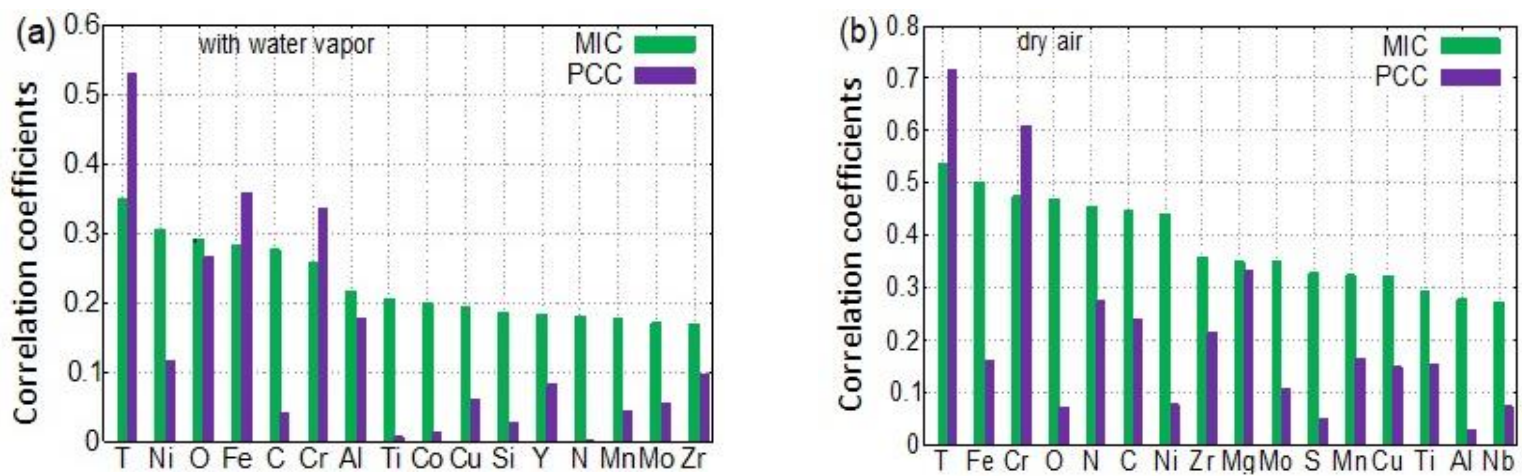

Figure I.1.4.1. The top 15 features determined by correlation analysis between all input features (i.e., oxidation-testing temperature, $T$, and the mass fraction of each element) and pkp_kp using (a) database A; and (b) database $B$.

Source: ORNL.

Figure I.1.4.2 shows the average accuracy of the models and their standard deviation as a function of the numbers of top-ranking features. As shown in Figure I.1.4.2 (a), using database A and increasing the numbers of top-ranking features from five to ten can significantly improve the performance of the BR and SVM models. The SVM model outperforms the other models. With the top 10 or more features, its maximum accuracy can reach 0.58. For database B (see Figure I.1.4.2 (b)), most models achieved the best performance with the top five or ten features. The maximum accuracy of the SVM models is $\sim 0.78$, which is considerably higher than the remaining models. We observed that considering more features in the machine-learning process led to a lower accuracy, which may correlate with the inclusion of more irrelevant features. For database B, the SVM model still has the best performance, while BR follows. 

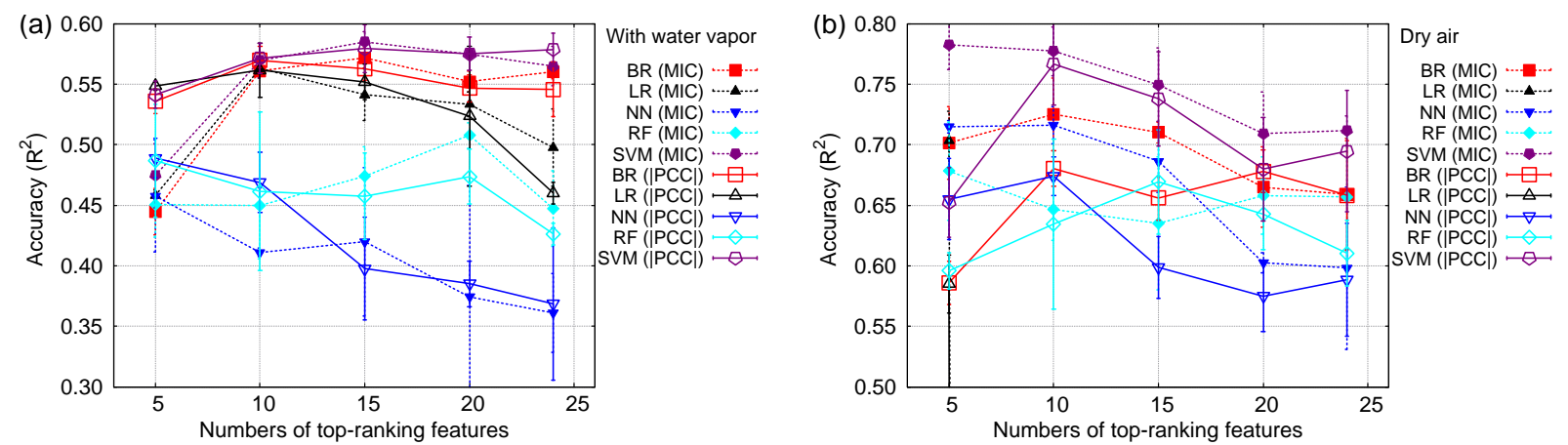

Figure I.1.4.2. Accuracy of five trained machine-learning models with: (a) database A; and (b) database B. The lines connecting the data points are only for visual purposes. Source: ORNL.

\section{Physics-based Alloy Oxidation Modeling}

Figure I.1.4.3 shows the comparison between the measured and calculated mass change for Nimonic ${ }^{\circledR} 80 \mathrm{~A}$ at $900^{\circ} \mathrm{C}$ in $1 \mathrm{~h}$ cycles dry air and $950^{\circ} \mathrm{C}$ in $1 \mathrm{~h}$ cycles air $+10 \% \mathrm{H}_{2} \mathrm{O}$. The model predictions are within the statistical variation of the measured mass changes.

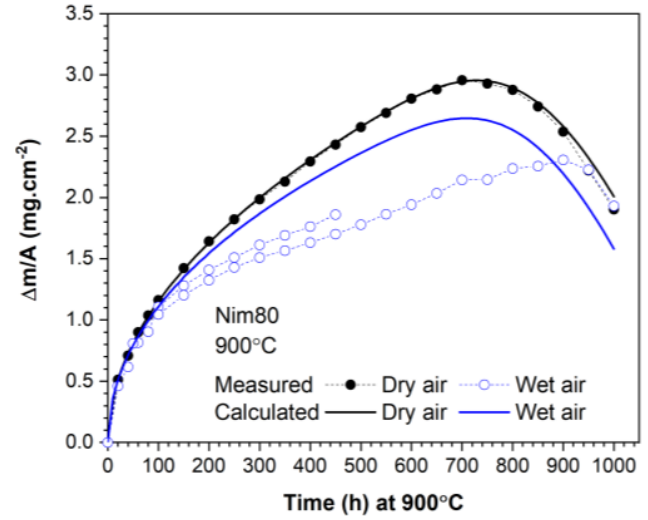

(a)

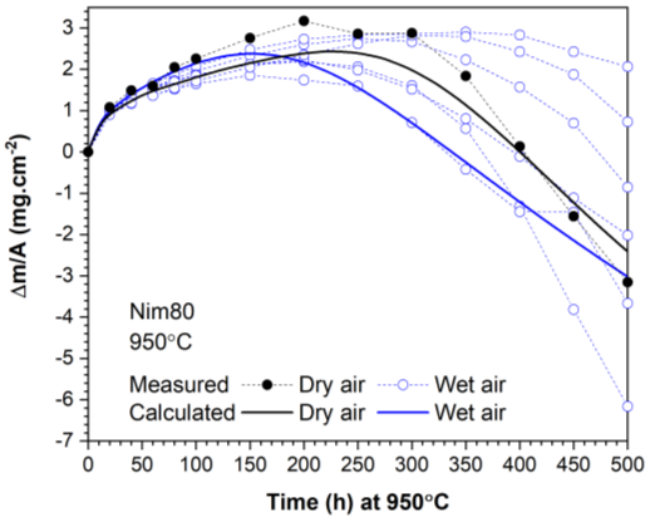

(b)

Figure I.1.4.3. Comparison between measured and calculated mass change for Nimonic ${ }^{\circledR} 80 \mathrm{~A}$ in $1 \mathrm{~h}$ cycles in dry air and air $+10 \% \mathrm{H}_{2} \mathrm{O}$ at: (a) $900^{\circ} \mathrm{C}$; and (b) $950^{\circ} \mathrm{C}$. Source: ORNL.

If the alloys are grouped according to the chemical compositions in Table I.1.4.1, and an attempt is made to predict the oxidation kinetics of the alloys outside the dataset employed to estimate the parameters of the oxidation model $\left(k_{p}, w_{o x, s p}\right)$, an approximate agreement is achieved with experimental observations, as seen in Figure I.1.4.4.

Table I.1.4.1. Grouping of Different NiCr Alloys Based on Their Composition in wt.\%.

\begin{tabular}{|c|c|c|c|c|c|c|c|c|c|c|c|}
\hline Alloy & Ni & Cr & Co & Fe & Al & Ti & Mo & Mn & Si & W & C \\
\hline N90 & Bal. & 19.6 & 16.2 & 0.5 & 1.5 & 2.4 & 0.3 & 0.04 & 0.12 & - & - \\
\hline H282 & Bal. & 19.5 & 10.5 & 0.2 & 1.6 & 2 & 8.4 & 0.06 & 0.05 & - & - \\
\hline R41 & Bal. & 18.4 & 10.4 & 0.08 & 1.6 & 3.3 & 9.8 & - & - & - & - \\
\hline U520 & Bal. & 18.67 & 11.95 & 0.51 & 1.94 & 3.02 & 6.21 & - & - & 1.05 & 0.039 \\
\hline
\end{tabular}




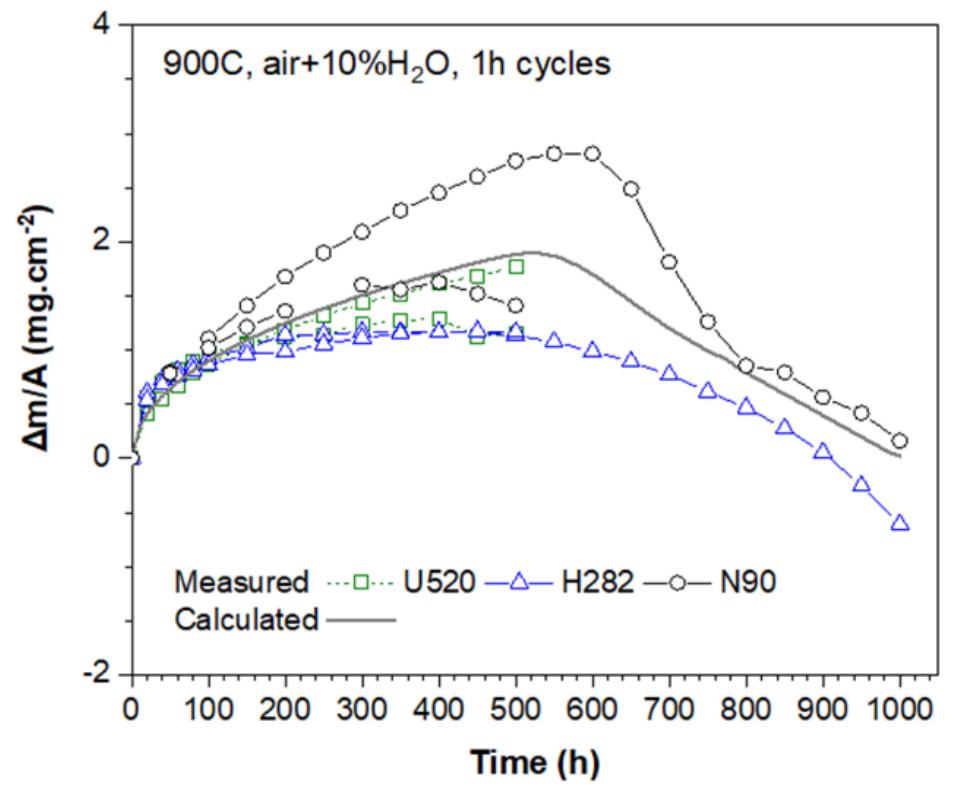

Figure I.1.4.4. Comparison between the measured and calculated mass change for Nimonic ${ }^{\circledR} 90, \mathrm{HAYNES}^{\circledR} 282^{\circledR}$, and Udimet 520 in $1 \mathrm{~h}$ cycles under air $+10 \% \mathrm{H}_{2} \mathrm{O}$ at $900^{\circ} \mathrm{C}$. Source: ORNL.

It has been shown previously that a protective external chromia formation can most likely not be sustained below a critical value of $\mathrm{Cr}$ concentration at the $\mathrm{O} / \mathrm{M}$ interface. The critical value was given to be $10 \mathrm{wt} \%$ for the Ni-base alloys $[4,12]$. The critical $\mathrm{Cr}$ concentration is a key descriptor to transition from protective to non-protective oxidation behavior.

A correlation between $\mathrm{Cr}$ depletion in the alloy and oxidation kinetics can be seen in the case of Nimonic ${ }^{\circledR} 80 \mathrm{~A}$ at $950^{\circ} \mathrm{C}$, as shown in Figure I.1.4.5. The model predicts that the $\mathrm{Cr}$ concentration will drop below $10 \mathrm{wt} . \%$ at $950^{\circ} \mathrm{C}$ in both dry and wet air after about $300 \mathrm{~h}$, as shown in Figure I.1.4.5 (a). This coincides with the time where the specimens experience significant mass loss, as shown in Figure I.1.4.5 (b).

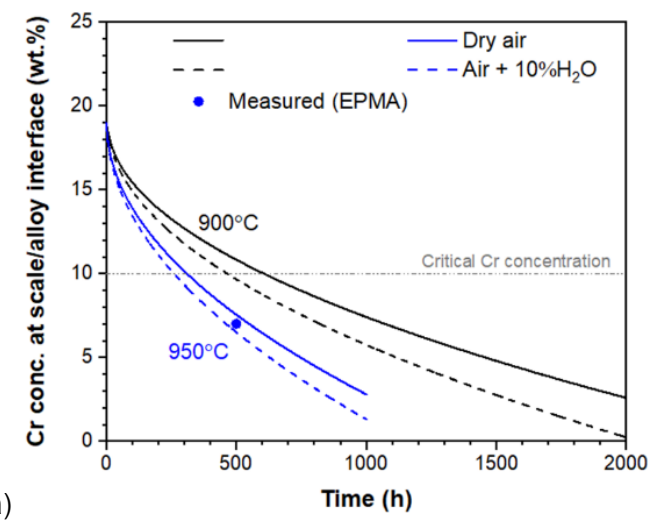

(a)

Figure I.1.4.5. Calculated $\mathrm{Cr}$ concentration at the scale/alloy interface for Nimonic ${ }^{\circledR} 80 \mathrm{~A}$ in $1 \mathrm{~h}$ cycles in dry air and air $+10 \% \mathrm{H}_{2} \mathrm{O}$ at $900^{\circ} \mathrm{C}$ and $950^{\circ} \mathrm{C}$. (a) Symbol represents measured (electron probe microanalysis) Cr concentration.

(b) Measured mass change/area for Nimonic ${ }^{\circledR} 80 \mathrm{~A}$ in $1 \mathrm{~h}$ cycles in air $+10 \% \mathrm{H}_{2} \mathrm{O}$ at $950^{\circ} \mathrm{C}$. Source: ORNL.

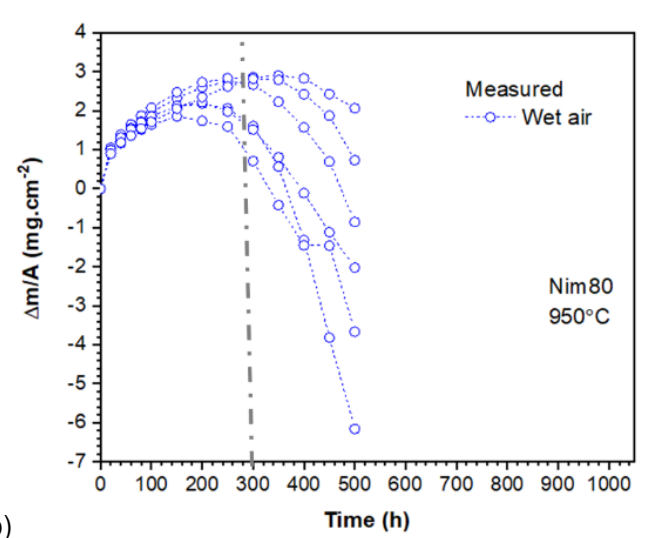

(b) 


\section{Conclusions}

\section{Data Analytics}

In this study, we attempted to represent the oxidation resistance with the parabolic rate constants from the $\mathrm{p}-\mathrm{k}_{\mathrm{p}}$ model and rank their performance on the training of machine-learning models. The performance of the correlation analysis and five machine-learning models was also evaluated. The results show that the highranking features from correlation analysis are in good agreement with the generally accepted oxidation mechanism. This demonstrates that the correlation analysis is an effective approach in evaluating the strength of correlation between the input features, such as oxidation-testing temperatures, compositions of the alloys, and the parabolic rate constant. The numbers of top-ranking features considered in the machine-learning process has a significant influence on the performance of the models. The SVM model outperforms the remaining models (i.e., LR, BR, NN and RF) and can reach an accuracy of $\sim 0.8$ for the database with experimental data collected in dry air. Parabolic rate constants from both oxidation models do not have a significant influence on the performance of the machine-learning models. Further analysis of the experimental data and the present results are planned to improve the performance of the machine-learning models.

\section{Physics-based Alloy Oxidation Modeling}

The developed physics-based oxidation model has provided promising results regarding the capability to predict oxidation kinetics of multicomponent $\mathrm{NiCr}$ alloys as a function of alloy chemistry, time, temperature, and atmosphere. Coupling this model with a thermodynamic-kinetic model enables the prediction of compositional changes in the alloy, thereby allowing a realistic evaluation of the alloy chemistry role on the oxidation-induced lifetime. Further work constitutes optimization of the oxidation model, performing calculations for different alloy chemistries and validating with experimental observations.

\section{References}

1. Poquillon, D., and D. Monceau, 2003, "Application of a simple statistical spalling model for the analysis of high-temperature, cyclic oxidation kinetics data," Oxid. Met., Vol. 59, pp. 409-431.

2. Tedmon, C. S., 1966, "The effect of oxide volatilization on the oxidation kinetics of $\mathrm{Cr}$ and $\mathrm{Fe}-\mathrm{Cr}$ alloys," J. Electrochem. Soc., Vol. 113, pp. 766-768.

3. Lowell, C. E., C. A. Barrett, R. W. Palmer, J. V. Auping, and H. B. Probst, 1991, “COSP: A computer model of cyclic oxidation," Oxid. Met., Vol. 36, pp. 81-112.

4. Duan, R., A. Jalowicka, K. Unocic, B. A. Pint, P. Huczkowski, A. Chyrkin, D. Grüner, R. Pillai, and W. J. Quadakkers, 2017, "Predicting oxidation-limited lifetime of thin-walled components of NiCrW Alloy 230," Oxid. Met., Vol. 87, pp. 11-38

5. Holcomb, G. R., 2008, “Calculation of reactive-evaporation rates of chromia," Oxid. Met., Vol. 69, pp. 163-180.

6. Pillai, R., W. G. Sloof, A. Chyrkin, L. Singheiser, and W. J. Quadakkers, 2015, “A new computational approach for modeling the microstructural evolution and residual lifetime assessment of MCrAlY coatings," Mater. High Temp., Vol. 32, pp. 57-67.

7. Reshef, D. N., Y. A. Reshef, H. K. Finucane, S. R. Grossman, G. McVean, P. J. Turnbaugh, E. S. Lander, M. Mitzenmacher, and P. C. Sabeti, 2011, "Detecting novel associations in large data sets," Science, Vol. 334, pp. 1518-1524.

8. Neter, J., M. H. Kutner, C. J. Nachtsheim, and W. Wasserman, 1996, Applied Linear Statistical Models, Irwin, Chicago.

9. Yu, K., V. Tresp, and A. Schwaighofer, 2005, "Learning Gaussian processes from multiple tasks," Proc. Int. Conf. Mach. Learn., Vol. 22, pp. 1012-1019. 
10. Cherkassky, V., and Y. Ma, "Comparison of model selection for regression," Neural Comput., Vol. 15, pp. 1691-1714.

11. Segal, M. R., 2004, "Machine-learning benchmarks and RF regression," Division of Biostatistics, University of California, San Francisco, CA, pp. 1-14. http://escholarship.org/uc/item/35x3v9t4.pdf.

12. Pillai, R., H. Ackermann, and K. Lucka, 2013, "Predicting the depletion of chromium in two hightemperature Ni-alloys,” Corros. Sci., Vol. 69, pp. 181-190.

\section{Acknowledgements}

The authors acknowledge the roles of our ORNL co-principal investigators: Rishi Pillai, Muralidharan Govindarajan, Bruce Pint, Jay Jun, and Jian Peng. 


\section{I.1.5 Multiscale Development and Validation of the Stainless Steel Alloy Corrosion Tool for High-Temperature Engine Materials (University of Florida)}

\section{Michael Tonks, Principal Investigator}

University of Florida

158 Rhines Hall

Gainesville, FL 32611

E-mail: michael.tonks@ufl.edu

\section{Jerry L. Gibbs, DOE Technology Development Manager}

U.S. Department of Energy

E-mail: jerry.gibbs@ee.doe.gov

Start Date: October 1, 2018

Total Project Funding: \$617,474
End Date: December 31, 2021

DOE share: $\$ 492,474$
Non-DOE share: $\$ 125,000$

\section{Project Introduction}

The environment within an engine cylinder during operation is extremely harsh, with high temperatures, combustion, and corrosive exhaust gases. This environment corrodes engine components, including EVs and EV seats. Current engine materials, such as stainless steels, resist corrosion during normal operation. However, as new engines are pushed to higher temperatures, the material can undergo microstructure evolution that sensitizes the material to corrosion and corrosion fatigue and can result in premature failure, as shown in Figure I.1.5.1.

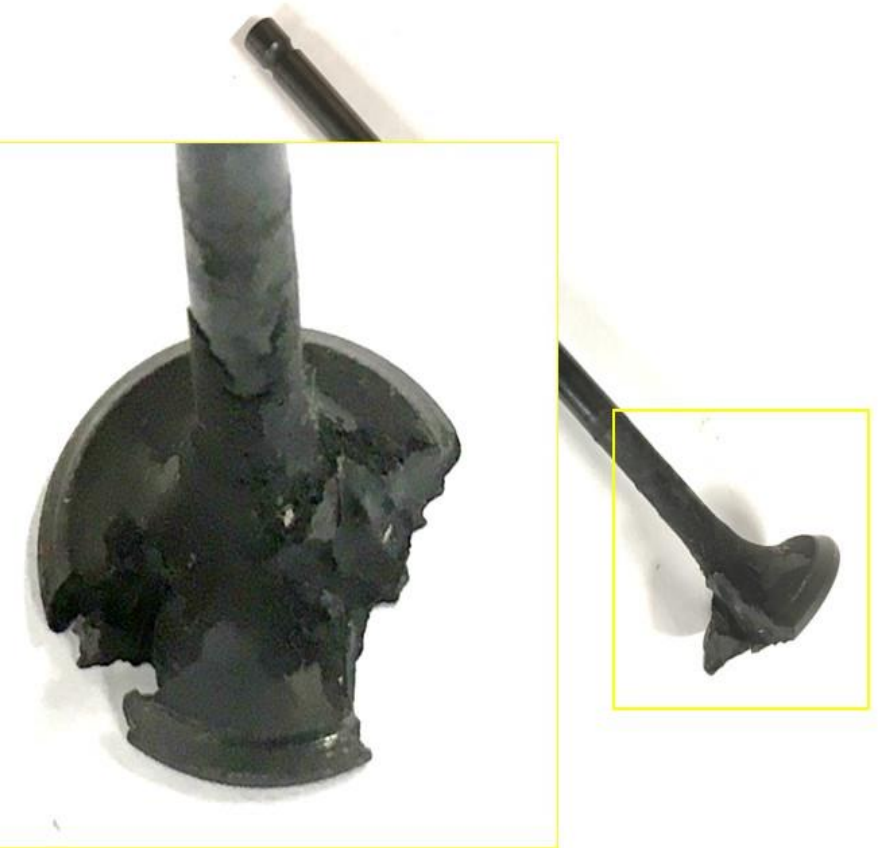

Figure I.1.5.1. Extreme environmental degradation to an alloy 21-4-3 valve head due to local temperature spikes resulting from improper seating. Source: Tenneco.

Companies that design and build engine parts do not typically employ models of corrosion; rather, they employ standard temperature ranges and allowable fuels for which each alloy can be employed when making material selection decisions for a specific part and customer. These standards are based on conservative 
physical properties of the alloy and on successful deployment history. This reliance on conservative standards can result in over-design and increased cost. In addition, if a new advanced engine design results in conditions significantly different from the deployment history, these standards may not be applicable. This can lead to a vastly increased time and cost of development.

\section{Objectives}

The goal of this project is to create a simulation tool to assist in part design, reducing costs by eliminating the need for conservative material selection, and enabling alloy optimization to improve corrosion resistance. Thus, it will help reduce costs for existing engines and will assist in the material and engine design for the engines of the future, reducing the development time by years.

To meet our stated goal, we will develop the SStAC tool, which will:

- Model corrosion of valve steels in an engine environment at temperatures up to $800^{\circ} \mathrm{C}$.

- Be able to run in one dimension for a fast estimate of the corrosion rate and in two or three dimensions for more detailed simulations that represent any part of the geometry and predict the precise location and rate of corrosion and its impact on the mechanical and thermal behavior.

- $\quad$ Be implemented using the Multiphysics Object Oriented Simulation Environment (MOOSE).

- Couple a corrosion model that describes the fundamental mechanisms of corrosion (including the impact of the microstructure and alloy composition) with mechanics and thermal transport models.

At the completion of the project, the SStAC tool will be able to predict the corrosion rate of engine valves with no more than $10 \%$ error.

\section{Approach}

The SStAC tool is being developed using a combination of macroscale model development, mesoscale, and atomic scale simulations to define the impact of microstructure on the alloy corrosion, and an experimental campaign to inform and validate the models. This approach is summarized in Figure I.1.5.2.

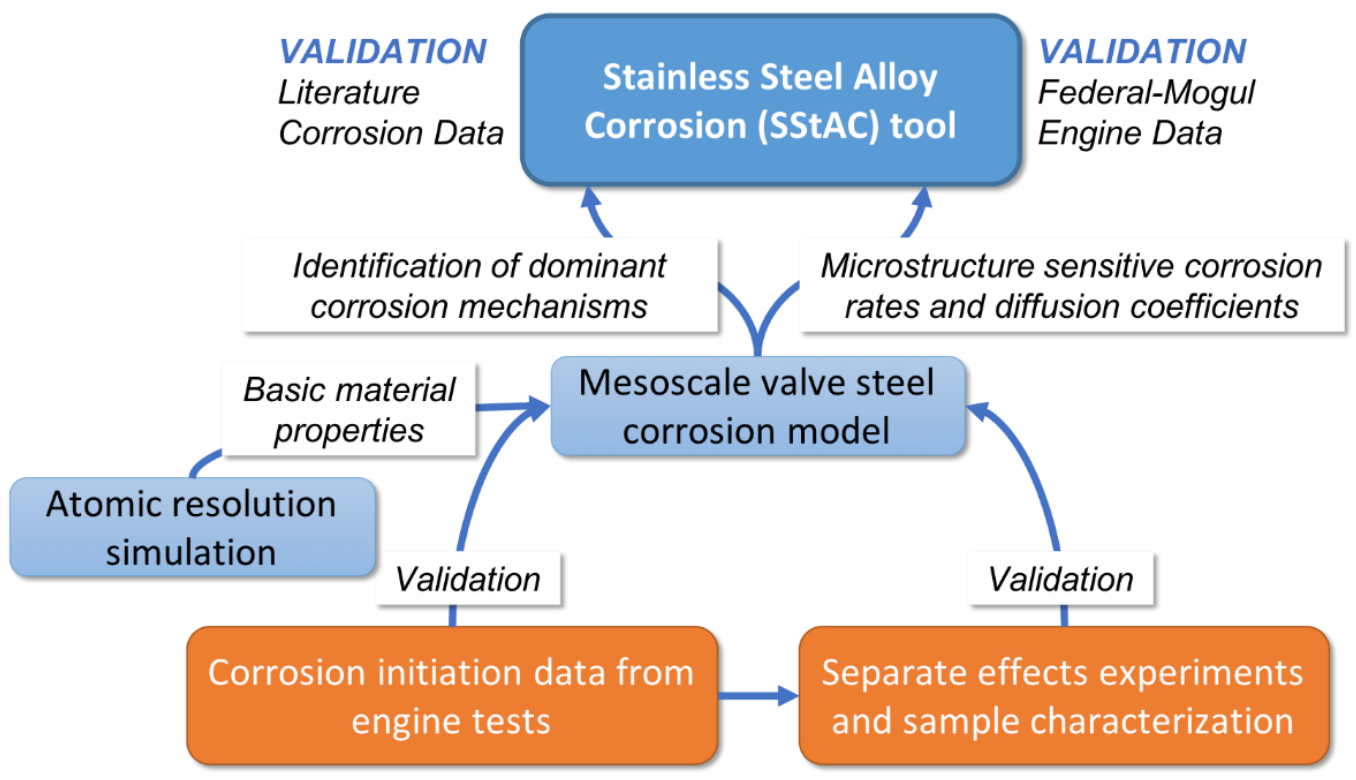

Figure I.1.5.2. Summary of the multiscale approach to be used to develop the SStAC tool. Note both the macro- and meso-scale tools will be built using the MOOSE framework. Source: University of Florida. 
The development of the SStAC tool has been divided into three tasks per year: the first task, to be accomplished in Budget Period (BP)1, is focused on the experiments; the second relates to the atomistic and mesoscale simulations to be completed in BP2; and the last will focus on the development of the macroscale SStAC tool in BP3. The tasks for the first year are:

- $\quad$ Task 1.1 - First Experimental Campaign: Samples of 21-2N and 23-8N valve steel alloys will be exposed to various gaseous compositions at $700^{\circ} \mathrm{C}$ and $800^{\circ} \mathrm{C}$ and corrosion data will be collected. Samples will be characterized before exposure.

- $\quad$ Task 1.2 - Phase-Field Model Development: The phase-field model will be developed, including diffusion, phase changes, and chemical reactions. Density functional theory simulations will be used to calculate steel surface energies and the formation energies of the surface point defects.

- $\quad$ Task 1.3 - Corrosion Model Development: A semi-empirical macroscale corrosion model will be developed by fitting to the experimental data. Two mechanistic models will also be developed - one assuming the transport of charged elements through the oxide layer (n-type) and the other assuming the transport of gas molecules through the oxide layer (p-type). One of these two models will be selected for further development.

\section{Results}

\section{Task 1.1 - First Experimental Campaign}

New experimental corrosion data will be collected in this project for validation of the SStAC tool. As such, a new experimental setup has been installed at the University of Wisconsin - Madison including a gold mirror transparent furnace equipped with a state-of-the-art control evaporation mixer used for well-controlled mixed carbon dioxide and water vapor corrosion experiments, as shown in Figure I.1.5.3. Corrosion experiments are being performed on two types of stainless steel (SS) alloys typically used in thermal engine valves and provided by Tenneco- $-21-2 \mathrm{~N}$ and $23-8 \mathrm{~N}$ — with compositions presented in Table I.1.5.1.

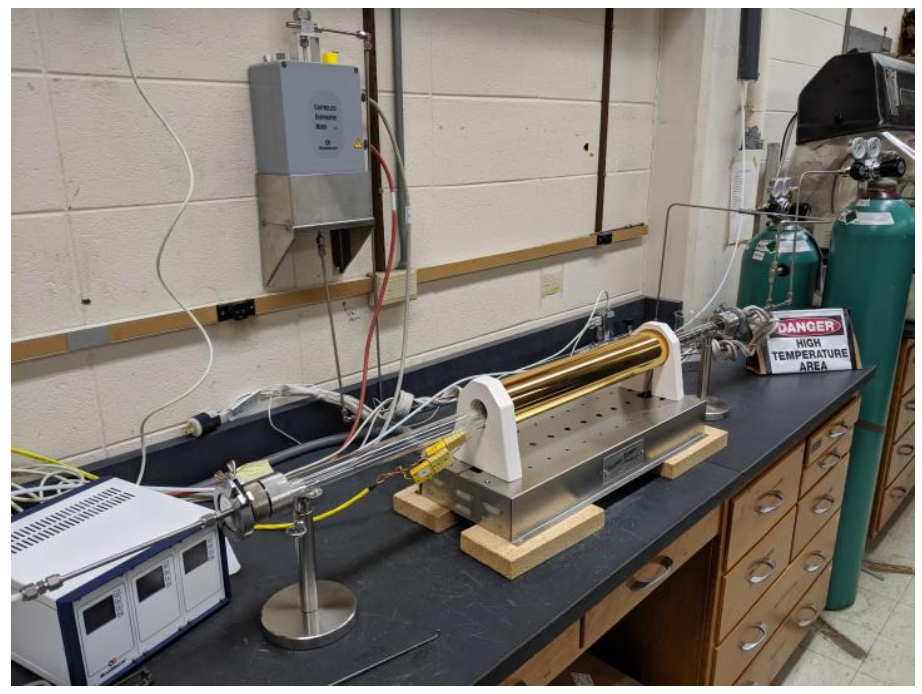

Figure I.1.5.3. High-temperature corrosion experiment. Source: University of Wisconsin-Madison.

Table I.1.5.1. Chemical Composition of the Two Different Studied Alloys 21-2N and 23-8N in wt.\% with Fe in Balance.

\begin{tabular}{|c|c|c|c|c|c|c|}
\hline & Cr & Mn & Ni & C & Mo & Si \\
\hline $\mathbf{2 1 - 2 N}$ & 20.25 & 8.5 & 2.13 & 0.55 & 0.5 & 0.25 \\
\hline $\mathbf{2 3 - 8 N}$ & 23 & 1.5 & 8 & 0.38 & 0.5 & 1 \\
\hline
\end{tabular}


Preliminary microchemical and structural characterization of the as-received SS alloys has been carried out using SEM-EDS and SEM-EBSD spectroscopy and an example is shown in Figure I.1.5.4 (a). Relatively large carbides with an average diameter of $4.02 \mu \mathrm{m}$ are present in the sample. Larger CrxCy carbides are identified by EDS, which is also shown in Figure I.1.5.4 (a). EBSD image quality maps of the two zones of the 21-2N are presented in Figure I.1.5.4 (b) and show the middle of the valve had a higher average grain size $(\sim 4 \mu \mathrm{m})$ than the upper neck $(\sim 1 \mu \mathrm{m})$. The corrosion tests on these two alloys will be conducted in pure carbon dioxide $\left(\mathrm{CO}_{2)}\right.$ and $\mathrm{CO}_{2}+20 \%$ water vapor at $700^{\circ} \mathrm{C}$ and $800^{\circ} \mathrm{C}$ for up to 3000 hours. The corroded samples will undergo the same detailed characterization as the initial samples.
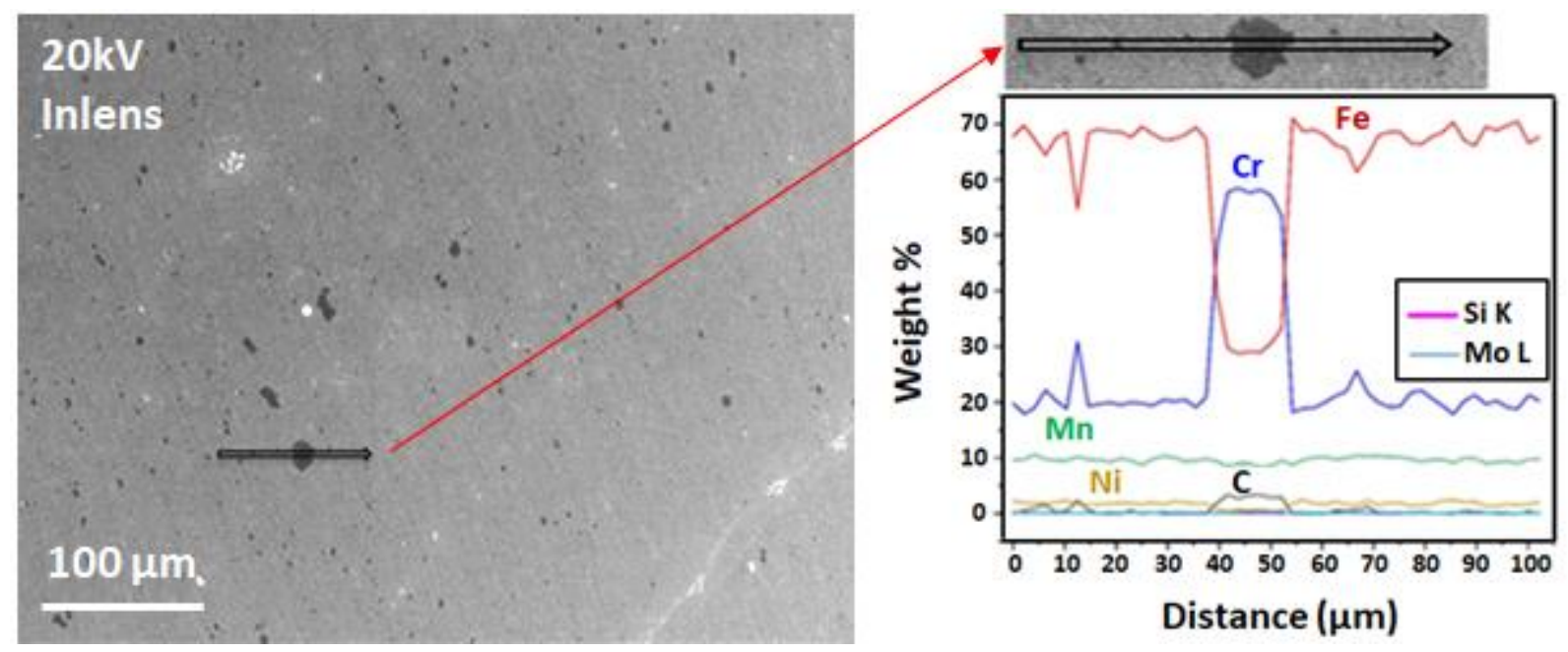

(a)
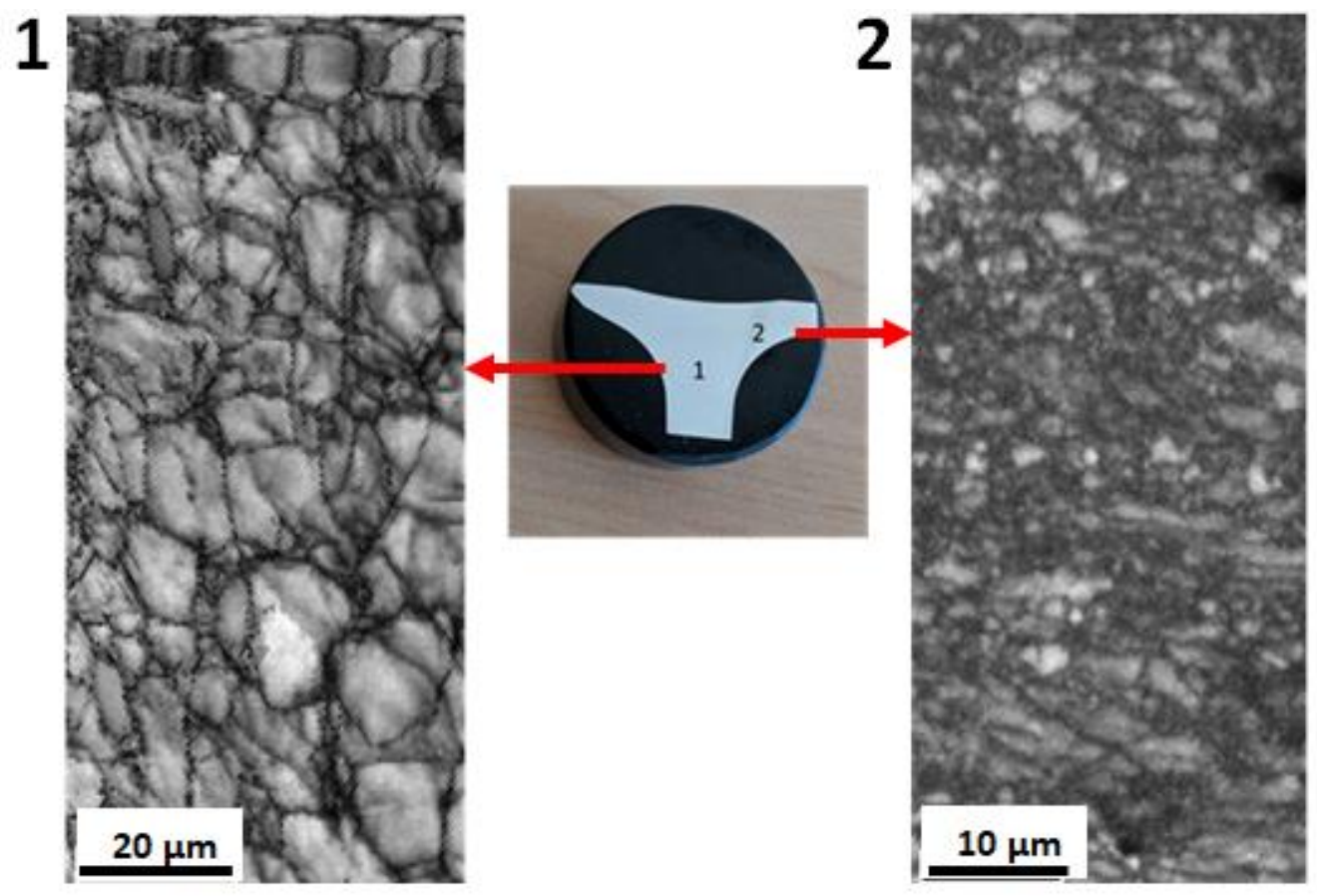

(b)

Figure I.1.5.4. (a) Low magnification SEM image with carbides particles and the corresponding EDS analysis; and (b) image quality maps on two regions of the 21-2N valve. Source: University of Wisconsin-Madison. 


\section{Task 1.2 - Phase-Field Model Development}

\section{Mesoscale Model Development}

A mesoscale phase-field model of the corrosion will explicitly resolve the material microstructure and will be used to quantify the impact of microstructure on the corrosion behavior.

A preliminary phase-field model for Fe corrosion in oxygen has been developed using the MOOSE framework. Three phases are included-Fe, iron oxide $(\mathrm{FeO})$, and the oxygen phase as shown in Figure I.1.5.5. Each phase is represented by a variable, which is equal to 1.0 in the corresponding phase and 0.0 in all other phases, and smoothly transitions between values at the interface between phases. The variables evolve to lower the free energy of the system including the interfacial and bulk energies. The interfacial free energy is a function of the interfacial energy for each interface. The bulk free energy of the metal and oxide phases is a function of the formation energy of oxygen atoms in both structures. The kinetics of the corrosion is controlled by the interface mobility (a function of the reaction rate) and the oxygen atomic mobility (a function of the diffusion coefficient). The interface mobility takes on different values for the various interfaces. Similarly, the diffusion rate takes on different values in the different phases.

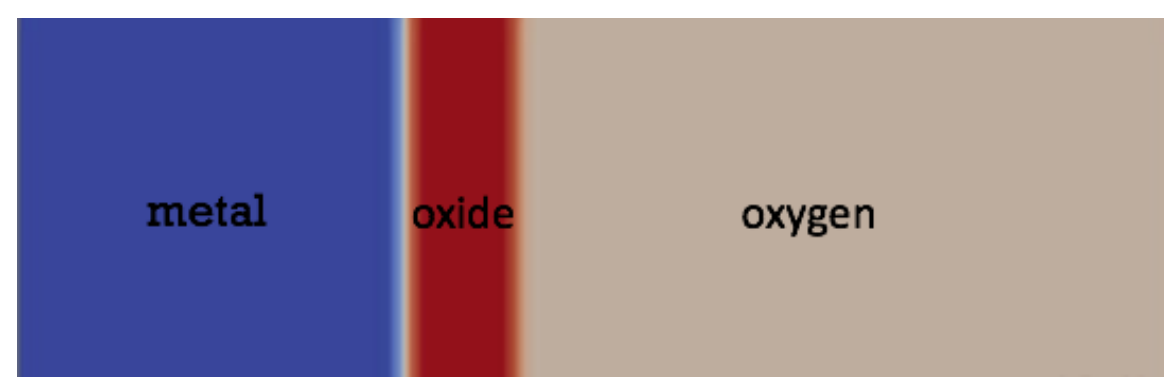

Figure I.1.5.5. Simulation domain of Fe corrosion in oxygen where the phases from left to right are metal, oxide, and oxygen. Source: University of Florida.

The thermodynamic behavior of the system was verified by running the simulations to equilibrium for different initial oxygen concentrations to determine if the equilibrium phase composition predicted by the model is the same as that from a common tangent construct using the free energy curves as shown in Figure I.1.5.6. With the initial oxygen concentration assigned to be between 0.64 and 0.86 , the simulations accurately predicted the $\mathrm{FeO}$ and oxygen phase fractions. We are currently developing a more advanced corrosion model that also includes chromium $(\mathrm{Cr})$ and $\mathrm{Cr}$ oxide. 


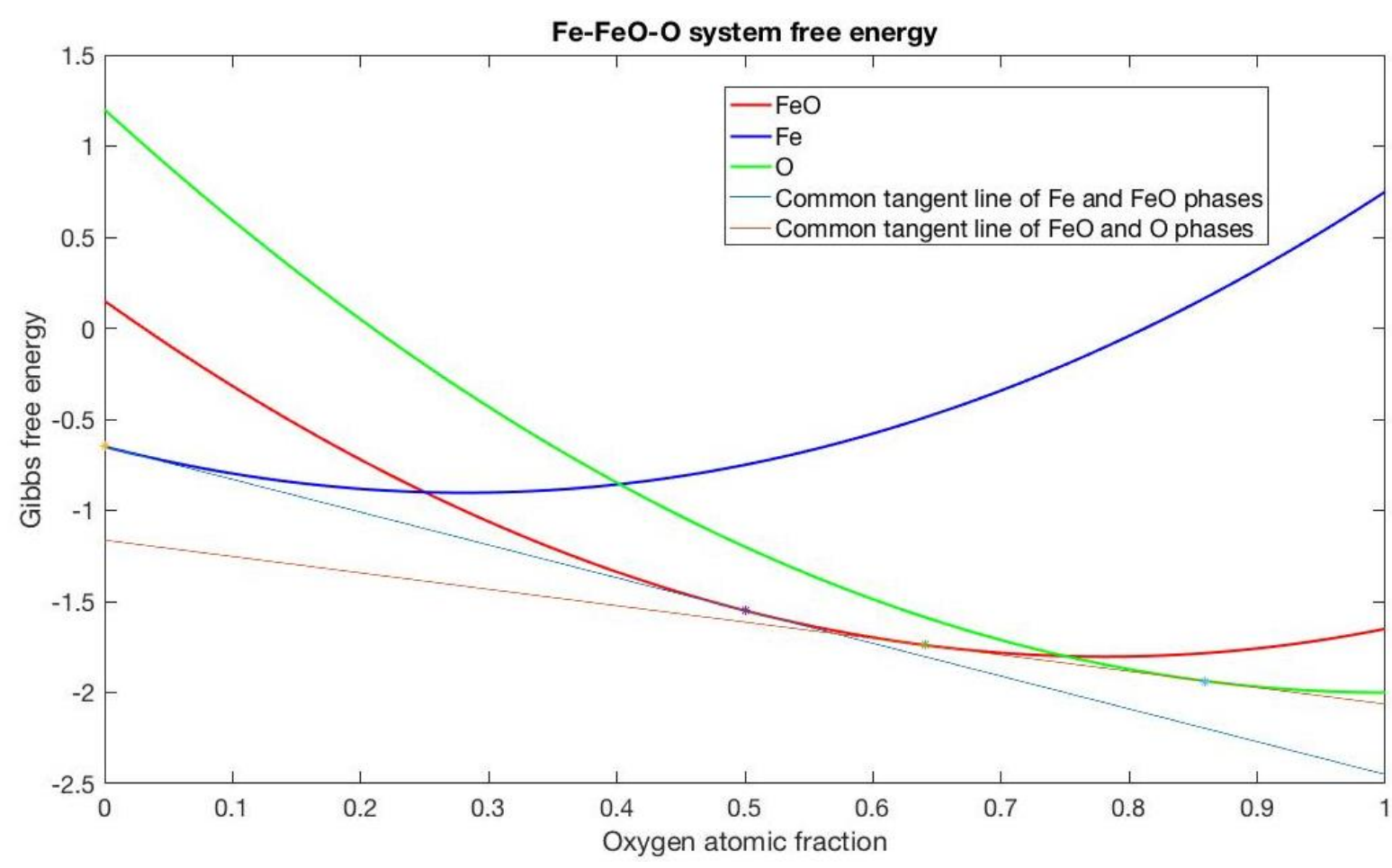

Figure I.1.5.6. Gibbs free energy plot of Fe-FeO-O system. The two tangent lines (colored orange and light blue, not specified in the legend) define five equilibrium regions from left to right: $\mathrm{Fe}, \mathrm{Fe}+\mathrm{FeO}, \mathrm{FeO}, \mathrm{FeO}+\mathrm{O}$, and $\mathrm{O}$.

Source: University of Florida.

\section{Atomic Scale Simulations}

In order for the phase-field model to quantitatively predict the corrosion, it is necessary to have accurate values for the diffusion coefficients of oxygen in the various phases, the interface energies, and the formation energies of oxygen in the different phases. It is also important to know how these values change for different alloy compositions. While some of these values are known (e.g., the diffusion coefficients), the others will be obtained using atomic scale simulations. The atomistic values will be validated by comparing to known values at the few conditions at which they are known.

In accordance with the project milestone schedule, surface energies of the low index planes of steel and its pure components have been calculated. These calculations were carried out using the reliable Vienna Ab-Initio Simulation Package (VASP) code and compared to relevant literature to ensure validity and the results are illustrated in Figure I.1.5.7. In addition to characterizing surface energies, the magnetic structure of facecentered cubic (FCC) Fe was investigated via spin-polarized calculations and compared to bcc Fe. These calculations indicate that an antiferromagnetic double-layered structure (AFM-D) is energetically favorable relative to a single layered (AFM-1) structure. 


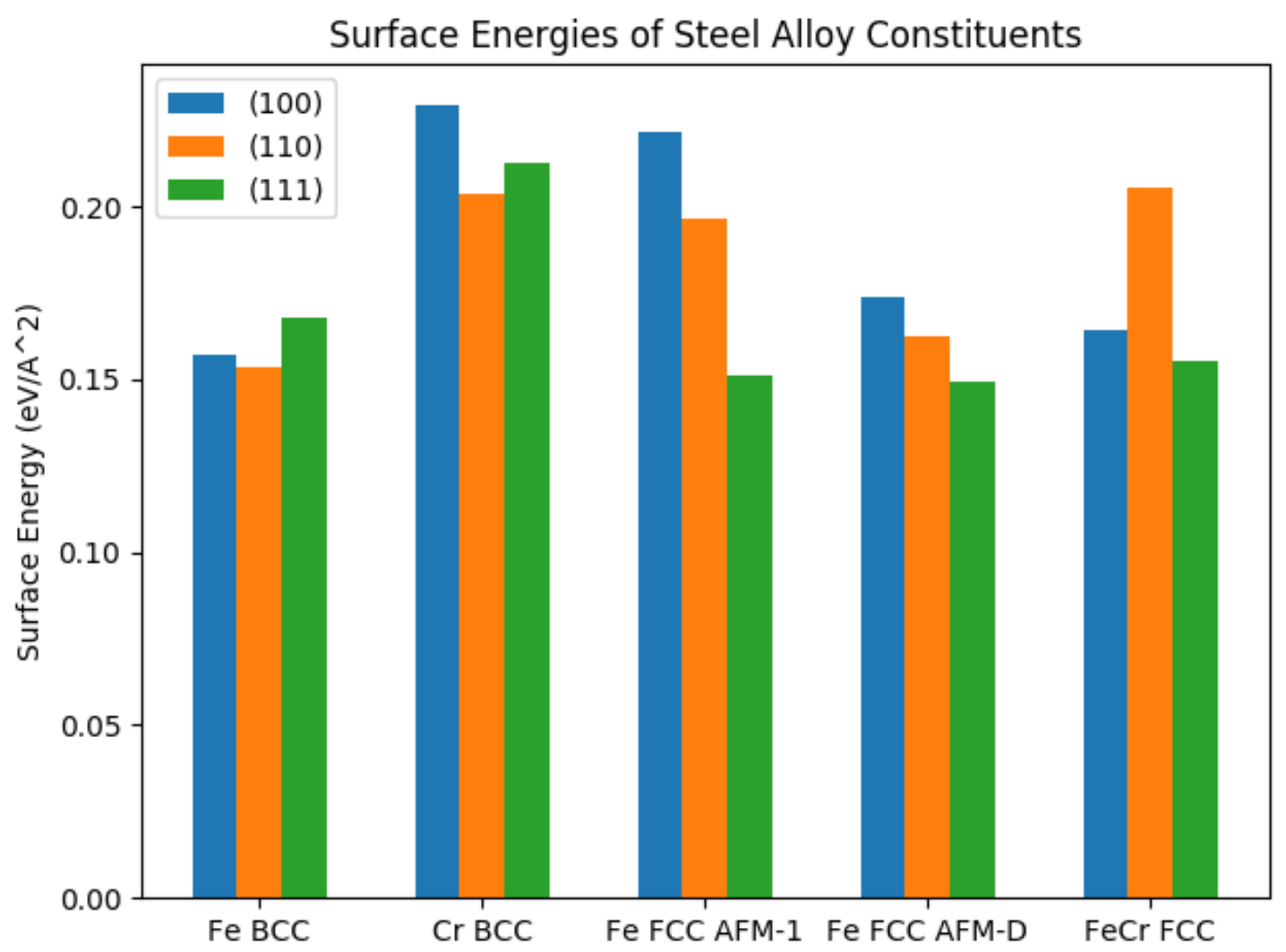

Figure I.1.5.7. Surface energy of each alloy constituent for FCC-Fe and BCC-Fe. Source: University of Florida.

\section{Task 1.3 - Corrosion Model Development}

A mechanistic model of chromium (III) oxide, $\mathrm{Cr}_{2} \mathrm{O}_{3}$, formation is being developed to simulate oxidation kinetics in our alloys of interest using the 'Coupled Current Charge Compensation' (C4) framework [1]. In this model, both n-type and p-type behaviors are being considered via charged species diffusion (i.e., oxygen and chromium vacancies, as well as electrons and holes) to form a $\mathrm{Cr}_{2} \mathrm{O}_{3}$ scale. Figure I.1.5.8 shows a brief description of the n-type $\mathrm{Cr}_{2} \mathrm{O}_{3}$ scale formation.

In order to calibrate the parameters for the $\mathrm{C} 4$ model, we fitted existing high $\mathrm{Cr} \mathrm{SS}$ alloy data at high temperatures [2-6] to the model. As an example, Figure I.1.5.9 shows that the C4 model reproduces corrosion kinetics of a high $\mathrm{Cr}$ SS alloy oxidized in air at $800^{\circ} \mathrm{C}$ for up to 1000 hours (shown in red). Other high Cr SS alloys are presented for smaller exposure times (in pink, green, violet, and blue). A literature search is in process for additional weight gain and oxide thickness measurements of high $\mathrm{Cr}$ SS alloys oxidized in relevant environments, such as $\mathrm{CO}_{2}$. However, literature data is scarce for high $\mathrm{Cr}$ SS alloys in an atmospheric pressure $\mathrm{CO}_{2}$ environment at $700^{\circ} \mathrm{C}$ and $800^{\circ} \mathrm{C}$. Once the model has been fitted to the literature data, the model predictions will be compared to the data from the experimental campaign of the $21-2 \mathrm{~N}$ and $23-8 \mathrm{~N}$ alloys. 


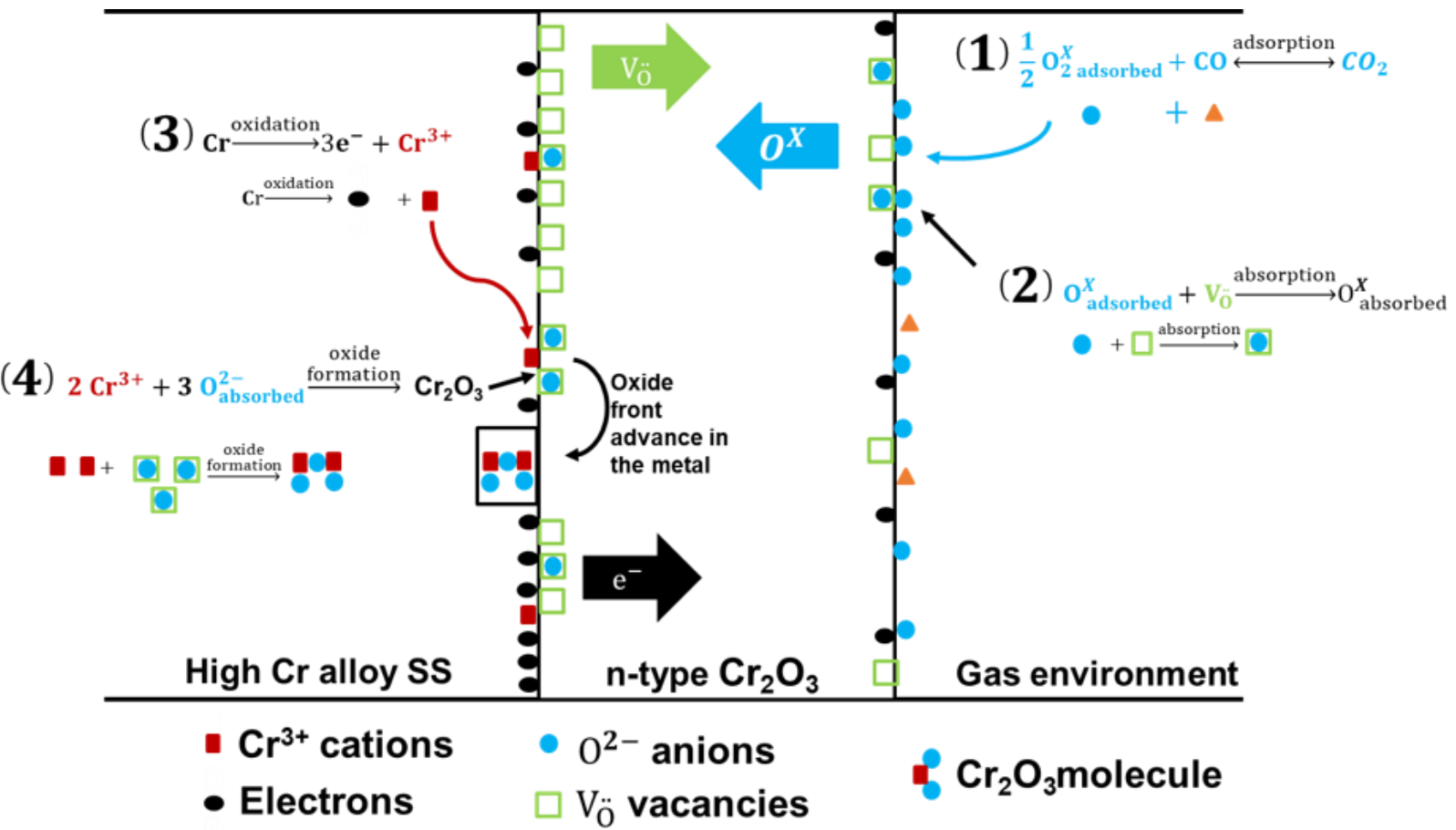

Figure I.1.5.8. Summary of the n-type $\mathrm{C}_{\mathrm{r} 2} \mathrm{O}_{3}$ formation mechanism. Source: University of Wisconsin-Madison.

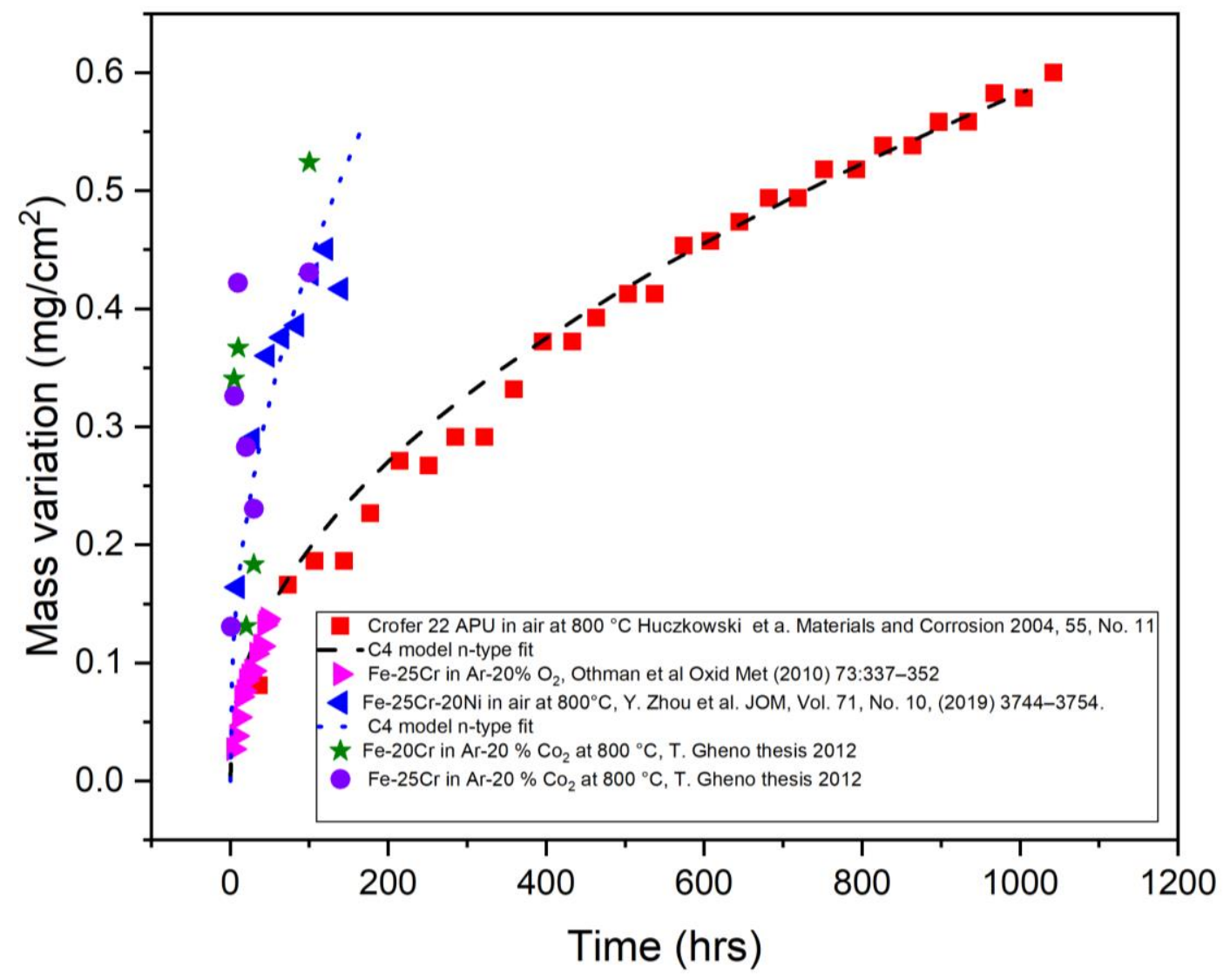

Figure I.1.5.9. An example of fitting existing data of high chromium SS with the C4 n-type model. Source: University of Wisconsin-Madison. 


\section{Conclusions}

The objective of this project is to develop the SStAC tool for predicting the corrosion of $21-2 \mathrm{~N}$ and $23-8 \mathrm{~N}$ stainless steel engine valves during engine operation. A mesoscale phase-field model of the corrosion is also being developed to provide microstructure and alloy element sensitive parameters for the SStAC tool and we are conducting new corrosion and engine tests to obtain new validation data. In the first year of the project, we have completed a new experimental setup for conducting laboratory corrosion tests, and we have characterized the initial microstructure of the valve steel samples. We have also created a preliminary phase-field model of the corrosion of $\mathrm{Fe}$ and verified that it correctly represents the thermodynamic behavior. Atomic scale simulations have calculated the surface energy of various Fe phases. We have calibrated the $\mathrm{n}$-type $\mathrm{C} 4$ corrosion model that will be used in the SStAC tool and we will validate it once the experimental data is ready.

\section{References}

1. Couet, A., A. T. Motta, and A. Ambard, 2015, "The coupled current charge compensation model for zirconium alloy fuel cladding oxidation: I . Parabolic oxidation of zirconium alloys," Corros. Sci., Vol. 100, pp. 73-84.

2. Nurul Atikah, S., J. Azman, S. Muhamad Izhar, and O. Norinsan Kamil, 2014, "Effect of hightemperature corrosion on austenitic stainless steel grade 304 in $\mathrm{CO}_{2}$ gas at $700^{\circ} \mathrm{C}$, Sains Malaysiana. Vol. 43, pp. 1069-1075.

3. Othman, N. K., J. Zhang, and D. J. Young, 2010, "Water vapor effects on Fe - Cr alloy oxidation," Oxid. Met., Vol. 73, No. 1-2, pp. 337-352.

4. Huczkowski, P., N. Christiansen, V. Shemet, and L. Singheiser, 2004, "Oxidation-limited life times of chromia-forming ferritic steels," Mater. Corros., Vol. 55, No. 11, pp. 825-830.

5. Zhou, Y., D. Zou, Y. Pang, T. Wei, W. E. I. Zhang, Y. Zhang, L. Tong, X. Lv, and W. Chen, 2019, "Comparative study on the oxidation behavior of austenitic and ferritic heat-resistant stainless steels at high temperatures," JOM, Vol. 71, pp. 3744-3754.

6. Gheno, T., 2012, "Oxydation et carburation d'alliages modèles chromino-formeurs dans le dioxyde de carbone," Université de Toulouse-Institut National Polytechnique de Toulouse.

\section{Acknowledgements}

All the work summarized in this report is due to the hard work of our research team composed of members from the University of Florida, University of Wisconsin-Madison, Tenneco, and Idaho National Laboratory:

- University of Florida: Simon Phillpot, Xueyang Zhang, Robert Seaton Ullberg

- University of Wisconsin-Madison: Adrien Couet, John Perepezko, Iman Abdallah

- Tenneco: Mark Carroll

- Idaho National Laboratory: Wen Jiang 


\section{I.2 Solid-State Processing and Metal-Matrix Composites}

\section{I.2.1 Direct Extruded High Conductivity Copper for Electric Machines (Pacific Northwest National Laboratory)}

\section{Glenn Grant, Principal Investigator}

Pacific Northwest National Laboratory

902 Battelle Boulevard

Richland, WA 99352

E-mail: Glenn.Grant@pnnl.gov

\section{Jerry L. Gibbs, DOE Technology Development Manager}

U.S. Department of Energy

E-mail: jerry.gibbs@ee.doe.gov

Start Date: October 1, 2017

Project Funding (FY19): \$1,150,000
End Date: September 30, 2019

DOE share: $\$ 600,000$
Non-DOE share: $\$ 550,000$

\section{Project Introduction}

This project is developing and demonstrating a high-power induction motor that utilizes a rotor "squirrel cage" (shorting bars plus end cap assembly) composed of a new high conductivity, copper-carbon composite material fabricated by Shear-Assisted Processing and Extrusion (or ShAPE ${ }^{\mathrm{TM}}$ ), a new manufacturing process being developed at PNNL. In this rotor, shown in Figure I.2.1.1, the shorting bars are linear blade-like parts that run the height of the rotor from end cap to end cap. The bars allow for current to flow between the end caps during motor operation. Higher conductivity in this bar will allow for greater torque to be generated, which can lead to potential improvements in performance or downsizing and lightweighting opportunities, depending on product drivers. The project is a 50/50 cost share with GM and will develop a motor application relevant to their commercial transportation market.

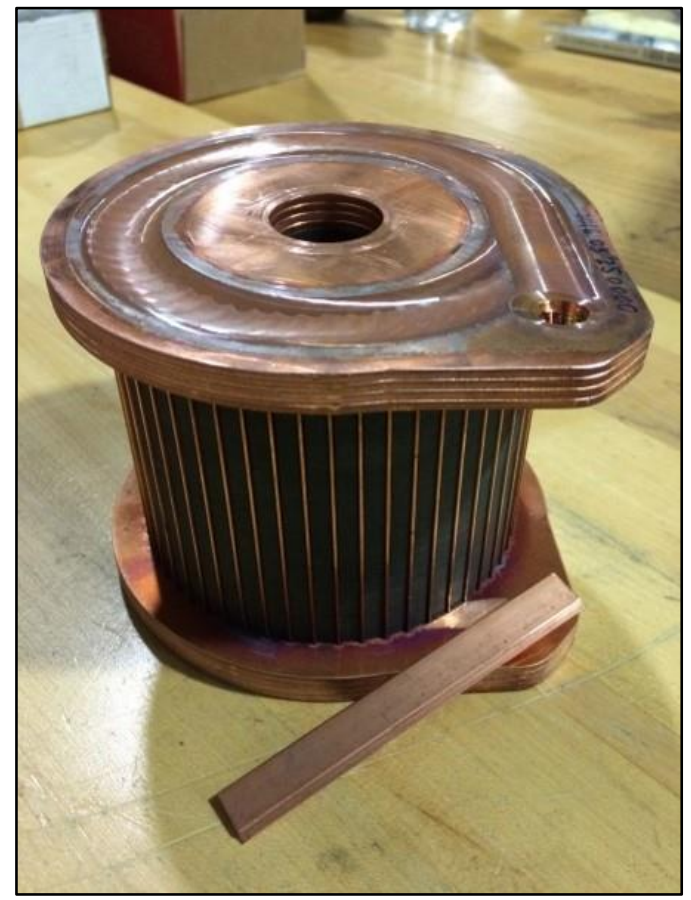

Figure I.2.1.1. Induction motor rotor and an example of a shorting bar. Source: PNNL. 
While copper-carbon composites are well-known in the research world for their enhanced conductivity and ampacity, scalable methods to fabricate composite conductors have been elusive. The ShAPE ${ }^{\mathrm{TM}}$ equipment comprises of a rotating tool with a through-cavity and extrusion container. The billet to be processed is inserted into the stationary extrusion container, while the flat face of the rotating die is abutted against it. Owing to the intense shear and frictional heat, the billet material at the tool interface is plasticized and flows through the cavity in the tool to form the extrudate. ShAPE ${ }^{\mathrm{TM}}$ can be used to manufacture tubular and solid extruded products with microstructures with homogeneous, refined grains. PNNL houses a custom-designed and built 100-ton force $\mathrm{ShAPE}^{\mathrm{TM}}$ equipment capable of applying $1000 \mathrm{ft} / \mathrm{lbs}$ of rotational torque at the billet face.

\section{Objectives}

In FY 2019, PNNL focused on developing copper-carbon composite forms demonstrating electrical conductivity greater than that of copper at room temperature using low-defect density crystalline nano-carbon additives.

\section{Approach}

In FY 2019, ShAPE was used to manufacture copper-carbon composite wires using monolayer graphene, based on previous work suggesting that the electrical performance of copper/graphene was dependent on additive defect density [1]. Low-defect density graphene procured as sheets that were chemically vapordeposited on C110000 copper alloy foils were consolidated with a solid copper billet and extruded to form wires using the ShAPE ${ }^{\mathrm{TM}}$ process. Copper alloy foils without graphene were also extruded into wires to manufacture the control samples. The composition of the different samples manufactured in FY 2019 is provided in Table I.2.1.1.

Table I.2.1.1 Composition of the Copper-Carbon Composite Samples Manufactured in FY 2019.

\begin{tabular}{|c|c|}
\hline Sample & Graphene content (wt.\%) \\
\hline A (control sample) & 0 \\
\hline B & 0.00015 \\
\hline C & 0.0002 \\
\hline D & 0.0006 \\
\hline
\end{tabular}

In FY 2018, accurate measurement of composite sample electrical performance was a challenge owing to the high conductivity of the samples and an inavailability of testing equipment. In FY 2019, electrical performance of the composite samples were measured by multiple third parties to ascertain the accuracy of their properties. Of those third parties, the least measurement error was provided by Ohio University [2]. Their setup, as shown in Figure I.2.1.2, comprises an Al base along which the wire samples are fixed in slight tension, a Keithley 2182A nanovoltmeter (accuracy $0.002 \% \pm 1 \mathrm{mV}$ ) connected to the sample wire in parallel, and a Keithley 2260B-30-72 bench power supply (accuracy $0.1 \% \pm 70 \mathrm{~mA}$ ) connected in series. Electrical conductivity of the samples was determined by measuring their resistivity using the four-point probe method as per ASTM B19316. Two pairs of electrical leads were connected in parallel across the wire sample. One pair of leads provided test current through the wire, and the other pair of leads was connected to the nanovoltmeter, whose readings were used to calculate sample resistance $(R)$ according to Equation 1.

$$
R=\frac{V}{I}
$$

where $V$ is the voltage measured across the sample length $l$ of the wire, and $I$ is the input current from the DC power supply. Electrical conductivity was calculated per Equation 2. 


$$
\sigma=\frac{4 l}{R \pi d^{2}}
$$

where $d$ is the average cross-sectional diameter of the wire. Prior to electrical conductivity testing, the diameter of the wire was accurately measured using a Keyence LS-7601 Optical Micrometer.

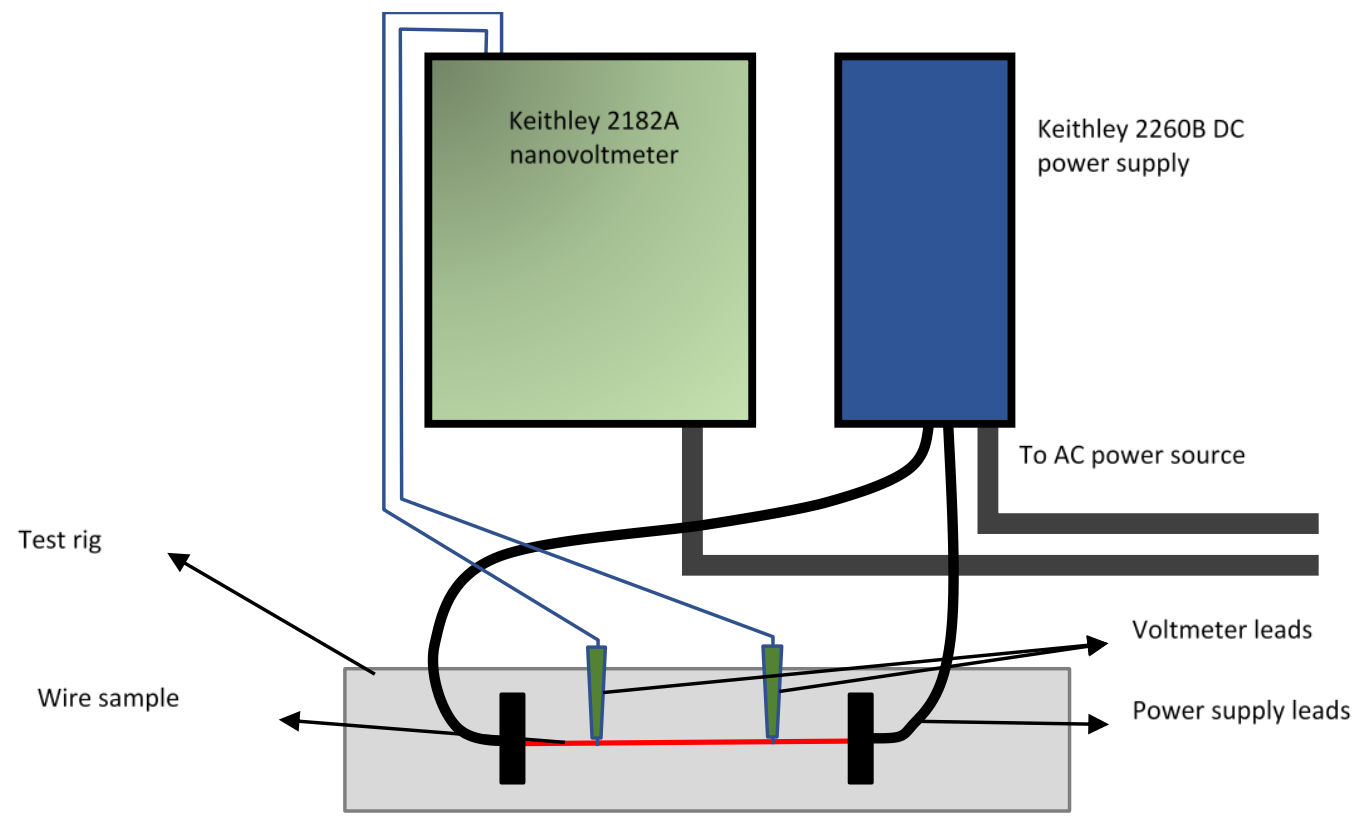

Figure I.2.1.2. Four-point probe method for measuring electrical conductivity and current density. Source: PNNL.

\section{Results}

Table I.2.1.2 shows the electrical conductivity of the copper-carbon composite wires measured using the fourpoint probe method. Results show a progressive increase in the conductivity of the wires with an increase in graphene content. Highest conductivity of $104.8 \%$ by the International Annealed Copper Standard (IACS), corresponding to an increase of $4 \%$, was measured for the wires with $0.0006 \mathrm{wt} . \%$ graphene.

Table I.2.1.2 Electrical Conductivity of the Copper-Carbon Composite Wires Measured by the Four-Point Probe Method.

\begin{tabular}{|c|c|c|c|}
\hline Sample & Graphene content (wt.\%) & $\begin{array}{c}\text { Electrical conductivity \% } \\
\text { by IACS }\end{array}$ & $\begin{array}{c}\text { Property improvement } \\
\text { with respect to control }\end{array}$ \\
\hline A (control sample) & 0 & $100.75 \pm 0.002$ & - \\
\hline B & 0.00015 & $103.57 \pm 0.001$ & $2.8 \%$ \\
\hline C & 0.0002 & $103.83 \pm 0.002$ & $3.1 \%$ \\
\hline D & 0.0006 & $104.8 \pm 0.003$ & $4.0 \%$ \\
\hline
\end{tabular}

Mechanical testing on sample ' $\mathrm{D}$ ' showed that its ultimate tensile strength was $220 \mathrm{MPa}$ which is very similar to that of annealed copper. Electrical resistivity testing of the copper-composite sample ' $\mathrm{B}$ ' at elevated temperatures was performed by Thermophysical Properties Research Laboratory, Inc. in Indiana. Four-point probe apparatus was set up in a furnace with a temperature capability of $1100^{\circ} \mathrm{C}$. Resistivity was reported with a 3\% standard error and values are presented in Figure I.2.1.3. Resistivity of sample ' $B$ ' was measured lower than that of the pure copper reference (obtained from literature) as well as sample 'A' (control sample with no graphene). 


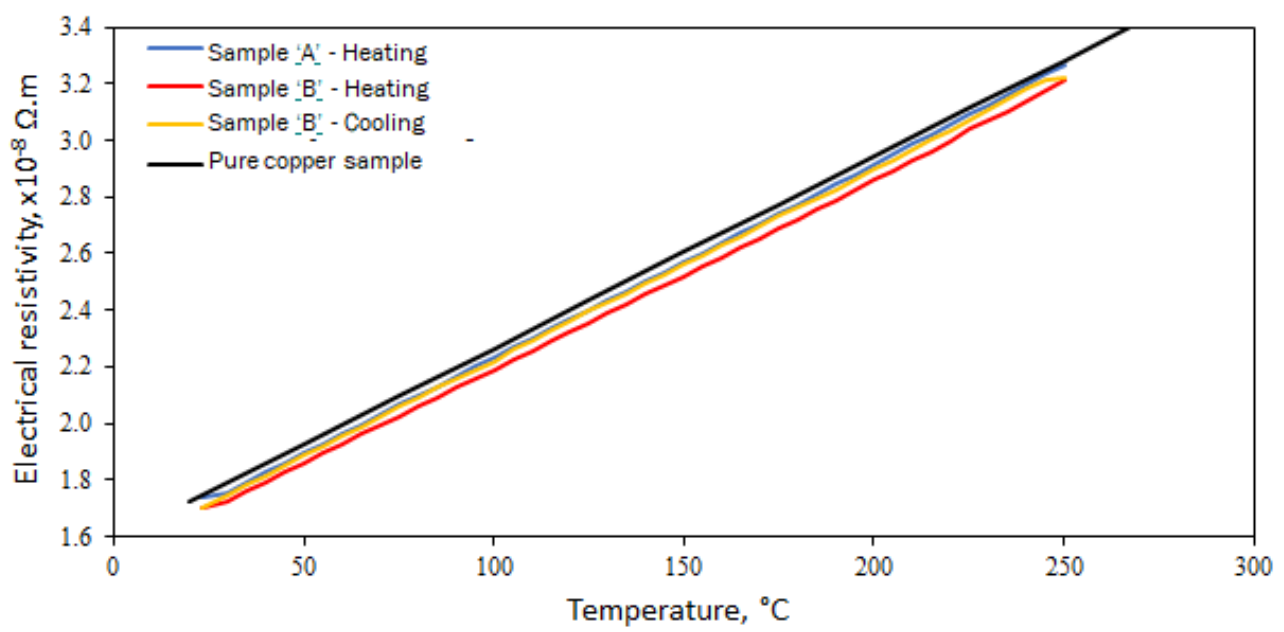

Figure I.2.1.3. Electrical resistivity of copper-composite with 'B' measured from $20-250^{\circ} \mathrm{C}$ using the four-point probe method in a furnace. Source: Thermophysical Properties Research Laboratory, Inc.

Optical imaging of the sample 'A', presented in Figure I.2.1.4 (a) shows that the grain size distribution in the microstructure resembled that of ShAPETM extrudate of similar dimensions made with a pure copper billet, presented in Figure I.2.1.4 (b). This demonstrates the ability of the ShAPETM technique to generate highperformance microstructures with high grain refinement and desired textures agnostic of the precursors used. The microstructure of sample 'D,' as shown in Figure I.2.1.4 (c), is similar to that of sample 'A.' This could be because of the very small quantities $(0.0006 \mathrm{wt}$.\%, equivalent to $6 \mathrm{ppm})$ of graphene used to manufacture the copper-composite wire.
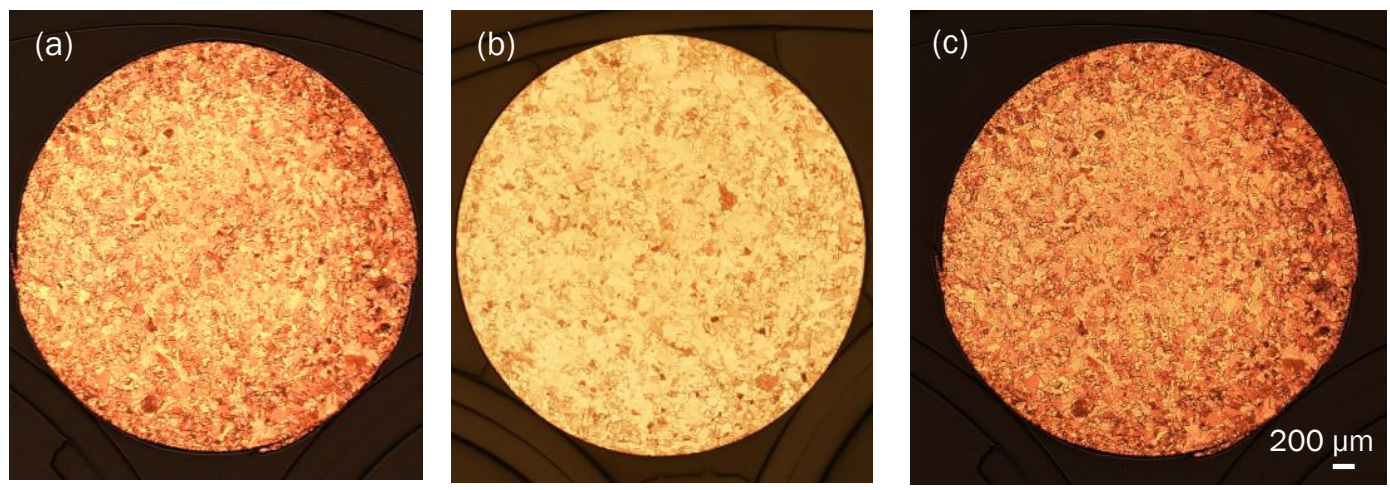

Figure I.2.1.4. Microstructure of: (a) sample 'A' (control) made with consolidated C110000 foils; (b) ShAPE'M extrudate manufactured using pure copper billet; and (c) sample 'C' containing 0.0006 wt.\% graphene. Source: PNNL.

Simultaneously, GM explored the welding and joining behavior of the copper-carbon composite wires with varying graphite content manufactured previously in FY 2018.

\section{Conclusions}

In FY 2019, ShAPETM technology was used to fabricate copper-carbon composite wires with $2.5 \mathrm{~mm}$ diameter and $>1 \mathrm{~m}$ length. Monolayer graphene with low-defect-density was used to manufacture the composite wires instead of reduced graphene oxide or graphite. Extrusion tools and dies, as well processing parameters, were determined to achieve fully compacted microstructures with adequate graphene distribution and no porosity. Electrical conductivity of the composite wires increased as a function of graphene content at room temperature as verified by third-party testing. At elevated temperatures, resistivity of the composite wires was seen to be lower than the control sample, as well as that of pure copper. Mechanical testing demonstrated that the 
composite wires behaved similar to annealed pure copper samples, plausibly owing to the very low graphene quantities used in the fabrication process. This project leverages a new manufacturing process to manufacture high conductivity wire by an unconventional method involving high-shear and plastic deformation. It could be disruptive to the way automotive traction drives are constructed, sized, or powered. The high conductivity wire for stator winding could spill over into permanent magnet motor space where there are also strong incentives to improve motor performance.

\section{Key Publications}

1. Kappagantula, K., X. Li, and G. J. Grant, 2019, "Development of ultraconductive materials using solidphase processing," Invited Lecture presented at the Chemical Engineering Department Seminar at Ohio University, Athens, OH, USA.

2. Kappagantula, K., X. Li, C. Zhou, H. Wang, J. G. Schroth, and G. J. Grant, 2019, "Solid-phase processing of conductive copper/graphene alloys via ShAPETM approach," MS\&T 2019, Portland OR, USA.

3. Li, X., N. L. Canfield, J. T. Darsell, C. Zhou, H. Wang, J. G. Schroth, and G. J. Grant, 2019, "ShAPE: A novel solid-phase processing of making bulk size metal-matrix-composite without agglomeration," $M S \& T$ 2019, Portland OR, USA.

4. Li, X., G. J. Grant, C. Zhou, H. Wang, T. A. Perry, and J. G. Schroth, 2019, "Copper-graphite composite wire made by ShAPE," TMS 2019, San Antonio TX, USA.

5. Li, X., N. R. Overman, T. J. Roosendaal, M. J. Olszta, C. Zhou, H. Wang, and T. A. Perry, 2019, "Microstructure and mechanical properties of pure copper wire produced by ShAPE," JOM. The Journal of the Minerals, Metals and Materials Society. PNNL-SA-147051.

\section{References}

1. Kappagantula, K., F. Kraft, 2019, "Ultraconductive metal composite forms and the synthesis thereof." U.S. Patent No. US20190267153A1, August 29, 2019.

2. Smith, J., 2018, "Electrical performance of ultraconductive copper-graphene nano-alloys," Master's Thesis. Ohio University, Athens, OH, USA. 


\title{
I.2.2 Metal-Matrix-Composite Brakes Using Titanium Diboride - LightMAT DFA1 (Pacific Northwest National Laboratory)
}

\section{Glenn Grant, Co-Principal Investigator}

Pacific Northwest National Laboratory

902 Battelle Boulevard

Richland, WA 99352

E-mail: Glenn.Grant@pnnl.gov

\section{Merritt Osbourn, Co-Principal Investigator}

Arconic Technical Center

100 Technical Drive

New Kensington, PA, 15069

E-mail: Merritt.Osbourn@arconic.com

\author{
Sam Morgan, Co-Principal Investigator \\ Pacific Northwest National Laboratory \\ 902 Battelle Boulevard \\ Richland, WA 99352 \\ E-mail: $\underline{\text { Sam.Morgan@ arconic.com }}$
}

\section{Jerry L. Gibbs, DOE Technology Development Manager}

U.S. Department of Energy

E-mail: jerry.gibbs@ee.doe.gov

Start Date: October 1, 2017

Project Funding (FY19): \$660,800
End Date: June 30, 2020

DOE share: $\$ 300,000$
Non-DOE share: $\$ 360,800$

\section{Project Introduction}

There is currently no viable, cost-competitive alternative to cast-iron brake rotors for the automotive market. While in past years many weight-savings materials and designs have been developed for other parts of a vehicle, the brake assembly remains tied to near fifty-year-old technology, as shown in Figure I.2.2.1. Metalmatrix composites (MMCs) present an opportunity to replace cast-iron brakes in certain vehicles. By replacing cast-iron rotors with lighter-weight MMCs, the opportunity exists to reduce the unsprung weight of the brake assembly and adjacent components, such as suspension arms and springs. A 50\% savings in mass in a rotating and unsprung location can lead to a $0.25-0.35 \mathrm{mpg}$ fuel savings in a mid-sized car. In addition, Al MMC brakes can provide combinations of high friction coefficients, particularly heat transfer characteristics and increased wear life that can favorably affect system life cycle cost. A 50\% increase in wear life can double the interval between rotor change-outs and affect the economics for those vehicles sensitive to down time. Lower wear also means fewer wear particles are generated during braking. These wear particles are the second largest source of particulate emissions from a vehicle. "In urban areas, around $55 \%$ of total non-exhaust particulate matter smaller than 10 micrometers emissions is from brake wear" [1]. 


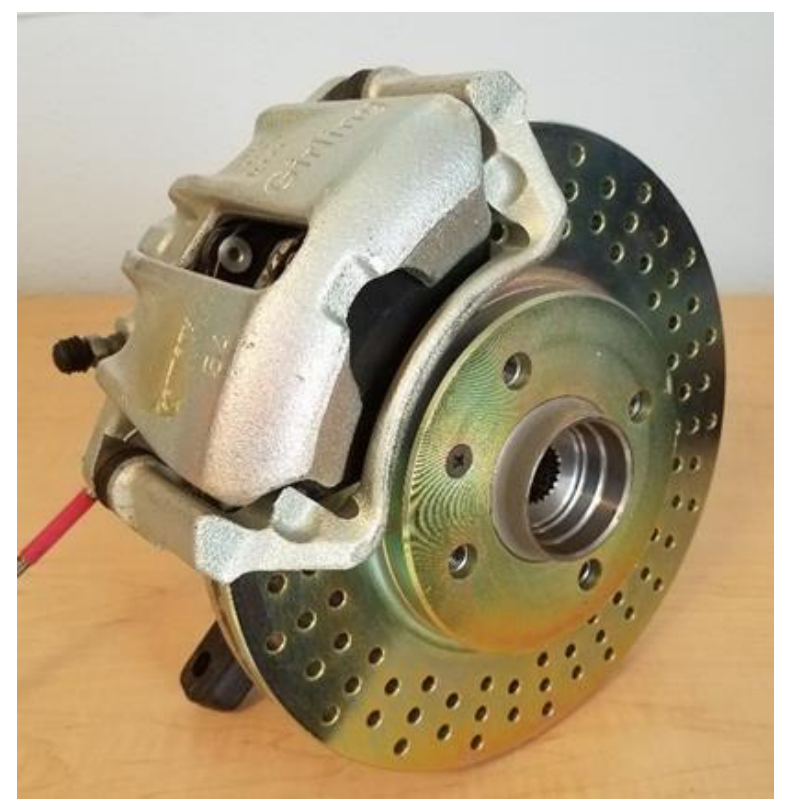

Figure I.2.2.1. Conventional Brake design utilizing an Al caliper and a cast-iron brake disc. Source: PNNL.

Al MMC brake rotors were investigated in past DOE-funded programs during the early 2000s. A commercial application of $\mathrm{Al} \mathrm{MMC}$ silicon carbide $(\mathrm{SiC})$ rotors was implemented in a limited number of Plymouth Prowler vehicles in 1998, while numerous manufacturers have implemented Al MMC rotors and drums in limited production vehicles (e.g., Toyota RAV4-EV, GM EV1, Lotus Elise, Volkswagen Lupo). But in all cases, cost avoidance could not be fully demonstrated replacing the cast-iron equivalent part.

The current automotive landscape has changed in the last 10 years, however. The changes that have been made may provide opportunities to look again at Al MMC brakes. One of the significant changes comes from electric/hybride vehicles. Energy harvesting in electric/hybrid vehicle operation decreases the amount of energy that must be dissipated by the mechanical brakes by as much as $40 \%$. This can allow for much lower front brake temperatures, enabling the use of lighter-weight, lower melting temperature alloys as rotors. Also, cast-iron rusts and the friction coefficent of rusty surfaces is not consistent, making it difficult to manage in brake-by-wire systems tasked with balancing mechanical brake force with energy harvesting. Consumers demand a smooth stop (i.e., driver feel and noise, vibration and harshness issues); corrosion-resistant and lowwear alloys (e.g., Al MMCs) may provide better control. Another factor is the coming change in duty cycle for a vehicle. The next-generation of personal transportation vehicles may need drastically improved durability and longer maintenance intervals if they are to be used in new mobility strategies like 24/7 ride-sharing or fleet ownership scenarios.

Barriers to more widespread use of MMCs for vehicle lightweighting include: (1) the costs of the feedstock, especially the insoluble reinforcement (e.g., particle, whisker, or fiber) usually $\mathrm{SiC}$; (2) the cost of combining the reinforcement with the matrix in production, which requires cost and effort to insure the SiC particles are "sized" or coated correctly to promote wetting in molten $\mathrm{Al}$; and (3) the cost of shaping/machining MMC components. One of the concepts to address these barriers is to develop an alternative reinforcing particle. Titanium diboride (TiB2) reinforcement offers an opportunity to improve wear resistance at a lower particle loading because of improved $\mathrm{C}$-Al bonding. $\mathrm{SiC}$ reinforcement is costly when prepared for inclusion in an $\mathrm{Al}$ composite (i.e., particle size fraction constraints, $\mathrm{SiO} 2$ coating, etc.). TiB2 has much improved wetting with $\mathrm{Al}$ allowing for finer particle size and better homogeneity, which may prove to provide a lower-cost overall due to the lower amount of particle loading required and reduced compositing time for the same friction and wear performance. 
Recent improvements to the TiB2 production process by Arconic may make it a more viable technical ceramic for MMCs than in the past. This LightMAT funded project will utilize TiB2 as a substitute for more traditional $\mathrm{MMC}$ efforts using $\mathrm{SiC}$ to improve performance, wear life, and cost in brake rotors.

\section{Objectives}

The objective of the project is to reduce the weight of brake rotors by $>50 \%$ over current cast-iron materials, and at the same time, improve brake performance, wear life, and life cycle costs over traditional cast-iron systems. The project will develop an Al MMC material that shows appropriate wear resistance, friction coefficient, and tribologic properties, and shows a potential for a cost/benefit ratio appropriate for commercial development.

\section{Approach}

The overall approach to the work will be to cast TiB2 reinforced $\mathrm{Al}$ brake rotors in several different reinforcement loadings and test for friction and wear performance. The data will be compared to a large DOEgenerated database of properties developed on $\mathrm{Al}-\mathrm{SiC}$ (and other) MMC brake materials in the early 2000s.

The project will proceed through the six tasks listed in Table I.2.2.1. The first task involves the production of a 50 vol. \% reinforced master alloy produced at the Arconic Technology Center. This master alloy is produced by liquid $\mathrm{Al}$ infiltration of a TiB2 preform. Eventually if wear-testing is favorable, it is envisioned that the final composite will be made by direct powder addition and rapid mixing in the melt state, and not by preform infiltration. However, this route gets the project to the initial wear test trials.

Table I.2.2.1. List of Tasks to be Accomplished by this Project.

\begin{tabular}{|c|c|c|c|c|c|c|c|c|c|c|c|c|}
\hline \multirow{2}{*}{$\begin{array}{l}\text { Task Number \& } \\
\text { Brief Description }\end{array}$} & \multicolumn{4}{|c|}{ FY 2017} & \multicolumn{4}{|c|}{ FY 2018} & \multicolumn{4}{|c|}{ FY 2019} \\
\hline & Q1 & Q2 & Q3 & Q4 & Q1 & Q2 & Q3 & Q4 & Q1 & Q2 & Q3 & Q4 \\
\hline $\begin{array}{l}\text { Task 1: Raw } \\
\text { Material Production } \\
\text { (Arconic Task) }\end{array}$ & & & & & & & & & & & & \\
\hline $\begin{array}{l}\text { Task 2: Casting of } \\
\text { MMC Billet }\end{array}$ & & & & & & & & & & & & \\
\hline $\begin{array}{l}\text { Task 3: Machining } \\
\text { of MMC Test Rotors }\end{array}$ & & & & & & & & & & & & \\
\hline $\begin{array}{l}\text { Task 4: Material } \\
\text { Characterization } \\
\text { (Arconic Task) }\end{array}$ & & & & & & & & & & & & \\
\hline $\begin{array}{l}\text { Task 5: Brake Wear } \\
\text { Testing }\end{array}$ & & & & & & & & & & & & \\
\hline Task 6: Final Report & & & & & & & & & & & & \\
\hline
\end{tabular}

In a second task, PNNL will use the Al-TiB2 master alloy along with $\mathrm{A} 356 \mathrm{Al}$ ingot to cast four MMC billets of MMC composition (i.e., 5, 10, 15, 20 vol. \% of TiB2) utilizing PNNL MMC stir-casting technology and equipment shown in Figure I.2.2.2 and Figure I.2.2.3. 


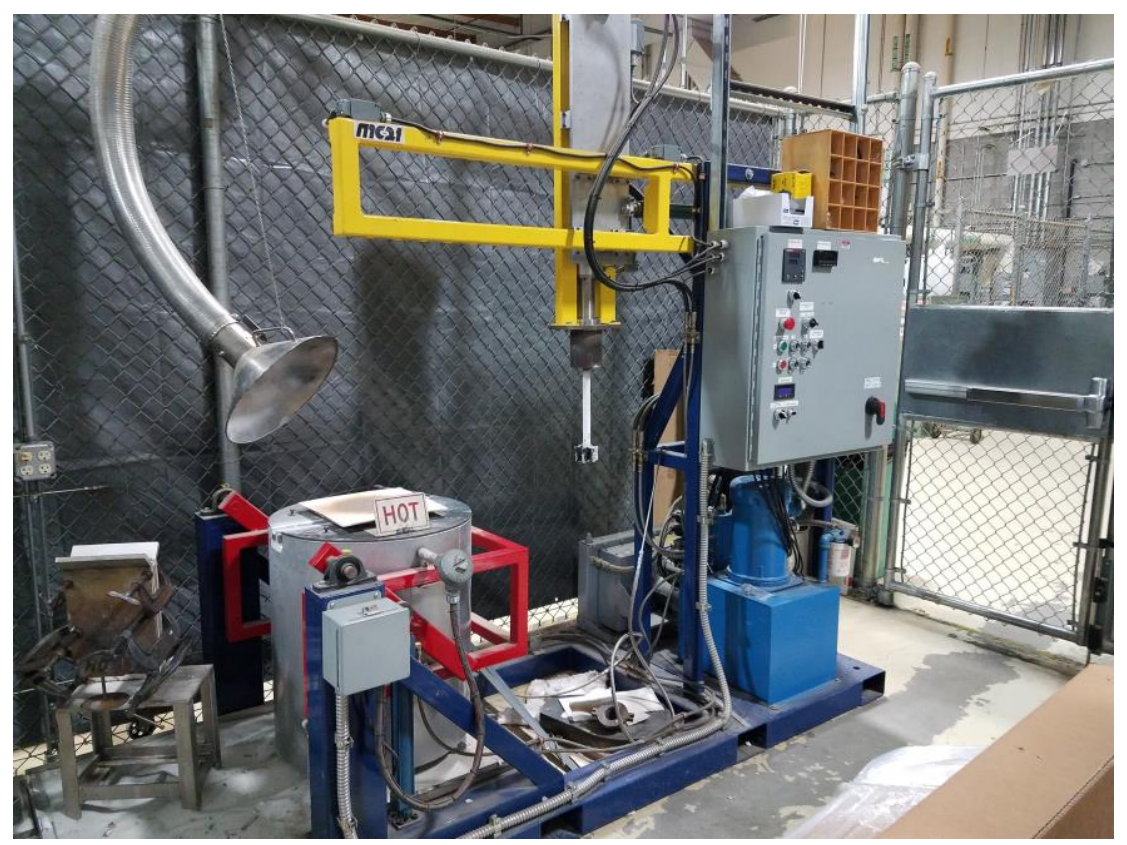

Figure I.2.2.2. PNNL tilt-pour stir-casting equipment used for the casting trials. Flat-bladed impeller installed in mixer head. Source: PNNL.

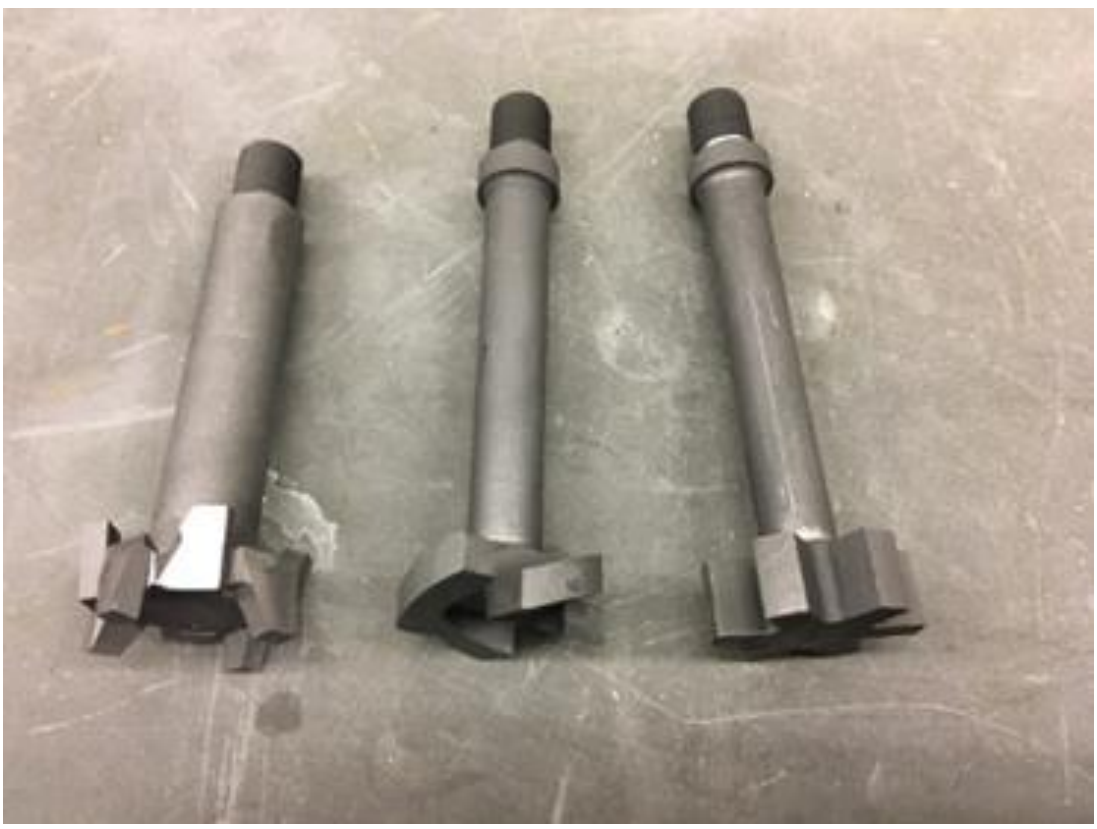

Figure I.2.2.3. Impeller designs for mixing trials. Source: PNNL.

These billets are then machined to subscale rotors for wear-testing, which is comprised of mounting the subscale rotors on an instrumented brake testing rig, as shown in the photographs of Figure I.2.2.4, capable of rotating the rotor or pad holder shown in Figure I.2.2.5, and applying clamping loads appropriate for automotive braking conditions. Several energy profiles are investigated, including low temperature, low-load continuous torque tests, and high-load, high-temperature transients. 

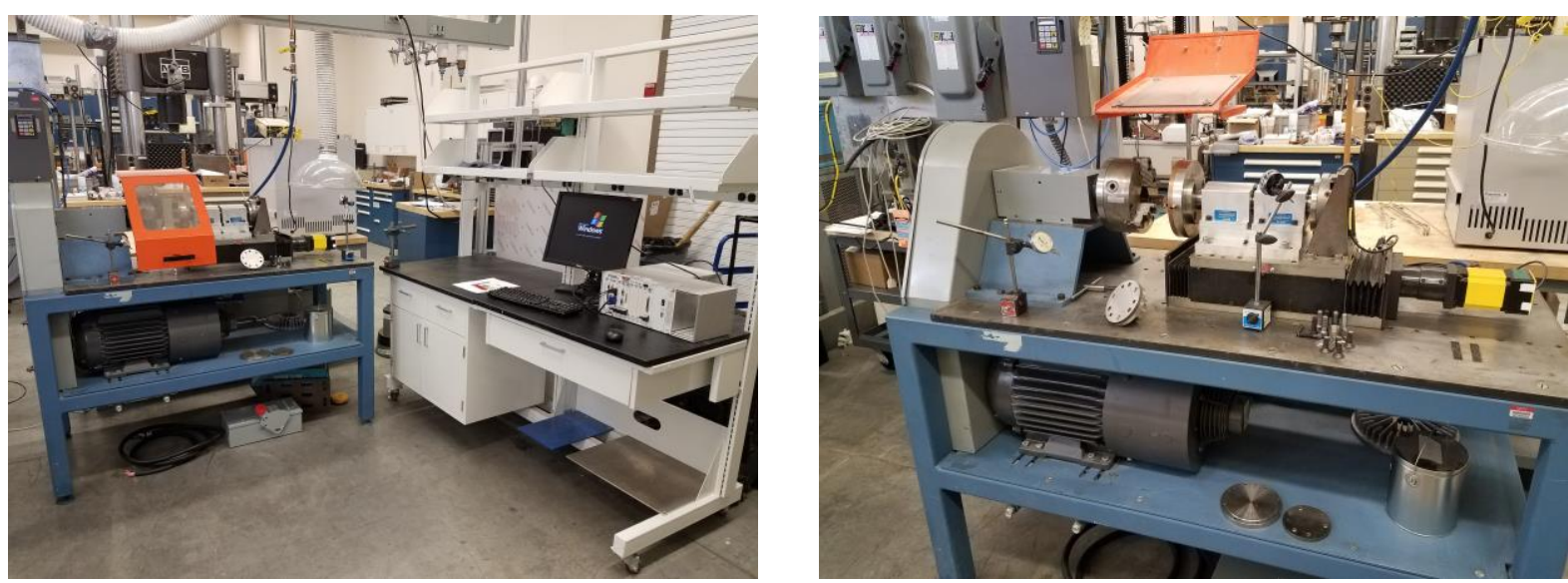

Figure I.2.2.4. Brake friction pair test stand at PNNL. Source: PNNL.
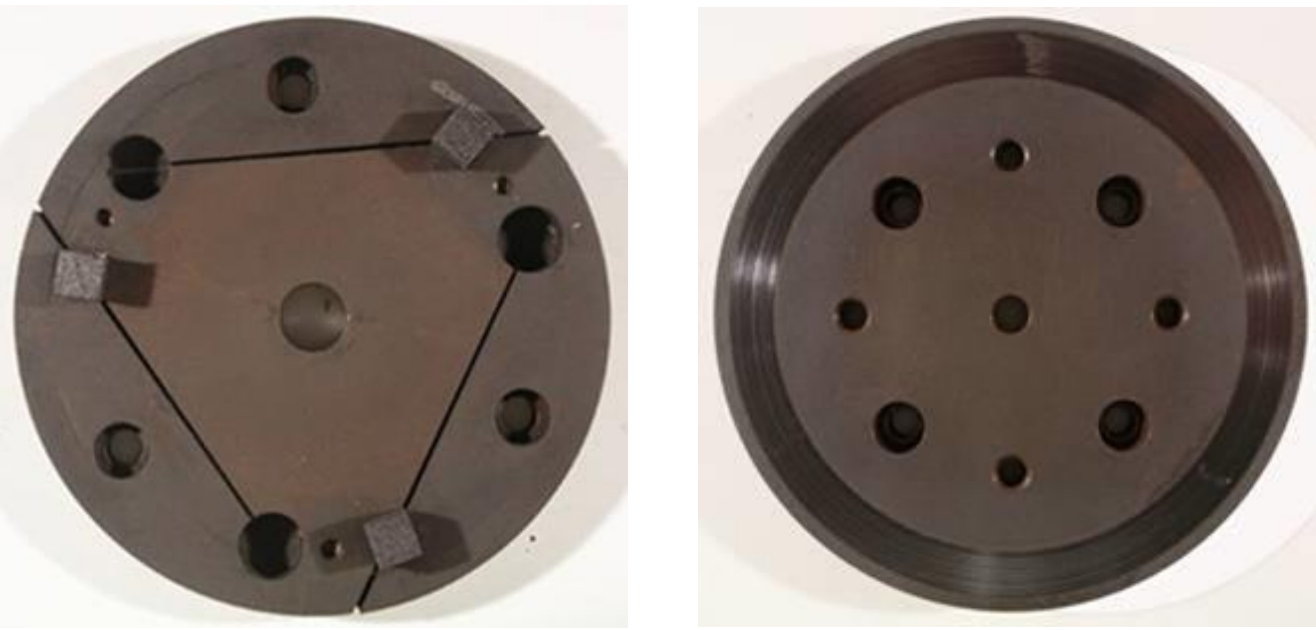

Figure I.2.2.5. Brake pad holder and tested subscale brake rotor. Source: PNNL.

After testing, the data on loads, torque, power, and temperature were compiled, friction coefficients were calculated, and the data was correlated to the test conditions. During the tests, the rotors were paired with several different pad compositions to measure the effect and to optimize the friction pair. Rotors and pads were measured and weighed to establish mass gain or loss. The rotor wear tracks were analyzed for geometry using the techniques of profilometry as shown in Figure I.2.2.6 (a) and transfer chemistry characterization as shown in Figure I.2.2.6 (b). The transfer layer, which usually formed on the rotor, was also analyzed for chemistry and pad reactions. This testing data will be utilized to evaluate the feasibility and advantages of using TiB2 as a reinforcement for an $\mathrm{Al} \mathrm{MMC} \mathrm{brake} \mathrm{rotor.}$ 


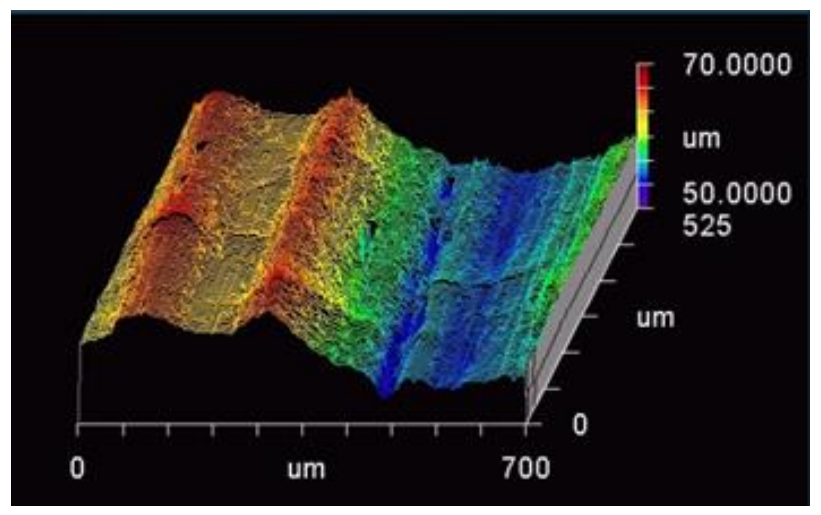

(a)
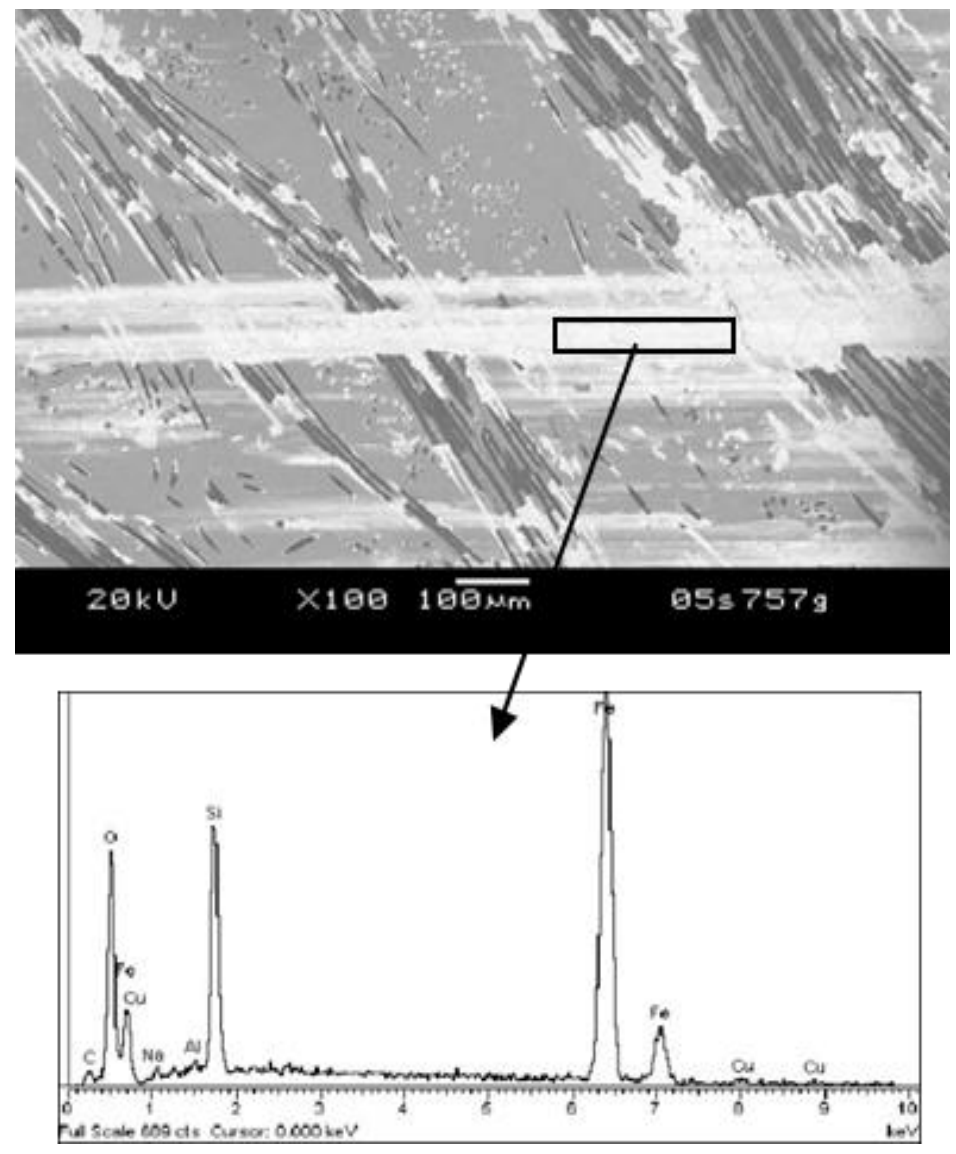

(b)

Figure I.2.2.6. (a) Wear track profilometry; and (b) transfer layer chemistry characterization. Source: Rockwell Science Center.

\section{Results}

During FY 2019, the stir-casting trials at PNNL were successful at producing stir cast plates in three different particle loadings. Master alloy and A356 ingot were combined in a crucible at appropriate ratios to produce 5, 10 , and $15 \mathrm{vol} . \%$ reinforced melts. A series of stir-casting trials were initiated to establish mixing times, melt temperatures, mixing paddle geometry, and mixing paddle speeds. The fixture for the stir-casting trials shown in Figure I.2.2.7 (a). After a sufficient mixing time, the melts were poured into open steel book molds to produce rectangular castings approximately 10 in. $\times 7$ in. $\times 1$ in, as shown in Figure I.2.2.7 (b). Initial 
microscopy on these castings revealed minor porosity < 5\%. Production Al MMC rotors would likely be fabricated by squeeze-casting or high-pressure die-casting where porosity would be minimized, so we decided to hot-roll the plates to better mimic a final product configuration. The as-cast plates were hot-rolled at $450^{\circ} \mathrm{C}$ for two passes with an approximate $30 \%$ reduction, and then solutionized and heat-treated to T7.

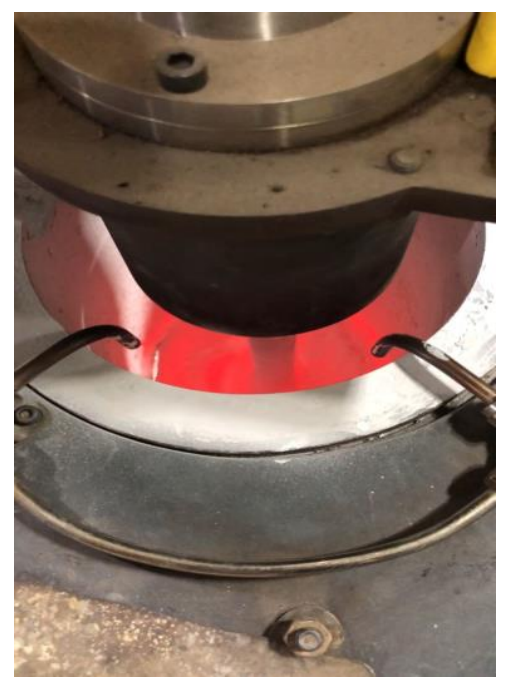

(a)

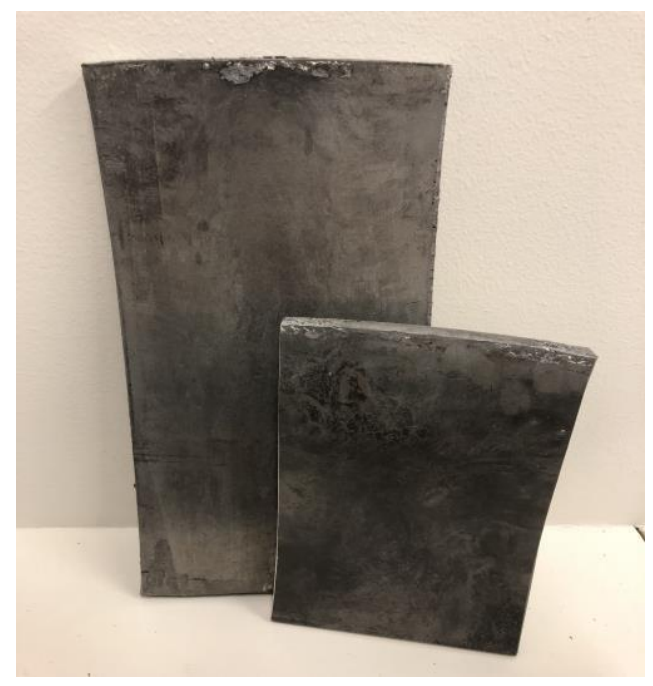

(b)

Figure I.2.2.7. (a) Fixture for stir-casting trials and (b) the gravity cast plates for 5, 10, and 15 vol. \% TiB2 reinforced A356 alloy. Source: PNNL.

The plates were then machined to the subscale rotor configurations for testing. Gravity cast plates show some variability in porosity, but we do not expect the small variations to affect the friction testing significantly. Approximately three rotors from each particle loading have been fabricated for wear-testing, as shown in Figure I.2.2.8.

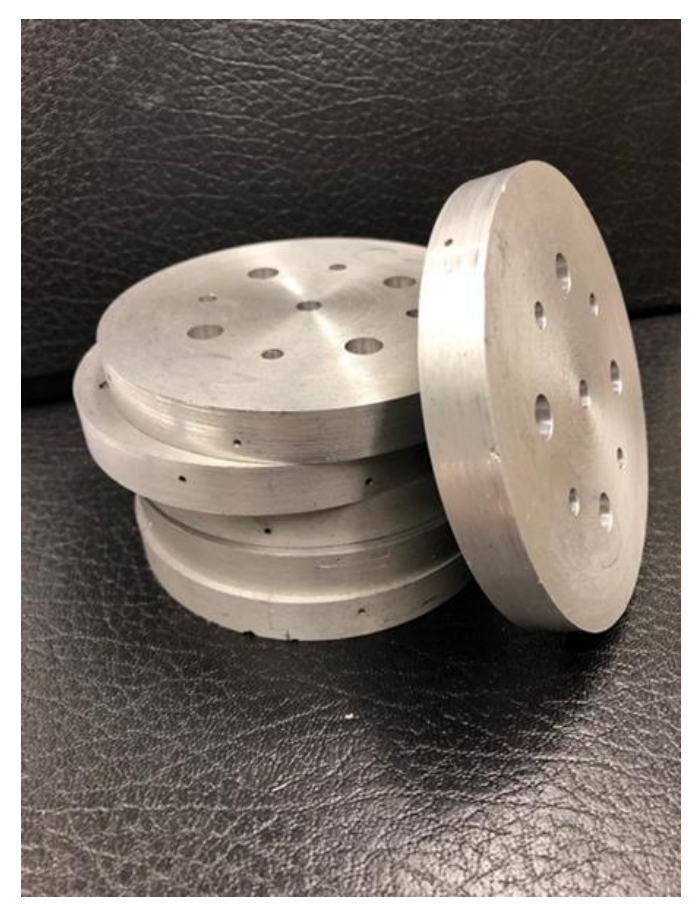

Figure I.2.2.8. Subscale rotors ready for friction/wear-testing. Source: PNNL. 
In addition, pad materials have been sourced and machined to fit in the pad holder shown in Figure I.2.2.5 (a). Pad materials have been selected to be a range of properties that may produce different behaviors when applied against $\mathrm{Al} \mathrm{MMC}$ rotors. It is not the intention to develop a fully optimized friction pair during this testing, but the pad compositions selected should give us a range of friction coefficients and performance. Pads include examples of the original Plymouth Prowler pads that were specified by Chrysler for the $\mathrm{Al} / \mathrm{SiC} \mathrm{MMC}$ rotors used in the 1980s, pads designed to run against $\mathrm{SiC}$ ceramic brakes found in several high-performance cars, phenolic pads used against cast-iron rotors in racing applications, and a conventional phenolic used in a midsized sedan. Friction pair testing will begin in the first quarter of FY 2020.

\section{Conclusions}

Future vehicle platforms have created opportunities to re-evaluate Al MMC brakes. Lightweight brake rotors have an opportunity to contribute to vehicle lightweighting efforts and offer the possibility of a $50 \%$ weightsavings in rotating and unsprung mass in some vehicle applications. This project will investigate a potentially lower-cost reinforcement than the more traditional $\mathrm{SiC}$. The project will test this concept by fabricating TiB2 reinforced subscale brake rotor components, test them for wear and tribologic performance, and compare this performance to a large dataset of performance characteristics generated by $\mathrm{DOE}$ projects on $\mathrm{Al} / \mathrm{SiC} \mathrm{MMCs}$ in the early mid-2000s. The results of this work will inform an opportunity for lightweighting this important contributor to vehicle mass.

\section{References}

1. Halonen, A., 2016, "Is now the time for lightweight brakes?" The Brake Report. Available at: https://thebrakereport.com/is-now-the-time-for-lightweight-brakes/ (accessed December 2, 2019). 


\section{I.3 Powertrain Materials Core Program}

\section{I.3.1 High-Temperature Lightweight Engine Materials - Thrust 1 (Oak Ridge National Laboratory)}

\section{I.3.1.1 New Aluminum Alloys with Improved High-Temperature Performance (1A) \\ I.3.1.1.1 Fundamental Studies of Complex Precipitation Pathways (1A1)}

\section{Amit Shyam, Co-Principal Investigator}

Oak Ridge National Laboratory

1 Bethel Valley Road

Oak Ridge, TN 37831

E-mail: shyama@ornl.gov

\section{Dongwon Shin, Co-Principal Investigator}

Oak Ridge National Laboratory

1 Bethel Valley Road

Oak Ridge, TN 37831

E-mail: shind@ornl.gov

\section{Jerry L. Gibbs, DOE Technology Manager}

U.S. Department of Energy

E-mail: jerry.gibbs@ee.doe.gov

Start Date: October 1, 2018

Project Funding (FY19): \$350,000
End Date: September 30, 2023

DOE share: $\$ 350,000 \quad$ Non-DOE share: $\$ 0$

\section{Project Introduction}

ACMZ alloys developed by ORNL using ICME tools are stable to $\sim 350^{\circ} \mathrm{C}$ and are strengthened with the $\theta^{\prime} \mathrm{Al}_{2} \mathrm{Cu}$ precipitates [1,2]. Engineering of key interfaces to increase the temperature capability of lightweight $\mathrm{Al}$ alloys will be the scientific driver for this subtask. The ORNL team recently discovered that $\theta^{\prime}$ and metastable $\mathrm{L}_{2}\left(\mathrm{Al}_{3} \mathrm{Zr}\right)$ co-precipitates can be stabilized. Co-precipitates will have a high-volume fraction (from $\mathrm{Cu}$ ), with the stabilizing influence afforded by an envelope of $\mathrm{L}_{2}$ type precipitates. These coprecipitates could underpin the development of new higher performance alloys (e.g., $375-400^{\circ} \mathrm{C}$ ). This section covers FY 2019 efforts for Subtask 1A1. Fundamental Studies of Complex Precipitation Pathways as part of Thrust 1A for High-Temperature Engine Materials.

\section{Objectives}

This subtask focuses on developing a fundamental understanding of the features that impart thermal stability to cast $\mathrm{Al}$ precipitate microstructures. This is a low technology readiness level subtask to understand and suggest pathways for $\mathrm{Al}-\mathrm{Cu} / \mathrm{ACMZ}$ alloys, including potential mechanisms for further improvement of thermal stability of favorable precipitates. Co-precipitates in $\mathrm{Al}-\mathrm{Cu}$ alloys are being explored for potential demanding powertrain applications.

\section{Approach}

The approach relies on an interplay of characterization and theoretical calculations of alloys of interest. Atom probe tomography (APT) characterization is utilized to examine compositions in the matrix, precipitates, and at matrix/precipitate interface, for different alloy compositions and under different aging conditions. Highresolution transmission electron microscopy (HRTEM) has revealed the structure of interfaces and the formation of $\mathrm{Al}_{3} \mathrm{Zr}$ co-precipitates at coherent $\theta^{\prime} /$ matrix interfaces. Density functional theory (DFT) 
calculations are used to understand interfacial energies, segregations tendencies, and solute-solute interactions that are important for ordering of interfacial solutes forming co-precipitates. Phase-field modeling provides an approach for examining the interplay between thermodynamic and kinetic effects of coarsening and phase transformations, and for different contributions to the driving force for phase transformations - including differences in bulk Gibbs free energies, interfacial energies in the presence of solute, and elastic interactions associated with precipitate morphology. We also apply synchrotron diffraction at the Advanced Photon Source to monitor phase evolution. Further, preliminary in situ HRTEM results of morphological transformations provide key insights and directions into the mechanisms of microstructural evolution.

These capabilities are utilized in close conjunction with each other under an overarching approach to alloy design illustrated by the flow diagram in Figure I.3.1.1.1.1. Structures observed in HRTEM provide key input for DFT calculations, which may then be used to examine segregation tendencies near such interfaces, as well as the energies of those interfaces. These quantities are necessary inputs into the phase-field modeling, particularly segregation tendencies where there are little or no quantitative experimental estimates of related energetics. The phase-field modeling provides information on the changing morphologies and solute distributions at different temperatures and can provide direct comparison with solute distributions from APT. Finally, APT provides much more quantitative information on solute segregation that HRTEM lacks, while HRTEM provides the atomistic and crystallographic resolution that is unavailable from APT. Eventually, the microstructure itself becomes input for the various properties of interest - particularly the mechanical properties. The three areas are referred to as topics in Figure I.3.1.1.1.1 and the alloy design becomes informed and informs these sequential activities in a feedback loop. The approach is expected to accelerate the powertrain alloy development cycle.

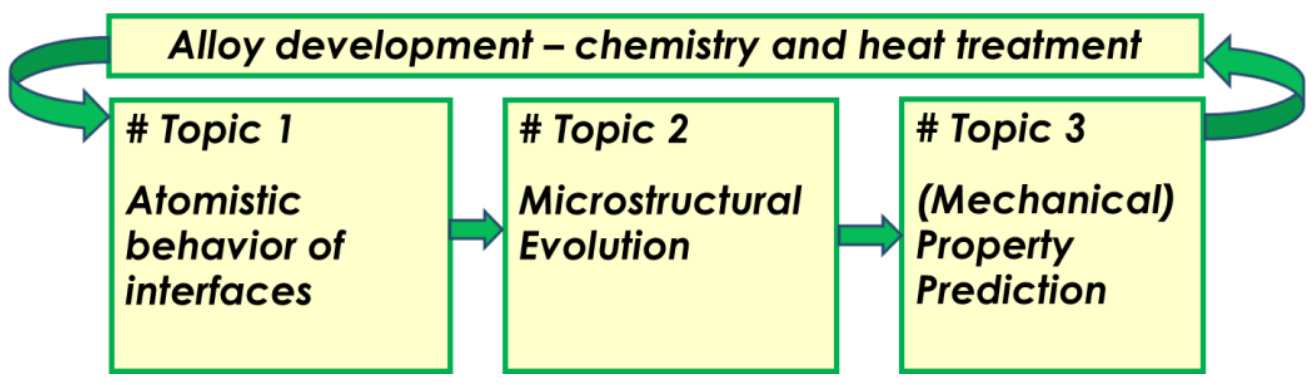

Figure I.3.1.1.1.1. Illustration of the approach for high-temperature alloy design in this effort. See text for details. Source: ORNL.

\section{Results}

\section{Atomistic Behavior of Interfaces}

Figure I.3.1.1.1.2 illustrates an example from Topic 1; i.e., the study of the atomistic behavior of key $\theta^{\prime} / \mathrm{Al}$ matrix interfaces. Here, high-resolution scanning transmission electron microscopy (HR-STEM) revealed the accurate atomic structure of the interface, which was then applied in DFT calculations to measure the interfacial energy of the semi-coherent $\theta^{\prime} / \mathrm{Al}$ matrix interface. In particular, a missing column of $\mathrm{Cu}$ atoms, as seen by the dashed circles in Figure I.3.1.1.1.2, was observed in HR-STEM images with a specific periodicity. Reproduction of the same missing columns in the DFT calculations led to an interfacial energy calculation that was more accurate. The interfacial energy values determine the coarsening resistance of $\mathrm{Al}-\mathrm{Cu}$ alloy microstructures. The results will be reported in upcoming publications. 


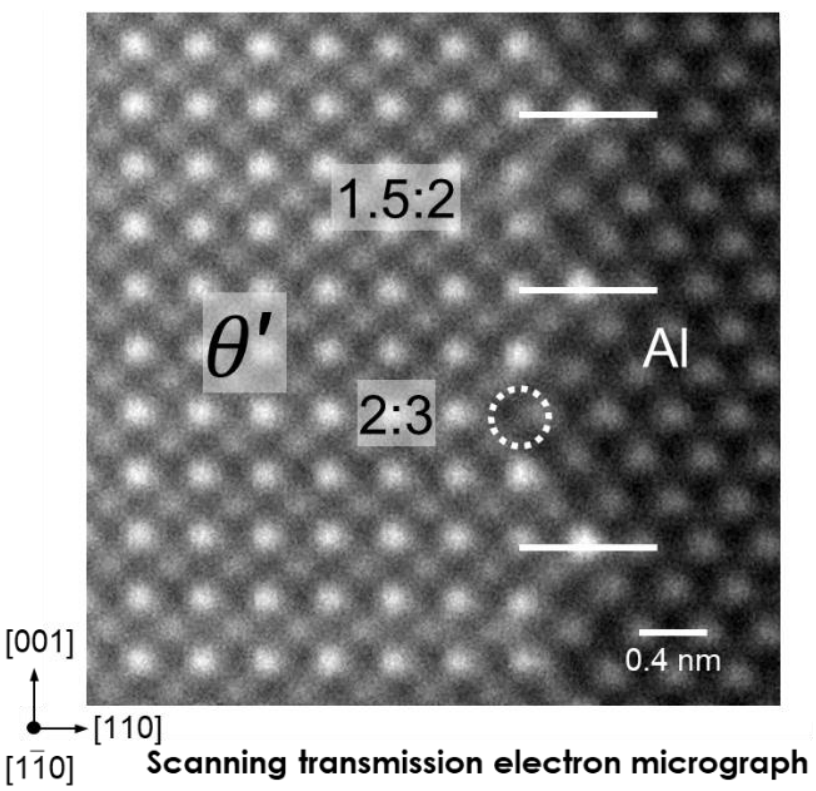

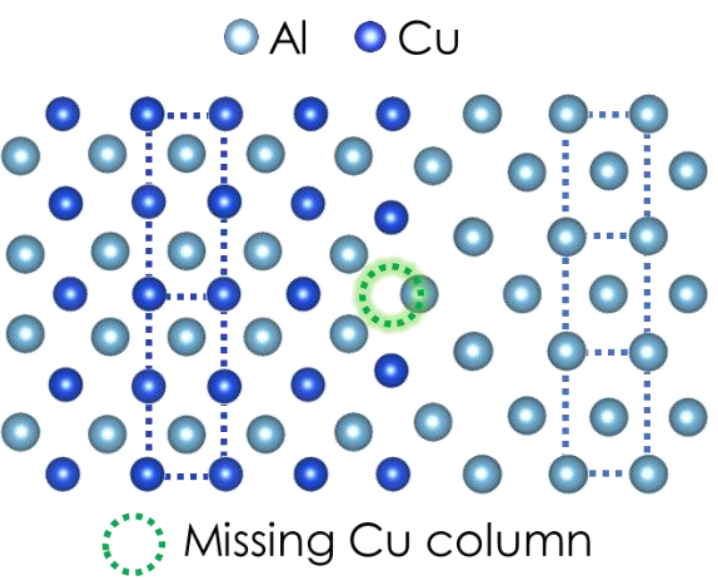

Density Functional Theory model

Figure 1.3.1.1.1.2. An example of advanced characterization (STEM) of key $\theta^{\prime} /$ matrix Al interface (left) guiding atomistic calculations by DFT (right). Source: ORNL.

\section{Microstructural Evolution - Solute Segregation}

Segregation of $\mathrm{Mn}$ and $\mathrm{Zr}$ solute atoms to specific $\theta^{\prime} / \mathrm{Al}$ matrix interfaces is an enabling feature for the thermal stabilization of ACMZ alloys. Figure I.3.1.1.1.3 illustrates solute segregation profiles predicted by phase-field simulations compared to empirical segregation profiles observed with APT [3-4]. The two-dimensional contour plot of Mn near the $\theta^{\prime}$ precipitate that was calculated from APT measurements with $1 \times 1 \times 30 \mathrm{~nm}$ bins is shown in Figure I.3.1.1.1.3 (a). The simulated solute segregation profile with only the lattice strain mechanism activated can be seen in Figure I.3.1.1.1.3 (b) where the greatest segregation occurs at the junction of the coherent and semi-coherent interfaces, similar to the segregation profile observed for $\mathrm{Zr}$ in the next image. The two-dimensional contour plot of $\mathrm{Zr}$ near the $\theta^{\prime}$ precipitate that was also calculated from APT measurements with $1 \times 1 \times 30 \mathrm{~nm}$ bins is shown in Figure I.3.1.1.1.3 (c). The simulated segregation profile with both the lattice strain and interfacial energy mechanisms activated is presented in Figure I.3.1.1.1.3 (d). There is extensive solute segregation to both interfaces, similar to the segregation profile observed for Mn in Figure I.3.1.1.1.3 (a). The purple and gold spheres shown in Figure I.3.1.1.1.3 (a) and Figure I.3.1.1.1.3 (c) are the $\mathrm{Mn}$ and $\mathrm{Zr}$ atoms, respectively. Note that the observed precipitate is approximately $8 \mathrm{~nm}$ thick and the simulated precipitates are approximately $3 \mathrm{~nm}$ thick. 


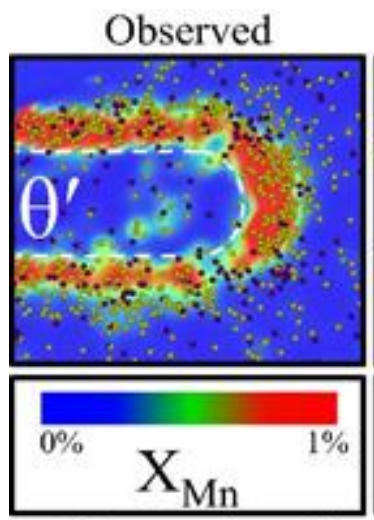

Mn segregation profile

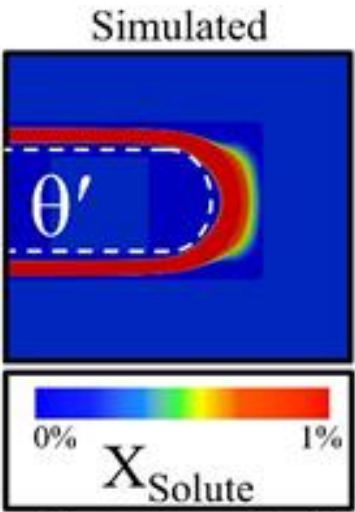

Segregation profile with solute drag and interfacial energy reduction

(a)

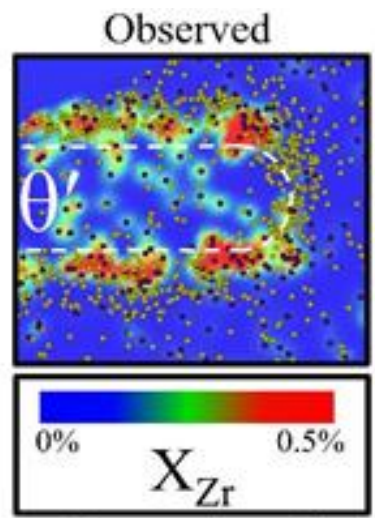

$\mathrm{Zr}$ segregation profile

(c)

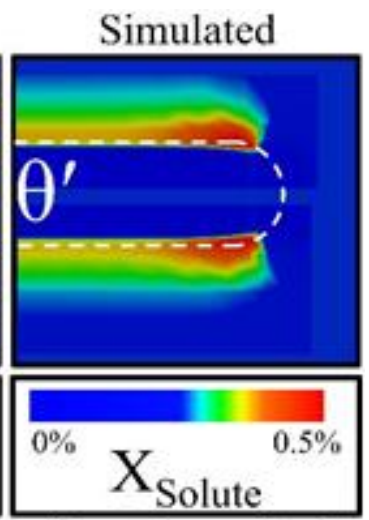

Segregation profile with lattice strain

(d)

Figure I.3.1.1.1.3. Solute segregation profiles predicted by phase-field simulations compared to empirical segregation profile. (a) 2D contour plot of $\mathrm{Mn}$. (b) The simulated solute segregation profile with only the lattice strain mechanism activated. (c) 2D contour plot of Zr. (d) The simulated segregation profile with both the lattice strain and interfacial energy mechanisms activated. Source: ORNL.

\section{Microstructural Evolution - Size and Aspect Ratio Dependence of Rapid $\theta^{\prime} \rightarrow \theta$ Transformations}

In situ microscopy shows that under certain conditions, $\theta^{\prime}$ precipitates may rapidly transform into $\theta$, which then destabilizes the microstructure. We have demonstrated that the conditions for rapid transformation depends on temperature, particle size, and aspect ratio, as observed in Figure I.3.1.1.1.4 (a). For small particles and large aspect ratios, phase-field simulations of small regions of $\theta$ will decrease in size and transform back into $\theta^{\prime}$ shown schematically in Figure I.3.1.1.1.4 (b). In contrast, for larger particles with small aspect ratios, small $\theta$ regions quickly grow and become more equiaxed. We developed an analytic theory for conditions for rapid transformation, which closely captures both phase-field results, observed microstructures and temperature dependencies as shown in Figure I.3.1.1.1.4 (c). The work points to the importance of controlling both size and aspect ratios to stabilize a favorable $\theta^{\prime}$ microstructure and may help identify the most promising approaches to enhancing high-temperature stability [5].

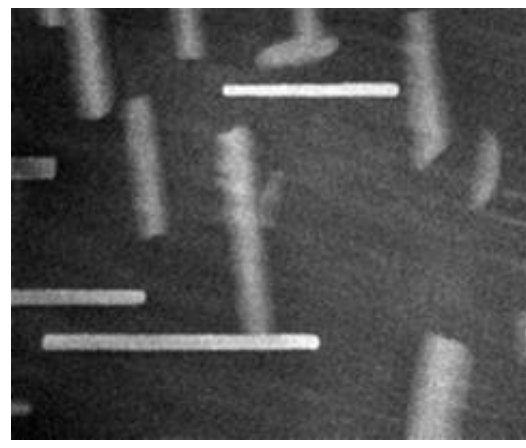

(a)

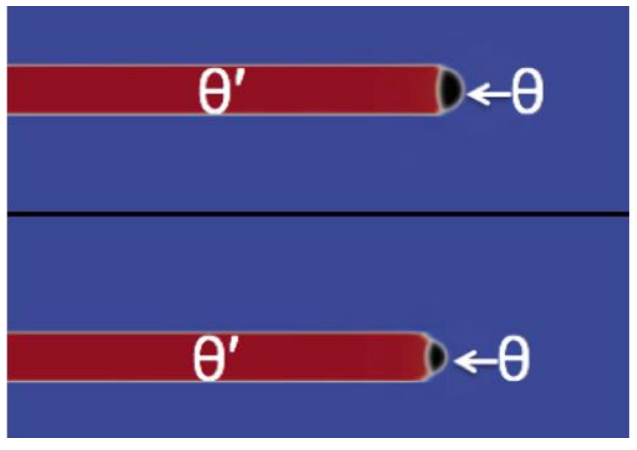

(b)

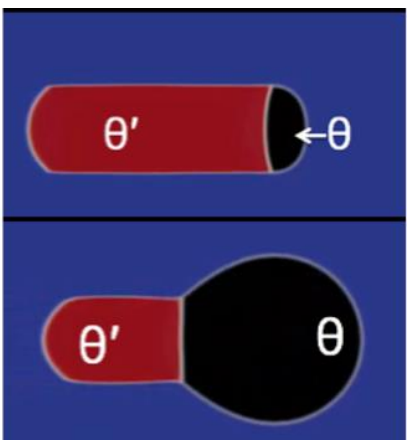

(c)

Figure I.3.1.1.1.4. (a) In situ HR-STEM results showing transformations from $\theta^{\prime}$ to $\theta$. (b) Phase-field simulations show that for small aspect ratios, small precipitates can rapidly transform the entire precipitate, but larger aspect ratios favor the $\theta^{\prime}$ phase. (c) Analytic theory demonstrates the temperature-dependent map of behaviors. Source: ORNL. 


\section{Co-Precipitation of $\mathrm{L1}_{2} \mathrm{Al} / 3 \mathrm{Zr}$ on $\boldsymbol{\theta}^{\prime}$ precipitates}

The HR-STEM results in Figure I.3.1.1.1.5 show that during high-temperature aging, the accumulation of $\mathrm{Zr}$ on the matrix / $\theta^{\prime}$ interface can lead to nucleation of an $\mathrm{Al}_{3} \mathrm{Zr}$ phase ( $\mathrm{L}_{2}$ structure) that is coherent with both the $\mathrm{Al}$ matrix and the $\theta^{\prime}$ precipitate. The $\mathrm{Al}_{3} \mathrm{Zr}$ phase is stable at higher temperatures, and the formation of this phase may stabilize the $\mathrm{Al}_{2} \mathrm{Cu} \theta^{\prime}$ precipitate structure, shown in Figure I.3.1.1.1.5 (a), to temperatures higher than $350^{\circ} \mathrm{C}$. Indeed, after higher temperature aging, the results shown in Figure I.3.1.1.1.5 (b) and Figure I.3.1.1.1.5 (c) indicate that the $\mathrm{Al}_{3} \mathrm{Zr}$ co-precipitate may remain even after the $\theta^{\prime}$ precipitate dissolves after full nucleation and higher temperature aging. Phase-field simulations have shown that the slow $\mathrm{Zr}$ diffusion rates may account for the formation of the remaining $\mathrm{Zr}$-rich regions, even as the $\theta^{\prime}$ precipitate dissolves during coarsening. Thus, the co-precipitate may help thermal stability by preserving the coherent interface and by hindering $\mathrm{Cu}$ diffusion from the precipitate into the matrix. Prior calculations [1] have shown that $\mathrm{Zr}$ preferentially segregates to this coherent interface. Recent DFT calculations show that $\mathrm{Zr}-\mathrm{Zr}$ solute interactions at the interface favor the formation of a single layer that has a characteristic $\mathrm{Zr}$ ordering that essentially templates the $\mathrm{L}_{2}$ phase. Moreover, DFT calculations of $\mathrm{L}_{2} / \theta^{\prime}$ interfacial energies show that the interfaces have a low energy, particularly for $\mathrm{Al}_{3} \mathrm{Zr}$ (in comparison with $\mathrm{Al}_{3} \mathrm{Ti}$ and $\mathrm{Al}_{3} \mathrm{Sc}$ ). Ti and $\mathrm{Sc}$ have similar segregation tendencies, but different diffusion rates in the matrix, and this observation provides an opportunity to both test the role of interfacial energy vs. diffusion kinetics, and to control the processes of coprecipitation for favorable high-temperature response.

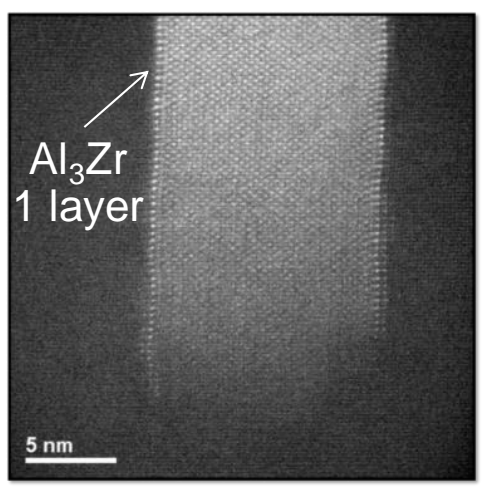

(a)

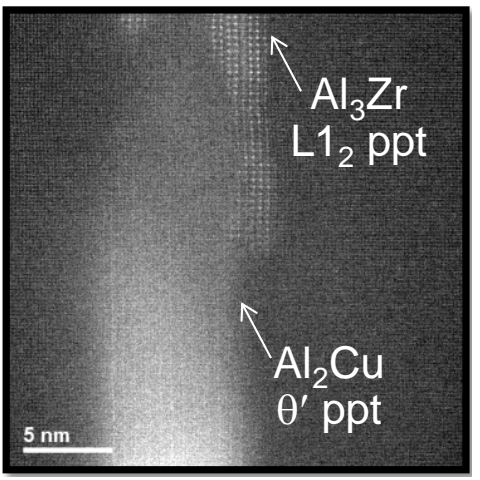

(b)

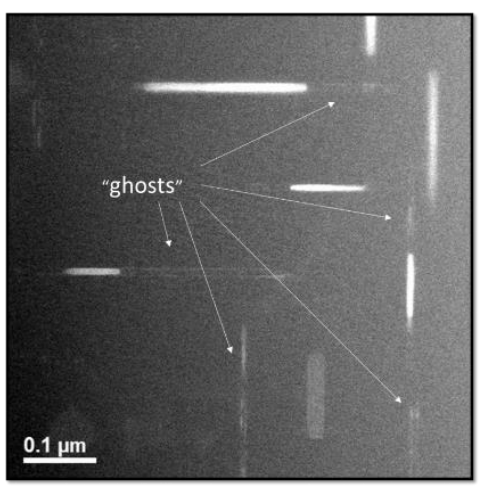

(c)

Figure I.3.1.1.1.5. (a) HR-STEM results showing a single layer of $\mathrm{Al}_{3} \mathrm{Zr}$ at the interface between the $\mathrm{Al}$ matrix and the $\mathrm{Al}{ }_{2} \mathrm{Cu}$ $\theta^{\prime}$ precipitate. (b) Full nucleation of $\mathrm{Al}_{3} \mathrm{Zr} \mathrm{L}_{1} 1_{2}$ on $\theta^{\prime}$ precipitate. (c) “Ghost” layers of $\mathrm{Al}_{3} \mathrm{Zr}\left(\mathrm{L} 1_{2}\right.$ structure) near the $\theta^{\prime}$ precipitates after higher temperature aging. Source: ORNL.

\section{Conclusions}

The following are the key outcomes of the present subtask:

- We have identified key thermodynamic and kinetic processes for solute-stabilized microstructures—and some of the synergy and competition between these processes - to identify key aspects of the enhanced stability of the ACMZ alloys.

- Co-precipitates of $\mathrm{Al}_{3} \mathrm{Zr}$ on $\theta^{\prime}$ precipitates have been observed to form at elevated temperatures and may provide stabilization of the favorable microstructure at higher temperatures.

- In situ microscopy shows process of development of $\theta$ phase from the $\theta^{\prime}$ phase. Phase-field simulations and analytic theory demonstrates conditions for rapid $\theta^{\prime}$ to $\theta$ transformation.

- HR-STEM results show the structure of the [110]-oriented $\theta^{\prime} /$ matrix semi-coherent interface, and DFT calculations show this has an interfacial energy comparable to the [100] orientation. 


\section{Key Publications}

1. Allard, L. F., D. N. Leonard, J. D. Poplawsky, M. F. Chisholm, B. D. Eckhart, A. Shyam, F. S. Walden, B. B. Larson, R. Kelly, A. Stokes, and W. C. Bigelow, 2019, "The utility of Xe-plasma FIB for preparing $\mathrm{Al}$ alloy specimens for MEMS-based in situ double-tilt heating experiments," Microsc. Microanal., Vol. 25, Supp. S2, pp. 1442-1443, 05 August 2019.

2. Shyam, A., S. Roy, D. Shin, J. D. Poplawsky, L. F. Allard, Y. Yamamoto, J. R. Morris, B. Mazumder, J. C. Idrobo, A. Rodriguez, T. R. Watkins, and J. A. Haynes, 2019, "Elevated temperature microstructural stability in cast AlCuMnZr alloys through solute segregation," Mat. Sci. Eng. A-Struct, Vol. 765, Art. \#138279, 23 September 2019.

3. Shower, P., J. R. Morris, D. Shin, B. Radhakrishnan, J. D. Poplawsky, and A. Shyam, 2019, "Mechanisms for stabilizing $\theta^{\prime}(\mathrm{Al} 2 \mathrm{Cu})$ precipitates at elevated temperatures investigated with phase-field modeling," Materialia, Vol. 6, Art. \#100335, June 2019.

4. Shower, P., J. R. Morris, D. Shin, B. Radhakrishnan, L. F. Allard and A. Shyam, 2019, "Temperaturedependent stability of $\theta^{\prime}$-Al2Cu precipitates investigated with phase-field simulations and experiments," Materialia, Vol. 5, Art. \#100185, March 2019.

5. Shower, P., J. R. Morris, D. Shin, L. F. Allard, B. Radhakrishnan, and A. Shyam, "Onset of $\theta$ ' to $\theta$ phase transformation in Al-Cu alloys," (in review - Mat. Sci. Eng. A ).

6. Samolyuk, G. D., M. Eisenbach, D. Shin, Y. N. Osetsky, A. Shyam, and J. R. Morris, "Equilibrium solute segregation to matrix- $\theta$ ' precipitate interfaces in Al-Cu alloys from first principles," (in review - Phys Rev Mat).

\section{References}

1. Shin, D., A. Shyam, S. Lee, Y. Yamamoto, and J. A. Haynes, 2017, "Solute segregation at the Al/ $\theta^{\prime}-$ $\mathrm{Al}_{2} \mathrm{Cu}$ interface in Al-Cu alloys," Acta Mater., Vol. 141, pp. 327-340, December 2017.

2. Shyam, A., S. Roy, D. Shin, J. D. Poplawsky, L.F. Allard, Y. Yamamoto, J. R. Morris, B. Mazumder, J. C. Idrobo, A. Rodriguez, T. R. Watkins, and J. A. Haynes, 2019, "Elevated temperature microstructural stability in cast AlCuMnZr alloys through solute segregation," Mat. Sci. Eng. A-Struct, Vol. 765, Art. \#138279, 23 September 2019.

3. Shower, P., J. R. Morris, D. Shin, B. Radhakrishnan, J. D. Poplawsky, and A. Shyam, 2019, "Mechanisms for stabilizing $\theta^{\prime}(\mathrm{A} 12 \mathrm{Cu})$ precipitates at elevated temperatures investigated with phase-field modeling," Materialia, Vol. 6, Art. \#100335, June 2019.

4. Shower, P., J. R. Morris, D. Shin, B. Radhakrishnan, L. F. Allard, and A. Shyam, 2019, "Temperaturedependent stability of $\theta^{\prime}$-Al2 $\mathrm{Cu}$ precipitates investigated with phase-field simulations and experiments," Materialia, Vol. 5, Art. \#100185, March 2019.

5. Shower, P., J. R. Morris, D. Shin, L. F. Allard, B. Radhakrishnan, and A. Shyam, "Onset of $\theta^{\prime}$ to $\theta$ phase transformation in Al-Cu alloys," (in review - Mat. Sci. Eng. A ).

6. Samolyuk, G. D., M. Eisenbach, D. Shin, Y. N. Osetsky, A. Shyam, and J. R. Morris, "Equilibrium solute segregation to matrix- $\theta$ ' precipitate interfaces in Al-Cu alloys from first principles," (in review - Phys Rev Mat).

\section{Acknowledgements}

The authors would like to acknowledge the contributions of the following individuals: James Morris, Patrick Shower, Lawrence Allard, Dana McClurg, Shane Hawkins, and Sumit Bahl for their contributions to this research. 


\section{I.3.1.1.2 New Higher Performance Aluminum Alloys (1A2)}

\section{Amit Shyam, Co-Principal Investigator}

Oak Ridge National Laboratory

1 Bethel Valley Road

Oak Ridge, TN 37831

E-mail: shyama@ornl.gov

\section{Dongwon Shin, Co-Principal Investigator}

Oak Ridge National Laboratory

1 Bethel Valley Road

Oak Ridge, TN 37831

E-mail: shind@ornl.gov

\section{Jerry L. Gibbs, DOE Technology Development Manager}

U.S. Department of Energy

E-mail: jerry.gibbs@ee.doe.gov

Start Date: October 15, 2018

Project Funding (FY19): \$400,000
End Date: September 30, 2023

DOE share: $\$ 400,000$
Non-DOE share: $\$ 0$

\section{Project Introduction}

New alloys (including with co-precipitates) will be developed with targeted increases in temperature capabilities and other improved attributes, for specific applications like LD pistons and turbo compressors. Attributes for these alloys will be properties - especially mechanical properties - that need to be optimized for specific component level engineering applications. An ICME approach with alloy design and evaluation was performed iteratively. This section covers FY 2019 efforts for Subtask 1A2. New Higher Performance Al Alloys as part of Thrust 1A for High-Temperature Engine Materials.

\section{Objectives}

This subtask focuses on a fundamental understanding of the features that impart thermal stability to cast Al precipitate microstructures. This is a low technology readiness level subtask, to understand and suggest pathways for $\mathrm{Al}-\mathrm{Cu} / \mathrm{ACMZ}$ alloys, including potential mechanisms for further improvement of thermal stability of favorable precipitates. Co-precipitates in $\mathrm{Al}-\mathrm{Cu}$ alloys are being explored for potential applications above $350^{\circ} \mathrm{C}$.

\section{Approach}

The approach in this subtask is like Subtask 1A1-Linking Experiments and Simulations-with the primary difference being an emphasis on the establishment of microstructure and mechanical property linkages of interest. The approach relies upon an interplay of characterization and theoretical calculations that lead to the development of improved high-temperature $\mathrm{Al}$ alloys of interest. These theoretical and experimental capabilities are utilized in close conjunction with each other.

Within the precipitation-hardened alloys, there is a short-term emphasis on utilizing the non-equilibrium interfacial solute segregation (NEISS) mechanism to improve the chemistry, heat-treatments, and elevated temperature capability of ACMZ alloys and variants. For the dispersion-hardened alloys in development, the chemistries to be examined include some alloys with improved elevated temperature properties under additive/rapid solidification processing conditions. 


\section{Results}

\section{NEISS Mechanism}

A team led by ORNL demonstrated that the operating temperature limit of Al-Cu alloys can be extended by stabilizing the strengthening $\theta^{\prime}$ phase to a higher temperature [1-2]. In this subtask, we identify a NEISS mechanism in $\mathrm{Al}-\mathrm{Cu}$ alloy microstructures that imparts increased thermal stability of the metastable $\theta^{\prime}$ phase over the thermodynamically stable $\theta$ phase, as shown in Figure I.3.1.1.2.1. The NEISS mechanism can be implemented in $\mathrm{Al}-\mathrm{Cu}$ alloy microstructures through microalloying additions that have diffusion coefficients lower than $\mathrm{Cu}$ and that have a driving force for segregating to critical interfaces between the $\theta^{\prime}$ and the matrix. APT calculations of interfacial $\mathrm{Zr}$ concentration show a linear dependence on the calculated diffusion distance of $\mathrm{Zr}$ for different temperatures and times as shown in Figure I.3.1.1.2.1 (a). Through examples from the ACMZ alloy system, where $\theta^{\prime}$ precipitates are stable up to $350^{\circ} \mathrm{C}$, it was demonstrated that the NEISS mechanism is effective in improving the thermal stability of $\theta^{\prime}$ precipitates. At long exposure times, an $\mathrm{L}_{2}$ phase forms on the coherent interface and the solute concentration corresponding to this $\mathrm{Al}_{3} \mathrm{Zr}$ phase as shown in Figure I.3.1.1.2.1 (b).

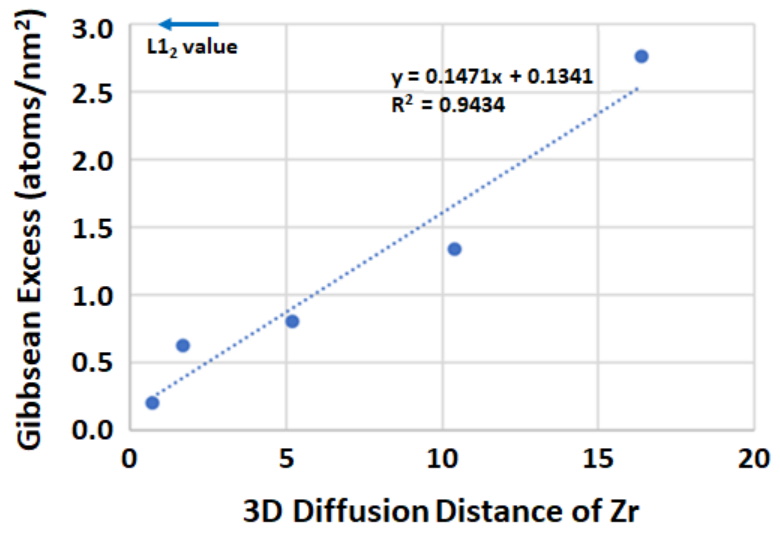

(a)

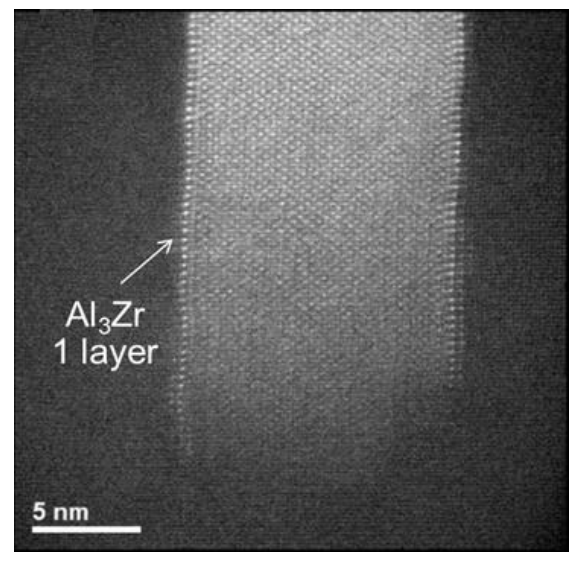

(b)

Figure I.3.1.1.2.1. The NEISS mechanism for stabilization of precipitation-hardened Al-Cu alloy microstructures using: (a) atom probe calculations of interfacial Zr concentration; and (b) an $\mathrm{L} 1_{2}$ phase on the coherent interface and the solute concentration corresponding to this $\mathrm{Al}_{3} \mathrm{Zr}$ phase. Source: ORNL.

Finally, the DFT calculated values of the interfacial energy for the semi-coherent, coherent interface of $\theta^{\prime}$ phase and the interfaces between the $\mathrm{Al}-\mathrm{Al}_{3} \mathrm{Zr}$ and $\mathrm{Al}_{3} \mathrm{Zr}_{\mathrm{Al}} \mathrm{Al}_{2} \mathrm{Cu}$ coherent interface, which are the coprecipitates of $\theta^{\prime}-\mathrm{Al}_{3} \mathrm{Zr}$, were calculated and are displayed in the bar chart of Figure I.3.1.1.2.2. Formation of the co-precipitate leads to a reduction in the interfacial energy of precipitates as seen in Figure I.3.1.1.2.1 (b) above and, therefore, more thermally stable microstructure. It was further demonstrated that DFT calculations are a useful tool in identifying elements possessing a thermodynamic driving force to segregate to the critical interfaces. Two areas of current research are: (i) the general nature of the NEISS mechanism; and (ii) whether the mechanism can be applied to progressively stabilize or "rejuvenate" precipitation-hardened alloy systems. 


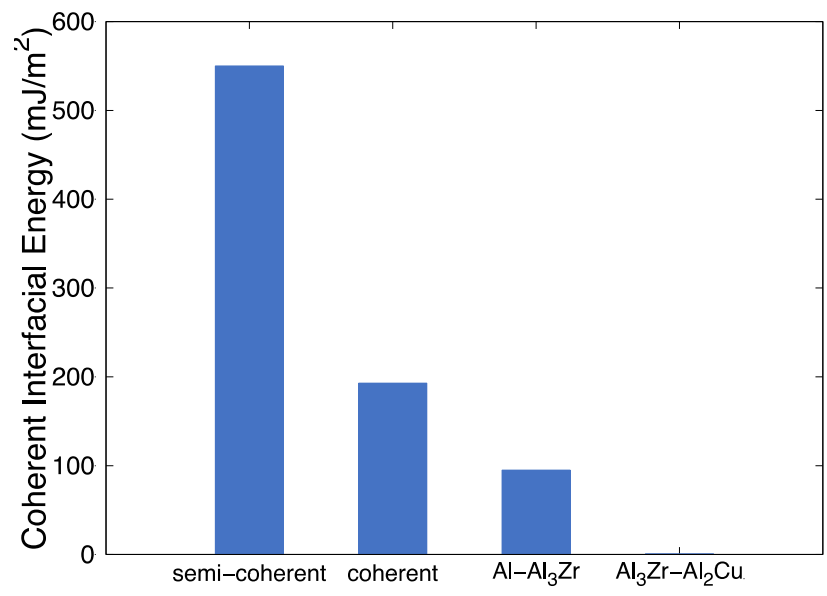

Figure I.3.1.1.2.2. DFT calculated values of interfacial energy for the semi-coherent, coherent interface of $\theta^{\prime}$ phase and the interfaces between the $\mathrm{Al}_{-} \mathrm{Al}_{3} \mathrm{Zr}$ and $\mathrm{Al}_{3} \mathrm{Zr}-\mathrm{Al} 2 \mathrm{Cu}$ coherent interface. Source: ORNL.

\section{Anisotropic Strain-Hardening in an Al-Cu Alloy}

Neutron diffraction was applied to determine the anisotropy in strain-hardening of an Al-Cu alloy with various heat-treatments such as solution-treated (or naturally aged) shown in Figure I.3.1.1.2.3 (a); $200^{\circ} \mathrm{C}$

preconditioned shown in Figure I.3.1.1.2.3 (b); and $300^{\circ} \mathrm{C}$ preconditioned shown in Figure I.3.1.1.2.3 (c).

Plastic anisotropy in the microstructure is determined by the microstructure following the aging treatment. The strain-hardening rate was strongly orientation-dependent and this orientation dependence was modeled based on dislocation precipitate interactions.

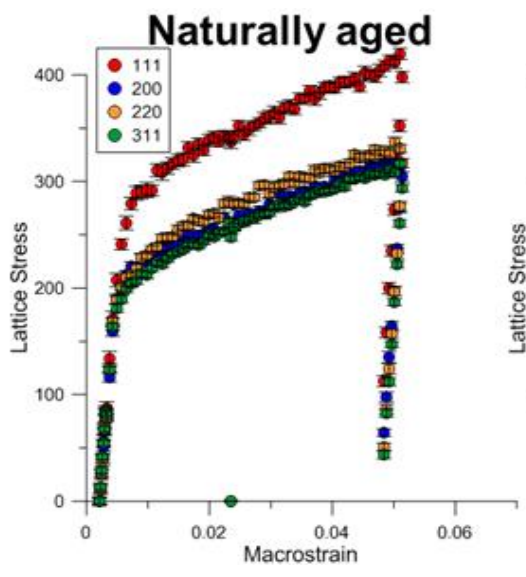

(a)

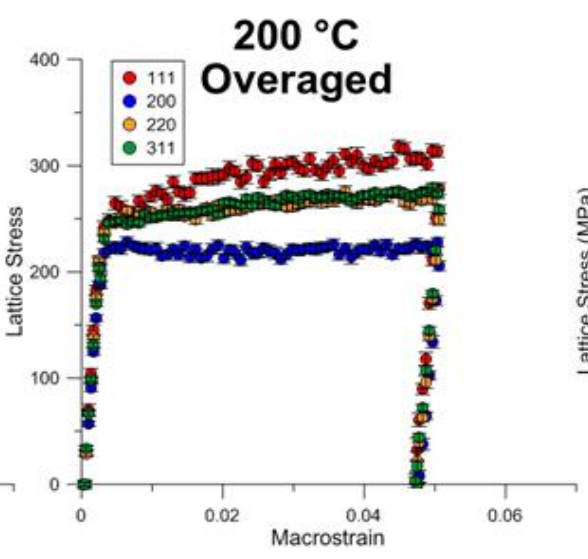

(b)

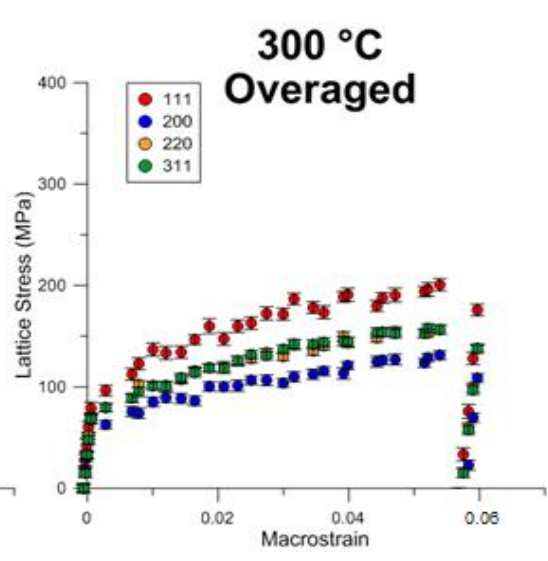

(c)

Figure I.3.1.1.2.3. In situ neutron diffraction during room temperature tensile testing of an Al-Cu alloy under various testing conditions. (a) Solution-treated (or naturally aged); (b) $200^{\circ} \mathrm{C}$ preconditioned; and (c) $300^{\circ} \mathrm{C}$ preconditioned. Source: ORNL.

Figure I.3.1.1.2.4 presents a schematic summary of the deformation mechanisms in the Al-Cu alloy of interest. There are three regions of the deformation curve in each of these aging conditions. The curves begin with elastic loading, where both the precipitate and matrix phases are being loaded by the applied stress, and little load transfer between grains or precipitates is occurring. Next, the bulk material yields. In the natural aged condition, the shearing of bulk grains occurs via precipitate shearing. In the $200^{\circ} \mathrm{C}$ and $300^{\circ} \mathrm{C}$ overaged conditions, the shearing occurs via Orowan looping. In the latter two conditions, the matrix will be straining plastically while the precipitates are straining elastically, which leads to high elastic strains - and therefore, stresses - in the precipitates. The final part of the curve occurs at a higher strain. At this point in all the aging 
conditions, the precipitates have built up enough stress that they undergo one of a handful of mechanisms, including shearing or fracture. The $200^{\circ} \mathrm{C}$ overaged condition shows significant orientation dependence due to orientation-dependent precipitate shearing. The shear strength of the $\theta^{\prime}$ precipitate was calculated from neutron diffraction data to be about $2 \mathrm{GPa}$, which is in line with the reported value according to molecular dynamics simulations. These results will be published at a later date. They support the development of improved ductility and low-cycle fatigue resistant Al alloys for cylinder head and other internal combustion engine applications.

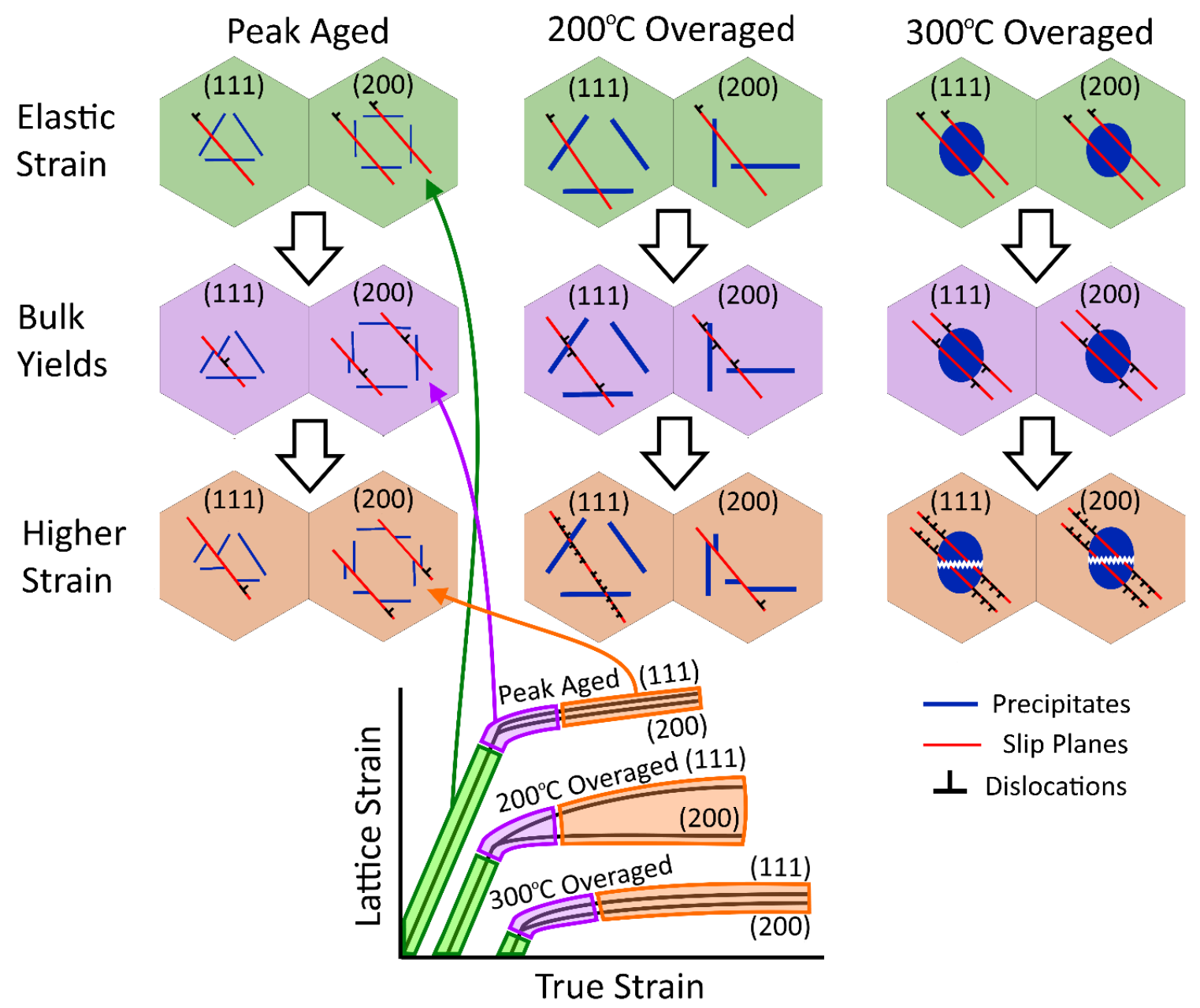

Figure I.3.1.1.2.4. Schematic displaying the stages of deformation-as well as their grain-and precipitate-scale deformation mechanisms for each aging condition. Source: ORNL.

\section{Conclusions}

The following are the key outcomes for this subtask:

- A NEISS mechanism was discovered in Al-Cu alloys, which is responsible for imparting thermal stability to these microstructures.

- $\quad$ Orientation dependence of the strain-hardening rate in $\mathrm{Al}-\mathrm{Cu}$ alloys with various heat-treatments was measured experimentally with neutron diffraction techniques and are being analytically modeled.

- Dispersion-hardened high-temperature $\mathrm{Al}$ alloys continue being developed. 


\section{Key Publications}

1. Shyam, A., et al., 2019, "Non-equilibrium interfacial solid solute segregation as a thermal stabilization mechanism in Al-Cu alloys," (in preparation for Acta Materialia).

2. Wang, J., Y. Nobakht, J. D. Blanks, D. Shin, S. Lee, A. Shyam, H. Rezayat, and S. Shin, 2019, "Machine-learning for thermal transport analysis of Al alloys with precipitate morphology," Adv. Theory Simul., Vol. 2, No. 4, Art. \#1800196, April 2019.

3. Wang, J., S. Shin, A. Y. Nobakht, and A. Shyam, 2019, "Structural deformation and transformation of $\theta^{\prime}$ $\mathrm{Al}_{2} \mathrm{Cu}$ precipitate in $\mathrm{Al}$ matrix via interfacial diffusion," Comp. Mater. Sci., Vol. 156, pp. 111-120, January 2019.

4. Milligan, B. K., S. Roy, L. F. Allard, C. S. Hawkins, and A. Shyam, 2019, "The effect of microstructural stability on the creep behavior of cast Al-Cu alloys at $300^{\circ}$ C," Mat. Sci. Eng. A-Struct, Vol. 765, Art. \#138697, 20 January 2020.

5. Milligan, B. K., D. Ma, A. Shyam, and A. J. Clarke. 2019, "Grain orientation-dependent strain-hardening in a precipitation-strengthened Al-Cu alloy," (in preparation for Acta Materialia).

\section{References}

1. Shin, D., A. Shyam, S. Lee, Y. Yamamoto, and J. A. Haynes, 2017, "Solute segregation at the Al/ $\theta^{\prime}$ $\mathrm{Al}_{2} \mathrm{Cu}$ interface in Al-Cu alloys," Acta Mater., Vol. 141, pp. 327-340, December 2017.

2. Shyam, A., S. Roy, D. Shin, J. D. Poplawsky, L.F. Allard, Y. Yamamoto, J. R. Morris, B. Mazumder, J. C. Idrobo, A. Rodriguez, T. R. Watkins, and J. A. Haynes, 2019, "Elevated temperature microstructural stability in cast AlCuMnZr alloys through solute segregation," Mat. Sci. Eng. A-Struct, Vol. 765, Art. \#138279, 23 September 2019.

\section{Acknowledgements}

The authors would like to acknowledge the contributions of the following individuals: James Morris, Patrick Shower, Lawrence Allard, Dana McClurg, Shane Hawkins, and Sumit Bahl for their contributions to this research. 


\title{
I.3.1.1.3 Intermediate Temperature Variants of Cast ACMZ Alloys (1B1)
}

\author{
Amit Shyam, Co-Principal Investigator \\ Oak Ridge National Laboratory \\ 1 Bethel Valley Road \\ Oak Ridge, TN 37831 \\ E-mail: shyama@ornl.gov
}

\section{Jerry L. Gibbs, DOE Technology Development Manager}

U.S. Department of Energy

E-mail: jerry.gibbs@ee.doe.gov

Start Date: October 15, 2018

Project Funding (FY19): \$300,000
End Date: September 30, 2023

DOE share: $\$ 300,000$
Non-DOE share: $\$ 0$

\section{Project Introduction}

The microstructure of ACMZ alloys remains stable up to temperatures as high as $350^{\circ} \mathrm{C}$, which is well beyond the $250^{\circ} \mathrm{C}$ limit of existing commercial cast $\mathrm{Al}$ alloys [1,2]. Aside from high-temperature microstructural stability, improvement in low to intermediate temperature ductility $\left(<150^{\circ} \mathrm{C}\right)$ is required to enhance the lowcycle fatigue life of $\mathrm{Al}$ alloys for cylinder head applications [3]. Room temperature elongation between $5 \%$ and $6 \%$ is targeted at higher copper contents (> 7 wt. \%) in ACMZ alloys. Higher copper content is required to maintain the much-needed hot tear resistance in ACMZ alloys [4]. Eventually, the low-cycle fatigue life of modified ACMZ alloys is targeted to exceed the low-cycle fatigue life of 319 and A356 + 0.5 Cu commercial cast Al alloys. This section covers FY 2019 efforts for Subtask 1B1. Intermediate Temperature Variants of Cast ACMZ Alloys as part of Thrust 1A for High-Temperature Engine Materials.

\section{Objectives}

The subtask aims to develop fundamental insights into the factors that affect ductility and low-cycle fatigue life of ACMZ alloys. Efforts will identify the key microstructural attributes that can be tailored for improving the ductility and low-cycle fatigue life of ACMZ alloys.

\section{Approach}

A typical ACMZ alloy microstructure consists of grain boundary intermetallic $\theta\left(\mathrm{Al}_{2} \mathrm{Cu}\right)$ particles. These particles form due to excess copper content above the solid solubility limit of copper in Al. Consequently, the formation of these particles is a function of the copper content. The particles are potent crack initiation sites and therefore, a key lever for managing ductility in ACMZ alloys. A series of five ACMZ alloys were prepared with increasing copper content. The copper contents of the five alloys in wt.\% were 6.0,6.6, 7.3, 8.0, and 9.0. Tensile properties of the as-aged alloys were measured at room temperature. Low-cycle fatigue tests are being performed at $150^{\circ} \mathrm{C}$ on the as-aged alloys and at $250^{\circ} \mathrm{C}$ on the as-aged alloys thermally exposed to $250^{\circ} \mathrm{C}$ for $100 \mathrm{~h}$. The aging condition for each alloy was $240^{\circ} \mathrm{C}$ for $5 \mathrm{~h}$. Finite element model is applied on real microstructures to simulate ductility of the alloys $[5,6]$.

\section{Results}

\section{Microstructures of As-Aged Alloys}

Figure I.3.1.1.3.1 (a)-(e) show micrographs of the as-aged ACMZ alloys with different copper contents captured with a SEM. The Al grains have an equiaxed morphology and the grain size is similar in all the alloys. The grain boundaries are decorated with $\theta$ particles that appear white in contrast. The volume fraction and size of grain boundary $\theta$ particles can be seen to increase with copper content and their values are 
summarized in Table I.3.1.1.3.1. Figure I.3.1.1.3.1 (f) shows a representative high magnification micrograph of the $\mathrm{Al}$ grains. The microstructure within the grains is comprised of strengthening $\theta^{\prime}$ precipitates and is invariant with the copper content. Therefore, any variation in the mechanical properties among the five alloys will be a function of only the grain boundary $\theta$ particles.
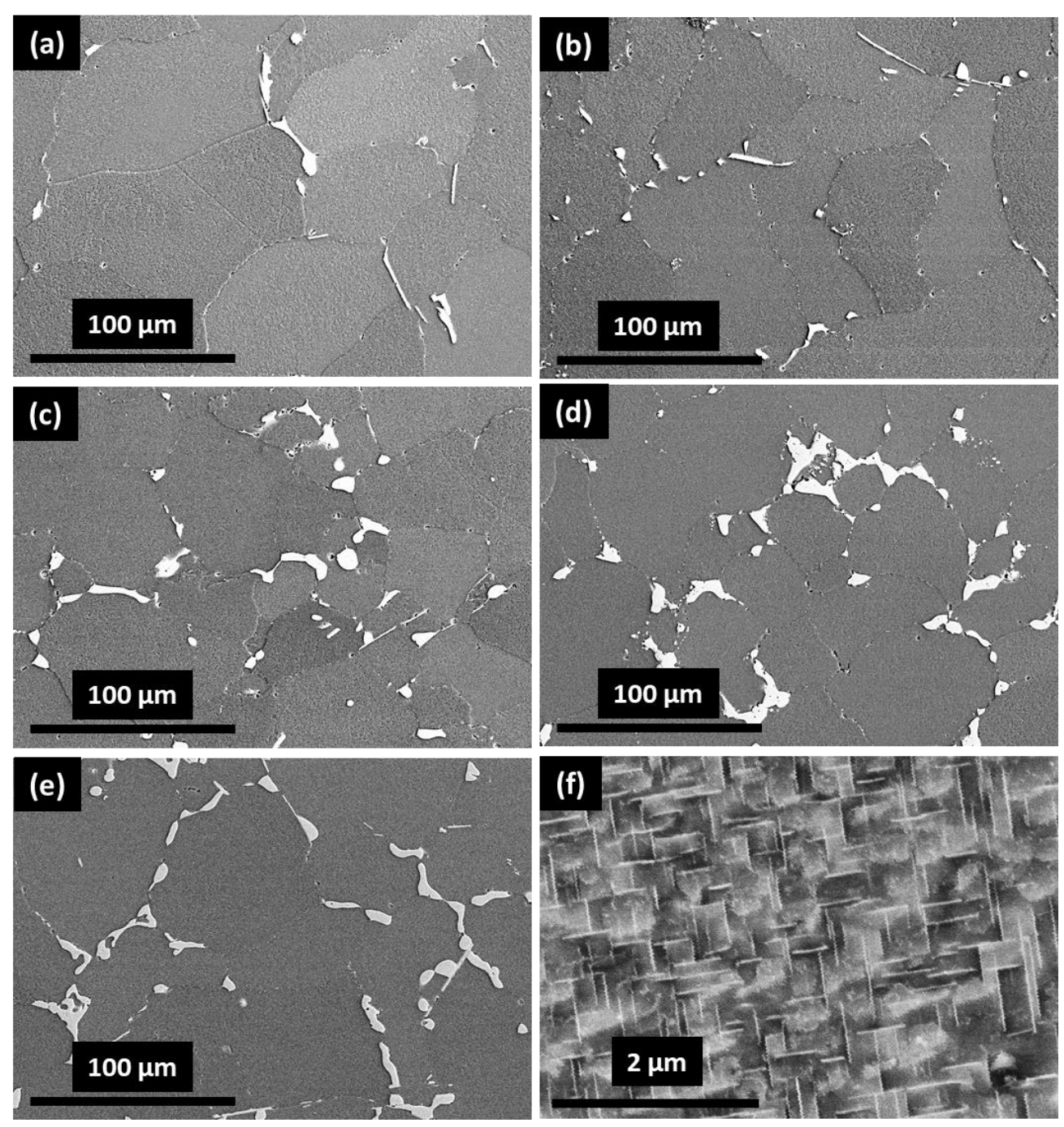

Figure I.3.1.1.3.1. Low magnification scanning electron micrographs of the as-aged alloys showing particles in (a) $6.0 \mathrm{Cu}$; (b) $6.6 \mathrm{Cu}$; (c) $7.3 \mathrm{Cu}$; (d) $8.0 \mathrm{Cu}$; and (e) $9.0 \mathrm{Cu}$ alloys. (f) Higher magnification scanning electron micrograph of $8.0 \mathrm{Cu}$ alloy showing $\theta^{\prime}$ precipitates. Source: ORNL.

Table I.3.1.1.3.1. Mean Values of Al Grain Size as Well as Area and Volume Fraction of Grain Boundary $\Theta$ Particles in the As-Aged ACMZ Alloys.

\begin{tabular}{|c|c|c|c|}
\hline Alloy & Grain Size $(\boldsymbol{\mu m})$ & Particle Area $\left(\boldsymbol{\mu m}^{2}\right)$ & Volume Fraction (\%) \\
\hline $6.0 \mathrm{Cu}$ & $67 \pm 6$ & $11 \pm 2$ & 0.3 \\
\hline $6.6 \mathrm{Cu}$ & $49 \pm 7$ & $20 \pm 2$ & 0.6 \\
\hline $7.3 \mathrm{Cu}$ & $48 \pm 6$ & $26 \pm 2$ & 2.3 \\
\hline $8.0 \mathrm{Cu}$ & $48 \pm 3$ & $32 \pm 3$ & 3.2 \\
\hline $9.0 \mathrm{Cu}$ & $60 \pm 6$ & $37 \pm 3$ & 4.8 \\
\hline
\end{tabular}




\section{Tensile Properties of As-Aged}

Figure I.3.1.1.3.2 (a) shows room temperature engineering stress-strain curves of the as-aged alloys. The yield strength, ultimate tensile strength, and tensile elongation values are summarized in Figure I.3.1.1.3.2 (b). The tensile elongation, as a measure of ductility, decreases monotonically with an increase in copper content. Yield strength, on the other hand, remains independent of the copper content. The similar yield strength among the five alloys with different amounts of copper is due to similar microstructures in terms of strengthening $\theta^{\prime}$ precipitates, as shown in Figure I.3.1.1.3.1 (f) above. Therefore, the variation in elongation with copper content is primarily a function of grain boundary $\theta$ particles.
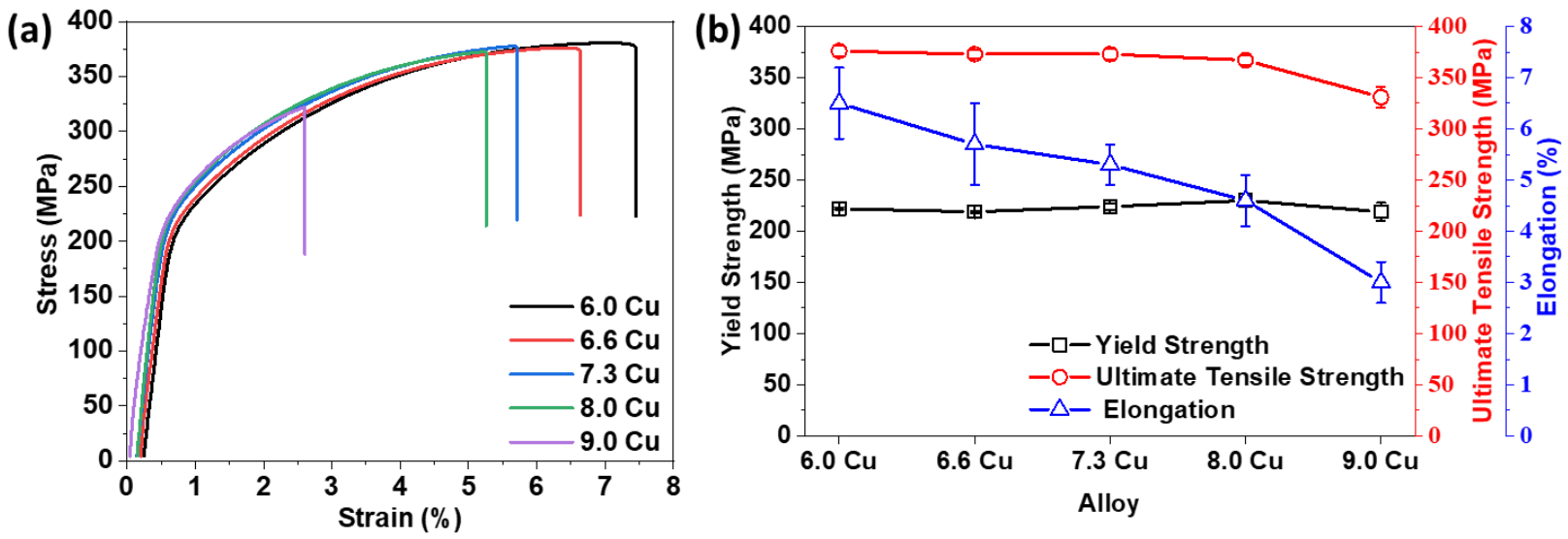

Figure I.3.1.1.3.2. (a) Room temperature engineering stress-strain curves of as-aged alloys; and (b) variation of yield strength, ultimate tensile strength, and elongation as a function of copper content. Source: ORNL.

Figure I.3.1.1.3.3 (a) shows a representative microstructure illustrating fractured grain boundary $\theta$ particles after tensile test highlighting their role in crack initiation. Figure I.3.1.1.3.3 (b) is a fractograph showing the fracture surface of a tensile tested ACMZ alloy captured in backscattered electron mode. The fracture surface is extensively covered with grain boundary $\theta$ particles implying their active role in the complete fracture process. Figure I.3.1.1.3.3 (c) and Figure I.3.1.1.3.3 (d) compare the mean area and circularity, respectively, of unfractured particles after tensile tests with the overall particle distribution in the un-tested alloy as a function of copper content. Circularity of a particle is given by $4 \pi$ (Area/Perimeter $\left.{ }^{2}\right)$. A value of circularity equal to 1 indicates a circle and below 1 indicates deviations from circular geometry. Circularity is an alternative to aspect ratio when particle shapes deviate significantly from ellipses, as in the present case. From Figure I.3.1.1.3.3 (d), the mean area of unfractured particles is smaller than mean area of overall particle distribution up to $7.3 \mathrm{wt} \%$ of copper. This observation implies that larger particles at higher copper contents are more prone to fracture. The increased propensity of particle fracture at higher copper level could lead to a concomitant decrease in the elongation of the alloy. The morphology of unfractured particles as defined by circularity is similar to the overall particle distribution suggesting its limited effect on elongation.

Figure I.3.1.1.3.4 (a) shows a finite element model for the alloy based on a montage prepared using real microstructures. The grain boundary $\theta$ particles are colored in dark green and the Al grains are colored in light green. A comparison between simulated and experimental values of elongation is presented in Figure I.3.1.1.3.4 (b). Simulation-derived crack initiation and crack propagation strain are also shown. 
(a)
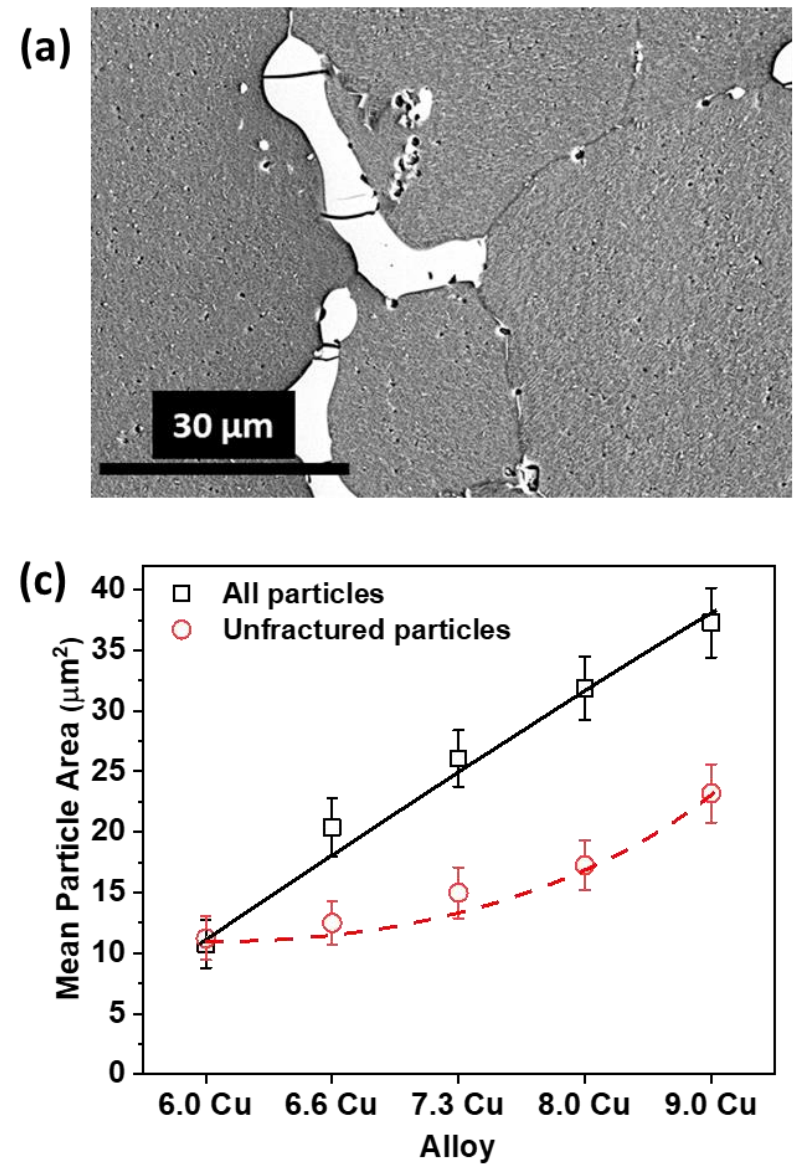

(b)

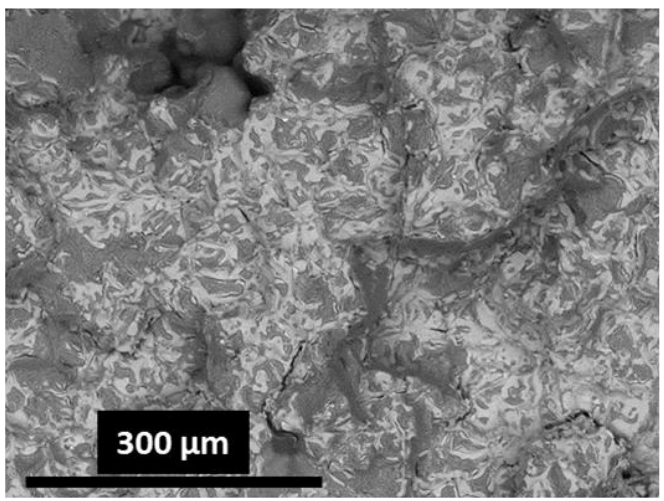

(d)

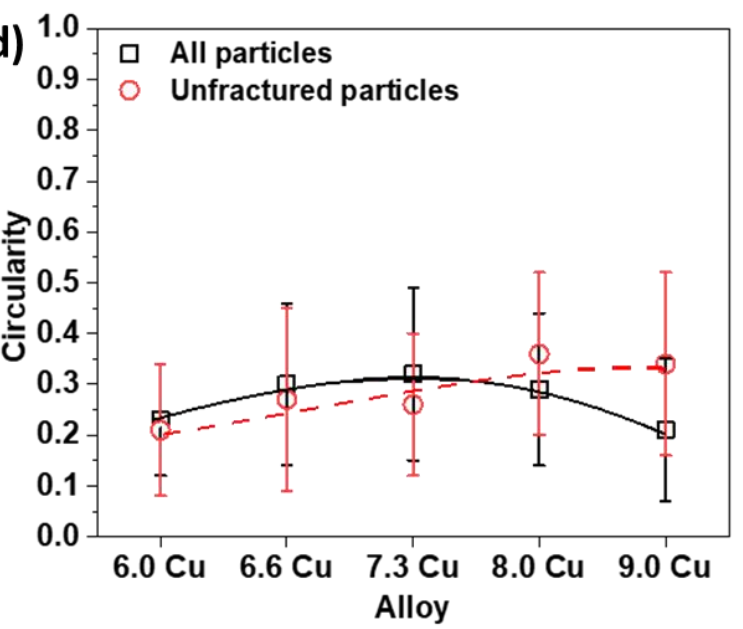

Figure I.3.1.1.3.3. (a) Representative micrograph of an ACMZ alloy after tensile test showing fractured grain boundary $\theta$ particles. (b) Fractograph of an ACMZ alloy illustrating extensive coverage of fracture surface by grain boundary $\theta$ particles.

Comparison between: (c) mean particle sizes; and (d) circularity of overall particle distribution before tensile test (all particles) and unfractured particle distribution after tensile test. Source: ORNL.

(a)

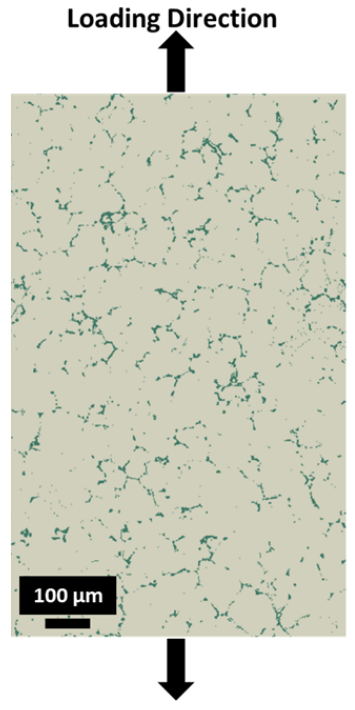

(b)

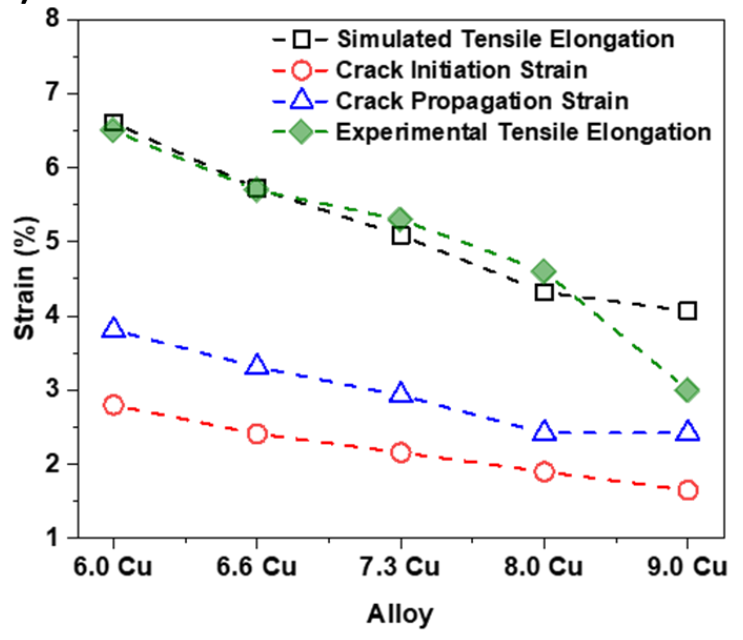

Figure I.3.1.1.3.4. (a) A finite element model of the alloy; and (b) a comparison between simulated and experimental values of elongation. Source: ORNL. 


\section{Low-Cycle Fatigue Properties}

The number of cycles to failure during low-cycle fatigue tests at $150^{\circ} \mathrm{C}$ and $250^{\circ} \mathrm{C}$ are summarized in Figure I.3.1.1.3.5 (a) and (b), respectively. The fatigue lives do not vary significantly with copper content at both $150^{\circ} \mathrm{C}$ and $250^{\circ} \mathrm{C}$ within the range of strain amplitudes tested here. Furthermore, the fatigue lives of the ACMZ alloys are comparable to or higher than 319 and A356 + 0.5 Cu baseline alloys.

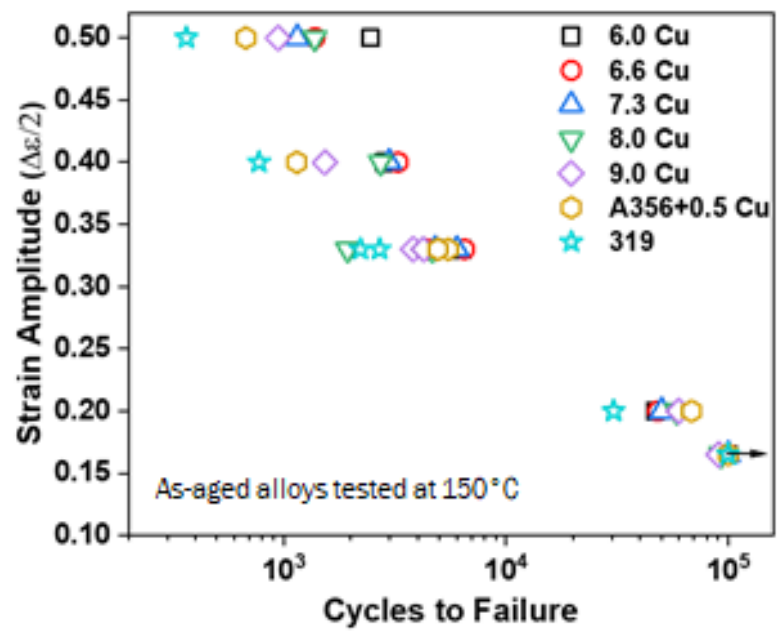

(a)

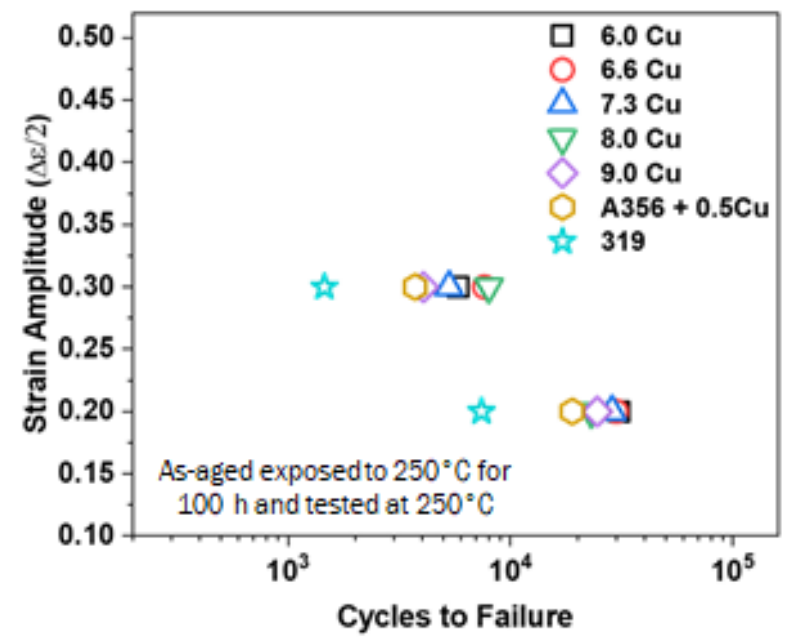

(b)

Figure I.3.1.1.3.5. Low-cycle fatigue lives of: (a) as-aged alloys tested at $150^{\circ} \mathrm{C}$; and (b) as-aged alloys exposed to $250^{\circ} \mathrm{C}$ for $100 \mathrm{~h}$ and tested at $250^{\circ} \mathrm{C}$. Source: ORNL.

\section{Conclusions}

The ductility of ACMZ alloys was found to be a function of copper content. The ductility decreases monotonically with increasing copper due to the formation of brittle grain boundary $\theta$ particles. Thus, controlling the formation of grain boundary particles is a means to develop ductile variants of ACMZ alloys. The comparable low-cycle fatigue properties of ACMZ alloys with 319 and A356 + 0.5 Cu is encouraging. Efforts are underway to identify the failure mechanisms in low-cycle fatigue and establish the ductility-lowcycle fatigue correlation in these alloys. The outcome of this effort would have an enabling effect towards setting the future course of action aimed at commercialization of ACMZ alloys.

\section{Key Publications}

1. Bahl, S., X. Hu, E. Hoar, J. Cheng, J. A. Haynes, and A. Shyam, 2019, "Effect of copper content on the tensile elongation of AlCuMnZr alloys: experiments and finite element simulations," Mat. Sci. Eng. AStruct, Vol. 765, Art. \#138801, 20 January 2020.

\section{References}

1. Shin, D., A. Shyam, S. Lee, Y. Yamamoto, and J. A. Haynes, 2017, "Solute segregation at the Al/ $\theta^{\prime}$ $\mathrm{Al}_{2} \mathrm{Cu}$ interface in Al-Cu alloys," Acta Mater., Vol. 141, pp. 327-340, December 2017.

2. Shyam, A., S. Roy, D. Shin, J. D. Poplawsky, L.F. Allard, Y. Yamamoto, J. R. Morris, B. Mazumder, J. C. Idrobo, A. Rodriguez, T. R. Watkins, and J. A. Haynes, 2019, "Elevated temperature microstructural stability in cast AlCuMnZr alloys through solute segregation," Mat. Sci. Eng. A-Struct, Vol. 765, Art. \#138279, 23 September 2019.

3. Javidani, M., and D. Larouche, 2014, "Application of cast Al-Si alloys in internal combustion engine components," Int. Mater. Rev., Vol. 59, No. 3, pp. 132-158. 
4. Sabau, A. S., S. Mirmiran, C. Glaspie, S. M. Li, D. Apelian, A. Shyam, J. A. Haynes, and A. F. Rodriguez, 2018, "Hot-tearing assessment of multicomponent nongrain-refined Al-Cu alloys for permanent mold castings based on load measurements in a constrained mold," Metall. Mater. Trans. B, Vol. 49, No. 3, pp. 1267-1287.

5. D.S.S. Corp, ABAQUS User Manual, 2017, Providence, RI, USA.

6. Hu, X. H., M. Jain, D. S. Wilkinson, and R. K. Mishra, 2008, "Microstructure-based FEA of strain localization behavior in AA5754 Al sheet," Acta Mater, Vol. 56, No. 13, pp. 3187-3201.

7. Wang, C., A. Soulami, Z. Xu, G. Cheng, S. Hu, D. Burkes, W. E. Frazier, K. S. Choi, X. Wang, and X. Hu, 2019, "Process Modeling of U-10 wt.\% Mo Alloys Using ICME," PNNL-28640, Richland, WA, USA.

\section{Acknowledgements}

The authors would like to acknowledge the contributions of the following individuals: James Morris, Patrick Shower, Lawrence Allard, Dana McClurg, Shane Hawkins, and Sumit Bahl for their contributions to this research. They would like to acknowledge S. A. Nemak for providing the cast alloys for this study. 


\section{I.3.2 Cost-Effective Higher Temperature Engine Alloys - Thrust 2 (Oak Ridge National Laboratory)}

\section{I.3.2.1 Advanced Affordable Wrought Engine Alloys (2A and 2B)}

\section{J. Allen Haynes, Principal Investigator}

Propulsion Materials

Oak Ridge National Laboratory

1 Bethel Valley Road

Knoxville, TN 37831

E-mail: haynes@ornl.gov

\section{Jerry L. Gibbs, DOE Technology Development Manager}

U.S. Department of Energy

E-mail: jerry.gibbs@ee.doe.gov

Start Date: October 1, 2018

End Date: September 30, 2022

Project Funding (FY19): $\$ 1,525,000 \quad$ DOE share: $\$ 1,525,000 \quad$ Non-DOE share: $\$ 0$

\section{Project Introduction}

This report describes the activities performed during the first year of the VTO Powertrain Materials Core Program. The goal of this program, which was launched in October 2018, is to rapidly develop and deliver new materials solutions, via an ICME framework, that enable both engine efficiency improvements and weight reductions over the full range of on-road vehicle classes (e.g., Class 1-8). This is being accomplished through five thrusts:

1. Cost-Effective Lightweight High-Temperature Engine Alloys.

2. Cost-Effective Higher Temperature Engine Alloys.

3. AM of Powertrain Alloys.

4. Advanced Characterization and Computation.

5. Emerging Technologies.

The following sections of this report outline the specific work conducted at ORNL, PNNL, and ANL for Thrust 2A, "Cost-Effective Higher Temperature Engine Alloys: Advanced Affordable Wrought Engine Alloys." Each task supports one or more goals within the VTO mission of developing new materials solutions to enable both engine efficiency improvements and weight reductions over the full range of on-road vehicle classes. 


\section{I.3.2.1.1 Oxidation-Resistant Valve Alloys for $900^{\circ} \mathrm{C}-950^{\circ} \mathrm{C}$ Operation (2A1)}

\section{Govindarajan Muralidharan, Co-Principal Investigator}

Oak Ridge National Laboratory

1 Bethel Valley Road

Oak Ridge, TN 37831

E-mail: muralidhargn@ornl.gov

\section{Bruce Pint, Co-Principal Investigator}

Oak Ridge National Laboratory

1 Bethel Valley Road

Oak Ridge, TN 37831

E-mail: pintba@ornl.gov

\section{Jerry L. Gibbs, DOE Technology Development Manager}

U.S. Department of Energy

E-mail: jerry.gibbs@ee.doe.gov

Start Date: October 1, 2018

Project Funding (FY19): \$400,000

End Date: September 30, 2022

DOE share: $\$ 400,000 \quad$ Non-DOE share: $\$ 0$

\section{Project Introduction}

Improving the engine efficiencies of passenger and commercial vehicles is a major goal of the VTO. One potential approach to achieving improved engine efficiency while reducing emissions results in retaining more heat in the exhaust gas, which results in higher exhaust gas temperatures, thus increasing the operational temperature requirements of components in the exhaust gas path including EVs. It has been projected that exhaust gas temperatures would increase from a current value of $760^{\circ} \mathrm{C}$ to values of at least $870^{\circ} \mathrm{C}$ and very likely reach as high as $1000^{\circ} \mathrm{C}$ in the long-term $[1,2]$.

High-performance EVs, currently used at temperatures up to $\sim 760^{\circ} \mathrm{C}$, are fabricated using Ni-based alloys such as Alloy 751. Other higher performance Ni-based alloys primarily developed for aerospace applications have the potential to operate at temperatures of $1000^{\circ} \mathrm{C}$ with desired strength but may be expensive for automotive applications and difficult to fabricate into desirable shapes. The focus of this subtask (2A1) is the development of lower-cost, improved wrought-processed Ni-based alloys for EVs. It is anticipated that the valves will be fabricated using the traditional forging route, followed by friction welding of valve stems. New alloys with the combination of yield and fatigue strengths, oxidation resistance, and cost have to be specifically developed for the operating characteristics and lifetime expectations for automotive valves. This project focuses on both chromia-forming and alumina-forming alloys, which have the potential for achieving the desired strength levels at a lower-cost than existing commercial alloys at temperatures. It is anticipated that chromia-forming alloys will achieve their oxidation resistance through the use of coatings at temperatures $\geq$ $900^{\circ} \mathrm{C}$, as shown in Table I.3.2.1.1.1, and is part of the research discussed later in this report in section I.3.2.1.3. 
Table I.3.2.1.1.1. Target Properties and Metrics Suggested by a Piston Manufacturer.

\begin{tabular}{|l|l|}
\multicolumn{1}{|c}{ Property } & \multicolumn{1}{c}{ Final Target } \\
\hline Yield Strength & $\geq 50 \mathrm{Ksi}$ at $950^{\circ} \mathrm{C}$. \\
\hline Fatigue Life & Fatigue life of new alloys $>$ fatigue life of Alloy 751 at $900-950^{\circ} \mathrm{C}$ at $30-50 \mathrm{Ksi}$. \\
\hline Oxidation Resistance & Mass change $\leq 2 \mathrm{X}$ commercial alumina-forming alloys at $950^{\circ} \mathrm{C}$. \\
\hline Cost & $\leq 1.25 \mathrm{X}$ of 751.
\end{tabular}

The critical challenge in this task is balancing high-temperature mechanical properties with oxidation resistance at a certain cost target. Two parallel approaches are being adopted in the design strategy:

- Chromia-forming, lower-cost Ni-based alloys: ORNL has recently developed new lower-cost alloys with excellent mechanical properties for use in EVs at temperatures up to $870^{\circ} \mathrm{C}$ through a synergistic approach that included computational modeling and selected experimental evaluation of properties [3, 4]. ORNL will identify a promising subset of alloys from this group of alloys that meet the mechanical property targets at $900^{\circ} \mathrm{C}$. Achieving good oxidation resistance in exhaust gases containing water vapor may be challenging for both commercial and newly developed alloys that depend on chromia scale formation for their oxidation resistance. Hence it is anticipated that improved oxidation resistance in this class of alloys will be achieved through the use of coatings described under the High-Temperature Coatings section later in the report.

- Alumina-forming Ni-based alloys: Alumina-forming alloys have superior oxidation resistance at $900^{\circ} \mathrm{C}$ and above, but existing commercial alumina-forming alloys do not possess adequate tensile and fatigue properties at $900^{\circ} \mathrm{C}$ and higher temperatures at target costs. The focus of this subtask is to develop new alumina-forming Ni-based alloys with good high-temperature strengths while achieving good oxidation resistance at target costs.

\section{Objectives}

The primary objective of this project is the identification/development of materials for EVs that could operate at temperatures up to $900-950^{\circ} \mathrm{C}$ and to demonstrate suitability for use in EVs in collaboration with industrial partners. These alloys should have the appropriate combination of oxidation resistance and high-temperature mechanical strength required for valve applications.

\section{Approach}

The overall approach to achieving Ni-based alloys with adequate high-temperature mechanical strength is through the use of stable $\gamma^{\prime}$ precipitates for strengthening in a $\gamma$-matrix at temperatures of interest. In addition to controlling the amount of $\gamma^{\prime}$, a fine distribution of these precipitates has to be achieved for optimum strengthening. One or more carbide phases, such as $\mathrm{M}_{23} \mathrm{C}_{6}, \mathrm{MC}$, and $\mathrm{M}_{7} \mathrm{C}_{3}$ (where $\mathrm{M}$ can be $\mathrm{Fe}, \mathrm{Cr}$, $\mathrm{Ni}$ or other), may also be present for grain size control. Since diffusion rates can be significant at $900^{\circ} \mathrm{C}$ and above, the key challenge in achieving long-term mechanical properties is to ensure that the $\gamma^{\prime}$ precipitates remain stable over the anticipated lifetime of the alloys with relatively low rates of coarsening. The approach used for the two classes of alloys are outlined below:

- Chromia-forming, lower-cost Ni-based alloys: Previous work at ORNL has shown that the strength in the heat-treated condition of the new lower-cost ORNL alloys is superior to that of Alloy 751. This subtask will identify alloys which can maintain their long-term strength at $900^{\circ} \mathrm{C}$ and above.

Microstructural evolution will be evaluated in a select group of alloys, followed by assessment of hightemperature fatigue properties. Prototype exhaust valve will be fabricated and tested in collaboration with industrial partners. Advanced characterization will be used to study $\gamma$ ' precipitate characteristics and how this affects high-temperature mechanical properties. Advanced modeling will be used to predict microstructural evolution and will be validated to enable long-term predictions. 
- Alumina-forming Ni-based alloys: The primary challenge in this class of alloys is achieving the hightemperature mechanical properties while maintaining the excellent oxidation resistance provided by the alumina scale. Computational thermodynamics will be used to design alloys with desired $\gamma^{\prime}$ contents while achieving good alumina scale formability at these temperatures. Kinetic modeling will be used to design alloys with appropriate $\gamma$ ' sizes. Modeling will be validated using TEM, APT, and ultra small angle/small angle scattering.

\section{Results}

\section{Chromia-forming alloys}

Key progress has been made in evaluating the microstructural stability and tensile properties after $250 \mathrm{~h}$ exposure at $900^{\circ} \mathrm{C}$ of the new, lower-cost chromia-forming alloys developed and patented at ORNL.

Figure I.3.2.1.1.1 compares the yield strength after $250 \mathrm{~h}$ exposure at $900^{\circ} \mathrm{C}$ of Alloy 751 with that of two new ORNL alloys-Alloy 163 and Alloy 200. Note that the new alloys have higher initial yield strengths at $900^{\circ} \mathrm{C}$ when compared to the commercial Alloy 751. Further, even after the $250 \mathrm{~h}$ exposure, these alloys demonstrate improved yield strength properties when compared to the baseline alloy. This result is the first demonstration of the long-term properties of the new ORNL alloys and clearly shows the feasibility of using the new alloys for long times at high temperatures without any undue loss of mechanical properties when compared to the baseline Alloy 751.

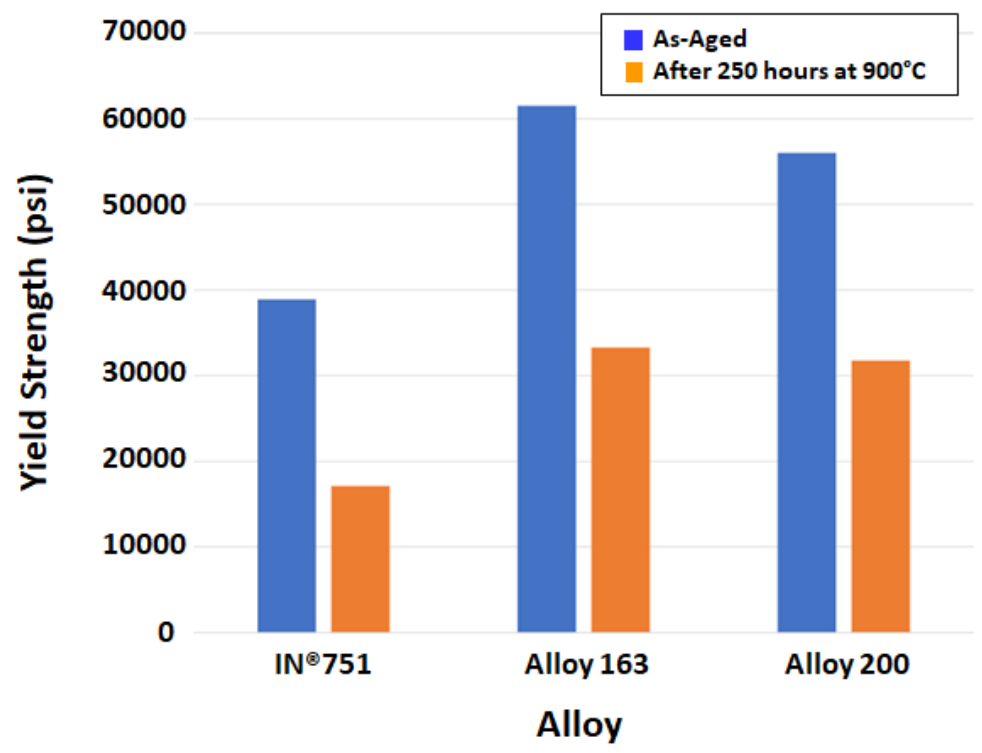

Figure I.3.2.1.1.1. Initial yield strengths and yield strengths after $250 \mathrm{~h}$ of aging of IN ${ }^{\circledR} 751$ and new ORNL Alloys 163 and 200. Source: ORNL.

Figure I.3.2.1.1.2 shows a typical optical and SEM micrograph from Alloy 163 after aging for $250 \mathrm{~h}$ at $900^{\circ} \mathrm{C}$. The higher magnification image confirms the presence of the $\gamma^{\prime}$ strengthening precipitates in Alloy 163 with an average size of $\sim 275 \mathrm{~nm}$. This confirms that the $\gamma$ ' strengthening precipitates are stable in this alloy at this temperature. Figure I.3.2.1.1.3 shows a plot of the high-cycle fatigue life at $900^{\circ} \mathrm{C}$ and $200 \mathrm{MPa}$ obtained from laboratory-scale heats of several lower Ni chromia-forming alloys developed at ORNL. Note that several alloys perform better than the baseline Alloy 751. This shows the potential for achieving improved performance using these alloys at this temperature. The lower fatigue rupture life in Alloy 163, in spite of a higher yield strength even after $250 \mathrm{~h}$ at $900^{\circ} \mathrm{C}$, suggests that further experiments at lower stresses are required. Since multiple specimens are required from the same heat for an appropriate comparison, larger (e.g., $50-100 \mathrm{lb}$.) vacuum induction melted heats of selected alloys are being prepared and will be tested in followon work. 


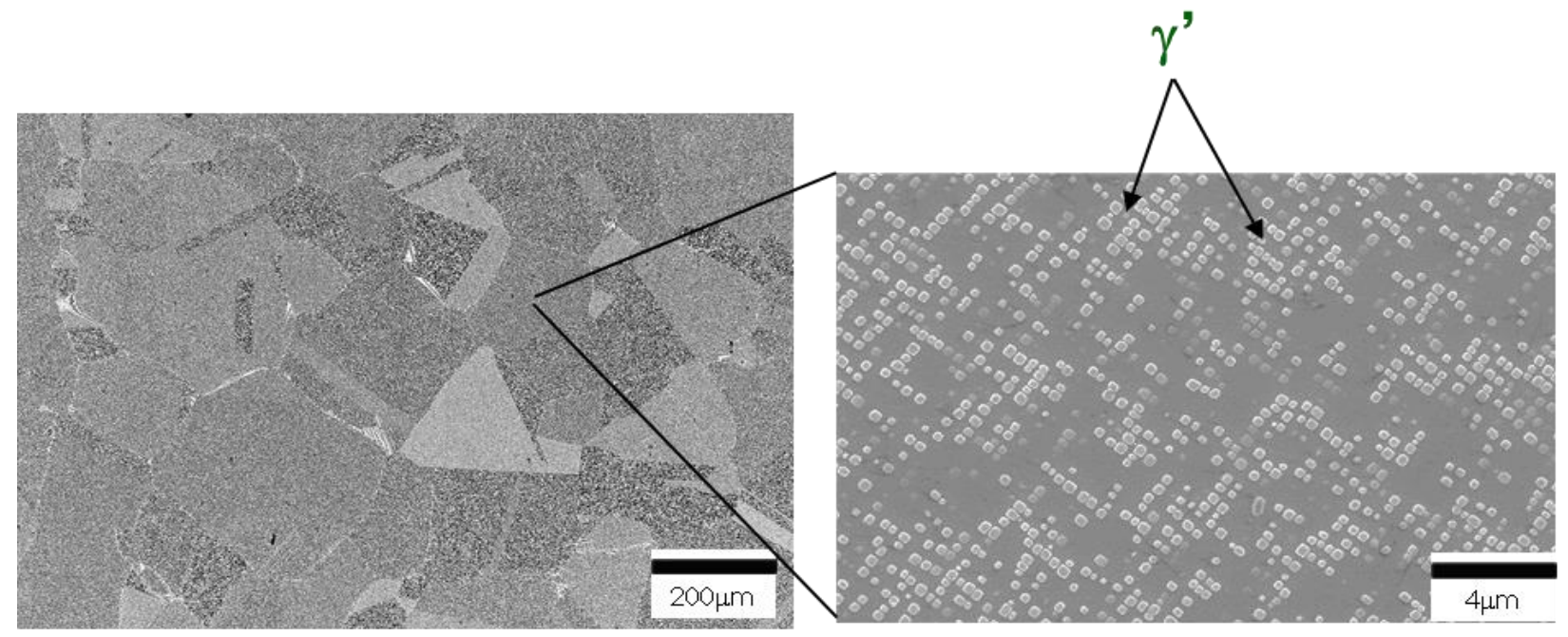

Figure I.3.2.1.1.2. SEM image showing the presence of $\gamma^{\prime}$ precipitates in Alloy 163 after $250 \mathrm{~h}$ aging at $900^{\circ} \mathrm{C}$. Source: ORNL.

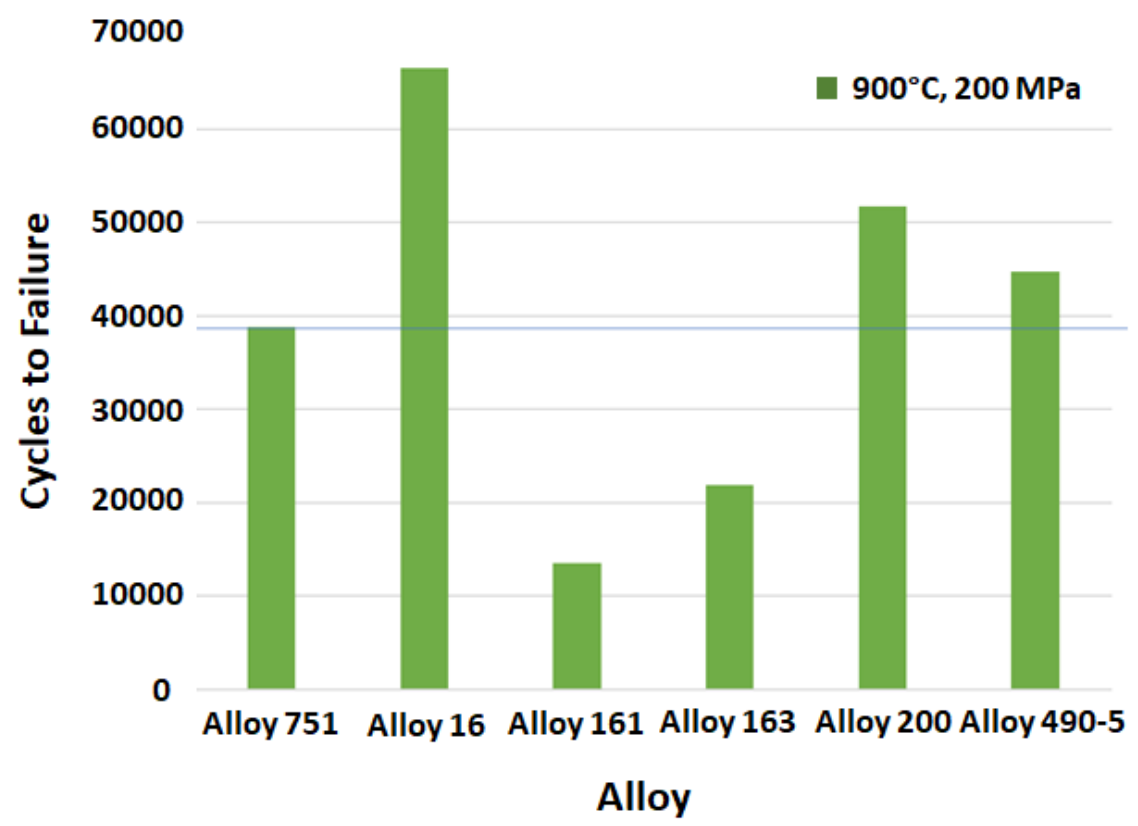

Figure I.3.2.1.1.3. High-cycle fatigue lives at $900^{\circ} \mathrm{C}$ and $200 \mathrm{MPa}$ of laboratory-scale heats for several ORNL alloys compared to the Alloy 751 baseline. Source: ORNL.

\section{Alumina-forming alloys}

Figure I.3.2.1.1.4 compares the mass change as a function of a number of one-hour cycles at $950^{\circ} \mathrm{C}$ in an air + $10 \%$ water vapor environment for several ORNL alloys with that of commercial alloy, Haynes ${ }^{\circledR} 214^{\circledR}$. The data shows that, up until about 1000 cycles, the ORNL alumina-formers show oxidation behavior comparable to that of the commercial alloy. All alumina-formers in the figure show oxidation behavior better than the chromia-former Udimet ${ }^{\circledR}$ 520. Figure I.3.2.1.1.5 shows the cross-section of a sample from ORNL Alloy 4K tested for 600 one-hour cycles with the mass change shown via the green line in Figure I.3.2.1.1.4. The presence of a thin oxide scale of Al suggests the formation of an Al-rich oxide in this condition. 


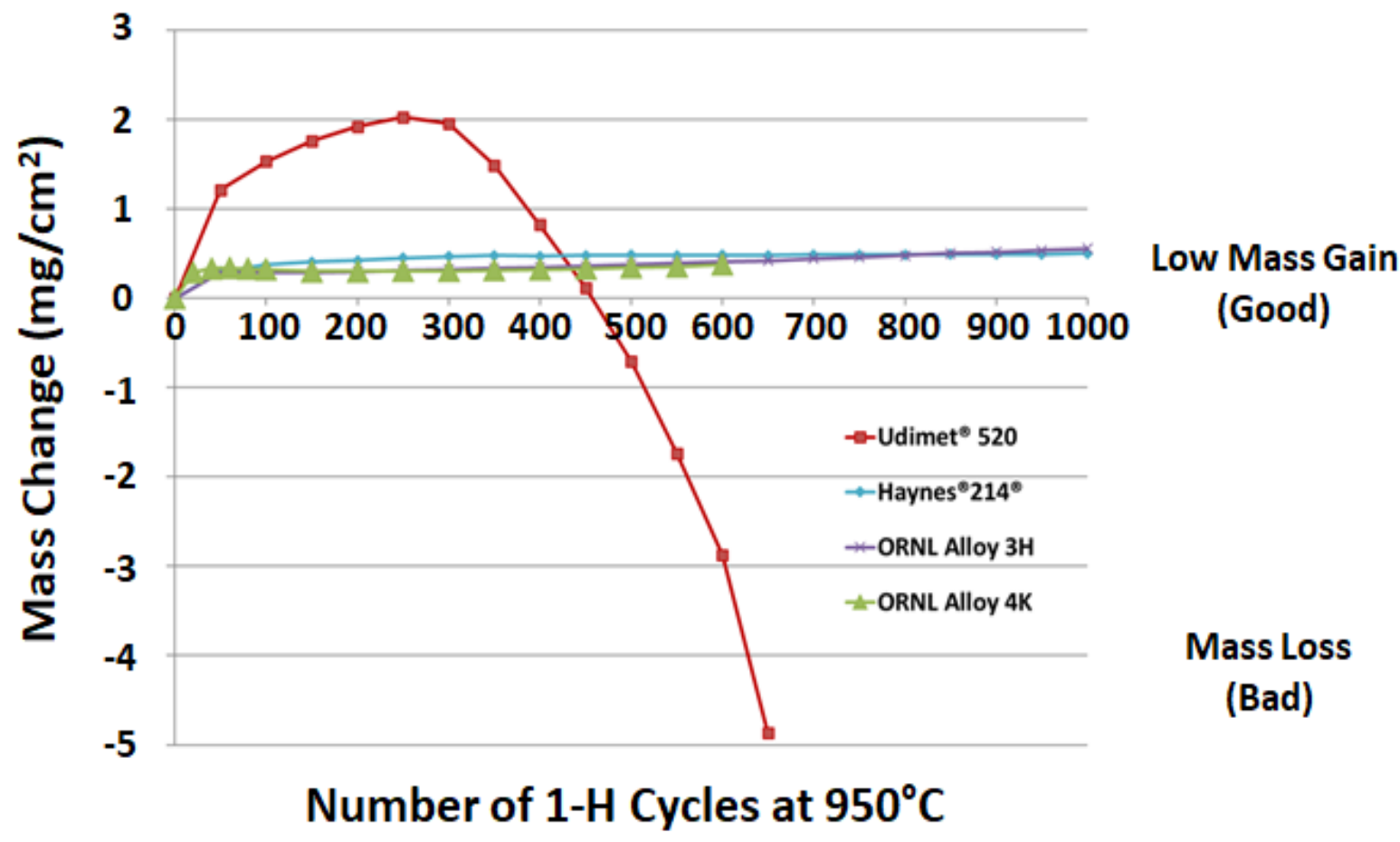

Figure I.3.2.1.1.4. Newly designed alumina-forming alloys showed oxidation behavior comparable to commercial aluminaforming Ni-based alloys at $950^{\circ} \mathrm{C}$ in air $+10 \%$ water vapor. The high rate of mass loss of the chromia-forming Udimet 520 alloy indicates poor oxidation resistance compared to the three alumina-forming alloys. Source: ORNL.

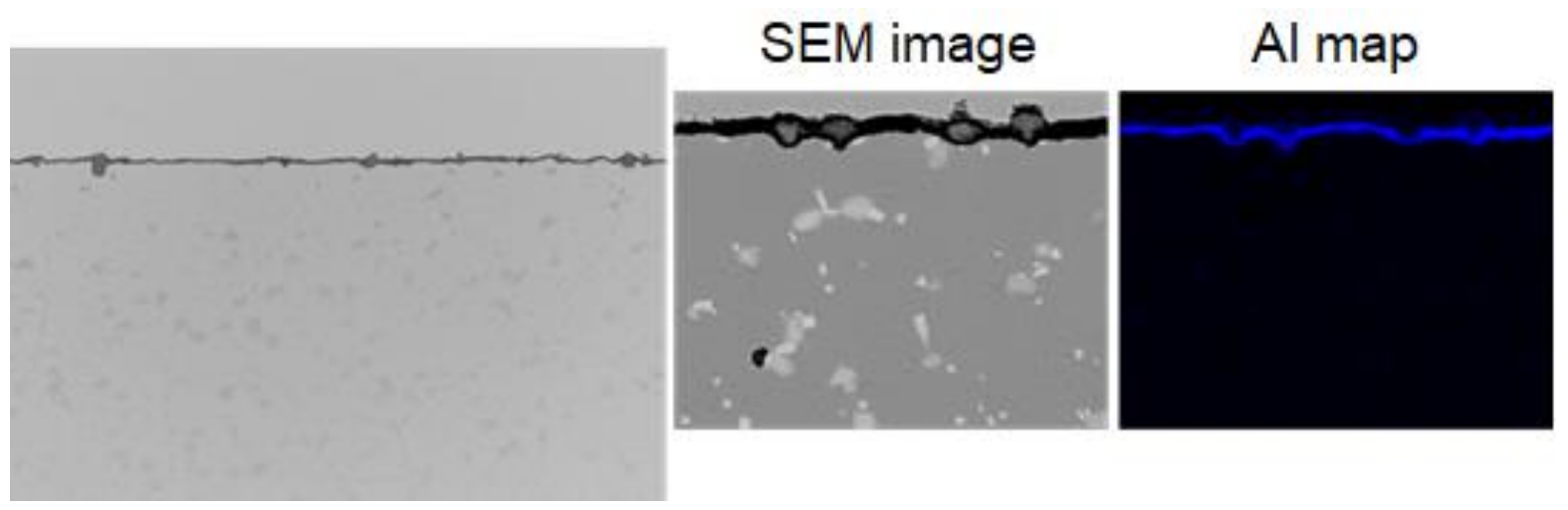

Figure I.3.2.1.1.5. Cross-sectional images of alumina-forming ORNL alloy $4 \mathrm{~K}$ shows the presence of an Al-rich oxide scale after $600 \mathrm{~h}$ at $950^{\circ} \mathrm{C}$, in air $+10 \%$ water vapor. Source: ORNL.

Figure I.3.2.1.1.6 shows the yield strength of new ORNL alloys developed in FY 2019 with those of commercial alumina-forming alloys. Note that substantial improvements have been achieved in yield strengths measured at $950^{\circ} \mathrm{C}$ and that the FY 2019 targets have been exceeded while achieving good oxidation resistance at $950^{\circ} \mathrm{C}$ in an air $+10 \%$ water vapor environment. Further improvements are needed to attain the targeted values for this project. The improvements achieved in FY 2019 suggest that the alloy development strategy is appropriate and will continue to be employed to explore further improvements. Advanced characterization is being performed in collaboration with researchers at ORNL, the ANL Advanced Photon Source and PNNL to understand precipitation in these novel alloys at the higher temperatures and to define paths for further improvements in strength. 


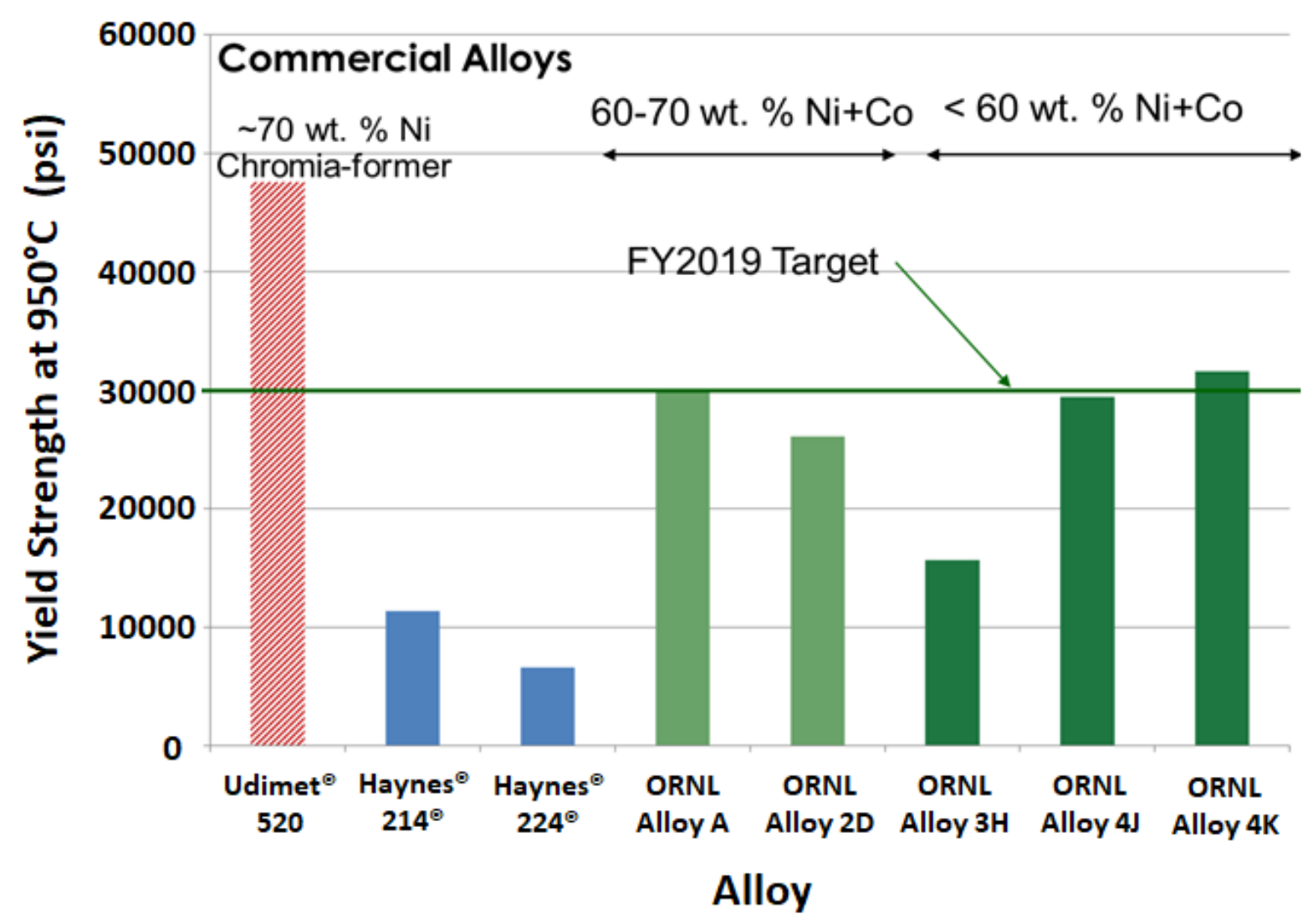

Figure I.3.2.1.1.6. Yield strengths of new alumina-forming alloys satisfied FY 2019 targets. Source: ORNL.

\section{Conclusions}

New ORNL-developed lower Ni chromia-forming alloys have better strengths compared to Alloy 751 after $250 \mathrm{~h}$ of exposure at $900^{\circ} \mathrm{C}$. Fatigue testing at $900^{\circ} \mathrm{C}$ shows promising results from these alloys. The long-term properties of the new ORNL alloys clearly show the feasibility of using the new alloys for long times at high temperatures without any undue loss of mechanical properties. New alumina-forming alloys with significantly improved strengths over commercially available alloys have been developed for operation at temperatures up to $950^{\circ} \mathrm{C}$. Further improvements in strength are needed to compete with high-strength, commercially available chromia-forming alloys. Advanced characterization is being performed in collaboration with researchers at ORNL, the ANL Advanced Photon Source and PNNL to highlight to understand precipitation in the novel alloys at the higher temperatures and to define paths for further improvements in strength.

\section{Key Publications}

1. Frith, M. G., J. Chiles, J. Poplawsky, J. Ilavsky, and G. Muralidharan, 2019, "Understanding interparticle interference in small angle-scattering pattern from a Ni-based alloy using APT," in preparation.

2. Muralidharan, G., D. Pierce, J. Poplawsky, J. Chiles et al, 2019, "On the nanoscale microstructure of a Ni-based alloy," in preparation. 


\section{References}

1. DOE Vehicle Technologies Office, 2013, "LD Vehicles Technical Requirements and Gaps for Lightweight and Propulsion Materials," DOE VTO Workshop Report No. DOE/EE-0868, February 2013.

2. DOE Vehicle Technologies Office, 2013, "Trucks and HD Vehicles Technical Requirements and Gaps for Lightweight and Propulsion Materials," DOE VTO Workshop Report No. DOE/EE-0867, February 2013.

3. Muralidharan, G., "Low-cost FeNiCr alloys for high-temperature valve applications," U. S. Patent No. 9,605,565, March 28, 2017.

4. Muralidharan, G., "Low-cost, high-strength FeNiCr alloys for high-temperature exhaust valve applications," U. S. Patent No. 9,752,468 B2, September 5, 2017.

\section{Acknowledgements}

The authors would like to acknowledge the support of J. Chiles, J. A. Haynes, and J. Poplawsky from ORNL; M. Frith, J. Ilavsky, S. Lapidus, and D. Singh from ANL; and B. Gwalani, A. Devraj, and L. Kovanik from PNNL. 


\section{I.3.2.1.2 Higher Temperature Heavy-Duty Piston Alloys (2A2)}

\section{Dean Pierce, Co-Principal Investigator}

Oak Ridge National Laboratory

1 Bethel Valley Road

Oak Ridge, TN 37831

E-mail: piercedt@ornl.gov

\section{Govindarajan Muralidharan, Co-Principal Investigator}

Oak Ridge National Laboratory

1 Bethel Valley Road

Oak Ridge, TN 37831

E-mail: muralidhargn@ornl.gov

\section{Jerry L. Gibbs, DOE Technology Development Manager}

U.S. Department of Energy

E-mail: jerry.gibbs@ee.doe.gov

Start Date: October 15, 2018

Project Funding (FY19): \$250,000

End Date: September 30, 2023

DOE share: $\$ 250,000 \quad$ Non-DOE share: $\$ 0$

\section{Project Introduction}

Quench and tempered martensitic steel 4140 and micro-alloyed steel 38MnVS6 are the primary materials used in heavy-duty diesel engine (HDDE) pistons. A major pathway to increase the efficiency of internal combustion engines involves increasing both the peak cylinder pressure and combustion gas temperatures. However, the aforementioned piston materials are operating at their limits in terms of mechanical and thermal loading. The 4140 and micro-alloyed steel alloys are limited to an operating temperature of $\sim 500^{\circ} \mathrm{C}$. As such, current piston materials are a major limitation to increasing engine efficiency.

\section{Objectives}

The objectives of this project are to investigate existing commercial alloys, as well as new developmental alloys for use in HDDE pistons, that have potential to enable increased operating temperatures and cylinder pressures but require only moderate cost increases. Specifically, the new alloys must meet the unique requirements of HDDE pistons, including high-temperature strength, high-cycle fatigue strength, cyclic oxidation resistance in a combustion environment, wear resistance, and microstructural stability. These phenomena can act synergistically to cause failure. The targeted peak piston operating temperature within this subtask is $\geq 600^{\circ} \mathrm{C}$. Further key targets include strength levels of $\sim 400 \mathrm{MPa}$ at peak temperature and oxidation resistance at $\geq 600^{\circ} \mathrm{C}$, which is equivalent or better than alloy 4140 at $500^{\circ} \mathrm{C}$.

\section{Approach}

Four major groups of alloys are being investigated to identify materials that are suitable from a performance and cost standpoint in next-generation HDDE pistons: (1) commercial martensitic stainless steels, (2) low-cost developmental alloys with medium levels of $\mathrm{Cr}$ and improved oxidation resistance, (3) developmental alloys with improved properties for thermal management to reduce piston surface temperatures and (4) advanced $9-14 \mathrm{Cr}$ alloys for operation at $650-700^{\circ} \mathrm{C}$. 


\section{Results}

\section{Commercial Alloys}

Two commercial alloys have been selected for evaluation at this stage: (1) martensitic stainless steels 418 (Fe-0.15C-0.25Mn-0.25Si-13Cr-2Ni-3W wt.\%) and (2) 422 (Fe-0.23C-0.75Mn-0.35Si-1Mo-11.5Cr-0.8Ni-1W wt. \%). The alloys have been subjected to relevant elevated temperature tensile tests at $600^{\circ} \mathrm{C}, 650^{\circ} \mathrm{C}$, and $700^{\circ} \mathrm{C}$ after quenching and double tempering, as well as after long-term aging for $500 \mathrm{~h}$ at $600^{\circ} \mathrm{C}$ and $650^{\circ} \mathrm{C}$. These tests were performed to understand how typical tempering processes, test temperature, and aging effect the strength of the alloys at high temperatures. Results for the yield strength are shown in Figure I.3.2.1.2.1 (a). The 422 maintains relatively high yield strength at temperatures up to $600^{\circ} \mathrm{C}$, even after aging at $600^{\circ} \mathrm{C}$ for $500 \mathrm{~h}$. The oxidation mass gain of 4140, T91 (a 9Cr alloy with widespread use in power generation applications), 418, and 422 were also measured during cyclic oxidation in $1 \mathrm{~h}$ cycles at temperatures of $500^{\circ} \mathrm{C}$, $550^{\circ} \mathrm{C}, 600^{\circ} \mathrm{C}$, and $650^{\circ} \mathrm{C}$ in air plus $10 \%$ water vapor. Water vapor is present in diesel combustion products and has a significant and detrimental effect on the oxidation behavior of steel. Thus, the furnace atmosphere during lab oxidation-testing includes a controlled volume of water vapor to more closely represent a combustion chamber environment. The results of oxidation-testing for T91, 418, and 422 at $600^{\circ} \mathrm{C}$ are shown in Figure I.3.2.1.2.1 (b). Up to 250 h, 418 and 422 exhibit relatively low mass gain and good oxidation resistance, while T91 (9Cr) exhibits relatively low mass gain up to $100 \mathrm{~h}$, but breakaway oxidation occurs thereafter. Microscopy and further evaluation of oxidation samples is ongoing to further understand the behavior.
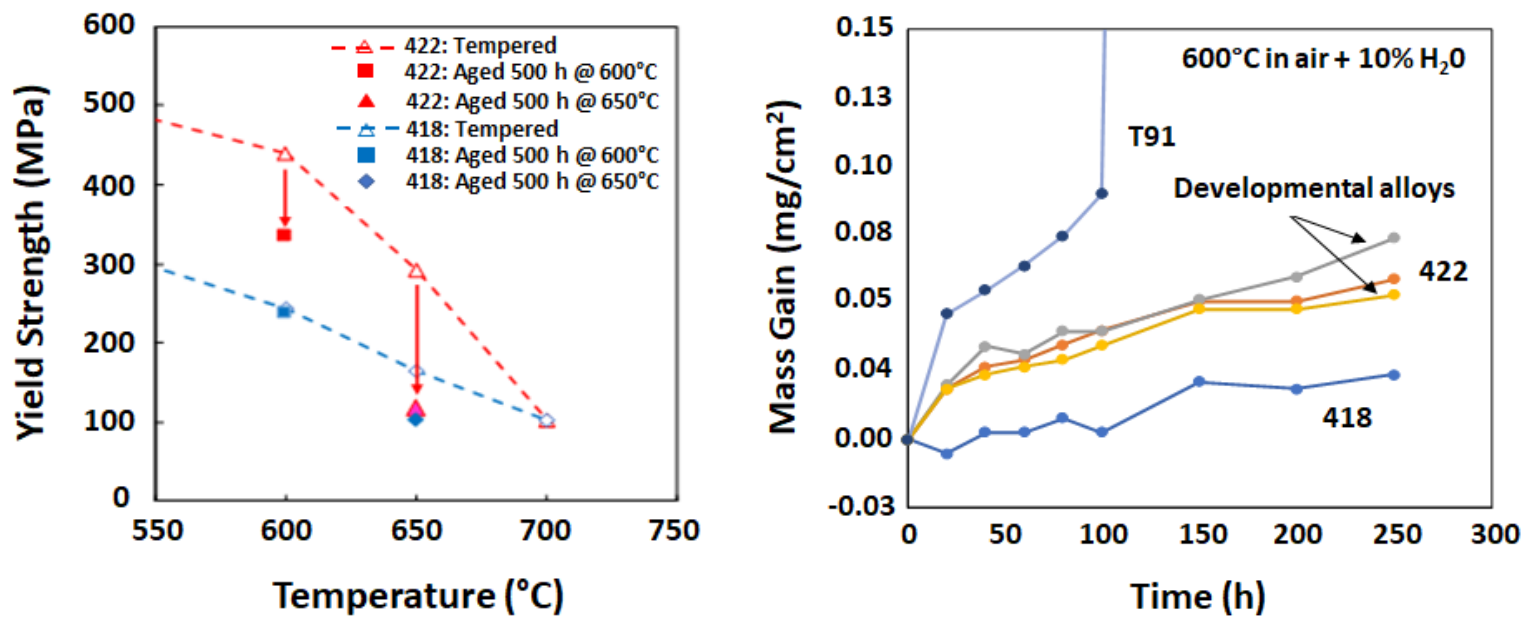

Figure I.3.2.1.2.1. (a) Yield strength of 418 and 422 steel at test temperatures of $600^{\circ} \mathrm{C}, 650^{\circ} \mathrm{C}$, and $700^{\circ} \mathrm{C}$, and at $600^{\circ} \mathrm{C}$ and $650^{\circ} \mathrm{C}$ after aging for $500 \mathrm{~h}$ at each temperature. (b) Oxidation mass gain of T91, 422, and 418 during cyclic oxidation testing $\left(1 \mathrm{~h}\right.$ cycles) at $600^{\circ} \mathrm{C}$ in air plus $10 \%$ water vapor. Source: ORNL.

Both 418 and 422 exhibit some potential for future HDDE piston materials-based on initial mechanical and oxidation-testing, but additional testing is planned to further evaluate their suitability. These additional tests will include longer-term oxidation evaluations and high-cycle fatigue (HCF). Next-generation pistons will be subjected to metal temperatures around $600^{\circ} \mathrm{C}$ for the life of the engine, which can exceed 10,000 hours of operating time. The oxidation behavior of ferritic/martensitic steels may undergo sudden and significant changes during oxidation, similar to that observed in T91, as shown in Figure I.3.2.1.2.1 (b), in part due to damage of the protective oxide layer and/or depletion of beneficial elements, such as $\mathrm{Cr}$ at the metal/oxide interface. These changes could lead to piston failure or engine damage if oxide intrudes into and weakens the base metal or thick oxides spall. Longer-term oxidation-testing will be employed to identify if 418 and 422 exhibit such changes in oxidation behavior not observed in the initial testing up to $250 \mathrm{~h}$. HCF testing will also be employed on 418 and/or 422 in order to approximate the rapid cyclic pressure pulses that the piston experiences during engine operation. The primary temperature range of interest for the HCF testing is from 
$550-600^{\circ} \mathrm{C}$. Currently, the target $\mathrm{HCF}$ strength (runout) at $600^{\circ} \mathrm{C}$ is approximately $400 \mathrm{MPa}$, which is the fatigue strength of 4140 measured at its maximum operating temperature of $500^{\circ} \mathrm{C}$. These $\mathrm{HCF}$ results from the commercial alloys will also serve as baseline data to compare with HCF results of developmental alloys.

\section{Low-Cost Medium Cr Alloys}

Alloy design efforts to achieve acceptable oxidation resistance at medium levels of $\mathrm{Cr}(\sim 3-7 \mathrm{wt} . \%)$ are ongoing in order to reduce alloy cost compared to higher Cr alloys, such as 418 and 422 (12Cr). Reducing Cr, a relatively high cost element, directly lowers the raw material cost and may allow alternative alloying strategies. These alloy design efforts have thus far been able to achieve oxidation resistance similar to 418 and 422 at significantly lower levels of Cr. Figure I.3.2.1.2.2 shows the mass gain due to oxidation of two developmental alloys, along with T91, 418, and 422. The developmental alloys exhibit similar oxidation resistance to 418 and 422 at significantly lower levels of $\mathrm{Cr}$ and significantly lower raw material cost. This is an important initial result as 418 and 422 martensitic steels represent a significant cost increase over existing piston materials, in part due to their high levels of $\mathrm{Cr}$, Mo, and W. Optimization of the compositions of these developmental alloys is ongoing to further enhance mechanical and oxidation properties.

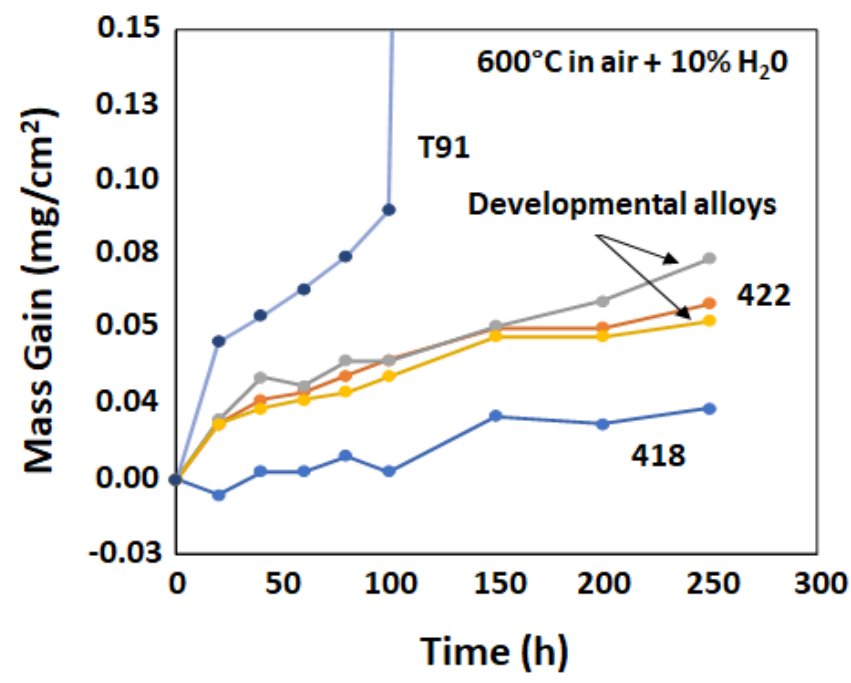

Figure I.3.2.1.2.2. Oxidation mass changes of two developmental alloys along with commercial alloys T91, 422, and 418 during cyclic oxidation testing ( $1 \mathrm{~h}$ cycles) at $600^{\circ} \mathrm{C}$ in air plus $10 \%$ water vapor. Source: ORNL.

\section{Alloys for Improved Thermal Management}

Alloy design concepts have also been developed to produce alloys with improved thermal management that may operate at significantly lower temperatures than typical high-temperature steels, while also costing significantly less. The purpose of these steels is to enable higher PCPs, but to keep the maximum piston surface temperature lower than would be possible with other costlier high-temperature steels. At lower temperatures, strength levels are generally greater and oxidation rates are lower. Figure I.3.2.1.2.3 shows the yield strength as a function of temperature of a developmental alloy "HTC-OR Plus" and 422 martensitic steel. The HTC-OR Plus alloy exhibits lower strength than 422, but its maximum surface temperature during operation is expected to be $\sim 50^{\circ} \mathrm{C}$ lower at the same engine operating conditions, due to its higher thermal conductivity compared to 422 . As such, the strength levels of HTC-OR Plus and 422 at their predicted maximum surface temperatures in next-generation pistons are similar, as observed in Figure I.3.2.1.2.3, but the HTC-OR Plus alloy has an $\sim 50 \%$ lower raw material cost. Initial tests have also shown significant improvement in the oxidation behavior of these alloys in comparison to 4140. However, further optimization of these alloys is needed and ongoing. 


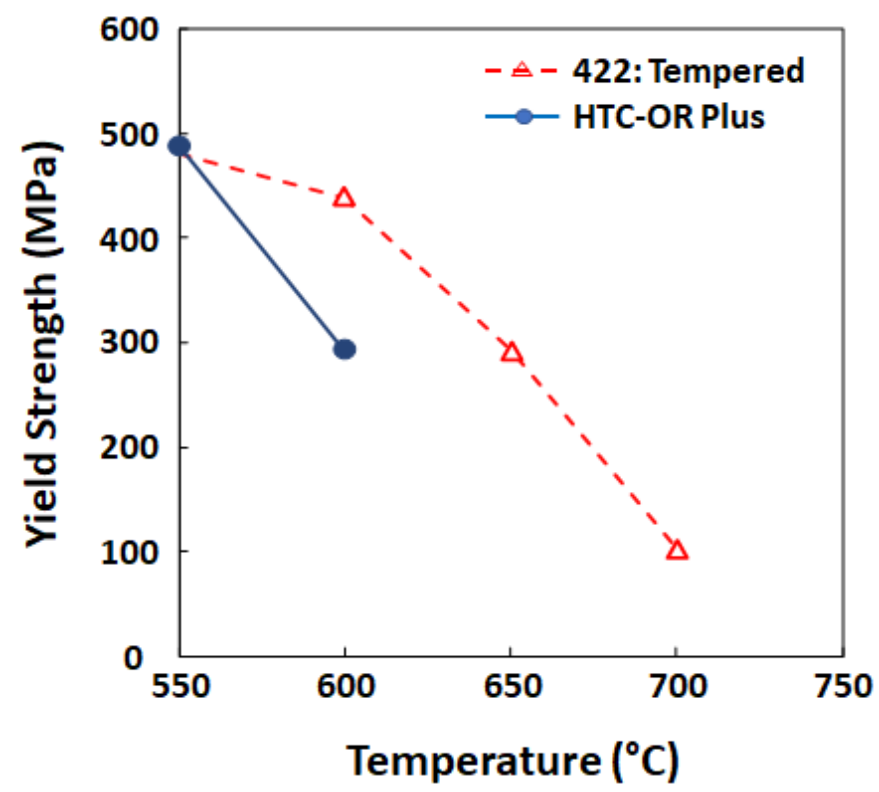

Figure I.3.2.1.2.3. Yield strength of developmental alloy HTC-OR and 422 steel at test temperatures of $550^{\circ} \mathrm{C}, 600^{\circ} \mathrm{C}$, $650^{\circ} \mathrm{C}$, and $700^{\circ} \mathrm{C}$. Source: ORNL.

\section{9-14Cr Martensitic Steels}

Alloy design concepts for advanced 9-14Cr martensitic steels have been developed for operating temperatures from $650-700^{\circ} \mathrm{C}$. These steels will be designed to possess advanced strength and cyclic oxidation resistance at $650-700^{\circ} \mathrm{C}$. Figure I.3.2.1.2.4 shows the ultimate tensile strengths at test temperatures from $550-700^{\circ} \mathrm{C}$ for some of the aforementioned steels, as well as developmental alloy HTCR-Plus, which exhibits ultimate strength levels $\sim 100 \mathrm{MPa}$ greater at $700^{\circ} \mathrm{C}$ when compared to commercial martensitic steels 418 and 422 . Further optimization of the strength of HTCR-Plus and similar alloys is ongoing.

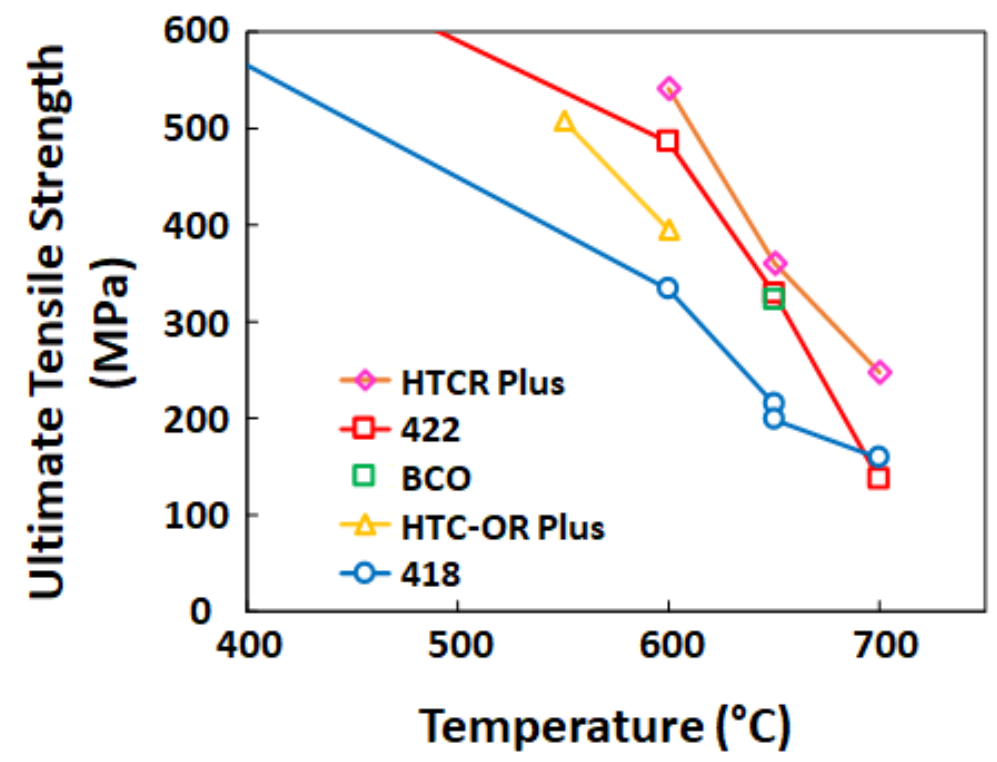

Figure I.3.2.1.2.4. Ultimate tensile strengths of commercial alloys 418,422 , as well as developmental alloys HTCR-Plus, $\mathrm{HTC}-\mathrm{OR}$, and $\mathrm{BCO}$ at test temperatures of $550^{\circ} \mathrm{C}, 600^{\circ} \mathrm{C}, 650^{\circ} \mathrm{C}$, and $700^{\circ} \mathrm{C}$. Source: ORNL. 


\section{Conclusions}

Initial investigations of two commercial alloys, 418 and 422 martensitic steels, as well as a series of developmental alloys for potential use as next-generation piston alloys have been performed. The commercial martensitic steels show some potential for use as piston alloys with operating temperatures near $600^{\circ} \mathrm{C}$, but additional testing is needed to confirm their adequacy. Several different alloy development concepts have been investigated with the goal of optimizing performance for piston applications while reducing cost relative to commercial alloys. Several of these concepts exhibit significant potential for improved performance at lowercost. These concepts will be optimized in year 2 , where significant improvements in performance are expected.

\section{Acknowledgements}

The following ORNL personnel are acknowledged for their contributions to this project: L. Allard, T. Muth, J. Poplawsky, A. Haynes, B. Pint, C. Carmichael, I. Stinson, H. Wang, E. Cakmak, and Y. Yamamoto. 


\title{
I.3.2.1.3 Advanced, Affordable Wrought Engine Alloys, High-Temperature Coatings (2A3)
}

\section{Sebastien Dryepondt, Co-Principal Investigator}

Oak Ridge National Laboratory

1 Bethel Valley Road

Oak Ridge, TN 37932

E-mail: dryepondtsn@ornl.gov

\author{
Beth Armstrong, Co-Principal Investigator \\ Oak Ridge National Laboratory \\ 1 Bethel Valley Road \\ Oak Ridge, TN 37932 \\ E-mail: armstrongbl@ornl.gov
}

\section{Jerry L. Gibbs, DOE Technology Development Manager}

U.S. Department of Energy

E-mail: jerry.gibbs@ee.doe.gov

Start Date: October 15, 2018

Project Funding (FY19): \$175,000

End Date: September 30, 2022

DOE share: $\$ 175,000 \quad$ Non-DOE share: $\$ 0$

\section{Project Introduction}

New high-temperature, high-strength valve alloys have been developed at ORNL for operating temperatures up to $\sim 950^{\circ} \mathrm{C}$, but their high-temperature oxidation resistance currently limits their use to $\sim 850^{\circ} \mathrm{C}$. Corrosionresistant coatings will be required to reach the required component lifetime. ORNL has previously demonstrated that aluminide and MCrAlY coatings can significantly improve valve Alloy $31 \mathrm{~V}(57 \mathrm{Ni}-23 \mathrm{Cr}-$ 13Fe-2Mo-2.3Ti-1.3Al-0.9Nb) cyclic oxidation resistance without impacting the alloy HCF behavior [1].

\section{Objectives}

This project aims at developing affordable, oxidation-resistant alumina-forming coatings to improve the durability of advanced high-strength valve alloys. Both slurry aluminide and thermal spray MCrAlY coatings will be deposited on selected high-strength alloys to study and understand system oxidation behavior and fatigue resistance.

\section{Approach}

Slurry aluminide coatings fabricated at ORNL and/or industrial partners were deposited on oxidation coupons and fatigue specimens. The slurry coating composition and heat-treatments were optimized based on the substrate alloy composition and recommended heat-treatment. Commercial MCrAlY thermal spray coatings were deposited at Stony Brook University based on previous work conducted on the valve alloy $31 \mathrm{~V}$, and then annealed according to the substrate heat-treatment. Cyclic oxidation testing was conducted at $\mathrm{T}>900^{\circ} \mathrm{C}$ in air $+10 \% \mathrm{H}_{2} \mathrm{O}$ to simulate combustion environments and $\mathrm{HCF}$ testing was conducted at temperatures below and above the ductile to brittle temperature transition of the coating (e.g., $500-770^{\circ} \mathrm{C}$ range) to ensure that the coating is not excessively brittle at low temperature. Extensive microstructural characterization is being conducted to understand the cyclic oxidation and HCF degradation mechanisms for the coated alloys.

In addition, a coupled thermodynamic and kinetic computational model is being employed to predict the coating microstructural evolution during fabrication and after high-temperature exposure [2]. This tool will support coating optimization, substrate selection, and component lifetime prediction. 


\section{Results}

\section{Coating Fabrication and Characterization}

Two of ORNL's new valve alloys were selected based on their excellent high-temperature tensile and fatigue properties, but limited oxidation resistance at $\mathrm{T}>850^{\circ} \mathrm{C}$. The composition of these alloys is compared in Table I.3.2.1.3.1 with the composition of commercial alloy 31V. Cylindrical coupons made of Alloys 2686, 2687 , and $31 \mathrm{~V}$ were dipped into an aluminide slurry and then heat-treated in a vacuum furnace based on the solution anneal recommended for these new alloys: $4 \mathrm{~h}$ at $1121^{\circ} \mathrm{C}$.

Table I.3.2.1.3.1. Alloy Composition in wt\%.

\begin{tabular}{|l|l|l|l|l|l|l|l|l|l|l|}
\hline & \multicolumn{1}{|c|}{$\mathbf{N i}$} & \multicolumn{1}{|c|}{$\mathbf{F e}$} & \multicolumn{1}{|c|}{$\mathbf{C r}$} & \multicolumn{1}{|c|}{$\mathbf{A l}$} & $\mathbf{T i}$ & \multicolumn{1}{|c|}{$\mathbf{C}$} & \multicolumn{1}{|c|}{$\mathbf{N b}$} & \multicolumn{1}{|c|}{ Mo } & Co & \multicolumn{1}{|c|}{$\mathbf{~ i ~}$} \\
\hline $\mathbf{3 1 V}$ & 57 & 13.5 & 23 & 1.3 & 2.3 & 0.04 & 0.9 & 2 & & 0.2 \\
\hline $\mathbf{2 6 8 6}$ & 39.7 & 34.6 & 19.2 & 1.2 & 3 & 0.03 & & 1.2 & 0.9 & 0.03 \\
\hline $\mathbf{2 6 8 7}$ & 47 & 27 & 18 & 1.8 & 3.8 & 0.03 & & 1.2 & 1 & \\
\hline
\end{tabular}

As can be seen in Figure I.3.2.1.3.1 (a)-(c), a 20-25 $\mu \mathrm{m}$ coating formed at the surface of the 2686 and 2687 coupon surface, as compared to $\sim 40 \mu \mathrm{m}$ for Alloy $31 \mathrm{~V}$. For all of the coupons, an $\sim 15-20 \mu \mathrm{m}$ interdiffusion zone was observed with larger $(\mathrm{Ni}, \mathrm{Al})$-rich precipitates for the 2686 and 2687 coupons. The coating thickness at the specimen surface was, however, quite inhomogeneous for the 2686 and 2687 alloys with very thin coating in some areas. Inconsistent wettability of the Al slurry on the 2686 and 2687 substrates was evident visually during the coating process prior to heat-treatment. Additional 2686 and 2687 coupons were encapsulated in a quartz ampoule backfilled with $\mathrm{Ar}+4 \% \mathrm{H}$ and then annealed according to the full substrate heat-treatment: $4 \mathrm{~h}$ at $1121^{\circ} \mathrm{C}$, fan air cooling followed by aging at $760^{\circ} \mathrm{C}$ for $16 \mathrm{~h}$, and fan air cooling again to determine if the resulting coating microstructure was repeatable with alternative heat-treatment conditions. Again, the resulting coatings were thin and quite inhomogeneous, which indicated that the low coating quality was related to the slurry melting and insufficient wettability at the substrate surface. Due to the low coating quality, only one 2687 coupon was oxidation-tested at $900^{\circ} \mathrm{C}$ in air $+10 \% \mathrm{H}_{2} \mathrm{O}$, for a $1 \mathrm{~h}$ cycle.

Figure I.3.2.1.3.1 (d) highlights the significant oxidation rate decrease for the coated 2687 coupon, as compared to the bare 2687 coupon, with the oxidation rate being similar to the oxidation rate for bare $31 \mathrm{~V}$. A better coating will, however, be needed for long-term applications due to the very limited $\mathrm{Al}$ reservoir in thin coatings. 


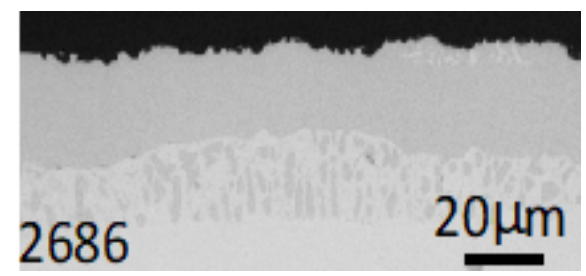

(a)

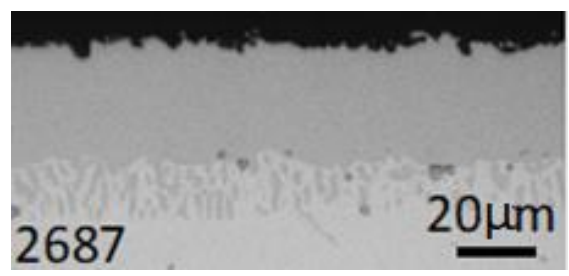

(b)

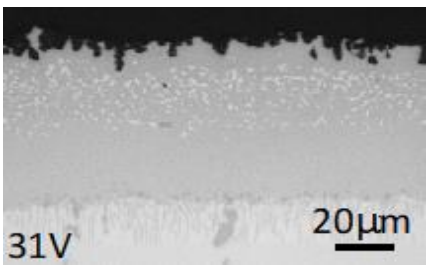

(c)

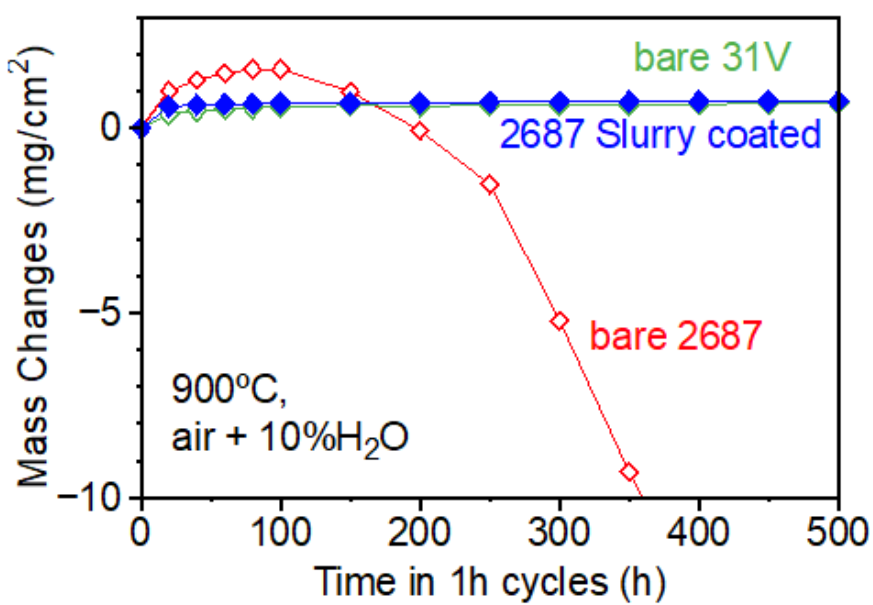

(d)

Figure I.3.2.1.3.1. Cross-section optical micrographs of aluminide slurry coated specimens annealed for $4 \mathrm{~h}$ at $1121^{\circ} \mathrm{C}$ in vacuum showing a $\sim 20-25 \mu \mathrm{m}$ coating formed at the surface of the (a) 2686, (b) 2687, and a $\sim 40$ um coating at the surface of (c) $31 \mathrm{~V}$. (d) Mass changes at $900^{\circ} \mathrm{C}$ in air $+10 \% \mathrm{H}_{2} \mathrm{O}$ with $1 \mathrm{~h}$ cycle for bare 2687 , bare $31 \mathrm{~V}$, and coated 2687 coupons showing a significant improvement of the oxidation behavior for the coated 2687 coupon compared to the bare coupon. Source: ORNL.

To improve the coating quality and uniformity, new commercial Al slurries were purchased from Ceral, Inc.one without activators (e.g., Ceral 10A green), one with an Si additive (e.g., Ceral 10 green), and one with a $\mathrm{Cr}$ additive (Ceral 10). Alloy 2687 coupons were coated with the Ceral series, but, as can be seen in Figure I.3.2.1.3.2, Ceral 10 and Ceral 10 green did not wet the 2687 coupons well. Further work is needed to improve the coating deposition and optimize the coating heat-treatment. A collaboration with Flame Spray, Inc. was initiated, and the company will deposit their own $\mathrm{Al}$ slurry on 2687 oxidation coupons and fatigue specimens, and then anneal the specimens in argon according to the standard heat-treatment for Alloy 2687. In addition, an $\mathrm{AlCl}_{3}$ activator was added to the ORNL in-house $\mathrm{Al}$ slurry composition. Alloys 2686 and 2687 specimens were coated; improved wettability was visually evident. Specimens are awaiting heat-treatment.

A coupled thermodynamic-kinetic model was employed to predict coating microstructure during aluminizing and high-temperature exposures as a function of time, temperature, and base alloy composition [2].

Figure I.3.2.1.3.3 shows the predicted phase distribution in the coating and interdiffusion zone (IDZ) based on the heat-treatment conditions. The XRD element maps and the EBSD spectroscopy phase map in Figure I.3.2.1.3.3 (b) and (c), respectively, are consistent with the model predictions.

The influence of different heat-treatments on the ORNL standard slurry aluminide coating microstructure on $31 \mathrm{~V}$ is evident in Figure I.3.2.1.3.4. A significantly thinner coating is observed after the single heat-treatment. Furthermore, the outer coating is precipitate rich after the single heat-treatment compared to the coating after the multi-step heat-treatment. The differing heat-treatments have also influenced the IDZ microstructure. 

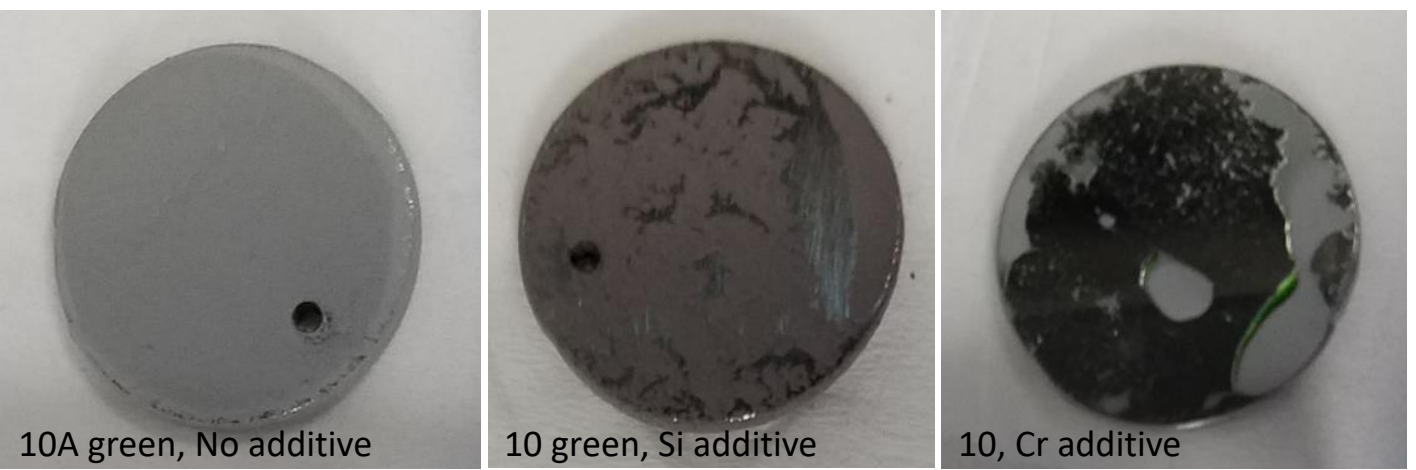

Figure I.3.2.1.3.2. Top view of 2687 coupons dipped in Al Ceral slurries showing that only the Ceral 10A green coating effectively wet the 2687 coupon. Source: ORNL.

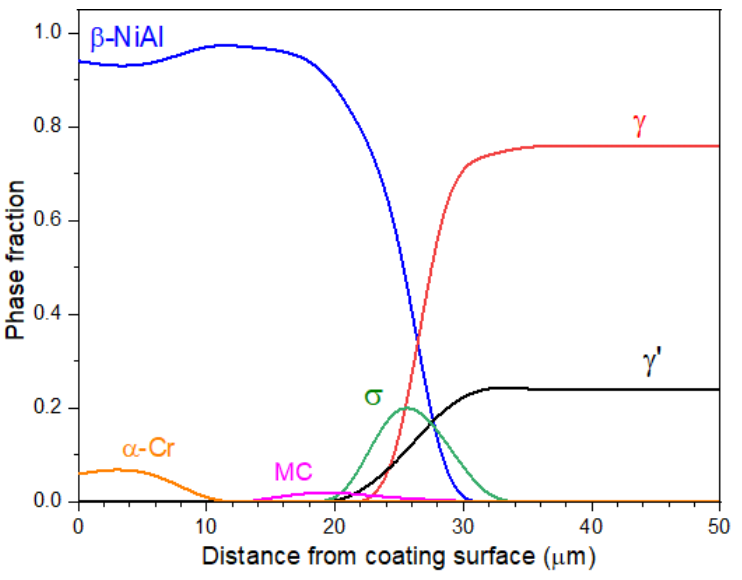

(a)

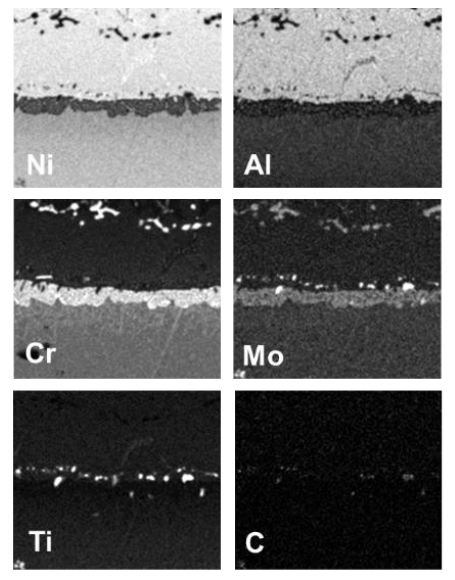

(b)

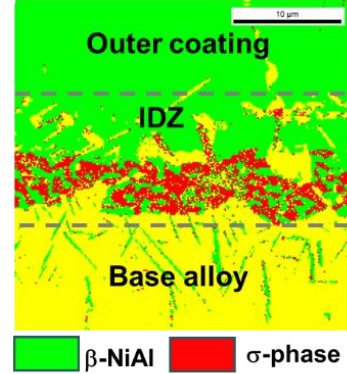

MC-type carbides Substrate (FCC)

(c)

Figure I.3.2.1.3.3. (a) Calculated phase distribution for the slurry aluminized $31 \mathrm{~V}$ specimen after aluminizing at $1100^{\circ} \mathrm{C}$ for $3 \mathrm{~h}$ followed by a heat-treatment at $850^{\circ} \mathrm{C}$ for $4 \mathrm{~h}$ and at $730^{\circ} \mathrm{C}$ for $4 \mathrm{~h}$. (b) Elemental mappings of the cross-section of the corresponding aluminized $31 \mathrm{~V}$ specimen and (c) EBSD spectroscopy phase map showing that the model predicted well the microstructure evolution in the coating and the substrate during the coating heat-treatment. Source: ORNL.

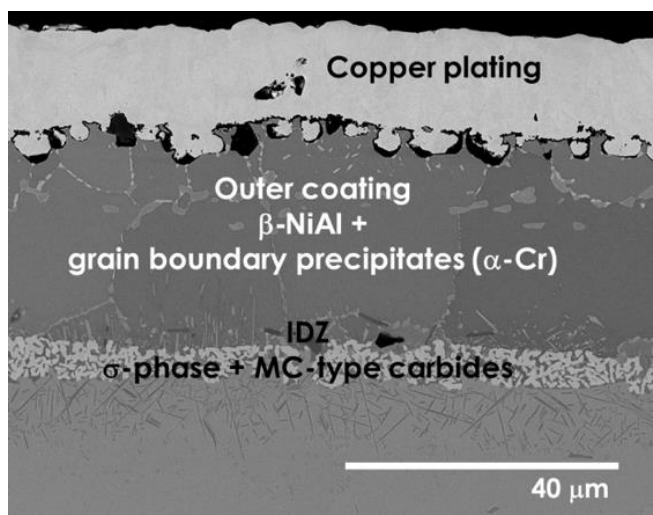

(a)

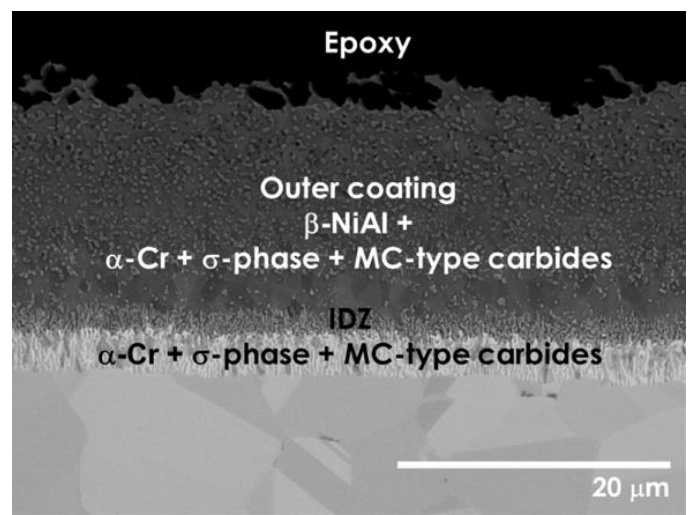

(b)

Figure I.3.2.1.3.4. (a) Backscattered electron image of the cross-section of the as-aluminized 31V specimen after a multistep heat-treatment for $3 \mathrm{~h}$ at $1100^{\circ} \mathrm{C}, 4 \mathrm{~h}$ at $850^{\circ} \mathrm{C}$, and $4 \mathrm{~h}$ at $730^{\circ} \mathrm{C}$ and after (b) a single-step heat-treatment for $16 \mathrm{~h}$ at $760^{\circ} \mathrm{C}$ highlighting the impact of the heat-treatment on the coating microstructure. Source: ORNL. 
The model was able to predict the influence of the varying heat-treatments on the coating microstructure, as shown in Figure I.3.2.1.3.5. The model predicted a much thinner coating (i.e., about $10 \mu \mathrm{m}$ ) after the singlestep heat-treatment, which is in reasonable agreement with the experimentally observed coating thickness (i.e., $15 \pm 3.5 \mu \mathrm{m}$ ). The predicted coating thickness after the multi-step heat-treatment was about $30 \mu \mathrm{m}$, which agrees well with the measured coating thickness (i.e., $32 \pm 4 \mu \mathrm{m}$ ). The higher fraction of precipitates in the outer coating after the single-step (i.e., $\sim 20 \% \alpha$-Cr $+3 \% \sigma$-phase) compared to the multi-step (i.e., about $7 \% \alpha$ $\mathrm{Cr}+2 \% \mathrm{MC}$-carbides) heat-treatment were also predicted by the model. The $\sigma$-phase enriched IDZ in the coating after the multi-step heat-treatment was well reproduced by the model.

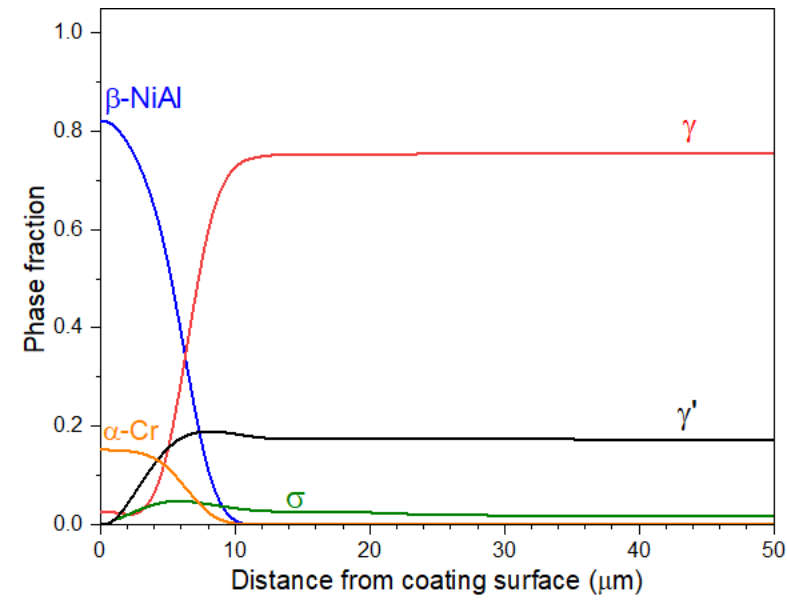

(a)

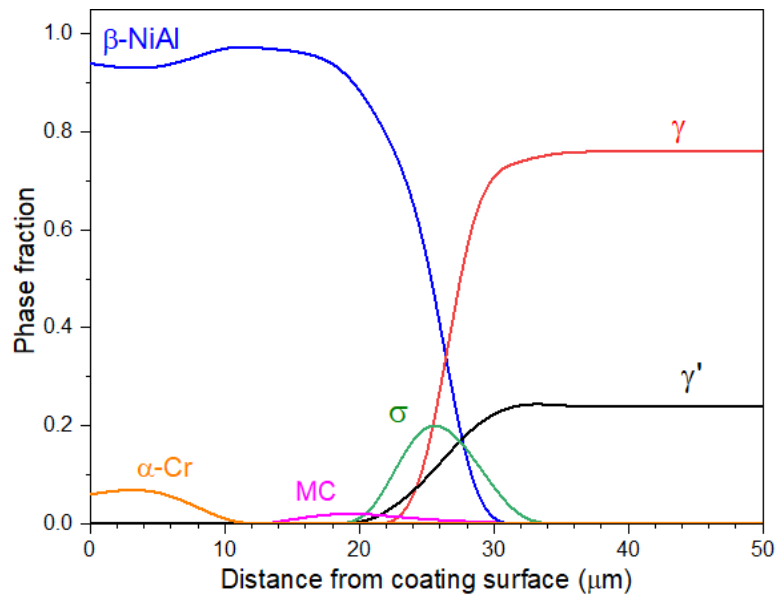

(b)

Figure I.3.2.1.3.5. Predictions of the influence of varying heat-treatments on the coating microstructure: (a) after the singlestep heat-treatment; and (b) after the multi-step heat-treatment showing that the impact of the heat-treatment on the coating/substrate microstructure is well captured by the model. Source: ORNL.

\section{Conclusions}

Initial results with the 2686 and 2687 alloys revealed that slurry coatings are a viable solution to significantly improve the cyclic oxidation performance of high-strength valve alloys. Ongoing work seeks to optimize the slurry chemistry and heat-treatment, as well as collaborate with industry partners to fabricate high-performance corrosion-resistant $\mathrm{Al}$ coatings. A coupled thermodynamic and kinetic computational model was successfully used to correlate the coating microstructures with the coating heat-treatments. Both $\mathrm{Al}$ and thermal spray coatings are being deposited on fatigue specimens to assess the coating impact on the alloys HCF performance.

\section{Key Publications}

A journal article is expected to be published in early 2020. One presentation was given at Minerals, Metals and Materials Society 2019 Conference, two presentations were given at the International Conference on Metallurgical Coatings and Thin Films 2019, one presentation was given at TMS 2019 Annual Meeting \& Exposition, and one invited presentation was given at Materials Science and Technology 2019 Technical Meeting and Exposition. 


\section{References}

1. Dryepondt, S., S. J. Jones, Y. Zhang, P. J. Maziasz and B. A. Pint, 2015, "Oxidation, creep, and fatigue properties of bare and coated $31 \mathrm{~V}$ alloy," JOM, Vol. 67, No. 1, pp. 68-76.

2. Pillai, R., W. Sloof, A. Chyrkin, L. Singheiser, and W. J. Quadakkers, 2015, “A new computational approach for modeling the microstructural evolution and residual lifetime assessment of MCrAlY coatings," Mater. High Temp., Vol. 32, No. 1-2, pp. 57-67.

\section{Acknowledgements}

The author would like to thank A. Rogers, M. Stephens, T. Lowe, T. Jordan, and M. Howell for assisting with the experimental work. 


\title{
I.3.2.1.4 Development of Cast, Higher Temperature Alloys (2B1)
}

\section{Michael P. Brady, Co-Principal Investigator}

Oak Ridge National Laboratory

1 Bethel Valley Road

Oak Ridge, TN 37831

E-mail: bradymp@ornl.gov

\author{
Yukinori Yamamoto, Co-Principal Investigator \\ Oak Ridge National Laboratory \\ 1 Bethel Valley Road \\ Oak Ridge, TN 37932 \\ E-mail: yamamotoy@ornl.gov
}

\section{Jerry L. Gibbs, DOE Technology Development Manager}

U.S. Department of Energy

E-mail: jerry.gibbs@ee.doe.gov

Start Date: October 1, 2018

Project Funding (FY19): \$275,000

End Date: September 30, 2022

DOE share: $\$ 275,000 \quad$ Non-DOE share: $\$ 0$

\section{Project Introduction}

Automotive exhaust components are anticipated to experience temperatures of $\geq 900-950^{\circ} \mathrm{C}$ in the near future in order to reach efficiency targets. The cast alloys currently used for turbocharger housings and exhaust manifolds are at their upper-temperature limit for creep and/or oxidation resistance. Therefore, new, costeffective alloys will be required to meet these increasing operating temperature requirements.

\section{Objectives}

The goal of this subtask is to increase the upper-temperature oxidation limit to $\geq 900-950^{\circ} \mathrm{C}$ for nextgeneration cast austenitic stainless steel exhaust gas components. Candidate alloys will utilize an $\mathrm{Fe}(\mathrm{Ni})$ base with $\leq 25-30 \mathrm{wt} . \% \mathrm{Ni}$ to remain cost-competitive, as well as minimize the use of other costly alloying additions, such as $\mathrm{Mo}, \mathrm{Nb}, \mathrm{W}$, etc. The cast stainless alloys being developed in this subtask are of a class referred to as alumina-forming alloys (AFAs), and have the distinction of being designed to form an $\mathrm{Al}_{2} \mathrm{O}_{3}$ protective oxide scale on the surface during oxidation vs the $\mathrm{Cr}_{2} \mathrm{O}_{3}$ oxide scales that form on most commercial stainless alloys. Alumina scales are typically slower growing and more protective than chromia scales, particularly at higher temperatures, but alloy design and the casting of Al-containing stainless alloys can be challenging.

\section{Approach}

Leveraging recent ORNL alloy development successes for wrought 25Ni-base AFA alloys for use up to 850$900^{\circ} \mathrm{C}$ and cast $35 \mathrm{Ni}$-base AFA alloys for use up to $1150^{\circ} \mathrm{C}$, design exploration of second generation cast AFA alloys with $\leq 25 \mathrm{wt} . \% \mathrm{Ni}$ has been pursued for use in exhaust components. Alloy design, development, and refinement in this Thrust 2 subtask are being guided by Thrust 4 complementary activities, including advanced characterization of oxide scale structures and nano-microscale strengthening precipitates, as well as Thrust 4 computational activities including CALPHAD and machine-learning studies. In FY 2019, several lab-scale heats of cast AFA type alloys (including the alloys prepared in FY 2018) were designed, manufactured, and are under evaluation for oxidation, mechanical, and thermal properties. 


\section{Results}

Oxidation-testing was completed at $900^{\circ} \mathrm{C}, 950^{\circ} \mathrm{C}$, and $1000^{\circ} \mathrm{C}$ in air $+10 \% \mathrm{H}_{2} \mathrm{O}$ for $1000,1 \mathrm{~h}$ cycles. All of the ORNL developmental lab arc-cast AFA alloys, listed in Table I.3.2.1.4.1, exhibited superior oxidation resistance, consistent with protective $\mathrm{Al}_{2} \mathrm{O}_{3}$ formation at $900^{\circ} \mathrm{C}$ and $950^{\circ} \mathrm{C}$, as shown in Figure I.3.2.1.4.1 (a) and (b), respectively. On the contrary, mass loss and oxide scale spallation were observed for benchmark commercial cast $\mathrm{Cr}_{2} \mathrm{O}_{3}$-forming alloys, such as 1.4826 (22Ni-12Cr base), $\mathrm{HK} 30 \mathrm{Nb}$ (25Cr-20Ni base, HK), and $\mathrm{HP} 40 \mathrm{Nb}(25 \mathrm{Cr}-35 \mathrm{Ni}$ base, HP). In contrast, lab arc-cast AFA1 through AFA5 all exhibited small positive mass gains consistent with protective $\mathrm{Al}_{2} \mathrm{O}_{3}$ formation, which was confirmed by cross-section analysis of the $950^{\circ} \mathrm{C}$ test samples. At $1000^{\circ} \mathrm{C}$, a transition to minor mass loss and oxide scale spallation was observed after 500-800 one-hour cycles, indicative of a transition to borderline $\mathrm{Al}_{2} \mathrm{O}_{3}$ formation and initiation of Fe-base oxide nodule formation.

Table I.3.2.1.4.1. Alloy Compositions of Cast Austenitic Stainless Steel in the Present Study.

\begin{tabular}{|c|c|c|c|c|c|c|}
\hline \multirow{2}{*}{ Heat } & \multicolumn{5}{|c|}{ Base alloy composition wt.\% } & \multirow{2}{*}{ Remarks } \\
\hline & $\mathrm{Fe}$ & $\mathrm{Ni}$ & $\mathrm{Cr}$ & $\mathrm{Al}$ & others & \\
\hline Gen. 2 AFA1 & 50.7 & 25 & 15 & 4 & \multirow{4}{*}{$\begin{array}{l}\text { With various combined additions of } \mathrm{Cu}, \mathrm{Mn}, \mathrm{Mo} \text {, } \\
\qquad \mathrm{Nb}, \mathrm{Si}, \mathrm{Ti}, \mathrm{W}, \mathrm{Zr}, \mathrm{Y}, \mathrm{C} \text {, and } \mathrm{B}\end{array}$} & \\
\hline Gen 2 AFA2 & 47.7 & 25 & 15 & 4 & & $\begin{array}{l}\text { Focused in } \\
\text { FY19 }\end{array}$ \\
\hline Gen. 2 AFA3 & 49.2 & 25 & 15 & 4 & & \\
\hline Gen. 2 AFA5 & 51.7 & 22 & 17 & 4 & & $\begin{array}{l}\text { Focused in } \\
\text { FY19 }\end{array}$ \\
\hline 1.4826 & 64.5 & 11 & 22 & - & $0.9 \mathrm{Mn}, 1.1 \mathrm{Si}, 0.4 \mathrm{C}, 0.06 \mathrm{~N}$ & Reference \\
\hline HK30Nb (HK) & 50.5 & 20.5 & 25 & - & $1 \mathrm{Mn}, 1.4 \mathrm{Nb}, 1.4 \mathrm{Si}, 0.3 \mathrm{C}$ & Reference \\
\hline HP4ONb (HP) & Bal. & 35 & 25 & - & $\begin{array}{c}<1.5 \mathrm{Mn}, 0.5 \mathrm{Mo}, 0.5-1.5 \mathrm{Nb}, 0.5-1.5 \mathrm{Si}, 0.38- \\
0.45 \mathrm{C}\end{array}$ & Reference \\
\hline
\end{tabular}

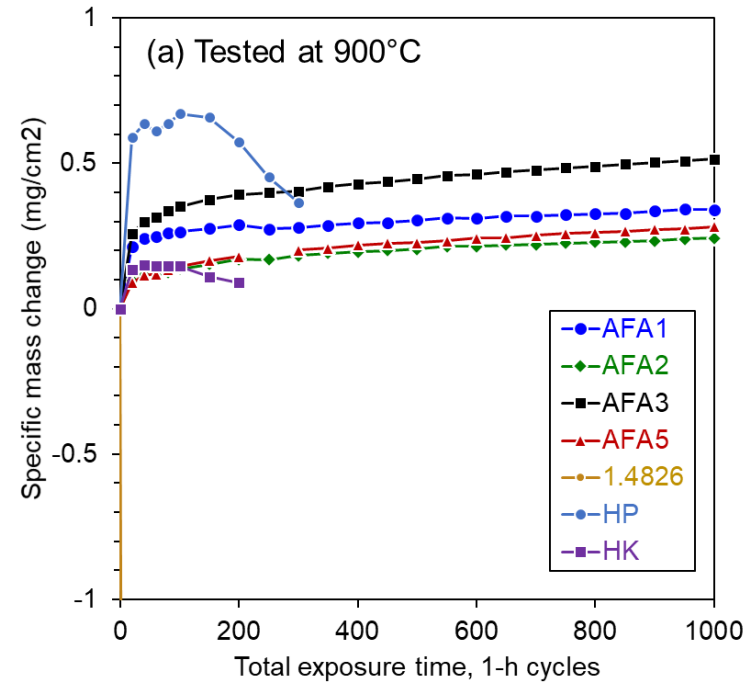

(a)

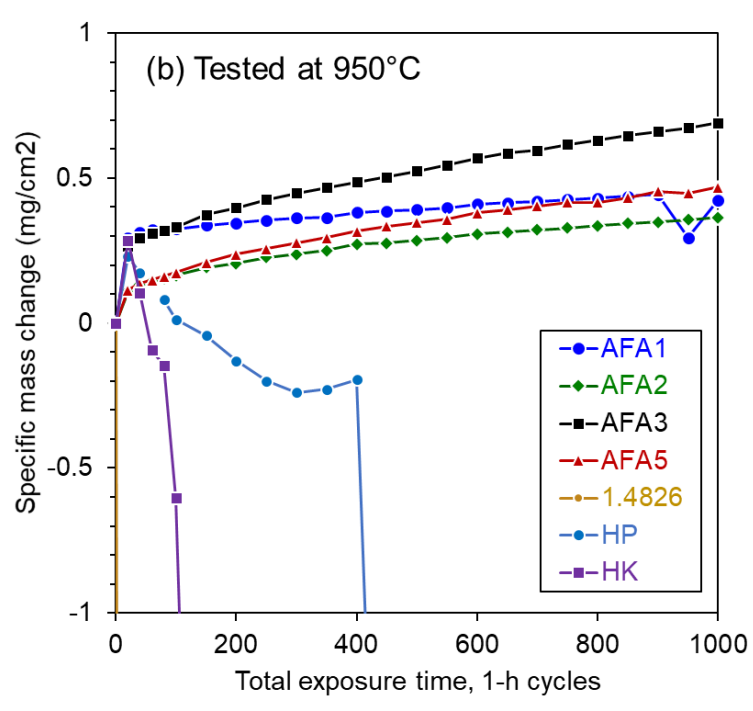

(b)

Figure I.3.2.1.4.1. Specific mass changes in various cast austenitic steels after cyclic oxidation with $1 \mathrm{~h}$ cycles at: (a) $900^{\circ} \mathrm{C}$ and (b) $950^{\circ} \mathrm{C}$ in air with $10 \% \mathrm{H}_{2} \mathrm{O}$ Specimens exhibiting rapid mass gains or losses indicate poor oxidation resistance. Source: ORNL. 
Cross-sectional characterization of the surface oxide layers and the substrate matrix in the AFA2 and AFA5 alloys after oxidation-testing has been summarized in Figure I.3.2.1.4.2 (a) and (b), respectively. Both samples were exposed in air $+10 \% \mathrm{H}_{2} \mathrm{O}$ at $950^{\circ} \mathrm{C}$ for a total of $1000 \mathrm{~h}$, with $1 \mathrm{~h}$ cycles. The SEM-backscattered electron (BSE) images illustrated that the surface was fully covered by a continuous, dark contrast layer, which corresponds to the $\mathrm{Al}_{2} \mathrm{O}_{3}$ scale formed during oxidation-testing, which protected the sample surface effectively. The matrix below the protective scale exhibited a B2-NiAl denuded zone, indicating that the B2$\mathrm{NiAl}$ precipitates (i.e., the light dark contrast particles observed lower part of the SEM-BSE images) act as the alloy $\mathrm{Al}$ reservoir to support the continuous growth of the $\mathrm{Al}_{2} \mathrm{O}_{3}$ scale layer. STEM-dark field images indicated an adherent, columnar $\mathrm{Al}_{2} \mathrm{O}_{3}$ grain structure with no detectable defects at the oxide-alloy interface, together with Al-rich transient oxides at the top of the scale. STEM-EDS identified the major components of the oxide layers are $\mathrm{Al}$ and $\mathrm{O}$, as defined by ORNL's Dr. Michael Lance under Thrust 4A, "Advanced Characterization," within this program.

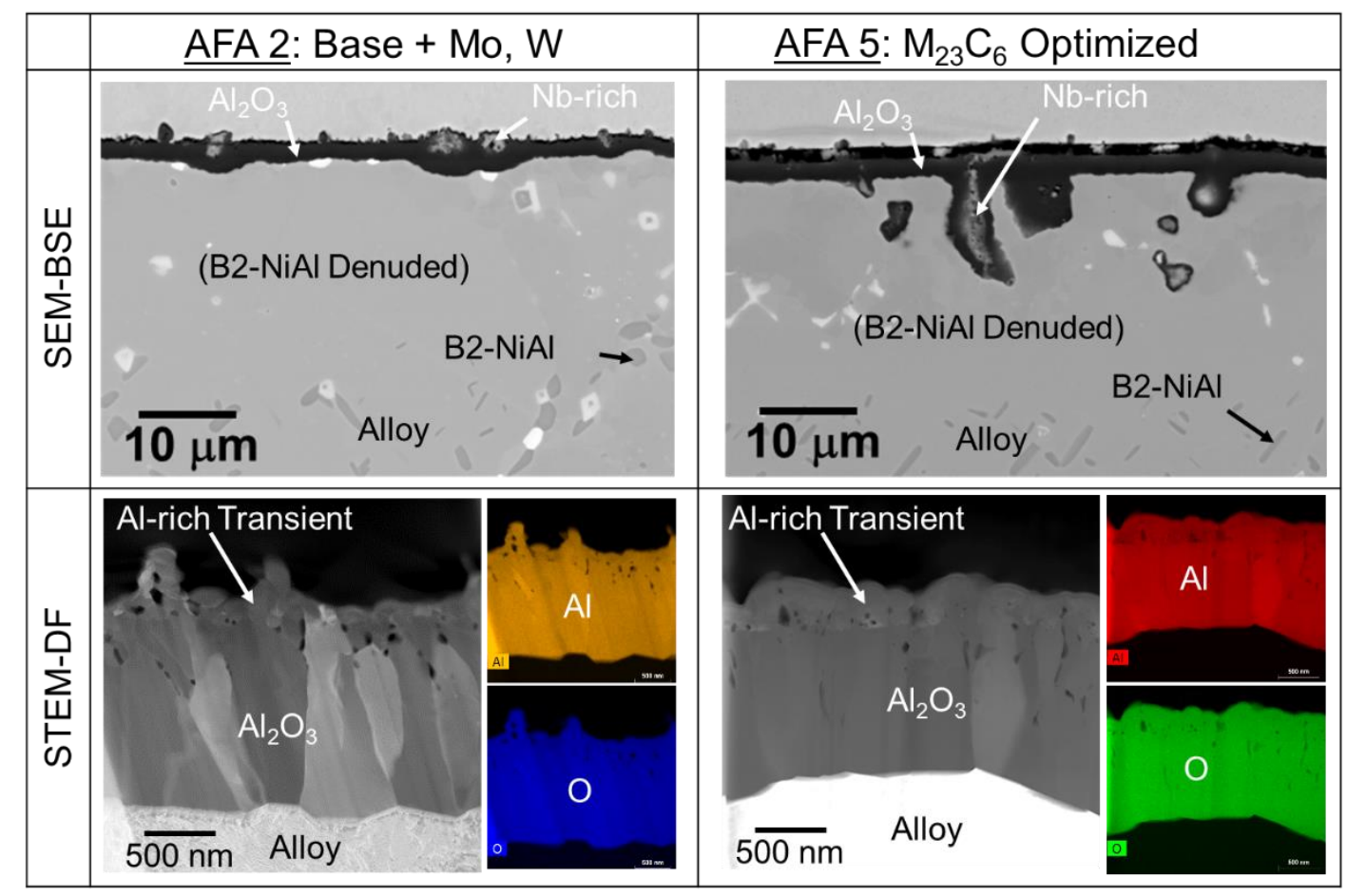

Figure I.3.2.1.4.2. Cross-sectional SEM-BSE images and STEM-dark field images, together with element distribution maps acquired by STEM-EDS, of as-cast AFA2 and AFA 5 after a total of $1000 \mathrm{~h}$ exposure time in $1 \mathrm{~h}$ cycles at $950^{\circ} \mathrm{C}$ in Air $+10 \% \mathrm{H}_{2} \mathrm{O}$. Source: ORNL.

Based on their excellent oxidation resistance at $950^{\circ} \mathrm{C}$, screening level tensile properties obtained at room temperature, $700^{\circ} \mathrm{C}$ and $900^{\circ} \mathrm{C}$ and estimated raw materials cost for the two AFA alloys-AFA2 and AFA5were down-selected for creep testing and initial trial commercial cast heats. Figure I.3.2.1.4.3 shows creep behavior for lab-scale heats of AFA2 (2 different Ni levels) and AFA5 (3 different Ni levels) at an accelerated test condition of $900^{\circ} \mathrm{C}$ and $50 \mathrm{MPa}$, relative to reference cast $\mathrm{Cr}_{2} \mathrm{O}_{3}$-forming austenitic stainless steels (estimated from literature data). Lab-cast AFA5 exhibited a $\sim 1200-1400 \mathrm{~h}$ rupture life, compared with $\sim 400 \mathrm{~h}$ for AFA 2 and less than $\sim 200 \mathrm{~h}$ for the reference stainless steels, indicating a strong advantage of the newly developed cast AFA steels in creep properties. It also indicated that the creep performance was not sensitive to the Ni variation, suggesting a wide compositional tolerance on the creep performance. Although the results are extremely promising, additional creep testing at lower loads and longer rupture times will also be needed to confirm the excellent creep resistance suggested by this accelerated test condition. 


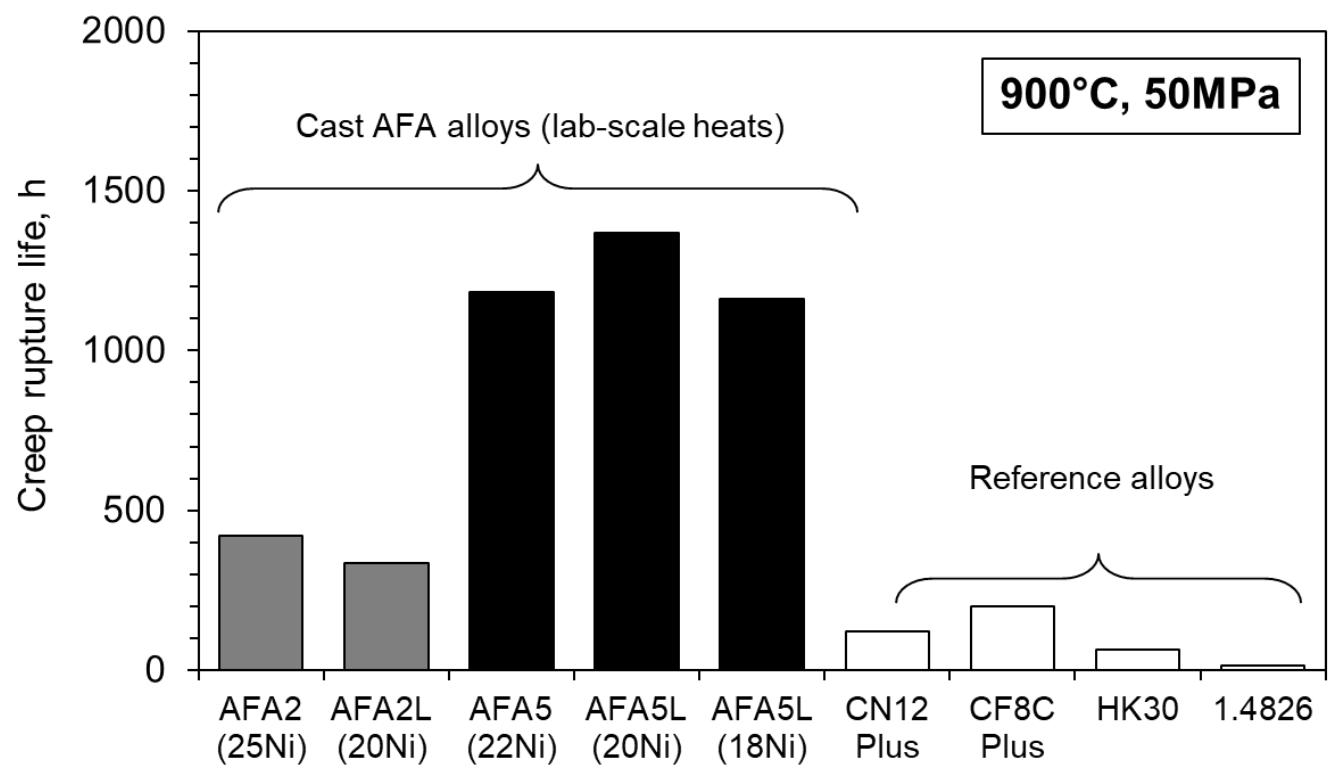

Figure I.3.2.1.4.3. Comparison of creep-rupture lives of various cast austenitic steels tested at $900^{\circ} \mathrm{C}$ and $50 \mathrm{MPa}$ including lab-scale heats based on AFA2 and AFA5 with a variation of low Ni contents. Source: ORNL.

The oxidation resistance showed a slightly different alloy composition trending result from the creep-rupture properties. Figure I.3.2.1.4.4 represents the specific mass changes of the lab-scale heats of AFA2 and AFA5 base cast alloys with a variation of Ni contents, together with the results of commercially produced cast AFA2 $(25 \mathrm{Ni})$ and AFA5 heats (22Ni) (Note: the details of these commercial heats can be found later in this report). The cast AFA alloys with 20Ni showed slow oxidation kinetics, similar to the high Ni containing heats for up to $\sim 700 \mathrm{~h}$ exposure, whereas the alloy with $18 \mathrm{Ni}$ started a significant mass loss after $\sim 400 \mathrm{~h}$ exposure, likely due to non-protective Fe-rich oxide formation and spallation. Lower Ni content is more advantageous for reducing the raw material costs, although this result indicates that more than $20 \mathrm{Ni}$ would be required to obtain the balanced properties of creep and oxidation resistance at the targeted elevated temperatures.

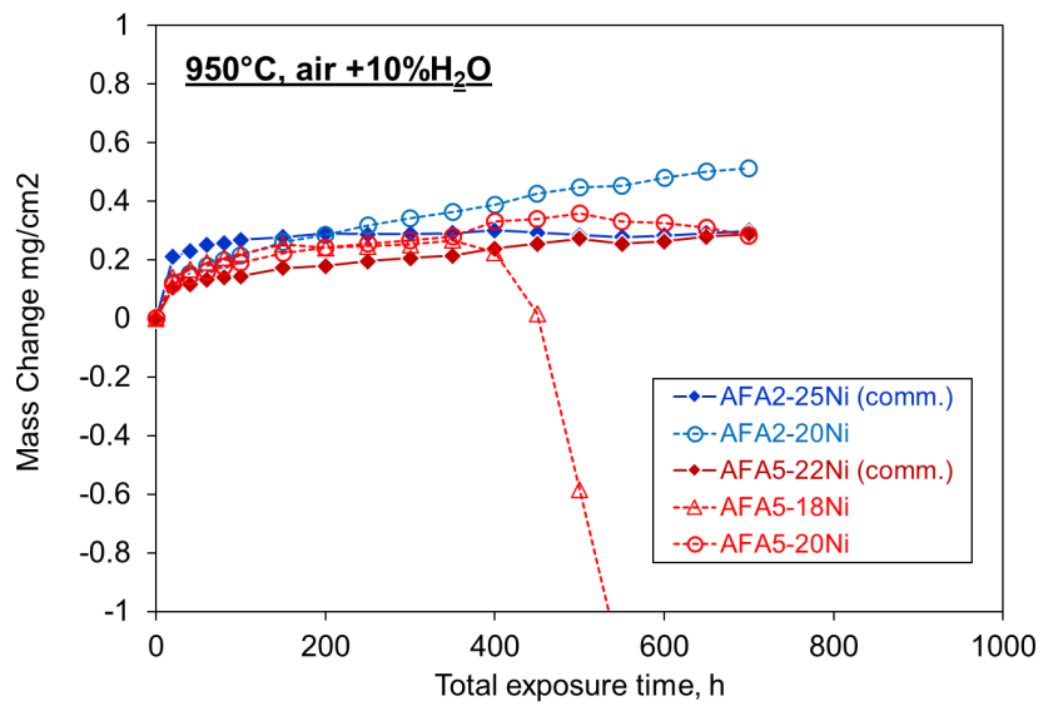

Figure I.3.2.1.4.4. Specific mass changes in cast AFA2 and AFA5 base alloys with a variation of Ni contents after cyclic oxidation with $1 \mathrm{~h}$ cycles at $950^{\circ} \mathrm{C}$ in air with $10 \% \mathrm{H}_{2} \mathrm{O}$, together with the results from the commercially produced cast AFA heats. Source: ORNL. 
Microstructure characterization of the creep-ruptured AFA2 and AFA5 tested at $900^{\circ} \mathrm{C}$ and $50 \mathrm{MPa}$ has been conducted as a collaborative effort with Drs. Bharat Gwalani and Arun Devaraj at PNNL under Thrust 4, "Advanced Characterization and Computation," of this program, targeting the identification of the secondphase precipitates and their distribution in the deformed matrix. Figure I.3.2.1.4.5 illustrates TEM bright field images showing the dislocation structure in the FCC-matrix interacted with B2-NiAl (and possibly Lavesphase) precipitates. Spot compositional analysis of the second-phase precipitates by TEM-EDS, as well as selected area diffraction pattern analysis, have been utilized for the phase identification, and then compared with thermodynamically predicted second phases. Detailed characterization for measuring the amount of each second-phase is currently in progress, which will be correlated with the creep deformation performance and the strengthening mechanism.

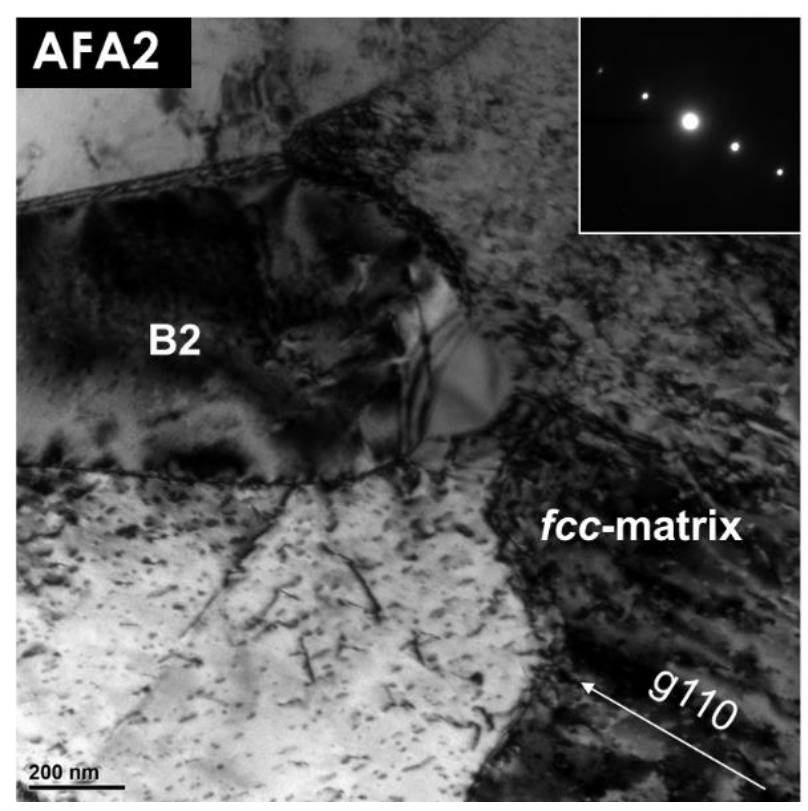

(a)

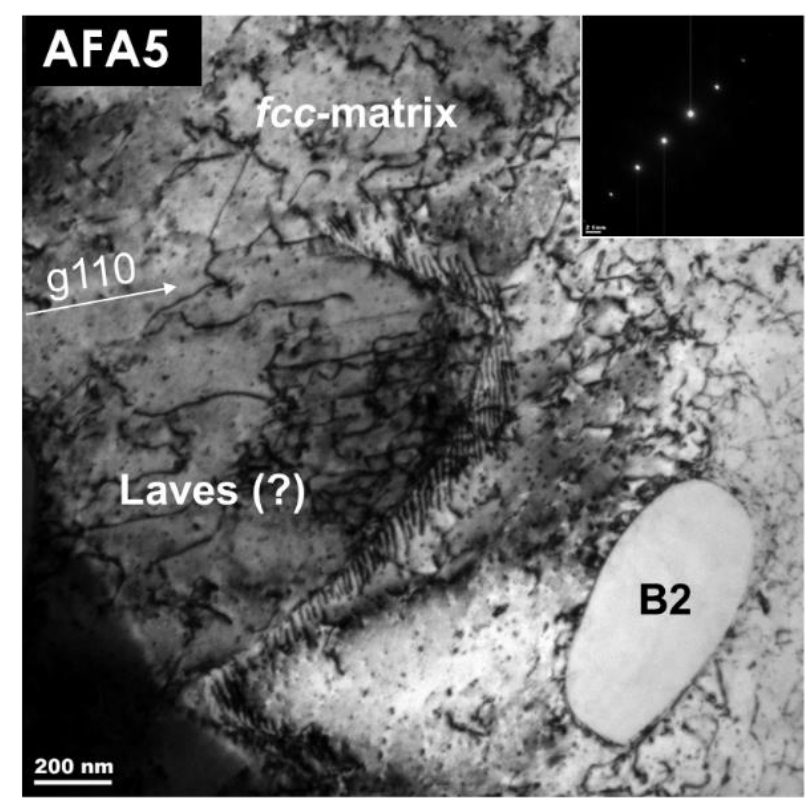

(b)

Figure I.3.2.1.4.5. Preliminary TEM characterization results of: (a) cast AFA2; and (b) AFA5, tested at $900^{\circ} \mathrm{C}$ and $50 \mathrm{MPa}$. Source: PNNL.

First scale-up cast production of AFA2 and AFA5 was completed through a commercial foundry (MetalTek International, Waukesha, WI). Three different forms of the ingots were delivered-plate, keel block, and round bar, as observed in Figure I.3.2.1.4.6. The cross-sectional macroscopic inspection indicated that the ingots had no visible defects attributed to the solidification process (e.g., porosity, inclusion, segregation, etc.). Chemical analysis indicated that the $\mathrm{Cr}$ content of AFA5 was higher than the maximum limit of the specification (20 vs. $17 \mathrm{wt} . \%$ ), so the first AFA5 heat was treated as an off-specification material. Some of the other elements (e.g., $\mathrm{B}, \mathrm{C}, \mathrm{Si}, \mathrm{V}$, and Y) were also deviated slightly from the target ranges, although the impact of these elements was considered negligible. The chemistry of the commercial heat on the AFA2 met the specification more accurately. 


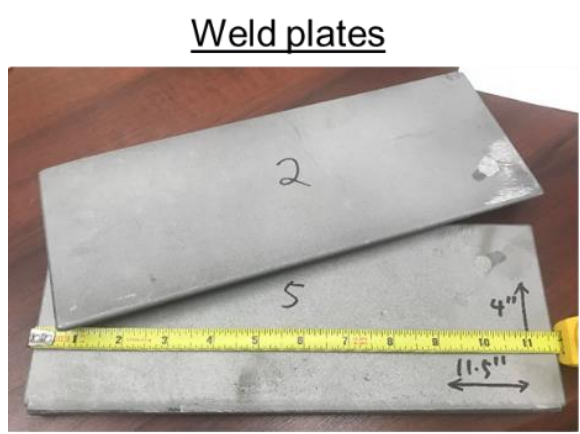

(Size: $0.5 "$ x 4" x 11.5")

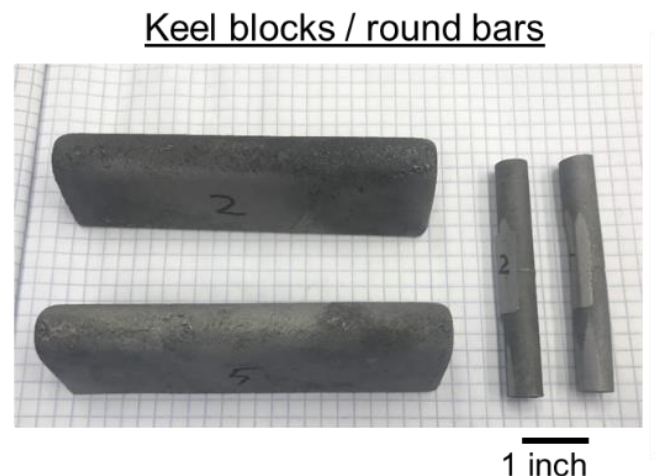

Cross-sectional view (\#190201, Keel block)

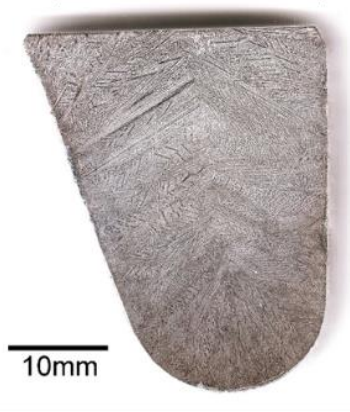

Figure I.3.2.1.4.6. Images of the first commercial heats of AFA2 and AFA5, together with a cross-sectional macrostructure of AFA5 (heat \#190201) showing no visible solidification defects. Source: ORNL.

Tensile properties of commercial AFA2/5 heats are summarized in Figure I.3.2.1.4.7, comparing the lab-scale heats of AFA2/5 and the other reference cast austenitic stainless steels. The high-temperature yield strengths of the commercial heats were comparable to that of the lab-scale heats, maintaining higher values than that of the reference cast austenitic steels at both $700^{\circ} \mathrm{C}$ and $900^{\circ} \mathrm{C}$. Commercial heat AFA5 also showed the room temperature (RT) ductility similar to the lab-scale heat and the other reference materials, whereas commercial heat AFA2 exhibited very little RT ductility. Although the mechanism of ductility reduction has not been identified yet, it was speculated that the higher $\mathrm{Nb}$ in AFA2 vs AFA5 might result in elemental segregation to degrade RT toughness in the larger scale cast production as compared to the smaller lab-scale heats.

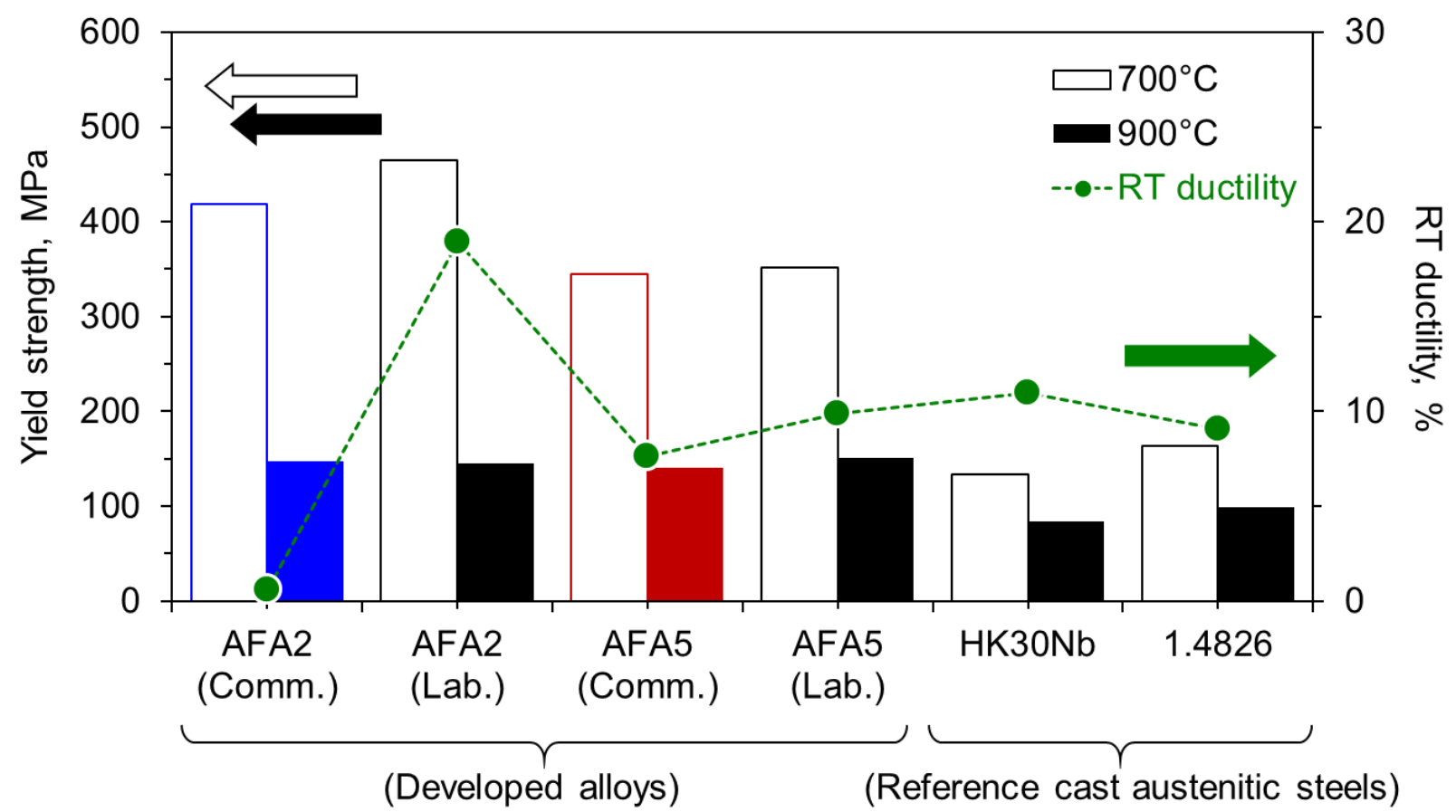

Figure I.3.2.1.4.7. Tensile properties of the cast austenitic stainless steels at $700^{\circ} \mathrm{C}, 900^{\circ} \mathrm{C}$ (yield strength), or RT (ductility), comparing the commercial and lab-scale heats of AFA2 and AFA5 together with the benchmark cast materials. Source: ORNL. 


\section{Conclusions}

Two of the second generation cast AFA alloys-AFA2 and AFA5 - were selected for detailed property evaluation and characterization in FY 19. Both alloys showed very slow oxidation kinetics in air $+10 \% \mathrm{H}_{2} \mathrm{O}$ at $950^{\circ} \mathrm{C}$ for up to a total of $1000 \mathrm{~h}$ exposure, which was attributed to the formation of a thin, continuous alumina scale on the sample surface during oxidation exposure. AFA5 base alloys with 20-22 wt.\% Ni were found to show one order-of-magnitude longer creep-rupture life at $900^{\circ} \mathrm{C}$ and $50 \mathrm{MPa}$ than that of the benchmark commercial chromia-forming austenitic stainless steels (HK/HP), in combination with very promising oxidation resistance. Based on the results, AFA5 was selected for further property evaluation and microstructure characterization in FY 2020, especially focusing on the long-term creep and oxidation performance, as well as the effect of minor alloying elements on the positive/negative impacts to these properties. For the scale-up efforts toward a scope of commercial production, it is also planned to secure industrial partners in the near future to guide alloy property targets and required data for commercialization.

\section{Key Publications}

ORNL invention disclosure submitted in October 2019.

\section{Acknowledgements}

The authors would like to thank G. A. Cox, D. T. Moore, D. C. Heidel, K. O. Hanson, G. Garner, M. S. Stephens, T. Lowe, T. Jordan, C. S. Hawkins, J. L. Moser, and K. L. Hedrick (ORNL) for helping with the experimental work. The authors would also like to acknowledge Drs. B. Gwalani and A. Devaraj of PNNL, and Dr. M. Lance of ORNL, for their collaborative support on the detailed microstructure and oxide characterization under Thrust 4, as well as Dr. G. Muralidharan of ORNL for his alloy design insights. 


\section{I.3.2.1.5 Selective Material Processing to Improve Local Properties (2B2)}

\section{Glenn Grant, Principal Investigator}

Pacific Northwest National Laboratory

902 Battelle Blvd.

Richland, WA 99354

E-mail: glenn.grant@pnnl.gov

\section{Jerry L. Gibbs, DOE Technology Development Manager}

U.S. Department of Energy

E-mail: jerry.gibbs@ee.doe.gov

Start Date: October 1, 2019

Project Funding (FY19): \$300,000
End Date: September 30, 2022

DOE share: $\$ 300,000 \quad$ Non-DOE share: $\$ 0$

\section{Project Introduction}

This project is Subtask 2B2 and is developing advanced hybrid manufacturing processes to locally improve the performance of parts and assemblies through selective microstructure modification. The concept includes the notion that, in general, strength requirements of an assembly are often driven by a limited number of highly loaded small areas on a complex part. Failure in these areas can drive global section thickness or strength requirements. If just those selected areas could be improved to the strength —or more importantly the fatigue and toughness - requirements, then overall material or cost-savings could be achieved.

This concept has the potential to allow lower-cost materials to achieve properties that meet or exceed current state-of-the-art materials. The concept is to apply a secondary process to selective areas of the part to locally improve the properties only where they are needed. The added cost of the secondary process is offset by the lower base material cost, with the balance designed to achieve a total cost no more than $10 \%$ higher than the current production process.

In addition, selective area modification could enable new high-performance alloys, local up-alloying, and improve the performance of parts made by advanced manufacturing techniques, such as laser or friction additive processes. In many cases, advanced alloys are designed to gain, for instance, higher temperature performance, but often at the expense of another property, such as castability, fracture toughness, or fatigue performance. For these new alloy opportunities, local property improvement through selective processing and engineering could enable economical and better bulk material performance, while still addressing the extreme performance requirements in localized areas.

The project will investigate and demonstrate advanced processes such as friction stir processing (FSP), cold spray (CS), and others that have the potential to produce enhanced properties on local areas of complex powertrain material castings, forgings, or additively manufactured materials. FSP, for example, has been shown to provide dramatic improvements in fatigue performance to cast materials across a wide range of alloy systems [1, 2], as observed in Figure I.3.2.1.5.1. 

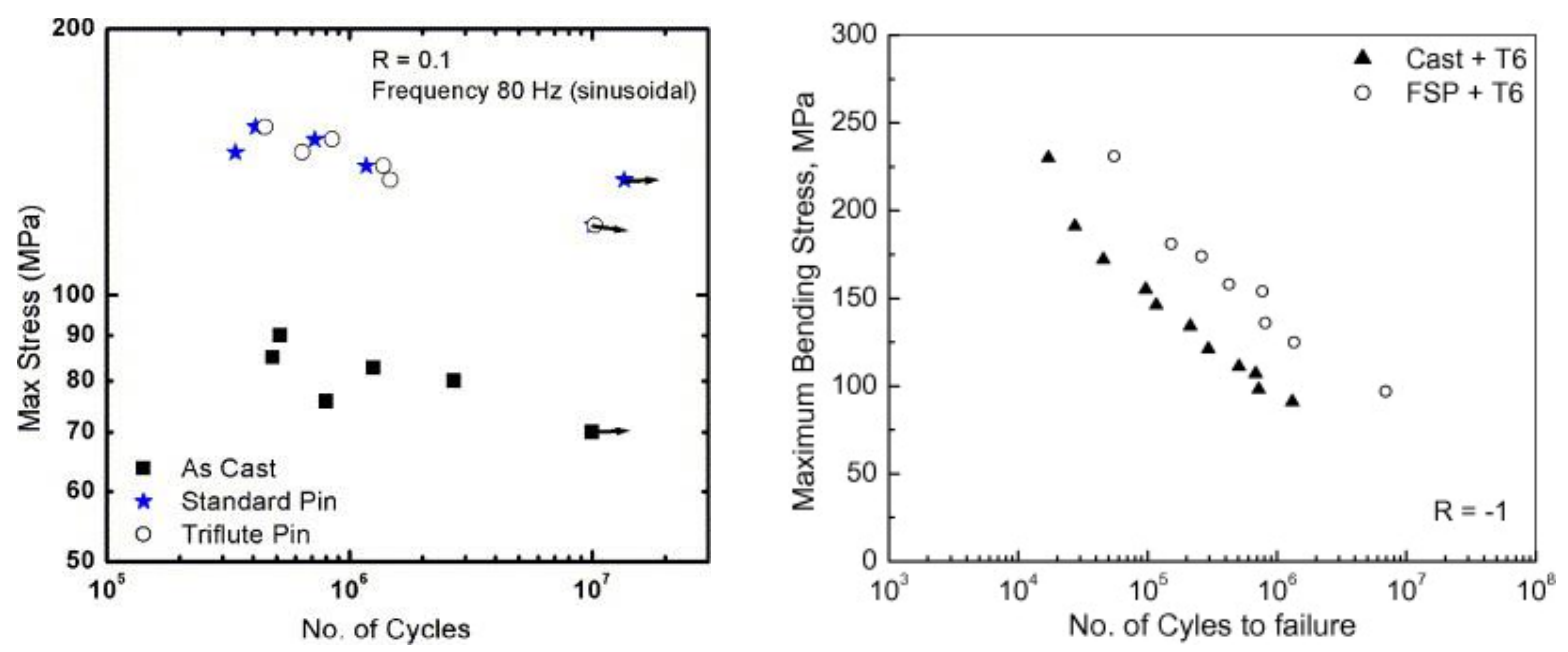

Figure I.3.2.1.5.1. Previous results of fatigue testing showing significant improvements in fatigue performance can be achieved when applying FSP to cast Al alloys. Source: PNNL.

Local property improvements can be especially important in enabling new high-performance alloys. In some cases, alloys designed for higher temperature operation arrive at their performance through complex alloy chemistries that can show coarse second-phase precipitates or castability issues leading to near surface casting defects. FSP has been shown to be able to refine microstructure, heal casting defects, improve alloy homogeneity, and produce a wrought microstructure. FSP has also been shown to produce significant improvement in the fatigue behavior of forged steel crankshaft materials where geometric stress concentrations have been created by machining or drilling. Figure I.3.2.1.5.2 shows an example where FSP of an advanced crankshaft steel can fully recover fatigue knockdown due to the stress concentration around a drilled oil hole in the crank journal. Selective microstructure modification can be used to improve local properties and remove the consequences of stress concentrations. 


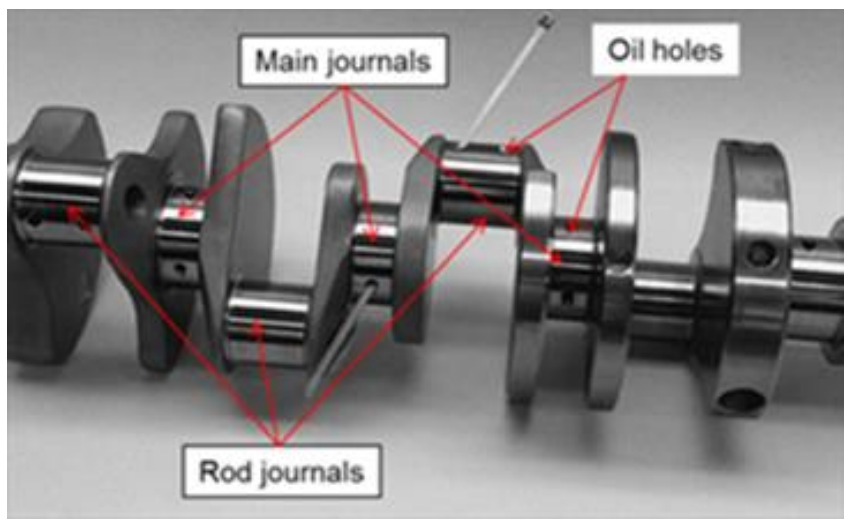

(a)

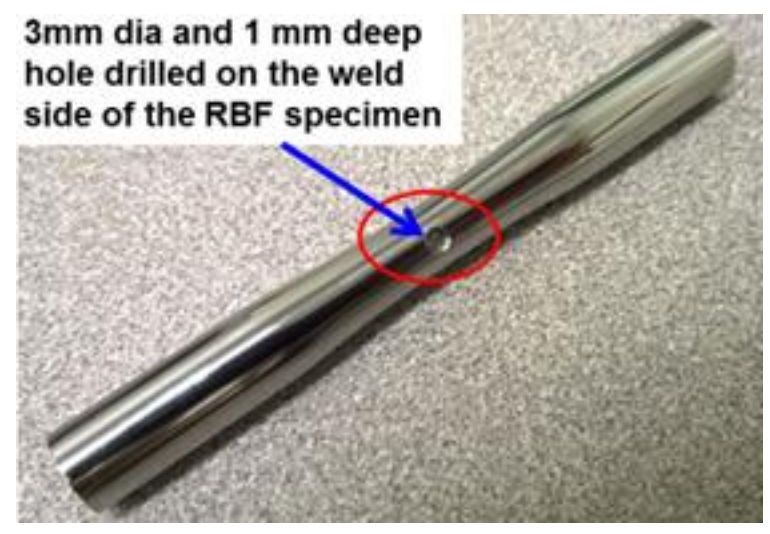

(b)

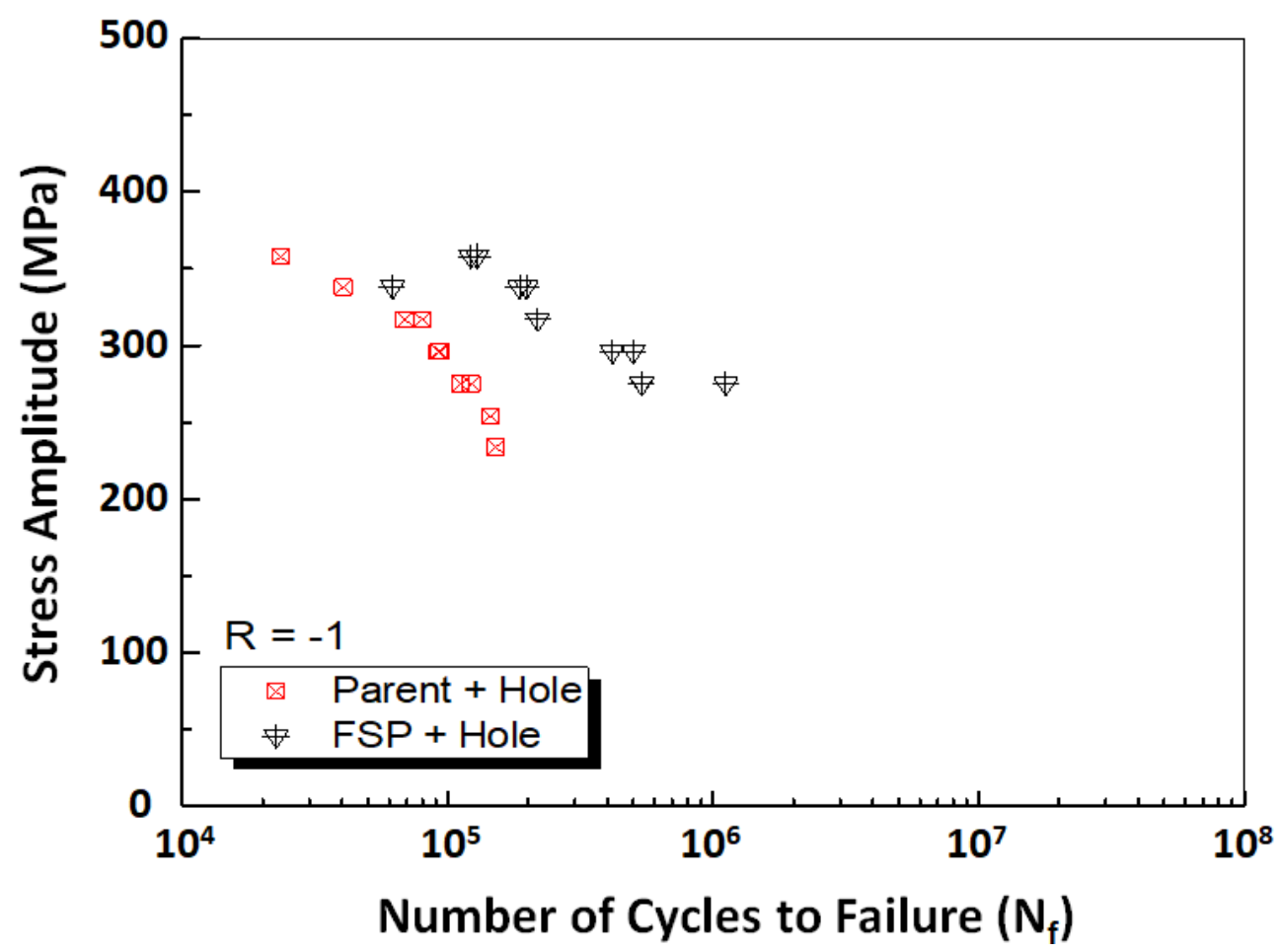

(c)

Figure I.3.2.1.5.2. FSP can be used to mitigate stress concentrations around: (a) oil holes in crankshaft steels; and (b) a rotating beam fatigue (RBF) test specimen by improving local fatigue properties. (c) Fatigue test results showing improved performance using FSP. Source: PNNL.

\section{Objectives}

The project will investigate and demonstrate advanced processes, such as FSP, CS, and others, which have the potential to produce enhanced properties on local areas of complex propulsion material castings, forgings, or additively manufactured materials. In some cases, it is expected that local secondary processing can enable lower-cost ferrous and non-ferrous alloys to achieve properties that meet or exceed the current state-of-the-art advanced materials. Additionally, local processing of next-generation materials may be able to push these materials to extraordinary properties. 


\section{Approach}

FY 2019 and FY 2020 tasks will demonstrate FSP as a process capable of improving the microstructure and performance of $\mathrm{Al}$ materials in blocks, cylinder heads, and pistons. These tasks focus on improving the local microstructure and performance of areas within a casting that will undergo drilling and threading operations to receive threaded fasteners. This work will demonstrate the concept of improving the microstructure, healing potential casting defects near cast-in holes, and provide data on the pullout strength of fasteners threaded in these holes.

The concept is to process the region where a threaded hole will be drilled and tapped, such that the microstructure created by FSP provides greater reliability for the later fastening operation. The requirement for casting integrity around fastener areas can be a driver of cost-forcing complex die design, and higher cost alloys. A secondary operation, such as FSP, just prior to drilling and tapping may be able to allow either lowercost alloys, lower-cost die design, or allow new alloys without as much consideration given to alloy fluidity and casting integrity around areas that will be later machined, such as features like threaded holes, or even deck or sealing surfaces. The deliverable of this task will be reports documenting microstructure, mechanical properties, and threaded fastener pullout strength as a function of processing parameters.

Later in FY 2020, the focus will broaden to include the modification of block areas near the top deck sealing surface where local stresses and high-temperature can cause issues with creep and mechanical overload. In addition, several areas on $\mathrm{Al}$ pistons will be considered for selective property improvement. As advanced cast alloys are developed by ORNL and others, components like pistons may require variable microstructures and local properties to optimize performance. For example, local properties of areas such as piston bowl rims or top ring lands may require additional alloy homogenization or microstructure optimization in order to achieve higher properties than the bulk material in order to avoid premature failure due to fatigue or local softening. In another example, it is anticipated that FSP may be a high value-added processing method for local areas of parts made by conventional casting/forging methods, or even advanced techniques like laser-AM to improve a common shortcoming of these materials with respect to anisotropic fracture toughness and fatigue strength. We anticipate the task will be highly coordinated with ORNL, resulting in the development and delivery of new materials that are hybrids of several advanced manufacturing processes. In addition to the $\mathrm{Al}$ alloy focus, the project during FY 2020 and FY 2021 will also focus on high-temperature materials.

In out years, the project will focus on the notion of using selective processing to create local compositional gradients or local alloying by CS and FSP. These two solid-phase alloying processes are currently being investigated in programs at PNNL as a way to produce local alloy chemistries and functionally graded materials. We propose to leverage this work and develop multi-material structures, different from coatings, that can produce high-performance local regions on potentially lower-cost bulk substrates. High peak cylinder pressure piston tops on lower-cost $\mathrm{Al}$ pistons are one example of the concept.

\section{Results}

The project started in mid-FY 2019 and focused primarily on obtaining Al materials on which to focus the FSP trials. The project is collaborating with General Motors Research and Development (GMR\&D) who are providing expertise and materials. GMR\&D has provided flat plate castings of a strontium-modified A356 alloy for use as base materials. These book mold castings have small secondary dendrite arm spacing and are representative of the kinds of casting microstructures seen in production cylinder heads. Twenty-five plates were received, approximately $20 \mathrm{in}$. long x 6 in. wide x 1 in. thick.

Also, during the second half of FY 2019, work began on modifying a test rig to test the pullout strength of a threaded fastener. The rig shown in Figure I.3.2.1.5.3 uses an RS Technologies Torque-tension measuring system with an Altas-Copco Nutrunner electric-drive torque wrench mounted in a frame that captures a small section of $\mathrm{Al}$ casting in which there is a threaded hole. A bolt is inserted in the threaded hole and the instrumented torque wrench records the torque required to either fail the fastener or the threads in the casting. 
The frame also incorporates a load cell so that the clamp force can also be recorded. This device can be used to quantify the effects of casting defects near threaded holes and be used to quantify the advantage of producing a friction processed region in the area that will be threaded for a fastener.

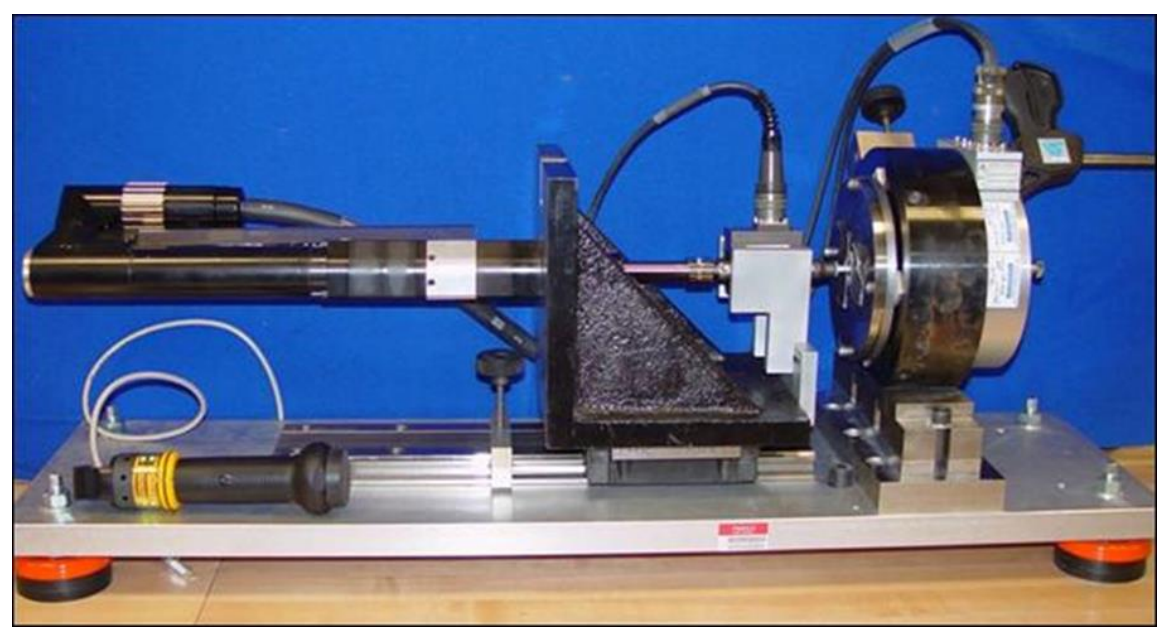

Figure I.3.2.1.5.3. Torque-tension measuring rig used to quantify the thread pullout performance of a FSP region. Source: PNNL.

Leading up to and during FY 2019, we received additional materials to test a concept shown in an earlier program where FSP can be used to improve the fatigue properties of crankshafts. We have received from GMR\&D ten crankshafts both machined and as-forged to use in FSP trials as can be seen in Figure I.3.2.1.5.4. This work anticipated to begin in FY 2020 will involve processing the area around oil holes (and possibly some fillets) on the as-forged piece then returning the cranks to GM for finish machining and testing.

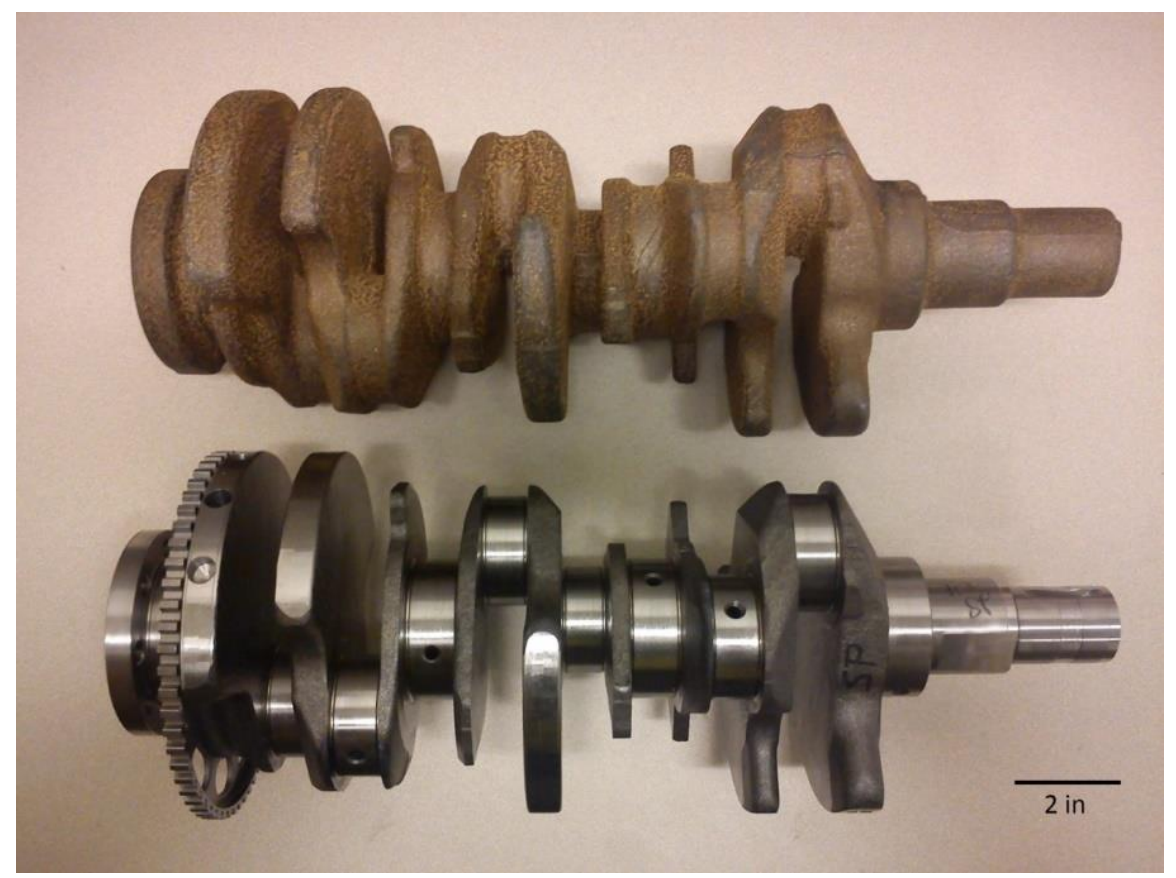

Figure I.3.2.1.5.4. Ten cranks were received from GMR\&D for processing trials. The top crank is as-forged and bottom crank is as-machined. Source: PNNL. 


\section{Conclusions}

During FY 2019 project work focused on obtaining both Al and steel materials for selective processing and building/modifying test equipment for measuring threaded fastener pullout strength. FSP work will begin in the first quarter of FY 2020. Table I.3.2.1.5.1 outlines applications potentially targeted for this project. FY 2019 and FY 2020 material and application focus areas are highlighted with an asterisk.

Table I.3.2.1.5.1. Targeted Applications of Selective Processing.

\begin{tabular}{|c|c|c|c|}
\hline Application & Technical Barrier & Material & Process Method \\
\hline *Engine Block & $\begin{array}{l}\text { Cast microstructural } \\
\text { defects. Head bolt thread } \\
\text { failure/pullout. }\end{array}$ & $\begin{array}{l}\text { Al } \\
\text { (also applicable to Mg) }\end{array}$ & - Selective FSP \\
\hline *Engine Block & $\begin{array}{l}\text { Deck surface near } \\
\text { cylinder bore, } \\
\text { strength/creep at high- } \\
\text { temperature }\end{array}$ & Al & $\begin{array}{l}\text { - FSP, and selective } \\
\text { alloying through FSP }\end{array}$ \\
\hline *Piston & $\begin{array}{l}\text { Cast or forged piston and } \\
\text { ring failures due to over- } \\
\text { loading/heating } \\
\text { associated with advanced } \\
\text { combustion schemes. }\end{array}$ & $\begin{array}{l}\text { Al } \\
\text { Al MMC }\end{array}$ & $\begin{array}{l}\text { - Selective FSP } \\
\text { - Selective friction additive }\end{array}$ \\
\hline Crankshafts & $\begin{array}{l}\text { Fatigue limit failures in } \\
\text { fillet areas and oiling } \\
\text { holes. }\end{array}$ & Steel, cast and forged & - Selective FSP \\
\hline $\begin{array}{l}\text { Shafts (Cams and } \\
\text { Counter-Shafts) }\end{array}$ & $\begin{array}{l}\text { Flexural fatigue and } \\
\text { surface hardness failures. }\end{array}$ & Steel, cast & $\begin{array}{l}\text { - Selective FSP } \\
\text { - CS }\end{array}$ \\
\hline $\begin{array}{l}\text { Valves and Valve } \\
\text { Seat/Bridge Area }\end{array}$ & $\begin{array}{l}\text { Low oxidation resistance } \\
\text { and premature failure } \\
\text { when operated at high- } \\
\text { pressure/temps. }\end{array}$ & Steel & - CS and FSP \\
\hline
\end{tabular}

\section{References}

1. Jana, S., R. S. Mishra, J. B. Baumann, and G. J. Grant, 2010, "Effect of FSP on fatigue behavior of an investment cast Al-7Si-0.6 Mg alloy,” Acta Mater., Vol. 58, No. 3, pp. 989-1003.

2. Jana, S., R. S. Mishra, and G. J. Grant, 2016. Friction stir casting modification for enhanced structural efficiency: A volume in the friction stir welding and processing book series, Butterworth-Heinemann (Elsevier), Oxford, England. ISBN: 9780128033593. 


\title{
I.3.3 Additive Manufacturing - Thrust 3 (Oak Ridge National Laboratory) \\ I.3.3.1 Additive Manufacturing of Powertrain Alloys (3A)
}

\section{Sebastien Dryepondt, Co-Principal Investigator}

Oak Ridge National Laboratory

1 Bethel Valley Road

Oak Ridge, TN 37831

E-mail: dryepondtsn@ornl.gov

\section{Ryan Dehoff, Co-Principal Investigator}

Oak Ridge National Laboratory

1 Bethel Valley Road

Oak Ridge, TN 37831

E-mail: dehoffrr@ornl.gov

\author{
Jerry L. Gibbs, DOE Technology Development Manager \\ U.S. Department of Energy \\ E-mail: jerry.gibbs@ee.doe.gov
}

Start Date: October 1, 2018

Project Funding (FY19): \$1,050,000

End Date: September 30, 2023

DOE share: $\$ 1,050,000$

Non-DOE share: $\$ 0$

\section{Overall Project Introduction}

Automotive companies are looking to apply the immense interest and potential for AM to further advance powertrain applications. AM offers unprecedented possibilities to fabricate unique and complex near-net-shape components, leading to significant savings, such as decreasing tooling and materials costs, accelerating prototype development, offering unique properties, or increasing system efficiency through advanced designs not achievable via conventional fabrication processes.

In-depth understanding of the relationship between microstructure and processing parameters could result in unique microstructures and enhanced or geometrically tailored properties for a wide range of powertrain materials and components. The goal of the subtasks in Thrust 3 (all of which are low to mid-TRL) is to address the specific challenges and opportunities associated with AM development for future LD and HD engine components assessing key factors, such as cost, influence of feedstock characteristics, volatilization of species, faster cooling rates, non-equilibrium phases, materials properties, non-uniform geometry-dependent thermal history, thermal cycling, and more for existing and new materials via both modeling and experimental studies. Alloy development and printing were performed in conjunction with Advanced Manufacturing Office capabilities and activities at ORNL, leveraging existing expertise in 3D printing and materials development. 


\section{I.3.3.1.1 Fundamental Development of Lightweight Alloys for Additive Manufacturing (3A1)}

\section{Amit Shyam, Co-Principal Investigator}

Oak Ridge National Laboratory

1 Bethel Valley Rd.

Oak Ridge, TN 37831

E-mail: shyama@ornl.gov

\section{Alex Plotkowski, Co-Principal Investigator}

Oak Ridge National Laboratory

1 Bethel Valley Rd.

Oak Ridge, TN 37831

E-mail: plotkowskiaj@ornl.gov

\section{Jerry L. Gibbs, DOE Technology Development Manager}

U.S. Department of Energy

E-mail: jerry.gibbs@ee.doe.gov

Start Date: October 1, 2018

Project Funding (FY19): \$250,000
End Date: September 30, 2019

DOE share: $\$ 250,000 \quad$ Non-DOE share: $\$ 0$

\section{Project Introduction}

$\mathrm{Al}$ alloys with improved specific strength are an obvious starting point for applying AM to lightweight engine applications. The primary focus in this task is employing higher temperature $\mathrm{Al}$ alloys in the following families: (1) precipitation-hardened alloys (e.g., ACMZ-type alloys), (2) dispersion-hardened alloys (e.g., $\mathrm{AlCeMn}$ ) and (3) combining the two concepts (e.g., ACMZ + Ce). The primary applications targeted for these alloys are powertrain components, such as cylinder heads, pistons, turbochargers, etc. Pistons with a reduced mass are a specific target application for AM in internal combustion engines, along with turbocharged engine components for higher power density.

\section{Objectives}

This subtask focuses on a fundamental understanding of the features that simultaneously impart printability and thermal stability to $\mathrm{Al}$ alloy microstructures. Subtask 3A1 is a low technology readiness level subtask that has the general technical target of improving upon the properties of current powertrain cast or wrought lightweight alloys. Specific targets, for example, for piston applications are measurable improvements (> 25\%) in alloy mechanical properties in the temperature range of interest.

\section{Approach}

The approach relies on an interplay of characterization and theoretical calculations of alloys of interest to realize the materials design strategy associated with high-temperature-capable rapid solidification processing (RSP) Al alloys [1,2]. It is noted that RSP leads to: (1) new phases with improved thermal stability; and (2) higher solubility of critical alloying elements, such as $\mathrm{Ce}, \mathrm{Mn}, \mathrm{Zr}$, etc. [3], which can both be favorable for elevated temperature powertrain applications. Traditional RSP parts are extremely expensive to manufacture, compared to conventional alloy processing, creating another opportunity for AM. The technical challenges, however, include the fact that RSP alloys typically have low fracture toughness and ductility at lower loading rates at elevated temperature. These challenges will need to be understood and addressed to help commercialize a new generation of additive $\mathrm{Al}$ alloys. 
The sequence of steps associated with the development of new additive $\mathrm{Al}$ alloys are as follows: (1) an assessment of the printability and possible strengthening mechanisms associated with an alloy chemistry is performed; (2) the specific alloy powders are synthesized or procured; (3) the alloy powder is printed by selective laser melting (SLM) processing, while the process parameters are optimized to reduce defect formation and improve the alloy mechanical properties; (4) the modeling of the relevant microstructural and mechanical properties is performed; and (5) the first four steps are repeated in a feedback loop to improve alloy chemistry and heat-treatment.

\section{Results}

\section{Influence of Alloying Additions on Hardness of AlCuCe Alloys}

Several $\mathrm{AlCuCe}$ based alloys were additively manufactured in budget period (BP) 1 - one alloy in the ternary system (e.g., Al-9Cu-6Ce), one with a 1 wt. $\% \mathrm{Zr}$ addition, and one with both $\mathrm{Zr}$ and $\mathrm{Mn}(0.5 \mathrm{wt} . \%)$ additions. The as-fabricated microstructure of these alloys was characterized, showing an extremely fine intermetallic structure. The RT hardness of the alloys was measured following progressive thermal exposure at $350^{\circ} \mathrm{C}$, as shown in Figure I.3.3.1.1.1 (a). The hardness of the alloys increases sharply for short thermal exposures, suggesting successful precipitation hardening, and then remains stable, within the measurement variability, out to $200 \mathrm{~h}$. The addition of $\mathrm{Mn}$ resulted in an approximate 15 Vickers hardness number increase, likely due to the effects of solid-solution strengthening in the $\mathrm{Al}$ matrix. Micrographs comparing the structures of both alloys are shown in Figure I.3.3.1.1.1 (b). Example piston geometries were printed using these alloys as a demonstration, also as shown in Figure I.3.3.1.1.1 (a). In particular, the AlCeCuZr alloy showed excellent printability and as-fabricated surface finish. Mechanical testing results are pending.

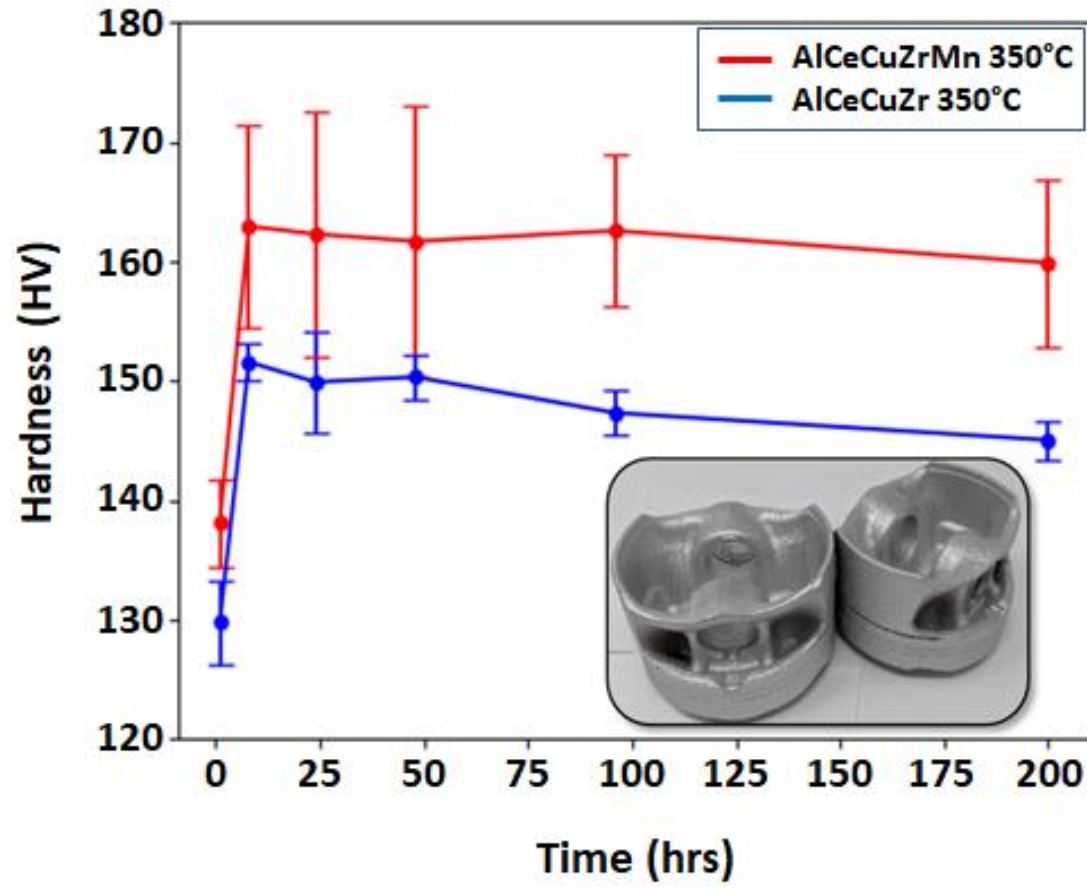

(a)
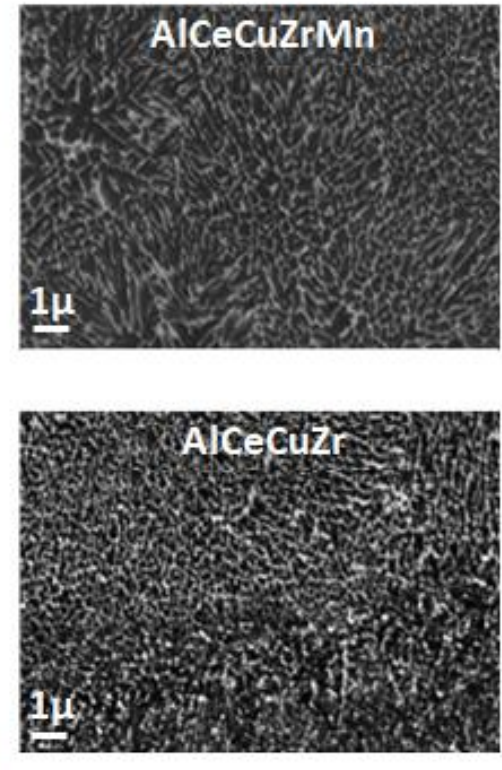

(b)

Figure I.3.3.1.1.1. (a) RT hardness of two Al-Ce-Cu-based alloys as a function of prior thermal exposure time at $350^{\circ} \mathrm{C}$ showing that the addition of small amounts of $\mathrm{Mn}$ has a significant increase for the hardness of the AICeCuZrMn alloy due to additional solid-solution strengthening. (b) Micrographs of the alloy structures. Source: ORNL. 


\section{AM of an ACMZ Alloy}

An ACMZ alloy was successfully additively manufactured through SLM. The as-fabricated structure exhibited some porosity and micro-cracking (likely hot-tears), but only for certain geometries. Tensile coupons appear to be crack-free. Characterization of the microstructure and a heat-treatment design of experiments study have been initiated. Mechanical testing for this alloy composition is planned for early FY 2020.

\section{Thermal Stability of AICe-based Alloys for Piston Applications}

Mechanical properties of additively manufactured AlCeMn and AlCeNi alloys have been measured at elevated temperatures and compared to common $\mathrm{Al}$ alloys used for $\mathrm{AM}$, as well as to forged alloy 2618, which is an alloy commonly used for high-performance Al pistons. Figure I.3.3.1.1.2 (a) shows the ultimate tensile strength (UTS) of these alloys as a function of temperature after $30 \mathrm{~min}$. exposure time. Both alloys exceed $\mathrm{AlSi} 10 \mathrm{Mg}$ and Scalmalloy which are two commercial Al alloys commonly used for AM.

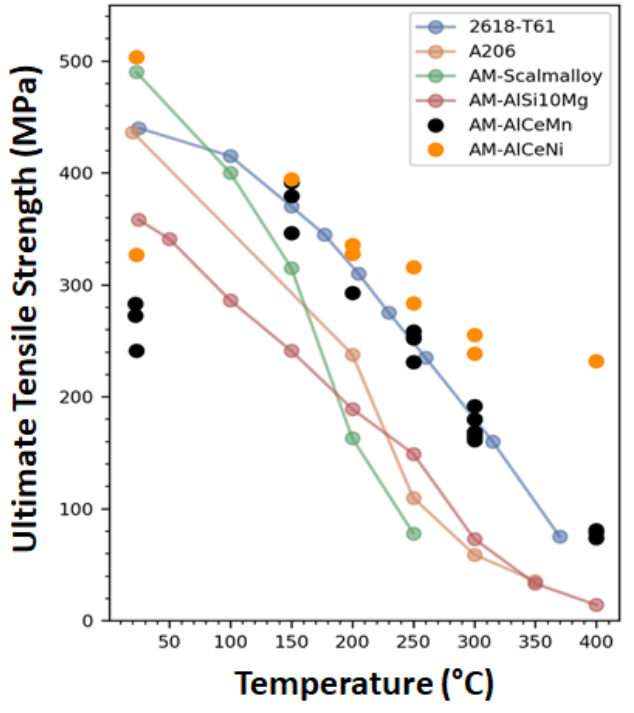

(a)

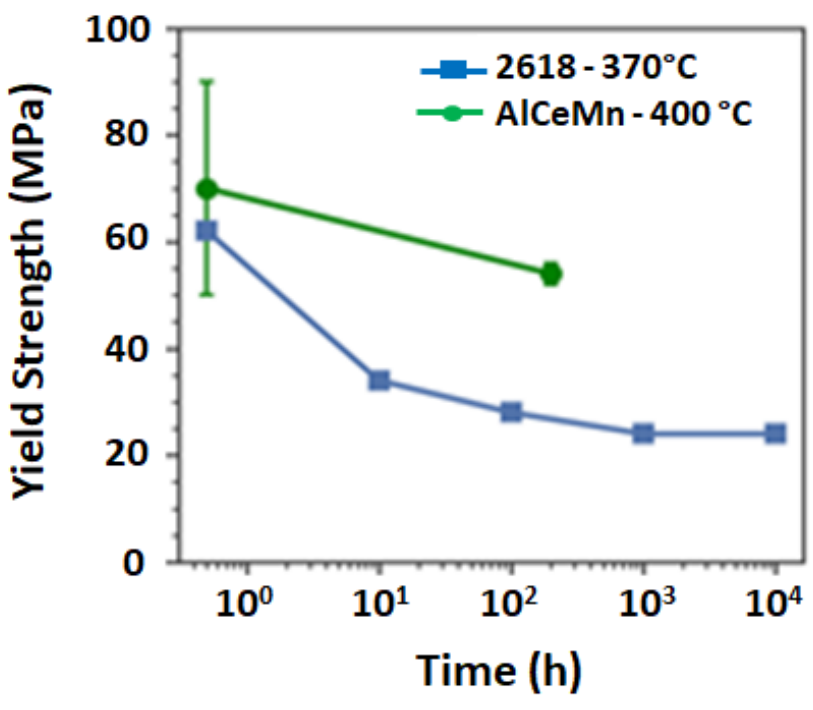

(b)

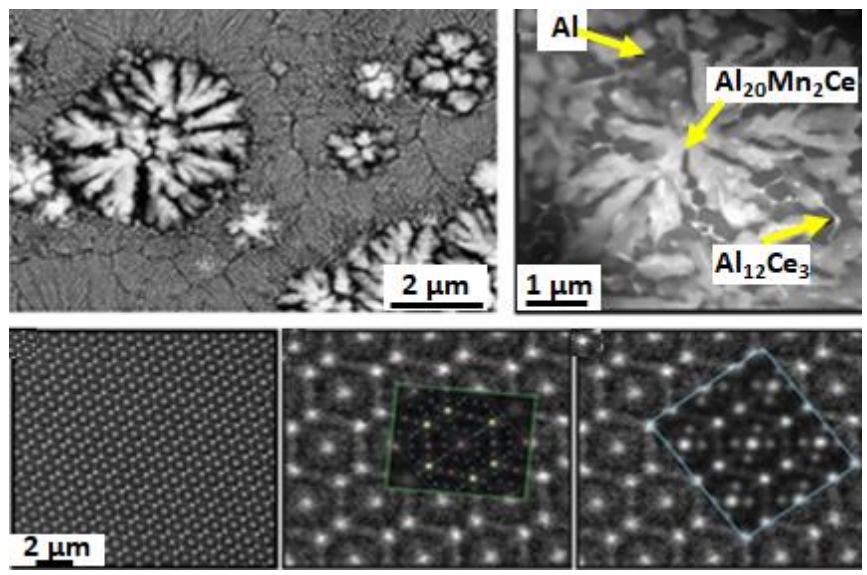

(c)

Figure I.3.3.1.1.2. (a) UTS for the AICeMn and AICeNi AM alloys following 30 min. exposure time as compared to similar exposure times for other Al alloys. (b) Yield strength of the AICeMn alloy as a function of time compared to 2618 showing much greater thermal stability for the AICeMn alloy. (c) Micrographs used for advanced characterization to identify the phase structure and strengthening mechanisms that enable the excellent high-temperature performance of the AICeMn alloy. Source: ORNL. 
The performance of the AlCeMn alloy is similar to 2618 as shown in Figure I.3.3.1.1.2 (b), although it lacks RT toughness. However, at longer exposure times, the thermal stability of the AlCeMn alloy is much greater than 2618. Advanced characterization (provided by Thrust 4 of the Core Program) images shown in Figure I.3.3.1.1.2 (c) has helped to understand the non-equilibrium microstructure evolution of the alloy during both the AM process and during thermal exposure. The AlCeNi alloy shows excellent strength at temperatures above $250^{\circ} \mathrm{C}$ compared to 2618 , even up to $400^{\circ} \mathrm{C}$. There is remarkable potential for these alloy concepts to enable future advanced piston designs for $\mathrm{LD}$ and $\mathrm{MD}$ engines.

\section{Conclusions}

We have identified a compositional space where alloys previously shown to have good elevated temperature properties under RSP conditions can be additively manufactured. This development allows the refinement of the compositions of the AlCeMn alloys and further development of other compositions with attractive elevated temperature properties. The temperature range over which the AlCeMn and $\mathrm{AlCeNi}$ alloys have improved tensile properties makes them an attractive alternative to conventional alloys for piston and other powertrain applications. AlCuCe alloys exhibit excellent printability and resistance to hot-tearing and appear to be precipitation-resistant, thereby achieving a hardness greater than cast $\mathrm{Al}-\mathrm{Cu}$ alloys.

\section{Key Publications}

1. Plotkowski, A., Y. Yang, K. Sisco, A. Shyam, P. Nandwana, L. Allard, S. Bahl, A. Rossey, and R. R. Dehoff, 2019, "Microstructure and properties of a high-temperature AlCeMn Alloy produced by AM," submitted to Acta Mater.

\section{References}

1. Gordillo, M. A., I. Cernatescu, T. T. Aindow, T. J. Watson, and M. Aindow, 2014, "Phase stability in a powder-processed Al-Mn-Ce alloy,” J. Mater. Sci., Vol. 49, No. 10, pp. 3742-3754.

2. Coury, F. G., W. J. Botta, C. Bolfarini, C. S. Kiminami, and M. J. Kaufman, 2015, "Reassessment of the effects of Ce on quasicrystal formation and microstructural evolution in rapidly solidified Al-Mn alloys," Acta Mater., Vol. 98, pp. 221-228.

3. Croteau, J. R., S. Griffiths, M. D. Rossell, C. Leinenbach, C. Kenel, V. Jansen, D. N. Seidman, D. C. Dunand, and N. Q. Vo, 2018, "Microstructure and mechanical properties of Al-Mg-Zr alloys processed by SLM," Acta Mater., Vol. 153, pp. 35-44.

\section{Acknowledgements}

The authors would like to thank K. Sisco, S. Bahl, D. McClurg, L. Allard, Y. Yang, and A. Marquez for their contributions to this research. 


\section{I.3.3.1.2 Hybrid Manufacturing of Additive Manufactured Interpenetrating Phase Composites (3A2)}

\section{Derek Splitter, Co-Principal Investigator}

Oak Ridge National Laboratory

1 Bethel Valley Rd.

Oak Ridge, TN 37831

E-mail: splitterda@ornl.gov

\section{Amit Shyam, Co-Principal Investigator}

Oak Ridge National Laboratory

1 Bethel Valley Rd.

Oak Ridge, TN 37831

E-mail: shyama@ornl.gov

\section{Jerry L. Gibbs, DOE Technology Manager}

U.S. Department of Energy

E-mail: jerry.gibbs@ee.doe.gov

Start Date: October 1, 2019

Project Funding (FY18): \$250,000
End Date: September 30, 2022

DOE share: $\$ 250,000$
Non-DOE share: $\$ 0$

\section{Project Introduction}

This subtask aims to understand how the combination of AM with casting (termed PrintCasting here) can be used to increase the energy absorption and prevent failures of the ringland area of automotive pistons without significantly affecting overall piston weight or thermal conductivity. A key advantage of PrintCasting is that it leverages AM control over the mesostructure. We can therefore precisely control the geometry and spatial distribution of the constituents to create composites with geometries that realize improved energy absorbing behavior. High levels of energy absorption can be achieved by creating a reinforcement geometry that converts the applied tensile load into local compression of the more brittle matrix. That is, we pattern the reinforcement geometry with a topology that is dominated by bending, which upon axial loading collapses, thus compressing the cast $\mathrm{A} 356 \mathrm{Al}$ matrix trapped in between the additive skeletal structure.

\section{Objectives}

This project will create PrintCast-metal-metal composites with high-energy absorption. We are interested in energy absorption under tensile loading for applications including impulse loading, blast containment, and load-bearing structures, which are conditions relevant to automotive pistons in boosted spark-ignited engines that are prone to knock/pre-ignition. We plan to understand the geometric factors, bonding, material selection, and processing aspects to develop a multi-material hybrid material system that is applicable to high-demand applications.

\section{Approach}

A powerful feature of metal AM is the ability to directly print net-shaped composites with high levels of architectural complexity. In general, local control over composition is achieved by changing the feedstock composition during printing. However, rapid mixing of the constituents inside the melt pool can lead to the formation of undesirable brittle intermetallic compounds making it challenging to print metallic composites using local variations in feedstock chemistry. As a proof-of-concept, this project fabricates composites with a skeletal structure of additively manufactured internal meshes of 316L austenitic stainless steel (or Ti-6Al-4V) 
overcast and infiltrated by a matrix of an $\mathrm{Al}$ alloy (alloy A356 in this study), which has a much lower melting point than the mesh material. The A356 alloys experience brittle failure under tension, but undergo very large plastic deformation under compression, whereas the AM 316L or Ti64 internal reinforcement can undergo very high-strength and tensile ductility.

However, the A356/316L or Ti64/Al material system is known to form brittle Fe-Al or Ti-Al intermetallic compounds when joined together by conventional welding processes. In this subtask, composites with different 316L/Ti64 reinforcement contents were manufactured by the PrintCasting technique. This novel hybrid processing strategy, combining AM with casting to eliminate liquid-phase mixing and overcome intermetallic formation, is highlighted in Figure I.3.3.1.2.1. The model interior mesh and external walls are (1) printed using a SLM process, (2) filled with lower melting point liquid-metal using centrifugal casting, and (3) postprocessed to optimize the properties.

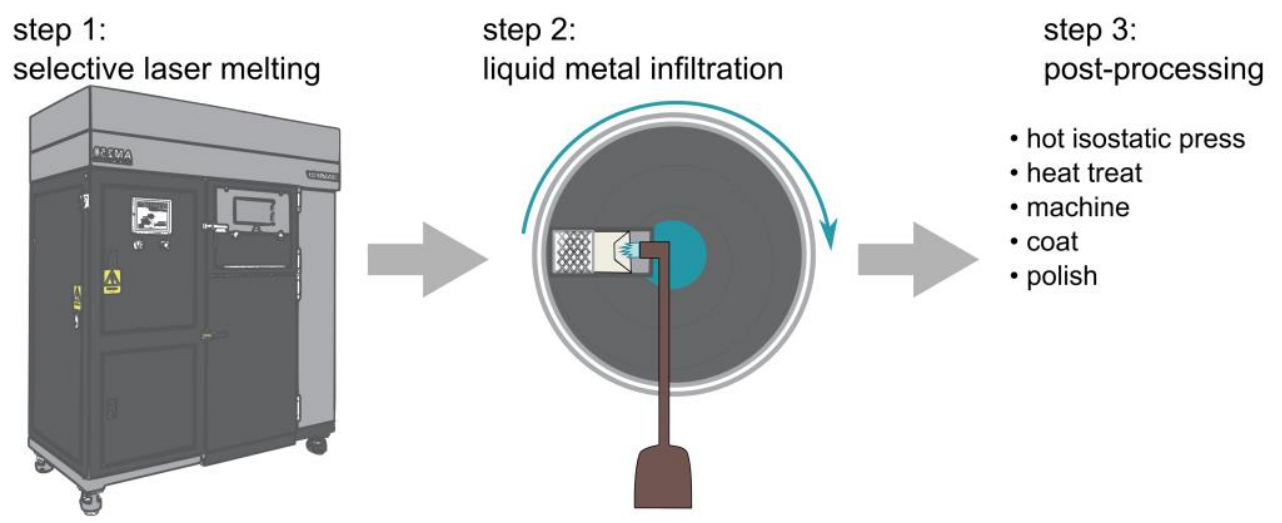

Figure I.3.3.1.2.1. Process for synthesizing interpenetrating phase composites using the PrintCasting technique. Source: ORNL.

The resulting part is an interpenetrating phase composite in which constituents form continuous and interconnected networks. It has been shown that an interpenetrating morphology can enable unique mechanical and thermal properties in a nonlinear blending manner from each of the constituents to the overall behavior of the composite. More specifically, it has been shown that the fracture toughness can be increased by interconnecting the more ductile phase inside a brittle/ductile matrix, which helps improve energy absorption.

\section{Results}

Progress included preform fabrication. The build plate of six different lattice geometries of PrintCast preforms fabricated by SLM using 316L steel (see Step 1 in Figure I.3.3.1.2.1) prior to infiltration are shown in Figure I.3.3.1.2.2. The 316L stainless steel AM produced preforms were then infiltrated with A356 Al via vacuum casting. In previous preliminary work in the VTO Materials Program, the infiltration technique used was centrifugal casting, which was found to infiltrate, but significant interfacial gaps between the two constituents were observed. However, the preliminary findings of vacuum infiltration A356 appear to have significantly reduced the gaps resulting from the centrifugal casting approach. 


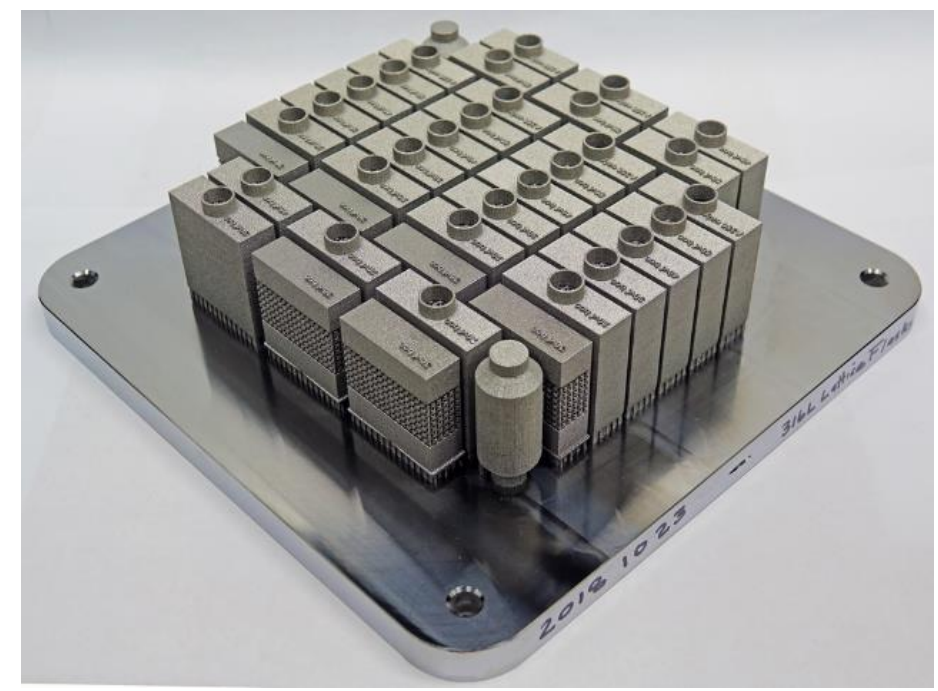

Figure I.3.3.1.2.2. Build plate of six different lattice geometries of PrintCast preforms fabricated by SLM using 316L steel prior to infiltration. Source: ORNL.

Along with the fabrication of new composites for testing, results in FY 2019 have consisted of significant data analysis and simulation efforts of previous data. The simulations are being used to not only understand the experimental results, but also to understand where design challenges and opportunities exist so that the next generations of metal-metal composite systems can be developed. Significant effort has been focused on understanding neutron diffraction data that was collected with 316L-A356 specimens in tension. Specifically, neutron diffraction results showed that, in tension, the reinforcement substructure of $316 \mathrm{~L}$ stainless steel bears much of the tensile loading, but it is supported by compression in the matrix material, A356 Al. This finding is significant as it highlights the unique material loading state that the additively manufactured interpenetrating phase composite system affords. Figure I.3.3.1.2.3 highlights our key findings, where asymmetry in the stressstrain behavior was found. Specifically, the reduced volume fraction of $316 \mathrm{~L}$ had significantly reduced strain to failures.

Figure I.3.3.1.2.3 (a) provides the experimental and simulated engineering stress-strain curves of PrintCast composites with different $316 \mathrm{~L}$ volume fractions showing good agreement between experimental results and modeling. A low-magnitude optical image of an as-tested 20 vol.\% $316 \mathrm{~L}$ composite showing $316 \mathrm{~L}$ in dark contrast and A356 in bright contrast is presented in Figure I.3.3.1.2.3 (b). In this case, the sample is showing localized damage. On the top of this image, a higher-magnitude cross-section of the simulated tensile specimen showing the Von Mises equivalent stress distribution is observed. At the bottom, the higher-magnitude secondary electron micrograph of the fracture surface showing $316 \mathrm{~L}$ failing at the ligament nodes can be seen. The same layout as observed in Figure I.3.3.1.2.3 (b) but for the as-tested 50 vol.\% 316L composite is shown in Figure I.3.3.1.2.3 (c). The transition from localized-to-delocalized damage is key for high-energy absorption. 


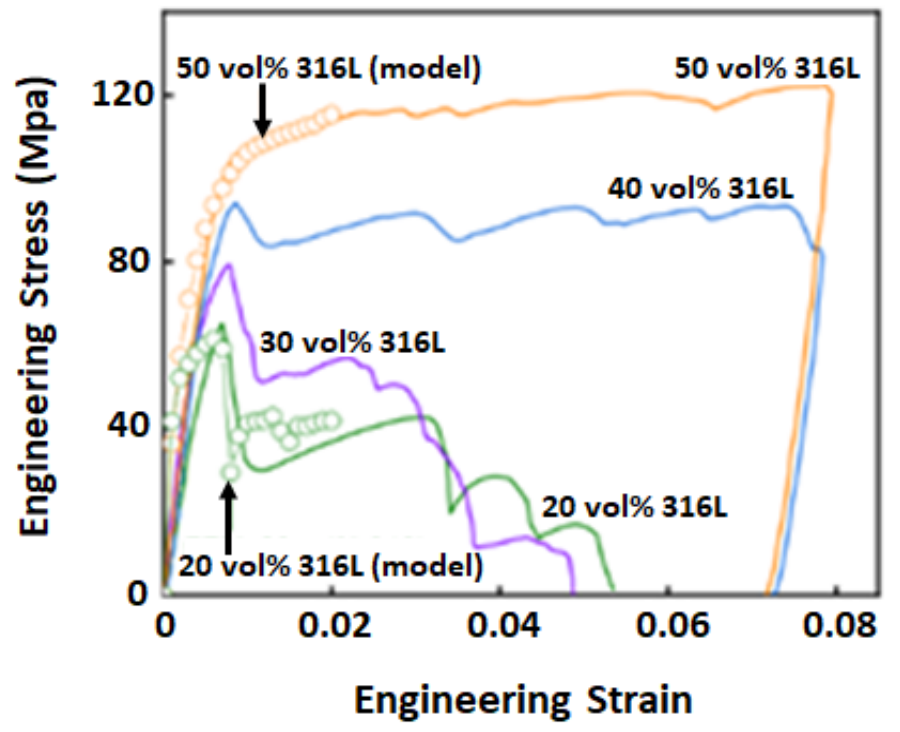

(a)

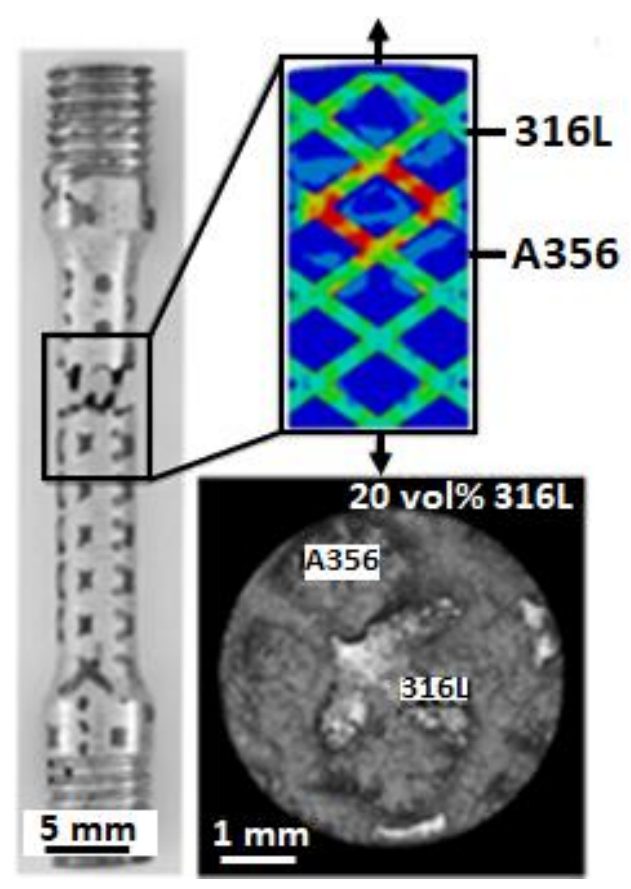

(b)

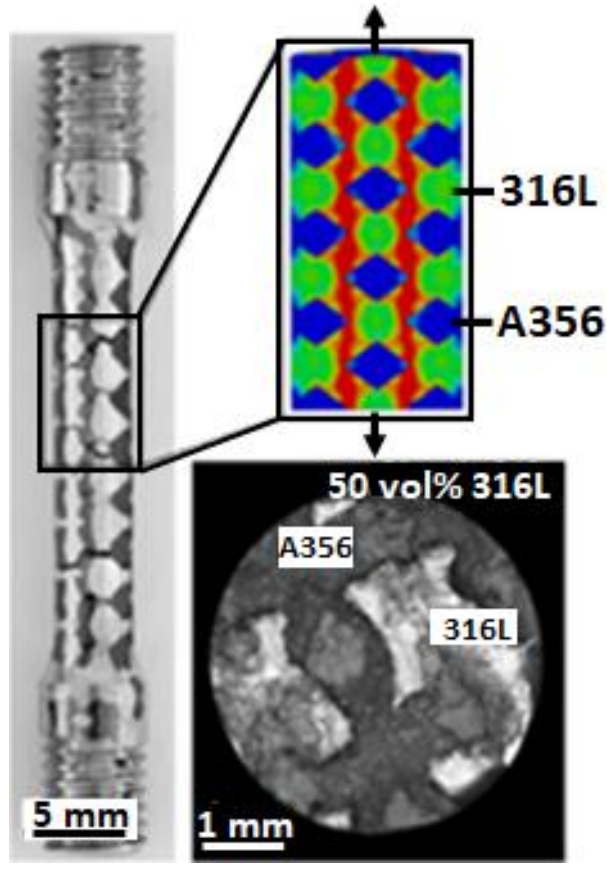

(c)

Figure I.3.3.1.2.3. (a) Experimental and simulated engineering stress-strain curves. (b) A low-magnitude optical image of an as-tested 20 vol.\% 316L composite with an insert showing stress distribution simulation and (c) A low-magnitude optical image of an as-tested 50 vol.\% 316L composite. Source: ORNL.

Using detailed FEA simulation tools coupled with this experimental data, it was found that the transition from localized-to-delocalized damage in Figure I.3.3.1.2.4 (a) (e.g., the ripple patterns in the plastic deformation portion of the data) represents the critical point at which high-energy absorption can be obtained. When damage is delocalized, the composite continues to deform, and considerable work is done by putting the brittle matrix under a state of near hydrostatic compression. 
To demonstrate this point, the averaged hydrostatic stress in both the $316 \mathrm{~L}$ reinforcement and A356 matrix from the measured lattice-strains is computed and the results plotted in Figure I.3.3.1.2.4 (b). The results in Figure I.3.3.1.2.4 (b) illustrate the experimental and simulated averaged hydrostatic stress in both the 316L and A356 as function of the applied engineering strains for the $50 \mathrm{vol} \% 316 \mathrm{~L}$ composite. The state of hydrostatic stress in the A356 is tensile up to $0.25 \%$ engineering strain and compressive thereafter. The $316 \mathrm{~L}$ on the other hand undergoes a state of tensile hydrostatic stress throughout the loading history. When unloaded the residual hydrostatic stresses remain tensile in the 316L reinforcement and compressive in the A356.

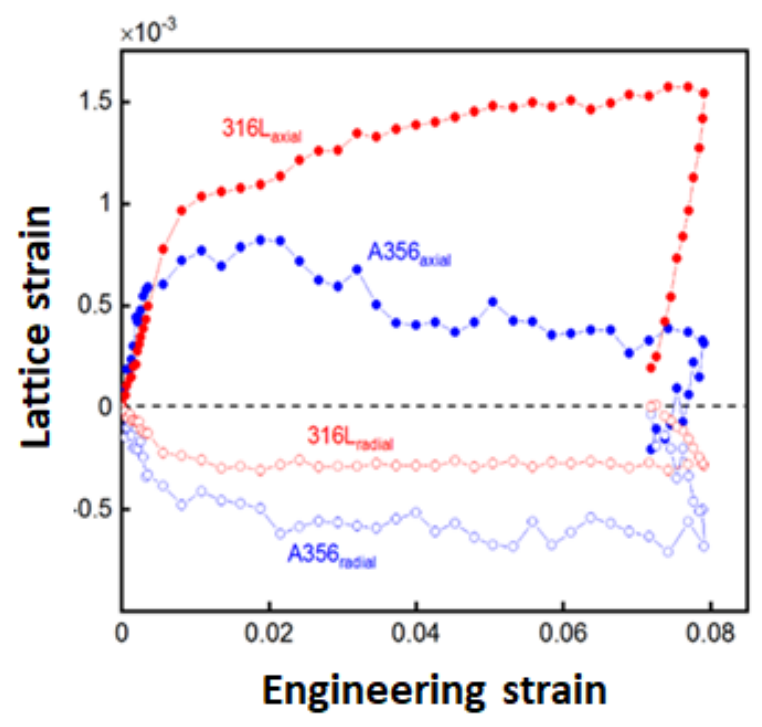

(a)

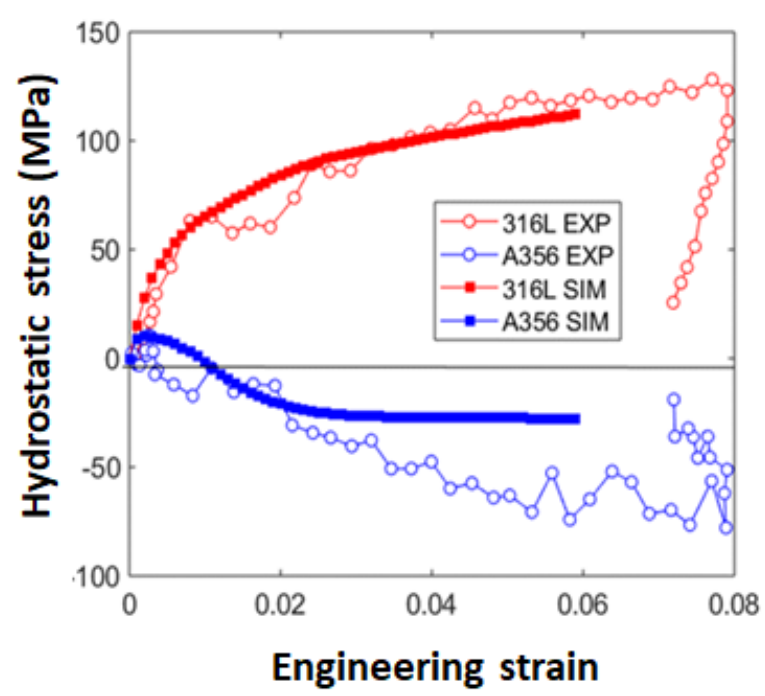

(b)

Figure I.3.3.1.2.4. (a) Averaged lattice-strains measured via in situ neutron diffraction as for 316L reinforcement and A356 matrix in the axial and radial direction to the loading axis used to estimate stress during tensile testing. (b) Experimental and simulated averaged hydrostatic stress for the 316 L reinforcement and the A356 matrix showing tensile stresses for 316L but mainly compressive stresses for A356. Source: ORNL.

In general, A356/316L PrintCast composites were found to effectively convert the applied tensile load into local compression of the brittle A356 matrix. Under compression, the A356 dissipates a large quantity of energy through plastic deformation regardless of its low tensile ductility. The total energy absorbed per unit volume for a 40 vol. $\% 316 \mathrm{~L}$ PrintCast composite is equal to $30 \mathrm{MJ} / \mathrm{m}^{3}$. This value is comparable with the value of chain composites with $44 \mathrm{vol} \% \%$ steel links embedded in an epoxy matrix. It is worth mentioning that damage delocalization for chain composites with an Al alloy matrix having strength like the A356 used in this work was not achieved. Rather, the composite failed in a localized manner. This clearly shows the potential for the PrintCast composite in achieving high levels of energy absorption in metal-metal systems.

A simultaneous effort to understanding lattice parameters and design has been initiated to understand how lattice geometry can affect the localized-to-delocalized stress state behavior. Currently, octahedral designs have been fabricated and infiltrated, as seen in Figure I.3.3.1.2.5 (a). Testing of this geometry is planned soon using digital image correlation, where the results will be coupled to FEA simulation of these lattice and other similar lattices, like fcc lattices, shown in Figure I.3.3.1.2.5 (b). Initial modeling analysis results suggest that reduced reinforcement volumes may be possible with such designs. The coupled approach of simulations and targeted experiments is planned to address the fundamental tradeoffs in the material performance of the PrintCast metal-metal composite system. 


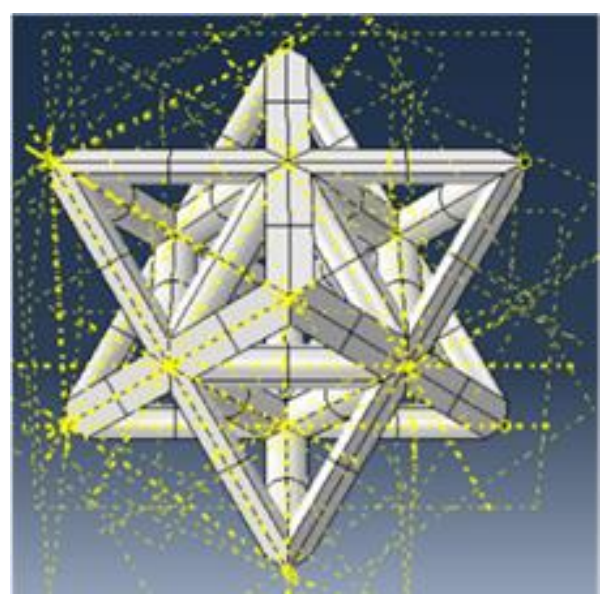

(a)

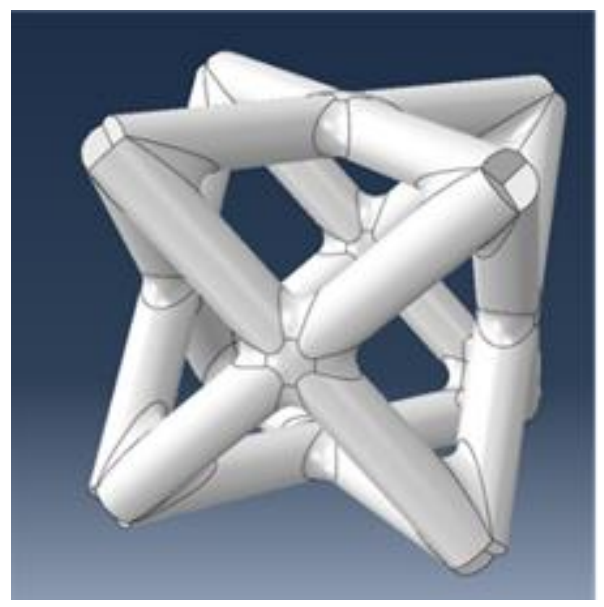

(b)

Figure I.3.3.1.2.5. Second generation of PrintCast for (a) octahedral and (b) fcc reinforcement designs. Source: ORNL.

\section{Conclusions}

In situ Neutron diffraction results showed that, in tension, the reinforcement of 316L stainless steel fabricated by SLM bears much of the tensile loading, but it is supported by compression in the A356 Al cast matrix. The transition from localized-to-delocalized damage was identified as the critical point at which high-energy absorption can be obtained. A coupled approach of simulations and targeted experiments is planned to address the fundamental tradeoffs in the material performance of the PrintCast metal-metal composite system.

\section{Key Publications}

1. Moustafa, A. R., J. Cheng, J. Allen, X. Hu, D. A. Splitter, A. Shyam, and Z. C. Cordero, 2019, "Energy absorption capabilities of PrintCast A356/316L composites," in preparation.

\section{References}

1. Pawlowski, A. E., D. A. Splitter, T. R. Muth, A. Shyam, K. Carver, R. B. Dinwiddie, A. M. Elliott, Z. Cordero, and M. French, 2017. "Producing hybrid metal composites by combining AM and casting," Advanced Materials and Processes, Vol. 175, No. 7, pp. 16-21.

\section{Acknowledgements}

The authors would like to thank J. Cheng, J. Allen, M. Gussev, and A. Moustafa of Rice University for helping with the experimental work. 


\section{I.3.3.1.3 Fundamentals of Austenitic Alloys Processing by Additive Manufacturing (3B1)}

\section{Sebastien Dryepondt, Principal Investigator}

Oak Ridge National Laboratory

1 Bethel Valley Road

Oak Ridge, TN 37831

E-mail: dryepondtsn@ornl.gov

\section{Jerry L. Gibbs, DOE Technology Manager}

U.S. Department of Energy

E-mail: jerry.gibbs@ee.doe.gov

Start Date: October 1, 2019

Project Funding (FY19): \$200,000

End Date: September 30, 2022

DOE share: $\$ 200,000$
Non-DOE share: $\$ 0$

\section{Project Introduction}

There is a growing interest in using AM to design complex high-efficiency, high-temperature combustion engine components. High-temperature cast austenitic steels, such as $\mathrm{HK} 30 \mathrm{Nb}$ or CF8C+, would be needed for components like turbocharger housing, but AM work on austenitic steels has been focused so far on few key alloys such as 304 and $316 \mathrm{~L}$ for low- to mid-range temperature use. For higher temperature applications, more expensive Ni-based alloys are currently being considered. There is, therefore, a need to assess the AM production feasibility of various high-temperature stainless steels, optimize the fabrication parameters for the most promising candidates, and generate the high-temperature data required to determine potential suitability for component operation.

\section{Objectives}

This lower technology readiness level project aims for the characterization of austenitic steels fabricated by AM for powertrain components operating at $600-900^{\circ} \mathrm{C}$. $316 \mathrm{~L}$ will first be studied as a reference for austenitic stainless steel, and then tensile, creep, and oxidation data will be generated at temperatures up to $\sim 650^{\circ} \mathrm{C}$.

Similar AM work will then be conducted on higher temperature austenitic steels, such as HK30Nb or ORNL's CF8C+. Extensive work will be carried out to optimize the AM process parameters to fabricate low-defect specimens with the required microstructure features (e.g., grain size, precipitates, etc.) for high-temperature mechanical strength and oxidation resistance up to $900^{\circ} \mathrm{C}$. Once correlations between the alloy chemistries, the processing parameters, and the resulting microstructures are established, new AM austenitic alloys will be developed. Most of the work will be performed using fusion-based AM techniques, such as SLM, but other emerging techniques like AM binder-jetting will be explored due to their lower-cost.

\section{Approach}

Commercial (e.g., 316L, HK30Nb, etc.) and advanced (e.g., CF8C+, CF8C+W/Cu, etc.) austenitic steels are being fabricated using power-bed fusion techniques. The microstructure stability of the alloys is being assessed by tensile, creep, and oxidation-testing at temperatures up to $900^{\circ} \mathrm{C}$ on as-fabricated and annealed specimens.

An appealing combination of strength and ductility at RT has been reported for 316L steel fabricated by SLM due to unique microstructure features, such as colonies of fine cells, but the stability of such features and their impact on the alloy high-temperature properties need to be evaluated [1]. 
In addition, the very rapid cooling inherent to powder-bed fusion techniques, such as SLM, results in unique microstructures, some of which may lead to superior properties as compared to cast and wrought alloys. To accelerate the selection/development of high-strength AM austenitic steels, experimental and computational tools are needed to reduce the dependence for custom-made powder and AM machine time. Single-track laser beam experiments are being combined with phase equilibrium and solidification path simulations to establish the relationship between alloy composition, process parameters, and resulting microstructure. The impact of various post heat-treatments on the alloy microstructures are being assessed using ORNL's Gleeble System, with a particular focus on the formation of brittle phases or strengthening precipitates. Based on these results, new powder chemistries will be identified to produce high-strength, high-temperature austenitic steels by SLM.

\section{Results}

Figure I.3.3.1.3.1 (a) shows the 316L build fabricated for this study by SLM with a Concept Laser M2 machine using the build parameters recommended by Concept Laser GmbH. Rods and large rectangular plates were fabricated to enable machining of tensile specimens along and perpendicular to the build direction, respectively. Some rods were annealed for $5 \mathrm{~h}$ at $500^{\circ} \mathrm{C}, 600^{\circ} \mathrm{C}$, and $800^{\circ} \mathrm{C}$, respectively, to simulate a stressrelease heat-treatment and assess the material microstructure stability. As can be seen in Figure I.3.3.1.3.1 (b), few defects were observed in the SLM316L rods; most of them are located near the specimen surface. The EBSD images presented in Figure I.3.3.1.3.1 (c) and Figure I.3.3.1.3.1 (d) highlight a "typical" as-fabricated SLM grain structure with elongated grains along the build direction due to the local thermal gradient and solidification conditions. Very similar EBSD maps were observed for the SLM316 material after annealing at $500-800^{\circ} \mathrm{C}$. The same metallographic mounts were etched and BSE-SEM images, shown in Figure I.3.3.1.3.1 (e), revealed, as expected, the presence of colonies of cells with different orientations and size ranging from $\sim 0.5-1.5 \mu \mathrm{m}$. Similar cell structures were observed after annealing for $5 \mathrm{~h}$ at $500^{\circ} \mathrm{C}$ and $600^{\circ} \mathrm{C}$, while Figure I.3.3.1.3.1 (f) shows that the cells were no longer present after annealing for $5 \mathrm{~h}$ at $800^{\circ} \mathrm{C}$.
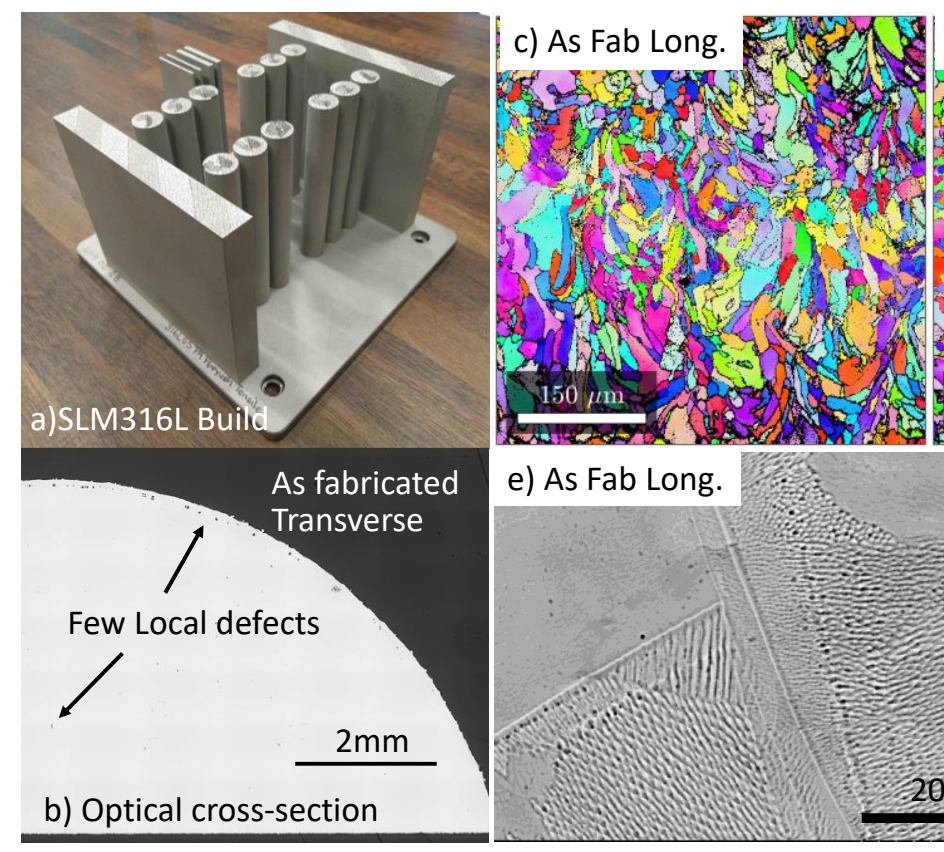

e) As Fab Long.

Figure I.3.3.1.3.1. As-fabricated SLM 316L: (a) build configuration; (b) optical cross-section micrograph of the rod showing very few large defects; (c) EBSD map highlighting elongated grains along the build direction with no preferential orientation;

(d) EBSD map transverse to the build direction; (e) etched BSE-SEM image for the as-fabricated SLM316L and (f) etched BSE-SEM image of SLM316L after annealing for $5 \mathrm{~h}$ at $800^{\circ} \mathrm{C}$. Source: ORNL. 
Figure I.3.3.1.3.2 (a) compares the SLM 316L RT tensile data generated at ORNL in the as-fabricated and annealed conditions with the SLM 316L literature data. Overall, the ORNL SLM 316L material exhibited high ductility and moderate strength, most likely due to the broad range of cell sizes observed, leading to a relatively low dislocation density for SLM materials. It is important to note that most of the literature data published on SLM316L were generated using small-scale specimens, which typically overestimate alloy ductility. ORNL results showed that the ductility of the small-scale SS3 tensile specimens was $\sim 20 \%$ higher as compared to standard-sized specimens. Testing both along and transverse to the build direction also revealed that the yield strength was slightly higher transverse to the build direction.

As expected, a progressive decrease of the yield strength and UTS was observed when temperature was increased from $20-700^{\circ} \mathrm{C}$. A significant decrease in ductility was also observed when testing at temperature $>200^{\circ} \mathrm{C}$, which is consistent with the results observed on wrought $316 \mathrm{~L}(\mathrm{~N})$. This reduction in ductility is due to a change of deformation microstructure, from twins and stacking faults at RT to dislocations at hightemperature [12]. In addition, Figure I.3.3.1.3.2 (b), Figure I.3.3.1.3.2 (c), and Figure I.3.3.1.3.2 (d) show that annealing for $5 \mathrm{~h}$ at $500-800^{\circ} \mathrm{C}$ resulted in a decrease of the yield strength and an increase of the elongation at rupture at all testing temperature, with the most significant changes being observed after annealing for $5 \mathrm{~h}$ at $800^{\circ} \mathrm{C}$

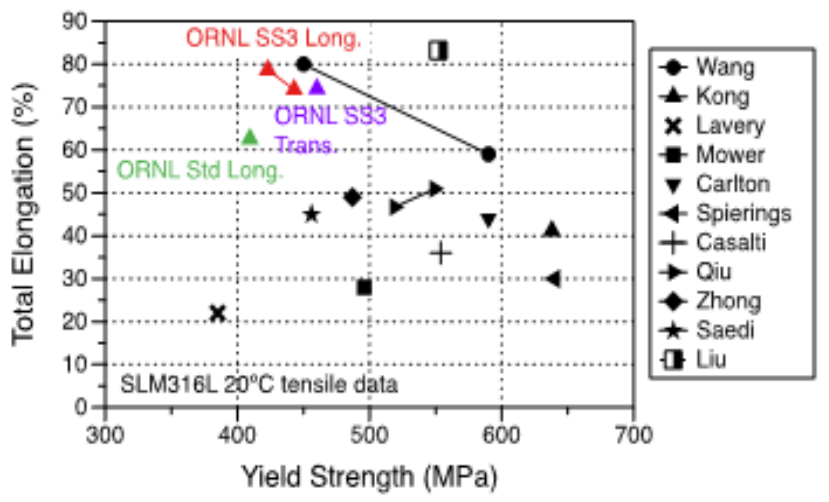

(a)

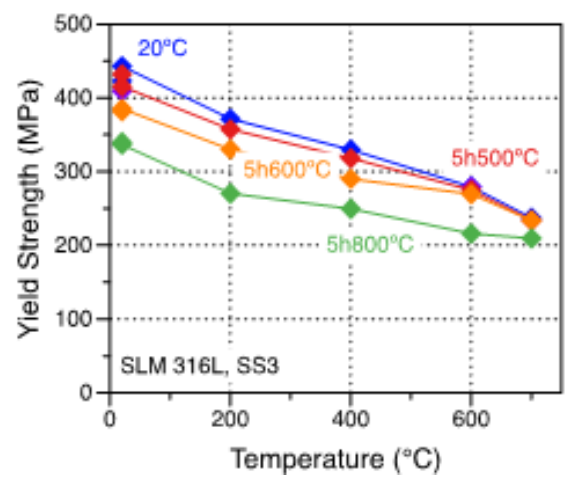

(c)

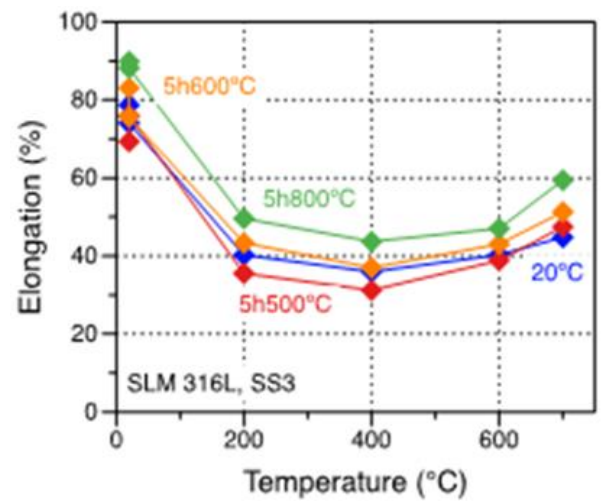

(b)

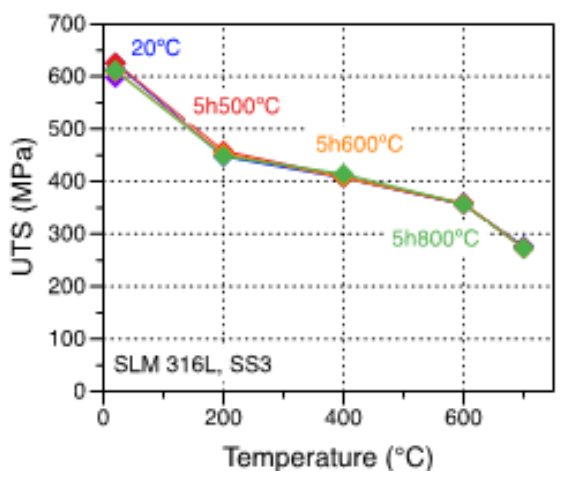

(d)

Figure I.3.3.1.3.2. (a) Comparison of the RT tensile properties of SLM 316L with literature data showing superior ductility but low yield strength for ORNL's SLM316L [1-11]. (b) Plastic elongation at rupture showing significant decrease of ductility at $\mathrm{T}>200^{\circ} \mathrm{C}$. (c) Yield strength results. (d) Ultimate tensile strength results. Source: ORNL.

In addition, two commercial and four ORNL CF8C+-type austenitic cast alloys were selected for single-track laser beam experiments using an SLM Renishaw 250 machine. The alloy chemical compositions are summarized in Table I.3.3.1.3.1. An example of the resulting microstructure for the CF8C+ steel is shown in Figure I.3.3.1.3.3 (a) with further magnification of the area at the end of the dotted line shown in 
Figure I.3.3.1.3.3 (b). In the laser-melted area, the larger carbides have disappeared, while the elongated cellular structures are present. Similar results were observed for all of the cast alloys, with only one crack detected for the HP40Nb steel. Advanced characterization will be conducted in FY 2020 to identify and quantify precipitates at the nanoscale and compare our experimental results with phase fraction prediction for rapidly cooled systems, as shown in Figure I.3.3.1.3.3 (c) for the CF8C+ steel.

Table I.3.3.1.3.1. Chemical Composition of the Cast Alloys Exposed to Laser Beam Single-Track Experiments.

\begin{tabular}{|c|c|c|c|c|c|c|c|c|c|c|c|}
\hline Alloy & Cr & Ni & Mn & Mo & Cu & Si & Nb & W & C & N & Fe \\
\hline HP40-Nb & 24.40 & 35.10 & 1.27 & 0.06 & - & 1.58 & 1.10 & - & 0.440 & - & - \\
\hline HP30-Nb & 25.15 & 19.35 & 1.18 & 0.27 & 0.05 & 1.55 & 1.16 & 0.02 & 0.290 & - & 50.72 \\
\hline CF8C+ & 19.77 & 12.74 & 3.45 & 0.28 & 0.56 & 0.52 & 0.91 & 0.02 & 0.100 & 0.269 & 61.19 \\
\hline CF8C+W/Cu & 19.64 & 12.31 & 3.47 & 0.30 & 2.96 & 0.53 & 0.92 & 1.10 & 0.0796 & 0.236 & bal \\
\hline CF8C+W/Cu 20/15 & 20.74 & 15.25 & 3.44 & 0.35 & 2.61 & 0.30 & 0.92 & 1.06 & 0.121 & 0.1925 & bal \\
\hline CF8C+W/Cu 21/17 & 22.15 & 17.41 & 3.70 & 0.35 & 2.59 & 0.46 & 0.91 & 1.01 & 0.089 & 0.200 & bal \\
\hline
\end{tabular}

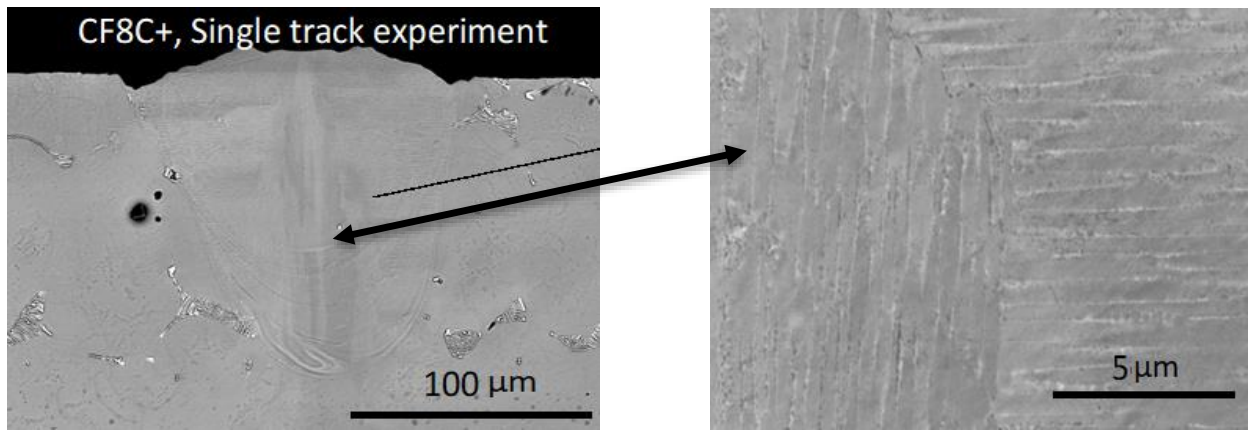

(a)

(b)

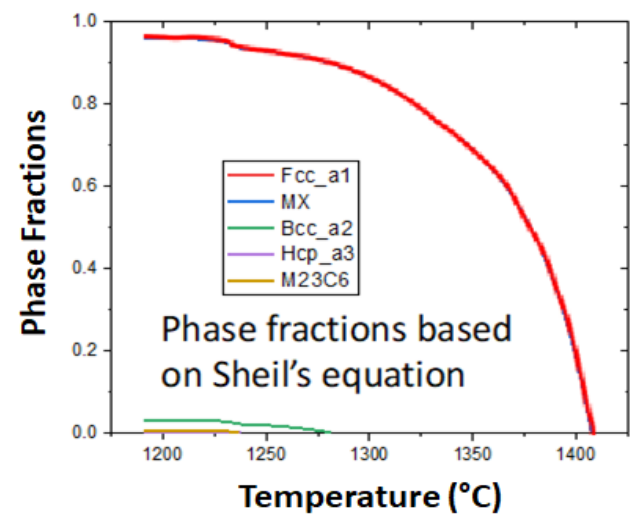

(c)

Figure I.3.3.1.3.3. (a) Cross-section BSE-SEM micrograph and (b) further magnification of the single-track laser-melted area for the $\mathrm{CF} 8 \mathrm{C}+$ steel highlighting the disappearance of coarse carbides and the presence of a cellular structure in the melted area. (c) Phase fraction in the CF8C+ steel based on the Sheil's equation to assess the impact of very fast cooling on the alloy microstructure. Source: ORNL.

\section{Conclusions}

High-temperature data have been generated for the first time for SLM 316 $\mathrm{L}$ and the properties are similar to widely used wrought $316 \mathrm{~L}$ after cold working. To assess the AM potential of high-temperature steels strengthened by carbonitrides, such as $\mathrm{HK} 30 \mathrm{Nb}$ and $\mathrm{CF} 8 \mathrm{C}+$, single-track experiments were conducted on 
commercial and CF8C+-type cast alloys. Initial SEM imaging showed the disappearance of coarse precipitates and advanced characterization will be required to detect the presence of nanoscale carbides or carbonitrides. Such a refinement of the steel microstructure could result in mechanical strength superior to similar cast steels if the microstructure is stable at high-temperature.

\section{Key Publications}

Publication expected in the first quarter of FY 2020 on the high-temperature properties of SLM 316L.

\section{References}

1. Wang, Y. M., T. Voisin, J. T. McKeown, J. Ye, N. P. Calta, Z. Li, Z. Zeng, Y. Zhang, W. Chen, T. T. Roehling, R. T. Ott, M. K. Santala, P. J. Depond, M. J. Matthews, A. V. Hamza, and T. Zhu, 2018, "Additively manufactured hierarchical stainless steels with high-strength and ductility," Nat. Mater., Vol. 17, pp. 63-73.

2. Kong, D., C. Dong, X. Ni, L. Zhang, J. Yao, C. Man, X. Cheng, K. Xiao, and X. Li, 2019, "Mechanical properties and corrosion behavior of selective laser-melted 316L stainless steel after different heattreatment processes," J. Mater. Sci. Technol., Vol. 35, No. 7, pp. 1499-1507.

3. Lavery, N. P., J. Cherry, S. Mehmood, H. Davies, B. Girling, E. Sackett, S. G. R. Brown, and J. Sienz, 2017, "Effects of hot isostatic pressing on the elastic modulus and tensile," Mat. Sci. Eng. A-Struct., Vol. 693, pp. 186-213.

4. Mower, T. M., and M. J. Long, 2016, "Mechanical behavior of additive manufactured, powder-bed laserfused materials," Mat. Sci. Eng. A-Struct., Vol. 651, pp. 198-213.

5. Carlton, H. D., A. Haboub, G. F. Gallegos, D. Y. Parkinson, and A. A. MacDowell, 2016, "Damage evolution and failure mechanisms in additively manufactured stainless steel," Mat. Sci. Eng. A-Struct., Vol. 651, pp. 406-414.

6. Spierings, A. B., T. L. Starr, and K. Wegener, 2013, "Fatigue performance of additive manufactured metallic parts," Rapid Prototyping J., Vol. 19, pp. 88-94.

7. Casati, R., J. Lemke, and M. Vedani, 2016, "Microstructure and fracture behavior of 316L austenitic stainless steel produced by SLM,” J. Mater. Sci. Technol., Vol. 32, pp. 738-744.

8. Qiu, C., M. Al Kindi, A. S. Aladawi, and I. Al Hatmi, "A comprehensive study on microstructure and tensile behavior of selectively laser-melted stainless steel," Sci. Rep., Vol. 8, Art. \#7585.

9. Zhong, Y., L. Liu, S. Wikman, D. Cui, and Z. Shen, 2016, "Intragranular cellular segregation network structure strengthening 316L stainless steel prepared by SLM," J. Nucl. Mat., Vol. 470, pp. 170-178.

10. Saeidi, K., X. Gao, F. Lofaj, L. Kverkova, and Z. J. Shen, 2015, "Transformation of austenite to duplex austenite-ferrite assembly in annealed stainless steel $316 \mathrm{~L}$ consolidated by laser melting," J. Alloy. Compd., Vol. 633, pp. 463-469.

11. Liu, L., Q. Ding, Y. Zhong, J. Zou, J. Wu, Y. L. Chiu, J. Li, Z. Zhang, Q. Yu, and Z. Shen, 2018, "Dislocation network in additive manufactured steel breaks strength-ductility trade-off," Mater. Today, Vol. 21, pp. 354-361.

12. Byun, T. S., N. Hashimoto, and K. Farrel, 2004, "Temperature dependence of strain-hardening and plastic instability behaviors in austenitic stainless steels," Acta Mater., Vol. 52, pp. 3889-3899.

\section{Acknowledgements}

The authors would like to thank K. Hedrick, S. Hawkins, K. Carver, and T Lowe for helping with the experimental work. 


\section{I.3.3.1.4 Development of Ferritic Alloys for Heavy-Duty Pistons by Additive Manufacturing (3B2)}

\section{Peeyush Nandwana, Co-Principal Investigator}

Oak Ridge National Laboratory

1 Bethel Valley Rd.

Oak Ridge, TN 37831

E-mail: nandwanap@ornl.gov

\section{Amy Elliott, Co-Principal Investigator}

Oak Ridge National Laboratory

1 Bethel Valley Rd.

Oak Ridge, TN 37831

E-mail: elliottam@ornl.gov

\section{Jerry L. Gibbs, DOE Technology Manager}

U.S. Department of Energy

E-mail: jerry.gibbs@ee.doe.gov

Start Date: October 1, 2019

Project Funding (FY19): \$250,000
End Date: September 30, 2022

DOE share: $\$ 250,000 \quad$ Non-DOE share: $\$ 0$

\section{Project Introduction}

Some common approaches to improving internal combustion engine efficiency require operating an engine at higher temperatures; however, this will also increase the demands on the piston materials. Thus, the need exists to develop new materials that have improved higher temperature performance over existing piston alloys, such as 4140 steel and micro-alloyed steels [1]. Conventional pistons are either cast or forged. As such, piston geometries are limited to those that can be easily fabricated with materials that are also easy to machine. AM offers a solution to fabricate near-net-shape components requiring minimal machining, thereby providing geometric flexibility and opening opportunities for new materials. Cost is an important consideration for piston fabrication. While fusion-based processes, such as laser powder-bed fusion and electron beam powder-bed fusion, can be quite expensive, binder-jet AM offers a potential lower-cost alternative [2]. An in-depth description of binder-jet AM can be found in the literature [3-5]. Binder-jet AM parts are quite fragile in an asdeposited condition, and typically require post-processing steps, such as sintering, for fabrication of a fully dense functional component. Multiple challenges need to be overcome to leverage the full cost potential of binder-jet AM. FY 2019 focused on developing an understanding of binder-jet AM processing of H13 steel with regards to densification and material properties. H13 was chosen as a model alloy for AM development on account of being a ferritic alloy that can be compared to 4140 steel, the most common commercial piston material for heavy-duty diesel engines. The $\mathrm{H} 13$ alloy is typically difficult to machine, which makes it a candidate for binder-jet AM. Further, being a chromium-molybdenum-based alloy, H13 is expected to provide better corrosion and oxidation resistance as compared to 4140 .

\section{Objectives}

To better understand some of the nuances of binder-jet AM with regards to the impact of powder feedstock and deposition parameters, the study focused on evaluating the impact of powder feedstock on green strength and dimensional accuracy. Green strength is defined as the flexural strength of the part in an as-printed condition. Based on these results, one of the powder feedstocks was down-selected for the development of sintering parameters to achieve maximum densification of H13, subjected to hot isostatic pressing (HIP), and tensile tested at RT to establish baseline properties. 


\section{Approach}

Green parts obtained after binder-jet AM are too fragile to be used for structural applications and require densification via sintering. Sintering can be classified as solid-state sintering (SSS) or supersolidus liquidphase sintering (SLPS) based on the dominant sintering mechanisms [6, 7]. During SSS of monolithic alloys, the components are sintered below the solidus temperature, thereby utilizing grain boundary diffusion and volume diffusion as the dominant materials transport mechanisms. On the other hand, SLPS is conducted just above the solidus temperature to introduce the presence of a liquid-phase to enhance the sintering kinetics by up to three orders of magnitude as compared to SSS. However, if the temperature, and hence, the liquid-phase fraction is not carefully controlled during SLPS, it can result in shape loss and distortions [8]. Thus, sintering parameters were developed for H13 while evaluating SSS and SLPS. The optimal parameters were then used to fabricate tensile bars, which were subjected to testing in as-sintered condition. HIP parameters and subsequent heat-treatment cycles were developed for further testing.

\section{Results}

Figure I.3.3.1.4.1 (a) compares the powder size distribution of $\mathrm{H} 13$ powders procured from Carpenter and Sandvik, with the Carpenter powders being finer than the Sandvik powders. Further, based on the micrographs in Figure I.3.3.1.4.1 (b) and Figure I.3.3.1.4.1 (c), the Carpenter powders have a larger size difference between the most coarse and most fine particles as compared to Sandvik powders. This translates to a slightly higher flexural strength of the Carpenter powders printed at the same binder saturation, as shown in Figure I.3.3.1.4.1 (d). Increasing binder saturation, i.e. higher binder content, for Sandvik powders increased the green strength by $\sim 3 \mathrm{x}$. Finally, both powders print to close tolerances with respect to the specified build dimensions with Sandvik powders being marginally better than Carpenter powders as shown in Figure I.3.3.1.4.1 (e).

Sandvik powders were down-selected for the development of sintering parameters. The sintering cubes were printed with binder saturations of $85 \%$ and $105 \%$ with higher saturations resulting in higher flexural strength, but with the potential to leave higher amounts of residual carbon in the material during sintering, as shown in Figure I.3.3.1.4.2 (a). Higher amounts of carbon can result in the formation of liquid at lower temperatures during sintering. The samples were subjected to SSS and SLPS between $1340-1400^{\circ} \mathrm{C}$, and density measurements were conducted using Archimedes method. Figure I.3.3.1.4.2 (b) shows that at $1400^{\circ} \mathrm{C}$, both samples show noticeable distortion regardless of binder saturation with $105 \%$ of the saturation sample showing a larger distortion possibly due to a larger liquid volume fraction resulting from higher carbon. For both saturation levels, the density maximum is achieved at $1380^{\circ} \mathrm{C}$ without any distortion. Thus, $1380^{\circ} \mathrm{C}$ was chosen as the optimal process temperature for sintering. XRD was conducted on the samples to determine the phase fractions via Reitwald analysis and results are shown in Figure I.3.3.1.4.2 (c). At each sintering temperature, the $105 \%$ saturation sample has a higher fraction of $\mathrm{M}_{23} \mathrm{C}_{6}$ type carbides and a similar fraction of $\mathrm{M}_{7} \mathrm{C}_{3}$ as compared to the $85 \%$ saturation sample. The retained austenite content increases with increasing sintering temperatures, regardless of binder saturation. 


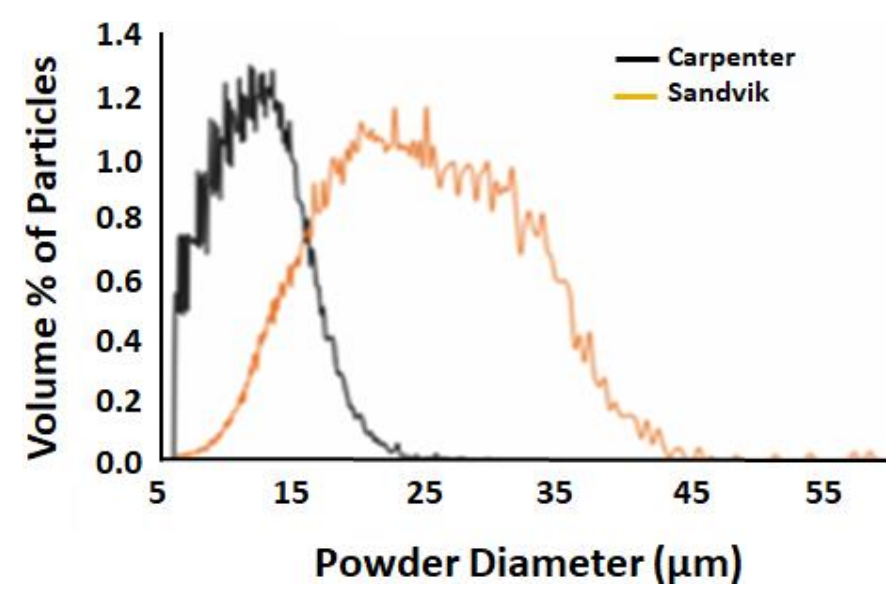

(a)

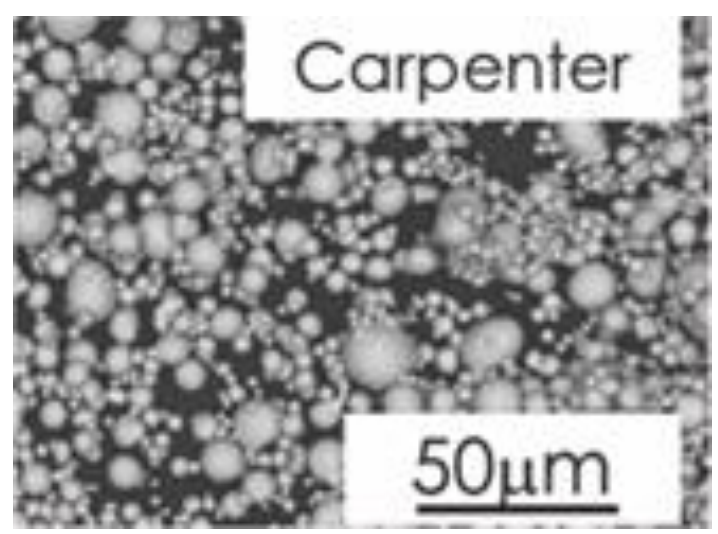

(b)

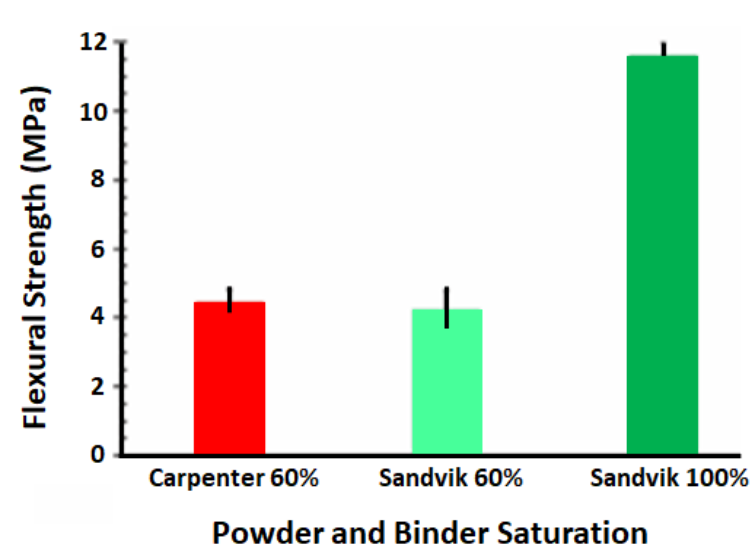

(d)

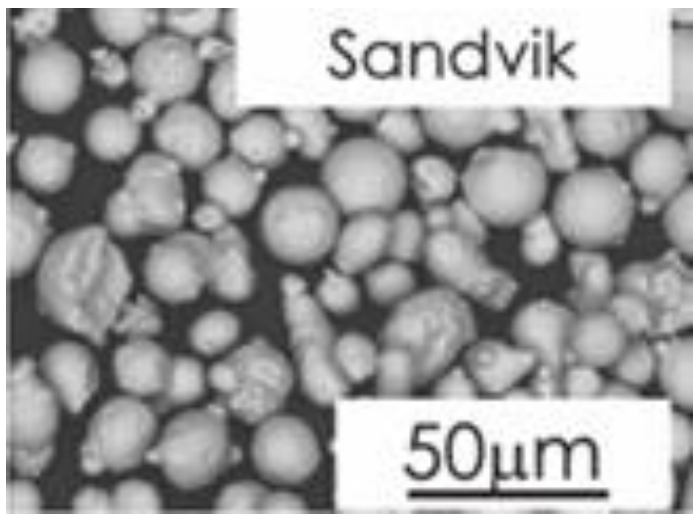

(c)

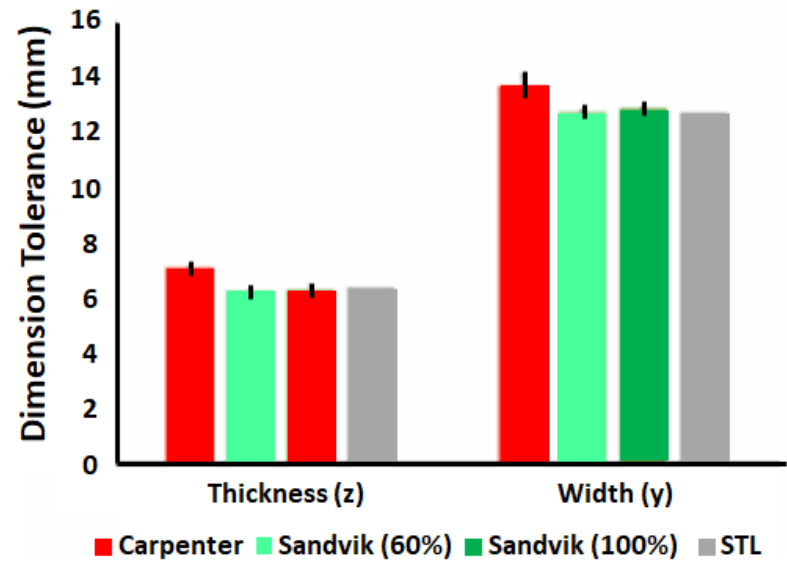

(e)

Figure I.3.3.1.4.1. (a) Powder size distribution; (b) micrograph of the Carpenter powder; (c) micrograph of the Sandvik powder; (d) flexural strength showing the impact of powder size and binder saturation on green part strength; and

(e) dimensional tolerance with respect to the specified dimensions for Carpenter and Sandvik powders highlighting good dimension tolerance. Source: ORNL. 


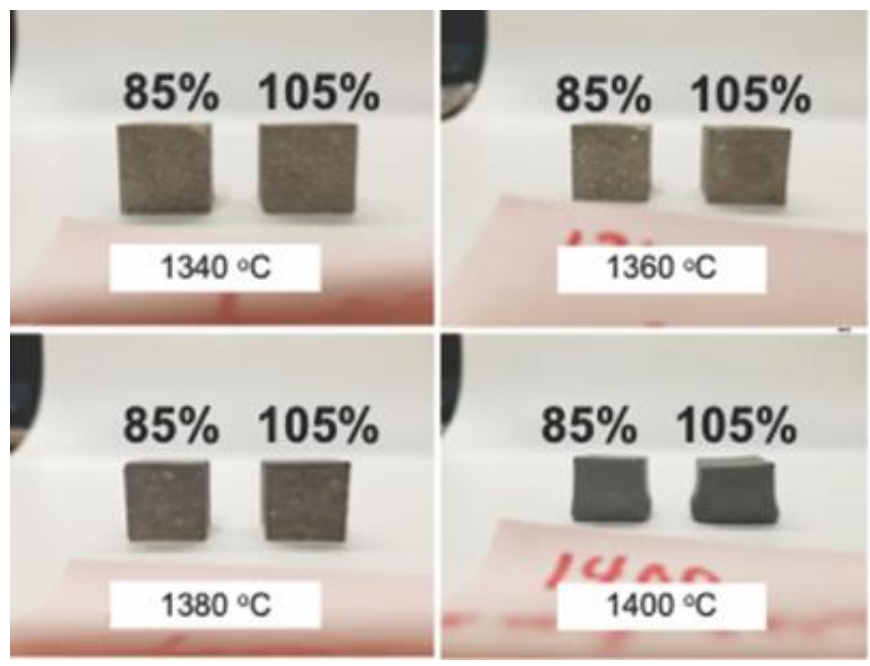

(a)

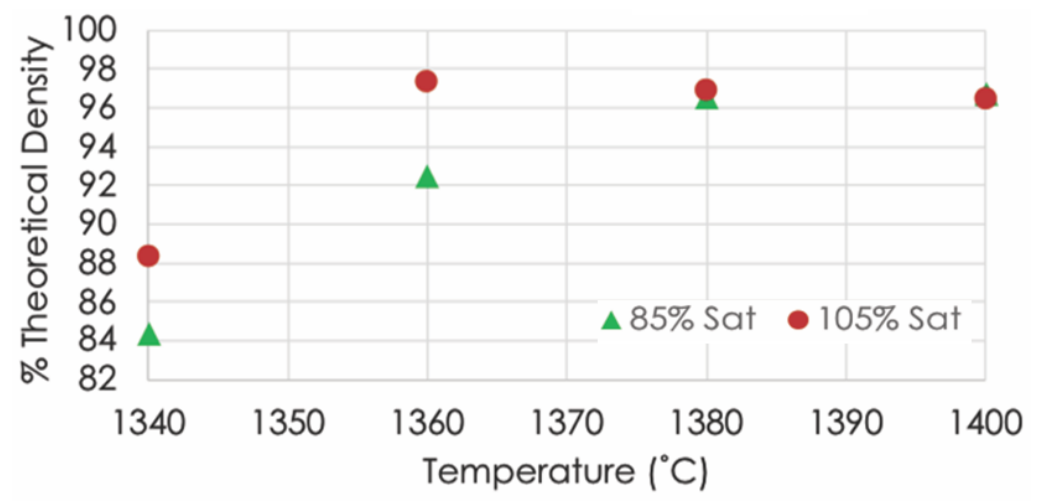

(b)

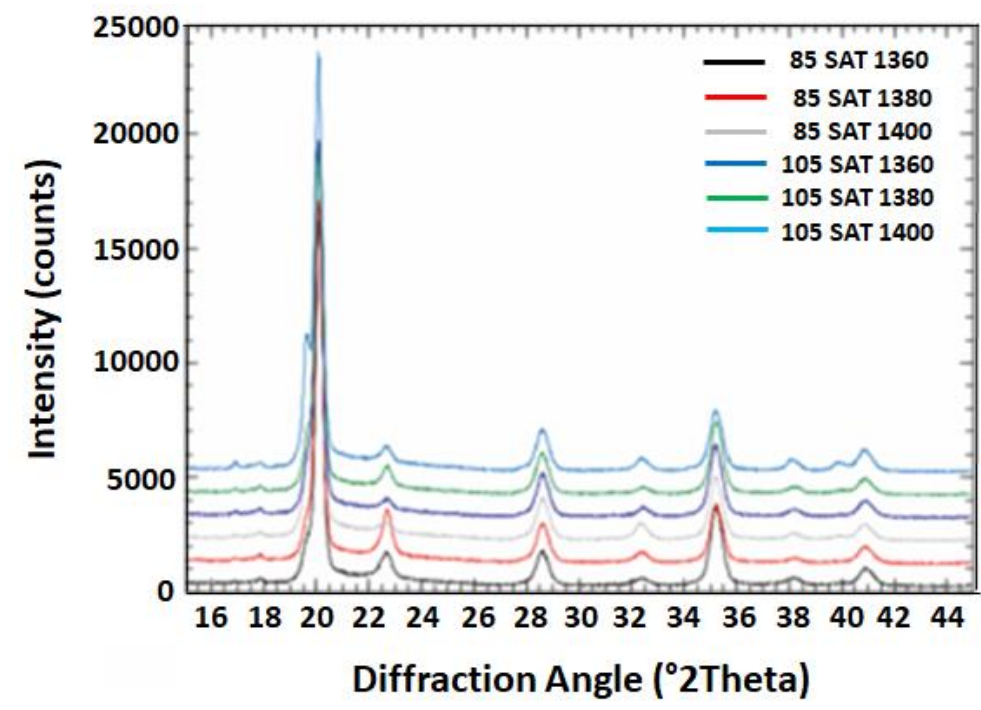

(c)

Figure I.3.3.1.4.2. (a) Optical images showing a lack of shape retention at $1400^{\circ} \mathrm{C}$ sintering temperature. (b) Densification in the phase fractions reaching a maximum at $\mathrm{T}>1380^{\circ} \mathrm{C}$. (c) X-ray diffraction analysis of sintered samples used to calculate phase fractions. Source: ORNL. 
Microstructural analysis was conducted on the $85 \%$ and $105 \%$ samples sintered at $1380^{\circ} \mathrm{C}$ in the as-sintered condition. Figure I.3.3.1.4.3 (a) shows the optical micrograph highlighting the spherical powders embedded within the re-solidified liquid post-SLPS. The high-resolution SEM image in Figure I.3.3.1.4.3 (b) shows the grain boundary carbides along with a pore at the grain boundary. The bulk microstructure in the powder zone consists of a mix of ferrite, austenite, and upper bainite. Thermocalc ${ }^{\mathrm{TM}}$ Scheil analysis as shown in Figure I.3.3.1.4.3 (c) was conducted on the liquid to understand the microstructural evolution during solidification and the precipitation of carbides.

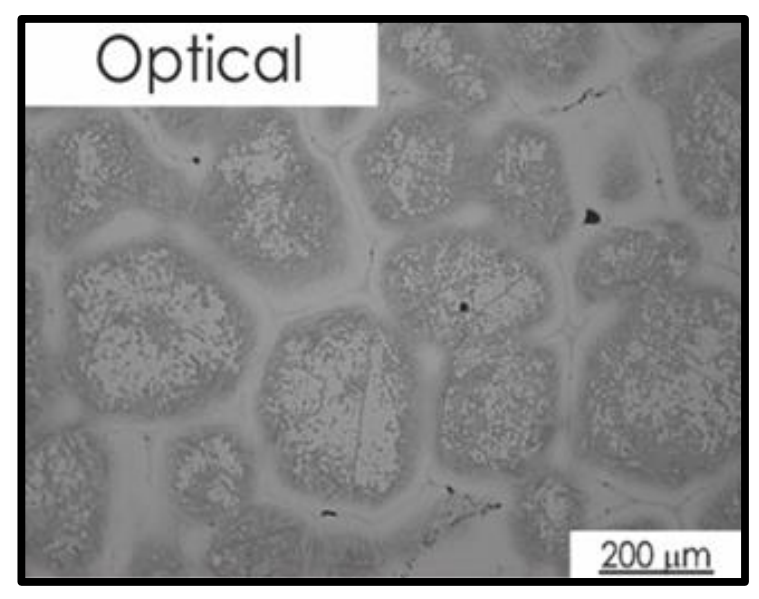

(a)

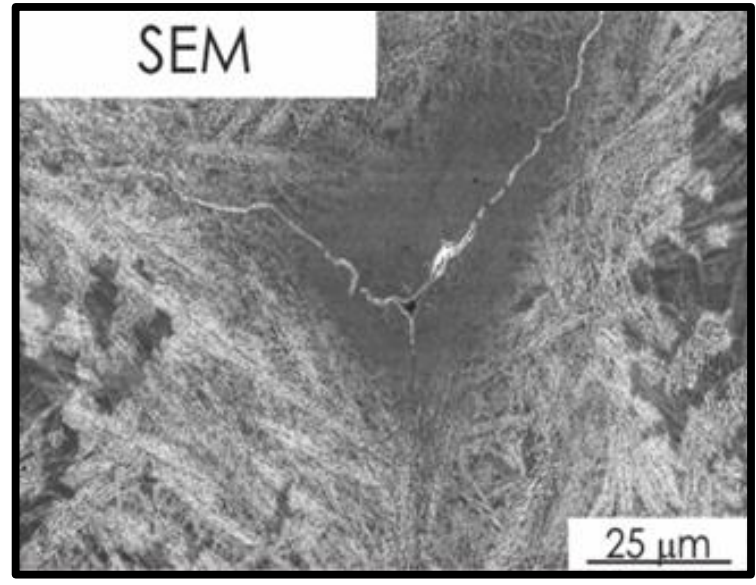

(b)

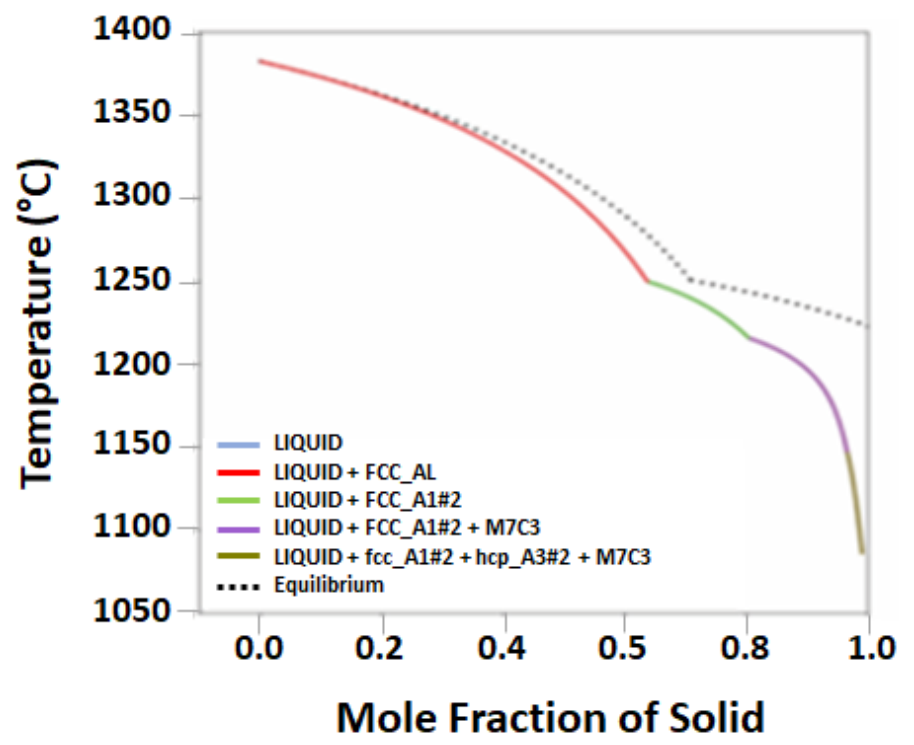

(c)

Figure I.3.3.1.4.3. (a) Optical micrograph showing embedded spherical powder particles, (b) SEM micrograph highlighting grain boundary carbides and (c) Scheil simulation for solidification of the liquid post-SLPS for the $105 \%$ sample sintered at $1380^{\circ} \mathrm{C}$ used to better understand microstructure evolution during solidification. Source: ORNL.

RT tensile testing was conducted on the SS3 tensile samples for the $85 \%$ and $105 \%$ samples sintered at $1360^{\circ} \mathrm{C}$ and $1380^{\circ} \mathrm{C}$, along with samples subjected to HIP followed by austenitizing and double tempering treatments. To be noted is the extremely brittle nature of the samples in the as-sintered condition. This data is summarized in Figure I.3.3.1.4.4. No clear trend in tensile strength as a function of binder saturation in the as-sintered condition can be seen. The lack of ductility can result from a combination of the presence of porosity, grain 
boundary carbides, and bainite. Significant improvement in ductility after HIP and heat-treatment (HT) was observed. The inset of Figure I.3.3.1.4.4 shows the porosity levels for the tensile samples generated using x-ray computed tomography. To be noted is the high levels of porosity in one of the samples post-HIP, which relates to the poor tensile performance. This high-level of porosity was observed in samples extracted closer to the surface. HIP is ineffective in closing interconnected surface porosity. The HIP+HT microstructure consisting of tempered martensite is also presented in the inset of Figure I.3.3.1.4.4. The HIP+HT samples exhibited much better ductility compared to the brittle as-sintered specimens. The UTS of the HIP+HT samples are within $10 \%$ of 4140 steel at RT. The UTS can be further improved by heat-treatments for refining the prior austenite grain size.

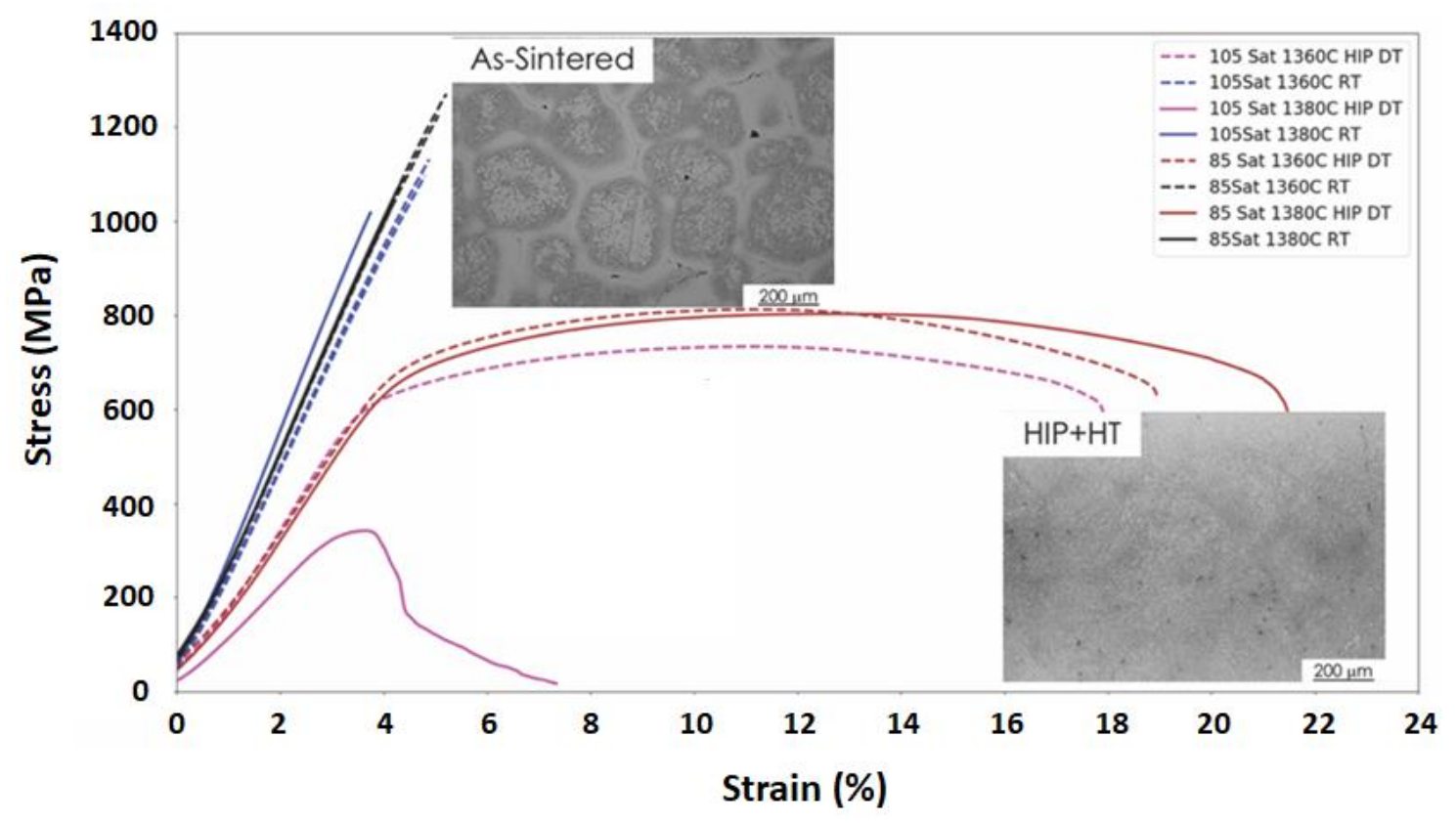

Figure I.3.3.1.4.4. Tensile behavior of the samples in the as-sintered and HIP plus heat-treatment conditions with the inset showing the representative micrographs for each condition along with porosity reconstructions for the HIP+HT samples Source: ORNL.

\section{Conclusions}

In summary, sintering parameters for binder-jet printed H13 steel powders were developed and it was concluded that SLPS results in maximum densification. The microstructure in the as-sintered condition was evaluated via optical and electron microscopy and rationalized based on thermodynamic simulations. Further, HIP parameters were developed to mitigate porosity and it was found that surface porosity in $\mathrm{H} 13$ steels is retained even after HIP. The post heat-treatments were successful in improving ductility. Future work will focus on retaining the strength by refining the prior austenite grains and conducting high-temperature tensile testing to be baselined against wrought alloy 4140 , which is the currently preferred commercial alloy for HDDE pistons. Finally, initial sintering trials on binder-jet printed 4140 steels have been initiated and parameters will be developed for maximum densification.

\section{Key Publications}

Manuscript in preparation for initial sintering and mechanical testing on H13. (Journal to be determined) 


\section{References}

1. Pierce, D., A. Haynes, J. Hughes, R. Graves, P. Maziasz, G. Muralidharan, A. Shyam, B. Wang, R. England, and C. Daniel, 2019, "High-temperature materials for heavy-duty diesel engines: Historical and future trends," Prog. Mater. Sci., Vol. 103, pp. 109-179.

2. Tofail, S. A. M., E. P. Koumoulos, A. Bandyopadhyay, S. Bose, L. O’Donoghue, and C. Charitidis, 2018, "Additive manufacturing: Scientific and technological challenges, market uptake, and opportunities," Mater. Today, Vol. 21, No. 1, pp. 22-37.

3. Nandwana, P., A. M. Elliott, D. Siddel, A. Merriman, W. H. Peter, and S. S. Babu, 2017, "Powder-bed binder-jet 3D printing of Inconel 718: Densification, microstructural evolution and challenges," Curr. Opin. Solid State Mater. Sci., Vol. 21, No. 4, pp. 207-218.

4. Wang, Y., and Y. F. Zhao, 2017, "Investigation of sintering shrinkage in binder-jetting AM process," Procedia Manuf., Vol. 10, pp. 779-790.

5. Enneti, R. K., K. C. Prough, T. A. Wolfe, A. Klein, N. Studley, and J. L. Trasorras, 2018, "Sintering of WC-12\% Co processed by binder-jet 3D printing (BJ3DP) technology," Int. J. Refract. Met. Hard Mater., Vol. 71, pp. 28-35.

6. Xu, X., W. Yi, and R. M. German, 2002, "Densification and strength evolution in SSS: Part IExperimental investigation," J. Mater. Sci., Vol. 37, No. 3, pp. 567-575.

7. German, R. M., 1997, "Supersolidus liquid-phase sintering of prealloyed powders," Metall. Mater. Trans. A, Vol. 28, No. 7, pp. 1553-67.

8. Liu, J., A. Lal, and R. M. German, 1999, "Densification and shape retention in supersolidus liquid-phase sintering," Acta Mater., Vol. 47, No. 18, pp. 4615-4626.

\section{Acknowledgements}

The authors would like to thank D. Siddel, K. Hedrick, and A. Marquez for their help with the experiments. 


\section{I.3.4 Advanced Characterization and Computational Methods - Thrust 4 (Oak Ridge National Laboratory)}

\section{Thomas R Watkins, Co-Principal Investigator}

Oak Ridge National Laboratory, Materials Science and Technology Division

1 Bethel Valley Road

Oak Ridge, TN 37831

E-mail: watkinstr@ornl.gov

\section{Dongwon Shin, Co-Principal Investigator}

Oak Ridge National Laboratory, Materials Science and Technology Division

1 Bethel Valley Road

Oak Ridge, TN 37831

E-mail: shind@ornl.gov

\section{Jerry L. Gibbs, DOE Technology Development Manager}

U.S. Department of Energy

E-mail: jerry.gibbs@ee.doe.gov

Start Date: October 1, 2018

Project Funding (FY19): \$1,650,000

End Date: September 30, 2019

DOE share: $\$ 1,650,000 \quad$ Non-DOE share: $\$ 0$

\section{Project Introduction}

The goal of the VTO Powertrain Materials Core Program (PMCP), which was launched in October 2018, is to rapidly develop and deliver new materials solutions, via an ICME framework, that enable both engine efficiency improvements and weight reductions over the full range of on-road vehicle classes (e.g., Class 1-8). There are four main tasks within the PCMP, each with subtasks. Tasks 1-3 are long-term alloy development tasks (briefly discussed in the Approach Section below).

This report describes the activities performed during the first year of Advanced Characterization and Computational Methods project, hereafter referred to as Task 4, within the VTO PMCP. Task 4 is a uniquely missioned and integrated task that is designed to provide advanced characterization and computational support to specific subtasks within the three long-term alloy development tasks. Subtask 4A, "Advanced Characterization," is a multi-laboratory effort involving ORNL, Pacific Northwest National Laboratory (PNNL), and Argonne National Laboratory (ANL). ORNL and PNNL conducted the advanced electron microscopy studies and APT studies, while high-energy diffraction and small angle scattering of synchrotron $\mathrm{x}$-ray analysis were conducted at ANL's Advanced Photon Source (APS). Neutron and thermophysical property measurement work will be conducted at ORNL's Spallation Neutron Source and High Flux Isotope Reactor and the Materials Science and Technology Division, respectively.

Subtask 4B, "Advanced Computation," provides a range of computational capabilities to the three long-term alloy development tasks including: computational thermodynamic and kinetic approaches (CALPHAD), highthroughput first principles DFT calculations, phase-field simulations, FEA, crystal plasticity analysis, and modern data analytics. Some of these computations are highly scalable and use DOE's high-performance computing (HPC) facilities within the Leadership Computing Facilities at ORNL and ANL. Subtask 4B is solely an ORNL effort. 


\title{
Objective
}

The overall objective of Task 4 is to combine advanced predictive ICME tools and advanced characterization techniques to accelerate alloy design from a Technology Readiness Level (TRL) of 1 to 4.

\begin{abstract}
Approach
Advanced Characterization and Computational Methods supports Tasks 1-3, each with multiple subtasks, providing needed information and insight into current and future alloys. A brief description of the tasks and supported subtasks follows. Task 1 investigates cost-effective, lightweight, high-temperature engine alloys and consists of two subtasks: (1) 1A1, fundamental studies of complex precipitation pathways; and (2) 1A2, development of new higher performance $\mathrm{Al}$ alloys exceeding $400^{\circ} \mathrm{C}$ for turbo and piston applications. Engineering of key interfaces to increase the temperature capability of lightweight $\mathrm{Al}$ alloys will be studied in subtask 1A1. In subtask 1A2, new high-performance Al alloys will be developed and tailored for targeted applications at temperature.
\end{abstract}

Task 2 investigates cost-effective higher temperature engine alloys and consists of five subtasks: (1) 2A1, oxidation-resistant valve alloys $\left(900-950^{\circ} \mathrm{C}\right)$; (2) $2 \mathrm{~A} 2$, higher temperature HD piston alloys $\left(>600^{\circ} \mathrm{C}\right)$; (3) 2A3, high-temperature coatings; (4) 2A4, high-temperature oxidation; and (5) 2B1, development of cast, higher temperature alloys. In subtask 2A1, new and cost-effective (lower $\mathrm{Ni}$ and $\mathrm{Co}$ ) AFAs for $900-950^{\circ} \mathrm{C}$ will be developed. In subtask 2A2, economical Fe-based alloys will be developed which balance properties to enable $>100^{\circ} \mathrm{C}$ increase in HD piston peak temperature capacity. In subtask $2 \mathrm{~A} 3$, protective coatings will be evaluated to enable $900-950^{\circ} \mathrm{C}$ adoption of cost-effective chromia-forming valve alloys. Subtask $2 \mathrm{~A} 4$ seeks to understand oxidation behavior of various commercial automotive exhaust valve alloys during high-temperature oxidation with laboratory-based cyclic oxidation testing in simulated exhaust environments. Subtask 2B1 will develop lower-cost cast chromia-forming alloys and cast alumina-forming austenitic steels for transportation and power generation.

Task 3 investigates opportunities of AM of powertrain alloys and consists of four subtasks: (1) 3A1, fundamental development of Al alloys for AM; (2) 3A2, AMIPCs and hybrid manufacturing/composites; 3B1, fundamentals of austenitic alloy processing by AM; and (4) 3B2, ferritic alloys for HD pistons via AM.

Subtask $3 \mathrm{~A} 1$ focuses on the development of the promising AlCuMnZr-Ce alloy for AM processing methods. Subtask 3A2 seeks to understand the composite behaviors of AMIPCs (e.g., stainless steel reinforced Al composite). In subtask 3B1, AM technologies will be explored to fabricate austenitic steels for piston, turbo, and exhaust applications at $600-850^{\circ} \mathrm{C}$ leading to locally tailored properties, microstructure or chemistry. Subtask 3B2 focusses on employing binder-jet AM technology to produce fully dense, dimensionally tolerant, high-strength, high-temperature ferritic steels for future HD pistons.

\section{Results}

Selected results of specific projects within subtasks 4A and 4B are presented as vignettes in the following pages to provide examples of progress and results in FY 2019.

\section{Subtask 4A: Advanced Characterization (ORNL, ANL, and PNNL) - Subtask PI: Thomas R Watkins}

Subtask 4A involved the following Co-Principal Investigators: J. Paplawski (ORNL), L. Allard (ORNL), D. Singh (ANL), L. Xiong, (ANL) A. Chuang, (ANL), J. Ilavsky (ANL), M. Firth (ANL), A. Devaraj (PNNL), B. Gwalani (PNNL), and L. Kovarik (PNNL). These investigators are identified with their appropriate subtasks, research capabilities, and contributions along with the location of the resources used and identified in the Acknowledgements with crosses (e.g., $t$ ). 


\section{APT - J. Poplawsky, ORNL† (see Acknowledgements)}

APT is a microscopy technique capable of reconstructing the 3D atomic locations within a small volume $\left(\sim 1 \times 10^{6}\right.$ to $\left.1 \times 10^{7} \mathrm{~nm}^{3}\right)$ with $<1 \mathrm{~nm}$ resolution and $10 \mathrm{ppm}$ sensitivity. Therefore, APT is an effective technique to measure matrix compositions, precipitate contents, and interfacial segregation profiles. APT has been utilized to better understand the nanostructures in alloys used for powertrain applications. The results for each subtask are summarized in the sections that follow.

\section{Fundamental Studies of Complex Precipitation Pathways}

These studies supported the subtask for fundamental studies of precipitation pathways (e.g., subtask 1A1). APT was employed to quantify the interfacial solute segregation to metastable strengthening precipitates $\left(\theta^{\prime}, \mathrm{Al}_{2} \mathrm{Cu}\right)$ within the ACMZ alloys. The ORNL-developed ACMZ alloy classes are lightweight cast $\mathrm{Al}-\mathrm{Cu}$ alloys with $\mathrm{Mn}$ and $\mathrm{Zr}$ additions and low $\mathrm{Si}$ content that retains strength beyond $350^{\circ} \mathrm{C}$. The $\mathrm{Mn}, \mathrm{Zr}$, Ti, and $\mathrm{Si}$ segregation behavior at the $\theta^{\prime}$ precipitate interfaces, within the $\theta^{\prime}$ phase in these alloys as a function of temperature and time, was studied and revealed the synergistic effect of $\mathrm{Mn}$ and $\mathrm{Zr}$ additions to $\mathrm{Al}-\mathrm{Cu}$ alloys for stabilizing the $\theta^{\prime}$ strengthening phase at temperatures up to $350^{\circ} \mathrm{C}$. Examples of cross-sectional $\theta^{\prime}$ 2D composition plots for ACMZ and the commercially available RR350 after exposure to $300^{\circ} \mathrm{C}$ for $200 \mathrm{~h}$ are shown in Figure I.3.4.1, which reveal the $\mathrm{Si}, \mathrm{Zr}$, and $\mathrm{Mn}$ interfacial segregation profiles. A second experiment used APT to study the effect of Si on the high-temperature stability of the ACMZ alloy system. Prior mechanical properties data shows that $\mathrm{Si}$ contents between 0.05 and $0.1 \mathrm{wt} . \%$ are necessary to have hightemperature stability. APT was performed on ACMZ alloys with low $\mathrm{Si}$, ideal $\mathrm{Si}$, and high $\mathrm{Si}$ additions in the as-aged state and after exposure to $350^{\circ} \mathrm{C}$ for $200 \mathrm{~h}$ to elucidate the role of $\mathrm{Si}$ on high-temperature alloy stability.

a)
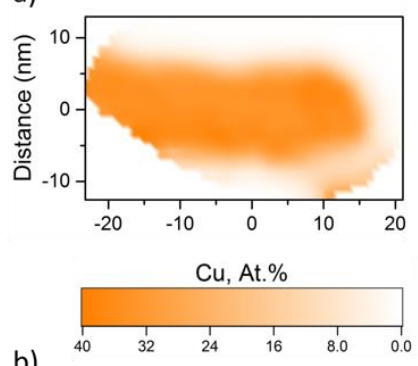

b)

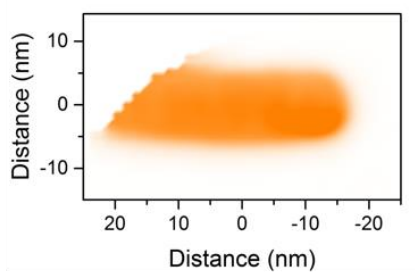

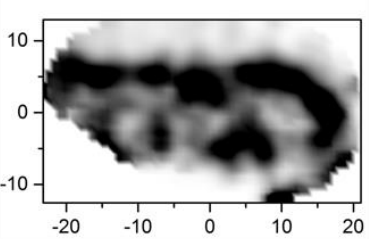

Si, At.\%
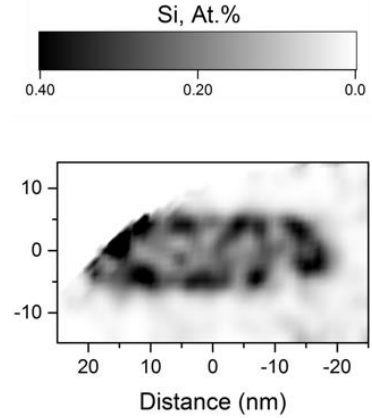
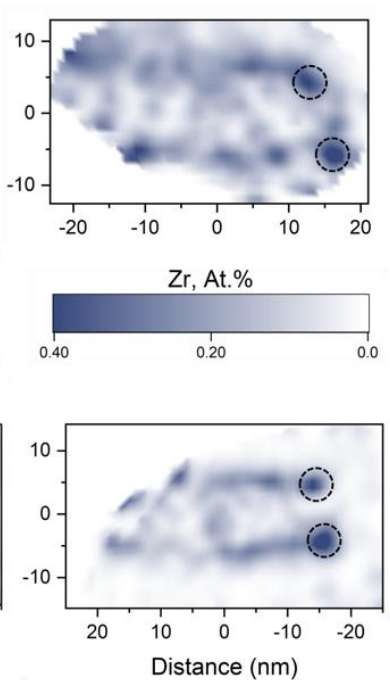
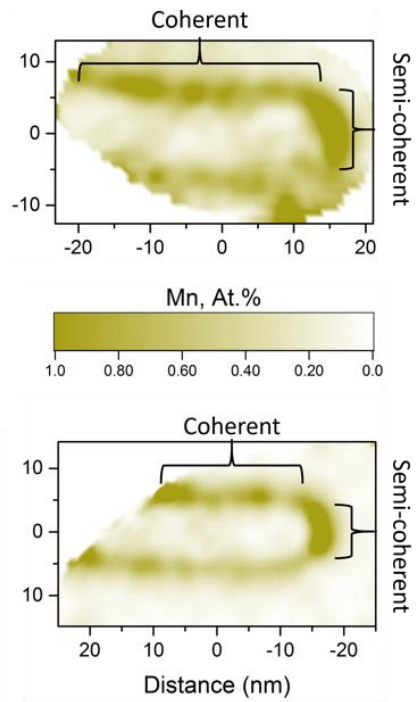

Figure 1.3.4.1. Cross-sectional $\theta^{\prime} 2 \mathrm{D}$ composition plots for: (a) ACMZ; and (b) RR350 exposed to $300^{\circ} \mathrm{C}$ for $200 \mathrm{~h}$ reveal the interfacial segregation profiles for $\mathrm{Si}, \mathrm{Zr}$, and $\mathrm{Mn}$. Si shows segregation to regions of the coherent and semi-coherent interfaces, Zr segregates only to the coherent interfaces with additional segregation to the coherent/semi-coherent intersection, and $\mathrm{Mn}$ segregates to the coherent and semi-coherent interfaces with additional segregation to the semicoherent interface [1]. Source: ORNL. 
Development of Low-Cost, Oxidation-Resistant Materials for Exhaust Manifolds and Turbo Housings This development supported the subtask $2 \mathrm{~A} 2$ for higher temperature HD piston alloys $\left(>600^{\circ} \mathrm{C}\right)$. The precipitate contents, spatial distribution, and sizes, as well as matrix $\mathrm{C}$ and $\mathrm{N}$ contents, are important for the strength of high-temperature piston alloys. APT was performed on a series of developmental and commercial iron-based alloys that may be suitable for next-generation HD diesel engines such as the example depicted in Figure I.3.4.2. Although not shown, the results revealed the matrix composition and precipitate distribution after various heat-treatments to help link the hardness data to microstructural changes.
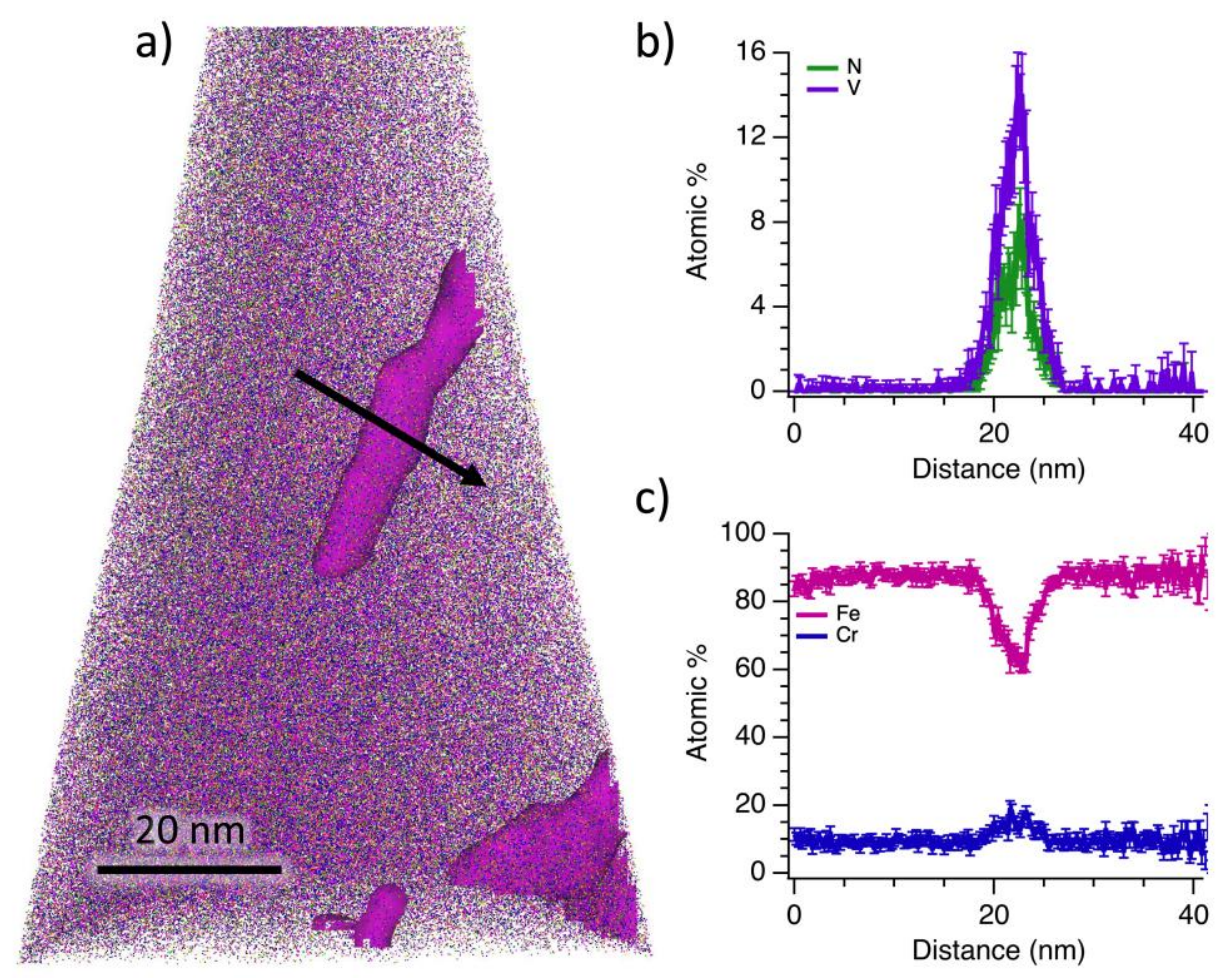

Figure I.3.4.2. APT data for a commercial 422 martensitic stainless steel double tempered at $650^{\circ} \mathrm{C}$. (a) APT atom map with $\mathrm{Fe}, \mathrm{Cr}, \mathrm{N}$, and $\mathrm{V}$ atoms displayed as magenta, blue, green, and purple dots, respectively. A 3 at. $\%$, the $\mathrm{V}+\mathrm{N}$ isoconcentration surface is displayed to highlight V-N particles. A 1D concentration profile was taken across a V-N particle as marked by the black arrow. (b) The $\mathrm{V}$ and $\mathrm{N}$ profile across the $\mathrm{V}-\mathrm{N}$ particle. (c) The Fe and Cr profile across the same V-N particle. Source: ORNL.

Development of High-Strength Alloys for Higher Temperature Capable Pistons

This effort supported the subtask 2B1 for the development of cast, higher temperature alloys. For the experimental procedure, oxidation was performed under an ${ }^{18}$ oxygen-rich environment to distinguish oxygen contamination during the APT experiment from oxygen in the material. The temperature-dependent oxygen infiltration into metal at the metal/oxide interface in oxidized model $\mathrm{Ni}, \mathrm{NiCr}$, and $\mathrm{NiCrAl}$ alloys at different temperatures was studied by APT with the results shown in Figure I.3.4.3. The results from these studies are being used to develop predictive models for complex alloy oxidation behavior, which can then be used to design new, affordable oxidation-resistant alloys for exhaust manifolds and turbo housings. 
a)

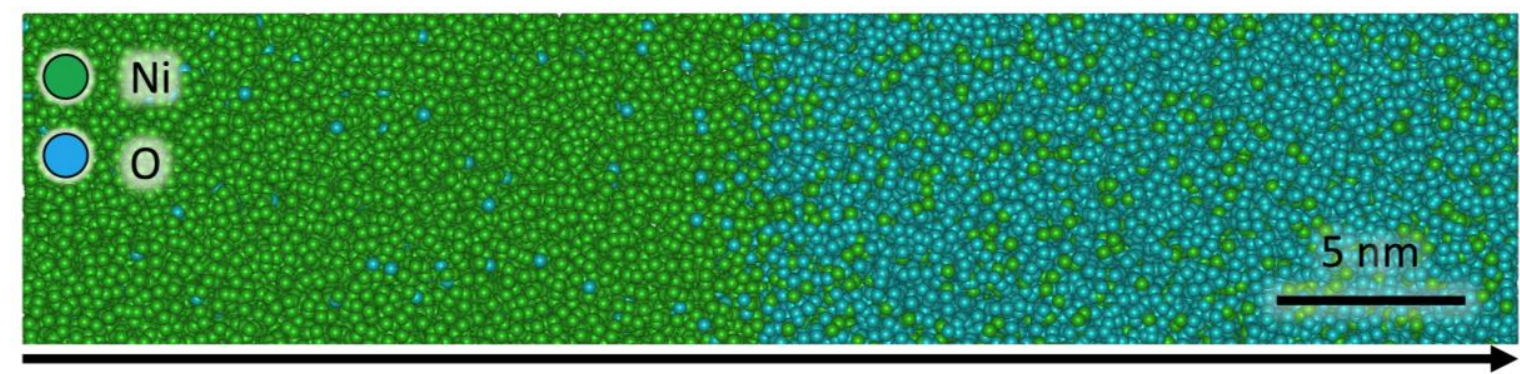

b)

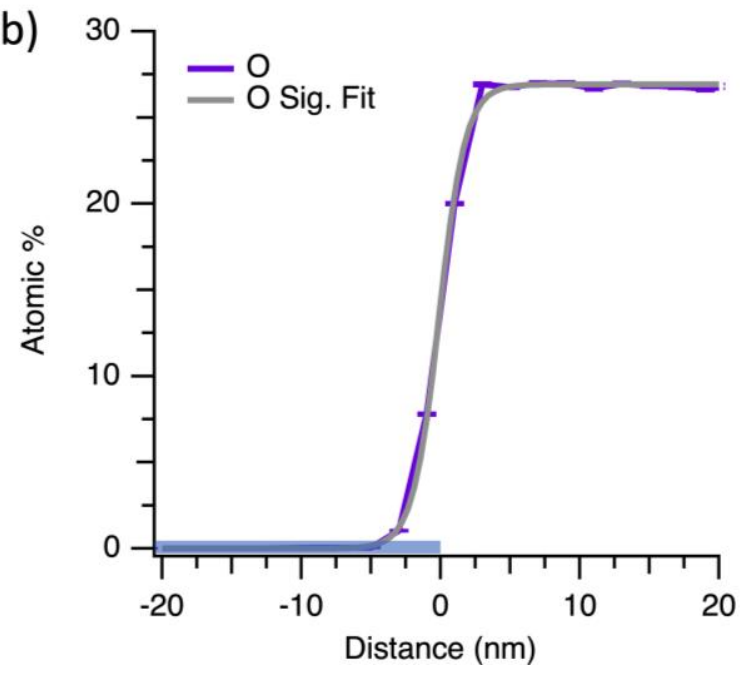

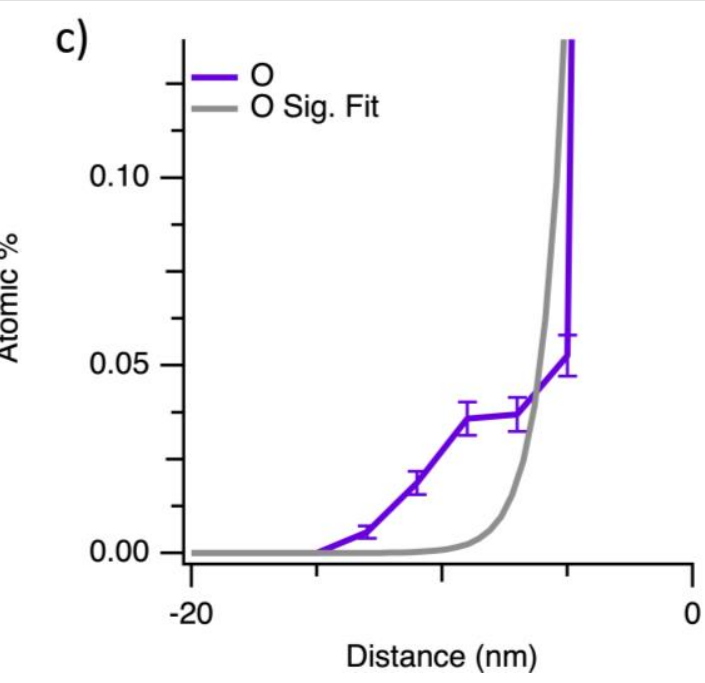

Figure I.3.4.3. A Ni/NiO interface for oxidized $\mathrm{Ni} 200$ at $1000^{\circ} \mathrm{C}$ for $24 \mathrm{~h}$. (a) APT atom map of the NiO/Ni interface with $\mathrm{Ni}$ and $\mathrm{O}$ ions shown as green and blue spheres, respectively. (b) An $\mathrm{O}$ concentration profile across the $\mathrm{NiO} / \mathrm{Ni}$ interface in the direction shown by the black arrow with a sigmoidal fit overlaid. (c) A magnified view of the 0 concentration profile highlighted by the blue rectangle in (b) showing $\mathrm{O}$ infiltration into the Ni metal. Control experiments are being conducted to determine the accuracy of the APT measurements of O solubility in Ni. Source: ORNL.

\section{AC-STEM with In Situ Heating Stage - L. Allard, ORNL}

\section{Fundamental Studies of Complex Precipitation Pathways}

Supporting subtask 1A1, microstructural results via aberration-corrected electron microscopy in the aberrationcorrected scanning transmission electron microscope (AC-STEM) have informed the development of computer models that have facilitated rapid improvements in alloys for powertrain applications (e.g., the new "ACMZ" cast alloy recently developed at ORNL). We undertook the first steps to utilize new technologies, a.k.a. an in situ heating stage as shown in Figure I.3.4.4, which offers promise to greatly expedite the discovery process significantly. By heating electron-transparent thin foils of a given alloy in situ, where the entire precipitation process can be studied with the microscope at the atomic scale in a "continuous" fashion, we aim to gain insights into the mechanisms of precipitate development and the atomic-level phenomena that control their behavior during advanced heating regimes.

The new capability for in situ precipitation studies has been facilitated first by the ability to affix a chosen thin section of an electropolished foil (e.g., an electron-transparent alloy grain with an appropriate crystallographic orientation) onto a MEMS-based heater device ${ }^{7}$ using the new technique of Xenon-Plasma Focused Ion Beam milling (Xe-PFIB). This FIB technique avoids the use of more standard Ga-based FIB milling procedures for which $\mathrm{Al}$ alloy studies are precluded due to the deleterious effects of gallium on $\mathrm{Al}$ [2]. A new double-tilt heating holder from Protochips ${ }^{7}$ was developed that enables the thin section on the heater to be precisely tilted into the desired orientation to allow the atomic structure to be imaged. The present example used an initial foil

${ }^{7}$ Microelectromechanical systems, Protochips Inc., Raleigh, NC. https://www.protochips.com/. 
with pre-existing precipitate structure, for the early tests. Later, we observed initial stages of precipitate nucleation and growth from the solution-treated starting foils; interesting new pre- $\theta$ ' structures have been seen for the first time. These newly developed capabilities for our studies will not only expedite new alloy development but also offers the possibility to observe new microstructural features that could lead to even more novel discoveries.
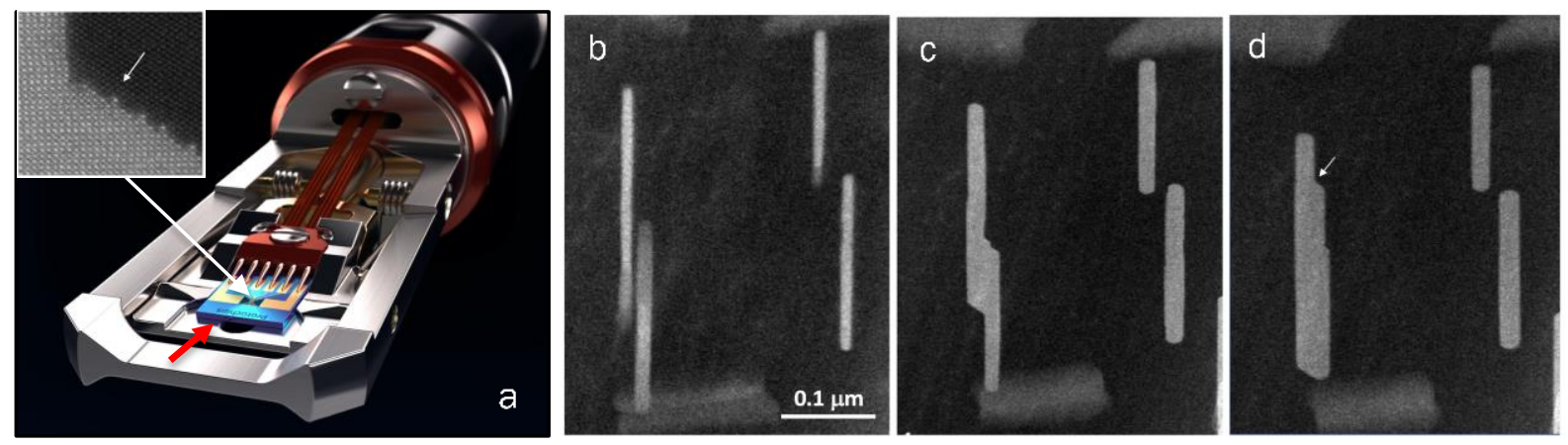

Figure I.3.4.4. (a) Tip of the double-tilt heating holder showing the Micro-Electro-Mechanical Systems (MEMS) heater at the red arrow installed with an inset image showing the atomic structure at the surface of an $\mathrm{Al}_{2} \mathrm{Cu}\left(\theta^{\prime}\right)$ precipitate from (d) and (b) through (c) the progression of the $\theta^{\prime}$ coalescence with heating at $200^{\circ} \mathrm{C}$. Source: ORNL.

\section{Synchrotron X-rays at the APS - D. Singh, L. Xiong, and A. Chuang, ANLT}

\section{Fundamentals of Interfacial Engineering in HT Aluminum}

These efforts supported the subtask for fundamental studies of complex precipitation pathways (e.g., subtask 1A1). Lightweight $\mathrm{Al}$ alloys that are capable of withstanding temperatures above $300^{\circ} \mathrm{C}$ are needed in the automotive industry for improved fuel efficiencies. In the widely used $\mathrm{Al}-\mathrm{Cu}-(\mathrm{Si})$ alloys, as-aged precipitates (metastable $\theta^{\prime}$ phase) with a high number of density and average aspect ratio strengthen the mechanical properties [3]. The thermal stability of the strengthening metastable $\theta^{\prime}$ phase determines the peak service temperature of $\mathrm{Al}-\mathrm{Cu}$ alloys because the metastable $\theta^{\prime}$ phase will transform into the equilibrium $\theta$ phase at elevated temperatures, which is deleterious to the mechanical properties. Therefore, quantification of $\theta^{\prime}-\theta$ phase transformation in $\mathrm{Al}$ alloys plays an important role in designing alloys with higher temperature capability.

Here high-resolution synchrotron XRD was performed to characterize the $\theta^{\prime}-\theta$ phase transformation in $\mathrm{Al}_{5} \mathrm{CuNi}$ (RR350) alloy and to quantify the phase fraction of $\theta^{\prime}$ and $\theta$ phases under various heat-treatment conditions. Diffraction patterns from 12 different locations in each sample were captured for representative sampling. Table I.3.4.1 shows the phase fraction of $\theta^{\prime}$ and $\theta$ phases under different heat-treated conditions from the Rietveld refinements. Background, scale factor, lattice parameters, peak position, shape and width were refined for the Rietveld refinements. While the equilibrium $\theta$ phase remains relatively constant throughout the varied conditions, the metastable $\theta^{\prime}$ phase increases with time at temperature as expected until it curiously decreases when exposed to $350^{\circ} \mathrm{C}$ for $200 \mathrm{~h}$. This information needs to be confirmed but would suggest an additional stabilizing stage for the beneficial $\theta^{\prime}$ phase in $\mathrm{Al}_{5} \mathrm{CuNi}(\mathrm{RR} 350)$ alloy and perhaps the ACMZ alloys.

Table I.3.4.1. Phase Fraction of Metastable $\theta^{\prime}$ and Equilibrium $\theta$ Phases of AI5CuNi (RR350) after Different Heat-Treated Conditions.

\begin{tabular}{|c|c|c|c|c|}
\hline Phase $\mid$ Condition & As-Aged & $300^{\circ} \mathrm{C}, 200 \mathrm{~h}$ & $300^{\circ} \mathrm{C}, 2113 \mathrm{~h}$ & $350^{\circ} \mathrm{C}, 200 \mathrm{~h}$ \\
\hline $\begin{array}{c}\text { Theta prime } \\
\text { fraction }(w \mathrm{w} \%)\end{array}$ & $2.96 \pm 1.4$ & $3.68 \pm 1.5$ & $4.34 \pm 1.4$ & $2.68 \pm 0.7$ \\
\hline Theta fraction $(w t \%)$ & $0.46 \pm 0.12$ & $0.52 \pm 0.25$ & $0.41 \pm 0.25$ & $0.44 \pm 0.26$ \\
\hline
\end{tabular}




\section{Synchrotron X-rays at the APS - J. Ilavsky, M. Firth, and D. Singh, ANLTt}

\section{Oxidation-Resistant Valve Alloys for Higher Temperatures}

These studies supported the subtask for development of oxidation-resistant valve alloys $\left(900-950^{\circ} \mathrm{C}\right)$ (e.g., subtask 2A1). We are utilizing unique in situ capabilities of APS ultra-small-, small-, and wide-angle x-ray scattering (USAXS/SAXS/WAXS) beamline, as well as select other APS instruments to obtain complementary information on the precipitate size, shape, spacing, and other characteristics that influence the hightemperature mechanical properties of the $\mathrm{FeNiCr}$ valve alloys developed by ORNL. ORNL is also performing other microstructure characterization, such as TEM and APT, to complement the USAXS/SAXS/WAXS data and provide input to better interpret the USAXS/SAXS/WAXS data. The APS USAXS/SAXS/WAXS beamline can characterize microstructures across critically important length scales (from Angstroms to microns) in 3 to 4 minutes while heating samples in a controlled atmosphere up to $1500^{\circ} \mathrm{C}$ along a temperature/time profile as needed. This year, multiple samples of new chemistries/microstructures at selected temperatures (i.e., $835^{\circ} \mathrm{C}, 900^{\circ} \mathrm{C}$, and $950^{\circ} \mathrm{C}$ ) were measured for up to $13 \mathrm{~h}$. Figure I.3.4.5 (a) shows different SAXS signals due to variation in precipitates volume, size, and structure in different Ni-based alloys. This enables designers to optimize alloy composition to create the optimal gamma-prime microstructure.

Figure I.3.4.5 (b) shows growth of the gamma-prime precipitates as a function of time at $900^{\circ} \mathrm{C}$, which enables optimization of manufacturing methods, as well as an estimation of the high-temperature life at different in-service conditions.

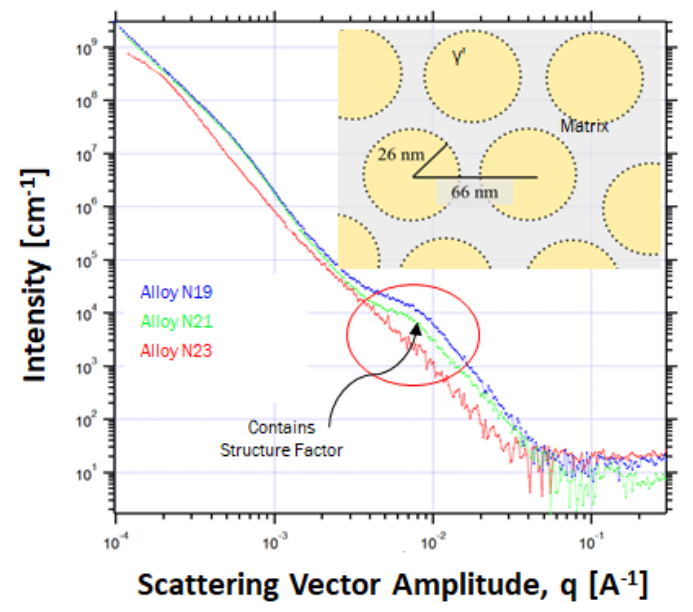

(a)

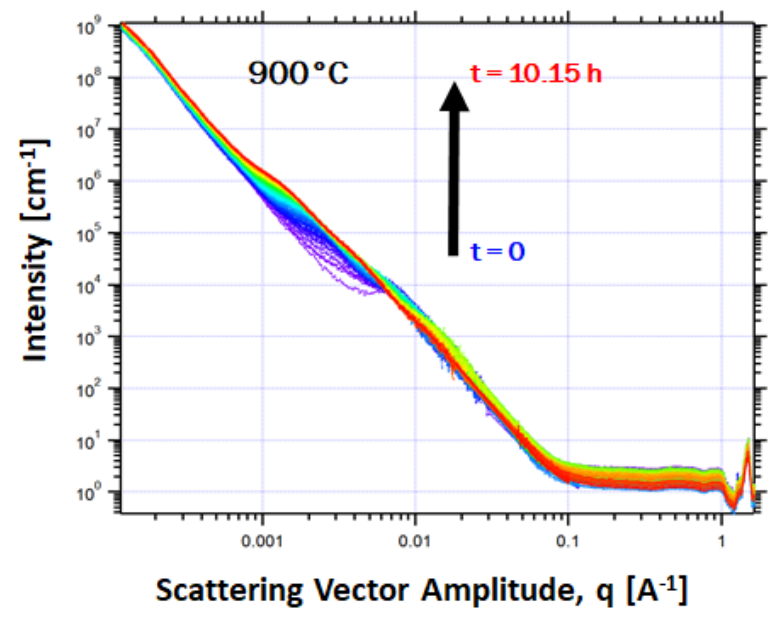

(b)

Figure I.3.4.5. (a) Tested alloy scattering variation caused by different microstructures as a function of q. (b) The scattering curves change as a function of time at $900^{\circ} \mathrm{C}$. Source: ANL.

\section{APT and AC-STEM - A. Devaraj, B. Gwalani, and L. Kovarik, PNNL†t†}

\section{Fundamentals of Interfacial Engineering in HT Aluminum}

These studies supported the subtask for the fundamental studies of complex precipitation pathways (e.g., subtask 1A1). The aim of this task was to conduct in situ APT annealing studies to analyze solute partitioning between $\theta^{\prime}$ and matrix it as a function of various heat-treatment temperatures and times using the setup shown in Figure I.3.4.6 (a). These in situ results would provide an understanding of continuous solute partitioning responsible for stability or instability of $\theta$ ' in different Al-Cu based alloys. In FY 2020, we initiated the work by using gallium FIB lift-out needles from a polished ACMZ alloy and conducted sequential in situ APT annealing at $300^{\circ} \mathrm{C}$ for 1 to $3 \mathrm{~h}$, which was followed by APT analysis capturing the time evolution of the solute partitioning. The results of these studies are shown in the images of Figure I.3.4.6 (b)-(g). Such understanding 
of solute partitioning in the same APT needle can now be correlated with extensive ex situ APT studies conducted at ORNL to reveal mechanistic insights on solute partitioning.
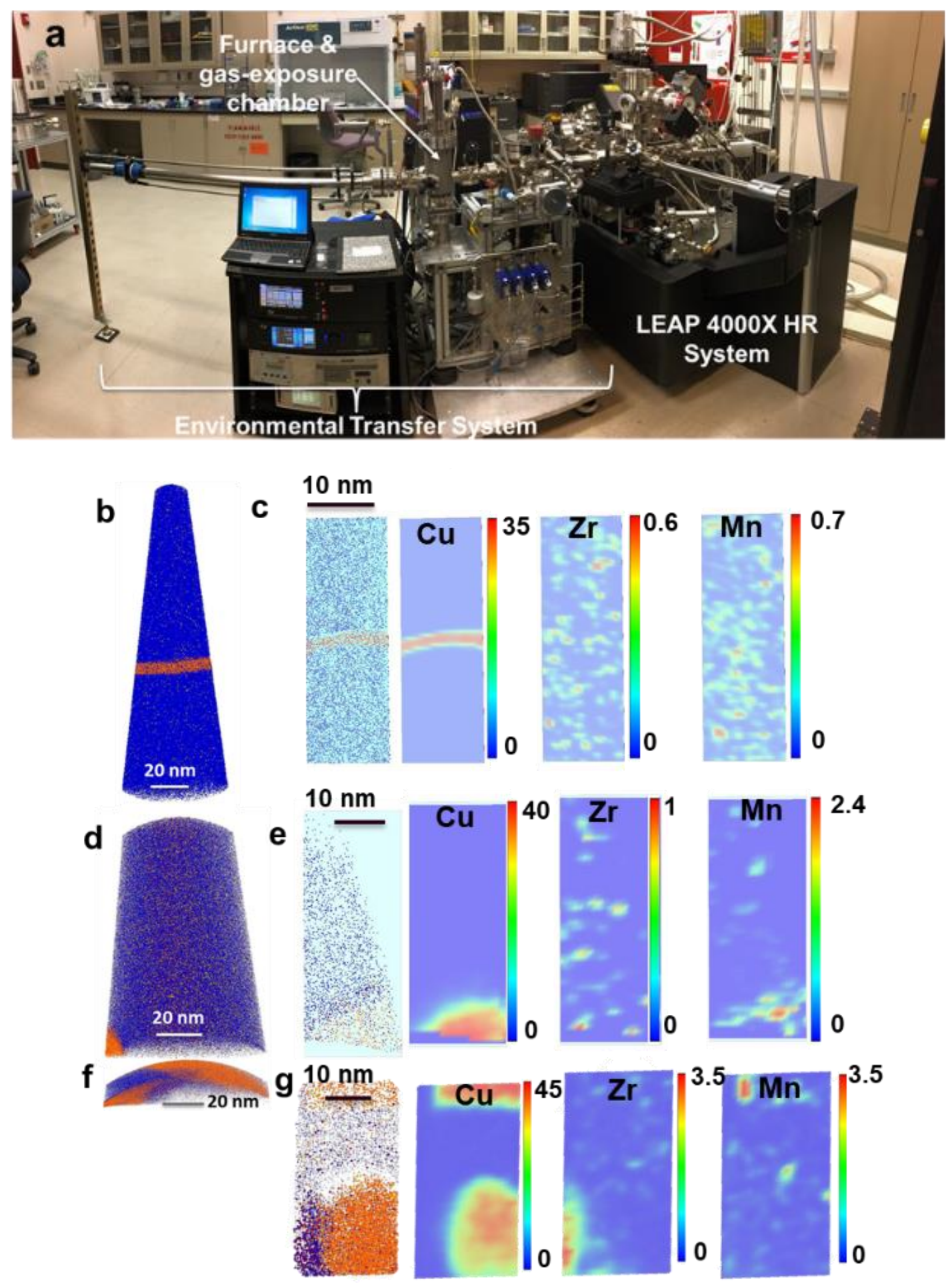

Figure I.3.4.6. (a) The environmental reactor chamber attached to APT at PNNL. (b) The APT run of as-received ACMZ alloy.

(c) $2 \mathrm{D}$ compositional analysis across precipitate and matrix. (d) Compositional analysis after $300^{\circ} \mathrm{C}$ for $1 \mathrm{~h}$. (e) $2 \mathrm{D}$ compositional mapping. (f) Compositional analysis after an additional $3 \mathrm{~h}$ of annealing. (g) 2D compositional map. Source: PNNL. 


\section{APT and AC-STEM - A. Devaraj, B. Gwalani, and L. Kovarik, PNNL†t†}

\section{Oxidation-Resistant Valve Alloys for Higher Temperatures $\left(900^{\circ} \mathrm{C}\right.$ to $\left.950^{\circ} \mathrm{C}\right)$}

In support of subtask $2 \mathrm{~A} 1$, the aim was to determine the precipitate structure, composition, shape, size, and size distributions in selected Ni-based alloys before and after fatigue testing and to understand the deformed microstructure of the alloys. A fundamental understanding of how the precipitates affect the strength and deformation characteristics in these alloys is required to understand the limitations of the lower Ni-alloys in achieving the target fatigue strengths of high Ni-alloys (such as Alloy 751) traditionally used for this application. The specific alloys studied under this task are industrially manufactured versions of alloys patented by ORNL. TEM and APT analysis of the microstructure of the alloys before deformation was conducted to first understand the phase equilibria. The SEM, STEM, and APT results for the microstructural characterization and precipitate structures of one such alloy received are shown in Figure I.3.4.7 (a)-(f).
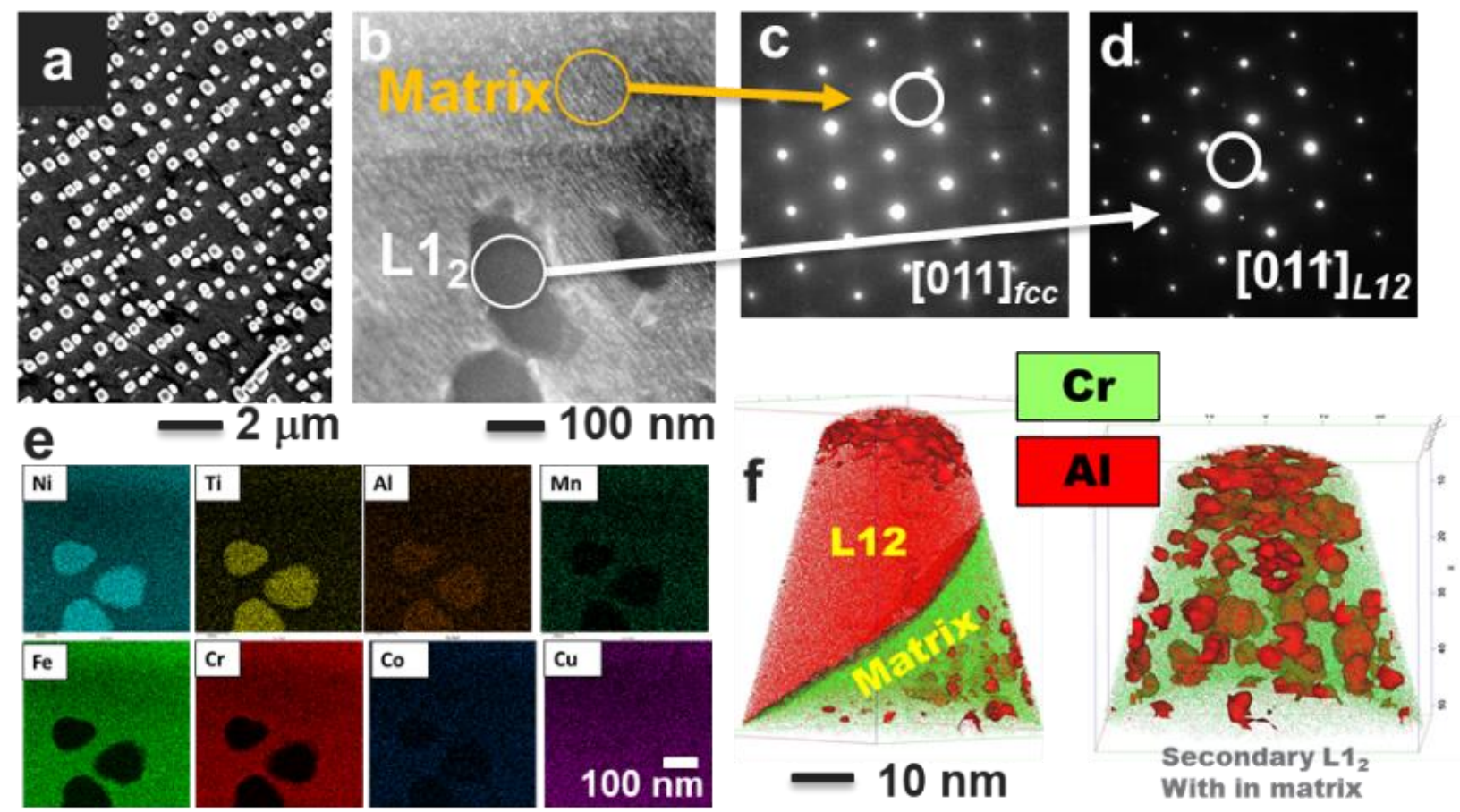

Figure I.3.4.7. Microstructural characterization results of HCC1163-2018-3 Aged $+250 \mathrm{~h}$ at $900^{\circ} \mathrm{C}$. (a) SEM image of the overall microstructure. (b) STEM image of the alloy showing matrix and L12 precipitates from which selected area diffraction patterns given in (c) and (d) where obtained. (e) STEM-EDS maps showing element portioning. (f) APT results showing primary and secondary L12 precipitates. Source: PNNL.

\section{APT and AC-STEM - A. Devaraj, B. Gwalani, and L. Kovarik, PNNL†††}

\section{Development of Cast, Higher Temperature Alloys}

In support of subtask 2B1, this work focused on the detailed microstructural characterization of cast aluminaforming alloys (e.g., subtasks AFA5 and AFA2) of austenitic stainless steels that were creep-rupture tested at $900^{\circ} \mathrm{C}$ and $50 \mathrm{MPa}$. In these AFA alloys with high creep performance, multiple strengthening second-phase precipitates were identified in the FCC-Fe matrix. SEM imaging, SEM-EDS, STEM imaging, STEM-EDS, and selected area diffraction in TEM permitted the analysis of multiple phases present in these alloys as shown in the case of AFA5 alloy in Figure I.3.4.8 (a)-(f). 

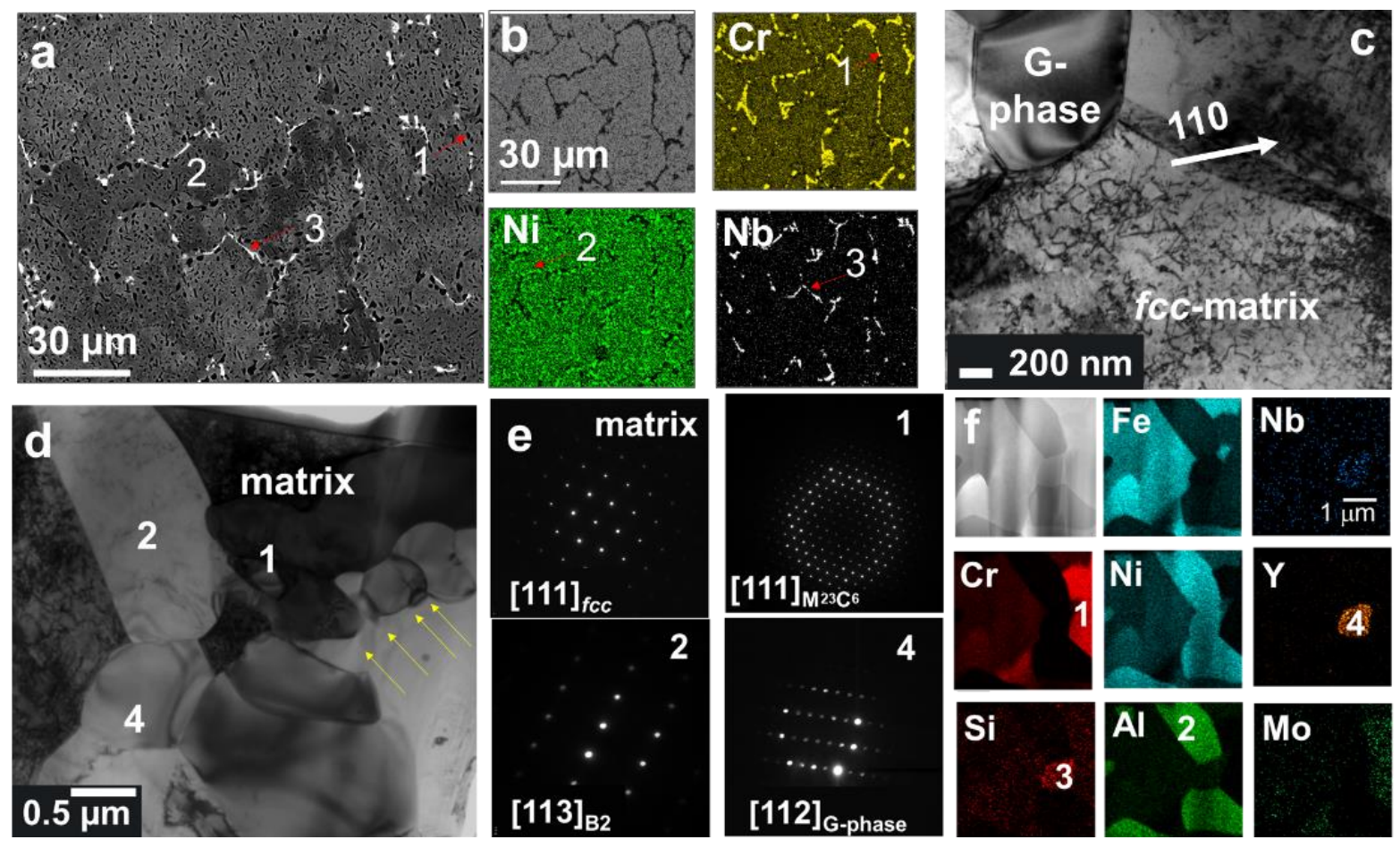

Figure I.3.4.8. (a) SEM image of the AFA5 alloy showing the different precipitates. (b) SEM-EDS image showing solute partitioning. (c) TEM weak beam dark field imaging identifying dislocation structures. (d) STEM image of dislocation structures. (e) Area diffraction from different phases in the alloy corresponding to the numbers in (d). (f) STEM-EDS mapping showing the compositional partitioning between phases. The different phases are numbered 1 , 2, 3, and 4 , respectively. Source: PNNL.

\section{Subtask 4B: Advanced Computation (ORNL) - Task PI: Dongwon Shin}

Task 4B involved the following Co-Principal Investigators at ORNL: Balasubramaniam Radhakrishnan, Xiahoua Hu, Rishi Pillai, Ying Yang, and Yousub Lee. These investigators are identified with their appropriate subtasks, research capabilities and contributions along with the location of the resources used and identified in the Acknowledgements $(\dagger)$.

\section{Phase-Field Modeling - B. Radhakrishnan, ORNL††††}

In support of subtask 3A1, the goal is to utilize HPC tools to perform phase-field simulations of the microstructure evolution in Al-Cu-X structural alloys during AM that includes the solidification microstructure, as well as the microstructures arising from solid-state precipitation during subsequent heattreatment. The simulations were performed using the Microstructure Evolution Using Massive Parallel Phasefield Simulations (MEUMAPPS) code developed in-house under ORNL funding through the internal Seed Program and later the ExaAM Exascale Computing Project. The code will capture the formation of columnar or equiaxed dendritic microstructure and the resulting solute micro-segregation in multicomponent $\mathrm{Al}-\mathrm{Cu}$ based alloys during AM as well as the morphological evolution of the $\theta^{\prime}$ and $\theta$ precipitates during post-AM heat-treatments. The phase-field simulations will be integrated with CALPHAD or DFT based descriptions of Gibbs free energies of evolving phases and with alloy-specific interfacial and kinetic parameters generated by appropriate lower length scale simulations. Preliminary simulations shown in Figure I.3.4.9 of the precipitation of the $\theta^{\prime}$ phase in Al-Cu using the MEUMAPPS code was demonstrated in Summitdev [4] using 256 cores Larger simulations are currently being performed in Summit. A user account was obtained in the Theta supercomputer at the Argonne Leadership Class Facility and simulations of $\theta^{\prime}$ precipitation and the formation of $\theta$ precipitates from $\theta^{\prime}$ with or without the presence of external strains will be performed. The ability of MEUMAPPS to perform full three-dimensional simulations of the formation of $\theta^{\prime}$ variants in Al-Cu binary has been demonstrated using HPC. 


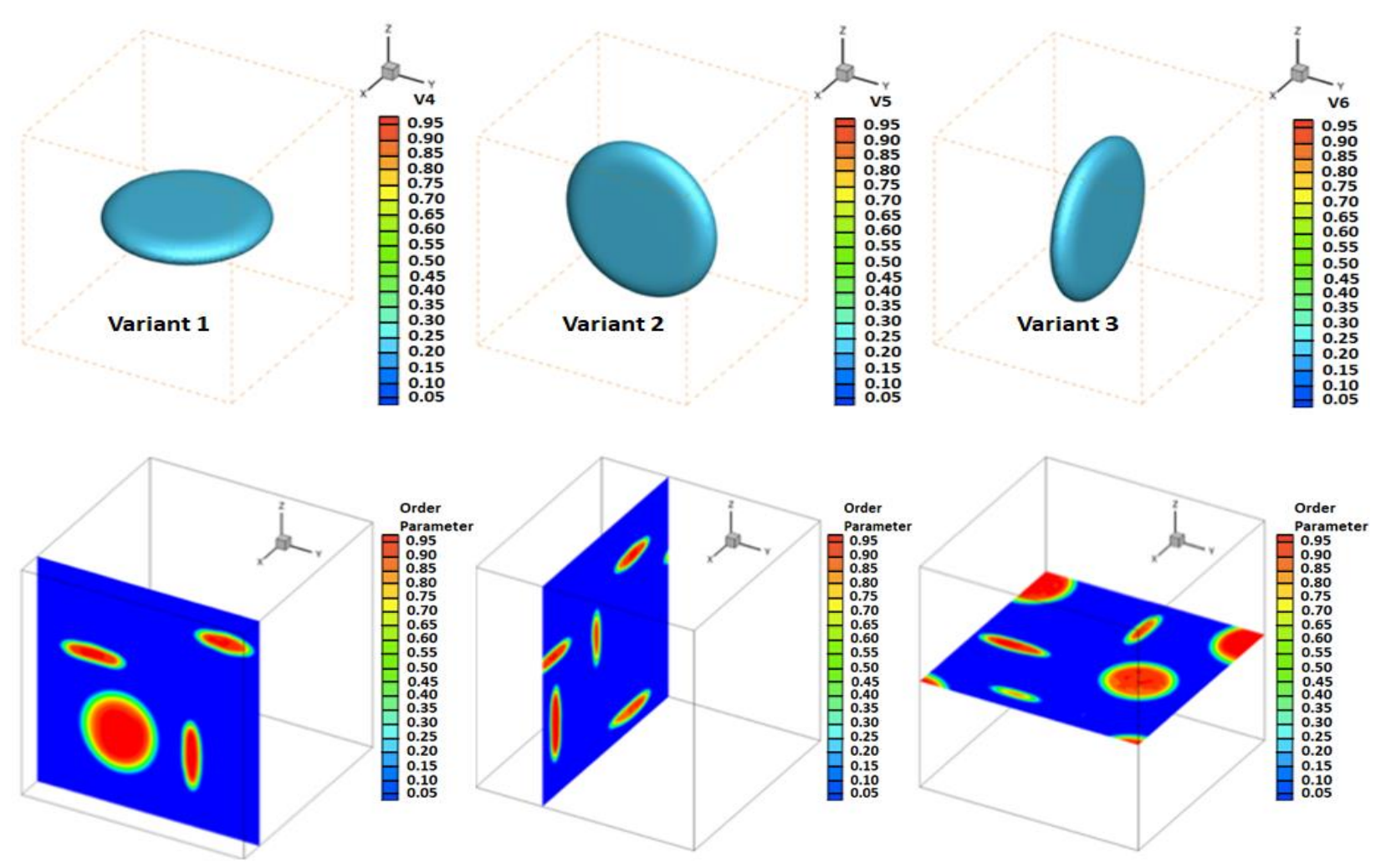

Figure I.3.4.9. Three-dimensional simulations of the precipitation of $\theta^{\prime}$ phase in Al-Cu binary alloy performed using MEUMAPPS in ORNL Summitdev [4]. Source: ORNL.

\section{Thermodynamic-Kinetic Simulations of High-temperature Coatings - R. Pillai, ORNL}

\section{High-Temperature Coatings}

In support of subtask 2A3, the goal of this subtask is the evaluation of the compatibility of protective coatings (e.g., overlay $\mathrm{NiCrAl}$ and diffusion slurry aluminides) as a solution to enable $900-950^{\circ} \mathrm{C}$ adoption of ORNL's cost-effective (lower $\mathrm{Ni} / \mathrm{Co}$ ) chromia-forming valve alloys, which possess outstanding strength to $950^{\circ} \mathrm{C}$, but have inadequate oxidation resistance for such extreme temperatures.

The objective of the computational effort is to predict the evolution of diffusion aluminide coatings during manufacturing and subsequent high-temperature exposure as a function of coating process parameters (e.g., $\mathrm{Al}$ activity, heat-treatment conditions) and exposure conditions (e.g., time, temperature, atmosphere, thermal cycling). This will enable the optimization of coating parameters and assist in the rapid evaluation of coating/substrate compatibilities.

Slurry aluminide coatings were deposited on Ni-base alloys $31 \mathrm{~V}$ and on two $\mathrm{NiCrFe}$ alloys developed at ORNL. Coupled thermodynamic-kinetic simulations were performed to predict the compositional evolution in the alloys during the coating manufacturing and during subsequent high-temperature exposures between $800-950^{\circ} \mathrm{C}$ under thermal cyclic conditions in air $+10 \% \mathrm{H}_{2} \mathrm{O}$.

A coupled thermodynamic-kinetic model was employed to predict the coating microstructure during aluminizing and during high-temperature exposures as a function of time, temperature, and base alloy composition. Figure I.3.4.10 (a) shows the predicted phase distribution in the coating and IDZ. The element maps and EBSD phase map in Figure I.3.4.10 (b) are consistent with the model predictions. 


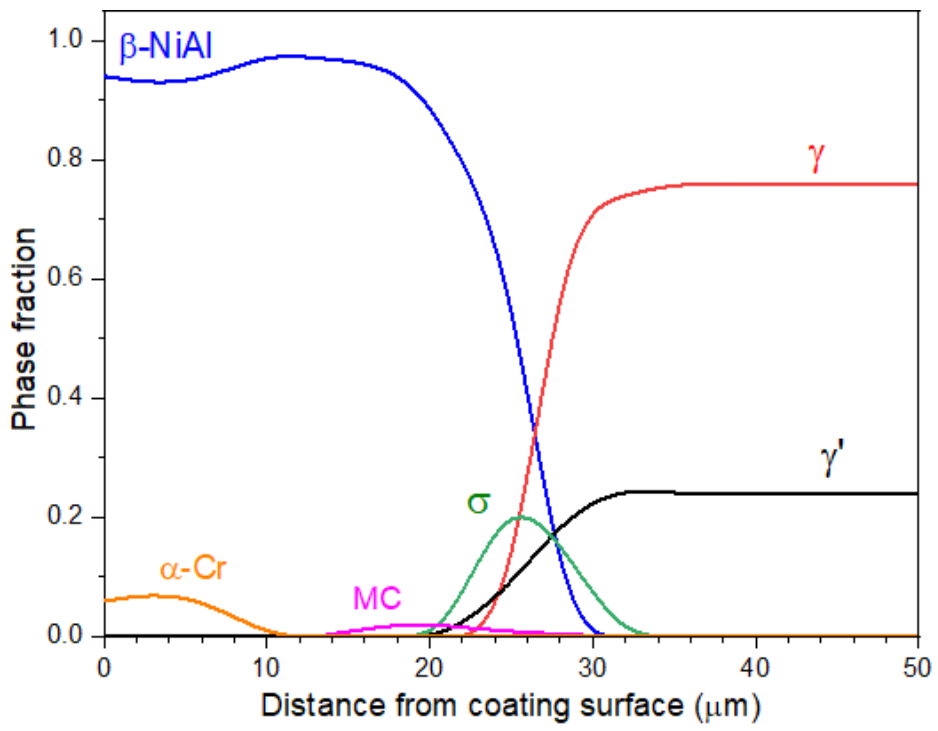

(a)
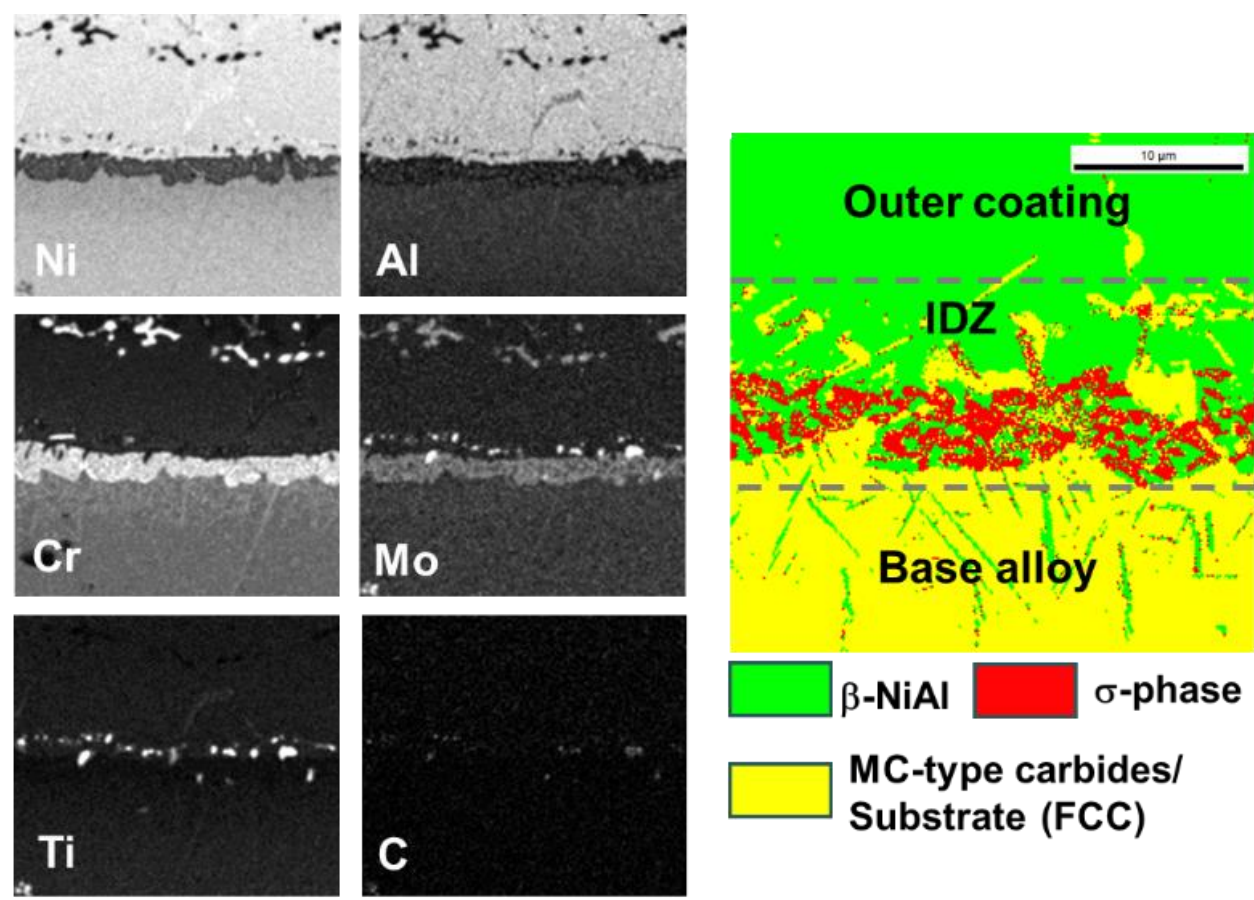

(b)

Figure I.3.4.10. (a) Calculated phase distribution for the aluminized $31 \mathrm{~V}$ specimen after aluminizing at $1100^{\circ} \mathrm{C}$ for $3 \mathrm{~h}$ followed by a heat-treatment at $850^{\circ} \mathrm{C}$ for $4 \mathrm{~h}$ and at $730^{\circ} \mathrm{C}$ for $4 \mathrm{~h}$ and (b) elemental and phase mappings of the crosssection of the corresponding aluminized 31V. Source: ORNL.

The model was able to predict the coating microstructure during aluminizing of different alloys for different heat-treatment conditions. The simulation with different heat-treatment conditions and alloy chemistries will be performed to optimize the coating process and predict detrimental phase formations (i.e., potential impact on mechanical properties) during manufacturing and high-temperature exposures. 


\section{Microstructure-based FE models of ACMZ - X. Hu, ORNL}

\section{Intermediate Temperature Variants of Cast ACMZ Alloys}

These studies supported the subtask $1 \mathrm{~A} 2$ for new higher temperature performance of $\mathrm{Al}$ alloys $\left(>400^{\circ} \mathrm{C}\right.$ for turbo and piston applications). The objective of this simulation effort is to understand the contributions of the theta phase particles to the ductility of the ACMZ alloys with different $\mathrm{Cu}$ content through a microstructurebased FE model. The ABAQUS/Explicit FE package is used, and the model is based on the stitched SEM montage micrographs of the ACMZ alloys. The micrographs with dimensions of $1.15 \times 0.73 \mathrm{~mm}^{2}$ shown in Figure I.3.4.11 (a) are directly mapped into the FE model shown in Figure I.3.4.11 (b).

In the model, the matrix is assumed to be isotropic elastoplastic with the experimentally measured Young's modulus of $65 \mathrm{GPa}$. The flow behavior follows a power law:

$$
\bar{\sigma}=677 \bar{\varepsilon}^{0.178}
$$

where $\bar{\sigma}$ is the flow stress and $\bar{\varepsilon}$ is the equivalent plastic strain. The parameters in this equation are determined by fitting to the stress-strain curve of $6.0 \mathrm{Cu}$ alloy. The critical value of strain for the local matrix fracture was calibrated by extensive parametric studies and determined to be 0.18 . The particles are assumed to be pure elastic with a Young's modulus (E) of $120 \mathrm{GPa}$. Brittle fracture is considered for the particles as determined experimentally and the element removal is determined by the criterion of whether the hydrostatic tensile stress reached a critical value. The value was chosen to be $710 \mathrm{MPa}$ after the parametric studies.

The FE simulations of different $\mathrm{Cu}$ contents ranging from $6 \%$ to $9 \%$ were performed, as seen in Figure I.3.4.11 (c), which indicate that the ductility of the materials decreases with $\mathrm{Cu}$ content are consistent with experimental results. The lower ductility in higher $\mathrm{Cu}$ alloy is attributed to the high-volume fraction, larger sizes, and complex shape of the hard and brittle particles. The larger particle tends to crack early and there is a smaller distance of crack propagation between particles with high particle volume fractions.
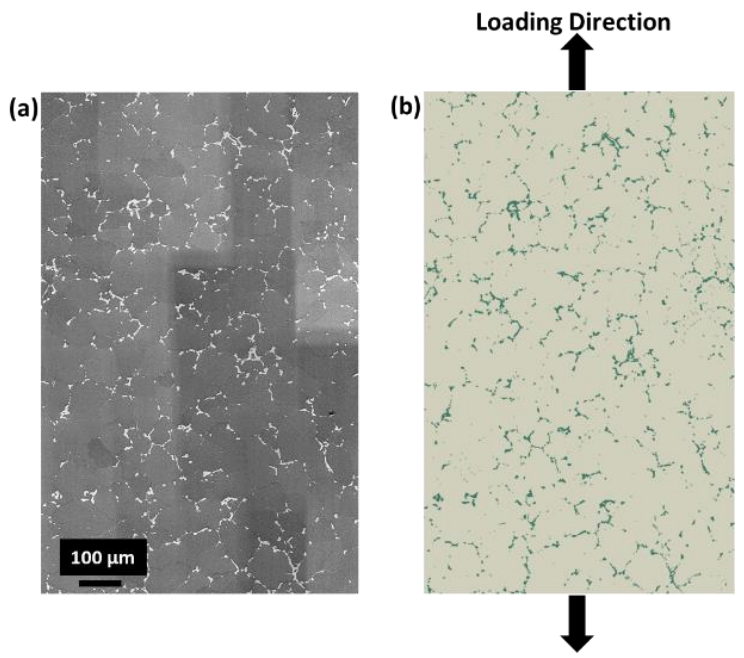

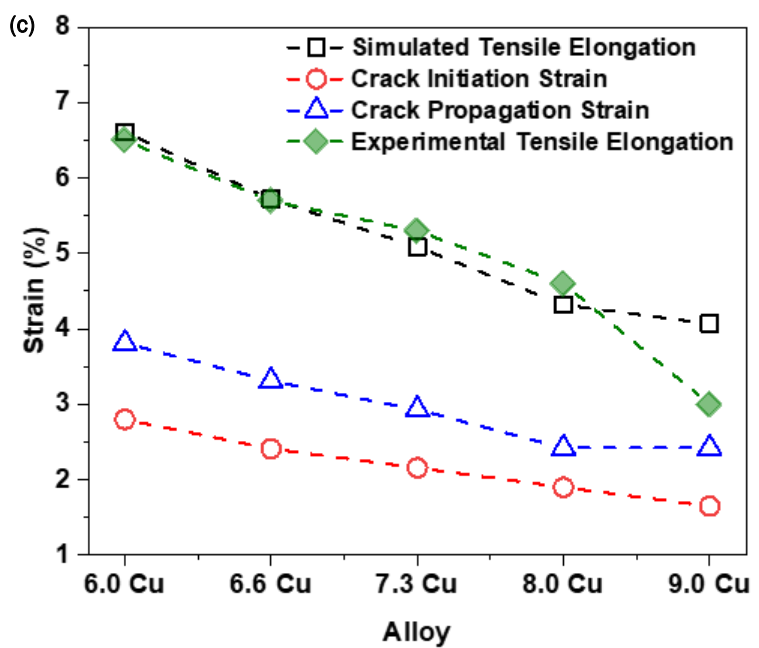

Figure I.3.4.11. (a) The stitched SEM images of a 9\% Cu AZMZ alloy. (b) The corresponding microstructure-based FE model. (c) The comparison of the simulation results of engineering strain at failure with those of the experiments. Source: ORNL.

\section{FE modeling of AMIPC - X. Hu, ORNL}

Development of AMIPCs

In support of subtask 3A2, A356/316L interpenetrating phase composites (AMIPCs or hybrid manufacturing/composites) were fabricated by infiltrating additively manufactured 316L lattices with A356 
cast infiltration and have shown diverse mechanical properties controllable by adjusting the volume fraction and/or topology of $316 \mathrm{~L}$ reinforcement. For example, under uniaxial tension, the composite versions with 40 vol\% 316 showed two times higher ductility and strength compared to the 20 vol\% 316L sample at the cost of $27 \%$ density increase. Transition from localized deformation and fracture to delocalized damage is accompanied with four times the increase in energy absorption capacity. Understanding the transition mechanism and designing composite systems with optimized properties is a key goal, with high-fidelity numerical analysis and simulations as key enablers. The objective of this work is to develop a FE-based mechanical model to capture the properties of AMIPC at different structures, volume fractions, and microstructures. The validated simulation also makes possible the fabrication of hybrid structural components with tailored properties and weights by printing spatially varying $316 \mathrm{~L}$ reinforcement.

Considering the individual properties of A356 and 316L, as well as their interactions at the interface, FE models were conducted for the elastoplastic deformation and cracking of AMIPC. The simulation results are being compared to experiments, including the neutron diffraction results of micro stress-strain and in situ digital image correlation to study the mechanisms that control composite properties.

The simulation effectively captures the transition from low ductility due to localized cracks in low 316L lattice volume fraction samples to higher ductility (i.e., delocalized cracks) at higher 316L lattice volume fraction samples. Very localized stress concentration and crack formation is observed for a $20 \% 316 \mathrm{~L}$ volume fraction model shown in (a)

Figure I.3.4.12 (a). Higher ductility with delocalized cracks formation in the homogeneous (along loading axial) stress distribution is captured in 50\% 316L volume fraction model shown in (a) Figure I.3.4.12 (b). Comparison of macroscopic stress-strain curves are well matched between the experiment and the simulation and is shown in (b)

Figure 1.3.4.12 (c). A FE analysis indicates the brittle A356 matrix fracturing at a very early stage triggers the crack initiation. The stress will then concentrate around the initial crack. If the strain-hardening rate from the lattice ligament is higher than the increased stress concentration (i.e., $\mathrm{d} \sigma / \mathrm{d} \varepsilon \geq \sigma$ ), the deformation is stable and remains uniform, then further cracks at the other location will occur without breaking the material. If the volume fracture of $316 \mathrm{~L}$ lattice is low, its hardening rate is not able to keep up with the stress concentration, thus leading to concentrated deformation and a localized crack.

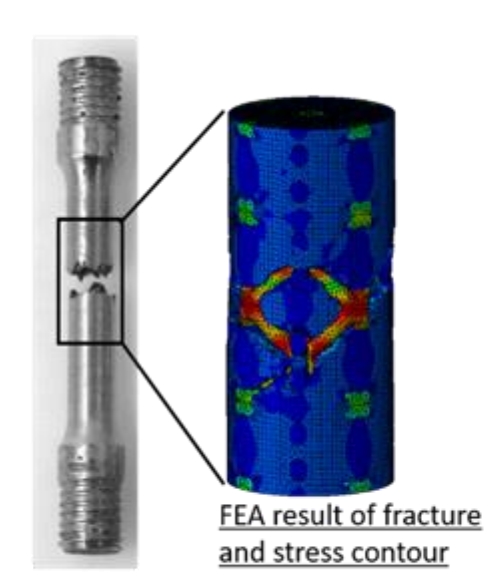

AMIPC sample of 20 vol\% $\underline{316 \mathrm{~L} \text {, after fracture }}$

(a)

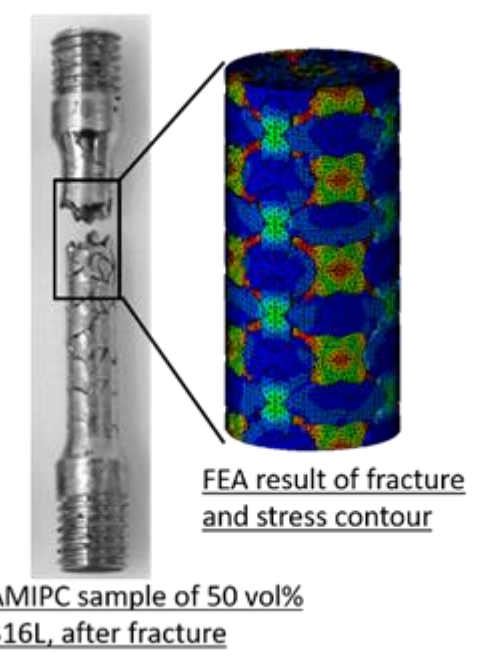

(b)

$\frac{\text { AMIPC sample of } 50 \text { vol\% }}{316 \mathrm{~L}, \text { after fracture }}$

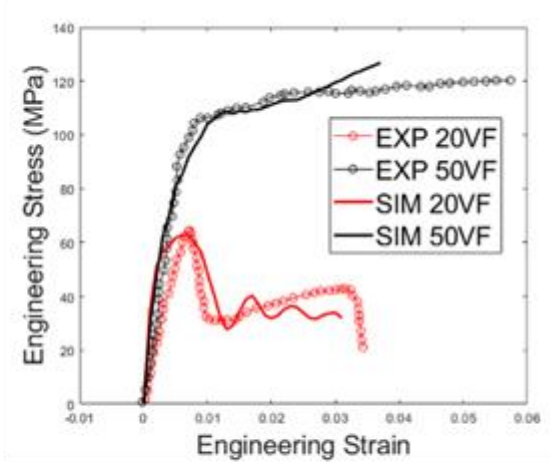

Comparison of stress-strain curves between simulation and experiment, well matched. (c)

Figure I.3.4.12. Comparison of the FE analysis and experimental results for body-centered lattice structure AMIPC at (a) 20 vol\%; and (b) 50 vol\%. (c) Stress-strain curves for simulated and experimental results. Source: ORNL. 
The comparison of micro stress-strain and hydrostatic stress in each individual phase are also well matched. The results show that the $316 \mathrm{~L}$ lattice is mainly withholding the tensile load, while the A356 matrix is actually in a compressive state, which helps to absorb the energy, therefore giving the material higher energy absorption properties.

\section{Data Analytics for Machine-learning to Accelerate Alloy Development - M. Lee, ORNL}

\section{Development of Cast Higher Temperature Austenitic Alloys}

These efforts supported the subtask 2B1 for development of cast, higher temperature alloys. We aimed to better understand oxidation behavior using data science and machine-learning techniques. First, we performed correlation analysis with existing alumina-forming austenitic alloy oxidation datasets using various correlation coefficients, such as PCC [5], MIC [6] and Spearman's Coefficient. One example for the PCC is shown in Figure I.3.4.13. The analysis has been performed with and without computational thermodynamics features. The results have been closely reviewed by domain experts. Second, we developed a web-based interactive and exploratory oxidation data analysis tool that allows users to explore various scientific features and their correlation with oxidation. The tool specifically provides various criteria to filter out and/or rank features so that domain experts can quickly identify important scientific features that need to be further investigated.

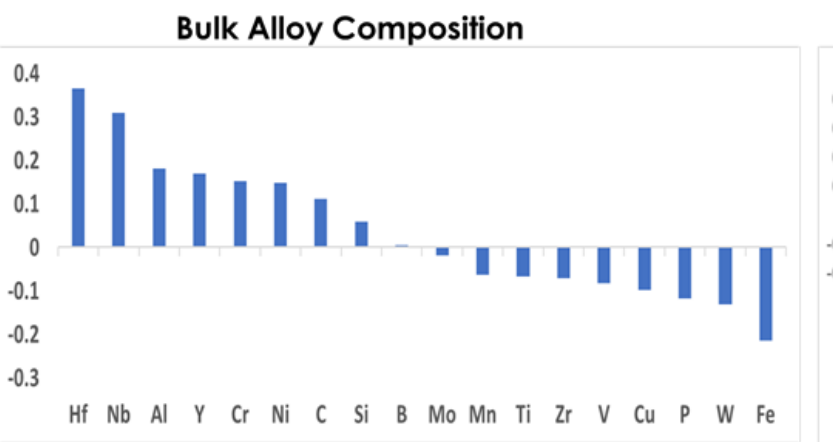

(a)

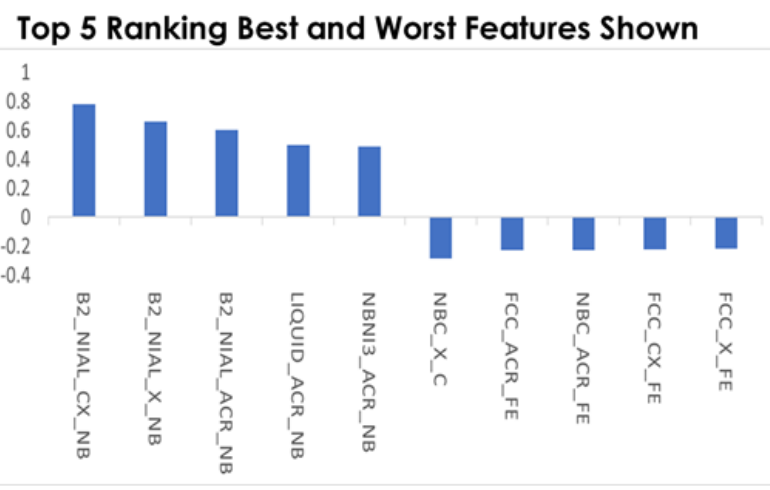

(b)

Figure I.3.4.13. Pearson Correlation Coefficient plots for: (a) bulk alloy composition; and (b) the top five best and worst computational features. Source: ORNL.

In Figure I.3.4.13 (a), computational thermodynamics features associated with $\mathrm{Nb}$ rank high (better oxidation) and with Fe rank low (worse oxidation), which confirms that the correlation analysis result nicely picks up bulk alloy composition oxidation trends. In Figure I.3.4.13 (b), the results show there are multiple scientific features that have not yet been closely investigated but correlate highly with oxidation (such as phase fraction, solubility of alloying elements in various phases, etc.), which can provide insights for better understanding oxidation mechanisms.

\section{Computational Thermodynamics for AM of Aluminum Alloys - Y. Yang, ORNL}

\section{Fundamental Development of Aluminum Alloys for AM}

In support of subtask 3A1, this task aims to develop thermodynamic models for the Al-Ce- $\mathrm{X}$ (X=Mn, $\mathrm{Mg}, \mathrm{Cu}$, $\mathrm{Ni}, \mathrm{Fe}$ ) system and then calculate phases presented in Scheil-solidification and equilibrium microstructure using computational thermodynamics tools to help design and understand the microstructure of AM novel Al$\mathrm{Ce}-\mathrm{X}$ alloys being developed under Task 3 within this program. 
Using the developed tools, we designed a series of Al-Ce-X alloys. Figure I.3.4.14 (a)-(c) shows one example. In this figure, $\mathrm{Al}-\mathrm{Ni}-\mathrm{Ce}, \mathrm{Al}-\mathrm{Fe}-\mathrm{Ce}$ and $\mathrm{Al}-\mathrm{Fe}-\mathrm{Ni}-\mathrm{Ce}$ alloys with $25 \%, 50 \%$, and $75 \%$ eutectic composition were designed, respectively. The calculated alloy compositions and melting temperature were provided to alloy developers together with the phase amount present in these alloys if aged at $500^{\circ} \mathrm{C}$. Computational thermodynamics tools were used to identify the eutectic reactions and their volume fractions, which were then used to guide the design of Al-Ce-X alloys appropriate for AM feedstock.

$\mathrm{Al}-\mathrm{Fe}-\mathrm{Ce}$

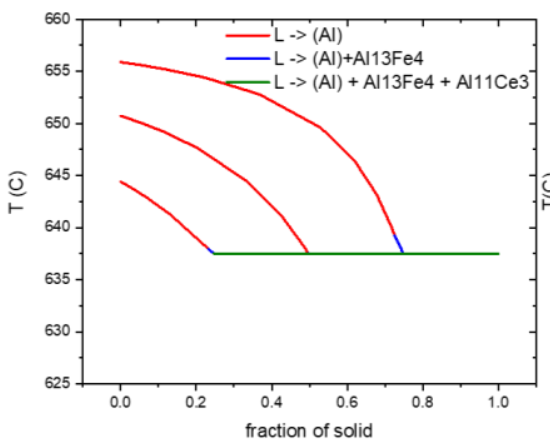

(a)
$\mathrm{Al}-\mathrm{Ni}-\mathrm{Ce}$

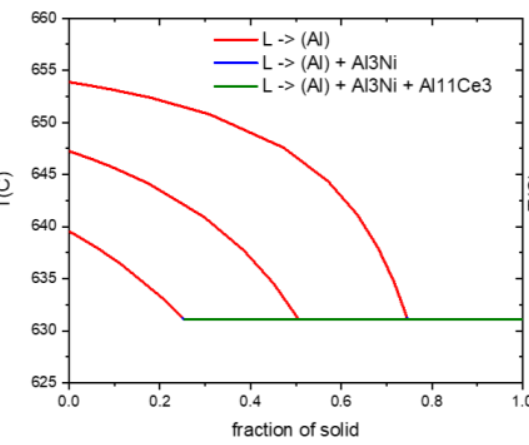

(b)
Al-Ni-Fe-Ce

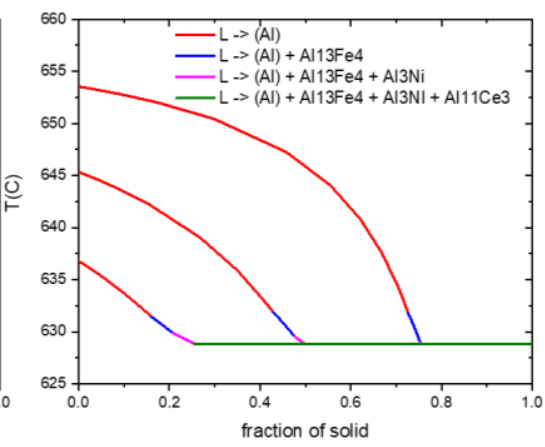

(c)

Figure I.3.4.14. Solidification path simulation of: (a) Al-Fe-Ce; (b) Al-Ni-Ce; and (c) Al-Fe-Ni-Ce showing 25, 50, and 75 mol\% eutectic in as-solidified microstructure. Source: ORNL.

\section{Scheil-Solidification Simulations for AM - Y. Yang, ORNL}

Fundamentals of Austenitic Alloy Processing by AM

In support of subtask 3B1, this computational activity performed Scheil-solidification and equilibrium microstructure simulations for various steels using computational thermodynamics tools to help understand the microstructure of various steels processed via AM. We calculated the solidification path and equilibrium microstructure for the alloys listed in Table I.3.4.2 using an in-house steel database. Typical calculation results are shown in Figure I.3.4.15 (a)-(c) using CF8C+ as an example. Computational thermodynamics tools have been used in helping the selection of solidification temperature and AM of austenitic steels.

Table I.3.4.2. Chemical Compositions of Alloys for Solidification and Phase Equilibrium Simulation.

\begin{tabular}{|c|c|c|c|c|c|c|c|c|c|c|c|c|c|c|c|}
\hline & $\mathrm{Cr}$ & $\mathrm{Ni}$ & Mn & Mo & $\mathrm{Cu}$ & Si & $\mathrm{Nb}$ & W & C & $\mathbf{N}$ & $P$ & $\mathbf{S}$ & $\mathrm{Fe}$ & Al & B \\
\hline CF8C+ & 19.5 & 12.6 & 3.9 & 0.31 & 0.49 & 0.45 & 0.91 & & 0.09 & 0.25 & 0.019 & 0.006 & bal & & \\
\hline $\mathrm{CF} 8 \mathrm{C}+\mathrm{W} / \mathrm{Cu}$ & 19.5 & 12.1 & 3.8 & 0.3 & 3.4 & 0.53 & 0.94 & 0.97 & 0.08 & 0.3 & 0.004 & 0.005 & bal & & \\
\hline $\begin{array}{c}\text { CF8C+W/Cu } \\
20 / 15\end{array}$ & 20.3 & 14.9 & 3.8 & 0.34 & 3 & 0.53 & 0.94 & 0.98 & 0.07 & 0.1 & 0.018 & 0.004 & bal & & \\
\hline $\begin{array}{c}\text { CF8C+W/Cu } \\
21 / 17\end{array}$ & 22.2 & 17.1 & 3.7 & 0.32 & 2.1 & 0.65 & 0.81 & 0.96 & 0.1 & 0.2 & 0.024 & 0.005 & bal & & \\
\hline AFA/OC4 & 14 & 25 & 2 & 2 & & & 2.5 & 1 & 0.1 & & & & bal & 3.5 & 0.01 \\
\hline
\end{tabular}


Equilibrium phases

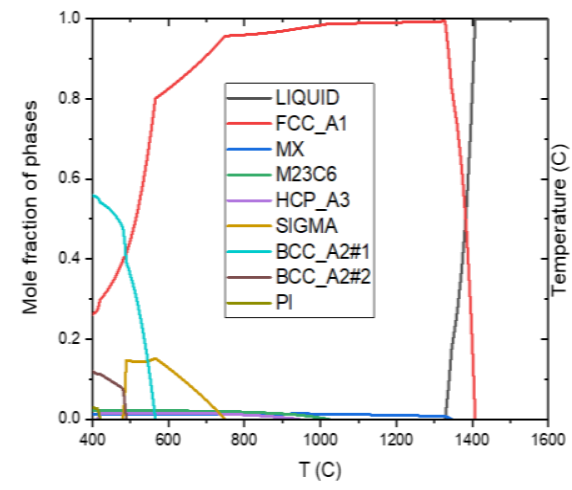

Solidification path

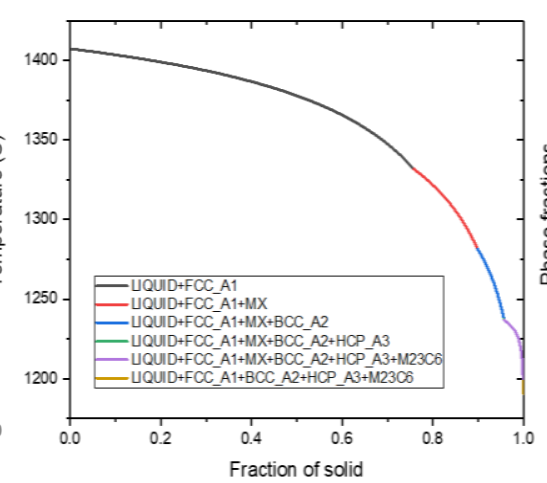

Solidification phases

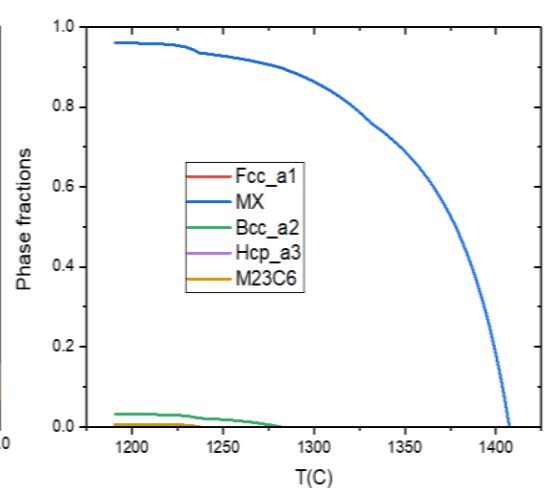

Figure I.3.4.15. The Scheil-solidified microstructure and equilibrium microstructure of a CF8C-Plus alloy for (a) the equilibrium phases; (b) the solidification path; and (c) the solidification phases. Source: ORNL.

FE Analysis of Ferritic Alloys for HD Piston via AM - Y. Lee, ORNL

Ferritic Alloys for HD Pistons Using AM

Interest in binder-jetting is growing, but the widespread use of this technology is hindered by the lack of material properties and modeling tools available to accurately predict a part shrinkage during sintering up to $40 \%$. In support of subtask 3B2, this modeling work is key to demonstrate the industrial potential of binderjetting. The objective of this task is to understand the part shrinkage mechanism during sintering and predict the final part geometry fabricated by binder-jetting.

ORNL has made significant advances toward a fundamental understanding of the sintering process, as well as FE simulation to predict component distortion. The approaches used for this task are to: (1) down-select and develop an applicable constitutive model for binder-jetting ferritic steels; (2) construct a FE modeling framework using our constitutive model; and (3) determine the most critical subcomponent model to accurately predict the shape and density of the final product.

Part shrinkage was effectively predicted using FE modeling. The cubic sample has dimensions of $20 \mathrm{~mm} \times$ $20 \mathrm{~mm} \times 20 \mathrm{~mm}$ in the green part before densification. The part shrank uniformly by $16 \%$ at all cube edges after sintering. Figure I.3.4.16 (a) shows the shrinkage in the FE model along the z-direction in comparison of the original to sintered geometry. The dimensional change of the cube in the green and sintered part was illustrated in Figure I.3.4.16 (b). About $3 \mathrm{~mm}$ of shrinkage in all three directions occurred during sintering. The simulation predicts the shrinkage with less than $0.5 \%$ error in the $y$ - and z-directions, but $1.7 \%$ in the $\mathrm{x}$-direction in Figure I.3.4.16 (c). The results show that the phenomenological simulation reasonably well predicts the part shrinkage for the simple cube geometry. The simulation predicts the deformation reasonably well within approximately $2 \% \sim 7 \%$ error at the temperature ranges of $1340^{\circ} \mathrm{C}$ to $1380^{\circ} \mathrm{C}$. However, the model overpredicted it by about $15 \%$ at $1400^{\circ} \mathrm{C}$. The same trend is observed in the relative density prediction in Table I.3.4.3. The predicted relative density is well aligned with the measured one at $1340^{\circ} \mathrm{C} \sim 1380^{\circ} \mathrm{C}$ but underpredicted only at $1400^{\circ} \mathrm{C}$. The error may be attributed to inhomogeneous packing density at the upper and lower regions in the green part that results in a non-uniform sintering rate. 
(a)

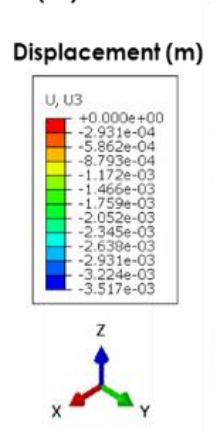

(b)

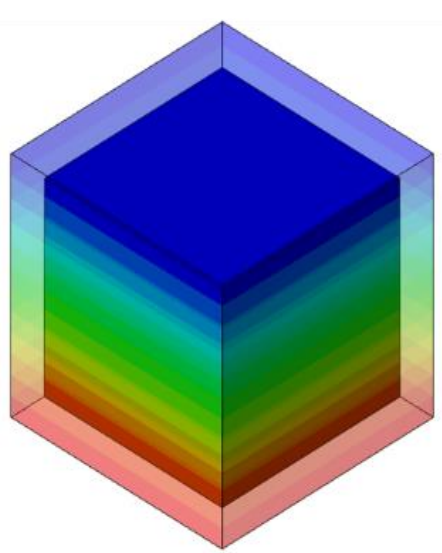

(c)

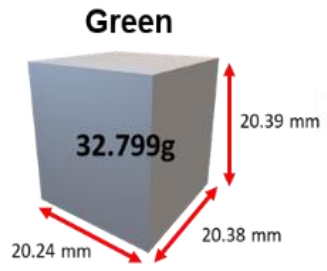

Sintered

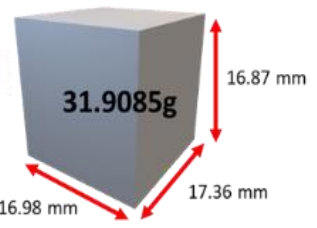

\begin{tabular}{|c|c|c|}
\hline Exp. & Sim. & \%Error \\
\hline 16.98 & 17.06 & $0.48 \%$ \\
\hline 17.36 & 17.06 & $-1.72 \%$ \\
\hline 16.87 & 16.80 & $-0.40 \%$ \\
\hline
\end{tabular}

Figure I.3.4.16. (a) Predicted part shrinkage for the cube shape. (b) The part shrinks about $3 \mathrm{~mm}$ in x-, y-, and z-direction. (c) The phenomenological model predicts the shrinkage within minor error. Source: ORNL.

Table I.3.4.3. Comparison of the Predicted Relative Density to the Experiment after Sintering.

\begin{tabular}{|c|c|c|c|}
\hline Temperature $\left({ }^{\circ} \mathbf{C}\right)$ & Experiment (\%) & Prediction (\%) & Error (\%) \\
\hline 1340 & 82.8 & 81.1 & -2.05 \\
\hline 1360 & 89.6 & 83.1 & -7.25 \\
\hline 1380 & 88.5 & 84.9 & -4.07 \\
\hline 1400 & 100.3 & 85.3 & -14.9 \\
\hline
\end{tabular}

The FE model simulation successfully predicted the part distortion and relative density in most cases within approximately $3 \%$ and $7 \%$ error range, respectively. However, the distortion and relative density was underpredicted at $1400^{\circ} \mathrm{C}$. This discrepancy is potentially attributed to non-uniform packing density over the green part. Further study is required to improve the model accuracy above $1400^{\circ} \mathrm{C}$.

\section{Conclusions}

The advanced characterization and computation efforts have supported the three long-term alloy development tasks. Advanced microscopy and APT of Al- and Ni-based alloys have located key strengthening precipitates and interfaces and identified their crystallographic orientations, morphologies and chemistries. Synchrotron $\mathrm{x}-$ rays were used to identify precipitate phases and sizes using diffraction and small angle $\mathrm{x}$-ray scattering.

Modeling and simulations predicted and guided alloy research efforts and understandings, particularly in area of AM. Phase-field, thermodynamic and kinetic simulations predict the microstructural features and evolution in Al- and Ni-based alloys, critical to save processing steps and time. FE and crystal plasticity models predict the mechanical behavior of composites and hybrid structures made via AM to guide future builds and for comparison to mechanical characterizations.

\section{Key Publications}

1. Allard, L. F., D. N. Leonard, J. D. Poplawsky, M. F. Chisholm, B. D. Eckhart, A. Shyam, F. S. Walden, B. B. Larson, R. Kelly, A. Stokes, and W. C. Bigelow, 2019, "The utility of Xe-plasma FIB for preparing $\mathrm{Al}$ alloy specimens for MEMS-based in situ double-tilt heating experiments," Microsc. Microanal., Vol. 25 (Suppl. 2), pp. 1442-1443. 


\section{References}

1. Shyam, A., S. Roy, D. Shin, J. D. Poplawsky, L.F. Allard, Y. Yamamoto, J. R. Morris, B. Mazumder, J. C. Idrobo, A. Rodriguez, T. R. Watkins, and J. A. Haynes, 2019, "Elevated temperature microstructural stability in cast AlCuMnZr alloys through solute segregation," Mat. Sci. Eng. A-Struct., Vol. 765, Art. \#138279.

2. Unocic, K. A., M. J. Mills, and G. S. Daehn, 2010, "Effect of gallium FIB milling on preparation of Al thin foils," J. Microsc., Vol. 240, No. 3, pp. 227-238.

3. Shower, P., J. Morris, D. Shin, B. Radhakrishnan, J. Poplawsky, and A. Shyam, 2019, "Mechanisms for stabilizing precipitates at elevated temperatures investigated with phase-field modeling," Materialia, Vol. 6, Art. \#100335.

4. ORNL, 2019, “Summitdev Quickstart Guide,” https://www.olcf.ornl.gov/for-users/system-userguides/summitdev-quickstart-guide/.

5. Benesty, J., J. Chen, Y. Huang, and I. Cohen, 2009, "Noise reduction in speech processing," in Pearson Correlation Coefficient, Springer, Berlin, Heidelberg, pp. 1-4.

6. Kinney, J. B., and G. S. Atwal, 2014, "Equitability, mutual information, and the MIC," Proc. Natl. Acad. Sci., Vol. 111, No. 9, pp. 3354-3359.

\section{Acknowledgements}

$\uparrow$ This research used the Center for Nanophase Materials Sciences (CNMS) resources at ORNL, which is a U.S. DOE Office of Science User Facility.

$\dagger \uparrow$ This research used the APS resources at ANL, a DOE Office of Science User Facility under Contract No. DE-AC02-06CH11357.

$+\dagger \dagger$ The research was performed at the Environmental Molecular Sciences Laboratory, a national scientific user facility sponsored by the DOE Office of Biological and Environmental Research and located at PNNL.

$+1 \dagger \dagger$ This research used resources of the Oak Ridge Leadership Computing Facility, which is a DOE Office of Science User Facility supported under Contract DE-AC05-00OR22725. 


\title{
I.3.5 Exploratory Research - Thrust 5 (Oak Ridge National Laboratory) \\ I.3.5.1 Novel Materials for Polymer Composite Engine Blocks (Oak Ridge National Laboratory)
}

\author{
Chemical Sciences Division \\ 1 Bethel Valley Road \\ Oak Ridge, TN 37831 \\ E-mail: naskarak@ornl.gov
}

Amit K Naskar, Co-Principal Investigator

\author{
Vlastimil Kunc, Co-Principal Investigator \\ Energy and Transportation Science Division \\ 1 Bethel Valley Road \\ Oak Ridge, TN 37831 \\ E-mail: kuncv@ornl.gov
}

\author{
Brian Knouff, Co-Principal Investigator \\ Energy and Transportation Science Division \\ 1 Bethel Valley Road \\ Oak Ridge, TN 37831 \\ E-mail: knouffbj@ornl.gov
}

\section{Jerry L. Gibbs, DOE Technology Development Manager}

U.S. Department of Energy

E-mail: jerry.gibbs@ee.doe.gov

Start Date: April 1, 2019

Project Funding (FY19): \$150,000
End Date: March 31, 2020

DOE share: $\$ 150,000 \quad$ Non-DOE share: $\$ 0$

\section{Project Introduction}

This PMCP Thrust 5 research effort conducts preliminary R\&D to develop novel PMC systems for LD internal combustion engine block applications targeting a 40\% reduction in component weight, a 30\% reduction in vehicle emissions, and a 50\% reduction in engine noise (noise, vibration and harshness) relative to conventional cast metal castings. Although PMC engine blocks are expected to exhibit promising results, there are no material systems specifically designed for this application. Thermomechanical properties of most polymer materials and second-phase reinforcements are not suited to engine block requirements. Typical PMC materials are particularly challenged by elevated temperature creep and fatigue operation, as well as by demands on efficient heat transfer. It is likely the PMC engine block will have to contain some metallic or ceramic structures, primarily in areas of high wear.

Experiments with PMC engine blocks focused primarily on race car requirements and leveraged existing products, such as Torlon or Bakelite polymer with commercially available fibers [1]. An engine block not only has to withstand demanding operating temperatures, it also must enable the heat to transfer away from the cylinders. The measurement of this ability to conduct heat is called thermal conductivity, and is defined by Fourier's Law: $\mathrm{q}=\mathrm{k} \Delta \mathrm{T}$, where $\mathrm{q}$ is the heat flux (flow of energy per unit time per unit area) and $\mathrm{k}$ is the thermal conductivity (usually expressed in units of watts per meter-Kelvin (W/mK)). Systematic R\&D of composites and constituent materials is needed to realize the potential benefits of PMC engine blocks for passenger vehicles with long-term service requirements and cost limitations. 


\section{Objectives}

This one year exploratory project addresses current issues with Bakelite embrittlement with continued thermal exposure and debonding from metal inserts. The objective of this work is to develop materials with high toughness, acceptable temperature resistance, cost-affordability, and rapid heat dissipation through a percolated network of thermally conducting reinforcing 'fibers,' while maintaining adhesion to metals. For proper development and design of the materials, this work also aims to develop the working environments of the PMC blocks. It includes the temperature profile or envelope during operation, mechanical loads, and vibrational analyses. The initial targets include fabrication of polymer matrix composites of CFs and demonstration of acceptable performance, such as $100 \mathrm{MPa}$ mechanical strength equivalent and retention of properties by $50 \%$ after continued exposure to $200^{\circ} \mathrm{C}$ for $4 \mathrm{~h}$.

\section{Approach}

Designing a composite material system capable of satisfying engine block operation requirements-at minimum potential cost and manufacturing complexity - would consist of a suitable polymer, a fiber reinforcement, a particulate phase with a tailored interface, and toughening agents and additives.

Thermosetting networked polymers offer the greatest potential for creep and fatigue resistance. While phenolic resins (Bakelite) have been evaluated in engine block applications, there are a variety of other potentially suitable candidates, including high-temperature epoxies, polyimides, bis-maleimides [2], cyanate esters [3], and benzoxazines [4]. However, many of these chemistries are currently cost prohibitive, since hightemperature formulations are currently produced in small volumes primarily for the aerospace industry. Variant phenolic resin chemistries that were developed earlier by ORNL may offer the potential for rapid manufacturing and high service loads above their glass transition temperature, while being reinforced with a moderate volume fraction of second-phase reinforcement. These variant phenolic resins are rubber-toughened Novolac and a sustainable lignin derivative — acrylonitrile-butadiene-lignin polymer [5, 6].

Reinforcements evaluated to date included low-cost glass fibers and PAN-based CFs. While these reinforcements are readily available and well-understood, their thermal conductivity (i.e., 2-13 W/mK) [7, 8] and mechanical properties may not be ideal for engine block applications. Composites containing pitch-based CFs [9] with a thermal conductivity exceeding $800 \mathrm{~W} / \mathrm{mK}$, and even higher mechanical stiffness than polyacrylonitrile $\mathrm{CF}$, offer greater freedom to tailor composite thermomechanical properties and will be evaluated in this project. The thermomechanical property dependence on fiber loading, aspect ratio, orientation and presence of additional fillers will also be evaluated. It has also been reported that nanomaterials like graphene can be used in the sizing of CFs or added to the base polymer to provide better thermomechanical properties $[10,11]$. Further improvements to the thermomechanical properties of the selected composite may be made through toughening of the matrix. Tailored chemistry between functionalized high-performance thermoplastics reacted with thermosetting resins have been reported to increase the overall fracture toughness and glass transition temperature, while decreasing thermal expansion [12, 13]. Also, while not part of the proposed Year 1 project, joining to metallic components would need to be taken into consideration in future applications to enable a multi-material system.

The ORNL Manufacturing Demonstration Facility has demonstrated significant advancements in high rate deposition and high quality component production using AM, which gives the unique capability to fabricate complex and variably sized parts in a relatively short amount of time, and this includes both polymeric and metallic AM. This ability may serve useful when fabricating prototypes as it alleviates the need for die-cast tooling for any metal parts or inserts that may be required in this or future projects that include more complex metal/polymer hybrid systems. Most importantly for this project application, AM can fabricate complex shapes 
of variable size, which will be crucial to optimize the design and capability of internal metallic components. Our research plan includes the following steps:

- $\quad$ Evaluation and down-selection of base polymer chemistries. Up to three polymers will be physically evaluated to obtain experimental data for the most promising crosslinked, amorphous, and semicrystalline chemistries. Their suitability for engine block use, as well as cost and manufacturing considerations, will be documented.

- Identification and evaluation of fiber and particulate reinforcements, including CF and graphene. Ongoing and potential efforts on cost-reduction and physical-property improvement will be documented.

- Development with down-selected polymer matrices, reinforcements with compatible sizing, and selected tougheners or modifiers.

- $\quad$ Establish suitable test protocols for the evaluation of time-dependent stiffness, thermal conductivity, and heat capacity of PMCs at temperatures up to $200^{\circ} \mathrm{C}$.

- Investigate the effects of elevated temperatures on mechanical properties.

- Exploratory thermal conductivity and heat capacity evaluation.

- Identification of technical challenges and opportunities.

- Identification of cost and manufacturing challenges and opportunities.

\section{Results}

\section{Resin Selection and Modification}

The team concluded an investigation of different candidates for the matrix material, including a variety of thermosetting networked polymers, and semi-crystalline and amorphous thermoplastics. Thermosetting phenolics were selected as the base chemistry for development mainly due to reduced cost. The early stage data addresses current issues with Bakelite embrittlement with continued thermal exposure and debonding from metal inserts. The toughened composition with tensile stress-strain profile, as shown in Figure I.3.5.1.1 (a), was further used for CFRP manufacturing. The processability and heat dissipation rate are to be optimized in this continued work. The processability (moldability) of the composites will be analyzed by melt-rheology and RT dynamic performance of the composites. ORNL's recent work shows toughened resin from the modified phenolics. The data shown in Figure I.3.5.1.1 (b) meets initial milestones for the project with developing protocol for resin composite fabrication from a selected toughened polymer. Thus, use of 4-12 wt.\% CFs delivered compositions with 60-100 MPa tensile strength and with $12 \mathrm{wt} \% \mathrm{CF}$, the toughened composition exhibits 1.6\% strain to failure as shown in Figure I.3.5.1.1 (c). 


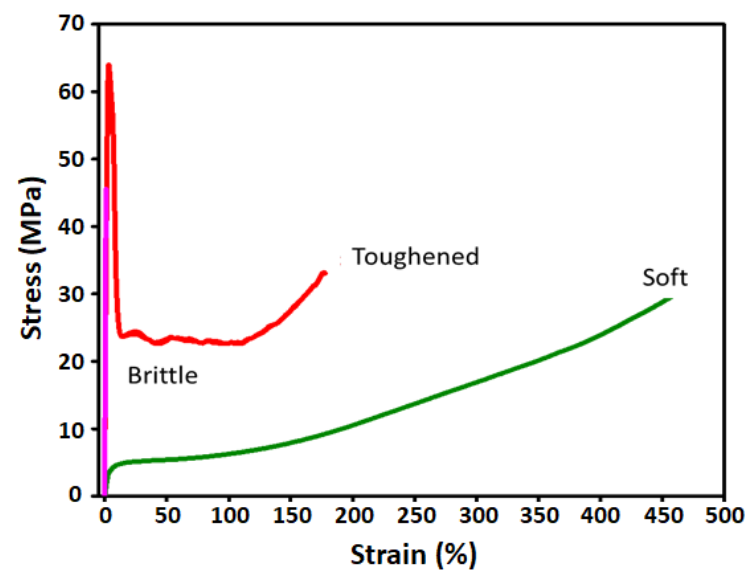

(a)

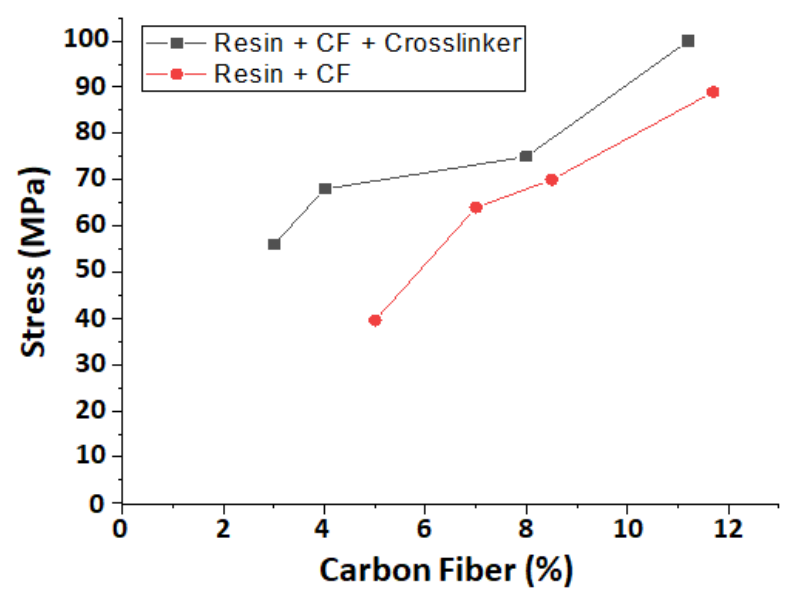

(b)

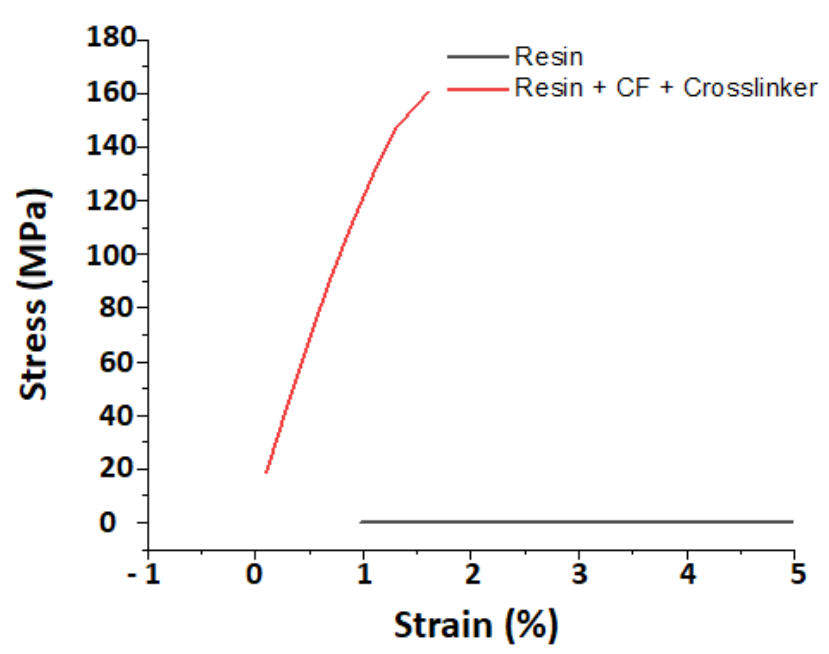

(c)

Figure I.3.5.1.1. (a) Temperature-resistant phenolics are preferred material for engine block applications. However, these compositions are very brittle (magenta colored data). (b) Use of crosslinker (both matrix and fiber surface modifier) changes in failure mode from fiber/matrix debonding to fiber breakage. (c) With $12 \mathrm{wt}$.\% CF, the toughened composition exhibits $1.6 \%$ strain to failure. Source: ORNL. 
Because phenolic resins are expensive in the current market, an alternative cross-linkable phenolic oligomer alloy - acrylonitrile-butadiene-lignin (ABL) previously developed at ORNL — was studied. The cost of this partially renewable material can be less than $<\$ 1.5 / \mathrm{lb}$. However, the most inexpensive material may not have good thermomechanical or dimensional stability. Therefore, we developed crosslinking and nano-

reinforcement chemistry of this resin system. The mechanical properties of those resin systems are displayed in Figure I.3.5.1.2. The dispersion of carbon nanoparticle (CNP) in ABL does not affect either the strength or the modulus of the ABL resin. However, crosslinking the ABL resin using a new crosslinking chemistry involving a difunctional linker (abbreviated as DL) doubled the tensile strength, shown in Figure I.3.5.1.2 (a) and modulus, shown in Figure I.3.5.1.2 (b). Dispersing the nanoparticle at that crosslinking stage does not further enhance the tensile strength but does enhance the tensile modulus significantly ( 70\%). In addition, it was observed that dynamic crosslinking (crosslinking under shear mixing) of the components (rubber or toughening agent and the resin) is significantly influenced by the time of the mixing cycle. We have studied crosslinker types affecting the glass transition temperature, $\mathrm{Tg}$, of the toughening agent. Some crosslinker could effectively increase the $\operatorname{Tg}$ by $5-8^{\circ} \mathrm{C}$, while enhancing the toughness significantly (50\%). This is a key finding for resin development work.

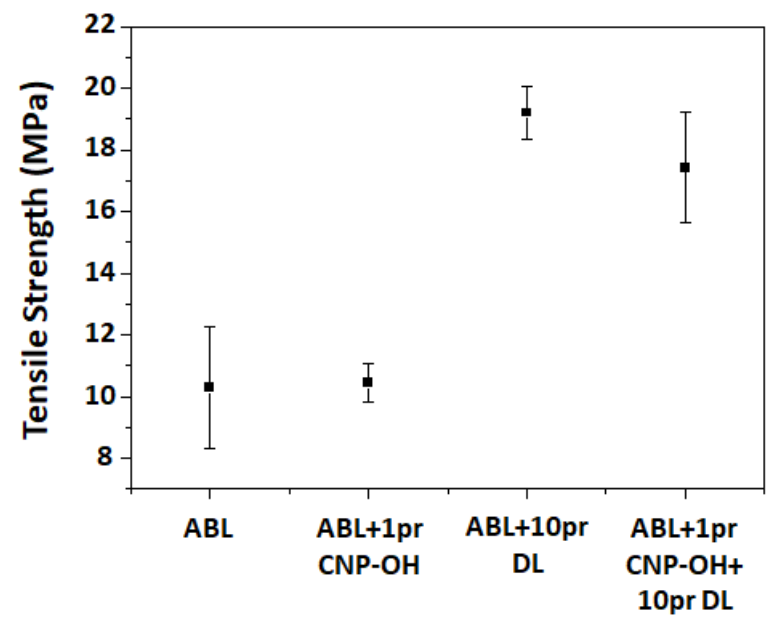

(a)

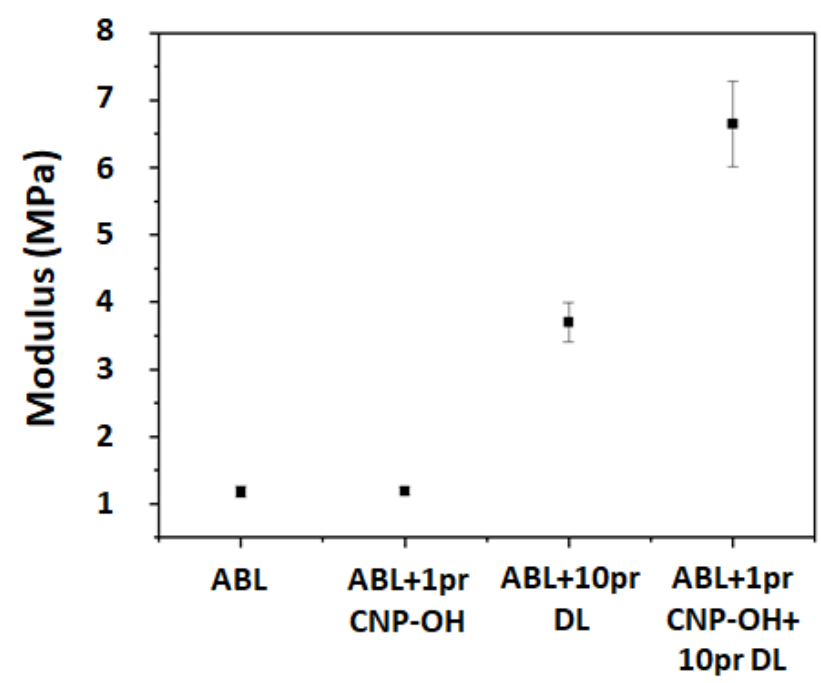

(b)

Figure I.3.5.1.2. Mechanical properties for (a) tensile strength and (b) modulus of a renewable variant of toughened phenolic resin. Source: ORNL. 


\section{Performance of Composites of the Resin}

To understand the use of the resins as a composite matrix with the appropriate chemistry of the interfacial bonding, we have deployed the same crosslinker as the interfacial bond promoter without the use of a conventional sizing agent. Based on the protocol developed in the bench-scale process using a hand-laying method, we have prepared composite laminate containing $12 \% \mathrm{CFs}$ with the unidirectional tensile properties shown in Figure I.3.5.1.3. The interfacial engineering using various resin crosslinker itself as a coupling agent works well to increase the mechanical properties by about 25-50\%. It may be noted that the role of the CNP with strength enhancement of the composite is not very significant compared to an optimal system that uses a trifunctional crosslinker abbreviated as TL (bar \#5). Nonetheless, CNP does provide some strength enhancement for composition with difunctional crosslinker (DL+CNP, bar \#3, vs DL alone in bar \#2). However, hydroxyl terminated CNP (CNP-OH, bar \#4) does not give a significant strength enhancement. The presence of CNPs can enhance thermal conductivity of the resin to be measured in FY 2020. Thus, we deployed a resin chemistry and developed composite fabrication protocol that may give us good engine blockgrade low-cost partially renewable composite. We are currently evaluating chopped carbon fiber-reinforced extrudable composite materials from these resins system, as that would enable rapid molding and prototyping of the components.

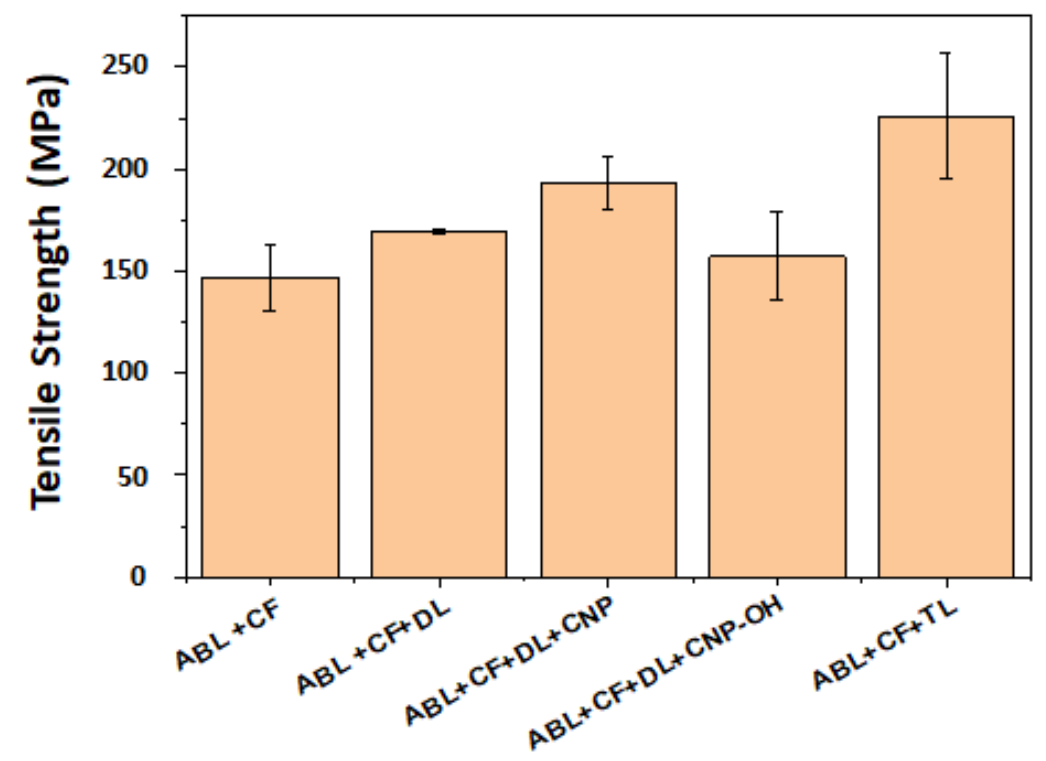

Figure I.3.5.1.3. Tensile strength of unidirectional renewable composite containing $\sim 12 \%$ CF. Source: ORNL.

The thermal degradation characteristics of the resin and composite system was analyzed by thermogravimetry (under nitrogen) and the data shown in Figure I.3.5.1.4 suggest no degradation up to $200^{\circ} \mathrm{C}$, and only $3 \%$ to $5 \%$ mass loss in the composite at $300^{\circ} \mathrm{C}$. The data confirm the initial feasibility of the usage of this product for engine block materials. Processability of the chopped fiber composite and mechanical properties of the aged materials will be tested in FY 2020. The char forming characteristics in this resin are visible from the $>30 \%$ charred residue, even in neat $\mathrm{ABL}$ resin at $600^{\circ} \mathrm{C}$. The crosslinked composites exhibit even more charred residue compared to the control composite. The charring behavior of this toughened resin is important for its thermal-oxidative resistance, should they become exposed to air. The charred layer acts as a barrier to the oxygen and protects the material. 


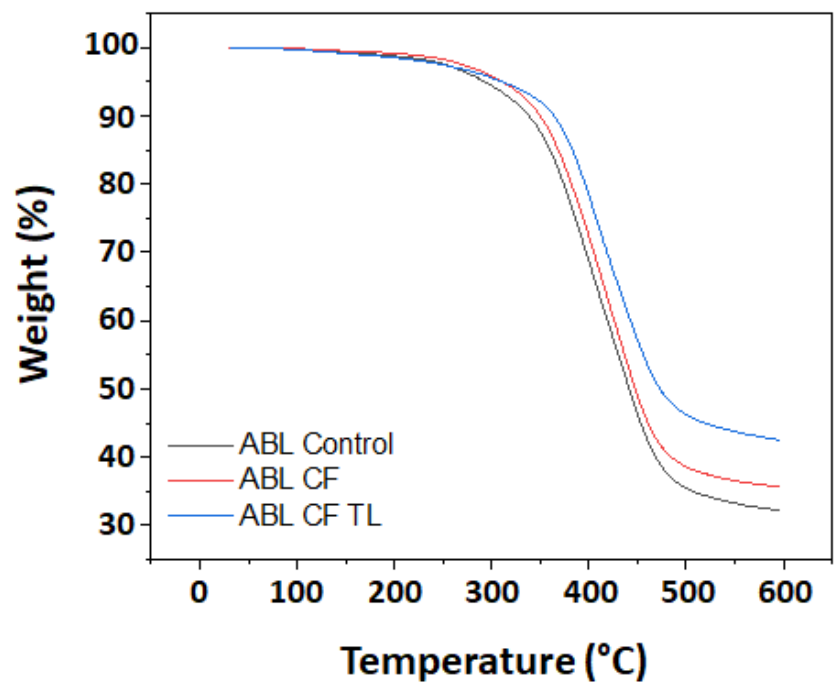

Figure I.3.5.1.4. Thermogravimetric analysis of the neat resin and its two composites. Source: ORNL.

\section{Parametric Studies and Virtual Simulations}

A single cylinder piston 2-stroke engine, as observed in Figure I.3.5.1.5, was purchased from Pegasus Aeronautics to develop the process parameters that will be used as a starting point to develop design standards for our PMC internal combustion engine application. The team will use the engine operation for temperature, mechanical, and vibrational analyses. This is a steppingstone to larger automotive engines. We expect these parameters to vary as we delve into automotive engines, but this was chosen as our starting point to match our budget and timeline. These parameters will then be used as resources for the virtual simulation, as well as being fed back into the development of the materials.
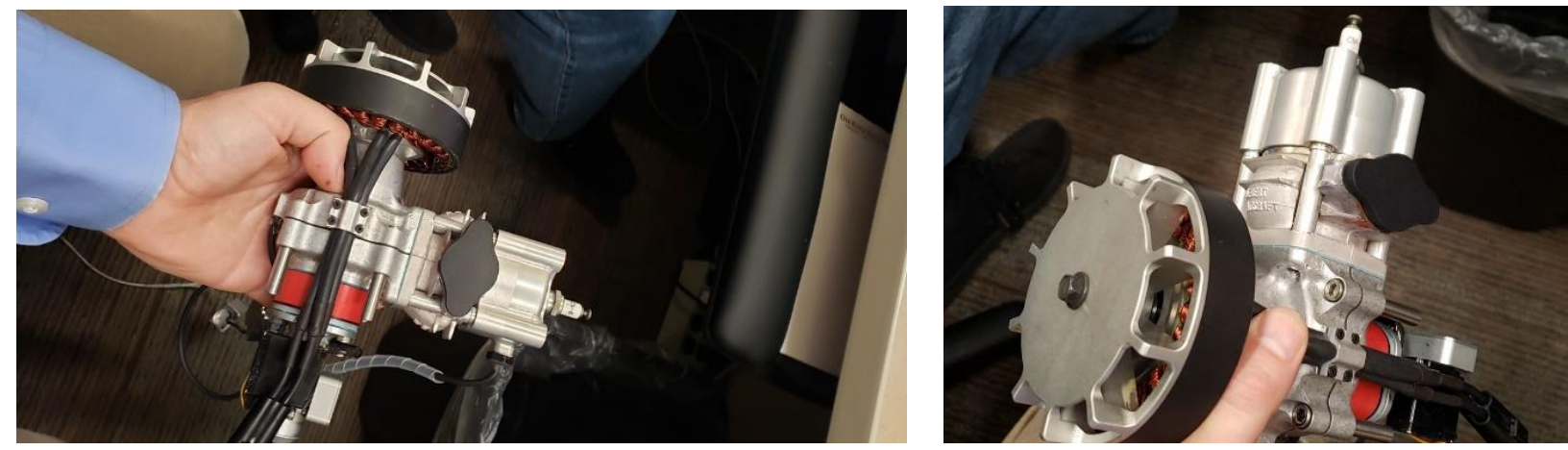

Figure I.3.5.1.5. Two views of the single cylinder 2-stroke GE35 engine purchased for parametric studies. Source: ORNL.

Computer-aided design files were also obtained from Pegasus Aeronautics and the geometry of the GE-35 engine is shown in Figure I.3.5.1.6. The geometries will be meshed and preprocessed for FEA, which will be used to determine the appropriate boundary conditions (i.e., loads and constraints) of the current engine design. Once completed, these boundary conditions will be used to aid in the design and optimization of the composite engine block and other appropriate components. Components that will be considered for composite designs include the cylinder block, crankcases (e.g., rear and front), throttle body, water jacket, generator drive cover, and gear train box. 


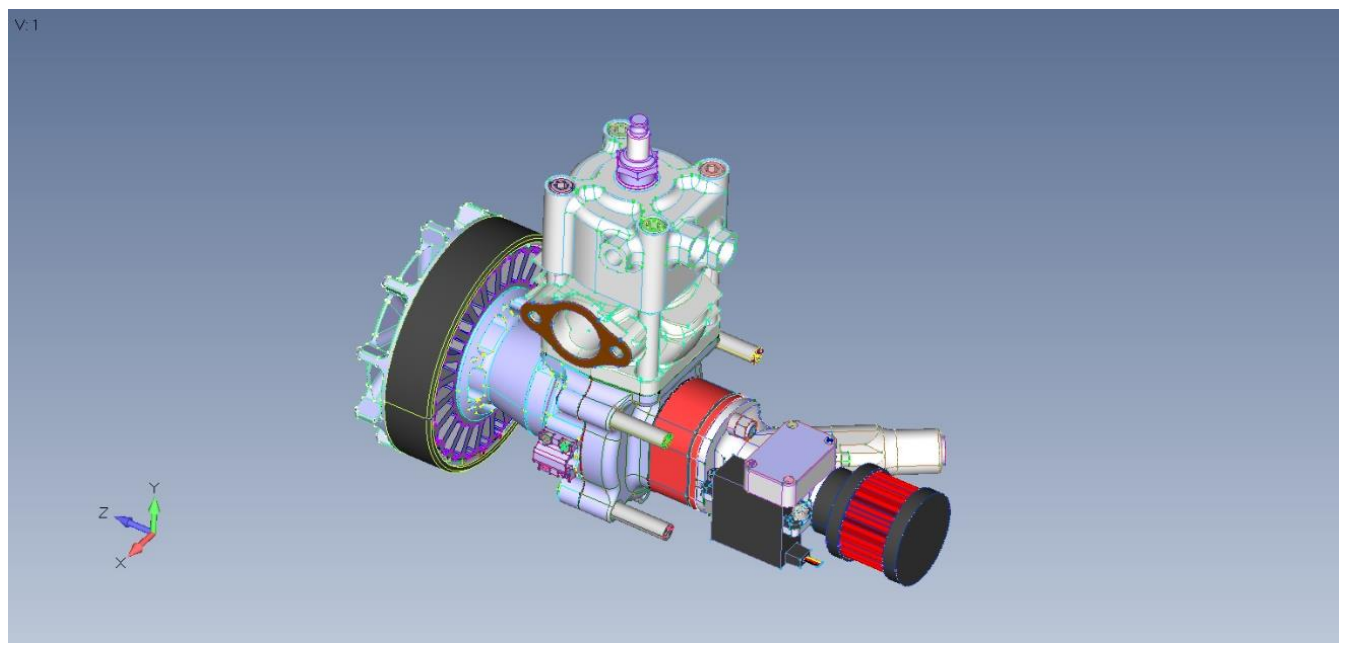

Figure I.3.5.1.6. Computer-aided design geometry of the Pegasus GE-35 engine that was imported into a preprocessor for the FEA. Source: ORNL.

It must be stressed that design must be developed with processing in mind. The best design cannot be used if it cannot be fabricated into a functional part. For the components mentioned, we will consider injection-molding of CFRCs (e.g., gear train box, water jacket, and drive cover) and compression molding for higher temperature applications (e.g., crankcases and cylinder blocks). Each of these processes and associated materials offer a different set of material properties which will be used in the optimization.

\section{Conclusions}

We have identified methods to toughen phenolic resins and prepared a renewable variant that is tough and provides an excellent matrix for superior fiber-reinforced composite performance. Appropriate fiber-matrix bonding methodology has been developed for these resin systems. We have fabricated composites from toughened phenolic resin systems and demonstrated $>100 \mathrm{MPa}$ strength equivalent in the composite materials. In the next steps, the rheological and mechanical properties of discontinuous fiber composites will be developed using the established methodology and mechanical property retention after thermal aging will be measured. A single cylinder piston 2-stroke engine has been obtained and it will be used as a starting point to develop design standards for our PMC internal combustion engine application. A FEA will be used to determine the appropriate boundary conditions (e.g., loads and constraints) of the current engine design and parameters will be considered for the property optimization of the composite material.

\section{Key Publications}

1. Naskar, A. K., V. Kunc, and B. Knouff, 2019, "Novel materials for polymer composite engine blocks," Informal presentation at EERE-Vehicle Technologies Program Annual Merit Review, June 13, 2019.

\section{References}

1. Magda, M., 2015, "Plastic race engine returns as Polimotor 2 project underway," Enginelabs, Available at: https://www.enginelabs.com/news/plastic-race-engine-returns-as-polimotor-2-projectunderway/, accessed November 27, 2019.

2. Sava, M., I. Sava, V. Cozan, and F. Tanasa, 2007, "Preparation and polymerization of bismaleimide compounds," J. Appl. Polym. Sci., Vol. 106, No. 4, pp. 2185-2191.

3. Hamerton, I., and J. N. Hay, 1999, "Recent developments in the chemistry of cyanate esters," Polym. Int., Vol. 47, No. 4, pp. 465-473. 
4. Yagci, Y., B. Kiskan, and N. N. Ghosh, 2009, "Recent advancement on polybenzoxazine-A newly developed high-performance thermoset," J. Polym. Sci. A1, Vol. 47, No. 21, pp. 5565-5576.

5. Tran, C. D., J. Chen, J. K. Keum, and A. K. Naskar, 2016, "A new class of renewable thermoplastics with extraordinary performance from nanostructured lignin-elastomers," Adv. Funct. Mater., Vol. 26, No. 16, pp. 2677-2685.

6. Naskar, A. K., J. K. Keum, and R. G. Boeman. 2016, "Polymer matrix nanocomposites for automotivestructural components," Nat. Nanotechnol., Vol. 11, No. 12, pp. 1026-1030.

7. Katzman, H. A., P. M. Adams, T. D. Le, and C. S. Hemminger, 1994, "Characterization of low thermal conductivity PAN-based CFs," Carbon, Vol. 32, No. 3, pp. 379-391.

8. McIvor, S. D., M. I. Darby, G. H. Wostenholm, B. Yates, L. Banfield, R. King, and A. Webb, 1990, "Thermal conductivity measurements of some glass fibre- and carbon fibre-reinforced plastics," J. Mater. Sci., Vol. 25, No. 7, pp. 3127-3132.

9. Huson, M. G., 2017, "High-performance pitch-based CFs," In: Bhat, G., (Ed.), Structure and Properties of High-Performance Fibers, pp. 31-78, Oxford: Woodhead Publishing.

10. Saleem, H., A. Edathil, T. Ncube, J. Pokhrel, S. Khoori, A. Abraham, and V. Mittal, 2016, "Mechanical and thermal properties of thermoset-graphene nanocomposites," Macromol. Mater. Eng., Vol. 301, No. 3, pp. 231-259.

11. Di Pasquale, G., O. Motto, A. Rocca, J. T. Carter, P. T. McGrail, and D. Acierno, 1997, "New highperformance thermoplastic toughened epoxy thermosets," Polymer, Vol. 38, No. 17, pp. 4345-4348.

12. Chen, H., V. V. Ginzburg, J. Yang, Y. Yang, W. Liu, Y. Huang, and B. Chen, 2016, "Thermal conductivity of polymer-based composites: Fundamentals and applications," Prog. Polym. Sci., Vol. 59, pp. 41-85.

13. Lin, C. H., J. C. Chen, C. M. Huang, and T. Y. Juang, 2014, "High-performance thermosetting films based on an amino-functionalized poly(ether sulfone)," J. Appl. Polym. Sci., Vol. 131, No. 21, 5 November 2014.

\section{Acknowledgements}

The authors acknowledge the guidance provided by Dr. Rich Davies (ORNL Sustainable Transportation Program Deputy Director), Mr. Fue Xiong, and Dr. Nihal Kanbargi for leading the laboratory work with the preparation and testing of composite specimens. 


\title{
I.3.5.2 Advanced Anticorrosion Coatings on Lightweight Magnesium Alloys by Atmospheric $\mathrm{CO}_{2}$ Plasma Treatment (Oak Ridge National Laboratory)
}

\author{
Oak Ridge National Laboratory \\ 1 Bethel Valley Road \\ Oak Ridge, TN 37831 \\ E-mail: jangg@ornl.gov
}

Gyoung Gug Jang, Principal Investigator

\section{Jerry L. Gibbs, DOE Technology Development Manager}

U.S. Department of Energy

E-mail: jerry.gibbs@ee.doe.gov

Start Date: April 1, 2019

Project Funding (FY19): \$157,500
End Date: March 31, 2020

DOE share: $\$ 150,000 \quad$ Non-DOE share: $\$ 7,500$

\section{Project Introduction}

ORNL, together with an industrial partner, initiated a one year exploratory research project funded by the VTO PMCP in mid-FY 2019 to develop a novel plasma-based surface treatment to protect lightweight $\mathrm{Mg}$ alloys. Powertrain application of $\mathrm{Mg}$ alloys will demand adequate corrosion protection. Generally, $\mathrm{Mg}$ and $\mathrm{Mg}$ alloys have a poor corrosion resistance due to their high chemical reactivity and unprotective surface film composed of magnesium hydroxide $\left(\mathrm{Mg}(\mathrm{OH})_{2}\right)$ and magnesium oxide $(\mathrm{MgO})$. The state-of-the-art in $\mathrm{Mg}$ corrosion protection is presently achieved by a multilayer coating via a series of processes, including pretreatment, chemical or electrochemical conversion, primer, and topcoat. This results in complexity, high costs, and an inadequate degree of corrosion protection to date to permit expanded use in automotive applications.

Recently, a possible breakthrough in anticorrosion protective surface treatments for $\mathrm{Mg}$ was reported. A protective magnesium carbonate $\left(\mathrm{MgCO}_{3}\right)$ layer on the surface of $\mathrm{Mg}$ was formed via direct reaction of an already existing surface oxide film with excited carbon dioxide $\left(\mathrm{CO}_{2}\right)$ under electron beam irradiation [1]. It was concluded that the naturally formed oxide/hydroxide film with hydrate corrosion products on $\mathrm{Mg}$ was carbonized into a smooth, compact $\mathrm{MgCO}_{3}$ protective surface layer via reaction with $\mathrm{CO}_{2}$ species activated by either the high-energy electron beam or glow discharge. The adhesive, dense $\mathrm{MgCO}_{3}$ layer resulting from this simple process could reduce $\mathrm{Mg}$ corrosion by retarding the access of aqueous phase into the underlying substrate. The corrosion current of a $\mathrm{Mg}$ specimen in $3.5 \mathrm{wt} . \% \mathrm{NaCl}$ decreased approximately by two orders with the presence of a $\mathrm{MgCO}_{3}$ layer. This discovery needs to be further studied and developed with lower-cost processes amenable to high-volume $\mathrm{Mg}$ manufacturing for vehicle production.

\section{Objectives}

Inspired by the $\mathrm{MgCO}_{3}$ layer formation under $\mathrm{CO}_{2}$ driven electron beam/plasma irradiation, this project team proposed to develop and demonstrate the formation of a protective $\mathrm{MgCO}_{3}$ surface film via RT atmospheric plasma treatment with a target of greater than 10 -fold reduction in corrosion rate on cast and/or rolled $\mathrm{Mg}$ alloy(s) as evidenced by advanced corrosion and characterization tests.

\section{Approach}

The approach is to form a $\mathrm{MgCO}_{3}$ nanocrystalline surface layer from native $\mathrm{Mg}(\mathrm{OH})_{2}$ and $\mathrm{MgO}$ layers via an atmospheric $\mathrm{CO}_{2}$ plasma and to then understand/evaluate the anticorrosion performance of the engineered $\mathrm{MgCO}_{3}$ protective layer. We are pursuing proof-of-principle assessments of the potential of this concept with an industrial partner, Atmospheric Plasma Solutions, Inc., Cary, NC. APS, Inc. has successfully developed a commercialized atmospheric plasma technology for rapid, low-cost, open-air (no vacuum chamber required) 
removal of paints containing organic/inorganics to prepare surfaces for recoating. Their approach utilizes a non-thermal, but chemically active, plasma of atomic nitrogen and oxygen from air to react with the surface. Surfaces can be treated manually or with automated batch or continuous systems and can exhibit low levels of roughness post-treatment. Using the $\mathrm{CO}_{2}$, rather than air, as the source feedstock gas, the potential exists to use the atmospheric plasma approach as a method to introduce activated $\mathrm{CO}_{2}$ into the naturally present $\mathrm{Mg}$ oxide/hydroxide layer on a $\mathrm{Mg}$ surface in order to produce the protective $\mathrm{MgCO}_{3}$ layer that provides the intended aqueous corrosion resistance.

\section{Results}

The project began in April 2019 and made steady early stage progress in developing protective oxide and carbonate films on pure $\mathrm{Mg}$ via a $\mathrm{CO}_{2}$-based atmospheric plasma (AP). Figure I.3.5.2.1 (a)-(c) provides photos and a schematic of this process. The $\mathrm{CO}_{2}$-AP treatment was carried out in a custom-designed $\mathrm{CO}_{2}$-enriched cover gas chamber to increase $\mathrm{MgCO}_{3}$ formation, shown in Figure I.3.5.2.1 (a). In order to better understand potential routes to $\mathrm{MgCO}_{3}$ formation shown schematically in Figure I.3.5.2.1 (b), we introduced two types of Mg specimens: (1) bare $\mathrm{Mg}$; and (2) pretreated $\mathrm{Mg}$ surfaces made of nano-porous $\mathrm{MgO}-\mathrm{Mg}(\mathrm{OH})_{2}$ formed via deionized (DI) water immersion prior to AP treatment. The results of surface characterization and corrosion rate measurement in $3.5 \mathrm{wt} . \% \mathrm{NaCl}$ solution were encouraging, as the $\mathrm{Mg}$ with plasma treatment formed a protective layer with $1 \sim 2 \mu \mathrm{m}$ thickness $\left(\mathrm{MgO} / \mathrm{MgCO}_{3}\right.$ mixture), as shown in Figure I.3.5.2.1 (c), exhibiting significantly improved corrosion resistance with greater than 10 fold rate reduction. However, additional quantitative testing and understanding of the corrosion resistance mechanism is pending. Further deposition parameters along with the pretreatments are yet to be investigated to further improve the protective layer.

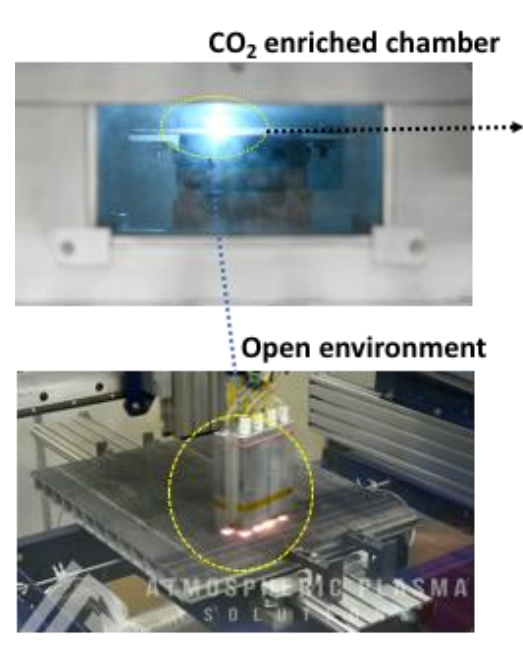

(a)

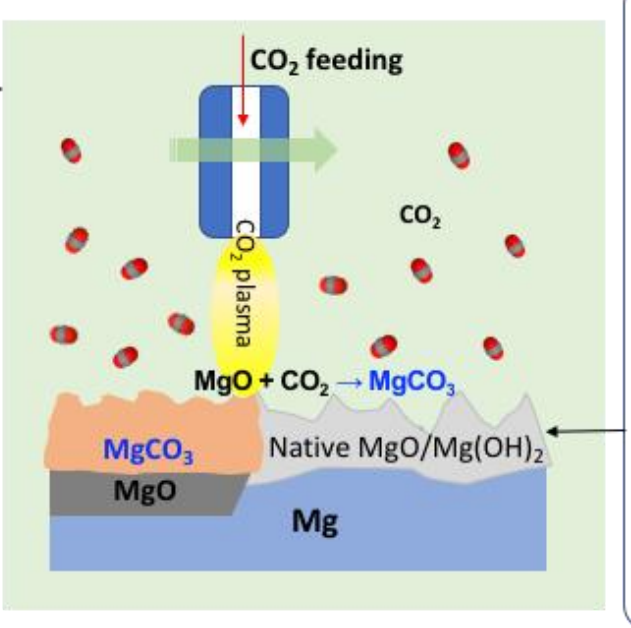

(b)

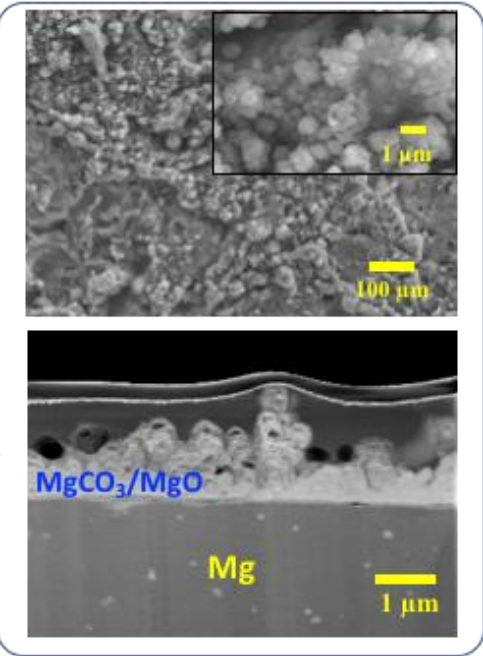

(c)

Figure I.3.5.2.1. (a) System for the inline $\mathrm{CO}_{2} \mathrm{AP}$ treatment on a $\mathrm{Mg}$ specimen inside a $\mathrm{CO}_{2}$ enriched chamber. (b) The proposed approach where the $\mathrm{CO}_{2}$ plasma converts the surface to an $\mathrm{MgCO}_{3}$ protective layer. (c) SEM and cross-sectional TEM images showing a $\mathrm{CO}_{2}$ plasma-driven protective layer that consisted of particle deposition on a dense layer with $\sim 1.5$ $\mu \mathrm{m}$ thickness. Source: (a) Atmospheric Plasma Solution (APS); (b) and (c) ORNL.

The $\mathrm{CO}_{2}$-AP treatment induced significant surface morphology changes due to $\mathrm{Mg}$-O-rich particulate deposition and a few hundreds of micron cavities formation (e.g., $\sim 200 \mu \mathrm{m}$ diameter) on the surface, as observed from the SEM analysis in Figure I.3.5.2.2 (a). For the pretreated Mg, DI-Mg, the larger and deeper cavity lines were formed along the $\mathrm{CO}_{2}$ - $\mathrm{AP}$ beam alignment, and nanoparticles were deposited on the preformed $\mathrm{MgO}-\mathrm{Mg}(\mathrm{OH})_{2}$ porous layer outside the lined areas. These results suggest that the process will require further operational optimization to resolve the non-uniform surfaces of the initial films. 

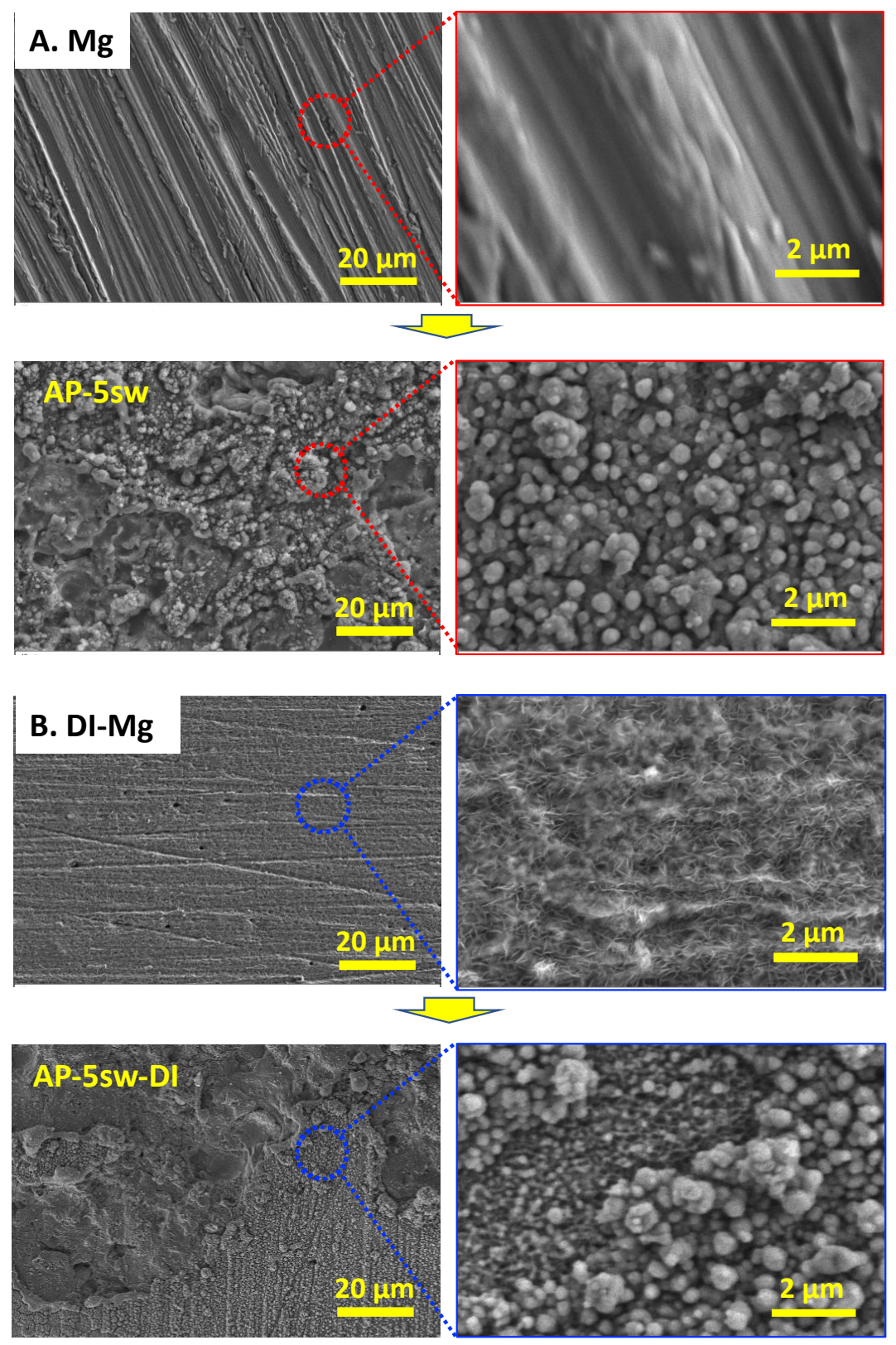

Figure I.3.5.2.2. Formation of protective layers on various $\mathrm{Mg}$ specimens via $\mathrm{CO}_{2}$-AP treatment (i.e., 5 sweeps, 0.15 in. height, $300 \mathrm{in./min,} 0.025 \mathrm{in.} \mathrm{spacing)} \mathrm{showing} \mathrm{microscopic} \mathrm{surface} \mathrm{morphology} \mathrm{changes} \mathrm{of} \mathrm{(a)} \mathrm{AP-treated} \mathrm{Mg} \mathrm{and} \mathrm{(b)} \mathrm{DI}$ water pretreated Mg (DI-Mg) specimens. Source: ORNL.

The $\mathrm{CO}_{2}-\mathrm{AP}$ treatment on $\mathrm{Mg}$ and DI-Mg at this stage showed excellent corrosion mitigation even though further process optimization is required. Figure I.3.5.2.3 (a) shows the surfaces of the $3.5 \mathrm{wt} \% \mathrm{NaCl}$ saltwater immersed specimens, which were fabricated by an increasing number of treatments. The AP- 5 sweep sample (AP-5sw) has much smaller areas of corrosion attack, compared to untreated $\mathrm{Mg}$ and other low-number APtreated $\mathrm{Mg}$ specimens. Electrochemical corrosion measurement showed that the corrosion current density $\left(i_{\text {corr }}\right)$ of the AP-5sw sample was significantly lower (e.g., approximately by seven orders of magnitude) as compared to the bare Mg sample, as observed in Figure I.3.5.2.3 (b). The extremely low current of AP-5sw-Mg sample in the first cycle is seemingly attributed to a hydrophobic characteristic which will be described later in this section. It is worth noting that even after three cycles of measurement, the corrosion current is greater than 10 fold lower than the bare $\mathrm{Mg}$ (i.e., one cycle). 


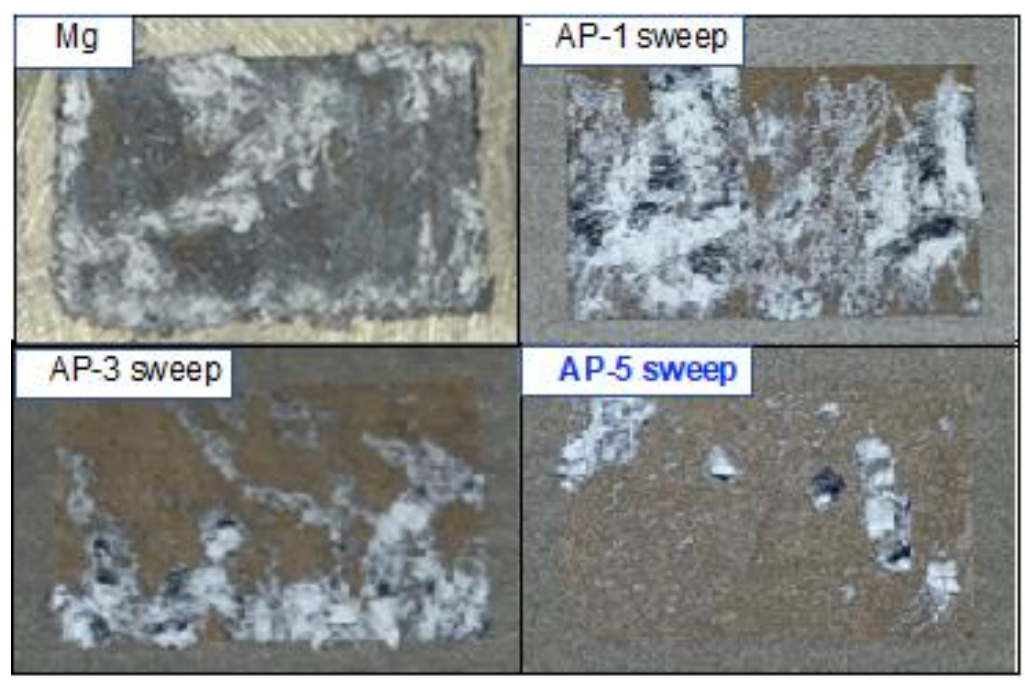

(a)

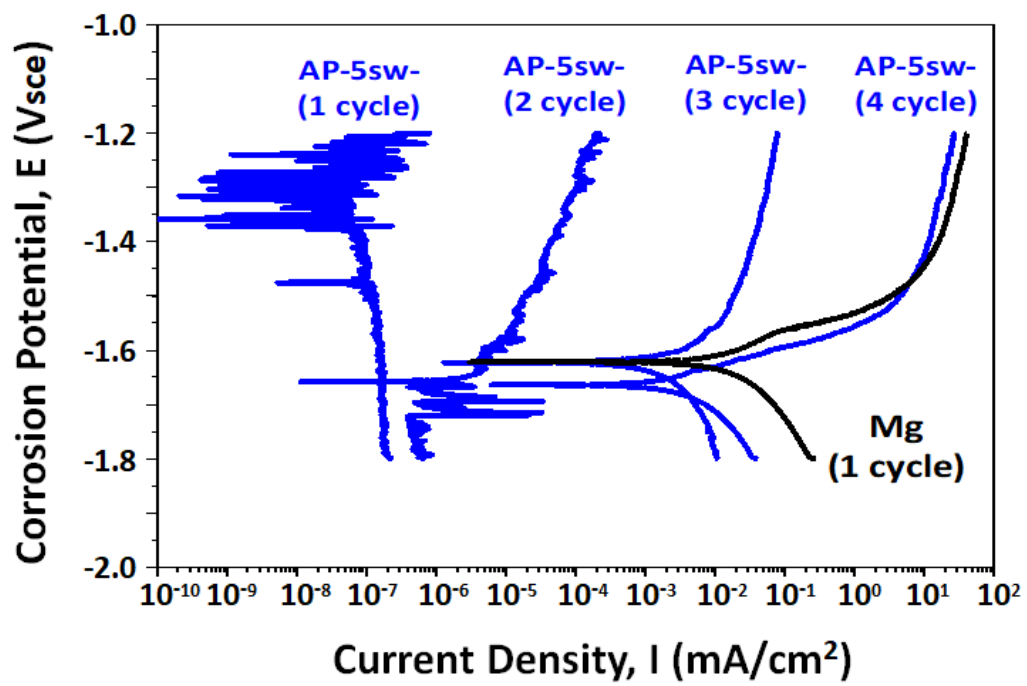

(b)

Figure I.3.5.2.3. Anticorrosion effects of $\mathrm{CO}_{2}$ AP-driven protective layer on Mg specimen: (a) AP-treated Mg specimens after a $20 \mathrm{~h}$ immersion corrosion test in $3.5 \mathrm{wt}$.\% NaCl solution; and (b) the electrochemical corrosion rate measurement on the AP-driven protective layer on the $\mathrm{Mg}$ surface where the potentiodynamic polarization measurement was in $3.5 \mathrm{wt} . \% \mathrm{NaCl}$

solution. Source: ORNL.

The hydrogen collection test also showed that the AP treatment on $\mathrm{Mg}$ and pretreated $\mathrm{Mg}$ samples significantly reduced the corrosion rate, as shown in Figure I.3.5.2.4 (a). For example, the AP treatment (i.e., five sweeps on bare $\mathrm{Mg}$ ) reduced corrosion rate by $\sim 5.5$ times vs. the bare $\mathrm{Mg}$ sample. Note that this simple test simulated a highly aggressive environment to evaluate the corrosion resistance for long-time exposure. The results of hydrogen collection agreed with the subsequent electrochemical corrosion rate measurement supported by the data in Figure I.3.5.2.4 (b). AP-5sw-DI and AP-1 sw-DI exhibited lower current densities, which decreased by approximately four and two orders of magnitude, respectively, as compared to the DI-Mg. The results suggest that the $\mathrm{CO}_{2}$ - $\mathrm{AP}$ treatment can provide anticorrosion performance on $\mathrm{Mg}$ with more than 10 times the corrosion reduction under these test conditions. 


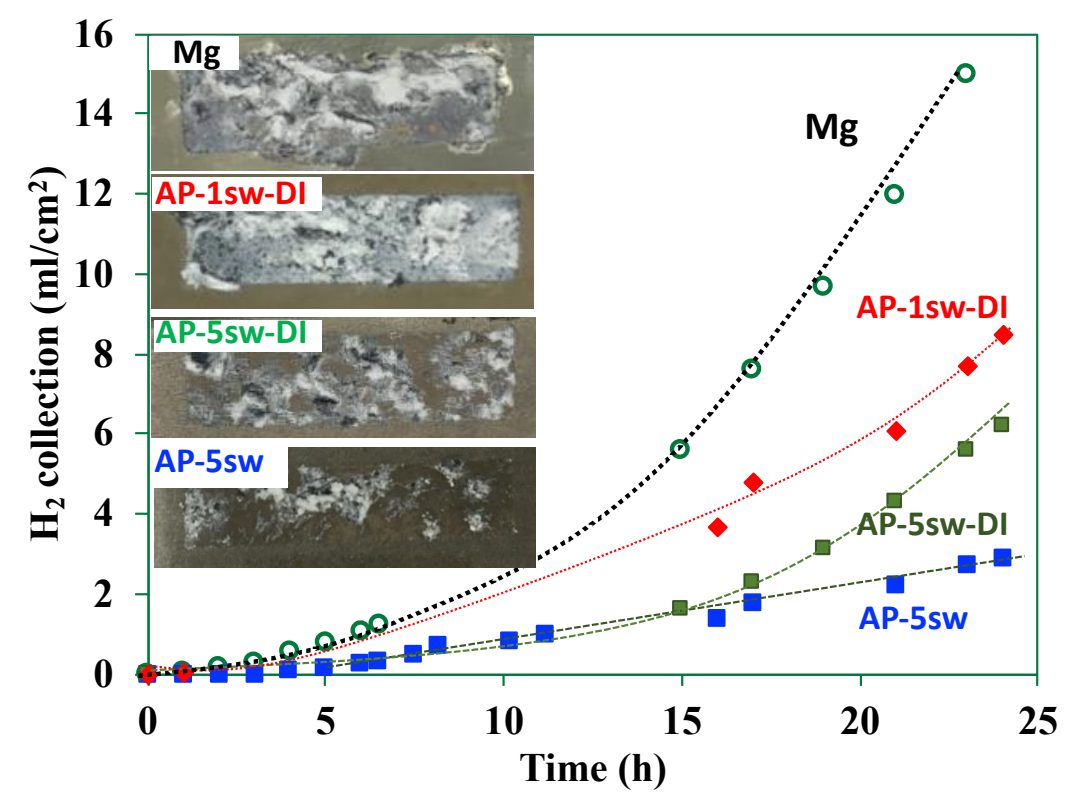

(a)

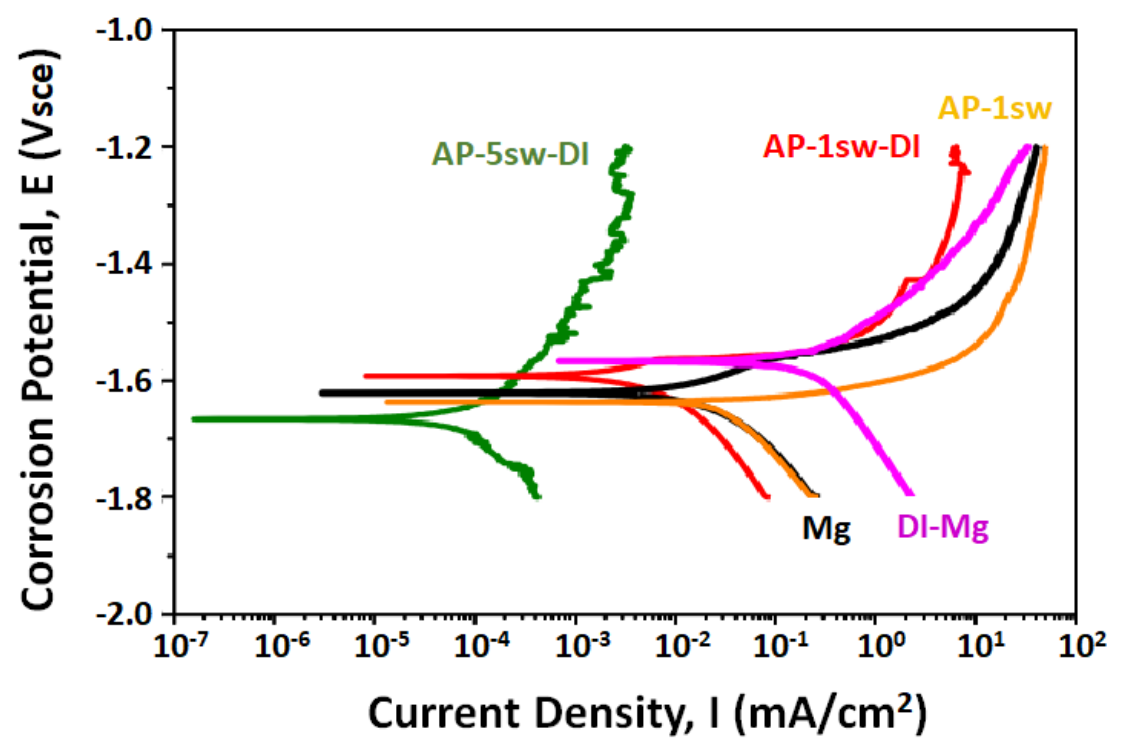

(b)

Figure I.3.5.2.4. (a) Hydrogen collection test on various Mg surfaces in $3.5 \mathrm{wt} . \% \mathrm{NaCl}$. Inset pictures are corroded $\mathrm{Mg}$ and AP-Mg surfaces after $24 \mathrm{~h}$ immersion. (b) The potentiodynamic polarization measurements of various $\mathrm{CO}_{2} \mathrm{AP}$-treated $\mathrm{Mg}$ specimens with different sweeping numbers and pretreatment. Source: ORNL.

Table I.3.5.2.1 compares the anticorrosion properties of $\mathrm{CO}_{2}$ AP-driven protective layer with other protective coatings formed by $\mathrm{CO}_{2}$ arc plasma, anodization, or micro-arc oxidation. The corrosion current density ( $\mathrm{i}_{\text {corr }}$ ) shown in bold font in Table I.3.5.2.1 was estimated using the intersection point of horizontal zero current potential line and cathodic slope in each potentiodynamic curve. We can see that the anticorrosion capabilities of the AP-driven protective layers are comparable with other state-of-the art coating approaches. Certainly, in order to obtain the optimized anticorrosion properties of AP-driven protective layer, more experiments should be done to obtain the optimal pretreatment time and AP treatment condition. 
Table I.3.5.2.1. The Results of Potentiodynamic Corrosion Tests in 3.5 wt.\% NaCl Solution and Comparisons with State-of-the Art Mg Anticorrosion Coating Methods.

\begin{tabular}{|c|c|c|}
\hline Sample & Zero Current Potential $\left(\mathbf{V}_{\text {sce }}\right)$ & $\mathbf{i}_{\text {corr }}\left(\boldsymbol{\mu A} / \mathbf{c m}^{2}\right)$ \\
\hline Mg & -1.62 & 28.55 \\
\hline DI water pretreated Mg & -1.56 & 243.9 \\
\hline AP-1sw & -1.62 & 30.67 \\
\hline AP-1sw-DI & -1.59 & 6.64 \\
\hline AP-5sw-DI & -1.66 & 0.058 \\
\hline AP-5sw (2/3/4 cycles) & $-1.66 /-1.62 /-1.66$ & $0.00222 / 2.09 / 6.02$ \\
\hline Mg [1] & -1.58 & 196.6 \\
\hline DI water pretreated Mg [1] & -1.59 & 251.3 \\
\hline CO2 Arc Plasma [1] & -0.73 & 0.22 \\
\hline Anodizing Coating [2] & -1.48 & 27 \\
\hline Composite Coating by Micro-Arc Oxidation [3] & -1.49 & 0.69 \\
\hline Micro-Arc Oxidation Coating [4] & -1.69 & 0.17 \\
\hline
\end{tabular}

We anticipate that the excellent corrosion mitigation effect is associated with the non-wetting surface properties (superhydrophobicity), chemical composition, and crystalline changes $\left(\mathrm{MgCO}_{3} / \mathrm{MgO}\right.$ mixture) resulting from the $\mathrm{CO}_{2}$-AP treatment. Figure I.3.5.2.5 (a) shows that an as-received $\mathrm{CO}_{2}-\mathrm{AP} \mathrm{Mg}$ specimen exhibited superhydrophobic surface characteristics with a water contact angle of $150^{\circ}$, as compared to the untreated bare $\mathrm{Mg}$ specimen (i.e., 600 grit polished), which wetted readily.

Interestingly, we observed a thin layer of air trapped in the AP-treated $\mathrm{Mg}$ surface when immersed in an aqueous salt solution as can be seen in Figure I.3.5.2.5 (b). When a superhydrophobic surface is immersed in water, it appears shiny due to the total internal reflection of light from a thin layer of air (plastron) trapped on their surface [5]. In general, a superhydrophobic surface can be formed by two requirements: (1) nanoscale surface roughness; and (2) low surface energy material coatings, such as carbon and fluorine. One plausible explanation for this unique AP-treated $\mathrm{Mg}$ surface feature is that the $\mathrm{CO}_{2}-\mathrm{AP}$ treatment induced nanoparticle (e.g., the $\mathrm{MgO} / \mathrm{MgCO}_{3}$ mixture) deposition or formation on the surface, the chemically active $\mathrm{CO}_{2}$ plasma molecules adsorbed on the $\mathrm{MgO}$ nanoparticle surfaces simultaneously, and then formed carbonated layers. The $\mathrm{MgO}$ nanoparticles have diverse basic sites where acidic $\mathrm{CO}_{2}$ molecules chemically/physically adsorbed on the surface, resulting in various carbonate surface species including bicarbonate, bidentate, and unidentate carbonates [6]. 

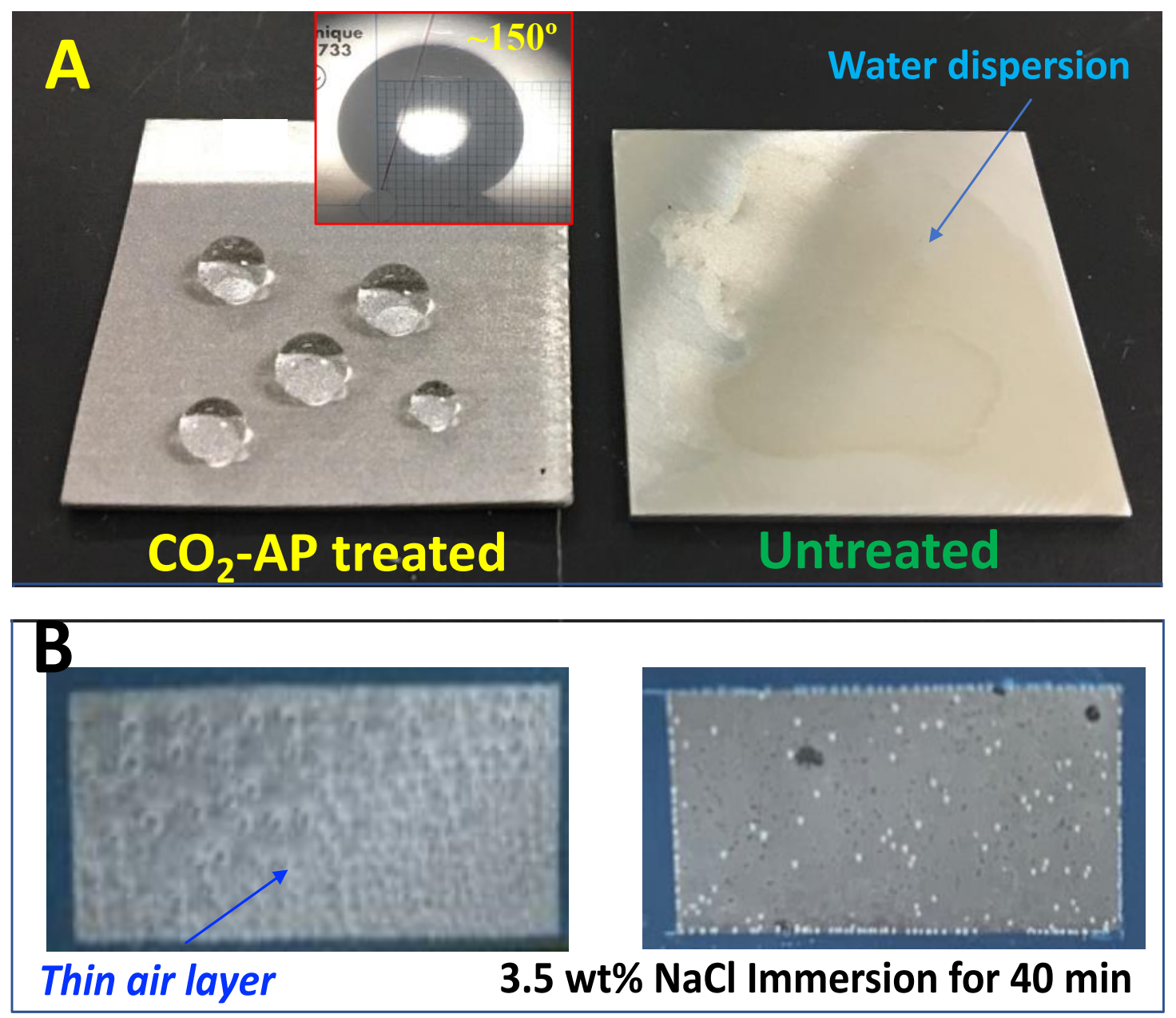

Figure I.3.5.2.5. (a) Photo-image of the $\mathrm{CO}_{2}$-AP-treated $\mathrm{Mg}$ surface showing superhydrophobicity and (b) photo-images of immersed Mg samples under 3.5 wt.\% NaCl solution. Source: ORNL.

Surface films after $\mathrm{CO}_{2}$-AP treatment were characterized by cross-sectional high-angle annular dark field (HAADF) STEM with EDS analyses. The elemental mapping of $\mathrm{CO}_{2}$-AP-treated surface layer with 1 2 $\mu \mathrm{m}$ thickness, shown in Figure I.3.5.2.6 (a), indicates a chemical composition of $\mathrm{Mg}$, O, and C. All elements were dispersed throughout the layer, verifying the coexistence of $\mathrm{MgO}$ and $\mathrm{MgCO}_{3}$. Also, XRD measurements, as observed in Figure I.3.5.2.6 (b) confirmed that the phase content of resultant layer was primarily $\mathrm{MgO}$ and $\mathrm{MgCO}_{3}$. XRD patterns of untreated $\mathrm{Mg}$ were measured at two angles $\left(0^{\circ}\right.$ and $\left.90^{\circ}\right)$ to avoid the crystalline directional effect. 

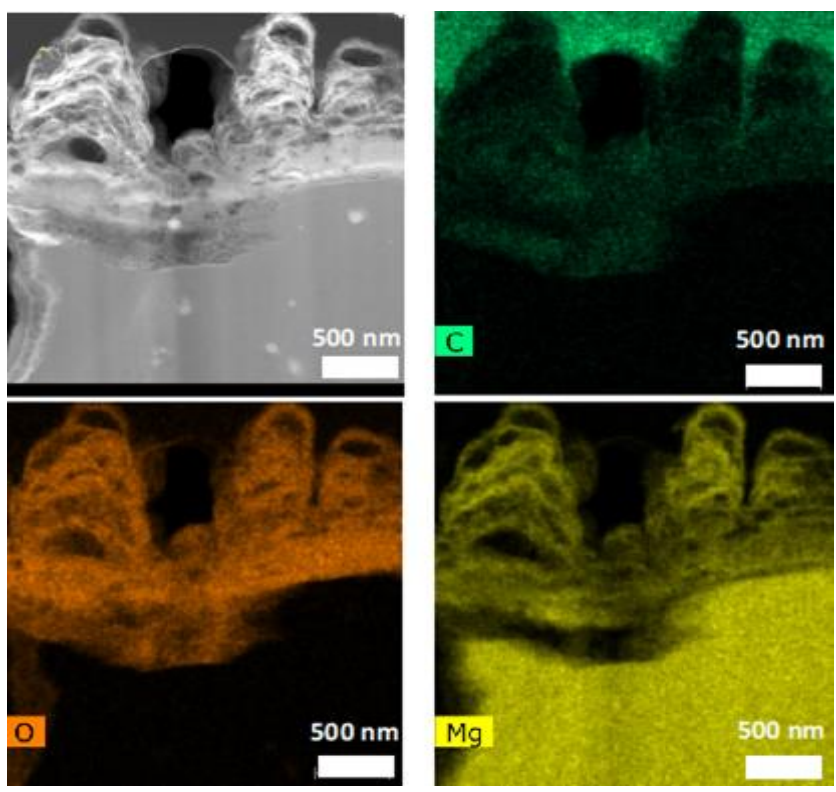

(a)

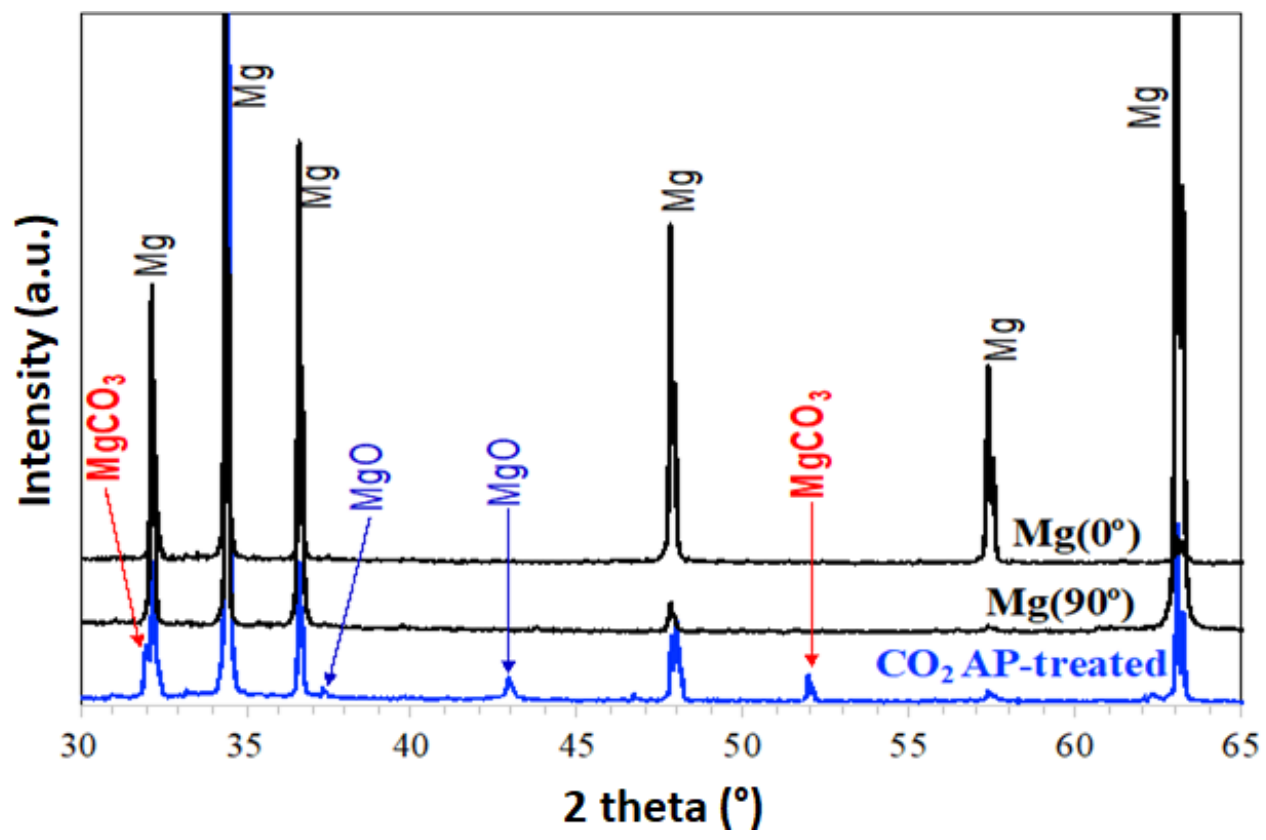

(b)

Figure I.3.5.2.6. (a) High magnification cross-sectional HAADF STEM images of $\mathrm{CO}_{2}$-AP-treated Mg along with EDS elemental mapping showing the distribution of $\mathrm{C}, \mathrm{O}$, and $\mathrm{Mg}$ in the treated layer. (b) EDS pattern for the $\mathrm{CO}_{2}$-AP-treated $\mathrm{Mg}$ (five sweeps). Source: ORNL.

The first generation of $\mathrm{CO}_{2}$-AP treatment on alloy AZ31B exhibited corrosion reduction such as low $\mathrm{H}_{2}$ collection rate shown in Figure I.3.5.2.7 (a) and low cathodic current density as can be seen in Figure I.3.5.2.7 (b); however, the corrosion rate mitigation effect was not as notable as the Mg samples. Depending on $\mathrm{Mg}$ alloy types with different thermal-mechanical properties and reactivity, each operational condition must be adjusted to ensure the effective protection in the future work. 


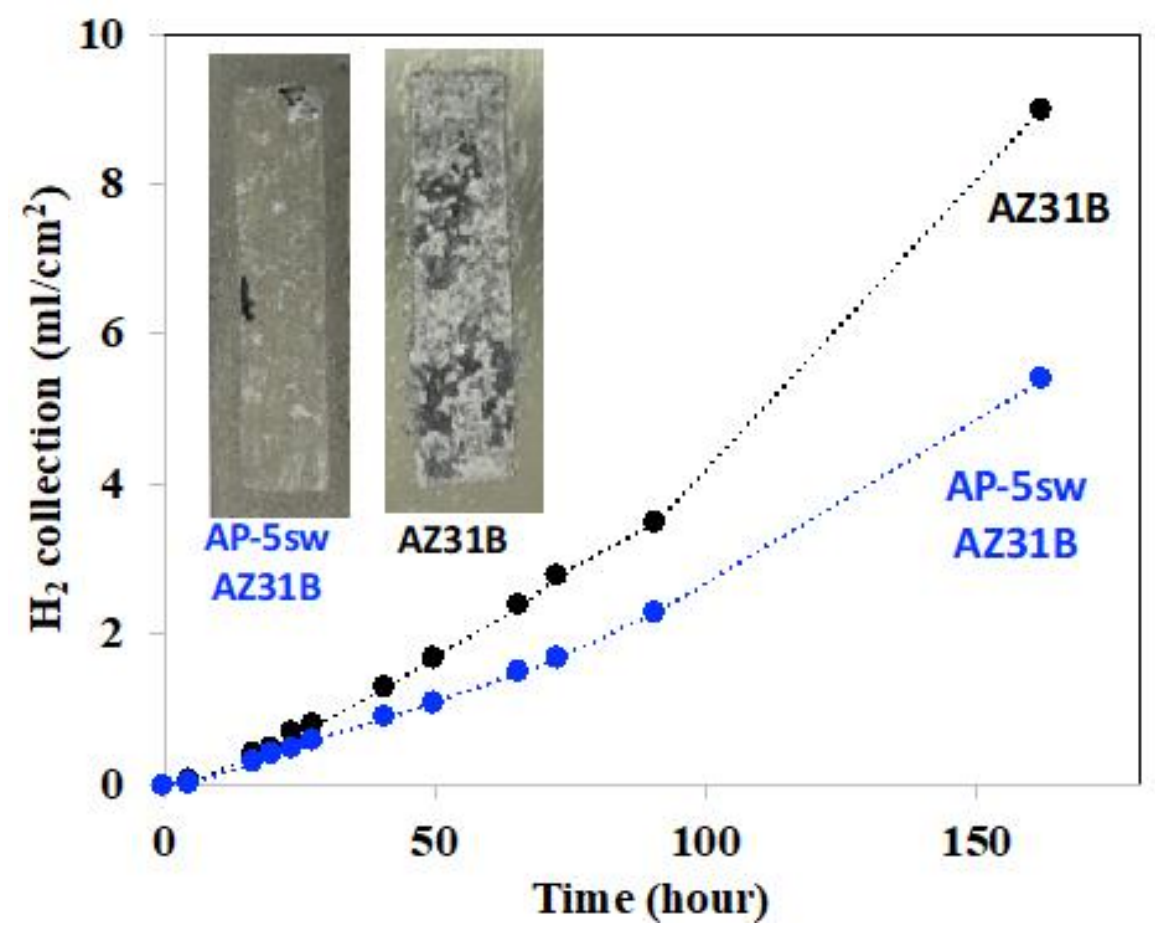

(a)

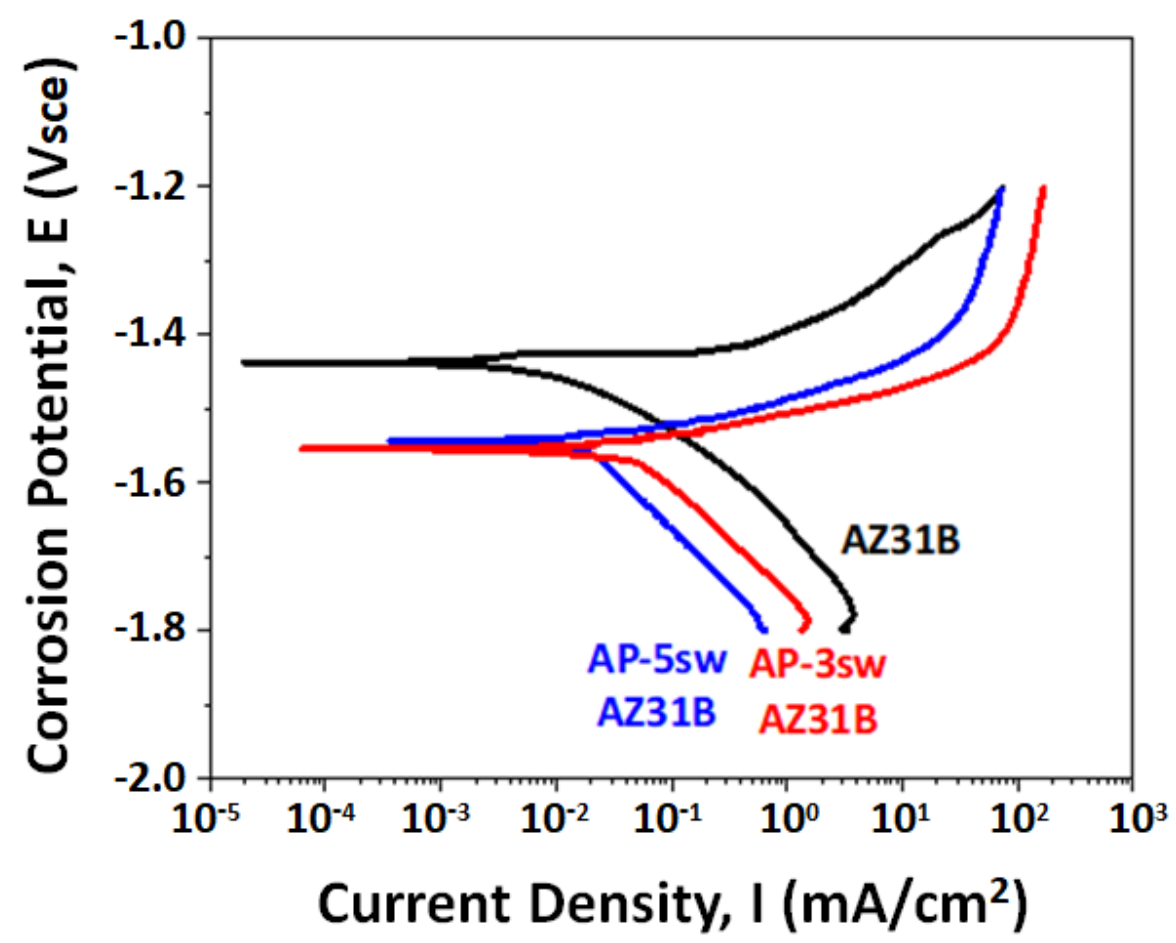

(b)

Figure I.3.5.2.7. (a) Hydrogen collection test of the $\mathrm{CO}_{2}-\mathrm{AP}-5$ sweep on AZ31B, compared with an untreated sample. The reduced $\mathrm{H}_{2}$ evolution indicates lower rate of corrosion in the $3.5 \mathrm{wt} \% \mathrm{NaCl}$ immersion test. Insets are surface features of $\mathrm{CO}_{2}$-AP-treated AZ31B after $160 \mathrm{~h}$ immersion test. (b) The potentiodynamic polarization measurement on various APtreated AZ31B surfaces in 3.5 wt.\% NaCl solution. Source: ORNL. 


\section{Conclusions}

The $\mathrm{CO}_{2}$-AP surface treatment demonstrated the formation of an effective anti-corrosive layer on $\mathrm{Mg}$. The protective layer was evaluated by potentiodynamic polarization measurements and hydrogen bubble collection in $3.5 \mathrm{wt} . \% \mathrm{NaCl}$ solution. The surface layers exhibited more than a 10-times reduction in the corrosion current density $\mathrm{i}_{\text {corr }}$ (the corrosion rate) compared to untreated $\mathrm{Mg}$. Also, the hydrogen collection tests indicated the APS-treatment reduced corrosion rate by $\sim 5.5$ times on the $\mathrm{Mg}$ specimen in a very aggressive immersion test. The significant corrosion mitigation was associated with the formation of $\mathrm{MgCO}_{3}$ and $\mathrm{MgO}$ layer and hydrophobic surface features. Surface characterization results with SEM, TEM-EDS, and XRD analysis suggested a protective, non-wetting $\mathrm{MgCO}_{3}$ layer formation, but duplication via additional experiments and characterization is required for full confirmation. Also, the AP process will require further operational optimization to resolve the non-uniform surfaces. Depending on $\mathrm{Mg}$ alloy types with different thermal-mechanical properties and reactivity, each operational condition and pretreatments must be studied to ensure effective protection.

\section{References}

1. Wang, Y., B. Liu, X. Zhao, X. Zhang, Y. Miao, N. Yang, B. Yang, L. Zhang, W. Kuang, J. Li, E. Ma, and Z. Shan, 2018, "Turning a native or corroded Mg alloy surface into an anticorrosion coating in excited $\mathrm{CO}_{2}$," Nat. Comm., Vol. 9, Art. \#4058.

2. Xue, D., Y. Yun, M. J. Schulz, and V. Shanov, 2011, "Corrosion protection of biodegradable Mg implants using anodization," Mater. Sci. Eng. C-Mater., Vol. 31, pp. 215-223.

3. $\mathrm{Mu}, \mathrm{W}$., and Y. Han, 2008, "Characterization and properties of the $\mathrm{MgF}_{2} / \mathrm{ZrO}_{2}$ composite coatings on $\mathrm{Mg}$ prepared by micro-arc oxidation," Surf. Coat. Tech., Vol. 202, pp. 4278-4284.

4. Zhao, L., C. Cui, Q. Wang, and S. Bu, 2010, "Growth characteristics and corrosion resistance of microarc oxidation coating on pure Mg for biomedical applications," Corros. Sci., Vol. 52, pp. 2228-2234.

5. Panchanathan, D., A. Rajappan, K. K. Varanasi, and G. H. McKinley, 2018, "Plastron regeneration on submerged superhydrophobic surfaces using in situ gas generation by chemical reaction," ACS Appl. Mater. Interfaces, Vol. 10, No. 39, pp. 33684-33692.

6. Hu, Y., Y. Guo, J. Sun, H. Li, and W. Liu, 2019, "Progress in $\mathrm{MgO}$ sorbents for cyclic $\mathrm{CO}_{2}$ capture: A comprehensive review," J. Mater. Chem. A, Vol. 7, pp. 20103-20120. 


\section{I.3.5.3 Novel Aluminum Matrix Composite for Powertrain Applications (Oak Ridge National Laboratory)}

\section{Zhili Feng, Principal Investigator}

Oak Ridge National Laboratory

1 Bethel Valley Road

Oak Ridge, TN 37831

E-mail: fengz@ornl.gov

\section{Jerry L. Gibbs, DOE Technology Development Manager}

U.S. Department of Energy

E-mail: jerry.gibbs@ee.doe.gov

Start Date: April 1, 2019

Project Funding (FY19): \$100,000
End Date: March 31, 2020

DOE share: $\$ 100,000$
Non-DOE share: $\$ 0$

\section{Project Introduction}

This 12-month exploratory research effort at ORNL began in April 2019 and was funded under Thrust 5 of the VTO PMCP.

There are several fundamentally distinctive ways to strengthen materials for elevated temperature service. Solid-solution strengthening and precipitation/aging hardening are commonly used in a variety of $\mathrm{Al}$ alloys, stainless steels, and nickel-based superalloys. Dispersion strengthening presents an additional attractive strengthening strategy by dispersing fine particles that are thermally stable, nearly insoluble, and exhibit very low diffusivity in the matrix to enhance elevated temperature strength. Well-known examples include oxidedispersion-strengthened steels and nickel alloys that can have even higher high-temperature strength than precipitation-based counterparts. Dispersion strengthening has also been extensively investigated and developed for $\mathrm{Al}$ alloys to enhance their elevated temperature strength, largely for aerospace and automotive applications [1]. MMCs are one of the examples of dispersion strengthening that have been used commercially in the automotive market for nearly 20 years, including powertrain applications such as cylinder liners, pistons, and connecting rods [2]. As the auto industry is highly sensitive to cost, wider application of MMCs will require performance improvements coupled with novel or innovative process improvements to drastically lower overall cost.

For the same volume fraction, it is well-accepted that nano-sized particles are much more effective than micron-sized particles in strengthening the material due to reduced interparticle spacing and the Orowan hardening effect [3]. However, achieving the uniform dispersion of nano-sized particles in a molten metal and subsequent solidified metal-matrix has been very difficult. Due to the surface tension effect and density differences, the nano-sized oxide particles have a much greater tendency to agglomerate and float to the surface during metal casting. Attempts to apply external energy fields - such as ultrasonic energy and electromagnetic energy - to break down the agglomerates and mix the nanoparticles uniformly in the molten metal have been experimented with at the laboratory-scale with limited success and have had little opportunity to demonstrate success in large quantity production. For other functional additives, chemical reactions with the molten matrix material during casting or liquid-metal infiltration would decompose or degrade the additives and cause weak bonding between the additives and the matrix. Therefore, the solid-state-based dispersion processes proposed here offer a new manufacturing process route to engineer and synthesize stronger and lighter MMCs for a number of applications, including powertrain components. 
As part of the DOE VTO PMCP Emerging Concepts task, ORNL and PNNL will jointly explore the technical feasibility of using FSP, friction stir extrusion (FSE), and ShAPE, to produce Al matrix composites (AMCs) for powertrain applications. Whereas FSP/E and ShAPETM share the same fundamental principles of solid-state material processing that rely on frictional heating and extensive shearing-dominated deformation to mix, consolidate, and produce materials, the capability and equipment at ORNL and PNNL differ. This collaborative effort enables the two National Laboratories to combine their collective and complementary expertise and equipment capability to effectively carry out the proposed research to achieve the proposed project goal and deliverables. This report primarily describes the research efforts at ORNL.

\section{Objectives}

The goal of the joint research is to demonstrate the technical feasibility of using FSP/E and ShAPETM processes to produce AMCs with additions of graphene, other forms of carbon, and other nano-sized additives such as carbide oxide particles to significantly improve the strength of Al-based alloys under elevated temperature relevant to powertrain applications. It aims for a minimum of $20 \%$ increase in strength at RT and $300^{\circ} \mathrm{C}$, compared to the baseline 7xxx series alloys, or alternatively, ORNL's AlCuMnZr (ACMZ) family of alloys available to the project. It will also investigate performance improvements in wear resistance, and/or thermal conductivities that are important to certain powertrain applications.

\section{Approach}

At ORNL, we proposed to explore the feasibility of a novel solid-state processing route to synthesize metalmatrix-composite materials for powertrain applications. We are attempting to combine several innovations in FSP and FSE patented by ORNL [4-7] to stir, consolidate, and extrude powders, chips, and other feedstock materials into useable product forms. Our past research $[8,9]$ has shown that FSP and FSE are highly effective to uniformly disperse high-volume fractions of nano-sized additives in the metal-matrix, which has been a major technical barrier in synthesizing nanocomposites especially by the molten casting processing routes [10].

This exploratory work focuses on Al-based MMCs for potential powertrain applications in cylinder liners, pistons, driveshaft, and connecting rods. Candidate nano additives include ceramic-based, carbon-based, and other types of functional additives. The primary objective is to synthesize purposely designed additives by the proposed solid-state processing route to determine whether they can significantly enhance the static and creep strength, wear resistance, reduced friction, and thermal conductivity of ACMZ cast alloys and 7xxx Al alloys. The synthesized materials will be tested for their materials properties and compared to key reference materials. Considerations to scale-up the technology for future development will be identified as part of the proposed work. One potential scale-up approach is friction stir AM, which is under development at ORNL for hightemperature materials for nuclear industry applications.

The Research Plan includes the following activities/steps:

- Identify and down-select potential nano additives for MMCs that may lead to creep strength, wear resistance, and thermal property improvement.

- Identify matrix materials having the most impact for powertrain applications with the proposed MMC approach. Potential candidate materials include two emerging high-strength Al alloys-ORNL's ACMZ $\mathrm{Al}$ alloy, and the $7 \mathrm{xxx}$ alloy, which is the highest strength wrought $\mathrm{Al}$ alloy for automotive applications.

- Design, engineering and processing for nano additives for simultaneous strength, wear resistance, and thermal property improvement.

- Develop and refine friction stir-based process to produce sample materials for testing and evaluation.

- Explore friction stir AM for MMC in auto powertrain applications, such as sample blocks for potential cylinder liners. 
- Characterize the microstructure, mechanical property, and functional properties on the MMC samples and baseline reference materials to determine the feasibility for property enhancement.

- Identify potential scale-up production approaches for complexity and cost.

\section{Results}

For this reporting period, we focused on developing and refining a high-throughput rapid screening method to add nano additives to the Al matrix based on the FSP and FSE process systems available at ORNL. The goal is to have a rapid screening process to produce approximately $3-4 \mathrm{in}$. long $\times 1.5-2 \mathrm{in}$. wide $\times 0.25 \mathrm{in}$. thick AMC material blocks for testing and evaluation.

The initial development of the rapid screening process has been successful. Figure I.3.5.3.1 shows the top surface appearance of the processed Al matrix with nano additives in the processed region. AA6061 and AA7075 machined chips and powders were used as the initial feedstock matrix materials, as observed in Figure I.3.5.3.2. Both ceramics- and intermetallic-based additives were evaluated in these preliminary efforts. Figure I.3.5.3.3 provides examples of progressive process refinement to achieve fully dense consolidated material blocks for property testing. The cross-sectional view of the top sample revealed holes and unconsolidated regions in the processed materials, whereas the bottom sample achieved full consolidation through process refinement.

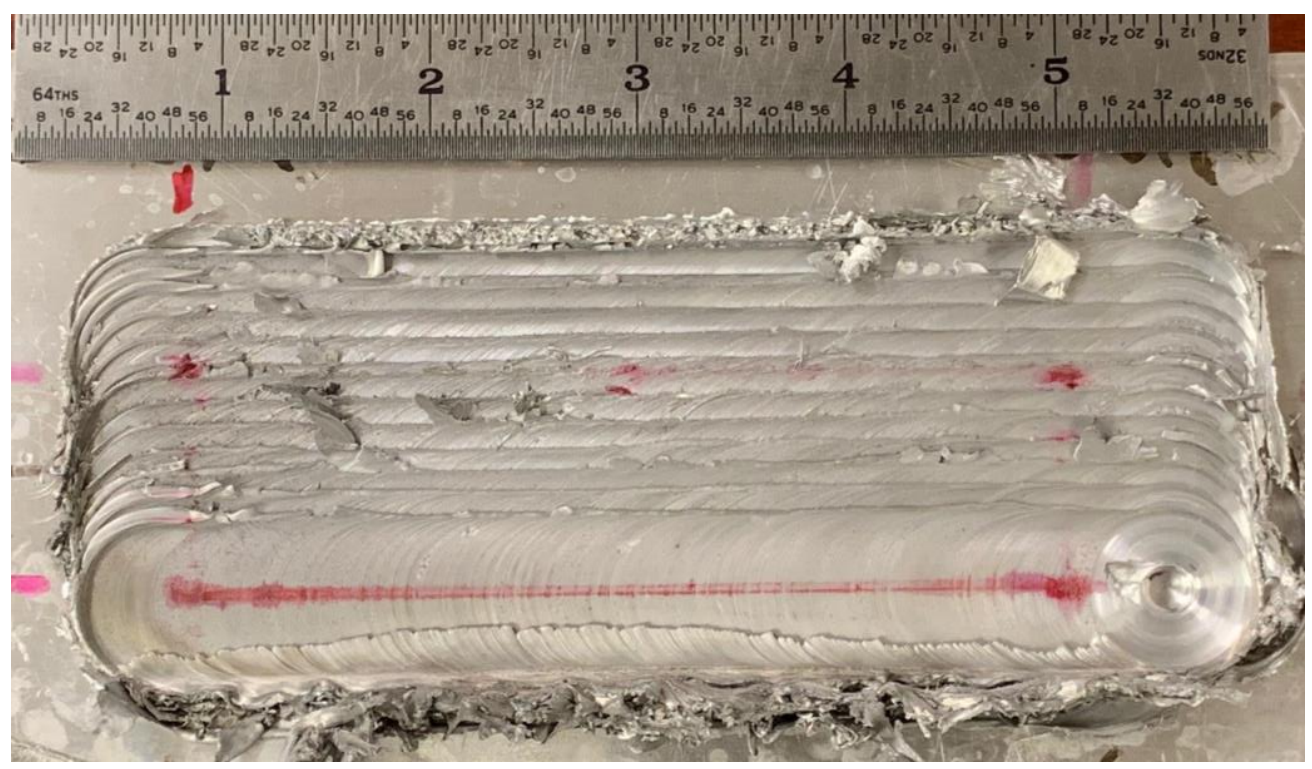

Figure I.3.5.3.1. Friction stir-processed AA6061 block with nano additives in the processed region. Source: ORNL. 


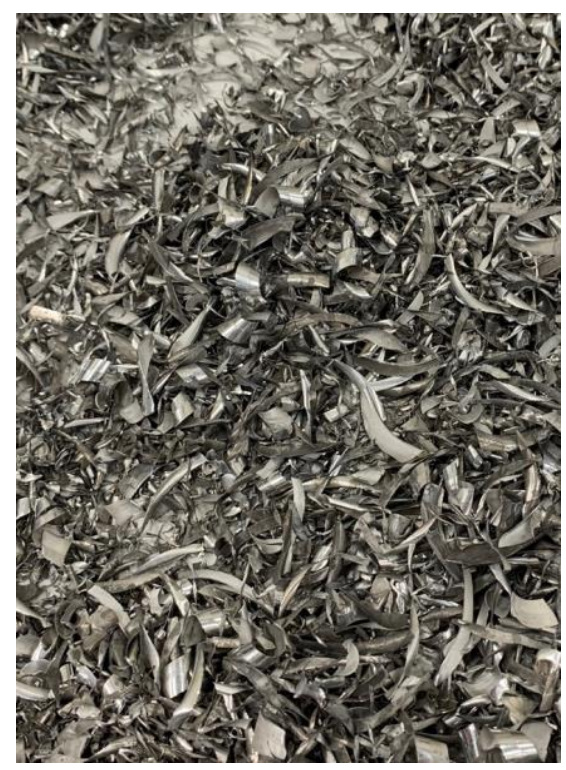

Figure I.3.5.3.2. AA7075 machined chips as feedstock material. Source: ORNL.

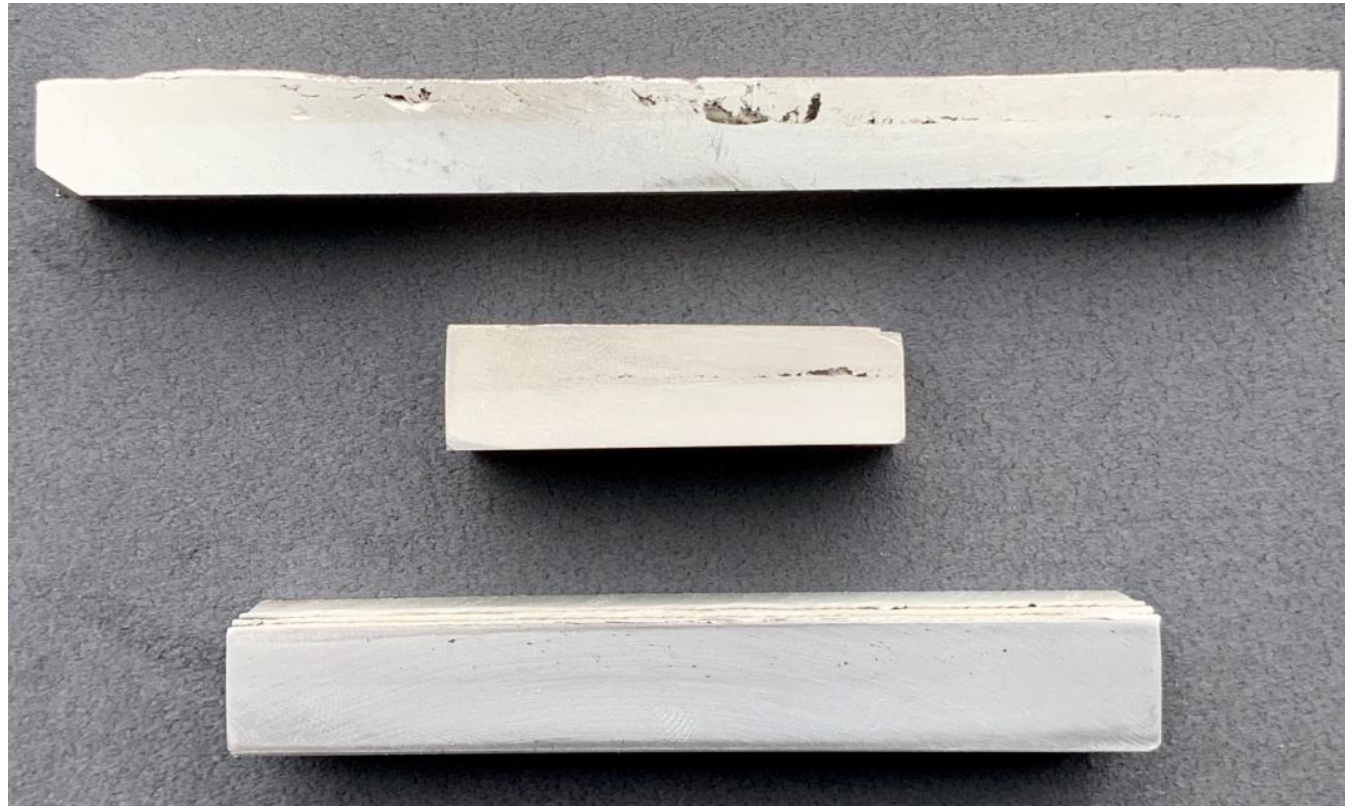

Figure I.3.5.3.3. Progressive process refinement to achieve fully dense consolidated material blocks for property testing. Source: ORNL.

The microhardness measurement results of different nano-additive Al composites from our initial experimental trials by the high-throughput FSP process are shown in Figure I.3.5.3.4. AA6061 powders were used as the feedstock material in the initial experimental trials. We note that the process is still being refined, so the results presented in the figure only represent the preliminary results obtained thus far. 


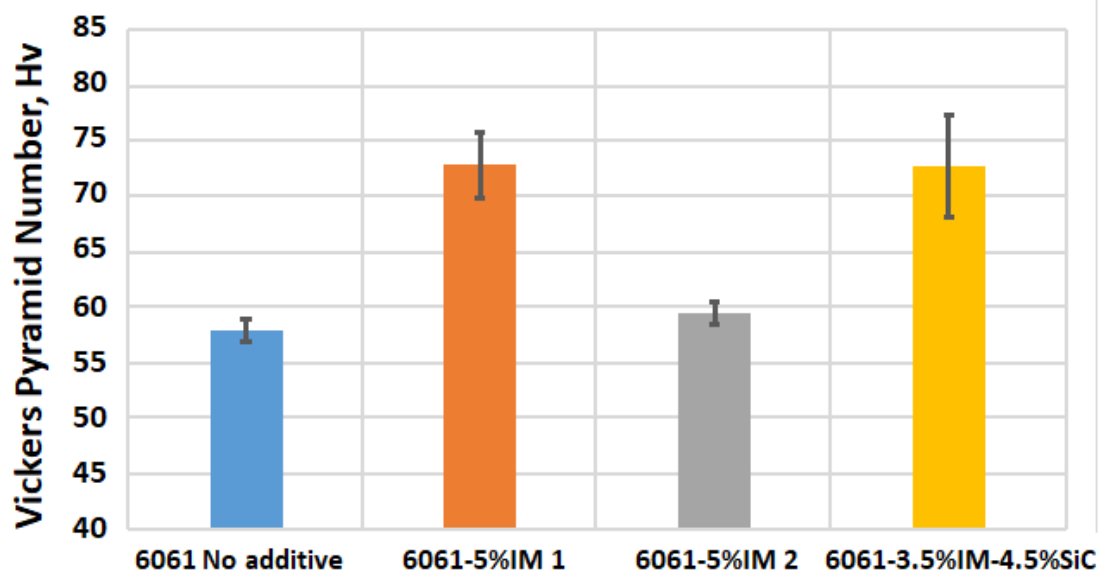

Figure I.3.5.3.4. Microhardness results by adding IMs and SiC to AA6061 matrix. Source: ORNL.

The microhardness was measured on the as-processed conditions, which is expected to be between the $\mathrm{O}$ and T4 condition. For the reference AA6061 without additives, the as-processed micro Vickers hardness was averaged at $57 \mathrm{Hv}$. With $5 \%$ of intermetallic (IM) additive, the hardness increased to $73 \mathrm{Hv}$ in one case, and $59 \mathrm{Hv}$ in the second case. The hardness increased to $72 \mathrm{Hv}$ with $3.5 \%$ of intermetallic and $4.5 \% \mathrm{SiC}$ additives. The preliminary results are promising, suggesting the feasibility of $20 \%$ to $30 \%$ increase of hardness and strength at ambient temperature.

ORNL and PNNL have maintained close collaboration during the course of the research. We collaborated on a selection of matrix materials and additives. The research at ORNL focused on the FSP/FSE high-throughput route, whereas PNNL focused on the ShAPETM route, to effectively utilize the capabilities and equipment at the two National Laboratories. ORNL also provided three different ACMZ alloy variants to PNNL as a matrix material to explore the possibility to further enhance the performance of ACMZ alloys through the addition of nano additives.

\section{Conclusions}

The research in the first 6 months of the project at ORNL has yielded promising initial results to produce AMC with nano additives through the solid-state FSP route. The progress so far has laid a foundation to further refine and improve a FSP/FSE-based solid-state processing route for high-throughput experiments. More detailed microstructure characterization and property testing are planned for the next reporting period of the project.

\section{Key Publications}

1. Feng, Z., 2019, "Novel AMCs for powertrain applications," 2019 VTO Annual Merit Review, VTO Powertrain Emerging Technologies, 10-13 June 2019, Arlington, VA, USA.

\section{References}

1. Bloch, E. A., 1961, “Dispersion-strengthened Al alloys,” Metallurgical Reviews, Vol. 6, No. 1, pp. 193-240.

2. Hunt, W. H., and D. B. Miracle, 2001, "Automotive applications of MMCs," in: Miracle, D. B, and S. L. Donaldson (Ed.), ASM Handbook, Vol. 21. OH: ASM International, pp. 1029-1032. 
3. Kouzeli, M., and A. Mortensen, 2002, "Size-dependent strengthening in particle-reinforced Al," Acta Mater., Vol. 50, pp. 39-51.

4. Feng, Z., S. A. David, and D. A. Frederick, 2011, "Friction stir method for forming structures and materials," U.S. Patent No. 8061579 B2, November 22, 2011.

5. Manchiraju, V. K., Z. Feng, A. David, and Z. Yu, 2017, "Providing plastic zone extrusion," U.S. Patent No. 9,616,497 B2, April 11, 2017.

6. Manchiraju, V. K., Z. Feng, A. David, and Z. Yu, 2014, "System for and method of plastic zone extrusion," European Patent No. EP2777837A1, October 15, 2014.

7. Manchiraju, V. K. and Z. Feng, 2015, “提供塑形区挤出 (translated to 'Providing plastic zone extrusion,"” Chinese Patent No. CN102371286B, July 29, 2015.

8. Qu, J., H. Xu, Z. Feng, D. A. Frederick, L. An, and H. Heinrich, 2011, "Improving the tribological characteristics of Al 6061 alloy by surface compositing with sub-micro-size ceramic particles via FSP," Wear, Vol. 271, No. 9-10, pp. 1940-1945.

9. Yu, Z, Z. Feng, V. Manchiraju, and S. A. David, 2013, "FSE of Al alloys," in: Marquis, F. (Ed.), Proceedings of the 8th Pacific Rim International Congress on Advanced Materials and Processing (PRICM 8), 4-9 August 2013, Waikoloa, HA, USA.

10. Yang, Y., J. Lan, and X. Li, 2004, "Study on bulk Al matrix nano-composite fabricated by ultrasonic dispersion of nano-sized SiC particles in molten Al alloy," Mat. Sci. Eng. A-Struct., Vol. 380, No. 1-2, pp. 378-383.

\section{Acknowledgements}

The principal investigator would like to recognize S. Zhang, D.A. Frederick of ORNL for their support and assistance with this project. 


\title{
I.3.5.4 High-Strength Aluminum-Graphene Composite for Powertrain System (Pacific Northwest National Laboratory)
}

\author{
Xiao Li, Principal Investigator \\ Pacific Northwest National Laboratory \\ 902 Battelle Boulevard \\ Richland, WA 99352 \\ E-mail: xiao.li@pnnl.gov
}

\section{Jerry L. Gibbs, DOE Technology Development Manager}

U.S. Department of Energy

E-mail: jerry.gibbs@ee.doe.gov

Start Date: April 1, 2019

Project Funding (FY19): \$100,000
End Date: March 31, 2020

DOE share: $\$ 100,000 \quad$ Non-DOE share: $\$ 0$

\section{Project Introduction}

The next-generation of powertrain material demands high-strength and lightweight. $\mathrm{Al}$ and its alloys, with a density of approximately one-third that of steel and cast-iron, has the potential of reducing weight by at least $40 \%$ in properly designed structures and components for powertrain systems. Barriers to the use of $\mathrm{Al}$ include limited strength, fatigue performance, lack of formability, and joining and corrosion issues. For example, the failure of an $\mathrm{Al}$ connecting rod-like the one shown in Figure I.3.5.4.1—usually called 'throwing a rod'-is one of the most common causes of catastrophic engine failure in vehicles, frequently putting the broken rod through the side of the crankcase and thereby rendering the engine irreparable.

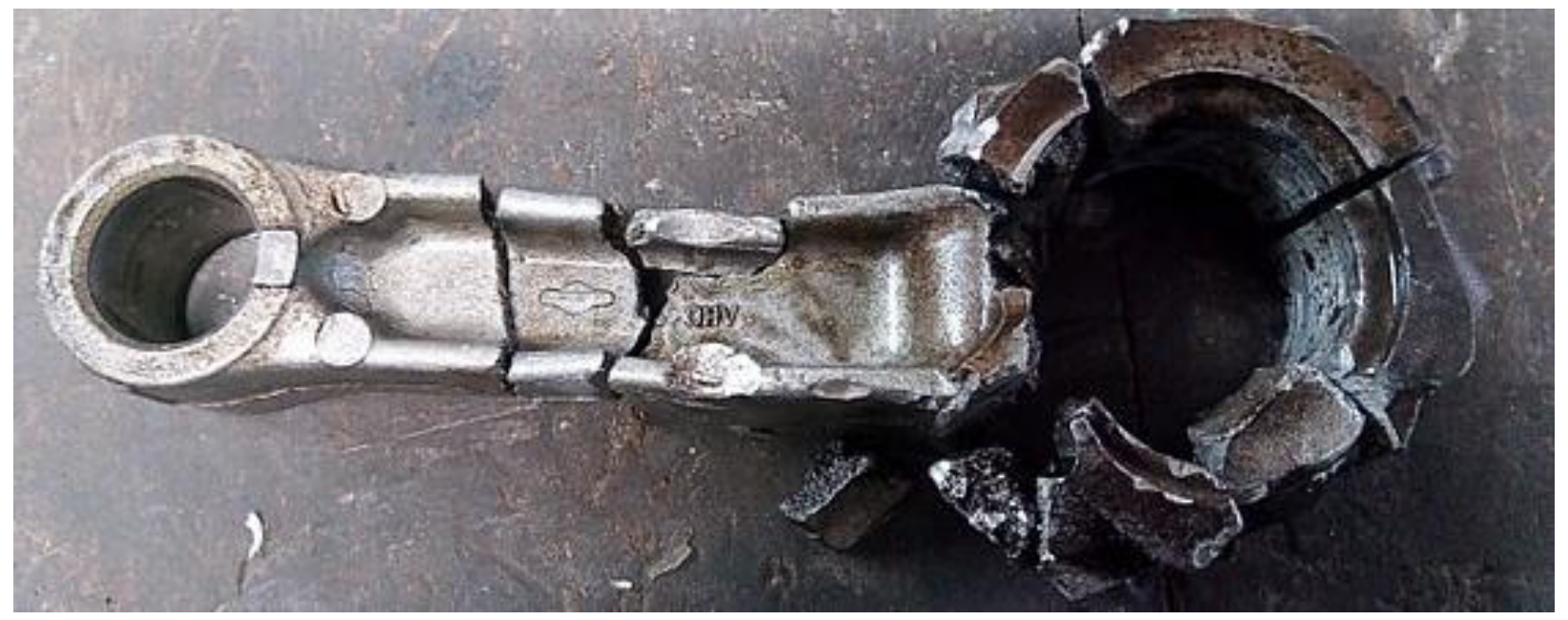

Figure I.3.5.4.1. Al connecting rod for a 4-stroke engine failed by fatigue, which subsequently impacted the crankshaft and caused catastrophic engine damage. Source: Wikipedia.

The application of an Al-graphene composite can have outstanding mechanical properties and can be a promising candidate material for next-generation powertrain components, if manufacturing methods can be developed to take full advantage of the novel composite. Recent work on manufacturing bulk size (e.g., $>100 \mathrm{~mm}$ ) graphene-reinforced metal-matrix material has been limited by two major issues: (1) an agglomeration of particles using powder metallurgy or melting process methods due to poor particle dispersion and (2) the processing temperatures being too high, and therefore, forming weak $\mathrm{Al}-\mathrm{C}$ intermetallics, which 
degrade the quality of the composite. To achieve the best mechanical properties, graphene must be homogeneously dispersed in the metal-matrix without significant detrimental thermal effects.

As part of the DOE VTO Core Powertrain Materials Program, PNNL is exploring the technical feasibility of using FSP and ShAPE - an advanced scalable form of friction extrusion - to produce AMCs for powertrain applications. Whereas FSP and ShAPETM share the same fundamental principles of solid-phase material processing described by extensive shear-dominated deformation, they can be applied differently for either selective-local reinforcing or to produce a bulk structure, respectively.

\section{Objectives}

This conceptual project is being worked on in collaboration with ORNL, which is conducting complimentary experiments using friction extrusion techniques to screen the material performance impact of adding other dispersion phases, besides carbon (C), to Al. The goal is to demonstrate the potential of using friction stirbased processes to produce AMCs with additions of graphene, other forms of $\mathrm{C}$, and other nano-sized additives, such as carbide oxide particles to significantly improve the strength of Al-based alloys under elevated temperatures relevant to powertrain applications. The goal is to achieve a minimum of $20 \%$ increase in strength at $\mathrm{RT}$ and $300^{\circ} \mathrm{C}$, as compared to the baseline $7 \mathrm{xxx}$ series alloys, or alternatively relative to ORNL's new AlCuMnZr (ACMZ alloy).

\section{Approach}

ShAPETM is a novel manufacturing process with a significant difference from the traditional process of extrusion, where the die rotates against the billet so that frictional heat softens the material, as shown in Figure I.3.5.4.2. The high-shear deformation leads to a refined grain structure and highly textured crystallographic orientation, which enables improved strength and ductility. In addition, ShAPETM perfectly addresses the two challenges discussed above; that is, (1) it is a solid-phase processing that mixes, consolidates, and extrudes material with the aid of high-shear deformation and (2) compared to melting methods, the processing temperature of ShAPE ${ }^{\mathrm{TM}}$ is much lower. Thus, thermal degradation can be largely reduced or even completely avoided.

In order to demonstrate the ability for ShAPETM to mix and homogenously distribute graphene throughout an $\mathrm{Al}$ matrix in the experimental setup, a series of Al-graphene composite wires were produced using the following procedure:

- Machined an Al alloy to a cylindrical billet with a 1 in. diameter and a 0.5 in. length.

- Drilled a pattern of holes with a 0.45 in. depth across the billets, in which the graphene nano-powder was filled, as shown in Figure I.3.5.4.3.

- $\quad$ The precursor billet was loaded into the ShAPETM machine and processed.

- Process temperature was controlled via active chilling by water applied around the billet on the container and helium gas used to increase convective heat transfer around the die face, as shown in Figure I.3.5.4.4.

- A scrolled-face die design tool with a 1 in. outside diameter and 0.1 in. hole diameter were used; thus, the extrusion ratio is 100:1, as also pictured in the inset in Figure I.3.5.4.4. 


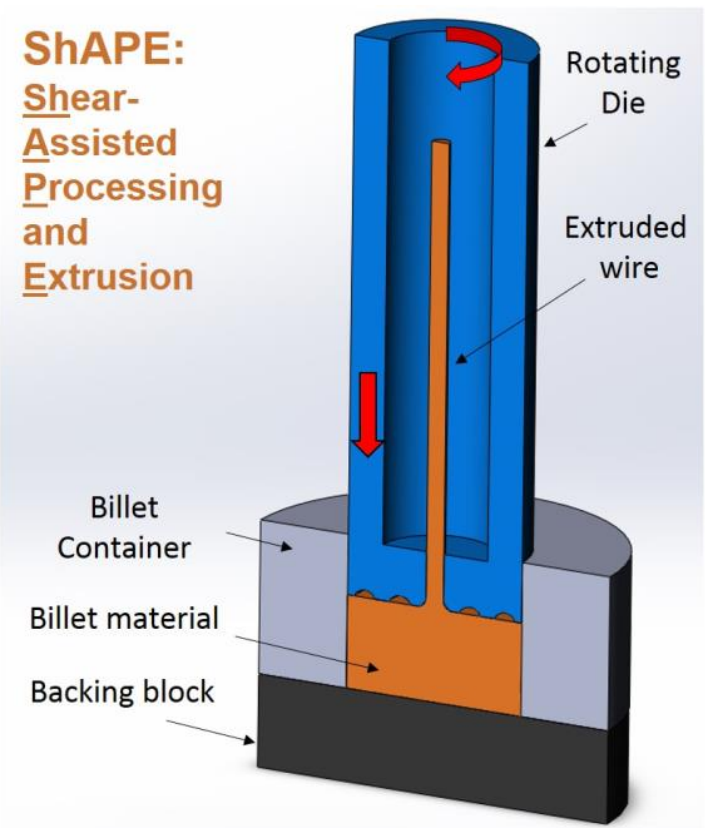

Figure I.3.5.4.2. Schematic diagram of the ShAPETM process. Source: PNNL.

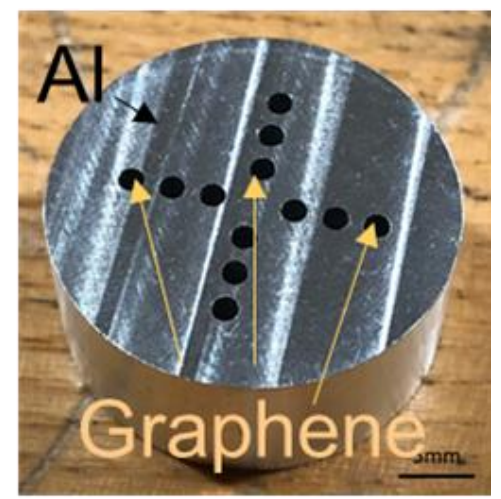

Figure I.3.5.4.3. Al billet loaded with graphene powders as a precursor of ShAPETM. Source: PNNL.

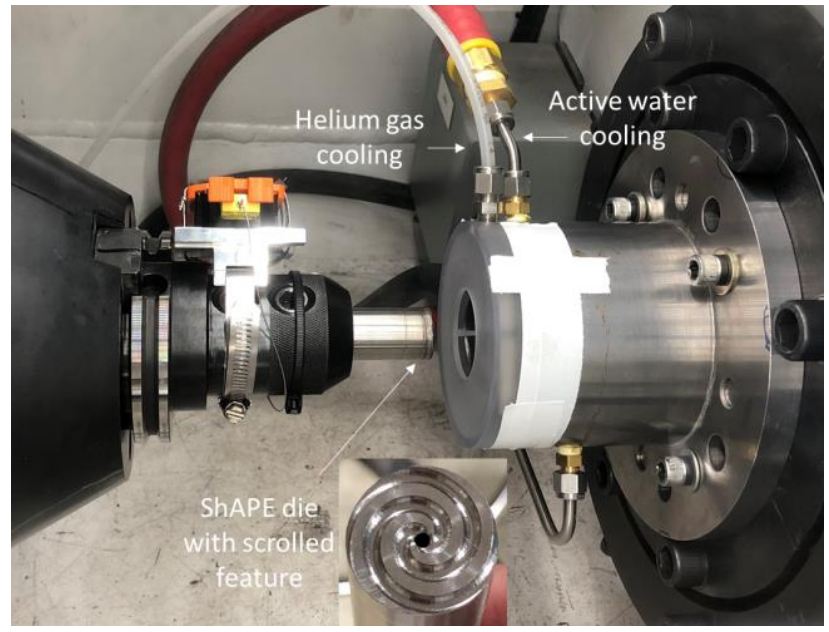

Figure I.3.5.4.4. Experimental setup of ShAPETM process. Source: PNNL. 


\section{Results}

Through a design of experiment approach, effective processing parameters were determined to be as follows:

- $\quad$ ACMZ alloy - $150 \mathrm{rpm}$ die rotational speed and $4 \mathrm{~mm} / \mathrm{min}$ die feed rate.

- $\quad$ AA7075 - $100 \mathrm{rpm}$ die rotational speed and $1 \mathrm{~mm} / \mathrm{min}$ die feed rate were selected.

High quality Al-graphene composite wires with about a 1 meter length, sound straightness, and a smooth surface were produced as can be seen in Figure I.3.5.4.5 (a).

The cross-sections of the composite wires were inspected by optical microscope. The results indicate the wires are void-free, uniform, and have no obvious phase segregation. Figure I.3.5.4.5 (b) shows the cross-section of the AA7075-G ShAPETM processed wire, as an example.

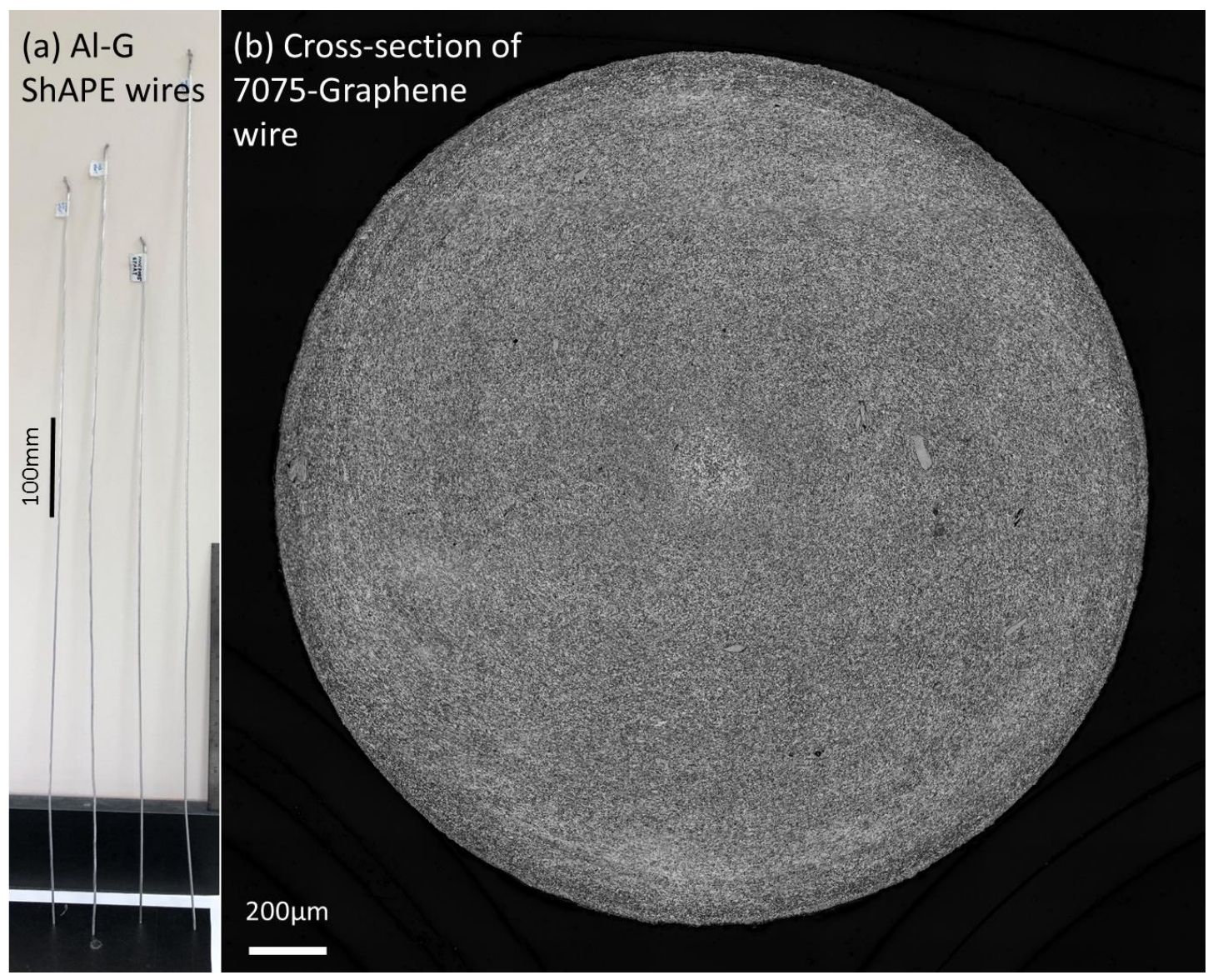

Figure I.3.5.4.5. (a) Al-graphene composite wires produced by ShAPETM. (b) A cross-section of 7075-graphene ShAPE'M wire. Source: PNNL.

Electron microscopy was utilized to further analyze the distribution of the graphene in the composite wire. Figure I.3.5.4.6 (a) is a backscatter electron image; the grey area is $\mathrm{Al}$ and white regions are copper in the ACMZ alloy. The EDS image is shown in Figure I.3.5.4.6 (b) from the same area, which indicates the graphene addition was very uniformly dispersed in the metal-matrix. In addition, the grain size of AA7075 was refined to $\sim 7 \mu \mathrm{m}$, while the particle size of the graphene powder was refined to submicron. 


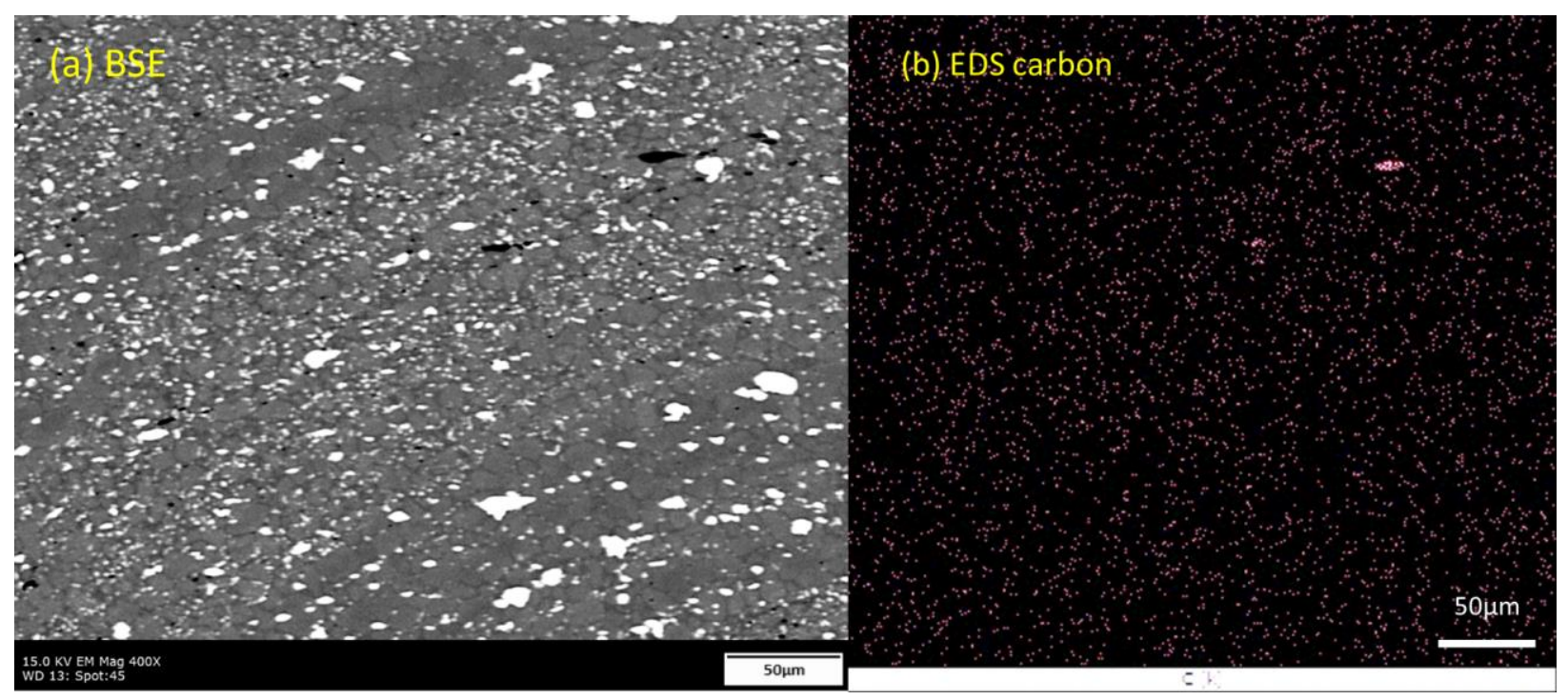

Figure I.3.5.4.6. (a) Backscatter electron image of Al-graphene ShAPETM wire. (b) EDS image shows uniform carbon dispersion. Source: PNNL.

Figure I.3.5.4.7 shows the die face temperature at the location where shear deformation occurs, which is considered to be the peak temperature achieved during processing. This temperature profile confirms adequate thermal management was achieved, while the processing temperature of ShAPETM was maintained in the $350-400^{\circ} \mathrm{C}$ range, which is much lower than that of other compositing processes, e.g., melting $\left(>600^{\circ} \mathrm{C}\right)$ or sintering $\left(450-600^{\circ} \mathrm{C}\right)$. Thus, the quality of the graphene is maintained, and the formation of brittle Al-carbides is avoided. Further analysis of the $\mathrm{Al}$-graphene composite product is ongoing.

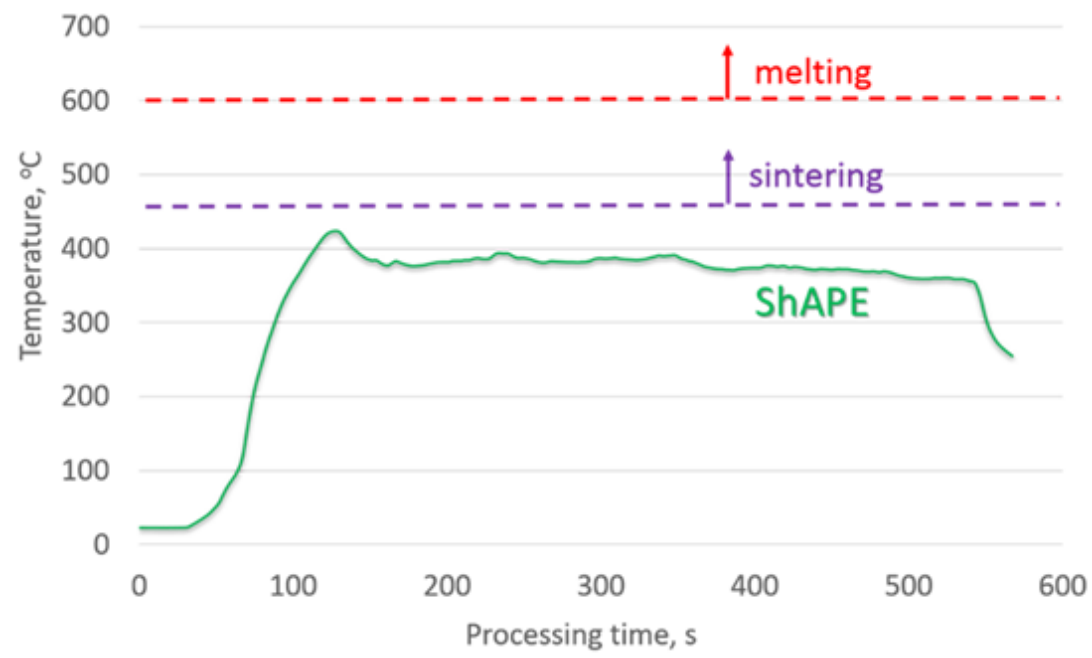

Figure I.3.5.4.7. ShAPE-processing temperature of making 7075-G composite, as compared to other processes. Source: PNNL.

\section{Conclusions}

ShAPE ${ }^{\mathrm{TM}}$ can successfully produce an Al-graphene composite by mixing and extruding an Al alloy with graphene via high-shear deformation. The high-shear deformation not only uniformly disperses the addition material, but also leads to refined grain structure and highly textured crystallographic orientation, which enables outstanding strength and ductility. 
With the successful completion of the concept trials, it was demonstrated that void-free uniformly dispersed AGC can be achieved by ShAPE ${ }^{\mathrm{TM}}$. Ongoing analysis is characterizing the mechanical properties of the composite material at RT and elevated temperature to prove strength improvement. Further inspection of phase identification will prove definitively that $\mathrm{Al}-\mathrm{C}$ formation, and other intermetallics, were avoided during the process.

Future proposed efforts aim to experimentally investigate the effect of: (1) the processing parameters; and (2) the type of graphene on the performance of the composite.

\section{Key Publications}

1. Li, X., 2019, "ShAPE: A novel solid-phase processing of making bulk size metal-matrix-composite without agglomeration," Multifunctional Ceramic and MMCs: Processing, Microstructure, Properties and Performance, Materials Science \& Technology 2019, 29 September - 3 October 2019, Portland, OR, USA.

\section{Key Publications}

1. Li, X., D. Baffari, and A. P. Reynolds, 2018, "Friction stir consolidation of Al machining chips," Int. J. Adv. Manuf. Tech., Vol. 94, No. 5-8, pp. 2031-2042.

2. Hwang, J., T. Yoon, S. H. Jin, J. Lee, T.-S. Kim, S. H. Hong, and S. Jeon, 2013, "Enhanced mechanical properties of graphene/copper nanocomposites using a molecular-level mixing process," Adv. Mater., Vol. 25, No. 46, pp. 6724-6729.

3. Sun, Y., C. Zhang, B. Liu, Q. Meng, S. Ma, and W. Dai, 2017, "Reduced graphene oxide-reinforced 7075 AMCs: Powder synthesis and mechanical properties," Metals, Vol. 7, No. 11, Art. \#499.

4. Renguo, G., L. Chao, Z. Zhanyong, C. Runze, and L. Chunming, 2012, "Study on preparation of graphene and Al-graphene composite," Rare Metal Mat. Eng., Vol. 41, pp. 607-611. 


\section{I.3.5.5 Development of High-Temperature Sample Environment for Advanced Alloy Characterization Utilizing High-Speed, Micron Resolution X-ray Imaging Techniques (Argonne National Laboratory)}

\section{Andrew Chuang, Principal Investigator}

Advanced Photon Source

Argonne National Laboratory

9700 S. Cass Ave.

Lemont, IL 60439

E-mail: cchuang@anl.gov

\section{Jerry L. Gibbs, DOE Technology Development Manager}

U.S. Department of Energy

E-mail: jerry.gibbs@ee.doe.gov

Start Date: February 1, 2019

Project Funding (FY19): \$100,000
End Date: September 30, 2020

DOE share: $\$ 100,000$
Non-DOE share: $\$ 0$

\section{Project Introduction}

The High-Energy X-Ray Microscope (HEXM) is a long beamline that is approved and will be built as a part of the Advanced Photon Source Upgrade (APS-U) project. The APS-U project will increase the brightness of the APS high-energy X-ray beams by two orders of magnitude, enabling researchers to make groundbreaking discoveries and innovations that create new products and industries. The HEXM will investigate structure and evolution within bulk materials, often in extreme environments, with both the enhanced high-energy x-ray scattering techniques used today (e.g., at 1-ID of the APS) and with novel coherence-based techniques enabled by the low-emittance APS-U multi-bend-achromat lattice.

Advanced material characterization plays an important role in the alloy development process, as observed in Figure I.3.5.5.1. It allows researchers to connect process parameters to performance by providing detailed information on the underlying microstructure. Among existing techniques, high-energy X-ray imaging has the unique combination of being fast, nondestructive, multiscale ( $\mathrm{mm}$ to submicron), and well-suited to in situ studies, such as mechanical and thermal loading. The context for this research is in situ studies of microstructure evolution (i.e., defect/secondary phase formation and/or annihilation) in select engineering alloys at elevated temperatures by high-energy X-ray imaging techniques. In order to have mechanistic understanding of the microstructures that govern the performance of materials at the mesoscale, we need direct observation of microstructure changes under operating conditions (at elevated temperatures in this case). In order to perform theoretical computations to model such processes, we need both images of the secondary phases and porosity, and microstructure information (e.g., crystal structure, texture). The goal is for real-time monitoring of the microstructure with submicron resolution at service environments (in terms of temperature and chemistry). The project aims to develop characterization capability at high-temperature for the material development community. 


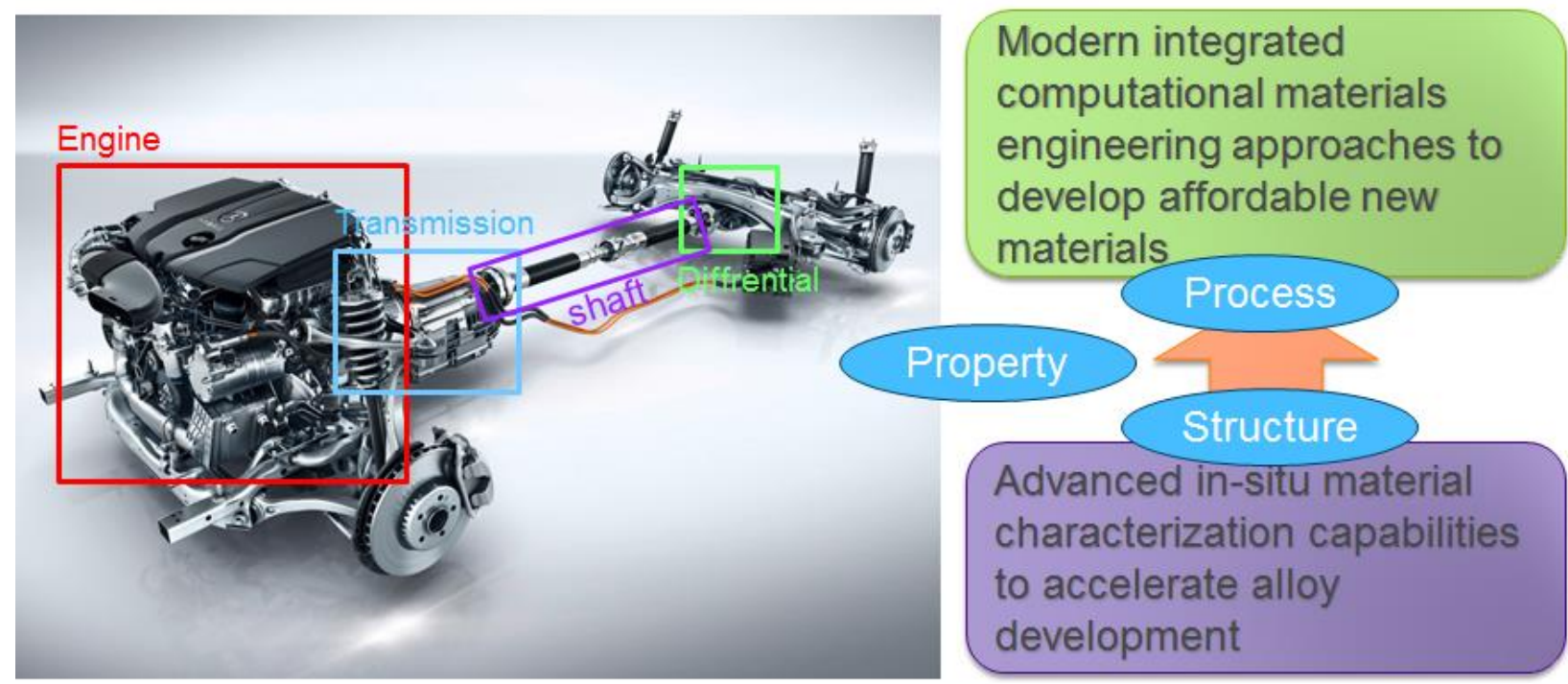

Figure I.3.5.5.1. Advanced in situ material characterization helps the accelerating alloy development process for automotive applications. Source: ANL.

\section{Objectives}

The objective of this work is to develop a high-temperature sample environment for use in a new high-energy $\mathrm{X}$-ray beamline that provides zoom-in and zoom-out X-ray imaging capability of mm-sized specimens with resolutions down to $100 \mathrm{~nm}$. This novel in situ capability will enable studies of time-dependent material phenomenon - including rapid heating and cooling - and generate vital data required for alloy development and performance improvement. The proposed system will utilize induction heating technology that can precisely and rapidly control the heating and cooling process of a specimen. In addition, its high-heating efficiency minimizes the heat load to the environment, which will significantly improve the thermal stability of the system end enable submicron spatial resolution of the image. The developed setup will improve accessibility and flexibility of the sample environment, avoid duplicate efforts within the community, enhance scientific productivity, and enable the expansion of in situ testing capabilities.

\section{Approach}

In this project, we developed a high-temperature environmental cell that can be used in conjunction with a high-resolution full-field X-ray imaging instrument, which is part of the newly constructed HEXM beamline in the APS-U project. The imaging instrument will use 40 120 keV synchrotron X-rays to provide the real-time zoom-in and zoom-out capability to study materials at different length scales (e.g., from millimeter to submicron). The spatial diffraction limit for the imaging system is expected to be $100 \mathrm{~nm}$ at the sample. The instrument can zoom-out to characterize the material with up to a $4 \mathrm{~mm}$ large field of view and resolution of $\sim 2 \mu \mathrm{m}$. After identifying the region of interest, one can zoom-in to study the detailed features with resolution down to $\sim 100 \mathrm{~nm}$. The zoom-in-out imaging setup, as illustrated in Figure I.3.5.5.2, is designed for RT measurement. However, applications of many propulsion materials are for a high-temperature environment. To study the material in its service environment, a high-temperature sample environment is necessary. 


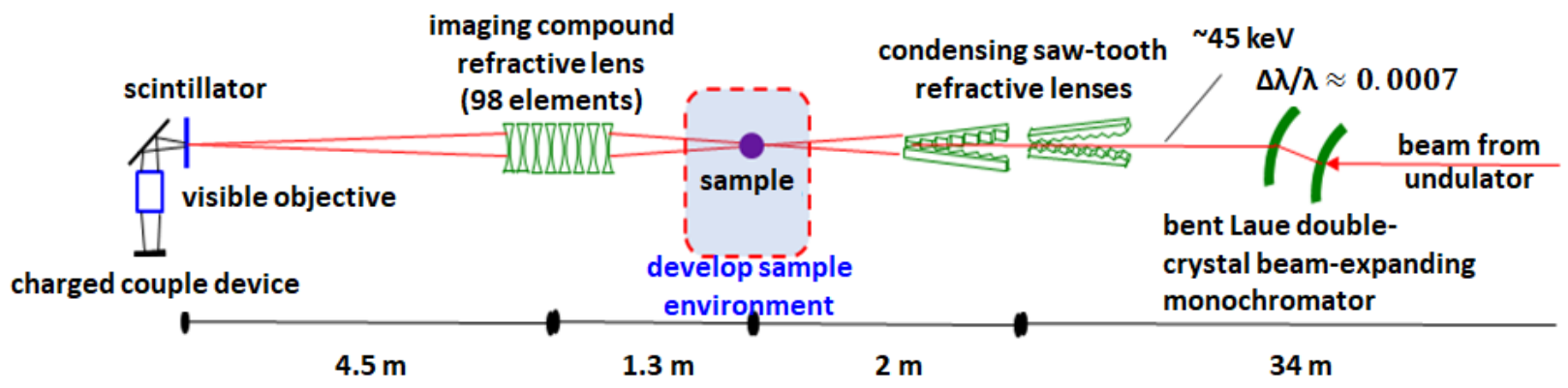

Figure I.3.5.5.2. Schematic diagram of zoom-in-out imaging setup for the future HEXM beamline (APS-U project). The project will develop a high-temperature environment for the imaging setup. Source: [1].

The current furnace available at 1-ID-E of the APS can provide temperatures up to $1100^{\circ} \mathrm{C}$. However, it is based on resistive heating, which generates significant heat load to the environment. The heat load affects the resolution of images by requiring large sample-detector distances and thermal instability to the entire imaging setup. To partially combat this, sophisticated cooling devices are used to protect the surrounding equipment, such as detectors and stages, which increases the complexity of the experiment, but does not entirely eliminate instabilities. The newly proposed furnace will utilize induction-based heating to provide energy-efficient, noncontact heating and heat up the specimen only, thus minimizing the heat load to the environment. Since highresolution imaging equipment developed in HEXM is very sensitive to the temperature of the environment, the minimized heat load will significantly increase the thermal stability of the apparatus and improve image quality. The proposed induction-based heating station can also provide precise temperature control with a highheating rate through a closed-loop temperature controller and increase the temperature limit to $1400^{\circ} \mathrm{C}$. The temperature measurement can be conducted through a thermocouple attached to the specimen or using a noncontact high-speed FLIR thermal camera, or perhaps both. A sample environment chamber will also be developed, based on a quartz container, to allow heating to be operated in a variety of relevant environments including air, inert gas, and vacuum.

\section{Results}

To demonstrate the concept of using a susceptor for induction heating, we fabricated the first prototype using an Inconel-718 tube and were able to push the temperature to $1300^{\circ} \mathrm{C}$ with a heating rate of $30^{\circ} \mathrm{C} / \mathrm{sec}$. Subsequently, a closed-loop temperature control system (Proportional-Integral-Digital control) was developed that provided the capability of having a programmable temperature profile. The integration of the setup to the existing beamline control interface and the functionality of the system was demonstrated in March 2019 for a user experiment studying spinodal behavior of $\mathrm{Cr}$-Fe alloy at $1250^{\circ} \mathrm{C}$.

During the test of the first setup, it was found that a reliable and durable susceptor is required for temperatures $>1000^{\circ} \mathrm{C}$. The Inconel-718 used in the first prototype was severely oxidized and degraded after several hours of use at temperatures $>1200^{\circ} \mathrm{C}$. In search of better susceptor material, $\mathrm{SiC}$ was selected due to its exceptional properties at high temperatures up to $1600^{\circ} \mathrm{C}$ and compatibility with induction coils. The heating and cooling characteristics of the $\mathrm{SiC}$ material have been tested, as shown in Figure I.3.5.5.3. We have tested combinations of different shapes and sizes of the material, as well as the coil geometries. It was demonstrated that the temperature can reach up to $1300^{\circ} \mathrm{C}$ in ambient atmosphere and the material remains intact without any sign of degradation or oxidation. 

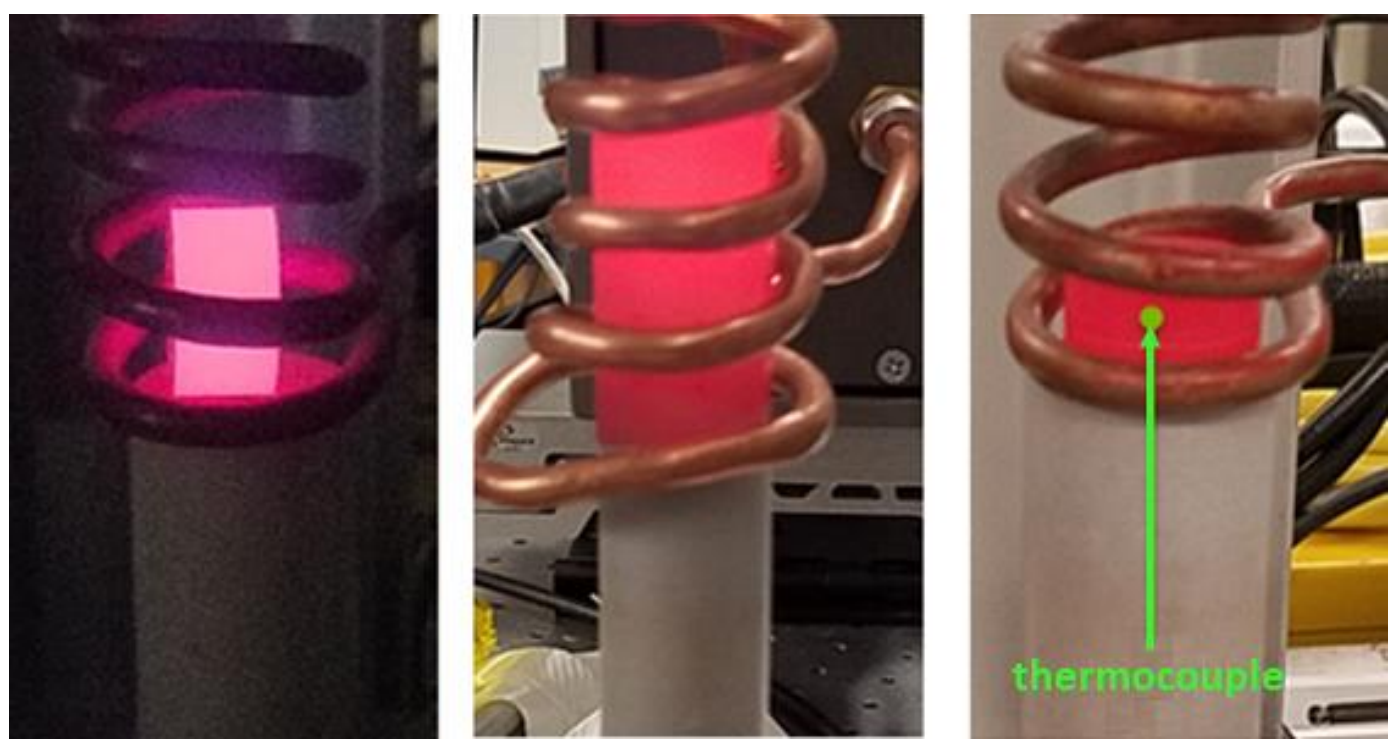

Figure I.3.5.5.3. Test of heating characteristic of SiC under various dimensions and coil geometry. Source: ANL.

A 3D model of the second prototype is shown in Figure I.3.5.5.4. The device consists of a SiC susceptor and a ceramic tube to hold the susceptor in place. There are holes on the side of the wall to clear the path for incident and diffracted X-ray. The center through hole is designed to insert a sample into the center of the heating cell. A lock pin is used to position the cell in a fixed orientation. Four turn coils were used for the setup.

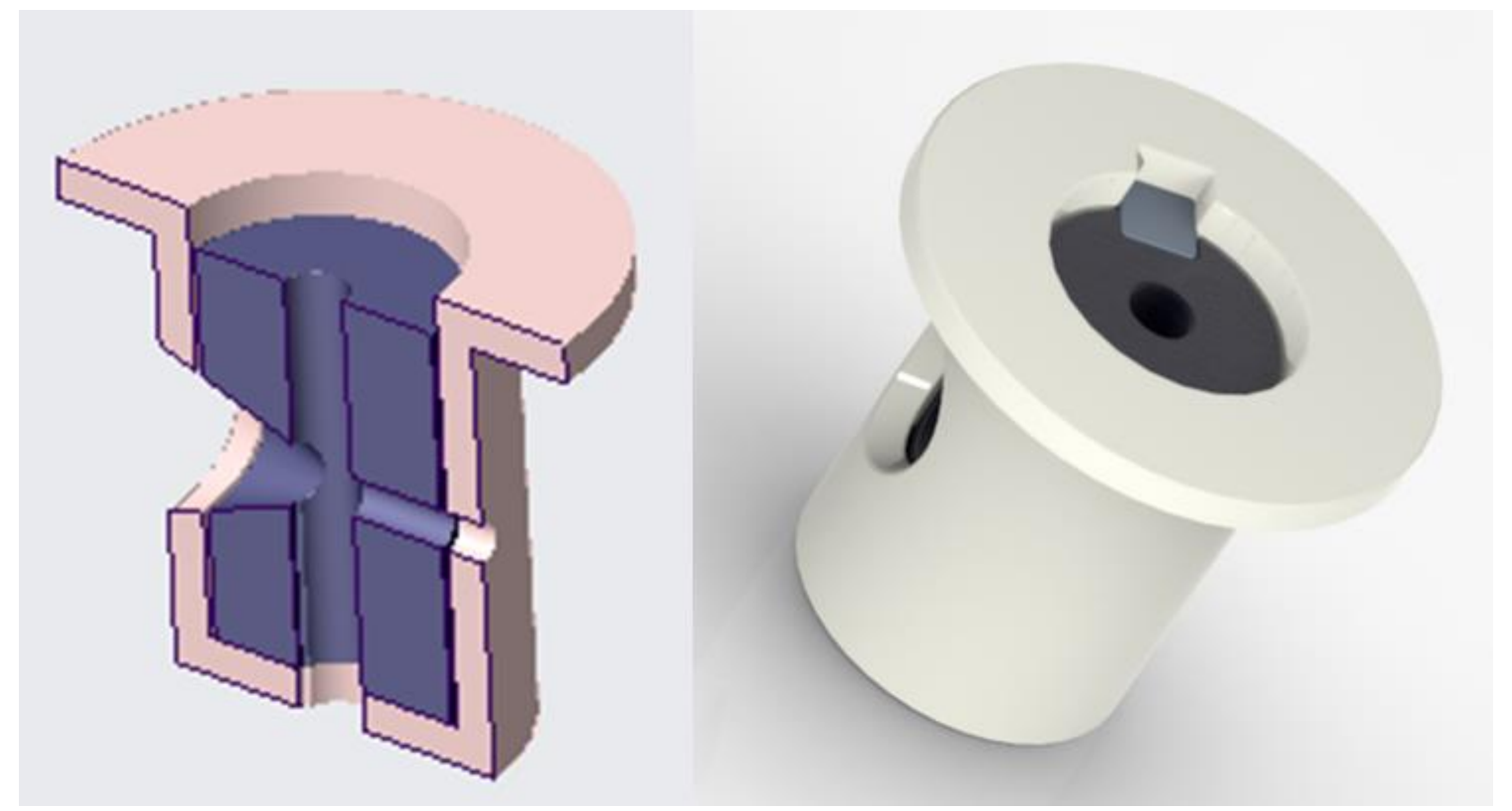

(a)

(b)

Figure I.3.5.5.4. (a) 3D model of high-temperature cell. (b) The setup consists of a SiC susceptor and ceramic holder. Source: ANL.

Heating and cooling characteristics of the device are shown in Figure I.3.5.5.5. The highest temperature tested in the laboratory was $1300^{\circ} \mathrm{C}$, but the system has the potential to go up to $1550^{\circ} \mathrm{C}$ with the full capacity of the power supply at the beamline. Figure I.3.5.5.5 (a) shows the temperature profiles recorded using a FLIR SC6100 infrared camera in three locations and a k-type thermocouple in one location. The heating rate during 
the test can reach $15^{\circ} \mathrm{C}$ per second above $800^{\circ} \mathrm{C}$ when the temperature increment is $<150^{\circ} \mathrm{C}$ as shown in Figure I.3.5.5.5 (b). The temperature stability is within $\pm 2{ }^{\circ} \mathrm{C}$ when the temperature is $>1000^{\circ} \mathrm{C}$. Due to the low thermal mass of the system (i.e., only the susceptor is being heated), the whole system cools very fast when the power is turned off. The cooling rate can reach $20 \sim 25^{\circ} \mathrm{C} / \mathrm{sec}$ under natural cooling condition. To push the cooling rate higher, a forced-cooling system (e.g., fan) can be added. The commissioning of the system with the real X-ray experiment to characterize the image stability and quality is scheduled in 2019 Q4 at 1-ID beamline of the APS.

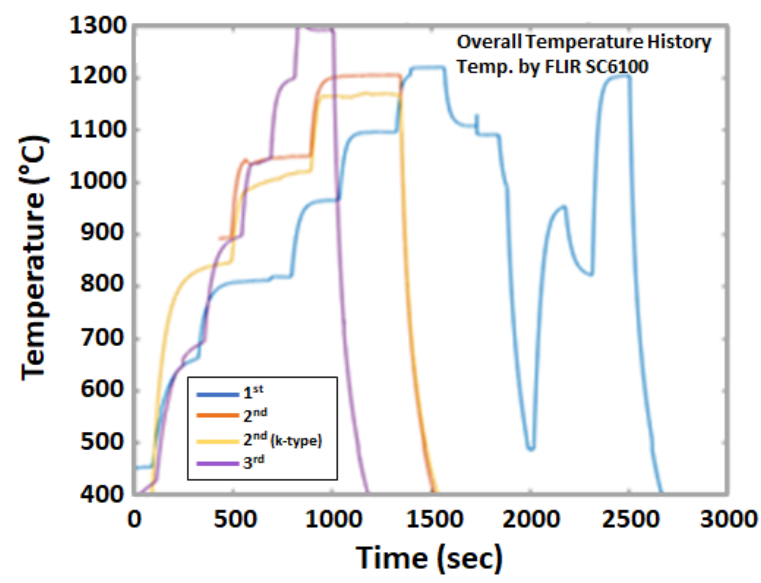

(a)

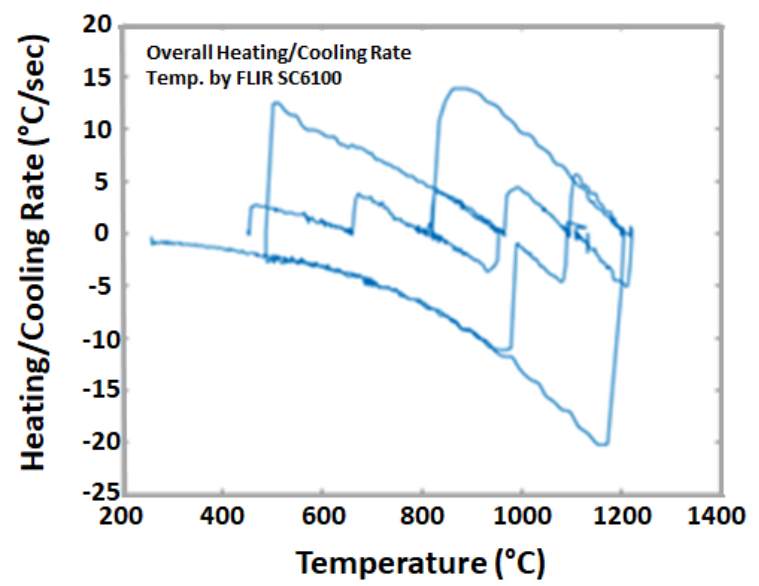

(b)

Figure I.3.5.5.5. (a) Overall temperature profile and (b) heating and cooling rates that are characteristic of the hightemperature cell. Source: ANL.

\section{Conclusions}

We have successfully developed a high-temperature heating cell that is capable of reaching $1400^{\circ} \mathrm{C}$ with heating and cooling rates of $25^{\circ} \mathrm{C}$ per second. The system utilizes the induction heating technology and ceramic susceptor to heat both conductive and non-conductive materials. It can operate in ambient atmosphere and provide a flexible heating capability for $\mathrm{x}$-ray imaging experiments. The system will be commissioned by early 2020 and become available to serve the material development community.

\section{Key Publications}

1. Chuang, A., and P. Kenesei, 2019, "High-temperature cell for high-energy X-ray imaging applications," in preparation.

\section{References}

1. Shastri, S. D., P. Kenesei, and R. M. Suter, 2015, "Refractive lens based full-field x-ray imaging at 45-50 keV with submicron resolution,” Proc. SPIE, X-Ray Nanoimaging: Instruments and Methods II, Vol. 9592, 95920X (18 September 2015).

\section{Acknowledgements}

The author would like to acknowledge the members of ANL's APS Material Physics and Engineering Group, X-ray Science Division, for their assistance during the development of the device. He also thanks Dr. Dileep Singh of ANL's Applied Materials Division for his fruitful discussion and guidance during the project. This project used resources of the APS, a DOE Office of Science User Facility, operated for the DOE Office of Science by ANL under Contract No. DE-AC02-06CH11357. 


\title{
II Lightweight Materials
}

\section{II.1 Automotive Metals}

\section{II.1.1 Non-Rare Earth Magnesium Bumper Beam (Pacific Northwest National Laboratory/Magna International CRADA)}

\author{
Scott Whalen, Principal Investigator \\ Pacific Northwest National Laboratory \\ 902 Battelle Blvd. \\ Richland, WA 99354 \\ E-mail: scott.whalen@pnnl.gov

\section{Tim Skszek, Co-Principal Investigator} \\ Magna International \\ 750 Tower Dr. \\ Troy, MI 48098 \\ E-mail: $\underline{\text { tim.skszek@magna.com }}$
}

\section{Sarah Kleinbaum, DOE Technology Development Manager}

U.S. Department of Energy

E-mail: sarah.kleinbaum@ee.doe.gov

Start Date: January 1, 2019

Project Funding (FY19): \$2,005,000
End Date: September 30, 2022

DOE share: $\$ 1,005,000 \quad$ Non-DOE share: $\$ 1,000,000$

\section{Project Introduction}

A lightweight bumper beam made from $\mathrm{Al}$ or $\mathrm{Mg}$ offers a $25 \%$ to $60 \%$ weight-savings, respectively, as compared to a high-strength steel state-of-the-art component. For a new Cooperative Research and Development Agreement (CRADA) between the PNNL and Magna International, this lightweight bumper beam was chosen as the component to target because its geometry is fairly simplistic when demonstrating direct manufacturing capability utilizing a novel advanced extrusion process developed by PNNL called ShAPE. The bumper component offers commercial value by providing mass reduction in the front of the vehicle along with ride and handling benefit.

Preliminary experiments performed at PNNL demonstrated the ability to ShAPETM process non-RE Mg tubing with high-strength, ductility, and energy absorption. ShAPETM has shown that extruded ZK60 and AZS312 tubing with a 0.30-in diameter and 0.03-in wall thickness have a similar energy absorption to AA6061-T6, which can be attributed to significant grain refinement, breakdown, and dispersion of second phases, as well as the ability to eliminate anisotropy in compressive/tensile strength [1-3]. This enables $\mathrm{Mg}$ to be considered as an option for further mass reduction, beyond what can be achieved with $\mathrm{Al}$; however, early market penetration using $\mathrm{Al}$ is a near-term opportunity. Al bumpers represent approximately $54 \%$ of the North American lightduty vehicle market. Should this research effort be successful in providing a 30\% mass reduction at equal cost relative to $\mathrm{Al}$ bumpers, it is anticipated that a ShAPETM processed $\mathrm{Al}$ bumper could reach $25 \%$ market penetration over the next five years and 50\% market share by 2030. Since Magna International, a tier one supplier, currently produces nearly all of the Al bumpers sold into the North American market, there is substantial opportunity to successfully commercialize this technology based on equal performance, reduced mass, and lower-cost equivalent bumpers. 


\section{Objectives}

The goal of this Magna/PNNL CRADA is to demonstrate the feasibility of using PNNL's ShAPETM technology for producing tubular extrusions, which are typically used in the fabrication of automotive subassemblies such as bumper beams.

\section{Approach}

The scope of this work focuses on two discrete thrusts: (1) using the ShAPETM process on Al to determine the process rate potential and limits; and (2) using the ShAPETM process on Mg for demonstrating the feasibility of producing a rectangular cross-sectional extrusion. Applicable time will also be dedicated to the design and development of die sets and process parameters for each extrusion material- $\mathrm{Mg}$ and Al. PNNL and Magna will perform microstructural analysis and mechanical property testing of the extrusions in order to assess the quality of extruded products and structural attributes. Figure II.1.1.1 provides a close-up view of PNNL's ShAPETM machine, as well as an example of extruded ZK60 tubing.

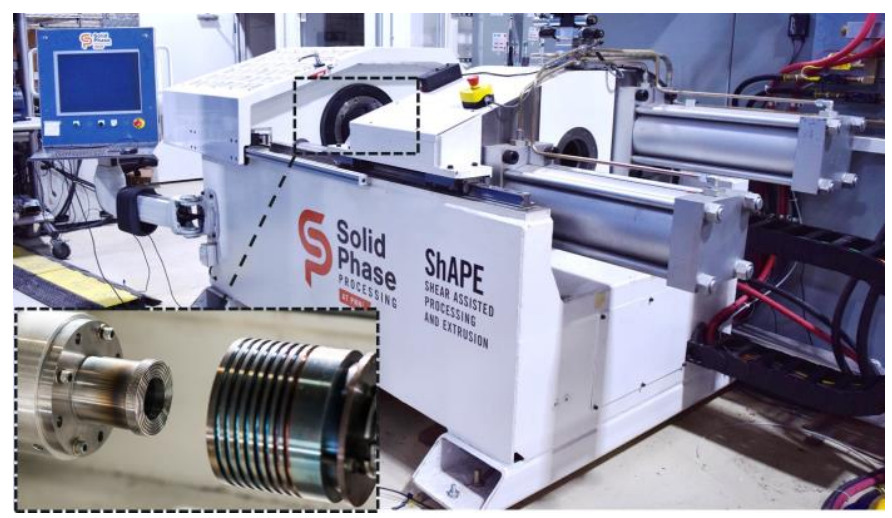

(a)

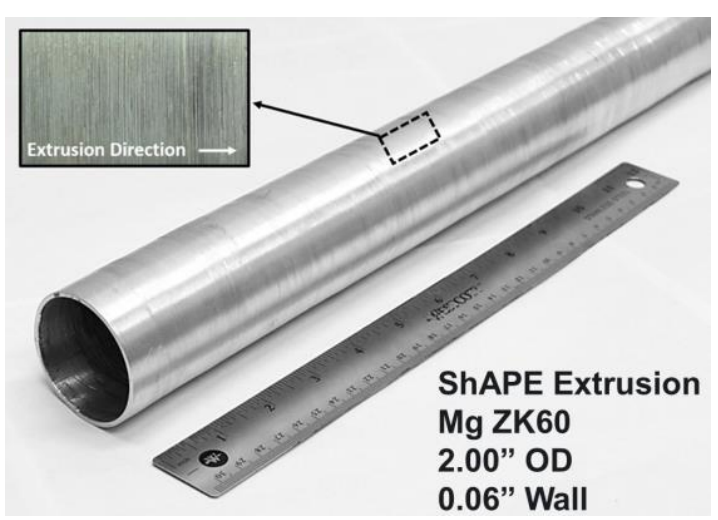

(b)

Figure II.1.1.1. (a) ShAPETM machine installed in PNNL's Solid-Phase Processing Laboratory and (b) thin-walled ZK60 circular tubing extruded by ShAPETM. Source: PNNL.

An initial work scope was established that focused on adapting existing tooling and process parameters to produce several rectangular cross-sections, going beyond what had been achieved and reported on previously [4]. To accomplish this, a ShAPE ${ }^{{ }^{T M}}$-compatible portal bridge die design approach was developed to fabricate rectangular profiles. Preliminary ShAPETM trials were performed, which is reported in the Results section.

Subsequent to the start of the project, the work scope was expanded to include ShAPETM on Al to study the rate at which ShAPETM can produce extrusions. The selection of $\mathrm{Al}$ over $\mathrm{Mg}$ for this thrust is motivated by potential opportunities for early ShAPETM technology adoption into Al bumper beams or similar Al components, while $\mathrm{Mg}$ tubular components are viewed as a longer-term potential and will likely require additional maturity before commercial applications will be ready. Both $\mathrm{Al}$ and $\mathrm{Mg}$ ShAPETM thrusts will be pursued in this project.

\section{Results}

During the initial proof-of-principal phase for this project, PNNL performed preliminary design work (i.e., FE modeling simulations and experimental material flow experiments) on a portal bridge die approach that could be integrated into the ShAPETM machine for extruding a 0.5 -in $x$ 0.5-in square cross-section tubing with 0.06in wall thickness. Figure II.1.1.2 shows an image of a ZK60 billet mounted on the rotating spindle of the ShAPETM machine within a clamshell assembly, which acts as the container akin to that found in conventional extrusion processes. This billet/container assembly is rotated and plunged into a specially designed portal bridge die. An internally cooled, two-piece die assembly was designed with scrolled features on the die face to 
plasticize the leading edge of the billet and encourage material flow through four distinct portals. Softened material flows down though these portals into a weld chamber where the four individual streams forge together, and then extrude through an orifice around a square mandrel. An image of the preliminary die set is shown in Figure II.1.1.3. The result of an initial extrusion trial confirmed that the portal bridge die was effective at: (1) separating the material into four distinct streams; and (2) recombining the streams within the weld chamber, as shown in Figure II.1.1.4. Compare Figure II.1.1.3 (c) with Figure II.1.1.4 to see the material that re-combined within the weld chamber.

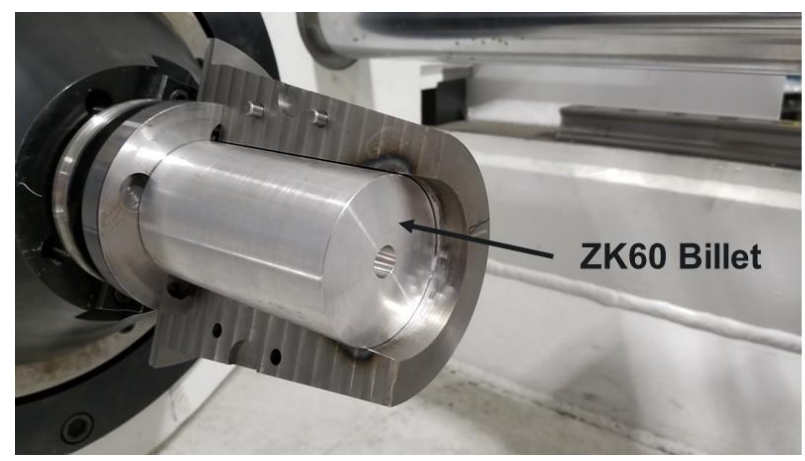

(a)

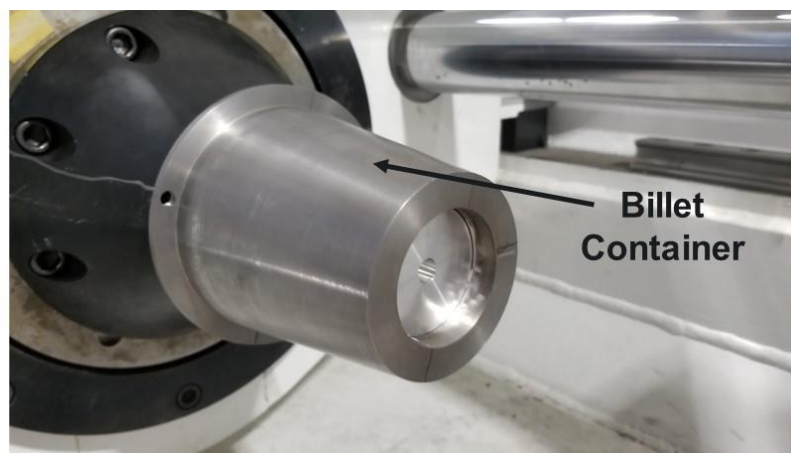

(b)

Figure II.1.1.2. (a) ZK60 billet mounted in rotating spindle of ShAPETM machine and (b) with the clamshell container assembly. Source: PNNL.

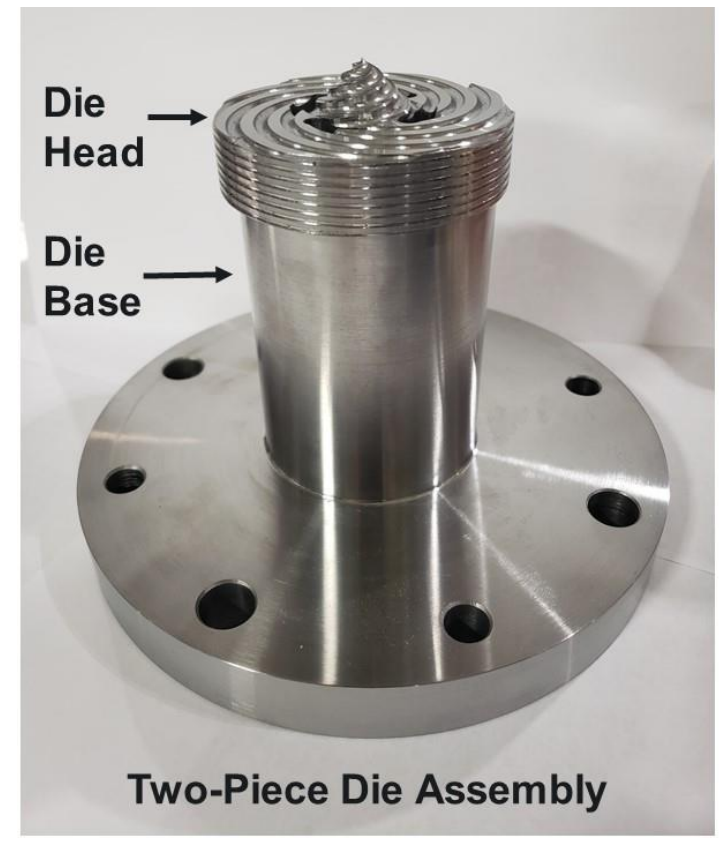

(a)

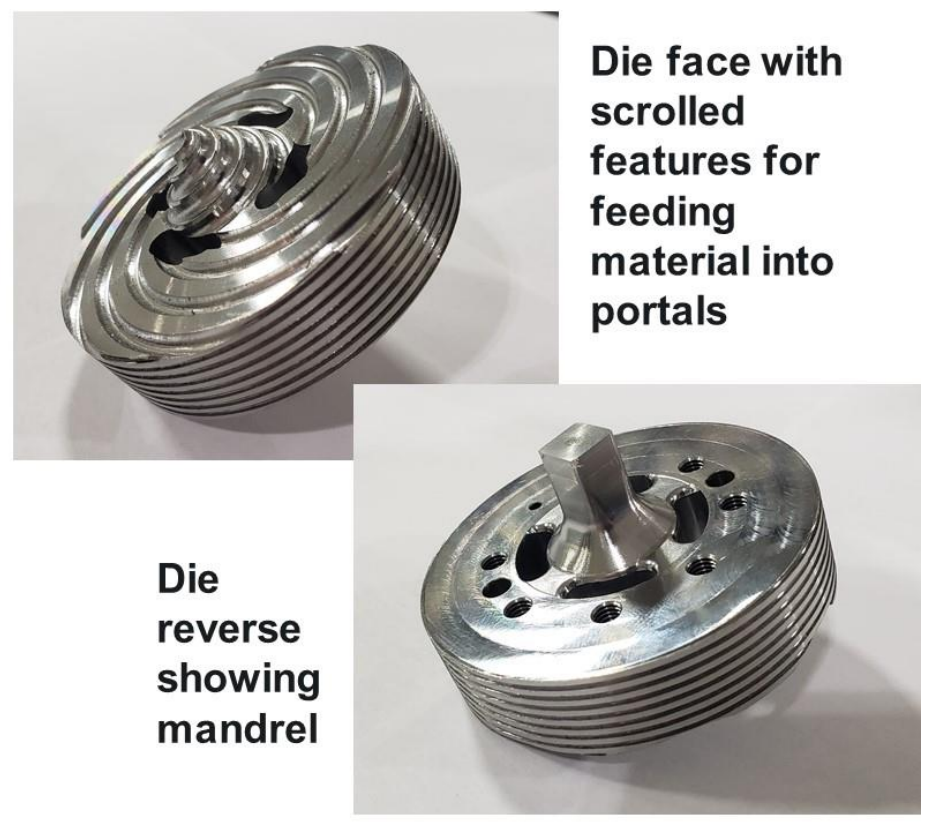

(b)

(c)

Figure II.1.1.3. (a) Two-piece portal bridge die design showing: (b) scrolled-face (front side); and (c) mandrel (back side). Source: PNNL. 


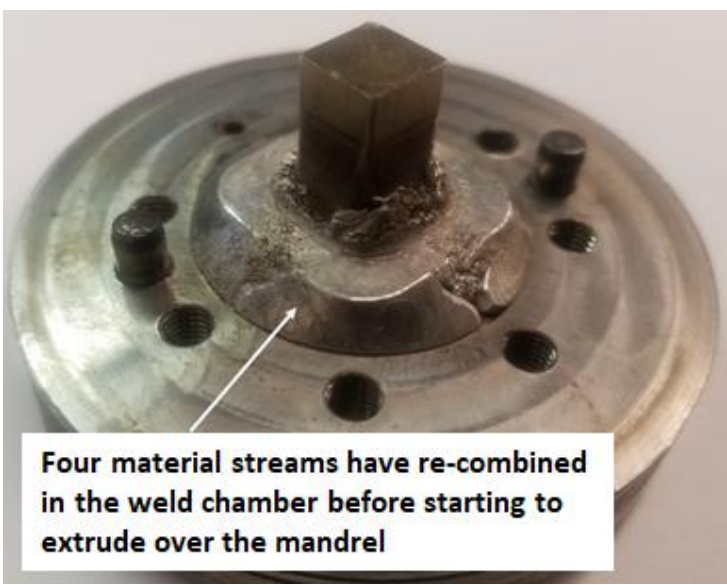

(a)

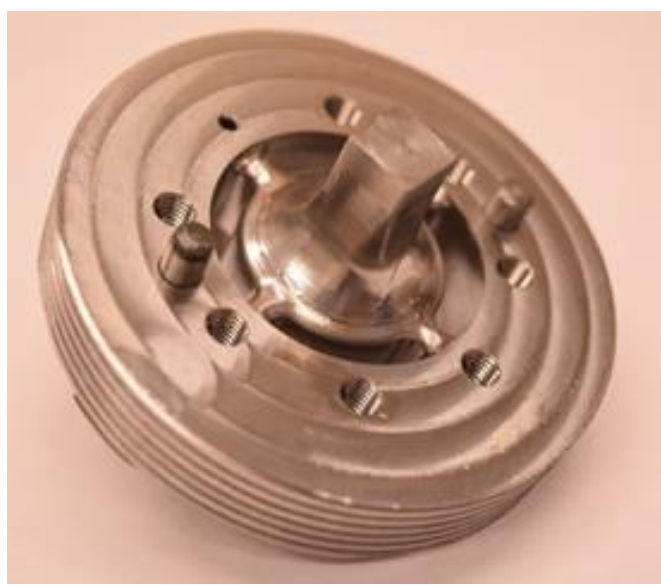

(b)

Figure II.1.1.4. (a) Back side of die head after an initial extrusion trial showing re-combination of four material streams in the weld chamber and (b) bare mandrel before extrusion. Source: PNNL.

\section{Conclusions}

A preliminary design was completed on a two-piece portal bridge die for extruding non-circular tubular profiles using the ShAPETM process. An initial experimental trial confirmed that the portal bridge die was effective at: (1) separating material into four distinct streams; and (2) recombining the streams within the weld chamber. Future planned tasks include analyzing tool and die geometries in order to optimize material flow behavior, and subsequently demonstrate the continuous extrusion of a non-circular Mg tubular product. Additionally, exploring the rate at which ShAPETM can produce product will be investigated and developing the process parameters.

\section{References}

1. Lavender, C., V. Joshi, G. Grant, S. Jana, S. Whalen, J. Darsell, and N. Overman, 2019, "System and process for formations of extrusion products," U.S. Patent No. 10,189,063, January 29, 2019.

2. Whalen, S., V. Joshi, N. Overman, D. Caldwell, C. Lavender, and T. Skszek, 2017, "Scaled-up fabrication of thin-walled Mg ZK60 tubing using ShAPE," Magnesium Technology 2017, Part of The Minerals, Metals \& Materials Series. Springer, pp. 315-321.

3. Whalen, S., N. Overman, V. Joshi, T. Varga, D. Graff, and C. Lavender, 2019, "Mg alloy ZK60 tubing made by ShAPE," Mat. Sci. Eng. A, Vol. 755, No. 7, pp. 278-288.

4. Joshi, V., S. Whalen, and C. Lavender, 2017, “Cost-effective Mg extrusion,” LM-112, Annual Merit Review 2017, 5-9 June 2017, Washington, D.C., USA.

\section{Acknowledgements}

PNNL would like to acknowledge Magna International as an excellent project partner. The collaboration with Magna thus far has been outstanding, already providing value-added contributions in the form of tooling fabrication, extrusion expertise, modeling support, onsite visits to participate in the research, suggestions for tooling design improvements, and the planning of extrusion parameter experiments. 


\title{
II.1.2 Low-Cost Mg Sheet Component Development and Demonstration Project (Fiat Chrysler Automobile, U.S., LLC)
}

\author{
Randy Gerken, Principal Investigator \\ Fiat Chrysler Automobile, U.S., LLC \\ 800 Chrysler Drive, CIMS: 485-00-15 \\ Auburn Hills, MI 48326 \\ E-mail: randy.gerken@fcagroup.com
}

\section{Sarah Kleinbaum, DOE Technology Development Manager}

U.S. Department of Energy

E-mail: sarah.kleinbaum@ee.doe.gov

Start Date: October 1, 2016

Project Funding (FY19): \$3,196,433
End Date: February 28, 2021

DOE share: $\$ 2,081,496$
Non-DOE share: $\$ 1,114,937$

\section{Project Introduction}

The value of the Low-Cost Magnesium Sheet (LCMS) Project to original equipment manufacturers lies in the potential availability of a lower-cost, more formable $\mathrm{Mg}$ sheet alloy, and reduced cost of using a $\mathrm{Mg}$ sheet in automotive applications due to (a) improved formability at reduced temperatures; and (b) improved corrosion protection and reduced coating costs as a result of improved forming lubricant and coating technologies.

\section{Objectives}

The objective of this project is to research, develop, and demonstrate at least one large, challenging $\mathrm{Mg}$ sheet component on a model year 2013 or newer vehicle at a manufacturing cost of less than $\$ 2.50$ per pound of weight saved.

\section{Approach}

The U.S. Automotive Materials Partnership, LLC (USAMP), has employed a technical approach that integrates experimental, computational, and data tools that can accelerate the development and deployment cycle of low-cost Mg sheets. To accomplish the project objective, USAMP proposed to research, develop, test, and evaluate at least one $\mathrm{Mg}$ alloy and commensurate processing suitable for rolling automotive appearance grade sheet, and forming inner and outer door panels based on the 2013 Ford Fusion (see Figure II.1.2.1) to validate the results.

This recently extended 52-month LCMS Project consists of the following major activities conducted over seven technical tasks: (1) technical cost guidance to identify key cost drivers associated with current $\mathrm{Mg}$ component production; (2) material characterization and modeling studies; (3) rolling trials on ingots; (4) pretreatment, coating application, and lubrication studies; (5) forming studies; (6) scaling to warm-forming of large components; and (7) joining studies. Many of the research tasks in this project, therefore, occur concurrently over three BPs. Public data and code developed as part of the project will be curated and hosted in the DataHUB repository developed and maintained by the LightMat Consortium. 


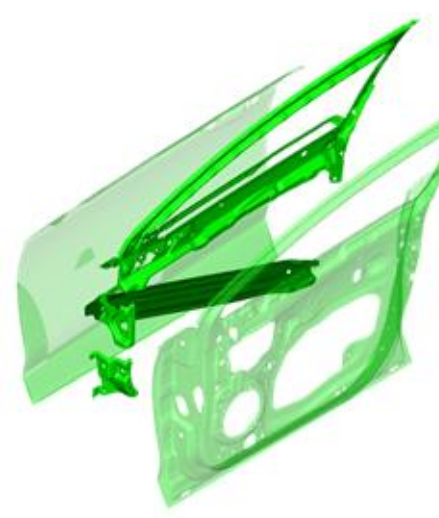

2013 Fusion

Assembly $=16.340 \mathrm{~kg}$

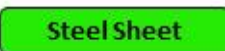

Hot Stamp Steel

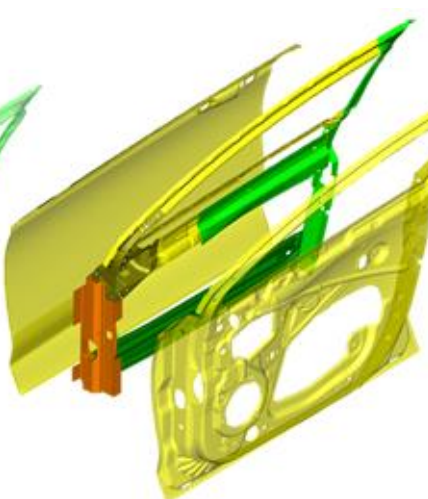

Mach 1

Assembly $=11.107 \mathrm{~kg}$

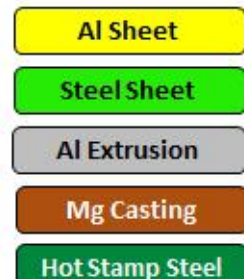

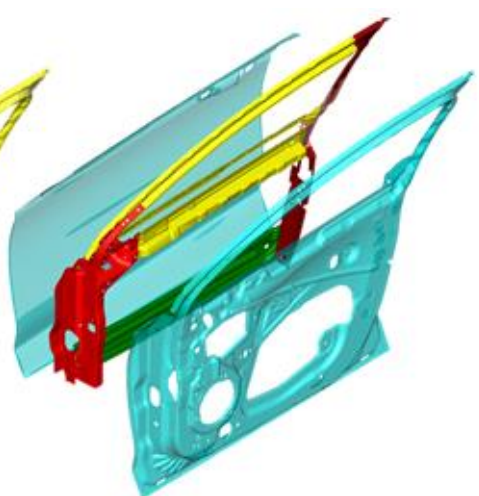

USAMP Mg Door

CDO-0008

Assembly $=9.520 \mathrm{~kg}$

Mg Sheet

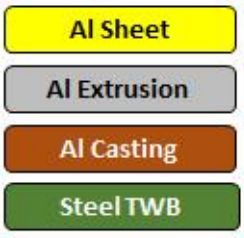

Hot Stamp Steel

Figure II.1.2.1. Illustration of the 2013 Ford Fusion door showing the progression from baseline steel to Multi-Material Lightweight Vehicle Al to an Al-intensive door with $\mathrm{Mg}$ inner and outer panels selected for demonstration in warm-forming. Source: Ford Motor Company.

The project tasks are organized to emphasize two key aspects for a comprehensive research approach to achieving the research objective:

1. In the first aspect, the practical understanding of $\mathrm{Mg}$ alloys is greatly expanded with the development of detailed material property data at elevated temperatures, and their implementation in computational modeling tools, thereby spurring technology for virtual development and characterization of new alloys, which are better optimized for complex automotive manufacturing systems.

2. The second aspect focuses on the improvement of downstream rolling and joining processes, pretreatments, high-temperature lubricants, and corrosion coatings to enable greater use of existing $\mathrm{Mg}$ alloy sheet materials.

The LCMS Project participants are listed below:

- $\quad$ Sub-awardees: Applied Engineering \& Technology (AET) Integration, Inc.; Fuchs Lubricants Co.; Henkel Corporation; PPG Industries, Inc.; Quaker Chemical Corporation; Vehma International of America, Inc.; Xtalic Corporation; The Ohio State University (OSU); University of Florida (UF); University of Illinois at Urbana-Champaign (UIUC); University of Michigan (UM); and University of Pennsylvania (UP).

- $\quad$ Vendors: Bucciero \& Associates, LLC; Camanoe Associates; M-Tech International, LLC; POSCO; Korea Mg Industry; Edison Welding Institute (EWI); FADI-AMT LLC; and Inal Tech, Inc.

- $\quad$ DOE National Labs: PNNL and ORNL. 
The project is divided into 11 milestones to be achieved over the three BPs. Table II.1.2.1 lists the percentage completion status of each milestone (at the end of FY 2019).

Table II.1.2.1. Status of Project Tasks and Milestones at the End of FY 2019.

\begin{tabular}{|c|c|c|c|c|c|}
\hline BP & $\begin{array}{l}\text { Milestone } \\
\text { Number }\end{array}$ & $\begin{array}{l}\text { Milestone } \\
\text { Type }\end{array}$ & Task & Description & Status \\
\hline \multirow{3}{*}{1} & 1 & Go/No Go & $\begin{array}{l}\text { Task 0: Project } \\
\text { Management/Contracting }\end{array}$ & $100 \%$ of purchase orders issued to subs. & Complete \\
\hline & 2 & Technical & $\begin{array}{l}\text { Task 1: Technical Cost } \\
\text { Guidance }\end{array}$ & $\begin{array}{l}\text { Baseline cost model for } \mathrm{Mg} \text { sheet } \\
\text { complete. }\end{array}$ & Complete \\
\hline & 3 & Technical & $\begin{array}{l}\text { Task 2: Alloy and Sheet } \\
\text { Processing Development }\end{array}$ & $\begin{array}{l}\text { New Mg alloy sheet composition(s) } \\
\text { identified. }\end{array}$ & Complete \\
\hline \multirow{4}{*}{2} & 4 & Technical & $\begin{array}{l}\text { Task 2: Alloy and Sheet } \\
\text { Processing Development }\end{array}$ & $\begin{array}{l}\text { Constitutive model for textured Mg alloy } \\
\text { completed and ideal texture suggested. }\end{array}$ & $60 \% *$ \\
\hline & 5 & Technical & $\begin{array}{l}\text { Task 2: Alloy and Sheet } \\
\text { Processing Development }\end{array}$ & $\begin{array}{l}\text { Forming analysis completed on medium } \\
\text { sheet. }\end{array}$ & $50 \% *$ \\
\hline & 6 & Technical & $\begin{array}{l}\text { Task 3: Sheet Coatings } \\
\text { and Lubricant Evaluation } \\
\text { and Development }\end{array}$ & Forming lubricant composition identified. & Complete \\
\hline & 7 & Go/No Go & $\begin{array}{l}\text { Task 5: Mg alloy Sheet } \\
\text { Production }\end{array}$ & $\begin{array}{l}\text { Manufacture and deliver experimental } \\
\text { medium-width sheets. }\end{array}$ & Complete \\
\hline \multirow{4}{*}{3} & 8 & Technical & $\begin{array}{l}\text { Task 3: Sheet Coatings } \\
\text { and Lubricant Evaluation } \\
\text { and Development }\end{array}$ & $\begin{array}{l}\text { Evaluation of corrosion protection } \\
\text { coating completed. }\end{array}$ & $70 \%$ \\
\hline & 9 & Technical & $\begin{array}{l}\text { Task 5: Mg alloy Sheet } \\
\text { Production }\end{array}$ & Delivery of wide sheet. & Complete \\
\hline & 10 & Technical & $\begin{array}{l}\text { Task 6: Mg alloy Large } \\
\text { Body Component } \\
\text { Production }\end{array}$ & Mg alloy panels formed to specifications. & $25 \%$ \\
\hline & 11 & Technical & $\begin{array}{l}\text { Task 7: Component(s) } \\
\text { Demonstration }\end{array}$ & $\begin{array}{l}\text { Final delivery and performance } \\
\text { evaluation completed. }\end{array}$ & $10 \%$ \\
\hline
\end{tabular}

NOTE: “*” Milestone expected to be achieved in BP3.

\section{Results}

USAMP achieved six of the 11 defined project milestones during FY 2018 and FY 2019 and made substantial progress on the remaining ones as described below, while addressing several technical challenges encountered, requiring alternate approaches and new task partners. Delays were encountered in completing experimental work on an extensive material test matrix for obtaining a minimum set of material property data for the selected Mg alloy at room and elevated temperature (e.g., tension tests, in-plane compression tests, interrupted strain tests, and limiting dome height tests). This level of testing was necessary for development of material cards for FEA and warm-forming process simulations, as well as for the validation of the ICME approach. The addition of EWI (for experimental warm-forming tests), Inal Tech Inc. (supporting modeling efforts), and FADI-AMT LLC (supporting material testing) enabled the USAMP team to recover lost schedule. 
While baseline simulations could not be completed in FY 2019 (BP2) as planned, USAMP expects to complete them in early FY 2020 with the availability of new material cards for E-Form Plus alloy. The team will work with PNNL to validate ICME predictions for formability and primary and secondary mechanical properties by using the constitutive model for the textured $\mathrm{Mg}$ alloy and recommend an ideal texture (Milestone 4) for the new Mg alloys.

\section{Development of Experimental Alloy 2}

In FY 2019, OSU designed a new USAMP alloy “Alloy 2" [ZXEM2000 (Mg-2Zn-0.3Ca-0.2Ce-0.1Mn)] for Mg sheet applications. Figure II.1.2.2 (a) shows that at RT, the USAMP Alloy 2 has a higher ductility than the E-Form Plus sourced from POSCO. Figure II.1.2.2 (b) shows that Alloy 2 can achieve significantly higher strength or ductility for various applications under different thermomechanical processing conditions and that it is slightly better than the best $\mathrm{Mg}$ sheet alloys described in the literature [1,2].

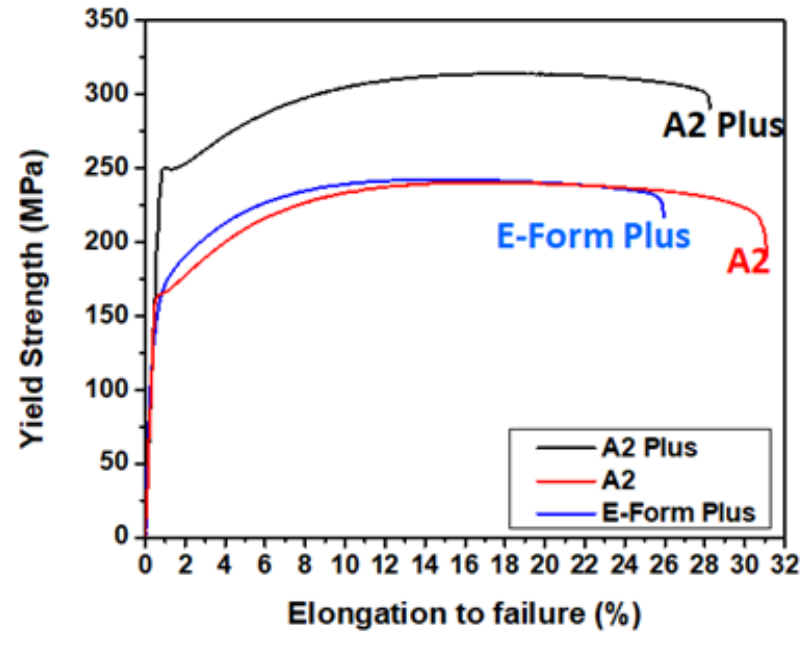

(a)

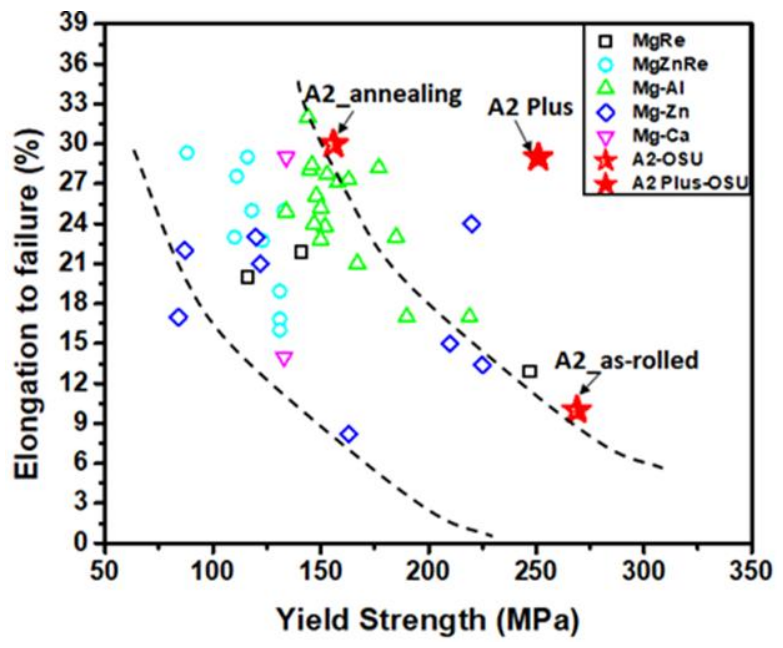

(b)

Figure II.1.2.2. (a) Tensile test results of USAMP Alloy 2 Plus at annealing condition; and (b) comparison of Alloy 2 Plus with other Mg sheet alloys reported in literature. Source: OSU.

OSU has since developed a new Mg alloy, Alloy 2 Plus [ZAXEM11100 (Mg-1Zn-1Al-0.3Ca-0.2Ce-0.4Mn)]. The new alloy possesses a better combination of tensile yield strength (245 MPa), elongation (29\%) and formability (Erichson index $7.7 \mathrm{~mm}$ from $20 \mathrm{~mm}$ cup test) at RT, compared with commercial alloys or other experimental alloys reported in literature. CALculation of PHAse Diagram (CALPHAD) modeling was used to optimize the addition of alloying elements by controlling solute concentration and precipitate in the $\mathrm{Mg}$ matrix. Additionally, CALPHAD and kinetic modeling was used to develop a new homogenization process for the new alloy which achieves complete dissolution of alloying elements without incipient melting.

The comparison of Alloy 2 Plus with the best existing Mg sheets for strength and RT formability using the Erichsen Index is shown in Figure II.1.2.3, and it has the best performance over all others. 


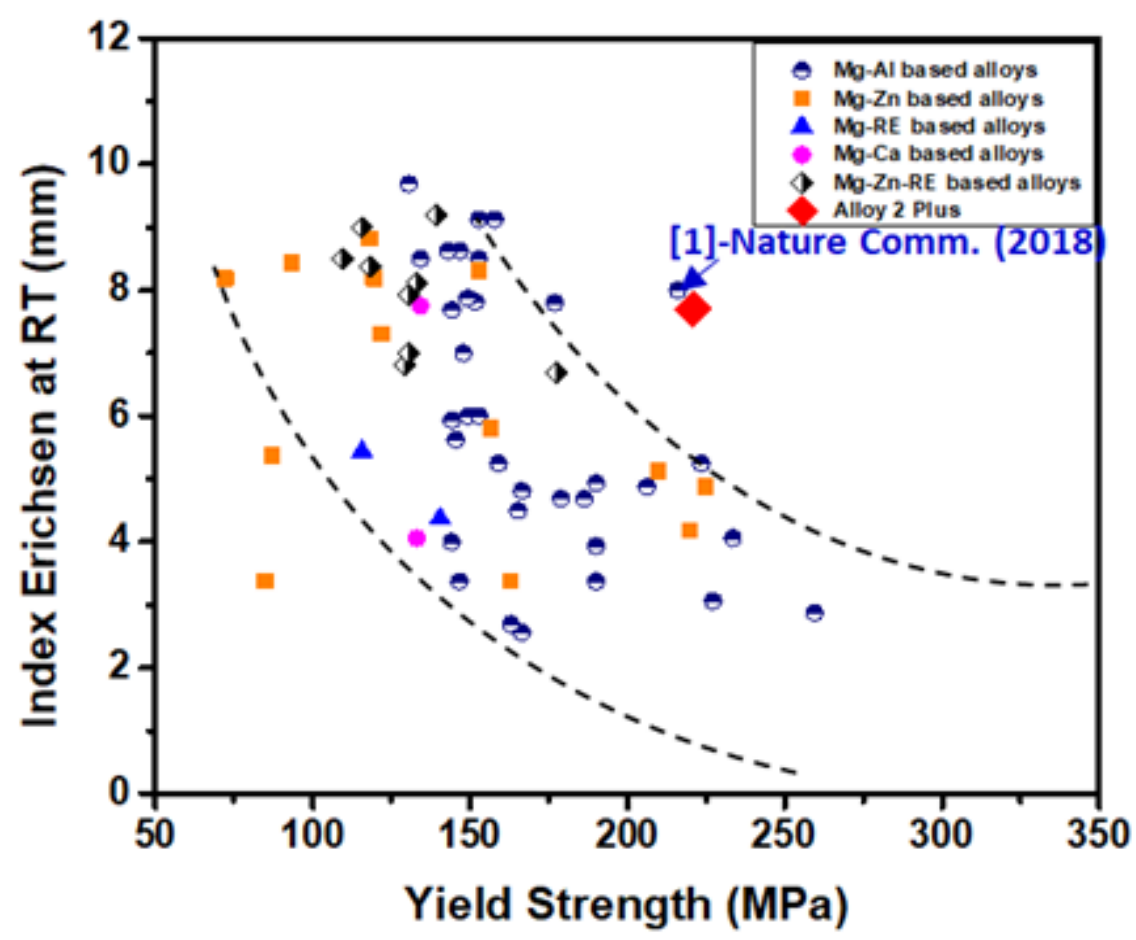

Figure II.1.2.3. Mechanical properties of USAMP Alloy 2 Plus sheet and summary of formability and strength of the existing Mg sheets. Source: OSU.

These results indicate that, at the lab-scale, an excellent combination of high-strength, high ductility of the new USAMP sheet alloy (Alloy 2 Plus) is achievable.

Evaluations by the university team for elevated temperature formability indicated that while superior to Alloy 1, Alloy 2 still could not meet the USAMP's formability and texture requirements for the sourcing of intermediate width sheets and scaling to wide sheets for the demonstration door inner component. Erichsen deep draw cup testing showed that only the new Mg alloy branded as E-Form Plus by POSCO was capable of forming a full cup below 250C; hence, a decision was made by the USAMP team early in BP2 (FY 2019) to select POSCO's E-Form Plus alloy for detailed characterization testing and production demonstrations, as it was available in commercial quantities. This critical material selection decision allowed USAMP to proceed with sourcing both, intermediate size and wide width sheets of E-Form Plus for further testing/characterization and eventually for formability trials steered by the ICME and computer-aided engineering (CAE) modeling activity. Hence, Milestone 7 (manufacture and deliver experimental medium-width sheets) and Milestone 9 (delivery of wide sheet) were both achieved in FY 2019, which enabled the detailed characterization, formability testing and the development of material cards for simulation studies to proceed.

The key to achieving low temperature formability will depend on the ability to decrease the intensity of the basal texture through alloy composition modification and rolling process development. Under the LightMAT award, ORNL was tasked with developing the rolling process to achieve the desirable texture in the experimental alloys.

Several ingots of USAMP Alloy 2 and Alloy 2 Plus were received from OSU in an as-cast condition. Initial ingot dimensions were $110-\mathrm{mm} \times 110-\mathrm{mm} \times 18-\mathrm{mm}$ or $(4.3 \times 4.3 \times 0.7 \mathrm{in}$.). Two ingots of Alloy 2 and one ingot of Alloy 2 Plus were homogenized in a flatbed infrared furnace. Table II.1.2.2 shows the homogenization and rolling conditions used by ORNL based upon the suggestions provided by OSU. 
Table II.1.2.2. Summary of Processing Parameters of USAMP Alloy 2 and Alloy 2 Plus.

\begin{tabular}{|l|l|l|l|l|}
\hline \multicolumn{1}{|c|}{ Processing/Alloy } & & Alloy $\mathbf{2}$ & \multicolumn{2}{|c|}{ Alloy 2 Plus } \\
\hline & $(1)$ & $275^{\circ} \mathrm{C}, 4 \mathrm{~h}$ & $(1)$ & $320^{\circ} \mathrm{C}, 12 \mathrm{~h}$ \\
\hline Homogenization & $(2)$ & $375^{\circ} \mathrm{C}, 12 \mathrm{~h}$ & & \\
\hline & $(3)$ & $420^{\circ} \mathrm{C}, 2 \mathrm{~h}$ & $(2)$ & $440^{\circ} \mathrm{C}, 32 \mathrm{~h}$ \\
\hline Rolling & $(4)$ & $510^{\circ} \mathrm{C}, 1 \mathrm{~h}$ & & \\
\hline & $(5)$ & $400^{\circ} \mathrm{C}, 5 \mathrm{~min}$ & (3) & $450^{\circ} \mathrm{C}, 5 \mathrm{~min}$ \\
\hline
\end{tabular}

The homogenized 0.7 in. thick ingot was cut into three $\sim 0.2 \mathrm{in}$. thick slices. The $4.3 \mathrm{in}$. wide slice was then cut into three $\sim 1.4$ in. wide strips. Rolling was initiated using symmetric rolling and preheating at the temperature provided by OSU, but early trials using Alloy 2 resulted in edge cracking or surface cracking. Following these initial failures, variations of time at preheat temperature, rolling speed, and reductions per pass were attempted during rolling without success. ORNL then decided that a modified experimental matrix that varied the preheat temperature, preheat time, reduction per pass, and rolling speed would be executed with narrow strips in an attempt to define the process window. Slices from both, Alloy 2 and Alloy 2 Plus ingots were used in this process development effort. Reductions per pass from 5\% to $15 \%$ were attempted to ensure that large overall reductions could be achieved with a minimum number of passes. Preheat times of 15-30 min were experimented with resulting in fairly long times to perform multiple experimental trials limiting the process window that could be sampled. ORNL's results from this experimental matrix showed that reductions of $\sim 12 \%$ at a preheat temperature of $360^{\circ} \mathrm{C}$ resulted in acceptable rolling behavior with limited edge cracking and no surface cracking. Once acceptable rolling parameters were identified, no further optimization was attempted to optimize resource utilization. Rolling experiments with Alloy 2 Plus resulted in much better quality rolled sheets with minimum edge cracking, as seen in Figure II.1.2.4. Final experiments were performed only with the Alloy 2 Plus ingots.

The aim of the final experiments was to use symmetric-only and symmetric + asymmetric rolling to achieve a comparable $\sim 1$-mm final thickness from a starting thickness of $\sim 5-\mathrm{mm}$. A slice of $\sim 4.3 \mathrm{in}$. $\mathrm{x} 4.3 \mathrm{in} . \mathrm{x} 0.2 \mathrm{in}$. was rolled using passes of $\sim 12 \%$ reduction to achieve a thickness of $\sim 1.65-\mathrm{mm}$. Figure II.1.2.4 shows the rolled sheet at this stage. This sheet was then cut into two pieces $\sim 2.1$ in. wide (i.e., half width). One of these two pieces was then selected and cut into two pieces normal to its longest direction. One was subsequently used for symmetric rolling to $\sim 0.05 \mathrm{in}$. thick while the other was used for asymmetric rolling using dissimilar sized rolls as shown in Figure II.1.2.5. Asymmetric rolling was performed using the same preheat temperature and reductions/pass. The final thickness of the asymmetric rolled sheet was $1.16-\mathrm{mm}(0.046 \mathrm{in}$.) while the symmetric rolled sheet was $1.27-\mathrm{mm}(0.05$ in.) thick. Pieces were cut from these sheets and sent to OSU for characterization and testing as shown in Figure II.1.2.6. Note that the asymmetrically rolled sheet shows some bending due to the two surfaces being rolled at different linear velocities. This bending can be reversed during shear rolling but was not attempted in this work so that the effect of shear rolling alone on the properties and texture could be evaluated. 


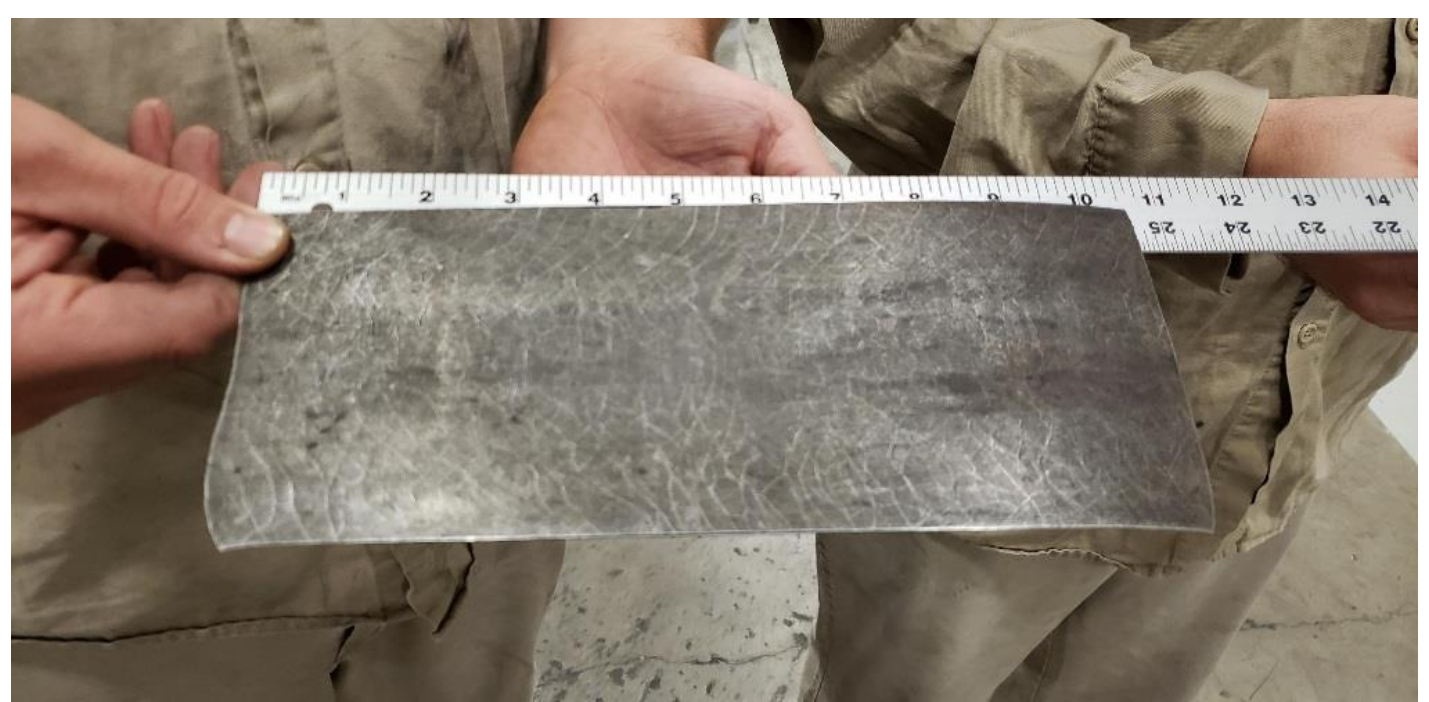

Figure II.1.2.4. ORNL-rolled Alloy 2 Plus sheet (size is $\sim 1.65-\mathrm{mm}$-thick and $\sim 11-\mathrm{cm}$ wide). Source: ORNL.

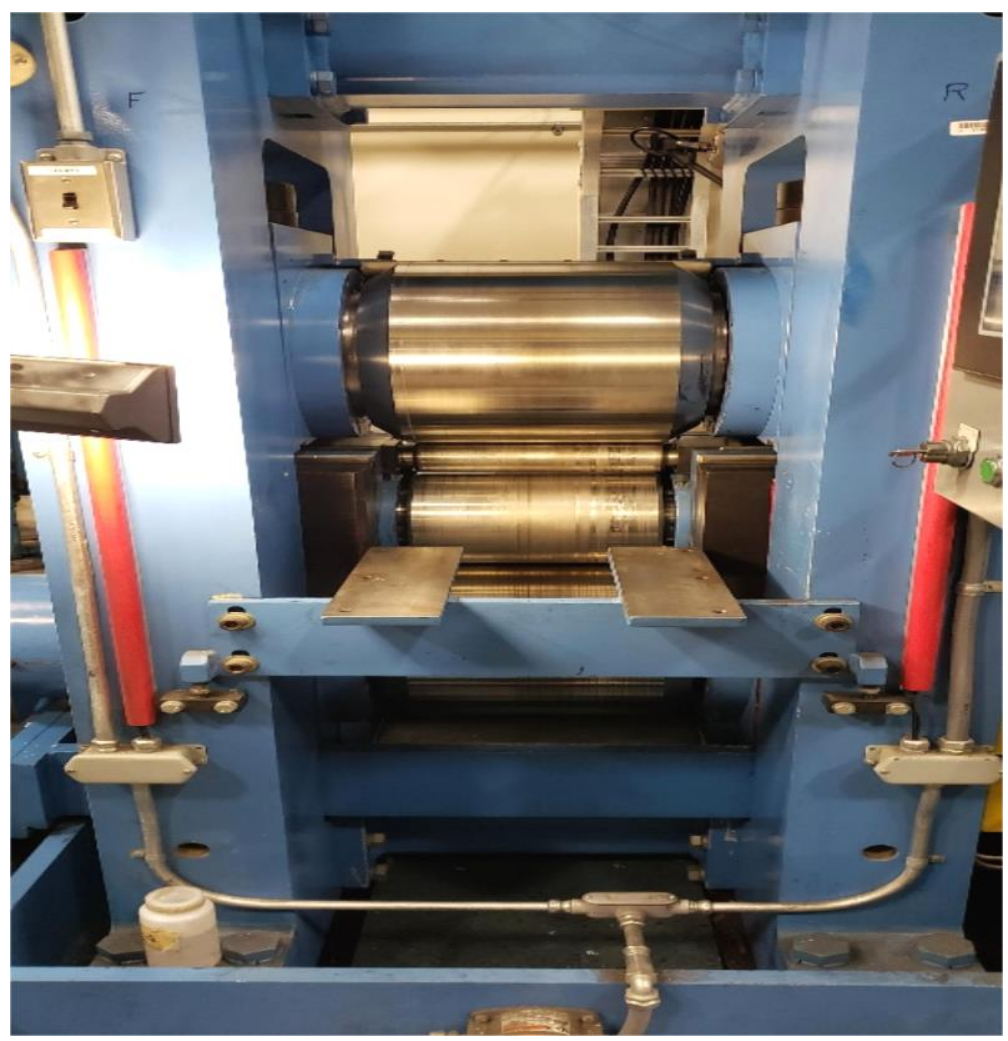

Figure II.1.2.5. Shear roll arrangement for asymmetric rolling. Source: ORNL. 


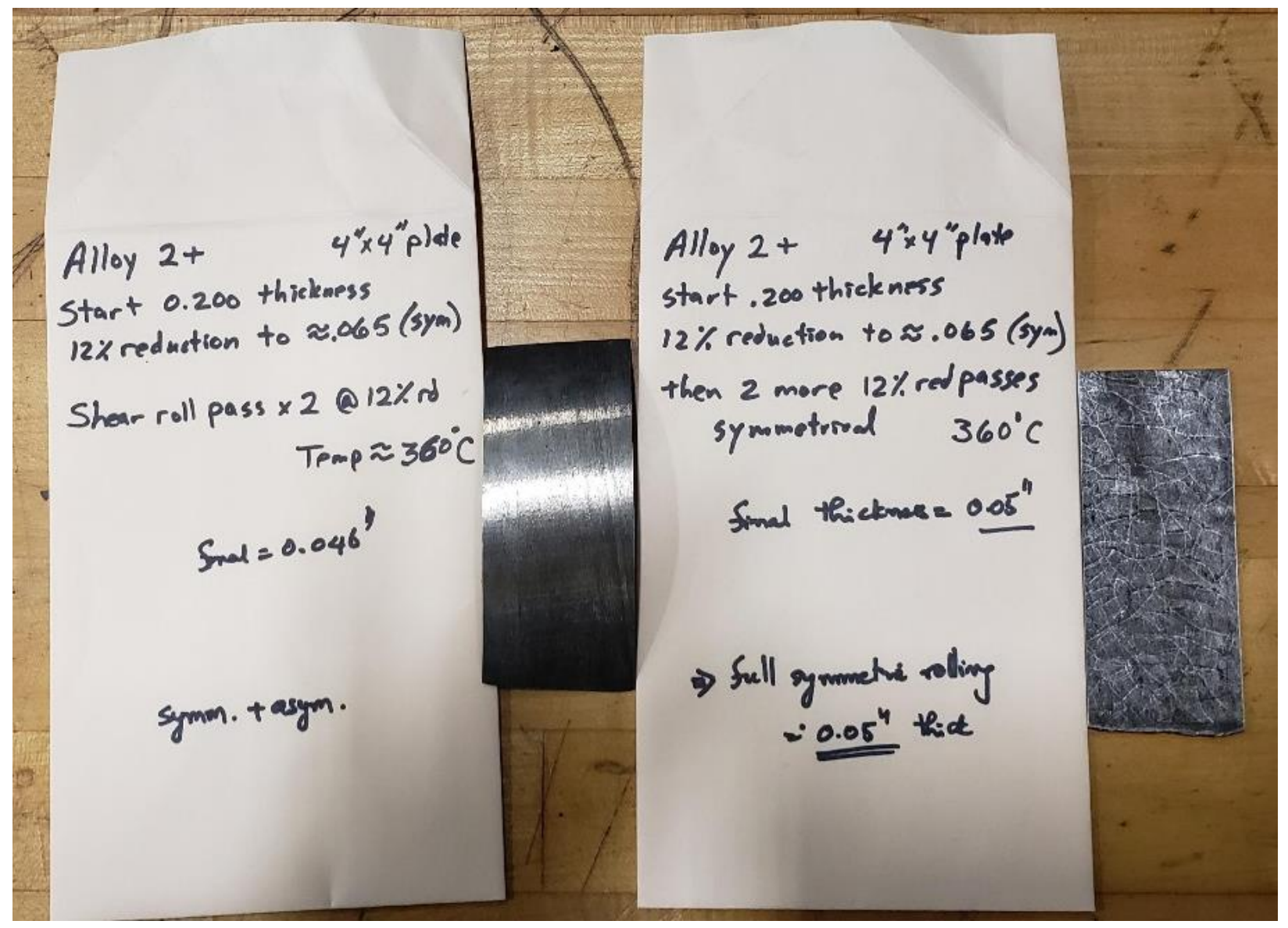

Figure II.1.2.6. Sections of symmetric + asymmetric rolled Mg sheet (left) and symmetric rolled Mg sheet (right) sent to OSU for analyses. Source: ORNL.

\section{Modeling to Support Alloy Development}

The low ductility of Mg alloys at RT is a direct consequence of the anisotropic response of the hexagonal close packed crystal structure to loading where the slip of basal dislocations occurs at a much lower critical stress as compared to non-basal dislocations. Deformation of polycrystalline $\mathrm{Mg}$ is therefore dominated by basal slip resulting in too few independent slip systems available at low temperatures for appreciable ductility. A promising approach for improving the ductility of $\mathrm{Mg}$ is adding solutes that interact with the dislocations to potentially reduce the difference in the stresses required to move basal and non-basal dislocations. The University of Illinois-Urbana Champaign has used DFT to compute inputs for solid-solution strengthening

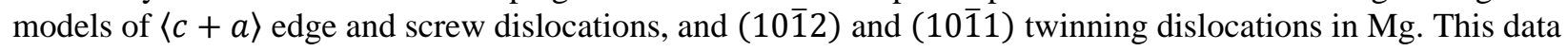
will serve as the input for predictive models of critical resolved shear stress (CRSS) as functions of alloy composition, temperature, and strain-rate. The key inputs to the solution strengthening models are (a) accurate dislocation geometries for computing solute-dislocation interaction energies from either direct substitution of solutes into core sites or from the local strain and slip distributions in the dislocation cores, (b) solute volumetric size misfits that determine the interaction strengths of solutes with the dislocation strain field and (c) solute chemical misfits that determine the interaction strengths of solutes with either stacking faults or twin boundaries [4-6].

UIUC has continued to work on solid-solution strengthening predictions for pyramidal II $\langle c+a\rangle$ edge and screw dislocations in Mg. Previously, the core structures of the $\langle c+a\rangle$ dislocations were optimized using DFT with lattice Green function-based flexible boundary conditions and size and chemical misfits computed for Al, $\mathrm{Ca}, \mathrm{Mn}, \mathrm{Sn}$, and $\mathrm{Zn}$ solutes in $\mathrm{Mg}$. Recent work on solution strengthening of $\langle c+a\rangle$ edge dislocations in $\mathrm{Mg}$ 
due to Y solutes has shown that Y causes large changes in the dislocation core geometry [6] and that the local strain and slip distributions from the changed dislocation core must be used to compute accurate solutedislocation interaction energies. UIUC directly substituted $\mathrm{Ca}$ into 24 different sites in the edge dislocation core which resulted in only minor changes in the core geometry. They also achieved similar results for $\mathrm{Al}, \mathrm{Mn}$, $\mathrm{Sn}$, and $\mathrm{Zn}$ at a smaller number of sites in the core. In contrast to the study on $\mathrm{Y}$, the findings show that the strain and slip distributions for the pure $\mathrm{Mg}$ dislocation geometry can be used to accurately predict solutedislocation interactions. This results in a dramatic reduction in computational cost and expedites the study of the strengthening effects of many different solute species. The dislocation geometry and solute misfit data serves as input into solution strengthening models [6] to predict changes in yield stress as a function of solute concentration, temperature, and strain-rate. Figure II.1.2.7 (a)-(c) show changes in the yield stress to move $\langle\mathrm{c}+\mathrm{a}\rangle,(10 \overline{1} 2)$ twinning, and(1011) twinning edge dislocations as a function of solute concentration at RT [4].

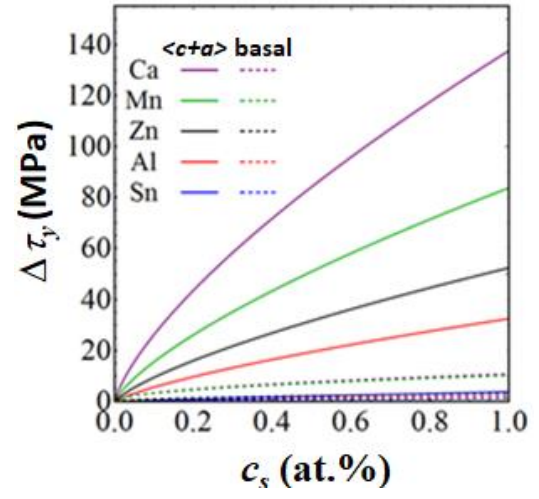

(a)

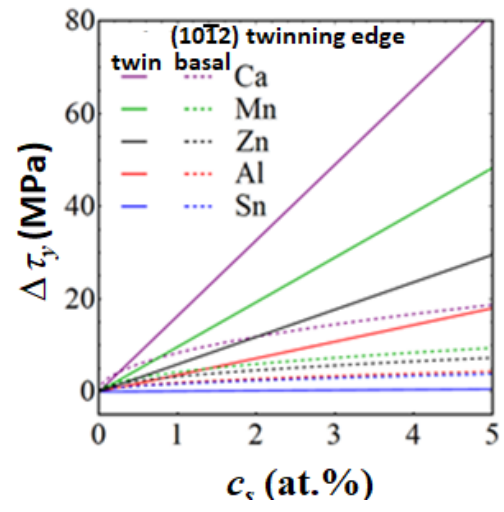

(b)

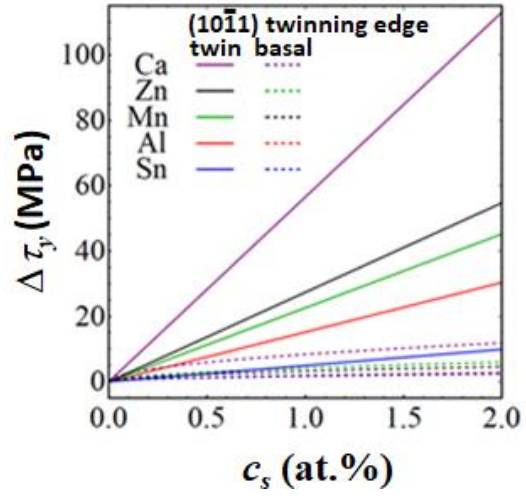

(c)

Figure II.1.2.7. Effect of solutes on the yield stress required to move (a) basal and $\langle c+a\rangle$ or (b) and (c) twinning edge dislocations at RT. Source: UIUC.

Work by UIUC also continued on solid-solution strengthening predictions for (1012) and (1011) twinning edge dislocations in Mg. A previous DFT study on solution strengthening of (1012) twinning dislocations due to $\mathrm{Al}$ and $\mathrm{Zn}$ solutes [5] showed that the volumetric strain contributions to the solute-dislocation interaction energies for $\mathrm{Zn}$ can be accurately determined by scaling directly computed $\mathrm{Al}$ interaction energies by the $\mathrm{Zn}$ volumetric size misfit, and that the chemical contributions to the interaction energies can be computed from

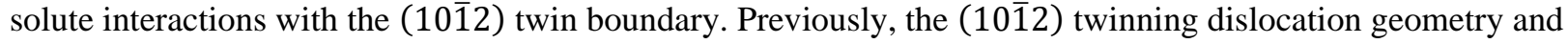
the direct $\mathrm{Al}$ interactions were obtained from reference [5] and UIUC computed the interaction energies of $\mathrm{Al}$,

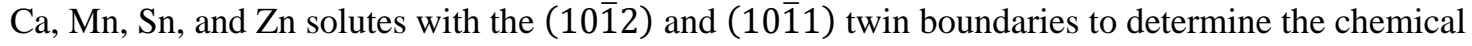
contributions to the solute-twinning dislocation interactions. Recently, DFT was used to optimize the core geometry of the (1011) twinning edge dislocation in $\mathrm{Mg}$ and to directly compute the interaction of $\mathrm{Al}$ solutes with this dislocation by substituting $\mathrm{Al}$ in several sites in the core. The team verified that the interaction energies of Ca solutes with the (10 $\overline{1} 1)$ twinning dislocation can be accurately computed by scaling the $\mathrm{Al}$ interactions by the Ca size misfit and shifting them by the chemical contribution computed from the $\mathrm{Ca}$ interactions with the (1011) twin boundary. Like the $\langle c+a\rangle$ dislocations, the pure Mg twinning dislocation geometries and solute misfit allow the team to efficiently compute the strengthening effects of many different solute species.

Solute size and chemical misfits are key inputs to the computationally efficient models for the solutedislocation interactions discussed above. UIUC researchers have computed volumetric size misfits for 63 substitutional solute species in $\mathrm{Mg}$, as shown in Figure II.1.2.7 above and are currently computing the chemical misfits for the $\langle c+a\rangle$ and twinning dislocations. It was found that the solute-dislocation interactions are dominated by the volumetric strain contributions, so the size misfits provide a good qualitative measure of solute strengthening potency. Solutes with the greatest size mismatch from $\mathrm{Mg}$ will have the strongest 
interactions with the $\langle c+a\rangle$ and twinning dislocations and are expected to have the largest strengthening effects on these deformation modes. Once the remaining chemical misfit calculations are complete, UIUC will input the data into the solution strengthening models to make quantitative strengthening predictions for the 63 solute species.

Two Mg-0.2Ca and Mg-1.5Sn (at.\%) binary alloy ingots were cast at UF as part of the solute strengthening model developed at UIUC. Experimental observations from these samples will serve as empirical inputs into the DFT model. The alloy compositions - $0.27 \mathrm{Ca}$ and $1.71 \mathrm{Sn}$ (at.\%), respectively-were selected according to the maximum solubility of $\mathrm{Ca}$ and $\mathrm{Sn}$ in $\mathrm{Mg}$ at $450^{\circ} \mathrm{C}$. Both, UIUC and PNNL collaborators were consulted for their input on composition selection.

Sheets measuring 60-mm-long x 2-mm-thick x 60-mm wide were obtained from ingots 80-mm-long x 20-mm wide $\mathrm{x} 80-\mathrm{mm}$ high of $\mathrm{Mg}-0.2 \mathrm{Ca}$ and $\mathrm{Mg}-1.5 \mathrm{Sn}$. All sheets were homogenized at $475^{\circ} \mathrm{C}$ for 50 hours, cold worked to $20 \%$ and $25 \%$ thickness reduction, and subsequently annealed at 350 and $400^{\circ} \mathrm{C}$ for different times to obtain complete recrystallization. Complete recrystallization was observed on $20 \%$ cold worked sheets of $\mathrm{Mg}-0.2 \mathrm{Ca}$ and $\mathrm{Mg}-1.5 \mathrm{Sn}$ annealed at $400^{\circ} \mathrm{C}$ for 120 hours, with an average grain size of $168 \pm 54$ and $199 \pm$ $83 \mu \mathrm{m}$, respectively, as shown in Figure II.1.2.8 (a) and Figure II.1.2.8 (b). Four sheets - two as-rolled and two fully recrystallized-were shipped to PNNL for further investigation. Basal texture intensity of $\mathrm{Mg}-0.2 \mathrm{Ca}$ and $\mathrm{Mg}-1.5 \mathrm{Sn}$ is expected to vary according to the applied annealing time.

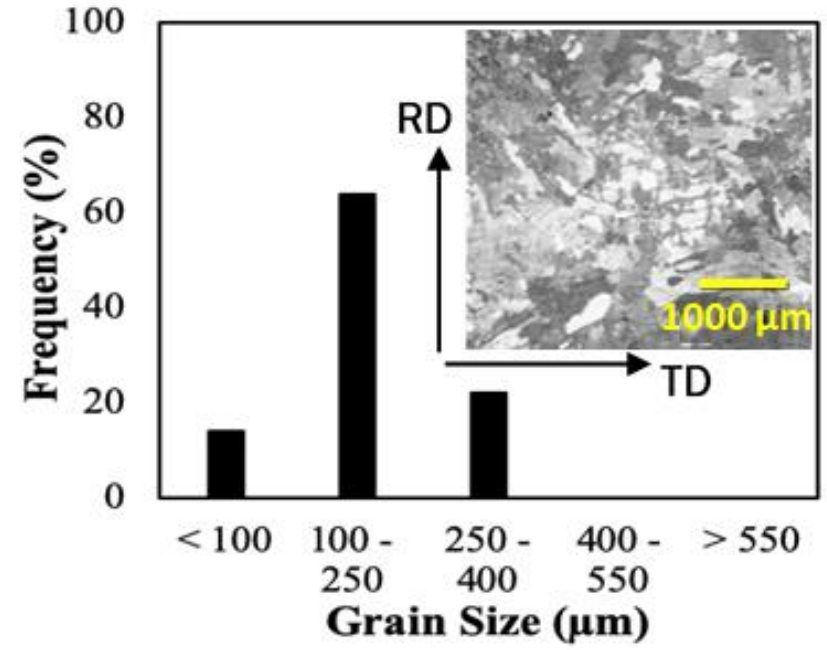

(a)

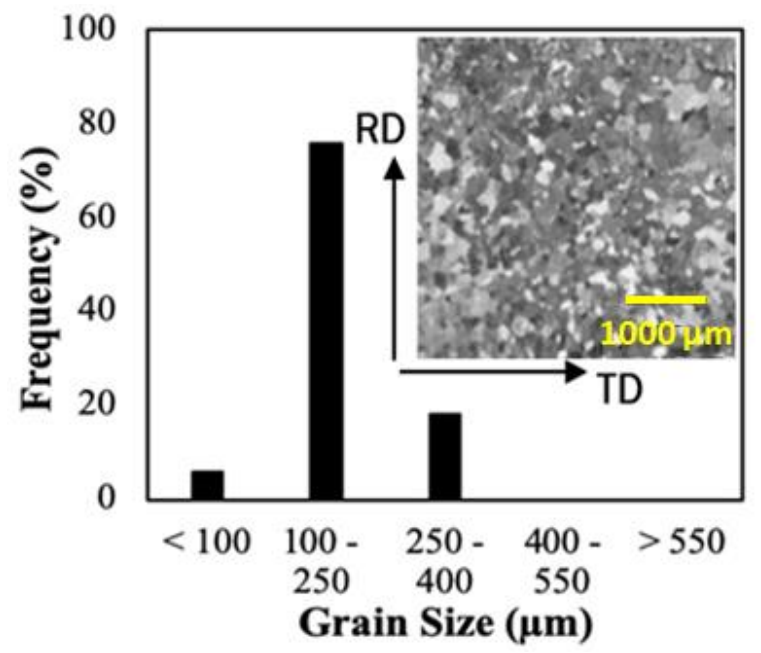

(b)

Figure II.1.2.8. Grain size distributions and optical micrographs (inset) showing the rolling direction (RD) and transverse direction (TD) of (a) Mg-0.2Ca and (b) Mg-1.5Sn sheets with $20 \%$ thickness reduction annealed at $400^{\circ} \mathrm{C}$ for 120 hours.

Source: UF.

\section{Development of Experimental Alloy 3}

The development of a USAMP Experimental Alloy 3 consisted of an investigation of binary and ternary alloys with a range of alloying elements of $\mathrm{Ca}$ (from $0.1-1 \mathrm{wt} \%$ ) and $\mathrm{Zn}$ (from $0-4 \mathrm{wt} \%$ ). The UF cast and delivered all $\mathrm{Mg}-\mathrm{Ca}$ and $\mathrm{Mg}-\mathrm{Ca}-\mathrm{Zn}$ based alloys requested by the UM. Prior to delivery, the verification of nominal composition from a few of the as-cast ingots was performed using inductively coupled plasma mass spectrometry. Additionally, nondestructive testing and nano-computed tomography were performed on the ingots to characterize defect size and distribution in the castings before delivery. No major defects were observed in 24 of these castings. 
Microstructure characterization of all homogenized $\left(475^{\circ} \mathrm{C} / 50 \mathrm{hrs}\right)$ samples were completed. Optical micrography and grain size distributions observed along all three directions from the 200 grams ingots of $\mathrm{Mg}$ $0.1 \mathrm{Ca}, \mathrm{Mg}-0.35 \mathrm{Ca}, \mathrm{Mg}-0.1 \mathrm{Ca}-0.5 \mathrm{Zn}$, and $\mathrm{Mg}-0.35 \mathrm{Ca}-0.5 \mathrm{Zn}$ (wt.\%) have been completed. No peaks corresponding to the $\mathrm{Mg} 2 \mathrm{Ca}$ secondary phase were detected in the XRD pattern of any of the homogenized compositions as observed in Figure II.1.2.9 (a). However, SEM and EDS performed on homogenized Mg-Ca binary and Mg-Ca-Zn alloys, shown in Figure II.1.2.9 (b), showed evidence of the Mg2Ca phase within the grain boundaries. However, no secondary particles were observed in the $\mathrm{Mg} \alpha$-matrix for all the compositions.

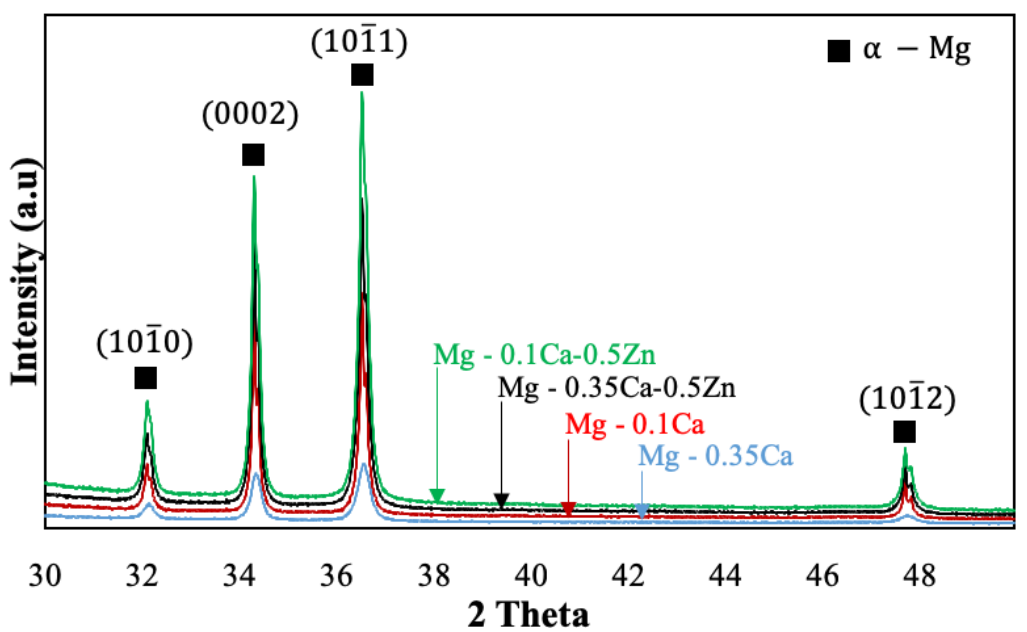

(a)

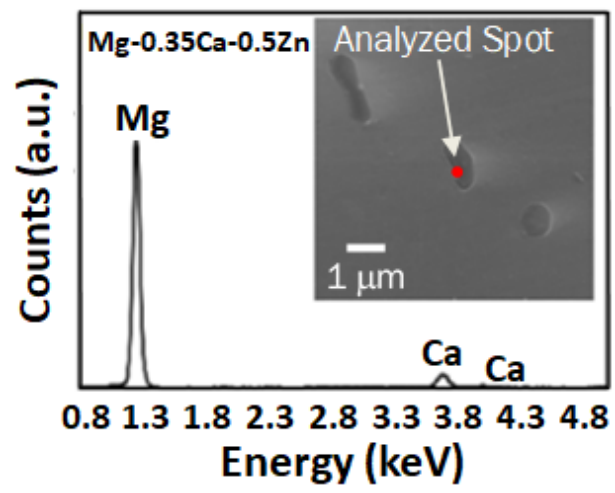

(b)

Figure II.1.2.9. (a) X-ray pattern obtained from the homogenized alloys. (b) Spot EDS spectrum of a Mg2 Ca particle from homogenized Mg-0.35Ca-0.5Zn alloy with the inset showing the particle and location diffraction from which the spectrum was obtained. Source: UF.

Dynamic strain aging (DSA) experiments on $\mathrm{Mg}$-Ca and $\mathrm{Mg}$-Ca-Zn alloys at $200^{\circ} \mathrm{C}$ and $250^{\circ} \mathrm{C}$ were completed. It is hypothesized that deformation of $\mathrm{Mg}$-Ca-based alloys under conditions at which DSA occurs can enhance formability via texture modification [3]. DSA is observed as serrated flow curves during hightemperature compression testing and under conditions at which the strain-rate sensitivity (SRS) is negative [3]. Figure II.1.2.10 (a) gives the stress-strain curves of $\mathrm{Mg}-0.35 \mathrm{Ca}-0.5 \mathrm{Zn}$ compressed at $250^{\circ} \mathrm{C}$ at $10-3 \mathrm{~s}-1,10-2 \mathrm{~s}-$ 1, and 10-1 s-1 showing serrations at 10-3 s-1. Serrations indicative of DSA were observed for all stress-strain curves at 10-3s-1. However, no serrations were observed on samples compressed at 10-1 s-1. Furthermore, $\mathrm{Mg}-0.1 \mathrm{Ca}$ was characterized by positive values of SRS; thus, no DSA was seen to occur at the tested conditions. 
This can be related to the low amount of $\mathrm{Ca}$ in the $\mathrm{Mg}$ matrix of $0.1 \mathrm{wt} . \%$. On the other hand, compositions of higher amounts of $\mathrm{Ca}$ (i.e., $0.35 \mathrm{Ca}$ wt.\%) were characterized by negative $\mathrm{SRS}$ at $200^{\circ} \mathrm{C}$ and $250^{\circ} \mathrm{C}$ from $10-3$ to $10-2 \mathrm{~s}-1$ as shown in Figure II.1.2.10 (b). Mg-0.35Ca was the only alloy that showed negative SRS values at $200^{\circ} \mathrm{C}$ from $10-2$ to $10-1 \mathrm{~s}-1$. These results correlate to findings demonstrating that increasing the solute content broadened the range of conditions at which DSA occurs [3].

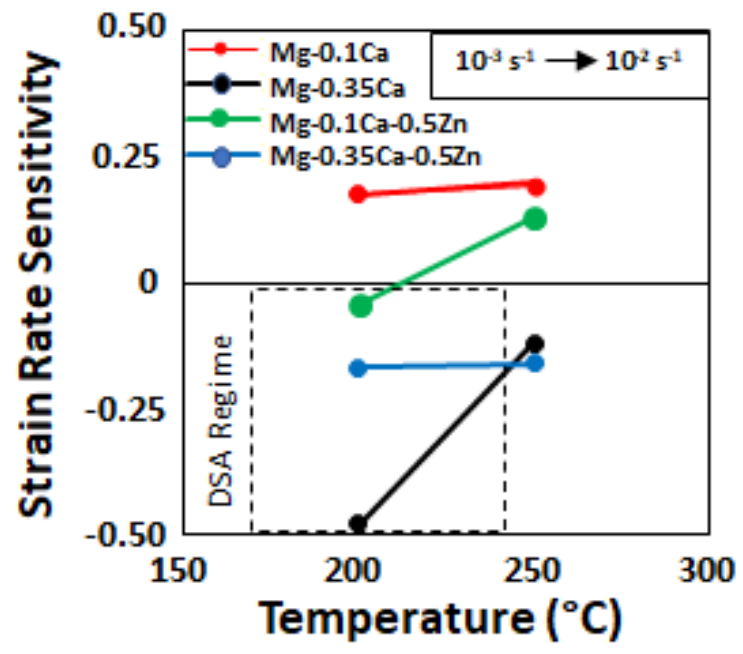

(a)

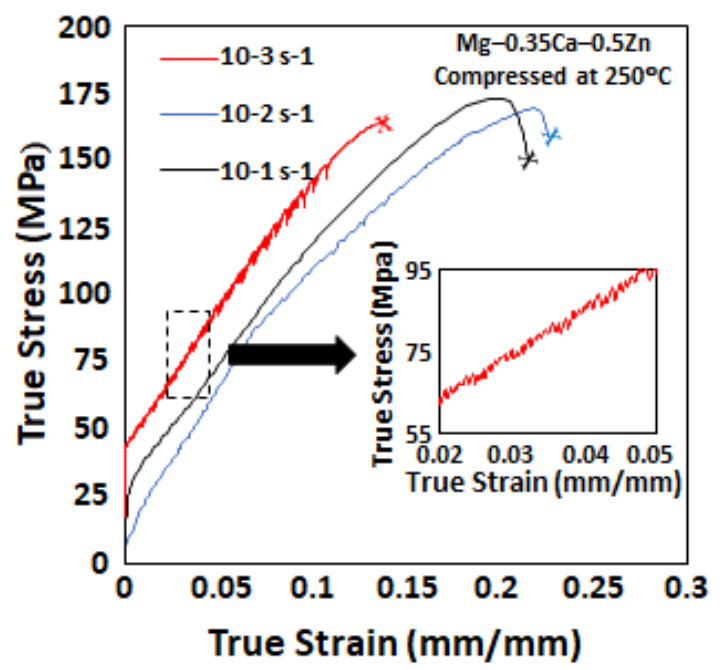

(b)

Figure II.1.2.10. (a) Representative stress-strain curves of Mg-0.35Ca-0.5Zn compressed at $250^{\circ} \mathrm{C}$ showing serrations at $10^{-3} \mathrm{~s}^{-1}$. "X" represents the fracture point of the sample. (b) SRS changes as a function of temperature from $10^{-3}$ to $10^{-2} \mathrm{~s}^{-1}$ for Mg-Ca binary and Mg-Ca-Zn ternary alloys showing the DSA regime. Source: UF.

Table II.1.2.3 summarizes the DSA regime obtained of all compositions at $200^{\circ} \mathrm{C}$ and $250^{\circ} \mathrm{C}$. Future experiments will be performed at $300^{\circ} \mathrm{C}$. Texture analyses will also be performed on samples showing DSA.

Table II.1.2.3. DSA Regime for Mg-Ca Binary and Mg-Ca-Zn Ternary Alloys Compressed at $200^{\circ} \mathrm{C}$ and $250^{\circ} \mathrm{C}$.

\begin{tabular}{|c|c|c|c|c|c|}
\hline \multirow{2}{*}{ Temperature } & \multirow{2}{*}{$\begin{array}{l}\text { Strain Rates } \\
\qquad\left(\mathbf{s}^{-1}\right)\end{array}$} & \multicolumn{4}{|c|}{ Composition (wt.\%) } \\
\hline & & $\mathrm{Mg}-0.1 \mathrm{Ca}$ & Mg-0.35Ca & Mg-0.1Ca-0.5Zn & Mg-0.35Ca-0.5Zn \\
\hline \multirow{3}{*}{$200^{\circ} \mathrm{C}$} & $10^{-3}$ & $x$ & DSA & DSA & DSA \\
\hline & $10^{-2}$ & $x$ & DSA & $x$ & DSA \\
\hline & $10^{-1}$ & $x$ & $X$ & $X$ & $x$ \\
\hline \multirow{3}{*}{$250^{\circ} \mathrm{C}$} & $10^{-3}$ & $x$ & DSA & $x$ & DSA \\
\hline & $10^{-2}$ & $x$ & DSA & $X$ & $x$ \\
\hline & $10^{-1}$ & $x$ & - & $x$ & $x$ \\
\hline
\end{tabular}

NOTES: “X” represents no DSA observed.

"-" represents a combination for which a future test is planned.

The development of a USAMP Experimental Alloy 3 candidate (e.g., the ternary Mg-Zn-Ca alloy) with a weak crystallographic texture was initiated by exploring the texture evolution and grain refinement that occurs during the plane-strain compression of ZX30 (e.g., Mg-3Zn-0.1Ca). This alloy was chosen to facilitate comparison with the rolled sheet available in the literature [7-8]. As the feed-through rate used to produce the sheets relating to the strain-rate was not given in the literature, the effect of strain-rate on the texture evolution was an important variable to explore. A Gleeble thermomechanical processing simulator was used to simulate 
the rolling process. The impact of strain-rate, number of deformations passes (total strain), and duration of intermediate annealing on the final microstructure of and texture on ZX30 was explored. Of all the variables studied, the most significant variable was the number of compressive passes (i.e., the total final strain). Though all samples showed refinement in the compressed region, as shown in Figure II.1.2.11, several larger grains were present in the reduced section of specimens subjected to only five passes.

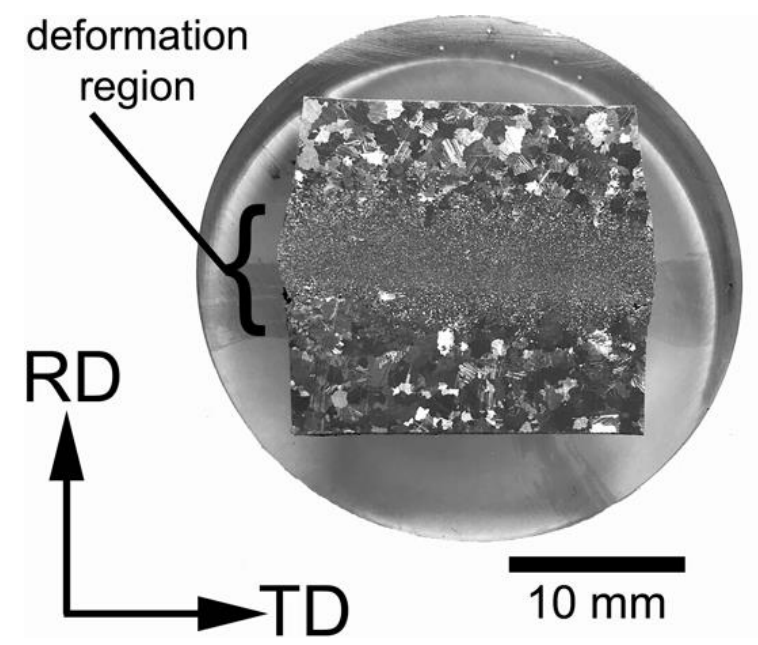

Figure II.1.2.11. Photograph of a ZX30 sample subjected to plane-strain compression. The top and bottom of the sample was unconstrained, and the middle of the specimen is the deformed region with a refined microstructure. Source: UM.

Specimens subjected to ten or more passes exhibited a more uniform grain size being refined to approximately $30 \mu \mathrm{m}$ in diameter as shown in Figure II.1.2.12 (a). Increasing the strain-rate from $0.1 \mathrm{~s}-1$ to $0.5 \mathrm{~s}-1$ resulted in a more diffuse basal texture. The TD spreading in the basal pole figures of ZX30 sheets presented in the literature and shown in Figure II.1.2.12 (b) was not observed in the Gleeble samples. It is unclear whether the difference in texture is due to being outside the thermomechanical processing window the sheets experienced during rolling or whether shearing is needed to replicate the texture evolution in the $\mathrm{Mg}$ alloy sheets.

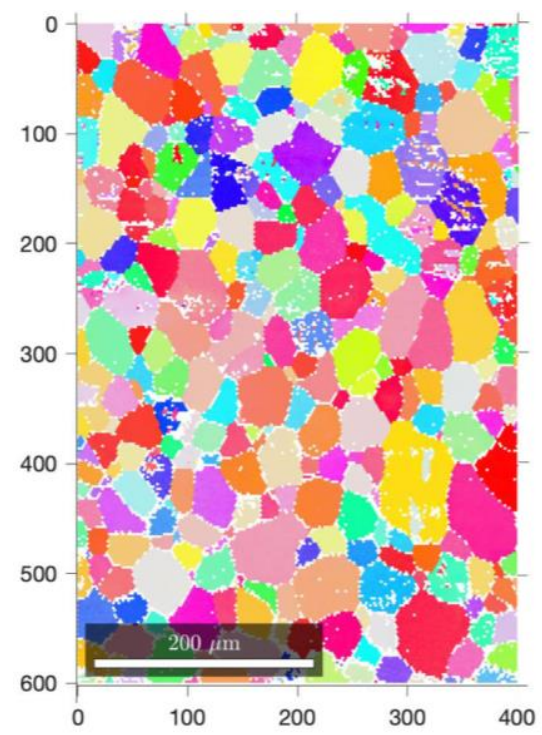

(a)
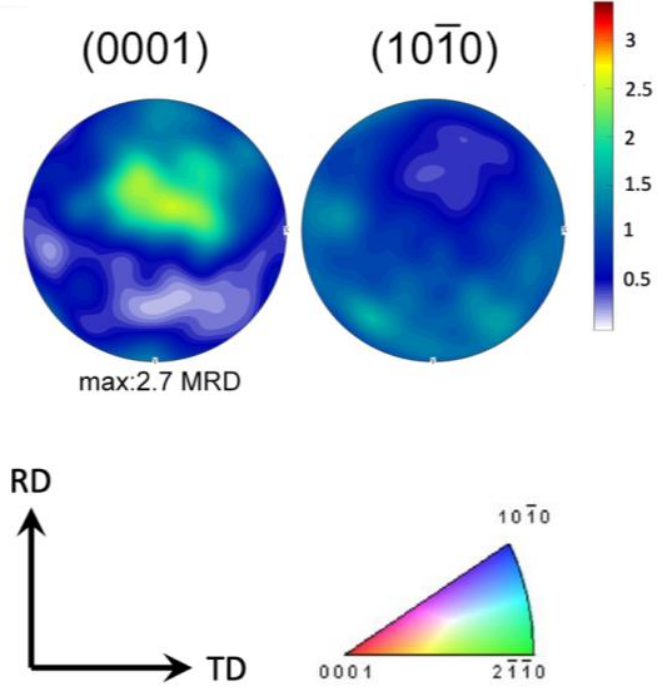

(b)

Figure II.1.2.12. (a) EBSD inverse pole figure map and (b) pole figures of a ZX30 Gleeble sample subjected to ten passes of 0.2 true strain with a strain-rate of $0.5 \mathrm{~s}^{-1}$. Source: UM. 
Rolled samples of two binary $\mathrm{Mg}$ alloys- $\mathrm{Mg}-0.2 \mathrm{Ca}$ (at. \%) and $\mathrm{Mg}-1.5 \mathrm{Sn}$ (at. \%) -were received at PNNL from UF under Subtask 2.3 where the wrought samples were rolled to a thickness reduction of $20 \%$ at RT, and then recrystallized by annealing at $400^{\circ} \mathrm{C}$ for $120 \mathrm{~h}$. Two tensile specimens were cut from the center of each four sheets as shown in Figure II.1.2.13. The longitudinal direction of tensile specimens is along the RD. The region between the two tensile specimens was metallographically polished for microstructure observation and texture analysis using SEM and EBSD. MATLAB toolbox Mtex was used to calculate pole figures and inverse pole figures (IPFs) from EBSD data.

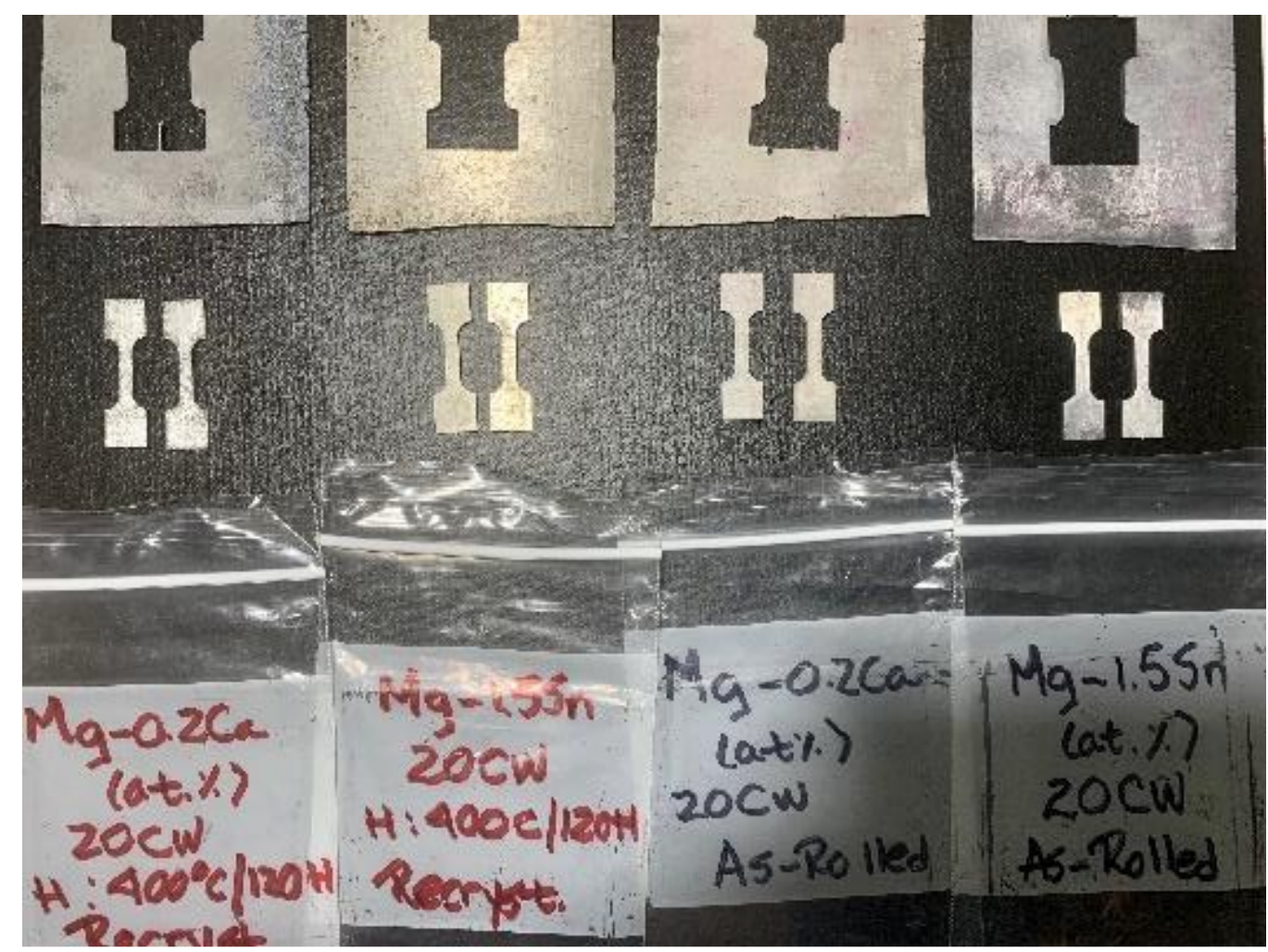

Figure II.1.2.13. The Mg alloy sheets and tensile specimens. Source: PNNL.

The image quality (IQ) maps, IPF maps, and the $\{10-10\},\{0001\}$, and $\{11-21\}$ pole figures show the microstructures of as-rolled $\mathrm{Mg}-0.2 \mathrm{Ca}$ as presented in Figure II.1.2.14 (a)-(c) and recrystallized $\mathrm{Mg}-0.2 \mathrm{Ca}$ as presented in Figure II.1.2.14 (d)-(f). The IQ map in Figure II.1.2.14 (a) shows severe local strain. The fraction of the nonindexed phase in black in Figure II.1.2.14 (b) is estimated to be 79.8\% resulting from large plastic deformation after cold-rolling. The $\mathrm{X}$ direction in the pole figures is along the $\mathrm{RD}$ and the pole axis is along the normal direction (ND) of the rolling sheet. In the as-rolled $\mathrm{Mg}-0.2 \mathrm{Ca}$ alloy, the $\{0001\}$ pole figures evidently show that a basal texture developed after cold-rolling. However, the basal fiber is $10-15^{\circ}$ away from ND. In as-rolled $\mathrm{Mg}-0.2 \mathrm{Ca}$, six-fold rotation symmetry was observed on $\{10-10\}$ pole figures and $\{11-21\}$ pole figures as shown in Figure II.1.2.14 (c). After annealing Mg-0.2Ca at $400^{\circ} \mathrm{C}$ for $120 \mathrm{~h}$, the deformed grains recrystallized as shown in Figure II.1.2.14 (d)-(e), where one abnormally large grain was observed in the EBSD area. Except for this very large grain, the other nearly equiaxed grains indicate the recrystallization with an average grain size of about $49 \mu \mathrm{m}$. The severe local strain is mostly relieved by recrystallization. After recrystallization, change of crystallographic texture is observed where the $\{0002\}$ basal texture was found to be conserved but the peak intensity weakened. Besides, the feature of six-fold rotation symmetry vanished as shown in Figure II.1.2.14 (f). 

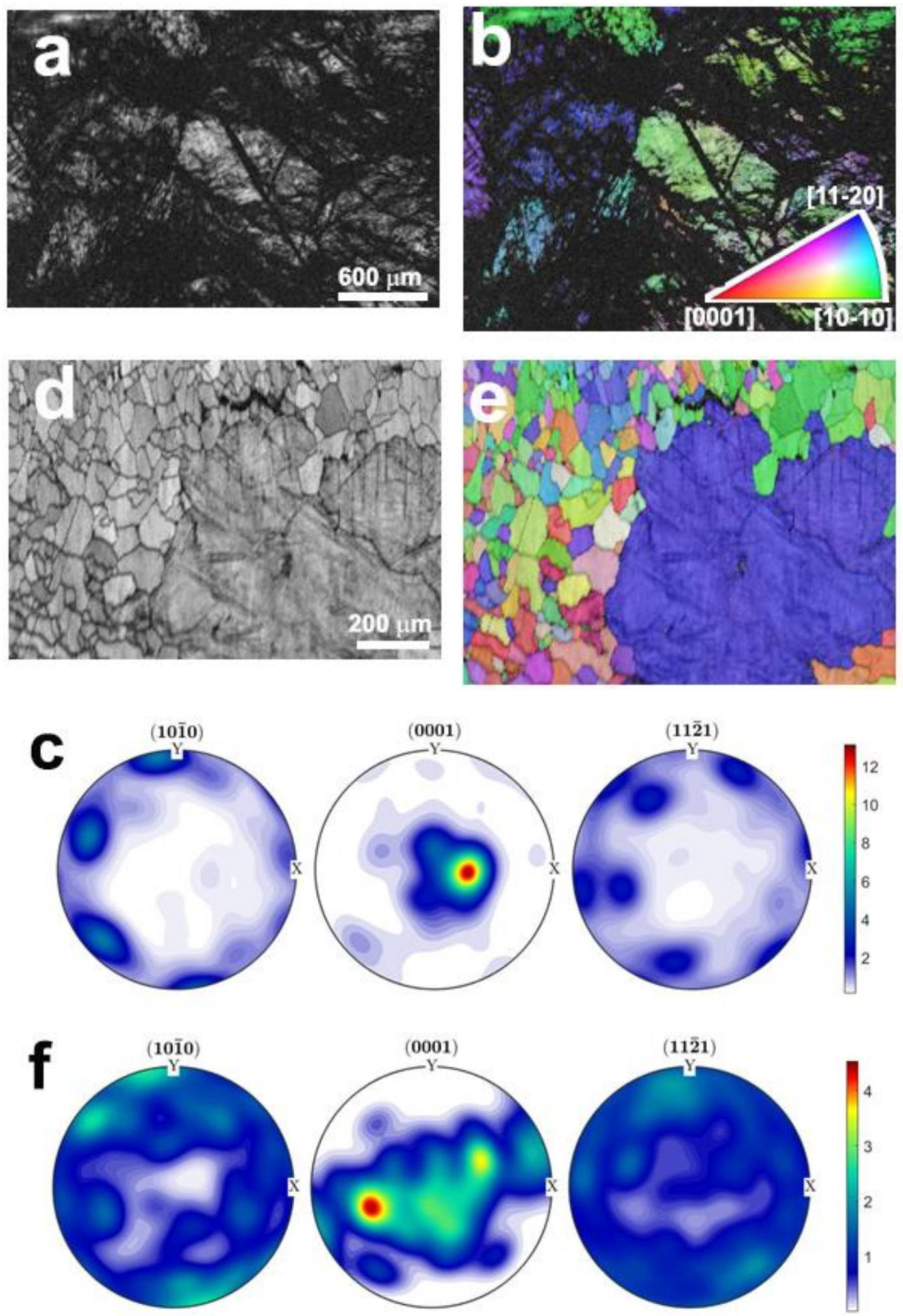

Figure II.1.2.14. EBSD results from as-rolled Mg-0.2Ca (a-c) and recrystallized Mg-0.2Ca (d-f). (a, d) The IQ map for the IPF map (b, e). The inserted color scale is projected along the RD. The nonindexed phase in the IPF map is black. (c, f) The pole figures. Three plots in each figure are set to equal color scale. The $X$ direction is along the RD, while the pole axis is along the ND of the rolling sheet. The pole figures were calculated by choosing the EBSD area in (b, e); however, the largest grain in (e) was not considered. Source: PNNL. 
Figure II.1.2.15 (a)-(f) show the IQ maps, IPF maps and $\{10-10\},\{0001\}$ and $\{11-21\}$ pole figures of asrolled $\mathrm{Mg}-1.5 \mathrm{Sn}$ and recrystallized $\mathrm{Mg}-1.5 \mathrm{Sn}$, respectively. With the same thickness reduction compared to the $\mathrm{Mg}-0.2 \mathrm{Ca}$ alloy, the local strain is much lower in as-rolled $\mathrm{Mg}-1.5 \mathrm{Sn}$. After recrystallization, the Mg-0.2Sn shows a nearly equiaxed grain structure with an average grain size of $65 \mu \mathrm{m}$. From the pole figures given in Figure II.1.2.15 (c) and (f), Mg-1.5Sn also shows a strong $\{0002\}$ basal texture after rolling, which weakened after recrystallization, in addition to the disappearance of 6-fold symmetry of the $\{10-10\}$ and $\{11-21\}$ poles.
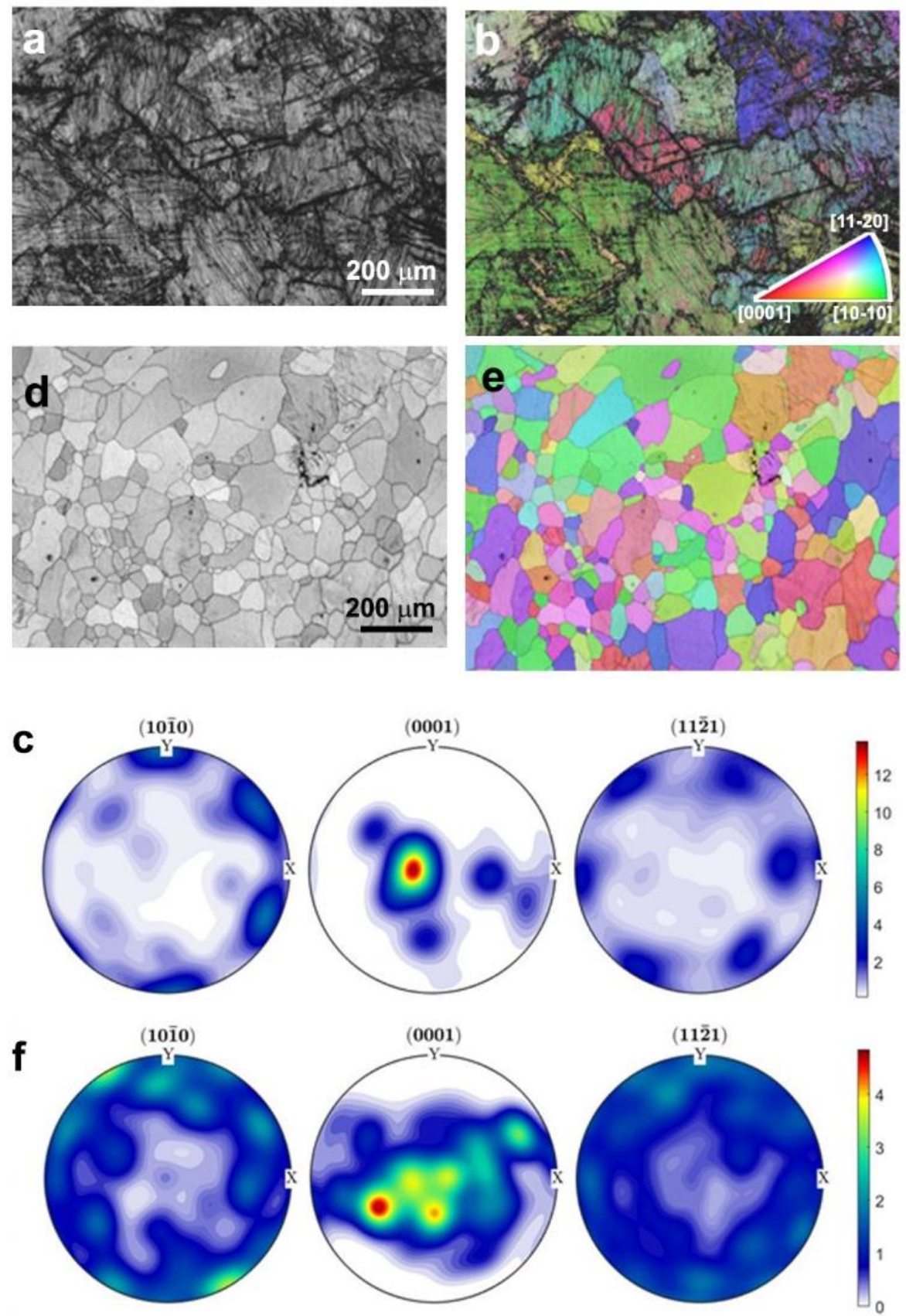

Figure II.1.2.15. EBSD results from as-rolled Mg-1.5Sn (a-c) and recrystallized Mg-1.5Sn (d-f). (a, d) The IQ map for the IPF map (b, e). The inserted color scale is projected along the RD. The nonindexed phase in the IPF map is black. (c, f) The pole figures. Three plots in each figure are set to equal color scale. The X direction is along the RD, while the pole axis is along the ND of the rolling sheet. The pole figures were calculated by choosing the EBSD area in (b, e); however, the largest grain in (e) was not considered. Source: PNNL. 


\section{Modeling for Predicting Formability of E-Form Plus}

The technical activity led by UP was supported by Inal Tech Inc. and centered on developing ICME methods coupled with experimental studies and data tools to simulate thermomechanical processing for improved and cost-effective formability.

A continuum model is being developed for textured $\mathrm{Mg}$ polycrystalline sheet materials that deform by slip and twinning mechanisms to be used in simulations of warm-forming. Primary features of the anisotropic elasticplastic model account for a continuous evolution of (1) the anisotropy differential (e.g., the strength in the principal direction of anisotropy relative to the strength in the TD), (2) the material symmetry (e.g., the orientation orthotropic triad that characterizes the symmetry of the microstructure) and (3) the strength differential (e.g., the tension/compression asymmetry). For non-uniform deformations, such as those found in forming a car door panel, those properties will vary continuously throughout a component. In previous studies, UP has shown that anisotropy and microstructural evolution has a very strong influence on microscopic and macroscopic behaviors associated with forming limits.

The ICME framework was developed to generate a continuum model that is based on the LS-DYNA *MAT233 material card. This card is based on an orthotropic yield criterion for hexagonal closed packed metals that was proposed by reference [9] and based on the already existing codes/models/subroutines developed by others [10-13]. The framework has been verified with the RT data initially provided by USAMP to Inal Tech.

A summary of the procedural steps in this ICME framework for the E-Form Plus alloy is as follows:

1. Perform Experimental Measurements: The uniaxial stress-strain responses and initial EBSD measurement for the material was obtained and is shown in Figure II.1.2.16 (a) and (b), respectively. The number of different uniaxial tensile experiments are dependent on the complexity of the underlying physics in the material and the following parameters apply:

- Material data: GM R\&D

- $\quad$ Material: POSCO Batch 3 E-Form Mg sheet

- Tensile data measured with digital image correlation

- Directions:

$0^{\circ}$ rolling direction (RD), $45^{\circ}$ diagonal direction (DD), $90^{\circ}$ transverse direction(TD)

- Temperature: RT

- Nominal strain-rate: $0.0039 / \mathrm{s}$.

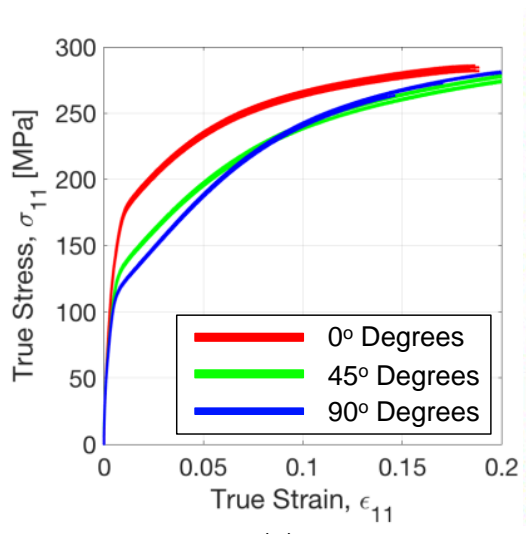

(a)

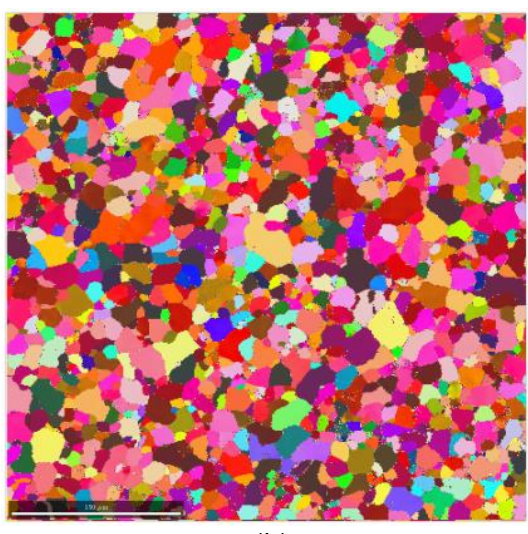

(b)

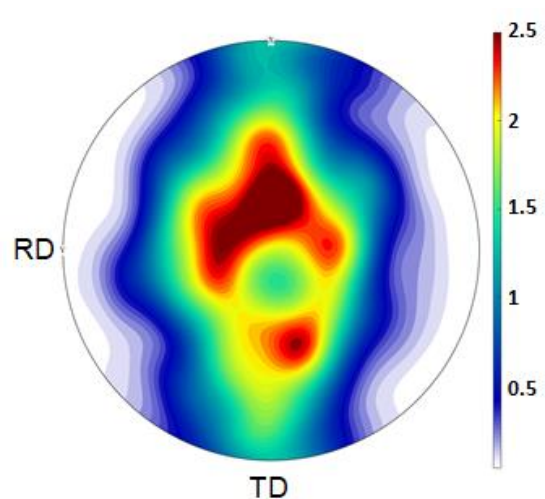

(c)

Figure II.1.2.16. (a) Uniaxial stress-strain responses; (b) initial EBSD measurement; and (c) the pole figure measurement for E-Form Plus Mg sheet. Source: Inal Tech Inc. 
As part of the LightMAT award during FY 2019, PNNL undertook an experimental approach to material characterization property measurements for use in phenomenological modeling being conducted by UP. Specifically, PNNL began performing limiting dome height (LDH) and tension tests on the USAMPsupplied E-Form Plus sheet to understand the formability and stress-strain behavior, respectively. LDH tests in equi-biaxial geometry were conducted at RT and in the $90^{\circ}$ direction to the RD; the TD was identified as the "limiting direction." Additional RT LDH tests in plane-strain geometry were performed in the TD and the major and minor strains near the failure location were also determined. LDH tests at elevated temperatures of $200^{\circ} \mathrm{C}$ and higher were also initiated. Tension tests were performed at RT that verified a Young's modulus of $\sim 45 \mathrm{GPa}$. A phenomenon termed as "micro-yielding" was also observed during RT tension testing (i.e., the alloy seemed to undergo yielding at a very low stress of $\sim 50 \mathrm{MPa}$ and at a strain of $\sim 0.1 \%$ ). PNNL also performed LDH tests at elevated temperatures to identify a suitable forming temperature for E-Form Plus. New non-standard tension test procedures needed to be developed to investigate the micro-yielding behavior of the E-Form Plus sheet. These tests are 50\% completed and will continue into FY 2020.

2. Build a Crystal Plasticity (CP) Model with the EBSD: Typically, a 2D EBSD is sufficient to represent the microstructure of sheet material as shown in Figure II.1.2.17 [14]. Taylor-type Mg CP formulation [11] that captures twin volume fraction evolution was used to model the E-Form Plus sheet behavior.

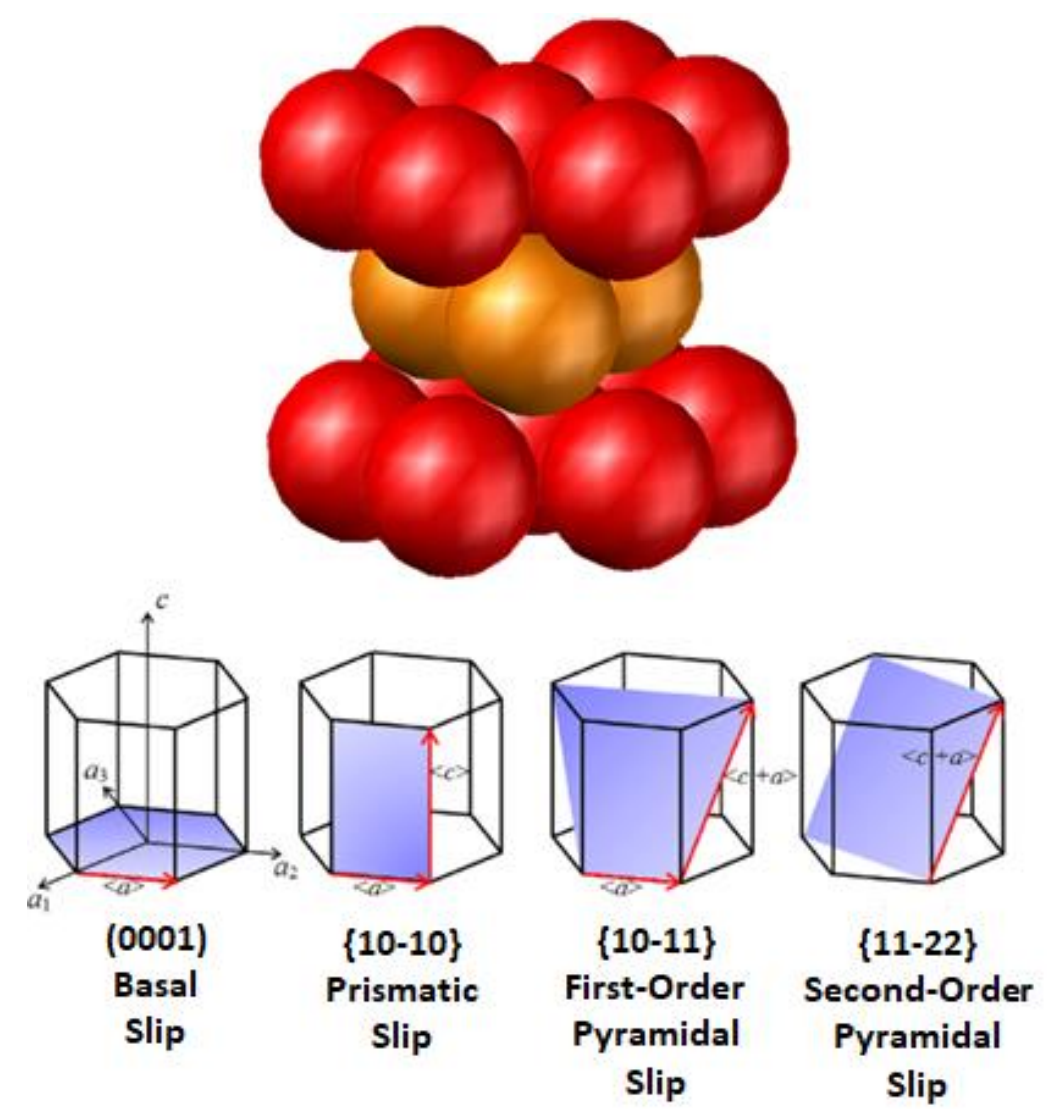

Figure II.1.2.17. Taylor-type Mg CP formulation [14].

3. Calibrate CP Model to Uniaxial Stress-Strain Responses: The observed experimental data shows a distinctive difference in the hardening rates in the different directions as shown in Figure II.1.2.18. This difference is caused by the microstructural dependence of the extension twinning mechanism resulting from the sheet texture. This means that at least two different experiments are required for calibration to capture this phenomenon. This framework uses the $0^{\circ} \mathrm{RD}$ and $90^{\circ} \mathrm{TD}$ stress-strain response for 
calibration. The stress-strain response in the $45^{\circ} \mathrm{DD}$ will be predicted and used as a verification. The following conditions applied:

- $\quad$ Calibrated using $0^{\circ} \mathrm{RD}$ and $90^{\circ} \mathrm{TD}$ :

○ In-house artificial intelligence-based differential evolution scheme for multi-parameter identification.

- Minimization of integrated root mean square (IMSE) function:

$$
\min (\mathrm{IMSE})=\sum_{i=1}^{n-1} \frac{\left(\sigma_{i+1}^{C P}-\sigma_{i+1}^{E x p}\right)^{2}+\left(\sigma_{i}^{C P}-\sigma_{i}^{E x p}\right)^{2}}{2}\left(\varepsilon_{i}^{C P}-\varepsilon_{i}^{E x p}\right)
$$

- Preliminary results after 10 iterations:

○ Captures the initial yield well:

- Minor deviation of $90^{\circ}$ after $10 \%$ strain

- Requires more iterations for better accuracy (generally around 50).

- $\quad$ Prediction of $45^{\circ} \mathrm{DD}$ :

- Captures the twinning and differential hardening.

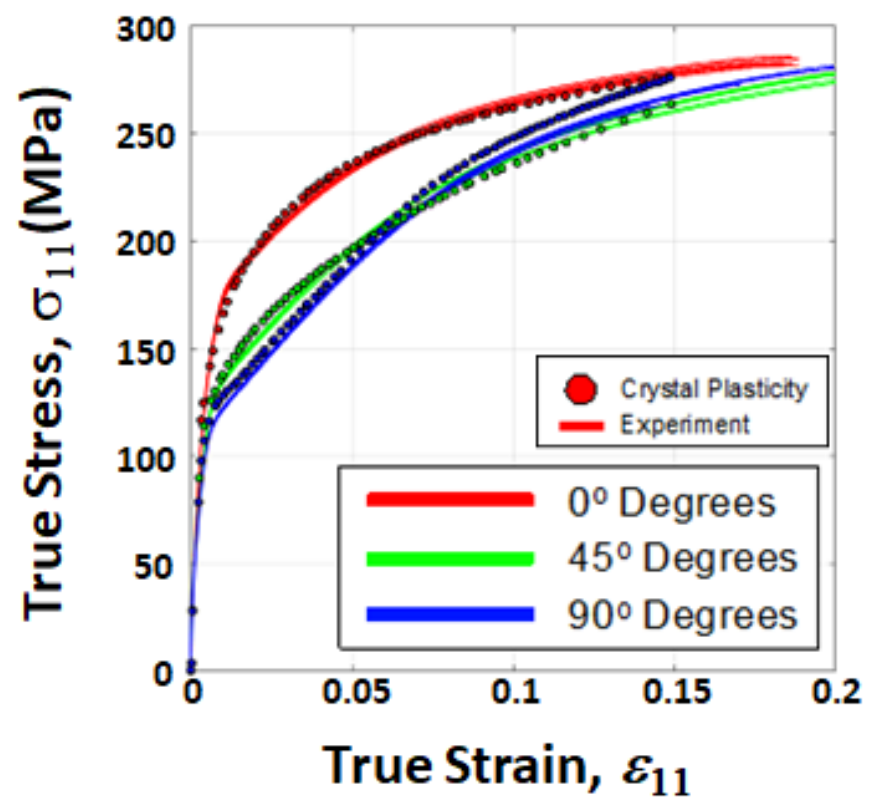

Figure II.1.2.18. Experimental data shows a distinctive difference in the hardening rates in the different directions.

Source: Inal Tech Inc.

4. Conduct Virtual Experiments using CP Formulation: Different strain $(\sigma)$ paths were simulated to produce anisotropic measurements to calibrate a desired yield function [9] as shown in Table II.1.2.4 and Figure II.1.2.19. The following virtual experiments were performed: (1) three uniaxial tensile (UT) tests at $0^{\circ}, 45^{\circ}$ and $90^{\circ}$; (2) three plane-strain (PS) tensile tests at $0^{\circ}, 45^{\circ}$ and $90^{\circ}$; (3) one biaxial tension (BT) test at $0^{\circ}$; (4) one simple shear test at $0^{\circ}$; and (5) one uniaxial compression (UC) test at $0^{\circ}$. Calibration of the yield surface is based on the plastic work calculated at $5 \%$ uniaxial tension at $0^{\circ}$. 
Table II.1.2.4. Loading Conditions for the CP Formulation Virtual Experiments.

\begin{tabular}{|c|c|c|c|c|}
\hline $\begin{array}{c}\text { Loading } \\
\text { Condition }\end{array}$ & $\begin{array}{c}\text { Direction } \\
{\left[{ }^{\circ}\right]}\end{array}$ & $\begin{array}{c}\boldsymbol{\sigma}_{\mathbf{1 1}} \\
{[\mathrm{MPa}]}\end{array}$ & $\begin{array}{c}\boldsymbol{\sigma}_{\mathbf{2 2}} \\
{[\mathbf{M P a}]}\end{array}$ & $\begin{array}{c}\boldsymbol{\sigma}_{\mathbf{1 2}} \\
{[\mathbf{M P a}]}\end{array}$ \\
\hline UT & 0 & 236.1 & 0.0 & 0.0 \\
\hline UT & 45 & 203.6 & 0.0 & 0.0 \\
\hline UT & 90 & 206.8 & 0.0 & 0.0 \\
\hline PS & 0 & 281.4 & 121.5 & 0.0 \\
\hline PS & 45 & 271.7 & 148.7 & 0.0 \\
\hline PS & 90 & 264.6 & 163.0 & 0.0 \\
\hline BT & 0 & 246.1 & 246.1 & 0.0 \\
\hline UC & 0 & -196.9 & 0.0 & 0.0 \\
\hline Shear & 0 & 64.8 & 18.7 & 102.6 \\
\hline
\end{tabular}

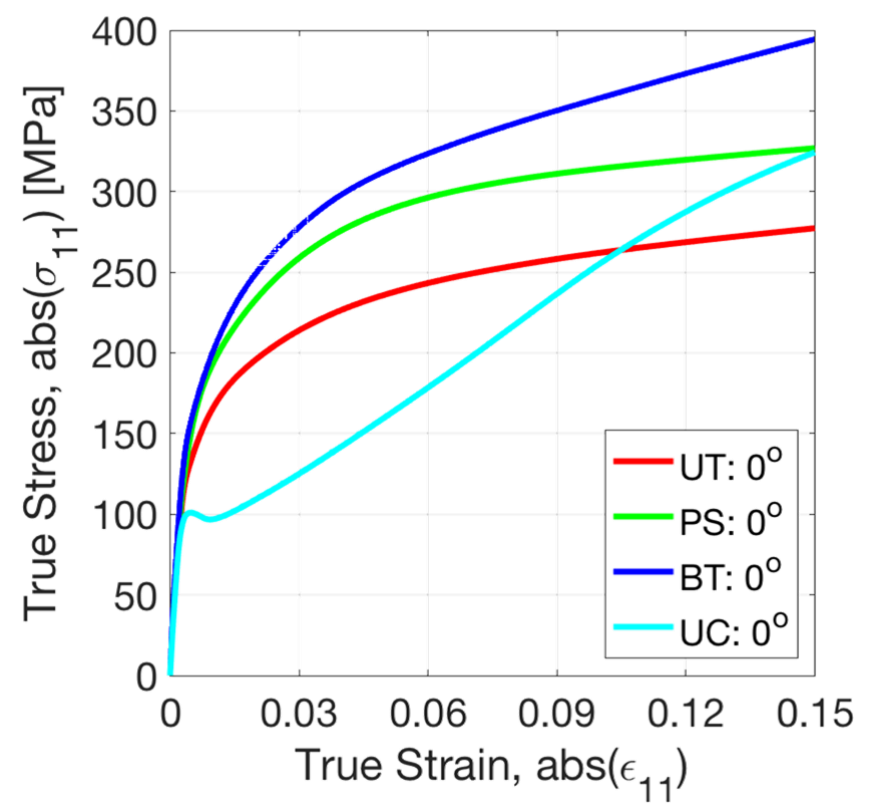

Figure II.1.2.19. Different strain paths are simulated to produce anisotropic measurements to calibrate a desired yield function. Source: Inal Tech Inc.

5. Calibrate Desired Phenomenological Material Model: The Cazacu-Plunkett-Barlat yield function is the desired model for calibration [9]. This model operates on a single transformation model with tensioncompression asymmetry. A total of nine coefficients (including asymmetry and yield surface exponent) are required for calibration.

6. Generate Material Card: Produce the LS-DYNA formatted material card with the populated values as shown in Figure II.1.2.20. This was provided by the Livermore Software Technology Corporation (LSTC) as a voluntary service which enables the new material model to be computationally compatible with their LS-DYNA software which is licensed by the OEMs and suppliers. 


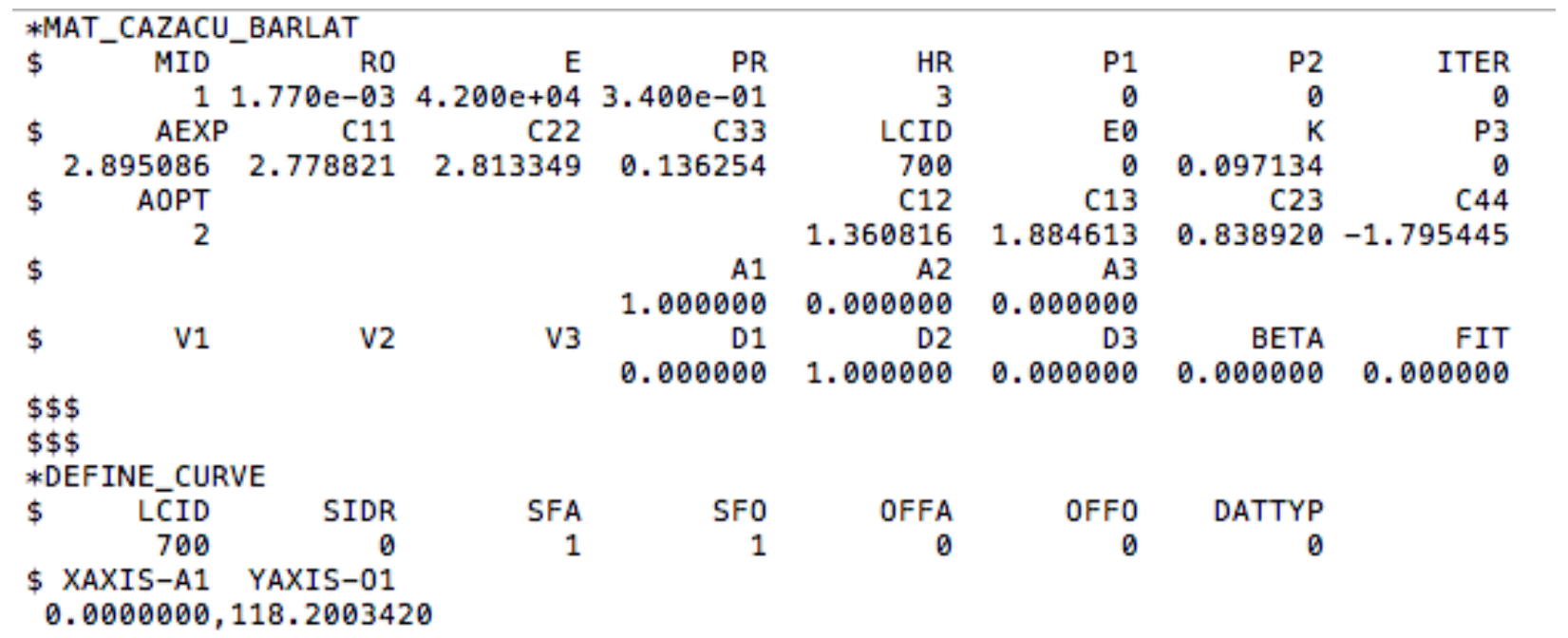

Figure II.1.2.20. Example of LS-DYNA formatted material card with the populated values. Source: LSTC.

\section{Coil-applied Anticorrosion Treatments}

The LCMS project has two sub-recipients, Xtalic and Henkel, pursuing coil-applied coatings to protect the Mg sheet from corrosion prior to being formed. The USAMP team evaluated the performance of Xtalic's Zncoated sheets and those that contained nanostructured $\mathrm{Al}$ over $\mathrm{Zn}$ and found that the performance attributes of the $\mathrm{Zn}$-coating alone were likely sufficient and more cost-effective than the nano-Al-coating. At the request of USAMP, Xtalic has ceased all work on the Al-coating program and redirected all program resources towards application of the $\mathrm{Zn}$-coating. A Zn-only coating may also more easily fit into the existing manufacturing operations utilizing coil-coated sheet in metal-forming.

Xtalic scaled the Zn pretreatment and Zn-plating process up to larger 5-in. x 5-in. (125-mm x 125-mm) samples. Twelve such samples were produced for cup-draw formability testing and shipped to EWI. The Zncoating was qualified through tape testing, adhesion checks (an example of good adhesion is shown in Figure II.1.2.21), cross-section verification of thickness $(5 \mu \mathrm{m})$ and dome formability testing. The formability was found to be on par with bare E-Form Plus sheet.

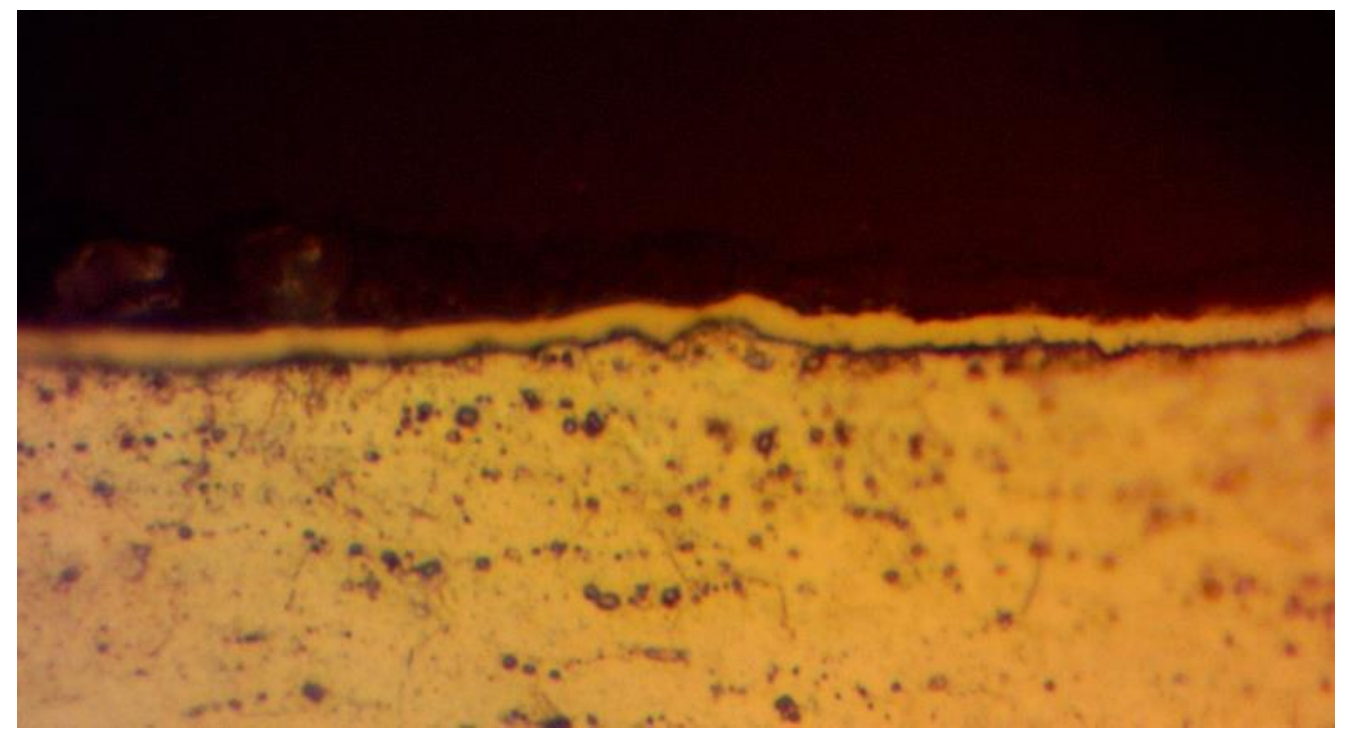

Figure II.1.2.21. Cross-section micrograph showing good adhesion in a 2- $\mathrm{mm}$ thick Zn-coating on E-Form Plus. Source: Xtalic. 
Xtalic collaborated with Henkel to harmonize the corrosion test method to be ASTM D2247, $40^{\circ} \mathrm{C}$ at $100 \%$ $\mathrm{RH}$ for a 24 to 72 -hour duration. This test method should provide adequate acceleration to mimic the corrosion performance required for storage between the mill and the forming stations. Zn-coating on an $\mathrm{Mg}$ sheet shows no corrosion in this test through a test duration of 72 hours at a $\mathrm{Zn}$ thickness of $4 \mu \mathrm{m}$.

Xtalic further scaled the Zn-plating technology onto its pilot-production line which includes larger 200-liter tank sizes. Each of the plating baths and cleaning tanks were cleaned and made up with fresh chemistry. Each of the process steps in the flow were verified for the chemical composition of the bath. The Zn-plating tank is the most crowded but can accommodate a 19 in. x 26 in. size panel, as shown in Figure II.1.2.22, which produces 12 samples that are 6 in. $x 6$ in. from every production run.

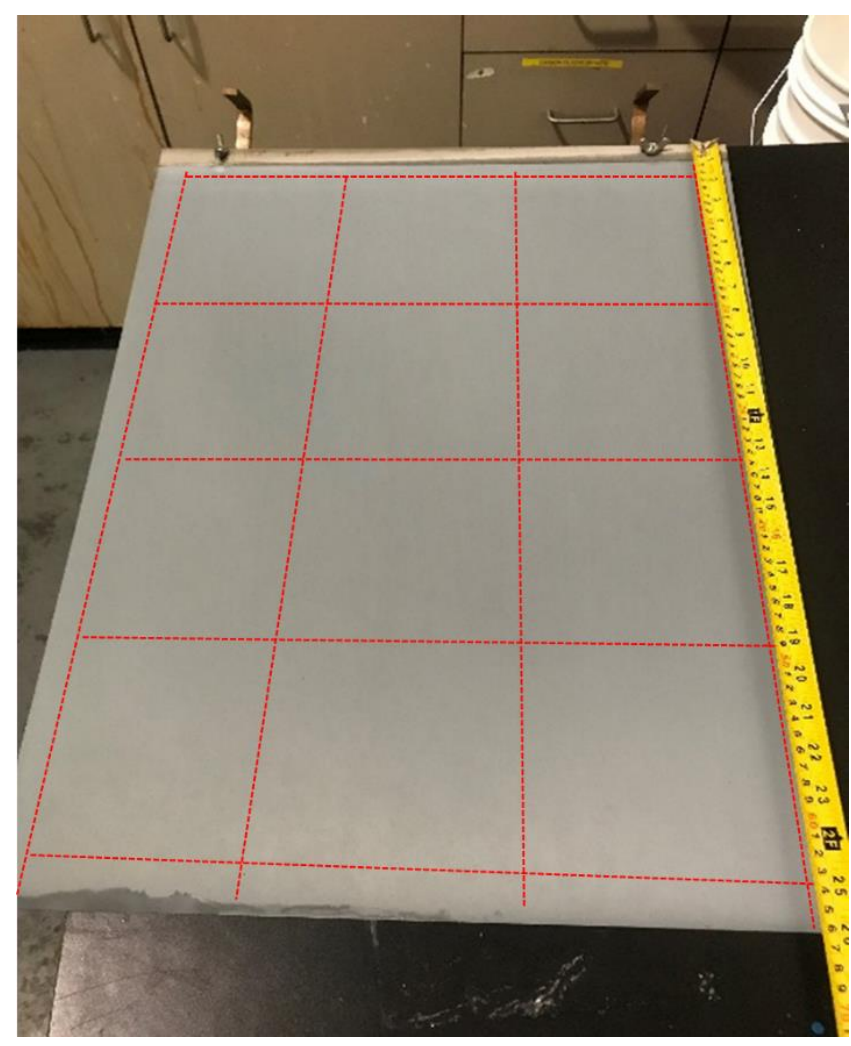

Figure II.1.2.22. Larger 19 in. x 26 in. Zn-plated panels with a superimposed dicing pattern. Anode connections can be seen at the top of the image. Source: Xtalic.

Xtalic plated several sheets in the full 19 in. x 26 in. size to verify the thickness and quality of the plated part. Thickness was measured by optical and electron microscopy on cross-sections taken from various points on the large sheet. Plating quality was confirmed using visual inspection, tape test for adhesion and 24-hour exposure to $40^{\circ} \mathrm{C} / 90 \% \mathrm{RH}$. The color is generally uniform and some slight thickness build can be seen along the edges. The edges will be trimmed before plated samples are shipped to USAMP team partners. In a production setting, the roll-to-roll sheet can be shielded to make the coating completely uniform across the sheet.

Large-form factor sheets were plated with $\mathrm{Zn}$, diced into 150-mm x 150-mm panels, and 76 of these samples were delivered to Fuchs, Quaker, and AET for their Task 3 and Task 4 developments. Henkel also supplied approximately 200 E-Form Plus panels pretreated using process "D" to the Task 3 team members-Fuchs, Quaker, PPG and AET. Xtalic and Henkel also provided pretreated samples for the EWI cross-form test experiments. 
AET observed poor performance of the Henkel pretreated sheets during welding evaluation, prompting further investigation. The organic component of the process " $\mathrm{D}$ " pretreatment was suspected to be creating the poor welding results. Henkel then developed process "E" which would deposit the protective/passivating component without the organic component. AET performed a subsequent evaluation comparing weldability of pretreatment processes "C," "D," and "E" and process "D" provided the best results.

In addition, Henkel performed lap-shear strength testing on triplicate sets of E-Form Plus coupons pretreated with Processes "D" and "E". Process "D" gave an average lap-shear strength of $11.49 \mathrm{MPa}$, which was higher than process "E" with a strength of $10.31 \mathrm{MPa}$. The decision was made to move forward with process "D."

Bend testing was performed to evaluate resistance to cracking of the pretreatment during forming. Henkel applied a modified version of "ASTM D522-93a Test Method B" on 4-in. x 6-in. E-Form Plus panels by bending them over a 3/8th-inch diameter cylindrical mandrel. The procedure was modified because the test is designed for material with a full paint system applied, not thin films. Henkel's coating is on the nanoscale, so cracking is not visible to naked eye. Instead of bending the samples over increasingly smaller diameter cylinders, Henkel used the smallest available cylinder size, then performed subsequent evaluations including field emission scanning electron microscopic (FE-SEM) analysis, confocal microscopy and humidity testing. Humidity testing at $100 \% \mathrm{RH}$ and $100^{\circ} \mathrm{F}$ resulted in no apparent increase in oxidation as can be seen by the images in Figure II.1.2.23.

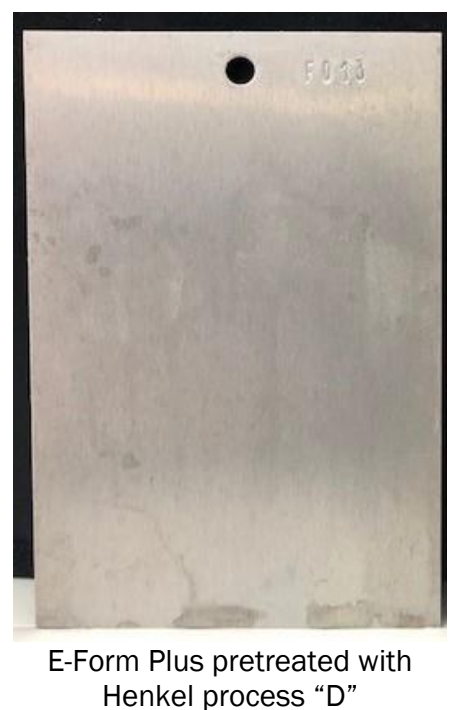

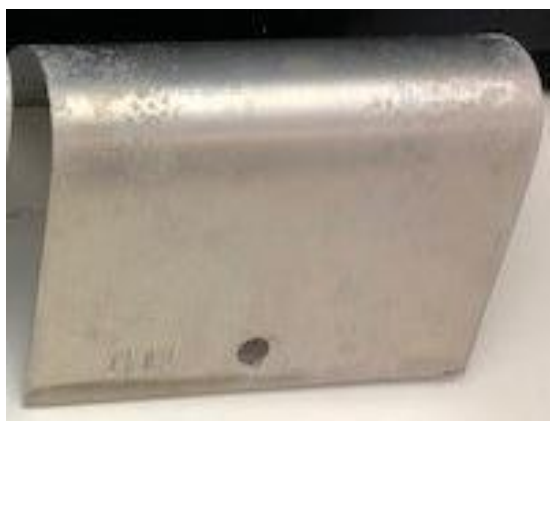

E-Form Plus pretreated with Henkel process “D”

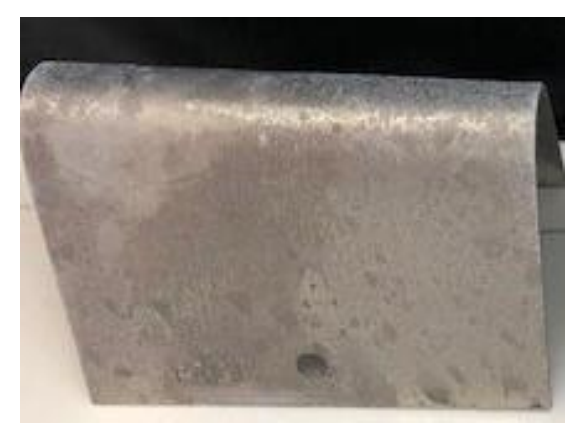

E-Form Plus - As-received (no pretreatment)

Figure II.1.2.23. Bend test after humidity exposure showing no apparent increase in oxidation. Source: Henkel.

Confocal microscopy was performed to measure the surface roughness parameters on the control and bent panels. These parameters include Ra, the average roughness, Rp, the maximum peak height, Rv, the maximum valley depth; Rz, the maximum height of the profile; Rt, the total roughness; and Rq, the root mean squared. Table II.1.2.5 shows the bent panel is slightly rougher than the control panel across all the profile roughness parameters measured.

Table II.1.2.5. Summary of Surface Roughness Measurements on Control and Bent Coated E-Form Plus Samples.

\begin{tabular}{|c|c|c|c|c|c|c|}
\hline & $\mathbf{R a}$ & $\mathbf{R p}$ & $\mathbf{R v}$ & $\mathbf{R z}$ & $\mathbf{R t}$ & $\mathbf{R q}$ \\
\hline Control & $0.767 \pm 0.0$ & $2.27 \pm 0.08$ & $5.00 \pm 0.5$ & $7.28 \pm 0.6$ & $5.56 \pm 4.5$ & $0.999 \pm 0.0$ \\
\hline Bent Panel & $0.877 \pm 0.01$ & $3.11 \pm 0.6$ & $5.38 \pm 1.1$ & $8.49 \pm 0.8$ & $11.3 \pm 0.8$ & $1.12 \pm 0.02$ \\
\hline
\end{tabular}


An FE-SEM analysis of the bent and unbent panels revealed a moderate amount of cracking on the surface of the bent panel. No flaking or loss of adhesion were apparent, however, as seen in Figure II.1.2.24.

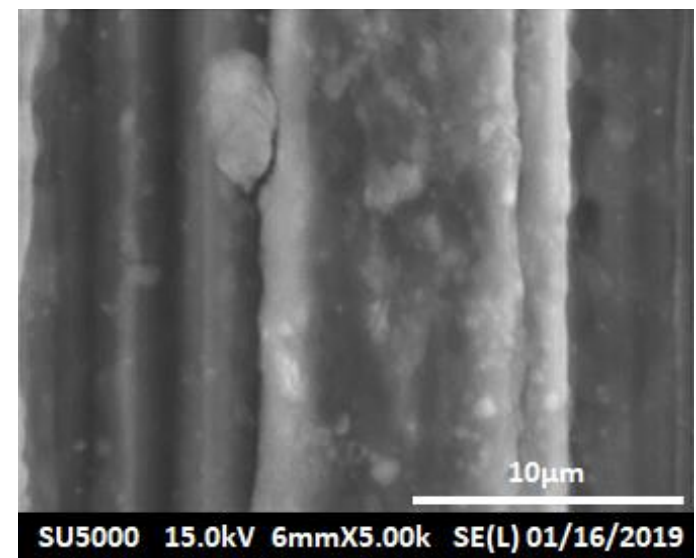

(a)

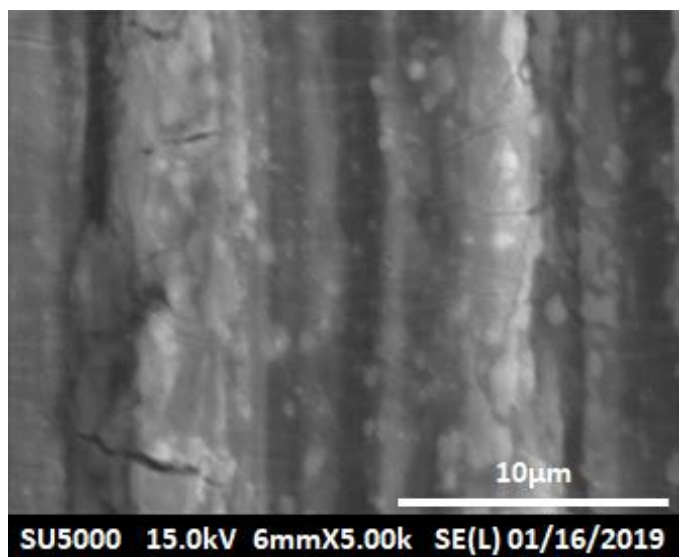

(b)

Figure II.1.2.24. FE-SEM of coated E-Form Plus Mg showing (a) the control and (b) bent at 5000x magnification showing moderate cracking on the surface. Source: Henkel.

Aging studies of the process " $\mathrm{D}$ " baths are in progress. Henkel's pretreatment process " $\mathrm{D}$ " is a multistage bath process which includes separate cleaner, rinse, etch and pretreatment stages. A variety of bath properties can change as chemical reactions and bath contamination takes place during processing of $\mathrm{Mg}$. For example, the $\mathrm{pH}$ of the cleaner stage changes based on the surface area E-Form Plus cleaned. In addition, oils and other contaminants may build-up in a treatment bath that would require replacement or replenishment. Bath aging was studied to establish bath replenishment schedules in order to maintain consistent bath parameters and help predict cost.

\section{Lubricants}

The project also has two sub-recipients developing lubricants for the Mg sheet forming process; Fuchs and Quaker. In FY 2019, Fuchs focused its testing on E-Form Plus Mg samples that had been coated with Henkel pretreatments designated as " $\mathrm{C}$ " and " $\mathrm{D}$ ". Fuchs performed testing in the Anton Parr rheometer at $100^{\circ} \mathrm{C}$ and $200^{\circ} \mathrm{C}$, concluding that the tribology results using pretreatment " $\mathrm{D}$ " coated $\mathrm{Mg}$ were consistent with previous tests on the bare E-Form Plus and F-Form Plus with Henkel pretreatment " $C$ ". It was determined that the pretreatment did not create advantageous or adverse effects regarding forming, and that the best results were observed using Renoform $1102 \mathrm{ALWF}$ at $200^{\circ} \mathrm{C}$. These results were based on the overall coefficient of friction and its duration through the sliding velocity, and consistency.

Following Quaker's positive findings regarding Formula 4A reported in BP1, additional lubricant formulations were generated with slight modifications to Formula $4 \mathrm{~A}$ and evaluated for use on AZ21 at $250^{\circ} \mathrm{C}$. Formula $4 \mathrm{C}$ was determined to offer the best lubrication performance on the AZ21 alloy. Formula 4C was named "QUAKERDRAW ${ }^{\circledR} 450$ MAG," and subsequent testing determined that it offers corrosion protection on AZ21 equivalent to current automotive industry standards. Cleanability performance was measured against current automotive industry standards as well which revealed that QUAKERDRAW® 450 MAG has a slightly longer time-to-water-break-free result than standard lubes. Varnish/oxidation-testing was performed at $250^{\circ} \mathrm{C}$ and $270^{\circ} \mathrm{C}$ where the lubricant is stable at $250^{\circ} \mathrm{C}$ with no oxidation and no sign of oxidation can be seen when maintained at $270^{\circ} \mathrm{C}$ after $10-25$ minutes. The optimum coating weight of QUAKERDRAW 450 MAG was evaluated by testing the lubricant from $1.0-3.0 \mathrm{~g} / \mathrm{m} 2$ by TCT on AZ21 with Henkel pretreatment "C" at $250^{\circ} \mathrm{C}$. Higher coating weights offered better lubrication in this evaluation. 
Four new lubricity additives were evaluated for use in QUAKERDRAW® 450 MAG utilizing a Design of Experiment (DOE) tool. Thirty-one formulations were generated and tested to measure the individual and combined impacts of the additives on lubrication. Three new formulations were generated for optimum performance based on the DOE results. These three formulations were then tested for varnishing performance at $220^{\circ} \mathrm{C}$, product stability and lubrication performance against QUAKERDRAW® $450 \mathrm{MAG}$ by TCT at $220^{\circ} \mathrm{C}$ and 3,000 PSI on AZ21 with Henkel pretreatment "D". QUAKERDRAW ${ }^{4} 450$ MAG outperformed the three DOE lubricant formulas. QUAKERDRAW® 450 MAG and the three DOE formula lubricants proved to be stable and passed a 30-minute oxidation test.

AZ21 E-Form Plus sheet with Henkel pretreatment "D" also became available during this reporting period, so QUAKERDRAW® 450 MAG was compared on bare AZ21, AZ21 with Henkel pretreatment "C" and AZ21 with Henkel pretreatment "D." These tests determined that the pretreatments did not significantly impact the performance of the lubricant.

A key accomplishment of this task team in FY 2019 was the evaluation of lubricants in warm-forming trials on the EFP (AZ21) Mg alloy sheet. In April 2019, USAMP contracted EWI to evaluate the two recommended lubricants with E-Form Plus (1.2 mm sheet thickness) using the B2 Cup Deep Draw test at elevated temperatures of $150^{\circ} \mathrm{C}, 200^{\circ} \mathrm{C}$ and $250^{\circ} \mathrm{C}$. The two lubricants provided to EWI were Fuchs Renoform 1102 ALWF (for Al warm-forming) and Quaker QUAKERDRAW® 450 MAG. Fuchs applied the Renoform 1102 ALWF to both sides of the blanks at a ratio of 4:1 (20\%) with water using a sponge brush. Once dried, the coating weights were determined to be $\sim 0.5 \mathrm{~g} / \mathrm{m} 2$. The results from the cup-draw tests concluded that the Fuchs lubricant consistently yielded acceptable results across a broader range of test conditions.

In August 2019, EWI was tasked with studying the sensitivity of the warm-forming process parameters to material formability using an intermediate size cross-form part geometry for blanks coated with recommended lubricants from Fuchs and Quaker as well as blanks pretreated by Henkel and Xtalic and subsequently coated with lubricants from Fuchs and Quaker. EWI conducted the cross-form trials at three temperatures and seven blank holder force levels for determining a viable process window and for the calibration of simulation models prior to full-scale door inner forming demonstrations planned for FY 2020. The physical testing performed in EWI's warm-forming test cell was successful in determining the forming limits of the E-Form Plus sheet material - from an analysis of wrinkling and splitting on cross-forms - and assessing the sensitivity of parameters to process variation. This work was also successful in determining a forming depth that sufficiently challenged material formability; wrinkles were seen at a low blank holder force and splits were seen at a higher blank holder force. A temperature of $200^{\circ} \mathrm{C}$ was found to be the best forming temperature for the cross-form part. In both cup-draw and cross-form tests, the Fuchs lubricant proved to be more successful than the Quaker product in forming quality parts. Henkel and Xtalic pretreatments did not have a major impact on formability.

Argus strain distribution measurements were made on cross-form parts for calibration of CAE simulation models. The data provided by this experimental forming work will be used in FY 2020 to establish correlations between FEA and physical testing. Material variation in E-Form Plus sheets sourced from different batches caused inconsistent results at the material formability limit.

\section{Joining Process Evaluation and Development}

AET is evaluating commercially available joining methods (RSW, Arplas Welding, and Laser Welding) and developing welding process parameters to join AZ21 Mg-based alloys, such as E-Form Plus, in bare and pretreated conditions with and without forming lubricants. Most of AET's effort in FY 2019 focused on the measurement and evaluation of electrical contact resistance and RSW weldability studies of E-Form Plus (AZ21) sheet material, although some preliminary investigations were conducted on Arplas and laser welding processes as well. 
Specifically, AET evaluated RSW with commonly used, commercially available electrodes for the surface conditions of bare AZ21 with and without Quaker forming lubricant, AZ21 pretreated with Henkel's Process D with no forming lubricant, and AZ21 pretreated with Xtalic's Zn-plating process with no forming lubricant.

The specific electrode types evaluated were:

- $\quad 50$-mm radius F-type welding electrodes

- $\quad$ 75-mm radius F-type welding electrodes

- 6-mm ball nose welding electrodes

- 6-mm truncated welding electrodes

- $\quad$ Multi-Ring-Dome (MRD) electrodes.

In these studies, the F-type welding electrodes showed better promise than the ball nose and truncated electrodes for robust welding process development for bare AZ21 material both, with and without the Quaker lubricant applied, and for the Xtalic Zn-plated material with no lubricant. Welding of the Henkel pretreated material proved to be a substantial challenge with the F-type, ball nose, and truncated electrodes due to the high-level of electrical contact resistance (the pretreatment " $\mathrm{D}$ " is a polymer which provides a level of electrical insulation), so the MRD-type electrode was evaluated for this material/coating configuration. This electrode design incorporates grooved rings that cut through the polymer coating to achieve direct contact with the $\mathrm{Mg}$ metal underneath the polymer, thereby providing an advantage for this pretreatment application.

AET also investigated the effect of electrode cap life on RSW weld quality as a function of the number of welds produced. Preliminary evaluation on bare AZ21 with 75-mm radius F-type electrodes indicated the electrode caps would need to be replaced every 15-20 welds in order to maintain weld quality. However, cleaning the electrode tips with a cleaner/deoxidizer significantly reduced weld quality degradation as a function of the number of welds.

Additionally, AET conducted a preliminary investigation into Arplas welding of bare AZ21 and Henkel pretreated AZ21. A key challenge for implementing this process with AZ21 is the ability to form the projection "dimple" in the material without developing forming cracks which could negatively affect the weld strength. At this time, Arplas has developed dimple stone geometries for high-strength steels and Al alloys, but none for $\mathrm{Mg}$ alloys. Despite the difficulties in forming the dimple without inducing cracks, several good welds have been achieved with this process on bare AZ21. Again, as in RSW, welding of the Henkel pretreated material proved to be a challenge and may not ultimately be feasible without significant changes to the process. One potential advantage of the Arplas process (in addition to being able to weld on narrow flanges) is that the electrodes showed substantially reduced tip erosion, potentially leading to increased service life, when compared to RSW.

Finally, AET conducted an initial feasibility investigation of laser welding with bare AZ21 material. Although some pores and micro cracks were observed in and outside the weld, this early study demonstrated that laser welding of this material should be feasible. Further optimization and refinement will be conducted with bare AZ21 and the study will be expanded to include pretreated materials, both with and without forming lubricants.

\section{Preparing for E-coating Mg Assemblies}

After part forming and joining into an assembly, PPG Industries approached the problem of metal pretreatment holistically considering the entire process from cleaning and surface preparation to pretreatment formulation to sealers and other post-rinses for maximum corrosion protection. A typical pretreatment line sequence is shown in Figure II.1.2.25. 


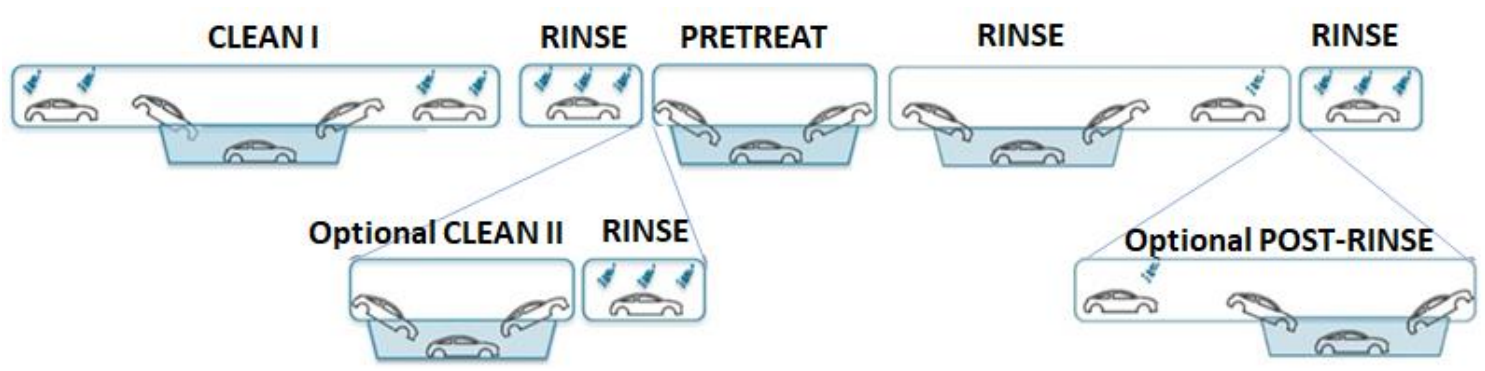

Figure II.1.2.25. A typical automotive pretreatment sequence before applying the electrocoat. Source: PPG.

PPG evaluated the effect of immersion time of the E-Form Plus in Cleaner 2 on corrosion performance to learn if the standard two-minute immersion time is optimal or if shorter times are possible. It was found that some additional corrosion protection is provided after 2-3 minutes of immersion, but most of the corrosion protection is imparted after just one-minute of immersion. This allows shortening of the coating process with a small loss in performance, if desired. PPG also performed x-ray photoelectron spectroscopic depth profiling to determine the relative forming rate of the two protective layers that form due to Cleaner 2. Figure II.1.2.26 shows the decrease in $\mathrm{Mg}$ and $\mathrm{O}$ over time which indicates formation of a conversion layer.

Ten Cleaner 2 formulations were designed to further improve corrosion performance. These cleaners were applied without additional pretreatment using a compact process. Cleaner 2 and three additional modified formulations (i.e., Cleaners 2.5, 2.6, and 2.7) were the top-performing pretreatments.
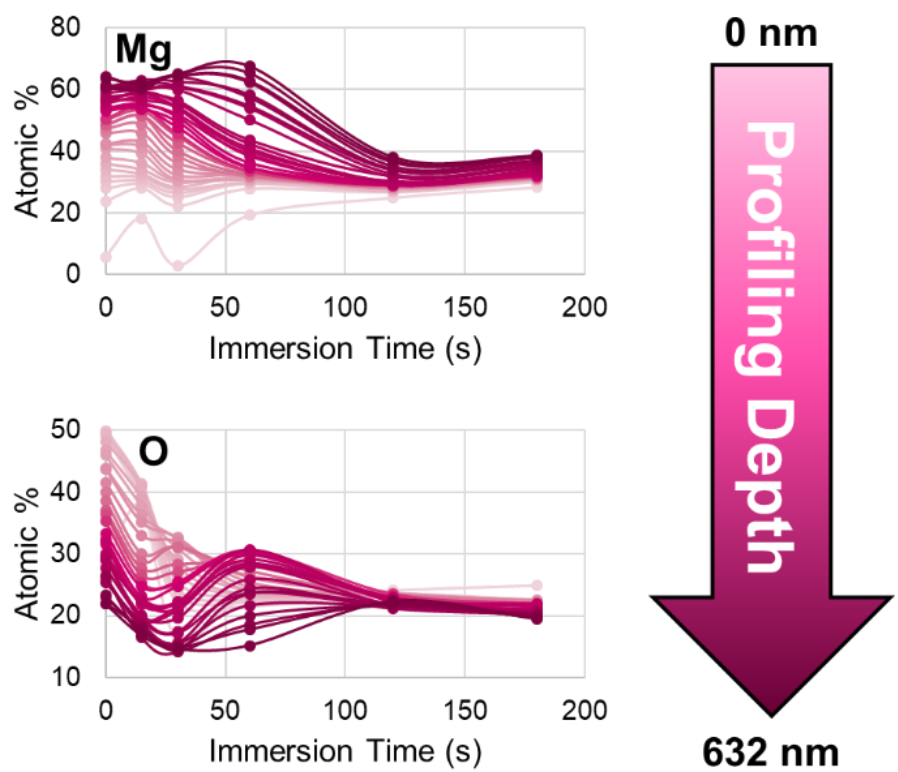

Figure II.1.2.26. X-ray photoelectron spectroscopic depth profiles of E-Form Plus immersed in an alkaline cleaner, then in Cleaner 2 for various lengths of time. The concentration of each element is shown changing in both time (along the x-axis), as well as at sample depth (light pink $\rightarrow$ dark pink). The decrease in oxygen content is clearly observed over time, which is attributed to the conversion of $\mathrm{Mg}(\mathrm{OH})_{2}$. Source: PPG.

Eight pretreatments utilizing small molecules and inorganic additives were developed. PPG observed strongly positive corrosion performance from a proprietary "Pretreatment 13" which was equivalent to their current top inorganic treatment, Cleaner 2.4. It also performed well in wet crosshatch testing. From Pretreatment 13, Pretreatment 21 was developed which improved off-scribe corrosion performance of E-Form Plus. These systems did not show any statistical improvement in corrosion or adhesion performance for $\mathrm{Al} 3003$ 
(AA3003). Preliminary results from other testing showed possible inconsistencies in Pretreatment 21's performance which could be from lot-to-lot variation of the starting material. These possible differences are being evaluated and ways to mitigate them are being pursued.

PPG also evaluated the compatibility of the cleaners and pretreatments developed with other Task 3 member coatings. Panels were prepared by Quaker (one lubricant), Fuchs (one of two lubricants), or Henkel (one coil pretreatment), and then sent to PPG. The panels underwent one process as shown in Table II.1.2.6. The Quaker lubricant required two runs through the alkaline cleaner to achieve a water break-free surface after rinsing, whereas both Fuchs lubricants were water break-free after only one cleaning step.

Table II.1.2.6. Processes used to Clean and Pretreat Panels Treated with Each Task 3 Team-Member's Coating.

\begin{tabular}{|c|c|c|c|c|}
\hline Process ID & Step 1 & Step 2 & Step 3 & Step 4 \\
\hline A & Cleaner 1 & Cleaner 2.8 & - & - \\
\hline B & Cleaner 1 & Cleaner 2.8 & Pretreatment 8 & - \\
\hline C & Cleaner 1 & Cleaner 5 & Cleaner 2.8 & - \\
\hline D & Cleaner 1 & Cleaner 5 & Cleaner 2.8 & Pretreatment 8 \\
\hline E & Cleaner 1 & Pretreatment 9 & - & - \\
\hline
\end{tabular}

To evaluate the compatibility of PPG's processes with a multi-metal system, non-joined samples were first tested and run in sequence. The metals evaluated in this matrix are cold-rolled steel (CRS), hot-dipped galvanized E-coated (HDGE) steel, Al 6111 (AA6111), and E-Form Plus Mg. Eight treatment processes were evaluated. The AA6111 panels performed the best in cyclic corrosion testing. The HDGE steel and E-Form Plus were middle-of-the-road performers while the CRS fared the worst of all the metals. Of the treatment systems studied with cyclic corrosion testing, Cleaner 2.4 followed by Pretreatment 1 showed the best performance for the cyclic corrosion testing. Pretreatment 24 followed by Pretreatment 1 was also promising, although some variability was observed in the E-Form Plus samples which will be further investigated with an aging study.

PPG researchers did not observe a single pretreatment step that can protect all substrates from corrosion but have identified three good options that use two advanced cleaning or pretreatment steps. The pretreatment and electrocoat baths were evaluated before and after the various substrates were run through them using inductively coupled plasma spectrometry. No unexpected changes to bath chemistry were observed. In cross hatch testing, AA6111 showed the best dry cross hatch adhesion performance, but there was not much difference seen between the substrates or the treatment systems used. To pass wet adhesion testing for all the substrates, Pretreatment 24 followed by Pretreatment 1 performed the best.

The second step of this work used self-piercing rivets to join samples together prior to cleaning or pretreating. The joined samples include identical couples of CRS, HDGE, AA6111, and E-Form Plus, as well as combinations of each dissimilar metal. Figure II.1.2.27 shows the couples containing E-Form Plus after the first three weeks of corrosion testing. In the couples shown in Figure II.1.2.27, the substrate that will corrode more is shown on the bottom for easier visualization.

It has been challenging to identify a pretreatment solution that works well for all substrates in a single-step. Based on preliminary corrosion results, it appears that the two-step pretreatment solution of Cleaner 2.4 followed by Pretreatment 1 remains the most promising solution for mixed substrates as an overall performer because there was less variability in the corrosion results when compared to Pretreatment 24 followed by Pretreatment 1. 

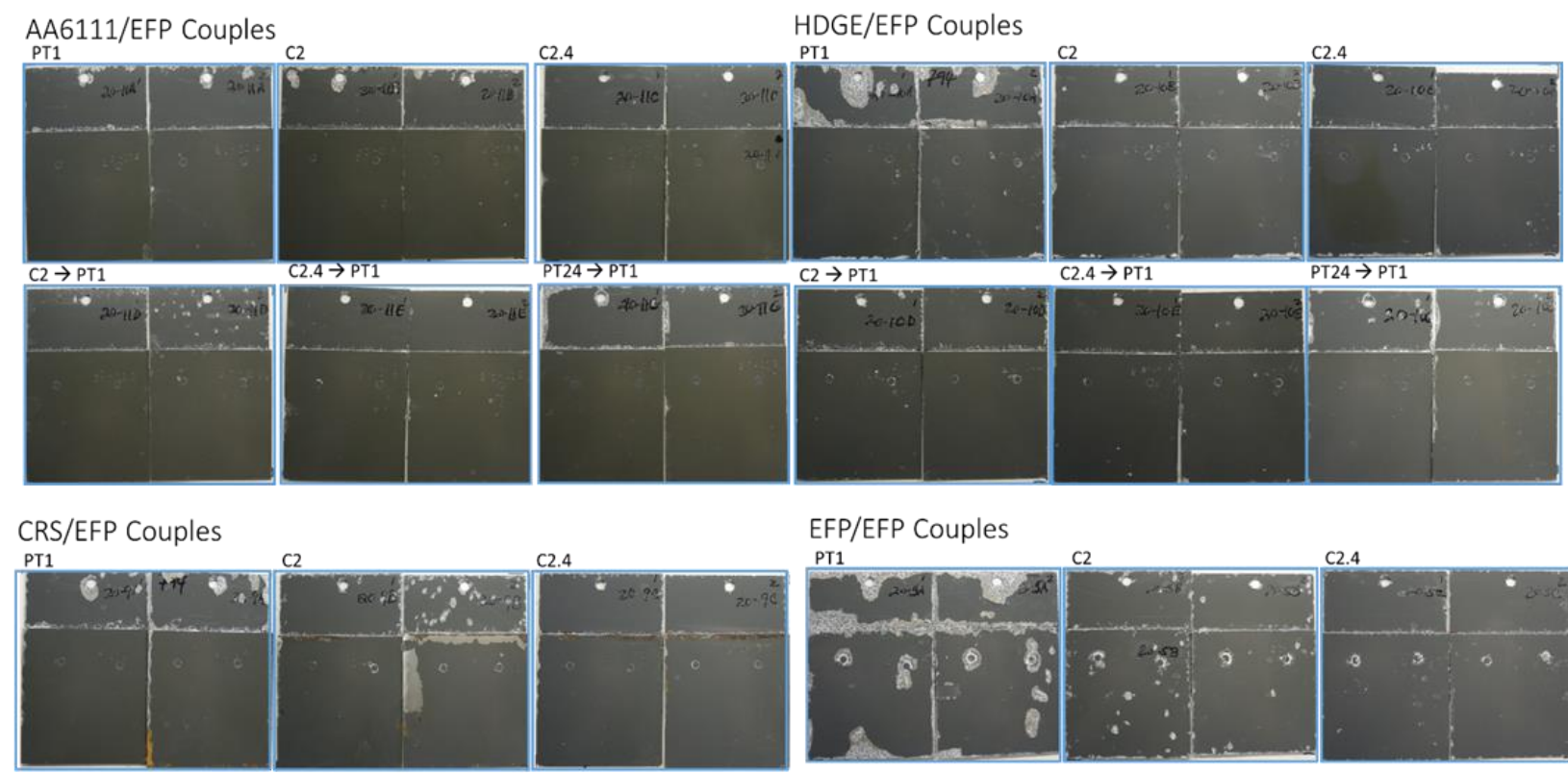

\section{EFP/EFP Couples}
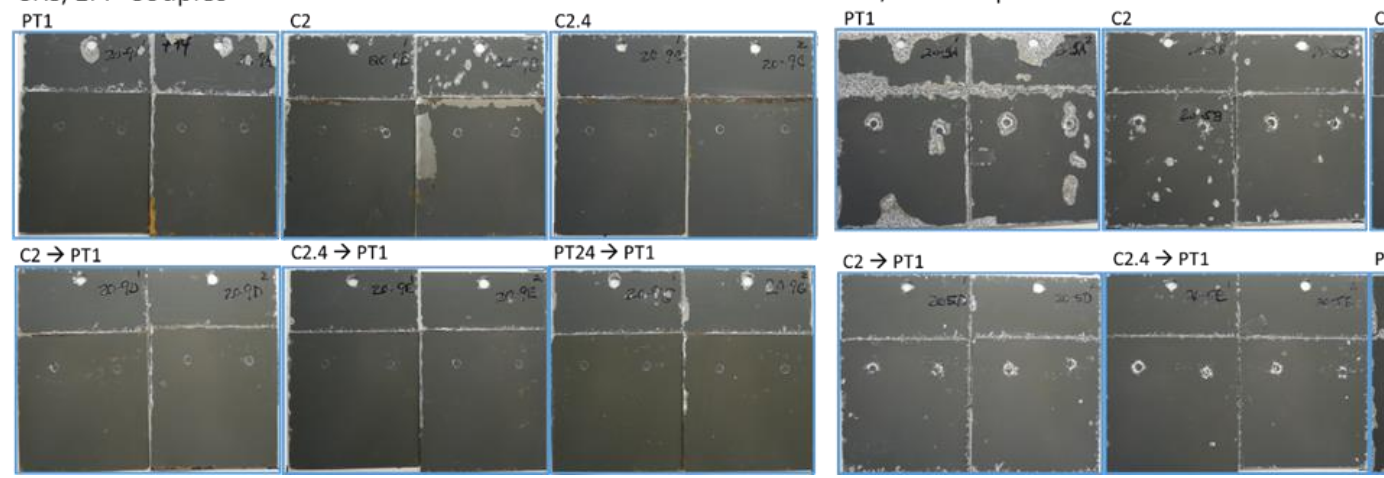

$\mathrm{C} 2.4 \rightarrow \mathrm{PT} 1$
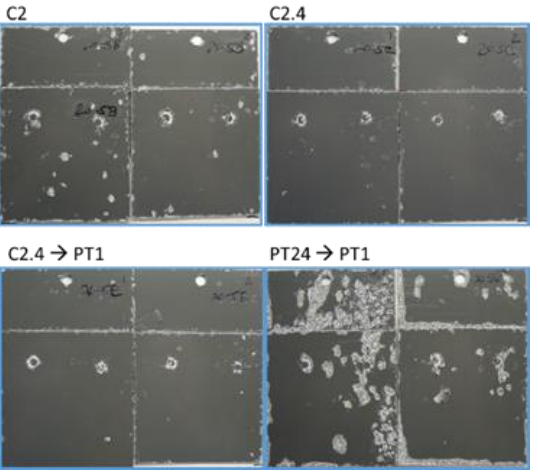

Figure II.1.2.27. E-Form Plus (EFP) joined to AA6111, HDGE, CRS, and itself using two self-piercing rivets and subjected to different cleaning and pretreating combinations after three weeks of filiform corrosion testing. Source: PPG.

\section{Production of Large Automotive Body Components}

During FY 2019, Vehma worked with the USAMP Task 6 team to select LS-DYNA as the preferred FEA simulation code for incorporating new material cards developed by the Task 2 team for EFP (AZ21) alloy sheet. Vehma also directed the LSTC code developers and Task 2 ICME modeling team to implement the material characterization test matrix requirements. The test matrix is nearly $75 \%$ complete and LSTC is presently finalizing the MAT36 card with EFP data after which the baseline cross-form (intermediate size part) simulations will be done in early FY 2020. Simultaneously, the USAMP Task 2 team (PNNL, FADI-AMT, UP and Inal Tech) are also generating the material property and anisotropy measurements required for application of CP in calibrating and validating the enhanced MAT233 card which Vehma will use for door forming simulations in FY 2020.

\section{Conclusions}

During FY 2019, USAMP focused on EFP (AZ21) Mg sheet as the material of choice for conducting detailed mechanical property testing, microstructure characterization, formability analysis and coating studies.

USAMP sourced three batches of EFP from POSCO. GM R\&D utilized inductively coupled plasma-atomic emission spectrometry to conduct elemental analysis of the three different batches to confirm that each batch was chemically consistent in composition. It was known that each batch was rolled on a different mill, thus USAMP is conducting several types of tests to assess potential variability in tensile anisotropy amongst the batches. 
Material testing and characterization of commercial EFP alloy will continue in FY 2020 for the development of new material cards that can be calibrated and used to simulate warm-forming of the cross-form and door inner part. USAMP's university team will continue computational modeling to predict viable alloy chemistries, produce and evaluate new RE-free alloys containing < $0.2 \mathrm{wt} . \% \mathrm{Zr}$ to accomplish grain refinement, and texture randomization without using strip-casting technology. The coatings research team will complete their evaluation of low-cost coil-applied pretreatments, corrosion coatings, and warm-forming lubricants for scale application in door inner forming. The project team will also complete evaluation of joining processes for Mg$\mathrm{Mg}$ sheets and fabricate 'mini-door' components for corrosion studies. Finally, with the USAMP's feedback on the demonstrated warm-forming process capability, technical cost modeling will be performed to estimate the impact of these technological advances.

\section{Key Publications}

1. Shi, R., J. Miao, Z. Li, and A. A. Luo, 2019, "A new Mg sheet alloy and its multistage homogenization for simultaneously improved ductility and strength at RT," Scr. Mater., Vol. 171, pp. 92-97.

\section{References}

1. Trang, T. T. T., J. H. Zhang, J. H. Kim, A. Zargaran, J. H. Hwang, B.-C. Suh, and N. J. Kim, 2018, "Designing a Mg alloy with high-strength and high formability," Nat. Commun., Vol. 9, Art. \#2522, pp. 1-6.

2. Bian, M. Z., T. T. Sasaki, T. Nakata, Y. Yoshida, N. Kawabe, S. Kamado, and K. Hono, 2018, "Bakehardenable Mg-Al-Zn-Mn-Ca sheet alloy processed by twin-roll casting," Acta Mater., Vol. 158, pp. 278-288.

3. Wang, T., L. Jiang, R. K. Mishra, and J. J. Jonas, 2014, "Effect of Ca addition on the intensity of the RE texture component in extruded Mg alloys," Met. Mater. Trans. A, Vol. 45, No. 10, pp. 4698-4709.

4. Yasi, J. A., L. G. Hector Jr., and D. R. Trinkle, 2010, "First principles data for solid-solution strengthening of Mg: From geometry and chemistry to properties," Acta Mat., Vol. 58, No. 17, pp. 5704-5713.

5. Ghazisaeidi, M., L. G. Hector Jr., and W. A. Curtin, 2014, "Solute strengthening of twinning dislocations in Mg Alloys," Acta Mat., Vol. 80, pp. 278-287.

6. Buey, D., L. G. Hector Jr., and M. Ghazisaeidi, 2018, "Core structure and solute strengthening of secondorder pyramidal $\langle c+a\rangle$ dislocations in Mg-Y alloys," Acta Mat., Vol. 147, pp. 1-9.

7. Chino, Y, T. Ueda, Y. Otomatsu, K. Sassa, X. Huang, K. Suzuki, and M. Mabuchi, 2011, "Effects of Ca on tensile properties and stretch formability at RT in Mg-Zn and Mg-Al alloys," Mater. Trans., Vol. 52, pp. 1477-1482.

8. Lee, J. Y., Y. S. Yun, W. T. Kim, and D. H. Kim, 2014, "Twinning and texture evolution in binary MgCa and Mg-Zn alloys," Met. Mater. Int., Vol. 20, pp. 885-891.

9. Cazacu, O., B. Plunkett, and F. Barlat, 2006, "Orthotropic yield criterion for hexagonal closed packed metals,” Int. J. Plasticity, Vol. 22, No. 7, pp. 1171-1194.

10. Inal, K., R. K. Mishra, and O. Cazacu, 2010, "Forming simulation of Al sheets using an anisotropic yield function coupled with CP theory," Int. J. Solids Struct., Vol. 47, No. 17, pp. 2223-2233.

11. Lévesque, J., K. Inal, K. W. Neale, and R. K. Mishra, 2010, "Numerical modeling of formability of extruded Mg alloy tubes," Int. J. Plasticity, Vol. 26, No. 1, pp. 65-83. 
12. Kohar, C. P., J. L. Bassani, A. Brahme, W. Muhammad, R. K. Mishra, and K. Inal, 2017, “A new multiscale framework to incorporate microstructure evolution in phenomenological plasticity: Theory, explicit FE formulation, implementation and validation," Int. J. Plasticity, Vol. 117, pp. 122-156.

13. Kohar, C. P., A. Brahme, F. Hekmat, R. K. Mishra, and K. Inal, 2019, "A computational mechanics engineering framework for predicting the axial crush response of Al extrusions," Thin Wall. Struct., Vol. 140, pp. 516-532.

14. Fan, H. and El-Awady, J.A., 2015. Towards resolving the anonymity of pyramidal slip in Mg. Materials Science and Engineering: A, 644, pp.318-324.

\section{Acknowledgements}

This project is a continuing collaboration with many researchers from Ford Motor Company, Fiat Chrysler Automobiles US LLC, GM, and sponsor/support/subrecipient/vendor organizations. The contributions of the following USAMP task leaders to this report are gratefully acknowledged: Leland Decker and Aslam Adam (FCA); Bita Ghaffari and Mei Li (Ford Motor); Lou Hector, Arianna Morales, and Anil Sachdev (GM R\&D); Aaron Yocum (DOE-NETL); Jon Carter and Manish Mehta (M-Tech International LLC); and Gloria Bucciero and Jenna Wiland (Bucciero and Associates). 


\title{
II.1.3 Mitigating Corrosion in Mg Sheet in Conjunction with a Sheet-Joining Method that Satisfies Structural Requirements within Subassemblies (Pacific Northwest National Laboratory)
}

\author{
Aashish Rohatgi, Co-Principal Investigator \\ Pacific Northwest National Laboratory \\ 902 Battelle Blvd. \\ Richland, WA 99352 \\ E-mail: aashish.rohatgi@pnnl.gov \\ Bill Kokosza, Co-Principal Investigator \\ Magna International - Stronach Centre for Innovation (SCFI) \\ 750 Tower Dr. \\ Troy, MI 48083 \\ E-mail: bill.kokosza@magna.com
}

\section{Sarah Kleinbaum, DOE Technology Development Manager}

U.S. Department of Energy

E-mail: sarah.kleinbaum@ee.doe.gov

Start Date: October 1, 2017

Project Funding (FY19): \$725,000

End Date: September 30, 2019

DOE share: $\$ 300,000$

Non-DOE share: $\$ 425,000$

\section{Project Introduction}

The push for lightweighting solutions for U.S. LD and HD vehicles have identified Mg alloy sheet materials as a possible candidate. The general consensus in the automotive industry is that the use of multi-material solutions is going to be the trend for future vehicles in order to achieve the desired weight reduction. A recent Multi-Material Lightweight Vehicle report [1] shows that $155 \mathrm{~kg}$ (47.5\%) body-in-white (BIW) mass reduction in a Multi-Material Lightweight Vehicle Mach-II design relative to a 2013 Fusion baseline vehicle, where Class A exterior body panels are warm-formed $\mathrm{Mg}$ sheets. The use of a Mg sheet up to $51 \%$ in closures-inwhite applications is proposed in the Mach-II design for a $48 \%$ mass reduction over the baseline vehicle. However, the report identified two major challenges related to the use of multi-material solutions:

(1) corrosion; and (2) joining. Additionally, for Mg alloys, the need for improved corrosion mitigation is a prime requirement, especially when coupled with a dissimilar metal because of a galvanic corrosion problem. Corrosion behavior of $\mathrm{Mg}, \mathrm{Mg} / \mathrm{Mg}, \mathrm{Mg} / \mathrm{Al}$, and $\mathrm{Mg} / \mathrm{steel}$ joints is being evaluated in this project through environmental corrosion chamber tests and microstructural characterization.

\section{Objectives}

The project goal is to develop strategies to join $\mathrm{Mg}$ with $\mathrm{Mg} / \mathrm{dissimilar}$ metals to meet structural requirements while simultaneously mitigating corrosion and achieving a Class A surface finish, with these objectives:

- Evaluate the corrosion behavior of two types of protective surface coatings on a Mg alloy sheet.

- Evaluate three different joining techniques for their respective corrosion behavior and structural strength in $\mathrm{Mg} / \mathrm{Mg}$ similar joints, and $\mathrm{Mg} / \mathrm{Al}$ and $\mathrm{Mg} / \mathrm{Steel}$ dissimilar joints.

- Determine the influence of the joining processes on the corrosion protection efficiency of the surface coatings. 


\section{Approach}

Magna initially identified three potential joining techniques for Mg-based similar and dissimilar joints. However, the corrosion behavior of such combinations of material systems and joint designs is unknown; hence, that is the focus of this work. The three initial joining techniques identified by Magna are: (1) Arplas RSW, (2) clinch lock and (3) breakaway stem rivet. Two types of proprietary coating-based corrosion protection schemes are being provided by Henkel and are labeled as Henkel pretreatment (HP) and Henkel HP + E-coated (HPEC). AZ31 has been selected as the representative Mg material.

Corrosion behavior of the joint coupons (i.e., similar/dissimilar) is determined according to American Society for Testing and Materials (ASTM) B117 standard salt (sodium chloride) spray (fog) test method. As per ASTM B117, corrosion coupons are subjected to a continuous exposure of $5 \%$ salt-fog at $35^{\circ} \mathrm{C}$ for a total duration of up to 1500 hours ( 60 days) at a pH of 6.5-7.2. During ASTM B117 testing, corrosion coupons are retrieved at regular intervals, gently washed under tap water, and immediately dried. Subsequently, the coupons are weighed to record their change in weight due to corrosion/corrosion product build-up.

The effectiveness of corrosion protection schemes (e.g., HP and HPEC, two different types of coating applied on an AZ31 alloy), and the effect of a corrosive environment on the mechanical strength of different joining techniques (e.g., Arplas RSW, clinch lock and breakaway stem rivet) are being evaluated in this study. Mechanical strength of $\mathrm{Mg} / \mathrm{Mg}$ similar joint coupons is determined through a RT standard lap-shear test. Joint coupons are pulled to failure in a uniaxial testing machine. A constant crosshead velocity of $1 \mathrm{~mm} / \mathrm{min}$ was maintained during testing. The effect of corrosion on joint strength has been determined by testing samples before and after corrosion chamber exposure. Post-mortem analysis of joint coupons using various microscopy techniques (e.g., stereo microscope and SEM) before and after corrosion testing provides valuable information about the nature of the protective coating, and its endurance behavior.

\section{Results}

\section{Mg AZ31 Similar Joint Fabrication}

In FY 2019, Mg/Mg (AZ31 alloy) similar joint coupons were tested using the ASTM B117 method. Mg/Mg similar lap joints were fabricated at the Magna facility and shipped to PNNL for corrosion testing. Lap joints were fabricated between $1.1 \mathrm{~mm}$ and $1.5 \mathrm{~mm}$-thick AZ31 sheets. Two types of joining methods were attempted: (1) Arplas RSW; and (2) breakaway stem rivet. For rivet joints, 3/16 in. all Al rivets were used. Additionally, two types of rivet hole configuration were evaluated: (1) rivet holes in deburred condition; and (2) rivet holes in non-deburred condition. In Arplas RSW joints, Mg sheets remained coated with HPEC. HPEC refers to a proprietary pretreatment by Henkel, topped by E-coating, a polymer-based organic coating. In the case of rivet joints, $\mathrm{Mg}$ sheets were coated with Henkel Alodine 5200, and topped by E-coating. Henkel Alodine 5200 is a common pretreatment method applied on Al, steel, etc. Through this study, we attempted to evaluate the effectiveness of a common pretreatment method in providing corrosion resistance in $\mathrm{Mg}$ alloys (AZ31) in comparison to the proprietary HP method. A joint fabrication study at Magna revealed that for a successful Arplas RSW in a lap configuration, the HP layer needs to be removed. The HP layer had to be removed from the top sheet on both sides, and on the mating side for the bottom sheet.

\section{Mg/Mg Similar Joint Corrosion Behavior}

Figure II.1.3.1 (a) shows the corrosion testing setup used for evaluating $\mathrm{Mg} / \mathrm{Mg}$ similar joint coupons. Each joint coupon measured 8.5 in. $\times 3$ in. and was vertically hung from two side bars as shown in Figure I.1.1.1 (b). The placement of test coupons in a vertical manner ensures that the front and back face of each joint is exposed to a similar environment inside the corrosion test chamber, minimizing sample placement configuration related artifacts. 


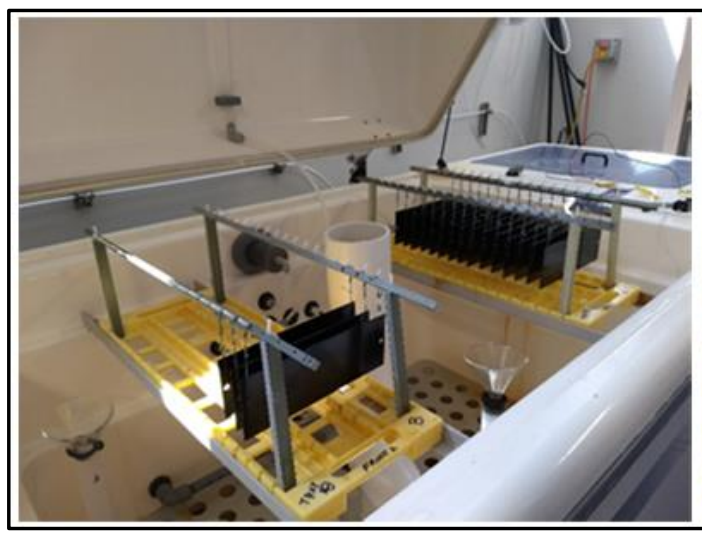

(a)

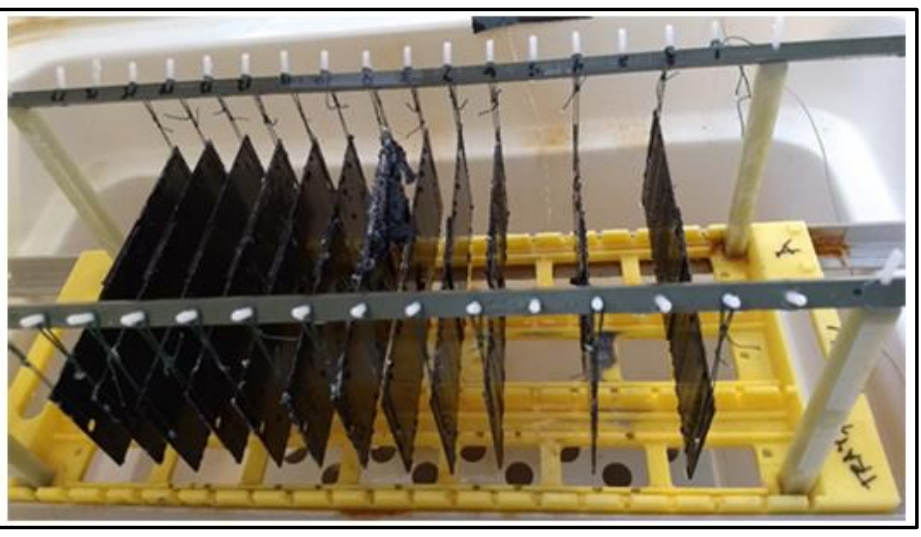

(b)

Figure II.1.3.1. (a) Corrosion test chamber for the ASTM B117 test where (b) the samples are vertically hung. Source: PNNL.

Figure II.1.3.2 shows the condition of joint coupons after $1500 \mathrm{~h}$ of continuous salt-fog testing. The condition of $\mathrm{Mg} / \mathrm{Mg}$ Arplas RSW joints is shown in Figure II.1.3.2 (a) and (b), capturing the appearance of the joint front and joint back sides, respectively. The appearance of the $\mathrm{Mg} / \mathrm{Mg}$ rivet joints (all $\mathrm{Al}$ rivets) after the corrosion tests is shown in Figure II.1.3.2 (c) and (d). The images of as-received joint coupons, without any corrosion testing, are also included to get an idea about the degree of the corrosion attack.

The formation of corrosion debris around the resistance spot weld mark could be noticed on the front face of the Arplas RSW joints, which is marked by a yellow circle as shown in Figure II.1.3.2 (a). In contrast, the back side of the same Arplas RSW joint does not show any such corrosion product build-up. In the case of rivet joints, an extensive corrosion attack could be observed on the front and back faces of the joints. In most of the rivet joint coupons, a portion of the top E-coat film peeled off during ASTM B117 testing, thus exposing the underlying metal surface. In summary, it appeared that the $\mathrm{Mg} / \mathrm{Mg}$ similar joints fabricated by the Arplas RSW method showed the least amount of surface corrosion in contrast to similar joints fabricated by the all $\mathrm{Al}$ rivet method. The relative change in the weight of corrosion coupons as a function of test duration also confirms that the Arplas RSW samples experience least amount of corrosion attack in comparison to rivet-based joint coupons, as shown in Figure II.1.3.3.

Visual examination of the tested joint coupons indicates that that the top E-coat does not have the required adherence with the underlying metal whenever the pretreatment is Alodine 5200-based. It could be the major reason behind higher degree of corrosion attack observed in $\mathrm{Mg} / \mathrm{Mg}$ rivet joints, since 5200 Alodine pretreatment was used there. Additionally, signs of corrosion attack could also be seen on the front face of Arplas RSW joints. For a successful Arplas RSW joint fabrication, HP layer removal on the front face of RSW joints had to be carried out. Absence of HP layer could be the reason behind observed corrosion attack in Arplas RSW joints. 


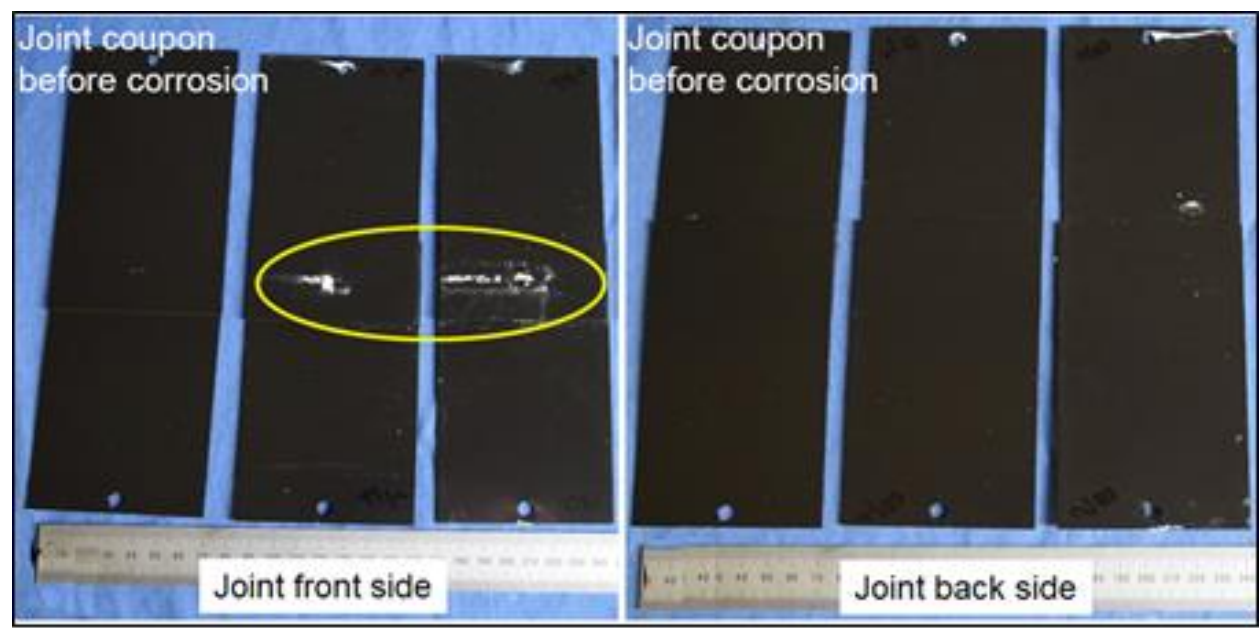

(a)

(b)

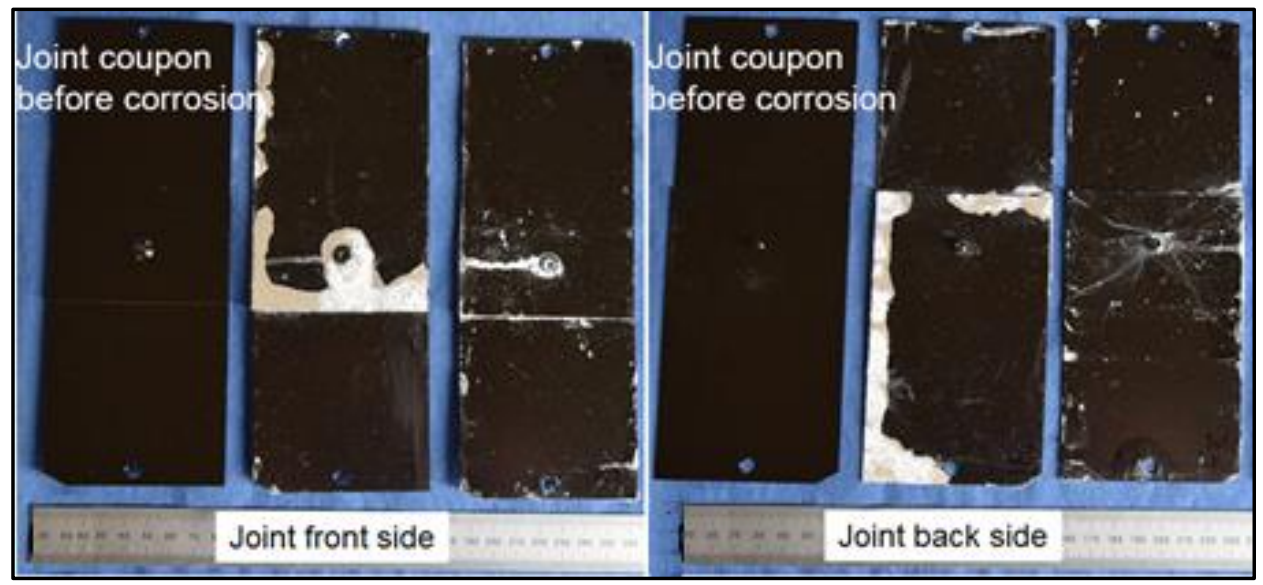

(c)

(d)

Figure II.1.3.2. The condition of joint coupons before and after $1500 \mathrm{~h}$ of ASTM B117 test: (a) Arplas RSW joint front side;

(b) Arplas RSW joint back side; (c) Al rivet joint front side; and (d) Al rivet joint back side. Source: PNNL.

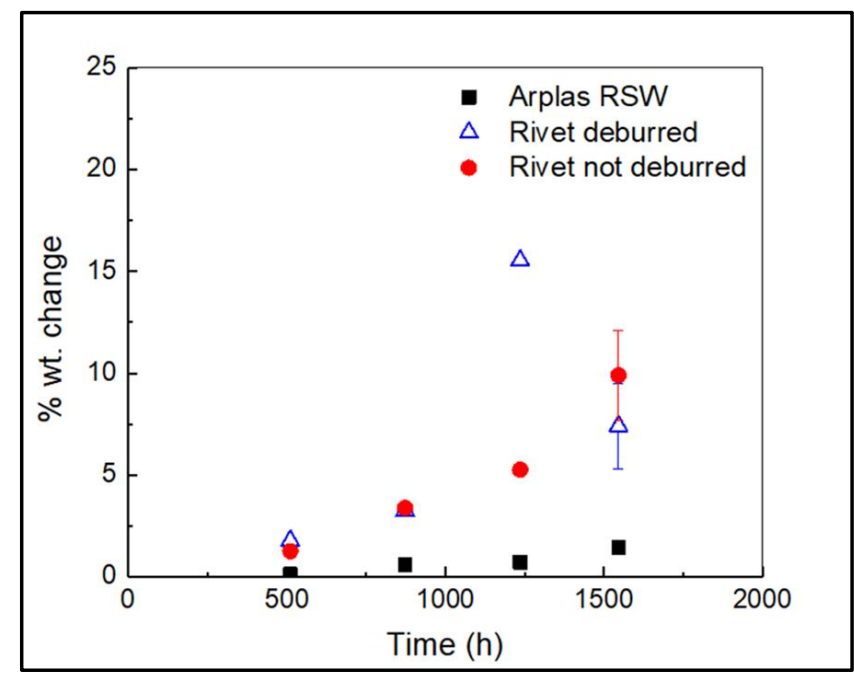

Figure II.1.3.3. Relative change in weight of corrosion coupons as a function test duration, ASTM B117 method, Arplas RSW goes through least amount of corrosion attack. Source: PNNL. 


\section{Mechanical Strength of $\mathrm{Mg} / \mathrm{Mg}$ Similar Joints}

RT mechanical strength of $\mathrm{Mg} / \mathrm{Mg}$ similar joints has been evaluated in our ongoing study. Lap-shear strength of the $\mathrm{Mg} / \mathrm{Mg}$ joints after $\sim 1500 \mathrm{~h}$ of corrosion test is summarized in Figure II.1.3.4. One joint sample each for the three different joint configurations currently investigated (e.g., Arplas RSW, Rivet with deburred hole, Rivet with non-deburred hole) were also tested in their as-received condition (i.e., without any exposure to the corrosion test chamber). Based on the limited lap-shear test data, it appears that the Arplas RSW joint has a higher maximum load-bearing capability $(\sim 125 \mathrm{kgf})$ than the rivet joints $(\sim 100 \mathrm{kgf})$, but with a lower ductility in its as-received condition, as shown in Figure II.1.3.4. However, exposure of the $\mathrm{Mg} / \mathrm{Mg}$ joints to a corrosion chamber environment has a detrimental effect on the Arplas RSW joint strength in comparison to the rivet joints. Maximum load-bearing capability of the two Arplas RSW joints after corrosion chamber exposure are $84 \mathrm{kgf}$ and $106 \mathrm{kgf}$, respectively, which is lower than in their as-received condition, $125 \mathrm{kgf}$, as seen in Figure II.1.3.4 (a). In comparison, lap-shear strength of rivet joints after corrosion testing is $\sim 100 \mathrm{kgf}$ for all of the four samples tested (e.g., two samples with rivet holes being deburred, and two samples with rivet holes being non-deburred), as observed in Figure II.1.3.4 (b) and Figure II.1.3.4 (c), which is the same as the rivet joint strength in the as-received condition.

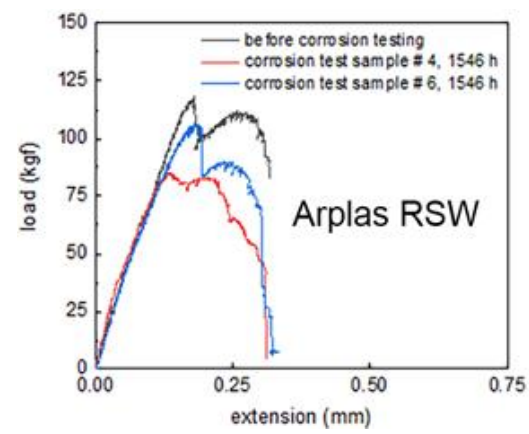

(a)

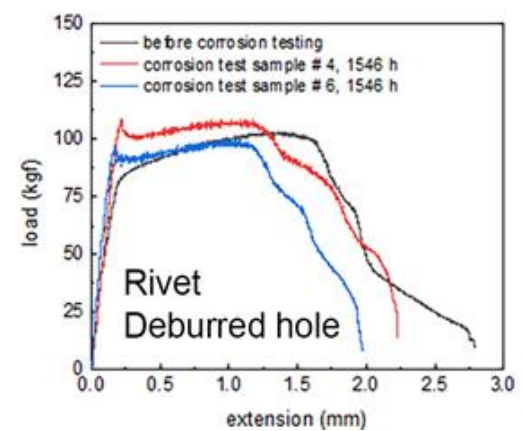

(b)

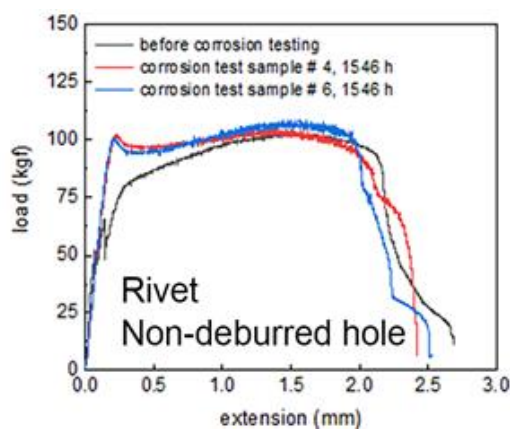

(c)

Figure II.1.3.4. RT lap-shear strength of Mg/Mg similar joints before and after exposure to corrosion test chamber (a) Arplas RSW; (b) rivet joint, deburred hole; and (c) rivet joint, non-deburred hole. Source: PNNL.

Since Arplas RSW involved spot welding, the joint interface will feature a solidified microstructure of parent AZ31 alloy. The chances of corrosion attack on a solidified Mg alloy is much higher, especially when the protective HP coating had to be removed for a successful joint fabrication. The formation of an inhomogeneous solidification microstructure at the joint interface together with the removal of a protective pretreatment layer could be the major reason behind localized corrosion and associated monotonic strength drop observed in Arplas RSW joints after the corrosion test. In comparison, rivet joints were fabricated with all $\mathrm{Al}$ rivets. Since $\mathrm{Al}$ is more noble than the parent $\mathrm{AZ} 31$ matrix, the galvanic corrosion would affect the surrounding AZ31 matrix and leave the Al rivets undamaged. However, the galvanic corrosion is highly influenced by the relative area of cathode (e.g., Al rivet) and anode (AZ31 matrix). The least amount of galvanic corrosion happens with a large anode to cathode area ratio, which is exactly the situation in the case of the rivet joints. As a result, we don't observe any strength reduction in rivet joints after corrosion testing. In summary, the rivet joints after corrosion testing maintain maximum load-bearing capability at par with its uncorroded counterpart. The effect of corrosion chamber exposure is shown by the Arplas RSW joints, in contrast. However, the overall surface appearance of the joints after $\sim 1500 \mathrm{~h}$ of test is exactly the reverse - the rivet joints showing increased surface degradation and higher weight gain in comparison to Arplas RSW joints, as observed previously in Figure II.1.3.2 and Figure II.1.3.3.

\section{Microstructural Characterization}

The joint interface of the corrosion coupons (after $\sim 1500 \mathrm{~h}$ test) followed by lap-shear testing has been studied using an optical stereo microscope. Lap-shear testing results in the separation of the top and bottom sheets of the lap joints, and, thus enables investigation of the joint interface. Figure II.1.3.5 is a compilation of low magnification stereo-micrographs obtained from the mating surface of the top sheet for the three different lap 
joints presently studied. The Arplas RSW joint interface, after $\sim 1500 \mathrm{~h}$ of corrosion testing, is shown in Figure II.1.3.5 (a). The resistance spot weld nugget, which shows some signs of corrosion and associated corrosion product build-up, is noticed at the center of the image. The adjoining AZ31 matrix away from the weld nugget shows some signs of corrosion as well (e.g., whitish product build-up, indicated by the arrows). In comparison, much heavier corrosion of the AZ31 matrix material is noted in the case of the rivet joints, as observed in Figure II.1.3.5 (b) for the rivet hole non-deburred and Figure II.1.3.5 (c) for the rivet hole deburred. Failed $\mathrm{Al}$ rivets could be seen at the center of both images and do not show any apparent signs of corrosion. Heavy corrosion of AZ31 matrix surrounding the Al rivets is due to galvanic coupling. Since AZ31 is electrochemically more active than Al, it suffers galvanic corrosion when $\mathrm{Al}$ rivets are used. Moreover, the lap-joint configuration itself leads to a crevice corrosion-type of attack. In summary, the microstructural observation indicates the corrosion attack mostly happening at the joint interface.

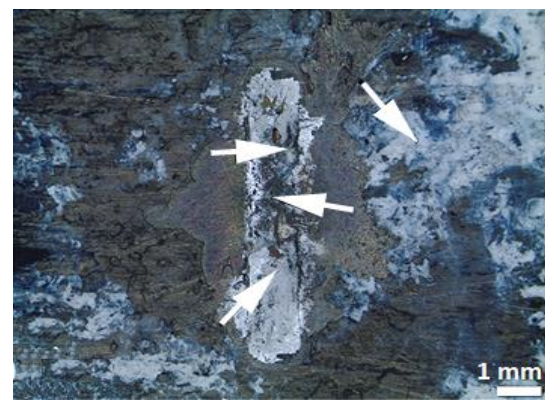

(a)

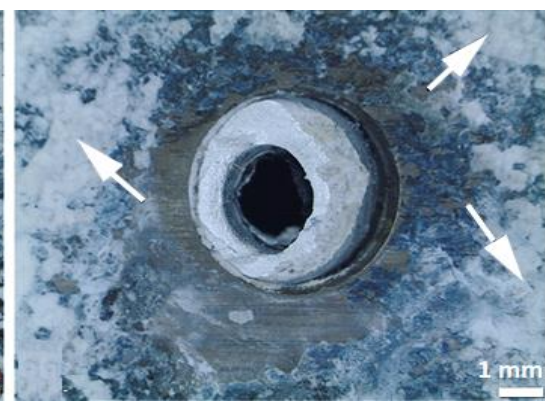

(b)

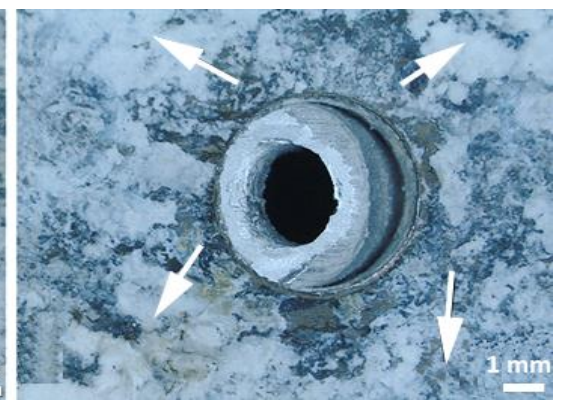

(c)

Figure II.1.3.5. Stereo micrograph of joint interface of corrosion coupons following a $1500 \mathrm{~h}$ exposure and subsequent lapshear test. The occurrence of corrosion is noted by the white arrows for: (a) the Arplas RSW; (b) the non-deburred hole rivet; and (c) the deburred hole rivet. Source: PNNL.

\section{Conclusions}

The evaluation of the corrosion behavior of AZ31 Mg/Mg similar joints has been completed in FY 2019. Two different joining techniques have been evaluated: (1) Arplas RSW; and (2) the Al rivet. ASTM B117 tests indicate a lower degree of corrosion attack in the case of the Arplas RSW joints in comparison to the rivet joints. However, the strength of Arplas RSW joints gets detrimentally affected by the exposure of joint samples to the corrosion chamber, which is attributed to the corrosion of resistance spot weld nuggets and the associated weakening effect. In contrast, $\mathrm{Mg} / \mathrm{Mg}$ rivet joints do not show any such weakening behavior after exposure to the corrosion test chamber since the $\mathrm{Al}$ rivets are more noble than the adjoining AZ31 matrix, and therefore, do not experience any galvanic corrosion. Additionally, the effectiveness of the Alodine 5200 pretreatment coating combined with the top E-coating towards corrosion prevention in $\mathrm{Mg}$ alloys has been investigated in the ongoing study. It appears that the Alodine $5200+$ E-coating combination does not protect underlying $\mathrm{Mg}$ matrix, in comparison to the $\mathrm{HP}+$ E-coating combination. In fact, the current study suggests that the HP is the most critical corrosion barrier layer for the Mg alloys.

\section{References}

1. U.S. Department of Energy, National Energy Technology Laboratory, 2015, "Demonstration Project for a Multi-Material Lightweight Prototype Vehicle as Part of the Clean Energy Dialogue with Canada," DOE Award \#DE-EE0005574. Available at: https://www.osti.gov/servlets/purl/1332277 (last accessed 4 December 2019).

\section{Acknowledgements}

Technical contributions of PNNL staff supporting this research is gratefully acknowledged; in particular, Dr. S. Jana for his role in leading the project tasks, Mr. M. Dahl for help with corrosion testing, Mr. N. Canfield for performing microstructural characterization, and Ms. E. Stephens for help with corrosion test procedures. 


\title{
II.1.4 Low-Cost Corrosion Protection Techniques for Magnesium Alloys (Pacific Northwest National Laboratory)
}

\author{
Aashish Rohatgi, Co-Principal Investigator \\ Pacific Northwest National Laboratory \\ 902 Battelle Blvd. \\ Richland, WA 99352 \\ E-mail: aashish.rohatgi@pnnl.gov
}

\section{Sarah Kleinbaum, DOE Technology Development Manager}

U.S. Department of Energy

E-mail: sarah.kleinbaum@ee.doe.gov

Start Date: December 1, 2018

Project Funding (FY19): \$350,000
End Date: December 31, 2019

DOE share: $\$ 350,000 \quad$ Non-DOE share: $\$ 0$

\section{Project Introduction}

Mg alloys, because of their excellent strength-to-weight ratio, have a high weight reduction potential, and are therefore attractive candidates for vehicle lightweighting and the resulting improved fuel efficiency and lowered greenhouse gas emissions. According to a report by U.S. Automotive Materials Partnership, greater use of $\mathrm{Mg}$ alloys (up to $350 \mathrm{lbs}$.) in place of steel and $\mathrm{Al}$ alloys per vehicle was forecast by 2020, leading to a $15 \%$ weight reduction [1]. However, the actual use of $\mathrm{Mg}$ alloys in the automotive sector is rather limited. Currently, 10-12 lbs. of Mg alloys are used as various components in an average North American vehicle [1]. Amongst other challenges, poor corrosion resistance of $\mathrm{Mg}$ alloys, especially in a chloride-containing environment, is one major reason behind its low penetration in the automotive sector. Corrosion mitigation strategies are, therefore, of very high importance to allow increased use of $\mathrm{Mg}$ alloys. Currently, several surface-coating technologies have been developed for $\mathrm{Mg}$ alloys to prevent/control corrosion. Some of the available coating methods are: (1) chemical conversion coatings; (2) anodizing; and (3) organic coatings. Powder coating and electrophoretic coating are the two most widely used organic coating technologies adopted in the automotive industry [2]. However, the use of hazardous chemical species, such as hexavalent chromium $\left(\mathrm{Cr}^{6+}\right)$, and other chemical baths during surface-coating of $\mathrm{Mg}$ alloys pose significant health and environmental risks. Therefore, development of non-chemical based surface treatment technologies for corrosion mitigation in Mg alloys could be a low-cost and environmentally benign alternative.

The present study investigates the effectiveness of a non-chemical bath-based surface processing technology in preventing aqueous-based environmental corrosion attack on an $\mathrm{Mg}$ alloy. The non-chemical surface processing technique involves the use of laser treatment called laser surface processing (LSP), which has been carried out on the AZ31 (Mg-3Al-1Zn) alloy to demonstrate its efficacy for corrosion prevention in a commercially available $\mathrm{Mg}$ alloy.

\section{Objectives}

Preliminary investigation during FY 2018 revealed a beneficial effect of LSP on the corrosion behavior of Mg alloys. Initial hypotheses to explain improved corrosion resistance due to LSP included microstructural modifications, such as reduction in grain size, reduced wettability of the surface, etc. Thus, the main objective of the present work was to identify the operative mechanism(s) of corrosion protection behavior exhibited by a surface-modified commercial AZ31B sheet. It is anticipated that an improved understanding of the protection mechanism(s) could help the development of low-cost corrosion protection strategies for Mg alloys, thus, overcoming the cost barrier to greater penetration of $\mathrm{Mg}$ alloys in the automotive market for lightweighting. 


\section{Approach}

In FY 2019, the experimental approach comprised LSP of a commercial AZ31 Mg sheet to demonstrate the "proof-of-concept" of this technology as an alternative non-chemical surface protection strategy for Mg alloys. The aim was to verify the improved corrosion resistance of the LSP through long-duration corrosion tests and to determine the differences in the surface and subsurface microstructures of LSP Mg relative to its nonsurface processed base metal (BM). Thus, the LSP and BM samples were subjected to standard corrosion testing and analytical characterization. In FY 2020, the project will develop and test hypotheses for the corrosion protection observed in the LSP Mg, and non-laser-based methods, which may enable a similar corrosion protection mechanism as LSP Mg to be explored. The research performed in FY 2019 is further detailed below.

In the present study, a commercially available $1 \mathrm{~mm}$-thick AZ31 Mg sheet alloy was processed (one sheet face only) using a neodymium-doped yttrium Al garnet - Nd:YAG - pulsed-laser with a spot size of $\sim 500$ microns.

LSP was performed at three different energies of $\sim 100, \sim 200$, and $\sim 300 \mathrm{~mJ}$ corresponding to a power of $\sim 1, \sim 2$ and $\sim 3 \mathrm{~W}$, respectively. Test coupons from the LSP sheets and one from the unprocessed BM sheared from their respective parent sheets and subjected to corrosion testing. Prior to corrosion testing, the non-LSP face (and sheared) edges of the LSP samples were coated with a black paint to eliminate any potential galvanic coupling between the LSP and non-LSP surfaces. The coupons were tested following ASTM B117 standard for a total of $1344 \mathrm{~h}$ (56 days) in an environmental chamber arranged as shown in Figure II.1.4.1 (a) and maintained at $35^{\circ} \mathrm{C}$ and in a salt-fog created by a $5 \mathrm{wt} . \%$ sodium chloride solution. During the testing, the coupons were periodically retrieved from the environmental chamber, dried and weighed to record the percent change in weight shown in Figure II.1.4.1 (b), and then returned to the environmental chamber to resume the corrosion test. Figure II.1.4.1 (c) provides images of the physical appearance of the BM and three different LSP coupons after 56 days of corrosion testing with the BM showing heavy corrosion and deposition of white colored corrosion products.

The as-processed LSP samples were examined using techniques including field emission SEM, TEM, glancing incidence angle XRD, x-ray photo-spectroscopy and secondary ion mass spectroscopy. The aim of the analytical characterization was to identify differences between the LSP and BM samples in their surface/subsurface microstructures with respect to grain size, chemistry, intermetallic phases, etc., and correlate these differences to the observed corrosion behavior. 

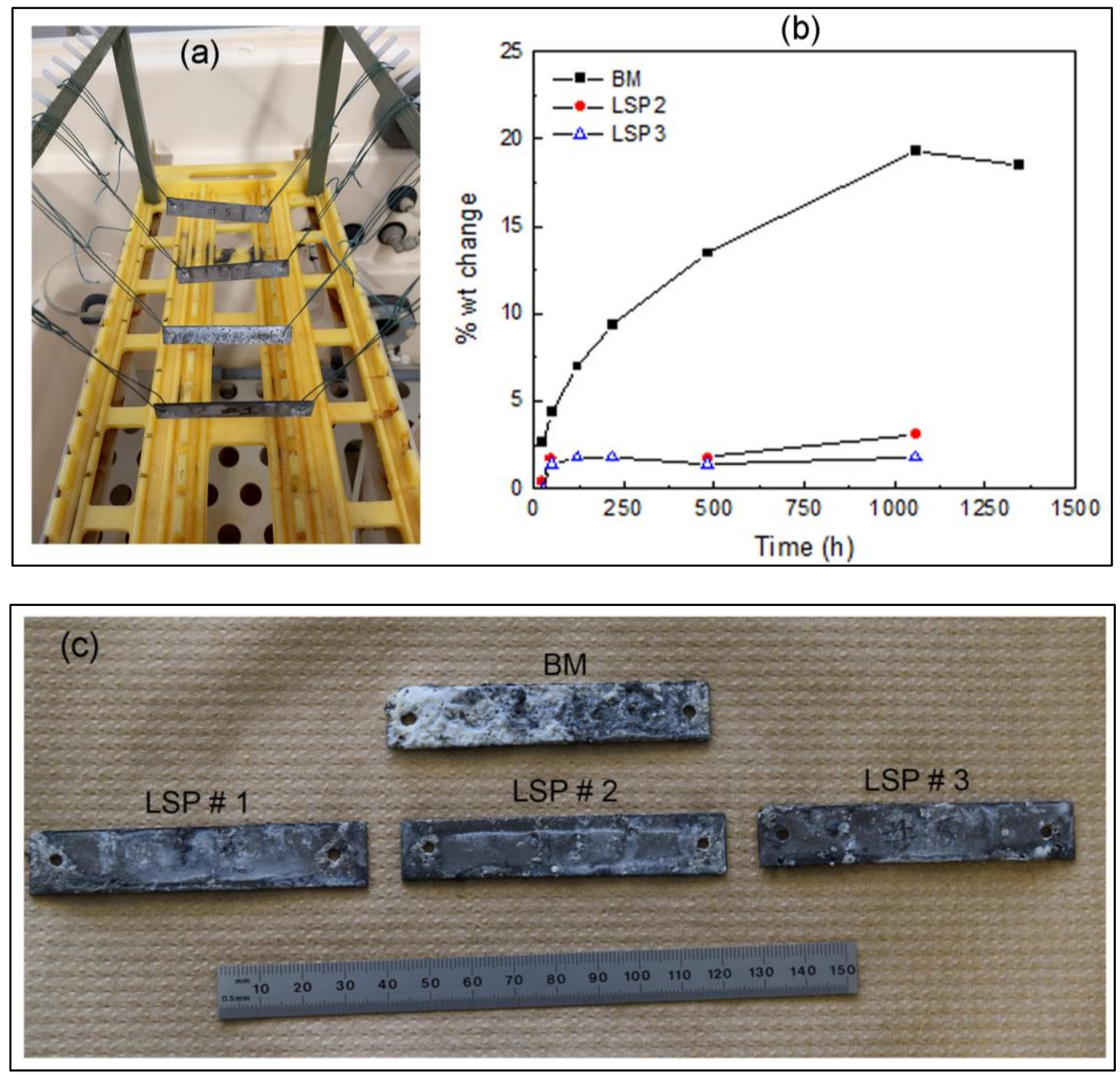

Figure II.1.4.1. (a) LSP and BM coupons hanging within the environmental test chamber for ASTM B117 test; (b) \% change in weight of LSP vs BM coupons as a function of test duration and (c) physical appearance of BM and three different LSP coupons after 56 days of corrosion testing with the BM showing heavy corrosion and deposition of white colored corrosion products. Source: PNNL.

\section{Results}

The results of the corrosion tests and analytical characterization of LSP and BM AZ31 are described in detail in the sections that follow.

Following the LSP treatment, the AZ31 sheet surface appearance remained uniform and showed a whitish color. In contrast, the unprocessed AZ31 BM had a much darker surface appearance that is generally seen in $\mathrm{Mg}$ alloy sheets due to natural oxidation. The relative weight change of corrosion coupons as a function of test duration is shown in Figure II.1.4.1 (b). Beneficial effect of LSP treatment for improved corrosion resistance in AZ31 is clearly evident in Figure II.1.4.1. The LSP coupons show a very slight initial increase in weight ( 2-3\%) after which there is no further weight gain. However, the non-LSP BM coupon shows a continuously increasing weight leading to an order-of-magnitude larger weight gain ( 20\%) by the end of 56 days of testing. As seen in Figure II.1.4.1 (c), the significant weight gain in the BM coupon can be attributed to the build-up of corrosion products while the surface appearance of the LSP coupons under identical corrosion testing as BM shows minimal corrosion and was very similar to each other. 
Backscattered electron imaging at a low accelerating voltage was employed to analyze the LSP surface. Figure II.1.4.2 (a) shows that the top surface of the LSP-treated AZ31 sheet has a rough, undulating surface while that of the non-LSP BM, shown in Figure II.1.4.2 (b), has a typical, relatively smoother, appearance of a rolled sheet. A cross-section SEM image of an LSP sample in the through-thickness direction is shown in Figure II.1.4.2 (c) and is characterized by the presence of a continuous $\sim 300-400 \mathrm{~nm}$ thick layer along the LSP-treated surface as shown by the red arrows. The undulating nature of the LSP surface is evident from the presence of multiple peaks and valleys, unlike the non-LSP BM surface seen in Figure II.1.4.2 (d) that is flatter in comparison and clearly devoid of any distinct layer. Additionally, bright, second-phase particles can also be noticed in the subsurface region of both the LSP and BM samples in Figure II.1.4.2 (c) and (d), respectively. Preliminary EDS analysis indicates these bright particles to be an Al-Mn rich intermetallic (IM) phase. However, the number density and size of the Al-Mn IM particles appear to be higher in the non-LSP BM sample than in the LSP sample.
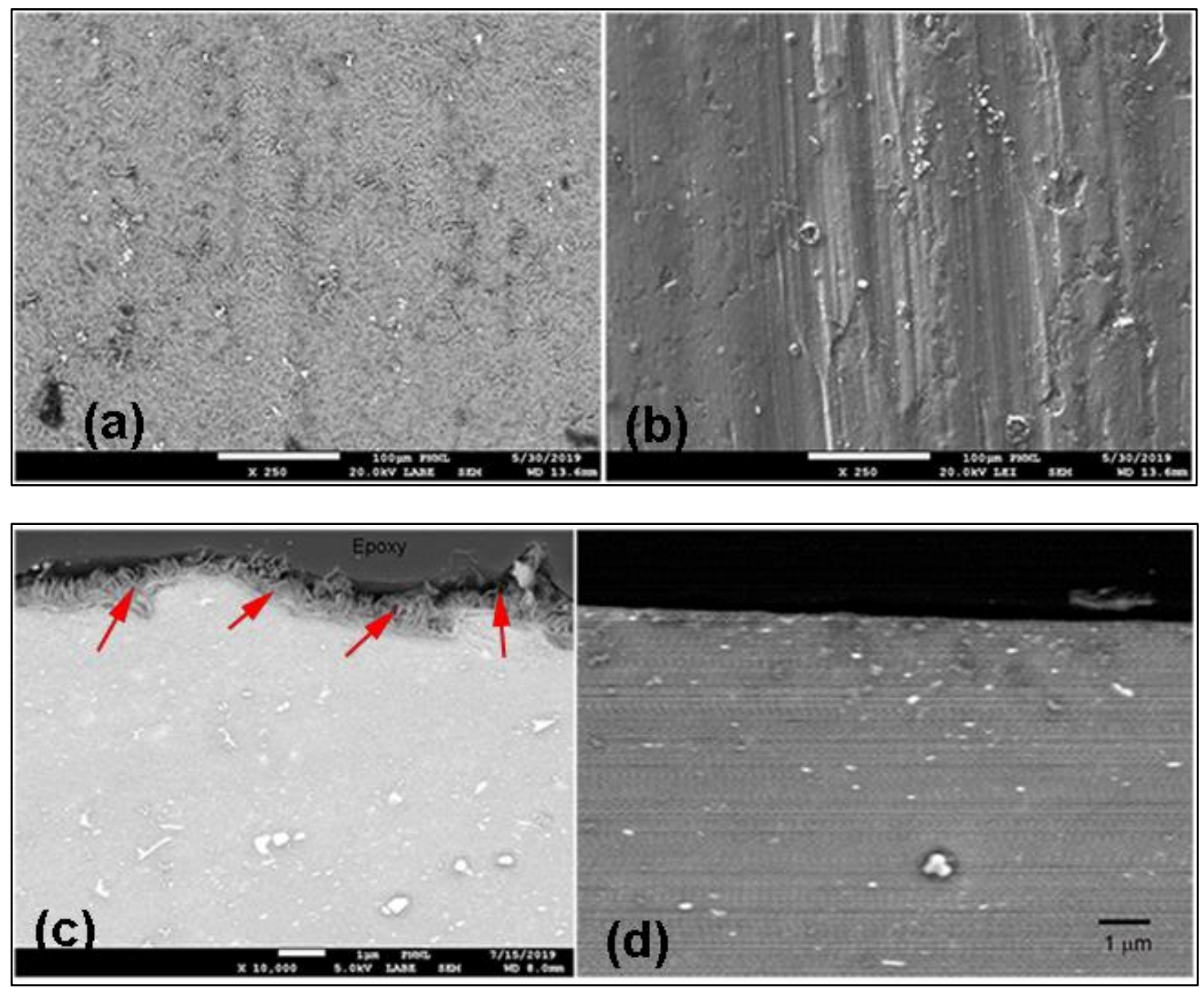

Figure II.1.4.2. SEM images showing the plan view of (a) the LSP surface and (b) the non-LSP BM surface. Through-thickness backscattered electron images of (c) the LSP sample showing the presence of a continuous layer indicated by red arrows and (d) the non-LSP sample showing the absence of any distinct surface layer. Source: PNNL.

Further information about the surface layer present along the LSP-treated side was obtained by preparing a sample in the sheet through-thickness direction by FIB milling technique and examining it in TEM.

Figure II.1.4.3 (a) shows the FIB image of the sample, while Figure II.1.4.3 (b) and Figure II.1.4.3 (c) show its corresponding bright field (BF) image and HAADF image, respectively. Presence of the continuous 300-400 $\mathrm{nm}$ thick surface layer on the LSP sample, as seen in the SEM image in Figure II.1.4.2 (c), was also confirmed from the BF TEM image, as indicated by the black arrows shown in Figure II.1.4.3 (b). Additionally, this 
surface layer is comprised of densely packed crystal-like features with conical morphology that appear to be strongly adherent to the underlying metal-matrix. Presence of a few submicron-sized second-phase particles with a dark contrast could also be observed in the BF image, as observed in Figure II.1.4.3 (b). It can clearly be seen that the darker contrast second-phase particles in Figure II.1.4.3 (b) appear brighter in the HAADF image in Figure II.1.4.3 (c). In the HAADF imaging mode, any phase/feature with a higher atomic number shows brighter contrast. AZ31, which contains 3 wt. $\% \mathrm{Al}$ and $\sim 0.2 \mathrm{wt} . \% \mathrm{Mn}$, is known to form Al-Mn-rich IM phase and was also observed in the SEM images seen in Figure II.1.4.3 (c). Since the Al-Mn IM phase has a higher Z value relative to an Mg matrix, it shows brighter contrast in the HAADF image seen in Figure II.1.4.3 (c).

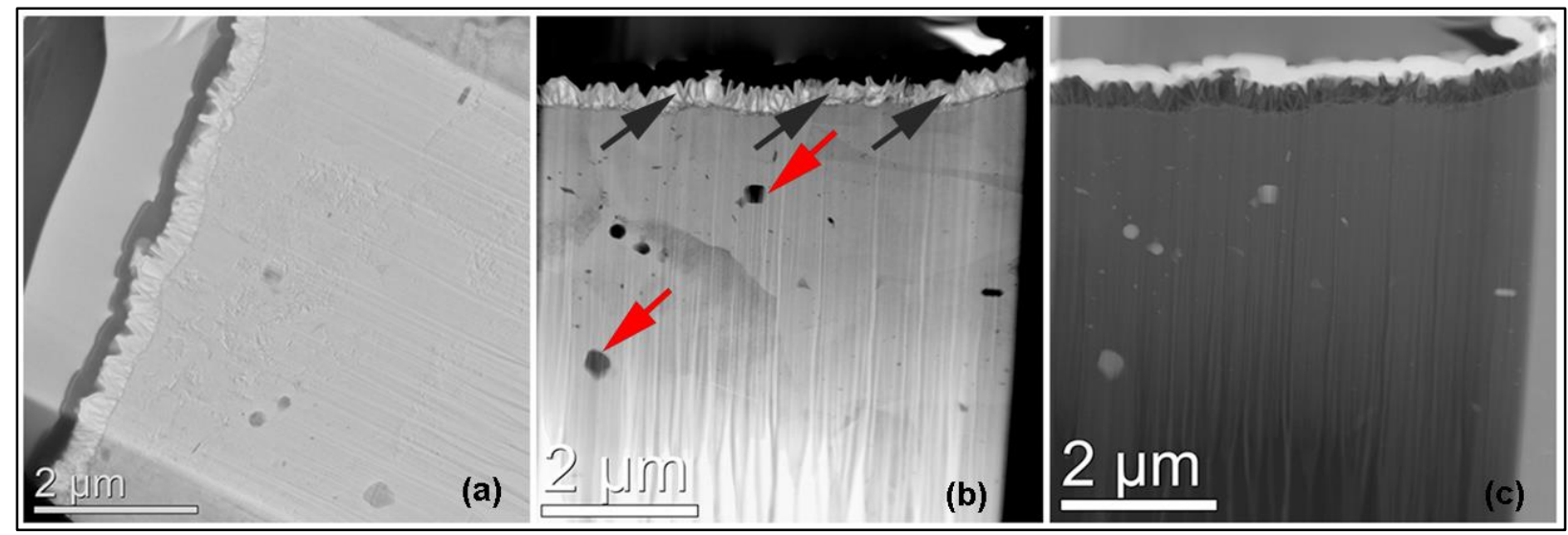

Figure II.1.4.3. (a) Through-thickness FIB image of LSP-treated AZ31 sample. (b) BF image from LSP-treated sample showing the top surface layer at black arrows and second-phase particles indicated by red arrows. (c) HAADF image from LSP sample, bright particles are an AI-Mn rich IM phase. Source: PNNL.

Chemical information about the surface film and various second particles were obtained by carrying out EDS analysis. Figure II.1.4.4 (a) shows a HAADF TEM image of the region of interest in the LSP-treated sample where the EDS analysis was performed. Specifically, the elemental concentration profile was obtained from the yellow arrow region, starting from the bottom of the image and ending at top of the image, as seen in Figure II.1.4.4 (a). The corresponding elemental concentration profile is shown in Figure II.1.4.4 (b), where it can be clearly seen that the big second-phase particle with the bright contrast is rich in $\mathrm{Mn}$ and Al, but lacks Mg. Thus, the EDS analysis further confirms the bright second-phase particles to be an Al-Mn IM phase. In addition, the surface film is noted to contain a significantly high-level of oxygen and $\mathrm{Mg}$. To get more information about the chemical composition of the surface film, a higher magnification HAADF image was captured, which is shown in Figure II.1.4.4 (c), and subsequently the EDS line profile analysis was completed along the yellow highlighted arrow region. The corresponding elemental concentration profile in Figure II.1.4.4 (d) shows that the film is indeed oxygen-rich. Additionally, the slight enrichment of $\mathrm{Al}$ is noted at the interface of the top surface film and the underlying AZ31 matrix. Thus, based on our EDS analysis, we believe LSP treatment results in some type of Mg oxide film on the surface, together with some type of $\mathrm{Al}$ oxide rich layer formation at the surface film/AZ31 matrix interface. $\mathrm{Mg}$ rich oxide film has a thickness of $\sim 300-400 \mathrm{~nm}$, while Al-rich oxide layer measures 20-30 nm. Improved corrosion resistance after LSP treatment is mainly associated with the formation of a mixed metal $(\mathrm{Mg}, \mathrm{Al})$-oxide surface film. 


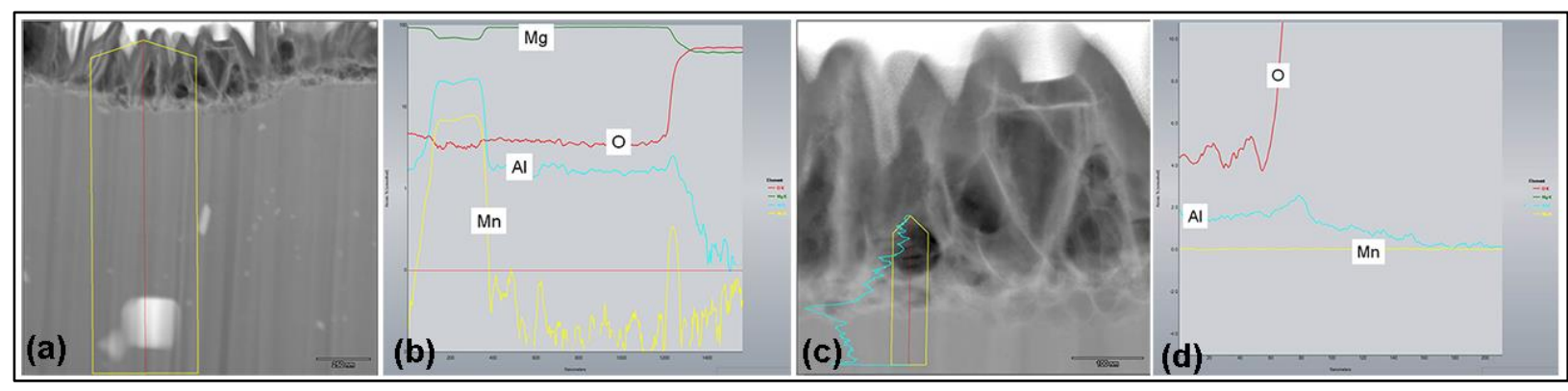

Figure II.1.4.4. (a) HAADF image of LSP-treated AZ31 sample showing the surface film and subsurface AZ31 matrix.

(b) Elemental concertation profile from the yellow arrow region shown in (a). (c) Higher magnification HAADF image of top surface film and adjoining AZ31 matrix. (d) Elemental concentration profile from the yellow arrow region shown in (c).

Source: PNNL.

\section{Conclusions}

A non-chemical laser-based surface processing technique has been employed on a $1 \mathrm{~mm}$-thick AZ31 alloy sheet. ASTM B177 testing indicates enhanced corrosion resistance in LSP-treated samples. The rate of corrosion attack is at least an order-of-magnitude lower in the case of the LSP samples in comparison to the non-LSP BM. Microstructural characterization using SEM and TEM technique reveals the presence of a continuous 300-400 nm thick surface film on the LSP-treated side. Chemical composition of the surface film, determined through the EDS technique, shows the presence of $\mathrm{Mg}$, Al, and O. Based on the EDS analysis, the surface film is believed to be a mixed metal $(\mathrm{Mg}, \mathrm{Al})$-oxide. Future work is directed at learning structural information about the surface film and determining the feasibility of developing a non-laser-based technique for a similar film formation.

\section{Key Publications}

1. Rohatgi, A., 2020, abstract submitted and accepted to TMS 2020, Coatings and Surface Engineering for Environmental Protection II, 23-27 February 2020, San Diego, CA, USA.

\section{References}

1. USCAR, “Mg vision 2020," 2006, Available at:

http://www.uscar.org/commands/files_download.php?files_id=240 (last accessed 4 December 2019).

2. Wang, J., X. Pang, and H. Jahed, 2019, "Surface protection of Mg alloys in automotive applications: A review," Mater. Sci., Vol. 6, No. 4, pp. 567-600.

\section{Acknowledgements}

The technical contribution of PNNL staff for performing this research is gratefully acknowledged. We also recognize Mr. R. Seffens for carrying out the corrosion test, Mr. A. Schemer-kohrn for the SEM imaging, and Dr. D. Edwards for the TEM imaging. 


\title{
II.1.5 Reducing Mass of Steel Auto Bodies Using Thin Advanced High-Strength Steels with Carbon-Reinforced Epoxy Coating (Idaho National Laboratory)
}

\author{
Gabriel Ilevbare, Co-Principal Investigator \\ Idaho National Laboratory \\ 2351 N. Boulevard St. \\ Idaho Falls, ID 83401 \\ E-mail: gabriel.ilevbare@inl.gov

\section{David Warren, Co-Principal Investigator} \\ Oak Ridge National Laboratory \\ 1 Bethel Valley Rd. \\ Oak Ridge, TN 37831 \\ E-mail: warrencd@ornl.gov
}

\section{Sarah Kleinbaum, DOE Technology Development Manager}

\section{U.S. Department of Energy}

E-mail: sarah.kleinbaum@ee.doe.gov

Start Date: April 1, 2018

Project Funding (FY19): \$639,000
End Date: March 31, 2020

DOE share: $\$ 300,000$
Non-DOE share: $\$ 339,000$

\section{Project Introduction}

Diversitak, a company based in Detroit, MI, has developed a proprietary, low specific gravity, carbon fiber reinforced epoxy (CFRE). Preliminary testing on this material conducted in collaboration with ArcelorMittal proved out the concept. A thin layer of a CFRE was applied to a stamped sheet of steel with residual stamping oils from a mill, in a time corresponding to automotive processing ( 15 secs $)$ and processed following automotive e-coat procedures (phosphating $+175-200^{\circ} \mathrm{C}$ heating), to complete the curing. No problems with adherence or performance were noted. While the CFRE does add weight to a thin gauge steel panel, it weighs much less than what is displaced by using thicker conventional mild steel gauges. The application of the coating showed a significant increased dent resistance, oil canning resistance, and part stiffness.

This project is designed to mature this new technology to near manufacturing readiness to reduce the weight of a vehicle and lower the cost of weight reduction. The process involves the use of thinner gauge steels than are currently used, stiffened by CFRE application on one side. The collaborative development team includes two industrial manufacturers, Diversitak and ArcelorMittal; LightMAT; and two National Laboratories, ORNL and Idaho National Laboratory (INL). The team is developing a new manufacturing process to reduce the weight of a vehicle and lower the cost of weight reduction. This team is also developing an understanding of how to apply the coating and how it will perform in service, as well as performing an in-depth study to determine the long-term durability of the materials manufactured using this technology.

\section{Objectives}

Recent steel industry advances have led to the development of AHSS, which are thinner gauge, and thus lighter, than conventional sheet steels, while supporting vehicle structural functions. Thinner gauge steel panels can potentially have lower in-service performance (due to reduction in structural stiffness), which may limit their application in auto body designs. Such limitations may include an increased potential for denting, vibration at high vehicle speeds, and "oil canning" (instability with a loud noise on application of load). Conventional autobody sheet-steel averages $1.1 \mathrm{~mm}$-thick, ranging from $0.65 \mathrm{~mm}$ to $2.5 \mathrm{~mm}$ depending on the location and application, but typical exterior panels are often $0.75 \mathrm{~mm}$-thick. Reducing total body panel thickness from $0.75 \mathrm{~mm}$ to $0.55 \mathrm{~mm}$ could reduce body mass from $130 \mathrm{~kg}$ to $95 \mathrm{~kg}(27 \%)$ [1]. 
The goal of this work is to develop CFRE technology so that AHSS body panels may be reduced in thickness from $0.75 \mathrm{~mm}$ to $0.55 \mathrm{~mm}$ to reduce component mass without sacrificing structural performance. To accomplish this goal, the optimal reinforcement fiber length and fiber concentration must first be determined. Following that phase, the CTE in all three directions must be determined to feed into manufacturing models and methods for rapidly and inexpensively applying the coating must be determined. This is being followed by panel level evaluations of the coating and steel combination and then followed by a full-part demonstration of the technology on door panels. The final step will conclude with corrosion testing of the parts.

\section{Approach}

This is a two-year effort. ORNL's tasks include optimization and selection of the best CFRE material. This includes a determination of the optimal fiber length, optimal fiber concentration, and a determination of the optimal coating thickness for best vehicle function and performance at the least cost. ORNL, along with the suppliers, developed a durable CFRE application process (e.g., gun material, design, robotic dispensing process) and identified the adhesion stability of the CFRE during process holding. An approach to ensure that application/curing timing conforms to conventional assembly line speed and plant cycle times is being determined. ORNL also determined the CTE of the material in all three directions and performed all material scanning electron microscopic analysis.

INL performed characterization of corrosion properties of steel panels coated with the optimized CFRE. The corrosion tests include coupons and body panels. Diversitak produced different epoxy formulations and determined the physical and chemical properties that are critical to mixing with CFs and produced a stable coating that can be cured in the time range to support automotive assembly processing ( 10-15 secs). They also mixed different amounts and types of $\mathrm{CF}$ with a range of epoxy formulations and measured the cured coating properties. ArcelorMittal also characterized several AHSS attributes (i.e., metallurgy heat-treatment for required AHSS properties versus sheet thickness, state of internal stress, and adhesion of the CFRE to the steel as a function of sheet rolling and stamping preparation).

\section{Results}

\section{Mass and Cost Comparison}

As the program progressed, the standard material being used by industry was still bake-hardenable steels, but they are now commonly used in the $0.70 \mathrm{~mm}$ and $0.65 \mathrm{~mm}$ thicknesses, so the focus of this work has now included a relative comparison to these materials used in industry. A baseline material thickness of $0.70 \mathrm{~mm}$ was used for this study and a revised bending stiffness test was conducted on the final material of interest in the final dimensions with the resulting flexural loads shown in Table II.1.5.1. Based on these tests, the $0.50 \mathrm{~mm}$ coated samples had a greater bending stiffness ( $36 \mathrm{~N}$ emphasized in red) than the $0.65 \mathrm{~mm}$ and $0.70 \mathrm{~mm}$ uncoated materials, and will, therefore, be used for the cost and mass comparison with other data presented for comparison purposes.

Table II.1.5.1. Flexural Load in Newtons (N) of DP500 with the CFRE Coating Applied with Varying Thicknesses of CFRE and Steel.

\begin{tabular}{|c|c|c|c|c|c|c|}
\hline & \multicolumn{5}{|c|}{ Steel Thickness } \\
\hline & & $0.50 \mathrm{~mm}$ & $0.55 \mathrm{~mm}$ & $0.60 \mathrm{~mm}$ & $0.65 \mathrm{~mm}$ & $0.70 \mathrm{~mm}$ \\
\hline \multirow{4}{*}{ 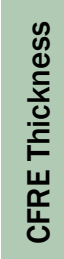 } & $0 \mathrm{~mm}$ & $22 \mathrm{~N}$ & $29 N$ & $32 \mathrm{~N}$ & $32 \mathrm{~N}$ & $35 N$ \\
\hline & $0.5 \mathrm{~mm}$ & $36 \mathrm{~N}$ & $48 N$ & $59 \mathrm{~N}$ & $\mathrm{~N} / \mathrm{A}$ & N/A \\
\hline & $1.0 \mathrm{~mm}$ & $50 \mathrm{~N}$ & $68 \mathrm{~N}$ & $79 \mathrm{~N}$ & N/A & N/A \\
\hline & $2.0 \mathrm{~mm}$ & $82 \mathrm{~N}$ & $104 \mathrm{~N}$ & $117 \mathrm{~N}$ & N/A & N/A \\
\hline
\end{tabular}


The steel had a density of $7.87 \mathrm{~g} / \mathrm{cm}^{3}$. While the door sizes vary greatly from car model to car model, the average door size is $1.0 \mathrm{~m}^{2}$, so that is the assumed door size for this study. The coating is an after-manufacture process, so manufacturing of the door is unchanged with or without the coating. Therefore, the steel manufacturing cost is not factored into this study. The CFRE had a density of $0.9 \mathrm{~g} / \mathrm{cm}^{3}$ and once-mixed costs $\$ 70 / \mathrm{gal}$. The dispensing equipment costs $\$ 150 /$ line. For applications where patches are needed, $200 \mathrm{~cm}^{2}$ of total area is required, so the per unit volume is calculated as $10 \mathrm{~cm} \times 20 \mathrm{~cm} \times$ CFRE thickness. Analysis has shown that $200 \mathrm{~cm}^{2}$ is the average necessary size of a "patch" for a door. The specific area will vary slightly depending upon the vehicle model and door geometry. For total coverage applications, the $1.0 \mathrm{~m}^{2}$ times the CFRE thickness was used as the volume. The cost and mass reduction of $20.3 \%$ weight saved and $\$ 8.11$ per kilogram of weight saved was calculated for full coverage of the area as shown in Table II.1.5.2. Values in red are for emphasizing the baseline values and the final cost and mass reduction values.

Table II.1.5.2. Full Coverage of DP490 Steel with 0.50 mm-thick CFRE with Mass and the Cost of Steel and CFRE Compared with the Baseline Materials of $0.70 \mathrm{~mm}$ Uncoated Steel.

\begin{tabular}{|c|c|c|c|c|c|c|c|c|}
\hline \multicolumn{3}{|c|}{ DP490 Steel } & \multicolumn{3}{|c|}{ CFRE } & \multicolumn{3}{|c|}{ Summary } \\
\hline Thickness & $\begin{array}{l}\text { Vol. } \\
\left(\mathrm{cm}^{3}\right)\end{array}$ & Wt (g) & $\begin{array}{l}\text { Vol. } \\
\left(\mathrm{cm}^{3}\right)\end{array}$ & Wt (g) & $\begin{array}{l}\text { Unit Cost } \\
\text { (\$) }\end{array}$ & $\begin{array}{l}\text { Final Wt } \\
\text { (g) }\end{array}$ & $\begin{array}{l}\% \mathrm{Wt} \\
\text { Saved }\end{array}$ & $\begin{array}{c}\text { Cost/Kg } \\
\text { Weight Saved }\end{array}$ \\
\hline $0.70 \mathrm{~mm}$ & 700 & 5460 & 0 & 0 & 0 & 5460 & Base & Base \\
\hline $0.65 \mathrm{~mm}$ & 650 & 5070 & 500 & 450 & 9.0 & 5520 & & $\mathrm{~N} / \mathrm{A}$ \\
\hline $0.60 \mathrm{~mm}$ & 600 & 4680 & 500 & 450 & 9.0 & 5130 & & $\$ 27.27$ \\
\hline $0.55 \mathrm{~mm}$ & 550 & 4290 & 500 & 450 & 9.0 & 4740 & & $\$ 12.50$ \\
\hline $0.50 \mathrm{~mm}$ & 500 & 3900 & 500 & 450 & 9.0 & 4350 & 20.3 & $\$ 8.11$ \\
\hline
\end{tabular}

\section{Environmental Testing}

Results reported previously included the determination of the optimum CF length and content for CFRE, optimum CFRE coating thickness for application on AHSS, flexural strength measurements, and accrued improvements in performance as a result of CFRE coating application, including dent testing, CTE testing, and an evaluation of the results on CFRE coating and a comparison with AHSS [2]. FY 2019 studies concentrated on the corrosion testing results only.

\section{Test Procedures}

INL tested 550 CFRE-coated coupons to three separate corrosion test protocols: (1) SAE J2334 Laboratory Cyclic Corrosion Test (April 2016), (2) Ford Laboratory Test Method (FLTM) BQ 104-07 Environmental Test Cycles, and (3) FLTM BQ 104-02 Humidity Resistance Testing. All salt application regimens were carried out in a Q-Fog Salt-Fog Chamber Model Cyclic Corrosion Testing 1100. All controlled relative humidity (RH) regimens below 100\% and all dry off regimens were carried out in a Thermotron SE-2000 environmental chamber.

SAE J2334, Laboratory Cyclic Corrosion Test (April 2016)

This test comprised a humid cycle $\left(50^{\circ} \mathrm{C}\right.$ at $100 \% \mathrm{RH}$ for $\left.6 \mathrm{~h}\right)$, a salt-fog application cycle $(0.25 \mathrm{~h})$, and a dry off cycle $\left(60^{\circ} \mathrm{C}\right.$ at $\left.50 \% \mathrm{RH}, 17.75 \mathrm{~h}\right)$ for a total of $24 \mathrm{~h}$ comprising one test cycle. The fog atomization was adjusted to generate a condensate collection rate of $2 \mathrm{~mL} / \mathrm{h}$ to $4 \mathrm{~mL} / \mathrm{h}$, as stipulated in SAE J2334, with the fog collection defined in ASTM D1735-14, "Standard Practice for Testing Water Resistance of Coatings using Water Fog Apparatus." The testing solution was $0.5 \% \mathrm{NaCl}, 0.1 \% \mathrm{CaCl}_{2}$, and $0.075 \% \mathrm{NaHCO}_{3}$. The first two cycles were conducted in a Q-Fog Salt-Fog Chamber, while the final dry off cycle was conducted in a humidity chamber where the humidity was generated using deionized water. 
FLTM BQ 104-02, Humidity Resistance Testing

This test comprised a constant humidity of $95 \%$ at $38^{\circ} \mathrm{C}$ for $24 \mathrm{~h}$.

FLTM BQ 104-07, Environmental Test Cycles (Procedure 11)

This test comprised a $24 \mathrm{~h}$, six-step freeze-thaw cycle in a Thermotron SE-1000 environmental chamber, as follows:

- Step 1: $3 \mathrm{~h}$ dwell time at $90^{\circ} \mathrm{C}$.

- Step 2: $2 \mathrm{~h}$ ramp down to $-40^{\circ} \mathrm{C}$.

- Step 3: $3 \mathrm{~h}$ dwell time at $-40^{\circ} \mathrm{C}$.

- Step 4: $1 \mathrm{~h}$ ramp up to $38^{\circ} \mathrm{C}$ and $95 \% \mathrm{RH}$.

- Step 5: $14 \mathrm{~h}$ dwell time at $38^{\circ} \mathrm{C}$ and $95 \% \mathrm{RH}$.

- Step 6: $1 \mathrm{~h}$ ramp up to $90^{\circ} \mathrm{C}$.

Three different types of coupons were tested: (1) lap-shear test coupons, (2) flexural strength test coupons and (3) corrosion evaluation test (CET) coupons. Samples of these coupons can be seen in Figure II.1.5.1. All coupons were made from AHSS DP500 coated with CFRE prior to being evaluated. Two steel thicknesses were tested $-0.6 \mathrm{~mm}$ and $0.5 \mathrm{~mm}$. Figure II.1.5.1 (a) shows the CFRE bonded lap-shear tensile test coupons that were 1 in. $\times 4$ in. strips with a 0.5 in. overlap and subjected to the SAE J2334 test only. Figure II.1.5.1 (b) shows the CFRE-coated flexural strength test coupons that were 1 in. $\times 6$ in. strips and subjected to the SAE J2334, FLTM BQ 104-02, and FLTM BQ 104-07 tests. Figure II.1.5.1 (c) shows the CFRE-coated CET coupon that is a 4 in. $\times 12$ in. plate and was subjected to the SAE J2334, FLTM BQ 104-02, and FLTM BQ 104-07 tests. Note that the scribe line in the middle of the CET coupon which is an optional coupon as prescribed by SAE J2334 to evaluate delamination. All the coupons were tested in the $0.5 \mathrm{~mm}$ and $0.6 \mathrm{~mm}$ thickness range at $4,8,20,30$, and 40 cycles. The CFRE coating thickness on the flexural and CET coupons was $0.5 \mathrm{~mm}$.

The lap-shear tensile coupons were tested for CFRE coating adhesion (to the metal) along the plane of adhesion after the SAE J2334 test. One hundred specimens were tested in total -50 at $0.5 \mathrm{~mm}$ and 50 at $0.6 \mathrm{~mm}$ AHSS thicknesses. The flexural strength coupons experienced the highest stress within the coating at the point of yield after all three tests. Three hundred specimens were tested in total-100 each for every test (J2334, humidity and environmental tests) and split evenly between the $0.5 \mathrm{~mm}$ and $0.6 \mathrm{~mm}$ AHSS thicknesses (e.g., 50 for each). The CET coupons were used to evaluate corrosion resistance of the CFRE coating. Test procedure SAE J2334 called for an optional scribe line through the CFRE coating on the CET coupons to evaluate the scribe creepback or delamination along the scribe line. As previously stated, the scribe is a controlled simulated damage site designed to represent a scratch or defect in the coating. The coupons for the two Ford test procedures did not have a scribe line machined into them. One hundred and fifty specimens were tested in total -50 each for each of the three test procedures and split evenly between the $0.5 \mathrm{~mm}$ and $0.6 \mathrm{~mm}$ AHSS thicknesses.

Coating adhesion in the lap-shear test results are presented in Figure II.1.5.2 (a) and the tensile strength decreased with the number of cycles, which approached $19 \%$ at 40 cycles after exposure to the SAE J2334 test. Flexural strength results are given by Figure II.1.5.2 (b) and the strength also decreased with the number of cycles, which reached approximately 9\% at 40 cycles after exposure to the SAE J2334 test. The corrosion evaluation panels did not have any corrosion impacting the CFRE coatings after 40 cycles of exposure to the SAE J2334 test. The only observed corrosion was on the bare metal of the scribe line of the CET coupons. The amount of corrosion observed on the scribe line increased slightly as the number of cycles increased. However, no coating delamination, blister, or creepback was observed in the analyzed specimens to this point. The specimen analysis is still ongoing. 


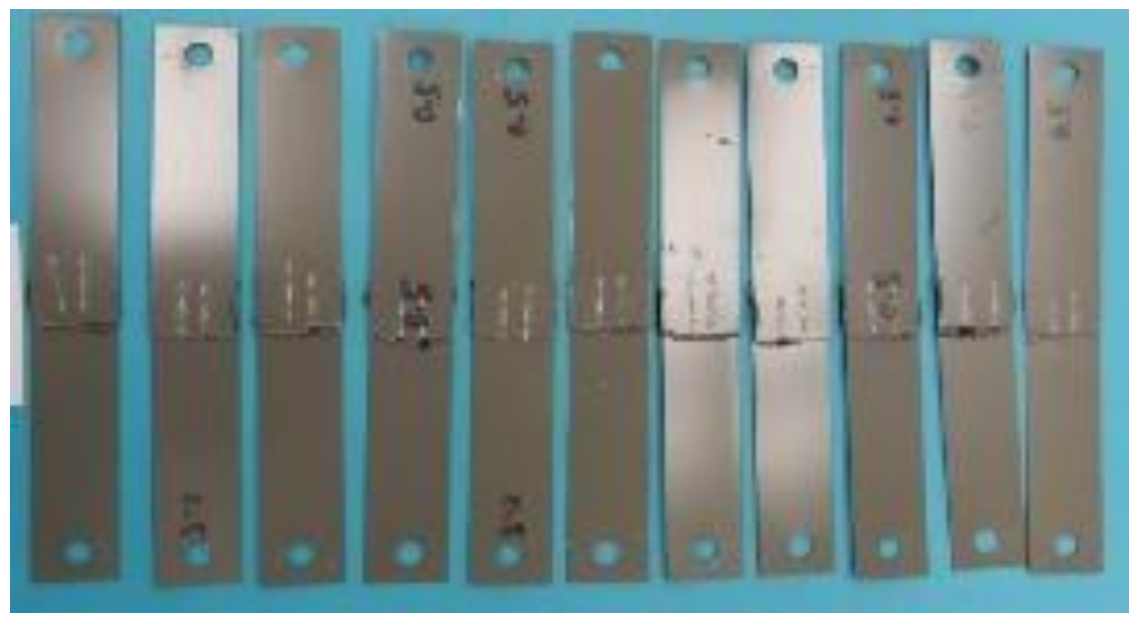

(a)

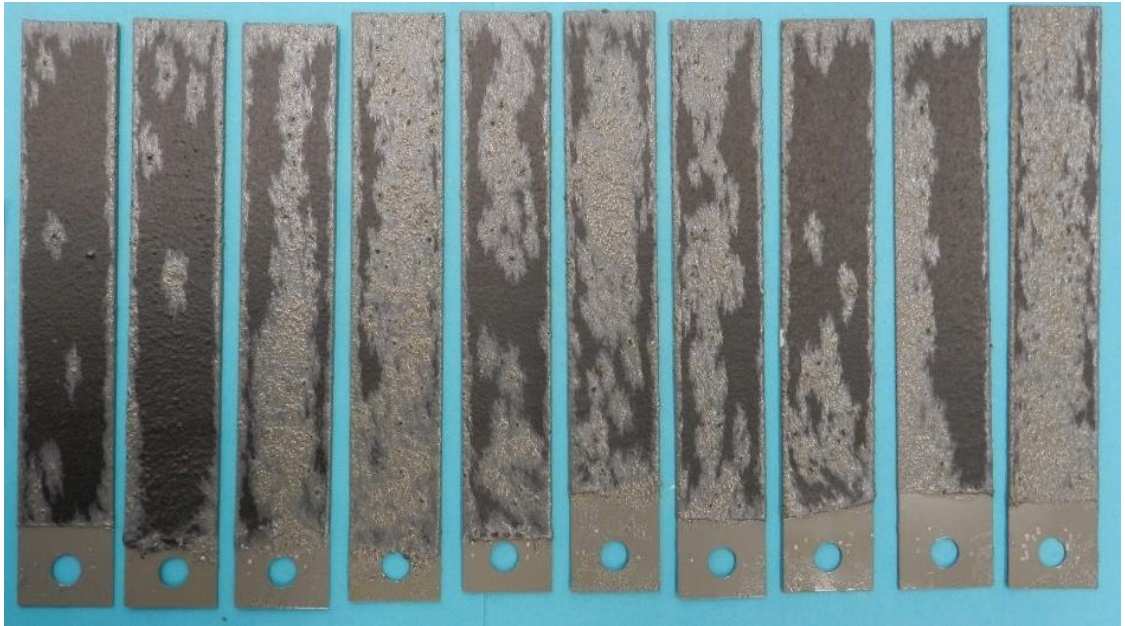

(b)

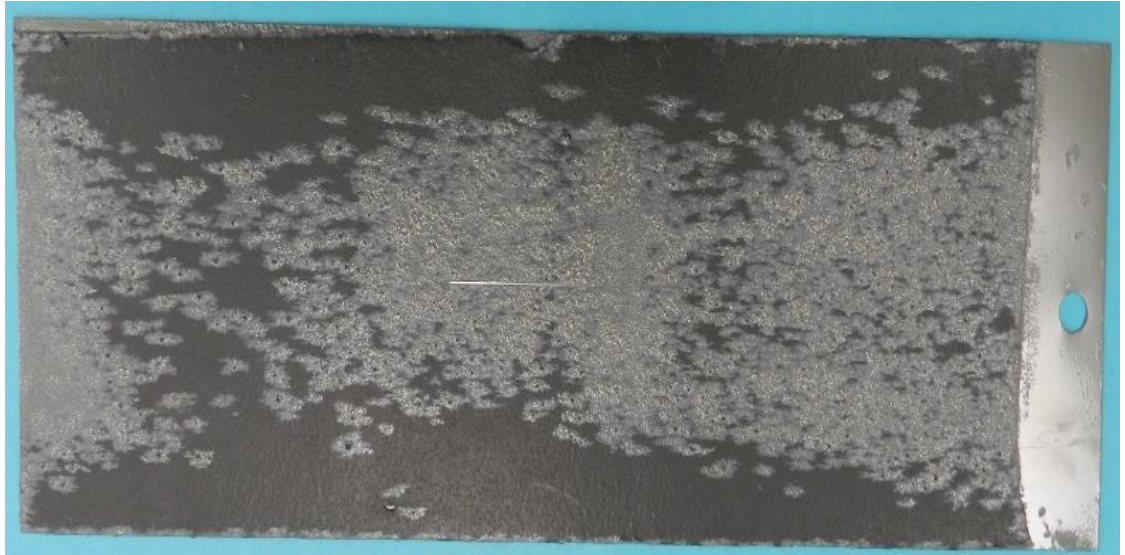

(c)

Figure II.1.5.1. Test coupons used for corrosion testing of CFRE-coated AHSS: (a) CFRE bonded lap-shear tensile test; (b) CFRE-coated flexural strength test coupons; and (c) a CFRE-coated CET coupon scribed in the middle to evaluate delamination. Source: INL. 


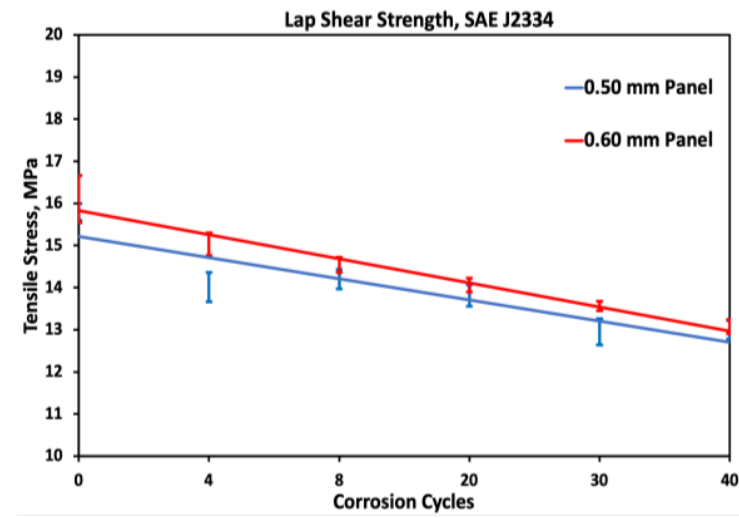

(a)

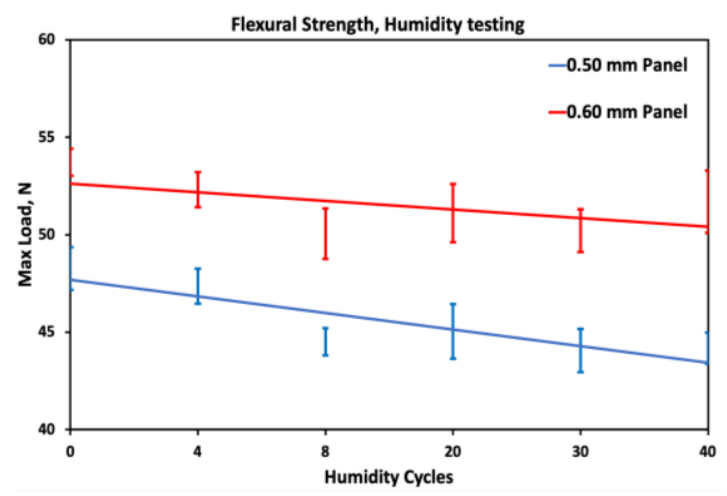

(c)

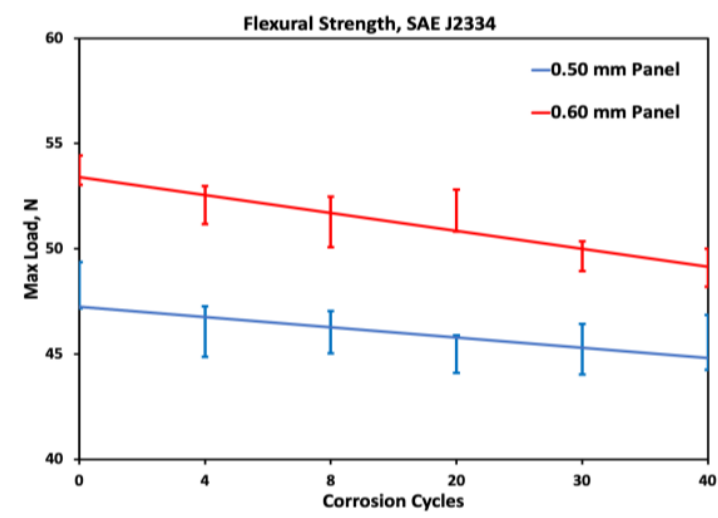

(b)

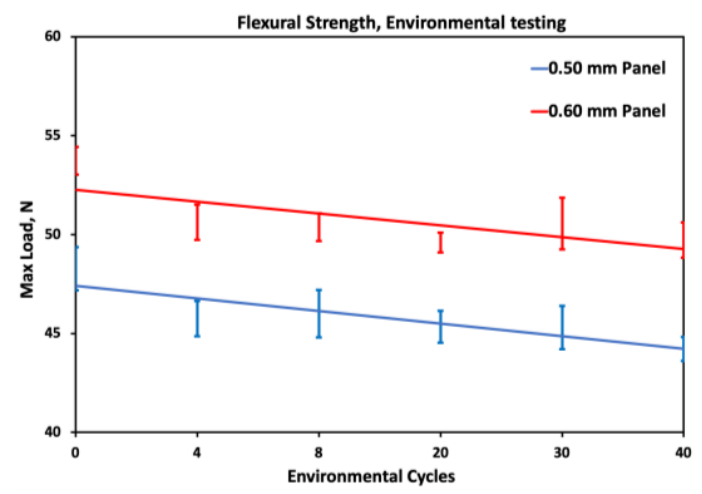

(d)

Figure II.1.5.2. (a) Coating tensile stress at yield point as a function of corrosion cycles after the SAE J2334 test. (b) Flexural strength at yield point as a function of corrosion cycles. (c) Flexural strength at yield point as a function of humidity cycles. (d) Flexural strength after environmental cycling at maximum loads. Each data point is an average of at least 10 values. All error bars are standard deviations. CFRE coating thickness on all specimens was $0.5 \mathrm{~mm}$. Source: INL.

No corrosion was observed on the CFRE coatings used in either the flexural strength or corrosion evaluation coupon tests after exposure to the FLTM BQ 104-02 and FLTM BQ 104-07 tests after 40 cycles. There were no blisters or other corrosion-related defects apparent. Additionally, no apparent delamination of the coating was observed anywhere on the coupons. In fact, no noticeable change was observed on these coupons after exposure up to 40 cycles when compared with the pre-exposure appearance. No scribe lines were made on any of the specimens used for these tests. Flexural strength decreased with an increasing number of cycles after exposure to the humidity test (e.g., FLTM BQ 104-02), as can be seen in Figure II.1.5.2 (c). There was an approximate decrease of $9 \%$ at 40 cycles for the $0.5 \mathrm{~mm}$-thick steel versus a $7 \%$ decrease at 40 cycles for the 0.6 mm-thick steel after exposure to the FLTM BQ 104-02 test.

Corrosion was not observed on the environmental cycling coupons after exposure to the FLTM BQ 104-07 test after 40 cycles. No corrosion-related defects, such as blisters or delamination caused by the exposure, were observed on any of the coupons. As with the coupons from the FLTM BQ 104-02 specimens, no noticeable change was observed on these coupons after exposure up to 40 cycles when compared with the pre-exposure appearance. No scribe lines were made on any of the specimens used for these tests. The flexural strength of coupons exposed to the FLTM BQ 104-07 test decreased with the number of cycles. There was an average decrease of approximately 8\% after 40 cycles for both thicknesses of AHSS as observed in Figure II.1.5.2 (d).

Figure II.1.5.3 (a) and Figure II.1.5.3 (b) displays the corrosion observed in the scribe line immediately after 30 cycles of SAE J2334 testing, and after the corrosion has been removed from the scribe line, respectively. The scribe line revealed no indication of creepback or delamination. 


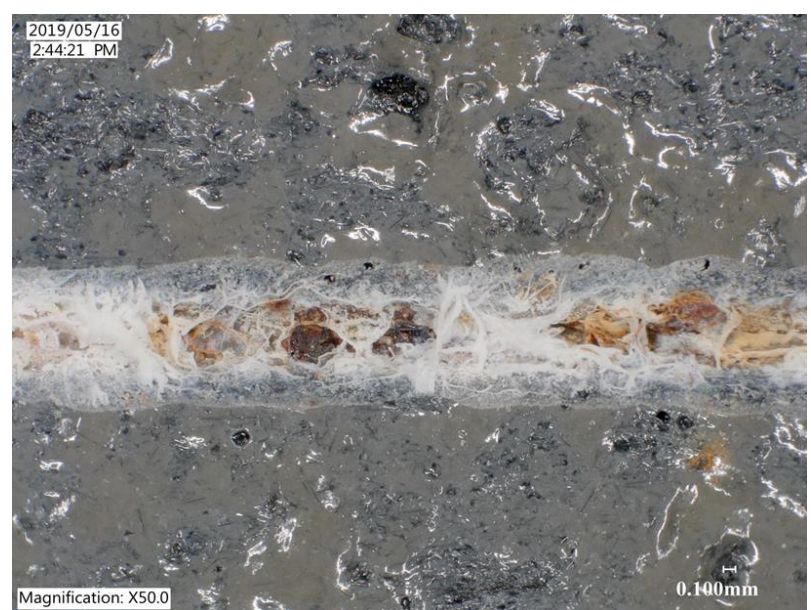

(a)

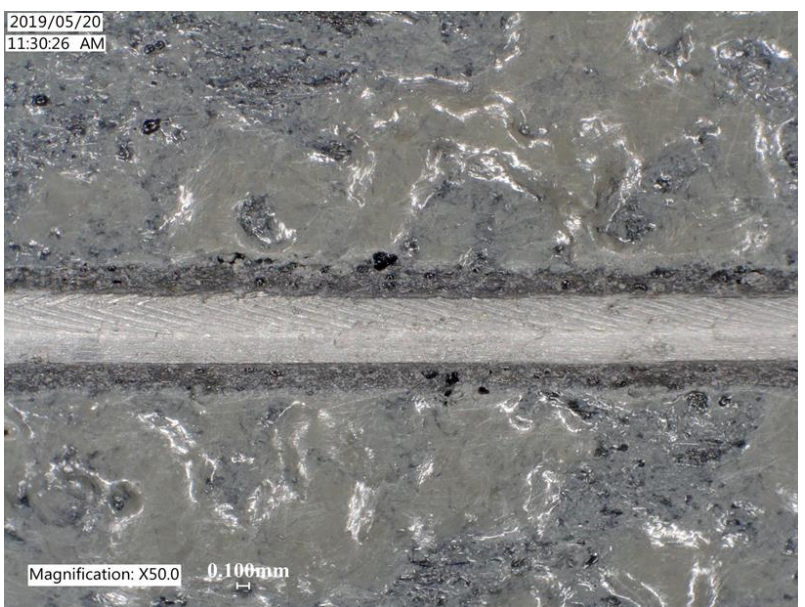

(b)

Figure II.1.5.3. Coupon J116 (a) immediately after 30 cycles of testing; and (b) after the corrosion has been cleaned out of the scribe line area. Source: INL.

\section{Conclusions}

No corrosion was observed with coatings on the flexural strength coupons, and no corrosion was observed on the coatings on the lap-shear strength coupons. All failure modes of the lap-shear coupons were cohesive failure. No corrosion was observed on the CET specimens without the center scribe that exposed bare metal. Minor corrosion was observed in the scribe line on the scribed CET coupons, but no coating delamination or creepback was observed. Further analyses of theses specimens continue. From a CFRE application standpoint, a typical steel door can be downgauged from $0.70 \mathrm{~mm}$ thickness to $0.50 \mathrm{~mm}$ thickness with the application of a $0.5 \mathrm{~mm}$-thick layer of CFRE. If the CFRE is applied for total coverage, the mass reduction can be $1.11 \mathrm{Kg}$ per door at a cost of $\$ 8.11$ per $\mathrm{kg}$ ( $\$ 9.00$ per door). If the CFRE is applied to specifically designated areas (patches), then the mass reduction can be $1.55 \mathrm{~kg}$ at a cost of $\$ 0.12$ per $\mathrm{kg}(\$ 0.19$ per door). Even if the patch thickness is increased to $1.0 \mathrm{~mm}$ as an additional performance margin, the mass reduction can be $1.54 \mathrm{~kg}$ at a cost of $\$ 0.23$ per $\mathrm{kg}(\$ 0.35$ per door $)$.

\section{Key Publications}

1. Sadagopan, S., M. Kuo, M. Lizak, R. Robison, R. Eadara, T. Brewer, J. M. Joseff, C. D. Warren, and G. Ilevbare, 2018, "Door Lightweighting Using Ultra-Thin AHSS Reinforced with Low Density CFRE Materials," Proceedings of the $17^{\text {th }}$ Annual Great Design in Steels Conference, 16-19 May 2018, Detroit, MI, USA.

\section{References}

1. Singh, H., and G. Coates, 2014, "Lightweighting with AHSS: Minimum Thickness Study \& Application Guidelines, Version 5.0," Proceedings of the $13^{\text {th }}$ Annual Great Design in Steels Conference, May 14, 2014, Livonia, MI, USA.

2. U.S. Department of Energy, 2019, “Materials 2018 Annual Progress Report,” VTO, DOE-EERE, DOE/EE-1827, pp. 214-222.

\section{Acknowledgements}

The significant technical contributions of the following individuals are acknowledged and appreciated: Timothy Yoder, INL; and Shuxiao Li and Philip Danquah, Diversitak. 


\title{
II.1.6 Optimizing Heat-Treatment Parameters for Third-Generation Advanced High- Strength Steels Using an Integrated Experimental-Computational Framework (Pacific Northwest National Laboratory)
}

\author{
Erin Barker, Co-Principal Investigator \\ Pacific Northwest National Laboratory \\ 902 Battelle Blvd. \\ Richland, WA 99352 \\ E-mail: erin.barker@pnnl.gov
}

\author{
Emmanuel De Moor, Co-Principal Investigator \\ Advanced Steel Processing and Products Research Center \\ Colorado School of Mines \\ 1500 Illinois St. \\ Golden, CO 80401 \\ E-mail: edemoor@mines.edu
}

\section{Jerry L. Gibbs, DOE Technology Development Manager}

U.S. Department of Energy

E-mail: jerry.gibbs@ee.doe.gov

Start Date: June 1, 2016

Project Funding (FY19): \$1,600,000

End Date: September 30, 2020

DOE share: $\$ 1,600,000 \quad$ Non-DOE share: $\$ 0$

\section{Project Introduction}

Medium Mn transformation-induced plasticity (TRIP) steels represent potential important products to achieve the performance requirements of third-generation AHSS in efforts to lightweight vehicles for improved fuel efficiency while meeting or exceeding safety regulations.

Medium Mn steels typically contain 4-10 wt.\% Mn and other alloying elements like those used in selected current AHSS grades. It is well-known that the mechanical properties of these steels are extremely sensitive to certain chemical compositions ( $\mathrm{C}$ and $\mathrm{Mn}$ content) and intercritical annealing (IA) temperatures, which in turn determine the volume fraction and thermodynamic stability of the retained austenite (RA) in the corresponding microstructures. Given a certain microstructure of the multiphase steel (i.e., volume fraction, morphology and stability of the RA), our team's prior work has established an integrated experimental and computational approach linking the microstructures to the corresponding mechanical properties [1-2].

However, traditional experimental heat-treatment and characterization techniques applied in optimizing the heat-treatment parameters for strength and ductility combinations are often laborious and time-consuming. Complete descriptions, efficient experimental characterization techniques and predictive capabilities, as well as the applications of these to describe relationships between chemical compositions, initial microstructures, heattreatment parameters and subsequent RA volume fraction, RA stability, and mechanical properties have not been established. 


\section{Objectives}

The objective of this project is to aide steel manufacturers and users in expediting the development-todeployment cycle by developing an integrated in situ and ex situ experimental and numerical modeling framework. The specific goal of the framework is to provide understanding and evaluation of the impact of the IA process parameters on medium Mn third-generation AHSS. By integrated the in situ and ex situ experimental results with numerical modeling, the framework will provide accurate thermodynamic parameters for models and aide in optimizing IA parameters for a given Mn content to achieve the desired/improved strength and ductility performance.

\section{Approach}

In this project, four focus objectives are proposed: (1) Develop an in situ characterization technique to determine the austenite formation kinetics of medium Mn TRIP steels on heating and during IA to enable the accelerated development of future third-generation AHSS; (2) Develop a phase-field-based modeling capability to predict the volume fraction, morphology (including grain size), and stability (C and Mn concentration) of the austenite formed during the IA process; (3) Link the predicted microstructures, including the austenite volume fraction retained after cooling, stability, and morphology to the mechanical properties; and (4) Optimize the strength and ductility of medium Mn TRIP steels by judicious IA temperature selection.

\section{Results}

Investigation of the microstructural evolution occurring during IA of medium Mn steels was continued with both experimental and simulation activities. High Energy X-ray Diffraction (HEXRD) assessments that were collected in situ during IA of a $0.19 \mathrm{C}-4.39 \mathrm{Mn}$ steel from the as-quenched (AQ) condition were correlated to microstructure analysis and compared to DICTRA simulations that predict austenite growth and Mn enrichment in austenite. Another medium Mn steel containing an ultra-low residual carbon $(\mathcal{C}$ concentration (i.e., 0.0005C-7.19Mn) was studied after processing to create three different conditions ranging in amount of prior cold deformation: (1) AQ; (2) cold-rolled to 50\% reduction (CR50); and (3) cold-rolled to 66\% reduction (CR66). The ultra-low C content enables a clearer assessment of the effects of prior cold deformation on Mn partitioning. Dilatometry assessments and microstructural analysis informed the development of phase-field simulations, which incorporated different amounts of austenite nucleation sites and Mn diffusivities in order to study the effect on the predicted austenite growth and Mn enrichment.

Temporal HEXRD assessments conducted in situ during IA of a 0.19C-4.39Mn steel are shown in Figure II.1.6.1. The results indicate that upon heating to the isothermal holding temperature, RA initially present in the AQ microstructure is decomposed, while cementite formation also occurs. Austenite was observed to form upon reaching the isothermal holding temperature; however, the presence of cementite did not diminish throughout the entire $1800 \mathrm{~s}$ isothermal hold at $650^{\circ} \mathrm{C}$. The microstructure assessments after IA, as shown in Figure II.1.6.2, indicated that austenite forms as an elongated, film-like constituent, while cementite forms intra-lath rod-like precipitates in martensite that are predominantly isolated from the austenite. 


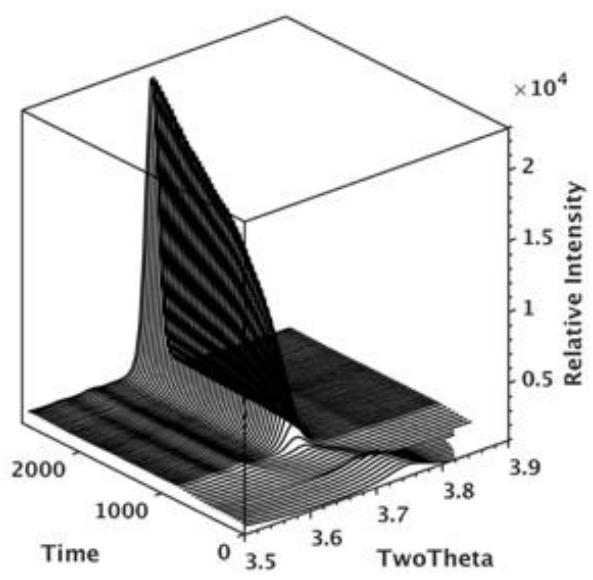

(a)

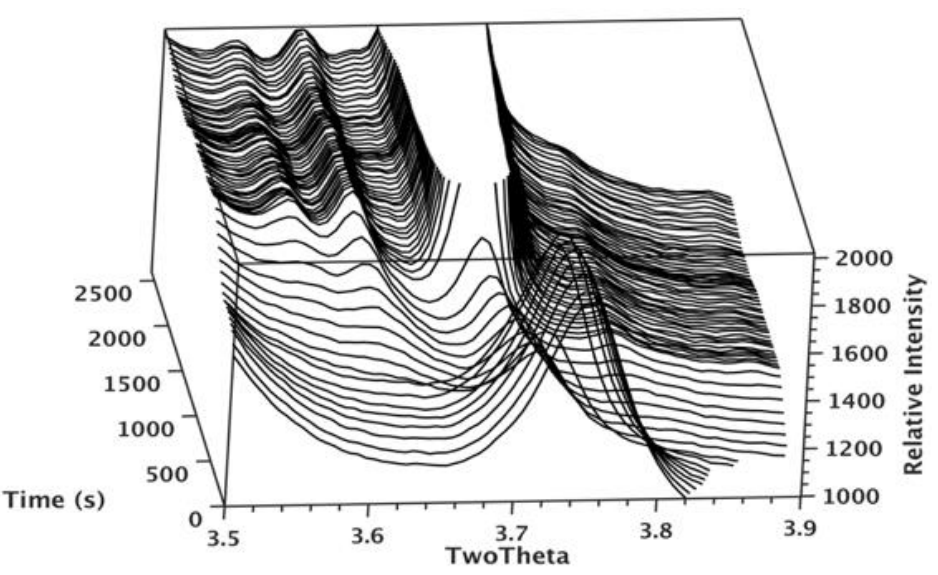

(b)

Figure II.1.6.1. Temporal HEXRD assessments for a portion of the $2 \theta$ range collected in situ during IA with a heating rate of $0.7625^{\circ} \mathrm{C} / \mathrm{s}$ and an isothermal holding at $650^{\circ} \mathrm{C}$ for $1800 \mathrm{~s}$ showing cementite $(\theta)$ and austenite ( $\mathrm{Y}$ ) diffraction peaks. The full y $\{200\}$ intensity is shown in (a), while the truncated intensity in (b) shows the $\theta$ peaks. Source: PNNL.

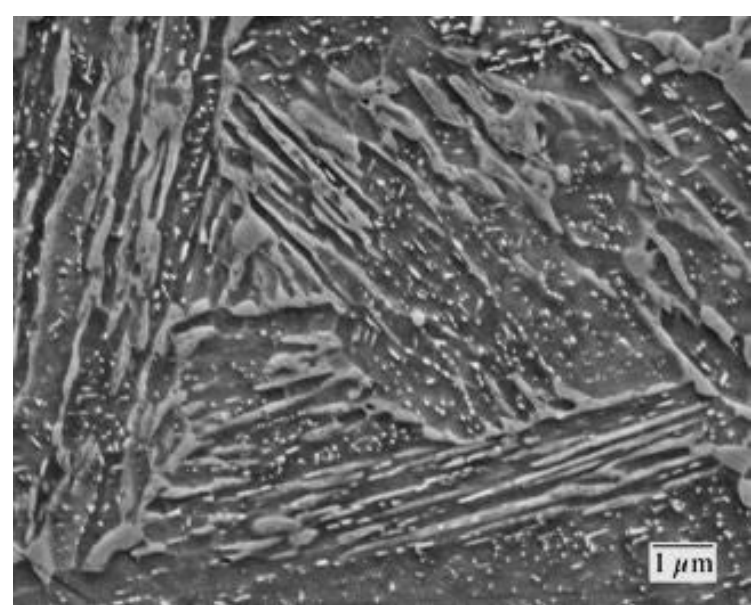

(a)

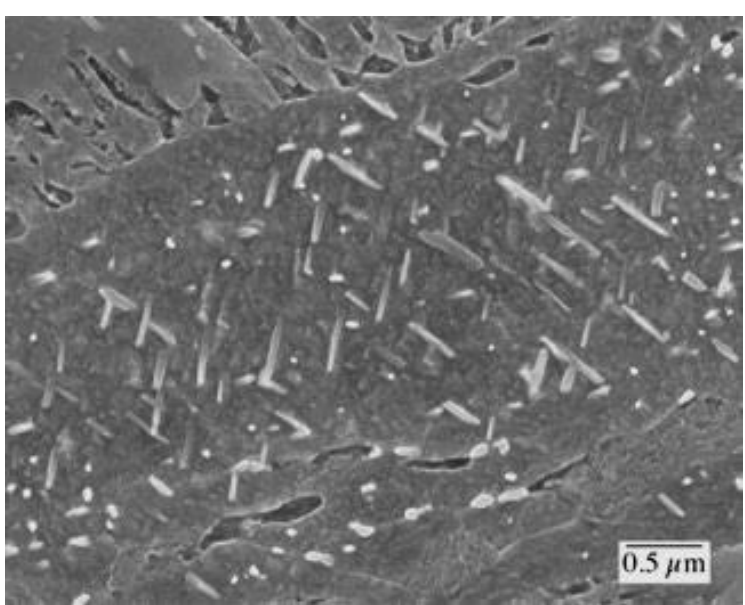

(b)

Figure II.1.6.2. Field emission SEM images of the microstructure produced from IA with a heating rate of $0.7625^{\circ} \mathrm{C} / \mathrm{s}$ and an isothermal hold at $650^{\circ} \mathrm{C}$ for (a) $1000 \mathrm{~s}$ and (b) $1800 \mathrm{~s}$. Image (a) exhibits the elongated austenite and fine cementite, while image (b) exhibits a higher magnification of intra-lath rod-like cementite. Source: Colorado School of Mines.

Two simulations were conducted using Thermo-Calc DICTRA to predict austenite growth during IA of the 0.19C-4.39Mn steel. Simulation 1 considered C to be in solution in martensite, while Simulation 2 considered the presence of intra-lath cementite. The predicted austenite fraction from the simulations are compared to the HEXRD assessment in Figure II.1.6.3. Simulation 1 predicts rapid austenite growth that far exceeds the HEXRD assessment, while Simulation 2 is in good agreement with the experimental results. Furthermore, the simulations predict very different Mn distributions for the austenite growth occurring during IA. Figure II.1.6.4 shows the Mn distribution near the austenite-ferrite interface for each simulation. In Figure II.1.6.4 (a) Simulation 1 predicts a Mn deficient austenite growth followed by Mn enriched austenite growth, while in Figure II.1.6.4 (b), Simulation 2 predicts Mn enrichment from the onset of austenite growth. This difference in Mn enrichment is the result of Simulation 1 predicting initial para-equilibrium growth of austenite with only $\mathrm{C}$ enrichment while Simulation 2 predicts Mn partitioning growth of austenite due to the $\mathrm{C}$ being tied up in the cementite. 


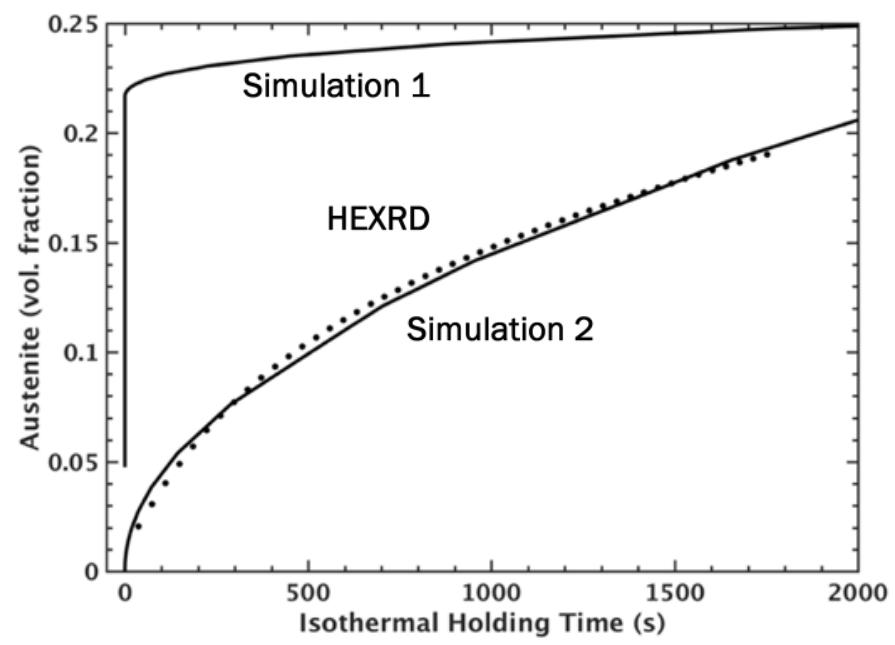

Figure II.1.6.3. Austenite volume fraction predictions from Simulations 1 and 2 for isothermal holding at $650^{\circ} \mathrm{C}$, as well as the austenite volume fraction assessments from the in situ HEXRD during isothermal holding at $650^{\circ} \mathrm{C}$.

Source: Colorado School of Mines.

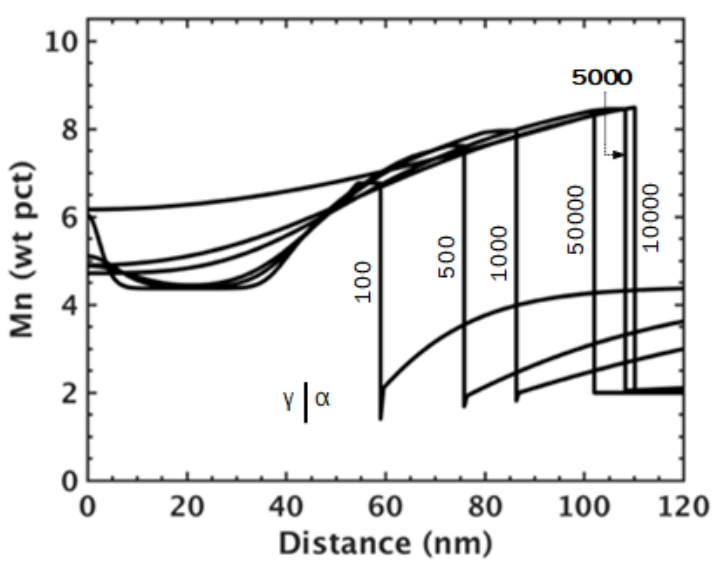

(a)

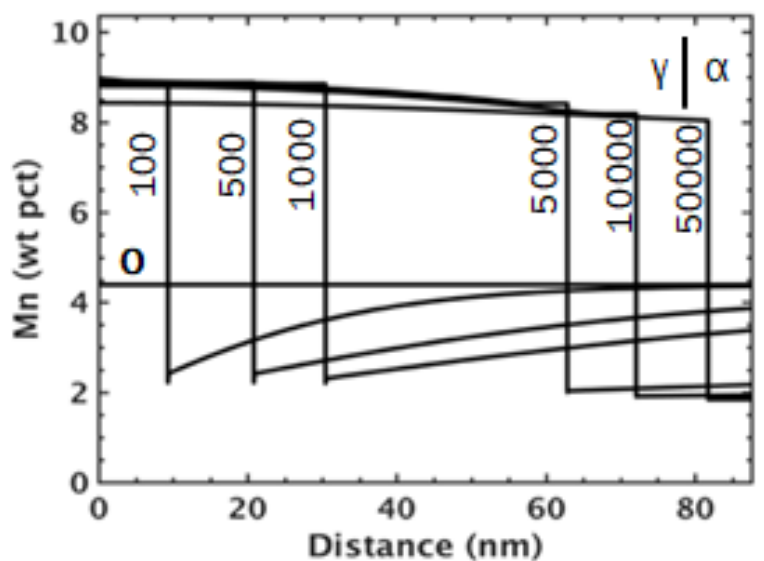

(b)

Figure II.1.6.4. Mn distribution predictions from (a) DICTRA Simulation 1 and (b) Simulation 2 for isothermal holding at $650^{\circ} \mathrm{C}$ and at isothermal hold times ranging from 100-50,000 s. Source: Colorado School of Mines.

Austenite volume fraction assessments from dilatometry for IA using two different heating rates (e.g., 0.05 and $96^{\circ} \mathrm{C} / \mathrm{s}$ ) and a $1000 \mathrm{~s}$ isothermal hold at $650^{\circ} \mathrm{C}$ for the three conditions of the $0.0005 \mathrm{C}-7.19 \mathrm{Mn}$ steel are shown in Figure II.1.6.5. Regardless of heating rate, increasing prior cold deformation resulted in an increased austenite volume fraction. Micrographs of the CR50 sample after heating to $650^{\circ} \mathrm{C}$ at $0.05^{\circ} \mathrm{C} / \mathrm{s}$ and quenching are shown in Figure II.1.6.6, and indicate that austenite forms on deformed ferrite grain boundaries, as well as recrystallized ferrite grains. 


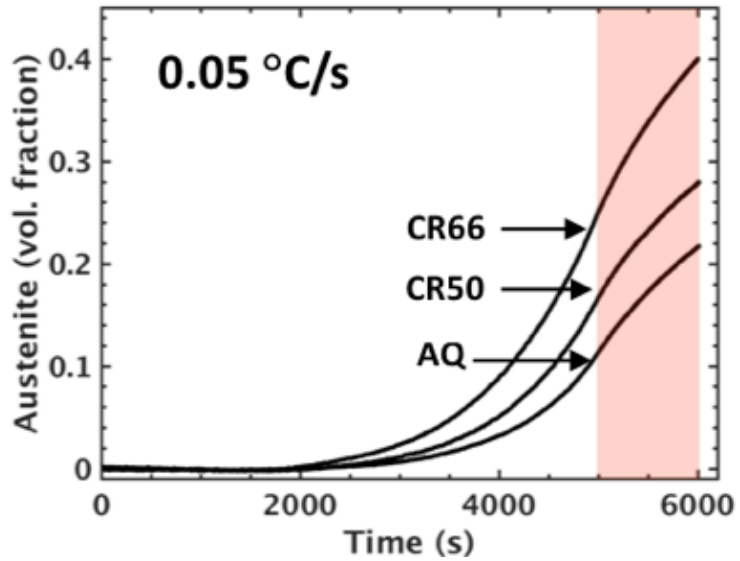

(a)

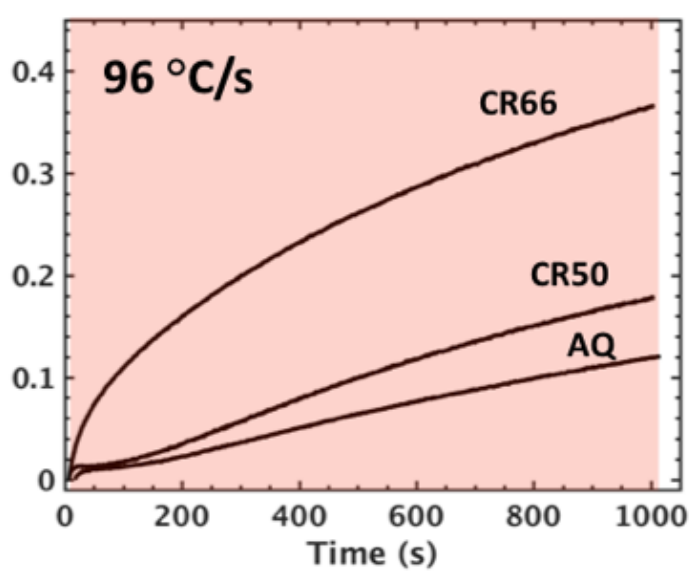

(b)

Figure II.1.6.5. Dilatometry assessments of austenite volume fraction from the three conditions of the $0.0005 \mathrm{C}-7.19 \mathrm{Mn}$ steel during IA with a $1000 \mathrm{~s}$ isothermal hold at $650^{\circ} \mathrm{C}$ and heating rates of: (a) $0.05^{\circ} \mathrm{C} / \mathrm{s}$; and (b) $96^{\circ} \mathrm{C} / \mathrm{s}$.

Source: Colorado School of Mines.
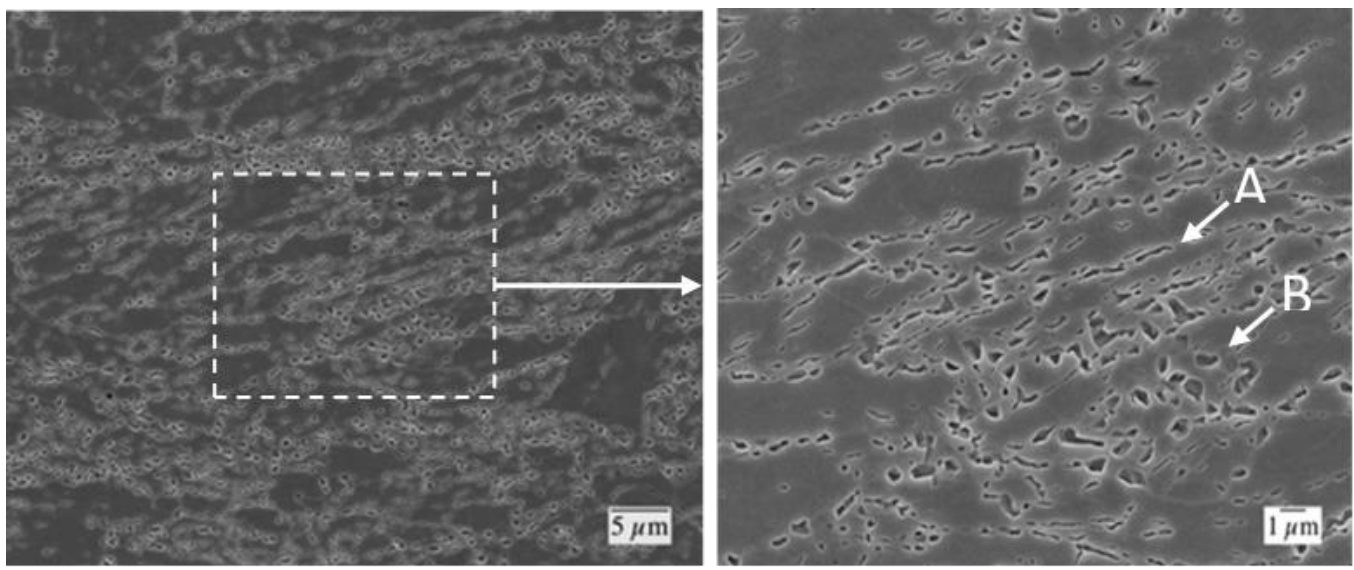

Figure II.1.6.6. Field emission SEM images of the microstructure produced from heating the CR50 condition of the $0.0005 \mathrm{C}-7.19 \mathrm{Mn}$ steel with a heating rate of $0.7625^{\circ} \mathrm{C} / \mathrm{s}$ to $650^{\circ} \mathrm{C}$ and quenching. Austenite is observed to form at elongated, deformed ferrite boundaries, labeled $\mathrm{A}$, as well as at recrystallized ferrite boundaries, labeled $\mathrm{B}$.

Source: Colorado School of Mines.

To simulate the microstructure evolution in the steel during IA, a phase-field simulation was constructed that reflects both austenite formation and ferrite recrystallization during IA. The initial structure of a simulation, as well as the results after $500 \mathrm{~s}$ of isothermal holding, are shown in Figure II.1.6.7. Timesteps are shown at 0 and $500 \mathrm{~s}$. The initial structure is composed of elongated deformed ferrite $\left(\alpha_{\text {def }}\right)$ with stored strain energy (SSE), while after isothermal holding, ferrite recrystallization $\left(\alpha_{\mathrm{rx}}\right)$ and austenite formation $(\gamma)$ occur, both of which have no SSE. The austenite is also predicted to form with Mn enrichment. In the phase maps of Figure II.1.6.7 (a), the simulation is shown to initially contain only deformed ferrite; after $500 \mathrm{~s}$ the phase map shows the presence of homogenously nucleated recrystallized ferrite and ferrite grain boundary nucleated austenite. The SSE maps, shown in Figure II.1.6.7 (b), show that the deformed ferrite has a distribution of SSE and that recrystallized ferrite and austenite have no SSE. The Mn distribution maps in Figure II.1.6.7 (c) show the simulation initially has a homogeneous distribution of $\mathrm{Mn}$ and that austenite growth occurs with an enriched Mn concentration. To test the effect of the amount of austenite nucleation sites and the Mn diffusivity on the predicted austenite volume fraction, simulations were conducted with different amounts of austenite nucleation sites ( $\gamma$ sites) and the diffusivity pre-factor for $\mathrm{Mn}$ in ferrite $\left(\mathrm{D}_{0}\right)$ were conducted. 


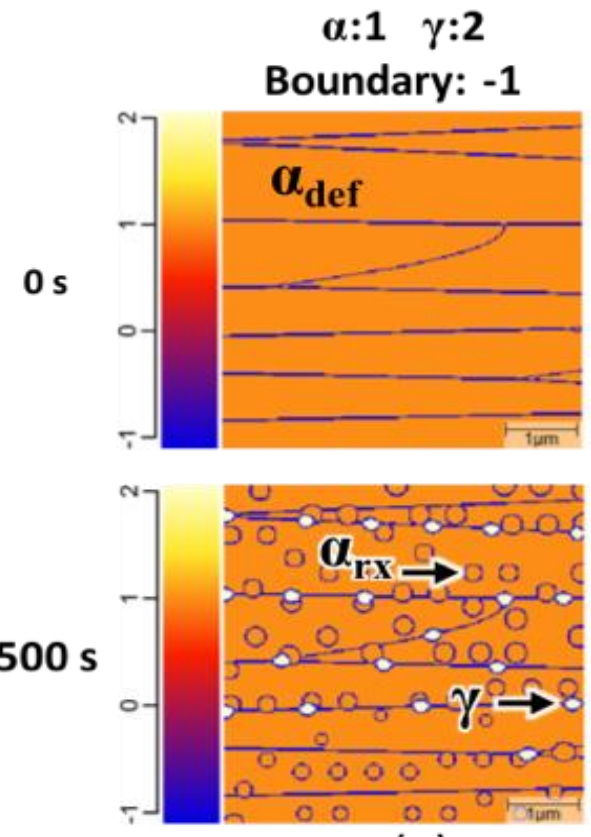

(a)

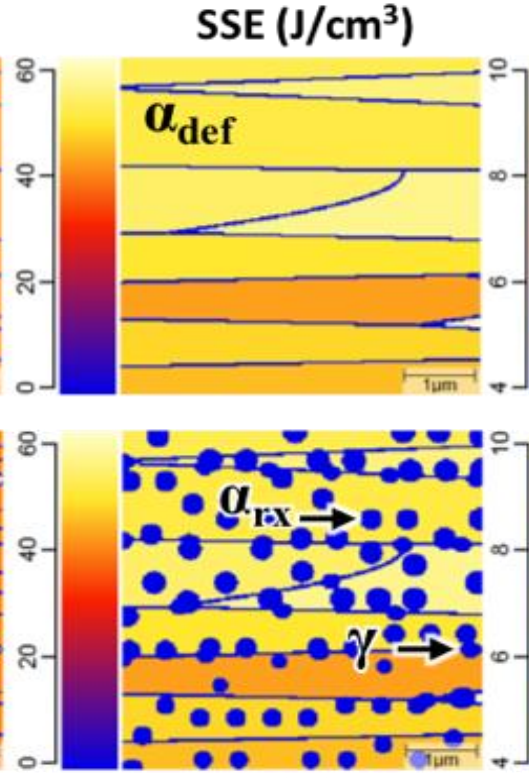

(b)

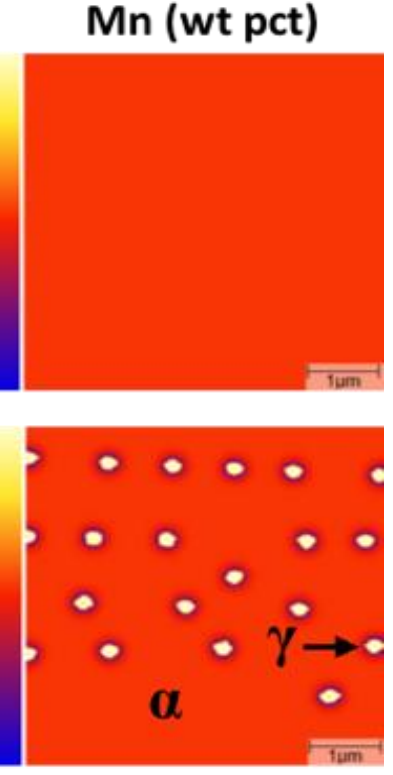

(c)

Figure II.1.6.7. Exemplary MICRESS ${ }^{\circledR}$ simulation results of IA with an isothermal holding temperature of $650^{\circ} \mathrm{C}$ and a heating rate of $96^{\circ} \mathrm{C} / \mathrm{s}$ for: (a) phase maps; (b) SSE maps; and (c) Mn distribution maps. Source: Colorado School of Mines.

Predicted austenite volume fraction for each of the simulations are shown in Figure II.1.6.8. As expected, increasing the amount of austenite nucleation sites increases the predicted austenite volume fraction. Increasing Mn diffusivity also increased the predicted austenite volume fraction; however, for large increases in diffusivity, there was a diminishing effect on the predicted austenite volume fraction. The Mn concentration across austenite-ferrite-interfaces for each of the simulations with different Mn diffusivities are shown in Figure II.1.6.9. Comparing the Mn gradient in ferrite for each simulation, it can be discerned that as the Mn diffusivity is increased, the Mn enrichment in austenite is increased. The austenite growth also transitions from diffusion-controlled to interface controlled growth with increasing Mn diffusivity.

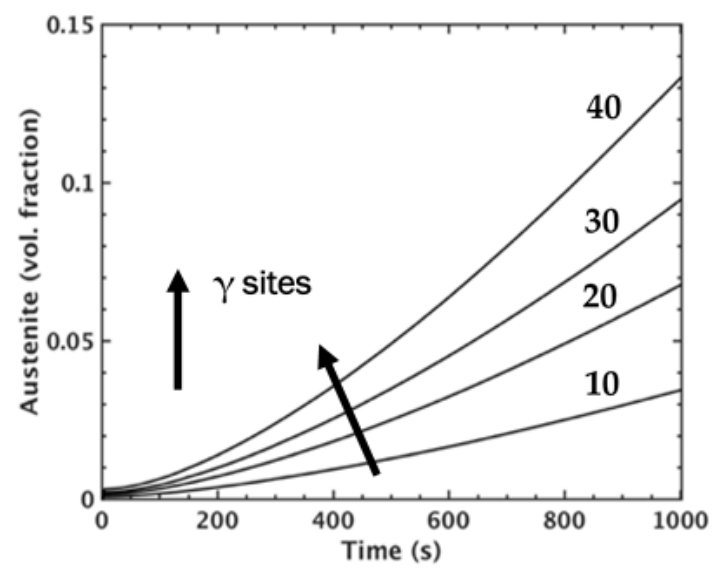

(a)

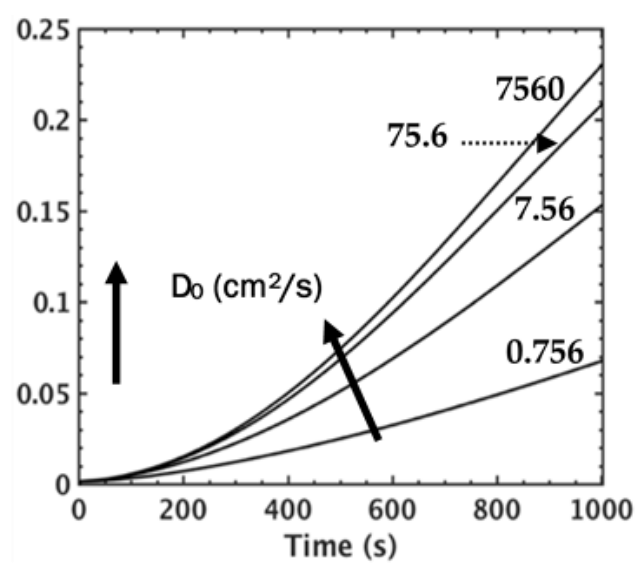

(b)

Figure II.1.6.8. Austenite volume fraction predictions during isothermal holding from phase-field simulations incorporating: (a) different amounts of austenite nucleation sites; and (b) diffusivity pre-factors for Mn in ferrite.

Source: Colorado School of Mines. 


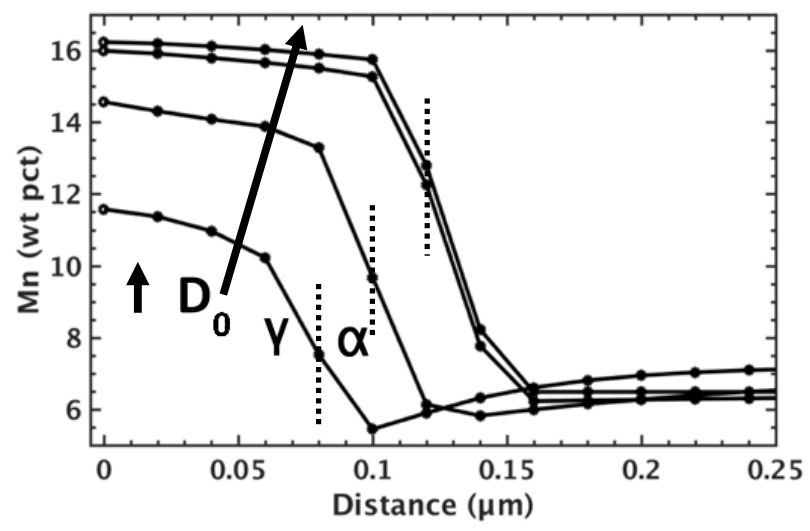

Figure II.1.6.9. Predictions for Mn distribution across the $\alpha-y$ interface after a $500 \mathrm{~s}$ isothermal hold at $650{ }^{\circ} \mathrm{C}$ for simulations incorporating different diffusivity pre-factors $\left(D_{0}\right)$ for $\mathrm{Mn}$ diffusivity in ferrite. Source: Colorado School of Mines.

\section{Conclusions}

Medium Mn TRIP steel was selected as the model steel for the development of an integrated experimental and modeling framework in optimizing the heat-treatment parameters for third-generation AHSS. In FY 2019, the mobility of $\mathrm{Mn}$ in ferrite/martensite was further explored in 5\% Mn steel through direct HEXRD measurements, DICTRA modeling, and phase-field modeling. Comparing 1D DICTRA models to experimental observations, showed that the model was not capturing the enhanced Mn mobility due to the increased defect density in the ferrite from cold-rolling. A mobility enhancement factor was developed to augment modeling results to account for increased mobility. Development of the phase-field modeling capability continued. Simulation results demonstrated the impact of initial microstructure and morphology on the distribution of austenite and chemical distributions. Insight from industry resulted in exploring the effect of heating rate on austenite growth and ferrite recrystallization. Once the model is fully developed, the research efforts will not only gain predictive understanding in the phase transformation and diffusion processes during IA process for medium manganese steels, but it is also expected to provide a general capability in highthroughput characterization and prediction of evolving phase boundaries and carbon concentration gradients during other thermomechanical processes such as the quenching and partitioning process.

\section{Key Publications}

1. Mueller, J. J., D. K. Matlock, J. G. Speer, and E. De Moor, 2019, “Accelerated ferrite-to-austenite transformation during IA of medium manganese steels due to cold-rolling," Metals, Vol. 9, No. 9, Art. \#926.

\section{References}

1. Choi, K. S., W.N. Liu, X. Sun, M. A. Khaleel, Y. Ren, and Y. D. Wang, 2008, “Advanced micromechanical model for transformation-induced plasticity steels with application of in situ highenergy XRD method," Metall. Mater. Trans. A, Vol. 39A, No. 13, pp. 3089-3096.

2. Choi, K. S., A. Soulami, W. N. Liu, X. Sun, and M. A. Khaleel, 2010, "Influence of various material design parameters on deformation behaviors of TRIP steels," Comp. Mater. Sci., Vol. 50, No. 2, pp. 720-730.

\section{Acknowledgements}

The authors would like to thank the VTO Technology Development Managers, Jerry L. Gibbs and Sarah Kleinbaum, for their continued sponsorship and support of this project. 


\title{
II.1.7 Overcoming the Barriers to Lightweighting by Enabling Low-cost and High- performance Structural Automotive Aluminum Castings (Pacific Northwest National Laboratory)
}

\author{
Aashish Rohatgi, Co-Principal Investigator \\ Pacific Northwest National Laboratory \\ 902 Battelle Boulevard \\ Richland, WA 99352 \\ E-mail: aashish.rohatgi@pnnl.gov

\section{David Weiss, Co-Principal Investigator} \\ Eck Industries, Inc. \\ 1602 North Eighth St. \\ Manitowoc, WI 54220 \\ E-mail: david.weiss@eckindustries.com
}

\section{Jerry L. Gibbs, DOE Technology Development Manager}

U.S. Department of Energy

E-mail: jerry.gibbs@ee.doe.gov

Start Date: July 25, 2018

Project Funding: $\$ 500,000$
End Date: September 30, 2020

DOE share: $\$ 250,000$
Non-DOE share: $\$ 250,000$

\section{Project Introduction}

The U.S. transportation industry is the biggest user of Al castings. In 2015, 17.84 million light vehicles (cars and trucks) were sold [1]. A typical vehicle contains $\sim 400 \mathrm{lbs} \mathrm{Al}$ ( $\sim 73 \%$ as castings), which represents a number that is expected to rise as high as $\sim 500 \mathrm{lbs}$ in 2025 [2]. Considering that a typical full-size sedan weighs anywhere between 2000-3000 lbs, greater use of low-cost and high-performance Al castings can contribute significantly to cost-effective vehicle weight reduction. However, cost and mechanical performance of $\mathrm{Al}$ castings are two key long-standing industry challenges that need to be overcome to enable greater lightweighting in mass-market vehicles. In other words, if the cost of Al castings could be reduced and their mechanical performance enhanced, even greater market penetration (e.g., by replacing ferrous components) is possible.

One way to lower the cost for $\mathrm{Al}$ castings is to use "secondary" Al alloys (i.e., those produced from recycled $\mathrm{Al}$ ), due to their $\sim 16 \%$ lower-cost than the "primary" Al produced via electrolytic reduction of alumina. However, many secondary $\mathrm{Al}$ alloys allow up to $0.60 \% \mathrm{Fe}$ (as compared to $0.1-0.2 \% \mathrm{Fe}$ in primary $\mathrm{Al}$ alloy) leading to the formation of iron-intermetallic phases in the microstructure. These intermetallics are detrimental to the tensile properties and elongation of the castings as compared to the primary $\mathrm{Al}$ alloy. Thus, to keep costs low, Al may be skipped in favor of cheaper, but heavier, cast-iron. Further, irrespective of the choice of primary versus secondary $\mathrm{Al}$ alloy, $\mathrm{Al}$ castings are often subjected to long-duration heat-treatments, such as solutioning and aging, to improve their strength and ductility. Such heat-treatments further add to the overall cost of the castings. Thus, techniques need to be developed that can help lower the cost of Al castings and enhance their performance (e.g., by enabling secondary $\mathrm{Al}$ alloys to overcome mechanical property limitations and by lowering the cost of heat-treatments). We started with low Fe containing $\mathrm{Al}$ and will work on the high Fe-containing $\mathrm{Al}$ alloys in FY 2020. 


\section{Objectives}

The goal of this project is to develop strategies to help lower the cost of $\mathrm{Al}$ castings by enabling secondary $\mathrm{Al}$ alloys to overcome the mechanical property limitations and by lowering the cost of heat-treatments. We propose to use two different processing techniques to achieve this goal. The first is an ultrasonic technique that enhances the mechanical properties by processing the molten secondary $\mathrm{Al}$ alloy, while the second uses a PNNL proprietary technique (in development) to shorten the solutionizing duration of the Al alloy. Thus, the project objectives are to:

- Develop process parameters for the ultrasonic technique leading to improvement in mechanical properties of the selected secondary Al alloy (i.e., containing "high" Fe \%).

- Develop an alternate heat-treatment (with a shorter duration and/or lower temperature) leading to similar mechanical properties as the conventional heat-treatment for the selected $\mathrm{Al}$ alloy.

\section{Approach}

Eck Industries has identified A356 Al and A206 Al as the alloys of interest for the purpose of ultrasonic process development and alternate heat-treatment development, respectively. The standardized chemistry of these alloys is listed in Table II.1.7.1 [3]. Both alloys were cast by Eck, and the subsequent experiments using these alloys are described in further detail in the sections that follow.

Table II.1.7.1. Standardized Compositions of the A356 Al and A206 Al Alloy.

\begin{tabular}{|c|c|c|c|c|}
\hline Alloy & \multicolumn{4}{|c|}{ Composition } \\
\hline A356 Al & $6.5 \%$ to $7.5 \% \mathrm{Si}$ & $0.05 \%$ to $0.25 \% \mathrm{Mg}$ & $0.1 \%$ to $0.2 \% \mathrm{Fe}$ & - \\
\hline A206 Al & $4.6 \% \mathrm{Cu}$ & $0.35 \% \mathrm{Mn}$ & $0.25 \% \mathrm{Mg}$ & $0.22 \% \mathrm{Ti}$ \\
\hline
\end{tabular}

Ingots of alloy A356 were cast with $\mathrm{Fe}<0.1 \%$ to mimic primary (low $\mathrm{Fe} \%$ ) version of this alloy. The ingots were cut into small pieces suitable to be placed inside a 1.5 in. diameter alumina crucible for melting inside a box furnace. Figure II.1.7.1 shows the benchtop setup developed for the ultrasonic experiments. Approximately $125 \mathrm{~g}$ of the A356 alloy were melted in the furnace at $\sim 650^{\circ} \mathrm{C}$ in an alumina crucible. An ultrasonic probe was inserted into the furnace via an opening in the top to interact with the molten metal to subject it to ultrasonic pulses. The key variables being studied in these experiments include the temperature for ultrasonic processing and the duration and power of the ultrasonic impulses. The molten metal, with or without ultrasonic processing, was then poured into a graphite crucible. The as-cast samples were polished by traditional metallography techniques and analyzed by optical and SEM.

The A206 Al alloy was die-cast in the form of tensile samples seen in Figure II.1.7.2 (a) with the idea of evaluating the mechanical performance as a function of heat-treatment. Some as-cast tensile bars were cut into $\sim 1 / 2$ in. thick sections and subjected to conventional T4 heat-treatment which comprises solution treatment for $12 \mathrm{~h}$ followed by RT aging for 5 days. Figure II.1.7.2 (b) shows the typical solutionization heat-treatment profile for cast A206 Al. For some T4 samples, the solution heat-treatment was interrupted at 2, 4, and 8 hours, followed by RT aging for 5 days. Some T4 heat-treated samples were further subjected to a T7 heat-treatment at $200^{\circ} \mathrm{C}$ for $4 \mathrm{~h}$. The heat-treated samples were characterized by hardness measurements to evaluate the influence of heat-treatment on the mechanical properties. Metallographic samples were prepared using standard techniques and imaged in SEM. ImageJ software was then used to analyze the SEM images to quantify microstructural features such as the volume fraction of different phases. 


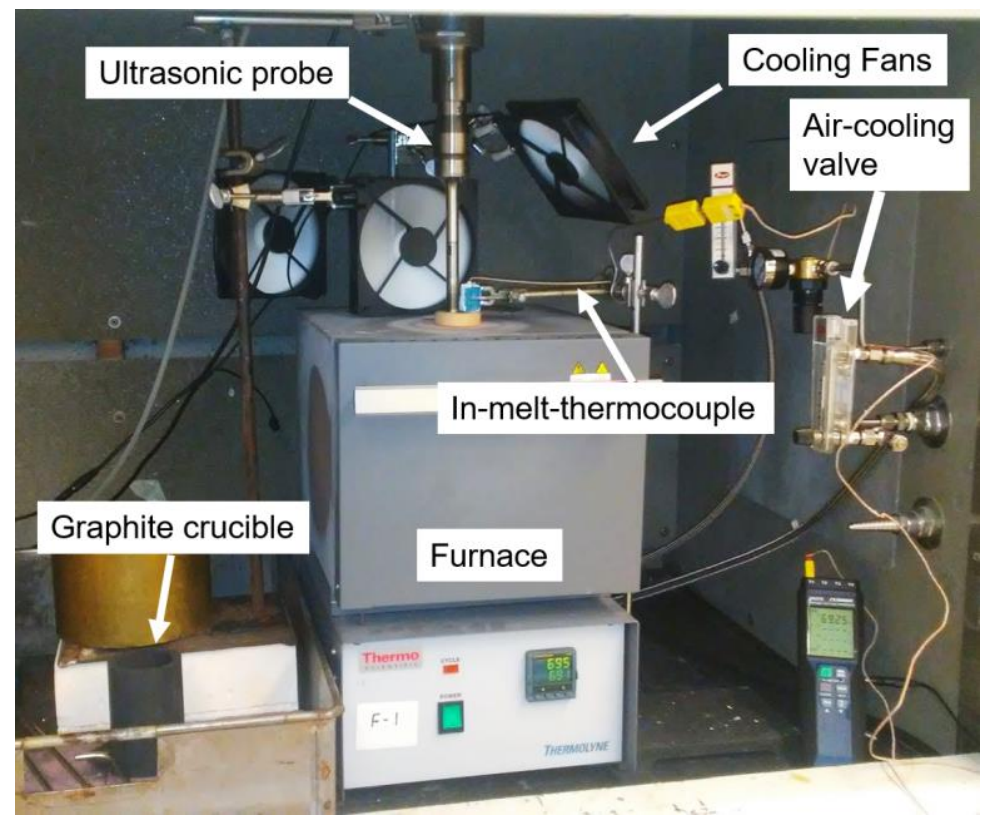

Figure II.1.7.1. Benchtop setup constructed at PNNL for ultrasonic melt-processing experiments. Source: PNNL.

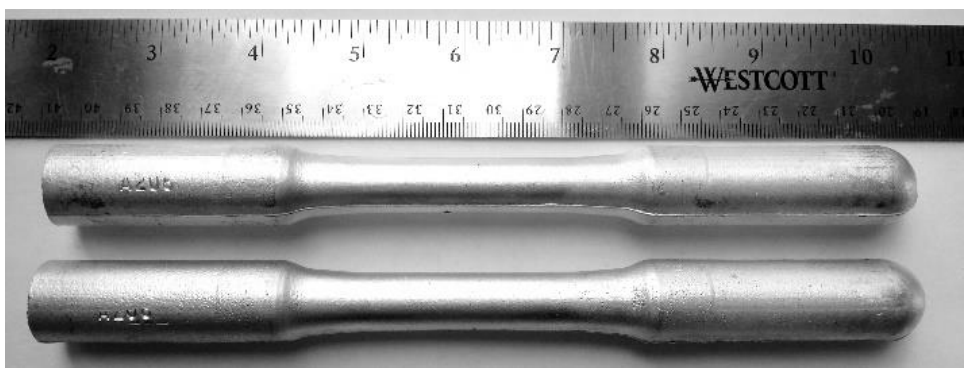

(a)

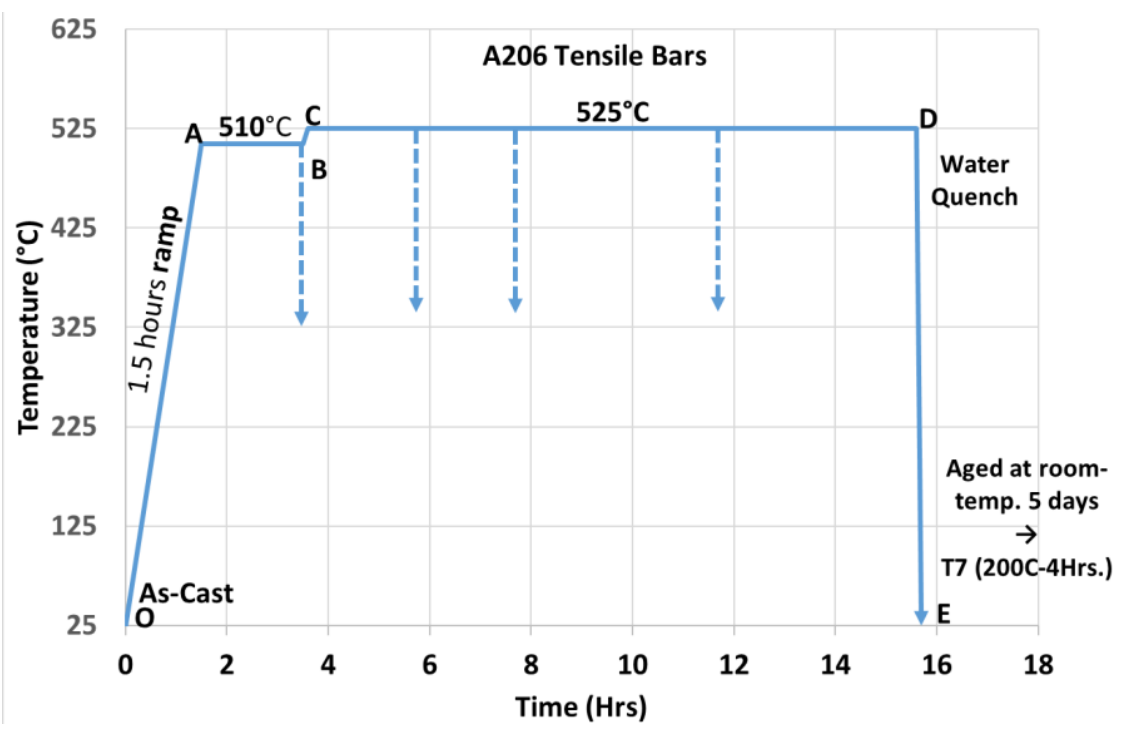

(b)

Figure II.1.7.2. (a) As-cast A206 Al alloy tensile bars; and (b) modified solutionization heat-treatment procedure implemented in the experiments. Source: PNNL. 


\section{Results}

Figure II.1.7.3 shows the microstructure of the A356 Al before and after ultrasonication. The as-cast microstructure without ultrasonic treatment is displayed in Figure II.1.7.3 (a), while the as-cast microstructure with ultrasonic treatment is shown in Figure II.1.7.3 (b). Ultrasonic pulse power of $20 \mathrm{~W}$ was applied for a duration of $2 \mathrm{~min}$ at the melt temperature of $\sim 620^{\circ} \mathrm{C}$ using a Ti-6Al-4V probe. These ultrasonication parameters, i.e. power and duration, correspond to the range of ultrasonic intensities and total input-energy usually encountered in ultrasonic processing of light alloy melts [4]. It can be readily observed that the microstructure after ultrasonication is significantly refined with smaller $\mathrm{Al}$ dendrites and intermetallic phases. Measurements show a decrease in Al-dendrite size from $73.2 \mu \mathrm{m}$ to $49.3 \mu \mathrm{m}$ and in average intermetallic-size from $4.4 \mu \mathrm{m}$ to $3.2 \mu \mathrm{m}$ after ultrasonication.
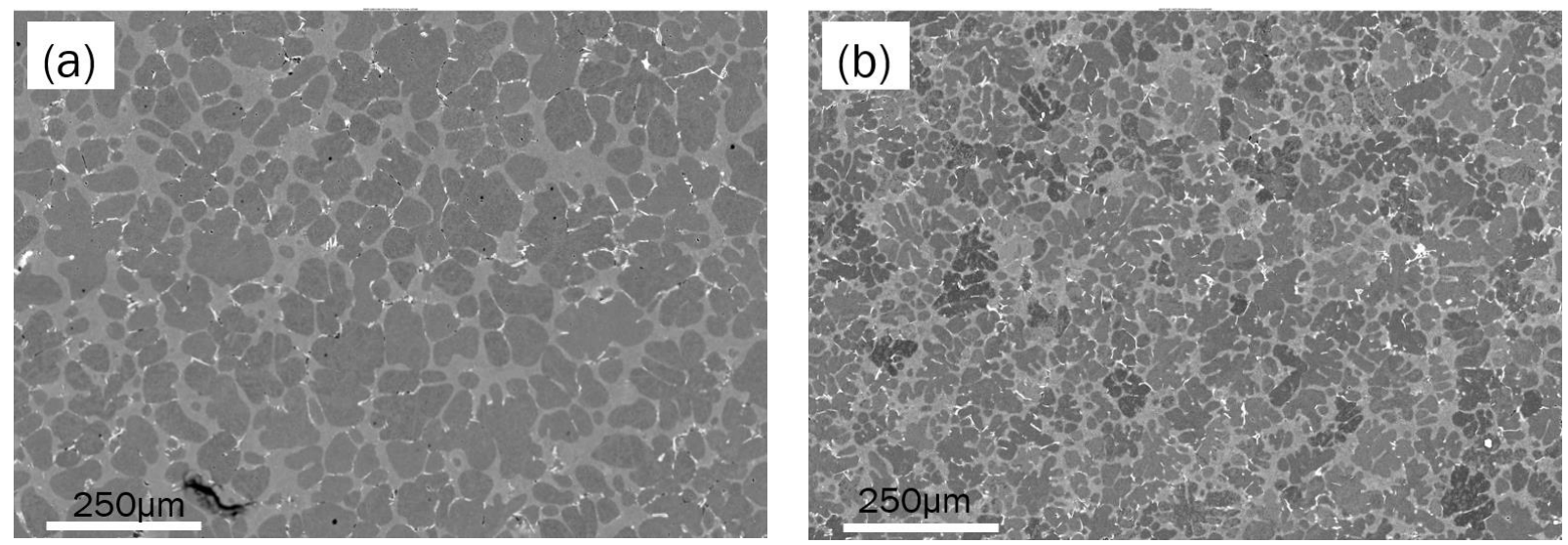

Figure II.1.7.3. As-cast SEM microstructure of A356 Al alloy: (a) without ultrasonication; and (b) with ultrasonication. Source: PNNL.

Figure II.1.7.4 shows EDS images of the A356 sample. Different phases present in the microstructure can be identified. The primary Al-dendrite phase, the Si-eutectic phase, and the intermetallic compounds, such as $\mathrm{Mg}_{2} \mathrm{Si}$ and Chinese-script like phases of composition $\mathrm{Al}_{8} \mathrm{Mg}_{3} \mathrm{FeSi}_{6}$, can be identified. There is no $\mathrm{Ti}$ or titanium aluminide phases observed in the microstructure indicating there was no transfer of Ti from the Ti$6 \mathrm{Al}-4 \mathrm{~V}$ probe during ultrasonication.
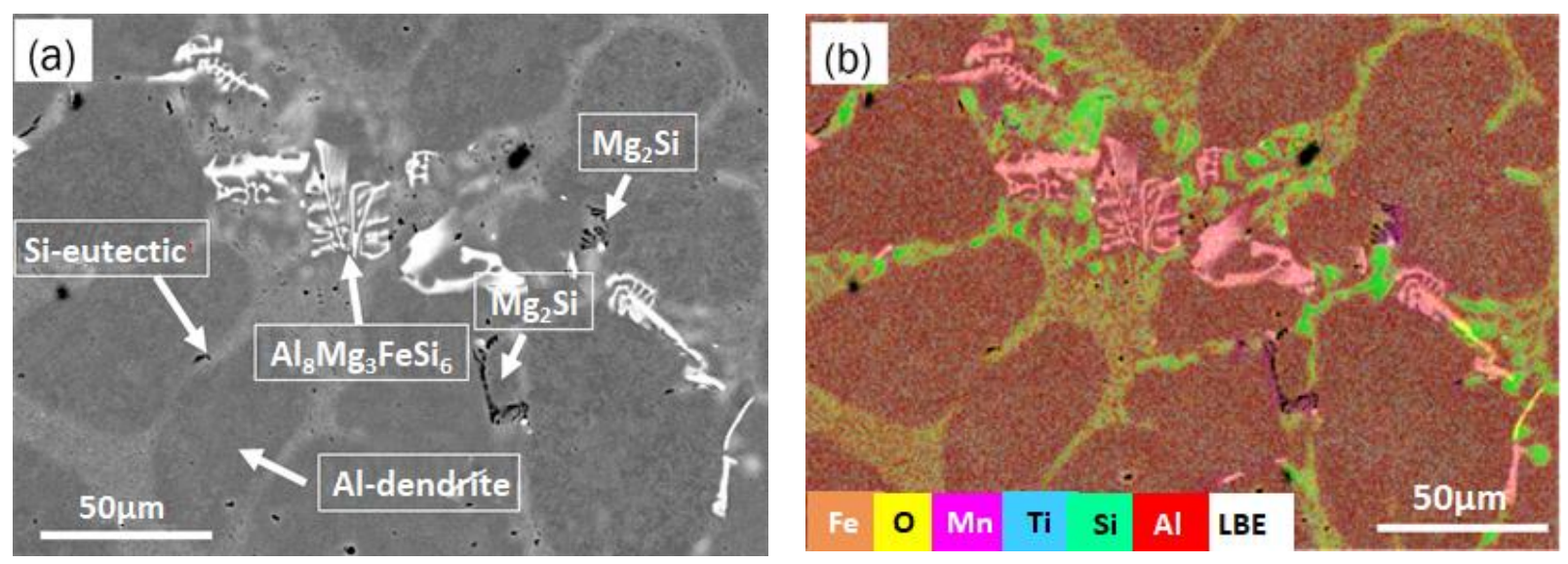

Figure II.1.7.4. (a) Phase identification in the A356 cast microstructure and (b) EDS mapping showing the evidence of the identified phases. No impurity elements, such as Ti from the ultrasonic probe, were detected. Source: PNNL. 
Figure II.1.7.2 previously showed the starting material and heat-treatment procedures carried out in the experiments for developing an alternate heat-treatment routine. The heat-treatment profile in Figure II.1.7.2 (b) was used by Eck Industries and included an intermediate hold temperature of $510^{\circ} \mathrm{C}$ before the sample is heated to the final solutionizing temperature of $525^{\circ} \mathrm{C}$. The samples quenched after the hold at $510^{\circ} \mathrm{C}$ (point $\mathrm{B}$ in Figure II.1.7.2 (b) correspond to "0" solutionization.

Figure II.1.7.5 shows the hardness of the samples for different solutionization times and subsequent natural aging/T7 heat-treatments. The data shows that the hardness of all the heat-treated samples was greater than in the as-cast condition. Hence, heating to $510^{\circ} \mathrm{C}$ (or higher) was enough to dissolve $\mathrm{Al}_{2} \mathrm{Cu}$ (as confirmed below in SEM images) and lead to strengthening relative to as-cast condition. It can be noticed that the effect of different solutionization duration on hardness is only reflected in the samples that are given $\mathrm{T} 7$ heat-treatment, whereas the hardness of the samples after 5 days of natural aging remains unaffected (or somewhat decreases) with increasing solutionization duration.

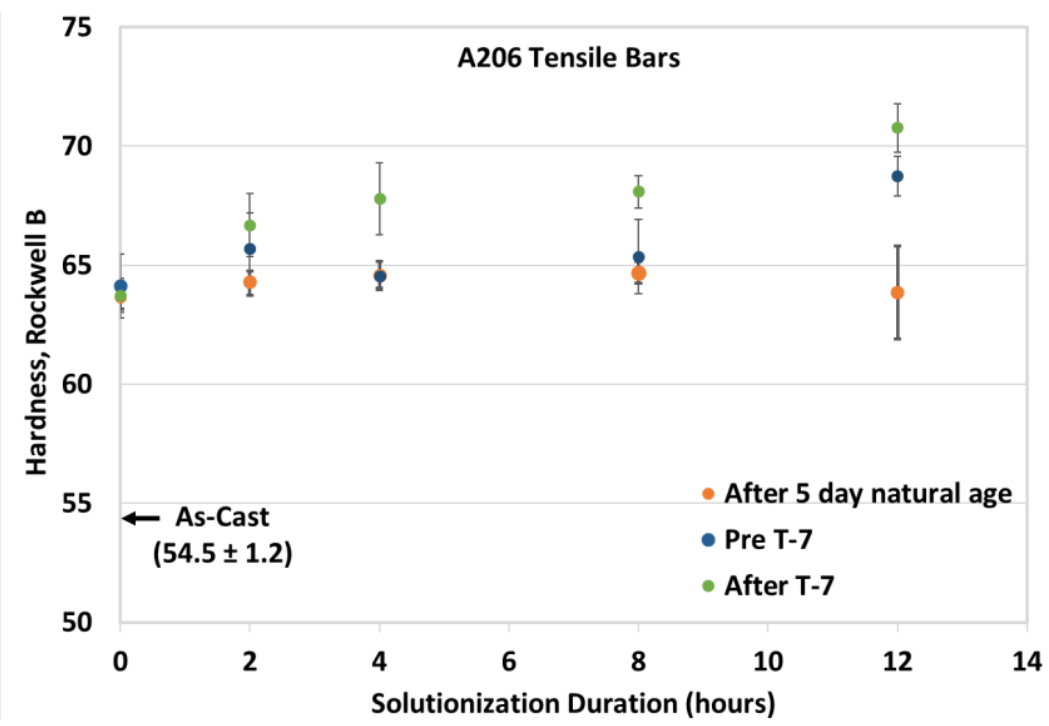

Figure II.1.7.5. Measured hardness in A206 after different solutionization durations and either natural aging or T7 conditions. Source: PNNL.

Representative SEM microstructures of the A206 alloy following different heat-treatments are shown in Figure II.1.7.6. Substantial dissolution of the second-phase $\left(\mathrm{Al}_{2} \mathrm{Cu}\right)$ can be observed not only in the $12 \mathrm{~h}$ solutionization case, but also in the $0 \mathrm{~h}$ solutionization heat-treatment sample implying that even a hold at $510^{\circ} \mathrm{C}$ (i.e. below $525^{\circ} \mathrm{C}$ ) was sufficient to dissolve the large $\mathrm{Al}_{2} \mathrm{Cu}$ precipitates.
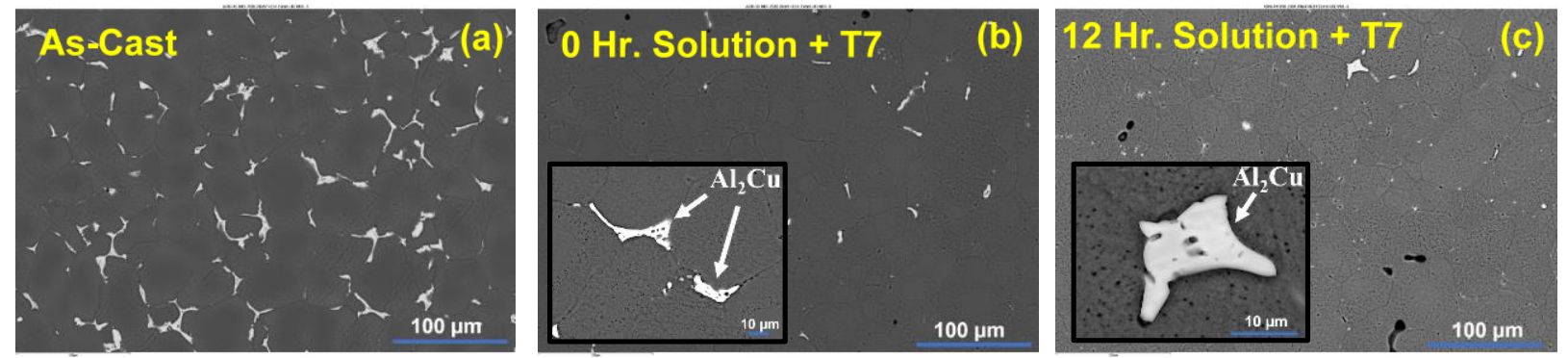

Figure II.1.7.6. SEM micrographs showing (a) as-cast microstructure and further effects of solutionization and T7 treatments for solutionization times of (b) $0 \mathrm{~h}$ and (c) $12 \mathrm{~h}$ in A206 Al alloy. Source: PNNL. 
Results from the microstructure are quantified in Figure II.1.7.7. Volume fraction and size of the second-phase $\left(\mathrm{Al}_{2} \mathrm{Cu}\right)$ was measured, where small volume fraction indicates more solute in the matrix and vice-versa. It was realized and confirmed that both the volume fraction and size of $\mathrm{Al}_{2} \mathrm{Cu}$ decreases as a result of an increase in the solutionization duration (i.e., from 0 to $12 \mathrm{~h}$.) which would imply relatively greater amount of $\mathrm{Cu}$ solute in the matrix (after $12 \mathrm{~h}$. solutionization) to produce precipitation strengthening during subsequent aging. Contrary to expectation, the average hardness for $12 \mathrm{~h}$. solutionization is somewhat lower than that for $0 \mathrm{~h}$. solutionization. It is likely that the precipitation kinetics of the fine-scale precipitates is too sluggish at RT to produce enough precipitation strengthening within 5 days. Some level of dispersion strengthening is also possible by the micron-sized $\mathrm{Al}_{2} \mathrm{Cu}$ particles, but any increase in strengthening due to their finer size (for $12 \mathrm{~h}$. solutionization) is likely countered by a corresponding reduction in the volume fraction. Hence, for the purposes of this project, it appears that the 5-day RT aging is not a suitable approach to differentiate between the effects of solutionization durations. Unlike RT aging experiments, the hardness after T7 heat-treatment showed a clear increase with an increase in solutionization duration. It is likely that the temperature-time $\left(200^{\circ} \mathrm{C}\right.$ for $4 \mathrm{~h}$.) used for the $\mathrm{T} 7$ treatment was enough to accelerate the kinetics of fine-scale precipitates and hence, the influence of solute content (on account of different solutionization durations) on precipitation strengthening was readily apparent. It is generally known in the literature that precipitation in the $\mathrm{Al}-\mathrm{Cu}$ system proceeds with the formation of Guinier-Preston zones and other intermediate phases ( $\theta$ ' and $\theta$ '") that are responsible for strengthening [5]. Since these phases can only be resolved in TEM, additional work would be needed to confirm the above analyses.

In summary, under the test conditions used in this project, solutionization time has negligible effect on the hardness of A206 Al alloy after natural aging for 5 days whereas a T7 heat-treatment showed a clear increase in hardness with increasing solutionization duration.

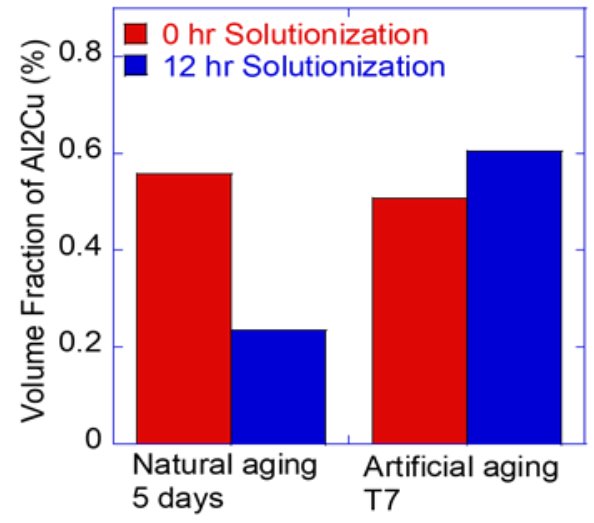

(a)

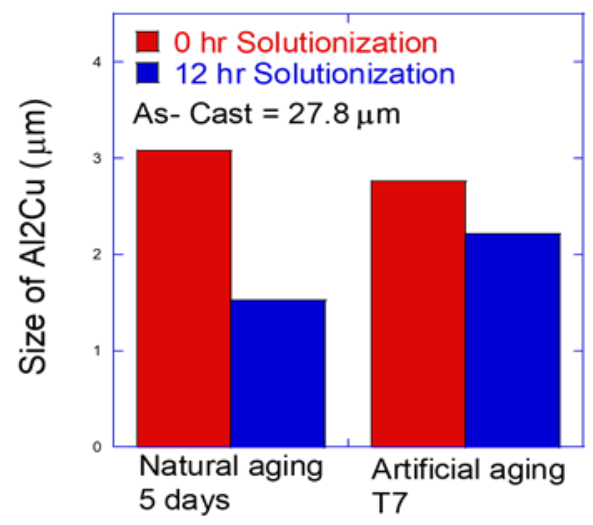

(b)

Figure II.1.7.7. Graphs showing the effect of different solutionization times (i.e., $0 \mathrm{~h}$ and $12 \mathrm{~h}$ ) followed by natural aging/ T7 treatments on: (a) volume fraction of $\mathrm{Al}_{2} \mathrm{Cu}$; and (b) size of $\mathrm{Al}_{2} \mathrm{Cu}$. Source: PNNL.

\section{Conclusions}

A molten metal processing approach is being developed where the molten metal is subjected to ultrasonic pulses prior to casting. A benchtop setup has been constructed and initial results on A356 Al alloy indicate improvement in the cast microstructure after ultrasonic treatment. Refinement in Al-dendrite arm spacing, and sizes of other intermetallic phases, were confirmed, which are expected to improve the mechanical properties of the casting. Additional experiments on quantifying the microstructure and mechanical properties of A356 alloy after different ultrasonic treatments are ongoing. In a parallel set of experiments, a modified heattreatment process is being developed for A206 Al alloy to shorten the duration and/or lower the solutionization temperature. It was found that in the conventional heat-treatment approach comprising solutionization followed by five-day natural aging, the post-aging hardness is generally unaffected by the duration of the solution heat-treatment. However, if the alloy is given a T7 heat-treatment following solutionization, then the 
hardness increases with increasing duration of the prior solutionization. Thus, the T7 heat-treatment can be used as a metric to compare the efficacy of the modified heat-treatment vs. the conventional approach. Further developments in the heat-treatment procedures and quantification of the effects of precipitates in $\mathrm{A} 206 \mathrm{Al}$ alloy will be completed by the end of FY 2020.

\section{References}

1. U.S. Geologic Survey (USGS), 2016, Minerals Yearbook 2015 - Volume I: Metals and Minerals, November 2016, https://doi.org/10.3133/mybvI.

2. Richman, D., 2015, "Driving innovation with automotive Al," Drive Aluminum website, available at: http://www.drivealuminum.org/research-resources/driving-innovation-with-automotive-aluminum-2/ (last accessed 10 December 2019).

3. Davis, J. R. (ed.), 1993, Aluminum and Aluminum Alloys - ASM Specialty Handbook, ASM International, Materials Park, OH, USA, p. 89.

4. Eskin, G. I., 1997, Principles of Ultrasonic Treatment: Application for Light Alloys Melt, Advanced Performance Materials, 4, pp. 223-232.

5. Davis, J. R. (ed.), 1993, Aluminum and Aluminum Alloys - ASM Specialty Handbook, ASM International, Materials Park, OH, USA, p. 291.

\section{Acknowledgements}

The technical contributions of PNNL staff supporting this research is gratefully acknowledged; in particular, Dr. Jens Darsell for his help in setting up the ultrasonic test capability, Dr. Tarang Mungole for performing the ultrasonic and heat-treatment experiments and associated analyses, Mr. Anthony Guzman for preparing metallographic samples, and Mr. Alan L Schemer-Kohrn for SEM Imaging. 


\title{
II.1.8 Room Temperature Stamping of High-Strength Aluminum Alloys (Pacific Northwest National Laboratory)
}

\author{
Aashish Rohatgi, Principal Investigator \\ Pacific Northwest National Laboratory \\ 902 Battelle Blvd. \\ Richland, WA 99354 \\ E-mail: aashish.rohatgi@pnnl.gov
}

\section{Sarah Kleinbaum, DOE Technology Development Manager}

\author{
U.S. Department of Energy \\ E-mail: sarah.kleinbaum@ee.doe.gov
}

Start Date: October 1, 2016

Project Funding: \$1,425,000
End Date: September 30, 2019

DOE share: $\$ 1,000,000$
Non-DOE share: $\$ 425,000$

\section{Project Introduction}

This project addresses the challenge that high-strength $\mathrm{Al}$ sheet alloys possess limited formability at $\mathrm{RT}$ and are, therefore, difficult to stamp into structural components with complicated geometries. In other words, the sheet metal is unable to deform uniformly and instead cracks during RT forming/stamping. Warm/hot-forming can increase the formability of $\mathrm{Al}$, but it is expensive due to the added costs of heated dies, forming lubricants, lubricant clean-up, etc. Unlike hot-stamped steels that undergo austenite to martensite phase transformation during quenching and achieve the high-strength at the end of the stamping process itself, Al alloy matrix does not undergo such phase transformation upon quenching. Hence, warm/hot-forming results in lower postformed strength such that heat-treatment, in addition to paint-bake, is needed (adding cost) to regain the strength. Thus, the use of an $\mathrm{Al}$ sheet is limited to lower strength $\mathrm{Al}$ alloys and/or simple-shapes (hood, deck lids, door inner, etc.) and many sheet components are still stamped in steel in the high-volume automotive industry. As such, the ability to form high-strength $\mathrm{Al}$ alloys cost-effectively (i.e., at RT), will expand $\mathrm{Al}$ use for structural components as well (crash beams, B-pillars, etc.) and has the potential to result in significant lightweighting. By further incorporating mathematical models pertaining to precipitation kinetics in highstrength $\mathrm{Al}$ alloys, we aim to predict the yield strength evolution before and after the stamping process; therefore, identify a favorable zone to stamp the high-strength $\mathrm{Al}$ sheet akin to industrial processing timescales.

\section{Objectives}

This project aims to develop processing techniques that will overcome the formability limitations of highstrength Al alloys and enable RT forming. Eventually, this knowledge must be leveraged to fabricate an automotive component. Thus, the project objectives are to:

- Identify automotive components that, if formed out of high-strength Al, can provide lightweighting, relative to if they were made of high-strength steels.

- Develop constitutive relations and perform stamping simulations to determine the feasibility of stamping a selected component out of a high-strength $\mathrm{Al}$ alloy.

- Integrate microstructure and mechanical property models for the selected $\mathrm{Al}$ alloy:

$\circ \quad$ Predict strength evolution during stamping and pre-/post-aging treatments using integrative modeling.

- Establish a process routine based on predictive modeling for effective RT stamping.

- Integrate microstructure and mechanical property models for the selected $\mathrm{Al}$ alloy. 


\section{Approach}

This project focuses on understanding the microstructure-formability-strength interplay in high-strength $\mathrm{Al}$ alloys with the expectation that microstructural control during processing could enable sufficient formability and strength in a prototypical automotive component. The project is structured to be performed in three phases.

In the first phase of the project (FY 2017), we performed a qualitative assessment of the commercial potential of stamped high-strength $7 \mathrm{xxx} \mathrm{Al}$ alloys. We surveyed the published literature and held discussions with industry partners. Consequently, multiple potential automotive components were identified that, if made from high-strength $\mathrm{Al}$, could provide a $30 \%$ to $50 \%$ weight-savings relative to high-strength steel. Based on the feedback from industry partners, a door side-impact beam was selected as the prototypical component for this project, and Al alloy AA 7075 was selected as the high-strength alloy.

In the second-phase of the project (FY 2017 to FY 2018), our goal was to determine the feasibility of achieving both high-strength and RT formability suitable for a side-impact beam made of AA 7075. An inproduction side-impact beam made of a hot-stamped steel, as seen in Figure II.1.8.1, was provided by GM as a prototypical component that could serve as an initial design for an Al side-impact beam. The mechanical property data of $\mathrm{Al}$ for these simulations was determined through mechanical testing at PNNL. The steel beam design was used as a basis by Magna to perform forming simulations using AA 7075 as the sheet material. It was established from simulations that the selected design made of AA 7075 is stampable.

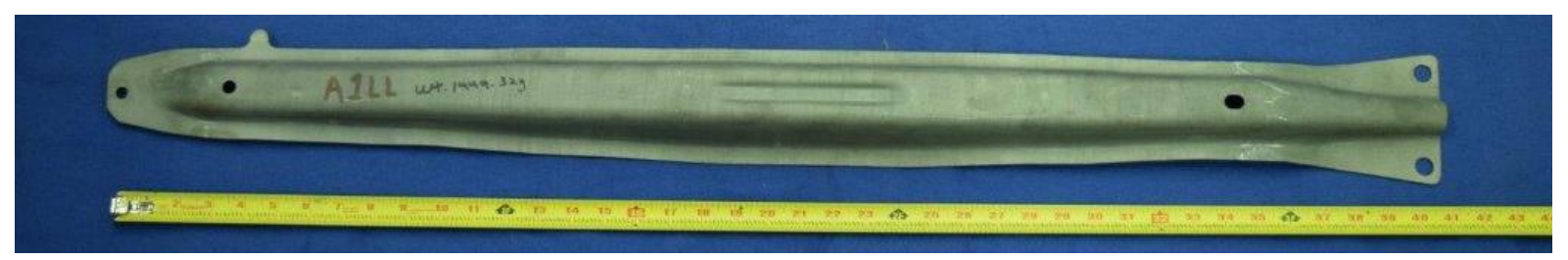

(a)

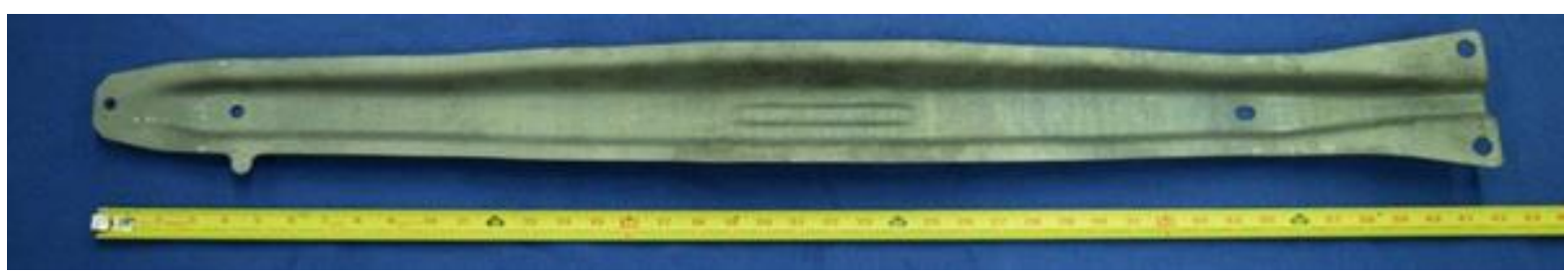

(b)

Figure II.1.8.1. Images of the steel side-impact beam: (a) top view, (b) bottom view. Source: PNNL.

In the third phase of the project (FY 2019), we are developing microstructure and mechanical property models of AA 7075 to study the evolution of its strength as a function of time after heat-treatment procedures (i.e., during natural/artificial aging). In our initial approach, we used age-hardening data of AA 7075 from experiments performed at PNNL, in conjunction with published literature data, and developed microstructure evolution models for a preliminary assessment of nucleation and growth kinetics of precipitates in terms of precipitate size and volume fraction. Subsequently, we evaluated methods to incorporate the effects of deformation, such as that imposed during stamping, on the resulting strength of AA 7075 sheet. The above initial approach allows us to show the applicability of our current mathematical model to predict essential features of strength evolution during natural aging and under stamping/straining conditions. We next performed in situ and ex situ small angle x-ray scattering (SAXS) and wide-angle x-ray scattering (WAXS) experiments at the National Synchrotron Light Source (NSLS), Brookhaven National Laboratory (BNL), to quantify the evolution of microstructure (e.g., precipitation kinetics) during natural aging of AA 7075 sheet being used in the project. The results of the above modeling work are described below. 


\section{Results}

It is generally understood that precipitation strengthening in AA 7075 proceeds through the nucleation and growth of Guinier-Preston (GP) zones, categorized as GP-I to GP-II zones, that transform into $\eta$ ' and $\eta$ precipitates with further growth [1]. Figure II.1.8.2 (a) shows an example of GP-I zone revealed via TEM in the as-received AA 7075-T6 sheet used in our work. The flow stress during natural aging and the modeling results obtained from microstructure evolution models and strength governing equations are shown in Figure II.1.8.2 (b) and Figure II.1.8.2 (c), respectively. Equations 1-2 describe classical mathematical relations that govern nucleation and growth kinetics of precipitates and are used here for modeling precipitation kinetics in AA 7075 during RT aging. Symbols used in the equations listed below are defined in Figure II.1.8.3.

$$
\begin{gathered}
\left.\frac{d N}{d t}\right|_{\text {mucleation }+ \text { growth }}=N_{0} Z \beta^{*} \exp \left(\frac{-\Delta G^{*}}{R T}\right)(1-\exp (-t / \tau) \\
\left.\frac{d R}{d t}\right|_{\text {nucleation }+ \text { growth }}=\frac{D_{s}}{R}\left(\frac{X_{s}-X_{e q}{ }^{i}}{X_{p p t}-X_{e q}{ }^{i}}\right)+\frac{1}{N} \frac{d N}{d t}\left(\alpha r^{*}-R\right)
\end{gathered}
$$

Subsequently, we estimate strength evolution during natural aging by applying Equations $3-5$ that essentially describe that the total strengthening is a combination of solid-solution strengthening, dislocation strengthening, and precipitation strengthening:

$$
\begin{gathered}
\Delta \sigma_{s s}=\frac{M G}{700} \varepsilon_{s s}^{3 / 2} \sqrt{c} \\
\Delta \sigma_{d}=M \alpha G b \sqrt{\rho} \\
\Delta \sigma=\Delta \sigma_{s s}+\sqrt{\Delta \sigma_{d}^{2}+\Delta \sigma_{p p t}{ }^{2}}
\end{gathered}
$$

(a)

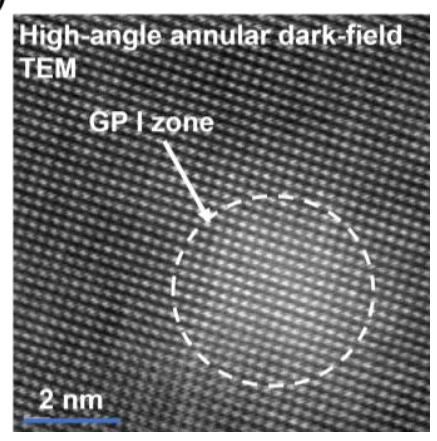

(b)

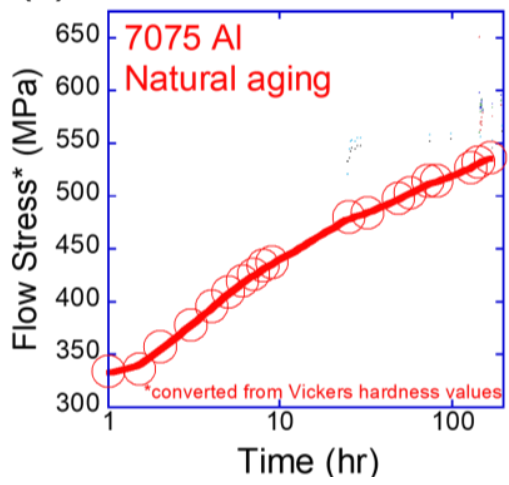

(c)

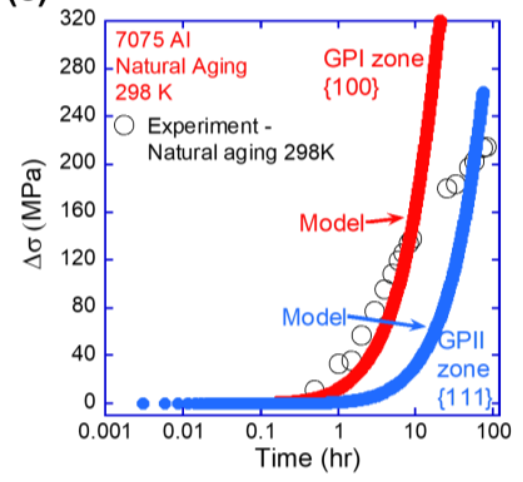

Figure II.1.8.2. (a) Dark field TEM image showing a GP-I zone. (b) Natural aging behavior of AA 7075 (flow stress vs time) after solutionization and quenching heat-treatment. (c) Modeling results obtained from microstructure evolution models and strength governing Equations 1-5, assuming strengthening only from GP-I zones (red curve) or only from GP-II zones (blue curve). Source: PNNL.

To consider the effects of stamping/deformation on precipitation kinetics, Equation 6 is combined with Equations 1-5 to include the coupling-effects of deformation on precipitation and vice-versa. Figure II.1.8.3 shows the physical meaning of all the constants used in Equations 1-6. 


$$
\frac{d \rho}{d \varepsilon}=k_{1} \sqrt{\rho}+\left(\frac{2 k_{d p}}{M \alpha G b}\right)\left(\frac{C_{S S}-C_{e q}}{C_{o}-C_{e q}}\right) \sqrt{\rho}-k_{2} \rho
$$

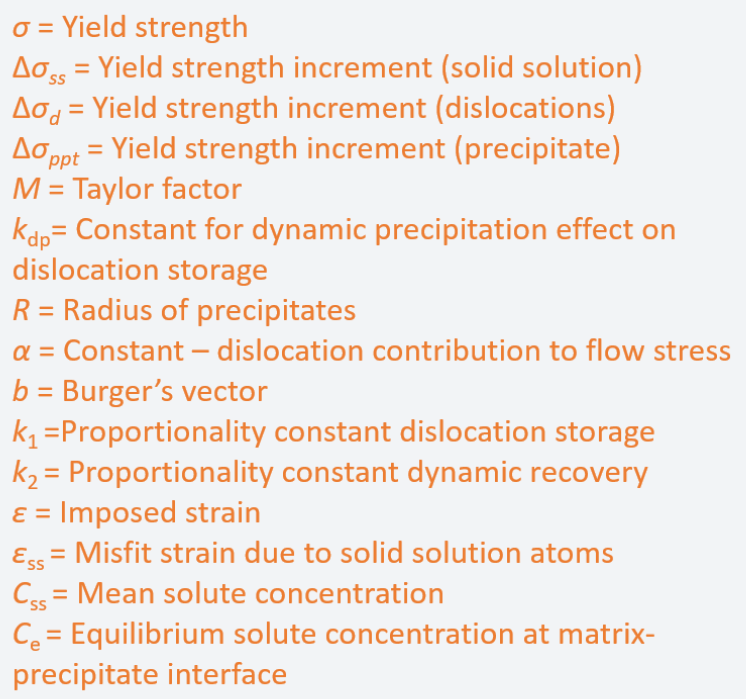

$C_{0}=$ Initial solute concentration

$\rho=$ Dislocation density

$N=$ Number of nuclei

$N_{0}=$ Potential nucleation sites per unit volume

$Z$ = Zeldovich's factor

$B^{*}=$ Attachment factor

$\Delta G=$ Gibb's free energy for nucleation

$\tau=$ Incubation time

$r^{*}=$ Critical radius for nucleation

$D_{\mathrm{s}}=$ Diffusivity of solute

$\alpha=$ Correction factor (used in eq. 2)

$X_{\text {eq }}{ }^{i}=$ Solute fraction in matrix at equilibrium

$X_{s}=$ Solute fraction in matrix

$X_{p p t}=$ Solute fraction in the precipitate

$c$ = Solute concentration in the matrix (for solutionstrengthening)

Figure II.1.8.3. Physical meaning of the constants used in Equations 1-6. Source: PNNL.

Figure II.1.8.4 shows the predicted yield strength behavior of AA 7075 where a strain of $6 \%$ is imposed during intermediate stages of aging to mimic the stamping procedure. Because our experiments and analyses to measure the microstructural evolution (e.g., precipitate radius and volume fraction) during deformation and natural aging were still in progress, which produced the graph in Figure II.1.8.5, we used the microstructural input parameters from SAXS/WAXS data from the literature [2]. In Figure II.1.8.4, our model is able to capture all of the important features of strengthening behavior in AA 7075, namely (1) increasing strength with aging time, (2) rapid increase in strength during straining, (3) a peak in strengthening vs. time curve and (4) strength decrease during over-aging.

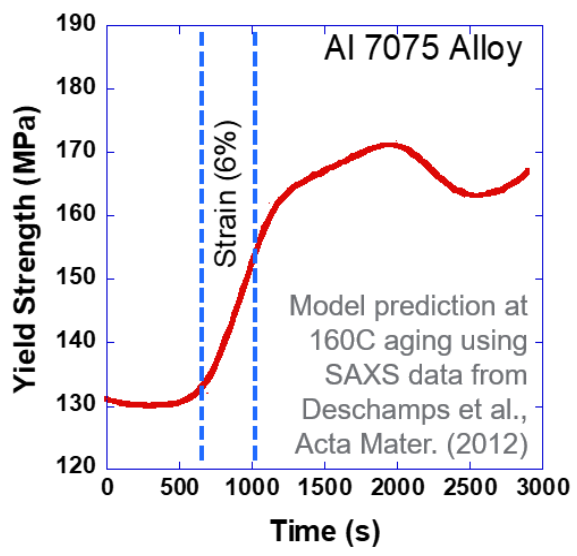

Figure II.1.8.4. Strength prediction in deformed AA 7075 during aging using the coupled deformation-precipitation model. Source: PNNL. 


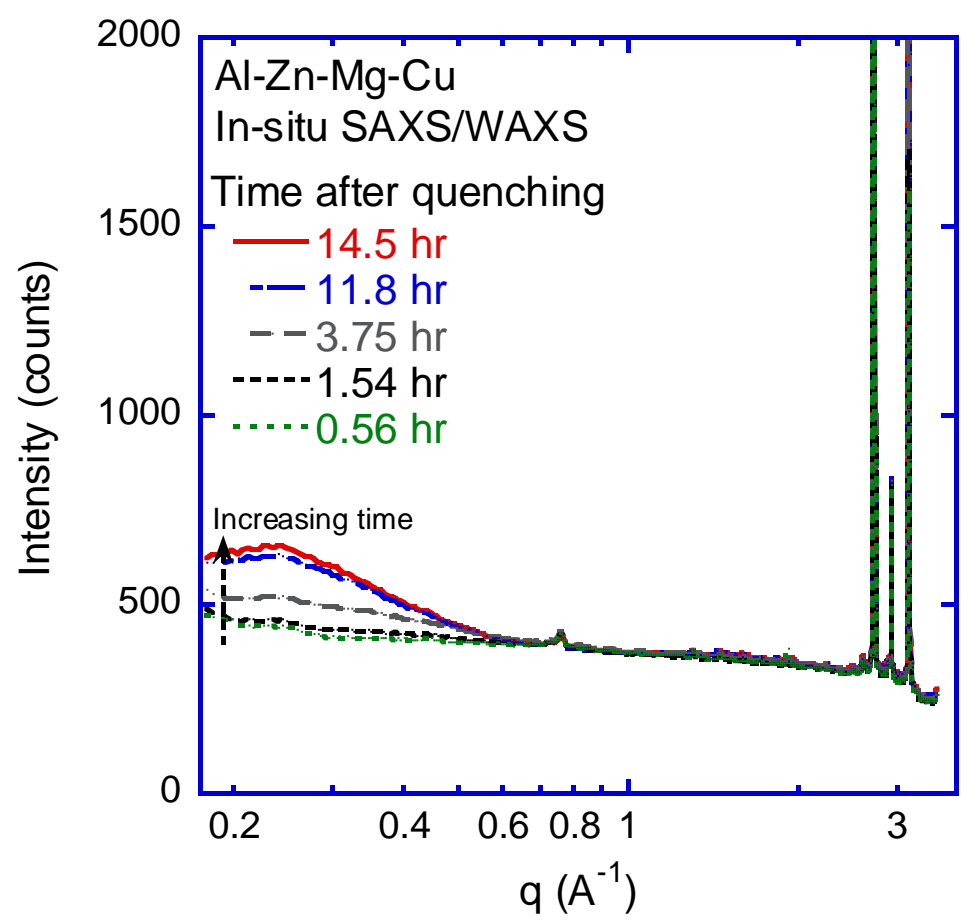

Figure II.1.8.5. Intensity (I) versus scattering-vector (q) plots from in situ SAXS/WAXS studies at NSLS-II, BNL, to study the precipitation kinetics during natural aging in AA 7075. Scattering at low angles (small q) is clearly visible owing to precipitate size and volume fraction evolution. Time is varied from $0.56 \mathrm{hr}$ to $14.5 \mathrm{hr}$ after solutionization and quench procedures. Source: PNNL/BNL.

Figure II.1.8.5 shows X-ray intensity (I) versus scattering-vector (q) plots from in situ SAXS/WAXS performed at NSLS-II, BNL on an AA7075 sheet. The I versus q plots at small q show changing scattering behavior (likely from GP zones) with time during natural aging immediately after solutionization and quenching procedures. In the first 15 hours, the low-angle scattering due to extremely small GP zones ( 1 nm) appears to change significantly. Additional analyses of this data are currently being performed and will be supplemented with data from experiments where the precipitation kinetics will be studied during in situ deformation of AA 7075.

\section{Conclusions}

The overall purpose of this project is to enable RT stamping of high-strength $\mathrm{Al}$ alloys to replace automotivestructural components that are typically made of steel. AA 7075 was selected as the representative highstrength Al alloy and a side-impact beam was selected as the prototype part to stamp at RT, demonstrating the feasibility of the proposed work. Our goal is to integrate microstructural and mechanical property models to help define appropriate thermomechanical processing conditions that will produce sufficient strengthening in the AA 7075 sheet without compromising its RT formability. Our preliminary modeling results suggest that the essential features of the age-hardening behavior of AA 7075 can be successfully captured. Our model also allows us to predict the pre- and post-forming strength, which will eventually allow us to optimize the stamping process. Further analyses on characterization of precipitation kinetics coupled with deformation, which will serve as input parameters in the model, are ongoing as part of the in situ experiments performed at x-ray beamlines. Finally, we will work with our industry partners to design a die and stamp a prototypical sideimpact beam in AA 7075 at RT. The stamped component will be characterized for mechanical behavior to evaluate the suitability of our stamping approach toward meeting the target properties. 


\section{References}

1. L. K. Berg, J. Gjønnes, V. Hansen, X. Z. Li, M. Knutson-Wedel, G. Waterloo, D. Schryvers, and L. R. Wallenberg, "GP zones in Al-Zn-Mg alloys and their role in artificial aging," 2001, Acta Mater., Vol. 49, No. 17, pp. 3443-3451.

2. A. Deschamps, G. Fribourg, Y. Bréchet, J. L. Chemin, and C. R. Hutchinson, "In situ evaluation of dynamic precipitation during plastic straining of an Al-Zn-Mg-Cu alloy," 2012, Acta Mater., Vol. 60, No. 5, pp. 1905-1916.

\section{Acknowledgements}

Technical contributions of the PNNL staff supporting this research is gratefully acknowledged. In particular, the authors are grateful to Dr. Tarang Mungole for developing mathematical models and securing synchrotron X-ray beamtimes; Mr. K. Mattlin, Mr. M. Dahl, Mr. T. Roosendaal, and Mr. M. Rhodes in the Mechanical Testing Lab for their help in conducting heat-treatments and mechanical tests; and Drs. M. Olszta and K. Kruska for their TEM analysis. We also acknowledge help from the beamline staff at NSLS-II, BNL; namely, Dr. Masafumi Fukuto and Dr. Ruipeng Li for conducting the SAXS/WAXS experiments and data analysis. This research used resources (11-BM) of the National Synchrotron Light Source, a DOE Office of Science User Facility operated for the DOE Office of Science by BNL under Contract No. DE-AC0298CH10886. Finally, we would like to acknowledge the technical support provided by Mr. B. Kokosza and his team at Magna-Stronach Centre for Innovation for their technical discussions and support, and the technical staff at GM R\&D — in particular, Mr. Jon Carter and Dr. Anil K. Sachdev, for their insightful comments and discussions and providing the steel impact beam for this research, as well as providing information on lightweighting analyses for automotive components. 


\title{
II.1.9 Graphene-Based Solid Lubricants for Automotive Applications (Argonne National Laboratory)
}

\author{
Anirudha V. Sumant, Principal Investigator \\ Argonne National Laboratory \\ 9700 S. Cass Ave. \\ Lemont, IL 60439 \\ E-mail: sumant@anl.gov

\section{Tim Skszek, Co-Principal Investigator} \\ Magna International \\ 750 Tower Dr. \\ Troy, MI 48098 \\ E-mail: tim.skszek@magna.com
}

Jerry L. Gibbs, DOE Technology Development Manager

U.S. Department of Energy

E-mail: jerry.gibbs@ee.doe.gov

Start Date: September 1, 2018

Project Funding (FY19): \$650,000
End Date: August 31, 2020

DOE share: $\$ 640,000$
Non-DOE share: $\$ 10,000$

\section{Project Introduction}

Stamped sheet metal components typically account for $30 \%$ to $35 \%$ of the total mass of a typical passenger vehicle. The BIW, chassis, and closure components define the structural integrity of the vehicle characterized by stiffness, durability, safety, and ride and handling parameters. Materials selection and design efficiency of stamped components significantly impact the vehicle's safety and mass-induced fuel efficiency.

Stamped Al and steel sheet metal components are typically formed at RT. RT metal stamping processes often include the use of water-based, oil-based, or solid lubricants to enable manufacturing of an efficient design by reducing the coefficient of friction between the stamping blank and the die surface, improving the formability of the material. The high coefficient of friction between the sheet metal blank and die surface during the forming operation results in a localized thinning condition, which compromises design efficiency and structural performance. Oil- and wax-based lubricants provide benefits up to approximately $250^{\circ} \mathrm{C}$.

To increase design efficiency, high-strength $7 \mathrm{xxx}$-series $\mathrm{Al}$ and ultra-high-strength steel alloys are used to increase energy absorption of safety components and enhance occupant safety of a passenger vehicle. Highstrength $\mathrm{Al}$ and ultra-high-strength steel alloys are processed at temperatures of $480^{\circ} \mathrm{C}$ and $930^{\circ} \mathrm{C}$, prohibiting the use of die lubricants used in RT forming processes. Solid lubricants, such as boron nitride, provide functional benefits at elevated temperatures up to $1,000^{\circ} \mathrm{C}$, but are not used due to costs associated with procurement, recovery, build-up on die surfaces, and secondary processing to enable adhesion of paint primer, sealants, and structural adhesives.

\section{Objectives}

1. Demonstrate a reduction in the friction coefficient between a 7-xxx Al sheet and a steel form die to enable cost-effective mass reduction of stamped Al safety components.

2. Demonstrate a reduction in the friction coefficient between a boron steel sheet and a steel form die to enable cost-effective mass reduction of stamped ultra-high-strength automotive-structural components. 
3. Demonstrate the ability to dispense a graphene-based lubricant in a cost-effective and environmentally safe manner.

4. Demonstrate the lack of build-up of the graphene-based lubricant on die surfaces.

5. Demonstrate the ability to cost-effectively remove the die lubricant from the surface of the formed component part.

\section{Approach}

Figure II.1.9.1 provides a look at our approach for the development of lubricants in this effort.

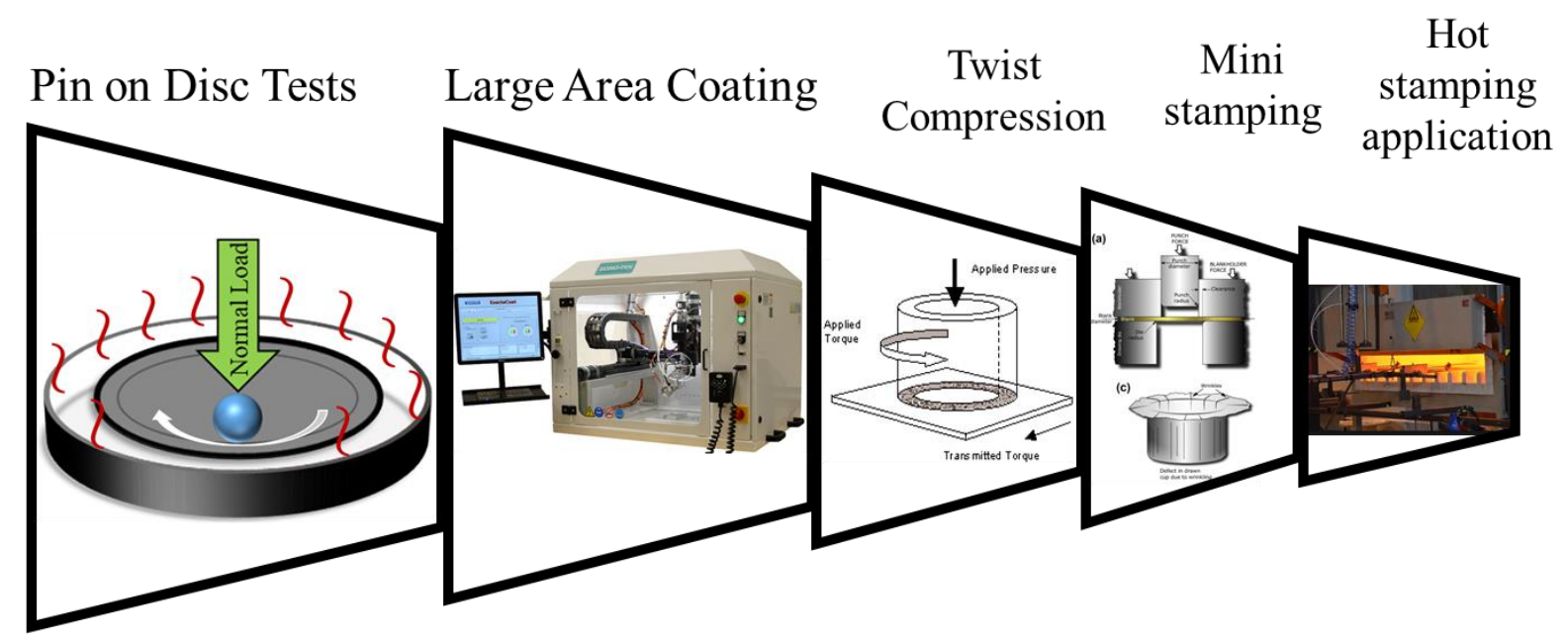

Months $1-8$

Months $9-16 \quad$ Months $17-24$

Figure II.1.9.1. Approach in the development of lubricants. Source: ANL.

\section{Pin-on-Disc Testing}

- Determine the friction and wear characteristics for various graphene-based solid lubricants applied to Al sheets using pin-on-disc testing apparatus. Multiple solid lubricant coatings are applied to the boron steel sheet surface and tested at RT and $275^{\circ} \mathrm{C}$.

- Determine the friction and wear characteristics of various graphene-based solid lubricants applied to boron steel sheets using pin-on-disc testing apparatus. Multiple solid lubricant coatings are applied to the boron steel sheet surface and tested at a RT and $275^{\circ} \mathrm{C}$.

\section{Twist-Compression Cup Testing}

- Determine the reduction in friction coefficient associated with the use of a graphene-based lubricant, $\mathrm{Al}$ sheets, and steel die surfaces at elevated temperatures using the twist-compression cup test apparatus. Evaluate the functional benefit of multiple solid lubricant coatings at temperatures of $230^{\circ} \mathrm{C}$ and $480^{\circ} \mathrm{C}$, respectively.

- Determine the reduction in friction coefficient associated with the use of a graphene-based lubricant, boron steel sheet, and steel die surfaces at elevated temperatures using the twist-compression cup test apparatus. Evaluate the functional benefit of graphene and other 2D materials-based solid lubricant coatings at a temperature of $930^{\circ} \mathrm{C}$. 


\section{Large Area Coating Apparatus}

- Characterize physical properties of graphene-based lubricant for dispensing application.

- Develop and demonstrate means to dispense solid lubricants efficiently onto a die surface.

\section{Deep Draw Testing (Planned)}

- Determine the functional benefit associated with the use of a graphene-based lubricants on the formability characteristics of $\mathrm{Al}$ sheets at elevated temperatures using the deep draw test apparatus. Evaluate the functional benefit of multiple graphene-based solid lubricants at blank temperatures of $230^{\circ} \mathrm{C}$ and $480^{\circ} \mathrm{C}$, respectively.

- Determine the functional benefit associated with the use of a graphene-based lubricant on the formability characteristics of $\mathrm{Al}$ sheets at elevated temperatures using the deep draw test apparatus. Evaluate the functional benefit of graphene and other 2D materials-based solid lubricants at blank temperatures of $930^{\circ} \mathrm{C}$.

\section{Results}

Two lubricants - Water-based High-Temperature Lubricants (WHTL1 and WHTL2) (suspended in water) were developed from the graphene-based 2D materials. While WHTL1 was developed for steel substrates, WHTL2 was suitable for the Al base. The lubricant volume coated onto the disc was about $1 \mathrm{~mL}$. The coated substrate and Raman spectra after coating are shown in Figure II.1.9.2. The pin-on-disc test carried out at $275^{\circ} \mathrm{C}$ revealed that the friction lowered from over 1.5 to 0.1 with a total reduction of $94 \%$. The wear track after test showed small scar on the surface. Relative to the bare substrate where the wear volume loss was $0.613 \mathrm{~mm}^{3}$, the wear loss was reduced to $0.456 \mathrm{~mm}^{3}$ due to the presence of the lubricant. For the case of highstrength Al, WHTL2 showed a reduction of $66 \%$ friction from bare substrate rubbing against the ball. The wear on the substrate was $1.115 \mathrm{~mm}^{3}$, whereas there was no calculable wear loss due to the presence of the lubricant. Both lubricants could be completely removed by subjecting them to a waterjet cleaning. The graphene-based 2D materials in the two lubricants are completely biodegradable and fully removable by waterjet cleaning, rendering the technology highly amenable for the application it is being targeted for. The results of the lubricant, Raman spectra, friction graph, and wear loss with and without the coating are shown in Figure II.1.9.3.

Following ascertaining the low friction metrics of the two lubricants, WHTL1 and WHTL2 were tested for their performance in twist-compression tests. The die cups in these tests were coated with the lubricants and the cups inserted into the cup holder. The cup was then pressed against steel or Al test blanks under a predetermined load as the cup was rotated while continuing to press against the blank. Friction was recorded as the cup rotated under the load. The friction trends were similar to the pin-on-disc tests, as shown in Figure II.1.9.4. The WHTL1 and WHTL2 showed significantly lower friction compared to bare metal-metal contact. Relatively, the friction was also lower compared to the state-of-the-art commercial lubricants currently being used in the industry. The bare substrate was observed to have a friction of about 0.35-0.4. Commercial lubricant lowered friction to 0.1 and both WHTL lubricants were consistently below 0.1 . 


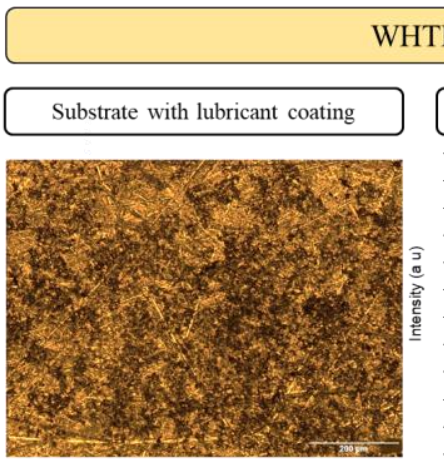

WHTL1 on STEEL tested at $275^{\circ} \mathrm{C}$
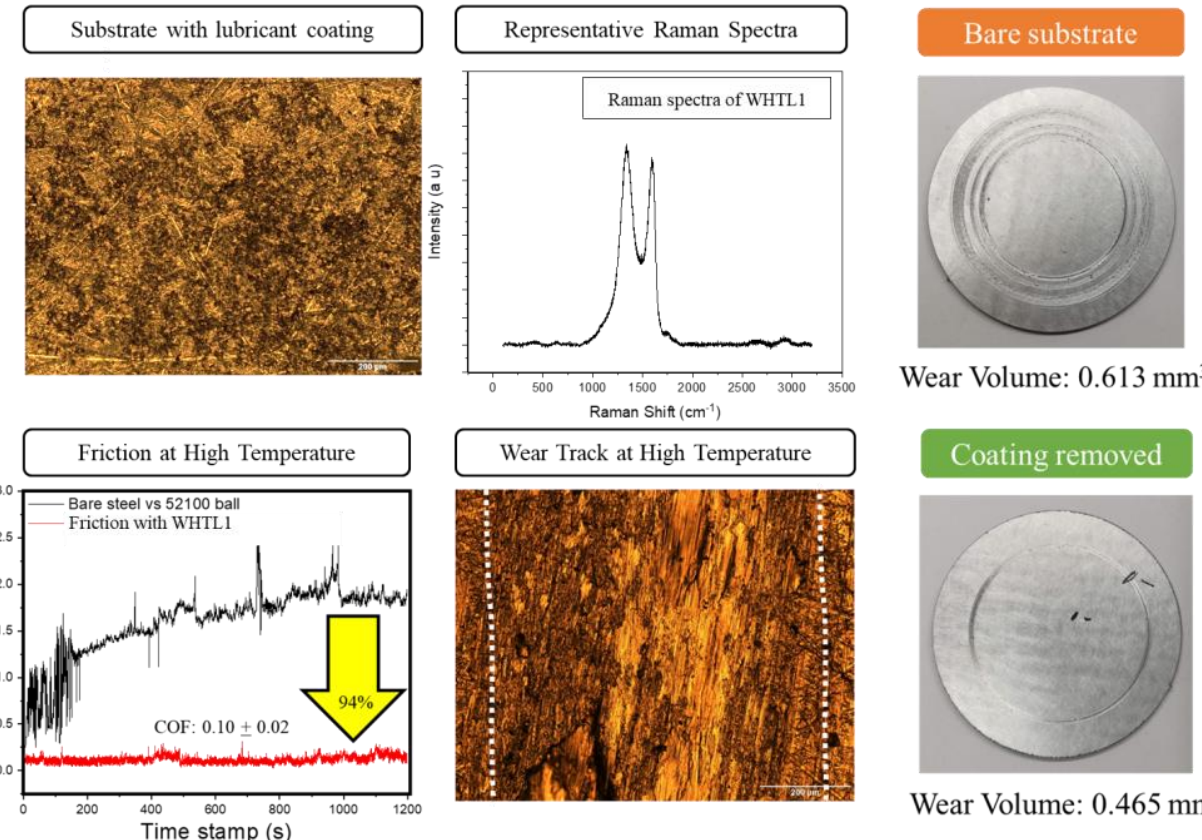

Wear Volume: $0.613 \mathrm{~mm}^{3}$

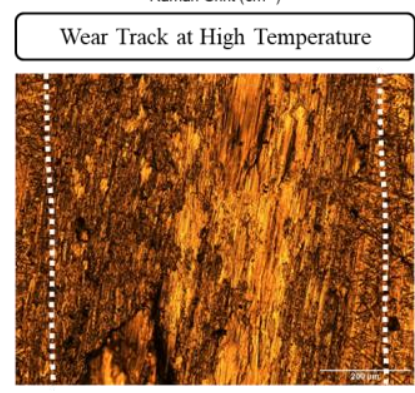

\section{Coating removed}

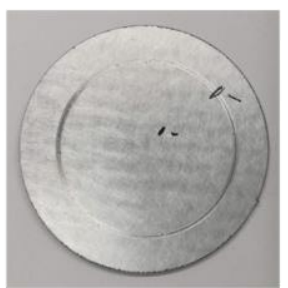

Wear Volume: $0.465 \mathrm{~mm}^{3}$

Figure II.1.9.2. (top) WHTL1 coating on the steel sample and Raman spectroscopy of the coating deposited on to the substrate with a wear volume on the bare disc of $0.613 \mathrm{~mm}^{3}$. (bottom) The graph of friction at a high-temperature with the lubricant down to 0.1 which was minimal and a wear volume after the coating was removed of $0.465 \mathrm{~mm}^{3}$. Source: ANL.
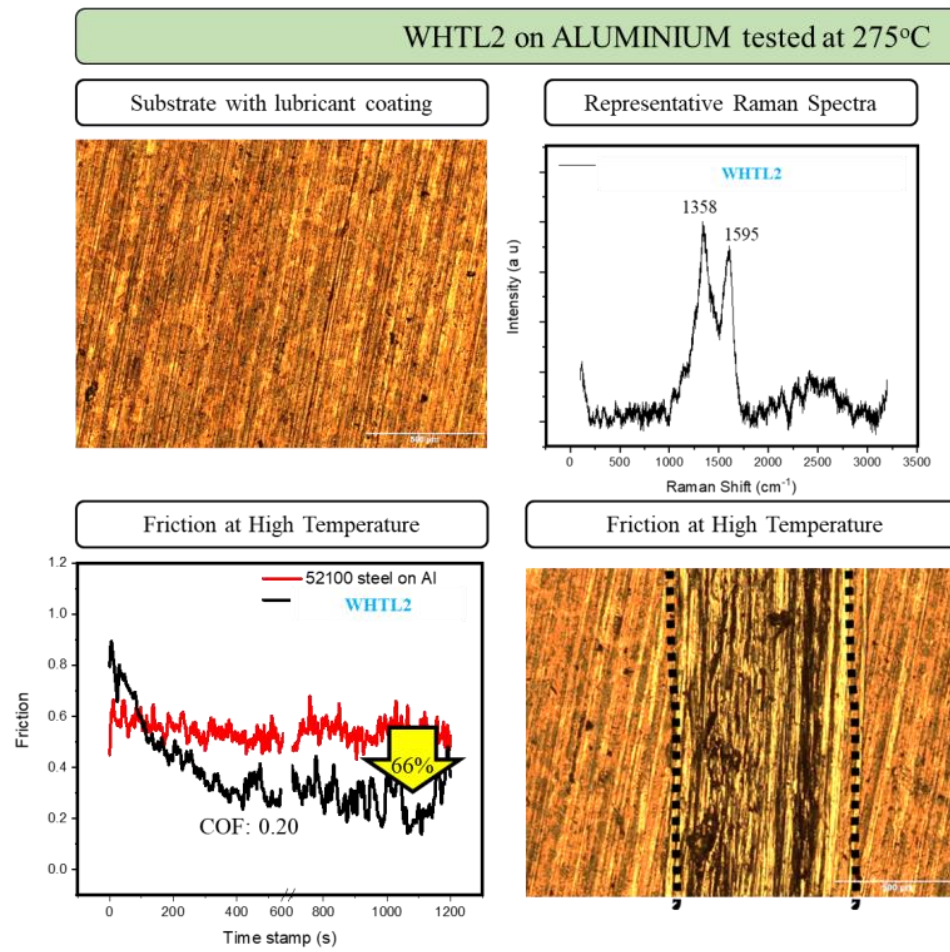

Bare substrate

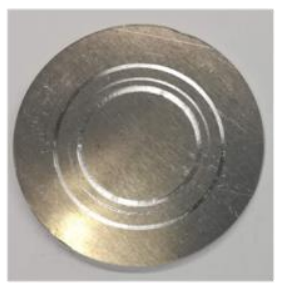

Wear Volume: $1.115 \mathrm{~mm}^{3}$
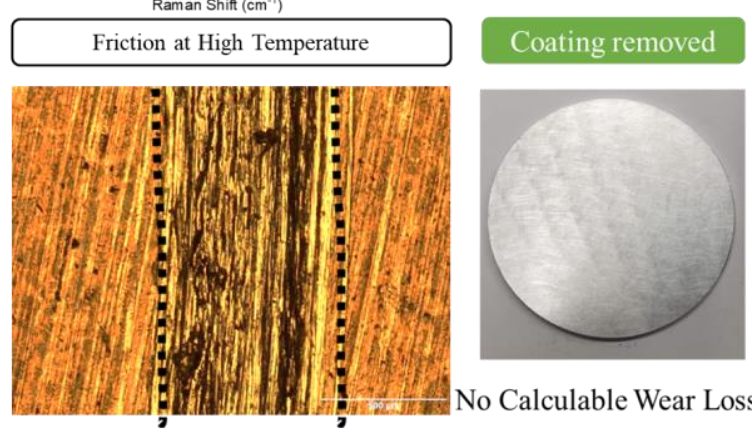

No Calculable Wear Loss

Figure II.1.9.3. (top) WHTL2 coating on the Al substrate and the Raman spectra of the lubricant showing characteristic peaks of the 2D materials present and a wear volume on the bare disc of $1.115 \mathrm{~mm}^{3}$. (bottom) A graph of the friction at a high-temperature showing $66 \%$ reduction in friction when compared to the lubricant-free substrate after wear-testing with the coating removed showing no calculable wear loss. Source: ANL. 


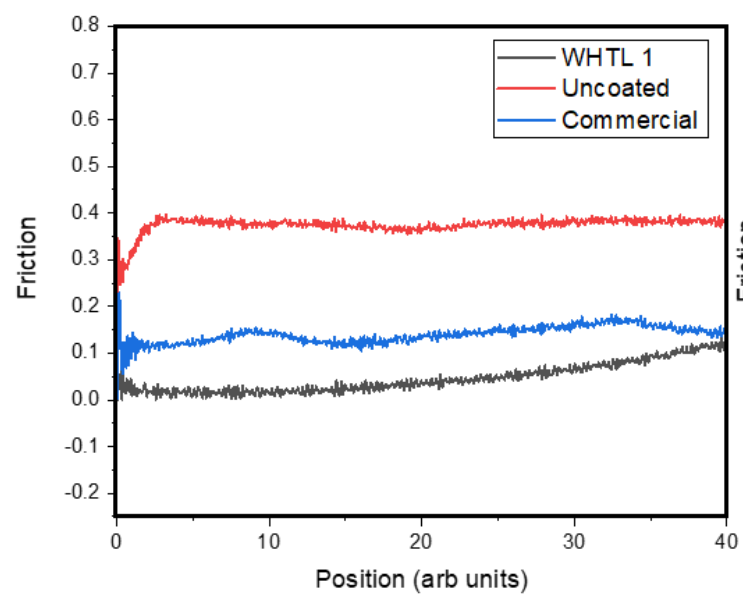

(a)

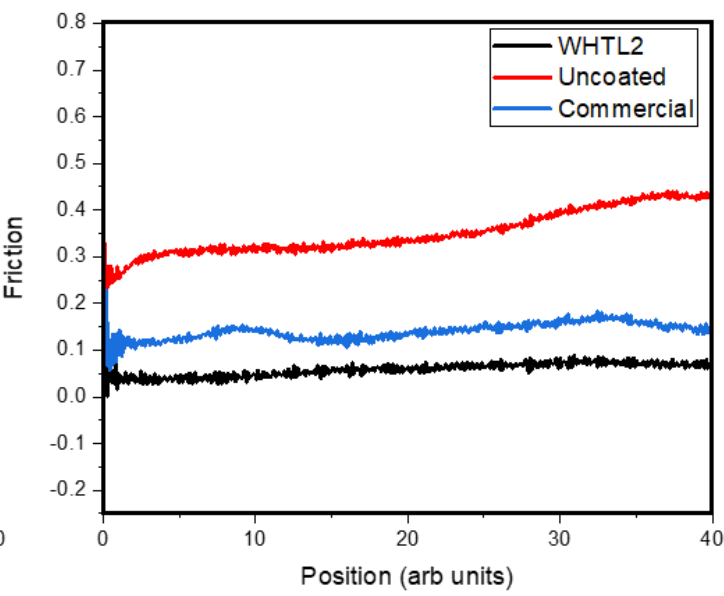

(b)

Figure II.1.9.4. Friction coefficient recorded on the twist-compression-testing machine with the developed lubricants during warm-forming in a temperature range of $275-480^{\circ} \mathrm{C}$. (a) The bare substrate was observed to have a friction of about 0.35-0.4. A commercial lubricant lowered the friction to 0.1 and WHTL1 produced a friction of 0.03-0.05. (b) WHTL2 produced a friction of 0.02-0.03. Source: ANL.

In comparison, WHTL1 produced a friction less than 0.03-0.05, whereas the WHTL2 showed 0.02-0.03 friction. In addition to these two lubricants used for 'warm' forming (temp range $275^{\circ} \mathrm{C}-480^{\circ} \mathrm{C}$ ), a third set of lubricants was developed to accommodate even higher temperature metal-forming operations (i.e., the blank temperature of $930^{\circ} \mathrm{C}$ and the temperature during contact to the blank of $790^{\circ} \mathrm{C}$ ). The area of high-temperature metal-forming is challenging from the materials and testing point of view. Very few selected materials can survive at high temperatures at which the hot-forming takes place, and secondly, state-of-the-art pin-on-disc machines lack the technical capability to achieve the relevant high temperatures. Hence, for this set of lubricants, the pin-on-disc tests were skipped, and a battery of tests were directly executed on twistcompression testing. The new combination of 2D solid lubricants (SL1) was tested on a steel substrate and showed a drastic reduction in friction from 0.35-0.10, as shown in Figure II.1.9.5. Attempts are underway to modify the SL1 materials to perform even better in terms of reducing the coefficient of friction to a lower value. Deep draw testing will be the next phase for further testing of these solid lubricants.

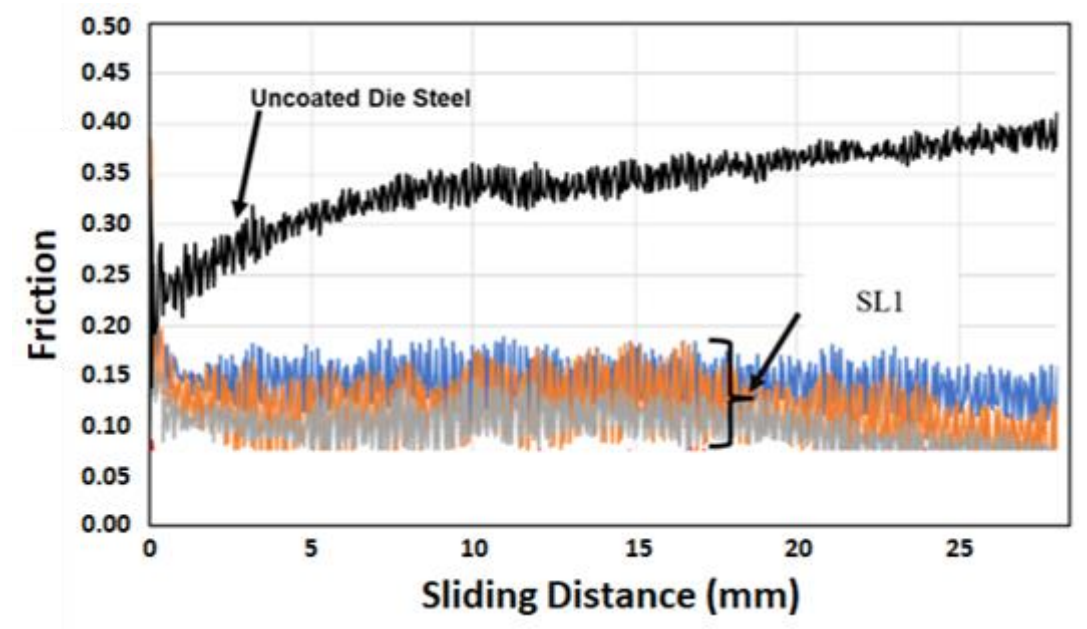

Figure II.1.9.5. Friction recorded on the twist-compression testing machine with lubricants for high-temperature $\left(790^{\circ} \mathrm{C}\right)$. The hot-forming lubricants (SL1) was observed to have a friction of 0.1-0.15 with whereas the commercial lubricant had shown a friction of 0.05 . The lubricant is seen to show consistent performance over three turns as shown in the graph.

Source: ANL. 


\section{Conclusions}

Three types solid lubricants developed for mitigating friction at warm and high temperatures have been shown to be effective. The friction was observed to lower by $94 \%$ and $66 \%$ for steel and Al, respectively, in pin-ondisc configuration, and the friction values were much lower at $0.02-0.05$ for both lubricants when tested in twist-compression testing. A new combination of 2D solid lubricants was also tested on a steel substrate and showed a drastic reduction in friction from $0.35-0.10$ with the potential for even lower friction reduction. The fact that these lubricants are applied to a die instead of metal foils and have shown satisfactory performance even for repeated cycles is very promising and is expected to help in reducing cost and improving the efficiency of the forming process.

\section{Key Publications}

One patent disclosure from Argonne (ANL-19-016) has been submitted.

\section{Acknowledgements}

Argonne would like to acknowledge excellent and productive collaboration with the Magna team and our university partners. The Magna team's support and suggestions during project planning, execution, and operation have been outstanding. Thus far, it has been fun to work with such an excellent team of professionals.

Funding from DOE, Technology Commercialization Grant TCF-17-13538. 


\title{
II.2 Carbon Fiber \& Polymer Composites
}

\section{II.2.1 Integrated Computational Materials Engineering Predictive Tools for Low-Cost Carbon Fiber for Lightweight Vehicles (University of Virginia)}

\section{Dr. Xiaodong (Chris) Li, Co-Principal Investigator}

University of Virginia

122 Engineer's Way

Charlottesville, VA 22904

E-mail: xl3p@ virginia.edu

\section{Dr. Leonid V. Zhigilei, Co-Principal Investigator}

University of Virginia

Wilsdorf Hall, Room 303D

Charlottesville, VA 22904

E-mail: 1z2n@virginia.edu

\section{Dr. Adri van Duin, Co-Principal Investigator}

Pennsylvania State University

240 Research Building East

University Park, PA 16802

E-mail: acv13@psu.edu

\author{
Dr. James W. Klett, Co-Principal Investigator \\ Oak Ridge National Laboratory \\ 1 Bethel Valley Road \\ Oak Ridge, TN 37831 \\ E-mail: klettjw@ornl.gov
}

\author{
Mr. Billy Harmon, Co-Principal Investigator \\ Solvay Composite Materials, Carbon Fibers \\ P.O. Box 849 \\ Greenville, SC 29602 \\ E-mail: billy.harmon@solvay.com
}

\section{Dr. H. Felix Wu, DOE Technology Development Manager}

U.S. Department of Energy

E-mail: felix.wu@ee.doe.gov

Start Date: October 1, 2017

End Date: September 30, 2020

Project Funding (FY19): \$1,163,965 DOE share: \$1,019,370 Non-DOE share: $\$ 144,595$

\section{Project Introduction}

In response to consumer demand for fuel efficient vehicles and stringent vehicle emission regulations, automotive manufacturers are searching for alternative lightweight, high-strength materials to replace conventional metal structures in vehicle designs. Even compared to high-strength metal alloys, CFRP composites are a promising alternative due to their superior strength-to-weight ratio. Recent estimates have predicted that automotive weight reductions of $50 \%$ or greater will be necessary, in addition to enhanced 
engine and drive train efficiencies, to meet national and international emission standards [1,2]. However, there are significant technical barriers that must be overcome to bring CFRP materials into widespread acceptance. CFRP materials are produced via a complex and expensive procedure and are often limited to small-scale production. A large portion of this cost is born by the precursor material preparation- $51 \%$ of the total CF cost may be attributed to the precursor fibers [3,4]. Therefore, this project aims to develop low-cost, alternative precursors and processing techniques through the implementation of an ICME framework to evaluate precursor conversion kinetics. This framework will be used to down-select precursors for laboratory-scale and pilot-scale production to validate resulting CFRP material properties and process cost-savings.

Currently, the highest quality CFs are produced from polyacrylonitrile (PAN) precursors, which have a high carbon yield and ideal polymer structure for conversion into CFs with excellent mechanical properties. Typical yields are $\sim 50 \% \mathrm{CF}$ for the quantity of PAN precursor at the start of conversion. In literature, the highest quality commercially available CFs achieve strengths of up to $7 \mathrm{GPa}(1000 \mathrm{ksi})[2,5,6]$; however, PANderived CFs are expensive due to their high precursor cost- and energy-intensive processing, with costs of $\sim 25 / \mathrm{kg}$ subject to petroleum price fluctuations. Although advanced manufacturing techniques may further lower the price toward $\$ 15 / \mathrm{kg}$, PAN-derived fibers will likely remain too expensive for widespread adoption in the automotive industry $[7,8]$.

Therefore, the two most promising approaches to reduce the cost of CFs are to (1) utilize new, low-cost precursors and (2) reduce the energy requirements of CF-processing. In FY 2019 of this work, we applied the ICME framework assembled in Year 1 to study alternative precursors, including polyethylene (PE), nylon, poly $(p)$-phenylene-2,6-benzobisoxazole (PBO), and others. From laboratory-scale experimentation, we have determined that both PE and nylon-based fibers can achieve the mechanical property targets. We concluded the FY 2019 with preliminary pilot-scale production of nylon CF with encouraging results.

\section{Objectives}

The first objective is to develop, integrate, and demonstrate an ICME framework and evaluate alternative precursors for suitability to manufacture low-cost CF. This ICME framework will predict CF properties, such as load-to-failure, failure mode, stiffness/deflection, dynamic performance, and microstructures, and it must be capable of minimum modeling element accuracies within $15 \%$ of measured properties, which will enable design, development, and optimization of precursor chemistry and kinetics associated with the CF conversion process. The ICME framework will also include methodologies to simulate the manufacturing process(es), including variability from both process and material. While the ICME framework was originally developed for the PAN precursor, it is being adapted for the down-selected alternative precursors over FY 2019 and FY 2020.

The second objective is to research, develop, manufacture, and demonstrate CF precursor technology and processing techniques, where $\mathrm{CF}$ is a material consisting of thin, strong multi-crystalline filaments of carbon used as a reinforcement material especially in resins, capable of achieving the requirements in Table II.2.1.1.

Table II.2.1.1. Project Parameters and Requirements.

\begin{tabular}{|c|c|c|}
\hline Parameter & \multicolumn{2}{|c|}{ Requirement } \\
\hline Cost & \multicolumn{2}{|c|}{$\leq \$ 5 /$ pound } \\
\hline Strength & $\geq 250 \mathrm{Ksi}$ & $\geq 1.725 \mathrm{GPa}$ \\
\hline Modulus & $\geq 25 \mathrm{Msi}$ & $\geq 172.5 \mathrm{GPa}$ \\
\hline Strain & \multicolumn{2}{|c|}{$\geq 1 \%$} \\
\hline
\end{tabular}


Project performance is planned to span three federal FY BPs and includes the following tasks:

1. Assemble an ICME framework to model the conversion of precursors into CF, accounting for initial polymer properties and the stabilization, carbonization, and graphitization processes.

2. Validate ICME accuracy on PAN precursors against literature results and lab-scale CF synthesis, using multiscale microstructural and mechanical property characterization techniques.

3. Extend the ICME to model and evaluate alternative precursors for low-cost CF.

4. Perform pilot-scale production to down-select precursors for low-cost CF, based on cost, performance, and properties.

5. Evaluate the performance of low-cost CF via multiscale mechanical testing, characterization, and modeling.

\section{Approach}

A closed-loop approach has been adopted to assemble the ICME framework over the course of the project period, in which a cyclic system of experimentation and computational modeling refines the framework to reduce error. The core of the framework development is computational modeling; these models input parameters such as precursor chemistry, microstructure, and process temperatures and duration to extrapolate the resultant chemistry via ReaxFF (reactive force field) simulations $[9,10]$ and mechanical properties via large-scale MD simulations $[11,12]$. The necessary input parameters and validation points are provided by experimental testing, which generates a wealth of mechanical properties and chemical characterization data at distinct stages during fiber conversion. The cyclic nature of running and refining the models with experimental results has achieved significant results in FY 2019 of the project.

The present ICME predictive tools for low-cost CF for lightweight vehicles project is organized into 11 tasks executed over a 36-month-period. An outline of the tasks carried out in FY 2019 follows:

- Task 2.1: Statistical analysis of alternative precursor oxidation and carbonization.

- Task 2.2: Understanding the chemical conversion of alternative CFs.

- Task 2.3: Model integration via fiber/matrix mechanics study.

- $\quad$ Task 2.4: Synthesis and evaluation of prototype CFs.

\section{Results}

The ICME framework was developed and validated around PAN-based CFs during FY 2018, and it can predict the properties of PAN-based CF within 15\% error. This critical milestone indicates a baseline level of success and reliability in the assembled framework, which was extended to analyze alternative precursors in the FY 2019. The ICME framework is constructed from ReaxFF MD simulations, which yield the fiber chemistry due to conversion, and large-scale MD simulations, which elucidate polymer matrix/fiber mechanics and properties. This framework requires key input parameters (i.e., precursor chemistry, chemical structure, and conversion parameters) and validation points (i.e., fiber chemistry, gas products, and mechanical properties), which are supplied by experimentation. Furthermore, experimentation has identified new conversion steps, such as mixing graphene additives within the polymer, ultraviolet (UV) radiation, microwave radiation, and nylon/CuCl treatments, which are in turn investigated by the ICME framework to elucidate the underlying chemical mechanisms. Thus, our project structure is optimized for closed-loop research feedback, in which each group receives and passes along information and data to the next as we investigated and optimized the conversion of low-cost CF. 
The following results, delineated by project task, were achieved FY 2019:

\section{Statistical Analysis of Alternative Precursor Oxidation and Carbonization}

In FY 2018, multiple alternative precursors were identified — primarily nylon, ultra-high molecular weight polyethylene (UHMWPE), PBO, and precursor blends of PAN with other polymers, all of which seemed most capable of meeting the VTO target properties of a tensile strength of $250 \mathrm{Ksi}(1.72 \mathrm{GPa})$, tensile modulus 25 Msi (172 GPa), as noted in Table II.2.1.2. At the start of FY 2019, UHMWPE and nylon (specifically Nylon-6, but referred to here as nylon) were down-selected for the focus of the research efforts due to their low-cost. The objectives of the statistical analysis of nylon and UHMWPE-based fibers were to (1) identify the conversion treatment necessary to achieve mechanical properties meeting specified targets, (2) investigate alternative treatments to reduce conversion costs, (3) optimize the conversion process to enhance performance and (4) generate experimental data with known conversion parameters, structure, and properties to validate the computational framework. A summary of the upper limit mechanical properties of lab-scale CF is presented in Table II.2.1.2. Results will be described in the following sections.

Table II.2.1.2. Summary of Key Lab-Scale Results (Upper Limits Only).

\begin{tabular}{|l|c|c|c|}
\hline \multicolumn{1}{|c|}{ Precursor } & Strength (GPa) & Modulus (GPa) & Strain (\%) \\
\hline Target & 1.73 & 172.5 & 1.0 \\
\hline UHMWPE (Lab-scale) & 1.77 & 179 & 1.19 \\
\hline Neat Nylon (Lab-scale) & 1.34 & 246 & 0.54 \\
\hline Nylon w/graphene (Lab-scale) & 1.86 & 192 & 1.05 \\
\hline Nylon w/graphene (Lab-scale, UV+microwave) & 1.78 & 184 & 0.98 \\
\hline
\end{tabular}

\section{Systematic Characterization of Nylon, Nylon/Polyvinyl Acetate (PVA), and Nylon/PVA/Graphene Fibers}

For Task 2.1 in FY 2018, we noted that added quantities of graphene would benefit the mechanical performance of PAN and PBO-based CF. This insight was applied to the nylon fibers FY 2019. We systematically characterized nylon, nylon/PVA, and nylon/PVA/graphene fibers to identify the role of PVA and graphene and elucidate the associated performance enhancement mechanisms. Here, the concentration of PVA and graphene was controlled at $0.1 \mathrm{wt} . \%$. The morphology, microstructure, and mechanical properties of the precursor, oxidized, and carbonized fibers were characterized with SEM, XRD, Raman spectroscopy, thermal gravimetric analysis (TGA), and single-filament tensile tests. The XRD patterns shown in Figure II.2.1.1 (d)-(f) of nylon, nylon/PVA, and nylon/PVA/graphene fibers before and after a $25 \mathrm{~h}$ oxidation revealed new diffraction peaks associated to the $\alpha_{1}(200)$ and $\alpha_{2}$ (002) phase of nylon, as well as diffraction peaks associated with $\mathrm{CuCl}$ (e.g., $\mathrm{CuCl}$ treatments are required prior to oxidation). Nylon/PVA and nylon/PVA/graphene fibers also exhibited diffraction peaks attributed to oxidized PVA. After carbonization, all samples featured a broad peak at 2-theta of $25^{\circ}$ shown in Figure II.2.1.1 (f), corresponding to the (002) peak indicative of graphitic structure. 

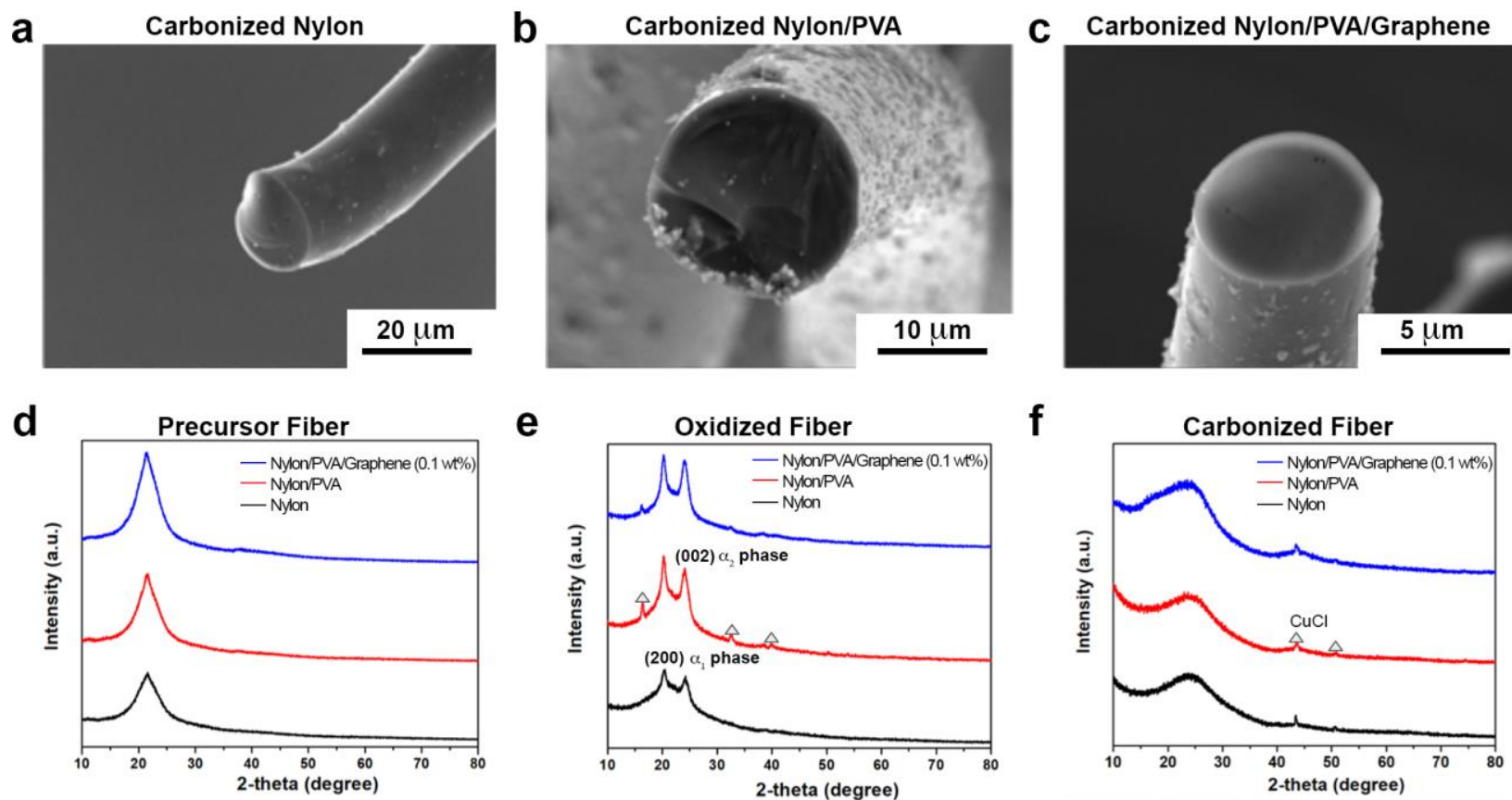

e

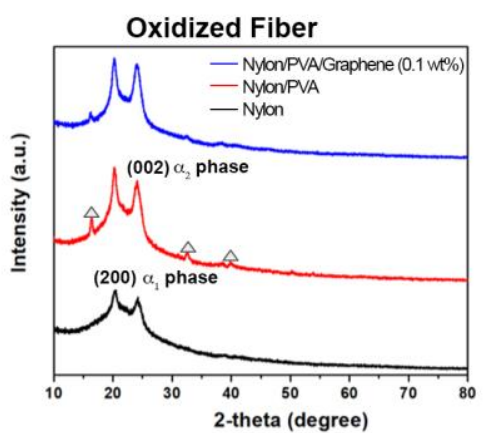

$f$

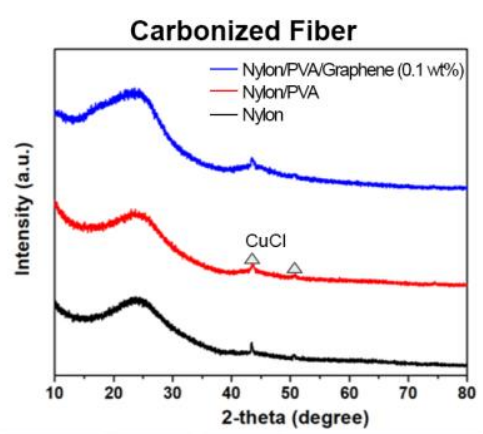

Figure II.2.1.1. Cross-sectional SEM micrographs of carbonized (a) neat nylon, (b) nylon/PVA and (c) nylon/PVA/0.1 wt.\% graphene. XRD patterns of neat nylon, nylon/PVA, and nylon/PVA/graphene (d) without any treatment, (e) after a 25-hour oxidation and (f) after carbonization. Source: University of Virginia.

Raman spectra of the carbonized fibers, as shown in Figure II.2.1.2 (a), indicated a sharper G band and an increased intensity ratio of the D and G band, IG/ID ratio, with the addition of PVA and graphene, which suggests more ordered graphitic structure inside the fibers. Interestingly, the oxidized nylon/PVA fiber also exhibited the highest weight retention based on the TGA curve, as shown in Figure II.2.1.2 (b), indicating improved thermal stability due to PVA. Tensile testing also indicated significantly enhanced mechanical properties of nylon/PVA and nylon/PVA/graphene samples compared to the neat nylon control sample, as observed in Figure II.2.1.2 (c). Strength, modulus, and strain were found to reach a maximum of $2.03 \mathrm{GPa}, 180$ $\mathrm{GPa}$, and $1.19 \%$, respectively, for the nylon/PVA/graphene sample, which meets our targets. These results suggest that PVA and graphene jointly enhance the mechanical properties and thermal stability of the composite fiber. 


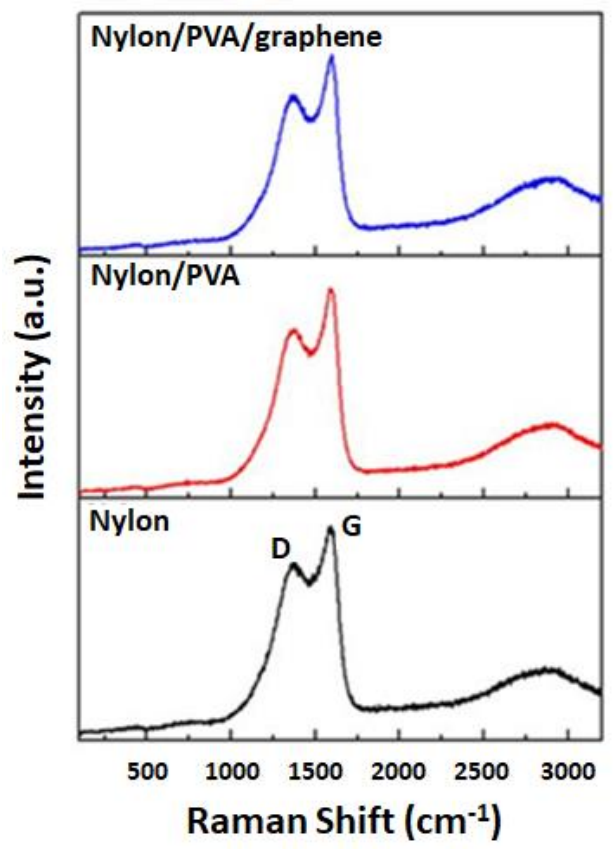

(a)

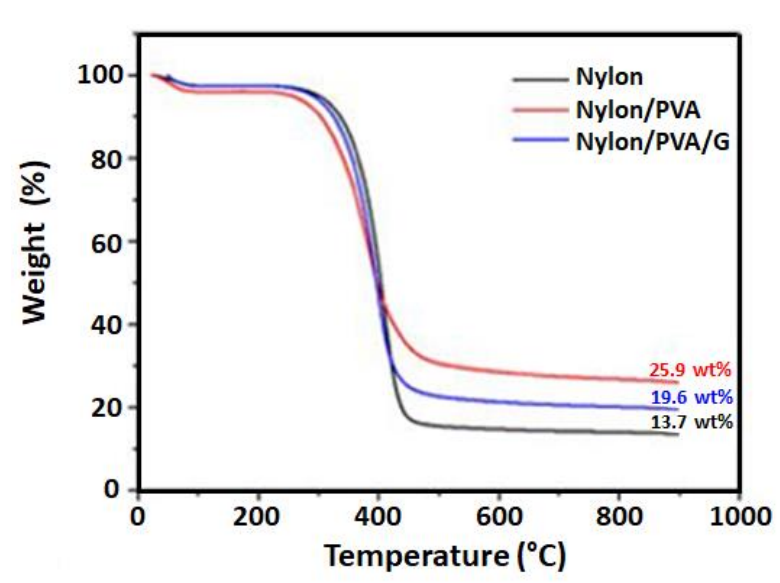

(b)

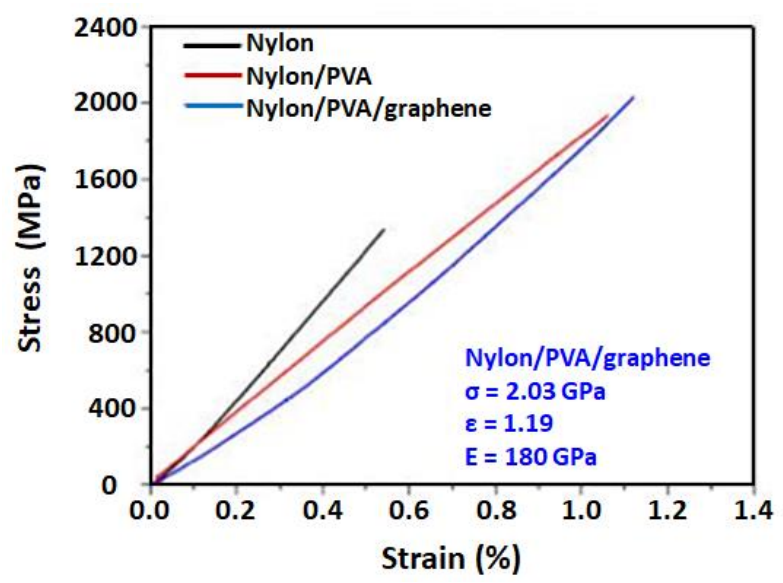

(c)

Figure II.2.1.2. (a) Raman spectra of carbonized nylon, nylon/PVA, and nylon/PVA/0.1 wt.\% graphene fibers. (b) TGA results of oxidized nylon, nylon/PVA, and nylon/PVA/graphene. (c) Stress-strain curves of carbonized nylon, nylon/PVA, and nylon/PVA/graphene. Source: University of Virginia.

\section{Stepwise Microwave-aided Thermal Stabilization}

The nylon/PVA/graphene fibers just described can meet the target properties, but conversion required using the conventional $25 \mathrm{~h}$ oxidation process, which is not practical for industrial production. To shorten the oxidation time, we introduced a novel technique-microwave irradiation. We found that the thermal stabilization time could be significantly reduced through a stepwise microwave treatment, as observed in Figure II.2.1.3 (a). Irradiation for a total of $10 \mathrm{~min}$ yielded fibers that met the target mechanical properties after only $5 \mathrm{~h}$ of oxidation, which is an $80 \%$ reduction in cycle time. The infrared image of the nylon/PVA/graphene during microwave irradiation is presented in Figure II.2.1.3 (b). The surface temperatures, measured with an infrared thermal camera, of the microwave-treated fibers with increasing treatment duration is plotted as a function of the total time that the microwave radiation was applied, and results are shown in Figure II.2.1.3 (c). 
Temperatures stabilized after about $10 \mathrm{~min}$. TGA results indicated that the microwave-treated fiber had a carbon retention of $12.4 \mathrm{wt} . \%$, lower than that of conventionally prepared fibers. While these results indicate that microwave irradiation is an effective methodology to accelerate the oxidation process, further work is required to shorten the oxidation period down to the $2 \mathrm{~h}$ target set by Solvay.

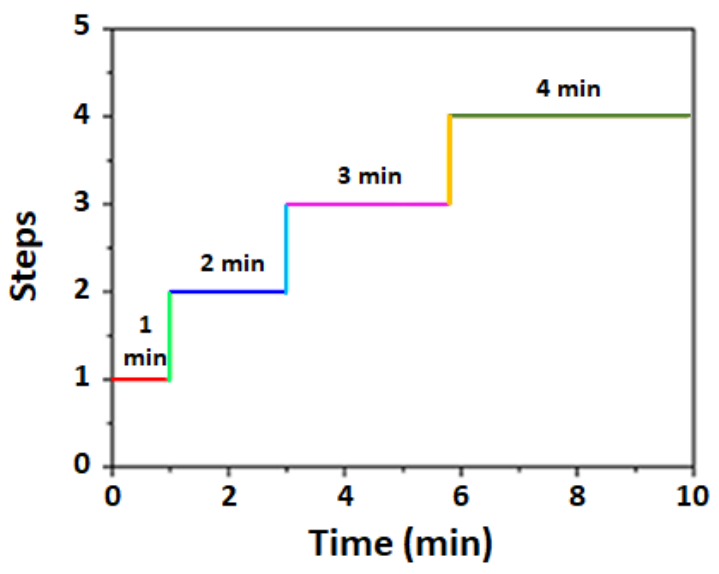

(a)

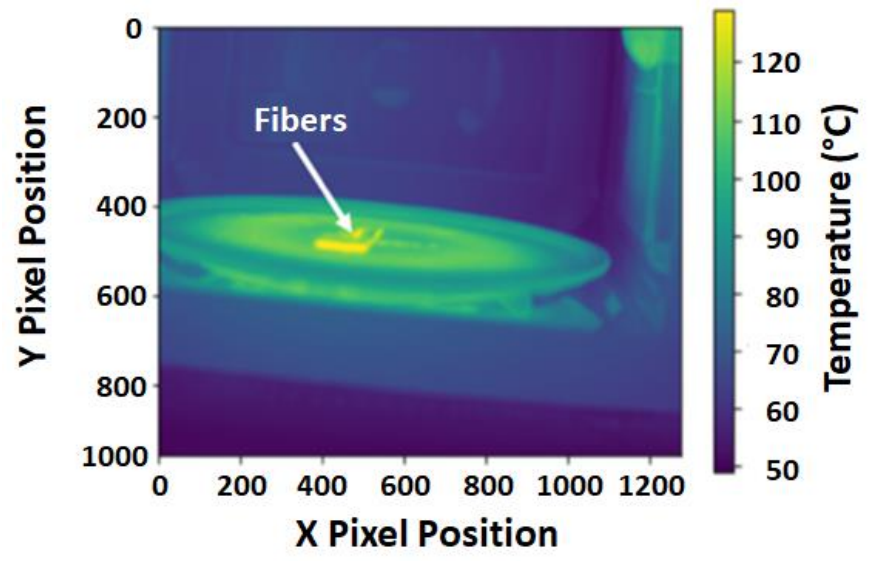

(b)

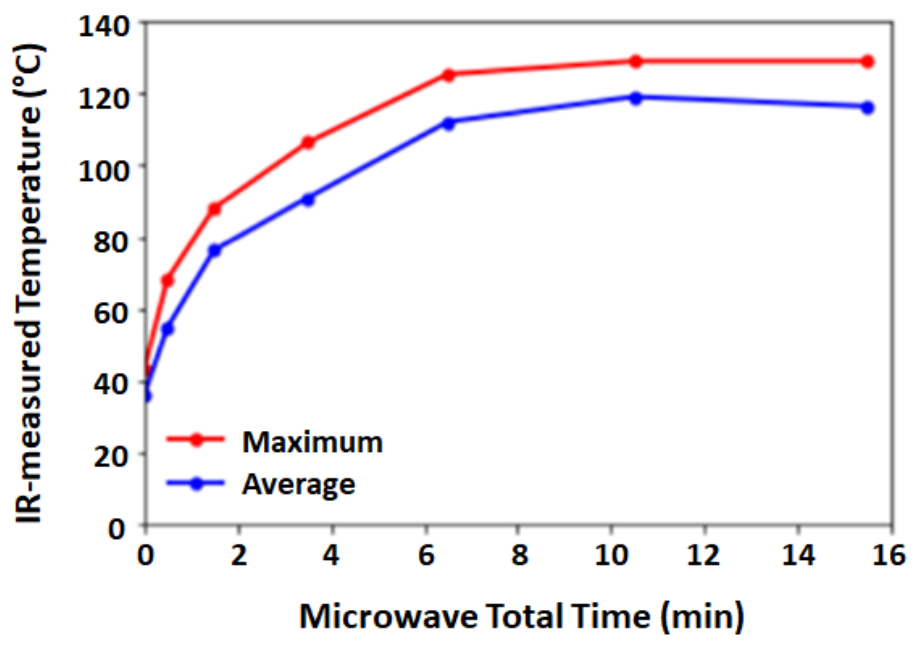

(c)

Figure II.2.1.3. (a) Graphic of the stepwise microwave heating time. (b) Infrared image of the nylon/PVA/graphene during microwave irradiation. (c) Surface temperature, measured with the thermal camera, of the microwave-treated fibers with increasing treatment duration. Source: University of Virginia.

\section{Polyethylene-derived CFs}

Lab-spun, high-density PE fibers could not be converted to CFs that met the target properties, but UHMWPE fibers were successfully converted to carbon fiber via sulfonation and carbonization processes. UHMWPE fibers required a sulfonation treatment at $145^{\circ} \mathrm{C}$ for $6 \mathrm{~h}$ prior to carbonization. Tensile testing was performed on the carbonized fiber, and the mechanical properties of the UHMWPE CFs met the targets with measured upper limit properties of strength, modulus, and ductility of $1.77 \mathrm{GPa}, 179 \mathrm{GPa}$, and $1.19 \%$, respectively. We will fine-tune these mixtures to further enhance the properties, in addition to characterizing the fibers after each conversion step to investigate reaction mechanisms. 


\section{Understanding the Chemical Conversion of Alternative CFs}

The key objectives of Task 2.2 in FY 2019 were to identify environmental dependencies during the carbonization of alternative precursors and to continue to enhance the accuracy of simulation results for alternative precursor chemistry to strengthen the overall ICME framework. This work was accomplished in several thrusts as described below in brief.

\section{Effect of Heating Rate on PBO Transformation}

In FY 2018, it was experimentally documented that faster heating rates generally translated to superior properties. We applied the ReaxFF framework to investigate this effect for PBO. We expect that the trend will likely be repeated for other precursors as well; therefore, we will update this work with the final down-selected alternative precursor. The evolution of a main reaction product, carbon monoxide, was compared for sample heating rates of $2 \mathrm{~K} / \mathrm{ps}$ and $700 \mathrm{~K} / \mathrm{ps}$, noting that the carbon monoxide release times are of the same order-ofmagnitude: 13.25 ps at $2 \mathrm{~K} / \mathrm{ps}$ and 20.06 ps at $700 \mathrm{~K} / \mathrm{ps}$, as shown in Figure II.2.1.4 (a) and Figure II.2.1.4 (b). The final structures of the initially considered fragments of the PBO chain are significantly different showing at the slower heating rate that only 6-membered, all carbon rings directly in contact with oxazole groups are disturbed. At the higher heating rate, all the 6-membered rings were disturbed, even those not directly connected to oxazole groups. Thus, we proposed a flexible, all carbon rings alignment mechanism is activated at faster heating rates, which results in a more disturbed, initial structure of the polymer chains and promotes the evolution of graphitic structure. This insight indicates that generally faster heating rates will benefit mechanical properties; indeed, lab-scale testing is capitalizing on this outcome, using faster heating rates to optimize fiber conversion.
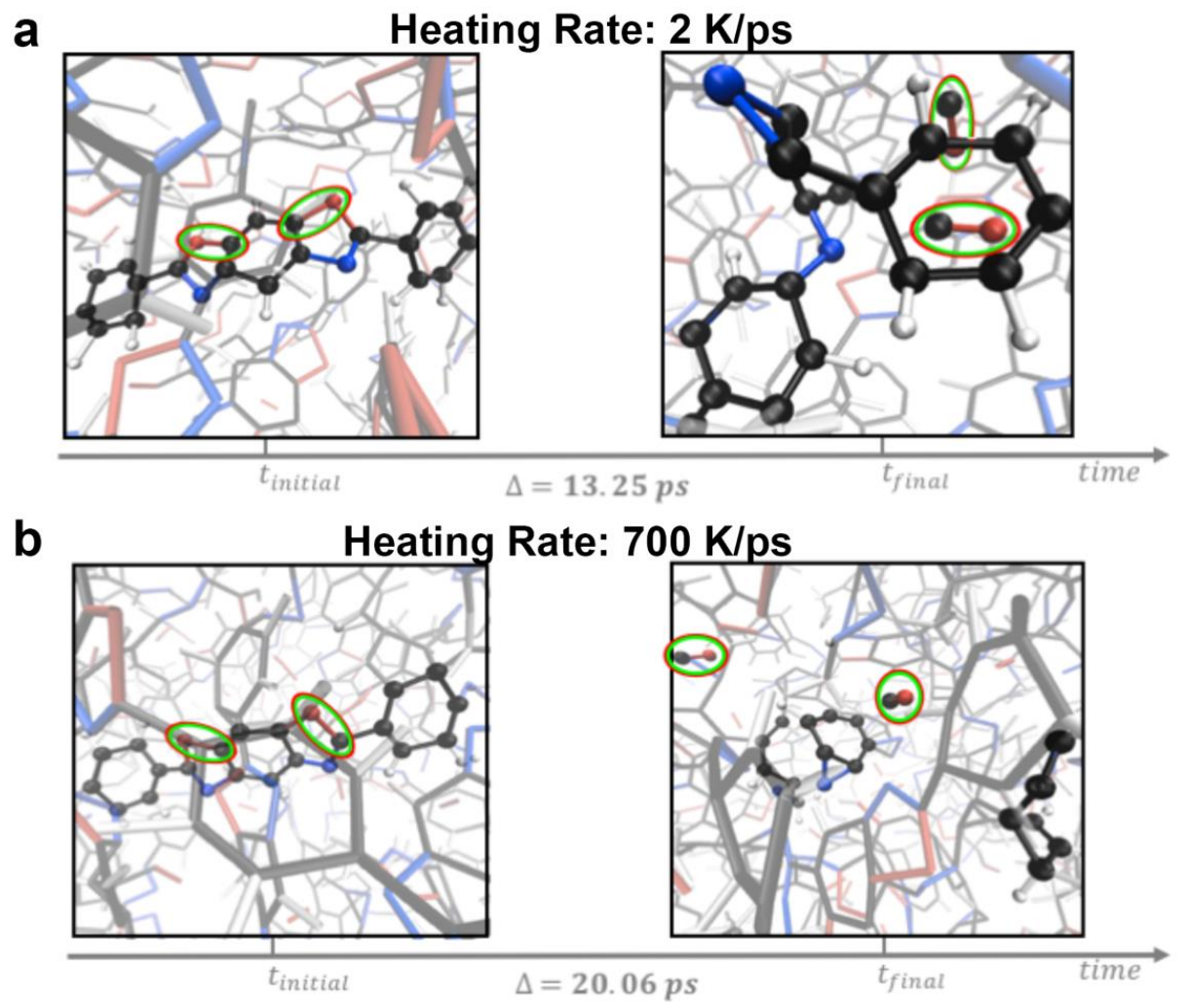

Figure II.2.1.4. Simulation snapshots representing carbon monoxide production for samples with (a) $2 \mathrm{~K} / \mathrm{ps}$ and (b) $700 \mathrm{~K} / \mathrm{ps}$ heating rate. Source: Pennsylvania State University. 


\section{ReaxFF Investigation of PE Sulfonation}

$\mathrm{PE}$ is an attractive, low-cost alternative CF precursor; however, PE cannot be directly converted to CF. Instead, PE must undergo a sulfonation procedure; we hypothesize sulfur atoms act as catalysts for the carbonization reaction. To identify the role of the $\mathrm{S}$ atoms in PE sulfonated derivatives, we applied the ReaxFF molecular dynamics echnique to simulate the heating and carbonization processes for sulfonated PE CF precursors. Using the recently developed ReaxFF force field, ReaxFF CHOSMoNiN-2012, which incorporates sulfur (S) atoms, we extracted the optimized parameters at the point where the total simulation error reached a target level and compared the bond dissociation energies and angle distortion energies between ReaxFF data and quantummechanical reference data [13], as shown in Figure II.2.1.5 (a)-(j). In most instances, the bond dissociation energies of ReaxFF are close to the quantum reference data, but some of the angle distortion energies diverge. In this way, the ReaxFF should give a good description when the C-S-C angle varies around the equilibrium angle. The new ReaxFF CHONS-2019 force field is still under development, and future efforts will be made into the validation of kinetics of S-containing groups and carbonization reactions of sulfonated PE derivatives.
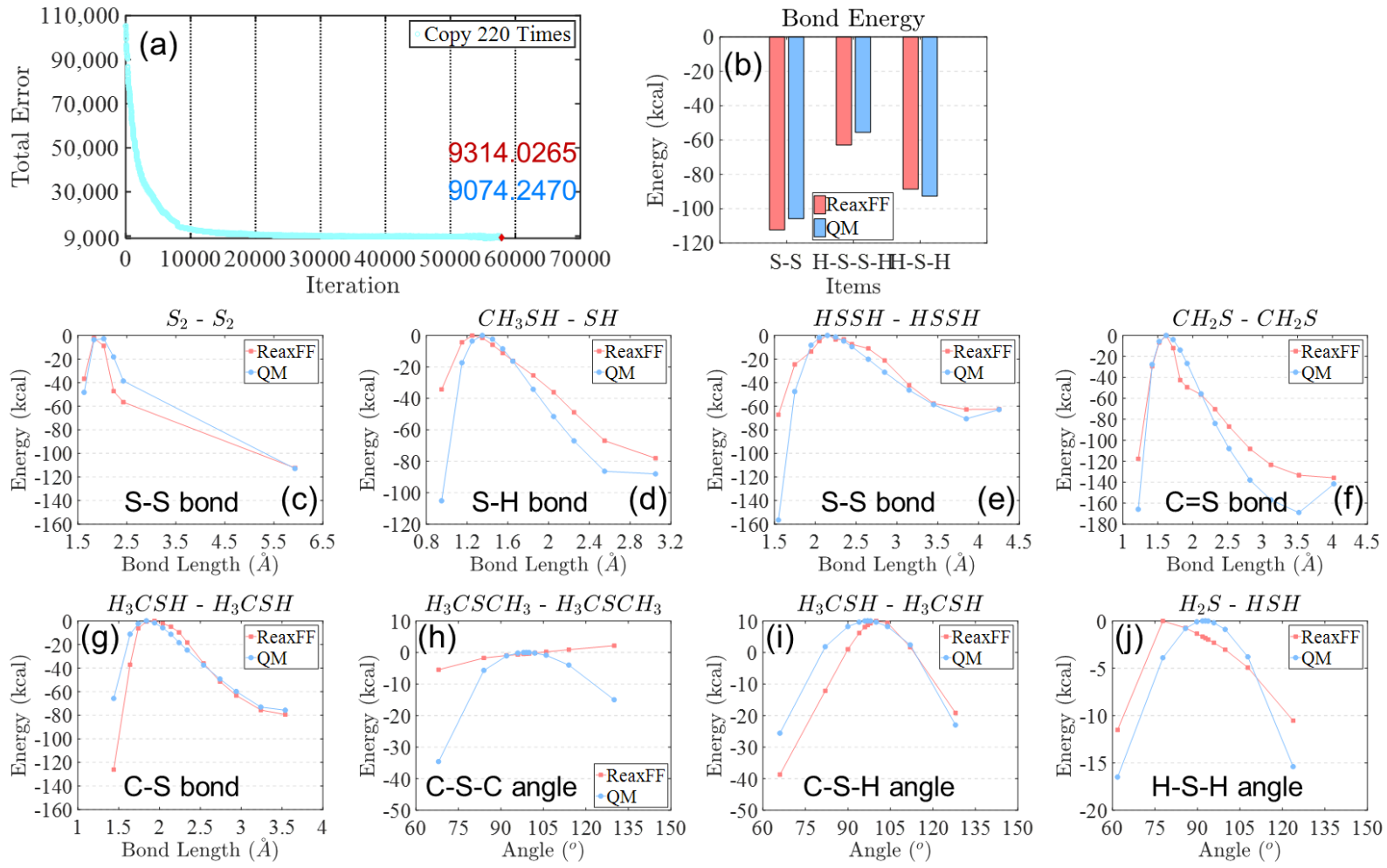

Figure II.2.1.5. Total error during parameterization and comparisons between ReaxFF and the quantum reference data after the force field training: (a) Total error versus iteration (220 times of the initial parameters) during parameterization;

(b) Comparisons of S-S, H-S-S-H, and H-S-H bond dissociation energies between ReaxFF and the quantum data; Comparisons of (c) S-S, (d) S-H, (e) S-S, (f) C=S, and (g) C-C bond dissociation energies between ReaxFF and the quantum data; Comparisons of (h) C-S-C, (i) C-S-H, and (j) H-S-H angle distortion energies between ReaxFF and the quantum data. Source: Pennsylvania State University.

\section{ReaxFF Simulations of Nylon - CuCl Treatment}

It has been shown experimentally that nylon precursor fibers must be treated with a $\mathrm{CuCl}$ solution for conversion, but the underlying mechanism was unknown; fibers produced without this treatment melt at low temperatures and cannot be oxidized. Thus, we performed ReaxFF simulations on three different systems of water solutions containing nylon polymer chains: (1) without any $\mathrm{CuCl}$ ions, (2) with randomly dispersed $\mathrm{CuCl}$ ions and (3) with $\mathrm{CuCl}$ ions aligned between the chains, as illustrated in Figure II.2.1.5 (a). We observed significant interactions of the $\mathrm{CuCl}$ ions with the polymer chains, as shown in Figure II.2.1.6. Ions placed in 
between the polymer chains created coordination complexes that resulted in better polymer chain alignment. When we performed the simulations within nylon polymer melt, there was a significant increase in system density with added $\mathrm{CuCl}$ content as seen in Figure II.2.1.6 (b). This density increased from $1.35-1.96 \mathrm{~g} / \mathrm{cm}^{3}$ with the addition of $140 \mathrm{CuCl}$ ions. This increase explains the experimentally observed increase in the melting temperature for nylon fibers after the $\mathrm{CuCl}$ treatment imposes a stable microstructure to the polymer chains.

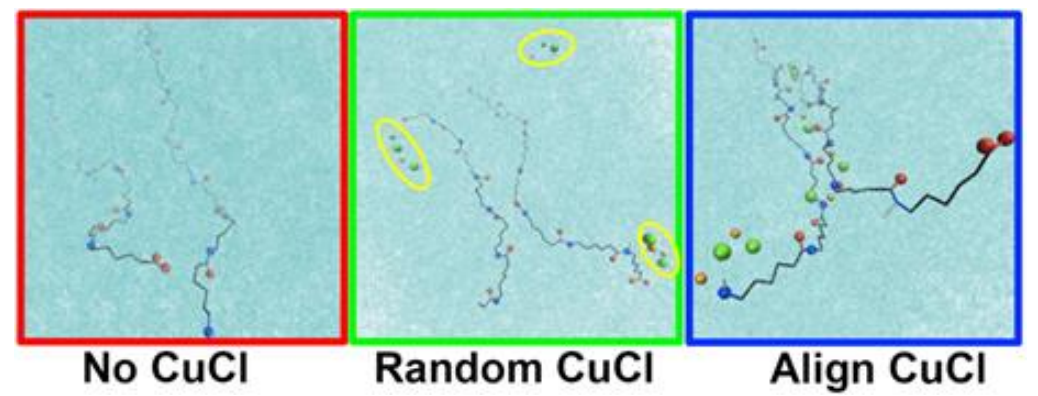

(a)

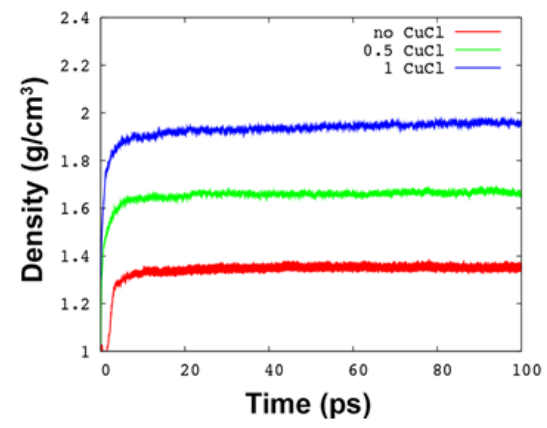

(b)

Figure II.2.1.6. (a) The nylon 6 with copper chloride ions in water solution simulations; and (b) densities for each solution. Source: Pennsylvania State University.

\section{ReaxFF Polymer Blend Simulations}

We previously identified that a precursor blend of PAN and PBO at a proper mixing ratio, PAN 25/PBO 25, could serve as an alternative $\mathrm{CF}$ precursor that has comparative all carbon ring productions (particularly 5- to 7-membered rings) to oxidized PAN. We also verified the roles of O-containing and $\mathrm{N}$-containing groups in the carbonization process; the O-containing groups are more efficient for initiating the reaction, whereas the Ncontaining groups assist with stitching and weaving together the polymer molecules and residuals of high molecular weight to form larger all carbon ring networks. This mechanism indicates that we could find a new, PAN-based polymer blend as a cost-effective CF precursor. We can apply this mechanism to mix PAN with an O-containing groups-enriched, inexpensive polymer - in particular amorphous cellulose (denoted as PAN/CL), for the design of a low-cost CF precursor. PAN/CL was found to achieve the highest 6-membered ring production via ReaxFF simulations. A least square exponential fitting method was used to derive the time and rate constants describing the one-phase association for 6-membered ring carbon conversion. Thus, we could predict the conversion duration over long periods of time despite the relatively small time-scales of the ReaxFF simulations ( $\sim 300 \mathrm{ps})$. From the calculations, we note that PAN/CL required the least time to reach $99 \%$ conversion into 6-membered rings, 12,666 ps. For comparison, pure PAN, pure PBO, and the PAN 25/PBO 25 blend required 17,327 ps, 20,334 ps, and 15,414 ps, respectively. The PAN/CL blend may thus be considered as a promising alternative, PAN-based precursor to reduce production costs by (1) reducing manufacturing costs via replacing expensive PAN with cheap amorphous cellulose and (2) having a higher 6-membered ring production within a short time to achieve a faster conversion rate reaching $100 \%$ 6-membered ring production. 


\section{Graphene Stacking Effect on CF Chemistry}

Experimental testing in FY 2018 has demonstrated that added quantities of graphene can be optimized to enhance the mechanical properties of PAN (FY 2018), PBO, and nylon (FY 2019) CF, but the cause of this effect was unknown. To investigate this effect, ReaxFF MD was implemented to study graphene stacking using PAN-based CF chemistry; this chemistry will be updated in FY 2020 with the final down-selected alternative precursor. The simulation found that 5/6/7-membered carbon rings formation occurred only at the graphene edges and that graphene inclusion does not significantly enhance gas molecule $\left(\mathrm{H}_{2} / \mathrm{H}_{2} \mathrm{O} / \mathrm{N}_{2}\right)$ production and rings formation, as observed in Figure II.2.1.7 (a). The quality of graphene (separated graphene sheets vs. stacked graphene layers) was not found to affect the carbon content, but increasing the carbonization temperature was associated with increasing the carbon content, as seen in Figure II.2.1.7 (b). The results demonstrate that graphene does not benefit the chemistry of conversion; instead, its benefits may be limited to its role aligning the polymer. We hypothesize that the same trends will extend to other precursors, but these trends will be confirmed with simulations of nylon and UHMWPE in FY 2020.

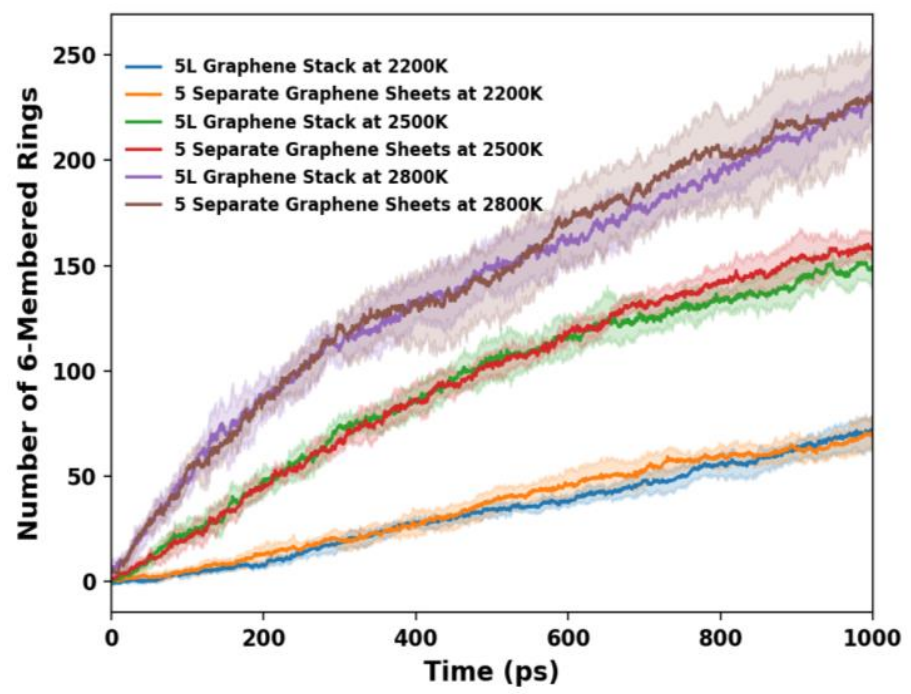

(a)

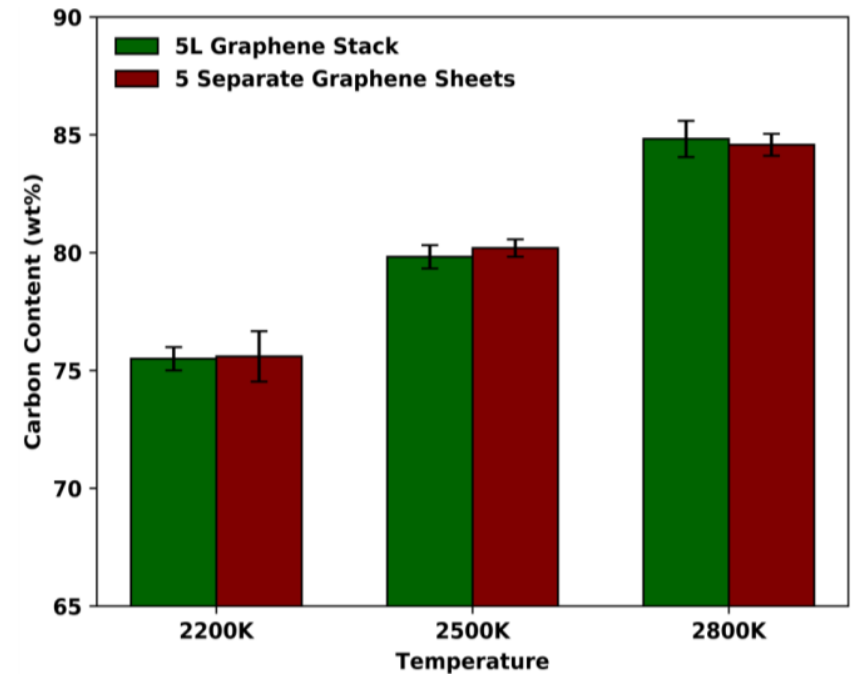

(b)

Figure II.2.1.7. (a) Six-membered, all carbon ring formation and (b) carbon content of 5-layered graphene/PAN for various carbonization temperatures. Source: Pennsylvania State University. 


\section{Hot-stretching ReaxFF Simulation}

A carbonization simulation at $2800 \mathrm{~K}$ was performed for the PBO melt at the number of particles in a system, the volume, and the temperature (NVT) ensemble. Two cases were considered - one with the simulation box remaining constant (i.e., mimicking the carbonization process with no external force applied to the fiber) and the other with the simulation box gradually increasing in the x-direction (i.e., mimicking the carbonization process with applied external force). The number of 6-membered rings that evolved after $1 \mathrm{~ns}$ of the carbonization simulation for the sample with the continuous box deformation that might correspond to the experimental hot-stretching is higher than for the sample with the box size kept constant throughout the same time of the carbonization simulation (320 versus 302). Also, the stress-strain data, as shown in Figure II.2.1.8 (a), clearly indicates an improvement in the mechanical response for the sample with the continuous box deformation; however, this trend was not consistent for all five considered samples. Further simulation of the carbonization process of PBO and the oxidized PAN is required to identify the simulation parameters that influence the possible observable differences for the final number of 6 -membered rings and their alignment in the case of the continuous box deformation.

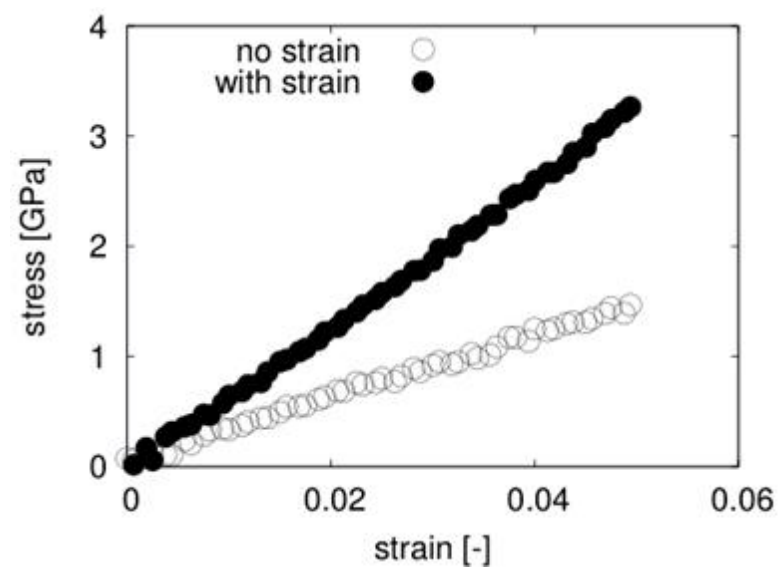

(a)

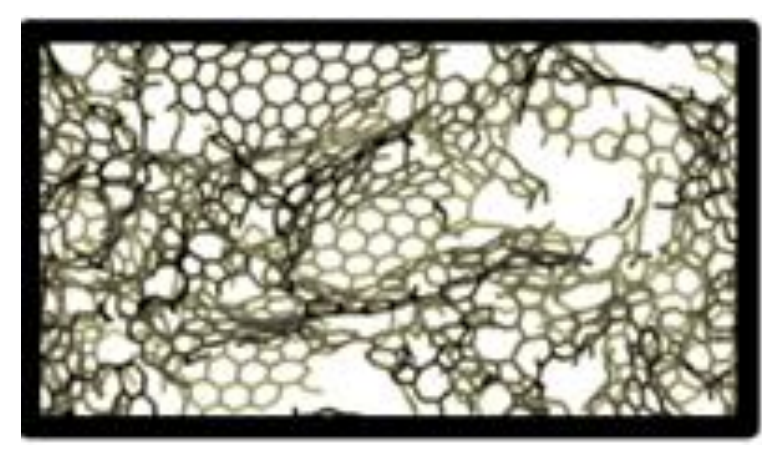

(b)

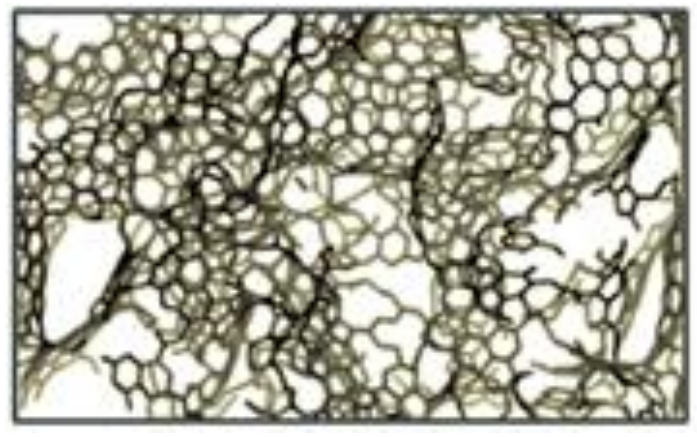

(c)

Figure II.2.1.8. (a) A comparison of the data from carbonization simulation for the PBO (b) with and (c) without box deformation on the x-direction during the carbonization simulation at $2800 \mathrm{~K}$. Source: Pennsylvania State University. 


\section{Design of Target Polymers ReaxFF Simulation}

A possible variation in the 6-membered ring production due to the structural differences of the monomers of the considered polymers was tested. As can be seen in Figure II.2.1.9 for only four possible variations of the cyclic-type monomer, the significant differences in the final number of the 6-member ring produced after 500 ps of the carbonization simulation are observed. In FY 2020, we will continue the simulations for alternative monomer structures to identify the potential synthetic polymer that could produce comparable number of 6membered rings oxidized PAN without prior oxidation step. Thus, in the future, we may be able to design a polymer optimized for low-cost, efficient CF conversion.

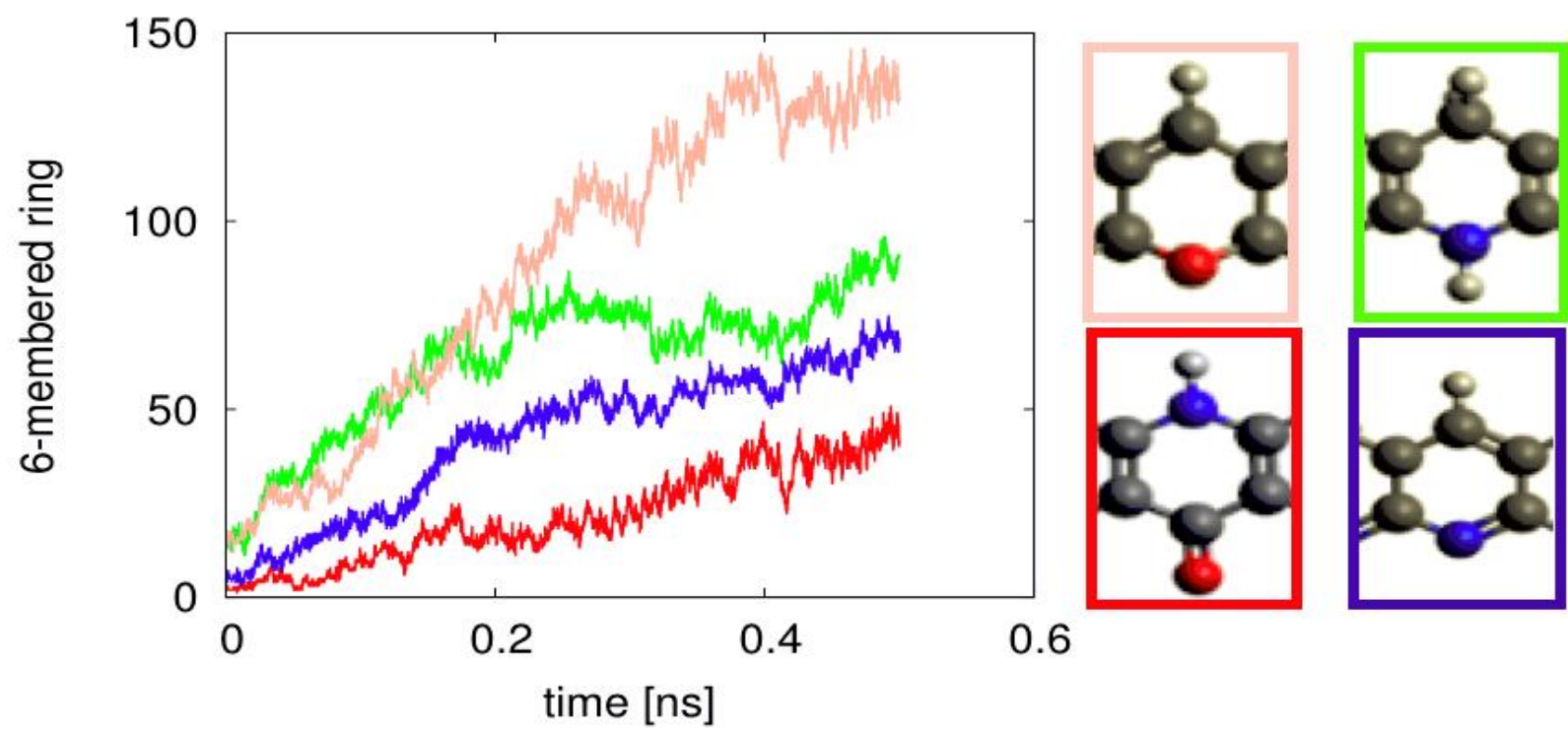

Figure II.2.1.9. A 6-membered ring production from the carbonization simulation at a temperature of $2800 \mathrm{~K}$ for the polymers with four monomers illustrated in the color boxes. Source: Pennsylvania State University.

\section{Exploratory UV Treatment Simulation with ReaxFF}

To support experimental findings that indicate a significant benefit to the precursor oxidation via UV treatment, we explored the possibility of a ReaxFF simulation of UV-light radiation with the use of a bondboosting technique. A small system of the aliphatic dicycloepoxide molecules was considered. NVT simulation at $900 \mathrm{~K}$ with and without bond-boosting were performed as well. For the system with no bondboost applied for the carbon-epoxy oxygen bond illustrated in Figure II.2.1.10 (a), no visible molecule crosslinking is observable; however, in the case of the system with the applied bond-boost for carbon-epoxy oxygen bond shown in Figure II.2.1.10 (b), we do observe molecule crosslinking. Further, this technique will be applied for nylon 6,6 NVT simulation to investigate possible molecules polymerization in FY 2020. 

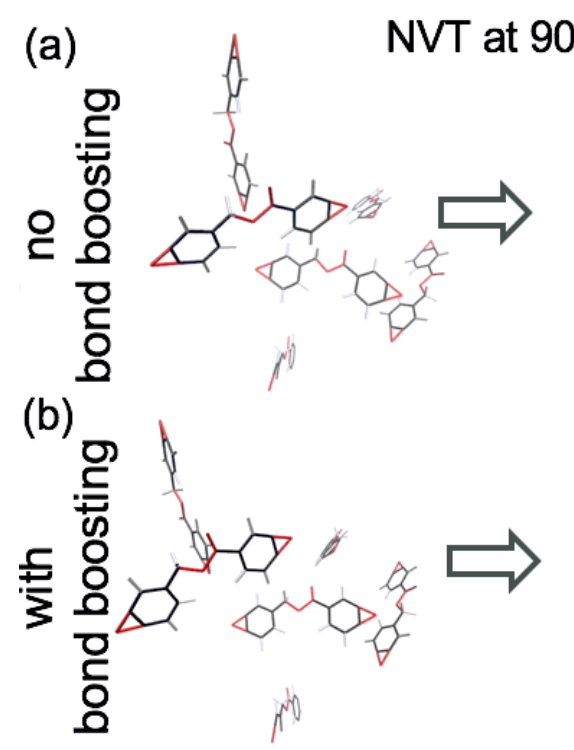
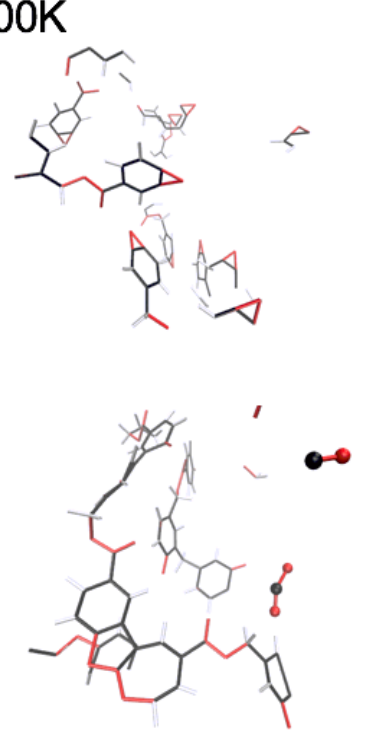

$>$ Some molecules fragmentation

\author{
$>$ Molecules \\ cross-linking \\ $>\mathrm{CO}, \mathrm{CO}_{2}$ \\ production
}

Figure II.2.1.10. A possible UV treatment simulation with ReaxFF. A system of 6 aliphatic dicycloepoxide molecules was consider without (a) and with (b) bond-boost applied to carbon-epoxy oxygen bond. Source: Pennsylvania State University.

\section{Model Integration via Fiber/Matrix Mechanics Study}

Building on the success of the nanoscale chemical simulations, large-scale MD simulations extended the structure of fibers produced by the ReaxFF simulations to conduct structural characterization and simulate mechanical testing for Task 2.3. This work was enabled by development in FY 2018 of a novel computational approach to generate realistic 3D microstructures. In FY 2019, this framework has been updated to elucidate the benefits of added graphene on fiber microstructure and to characterize local deformation of the microstructure.

\section{Effect of Added Graphene on Fiber Microstructure}

To investigate the effect of graphene on fiber microstructure, we performed large-scale atomistic simulations on a system containing PAN chains and a single layer graphene sheet, which corresponds to $2.5 \mathrm{wt} . \%$. A baseline system was also assembled without a graphene sheet. The system was annealed in a MD simulation performed with the non-reactive polymer consistent force field, as seen in Figure II.2.1.11. In contrast to ReaxFF, the polymer consistent force field is a non-reactive, all atom force field specifically parametrized for the simulation of polymetric materials [14]. To quantify the effect of the graphene sheet on chain alignment, we calculated Herman's Orientation Factor (HOF) for the orientations of carbon rings present in the PAN chains and graphene sheet, where a HOF value of 1.0 indicates perfect alignment of the PAN chains and 0.0 indicates random orientation. The HOF value at the beginning of both MD simulations was -0.05 , indicating random orientation of PAN chains. During the MD simulations, the PAN-only system exhibited very modest changes in the HOF value, indicating the PAN chains retain their random orientation as seen in Figure II.2.1.11 (c). In the presence of a graphene sheet, the HOF value of PAN chains increased to 0.53 as seen in Figure II.2.1.11 (b). Furthermore, the MD simulations revealed a thin layer (about $4 \mathrm{~nm}$ thick) of highly aligned chains (HOF > 0.75) formed in the vicinity of the graphene sheet, as seen in Figure II.2.1.11 (a). These results clearly demonstrate that the addition of graphene sheets enhance the overall alignment of PAN chains, which we previously demonstrated in FY 2018, and leads to a substantial improvement in the fiber mechanical properties. 
a

Isolated graphene sheet, $\mathrm{HOF}=0.94$

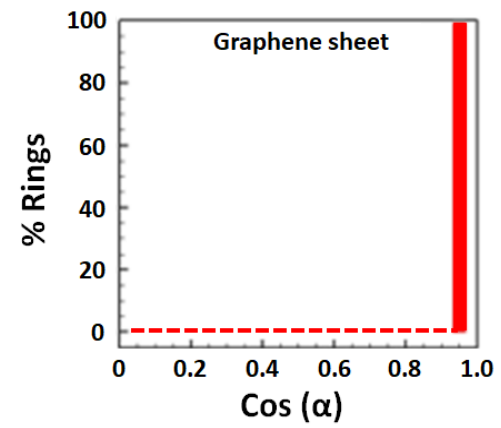

b
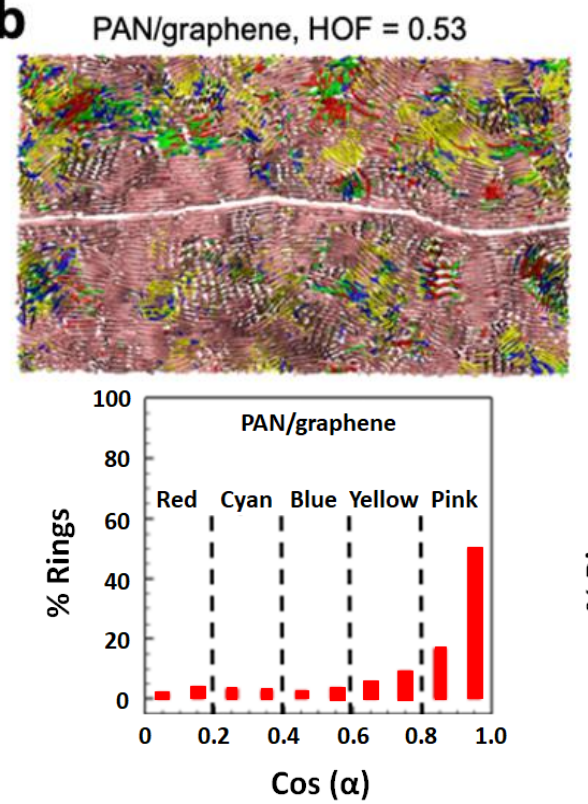
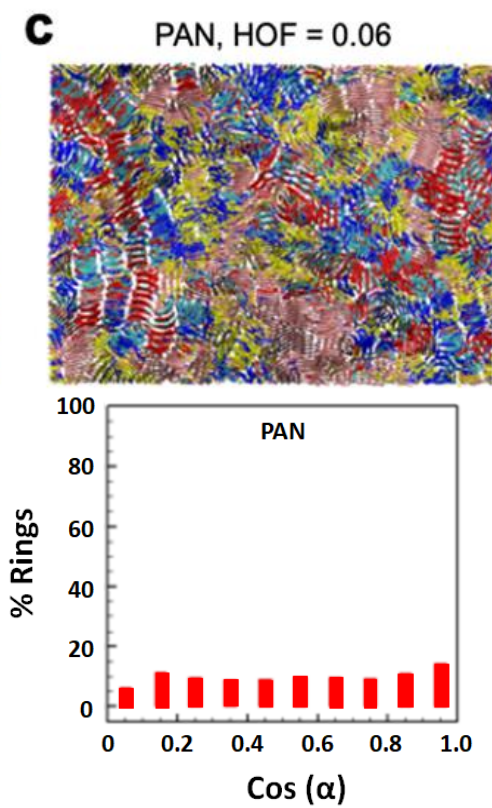

Figure II.2.1.11. (top) Visual representations of large-scale MD simulations of the effect of graphene sheet and (bottom) the accompanying distribution of ring normals. (a) An isolated graphene sheet represents maximum degree of alignment. (b) A system with graphene sheet obtains a high degree of alignment. (c) A system without graphene sheet maintains random alignment of polymers. Source: University of Virginia.

\section{Deformation of Local Microstructure}

In FY 2018, we presented our initial work towards predicting local deformation in CF microstructure by calculating the changes in C-C bond lengths. Such an approach, although simple and attractive, cannot fully capture effects of microstructure inhomogeneity and local sliding of carbon sheets/ladders. Hence, we are currently developing a new computational tool that can capture the evolution of local strain and local stress through calculation of atomic-level strain $\left(\epsilon_{i j}\right)$ and stress $(\sigma)$ using Equation 1.

$$
\epsilon_{i j}=\frac{1}{2}\left(\frac{\partial u_{i}}{\partial x_{j}}+\frac{\partial u_{j}}{\partial x_{i}}\right), \sigma=\frac{1}{V_{\text {ato } m}}(-m v \otimes v+r \otimes F)
$$

In this computational approach, the atomic-level strain is approximated using a central difference scheme, while the atomic-level stress is calculated from quantities directly obtained from MD simulations. To calculate the distribution of local strain and stress within the computational sample, we divided the system into a 3D grid of rectangular cuboids and calculated the local strain and stress values by averaging over all atoms within a cuboid. This computational approach is extended across the full range of applied strain during simulated tensile testing, as observed in Figure II.2.1.12 (a)-(c). Our analysis indicates that local strain is inversely correlated with the local degree of graphitization; hence, the cuboids with low graphitic content are likely to experience higher strain during the tensile loading as shown in Figure II.2.1.12 (d). This inverse correlation between local strain and degree of graphitization is observed for different global strain levels. This finding highlights the critical capability of our ICME framework to account for material heterogeneity and demonstrates the importance of selecting a good precursor and conversion procedure to induce a high degree of graphitization. 
a
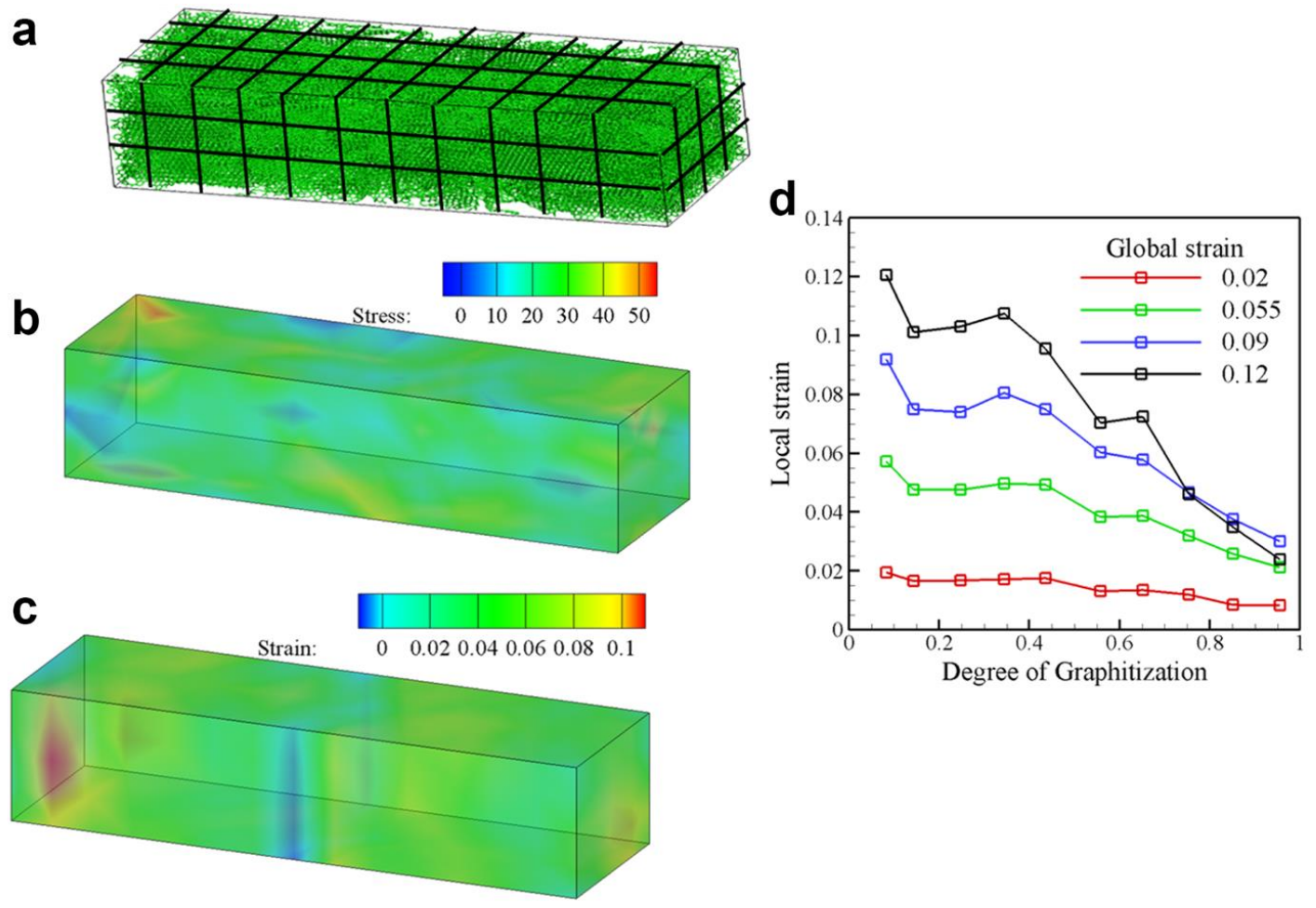

Figure II.2.1.12. Computational approach for identifying structure/mechanical properties relationships: (a) 3D grid of rectangular cuboids for a simulated microstructure; (b) local stress distribution; (c) local strain distribution; and

(d) correlation between local strain and degree of graphitization at different global strain levels marked on the panel. The contour plots shown in (b) and (c) corresponds to the global strain level of 0.04. Source: University of Virginia.

\section{Characterizing the Computational System Size Effect}

In FY 2018, we noted it was necessary to apply a Griffith's Law correction to the ICME framework property predictions $[15,16]$. Due to the size of the models, it was difficult to account for realistic defects (i.e., pore size was too large for the computational system) within the fiber. Thus, the previous iteration of the model tended to overpredict strength and strain. To investigate the computational system size effect on the mechanical properties of a simulated CF microstructure and refine the ICME predicted properties, we performed a largescale atomistic simulation on a system consisting of 3.8 million atoms. The starting configuration was built using non-stacked ladders of different lengths $(2.3-11 \mathrm{~nm})$. In the initial configuration, the polymer ladders were preferentially aligned along the fiber axis (x-axis). A reactive molecular simulation was then performed to generate a CF microstructure-based on this initial configuration of ladders.

The atomistic procedure used for generating larger microstructure is similar to the one used for smaller samples. Figure II.2.1.13 (a) shows the snapshot of the end microstructure, which is $54.3 \mathrm{~nm}$ long in fiber axis direction and has a cross-section of $24.3 \mathrm{~nm} \times 25.4 \mathrm{~nm}$. The microstructure contains $18.3 \%$ graphitic phase mostly in the form of small flakes of graphite, as observed in Figure II.2.1.13 (b). The void analysis seen in Figure II.2.1.13 (c) shows that the characteristic size of the biggest void in the large microstructure is nearly two times the corresponding size in a smaller microstructure, which was generated using non-stacked aligned ladders (L1_L2_xa). We also performed a tensile testing simulation on the larger microstructure using ReaxFF. Figure II.2.1.13 (d) compares the stress-strain curves of larger and smaller microstructures. It can be seen that the larger microstructure has a lower mechanical strength and fracture strain as compared to those of the smaller microstructure, which can be attributed to the presence of bigger voids. It must also be noted that we expect pore size in the actual fibers may be as large as $30 \mathrm{~nm}$ or more [17, 18], even larger than those simulated here. Thus, we can clearly see the link between the model predictions and experimental properties based on pore size. 
a
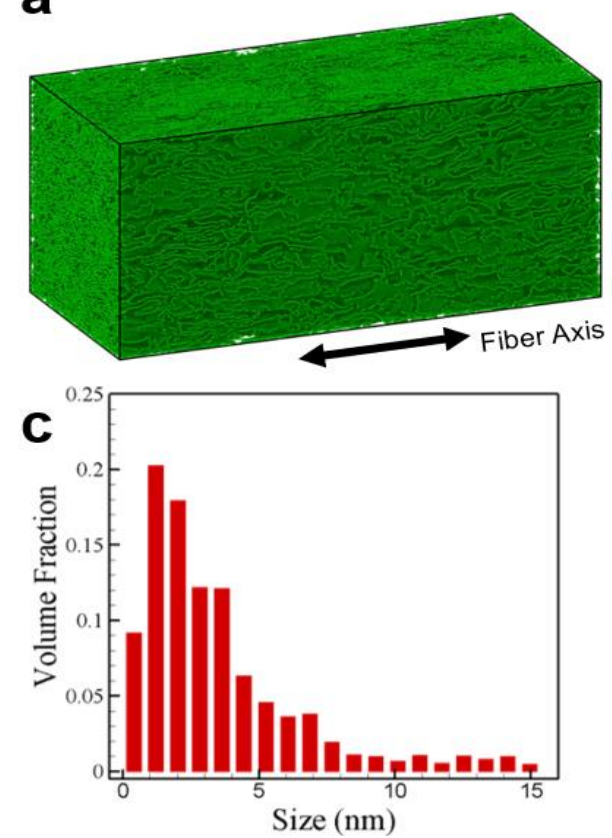
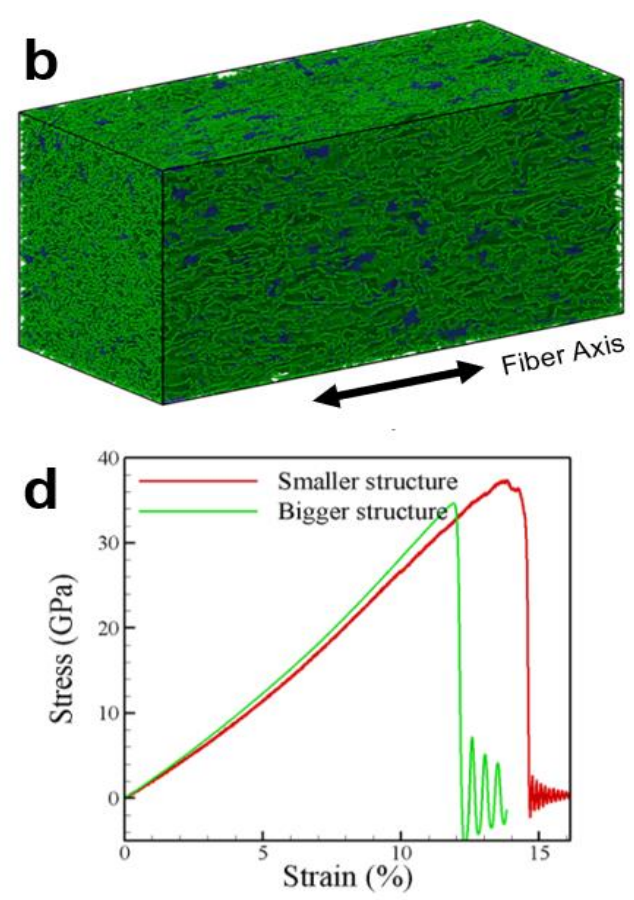

Figure II.2.1.13. Results of the atomistic simulations performed for a large (e.g., 3.8 million atoms) system representing CF microstructure. $(a, b)$ Snapshots of the 3D microstructure, where all carbon atoms are colored green in (a), while the graphitic phase is shown by blue in (b). (c) Void size distribution. (d) Stress-strain curves from ReaxFF tensile testing simulations performed for systems of two sizes. Source: University of Virginia.

\section{Synthesis and Evaluation of Prototype CFs}

Through collaboration with the LightMat consortium, the Task 2.4 testing at ORNL has been performed to address multiple fronts. ORNL provided critical technical and materials support for the university researchers, including access to furnace equipment for carbonization and graphitization of fibers. On the experimental front, ORNL has formed a bridge between the fundamental research and small-scale experimentation at the University of Virginia and the large-scale pilot runs currently underway. In tandem with the research efforts, ORNL has also pursued UHMWPE and Nylon precursors, helping to prepare, test, and analyze larger quantities of the materials than feasible at the University of Virginia. From the performed experimental work, we down-selected four nylon fiber recipes for initial pilot-scale production sponsored by Solvay: (1) neat nylon; (2) nylon with PVA; (3) nylon with PVA and 0.05 wt.\% graphene; and (4) nylon with PVA and 0.1 wt.\% graphene. While analysis of these fibers is ongoing, the initial findings are presented below.

\section{Experimental Evaluation of Nylon}

In FY 2018, ORNL demonstrated that nylon, which is available commercially at $0.2 \$ / \mathrm{lb}$, could be converted to CF. ReaxFF simulations conducted by the University of Virginia identified bonding within the nylon structure susceptible to $\mathrm{CuCl}$ treatments, thus forming a more stable chemical structure for the necessary oxidation and carbonization treatments for conversion. With this insight, ORNL developed a conversion procedure over FY 2018 and FY 2019 to produce nylon-based CF drawn to a diameter between 10 and $20 \mu \mathrm{m}$, as shown in Figure II.2.1.14. These fibers were used to conduct experimental analysis on the role of graphene and spinning nozzle size. While only two concentrations of added graphene were tested (i.e., 0.1 and 1.0 wt.\%), a clear distinction due to the addition of graphene could not be demonstrated, as seen in Figure II.2.1.15 (a). It was found that a smaller nozzle diameter resulted in a weaker fiber, which suggests that a larger fiber drawdown is beneficial for higher strength, as observed in Figure II.2.1.15 (b). We also conducted Raman spectroscopy on the fibers after the spinning, stabilization, and carbonization and the results are shown in Figure II.2.1.15 (c). There is a significant development of the I(D) and I(G) bands at 1330 and 
$1540 \mathrm{~cm}^{-1}$, indicative of significant development of graphitic structure during carbonization, but no clear distinction with increasing weight ratio of graphene. Furthermore, the mechanical testing with nylon fibers have drawn some contradictions. While testing at the University of Virginia indicates added graphene helps achieve the target properties, testing at ORNL has indicated that neat nylon alone without graphene, may be capable of achieving the desired properties with measured strength, modulus, and ductility of $2.41 \mathrm{GPa}, 179$ $\mathrm{GPa}$, and $1.35 \%$, respectively. Thus, it is critical to investigate the role of graphene further with multiple pilot runs.
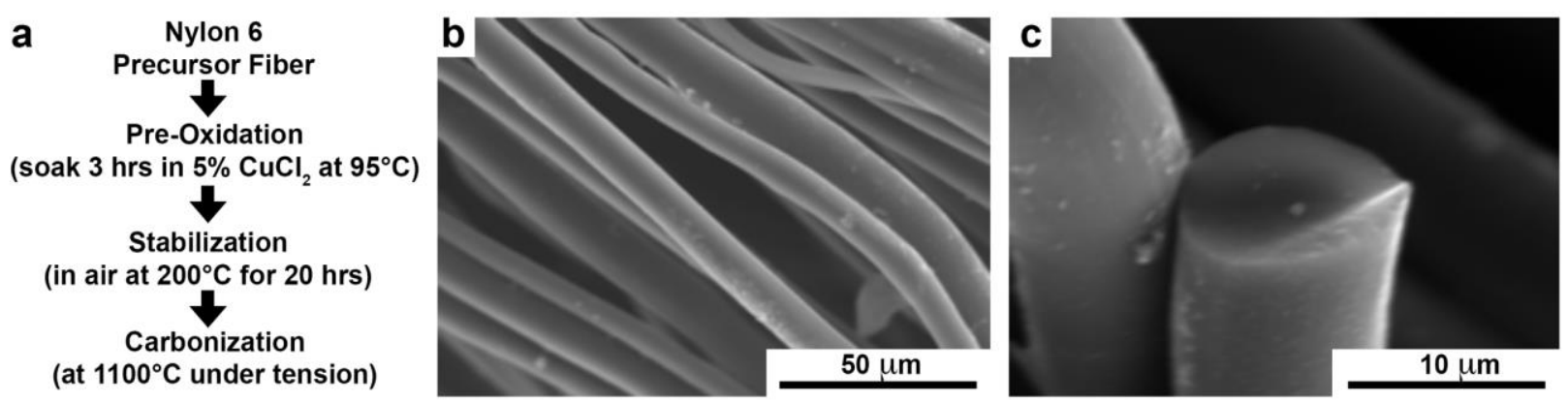

Figure II.2.1.14. (a) The preparation procedure for nylon CF developed at ORNL. (b, c) Micrographs of produced CF. Source: ORNL. 


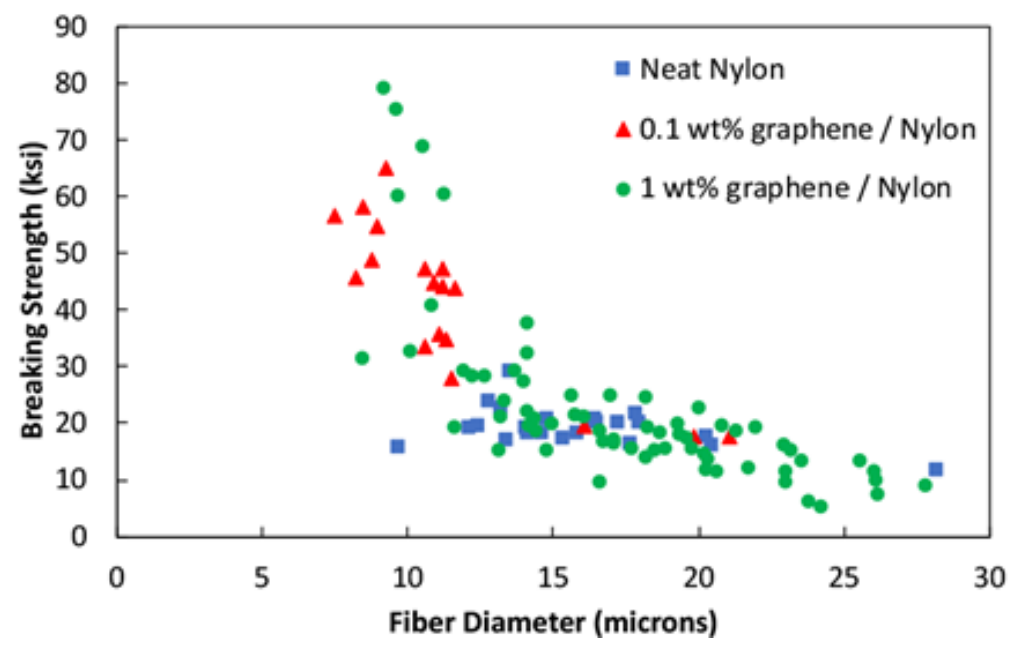

(a)

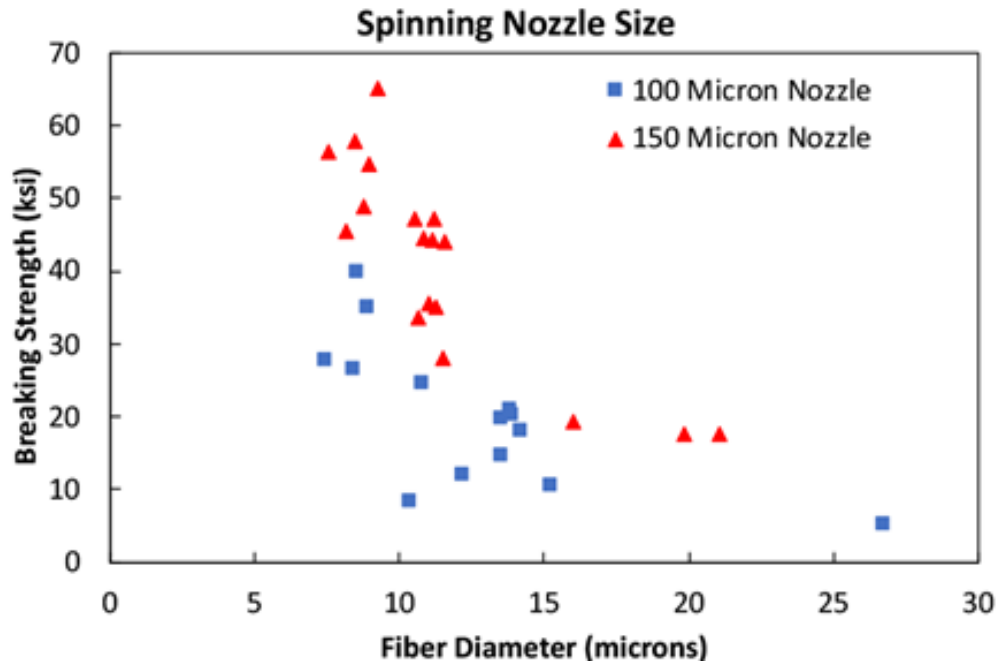

(b)

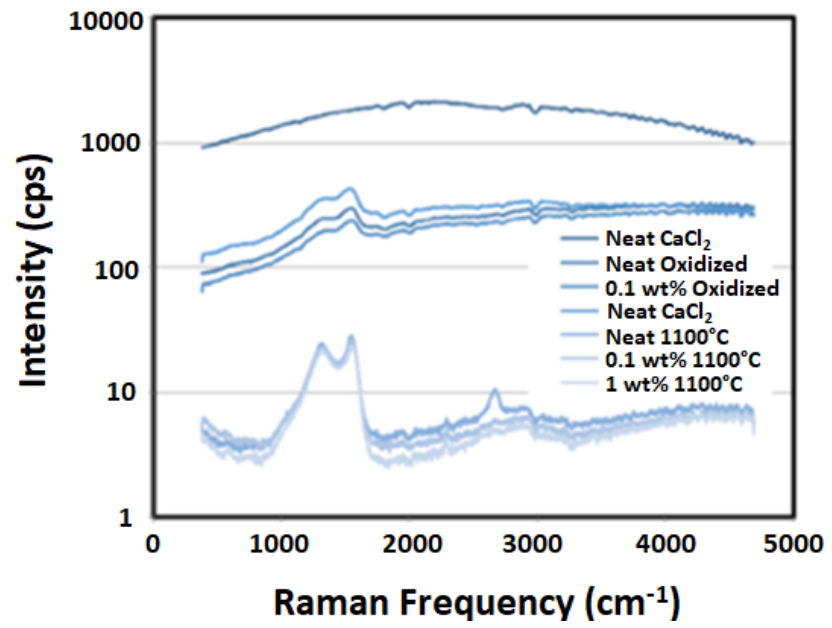

(c)

Figure II.2.1.15. Experimental testing with nylon fibers demonstrate the effect of: (a) graphene content; and (b) spinning nozzle diameter. (c) Raman spectra of fibers after spinning, stabilization, and carbonization. Source: ORNL. 


\section{Experimental Evaluation of UHMWPE}

At the University of Virginia, carbonized UHMWPE fibers were found to meet the target properties, but precursor UHMWPE fibers, if enhanced with graphene, may also be capable of achieving the targets without carbonization. ORNL is leveraging a connection with Honeywell, the manufacturer of Spectra ${ }^{\circledR}$ fibers, to test the benefits of graphene and graphene oxide in their commercial fibers. Honeywell has scheduled a UHMWPE fiber pilot trial, and specimens will be provided to our team for detailed characterization. Thus, we can demonstrate an immediate benefit to industry from the techniques derived in this project, namely the expertise in the integration of graphene within fibers in the desired weight ratio.

\section{Pilot-scale Production of Nylon-derived CFs}

Nylon 6 was selected for the first pilot-scale production run because nylon-based CF produced at the lab-scale met or exceeded DOE targets. Three test mixtures and one control were selected for pilot-scale production. The inclusion of graphene in small weight percentages was found in testing at the University of Virginia to increase the performance of PAN and nylon-derived CF, so two of the mixtures included graphene- one with $0.05 \mathrm{wt} . \%$ graphene and one with $0.10 \mathrm{wt} . \%$ graphene. The graphene was dispersed in a water/PVA solution to ensure that the graphene nanoparticles would not agglomerate during the pellet coating procedure. Therefore, a final mixture of nylon pellets with $0.10 \mathrm{wt}$.\% PVA and without graphene was included to control for the effect of PVA. Finally, a batch of neat nylon was spun as a reference to all test mixtures and to resolve conflicting results out of the University of Virginia and ORNL.

Testing of the pilot-scale fibers is ongoing. We are performing many tests to assemble a statistically significant dataset, typically consisting of about 50 filaments per mixture. Though this work is ongoing, it is possible to draw several critical insights:

1. The optimized conversion mixtures do not scale from the laboratory to the pilot-run. We note that applying the same conversion procedure used to convert the lab-scale fibers to the pilot-scale fibers did not yield proportional results as reflected by both the distribution and magnitude of measured properties for individual recipes collected thus far. Therefore, it will be necessary to optimize the conversion procedure (e.g., conversion temperature, duration, etc.) for fibers produced at a certain scale, with specific equipment. We hypothesize this lack of scalability may be attributed to the limitations of the laboratory conversion procedure and greater consistency of the pilot procedure. For example, on a pilotscale, fiber tension is much more regularly applied and maintained; this consistency is reflected in the relatively uniform precursor fiber diameter compared to lab-produced fibers. We also hypothesize the lab-scale fibers may contain a relatively high quantity of inherent defects (e.g., pores, cracks). In contrast, the pilot-scale fibers contain seemingly fewer inherent defects; thus, they are more sensitive to procedural deficiencies (e.g., misalignment during tension testing, inconsistent tension during conversion, etc.) during conversion, resulting in greater deviation of data for individual conversion recipes.

2. We can identify a mixture (though not yet fully optimized) that does convert the pilot-run fibers to CF capable of achieving the target mechanical properties. Further optimization is currently underway for the nylon pilot-scale fibers produced in FY 2019, but we have narrowed the conversion procedure and demonstrated these fibers can be optimized to achieve the mechanical property targets. A conversion procedure with a $2 \mathrm{~h}, 95^{\circ} \mathrm{C}$ pre-oxidation step; a $25 \mathrm{~h}, 200^{\circ} \mathrm{C}$ oxidation step; and a $0.5 \mathrm{~h}, 1000^{\circ} \mathrm{C}$ carbonization step produced nylon $\mathrm{CF}$ with measured upper limit strength, modulus, and a strain of $2.05 \mathrm{GPa}, 182 \mathrm{GPa}$, and $1.2 \%$, respectively, as observed in Table II.2.1.3. This upper limit reflects the greatest potential of this conversion mixture; the effects of procedural deficiencies or tensile misalignment of the specimen will only reduce the properties from this upper limit. A statistically significant dataset is currently being assembled to draw further conclusions and direct comparisons with other mixtures. 
Table II.2.1.3. Summary of Key Pilot-Scale Results (Upper Limits Only).

\begin{tabular}{|c|c|c|c|}
\hline Precursor & Strength $(\mathrm{GPa})$ & Modulus (GPa) & Strain (\%) \\
\hline Target & 1.73 & 172.5 & 1.0 \\
\hline Neat Nylon (Pilot-scale)* & 2.05 & 182 & 1.2 \\
\hline $\begin{array}{c}\text { Nylon w/PVA + 0.1 wt.\% } \\
\text { graphene (Pilot-scale)* }\end{array}$ & 1.51 & 168 & 1.1 \\
\hline * Testing is still ongoing, and results may change to reflect the latest findings.
\end{tabular}

3. There is a significant variation in measured fiber properties. While the results are not shared here (a statistically significant dataset is currently being assembled), we do note there is significant deviation among measured strength, modulus, and strain among fibers among a batch of the same conversion mixture. This deviation, on the order of $20-70 \%$, suggests there is significant conversion optimization still to perform. As previously mentioned, we attribute this deviation to inconsistencies inherent in laboratory procedures, such as variation in applied tension and angle of filament tension testing, which are well documented in literature to have severe implications for mechanical properties [19]. We also note that we typically report the upper limit mechanical properties; the upper limit represents the greatest potential properties a conversion mixture might achieve. Averages of the collected data would be skewed significantly by improperly handled specimens $[20,21]$. We are currently analyzing every test to characterize the specimen to remove data representing improperly treated fibers; thus, averages and standard deviation of properties may be reliably reported. We have also updated our tension testing alignment guide to improve fiber alignment and conducted slip calibration measurement via digital image correlation techniques [22]. In brief, we must ensure the statistical analysis reflects inherent variation within the fibers and conversion, not variation due to procedural discrepancies and test misalignment.

4. Additional pilot-production testing is necessary to reduce data deviation and improve consistency. As discussed, the pilot-run fibers are more uniform in contrast with the lab-scale fibers, which seems to correlate with reduced deviation of results. In accordance with our original plan for FY 2020, additional pilot-production testing, including later stage conversion (i.e., oxidation and carbonization), will be performed to reduce the data deviation and provide more reliable indicators of the properties of industrially produced $\mathrm{CF}$.

\section{Conclusions}

The work performed in FY 2019 has met the four project milestones planned for the year. First, statistical analysis of alternative precursors - namely UHMWPE and nylon - has been used to optimize lab-scale CF production and demonstrated fibers capable of achieve the mechanical properties targets. Second, ReaxFF simulations have been applied to probe the chemical conversion of these alternative precursors to expand the ICME framework beyond PAN and unveil the underlying mechanisms governing the conversion process (i.e., the role of the $\mathrm{CuCl}$ treatment, UV treatment, etc.). Third, large-scale MD simulations have extended the chemical structure to bulk mechanical properties; this approach has been significantly refined over the past year and will continue to be optimized in Year 3. Lastly, we have begun a scalability study of CF conversion, spanning the production capabilities at the University of Virginia, ORNL, and up to pilot-production facilitated by Solvay.

Towards the development and optimization of the ICME framework, we have continued to refine the computational tools to predict the chemical conversion, lattice structure evolution, and local strain, stress, and graphitization. This work will continue in FY 2020 as we explore the effect of $\mathrm{CuCl}$ treatments, UV treatments, and applied tension on fiber conversion. We will also add a new feature to calculate the matrix of elastic constants from atomic positions in an atomistic model of a CF microstructure to improve the mechanical property predictions of CF microstructure without performing actual tensile testing simulations. 
We are also continuing to perform atomistic simulations to understand the effect of void size distribution on the mechanical properties of CFs.

The key conclusion of FY 2019 was the identification of multiple CF mixtures, which can achieve the target mechanical properties. At the lab-scale, these mixtures include UHMWPE-based CF and nylon/PVA/graphene-based CF. However, these mixtures were found to not directly scale-up; on a pilotproduction scale, neat nylon fibers were found to significantly outperform nylon fibers treated with graphene. While analysis is ongoing, we hypothesize the lab-scale fibers may be more prone to defects (e.g., pores, cracks), which are somewhat mitigated by adding graphene. In contrast, the pilot-scale fibers contain seemingly fewer inherent defects, so added graphene disrupts the fiber microstructure and is itself a defect.

Furthermore, we note that applying the same conversion procedure used to convert the lab-scale fibers to the pilot-scale fibers did not yield proportional results. Thus, it will be necessary to optimize the conversion procedure (e.g., conversion temperature, duration, etc.) for fibers produced at a certain scale, with specific equipment. This optimization stage is currently underway for the nylon pilot-scale fibers produced in FY 2019, but we have narrowed the conversion procedure and demonstrated these fibers can be optimized to achieve the mechanical property targets. A conversion procedure with $2 \mathrm{~h}, 95^{\circ} \mathrm{C}$ pre-oxidation step; a $25 \mathrm{~h}, 200^{\circ} \mathrm{C}$ oxidation step; and a $0.5 \mathrm{~h}, 1000^{\circ} \mathrm{C}$ carbonization step produced nylon $\mathrm{CF}$ with a strength, modulus, and strain of $2.05 \mathrm{GPa}, 182 \mathrm{GPa}$, and $1.2 \%$, respectively.

A critical remaining task is to develop a detailed cost analysis of the fiber conversion. Preliminary estimates of the fiber conversion indicate a carbonized nylon fiber may cost $2.7-4.5 \$ / \mathrm{lb}$, but as the pilot-production continues, we will work with Solvay in FY 2020 to develop a more refined cost estimate.

\section{Key Publications}

1. Chowdhury, A., A. Vashisth, C. E. Bakis, and A. C. T. Van Duin, 2019, "Reactive molecular dynamics simulations of the atomic oxygen impact on epoxies with different chemistries," J. Phys. Chem. C, Vol. 123, No. 24, pp. 15145-15156.

2. Gao, Z., J. Zhu, S. Rajabpour, K. Joshi, M. Kowalik, B. Croom, Y. Schwab, L. Zhang, C. Bumgardner, K. Brown, D. Burden, J. Klett, A. van Duin, L. Zhigilei, and X. Li, 2019, "Graphene-reinforced CFs," submitted to Science Advances.

3. Joshi, K., M. I. Arefev, and L. V. Zhigilei, 2019, "Generation and characterization of CF microstructure in atomistic simulations," Carbon. Vol. 152, pp. 396-408.

4. Kowalik, M., A. Chowdhury, B. Damirchi, D. Akbarian, S. Rajabpour, and A. C. T. van Duin, 2019, "Atomistic scale analysis of the carbonization process for $\mathrm{C} / \mathrm{H} / \mathrm{O} / \mathrm{N}-$ based polymers with the ReaxFF reactive force field," J. Phys. Chem. B, Vol. 123, No. 25, pp. 5357-5367.

5. Ponomarev, I., A. van Duin, and P. Kroll, 2019, "Reactive force field for simulations of the pyrolysis of polysiloxanes into silicon oxycarbide ceramics," J. Phys. Chem. C., Vol. 123, No. 27, pp. 16804-16812.

6. Zhang, L., M. Kowalik, Z. Gao, A. Chowdhury, S. Rajabpour, C. Bumgardner, Y. Schwab, B. Damirchi, J. Zhu, D. Akbarian, J. Klett, A. van Duin, and X. L, 2019, "Converting PBO fibers into high-strength CF by ultrafast carbonization," submitted to Carbon.

7. Zhu, J., Z. Gao, M. Kowalik, K. Joshi, A. Chowdhury, M. Arefev, Y. Schwab, C. Bumgardner, K. Brown, D. Burden, L. Zhang, J. Klett, L. Zhigilei, A. van Duin, and X. Li, 2019, Submitted. "Unveiling carbon ring structure formation mechanisms in polyacrylonitrile-derived CF," submitted to ACS Applied Materials \& Interfaces. 


\section{References}

1. Mainka, H., O. Täger, E. Körner, L. Hilfert, S. Busse, F. T. Edelmann, and A. S. Herrmann, 2015, "Lignin - An alternative precursor for sustainable and cost-effective automotive CF," J. Mater. Res. Technol., Vol. 4, No. 3, pp. 283-296.

2. Park, S.-J., and G.-Y. Heo, 2015, Carbon Fibers. Carbon. Springer: Dordrecht.

3. Brown, S. F, 2013, “CF, light and strong, arrives where it's most needed," 2013, New York Times. New York. July 14, 2013. Available at: http://www.nytimes.com/2013/07/14/automobiles/carbon-fiber-lightand-strong-arrives-where-its-most-needed.html. Accessed 26 Nov. 2019.

4. Warren, C. D., 2007, "The development of lower-cost CF technologies for automotive applications," The Global Outlook for Carbon Fibers 2007, San Diego, CA, USA, 24 Oct. 2007.

5. Fitzer, E., 1989, "PAN-based carbon fibers-present state and trend of the technology from the viewpoint of possibilities and limits to influence and to control the fiber properties by the process parameters," Carbon, Vol. 27, No. 5, pp. 621-645.

6. Chen, J. C., and I. R. Harrison, 2002, "Modification of PAN CF precursor via post-spinning plasticization and stretching in dimethyl formamide (DMF)," Carbon, Vol. 40, No. 1, pp. 25-45.

7. Pichler, D., "“Give us affordable carbon fiber!,"” 2016, CompositesWorld, Available at: http://www.compositesworld.com/columns/give-us-affordable-carbon-fiber. Accessed 26 Nov. 2019.

8. Rao, S., T. G. A. Simha, K. P. Rao, and G. V. V. Ravikumar. 2015. "Carbon composites are becoming competitive and cost-effective." Infosys. Available at: https://www.infosys.com/engineeringservices/white-papers/Documents/carbon-composites-cost-effective.pdf. Accessed 26 Nov. 2019.

9. Senftle, T. P., S. Hong, M. M. Islam, S. B. Kylasa, Y. Zheng, Y. K. Shin, C. Junkermeier, R. EngelHerbert, M. J. Janik, H. M. Aktulga, T. Verstraelen, A. Grama, and A. C. T. van Duin, 2016, "The ReaxFF reactive force field: Development, applications, and future directions," Nat. Comput. Mater., Vol. 2, Art. \#15011.

10. Srinivasan, S. G., A. C. T. van Duin, and P. Ganesh, 2015, "Development of a ReaxFF potential for carbon condensed phases and its application to the thermal fragmentation of a large fullerene," J. Phys. Chem. A, Vol. 119, No. 4, pp. 571-580.

11. Joshi, K., M. I. Arefev, and L. V. Zhigilei, 2019, "Generation and characterization of CFs microstructure in atomistic simulations," Carbon, Vol. 152, pp. 396-408. https://doi.org/10.1016/j.carbon.2019.06.014.

12. Zhigilei, L. V., Z. Lin, and D. S. Ivanov. 2009. "Atomistic modeling of short pulse laser ablation of metals: Connections between melting, spallation, and phase explosion," J. Phys. Chem. C, Vol. 113, No. 27, pp. 11892-11906.

13. Castro-Marcano, F., A. M. Kamat, M. F. Russo, A. C. T. van Duin, and J. P. Mathews. 2012. "Combustion of an Illinois No. 6 coal char simulated using an atomistic char representation and the ReaxFF reactive force field," Combust. Flame, Vol. 159, No. 3, pp. 1272-1285.

14. Dinur, U., and A. T. Hagler, 1991. "New approaches to empirical force fields," Rev. in Comput. Chem., Vol. 2, pp. 99-164.

15. Griffith, A. A., 1921. "The phenomena of rupture and flow in solids," Philosophical Transactions of the Royal Society of London A: Containing Papers of a Mathematical or Physical Character, Vol. 221, pp. 163-198.

16. Yin, H., H. J. Qi, F. Fan, T. Zhu, B. Wang, and Y. Wei, 2015, "Griffith criterion for brittle fracture in graphene," Nano Lett., Vol. 15, No. 3, pp. 1918-1924.

17. Johnson, D. J., and C. N. Tyson, 1969, “The fine structure of graphitized fibres," J. Phys. D Appl. Phys., Vol. 2, No. 6, pp. 787-795. 
18. Ozcan, S., F. Vautard, and A. K. Naskar, 2014, "Designing the structure of CFs for optimal mechanical properties," Polymer Precursor-Derived Carbon, pp. 215-232.

19. Li, X., X. Wang, W.-C. Chang, Y. J. Chao, and M. Chang, 2005, "Effect of tensile offset angles on micro/nanoscale tensile testing," Rev. Sci. Instrum., Vol. 76, No. 3, Art. \#033904.

20. Andersons, J., R. Joffe, M. Hojo, and S. Ochiai, 2001, "Fibre fragment distribution in a single-fibre composite tension test." Compos. Part B-Eng, Vol. 32, No. 4, pp. 323-332.

21. Ilankeeran, P. K., P. M. Mohite, and S. Kamle, 2012, “Axial tensile testing of single fibres," Am. J. Mod. Mech. Eng., Vol. 2, No. 4, pp. 151-156.

22. Schreier, H., J. José Orteu, and M. A. Sutton, 2009, Image Correlation for Shape, Motion, and Deformation Measurements: Basic Concepts, Theory and Applications. Image Correlation for Shape, Motion and Deformation Measurements: Basic Concepts, Theory and Applications. Springer: US.

\section{Acknowledgements}

The PIs would like to recognize contributions from Co-PI Robert Hathaway and collaborator Jay Batten, who have provided valuable input and carbon materials to the team. This input has helped the project remain focused on industry production needs and key material requirements.

The PIs would like to recognize the contributions from Co-PI Billy Harmon, Quan Shi, and others at Solvay Composites, who facilitated the pilot-production run in the second FY. Dr. Shi and others from Solvay organized and attended the pilot-run. They were on-hand to provide guidance and immediate feedback. Solvay is also in the process of analyzing the prepared fiber, and we look forward to discussing the results in the upcoming quarter. 


\section{II.2.2 Consortium for the Production of Affordable Carbon Fibers in the United States (Western Research Institute)}

\section{Don Collins, Principal Investigator}

Western Research Institute

3474 North 3rd Street

Laramie, WY 82072

Email: Don.Collins@uwyo.edu

\section{Dr. H. Felix Wu, DOE Technology Manager}

U.S. Department of Energy

E-mail: felix.wu@ee.doe.gov

Start Date: October 1, 2017

Project Funding (FY19): \$1,174,629
End Date: September 30, 2020

DOE share: $\$ 821,245$
Non-DOE share: $\$ 353,384$

\section{Project Introduction}

Lightweight vehicles would produce several economic and environmental advantages - most notably, reduced fuel consumption and greenhouse gas emissions. Additional cascading benefits would include reductions in infrastructure costs and maintenance by virtue of less stress applied to roads and other transportation infrastructure, as well as far-reaching geopolitical impacts such as improved national security by reducing the dependency of the United States on foreign crude oil.

One way to reduce the weight of vehicles while not sacrificing strength and crashworthiness is to manufacture traditional stress-bearing metal components with lightweight advanced materials and composites, such as those comprising CF. These advanced materials can be engineered to have physical-strength properties greater than steel at a fraction of the weight. Additionally, by properly selecting the correct materials and applying appropriate manufacturing protocols, very strong and highly flexible materials can be produced. That is why CFs are used to produce materials as diverse as spaceship nosecones and the shafts of fly-fishing rods. This gives validity to pursuing CFs for future development of lightweight vehicles.

A difficulty in large-scale deployment of CF for the commercial-vehicle industry is the availability of large quantities of $\mathrm{CF}$ with appropriate physical properties at a reasonable cost. To address these limitations a consortium of academic, government, non-profit, and commercial entities has been assembled to study the feasibility of using large volume, low-cost natural resources to produce CFs of the appropriate quality and cost for use in the vehicle industry. Some of the most abundant natural resources are those from current biomass, such as sugars derived from agricultural sources or from ancient biomass, such as coal and petroleum. The ability to study several different feedstocks types and blends of these feedstocks provides for a robust roadmap for potential materials, while also reducing economic risks that can arise if industry becomes too dependent on any single material, which may itself be subject to climatic, environmental, geopolitical, and, ultimately, market variations. Therefore, within this work, no particular preference is being given to any one feedstock; CFs are being produced from coal-, petroleum-, and biomass-based feedstocks. 


\section{Objectives}

Investigate the current landscape of appropriate raw starting materials from petroleum-, coal-, and biomassbased feedstocks as CF feedstocks and precursors to produce low-cost CF materials for lightweight vehicles in the United States. This objective is divided into two complementary parts:

1. Develop, integrate, and demonstrate a suite of ICME modeling tools that predict CF properties, such as load-to-failure, failure mode, stiffness and deflection, dynamic performance, and microstructures capable of minimum modeling element accuracies within $\leq 15 \%$ of measured properties. This will enable the design, development, and optimization of precursor chemistry and the molecular structure associated with conversion into $\mathrm{CF}$ and evaluate alternative precursors for suitability to manufacture low-cost $\mathrm{CF}$. The ICME models will also include methodologies to simulate manufacturing processes, including variability from both process and material. All non-proprietary and non-business-sensitive public data and codesuch as technical data used to support published journal articles or research code used for simulationswill be provided to the LightMat Consortium for curation and hosting.

2. Develop, manufacture, and demonstrate CF precursor technology and processing techniques where $\mathrm{CF}$ is a material consisting of thin, strong, multi-crystalline filaments of carbon used as a reinforcement material, especially in resins, and capable of achieving the requirements in Table II.2.2.1.

Table II.2.2.1. Project Parameters and Requirements.

\begin{tabular}{|l|l|}
\hline \multicolumn{1}{|c|}{ Parameter } & Requirement \\
\hline Cost & $\leq \$ 5 /$ pound \\
\hline Strength & $\geq 250 \mathrm{Ksi}$ \\
\hline Modulus & $\geq 25 \mathrm{Msi}$ \\
\hline Strain & $\geq 1 \%$ \\
\hline
\end{tabular}

\section{Approach}

Researchers will study readily available large volume feedstocks from biomass, coal, and petroleum to produce precursors of appropriate qualities that lend themselves to the production of CF with the appropriate physical properties and cost to be used in the commercial-vehicle industry. The project will remove risk from this process by developing predictive models that can be used to guide the development of CF materials from new or blended feedstocks. To achieve this goal, the Consortium was assembled from partners that have significant experience working with these different feedstocks, as well as experts in CF production, mechanical testing, and modeling from the molecular-, micro- and macro-level. The team chosen for this project comprises:

- $\quad$ Western Research Institute (WRI): Prime-recipient

- $\quad$ ORNL: Funded by a field-work proposal (FWP)

- Grossman Group at Massachusetts Institute of Technology (GG-MIT): Subrecipient

- $\quad$ Southern Research Institute (SRI): Subrecipient

- $\quad$ University of Wyoming (UW): Subrecipient

- $\quad$ Advanced Carbon Products (ACP): Subrecipient

- Ramaco Carbon LLC (RAMACO): Subrecipient

- Solvay Composites (Solvay): Industrial advisor. 
Each member of the consortium brings a unique and complementary aspect to necessarily build-up fundamental scientific, and yet industrially relevant, understanding of the chemistry and molecular structure of different organic feedstocks and subsequent precursor formation while following the production chain through $\mathrm{CF}$ fabrication, mechanical characterization, and modeling. These individual aptitudes will be meshed using a database with high-level data mining through molecular dynamic models assisted by density function theory, machine-learning, and computational methods to deliver state-of-the-art, holistic, and robust models. The strengths of each consortium member are necessary for the wide scope set forth in the project and an imperative to produce minimum overlap and duplication of effort. Figure II.2.2.1 shows a high-level depiction of the consortium organization and the primary strengths and responsibilities for each consortium member.

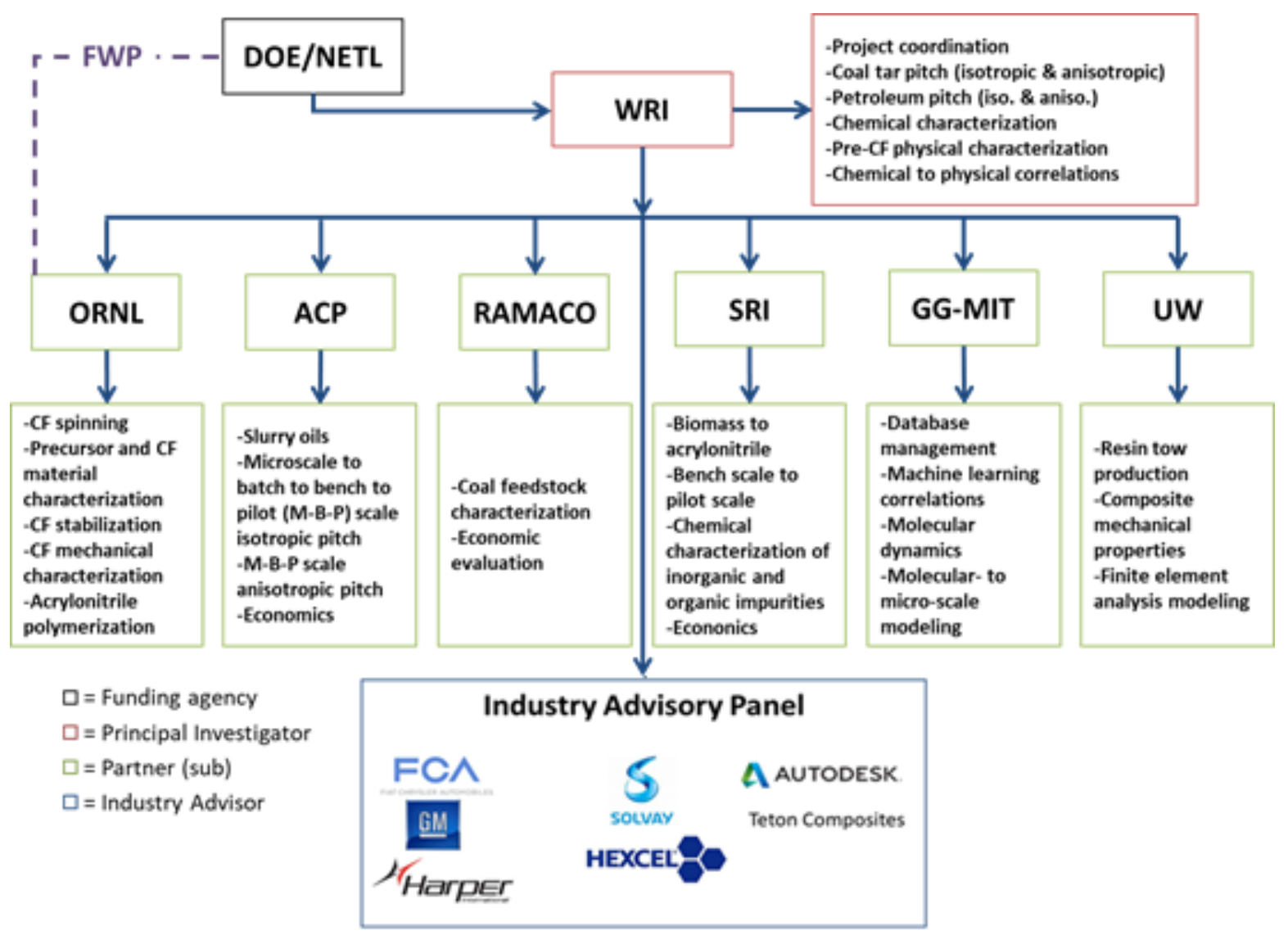

Figure II.2.2.1. Organization of the Consortium for Production of Affordable CFs in the United States. Source: WRI.

Overall, this project is being conducted in three BPs.

\section{BP1: CF Precursor Screening and Down-selection}

CF feedstocks and precursors are studied to down-select precursors capable of achieving the physical-property goals at $<\$ 5 / \mathrm{lb}$. The project entered into a no-cost extension from October 2018-December 2019 and all milestones were achieved by the end of the no-cost extension. CF produced from coal tar pitch (CTP), petroleum pitch (PP), and bio-based polyacrylonitrile (bio-PAN) meet the DOE targets for BP1 for both mechanical properties and cost from preliminary techno-economic analysis. 


\section{BP2: CF Tow-level Material System Properties and Analyses}

Scale-up of acceptable candidates from BP1 to produce larger batches of CF so that composite macro-level models will be created, and CF tow-level composites will be fabricated and tested at extreme temperatures for LD vehicle components to update micro-level and cost estimate models. Micro-level models are being developed to optimize CF properties and production-engineering cost trade-off studies. The milestones for BP2 are shown in Table II.2.2.2.

Table II.2.2.2. Milestones for BP2.

\begin{tabular}{|c|c|c|c|}
\hline Title & Type & Milestone Description & Verification \\
\hline Verify Macro-level FE Models & Tech & $\begin{array}{l}\text { Check predictions against tow uniaxial } \\
\text { creep and mechanical properties test data. }\end{array}$ & Accuracy is $\pm 15 \%$ \\
\hline Micro-Level Models Validated & Tech & Check predictions against data & Accuracy is $\pm 15 \%$ \\
\hline $\begin{array}{l}\text { CF Strength \& Cost Goals } \\
\text { Achieved }\end{array}$ & Go/No Go & $\begin{array}{l}\text { Check that higher volume precursor } \\
\text { production retains CF performance. }\end{array}$ & $\begin{array}{l}\text { Meets DOE strength } \\
\text { goals at }<\$ 5 / \text { lb }\end{array}$ \\
\hline $\begin{array}{l}\text { Establish CF Tow Strength- } \\
\text { Weight (S-W) Ratio }\end{array}$ & Tech & $\begin{array}{l}\text { Establish S-W ratio with LDV manufactures } \\
\text { to confirm weight-savings }\end{array}$ & $30 \%$ to $50 \%<$ steel \\
\hline
\end{tabular}

\section{BP3: Validation and Transfer of Models and Data to DOE ICME Program}

The most promising CF materials will be further evaluated for producibility and to improve macro-level models while building a comprehensive performance database up to the tow-level. Macro- and micro-level models will be integrated and validated for industry use. Models and data will be transferred to the DOE ICME program, and a final cost estimate will be produced.

\section{Results}

Several types of isotropic CTP materials, gilsonite, and PP were spun as fibers and evaluated as isotropic CF. As expected, CTP and PP materials can be spun into fibers after removal of any quinoline-insoluble materials. However, due to the low softening point (SP) of these isotropic materials, stabilization had to be performed at low temperatures and for long durations. In most cases, these materials produced $\mathrm{CF}$ that had strength values significantly below the DOE targets. One exception was CTP6, which has a strength of $205 \mathrm{Ksi}$ and a modulus of $17 \mathrm{Msi}$. These results are quite high for an isotropic pitch and warrant further investigation.

As expected, isotropic pitches must be converted to an anisotropic mesophase to increase the size of the aromatic molecules, the SP, and the carbon residue. Increasing the size of the aromatic molecules allows better alignment of these molecules, resulting in anisotropic domains. With proper fiber spinning, long-range structuring of the aromatic domains can align along the axis of the fiber along the entire fiber. This alignment produces stacking and interlocking aromatic sheets parallel to the fiber axis, resulting in high-strength CF. Isotropic CTP was converted to mesophase with various mesophase content and SPs. Figure II.2.2.2 shows different mesophase pitches produced from CTP6 under different reaction conditions. CTP6 mesophase with a $\mathrm{SP}$ of $320^{\circ} \mathrm{C}$ was successfully spun into precursor fiber and subsequently converted to $\mathrm{CF}$ that exceeded the DOE's target mechanical properties. 


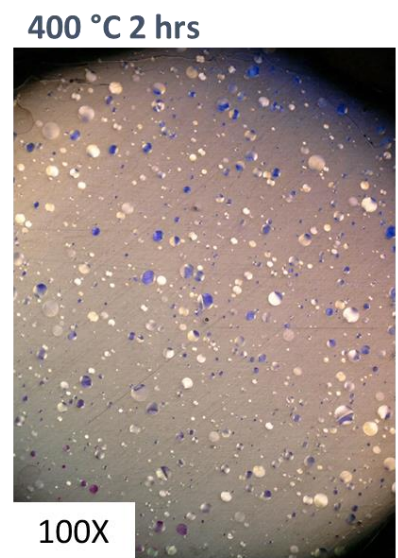

$\mathrm{SP}=$

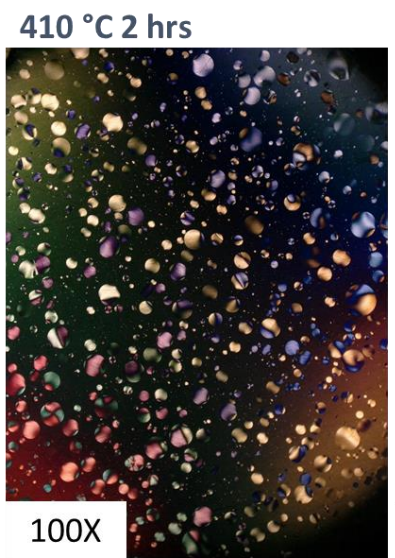

$237^{\circ} \mathrm{C}$

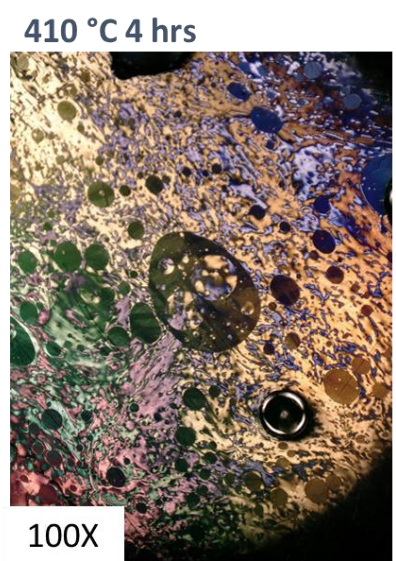

$320^{\circ} \mathrm{C}$

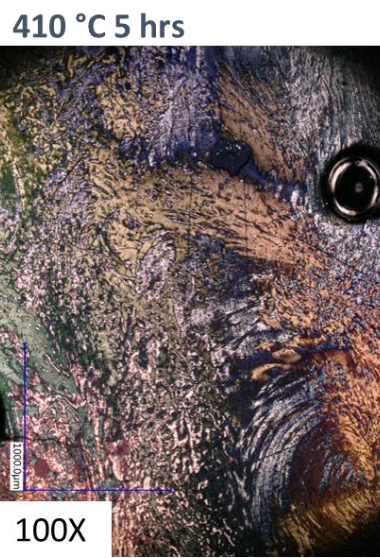

$333^{\circ} \mathrm{C}$

Figure II.2.2.2. Cross-polarized optical-microscopy micrographs for CTP6 under different reaction conditions (above the micrograph) to produce increasing mesophase content and SP (below the micrograph in green font). Source: WRI.

Cross-sections of CF produced from CTP6 mesophase showed both radial- and random-wave morphologies with good alignment of aromatic domains in the direction of the CF length, as seen in Figure II.2.2.3. The morphologies of CFs are being studied to provide links to the failure modes for the fibers. Some of the failure modes which were established result from inadequate stabilization (hollow fibers), interfilament fusing (surface defects or partial melting of fibers along the axis of the fibers) from lower-SP mesophase, and porous fibers produced from high SP $\left(>330^{\circ} \mathrm{C}\right)$ mesophase pitches, as seen in Figure II.2.2.4. For the high SP mesophase pitch, which must be spun at $>350^{\circ} \mathrm{C}$, it is assumed that the high spinning temperature produces devolatilization within the precursor fibers, resulting in porous domains.
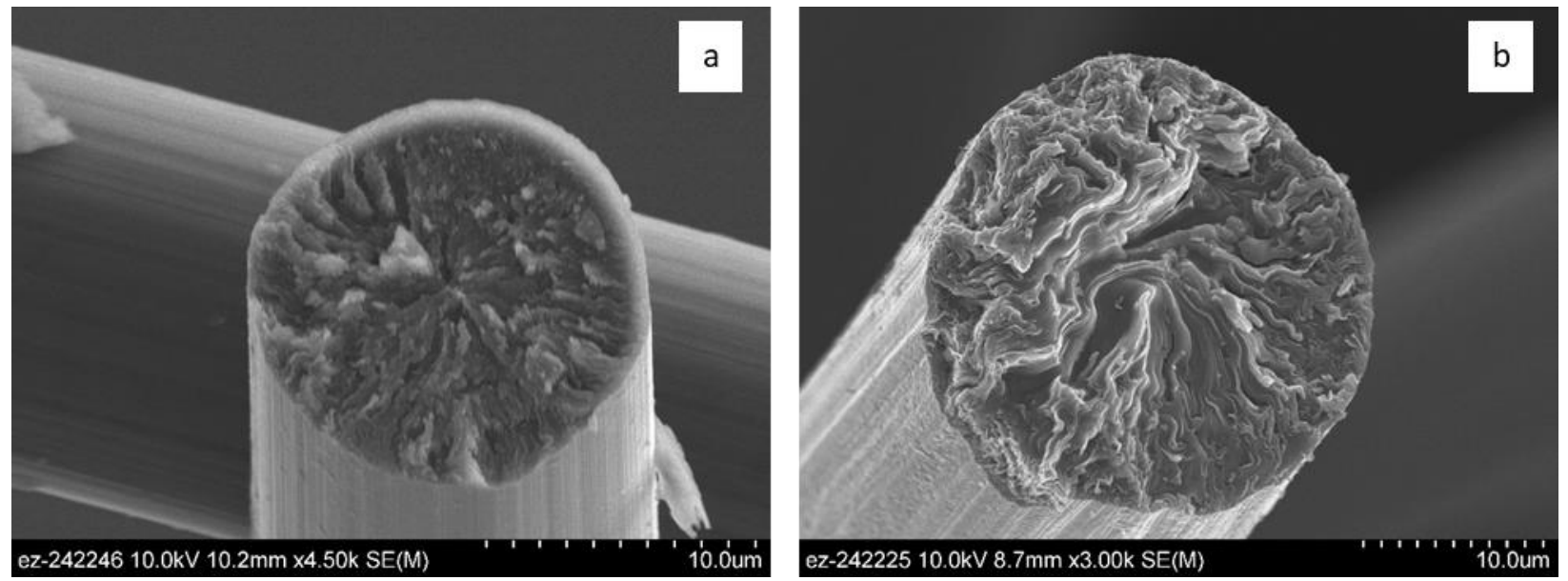

Figure II.2.2.3. Scanning electron micrographs of the well-stabilized CTPC mesophase CF at: (a) $10.2 \mathrm{~mm} \times$ 4.50k magnification; and (b) $8.7 \mathrm{~mm} \times 3.00 \mathrm{~K}$ magnification. Source: ORNL. 


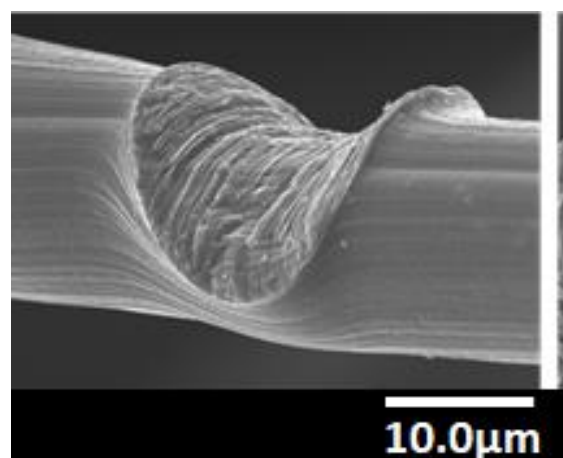

(a)

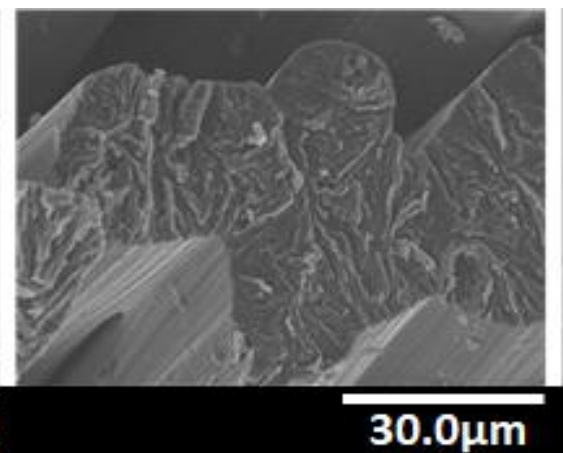

(b)

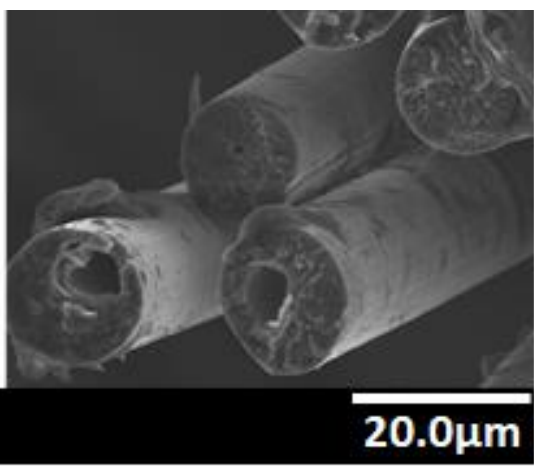

(c)

Figure II.2.2.4. Scanning electron micrographs of various fiber defects from an interfilament fusing: (a) at $10.0 \mu \mathrm{m}$; and (b) at $30.0 \mu \mathrm{m}$. (c) Poor stabilization is seen at $20.0 \mu \mathrm{m}$. Source: ORNL.

A sample of PP mesophase (>95\%) with a SP of $329^{\circ} \mathrm{C}$ was obtained through the ACP continuous-mesophase process. The ACP process takes fluidized catalytic-cracking slurry oil as the feed and converts it into isotropic (ACP 10) pitch, which is then converted into mesophase pitch (ACP 20). Prior to fiber spinning, the ACP 20 mesophase was filtered to remove residual catalyst fines. This filtered material produces CF that exceeds DOE mechanical properties.

Bio-based acrylonitrile (bio-acrylonitrile (ACN)), produced from biomass sugars, was copolymerized with methylacrylate to produce bio-PAN with molecular weights above 100,000 Daltons. This material was successfully solution-spun and produced adequate CF for BP1 requirements. For the overall goals of the project, the strength and modulus are acceptable, but the elongation is low for the final goals of the project. Table II.2.2.3 shows mechanical properties for down-selected precursors from CTP, PP, and bio-ACN that were obtained during BP1 and are planned for further study during BP2.

Table II.2.2.3. Mechanical Properties of Successful CF Candidates

Obtained During BP1 for Use during BP2.

\begin{tabular}{|c|c|c|c|}
\hline Precursor & Strength (ksi / MPa) & Modulus (Msi / GPa) & Strain (\%) \\
\hline \multicolumn{4}{|c|}{ Biomass-derived Precursors } \\
\hline BIO-PAN & $283 / 1948$ & $36 / 248$ & 0.86 \\
\hline \multicolumn{4}{|c|}{ Coal-derived Precursors } \\
\hline CTP 6 NMP & $227.0 / 1565.4$ & 21.2/146.1 & 1.02 \\
\hline CTP 6 MP SP320 & $361.3 / 2491.3$ & 26.5/182.5 & 1.17 \\
\hline \multicolumn{4}{|c|}{ Petroleum-derived Precursors } \\
\hline ACP MP Gen 2 & $347.9 / 2398.5$ & 25.2/173.9 & 1.18 \\
\hline ACP MP Gen 2 GRPH & 243.3/1677.3 & $36.6 / 252.6$ & .57 \\
\hline
\end{tabular}

BP2 is centered on scale-up of the down-selected CF precursors, shown in Table II.2.2.3, to obtain sufficient quantities of $\mathrm{CF}$ for the production of tow-level epoxy composites. These tow-level epoxy composites are being tested to model additional mechanical properties that will be used for advanced FEA methods developed by UW. The analysis of the tow-level composites and feedback loops for data sharing to develop models from the atomistic level through CF tow-level resin composites is shown in Figure II.2.2.5. 


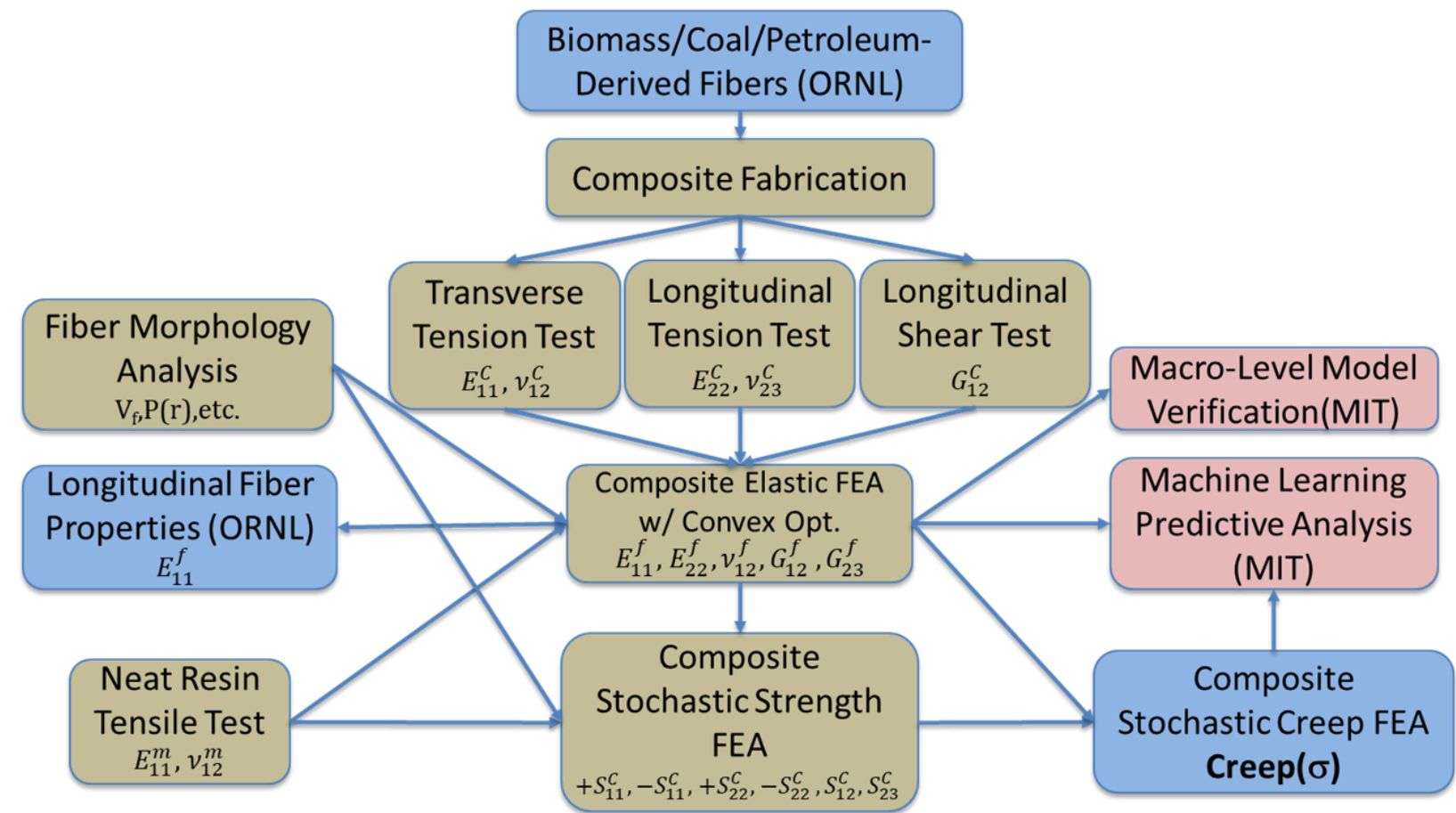

Figure II.2.2.5. Testing protocol developed by UW for tow-level resin composites and feedback loops that will be used for FEA and other modeling efforts. Source: UW.

At the time of this report, scaled batches of bio-PAN CF have been successfully fabricated and are being tested at the tow-level in epoxy resins. The bio-PAN CF that was produced for epoxy resin testing contained 1080 filaments in the tow, with $320 \mathrm{Ksi}$ strength, 33 Msi modulus, and elongation of 0.98 , which is within the DOE targets. The solution-spun precursor fibers exhibited 600-800 MPa tensile strength and $12 \%$ to $15 \%$ strain to failure.

Regarding the pitch materials, CTP6 was scaled-up by one order-of-magnitude to produce mesophase with a SP of $320^{\circ} \mathrm{C}$, which is of the same consistency as mesophase produced during BP1 that yielded CF with mechanical properties exceeding DOE requirements. At the time of this report, the scaled CTP6 mesophase was being produced as CF. Additionally, another CTP source (CTP7, with higher primary quinoline-insoluble content) was also scaled to produce larger batches of mesophase. CTP7 mesophase (80\% to $90 \%$ mesophase) with SPs of $328-340^{\circ} \mathrm{C}$ was spun successfully into fibers. However, $\mathrm{CF}$ from the SP340 material mesophase contained significant voids leading to CF failure, whereas the SP330 mesophase did not contain a void; nevertheless, the fibers lacked complete ordering or aromatic domains when characterizing the microstructure of the CF cross-sections. It is assumed that with current spinning equipment, the higher spinning temperatures needed to spin the CTP7 SP340 mesophase resulted in additional reactions within the pitch, liberating gases and producing the voids. The deficiency of well-ordered microstructure in the CTP7 SP330 mesophase has not been determined, but it may also be linked to the higher viscosity of the material, which is not allowing full alignment of aromatics domains during the spinning process. For the PP mesophase material, additional work is being performed to provide scaled-up filtration of the isotropic PP and slurry oil prior to mesophase formation so that larger batches of the filtered mesophase can be provided to ORNL for use on their pilot-scale continuous pitch-spinning line. 
A major outcome of this program was the development of a database, ${ }^{8}$ which contains chemical characterization of feedstocks, processing conditions, CF mechanical properties, morphologies, and tow-level epoxy composite data. These data were used to build models relating the chemistry and composition of the various stages of material development to $\mathrm{CF}$ and tow-level $\mathrm{CF}$ properties. Heat maps correlating various chemical and physical properties of the different materials with CF properties were generated to determine which measurements are cross-correlated and which are of most importance for modeling physical properties. Figure II.2.2.6 shows a heat map of various chemical and physical measurements from the characterization of isotropic pitch and CF properties of isotropic CF. The heat maps are used to guide the machine-learning (ML) effort to reduce overfitting and provide a targeted approach to determine more-exact chemical meaning from the models. Traditionally, most ML efforts are performed on entire data sets of multiple types of data (i.e., using a holistic approach), although this is a powerful way to build models with a high-level of accuracy, it may lead to overfitting, and many times the model becomes a black box where significant features in the data are not extracted or understood. In this study, both holistic and targeted approaches are applied with the intention of determining dominant chemical, molecular, and physical measurements in the feedstocks, intermediates, and precursors, and their relationship to CF properties. Of course, this becomes significantly stronger with the addition of CF-processing conditions and CF-characterization data. Figure II.2.2.7 shows a summary of how the targeted approach will be applied to gain various chemical and physical drivers that relate to $\mathrm{CF}$ performance.

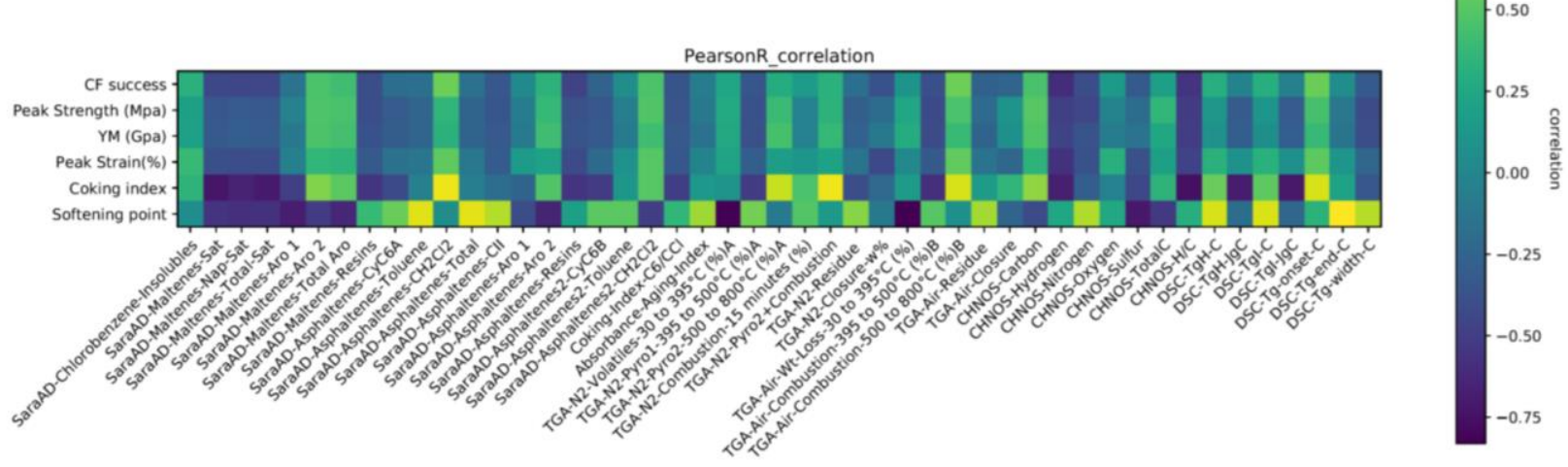

Figure II.2.2.6. Heat map showing positive and negative correlations according to the Pearson method for CF mechanical properties and chemical, compositional, and physical characterization of the corresponding isotropic and mesophase pitch. Source: UW.

8 http://carbonio.mit.edu. 


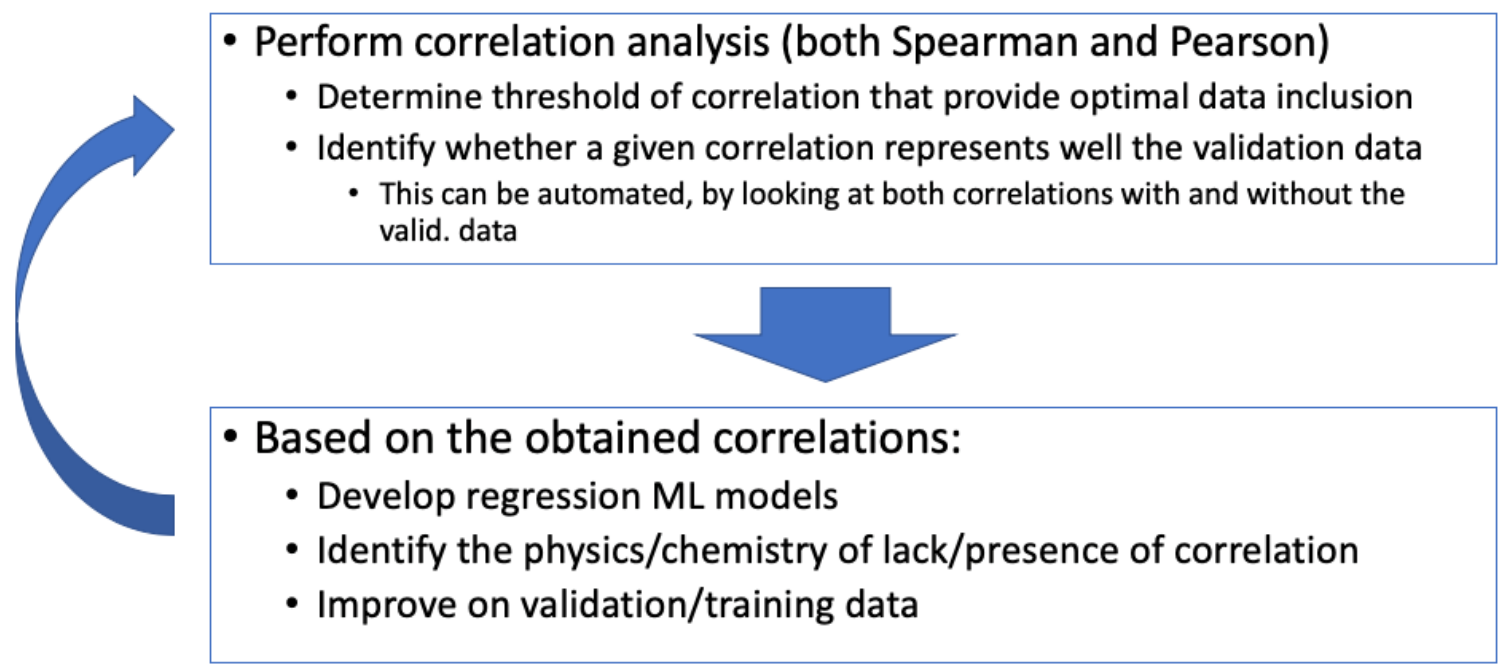

Figure II.2.2.7. The targeted approach that is being applied to data to generate ML predictive models using chemical and physical insights through correlation analysis. Source: MIT.

From the targeted approach, various relationships among the data were determined. These include the positive relationships between saturates, aromatics, resins-Asphaltene Determinator (SAR-AD) dichloromethanesoluble asphaltenes (most polar asphaltenes) and the thermogravimetric analysis fixed-carbon residue (coking index); SAR-AD total asphaltenes and the SP of the isotropic pitch; and the differential scanning calorimetry glass transition endpoint and the SP of isotropic pitch. Also, a negative correlation was determined between Fourier transform infrared spectroscopy aliphatic $\mathrm{CH} 2 / \mathrm{CH} 3$ bend at $1442 \mathrm{~cm}^{-1}$ with the SP of isotropic and mesophase pitch. These correlations will be used in conjunction with other correlations to bridge the gap between the composition and chemistry of the intermediates to precursors to CF. Figure II.2.2.8 shows some of the stronger correlations between various parameters. From these data, several ML models were developed that provide mean average error values less than 0.1 and which have greater than $95 \%$ accuracy. These models will continue to be refined when additional data become available. 

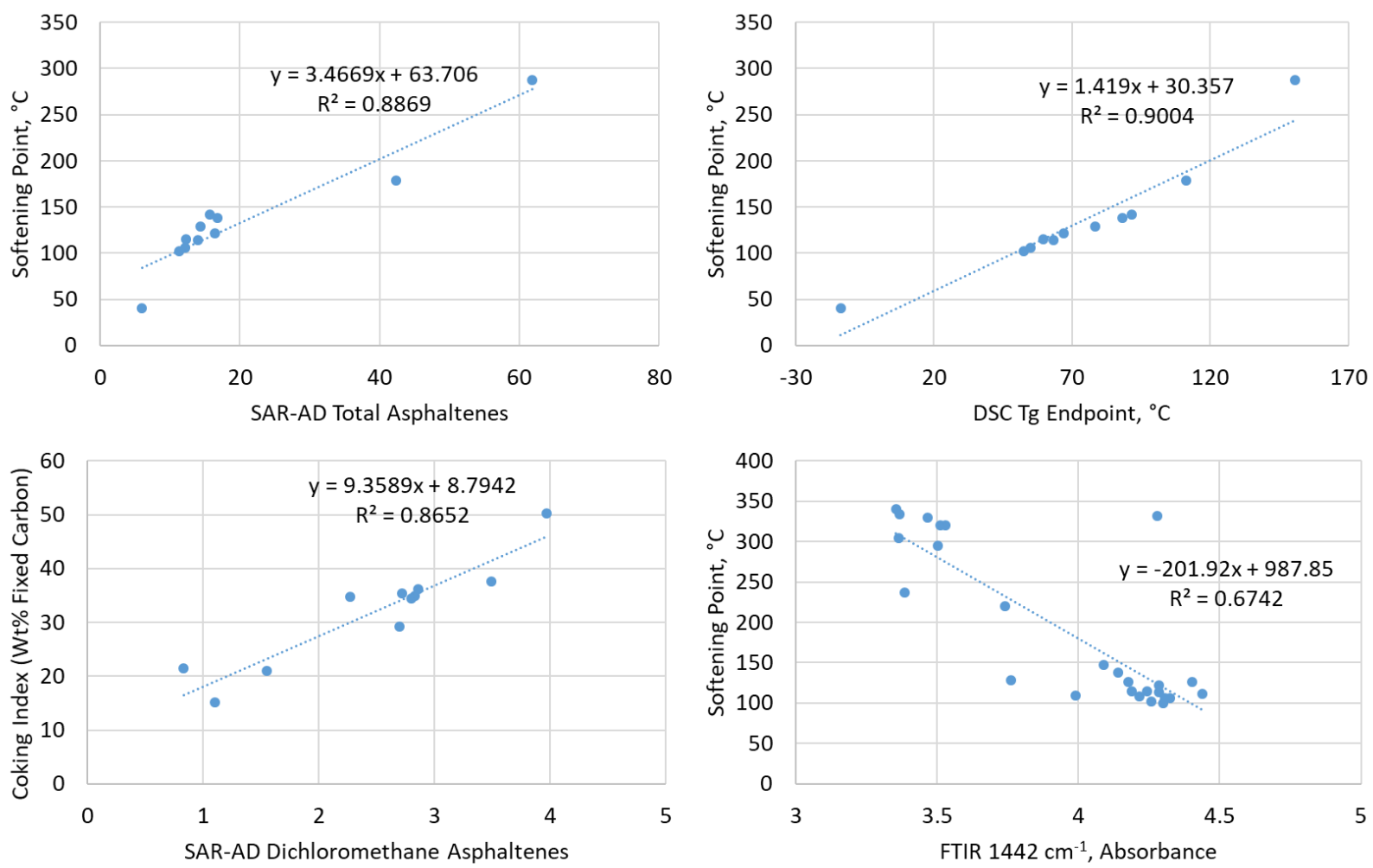

Figure II.2.2.8. Correlations between various chemical and physical properties of isotropic and mesophase pitch. Source: WRI.

At the molecular level, computational methods were used to illuminate the mechanisms through which aromatic CTP and PP precursors molecules are converted to intermediate mesophase mesogen molecules. Additional ongoing computations are being performed to show how the presence of substituents like methyl groups and oxygen heteroatom functional groups affect the formation of mesogens and their alignment. Initial aromatic growth was probed using a hydrogen abstraction followed by acetylene addition to polynucleararomatic-hydrocarbon (PAH) molecules to determine the most stable PAH isomers upon aromatic growth. Because aromatic growth during mesophase formation is generally believed to occur through dimerization of aromatic units, additional computations were performed using simple-model PAH molecules to determine energetically favorable pathways for dimerization, trimerization, and further oligomerization. Examples for these computational strategies are shown in Figure II.2.2.9.

Modeling for bio-PAN formation and bio-PAN CF necessitates a significantly different approach to modeling because each molecule of PAN has a molecular weight range from 100-150K Daltons, which would require significant computing power and significantly reduced throughput to track each atom for multiple PAN molecules. Therefore, a coarse-grained approach was developed and applied to minimize the number of atoms that must be tracked during simulations. Through this approach, the chemical evolution from ACN to bio-PAN through stretching, stabilization, carbonization, and graphitization have been successfully modeled. Correct modeling by this method required proper selection of the coarsening model to represent the chemical, molecular, and structural evolution without significantly sacrificing the computational response, especially regarding ladder formation. Figure II.2.2.10 shows the coarse-grained approach used to model bio-PAN CF formation from polymerization through stretching to form the fibers. 


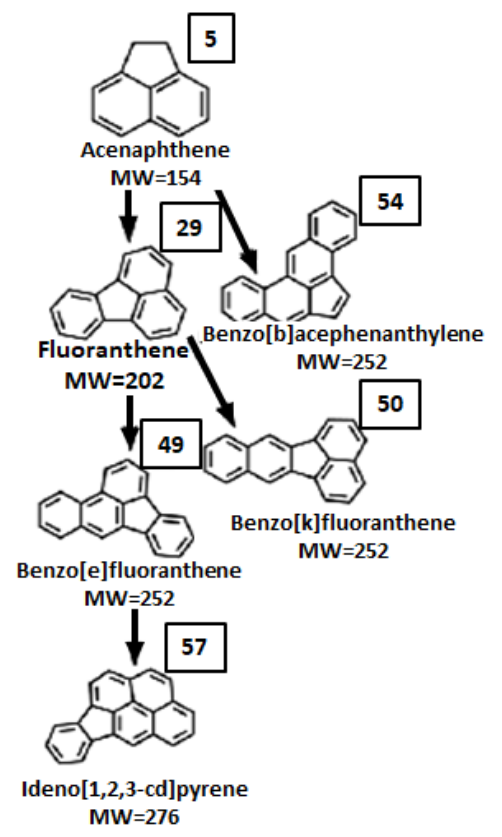

(a)

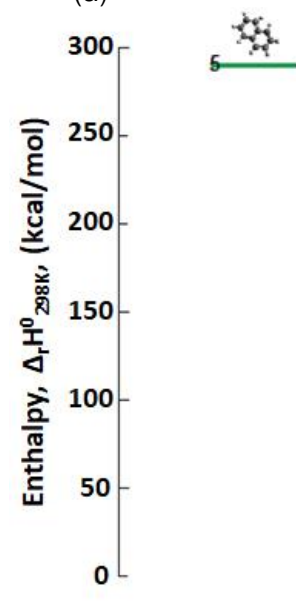

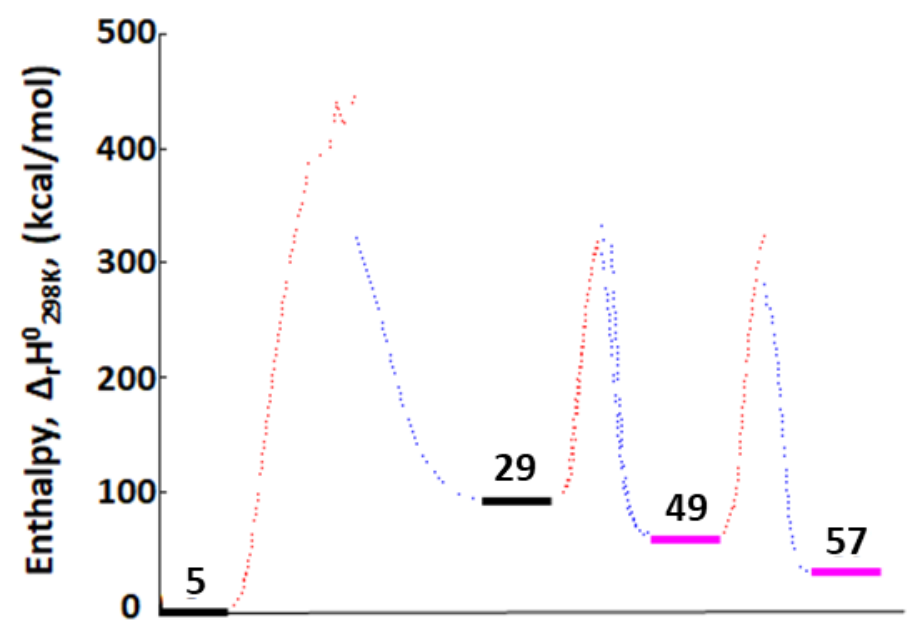

Chemical (see previous image)

(b)
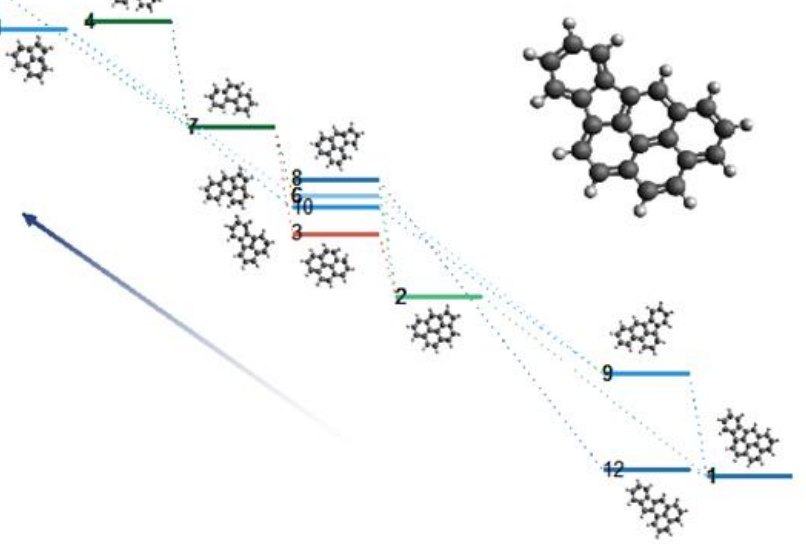

(c)

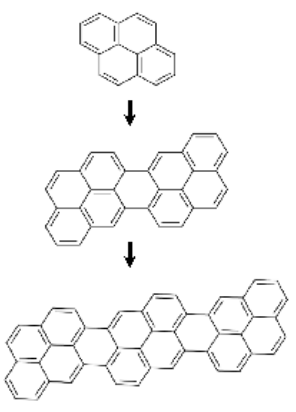

(d)

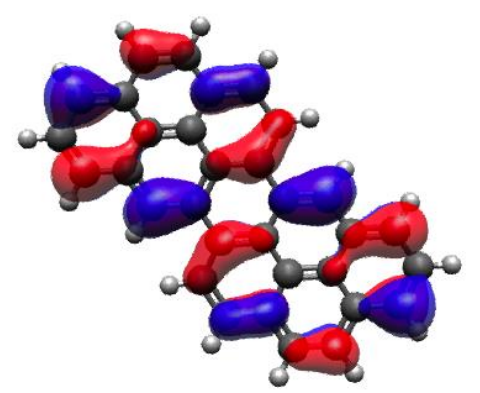

(e)

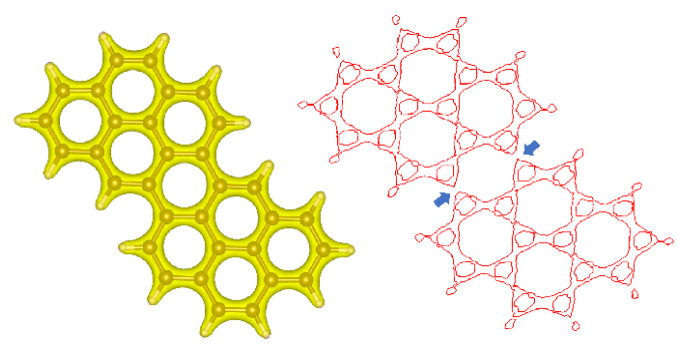

(f)

Figure II.2.2.9. First principles energetic calculations based on hydrogen abstraction acetylene addition mechanism. (a) A hypothesized pathway for indeno[1,2,3-cd]pyrene [1] is assessed regarding both (b) energetics and minimum energy path.

(c) The automatic enumeration of all possible pathways for indeno[1,2,3-cd]pyrene and (d) polynucleus condensation mechanism for pyrene dimerization and trimerization. (e-f) HOMO and total charge density of pyrene dimer. Source: MIT. 

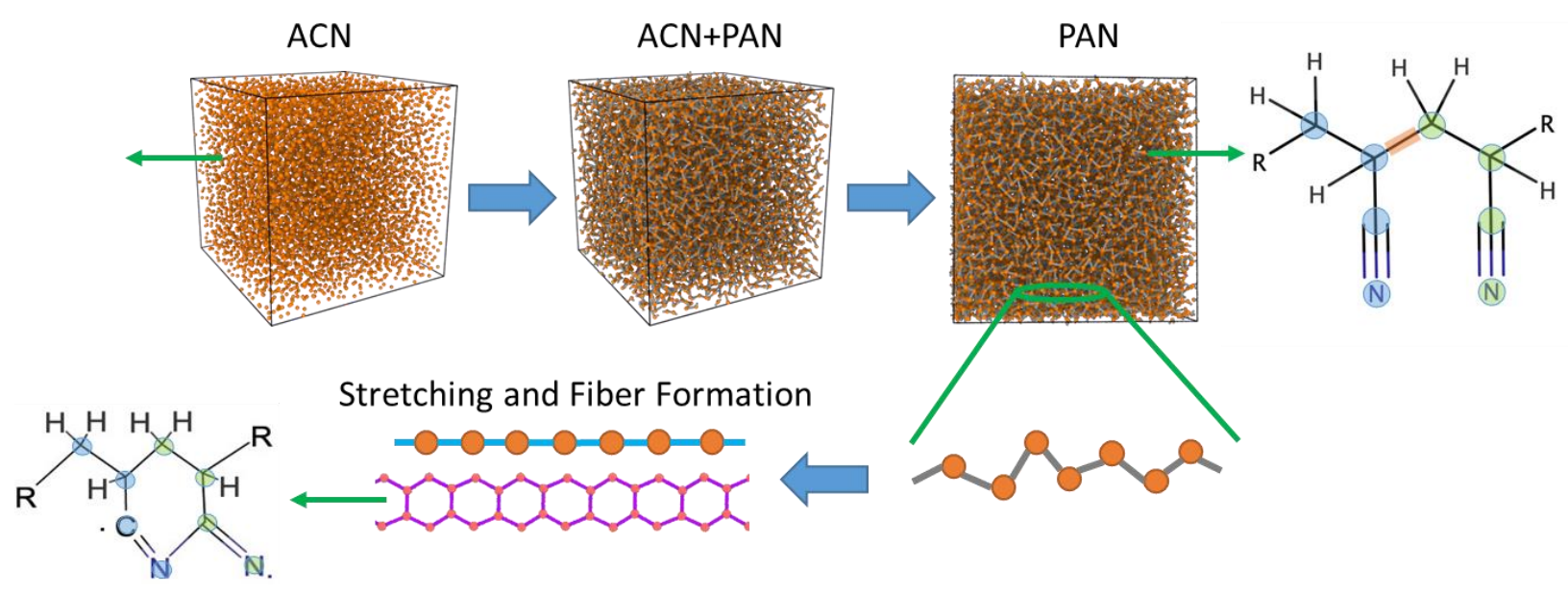

Figure II.2.2.10. Coarse-grained approach used to model the chemical and structural evolution of bio-ACN into bio-PANbased fibers. Source: MIT.

\section{Conclusions}

Through this consortium, CTP, PP, and bio-ACN precursors were successfully used to produce CF with a strength $\geq 250 \mathrm{Ksi}$, a modulus $\geq 25 \mathrm{Msi}$, and a strain of $\geq 1 \%$ at $\leq \$ 5 / \mathrm{lb}$. These precursors represent low-cost, high-volume materials that can potentially meet the demand for wider incorporation into lightweight vehicles. The precursors cannot be used directly in their raw form but must be converted into intermediate mesophase pitch or bio-PAN prior to spinning into fibers. Successful CF candidates are currently undergoing scaling under BP2 to generate larger batches of CF tows. These tows are being used in the construction of epoxy molds that are being used to determine non-trivial mechanical properties of the CF through FEA. Bio-PAN tow-level epoxy samples were constructed and testing of the epoxy resin composites is underway. Scale-up of CTP mesophase was successful, and several batches are being qualified for spinning into tows at ORNL. Scaled batches of ACP20 PP are awaiting hot-filtration prior to being sent to ORNL for spinning.

In the areas of computation and data-management, atomistic modeling of PAH molecules to generate understanding of mesophase formation from pitch materials is ongoing. Additional computations with methyl substituents and oxygen functional groups were also performed. Additional mechanistic computations are underway to model polyaromatic condensation for the growth of mesogen molecules. Oxidation reactions are being modeled to simulate stabilization. For the bio-PAN, a coarse-grained approach has been developed to allow reasonable computation of the large polymeric molecules. Through selection of an appropriate coarsegrained structure, the resultant models can predict alignment of bio-PAN polymers and proper reactivity for the formation of a condensed ladder structure. ML was applied to actual chemical characterization data sets and literature data sets to provide some level of confidence in predicting both process outcomes as well as CF properties. A database ${ }^{9}$ has been established by MIT to build predictive models from chemical, compositional, and physical data collected by the consortium members. As additional data become available, new models will be added, and refinements made.

The key objects for producing appropriate CF from petroleum, coal, and biomass feedstock, according to DOE specifications, were achieved. Additional scale-up of CF from the down-selected materials, modeling, FEA, and molecular modeling are ongoing to achieve a fully integrated ICME program.

$9 \underline{\mathrm{http}: / / \text { carbonio.mit.edu. }}$ 


\section{Key Publications}

1. Collins, D., "Consortium for the Production of Affordable Carbon Fibers (CPACF) in the U.S., ICME Predictive Tools for Low-Cost Carbon Fiber," 2019 U.S. DOE Vehicle Technologies Office Annual Merit Review, MAT125, 11 June 2019.

2. Collins, D., "Consortium for the Production of Affordable Carbon Fibers (CPACF) in the U.S., ICME Predictive Tools for Low-Cost Carbon Fiber," 2019 U.S. DOE Vehicle Technologies Office Annual Merit Review, MAT125 19 June 2018.

Public presentations were given regarding this work, and no other publications have been generated by this work, although several are currently being drafted.

\section{References}

1. Fan, X., Y. Fei, L. Chen, and W. Li, 2017, "Distribution and structural analysis of polycyclic aromatic hydrocarbons abundant in CTP," Energ. Fuel. Vol. 31, pp. 4694-4704.

\section{Acknowledgements}

The CPACF in the U.S. would like to acknowledge DOE Project Manager John Terneus for his administrative help and guidance during this project, as well as DOE Technology Manager Dr. H. Felix Wu for his useful feedback. The authors would also like to acknowledge those individuals who have contributed significantly to the technical success in several areas: Jeramie J. Adams at WRI for project coordination, writing this report, technical lead over the project and subrecipient activities, chemical characterization, molecular modeling, and mesophase conversion and characterization; Michael Elwardany at WRI for help with developing the targeted method for ML; Khalid Baig at WRI for analytical analysis, pitch filtration, and conversion of isotropic CTP to mesophase; Logan Kearney at ORNL for CF spinning, mechanical properties testing, and bio-ACN polymerization; Nicola Ferralis at MIT for spearheading the computational work at the molecular level, ML, and database; Don Malone at ACP for providing expert technical guidance on mesophase formation; Ray Fertig at UW for the work done on tow-level resin composites, mechanical testing of composites and FEA. 


\section{II.2.3 Ultralight Hybrid Composite Door Design and Rapid Manufacture (TPI Composites Inc.)}

\section{Nathan Gravelle, Principal Investigator}

TPI Composites Inc.

373 Market Street

Warren, RI 02885

E-mail: ngravelle@tpicomposites.com

\section{Dr. H. Felix Wu, DOE Technology Manager}

U.S. Department of Energy

E-mail: felix.wu@ee.doe.gov

Start Date: December 1, 2015

Project Funding (FY19): \$546,046.40
End Date: December 31, 2019

DOE share: $\$ 368,165.35 \quad$ Non-DOE share: $\$ 177,881.05$

\section{Project Introduction}

New corporate average fuel economy regulations require improved fuel efficiency of the future vehicle fleet. Weight reduction is key to achieving these targets. Replacing metallic body and chassis components with carbon fiber-reinforced composites (CFRC) offers the most weight reduction potential at up to $70 \%$. The introduction of the BMW i3 and i8 in 2014 required mass production processes to meet 20,000+ units per year. Preforming with High-Pressure Resin Transfer Molding (HP-RTM) has been implemented and meets rate, cost, and performance requirements. Our team members - Krauss-Maffei, Hexion, and Saertex ${ }^{\circledR}$ - were extensively involved in technology development (i.e., manufacturing, materials, and preforming) with BMW (only one of the manufacturers utilizing the technology) and brought this experience to our team, led by TPI, the vehicle original equipment manufacturer (OEM), and the University of Delaware Center for Composite Materials (UD-CCM). We will advance these technologies to develop an ultralight driver's side door for the vehicle with production rates of 80,000 units annually.

TPI has over 40 years of experience in the design, testing, prototyping, and production of lightweight composite structures and is leading the team of industry and academic partners with knowledge in all aspects of vehicle design and composite materials. The OEM will provide system requirements, integrate the ultralight door into the vehicle, and validate the design during vehicle testing. Krauss-Maffei, Hexion, Saertex, and Creative Foam will demonstrate their next-generation material and process solutions, while University of Delaware - Center of Composite Materials (UD-CCM) is world-renowned for their composite expertise in all aspects of composites R\&D. The team will implement the new composite door design and evaluate integration and manufacturing challenges to meet automotive rate and cost targets.

\section{Objectives}

The objectives for this project are to address the following targets and technology gaps:

Target: Reduce part count and full-system weight by a minimum of $42.5 \%$.

Gap: Current materials and methods utilize steel as the main structural component, adding mass to the overall structure, thereby reducing the vehicle fuel efficiency.

Target: Cost increase will not exceed $\$ 5$ per pound of weight saved.

Gap: One of the major lightweighting materials at our disposal $-\mathrm{CF}-\mathrm{is}$ upwards of $\$ 10-\$ 15 / \mathrm{lb}$. This material must be used judiciously to meet the cost targets. 
Target: Materials and processes will be demonstrated to meet the production rate and performance requirements (an approximate four- to five-minute cycle time is required to meet annual production rate).

Gap: Standard composite manufacturing processes can process these parts at a cycle time of about one-hour per part. New injection technologies and resin formulations have opened the possibility of faster cycle times.

\section{Approach}

Development of a vehicle BIW is a very complex and time-consuming process because various, oftenconflicting, functional requirements must be considered. Introducing new designs to reduce vehicle weight requires a systems approach where new designs can be quickly iterated and refined to evaluate their performance. This is particularly true when metals are replaced with composite materials because composite materials have significant potential to reduce weight when designs are fully optimized for parts consolidation and engineered properties using a variety of available material, fiber layups, and processing choices.

A typical automotive door is made from a combination of materials, including steel, plastic, and glazing. The structural materials are heavy, while the non-structural components do not contribute significantly to structural performance. Elements are joined together, increasing manufacturing and assembly cost and weight. We propose to replace all structural parts of the front side driver's door with continuous reinforced composites (with a weight-savings of up to 60\%), reduce part count and system weight through part consolidation, and evaluate alternative glazing materials. This approach has the potential to meet and exceed the goals of $42.5 \%$ system weight reduction as compared to the steel door baseline and to meet cost targets of $\$ 5$ per pound weight saved. The team will take a systems approach to meet the targets, as seen in the flow diagram in Figure II.2.3.1.

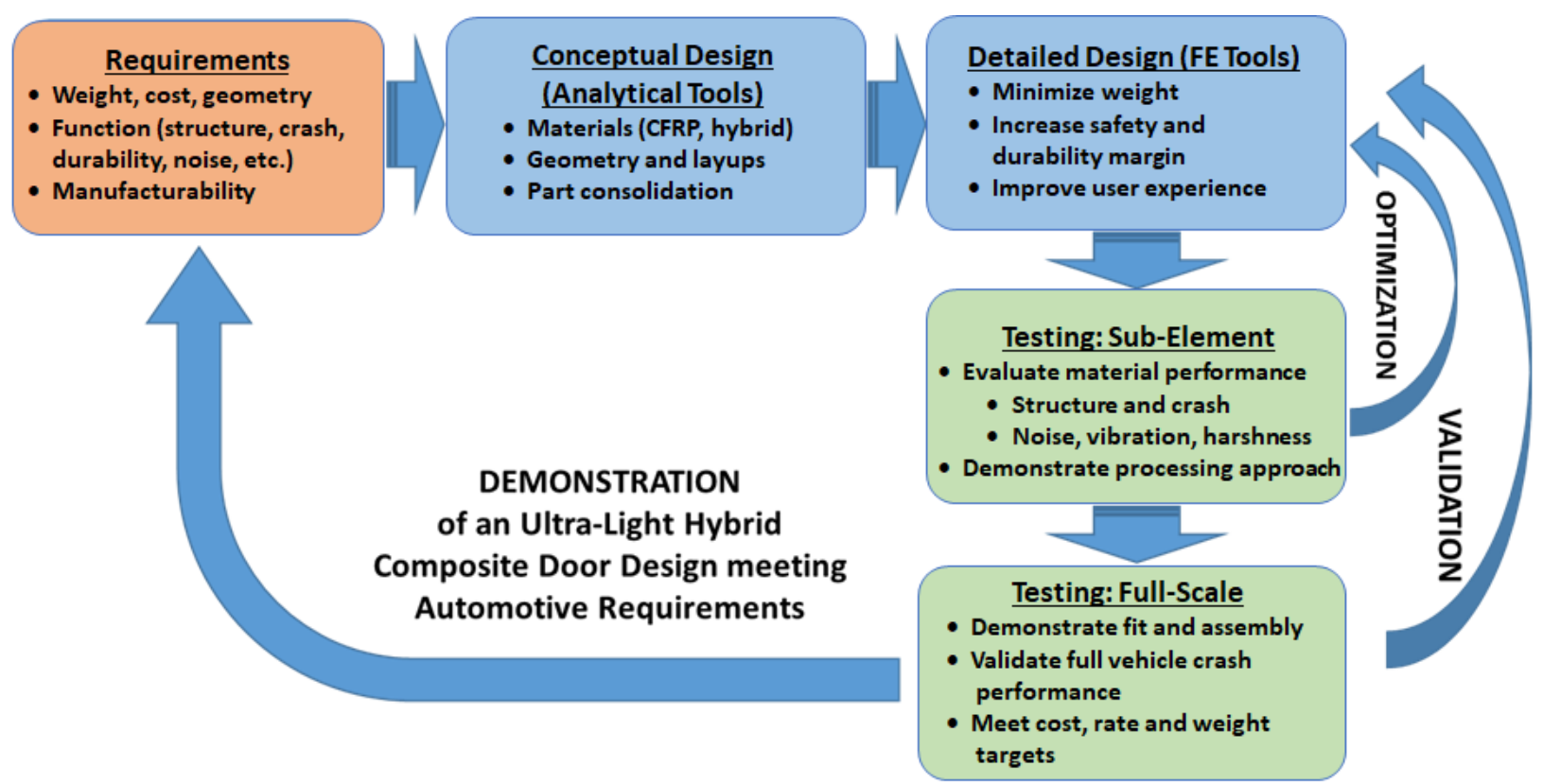

Figure II.2.3.1. Systems approach for reducing weight in complex automotive structures including the use of FE tools for detailed design. Source: University of Delaware.

This approach relies on the use of computational engineering analysis and simulation tools combined with sub-element testing to rapidly develop and evaluate design changes while full-scale testing is used to proof out the final design. The program will define the design requirements (such as weight and cost targets), functional and topology constraints, and consider the ability to manufacture the door at the required rate and performance. Cost; structural; crash; NVH; and manufacturing simulations exist and will be utilized. These individual 
simulation tools are state-of-the-art, commercially available, and have been validated on numerous occasions. Conceptual designs will be evaluated at the sub-element level to evaluate material performance (i.e., structure; crash; and $\mathrm{NVH}$ ) and to demonstrate that the processing approach meets rate and quality targets. Full-scale test articles will be manufactured to validate form, fit, function, and cost of all integrated structural and nonstructural components. A small number of design iterations may be required to optimize the various configurations.

The approach will allow (1) a shortened design cycle, resulting in reduced development time and costs; (2) elimination of trial-and-error process and part trials reducing tooling and manufacturing costs and (3) an optimum door configuration at minimum weight leading to a more cost-competitive product. The overall approach will be demonstrated on a composite door solution for the vehicle, but it is also applicable to a wide variety of automotive components. The comprehensive systems approach for designing, manufacturing, and validating a complex ultra-lightweight composite automotive component using a validated, multidisciplinary design tool with a small number of manufactured components for validation will reduce risk to convert metal structures to composites.

Predictive engineering tools guide material and design down-selection and are critical for eliminating trial-anderror and reducing cost and time. Figure II.2.3.2 shows the design environment the team will employ to evaluate the composite door structure.
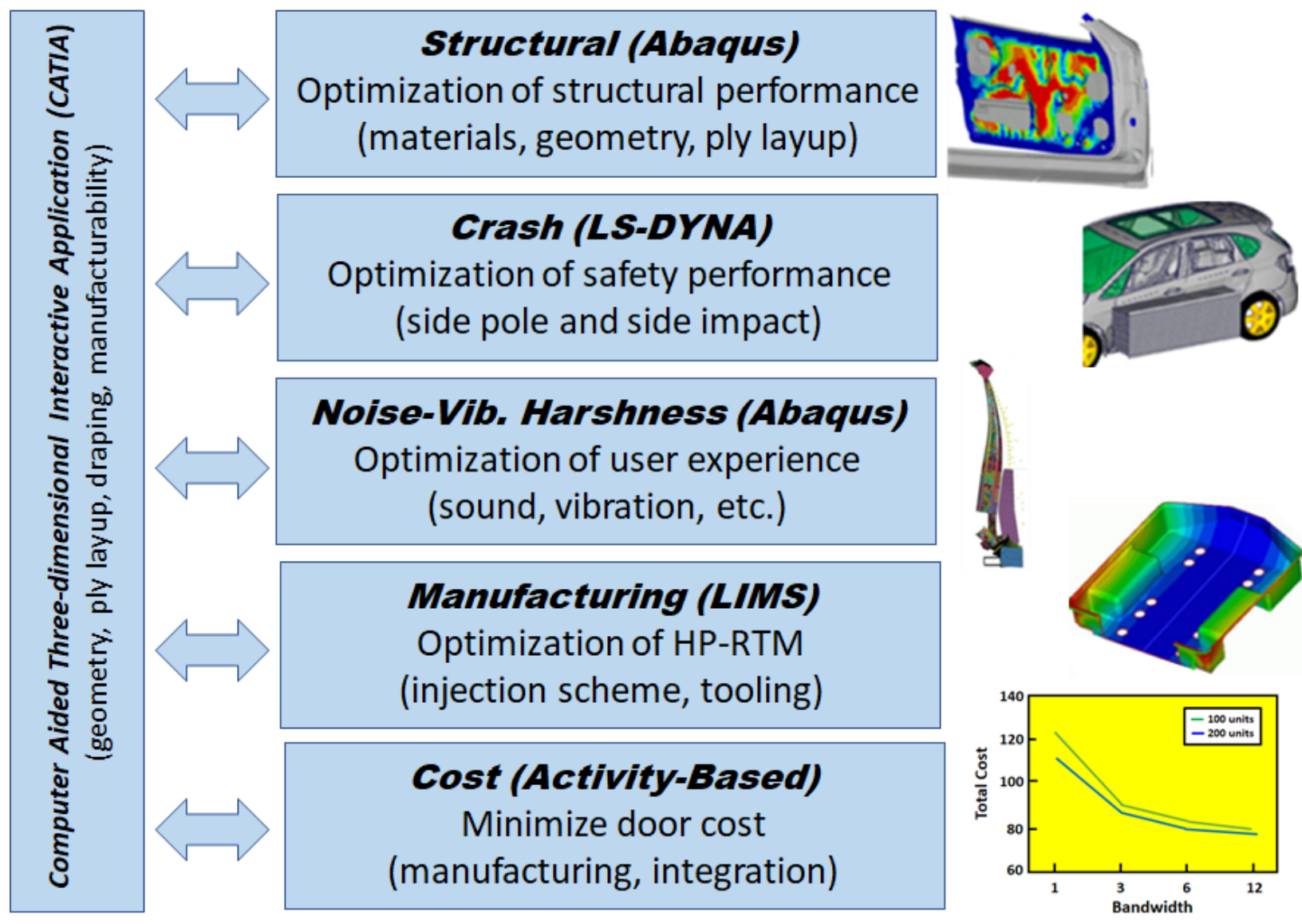

Figure II.2.3.2. Integrated predictive engineering environment. Source: University of Delaware. 
Dassault System's computer-aided three-dimensional interactive application product design solution is our product development platform that easily communicates with other simulation tools. This enables multiple disciplines to share geometry, ply layup, and manufacturing-induced fiber orientations. Thus, designs are developed in one environment and then evaluated in the specialist applications across all phases of the product development process. For example, an important aspect of composite manufacturing is the effect of draping the fiber layer onto the mold surface, resulting in changes of the local fiber orientation. This can affect the infusion behavior during resin injection and the structural and crash performance of the final part. Our approach captures manufacturing-induced variations in the design and feeds these properties into all sub-models. Another example is potential sandwich constructions where the design not only improves structural stiffness, but also noise attenuation (improving ride experience) with novel foam solutions. Integration of other nonstructural functional door items (e.g., speakers, glazing, and electronics) are captured in the design and are fed into the appropriate models and concepts.

The existing vehicle steel door is used as a baseline and the ability to reduce part count with a composite structure will be investigated. Part consolidation reduces weight and cost because a smaller number of parts must be manufactured. Assembly time and associated labor costs can be significantly reduced as well. The HPRTM process allows complex geometry part fabrication, which enables integration of features into one component. Figure II.2.3.3 illustrates the potential part count reduction of a steel door with an equivalent composite structure [1]. Part count reduction alone will not be able to meet our weight reduction goals of $42.5 \%$, but in combination with hybrid and/or CFRCs material replacement and lower weight window solutions, it will reduce the weight of the door structure to the required levels.

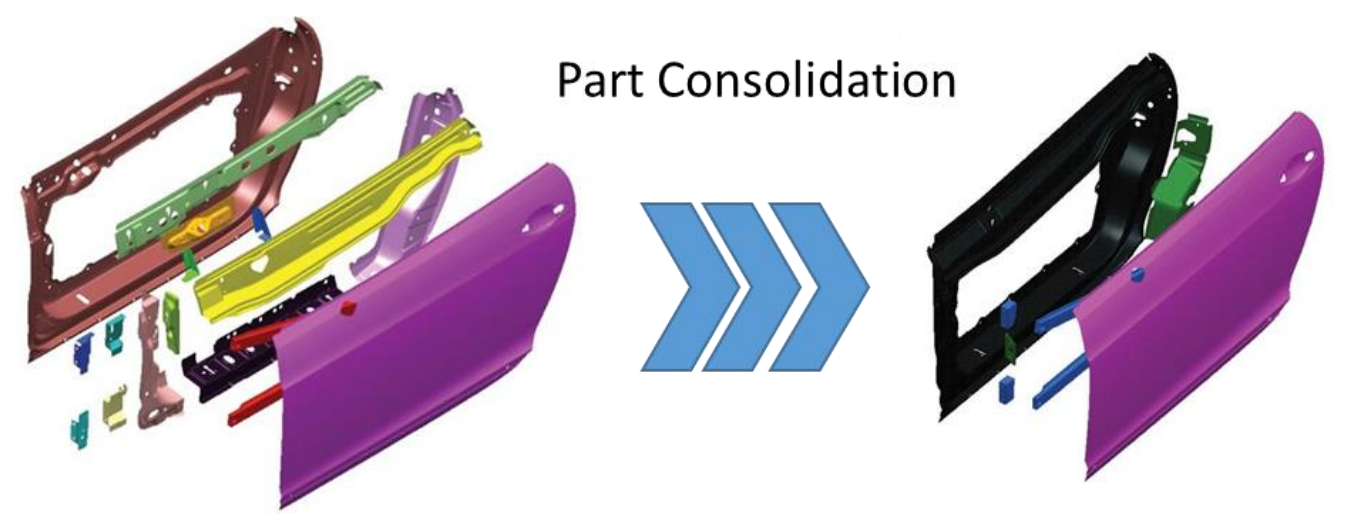

Figure II.2.3.3. Composites allow part consolidation, further reduces cost and weight. Source: Composites World [1].

Our hybrid solution will evaluate a variety of material solutions, including glass and CFs. Fiber modulus and strength depend on the fiber selection with specific properties being the highest for CFs. Design solutions without cost consideration will use $100 \%$ CFs and provide the best structural performance at the lowest weight. A hybrid design will incorporate alternative fiber solutions at a lower-cost and meet structural performance. Our optimal design will consider all options and will meet structural requirements and cost and weight targets.

Lower-density glazing (such as polycarbonate glazing) has been recently developed for automotive applications. Transparent polymers can be easily molded into complex shapes, and it offers a 50\% weightsavings compared to standard glass solutions. The new materials have been demonstrated in both concept and production cars, including the Chevrolet Volt, Hyundai European Design concept cars, the Mercedes SMART, and Toyota V (station wagon version of the Prius) vehicles [2]. New window solutions also address the requirement for improved cabin comfort. Because glazing thicknesses have been reduced to save weight, the noise level within the car has increased. Integrating transparent acoustic layers within the glazing can be used to increase damping performance and thinner and lighter-weight glazing can be employed without 
compromising cabin comfort or safety. Polycarbonate glazing enables new design concepts because complex geometry windows can be fabricated. Polycarbonate glazing with integral ribs lock the parts onto the vehicle or support other features. This program will evaluate a polycarbonate solution, which should not only impact the weight of the glazing, but the overall design of the composite door solution. This will simplify assembly and have the potential to lower the cost and weight of the total door solution.

All considered concepts will be evaluated at the component and full-door level using structural FEA tools. The composite laminate structure can be varied and will change the anisotropic stiffness and strength behavior of the part. The selection of fiber materials will impact performance and cost. Optimization of the layups, materials, and geometries needs to result in a manufacturable design at minimum weight while meeting all design requirements. The team has significant experience in design and analysis using commercially available structural static and dynamic FEA tools for vehicle structures that will be key for evaluating and optimizing the designs.

Crashworthiness will be evaluated using LS-DYNA, allowing simulation of the door and full vehicle under dynamic conditions. In particular, we will consider the crash performance under side-impact meeting Federal Motor Vehicle Safety Standard 214's protection requirements (other Funding Opportunity Announcement crash scenarios will be considered). The simulation will evaluate inward deflection as a function of time during impact for the baseline steel door and our composite solution. A conservative design goal would require the composite solution provide a deflection profile that stays below the transient intrusion levels of the steel baseline door. This would ensure the safety mechanisms (such as the side airbag) are able to be deployed in time and space and the passenger is protected in case of a side collision. UD-CCM has significant experience with crash predictions and under a current National Highway Traffic Safety Administration program evaluates composites for a steel B-pillar replacement. Strain-rate-dependent material properties for composites are available; however, additional properties for the HP-RTM resins and fibers may have to be determined using coupon and subscale element testing. The test data will provide the programs with a database of material properties for crash designs.

The program will implement the HP-RTM process to fabricate sub-elements and full-size components. The process has been proven to produce Class A finished structural components at automotive rates. Cycle times of less than ten minutes have been demonstrated in production on the BMW i3 and i8; this program will further reduce cycle time and performance using the most recent advances in resins and reinforcements developed by our team members (i.e., a four- to five-minute cycle time would meet current vehicle production rates). Our partner, Krauss-Maffei, has implemented a production cell to automate the process. Structural components, sidewall panels, floor pans, front-end carriers, crash boxes, and CF design components are applications that have been implemented via HP-RTM. Fiber mats or fabrics are preformed and then positioned in the mold. A variety of low-viscosity polymers (such as polyurethane, epoxy, and polyamide) can be used as matrix material. The material components are mixed and heated in a metering system and injected into the heated mold. The resin quickly cures in the closed tool and the part can be demolded. Trimming occurs onsite. The HP-RTM process can produce parts with fiber content up to $70 \%$. The process allows reuse of scrap material, improving material yield. Components manufactured using high-pressure RTM exhibit Class A surface quality and can produce high quality (low-defect) parts with an aesthetically pleasing $\mathrm{C}$ appearance. The procedure has been fully automated and is suited for series production from the manufacturing of preforms up to postmold processing. The program will use the existing HP-RTM as the baseline process, evaluate opportunities to reduce cycle time through innovative new materials (Saertex and Hexion), and evaluate new process improvements (Krauss-Maffei, UD-CCM, and TPI).

New resin materials are currently being developed at Hexion and will be optimized for this program. These resins (e.g., EPIKOTETM 05475) and appropriate curing agents have low initial viscosity (below 100 centipoise) and allow rapid infusion of reinforcement during the injection phase of the HP-RTM process [3]. The rheology of the EPIKOTE resin with three different curing agents is discussed in Hillermeier et al. [3] and 
shows the ability to control the viscosity profile, while ensuring rapid cure without significant exothermic reaction of the polymer. Recent advances show full property translation and rapid (i.e., snap) cure in less than two minutes at elevated temperature. The low-viscosity profile allows reduced injection pressure throughout the infusion cycle, relaxing the requirements of the preform, tool, mixer, and press. This, in turn, reduces preform distortion, cycle time, and capital cost.

Non-crimp fabrics (NCFs) provide the best fiber property translation and, using multiaxial systems, can be combined in a preform used in the production of large series vehicle components. These preforms are manufactured to the correct geometry and fiber layup, allowing rapid placement of the reinforcement into the HP-RTM tool. This enables minimum cycle time during the process, paired with the high quality of the final product. It is important to optimize the preform to reduce scrap material and lower material cost. Our partner, Saertex, is the worldwide leader in tailor-made NCF materials and they will support development of low-cost preforms for this program.

A key challenge of the HP-RTM is the design of the mold and preform to ensure full infusion of the polymer into the reinforcement. Tooling cost is a significant capital expense because applied pressures are high, and the tool is expected to last over the entire production run. UD-CCM is an expert in modeling the infusion behavior in liquid molding of hybrid preforms with complex geometry. The permeability and drapability of the reinforcement, as well as the rheology of the resin, are key material properties and are needed to allow optimization of the injection port locations and resin pressure cycle during infusion processing. We will evaluate the feasibility of the proposed designs to be manufactured and optimize the mold features for successful infusion, eliminating any required tool changes due to resin infusion issues. The program will ensure manufacturability of the proposed concept with HP-RTM and use virtual process tools to optimize tooling and infusion approaches. Tooling cost for HP-RTM is a significant investment and can only be amortized over a large production run. Conventional RTM processing of prototypes with equivalent part properties will be conducted as part of our risk reduction strategy.

The performance of our designs will be evaluated at the coupon, sub-element, and full-door component level. This will include structural performance testing (i.e., static and crash) and other functionalities such as durability to environmental exposures and NVH. Our team has existing testing capabilities in these areas and a comprehensive test plan will be developed as part of the program. Coupon testing is needed to characterize the mechanical and microstructural (i.e., void content and fiber volume fraction) properties of the hybrid composite design made with the Hexion resin and HP-RTM process. Other data (such as durability, acoustical damping, and environmental performance) may need to be generated and may require larger component testing.

\section{Results}

FY 2019 started with our waiting for new materials for improved door inner preform drapability. The preform materials will include a chain stitch, a tricot stitch — which allows for greater drapeability or shearability - and a braided broadgood with good properties, as shown in Figure II.2.3.4 (a)-(c). These new material forms should improve drapability and contouring and reduce the amount of wrinkling seen in the preform. 


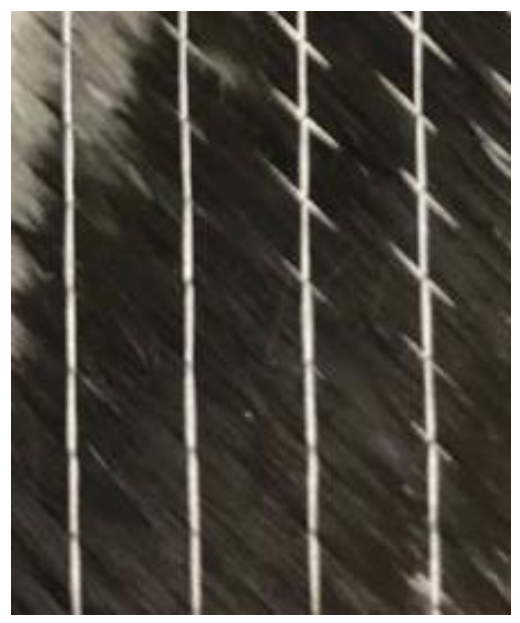

(a)

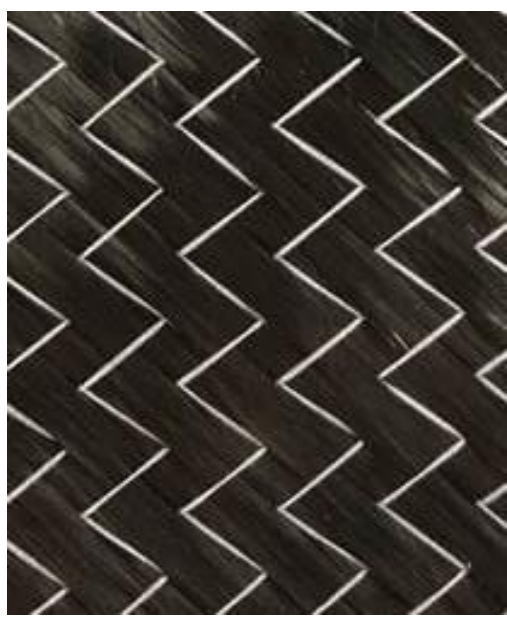

(b)

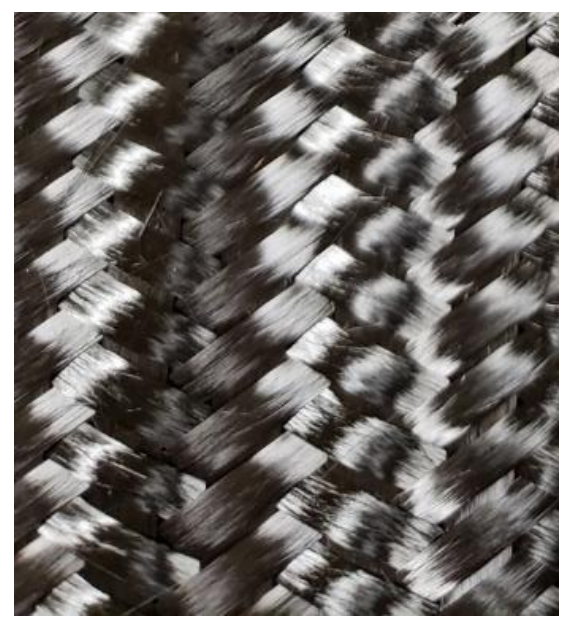

(c)

Figure II.2.3.4. Preform materials types: (a) chain stitch; (b) tricot stitch; and (c) braided broadgood fabric. Source: TPI Composites.

During the second quarter of 2019, the team worked on the following tasks: (1) applying draping models to model the preforming operation of the door inner; and (2) development of an optimization framework to maximize the structural performance of composites with minimum mass. The draping models developed in a previous DOE-funded project to GM (Contract No. DE-EE0006826) for developing NCFs were applied here. In parallel, a multi-variable optimization of a laminated composite beam subjected to a three-point bend test was investigated. The composite hat section beam layup was optimized with respect to two distinct requirements: (1) maximizing the peak load; and (2) maximizing the energy absorption (i.e., the area under the force-displacement curve). Subsequently, two layup designs optimizing the two requirements stated above were selected. The layup angles for these beams were modified slightly to match with the angles in the available fabrics. Composite beams were molded using these modified layups and experimentally evaluated. To validate the results, the predictions for the load versus displacement curves for these optimum solutions were compared with the experimental results. Good correlations were observed validating the developed models.

\section{Draping Simulations}

The draping simulation model used here involves modeling the fabric with independent behavior in three different modes, such as in-plane, bending and shear. Fabric characterization for bending and shear are essential for modeling. Following sections provide the details about the characterization tests.

\section{Bending Stiffness Evaluations}

The bending stiffness of a fabric is often measured using a cantilever beam bending apparatus, in which the fabric is allowed to bend as a cantilever beam under its own weight. ASTM D1388 provides a description of the method for making these measurements. The apparatus used for taking these measurements is shown in Figure II.2.3.5 (a). The bending stiffness can be calculated since it is related to the curvature of the deformed fabric as it bends under its own weight. To carry out the measurements, the fabric was cut into a $25 \mathrm{~mm} \times 229$ $\mathrm{mm}(1 \mathrm{in} . \times 9$ in.) strip and carefully placed onto the apparatus without disturbing the fibers. The fabric was then covered by the top movable sliding board. This board was then slid across the top plate to the predetermined overhang length and the sample was allowed to drape to its natural curve, as shown in Figure II.2.3.5 (b). After the target overhang length was reached, high-resolution images were captured in order to measure the draping curvature of the fabric at this overhang length. A $70 \mathrm{~mm}$ overhang length was selected for the measurements along the stitching direction, and $90^{\circ}$ to the stitching direction, while a $110 \mathrm{~mm}$ 
overhang length was selected for the measurements along the fiber direction. The bending stiffness could be determined using the equations available in the literature to describe the fabric draping curvature.

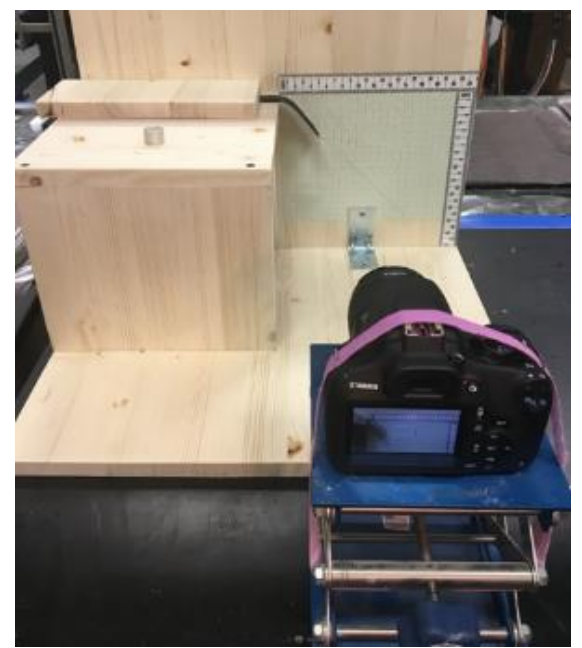

(a)

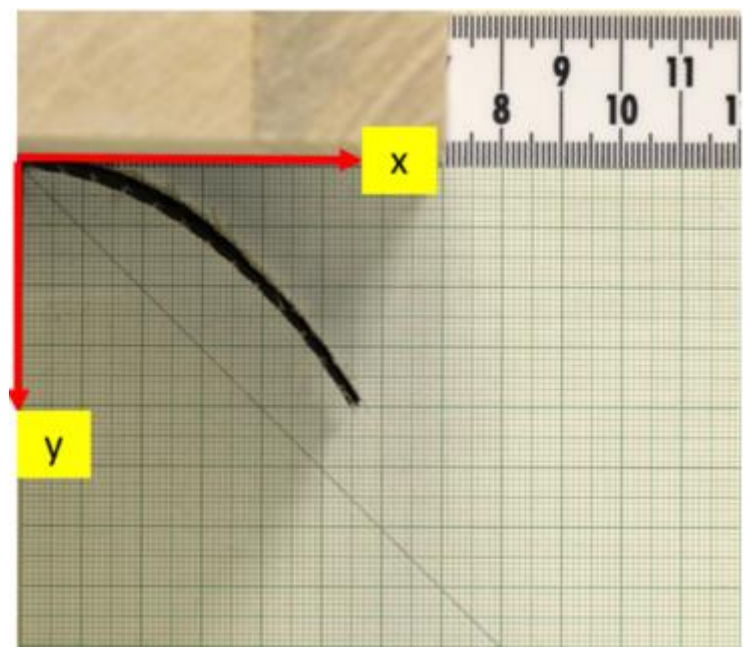

(b)

Figure II.2.3.5. (a) Bending stiffness measurement apparatus. (b) An example image used for measuring curvature and the extent of bending. Source: TPI Composites.

\section{Bias-Extension Experiments}

The bias-extension test measures the load required to stretch fabric that is prepared with the tows oriented at $+/-45^{\circ}$ with respect to the loading direction. The bias-extension test was carried out using specimens of both NCF fabrics. Data was collected for specimens that were $127 \mathrm{~mm}$ wide. This data was used in the calibration of the PAM-FORM material card. In order to simplify the analysis of the data, the length of the specimen was three times greater than the width. Therefore, the tested area of the fabric was $127 \mathrm{~mm} \times 381 \mathrm{~mm}$ ( 5 in. $\times 15$ in.). Since the specimens required an extra $38 \mathrm{~mm}$ at each end in order to mount them in the grips of the test frame, the actual specimen length was $457 \mathrm{~mm}$ for the $127 \mathrm{~mm}$ wide specimens. Physical testing was performed on an Instron 5582 load frame using series 2710-116 side action grips with $25 \mathrm{~mm} \times 76 \mathrm{~mm}$ grip faces. Great care was taken not to disturb the original fiber crossing angles of the test specimens as they were prepared and mounted in the testing frame. The grip separation rate for the $127 \mathrm{~mm}$ specimens was $30 \mathrm{~mm} / \mathrm{min}$.

The shear behavior of the fabric was measured with both of the stitches acting in compression and tension, respectively. When the stitches are in tension, the shear stiffness is significantly higher than the case with the stitches in compression. The nonlinear shear behavior illustrated in Figure II.2.3.6 was used in the model. 


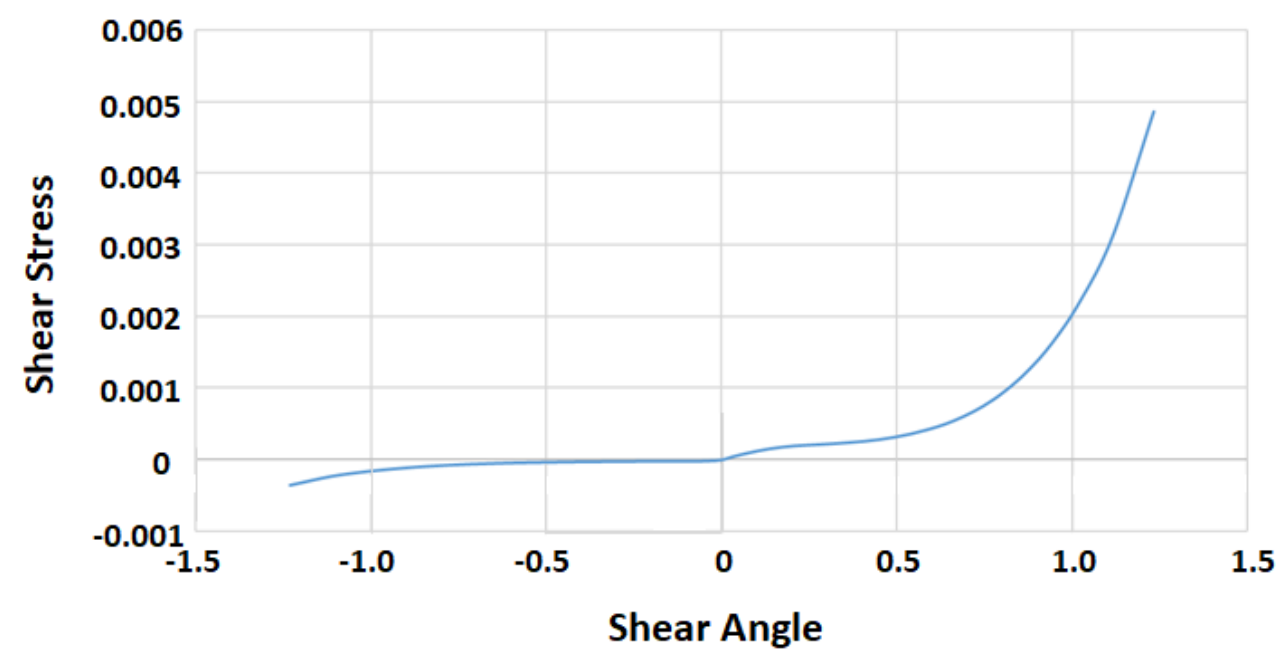

Figure II.2.3.6. Nonlinear shear behavior used in draping modeling. Source: OEM Partner.

\section{Draping Simulation Results - Comparison with Experiments}

A material model in PAM-FORM framework was developed using the fabric characterization tests performed above. Draping simulations were conducted and the simulations are compared with experiments for different preforms, as shown in Figure II.2.3.7 for preform 1 and Figure II.2.3.8 for preform 2.

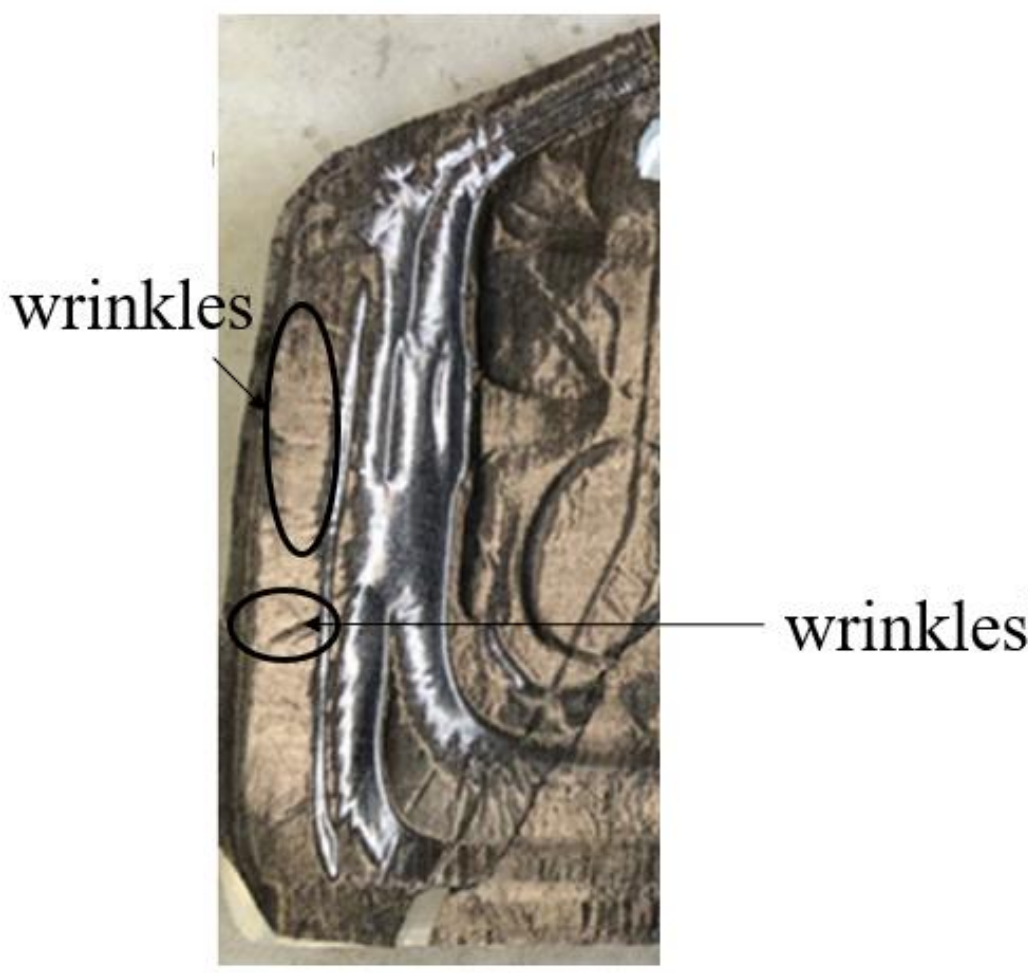

Experiment

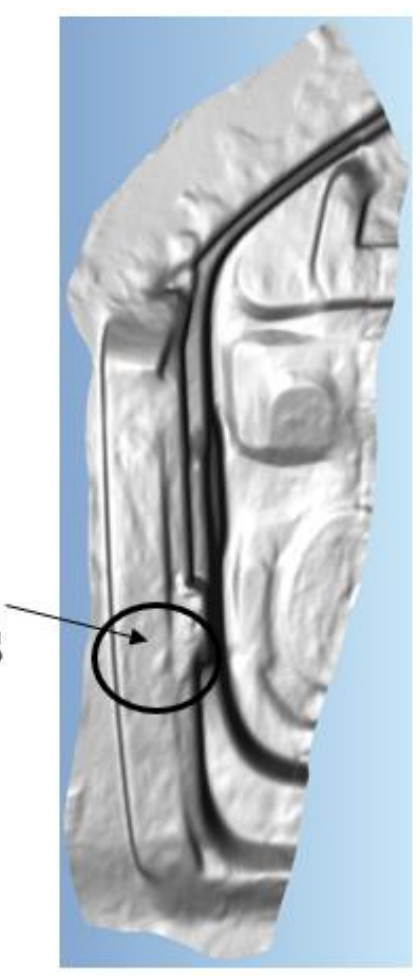

Simulation

Figure II.2.3.7. Comparison of draping experiment and simulation for the preform 1. Source: OEM Partner. 


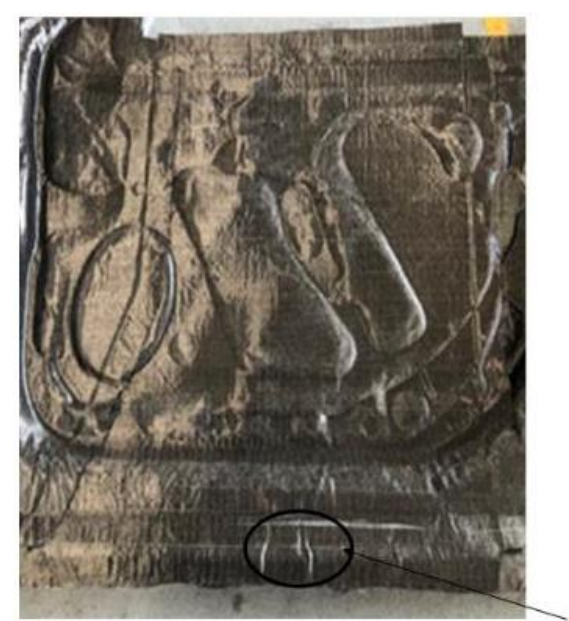

Experiment wrinkles

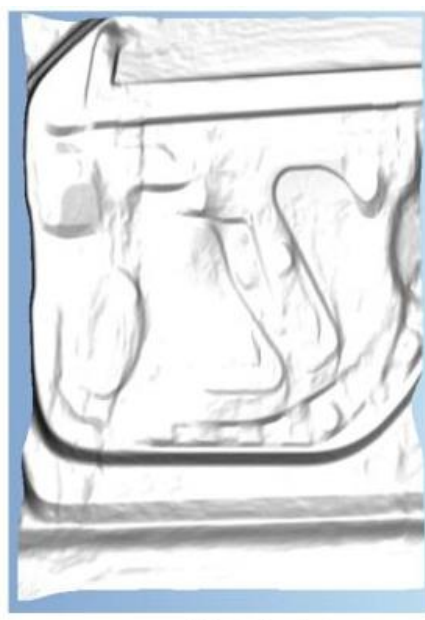

Simulation

Figure II.2.3.8. Comparison of draping experiment and simulation for preform 2. Source: OEM Partner.

Based on these preliminary results, no clear conclusions about the wrinkle correlations could be made. This could be due to the difference in blank size used for preforming, draping conditions (i.e., hand versus mating tools), material difference between the draped material and the material used for characterization.

\section{Simulation-Based Optimization}

Simulation-based optimization has become a powerful tool in the automotive industry to drive the safety designs virtually for automobiles. In the present work, the optimization framework was developed using Altair HyperStudy Software (a multidisciplinary design and optimization software) coupled with LS-DYNA as the FE solver. For the optimization, to avoid local optimum traps, the global response search method was adopted and its efficiency for the optimization of composite structures was assessed in this study. This method combines adaptive response-surface-based optimization with global searching capability. It is able to efficiently provide global or close-to-global optimum solutions in a highly nonconvex design problem. For this optimization, the composite hat section beam layup design for a three-point bending was optimized with respect to two objective functions: (1) maximize the peak load; and (2) maximize the energy absorption. Figure II.2.3.9 shows the top, back and end views of the composite beam used for the study.

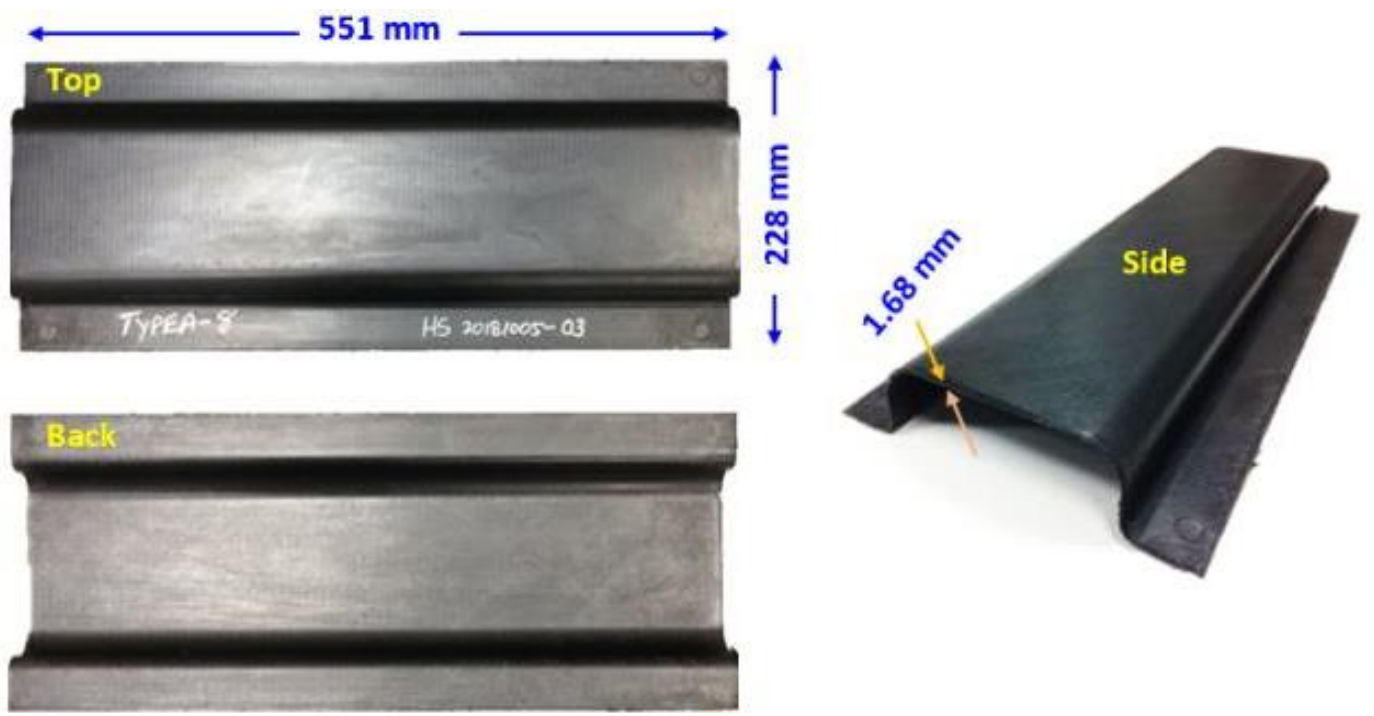

Figure II.2.3.9. Single hat section beam used for the study. Source: OEM Partner. 


\section{Optimization Results}

Two design points (i.e., design 1 and design 2), circled in Figure II.2.3.10, were selected as the extreme points of the objectives (e.g., one design point for each objective - peak load and energy absorption) to verify the optimization results through the use of three-point bend tests. The NCF material used in this study (i.e., C-PLY SP BX 240 T3.3 50K HS from Chomarat Inc.) was chosen for molding the beams. As only certain layup angle choices were available (i.e., 0/90, $+45 /-45$ and $-45 /+45$ ), designs 1 and 2 were adjusted to use the available fabric angles. The design 1 layup was altered from $[-84 / 6 /-68 / 22 / 90 / 180]_{S}$ to $\left[(90 / 0)_{3}\right]_{S}$ and denoted as Beam D. The design 2 layup was modified from $[-56 / 34 / 88 / 178 / 86 / 176]_{S}$ to $\left[\mp 45 /(90 / 0)_{2}\right]_{s}$ and denoted as Beam H. Table II.2.3.1 summarizes the experimental values and the simulation results obtained for Beams D and H, respectively. The simulations results were re-run using the altered layups.

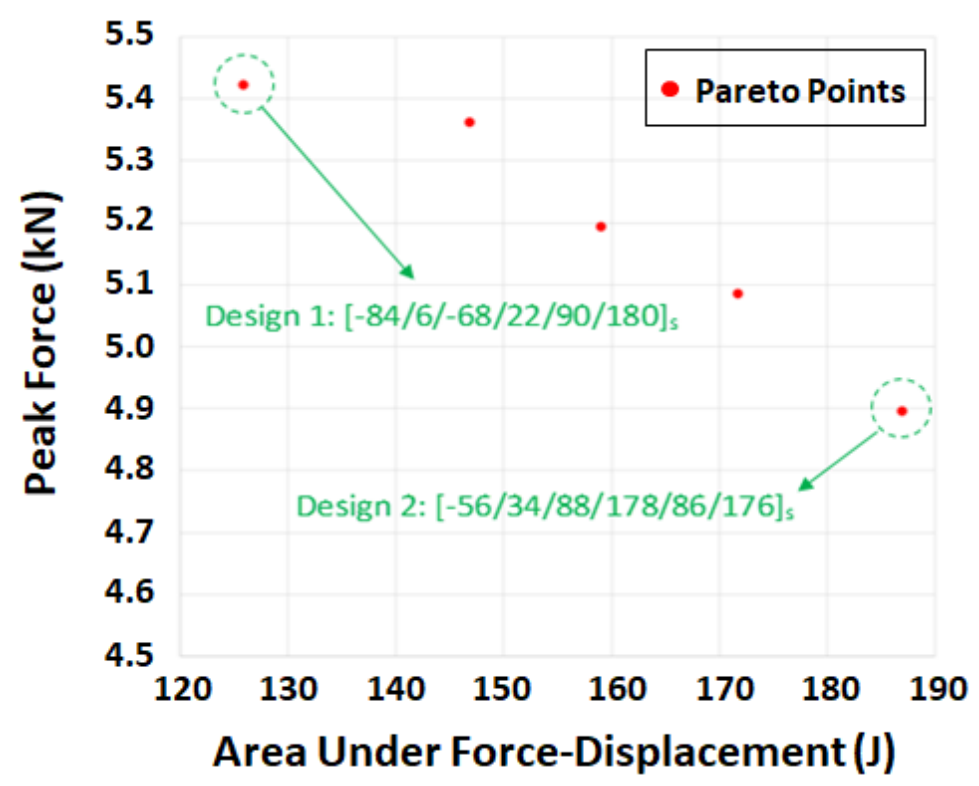

Figure II.2.3.10. Selected design points for conducting flexural tests. Source: OEM Partner.

Table II.2.3.1. Optimization Results.

\begin{tabular}{|c|c|c|c|c|}
\hline Design & Approach & Layup & $\begin{array}{l}\text { Peak Load } \\
(\mathrm{kN})\end{array}$ & $\begin{array}{l}\text { Area of force- } \\
\text { displacement } \\
\text { Curve }(J)\end{array}$ \\
\hline \multirow{3}{*}{ Design 1} & $\begin{array}{l}\text { Simulation-based } \\
\text { optimization }\end{array}$ & $(-84 / 6 /-68 / 22 / 90 / 180) \mathrm{s}$ & 5.4 & 126 \\
\hline & Simulation (Beam D) & $(90 / 0 / 90 / 0 / 90 / 0) s$ & 4.5 & 131 \\
\hline & Experiment (Beam D) & $(90 / 0 / 90 / 0 / 90 / 0) s$ & 4.7 & 127.6 \\
\hline \multirow{3}{*}{ Design 2} & $\begin{array}{l}\text { Simulation-based } \\
\text { optimization }\end{array}$ & $(-56 / 34 / 88 / 178 / 86 / 176) s$ & 4.9 & 187 \\
\hline & Simulation (Beam H) & $(-45 / 45 / 90 / 0 / 90 / 0) s$ & 4.15 & 184 \\
\hline & Experiment (Beam H) & $(-45 / 45 / 90 / 0 / 90 / 0) s$ & 4.4 & 164 \\
\hline
\end{tabular}




\section{Validation of Optimization Results}

Quasi-static flexural bending evaluations were carried out for the two different beam types-Beam D and Beam H. Five samples of each design were molded and tested. Figure II.2.3.11 (a) compares the average forcedisplacement curves and the typical failure patterns of Beams D and $\mathrm{H}$. Each representative curve is the average result of the three best tests, as some of them did not give good results due to warpage of the beams after molding because of unbalanced laminates, leading to the beams not conforming properly to the test fixture. It should be noted that for a given beam type, once the specimen fails, the data points following the failure are not considered in calculating the average curve. As expected, both peak force and initial stiffness were higher for the Beam D as compared with Beam H. Beam H exhibited more "ductile" behavior as compared with Beam D, due to the use of the biaxial layup and the resulting ductile shearing behavior of the resin. The energy absorption calculated from the area of the force-displacement curve for Beams D and $\mathrm{H}$ were 127.6 Joules and 164 Joules, respectively. These values were obtained by averaging the areas under the forcedisplacement curves of the three tests for a given beam type. It should be noted that both of the beam types failed into two pieces during the testing, as shown in Figure II.2.3.11 (b).

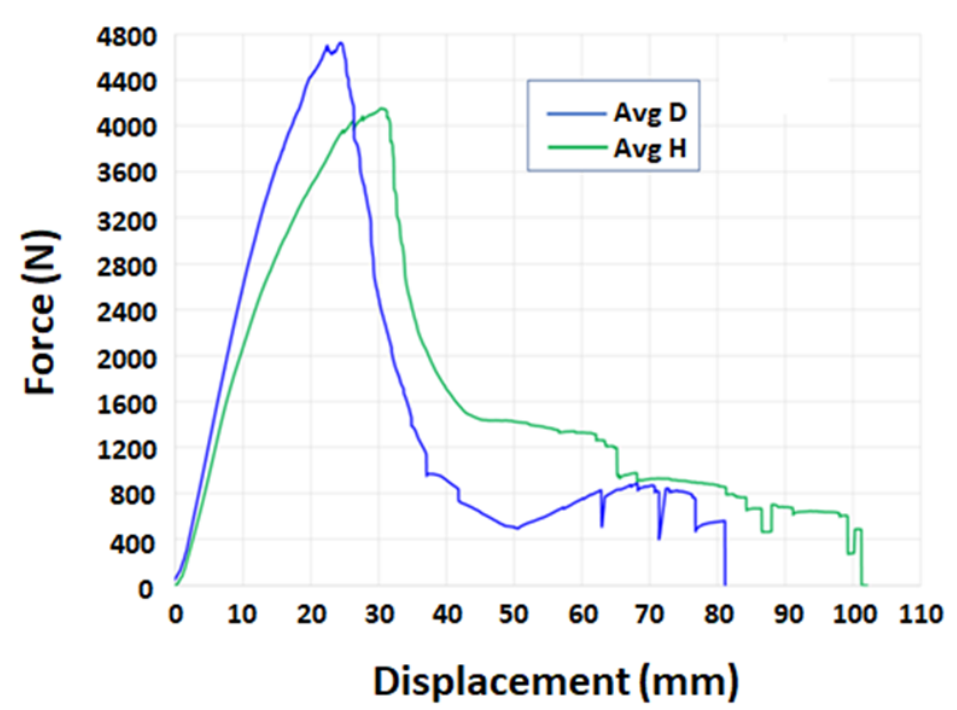

(a)
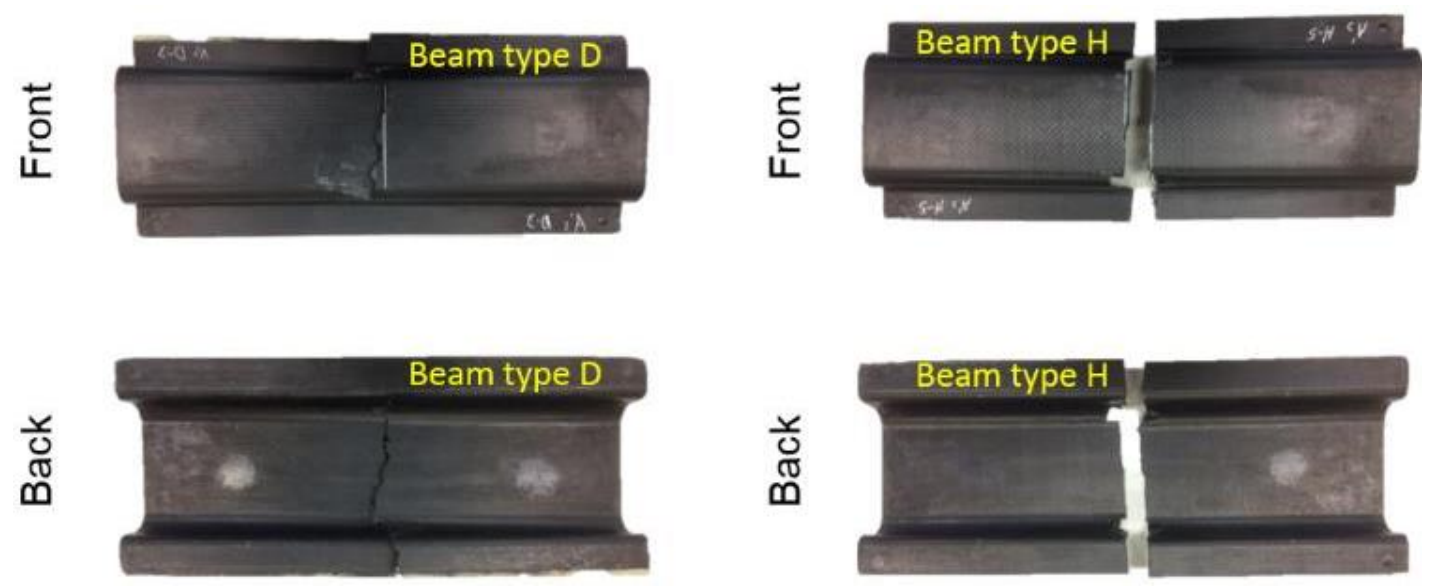

(b)

Figure II.2.3.11. Experimental three-point bend test results for the two beam types: (a) average force-displacement trends; and (b) damage pattern representatives. Source: OEM Partner. 
Figure II.2.3.12 and Figure II.2.3.13 compare the force-displacement trends and the damage patterns obtained from the simulations and the experiments for Beams D and $\mathrm{H}$, respectively. The force-displacement curve obtained from the experiment and the simulation showed close agreement for both of the beam types. The predicted peak load for Beam D was about $4.25 \%$ lower than the average experimental value, whereas it was about 5.6\% lower for Beam $\mathrm{H}$. The predicted damage pattern was in excellent agreement with the experiment for both beams, as shown in Figure II.2.3.12 (a) and Figure II.2.3.13 (a), respectively. For Beam D, the area under the force-displacement curve obtained from the prediction and experiment was 131J and 127.6J, respectively (e.g., a 2.6\% difference). For Beam $\mathrm{H}$, the area under the force-displacement curve between the prediction and the experiment was $184 \mathrm{~J}$ and $164 \mathrm{~J}$, respectively (e.g., a $10.9 \%$ difference). For Beam $\mathrm{H}$, this is because the damage in the simulation did not extend completely across the entire beam as was observed in the experiment. Figure II.2.3.13 (b) and Figure II.2.3.13 (b) show the damage patterns for Beam D and Beam H, respectively. However, this difference in results is deemed to be acceptable, given the challenges in predicting the energy absorption of the composites materials. Future work will be focused on fine-tuning the element deletion criteria for ductile laminates in order to improve the predictions.

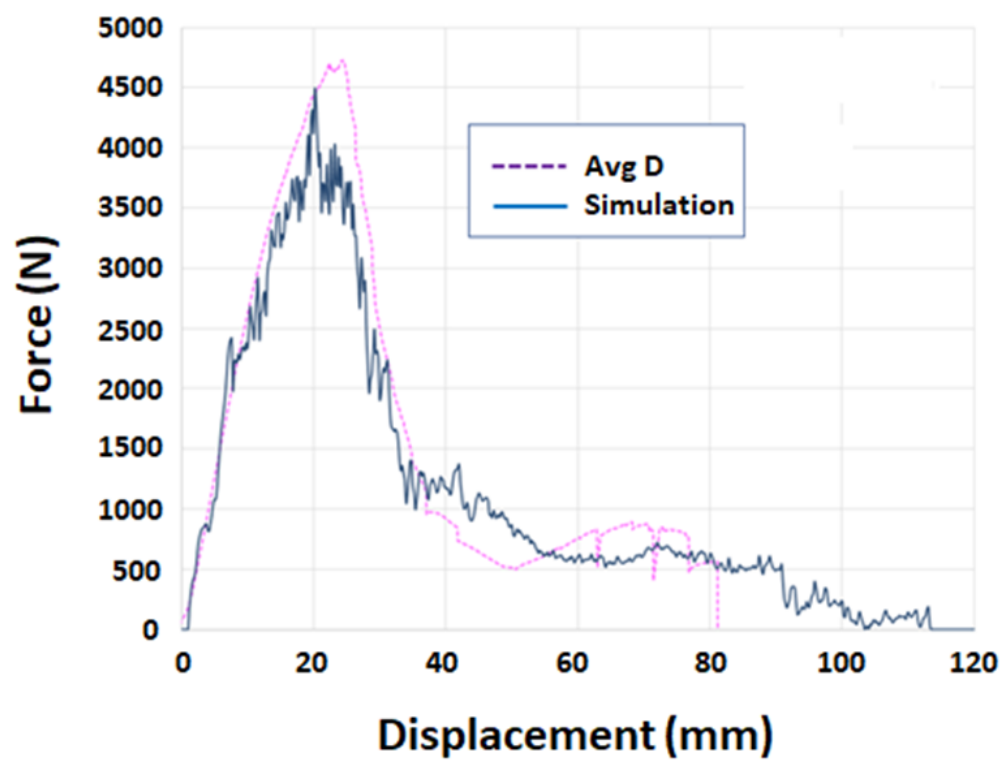

(a)
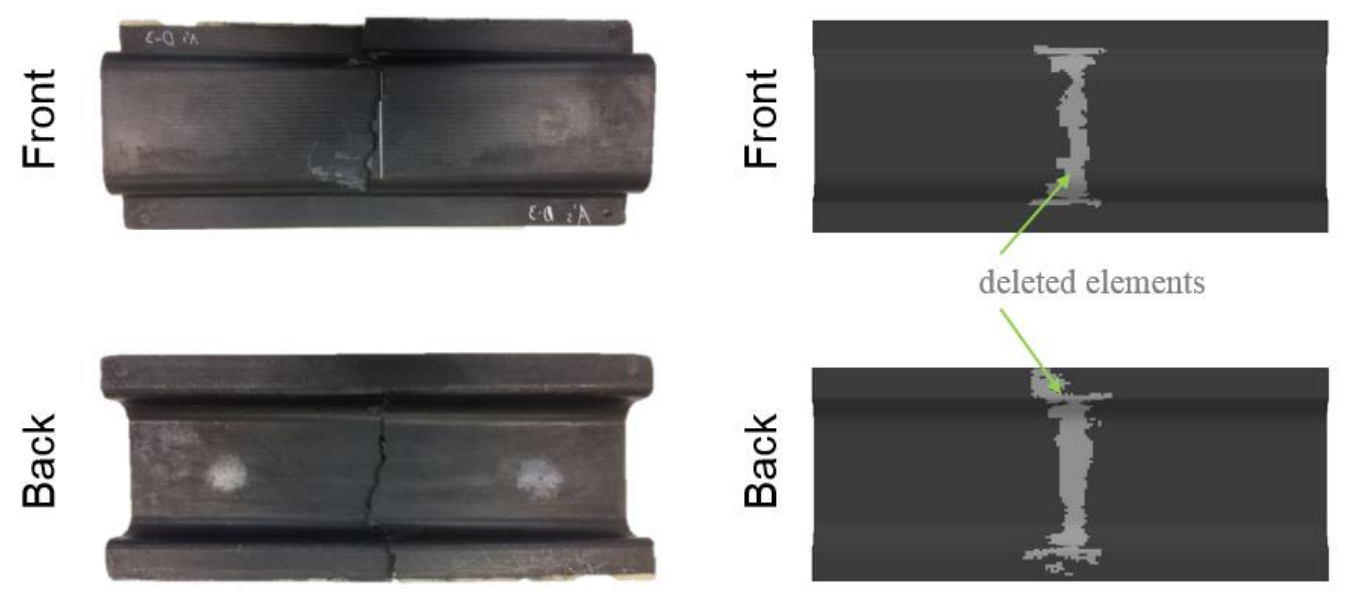

Experiment

(b)

Simulation

Figure II.2.3.12. Comparison of the flexural test and the simulation result for Beam D: (a) load-displacement trend; and (b) damage pattern. Source: OEM Partner. 


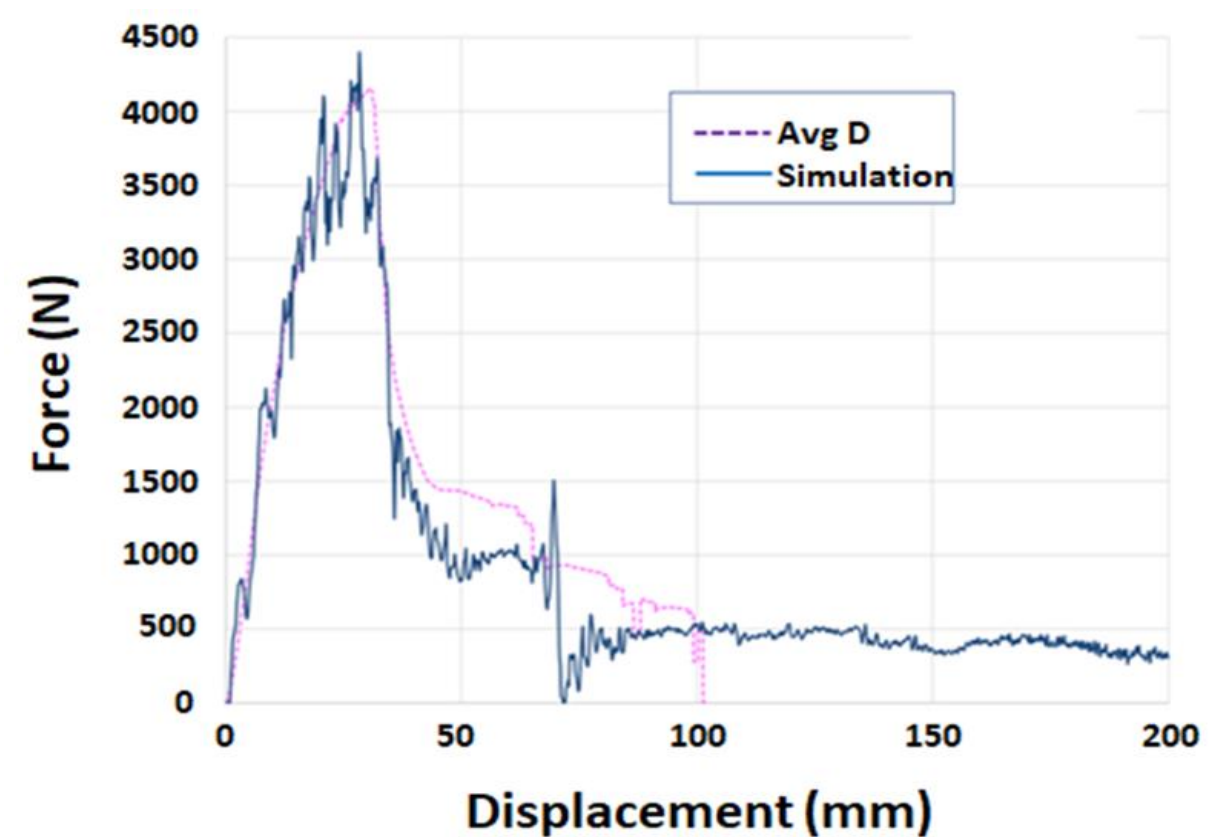

(a)
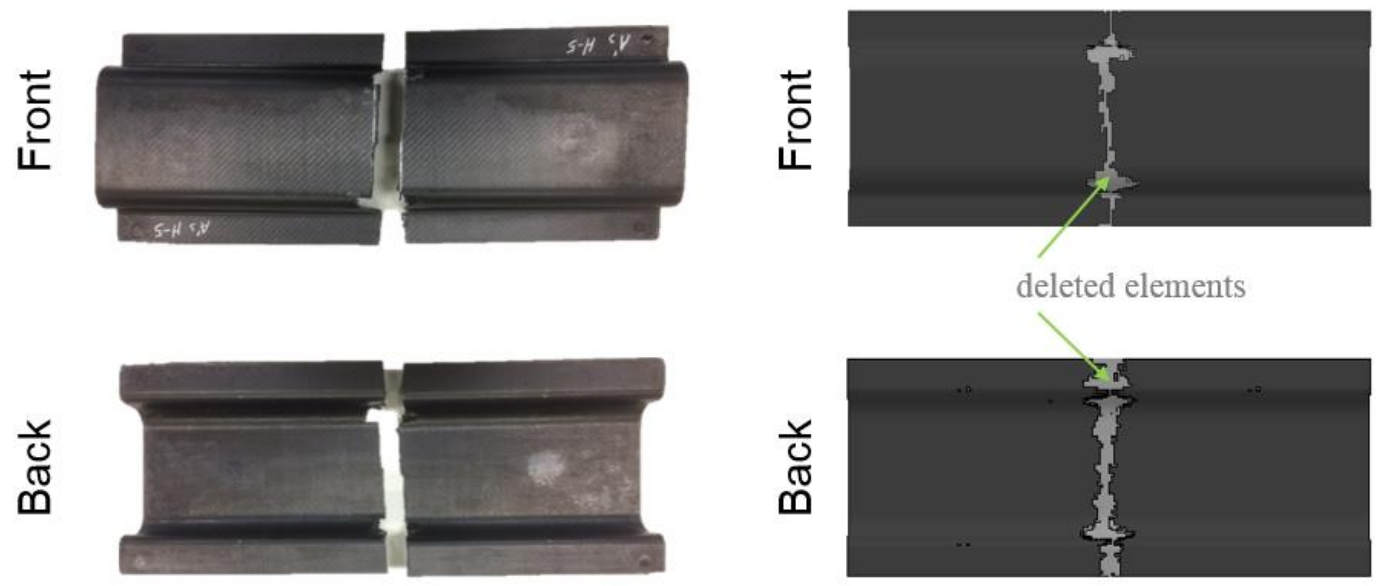

Experiment

(b)

Simulation

Figure II.2.3.13. Comparison of the flexural test and the simulation result for the Beam H: (a) load-displacement trend; and (b) damage pattern. Source: OEM Partner.

\section{Re-Manufacture of Door Inner}

Due to issues in design quality short-falls discussed previously during last year's annual report, the door inner parts were remade at Fraunhofer Project Centre for Composites Research in London, Ontario, Canada.

\section{Fabric Materials}

For the remake of the inner door panels, three different types of materials were utilized. The first was an NCF supplied by Chomarat, which has a thermoplastic veil attached to one side to act as a stabilization binder during preform consolidation. The second was made from the same Chomarat NCF material; however, the thermoplastic veil was replaced by a thermosetting power binder made by Hexion. The third was provided by A\&P Technology. This material is a woven carbon product that is then slit to produce a broadgood fabric. This material was also supplied with the Hexion thermosetting powder binder applied. 


\section{Preform Creation}

TPI cut all the materials for the door inner in advance at our Rhode Island facility and shipped them to the Fraunhofer Project Center in London, Ontario, Canada. The individual plies of various weights and angles were cut via an Eastman automated ply cutter. Then each laminate stack was created by hand to get the correct orientation of the stack. These plies were then laid on top of a heated $\mathrm{Al}$ tool with a silicone vacuum bag for the purpose of consolidating the fabric, as shown in Figure II.2.3.14 (a) and Figure II.2.3.14 (b). The Al preform tool was designed to create all five preforms required for the door inner.

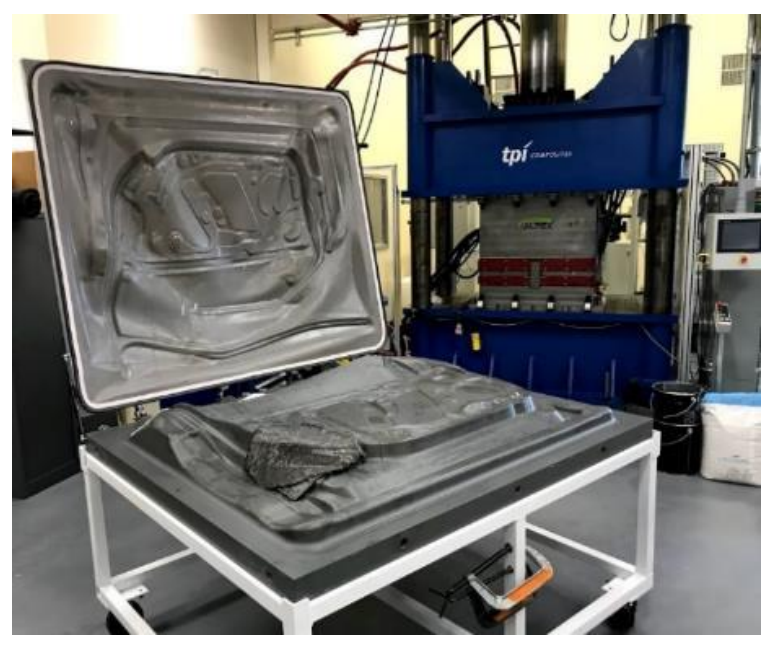

(a)

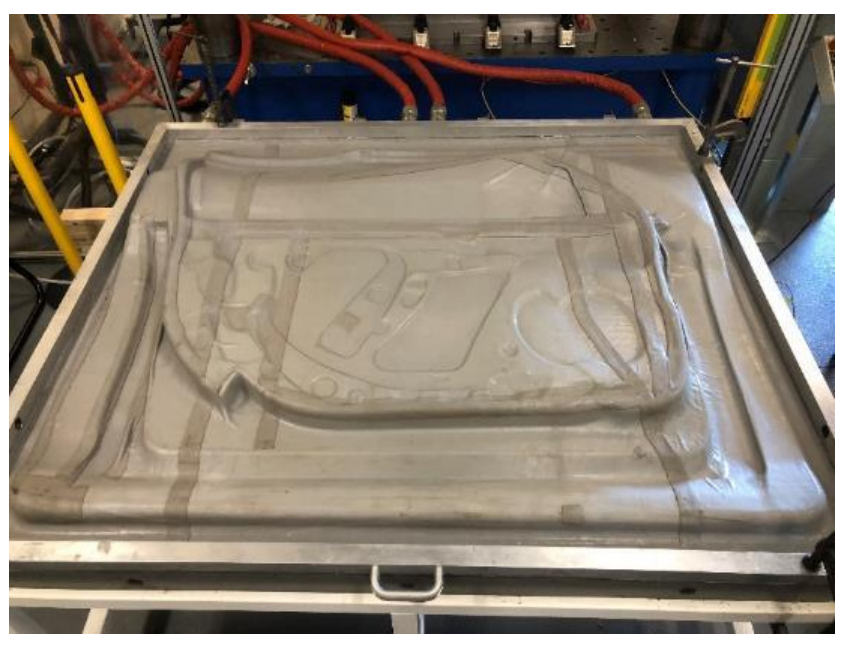

(b)

Figure II.2.3.14. Al preform tool with the silicone vacuum bag in (a) the open position and (b) the closed position. Source: TPI Composites.

The materials were placed onto the tool and smoothed by hand and darted where necessary to allow the material to lay flat. Then the silicone bag was closed, and a vacuum was applied. The tool was then heated above $265^{\circ} \mathrm{F}$ to allow the binder materials to hold the layers together. Photos taken during the preform fabrication process are shown in Figure II.2.3.15 (a) on the mold and Figure II.2.3.15 (b) after consolidation and trimming. Prior to removing the preforms, the tool was cooled to stabilize the material before removal. Unlike the previous preforms that were produced and molded, these preforms were all made using a separator fabric between certain layers to allow the separate preform to be lap-joined to each other. Previous preforms were only tab-joined, which led to unfavorable molded part results. The resulting preform and the inner door design are shown in Figure II.2.3.16 (a) and Figure II.2.3.16 (b), respectively. 


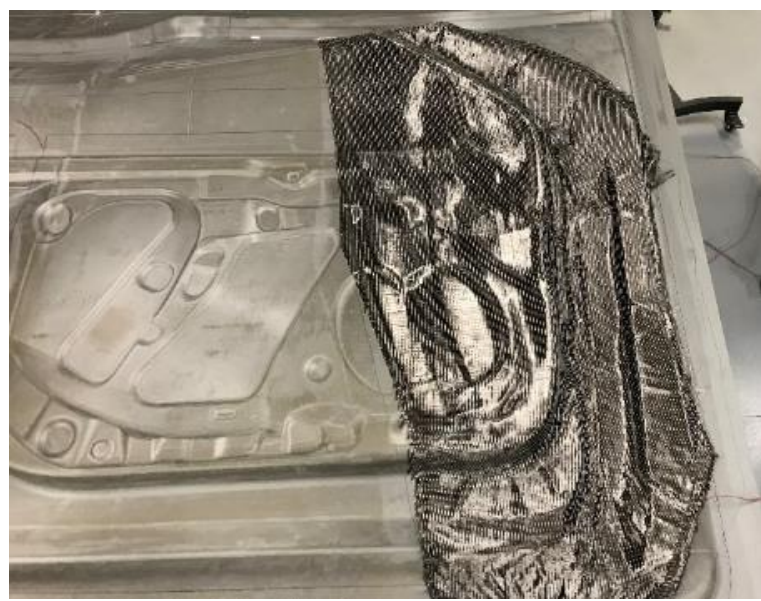

(a)

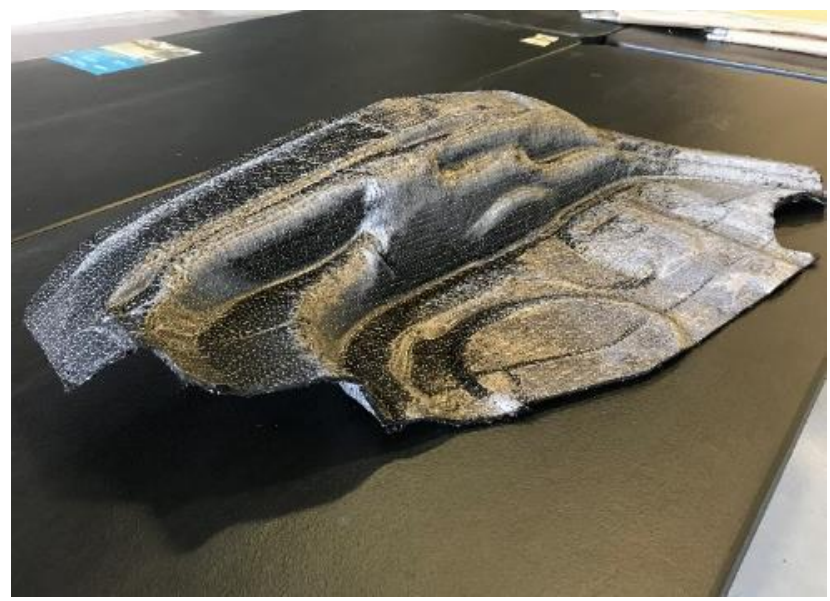

(b)

Figure II.2.3.15. Preform fabrication (a) on the mold and (b) after consolidation and trimming. Source: TPI Composites.

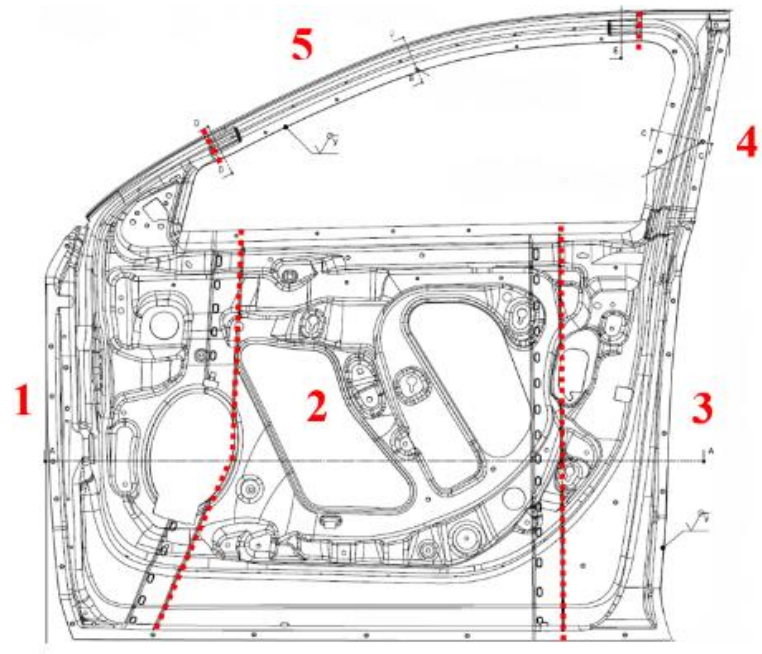

(a)

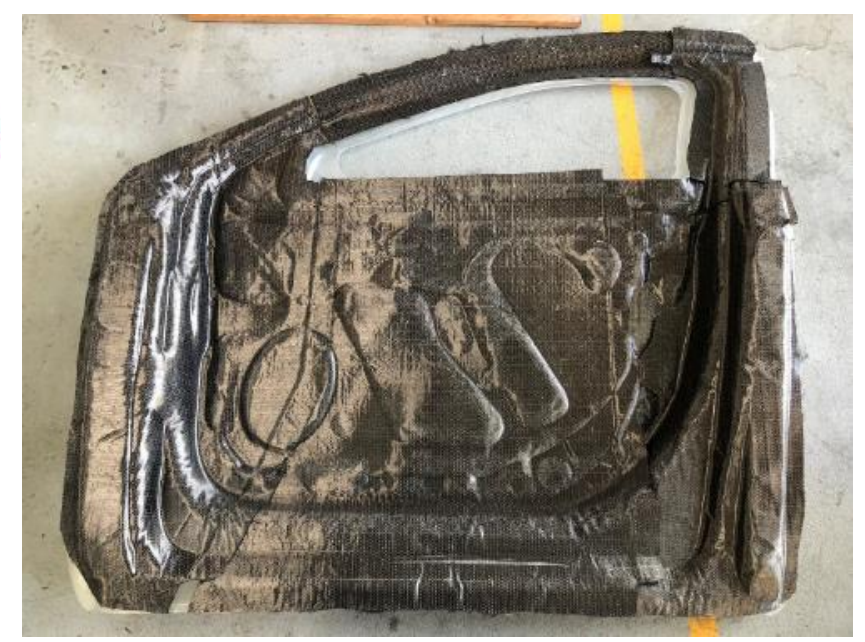

(b)

Figure II.2.3.16. Preform breakup (a) for the door inner (b). Source: TPI Composites.

\section{Molding}

The door inner mold was shipped to Fraunhofer Project Center in London, Ontario, Canada, pictured in Figure II.2.3.17. The Fraunhofer facility has a Dieffenbacher Compress Plus press combined with a KraussMaffei resin dosing system for manufacture of HP-RTM composite parts. TPI arrived on July 29, 2019, to begin molding the new preforms. At that point, the tool was preheated and connected to the press and we were ready for the trials. 


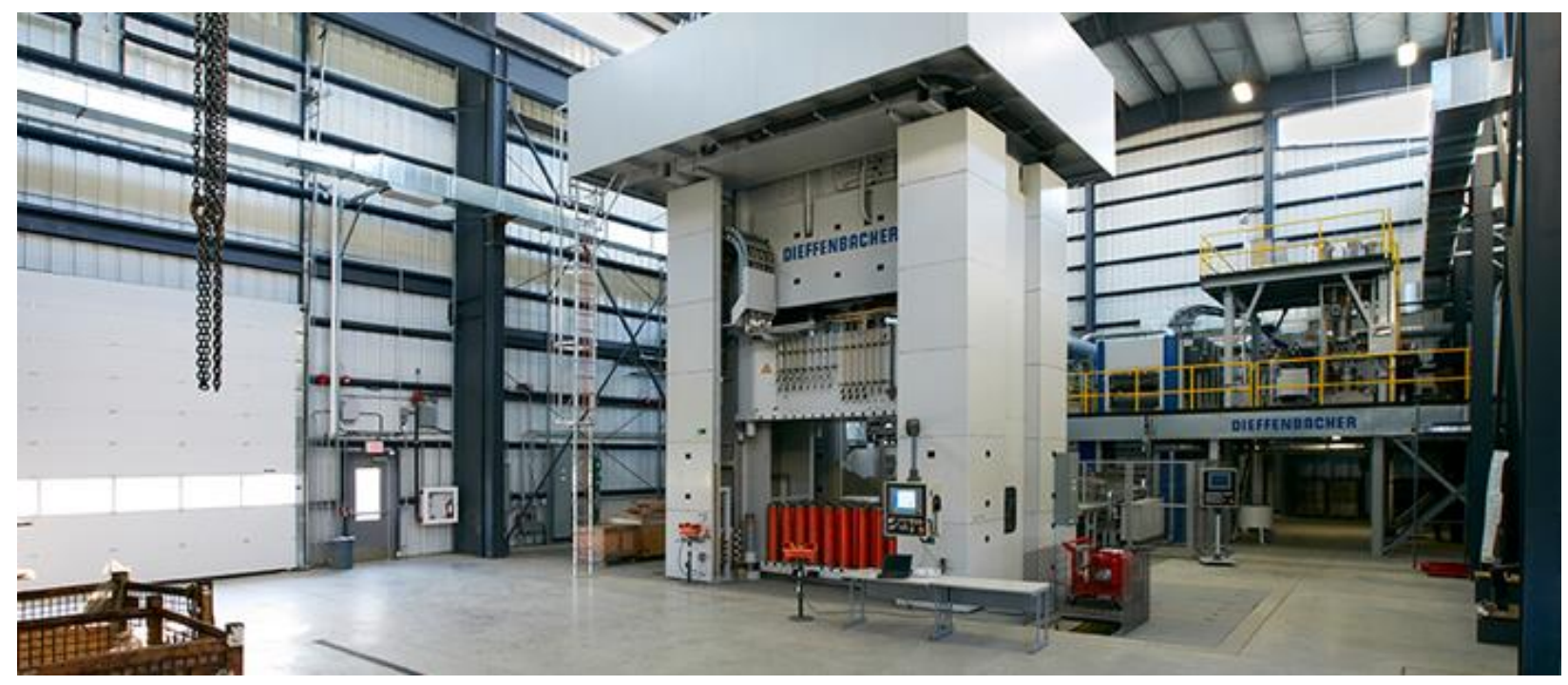

Figure II.2.3.17. The Fraunhofer facility. Source: Fraunhofer Project Center.

\section{Intrusion Beam Hats, Preform Creation and Molding}

Intrusion beam hats, as shown in Figure II.2.3.18, were produced via HP-RTM at TPI Composites Advanced Transportation Composites Center in Fall River, MA, USA.

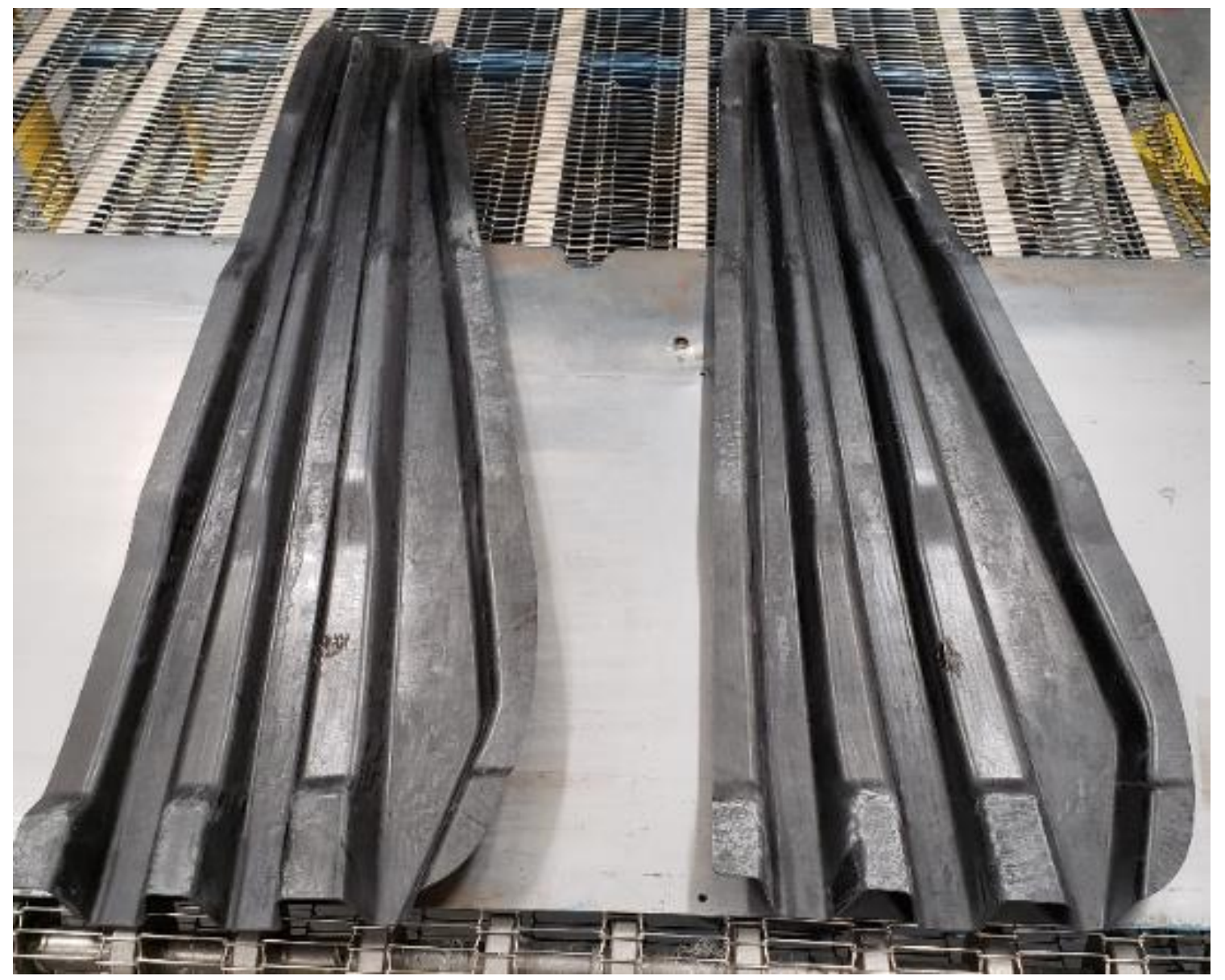

Figure II.2.3.18. HP-RTM molded intrusion beam hats. Source: TPI Composites. 
The preforms for the intrusion beams were made using an $\mathrm{Al}$ preforming mold, which allowed the laminate stack to be vacuum-bagged to contour. Once the fabric was contoured, the mold was shuttled through a conveyor oven to heat the preform/mold to allow the binder to fix the preform into the contour. These preforms were then hand-trimmed to fit into the HP-RTM mold cavity shown in Figure II.2.3.19.

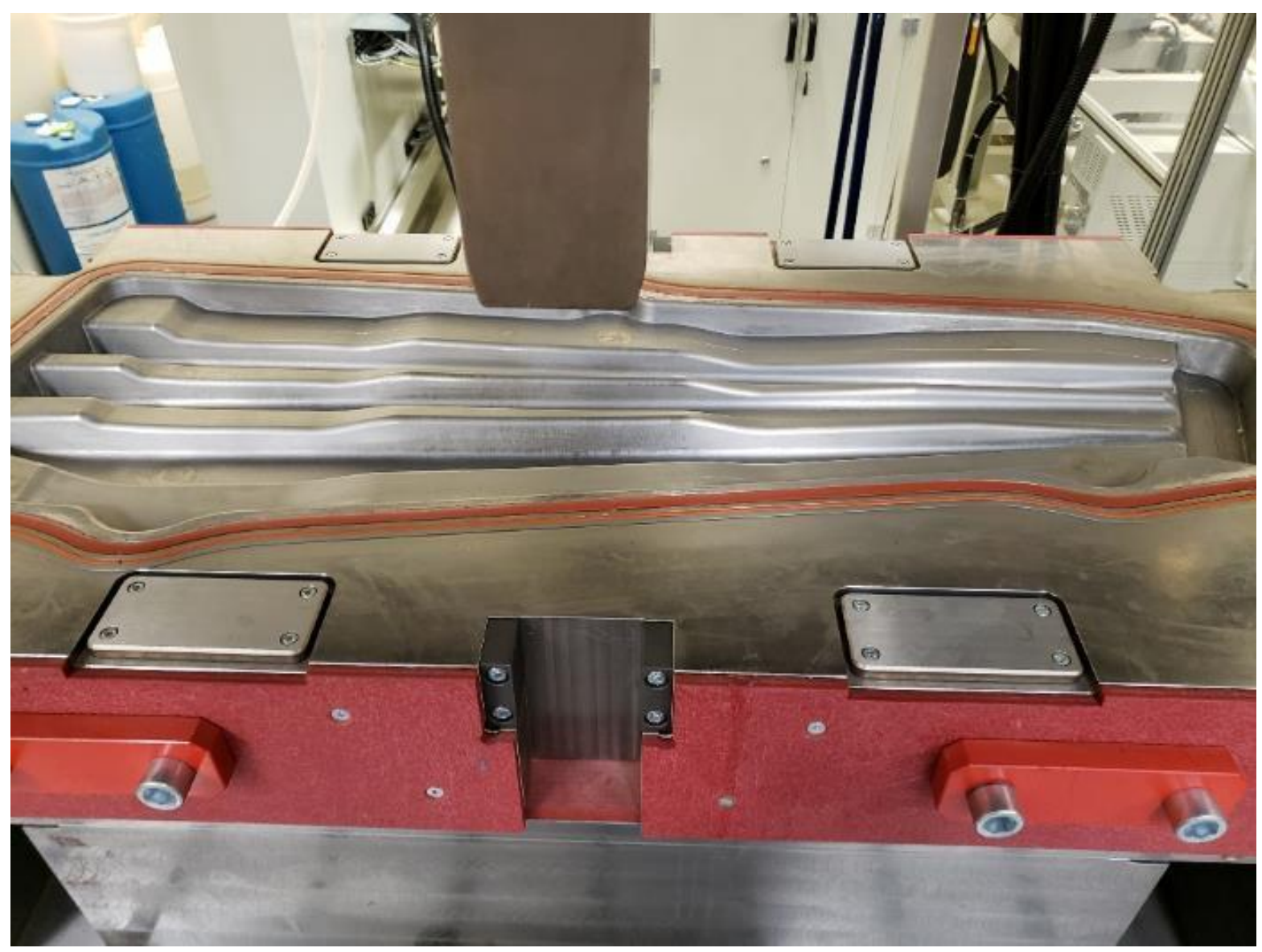

Figure II.2.3.19. The mold cavity for the HP-RTM intrusion beam hat. Source: TPI Composites.

The part-to-part cycle time was approximately six minutes, which included hand-loading the preforms, moldclosing, injection, cure, mold-opening, part extraction, and mold-cleaning.

\section{Issues, Risks, Mitigation}

\section{Dry Areas}

Although the parameters used to mold these new preforms were identical to those used to produce the previous parts, the molded part quality was not as good. Many of the produced parts had dry areas in the center of the part where the preforms had bunched, as observed in Figure II.2.3.20. Unfortunately, due to the limited number of preforms available, we were not afforded the ability to really make any process tweaks or refinement to try and improve the part quality. 


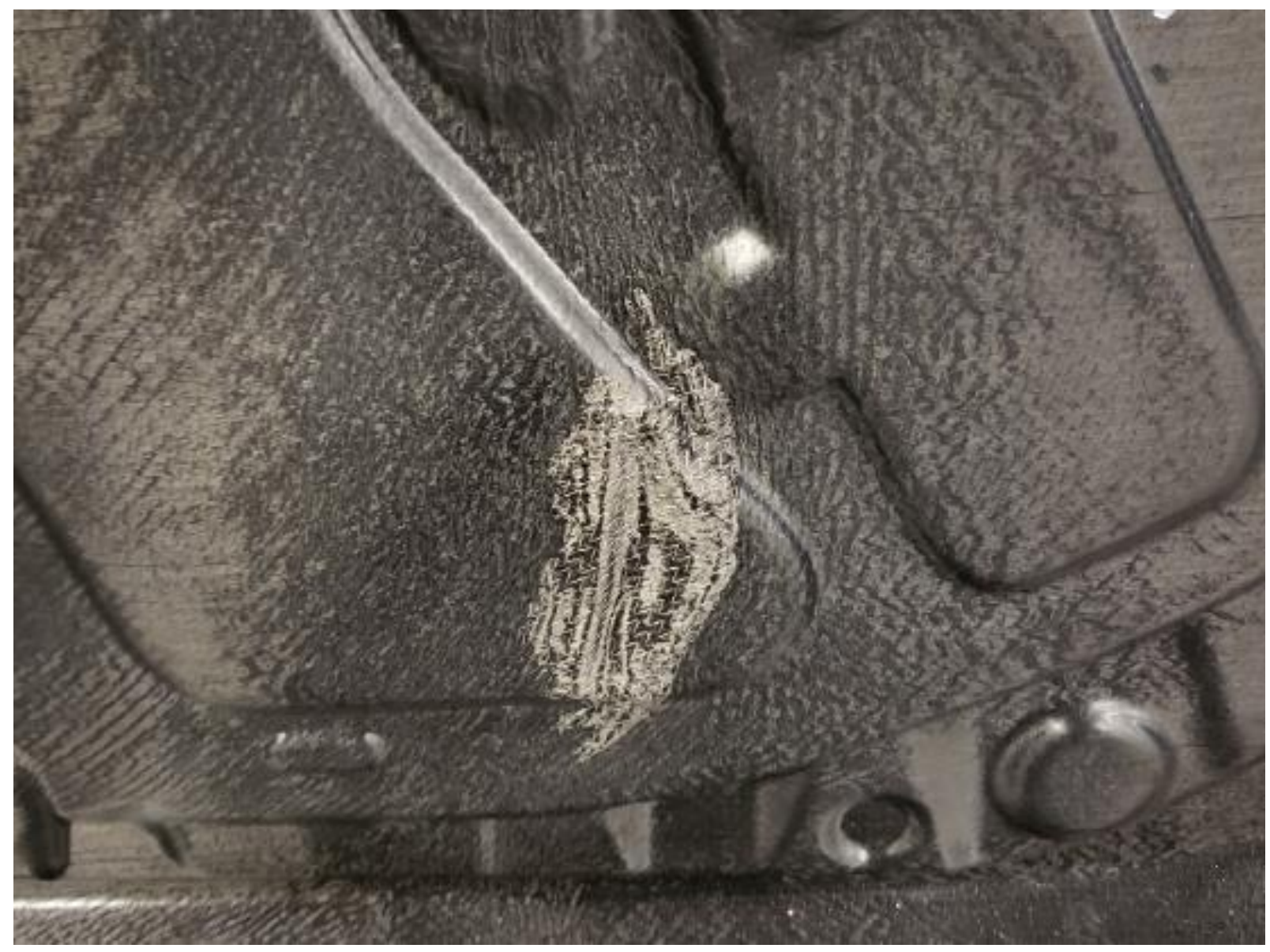

Figure II.2.3.20. Dry area. Source: TPI Composites.

\section{Preform Overlaps}

As was previously noted, the original tab-joined preform design caused issues with resin-richness and movement, as shown by the photographs in Figure II.2.3.21 (a) and Figure II.2.3.21 (b), respectively. The new lap-joined preform approach compared to the previous tab-joint approach as shown in Figure II.2.3.22 (a) and Figure II.2.3.21 (b), respectively, was very successful in eliminating these issues, possibly because the higher drapability of the woven material over the NCF was much better at shearing and conforming to the part details. 


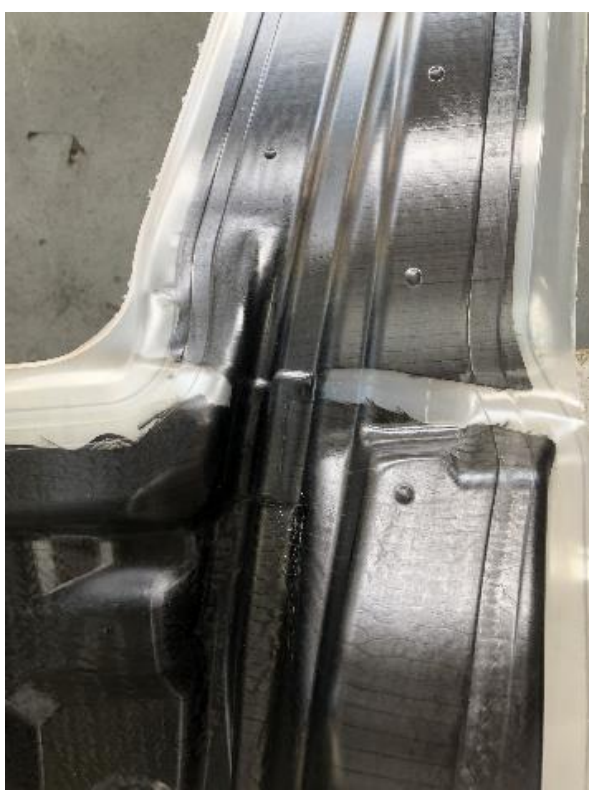

(a)

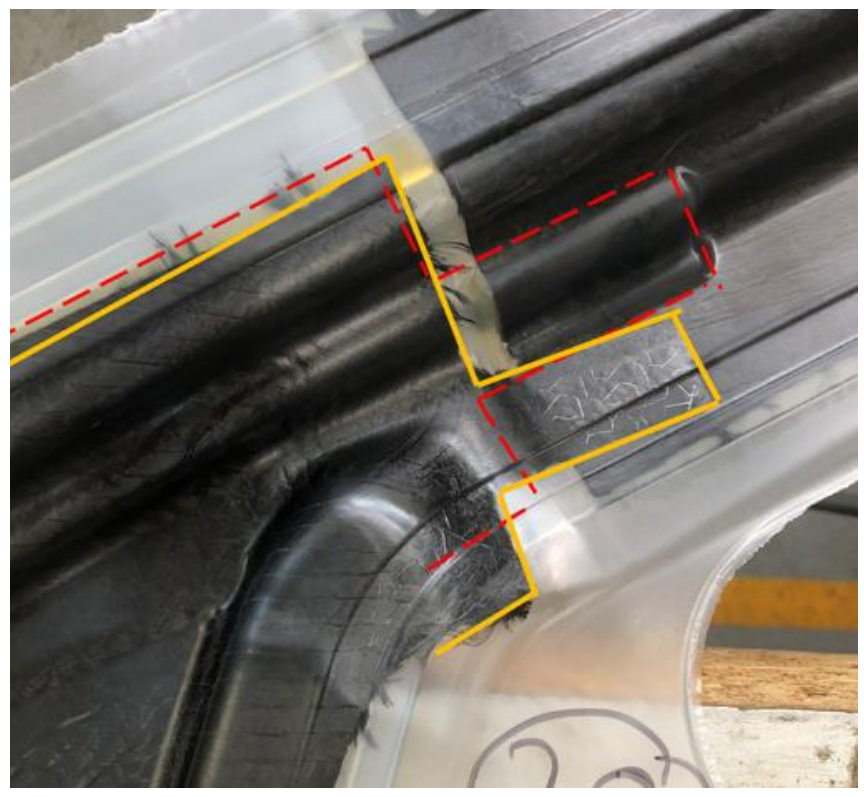

(b)

Figure II.2.3.21. Sliding preform at the mirror mount showing: (a) the resin-rich area; and (b) the preform out of position. Source: TPI Composites.

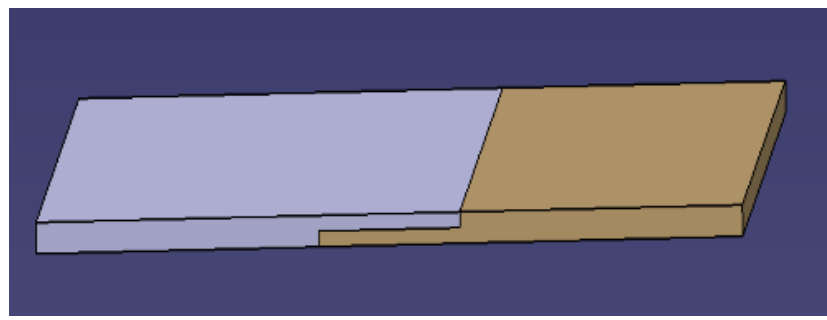

(a)

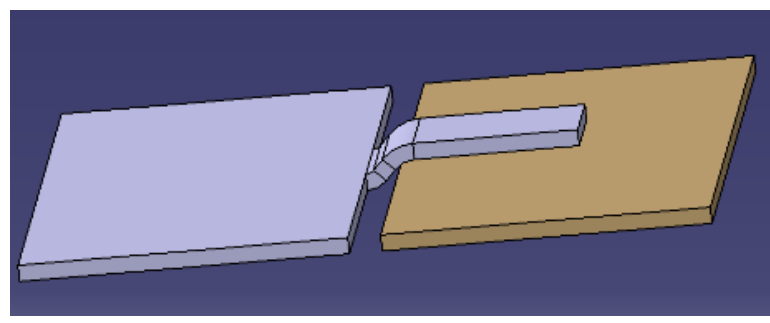

(b)

Figure II.2.3.22. Preform joint designs for: (a) the lap-joint for the new preforms; and (b) the tab-joint for the old preforms. Source: TPI Composites.

\section{General Preform Wrinkling}

Like the previous parts that were produced, wrinkling of the preform occurred across the part of the NCF preforms as shown in Figure II.2.3.23. The wrinkling was independent of the type of binder, thermoplastic veil, or thermoset powder, which was used to create the preform. However, the woven (braided) material did not exhibit any wrinkling of the preform. It is believed that due to the higher drapability of the woven material over the NCF, this material was much better at shearing and conforming to the part details. 


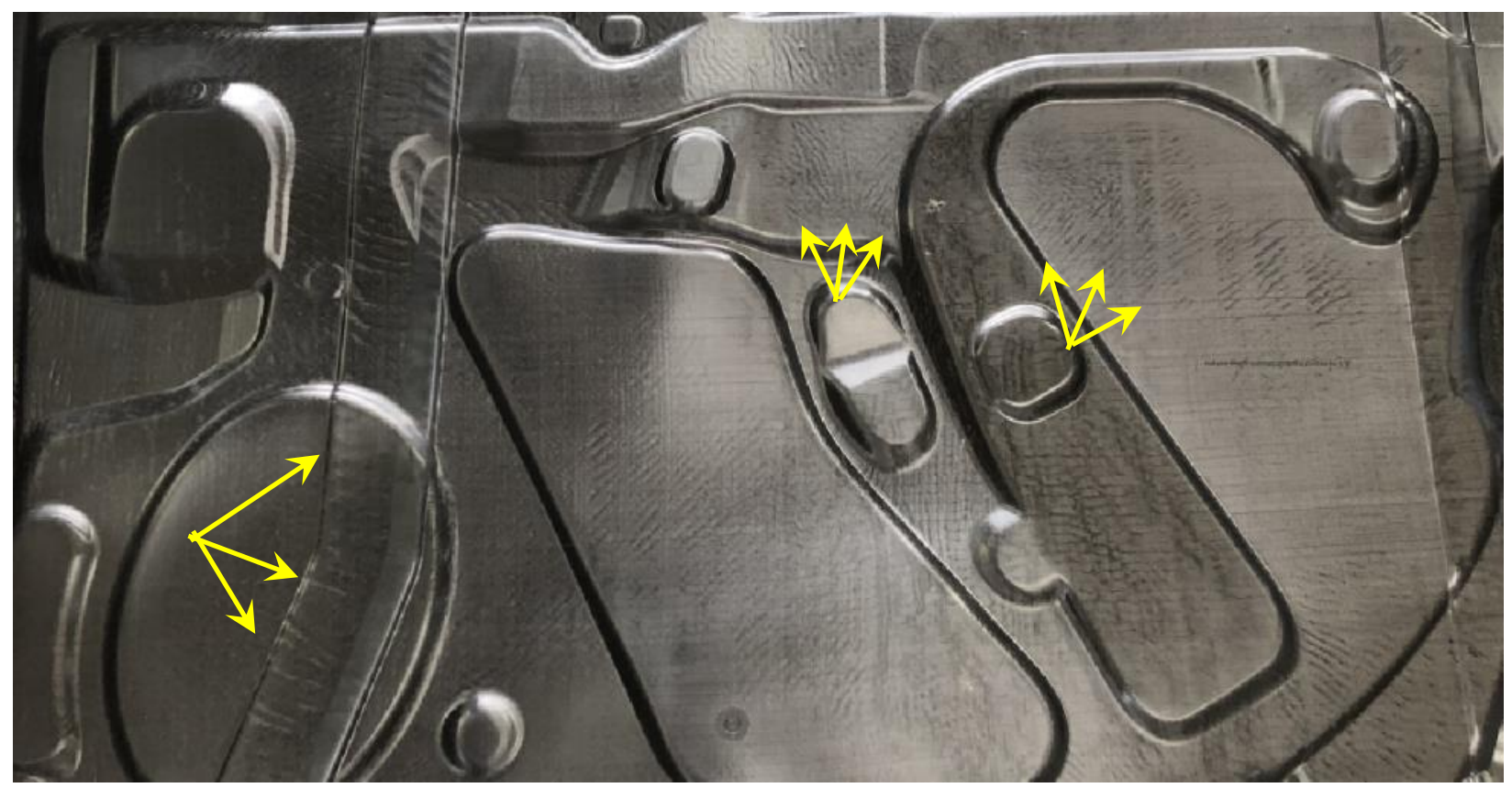

Figure II.2.3.23. Part wrinkling in the NCF preform. Source: TPI Composites.

The next steps are to have the molded inner panels machined. Following inner panel machining, the doors will be assembled and boned together into the final configuration. Fully assembled doors are scheduled to be completed and shipped for static testing with the dynamic impact testing to occur before the end of the project.

\section{Status to Target}

The design solutions presented in this report represented a cost of $\$ 715$ per door at a mass of $22.8 \mathrm{~kg}$ (or 50.2 lbs.) as depicted in the charts shown in Figure II.2.3.24 and Figure II.2.3.25 (a) and (b). This represents a savings of $13.7 \mathrm{~kg}$ (or $30 \mathrm{lbs}$.) over the existing baseline door design. The cost increase of $\$ 138$ per part and $30 \mathrm{lbs}$. of mass saved yields a $\$ 4.58$ cost increase per pound of weight saved, exceeding the program targets. The total mass saved is $38 \%$ going from $36.5 \mathrm{~kg}$ to $22.8 \mathrm{~kg}$. This target proves to be more difficult as the total mass of the door is included in this calculation. The window track/motor, latch, hinges, and other subassemblies represent $56 \%$ of the total mass of the door and they are harder to lightweight than the structure.

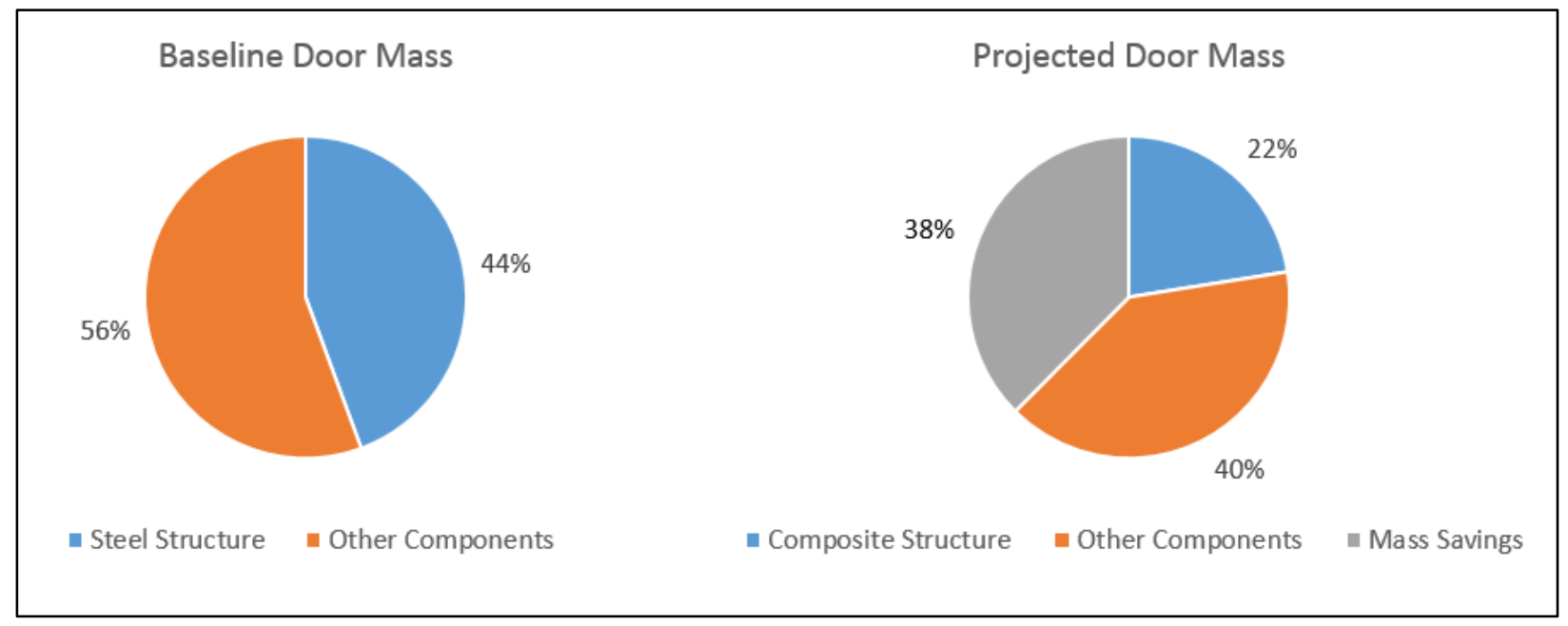

Figure II.2.3.24. Door mass breakdown. Source: TPI Composites. 


\begin{tabular}{|l|l|}
\hline Component & Cost \\
\hline Door Structure & $\$ 50.78$ \\
\hline Inner Panel & $\$ 67.08$ \\
\hline Door Mechanism & $\$ 57.33$ \\
\hline Window system & $\$ 122.37$ \\
\hline Sealing System & $\$ 21.92$ \\
\hline Hinges & $\$ 9.92$ \\
\hline Power System & $\$ 25.00$ \\
\hline Molding System & $\$ 25.69$ \\
\hline Mirror System & $\$ 46.45$ \\
\hline Other & $\$ 150.00$ \\
\hline TOTAL & $\$ 576.54$ \\
\hline
\end{tabular}

\begin{tabular}{|l|r|}
\hline Component & Current Mass [kg] \\
\hline Door Structure & 16.2 \\
\hline Inner Panel & 4.1 \\
\hline Door Mechanism & 1.7 \\
\hline Window system & 5.7 \\
\hline Sealing System & 2.6 \\
\hline Hinges & 1 \\
\hline Power System & 1.1 \\
\hline Molding System & 0.9 \\
\hline Mirror System & 1.6 \\
\hline Other & 1.6 \\
\hline TOTAL & $\mathbf{3 6 . 5}$ \\
\hline
\end{tabular}

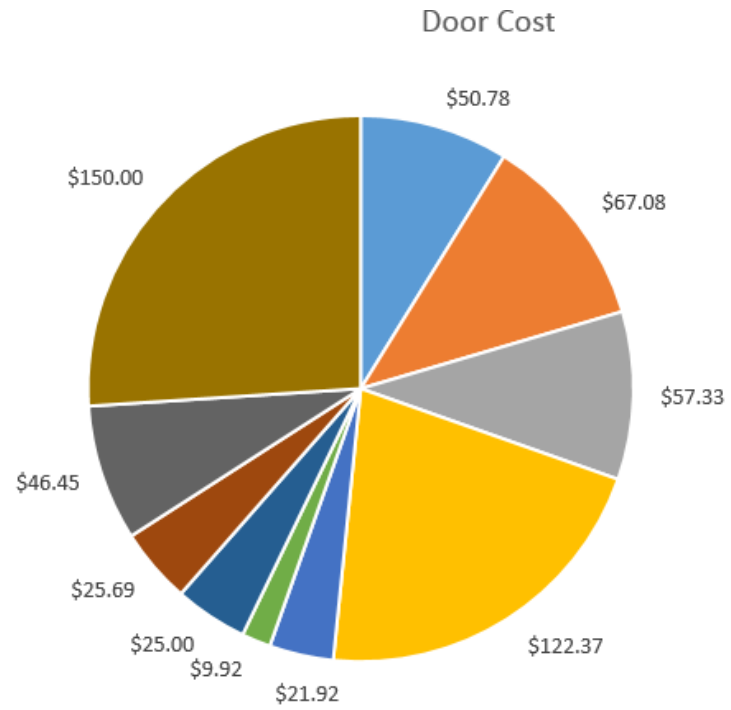

$$
\begin{aligned}
& \text { - Door Structure } \\
& \text { - Inner Panel } \\
& \text { - Door Mechanism } \\
& \text { - Window system } \\
& \text { - Sealing System } \\
& \text { - Hinges } \\
& \text { - Power System } \\
& \text { - Molding System } \\
& \text { - Mirror System } \\
& \text { - Other }
\end{aligned}
$$

(a)
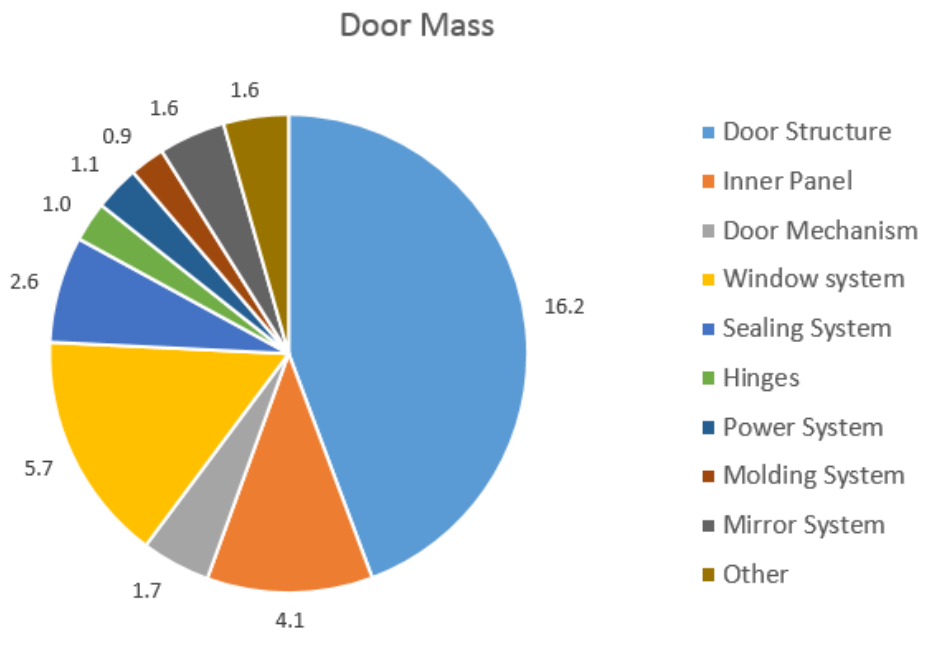

(b)

Figure II.2.3.25. Breakdown for: (a) the baseline door cost; and (b) the baseline door mass. Source: TPI Composites.

The optimized design as defined during the FY 2018 program yields a 38\% mass save and a $\$ 5.47$ cost increase per every pound saved. This is based on the input fiber, Zoltek Panex 35 , at a cost of $\$ 7.75$ per $1 b$. Should the low-cost CF from ORNL be commercialized and realize an input fiber cost of $\$ 4.75$, we could then exceed the cost target even further, getting down to as low as $\$ 4.33$ per pound saved, as shown in Table II.2.3.2. 
Table II.2.3.2 Status to Target.

\begin{tabular}{|l|c|l|c|}
\multicolumn{1}{|c|}{ Input CF Cost: \$7.75/lb } & \multicolumn{2}{c|}{ Input CF Cost: \$4.75/lb } \\
\hline \multicolumn{1}{|c|}{ Optimized Design } & \multicolumn{1}{|l|}{ ORNL Low-Cost Carbon Fiber Design } \\
\hline Weight Reduction (Ib.) & 30.3 & Weight Reduction (lb.) & 30.3 \\
\hline \% Reduction & $38 \%$ & \% Reduction & $38 \%$ \\
\hline Cost Increase & $\$ 165.72$ & Cost Increase & $\$ 131$. \\
\hline Dollars/lb. saved & $\$ 5.47$ & Dollars/lb. saved & $\$ 4.33$ \\
\hline
\end{tabular}

\section{Conclusions}

We are close to the major goals of reducing part count and full-system weight by a minimum of $42.5 \%$ (38\% achieved), the cost increase not exceeding $\$ 5$ per pound of weight saved ( $\$ 4.33$ achieved with low-cost carbon fiber), and materials and processes meeting the production rate and performance requirements.

\section{References}

1. Sloan, J., 2012, “Auto composites quest: One-minute cycle time?," Composites World (August), Available at: http://www.compositesworld.com/articles/auto-composites-quest-one-minute-cycle-time (last accessed 5 December 2019).

2. Motavalli, J., 2011, "Shattering glass: Low-weight plastic car windows are coming," CBS Money Watch (July), Available at: http://www.cbsnews.com/news/shattering-glass-low-weight-plastic-car-windowsare-coming/ (last accessed 5 December 2019).

3. Hillermeier, R., T. Hasson, L. Friedrich, and C. Ball, 2013, "Advanced thermosetting resin matrix technology for next-generation high-volume manufacture of automotive composite structures," $S A E$ Technical Paper 2013-01-1176. 


\title{
II.2.4 Functionally Designed Ultra-Lightweight Carbon Fiber Reinforced Thermoplastic Composites Door Assembly (Clemson University)
}

\author{
Dr. Srikanth Pilla, Principal Investigator \\ Department of Automotive Engineering \\ Department of Materials Science and Engineering \\ Clemson University \\ 4 Research Dr., Suite 340 \\ Greenville, SC, 29607 \\ E-mail: spilla@clemson.edu
}

\section{Dr. H. Felix Wu, DOE Technology Manager}

U.S. Department of Energy

E-mail: felix.wu@ee.doe.gov

Start Date: December 1, 2015

Project Funding (FY 19): \$1,424,056
End Date: November 30, 2020

DOE share: $\$ 643,239 \quad$ Non-DOE share: $\$ 780,817$

\section{Project Introduction}

One of the most promising routes for achieving the 2025 Corporate Average Fuel Economy standards involves decreasing vehicular weight by incorporating lightweight materials, coupled with component redesign, in order to improve overall fuel efficiency. Indeed, one recent study indicates that the simple replacement of current metallic doorframes with carbon fiber-reinforced polymer composites can reduce the overall weight of the component by nearly $58 \%$ [1]. The objectives of this project are to achieve a weight reduction of at least $42.5 \%$, as compared to the baseline door structure, at a cost of less than $\$ 5 / \mathrm{lb}$. while saving on energy metrics without compromising the fit, function, crash, and noise, vibration and harshness requirements. The strategy for achieving these targets involves a holistic systems approach through the integration of unique designs, novel materials, manufacturing technologies, and joining/assembly of subsystems to ensure the developed technologies are ready for commercialization.

\section{Objectives}

The objective of this project is to reduce the weight of a door assembly by at least $42.5 \%$ compared to a baseline driver's side front door with an expected cost increase of less than $\$ 5 / \mathrm{lb}$. in weight saved. A 2013 mid-sized sport utility vehicle's door from our OEM partner with an assumed production volume of 20,000 vehicles annually is the basis for design. These criteria will either meet or exceed the fit, function, crash, and $\mathrm{NVH}$ requirements of the baseline door.

The intent is to (1) enable a radical redesign of the baseline door via a holistic systems approach through the integration of unique designs, (2) use novel materials that render the door $100 \%$ recyclable and (3) investigate manufacturing technologies and joining/assembly of subsystems to ensure the developed technologies are ready for commercialization. The partner organizations listed in Table II.2.4.1 are providing highly leveraged knowledge expertise to ensure the success of this effort. 
Table II.2.4.1. Project Participants.

\begin{tabular}{|l|l|l|}
\hline \multicolumn{1}{|c|}{ Universities } & \multicolumn{1}{|c|}{ Industry Partners } & \multicolumn{1}{c|}{ Computation Partners } \\
\hline Clemson University & OEM & Altair Engineering \\
\hline & BASF, Polyone & Core-Tech Systems (Moldex 3D) \\
\hline \multirow{2}{*}{ University of Delaware } & Krauss-Maffei, Trexel Inc. & MSC Software (Digimat) \\
\hline & Corning & LS-DYNA \\
\hline
\end{tabular}

\section{Approach}

The project entails the use of a systems-level approach that begins with a systematic evaluation and benchmarking of the door and its subassemblies. In collaboration with our partnering companies and commercial suppliers, researchers are evaluating a variety of CF thermoplastic material forms for structural components (i.e., novel unidirectional and fabric prepregs, co-mingled fabrics, high aspect ratio discontinuous fibers, performance thermoplastic resins, novel fiber architectures, and localized reinforcements) and alternative solutions for glazing, trim, and other subcomponents. The initial focus involves creating the structural component and a materials database for all parts of the door structural assembly (i.e. outer shell, inner panel, carrier, and anti-intrusion beam(s)).

A two-phase integrated design and manufacturing optimization approach was adopted to obtain the optimal manufacturing process parameters of the thermoplastic materials and the optimal structural design parameters of the door. The Principal Investigators also conducted a top-level trade study to determine at least two candidate designs for optimization. The design parameters include: (a) thermoforming and injection-molding parameters (e.g., pressure, temperature); (b) fiber parameters (e.g., material, length, diameter, volume fraction); (c) matrix parameters (e.g., material, volume fraction); (d) structural wall thickness; and (e) material density distribution. The research team will use four analysis tools: (1) Moldex3D, (2) Digimat, (3) LS-DYNA Implicit and (4) LS-DYNA Explicit to construct the manufacturing-to-response analysis pathway [2-4]. The team will fabricate a door based on this optimal design for testing in accordance with OEM performance requirements.

\section{Results}

\section{Concept Development}

The team's focus in the fourth year was on designing for mass reduction, meeting static and dynamic crash targets, and improvement in manufacturability. In order to achieve the mass reduction target, the team performed three major revisions, as seen in Figure II.2.4.1, in composite door structural design, for which several composite optimization, static and dynamic tests were carried out over FY 2019. The first revision, as seen in Figure II.2.4.1 (b), included the removal of the injection-molded sash reinforcement part and its replacement with an inner beltline stiffener (IBS) extended into the sash region. The second revision, as seen in Figure II.2.4.1 (c) was carried out on outer panel stiffener (OPS) in order to attain maximum stiffness along with cost-effective manufacturability. The team conceptualized two different versions of OPS, viz. OPS version 1 and OPS version 2 by performing topology optimization. Figure II.2.4.1 (a), (b), and (c) shows the older composite door concept - along with the two new door designs - for which results are discussed in the sections that follow. The third major revesion involved design changes, as seen in Figure II.2.4.1 (d) on the inner panel in order to improve its manufacturability. The most important changes were increasing the overall draft angle by $2-4^{\circ}$ and removing complex features like the cavity for water bottle holder in order to simplify the design. 


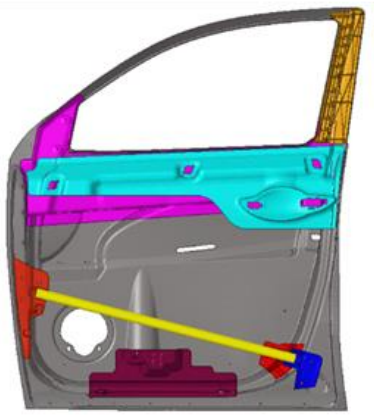

(a)

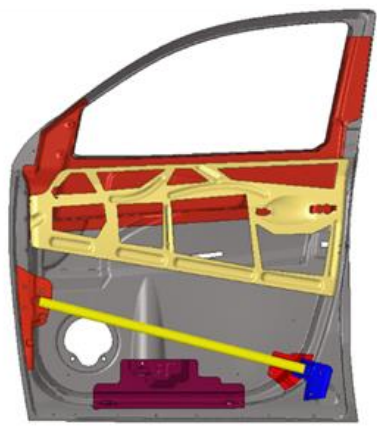

(b)

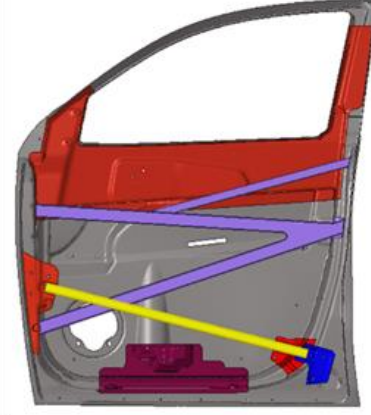

(c)

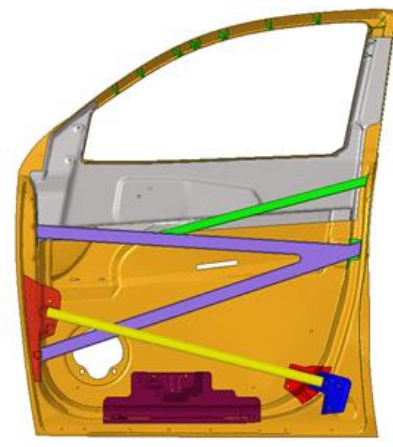

(d)

Figure II.2.4.1. Door concept comparison: (a) composite door with older OPS design; (b) newer composite door design with OPS version 1; (c) newer composite door design with OPS version 2; and (d) final composite door design with simplified inner panel geometry and OPS version 2 . Source: Clemson University.

\section{Summary of FEA Results}

The team assesed the existing unidirectional (UD) ply design, which included the extention of the IBS into the sash region and the extruded OPS design presented in Figure II.2.4.2 (a). Upon considering the thermoforming complexity of the UD ply setup, the team reassessed the design and came up with a mixed setup of woven and UD plies for inner panel. The new inner panel layup is presented in Figure II.2.4.2 (b), where the bottom-most 4-plies are woven (0/90 45/-45) and shaped like the inner panel minus the sash region, while the UD plies are laid over the woven plies in a quasi-isotropic manner. This layup would help in performing the thermoforming operation, since the four bottom woven plies are easily drapable and would require less darts and cuts, thus reducing the number of plies at the bottom. This will ensure that plies remain intact during the high-heating process. Considering this mixed-ply setup, several design iterations were performed to achieve the target weight while satisfying both linear and nonlinear load cases. Composite optimization was first undertaken for static load cases. These load cases were obtained both from the literature as well as from specific requirements provided by our OEM partner. Eleven static load cases and design constraints are prescribed on structural parts: (1) door sag (two subcases); (2) sash rigidity (two subcases); (3) door over opening; (4) beltline stiffness; (5) outer panel stiffness; (6) mirror mount rigidity (two subcases); (7) speaker mount rigidity; and (8) window regulator stiffness. Given the absence of OEM design constraint requirements for beltine stiffness, door over opening, and mirror mount stiffness, we obtained these requirements by applying the same load cases as on the baseline steel door. It is to be noted that the BIW component is eliminated in optimization runs to ensure a higher computational efficiency. Also, the effect of BIW deformation is incorporated by adjusting the door sag requirement via considering BIW deformation at the hinges.

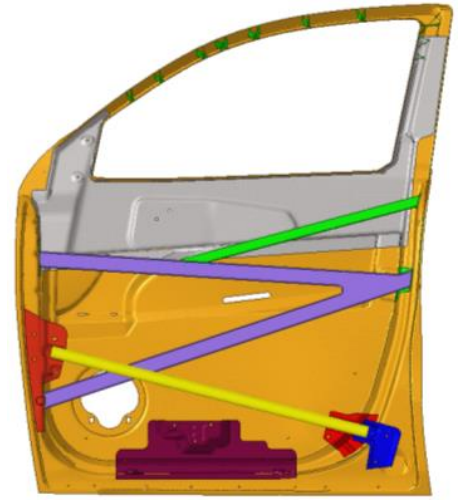

(a)

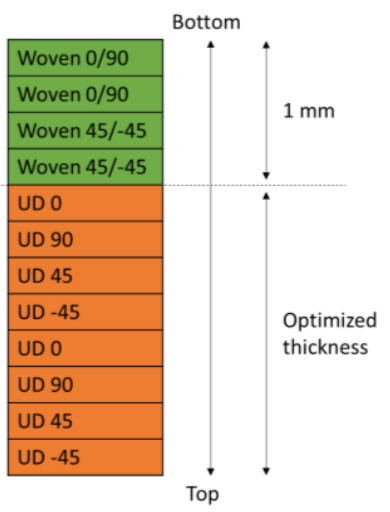

(b)

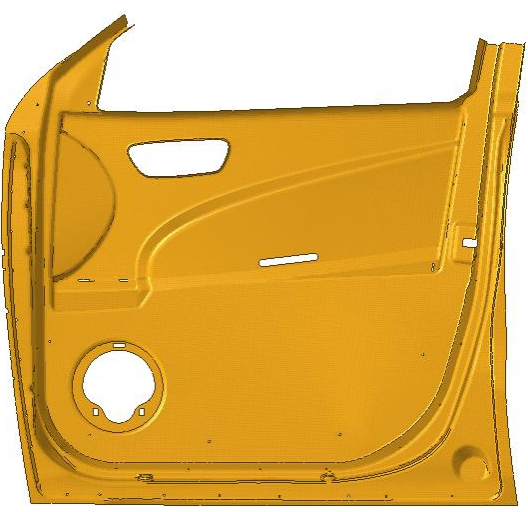

(c)

Figure II.2.4.2. (a) Latest door design; (b) new ply layup with mixed woven and UD plies; and (c) shape of bottom four woven plies. Source: Clemson University. 
The ply layup obtained from the drape simulation is used to setup the composite optimization problem in the Altair software suite. The design obtained from the initial optimization under linear static load cases is presented in Figure II.2.4.3. The weight of inner panel is $2.6 \mathrm{~kg}$, while its minimum thickness is $1.1 \mathrm{~mm}$. The IBS weighs $0.475 \mathrm{~kg}$ with a minimum thickness of $0.5 \mathrm{~mm}$. Since composite optimization is carried out only for static load cases, it still needs to be refined for dynamic load cases. In order to resolve this, a quasi-static pole (QSP) test is carried out first on the presented optimized design. Subsequently, regions that were failing early in the test were identified, thickness was manually increased, and the tests were repeated. After a few iterations, a final design was achieved for the two versions, such that it satisfied both linear and nonlinear load cases. The final design that satisfied both static and dynamic load cases is shown in Figure II.2.4.4. The weight of the IP is obtained as $3.57 \mathrm{~kg}$, while that of the IBS is $0.61 \mathrm{~kg}$. The minimum thickness of inner panel is $1.1 \mathrm{~mm}$, while its maximum thickness is $3.4 \mathrm{~mm}$ in some parts of the sash region. The minimum thickness of the IBS is obtained as $0.5 \mathrm{~mm}$, while its maximum thickness is found to be $4.8 \mathrm{~mm}$ in the mirror mount region. In order to satisfy the sash A and B load cases and constrain the maximum thickness (for ease of manufacturability), 1.2-mm steel ribs were placed in the sash region at ten locations that weigh $\sim 0.2 \mathrm{~kg}$. The static results with the final design are presented in Table II.2.4.2. It can be seen that all of the static load cases are satisfied with this design (values in bold green), and when compared with the baseline structure, the composite door structure weighs $56 \%$ less; however, the total weight of the door is lowered by $35 \%$, which is $7 \%$ higher than DOE's target.

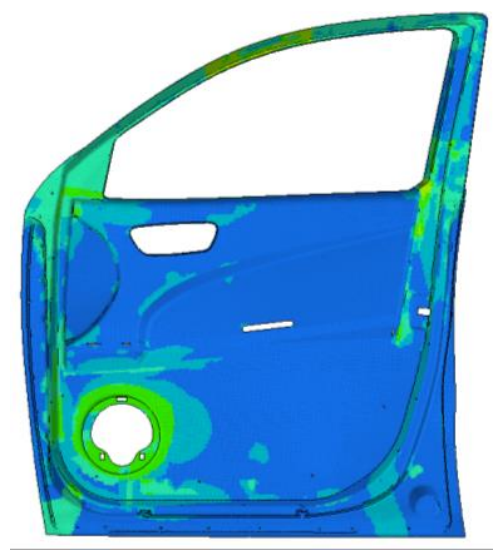

(a)

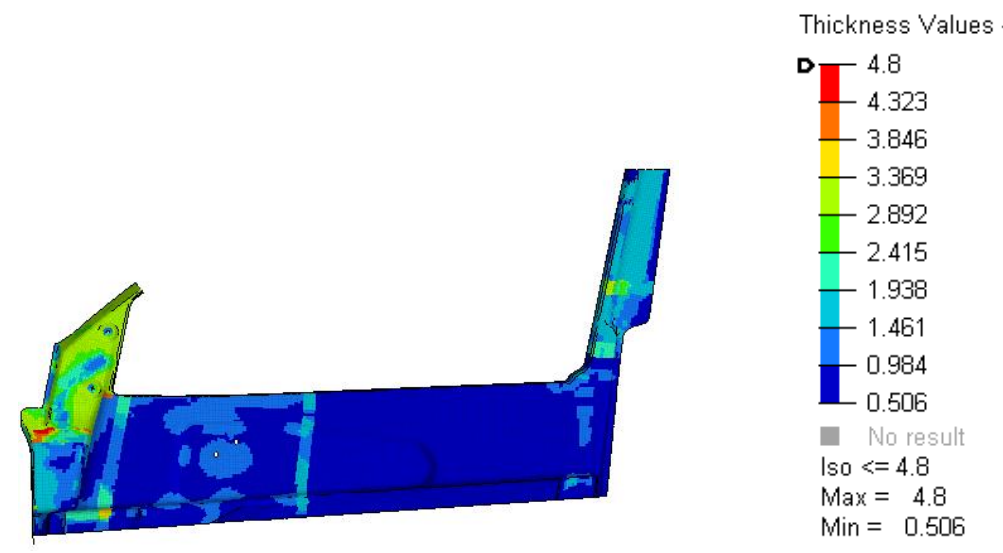

(b)

Figure II.2.4.3. Thickness distribution obtained after static optimization under linear load cases for the door components: (a) inner panel and (b) IBS. Source: Clemson University.

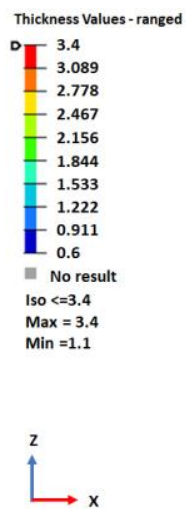

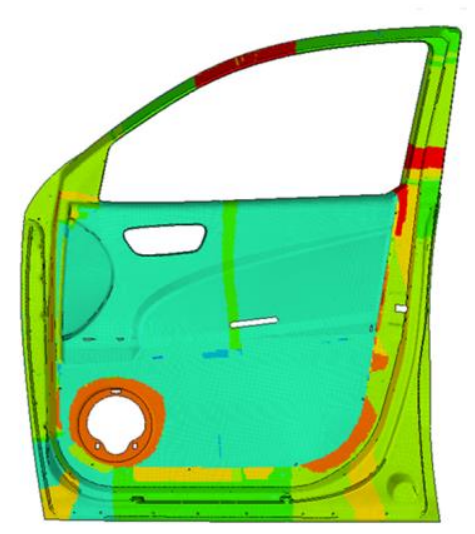

(a)
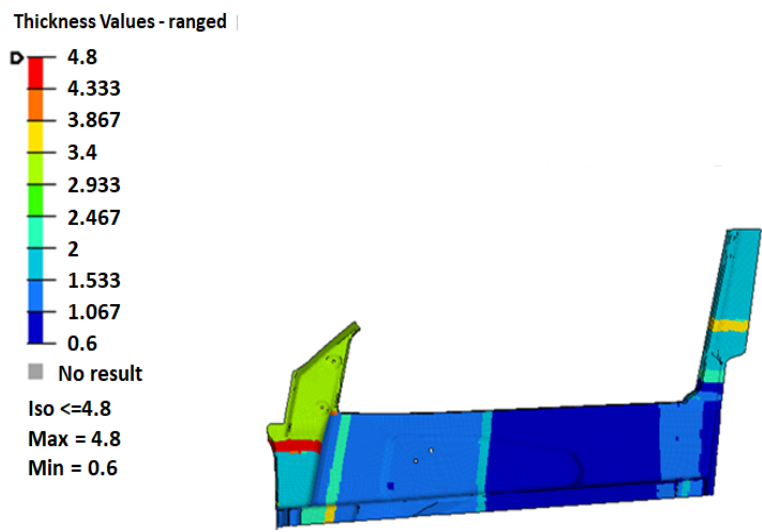

(b)

Figure II.2.4.4. Thickness distribution of the door components: (a) inner panel; and (b) IBS, which satisfies both linear and nonlinear load cases. Source: Clemson University. 
Table Il.2.4.2. Static Performance of Composite Door.

\begin{tabular}{|c|c|c|c|c|}
\hline $\begin{array}{l}\text { Serial } \\
\text { No. }\end{array}$ & $\begin{array}{l}\text { Target Category } \\
\text { Subcase }\end{array}$ & Target (Unit) & Target (Unit) & $\begin{array}{l}\text { \% Relative Difference } \\
\text { (Result-Target/Target) }\end{array}$ \\
\hline 1 & Mass Target & & & \\
\hline 1.1 & - & Structural Frame Mass & < Baseline (Kg) & $-56 \%$ \\
\hline 1.2 & - & Total Weight & < Baseline (Kg) & $-35 \%$ \\
\hline 2 & Frame Related & & & \\
\hline 2.1 & - & Door Sag - Fully Open & $<$ Baseline $(\mathrm{mm})$ & $-68 \%$ \\
\hline 2.2 & - & Sash Rigidity at Point $A$ & & $-4 \%$ \\
\hline 2.3 & - & Sash Rigidity at Point B & & $-35 \%$ \\
\hline 2.4 & - & Beltline Stiffness - IP & & $-63 \%$ \\
\hline 2.5 & - & Window Regulator (Normal) & & $-6 \%$ \\
\hline 2.6 & - & Mirror Mount Rigidity in X & & $-4 \%$ \\
\hline 2.7 & - & Mirror Mount Rigidity in $Y$ & & $-68 \%$ \\
\hline 2.8 & - & Door Over Opening & & $-6 \%$ \\
\hline 2.9 & - & Speaker Mount Stiffness & & $-3 \%$ \\
\hline
\end{tabular}

\section{Nonlinear Load Cases}

The composite door designs discussed in the previous section were further tested for nonlinear load cases to evaluate their crashworthiness. These load cases are (a) the QSP; (b) the full pole test (using the Japanese New Car Assessment Program AF05 female dummy) and (c) the deformable barrier test (Insurance Institute for Highway Safety Side-Impact Moving Deformable Barrier (IIHS SI MDB)) and the requirements of were provided by our OEM partner. The QSP test is evaluated by plotting the reaction force against pole displacement for each design. The area under the curve of this plot gives the energy absorbed during pole impact. Figure II.2.4.5 presents a plot comparing the baseline steel door and the composites door, which display the plot for the first stage (stroke $<6$ in.) and the second stage (stroke $<12$ in.). The initial average crush for the composite door is $22 \%$ higher than the actual OEM requirement. The intermediate crush (until 12 in.) is seen to be $85 \%$ higher than the actual OEM requirement. Figure II.2.4.5 presents the reaction force plot until the $18 \mathrm{in}$. of stroke for baseline and composite doors. It can be clearly seen from the plot that composite door satisfies the maximum peak crush requirement (horizontal dashed line in the plot) and is higher by $133 \%$. These results for the QSP load case are listed in Table II.2.4.3.

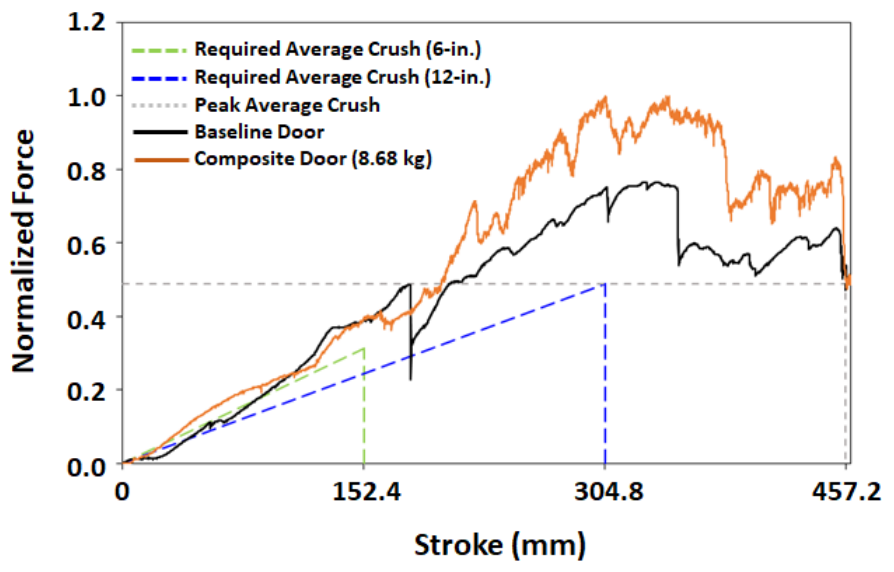

Figure II.2.4.5. Reaction force plot for the QSP test comparing the baseline door with the composite door. Source: Clemson University. 
Table II.2.4.3. Results for the QSP Test.

\begin{tabular}{|c|c|}
\hline Results & \% above OEM requirement \\
\hline Initial Average Crush & $122 \%$ \\
\hline Intermediate Average Crush & $185 \%$ \\
\hline Peak Crush & $233 \%$ \\
\hline Initial Average Crush & $122 \%$ \\
\hline
\end{tabular}

The dynamic simulations for the deformable barrier and the full pole load cases were then performed for both versions. To evaluate the performance of the doors, the team-after discussion with OEM partner-listed key performance indicators. Further, a gauging matrix was created to assess whether the composites door passed the OEM requirements. The gauging matrix was defined as: (a) successful (green), if the door response is better than the baseline; (b) tolerable (yellow), if the door response is lower than the baseline, but within a $10 \%$ margin; and (c) failure (red), if the door response is below 10\% from the baseline door. Table II.2.4.4 shows the results for the deformable barrier load case test, comparing the composite door with the baseline responses. The composite door response for the full pole (using the AF05 female dummy) is also presented and compared with the baseline door in Table II.2.4.4. The composite door responses either exceed or are within the tolerable range for both load cases.

Table II.2.4.4. Results For Full Pole and Deformable Barrier Dynamic Load Cases.

\begin{tabular}{|c|c|c|c|}
\hline Key Performance Indicator & Target & $\begin{array}{l}\text { FMVSS } 214 \text { Rigid Pole } \\
\text { (Response/Target) \% }\end{array}$ & $\begin{array}{c}\text { IIHS SI MDB } \\
\text { (Response/Target) \% }\end{array}$ \\
\hline Max center pillar intrusion & \multirow{7}{*}{ Baseline } & $-1 \%$ & $\mathrm{~N} / \mathrm{A}$ \\
\hline Max sill intrusion & & $-11 \%$ & $\mathrm{~N} / \mathrm{A}$ \\
\hline Max roof intrusion & & $-10.4 \%$ & $10.8 \%$ \\
\hline Max window-sill intrusion & & $-4.78 \%$ & $11.25 \%$ \\
\hline Front door dummy hip intrusion & & $-9.71 \%$ & $18.3 \%$ \\
\hline Max door lower intrusion & & $7 \%$ & $1 \%$ \\
\hline Safety survival space & & N/A & $2.46 \%$ \\
\hline \multicolumn{4}{|c|}{$\begin{array}{l}\text { Green (Success) - Below Baseline target values }(<b) \text {. } \\
\text { Yellow (Tolerable) - More than Baseline values but smaller than } 10 \% \text { difference }(>b,<b+10 \%) \text {. } \\
\text { Red (Failure) - More than } 10 \% \text { above Baseline value }(>b+10 \%) \text {. }\end{array}$} \\
\hline
\end{tabular}

\section{Subcomponent Manufacturing, Modeling, and Testing}

To verify crash simulations, a subcomponent test with validation was proposed and untertaken. A hat-stiffened panel was manufactured and tested to assess simulation predictions and compare with the experimental results. The tool that was used in the subcomponent fabrication is shown schematically in Figure II.2.4.6. The material selected was AS4 CF with nylon (PA6) having a quasi-isotropic orientation for both the hat and the flat section. This material was received in the form of a prepreg tape from Tencate and was used to fabricate the subcomponent. Both parts (hat and flat section) were manufactured separately with process conditions similar to those used to generate material property data, followed by bonding with the Plexus MA530 adhesive. The Plexus system has been characterized, while the adhesive material model is being implemented in crash simulations. 

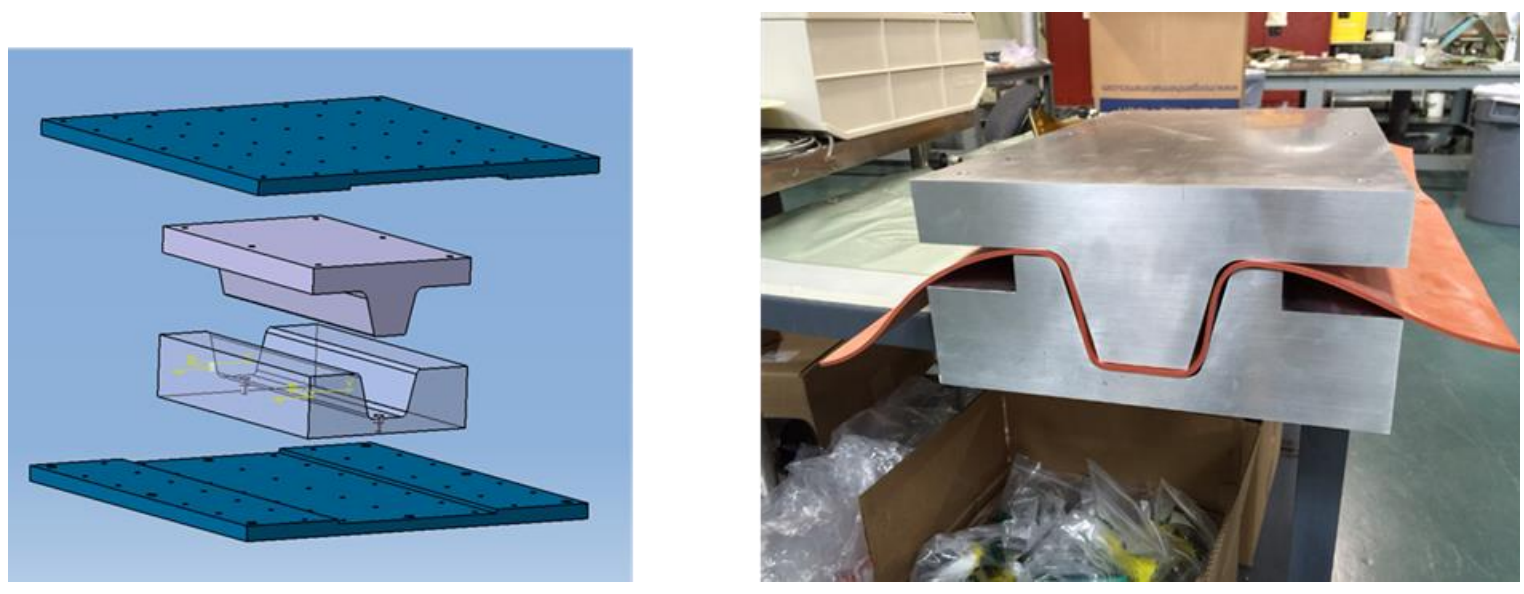

Figure II.2.4.6. Subcomponent tool for hat production. Source: University of Delaware.

The selected subcomponent geometry was comprised of two composite parts: (1) the hat and (2) the spine. The hat is a 16-layer laminate that was fabricated from $[45 /-45]_{\mathrm{s}}$ construction, with the spine fabricated from a quasi-isotropic construction. In both cases, flat panels were initially fabricated to appropriate geometries. The unidirectional AS4/nylon prepreg was cut and laid up to create the desired geometry and layup sequence in a flat laminate form. After layup, the prepreg stacks were autoclaved with the cycle shown in Figure II.2.4.7.

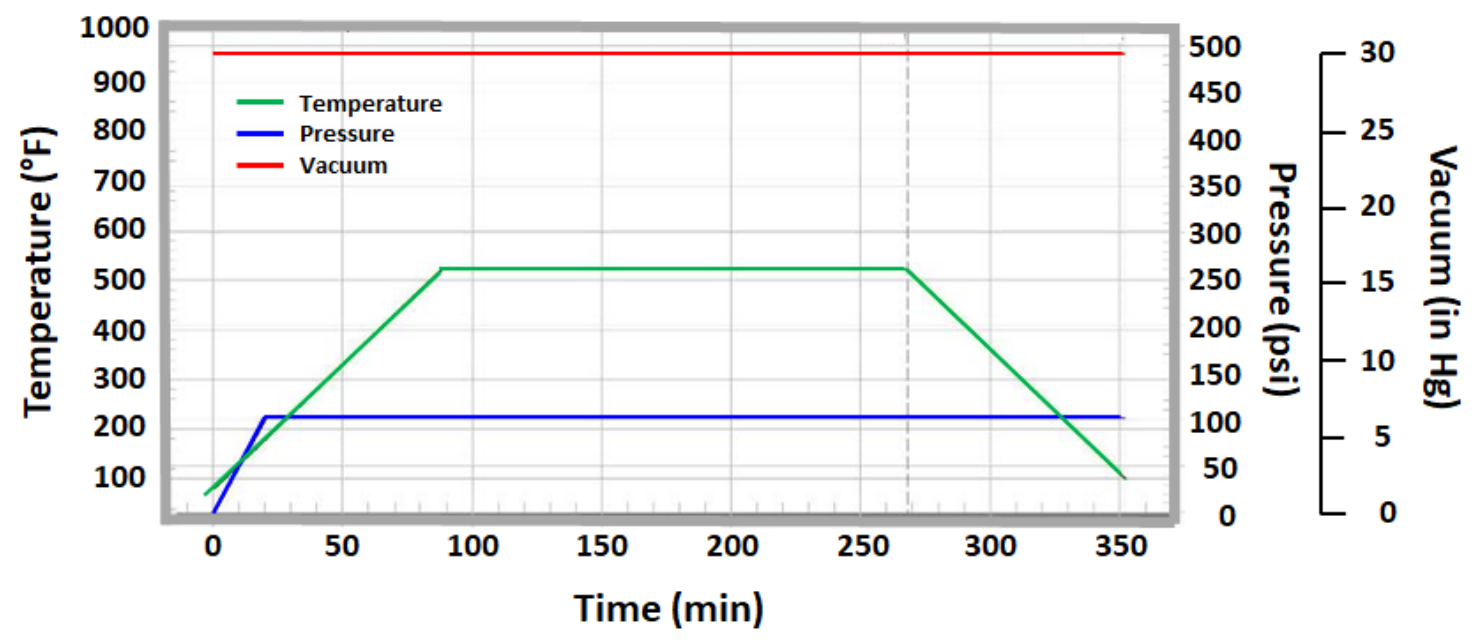

Figure II.2.4.7. Process conditions used to fabricate subcomponent. Source: University of Delaware.

A pressure of 100 psi was used to consolidate the prepreg sheets at a temperature of $520{ }^{\circ} \mathrm{F}$ and 28 in $\mathrm{Hg}$ vacuum for 190 min, based on the Tencate recommended process cycle shown in Figure II.2.4.8. Flat laminates were examined visually and with ultrasonic scans for quality assessment. The spine seen in Figure II.2.4.8 (a) is a flat laminate to close the hat geometry and no further processing was needed. The hat geometry seen in Figure II.2.4.8 (b) was formed from the flat laminate via the combination of temperature and pressure on an $\mathrm{Al}$ tool. The flat laminate was first vacuum-formed to shape in an oven at $450{ }^{\circ} \mathrm{F}$ to achieve the same shape of the tool, followed by autoclave cycle to re-consolidate the geometry to maintain laminate quality. An Al shim was used on the top surface for good surface finish and consistent thickness. The finished spine and the hat sections are shown in Figure II.2.4.8 (c). 


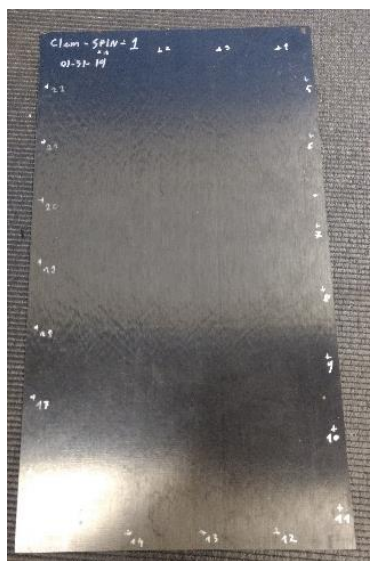

(a)

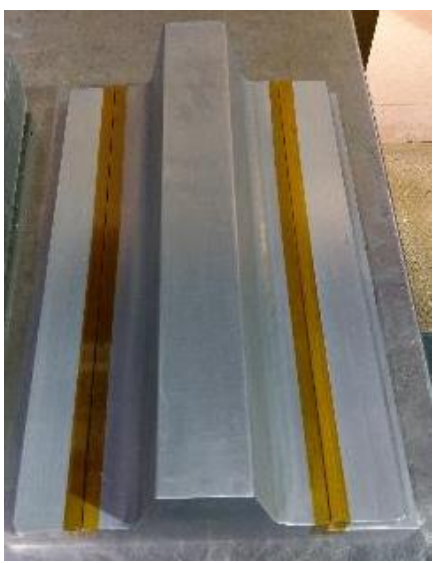

(b)

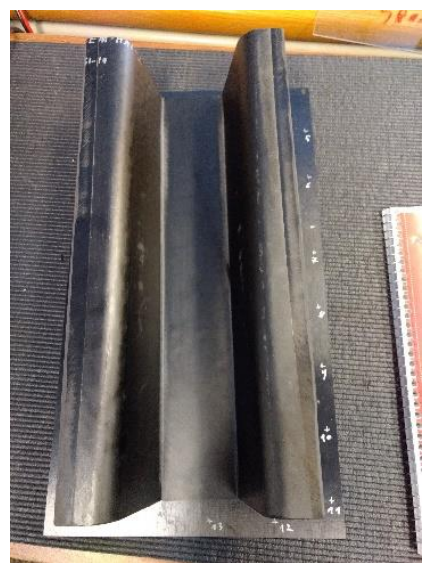

(c)

Figure II.2.4.8. (a) Fabricated spine; (b) hat geometry tool; and (c) finished hat section. Source: University of Delaware.

After fabrication of the hat and spine sections, thickness measurements were taken for each section at more than 22 locations. These locations are marked hat section in Figure II.2.4.9 (a) and for the spine section in Figure II.2.4.9 (b). The hat has an average thickness of $2.32 \mathrm{~mm}$ with a standard deviation of $0.54 \mathrm{~mm}$, while the spine has an average thickness of $2.26 \mathrm{~mm}$ with a standard deviation of $0.069 \mathrm{~mm}$. As mentioned above, these average thicknesses were used to determine the average ply thickness in the simulation.

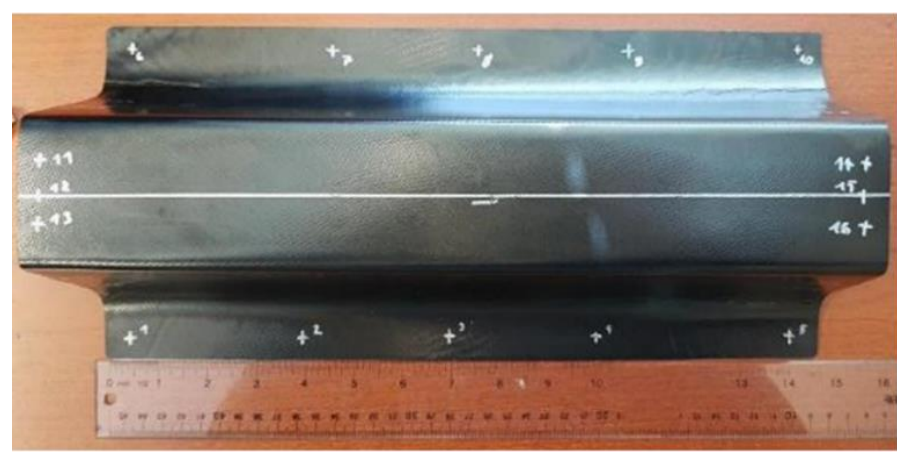

(a)

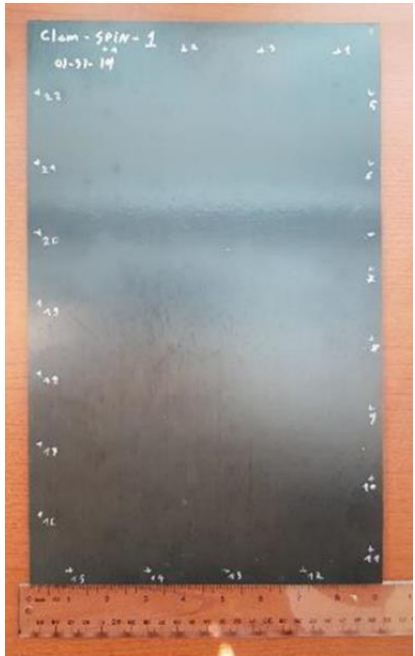

(b)

Figure II.2.4.9. Measurement points of: (a) hat; and (b) spine for thickness. Source: University of Delaware.

The assembly of the hat and spine was performed with adhesive-bonding. Plexus MA530 adhesive was chosen for this purpose based on prior testing of the adhesive and its performance assessment in coupon tests. Bond line thickness was chosen as $1 \mathrm{~mm}$ to provide a sufficient gap tolerance for full-scale door assembly. An adhesive cure schedule was developed by Clemson University during the adhesive performance assessment, and the same cure schedule was adopted here. The final bonded assembly was put together with 1-mm spacers at three locations along the length of the bond line. Surface preparation of the bond line interfaces were comprised of grit blasting with fine $\mathrm{SiC}$ of 120 grit size using a thin pencil blaster for good dimensional control and was followed by wiping with a cheesecloth. Paste adhesive was applied using a standard twocomponent adhesive applicator and was held in place with weights to provide sufficient pressure to ensure a 1$\mathrm{mm}$ bond line thickness. The completed subcomponent assembly can be seen in Figure II.2.4.10. 


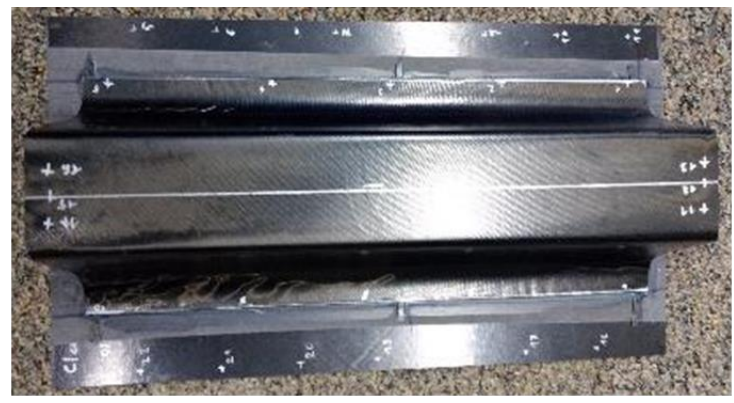

(a)

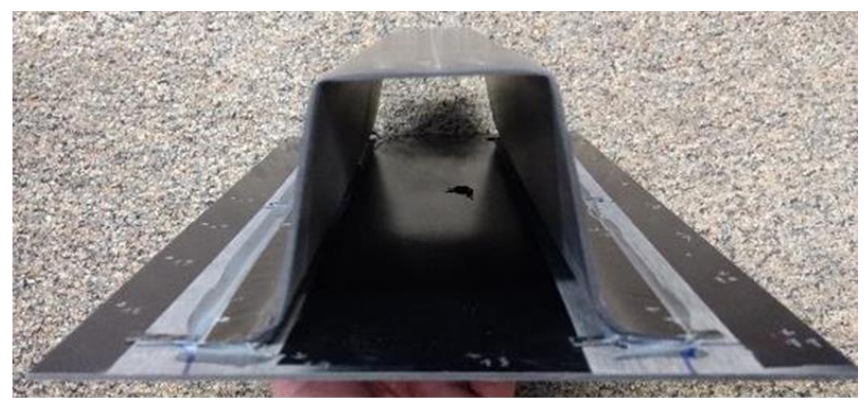

(b)

Figure II.2.4.10. Completed subcomponent assembly after adhesive-bonding: (a) top view; and (b) end view. Source: University of Delaware.

The subcomponent model consists of five-part definitions: (1) the support, (2) the impactor, (3) the hat (top half of the closed hat section), (4) the spine (base of the closed hat section) and (5) the adhesive used to bond the hat and the spine. The supports, as well as the punch, are modeled as rigid cylinders. The diameters for both support cylinders are one inch, while the punch is 2-in. The supports are constrained in all six degrees of freedom and the punch is only allowed to move in a global $z$-direction. The weight of the punch had been set to $80 \mathrm{~kg}$, which had an initial velocity of $2.8 \mathrm{~m} / \mathrm{s}$ that is equal to a fall distance of $15 \mathrm{in}$. and an impact energy of $300 \mathrm{~J}$. The hat and the spine are modeled as PART_COMPOSITE card with 16 layers. The layup sequence for the hat is $[45 /-45]_{\mathrm{s}}$ and for the spine is $[0 / 90 / 45 /-45]_{\mathrm{s}}$. To match the thickness of the hat and the spine, measurements were taken, and an average ply thickness was calculated for the actual parts fabricated. The adhesive was modeled with an element formulation 20 and material card MAT_138, which is a mixed-mode traction and separation law. The attachment between the composite parts and the adhesive had been modeled with a CONTACT_TIED_SHELL_EDGE_TO_SURFACE contact. The bond lines are 1-mm-thick $\times 1$-in. wide with this geometry maintained in the fabricated subcomponent. A summary of this setup is shown in Figure II.2.4.11.

Impactor:

- Round cylinder diameter: 2 in

- Property's: 80 kg rigid

- Constrains: $X, Y, R X, R Y, R Z$

- Initial velocity: $2.8 \mathrm{~m} / \mathrm{s}=$ fall from 15 in height

- Energy:300 J

Beam:

- Consist out of a head and a spine laminate which are bonded together

- Material: UD-AS4/NYLON-FVF=0.44 (MAT_54)

Head:

- Laminate: [45/-45]s 16 Layers

- Thickness: $2.32 \mathrm{~mm}$

Spine:

- Laminate: [0/90/45/-45]s

- Thickness: $2.26 \mathrm{~mm}$<smiles>[3H]C([3H])C([3H])[3H]</smiles>

Adhesive:

- Material: Plexus (MAT_138)

- Element formulation: 20

- Connected via: CONTACT_TIED_SHELL_EDGE_TO_SURFACE

Figure II.2.4.11. Subcomponent configuration, boundary conditions, and simulation summary.

Source: University of Delaware. 
The contact between the subcomponent and the supports, as well as the impactor, is modeled as the AUTOMATIC_SINGLE_SURFACE where the adhesive is excluded. In order to avoid the undesirable oscillation in contact, viscous damping is implemented at $20 \%$, which is the LS-DYNA recommended value for metallic and similar material contacts. This value is also used to define surface contacts in nonlinear load cases. Bulk viscosity and hourglass viscosity are set to a default value. The static and dynamic coefficients of friction between all parts that are included in the single surface contact is set to 0.08 . To speed up the simulation runtime, mass scaling was used, while the artificial mass increase was $<0.33 \%$ of the original mass, which accounts for a time step of $5.500 \mathrm{e}-07 \mathrm{~s}$.

Simulation results showed that the hat section of the beam will locally fail, but the structure itself will withstand the impact. No bond line failure was observed in the course of simulation. The maximal contact force between the impactor and the beam is predicted to be around $10 \mathrm{kN}$ at around $0.004 \mathrm{~s}$. The predicted force-time plot is shown in Figure II.2.4.12.

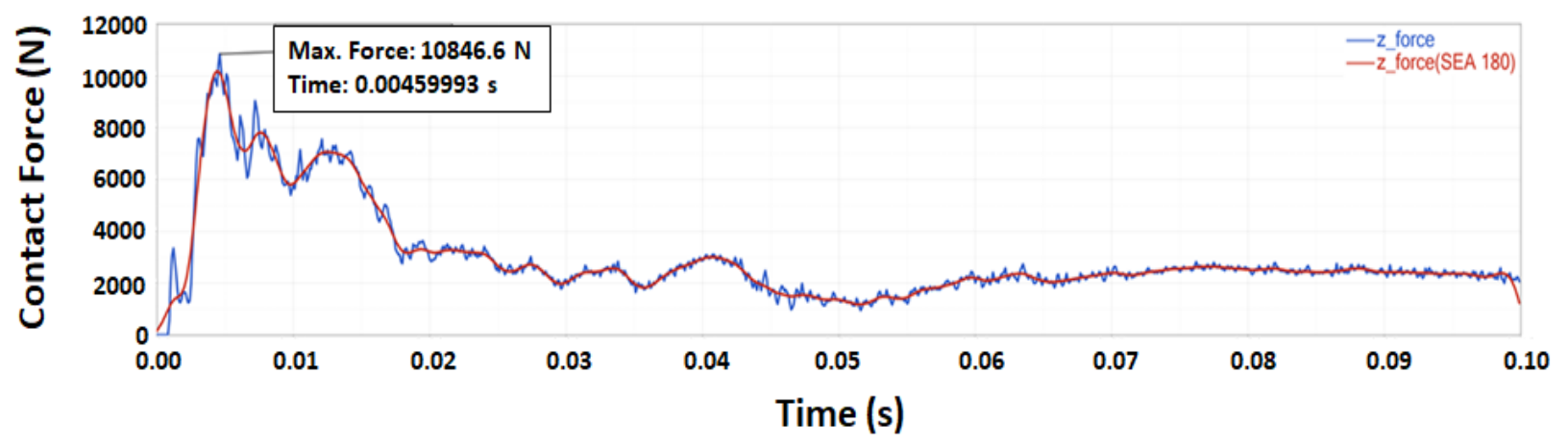

Figure II.2.4.12. Predicted contact force over time for the simulation conditions in Figure II.2.4.13. Source: University of Delaware.

Impact testing was performed at the University of Delaware, Center for Composite Materials, using their $1100 \mathrm{~J}$ drop tower and a 2-in. cylindrical impactor with 1-in. round cylindrical supports, as modeled. The subcomponent is simply supported on its supports, prior to an impact drop. The impact energy of 300J is selected by observing failure from initial numerical simulations. The impactor has a fixed weight of $80 \mathrm{~kg}$ while drop height is adjusted to 15 in. such that total impact energy matches $300 \mathrm{~J}$, which is the energy level where the initial simulations showed failure. The following were part of the test setup:

1. Strain gauges at key locations on the hat and the spine.

2. Load cell in the impactor for force response.

3. Spine center deflection is been measured with a laser displacement center.

4. High-speed video to capture dynamic deformation of the spine (side view).

Multiple biaxial strain gauges (two at the bottom and one on the side) were adhesively bonded onto the fully assembled subcomponent at locations that are predicted to experience higher strain determined from the simulations, as shown in Figure II.2.4.13. 


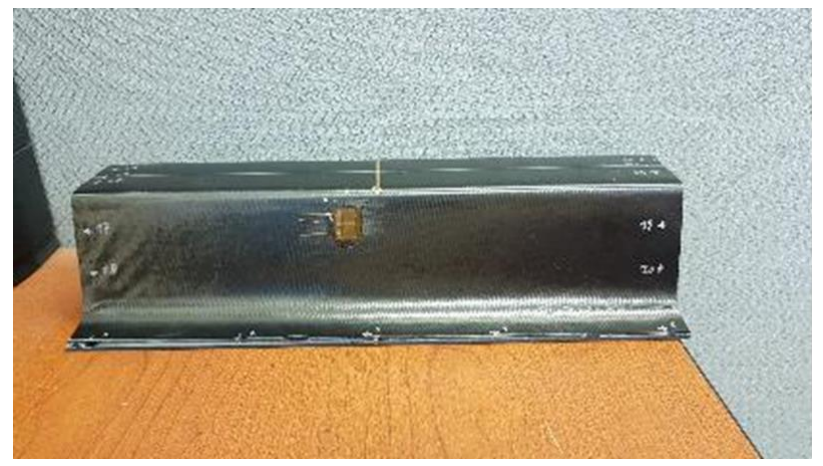

(a)

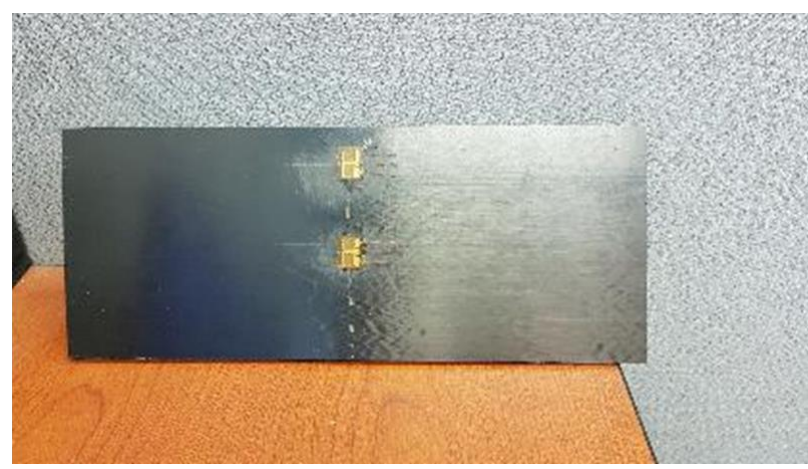

(b)

Figure II.2.4.13. Location of the bonded strain gauges on (a) the side and (b) bottom in order to record strain during impact. Source: University of Delaware.

The $1100 \mathrm{~J}$ drop tower with the $80 \mathrm{~kg}$ impactor and strain gauges were calibrated, and the test was carried out in accordance with the simulated test conditions. Excerpts from the high-speed camera are shown in Figure II.2.4.14, which show the impactor and subcomponent before the impact, at the moment of the impact, and after the impact.

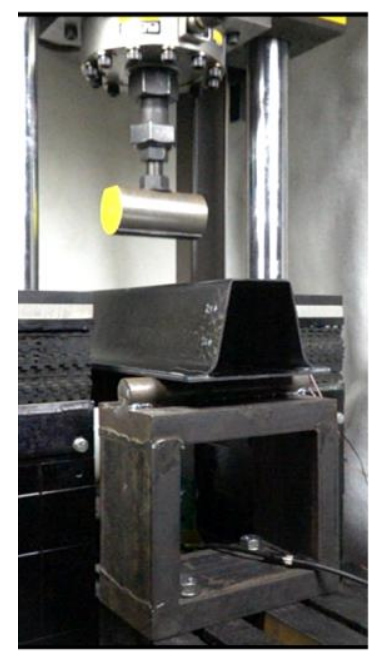

Before Impact

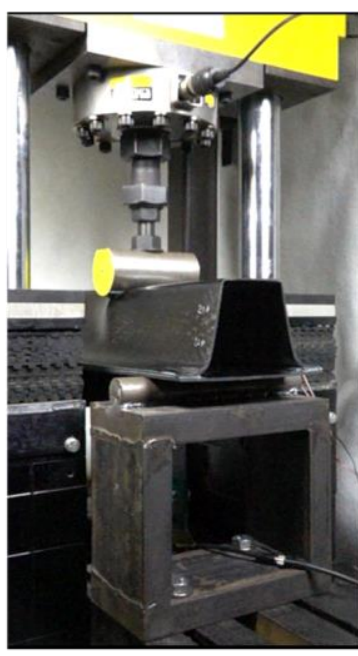

Moment of Impact

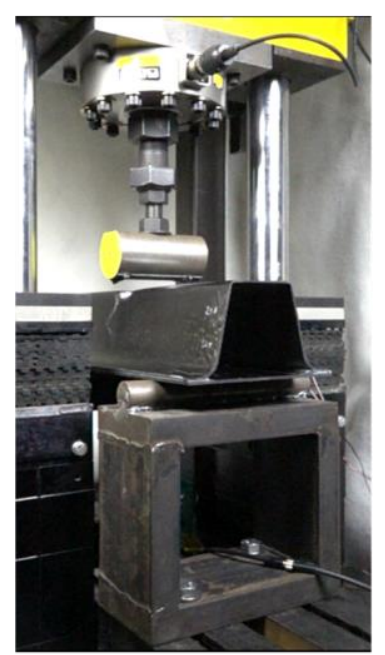

After Impact

Figure II.2.4.14. Subcomponent test progression showing the impactor and the subcomponent before the impact, at impact, and after the impact. Source: University of Delaware.

A preliminary comparison between the simulated and experimental results of the test revealed that the simulated contact force was lower than expected, as observed in Figure II.2.4.15 (a). One explanation for this could be that the model currently uses a material card where elements are deleted beyond a certain force and strain in the elastic regime. However, in reality, due to the ductile nature of the thermoplastic PA66 nylon matrix, we observe that the composite as a whole is able to absorb and withstand far higher loads without failing catastrophically if loaded in compression as can be seen by the pictures in Figure II.2.4.15 (b). The team is currently looking into the material model and will modify it and the simulation strategy to better reflect the experimental results. 


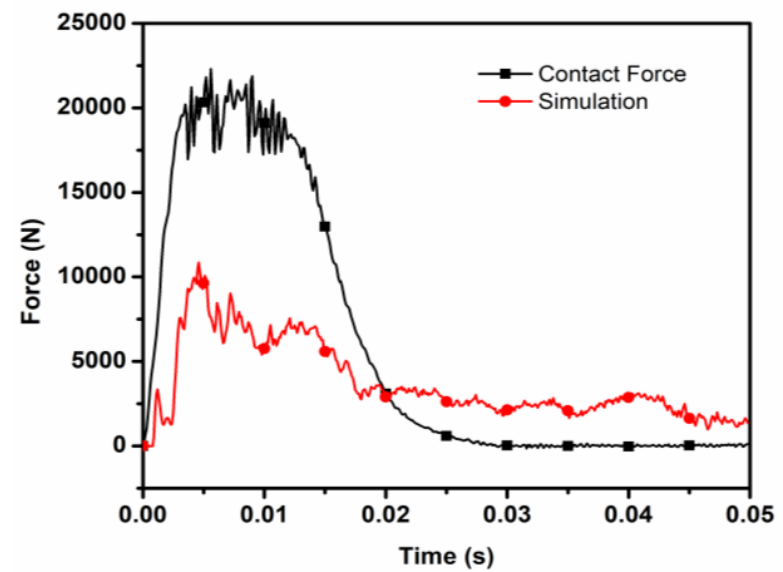

(a)

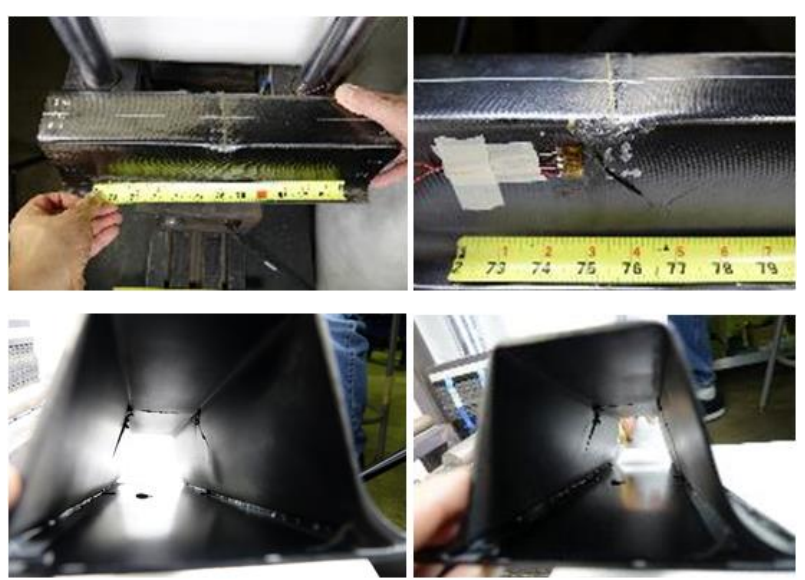

(b)

Figure II.2.4.15. (a) Comparison between the simulated and experimental results. (b) If loaded, the composite, as a whole, is able to absorb and withstand far higher loads. Source: University of Delaware.

\section{Fully Assembled Prototype Door Meeting Fit and Integration Requirements}

To validate the geometry of the ultralightweight composite door, the team decided to build a 1:1 door mockup to validate the fit and function of the current composite door. In addition, an important goal set by our OEM partner was to ensure that our design maintains the same sea-lining geometry as the baseline, while housing all of the baseline equipment. The inner panel is the most crucial component of the ultralightweight composite door, primarily because all of the interface points for vehicle structure are through the inner panel. A carbon fiber-reinforced epoxy door was built primarily using the vacuum infusion process for low-cost prototyping. To keep the fabrication cost down, a wooden cavity tool was procured and finished in-house. For all other internal parts, the team decided to 3D-print them using acrylonitrile butadiene styrene material.

The wooden tool was finished using dry sandpaper from 80 grit to 400 grit in increments. Once the tool was cleaned, two coats of a polyurethane tool coat were applied to a thickness of $\sim 0.5 \mathrm{~mm}$ and were allowed to cure for $48 \mathrm{~h}$. After this, the team sanded and polished the tool further to 800 grit in order to obtain a highly smooth surface. Four coats of chemical release agent were applied to the polished tool surface. Once the tooling coat was dry, the team placed dry CF fabrics similar to the ply design, in the actual door. The lines for the vacuum and the resin flow were then laid out to optimally fill the mold, which was bagged with breather cloth and resin flow media. Once the bag was properly sealed, the vacuum of $-28 \mathrm{in}$. $\mathrm{Hg}$ was applied and the resin was infused. The parts were allowed to cure for $48 \mathrm{~h}$ in the tool, and then demolded and further cured for an additional $12 \mathrm{~h}$ in the oven at $65^{\circ} \mathrm{C}$. The fully cured inner panel was then trimmed and painted.

The internal parts of the 3D-printed door were attached to the inner panel in addition to weather sealing. The assembled door was then installed onto the vehicle for fit and function validation. Below is the list of criteria used to verify that the designed door geometry and the 1:1 mockup fits and functions were as desired on the existing vehicle. Figure II.2.4.16 shows the manufacturing process for the 1:1 mockup with internal parts assembled on the existing vehicle. 


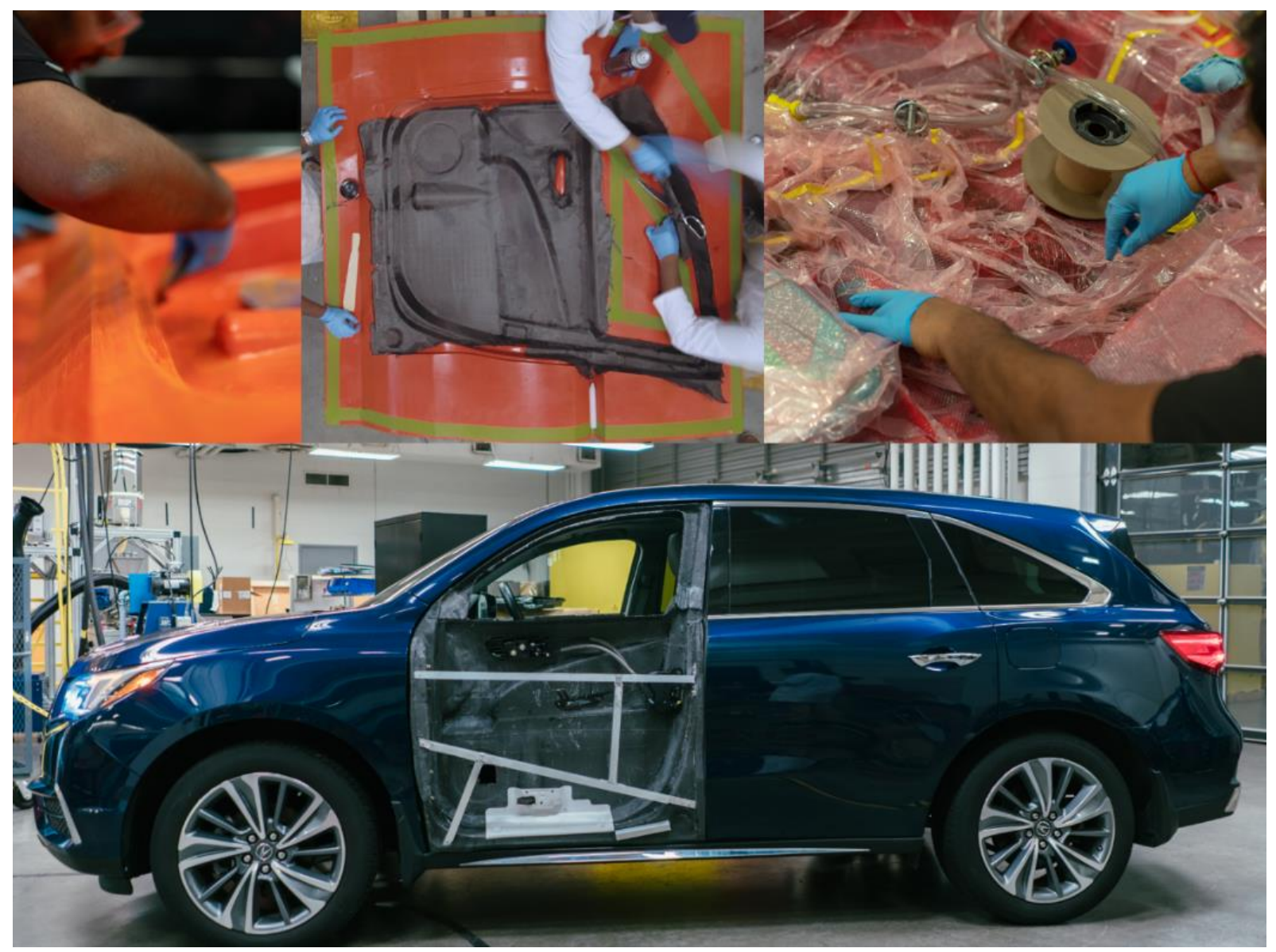

Figure II.2.4.16. Composite door manufacturing process. Source: Clemson University.

Door fit criteria:

- The team confirmed that the current design properly fits in the existing vehicle and properly aligns with all mounting points shown in Figure II.2.4.17.

- The weather sealing is properly compressed when the door is closed to achieve good sealing.

- The door frame has enough internal space for all of the components.

- The door properly aligns with the B-pillar and the dashboard.

- The door window frame is flush with the existing interior trim.

Door function criteria:

- The door frame properly opens and closes without any interference with the existing vehicle structure and rear door.

- $\quad$ The door securely latches with the existing latch loupe plate.

- The door has sufficient access for assembly, disassembly, and repair of the door internal components.

During this process, the team also identified minor design improvements for ease of assembly and repair. These changes are currently being incorporated into the final computer-aided design models. 


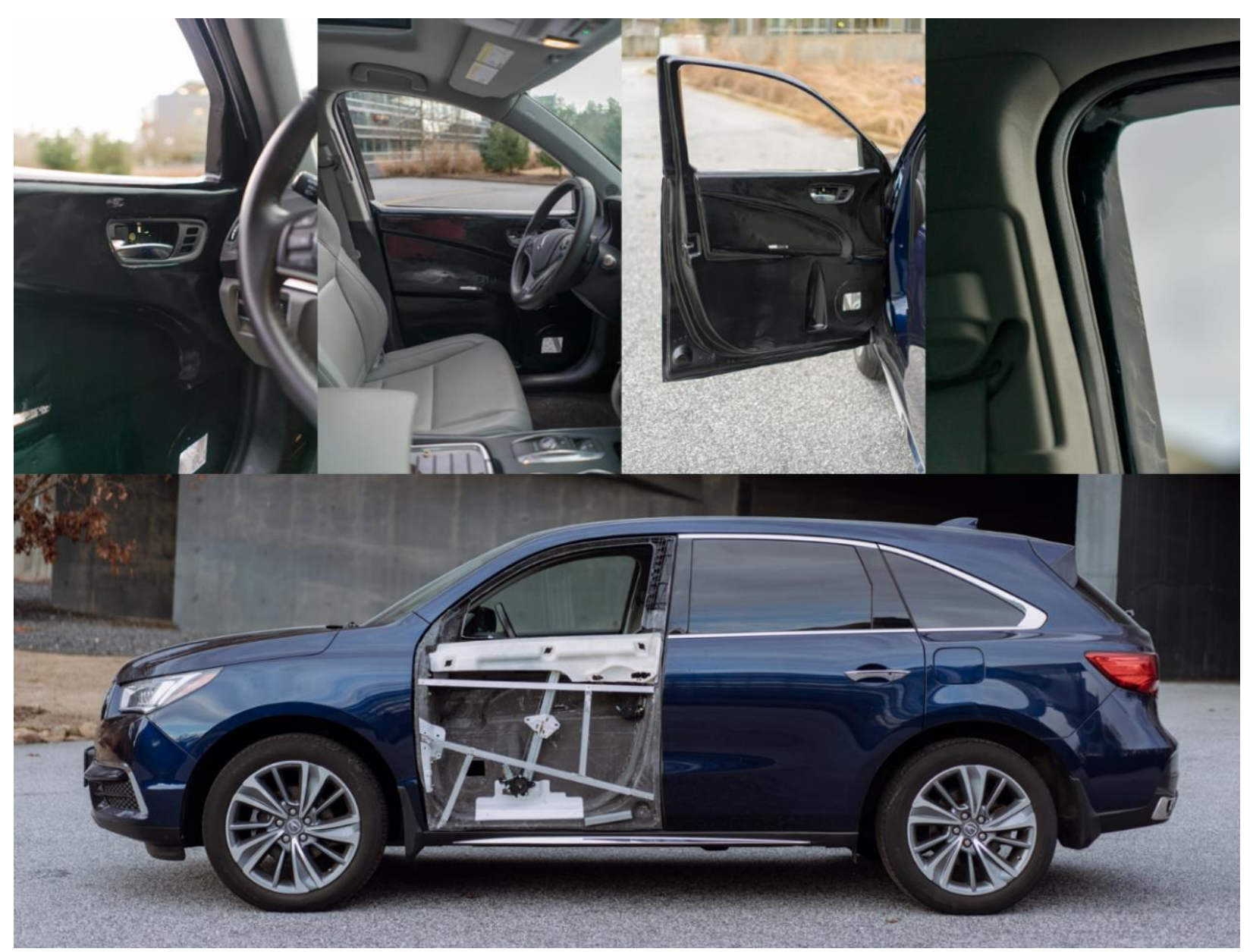

Figure II.2.4.17. Fully assembled door frame on the existing vehicle. Source: Clemson University.

\section{Cost Modeling Summary}

A generative cost model was developed to estimate the total cost of the latest design of the door, which was $\$ 989.79$. Cost of the inner panel is $\$ 379$, which accounts for $38 \%$ of the total cost of the door, as seen in Figure II.2.4.18. Material, tooling, equipment, labor, energy, building, maintenance, overhead, and capital were considered to calculate the cost of each individual part. 


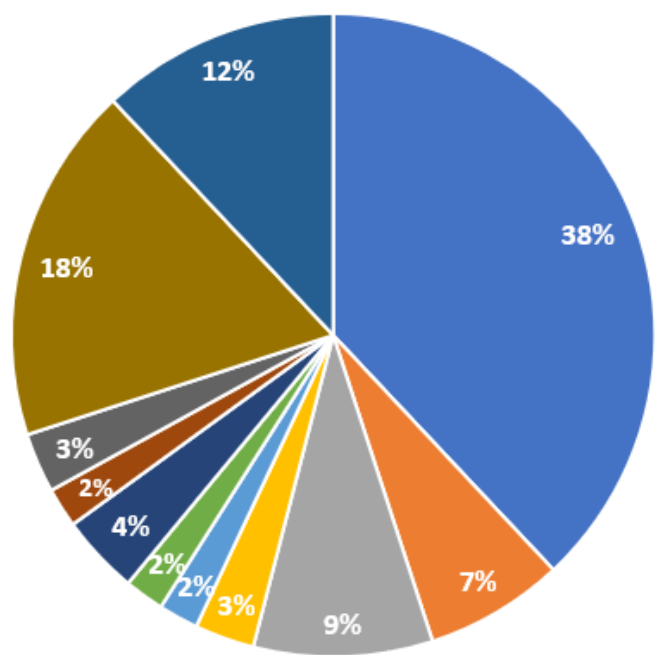

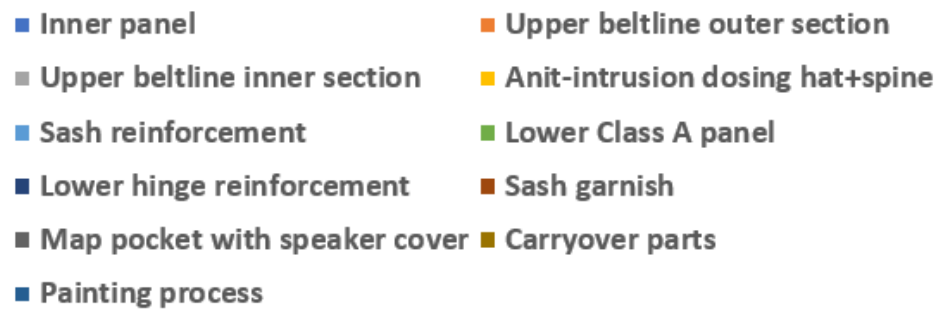

Figure II.2.4.18. Cost distribution of the individual parts of the door. Source: Clemson University.

After the development of a generative cost model, a parametric cost model was developed to find how individual parameters influenced the total cost. Parametric cost modeling was carried out in three steps: (1) development of cost estimating relationships; (2) parameterization of the door design; and (3) creation of the user interface to perform tradeoffs and estimate the cost of individual parts. The following assumptions were made for the development of parametric cost model:

1. Total cost is assumed to be a function of material, labor, equipment, tool, energy and overhead cost.

2. Parameters identified for cost estimation are independent of each other.

3. Data is collected and normalized using experience and a literature review.

4. Rate of overhead (18 24\% of total cost) is assumed by experience.

5. Production volume per year is assumed to be around 20,000 vehicles.

6. Cost of carry over parts $(\sim 183)$ is assumed to be constant.

7. Number of workers working on each machine are assumed to be four.

At first, individual parameters that influenced the overall cost of the door were identified, which are: (1) electricity cost per kWh, (2) scrap rate, (3) mold life, (4) equipment life, (5) labor wage, (6) production volume per year for 20,000 vehicles, (7) overhead rate and (8) material cost. Then, a relationship between these parameters with the total cost was determined using mathematical and statistical tools. The distribution plot for each of the identified parameters and the corresponding total cost was calculated and is presented in Table II.2.4.5. 
Table II.2.4.5 Statistical Distribution of Individual Parameters and Total Cost.

\begin{tabular}{|c|c|c|c|c|c|c|}
\hline $\begin{array}{l}\text { Serial } \\
\text { No. }\end{array}$ & Parameter & $\begin{array}{l}\text { Raw data } \\
\text { distribution }\end{array}$ & $\begin{array}{c}\text { Mean } \pm 2 \text { Std. } \\
\text { Dev. }\end{array}$ & $\begin{array}{l}\text { Total cost } \\
\text { distribution }\end{array}$ & $\begin{array}{l}\text { Total cost } \\
\text { (U.S.Dollars) }\end{array}$ & Probability \\
\hline 1 & $\begin{array}{c}\text { Electricity rate } \\
\text { (cents/kWh) }\end{array}$ & Log logistic & $7.5 \sim 15$ & Log normal & 988 991 & 0.94 \\
\hline 2 & Scrap rate (\%) & Log normal & $4 \sim 13$ & $\begin{array}{l}\text { Largest } \\
\text { extreme value }\end{array}$ & $974 \sim 1022$ & 0.95 \\
\hline 3 & $\begin{array}{l}\text { Mold life } \\
\text { (years) }\end{array}$ & Log logistic & $3.5 \sim 12.5$ & Logistic & $925 \sim 1136$ & 0.96 \\
\hline 4 & $\begin{array}{l}\text { Equipment life } \\
\text { (years) }\end{array}$ & Log normal & $5 \sim 13$ & Normal & $987 \sim 992$ & 0.94 \\
\hline 5 & $\begin{array}{l}\text { Labor wage } \\
\qquad(\$ / \mathrm{hr})\end{array}$ & Weibull & $15 \sim 25$ & Weibull & $981 \sim 1002$ & 0.95 \\
\hline 6 & $\begin{array}{c}\text { Production per } \\
\text { year }\end{array}$ & Weibull & $14500 \sim 26500$ & Log normal & $952 \sim 1048$ & 0.96 \\
\hline 7 & $\begin{array}{l}\text { Overhead rate } \\
\text { (\%) }\end{array}$ & Normal & $15 \sim 27$ & Normal & $956 \sim 1036$ & 0.95 \\
\hline 8 & $\begin{array}{l}\text { Material cost } \\
\text { (\$) }\end{array}$ & Weibull & $69 \sim 94$ & Log logistic & $978 \sim 1054$ & 0.95 \\
\hline
\end{tabular}

Furthermore, the total cost is determined by incorporating all the identified parameters. Assuming normal distribution, the total cost of the door has been determined to vary between $\$ 920$ and $\$ 1059$, as presented in Figure II.2.4.19.

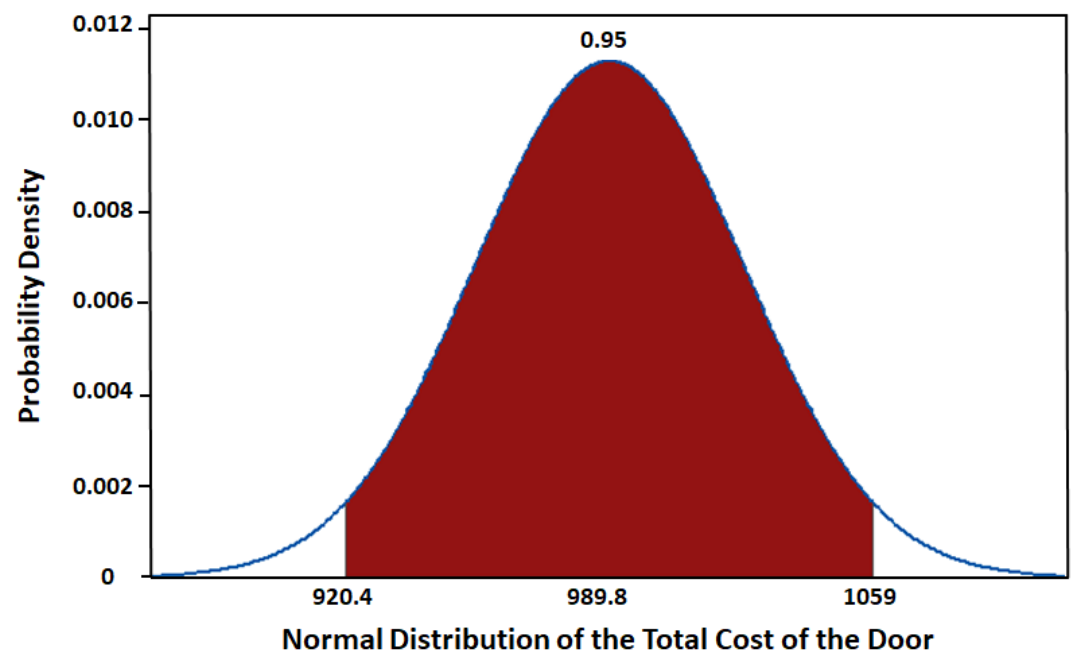

Figure II.2.4.19. Normal distribution of the total cost of the door. Source: Clemson University.

After developing the mathematical equations to determine the distribution of the total cost with a variation in identified parameters, our next step was to link the design geometry with the overall cost (i.e., parameterization of the door design). With a slight change in the geometry of the door, there will be a change in its weight, amount of raw material, and other resources used in its manufacturing, thus changing the overall cost of the door. In the last stage of efforts in FY 2019, we generated a user interface with a series of pop-up windows and dropdown menus that ask users for values of few parameters (or else, take default defined values). We could use this interface to perform tradeoffs (e.g., What-If analyses) to predict the total cost for a mix of possibilities. 


\section{Conclusions}

The research focus during FY 2019 involved the modification of door design by employing an iterative processing that involved feedback from static and crash simulations, cost analysis and manufacturing constraints, while trying to comply with the aggressive weight targets. The final door design met and exceeded expectations from the viewpoint of static and crash targets. This was further validated by experimental tests performed on a subcomponent. In addition, a fully functional low-cost prototype of the design was also manufactured in order to validate fit and function requirements. From a cost perspective, the door was slightly above the cost targets primarily due to high input material costs, and strategies are being evaluated in order to meet this requirement.

\section{Key Publications}

1. Limaye, M., A. Kothari, G. Dalal, S. Pilla, and G. Li, 2019, "Manufacturing process effects on crashworthiness analysis through a numerical simulation pathway," Society of Plastics Engineers ACCE Conference, 4-6 September 2019, Novi, MI, USA (awarded third place in graduate student poster competition).

2. Kothari, A., M. Limaye, G. Dalal, G. Li, and S. Pilla, 2019, "Design optimization for static and crash performance of an ultra-lightweight CF reinforced thermoplastic composite vehicle door assembly," Society of Plastics Engineers ACCE Conference, 4-6 September 2019, Novi, MI, USA.

3. Pradeep, S. A., T. Zheng, A. Yerra, and S. Pilla, 2019, "Lightweighting class A parts via supercritical fluid assisted (ScF) injection-molding of thermoplastic olefins (TPO)," Society of Plastics Engineers ACCE Conference, 4-6 September 2019, Novi, MI, USA (awarded second place in graduate student poster competition).

\section{References}

1. Kelly, J. C., J. L. Sullivan, A. Burnham, and A. Elgowainy, 2015, "Impacts of vehicle weight reduction via material substitution on life cycle greenhouse gas emissions," Environ. Sci. Technol., Vol. 49, No. 20, pp. 12535-12542.

2. Mi, H.-Y., X. Jing, J. Peng, L.-S. Turng, and X.-F. Peng, 2013, "Influence and prediction of processing parameters on the properties of microcellular injection-molded thermoplastic polyurethane based on an orthogonal array test," J. Cell. Plast., Vol. 49, No. 5, pp. 439-458.

3. Chang, S. H. and S. S. Cheon, 2006, "In-plane directional mechanical properties of carbon fabric skins in sandwich structures after thermoforming," Compos. Struct., Vol. 75, Nos. 1-4, pp. 577-581.

4. Yu, Y., J. Ye, Y. Wang, B. Zhang, and G. Qi, 2013, "A mesoscale ultrasonic attenuation FE model of composites with random-distributed voids," Compos. Sci. Technol., Vol. 89, pp. 44-51. 


\title{
II.2.5 Development and Integration of Predictive Models for Manufacturing and Structural Performance of CF Composites in Automotive Applications (General Motors)
}

\author{
Dr. Venkateshwar Aitharaju, Principal Investigator \\ Chemical Sciences and Material Systems Laboratory \\ General Motors R\&D Center \\ 30470 Harley Earl Blvd. \\ Warren, MI 48092-2031 \\ E-mail: venkat.aitharaju@gm.com
}

\section{Dr. H. Felix Wu, DOE Technology Manager}

U.S. Department of Energy

E-mail: felix.wu@ee.doe.gov

Start Date: May 1, 2015

Project Funding (FY19): \$2,270,476
End Date: April 30, 2019

DOE share: $\$ 1,589,333$
Non-DOE share: $\$ 681,143$

\section{Project Introduction}

GM was the first North American automotive OEM to develop and commercialize CF composites for Class A body panel (i.e., closure) applications. Going forward, our objective is to advance beyond closure components and implement $\mathrm{CF}$ composites in structural body panels that undergo the complex loading conditions represented in crash and durability. To enable these broader automotive-structural applications of CF composites, three key issues must be addressed. First, significant variability in material properties incurred during the manufacturing process need to be understood and predicted. Second, prediction of the structural performance of composites undergoing complex loading (i.e., crash) under varied environmental conditions is required. Third, the effect of manufacturing on the performance of the final components needs to be understood and predicted. Without a good understanding of these issues, automotive designs can be overly conservative, thereby increasing cost significantly and limiting the weight reduction potential of CF composites. The present project addresses all of these needs by developing a suite of ICME tools to predict manufacturing performance and structural performance of CF composites, including stochastic effects.

In FY 2018, major efforts were spent in designing a composite CF automotive assembly for a high-volume, medium-duty automobile currently made of steel using the integrated manufacturing and structural performance tools developed in this project. Various CF materials friendly to high-volume automotive manufacturing (e.g., non-crimp, woven, chopped systems) and manufacturing processes (e.g., such as RTM and compression molding) were used. Final designs of the assembly components were provided to the CSP for building the tools. The tools are expected to be delivered by the end of 2018 .

In FY 2019, the optimized CF automotive assembly will be certified, and the developed numerical models will be validated by comparing the numerical and experimental results for the performance of the assembly. Also, the optimized CF assembly design, weight, cost, and performance will be compared with the existing design. The availability of ICME tools to predict stochastic manufacturing and structural performance of structural composites to an OEM, like GM, can potentially eliminate significant bottlenecks that currently exist with large-scale implementation of CF composites in an automobile. 


\section{Objectives}

The objective of this project is to develop an integrated suite of state-of-the-art computational modeling tools that are critically needed to enable structural $\mathrm{CF}$ applications in automobiles. These tools help to predict the manufacturing and structural performance of $\mathrm{CF}$ composites, including stochastic effects. The project team is comprised of researchers from GM, Engineered Solutions, Inc. (ESI), Group (i.e., software company, owner of composite manufacturing tool PAM-COMPOSITES for composites, which include PAM-RTM for resin transfer molding and PAM-FORM for forming of composites), Altair (i.e., software company, owner of the multiscale structural performance predictive tool for composites), Continental Structural Plastics (CSP) (i.e., molder/material supplier for composite materials and GM-Corvette composite parts supplier), and the University of Southern California (i.e., leader in uncertainty quantification of DOE's Scientific Discovery through Advanced Computing Institute for Uncertainty Quantification). During the first phase of the project, both the manufacturing and structural performance tools, including a stochastic driver, were calibrated and validated against coupon and component level tests. The difference between predictions and experimental results was limited to less than $15 \%$. During the second-phase of the project, the manufacturing and performance tools will be integrated by mapping the manufacturing outcome (e.g., fiber angles, residual stresses, degree of cure, and defects [voids, dry patches, and wrinkles]) into structural models.

\section{Approach}

Four key components of probabilistic ICME will be at the core of our approach, namely model integration, optimization, validation, and design certification. The computational engine will be constructed by the integration of state-of-the-art tools and capabilities from specific domains, namely, composites manufacturing (from GM and CSP), process simulation software for composites (from ESI), integrated multiscale/multiphysics structural software (using the multiscale design system of Altair), probabilistic methods and probabilistic software (from the University of Southern California), and, finally, the largest U.S. automaker with considerable expertise and a record of accomplishment in the design and manufacturing of composite cars (e.g., the Chevrolet Corvette from GM) leads the project and integrates these modules. By model validation, we refer to making a prediction of the variables of interest and comparing the specific variables under the same conditions. By certification of a component, we mean the assurance that once designed, manufactured, and loaded (i.e., crash-tested), these components will perform satisfactorily up to preset specifications. Enabling model validation and component certification requires retooling of the deterministic scientific process, specifically by requiring evidential attributes to be attached to model-based predictions. During this project, we will identify the limitations of existing software components comprising the computational engine and offer alternative technologies and solutions that facilitate integration for design optimization. Once the computational engine has been developed and validated, the chosen CF assembly will be designed, optimized, tested, and certified, and the resulting composite component will be compared to current steel design.

The present CF ICME project is organized into ten tasks executed over a 48-month-period. An outline of the tasks carried out during FY 2019 is as follows:

- $\quad$ Task 1.0: Project planning, coordination, and reporting - ongoing.

- $\quad$ Task 2.0: Select the automotive assembly preliminary design - completed.

- $\quad$ Task 3.0: Identify preliminary CF material systems and manufacturing processes - completed.

- Task 4.0: Develop and validate the manufacturing and crash simulation engines - completed.

- $\quad$ Task 5.0: Integrate the manufacturing and structural models - completed.

- Task 6.0: Design and optimize automotive assembly for manufacturing and performance - completed.

- $\quad$ Task 7.0: Design and fabricate assembly and the test fixture - completed.

- $\quad$ Task 8.0: Manufacture the automotive assembly - started and close to completion. 
- $\quad$ Task 9.0: Certify the automotive assembly - started and close to completion.

- Task 10.0: Assessment of the assembly: Correlation of predictions with experimental results - started.

\section{Results}

In FY 2019, most of the work focused on the manufacturing of the CF composites panels for the rocker floor assembly, which was chosen to demonstrate the ICME technology developed in this project. Figure II.2.5.1 shows the design of the CF rocker floor assembly. Only half of the assembly will be built for the project as it is sufficient to evaluate the crashworthiness of the assembly under the critical load case of the side pole impact. The designed $\mathrm{CF}$ assembly is $30 \%$ lighter compared to the current steel design. The components of the assembly are manufactured using high-pressure resin transfer molding (HP-RTM), a state-of-the-art highvolume manufacturing process that is being studied with greater interest in the automotive industry. Three large tool sets were designed using ICME technology and process conditions were engineered using the fastcuring resin formulations to maximize the throughput. Further, the developed ICME math models will be validated by comparing the experimental results and predictions for both the manufacturing of individual components and crash-testing of the assembly. This report presents the results obtained in FY 2019 during the manufacturing of reinforcement and floor components of the CF underbody assembly.

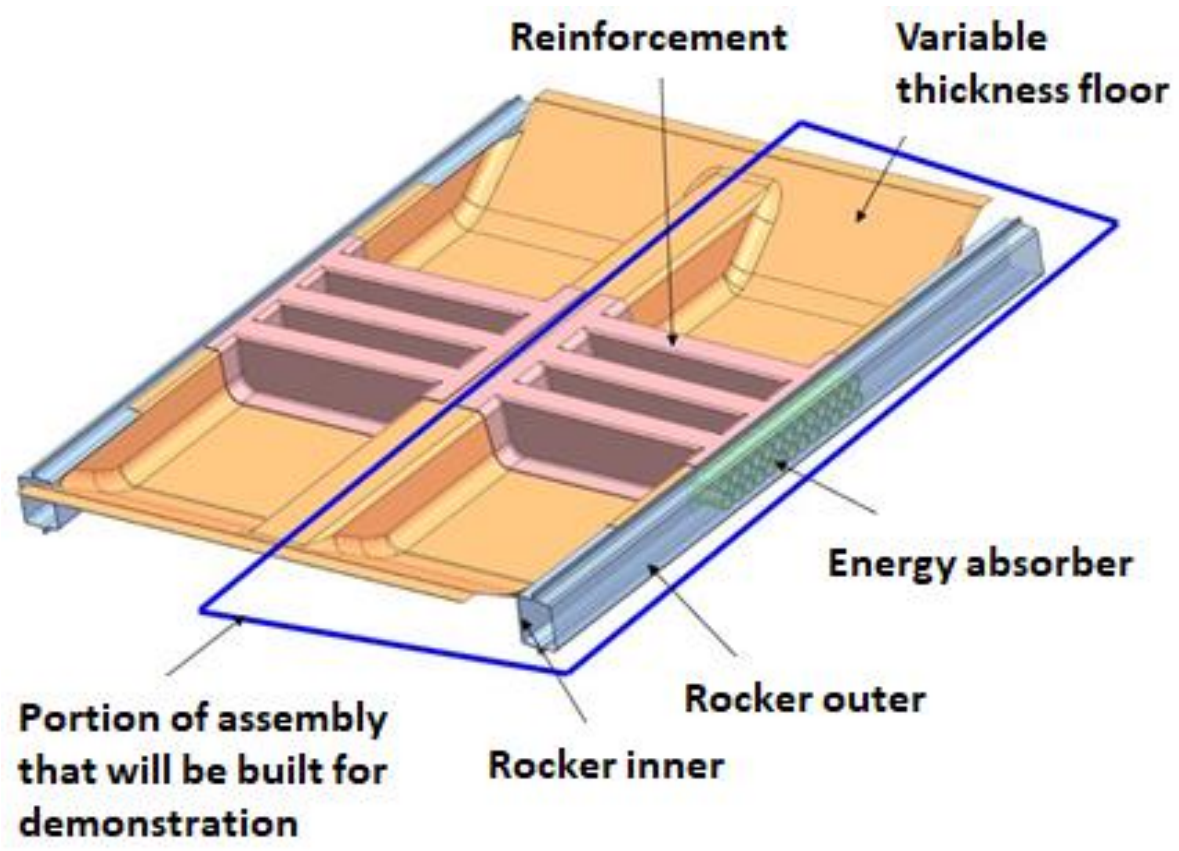

Figure II.2.5.1. CF composite rocker floor assembly built for the demonstration of ICME tools. Source: GM.

\section{Prototype Testing of Rocker Floor Assembly}

Following a successful first round of prototype testing in the previous year validating the crashworthiness design, a second round of testing was undertaken to explore potential design alternatives to improve the further structural performance, and thus mass-saving opportunities. A total of five additional assemblies were produced and tested. Among them, four of the assemblies were utilized to evaluate the design of a new honeycomb energy absorber as compared to the original cylinder design. Excellent results were obtained validating the honeycomb energy absorber design to proceed in implementing in the final assembly. The experiments on the prototype gave several ideas to improve the structural performance of the critical reinforcement component by strategically locating the slits needed for manufacturing. 


\section{CF Reinforcement Component}

Figure II.2.5.2 shows the molding tool for the reinforcement component that is engineered virtually and built for the project. In the figure, the location of the injection port, the vacuum ports, the pressure sensors, and the dielectric sensors on the tool are shown (i.e., $\mathrm{P \#}$ is the location of the pressure sensor and $\mathrm{C \#}$ is the location of dielectric curing sensor). A total of six sensors are designed and embedded in the reinforcement mold at strategic locations to capture valuable information regarding the state-of-the-resin, such as pressure, temperature, and curing degree. The experimental results obtained from the tool testing will help us to compare with numerical predictions in validating the computer models developed in the project. The injection port is designed to adapt both the CSP mixing head and the GM mixing head. The top and bottom molds of the reinforcement tool are made with $\mathrm{Al}$ material. The injection port is located at the center of the top half of the tool. A resin runner is engineered from the center injection port to the reinforcement flanges to strategically flow the resin to yield low-cycle time and high part quality without any dry areas. We have designed double seals for our HP-RTM tools. The fabric clamping seal allows holding the fabric at the perimeter to control the fabric deformation during the high-pressure injection. The resin seal helps to prevent low-viscosity resin from squirting out of the tool cavity. A channel outboard of the resin seal is designed to capture the extra resin. Simultaneously, a comprehensive die manufacturing standard for HP-RTM were developed and utilized in making these tools in the project. It should be noted that these standards are not yet developed in the United States as this technology is slowly evolving in this country as compared to Europe. Kistler pressure sensor type 6161 AA capable of measuring the cavity pressure up to 200 bar were also used. Finally, dielectric sensors from Lambient, Inc., were used to monitor the resin degree of cure, as well as in measuring the temperature of the resin during the molding process.

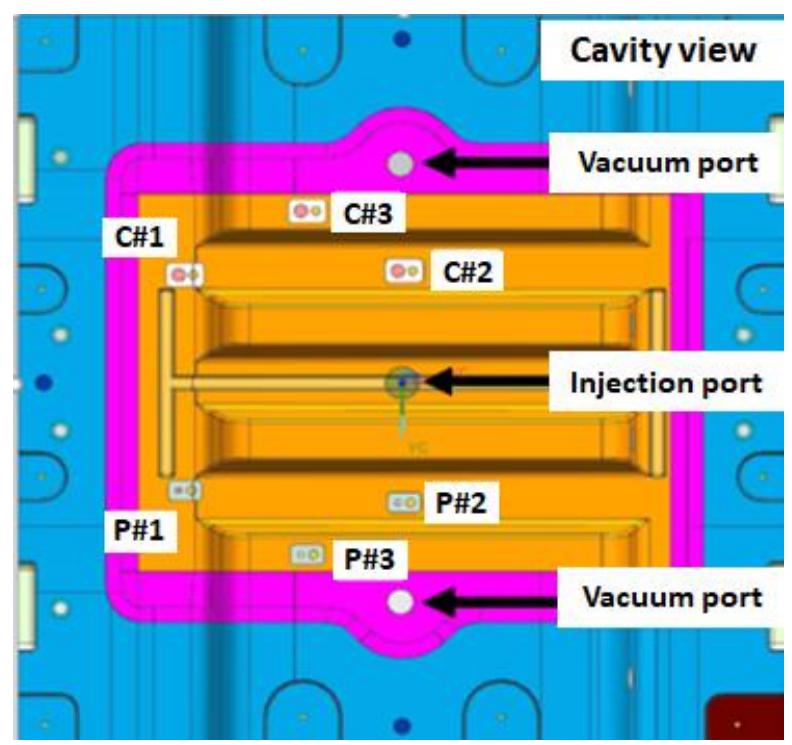

Figure II.2.5.2. Tool design of the reinforcement (P denotes pressure sensor location; $\mathrm{C}$ denotes dielectric sensors). Source: GM.

Draping and injection phases are very crucial steps in manufacturing this reinforcement component. During the draping, the 2D CF preform deforms to the 3D shape of the reinforcement, modifying the orientations of the CF preform. Prediction of these fiber direction changes are crucial in evaluating the structural performance, which is one of the primary challenges in the industry hindering the implementation of CF composites in the automotive industry. This challenge was addressed in the current project and this exercise of draping and injection experiments validates the numerical development. 


\section{Manufacturing of the Reinforcement}

The following sections provide the manufacturing details of the CF-reinforced part. The reinforcement component has complex 3D geometry and is one of the best examples for demonstrating the feasibility of a high-volume manufacturing aspect of CF composites. Draping, injection, and curing are the steps in manufacturing the component using HP-RTM, and they are discussed in the sections that follow.

\section{Draping Stage - 2D Blank Determination}

Figure II.2.5.3 shows the simulation workflow process that was developed to arrive at an accurate blank pattern design. The starting point is a rough blank pattern coming from kinematic draping simulation represented by the bottom image. From this initial pattern, a framework was developed to yield an accurate preform shape that can be used for manufacturing the components of the assembly.

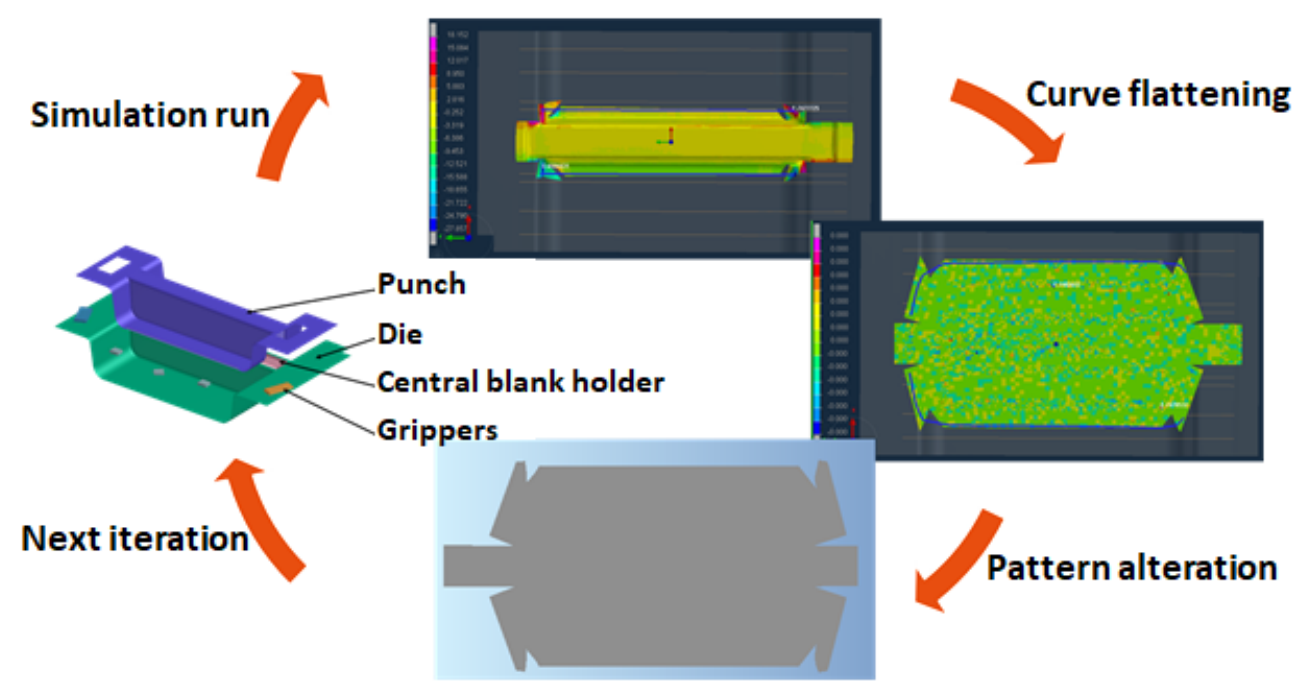

Figure II.2.5.3. Determining the 2D-flat blank for the reinforcement component. Source: GM.

\section{Blank Pattern Development}

In order to minimize material costs and layering/preforming time, the team made a strategic decision to use heavier fabrics (i.e., $600 \mathrm{gsm}$ vs. $240 \mathrm{gsm}$ ). This has helped to significantly reduce the number of plies in the reinforcement preform from 20 to 8 , and significantly reducing the manual effort and material costs.

A total of six blank patterns needed to be optimized for molding the reinforcement component. For each of the front, middle, and rear hat sections, there are two patterns that have different slit patterns to offset any gaps in the preforms. Each set of Patterns 1 and 2 are meant to be stacked on top of one another. The front and rear patterns are extended on one side to allow the preforms to extend out to the technical area and the vacuum ports. Rough representations of these six blank patterns are shown in Figure II.2.5.4. The six 2D final patterns that were developed are shown in Figure II.2.5.5.
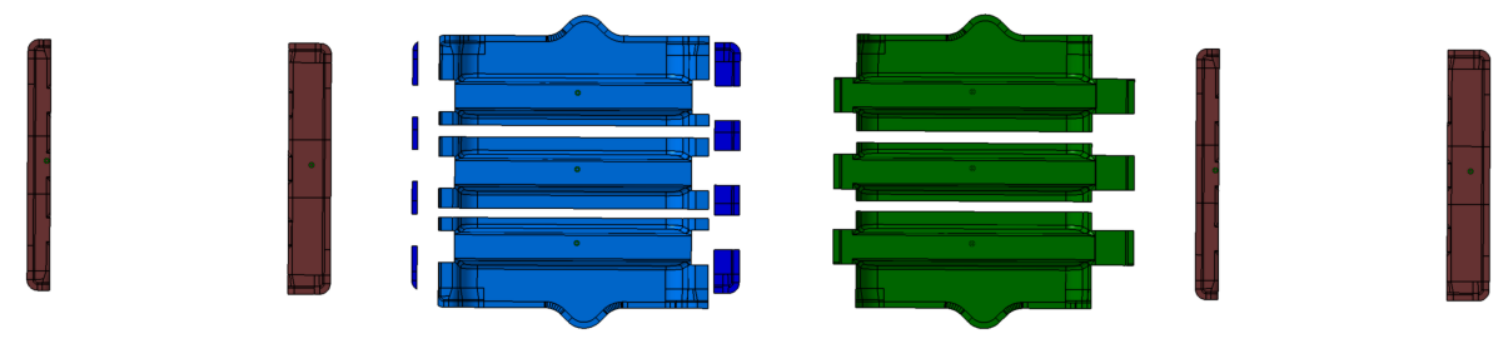

Figure II.2.5.4. Reinforcement preforms (Pattern 1 in blue, Pattern 2 in green, and the flange patches in brown and dark blue). Source: GM. 


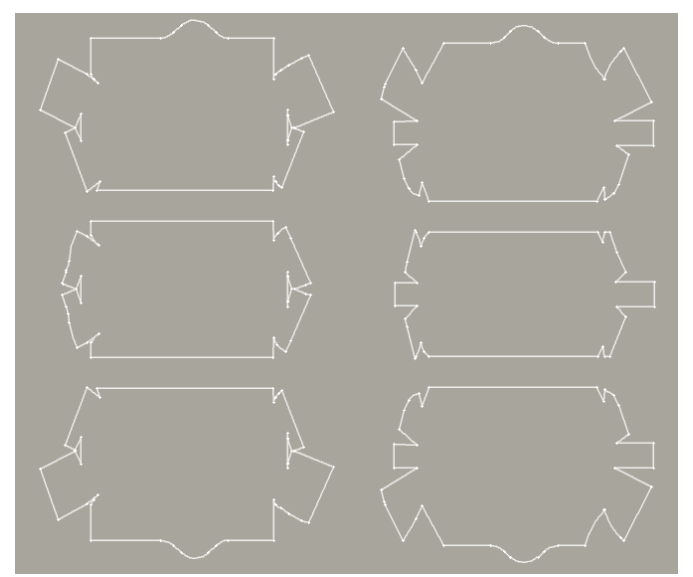

Figure II.2.5.5. The six preform 2D blank patterns used to produce the reinforcement. Source: GM.

An adjustable frame with clamps was designed and manufactured to handle the draping process of the reinforcement, as shown in Figure II.2.5.6. The frame can be adjusted to accommodate all of these six blank patterns that were developed. While the preform was being draped into the reinforcement geometry, the draw-in amounts were recorded and were compared with predictions to validate the draping models previously developed in this project.

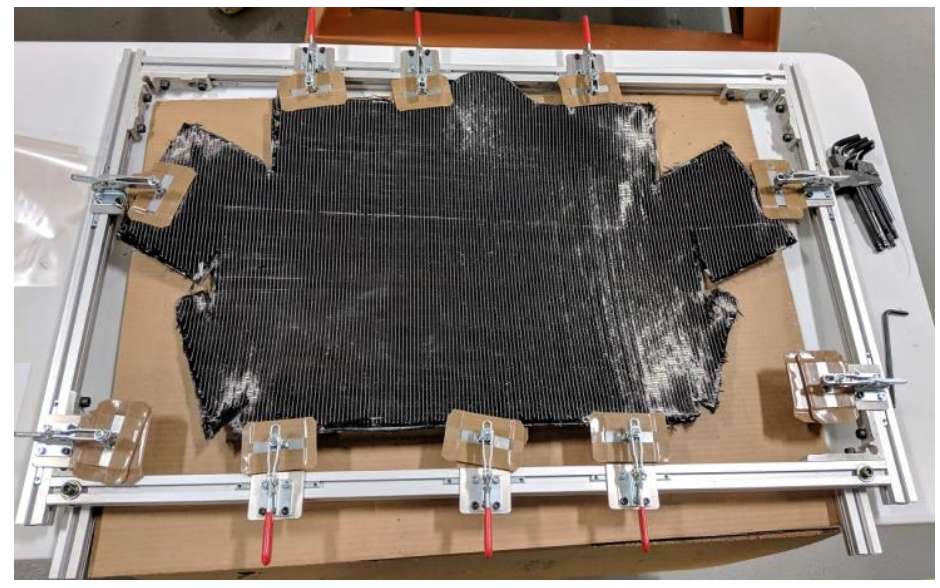

Figure II.2.5.6. Adjustable preforming frame with clamps. Source: GM.

Fiber Draw-In and Angle Changes in Molding of Reinforcement Component

The NCF used for these evaluations was obtained from Chomarat North America (Anderson, SC, USA). The designation for the fabric was C-Ply ${ }^{\mathrm{TM}}$ SP BT600 T3.3 50K HS, which has a fiber architecture of $0^{\circ} / 90^{\circ}$ referenced to the roll direction, roughly a $50 \%$ fiber volume fraction. The stitch gauge (spacing between lines of stitching) for this fabric was $5 \mathrm{~mm}$. The areal mass of the fabric was $600 \mathrm{~g} / \mathrm{m}^{2}$ and the thickness varied between $0.61 \mathrm{~mm}$ and $0.63 \mathrm{~mm}$.

Due to the complexity of the reinforcement geometry for draping, several slits were designed at strategic locations to allow the fabric to take the 3D shape without wrinkling. For the molding, two patterns were overlaid so the slit locations after molding were staggered through the thickness to reduce their effect on the structural performance. Detailed draping analyses were performed considering the process steps, and the fiber angle changes were calculated using the numerical models developed previously. Further, the fiber angles following draping were mapped onto the structural performance models to predict the structural performance of the reinforcement. 
The shear stiffness for the fabric was determined from bias-extension experiments following a previously established procedure. Additionally, the bending stiffness for this fabric was measured according to ASTM D1388. These parameters-shear stiffness and bending stiffness-were used to calibrate the draping models employed to model the change in fiber directions. The first parameter used to compare the simulations to the experimental results was the draw-in of the 2D pattern as it was draped into the 3D shape of the part. Several reference lines were added to the draping tool and in the simulation framework to provide consistent measuring points between the two. Three of the draping patterns were measured, as shown in Figure II.2.5.7. Up to 19 measurements were compared for each preform. Averages of the draw-in for the three separate preforms were used to provide the experimental data.

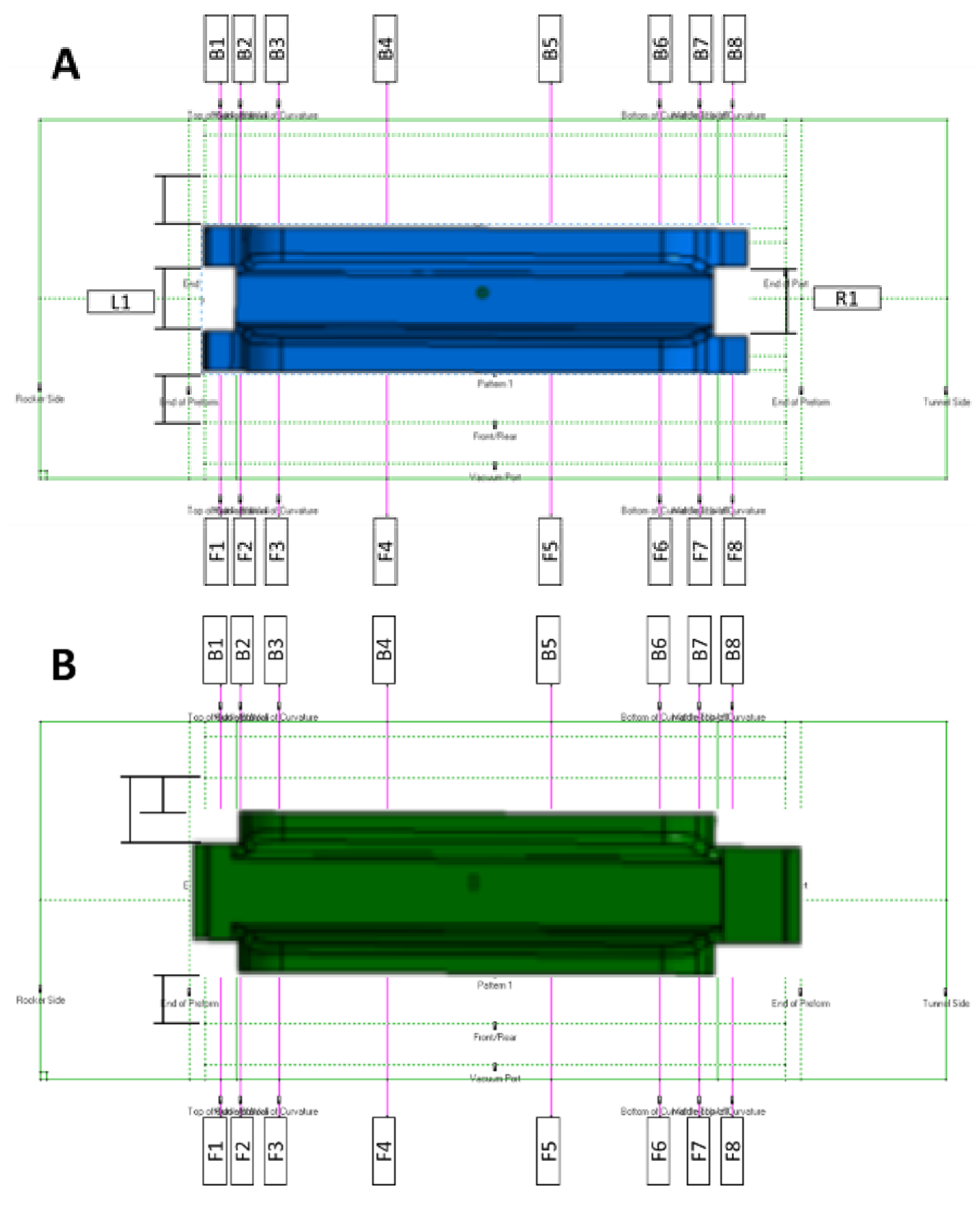



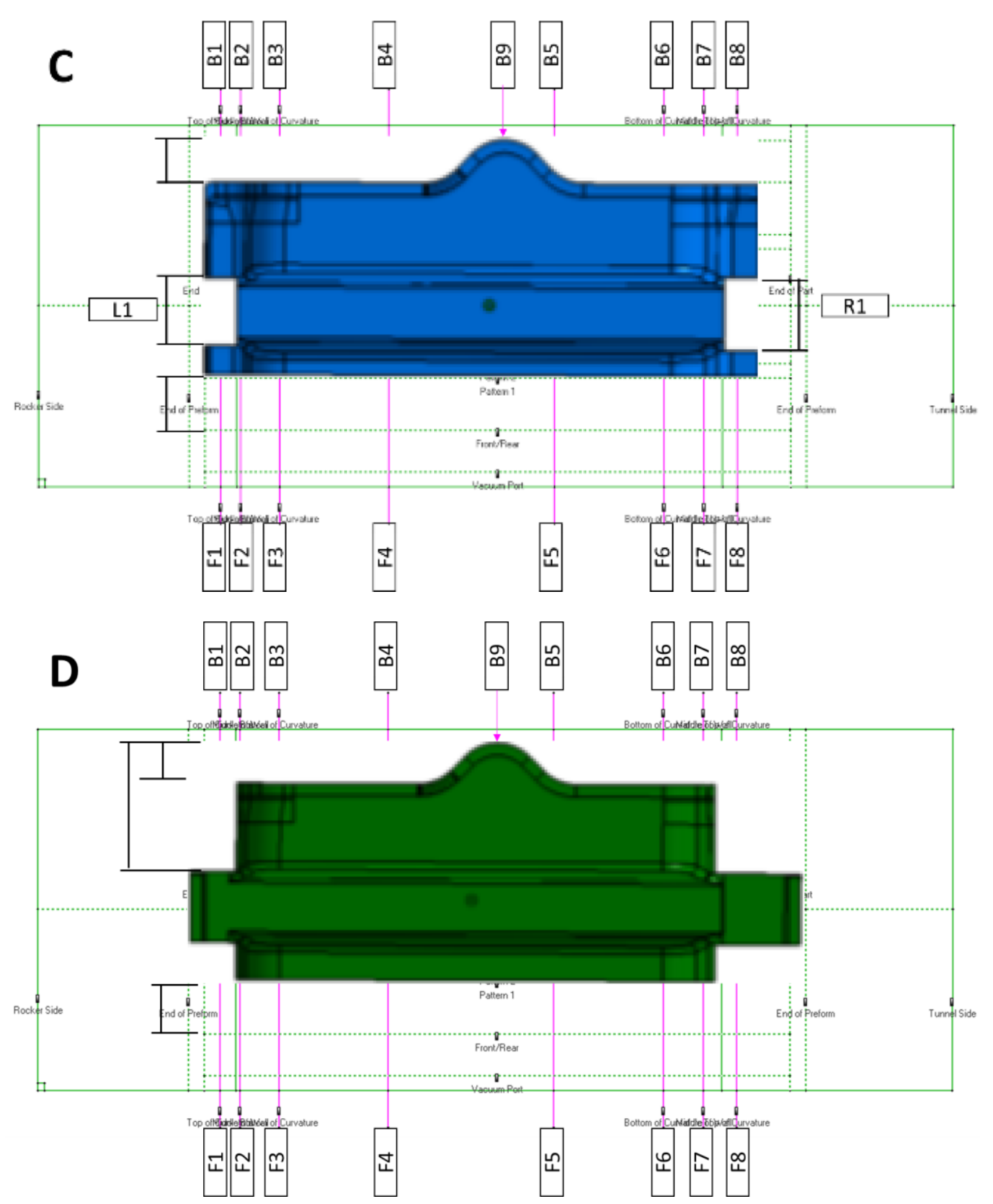

Figure II.2.5.7. Preform patterns after forming with measurement lines: (a) middle pattern 1; (b) middle pattern 2; (c) side pattern 1; and (d) side pattern 2. Source: GM.

Figure II.2.5.8 shows the results for the two patterns used for the middle hat section of the reinforcement. In both cases, this pattern is symmetrical around a horizontal centerline. Error bars represent $\pm 15 \%$ around the value, while the red symbols represent points where the simulation results are more than $20 \%$ away from the experimental measurements. It can be seen from Figure II.2.5.8 that only two points from the simulation are outside the desired error limits. Upon examining these points of discrepancy, they are found to be located between the "legs" of the pattern on either side. These "legs" are relatively thin, which tends to make them move somewhat uncontrollably. These points can be waived because of this. Overall, the correlation between the simulation and experiments for the draping stage is quite good. Similarly, for other patterns, a comparison of predicted and experimental draw-in are compared and an excellent correlation was observed in validating the draping models developed in this project. 

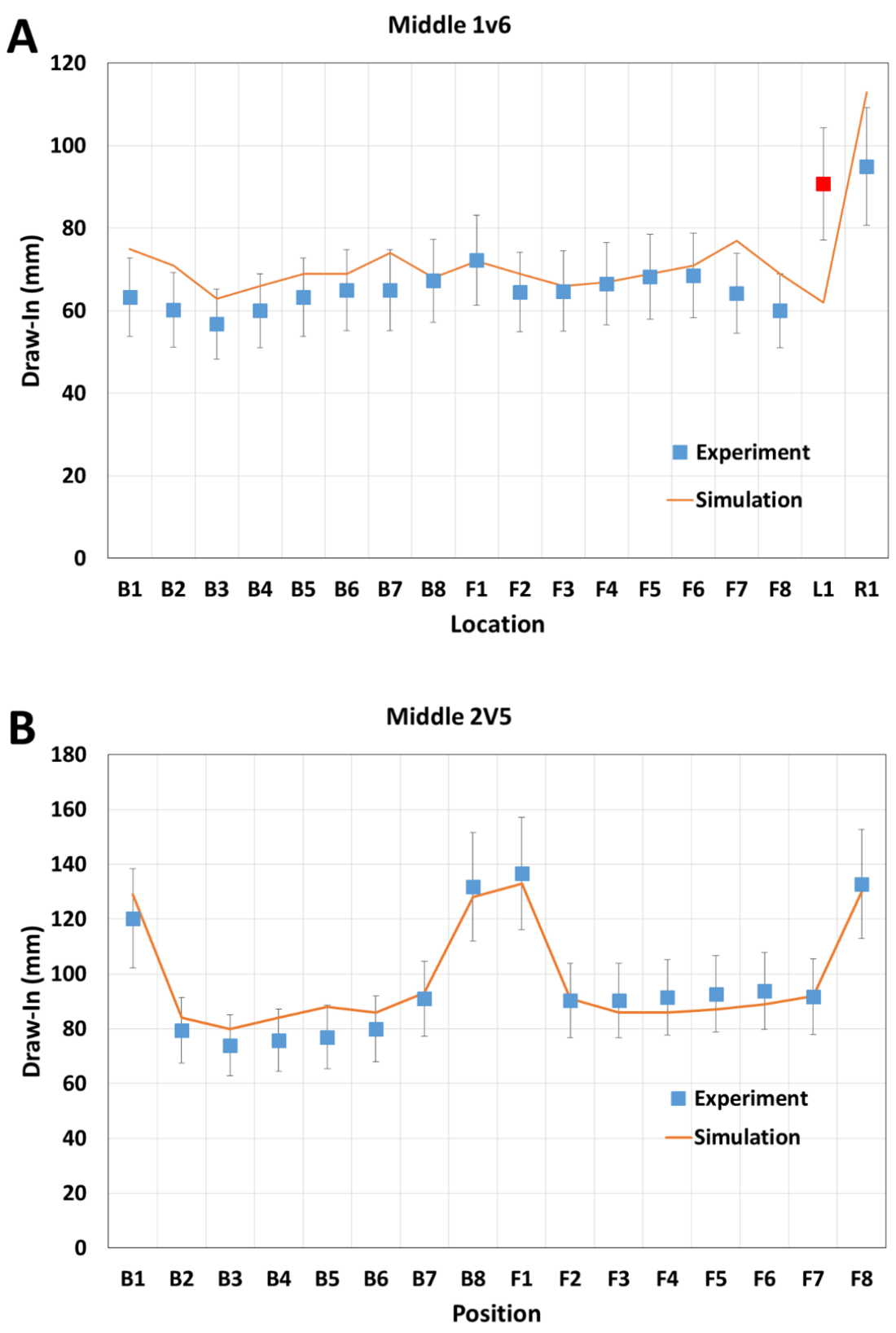

Figure II.2.5.8. Draw-in results for the middle patterns: (a) middle pattern 1; and (b) middle pattern 2. Source: GM.

\section{Reinforcement Component Molding}

Using the ICME models developed in the project previously, the process conditions such as the injection window and injection pressure can be programmed and controlled using the automation available in the GM R\&D HP-RTM press. The injection process is shown in Figure II.2.5.9. Once the press closes to the position that is 0.5 in. away from the final closing position, two vacuum ports were opened, and the vacuum was pulled from the entire mold cavity for 30 seconds. Meanwhile, the press top platen continues moving down to the final part thickness. Once the press closes to the final part thickness, it sends a handshake signal to the HP-RTM metering system in preparation for the resin injection. The injection duration is determined by the selected flowrate and amount of resin needed to completely fill the part via a tonnage of 600 tons equivalent to 1100 psi by using the projected area of the reinforcement part during the entire injection phase and resin cure phase. 


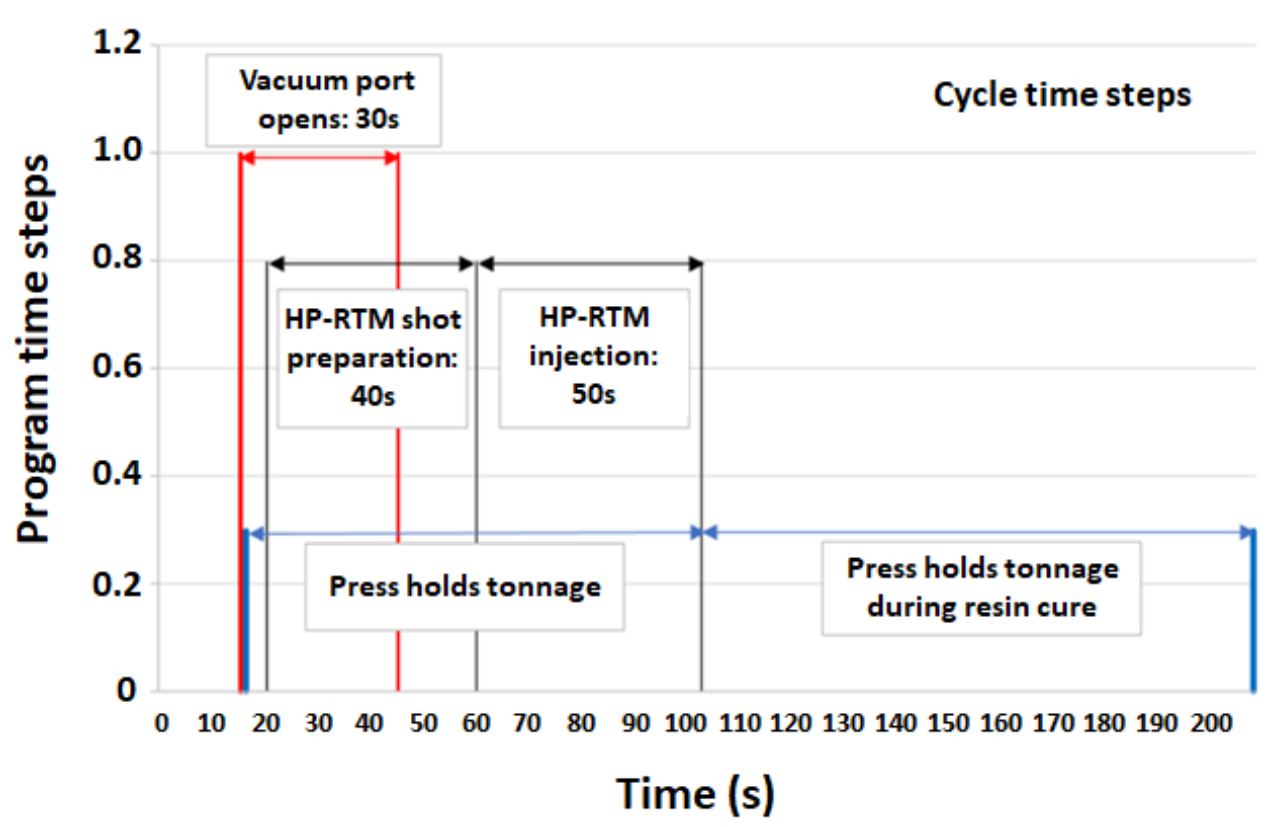

Figure II.2.5.9. Press and metering cycle timesteps control. Source: GM.

The metering system for the GM R\&D HP-RTM system was designed and manufactured by Krauss-Maffei, Inc., which is shown in Figure II.2.5.10. It is a 3-stream system capable of delivering three independent chemicals including resin, hardener, and in-mold release agent to the tool. Based on the system specifications, the flow rate range of these three streams are 30-160 g/s for the resin stream, $10-80 \mathrm{~g} / \mathrm{s}$ for the hardener stream, and $0.2-1.1 \mathrm{~g} / \mathrm{s}$ for the in-mold release stream. This system is capable of injection up to $6 \mathrm{~kg}$ of resin in 25 seconds. The injection pressure is adjustable in the range from 1500 psi to 3200 psi. The system constantly monitors real-time flow control parameters, such as flow rate, injection pressure, temperature, and status of the mix head for all three-material streams. It also integrates an enhanced data acquisition module to capture real-time process data from the in-mold pressure transducers and thermocouples.

To achieve a $50 \%$ of fiber volume fraction, the required amount of resin to inject into the reinforcement panel was determined to be $3500 \mathrm{~g}$ with some packing volume. For the phase-1 trials, we used Hexion EPIKOTE TRAC 06150 epoxy resin and EPIKURE TRAC 06150 cure agent, which has a mix ratio of 100:24. The resin stream was preheated to $60^{\circ} \mathrm{C}$ to lower the viscosity to around $40 \mathrm{mPa}-\mathrm{sec}$. The impingement mixing pressure for both streams were calibrated to 1800 psi to ensure a thorough mixing. 


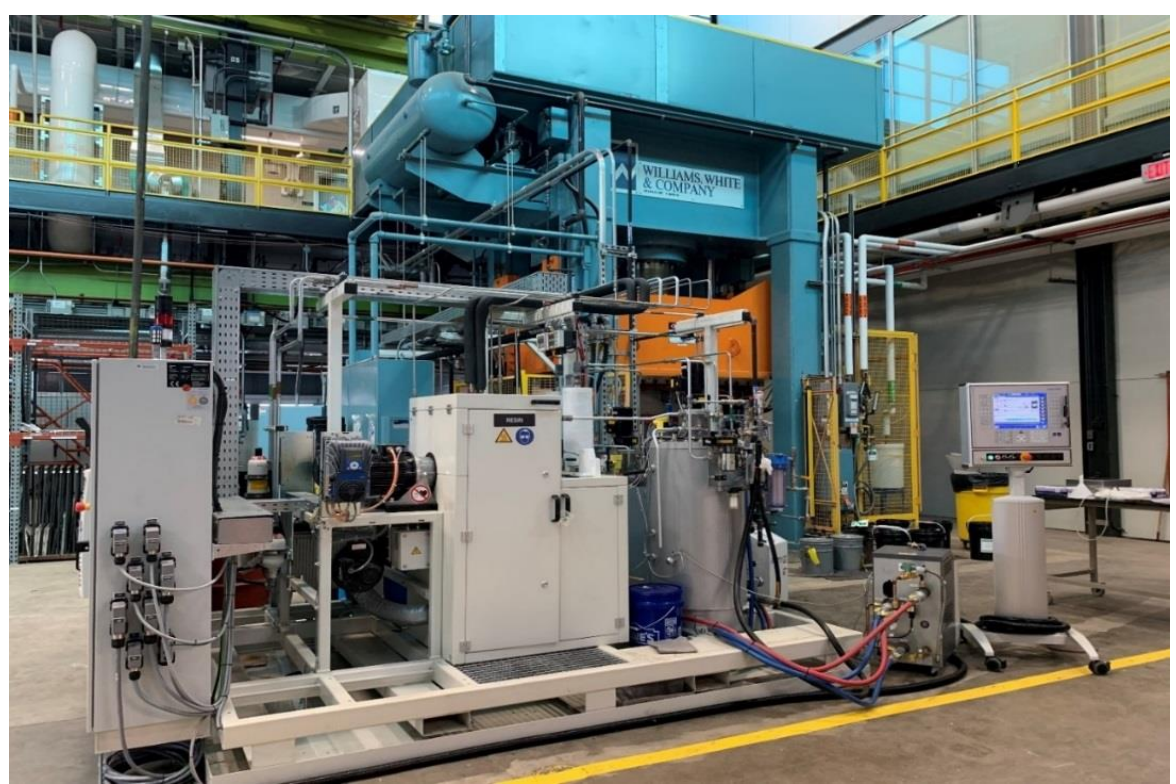

Figure II.2.5.10. HP-RTM metering system installed at GM R\&D for the project. Source: GM.

Using $70 \mathrm{~g} / \mathrm{s}$ flow rate, the total amount of $3500 \mathrm{~g}$ resin was injected into the closed mold cavity in 50 seconds. The reinforcement panel have been successively molded with minimum fiber wash and wrinkles. The final molded part with the part boundary marked in red is shown in Figure II.2.5.11 (a). As predicted in the simulation, two dry spots were observed that were located near the vacuum ports outside the part, showing a well-controlled molding process from both the press and metering system programing. All of the six in-mold sensors were accurately programmed and synchronized in the timesteps of each molding cycle using a GM R\&D developed data acquisition system.

The injection flow rate and pressure from both the resin and hardener streams plotted in Figure II.2.5.11 (b) and two vertical black dash lines represent the beginning and end of the injection. It reveals the metering system maintaining a steady-state constant flowrate in the entire injection phase on both streams. The injection pressure of the resin stream started from around 1900 psi and increased to approximately $2100 \mathrm{psi}$ at the end of the injection process due to the resin compaction near the vents. Meanwhile, the injection pressure of the hardener stream started from $1830 \mathrm{psi}$ and increased to $1954 \mathrm{psi}$ at the end of the injection process. Due to the lower viscosity, the hardener stream requires less injection pressure to deliver a relatively slower flowrate as compared to the resin stream. In addition to the injection pressure, three in-mold pressure sensor curves have been plotted out in Figure II.2.5.11 (b). The pressure sensor P1 is located on the flange of the reinforcement panel close to the end of the resin runner. The plot shows that the resin flow front arrived at the first pressure sensor around 17 seconds after the injection. It took 31.5 seconds for the resin stream to reach the second pressure sensor P2, and 38 seconds to reach the third pressure sensor P3. At the end of the injection phase, the in-mold pressure at these three locations all reached equilibrium with the injection pressure. Once the injection port was closed, the in-mold pressure remained at constant value before the resin chemical shrinkage took place due to the reaction. After the injection phase, the metering system turned back to the reduced circuit mode, the flowrate and mix head pressure dropped back to their original settings, and the system prepared itself for the next set of molding. 


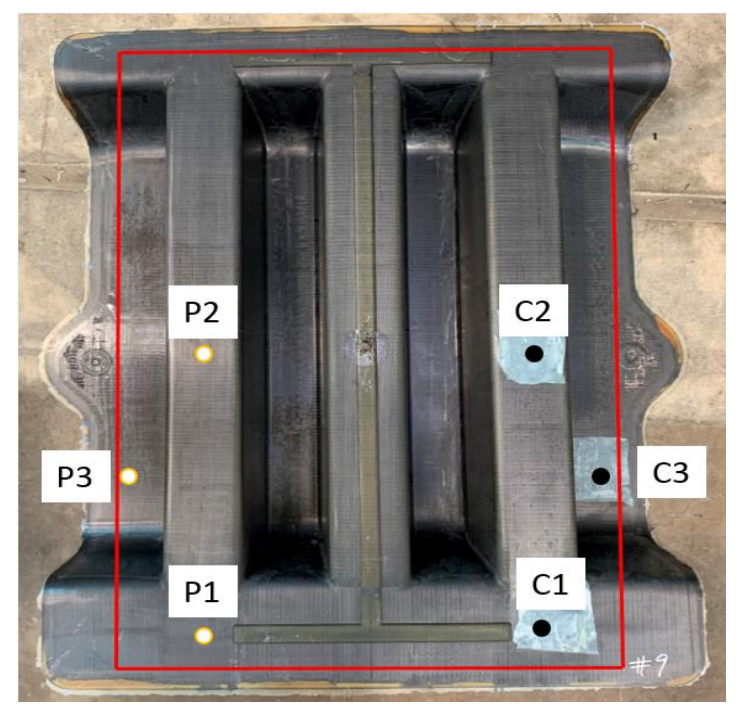

(a)

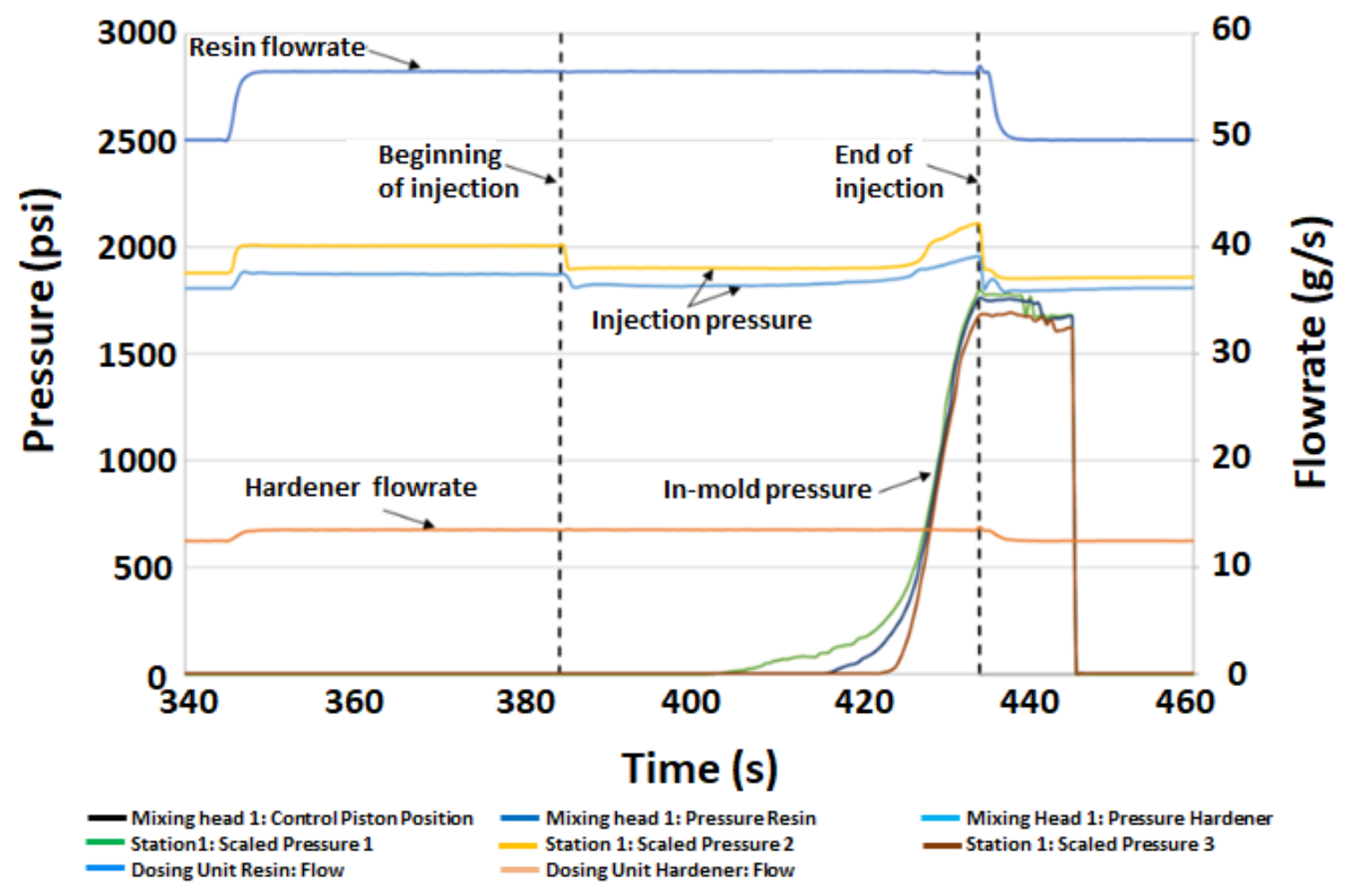

(b)

Figure II.2.5.11. (a) Molded reinforcement panel. (b) Process parameters including injection pressures and flow rates where in-mold pressures were measured from the pressure sensors. Source: GM.

During the resin curing phase, three in-mold dielectric cure sensors - $\mathrm{C} 1, \mathrm{C} 2$, and $\mathrm{C} 3$ - flush mounted to the tool cavity were used to measure both resin ionic viscosity and resin temperature. The evolution of temperature and ionic viscosity is shown in Figure II.2.5.12. Since the tool temperature in this case was at $125^{\circ} \mathrm{C}$, the epoxy was preheated to $60^{\circ} \mathrm{C}$, while the amine hardener was at $\mathrm{RT}$ of $25^{\circ} \mathrm{C}$. The first dielectric sensor, $\mathrm{C} 1$, revealed the temperature reduction once the resin arrived. Arrival time was around 10 seconds after the injection took 
place. The time delay for the resin to flow to the second dielectric cure sensor, $\mathrm{C} 2$, and the third dielectric cure sensor, C3, was 20 seconds and 32 seconds, respectively. Meanwhile, since the resin is electrically conductive, the measured resistivity or the ionic viscosity was reduced due to the liquid status of the resin during the injection phase. The ionic viscosity often differs to physical viscosity by a scaling factor, since the resistivity decreases as mobile ions experience less resistance to flow. Both viscosity and ionic viscosity increase as the material becomes more rigid and hardens. Resistivity will then go to a minimum once the resin arrives, and then increases due to a greater impediment to the flow of ions because of the polymerization and consolidation at elevated temperature. The Hexion 6150 indicated $90 \%$ degree of cure after 4 minutes at $125^{\circ} \mathrm{C}$ tool temperature.

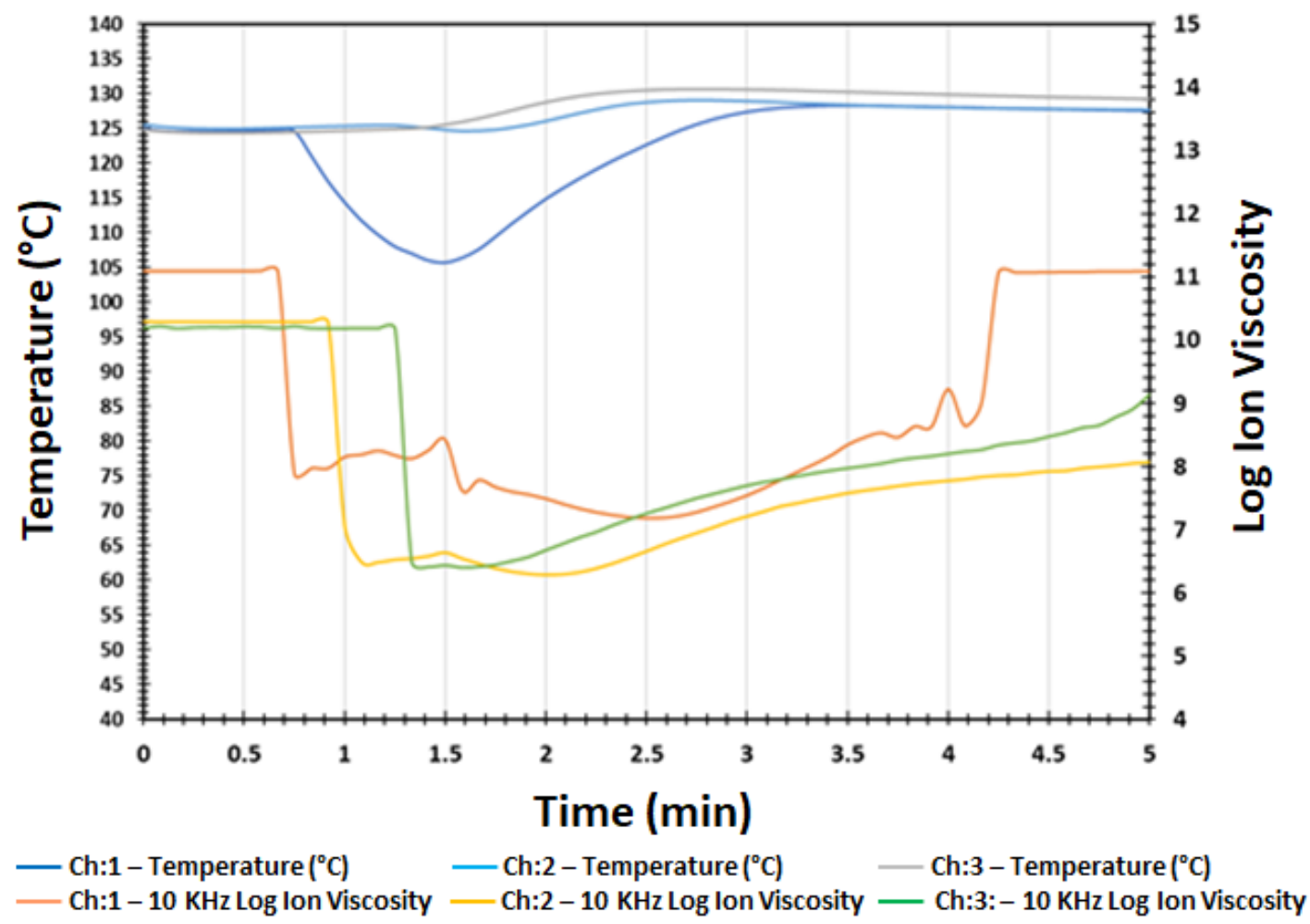

Figure II.2.5.12. In-mold temperature and log ion viscosity at C1, C2, and C3. Source: GM.

\section{Reinforcement HP-RTM Correlation}

Figure II.2.5.13 shows a comparison of pressure evolution curves from the simulation versus the experiment at the three pressure sensors in the tool. Very good agreement for the first 40 seconds can be observed. In the final stage of the injection, however, some discrepancies are observed. At this time, this was attributed to steep pressure rise during the final packing stage; therefore, it is believed that the simulation cannot accurately model this phenomenon. This explanation is yet to be confirmed through a deeper analysis.

Overall, good correlation between the two can be seen, given the complexity of modeling the flow field in the heterogeneous medium and via some uncertainty in the material parameters (e.g., permeabilities, thermal properties for resin). The process was initially designed virtually with strong support for simulation (e.g., injection philosophy, size of channel, location of vents, etc.) demonstrating here the capability of ICME models to accurately simulate the HP-RTM process. This capability allows us to drastically reduce the number of physical tests, time, and cost to production of CF composites. 


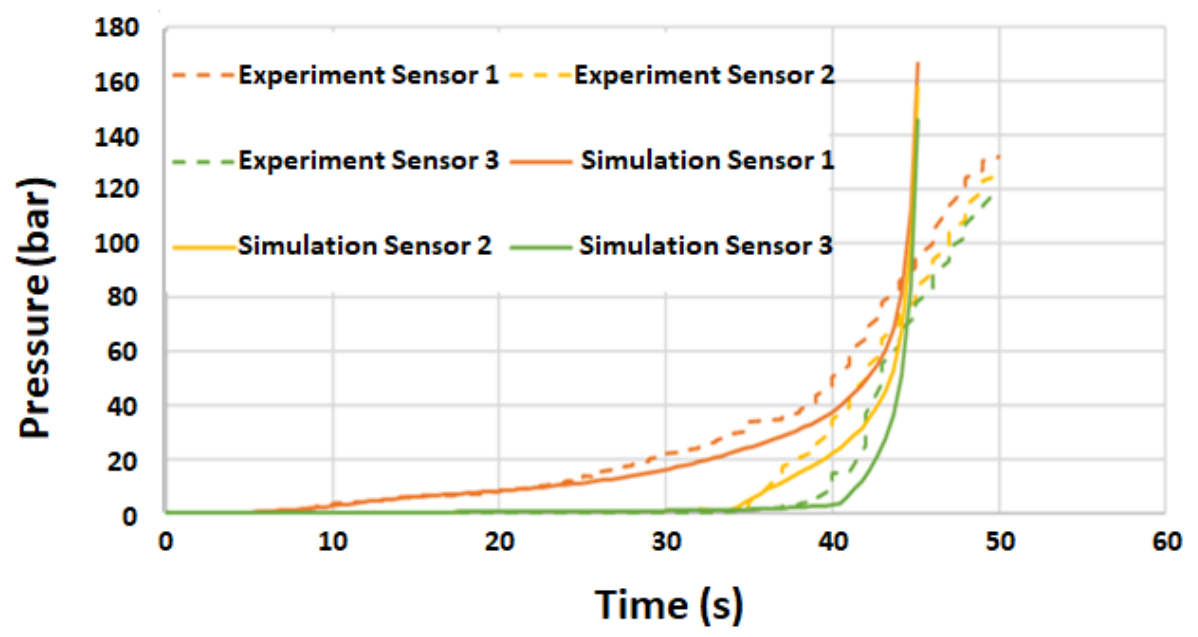

Figure II.2.5.13. Comparison of pressure profile between the experiment and the numerical prediction. Source: GM.

\section{Coats Preforming Experiments}

CSP has been working with a company called Coats on innovative solutions to drape complex 3D shapes, such as the reinforcement component in this rocker floor assembly. The Coats technology utilizes multidirectional fiber placement to maximize the structural performance and eliminate waste. This technology has been used to make new preforms for the reinforcement and will be studied side-by-side with the traditional NCF preforms.

Detailed side pole impact simulations of the reinforcement in the CF underbody assembly showed that the high stress areas are located on the flange radii at the rocker and tunnel side, as marked in red in Figure II.2.5.14 (a). Knowing the locations of these critical areas and load conditions, the preform slits needed for manufacturing were strategically arranged to avoid crack propagation in these areas and were staggered through the thickness for improved performance. The edges of the offset slits are shown in green and blue in Figure II.2.5.14 (b).
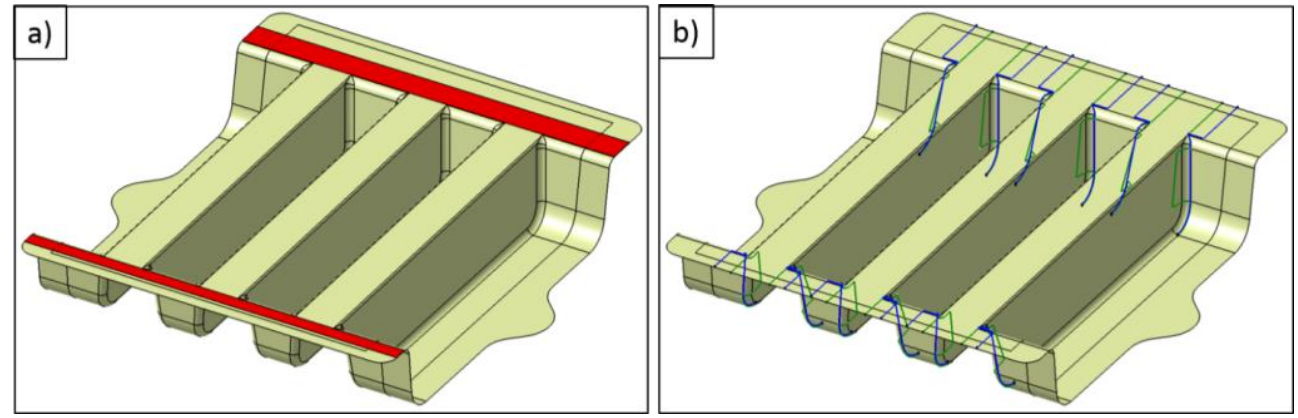

Figure II.2.5.14. (a) Critical areas during single pole-side impact crash (red). (b) Locations of slits (green and blue) in non-critical areas. Source: GM.

The current design features two patterns with offset slits, as shown in Figure II.2.5.15. After draping and consolidation, the resulting 3D preform is shown in Figure II.2.5.16. Several of these preforms were produced and used to mold parts at GM. 

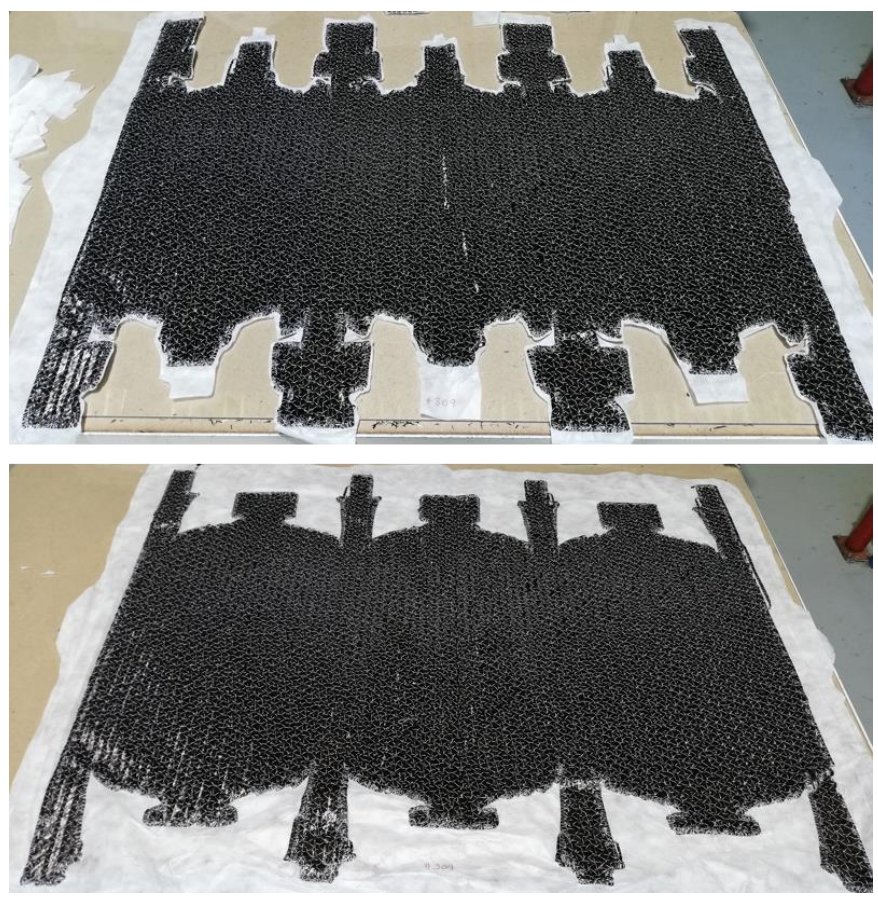

Figure II.2.5.15. Coats preforming patterns. Source: GM.

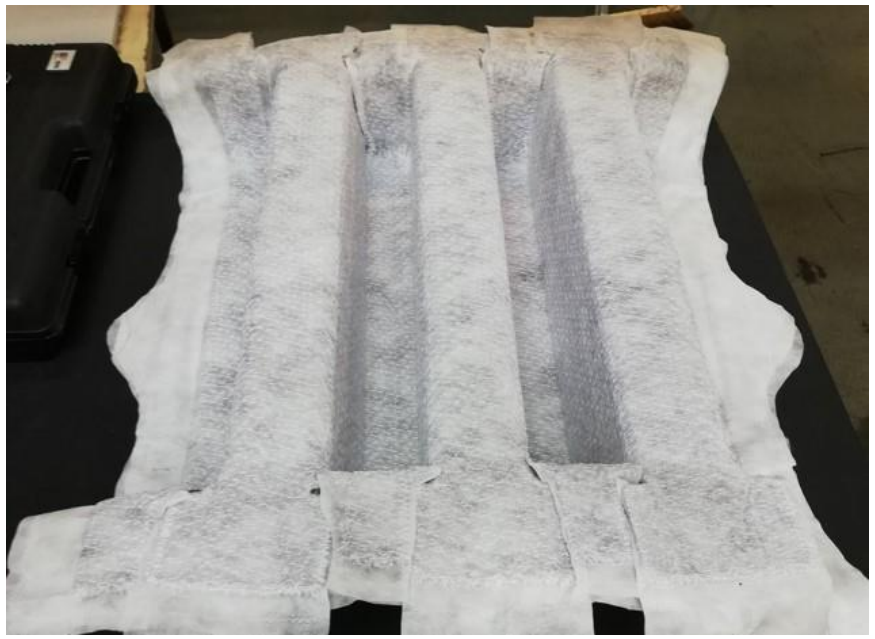

Figure II.2.5.16. Coats 3D preform. Source: GM.

The traditional approach of using NCFs can lead to significant waste due to the need to cut the 2D preform blanks from rolls. Due to the complex design of this part, $40 \%$ to $45 \%$ of the CF is wasted when cutting NCF preforms. The Coats Lattice ${ }^{\mathrm{TM}}$ process is an additive fiber placement approach that places fiber only where it is needed, thus eliminating this waste.

Since additional material around the part perimeter (i.e., the technical area) is needed to mold via HP-RTM, additional fiber is trimmed after the molding to produce the net shape part. Less-expensive glass fiber is used in this area in the Lattice ${ }^{\mathrm{TM}}$ preforms, as shown in Figure II.2.5.17, resulting in a further $13.5 \%$ reduction of CF waste. In total, our study estimates that only $47 \%$ of the CF needed to produce the NCF preforms is present in the net shape part, compared to virtually $100 \%$ in the case of the Coats Lattice ${ }^{\mathrm{TM}}$ preforms. Overall, 53\% of $\mathrm{CF}$ waste was eliminated. Figure II.2.5.18 shows the reinforcement panel molded with hybrid carbon and glass where glass is used outside the part. 


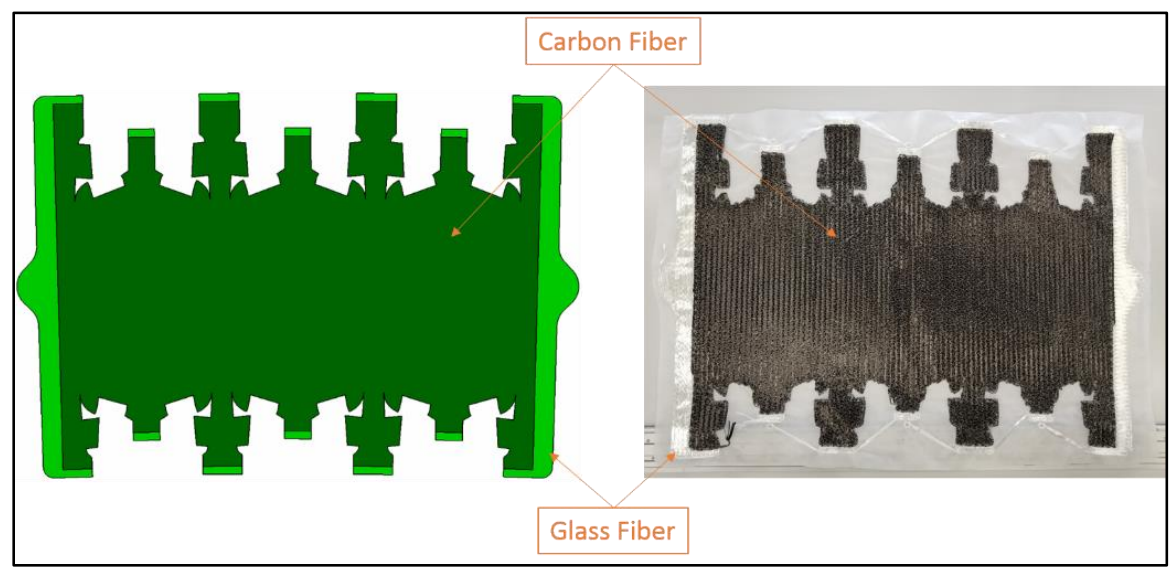

(a)

(b)

Figure II.2.5.17. Lattice ${ }^{\mathrm{TM}}$ preform with carbon and glass fibers selectively placed: (a) CAD generated and (b) finished product. Source: GM.

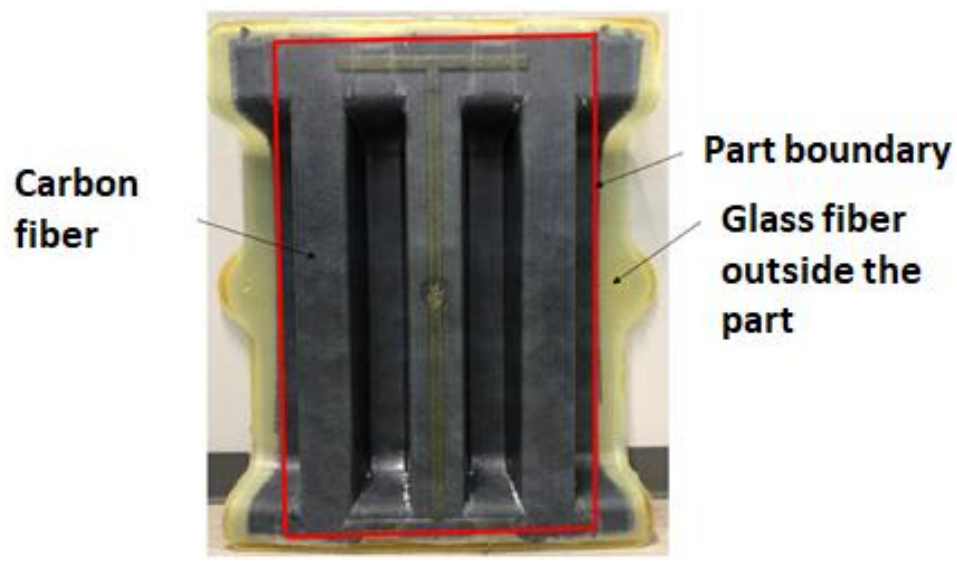

Figure II.2.5.18. Reinforcement panel molded with carbon and glass eliminating the scrap. Source: GM.

\section{CF Composite Floor}

The following sections provide an update on the tooling, draping, and molding progress in manufacturing the CF composite floor of this panel.

\section{Tooling}

Modifications to the floor tooling design continued iteratively based on the results from ESI's manufacturing simulations. The primary changes were to the size and shape of the resin injection channels. In general, these changes were made in order to reduce the resin filling time, and thus, the overall cycle time. Several changes made to the floor part design to improve manufacturability and avoid potential pitfalls also necessitated requisite changes to the tooling. Major changes to the tool designs are discussed below.

The peripheral injection channel on the floor tool remained in place; however, the cross-section was increased to accommodate more resin flow. A second injection port was added on the side opposite to the original port, which allows for higher flowrates by using two injection units. This will add a very small amount to the capital cost, but it allows molding the part using the fast-curing resin. Without adding this port, the resin cannot be injected in the short time that the fast-curing resins can be injected. The addition of patches to locally increase the part thickness altered the resin flow front and moved the area of the last filling. As a result, two vacuum ports were added along the centerline of the part. The port at the geometric center of the part was retained for optional C-RTM processing. The ports and sensor locations are detailed in Figure II.2.5.19. 


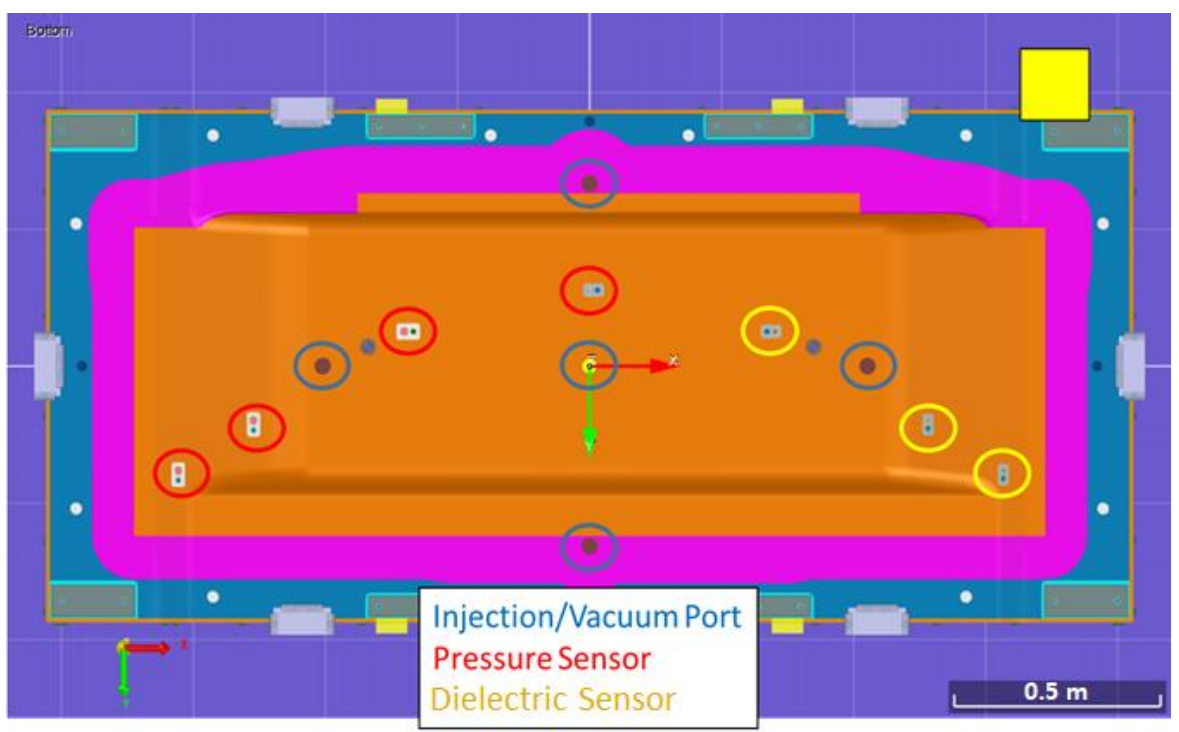

Figure II.2.5.19. Floor upper tool port and sensor locations. Source: GM.

Floor Draping Experiments

There are three preforming patterns for the floor: the main blank, a short patch, and a large patch. The short patch is a simple rectangular shape. The other two patterns are shown in Figure II.2.5.20. As with the reinforcement, the development of these patterns was driven by simulation. Several iterations of each pattern were draped experimentally. Good correlation was observed between the simulation and the experiment.

The 2D stack is produced in the same way as the reinforcement and is then draped in the RTM tool. The heated tool is then closed, and low tonnage is applied to fully form the preform and set the binder. An example of draped preform in the floor tool is shown in Figure II.2.5.21.

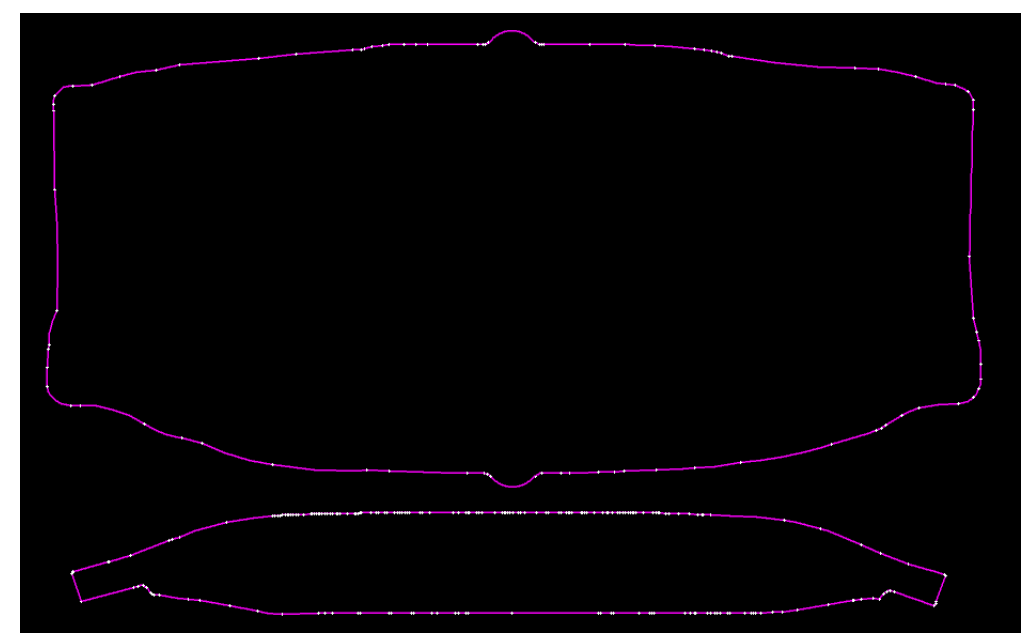

Figure II.2.5.20. Floor preforming patterns for the main blank (top) and large patch (bottom). Source: GM. 


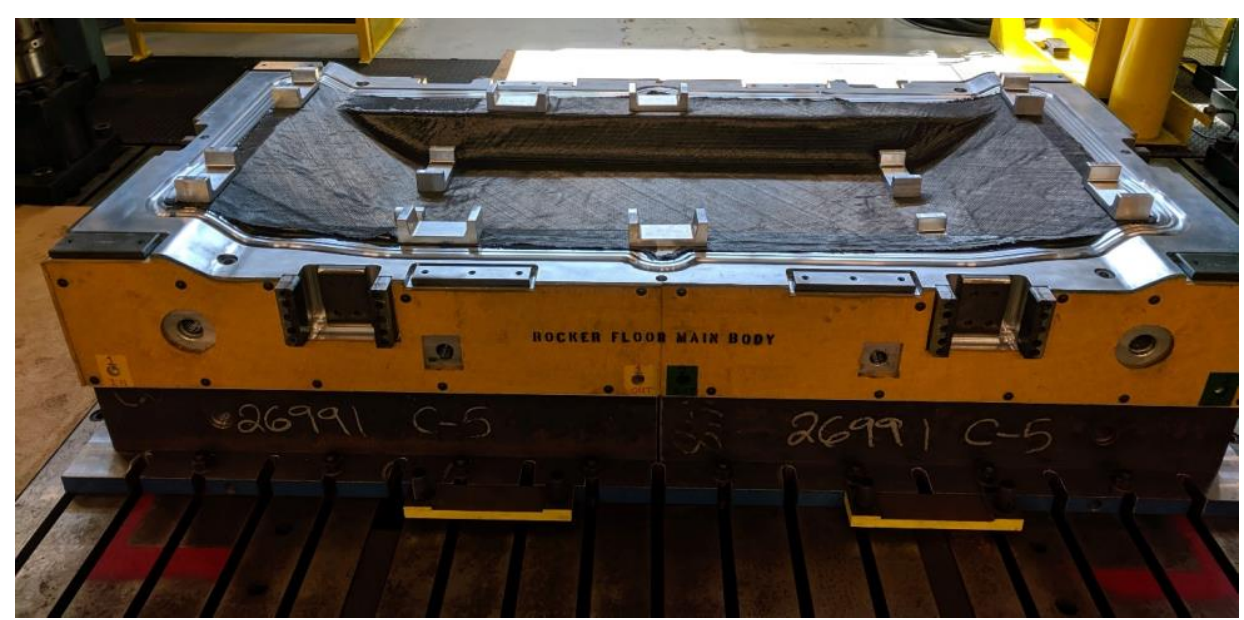

Figure II.2.5.21. Floor preforming pattern for the main blank, draped on the tool. Source: GM.

\section{Floor Molding}

Filling simulations of the floor were run by ESI to determine a good set of process conditions to start with. Several molding trials were undertaken to further refine the process parameters. Initial trials focused on producing good parts, without pushing the envelope on cycle time. A slower curing resin system was used for this initial round of molding. The full cycle time was on the order of 10 to 15 minutes. An example part is shown in Figure II.2.5.22. In the next phase of molding, two injection units will be used in tandem to rapidly fill the part with a faster curing resin system to demonstrate the feasibility of high-volume manufacturing.

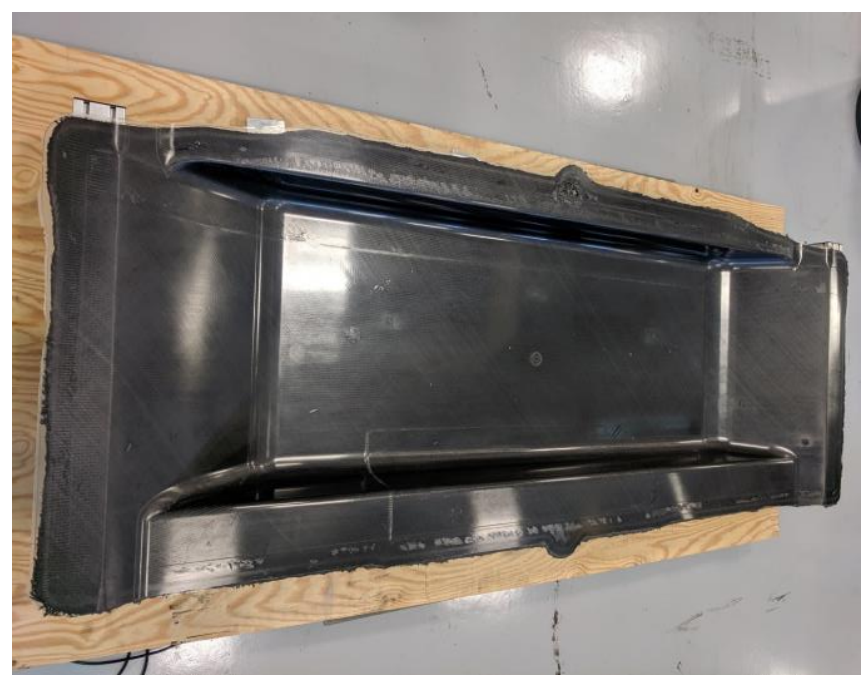

Figure II.2.5.22. Example of an untrimmed floor. Source: GM.

Rocker Outer, Rocker Inner and Rocker Assembly Impact Modeling

As on the floor tool, the cross-section of the resin runner was increased on the reinforcement and rocker tools. The locations of the ports and sensors were not changed. The only other changes were to accommodate part design alterations. The crashworthiness performance of the CF composite underbody structure with an energy absorber was studied using previously developed structural performance models. The LS-DYNA and multiscale designer modeler coupled simulations were performed considering material properties at the fiber tows and resin level. The side pole impact progression at various stages of the impact is shown in

Figure II.2.5.23, while the force-displacement predictions and deformation are shown in Figure II.2.5.24. It can be seen that the intrusion observed in the impact is only 4 in $(100 \mathrm{~mm})$. This design is attractive for future electric vehicle designs protecting battery integrity. 


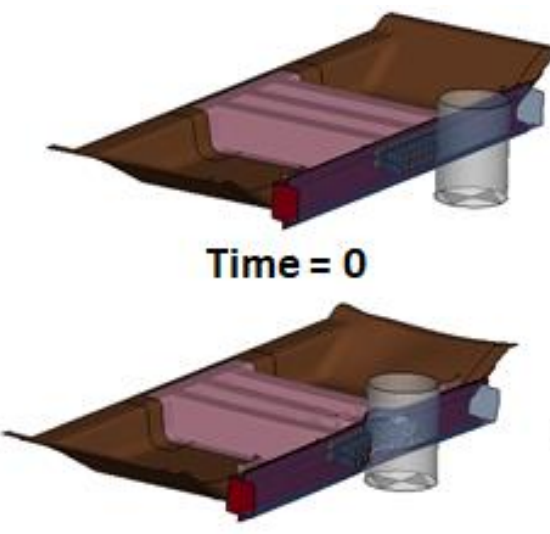

Time $=20$

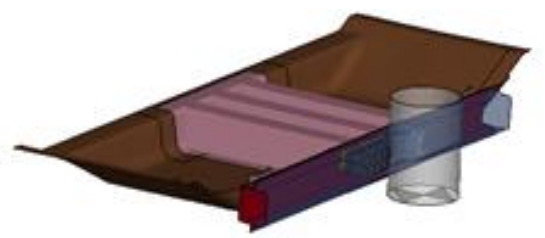

Time $=10$

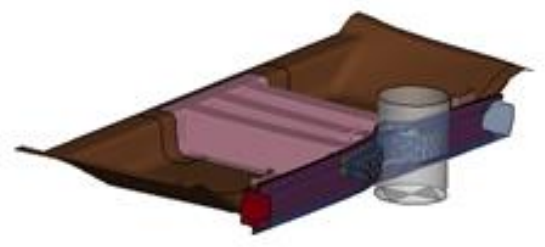

Time $=26$

Figure II.2.5.23. Viewgraph of pole-side impact simulation at different timesteps (in milliseconds). Source: GM.

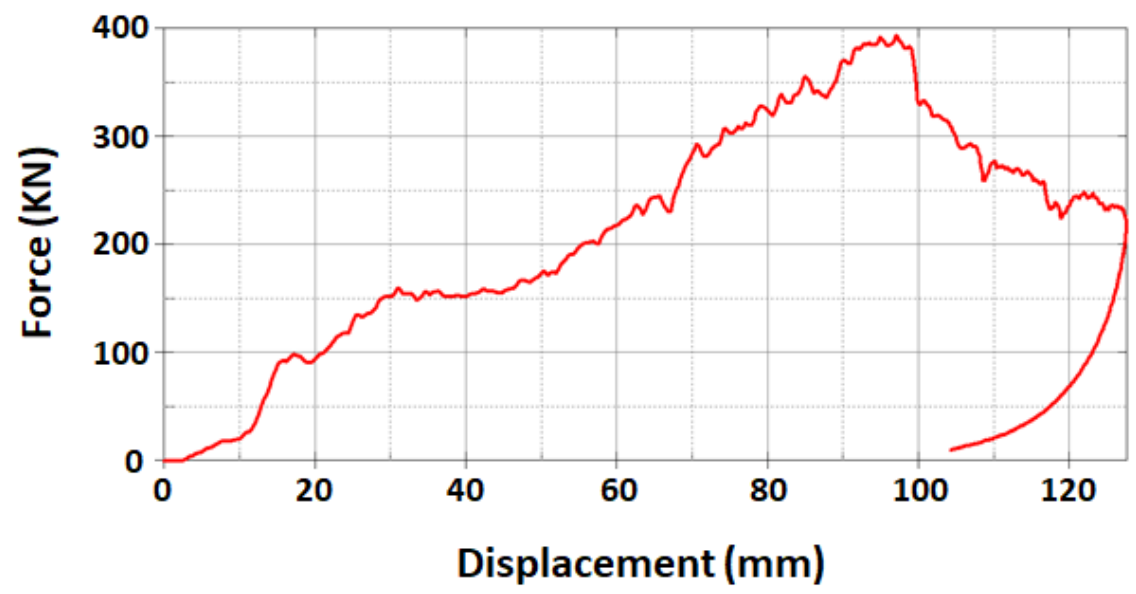

Figure II.2.5.24. Prediction for the force-displacement results. Source: GM.

\section{Stochastic Model Development}

During FY 2019, we developed a new ML approach for design optimization under uncertainty of parts and systems for the vehicle system. We also assessed various stochastic field models of permeability that are commonly used with resin transfer molding simulations.

Machine-Learning for Design Optimization

The results from 1000 simulation runs of the composite Multiscale Designer, developed in this project simulating at the microscale and upscaling it for macroscale simulation using the LS-DYNA computer program, are considered as a training set. Each of these runs provides data on fibers and resin properties, tow geometry, and up-scaled properties of tow, laminae, and laminates. Following this training exercise, our objective is the following: For a new design, when specifying only a subset of the features in the training set, can we infer anything about the remaining features? This is an important design problem usually faced by an industry and models developed in this project were extended to address this item for CF designs. Table II.2.5.1 shows the specific properties of the fiber/resin/twos used in the simulations. 
Table II.2.5.1. Input Parameters for Numerical Simulations of Microscale Simulations.

\begin{tabular}{|c|c|c|}
\hline Physical Component & Variable & Definition \\
\hline Fiber & $E_{f a}$ & fiber axial modulus \\
\hline Fiber & $E_{f t}$ & fiber transverse modulus \\
\hline Fiber & $G_{f a}$ & fiber shear modulus \\
\hline Fiber & $v_{f a}$ & fiber axial Poisson ratio \\
\hline Fiber & $v_{f t}$ & fiber transverse Poisson ratio \\
\hline Fiber & $\rho_{\text {fac }}$ & (compression modulus)/(axial modulus) \\
\hline Fiber & $\sigma_{y f a}$ & fiber axial yield strength \\
\hline Fiber & $\rho_{f t}=\sigma_{y t} / \sigma_{y a}$ & (transverse strength)/(axial strength) \\
\hline Fiber & $\rho_{f t C}=\sigma_{y a c} / \sigma_{y a}$ & (compressive strength)/(axial strength) \\
\hline Resin & $v_{m}$ & resin Poisson ratio \\
\hline Resin & $\sigma_{y m}$ & mean stress at damage initiation \\
\hline Resin & $K_{0 m}$ & yield strength \\
\hline Resin & $K_{1 m}$ & ultimate strength \\
\hline Resin & $H_{d}$ & linear term for hardening law \\
\hline Resin & $\delta$ & exponent for evolution law \\
\hline Tow & $D_{a}$ & major axis of tow's ellipsoid \\
\hline Tow & $D_{b}$ & minor axis of tow's ellipsoid \\
\hline Tow & $d_{a}$ & spacing between tows \\
\hline
\end{tabular}

For stochastic modeling, all of the variables shown in Table II.2.5.1 were assumed to have a symmetric beta distribution with a shape parameter equal to 1.5 and a range identified from the manufacturer's specifications and expert opinion. Experimental data on tow geometry, as identified by the parameters $D_{a}, D_{b}$, and $d_{a}$, is used to define a joint probability model for $D_{a}$ and $D_{b}$, with the probability density functions (PDFs) of $d_{a}$ independently estimated from its own data. The joint PDF of $D_{a}$ and $D_{b}$ is further constrained by the empirically measured volume fraction at the lamina scale. For the purpose of probabilistic ML, only a subset of the 18 random variables were treated as observables (namely, $E_{f t}, \rho_{f a c}, \sigma_{y f a}, \rho_{f t}, D_{a}, D_{b}, d_{a}$ ). This subset is identified in accordance with a sensitivity analysis developed previously. It should be noted that limiting the observables to this subset does not imply treating the remaining variables as deterministic, but rather as unspecified, and thus increasing the uncertainty in ensuing probabilistic inferences and widening the associated PDFs.

In addition to the model input variables, model output is measured in the form of stress-strain curves through the inelastic regime. The model is exercised in two different loading modalities - namely, a tension test and a three-point bending test. Further, 14 tensile and 11 bending experiments were performed in the GM R\&D Laboratory and their stress-strain histories recorded. Figure II.2.5.25 shows the stress-strain curves from these experiments together with numerical simulation results obtained through a sequence of upscaling procedure as described in the next section. The numerical results are shown as a band with a lighter dark color. It can be seen that the bending experiments 7, 8, 9, 10 and 11 in Figure II.2.5.25 (a) and the tensile experiments 1, 4, 5 and 13 in Figure II.2.5.25 (b) are on the fringes of the numerical predictions. 


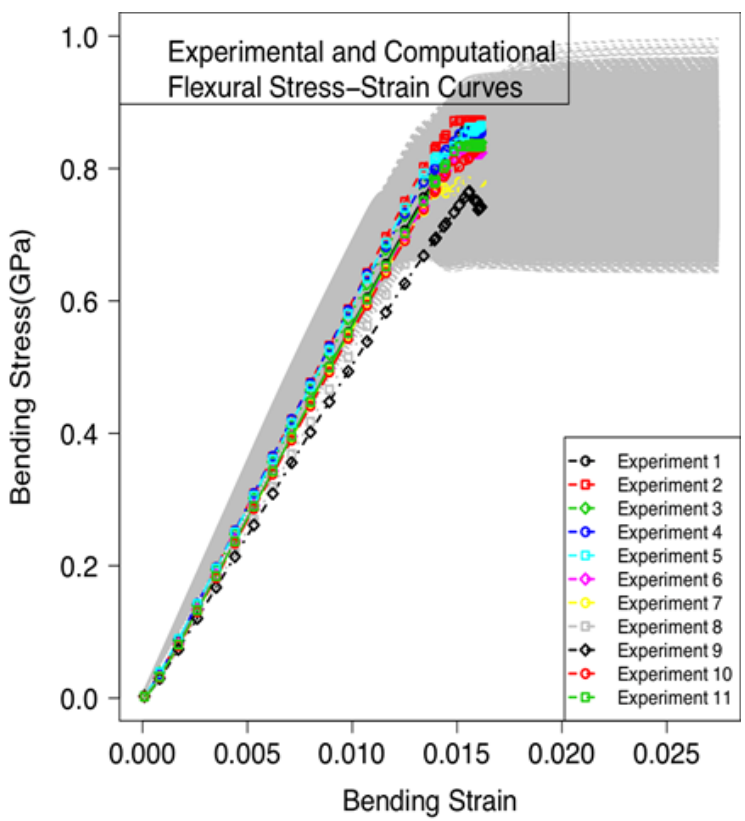

(a)

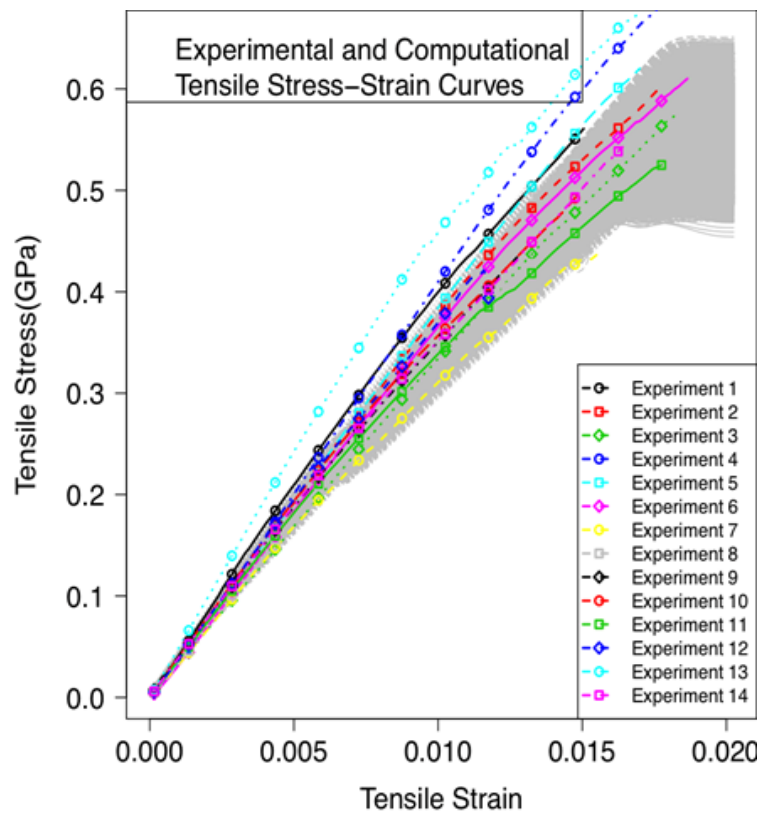

(b)

Figure II.2.5.25. Experimental stress-strain curves from (a) bending and (b) tensile tests. Source: GM.

We adopted the ML approach developed in Soize \& Ghanem [1]. In a first step, the data is treated through a principal component analysis to remove linear correlations. In a second step, the joint density function of the components from the principal component analysis are estimated through a kernel density estimation model. In a third step, a manifold is delineated within the dataset using diffusion map embedding, which provides an intrinsic constraint from within the data that we construe as describing all prevailing physics. A fourth step consists of augmenting the training dataset with a much larger number of samples generated from the kernel density estimation and having locus on the manifold. This is accomplished through a projected stochastic differential equation. With the much larger dataset, a last step consists of estimating the conditional expectation and conditional density functions of the objective function conditional on the control variables. The accuracy of this last step hinges on the availability of a very large dataset, thus motivating the first four steps. Figure II.2.5.26 shows the decay of the eigenvalues associated with the diffusion map algorithm, thus demonstrating the localization properties of the approach. 


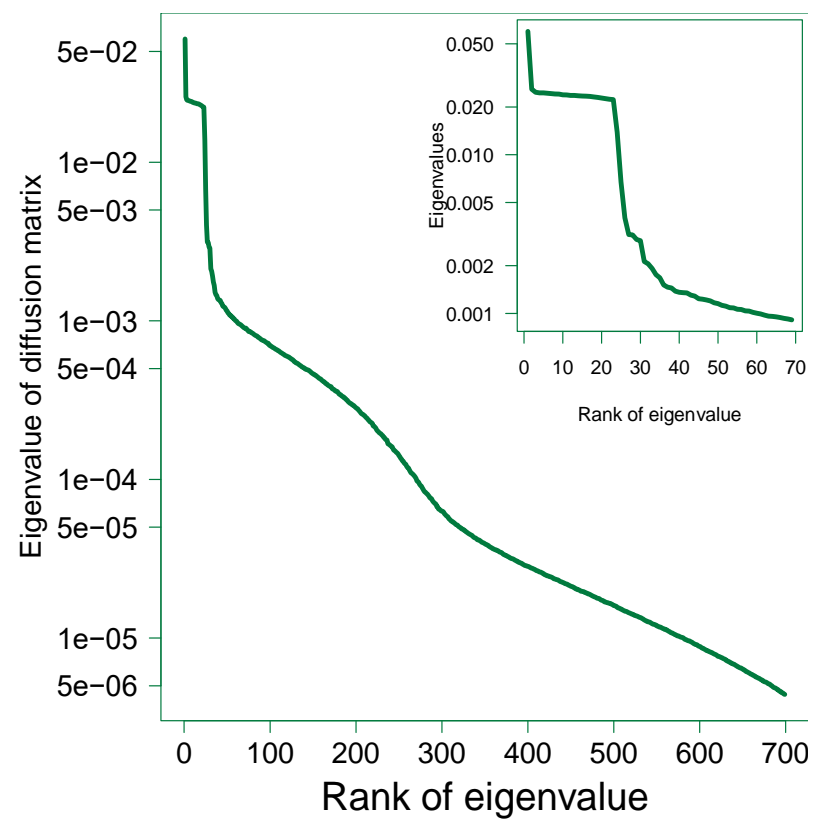

Figure II.2.5.26. Eigenvalues of diffusion. Source: GM.

Figure II.2.5.27 (a) and Figure II.2.5.27 (b) show several samples drawn from the training set and the augmented set for stress/strain curves under both bending and tensile loading.
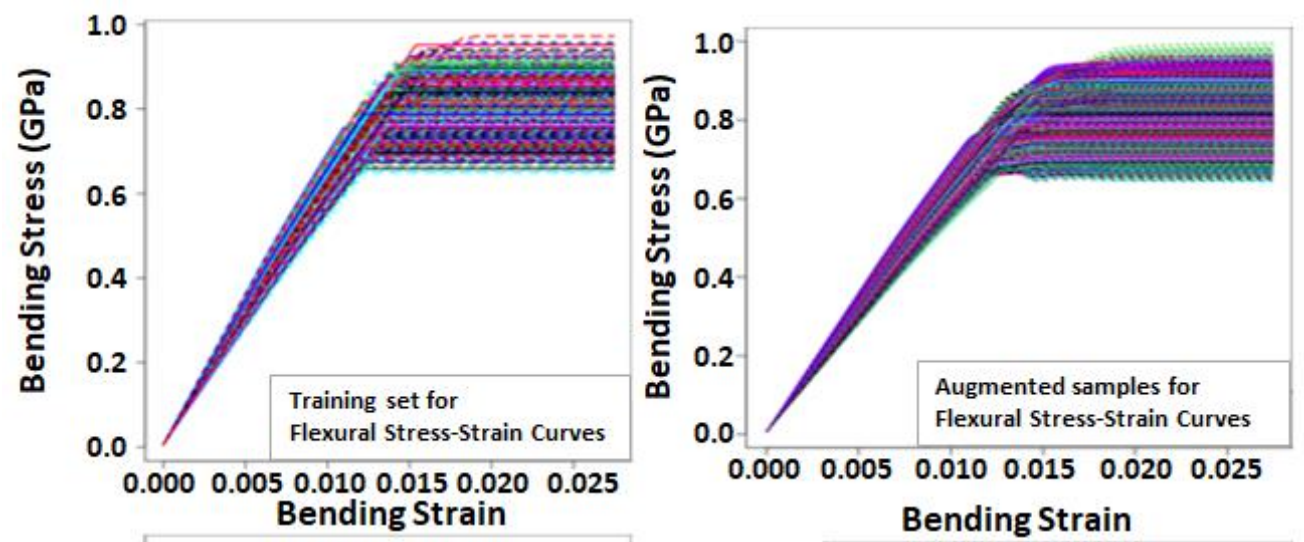

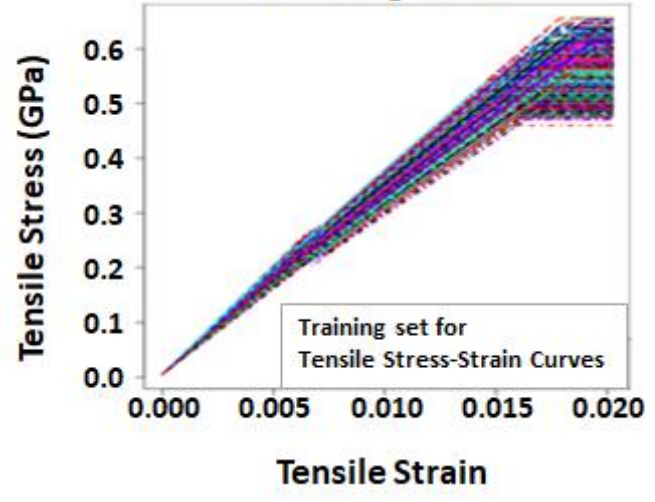

(a)

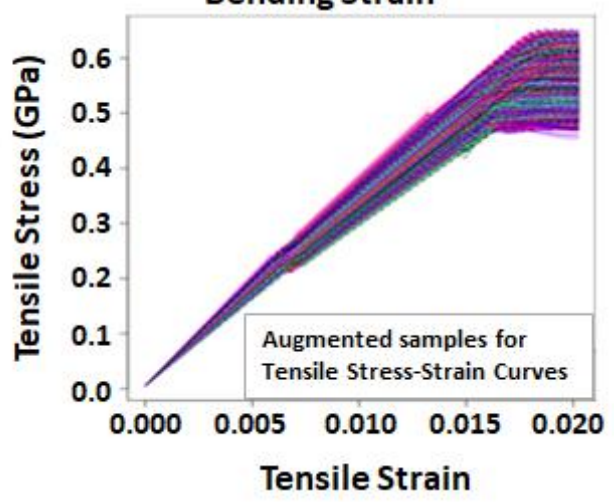

(b)

Figure II.2.5.27. Training sets (a) and augmented sets (b) for bending (top) and tensile (bottom) experiments. Source: GM. 
Figure II.2.5.28 (a) and Figure II.2.5.28 (b) show the PDFs of tensile and bending stresses at different strain levels, respectively. The solid lines in these figures depict the PDFs from the augmented set while the thin lines are associated with the training set. The datasets have clearly been augmented in a manner that is consistent with the available statistical evidence.
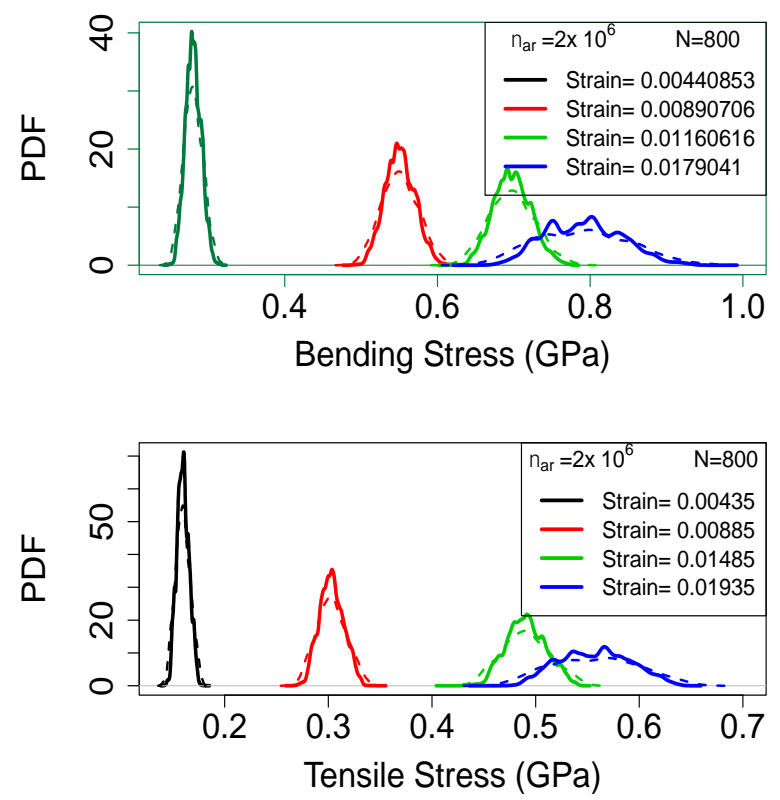

(a)
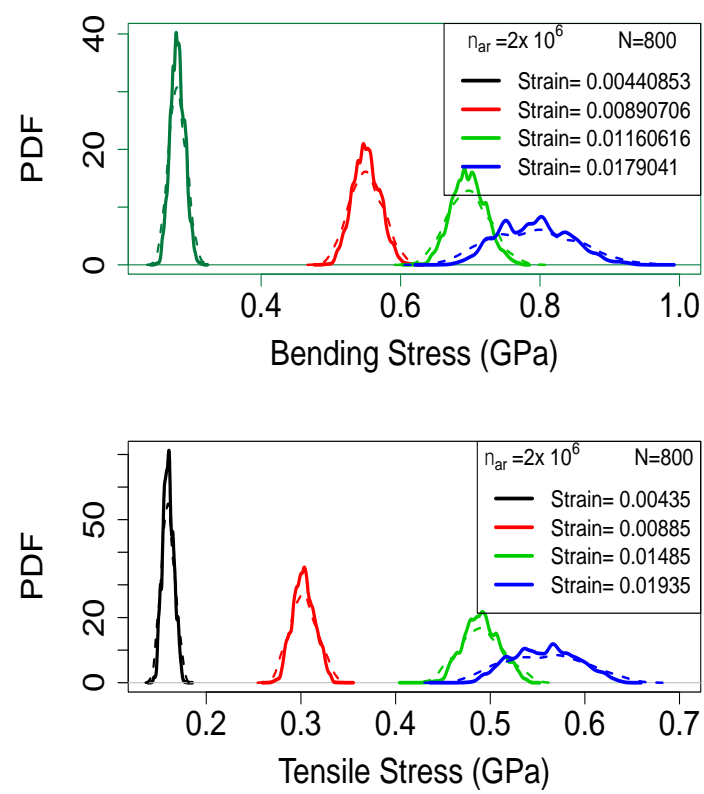

(b)

Figure II.2.5.28. PDF of the (a) flexural and (b) tensile stresses at different strain levels; leftmost curves are in the linear elastic regime while the rightmost curves are in the inelastic regime. Source: GM.

Figure II.2.5.29 shows the PDFs of stress at different strain levels when some fiber properties or some tow geometries are specified. PDFs are shown for stress levels in the linear and nonlinear regimes (the strain at which the stress is evaluated is indicated as a subscript on the stress symbol). 

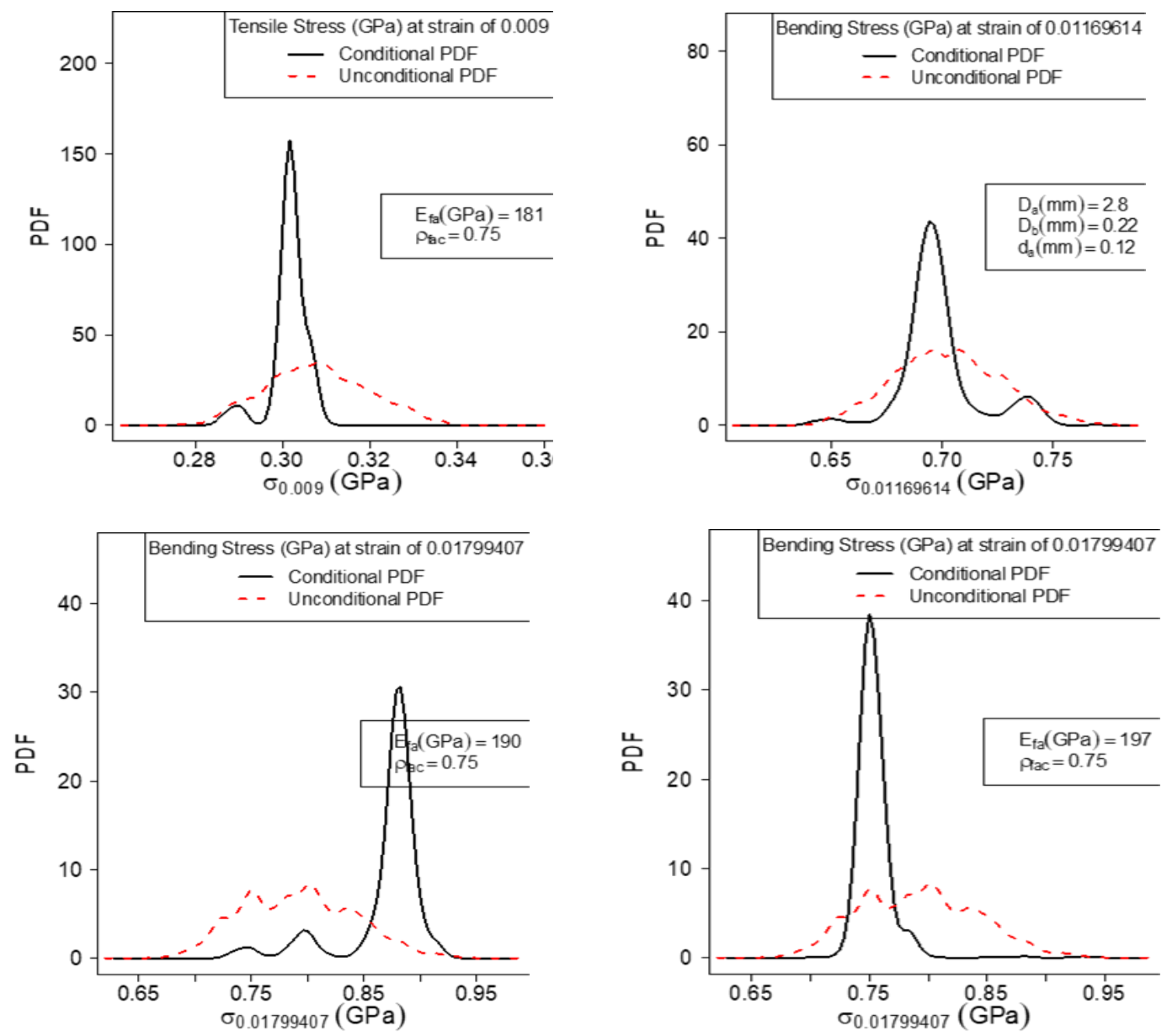

Figure II.2.5.29. PDF of stress at specified strain levels when conditioned on specific values of fiber properties or tow geometry. The legend on the right side of each graphic shows the conditioning state, while the legend at the top of each graphic indicates the quantity of interest being plotted. Source: GM.

Finally, Figure II.2.5.30 shows PDFs of the fiber elastic modulus when conditioned on specific values of stress and strain. In particular, the left subfigure in that figure shows conditioning on six stress/strain pairs, while the right subfigure shows conditioning on the full data in experiment \#6 shown in Figure II.2.5.25 above. 


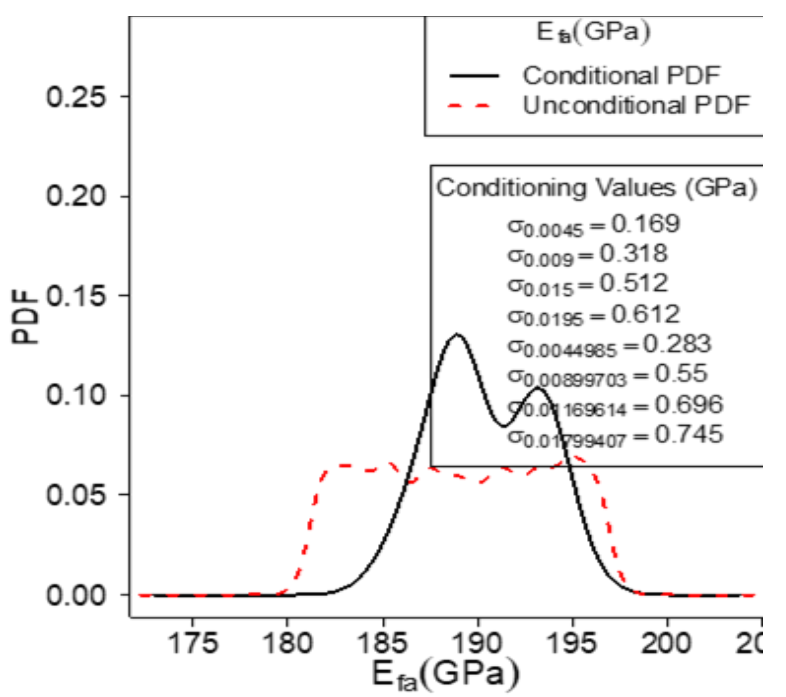

(a)

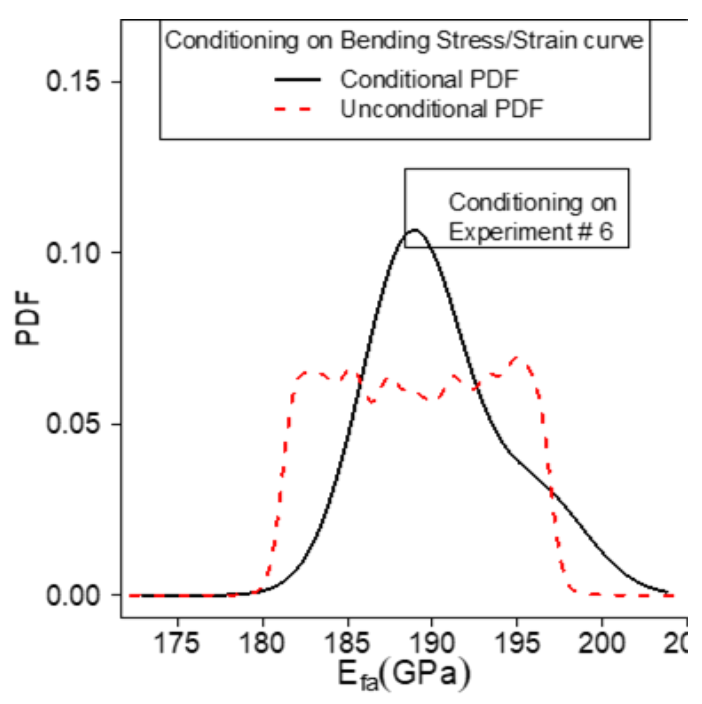

(b)

Figure II.2.5.30. PDF of fiber modulus (a) when conditioned on values of stress at strain levels or (b) when conditioned on the full experimental stress-strain curve. Source: GM.

Stochastic Field Model for Permeability of the Preform A second major effort during this year regarding stochastic modeling was focused on developing a random field model for permeability of the preform and assessing the predictive capability of various models from the literature. Through the forming process, we propagated uncertainties in fiber elastic and inelastic properties and sheet layup. This resulted in a spatially varying shearing angle that fluctuated across specimens. This spatial fluctuation was captured as a polynomial chaos of the shearing angle as a function of the fiber properties and orientations. A pointwise constitutive model was then used to transform the shearing angle into permeabilities, resulting in their polynomial chaos expansion (PCE) representation with respect to the same basic variables. A covariance function was deduced from this PCE.

Simultaneously, several realizations were also generated from this PCE and used to fit standard (and nonstandard) covariance models specified by their kernels. We specifically fitted to the data the following five kernel models - exponential, Gaussian, Matern, rational quadratic, and second-order autoregressive. The second-order autoregressive provides an oscillatory covariance function that reflects actual spatial fluctuations in the permeability and was observed to significantly outperform the other models using either a Frobenius norm of the error in the covariance matrix or the decay of the eigenvalues as a performance metric. All these kernel models, however, are presumed to be stationary in space with a spatially invariant PDF. The PCE-based covariance does not have that constraint. Spatial fluctuations of the PDF and boundary influence on the realizations were clearly visible in the PCE representation, but completely missing from all of the kernel models.

\section{Conclusions}

During FY 2019, the project team engineered three major tools to produce the components of the CF rocker assembly used to demonstrate the ICME technology developed in this project. The rocker floor assembly design baseline is the high-strength steel assembly currently produced for the 2016 GM Malibu. Using stateof-the-art ICME computational tools, the CF components were designed, manufactured, and assembled to compare the structural crashworthiness compared with the steel assembly. 
For the manufacturing studies, the components of the assembly were manufactured using HP-RTM, a potential game-changing high-volume composite manufacturing process for the automotive industry (e.g., lower cycle times, high-throughput). The injection strategy, vent locations, and process conditions (e.g., mold temperature, resin type, etc.) were engineered to minimize the cycle time, and thus, maximize the throughput. Our studies estimated that only $47 \%$ of the CF needed to produce the NCF preforms is present in the net shape part compared to virtually $100 \%$ in the case of the Coats Lattice ${ }^{\mathrm{TM}}$ preforms. Overall, $53 \%$ of CF waste was eliminated.

For the structural design, the $\mathrm{CF}$ assembly was designed for crashworthiness, which is a challenging load case for composites. The designed CF assembly is $30 \%$ lighter as compared to the current steel design. In addition to the mass savings, we have achieved significant part consolidation by designing the parts with variable thicknesses. Out of five large components in the CF rocker floor assembly, three components were molded successfully using the tools engineered in the project. For the reinforcement component, the draping and injection results from both the experiment and the simulation were compared and excellent correlations were obtained. This validates the demonstration of ICME technology developed in the manufacturing side. The results collected during the crash-testing of the assembly will be compared with the model predictions in FY 2020 to verify the accuracy of ICME stochastic structural models developed in this project. Further, a certification procedure will be developed to virtually certify the CF structural panels. We believe the project outcome will be a significant benefit to the entire automotive industry and will accelerate the implementation of CF composites for the next level of lightweighting of automobiles.

\section{Key Publications}

1. Dhaliwal, G., W. R., Rodgers, and V. Aitharaju, 2019, "Multiscale modeling of non-orthogonal twill weave composites," American Society of Composites 34th Technical Conference, 23-25 September 2019, Seattle, WA, USA.

2. Ghauch, Z., and R. Ghanem, 2019, "Comparison of multiscale and kernel-based correlations for stochastic permeability models in composites manufacturing," American Society of Composites 34th Technical Conference, 23-25 September 2019, Seattle, WA, USA.

3. Mehrez, L., and R. Ghanem, 2019, "Experimental validation of multiscale stochastic models for composites," American Society of Composites 34th Technical Conference, 23-25 September 2019, Seattle, WA, USA.

\section{References}

1. Soize, C., and R. Ghanem, "Data-driven probability concentration and sampling on manifold," 2016, J. Comput. Phys., Vol. 321, pp. 242-258.

\section{Acknowledgements}

The authors acknowledge Dr. H. Felix Wu, DOE Technology Manager and Mr. John Terneus, NETL Project Manager, for providing constant guidance and encouragement during the course of this project. 


\title{
II.2.6 Integrated Computational Materials Engineering Development of CF Composites for Lightweight Vehicles (Ford Motor Company)
}

\author{
Dr. Xuming Su, Co-Principal Investigator \\ Ford Research and Advanced Engineering \\ Ford Research and Innovation Center \\ 2101 Village Road \\ Dearborn, MI 48124 \\ E-mail: xsu1@ford.com
}

\section{Dr. Xuming Su, Co-Principal Investigator}

Ford Research and Advanced Engineering

Ford Research and Innovation Center

2101 Village Road

Dearborn, MI 48124

E-mail: dwagner6@ford.com

\section{Dr. H. Felix Wu, DOE Technology Manager}

U.S. Department of Energy

E-mail: felix.wu@ee.doe.gov

Start Date: May 1, 2015

Project Funding (FY19): \$2,270,476
End Date: April 30, 2019

DOE share: $\$ 1,589,333$
Non-DOE share: $\$ 681,143$

\section{Project Introduction}

Vehicle lightweighting is important for automotive manufacturers to meet the ever-increasing demand of fuel efficiency and to reduce greenhouse gas emissions and dependency on foreign oil. CFRP composites, with a density of $1.55 \mathrm{~g} / \mathrm{cm} 3$ and a tensile strength of about $2000 \mathrm{MPa}$ in the fiber direction, are among the promising candidates to replace the metals currently used for structural components. The goal of this project is to design, develop, and optimize, using CAE only, a LD vehicle CF composite intensive front subframe capable of achieving greater than a $25 \%$ weight reduction with a variable cost increase of less than $\$ 4.27$ per pound of weight saved when compared to the baseline technology to be replaced.

Unlike many metals typically used in automotive vehicles, the mechanical properties of CFRP are highly direction-dependent. The strength of the material in fiber-reinforced-direction could be an order-of-magnitude higher than in the direction perpendicular to it. It is important to note that the fiber orientation in a component is decided not only by the initial fabric layout, but also the preforming and molding processes. An optimized vehicle component design thus needs tools that are capable of predicting the performance of CFRP parts based on fiber architecture, molding process, and curing history, with considerations of uncertainties and probabilistic nature of materials, processes, and in-service conditions. The current project develops these ICME tools to meet the design challenge.

ICME tools developed in the project are robust, accurate, and reliable constitutive models for each constituent material, as well as the composite assembly under expected service conditions including high-strain rates utilizing physics-based models. The manufacturing process (including variability from both process and material) is simulated to predict the fiber orientation and other microstructural features of manufactured products. This information is passed to performance analysis. Material design, manufacturing simulation, and performance analysis are integrated. Local material properties are related to the composite architecture and microstructure by multiscale models. Design and manufacturing process are optimized simultaneously to achieve the most efficient usage of the material. 


\section{Objectives}

The project has two main goals: (1) to develop ICME techniques for CF composites that reduce developmentto-deployment lead time for lightweight CF composite components and (2) to create an initial design for a structural CF composite subframe to support immediate weight reduction in LD vehicles. Additionally, the project includes dissemination of results through multiple publications, conference papers and presentations as well as through the infrastructure and methods identified by the National Institute of Standards and Technology (NIST).

An ICME approach is utilized to develop, integrate and implement predictive models for CFRP composites that link the materials design, molding process, local material properties and final performance, with considerations of uncertainties and probabilistic nature of materials, processes and in-service conditions. The ICME techniques are used to design, develop and optimize an integrated CFRP composite intensive front subframe with equivalent performance to a baseline production subframe. At equivalent performance, the weight and cost are compared to the steel assembly.

The objective of the project was to produce an initial design of a LD vehicle CF composite subframe capable of achieving a $>25 \%$ weight reduction as well as $\leq \$ 4.27$ per pound of weight saved when compared to the baseline technology to be replaced. The subframe significantly contributes to the structural performance of the vehicle in driving and crash conditions. The developed CF composite subframe will achieve critical functions and packaging requirements of the baseline stamped steel subframe to be replaced.

\section{Approach}

The ICME tools relate performance requirements of a vehicle to material design and processes. While material performances are expressed at the continuum macroscale, they are decided by material and features at the microscale and again by molecular bonds and crosslinking at the nanoscale. The material microstructures and microstructural features, bonding, and crosslinking are results of material design and manufacturing processes. ICME tools are thus multiscale models and approaches that bridge different material scales. These models are based on physical principles, testing measurements, and observations. Test data are also needed in validating material models. Modeling and testing are always hand-in-hand in successful development of ICME tools. The project team consists of Ford Motor Company, an automotive manufacturer; Dow Chemical, a material manufacturer; Northwestern University, an institution of highly regarded academics; and the NIST, a worldrenowned test laboratory. As such, the team has all of the elements needed to make a breakthrough in developing ICME tools. The research team also includes software developers from LSTC (for LS-DYNA), Autodesk (for Moldflow), ESTECO (for modeFrontier), and HBM Prenscia (for nCode). The ICME tools developed in the project can be numerically implemented and made available through popular engineering software.

\section{Results}

There are four main project tasks as listed below. Each of the tasks had goals and accomplishments attained through this project. Table II.2.6.1 highlights these major project goals and lists the accomplishments. Major findings are discussed following the table. 
Table II.2.6.1. Task Goals and Accomplishments.

\begin{tabular}{|c|c|c|c|c|c|}
\hline \multirow{2}{*}{$\begin{array}{l}\text { Task } \\
\text { No. }\end{array}$} & \multicolumn{3}{|c|}{ Goal } & \multicolumn{2}{|c|}{ Accomplishment } \\
\hline & Description & Target & & Description & Metric \\
\hline 1 & $\begin{array}{l}\text { Material testing } \\
\text { and NIST } \\
\text { database } \\
\text { documentation }\end{array}$ & $\begin{array}{l}\text { Test UD, woven, and chopped } \\
\text { SMC CFRP epoxy composite } \\
\text { coupons characterizing } \\
\text { mechanical properties of the } \\
\text { constituents (fiber and resin) } \\
\text { and composite coupons at } \\
\text { quasi-static and elevated strain } \\
\text { rates, and from ambient to } \\
\text { elevated temperatures }\end{array}$ & $\begin{array}{l}\text { Three or more } \\
\text { repeats per test } \\
\text { condition }\end{array}$ & $\begin{array}{l}\text { Tested coupons to } \\
\text { determine charateristics } \\
\text { of constituents (fiber and } \\
\text { resin) }\end{array}$ & $\begin{array}{l}\text { Performed over } 700 \\
\text { coupon tests and included } \\
\text { all methods and results in } \\
\text { a NIST repository }\end{array}$ \\
\hline \multirow{3}{*}{2} & $\begin{array}{l}\text { Constituent } \\
\text { material } \\
\text { modeling (fiber/ } \\
\text { interphase/resin } \\
\text { and assembly of } \\
\text { such) }\end{array}$ & $\begin{array}{l}\text { Robust, accurate, and reliable } \\
\text { constitutive models for each } \\
\text { constituent material, as well as } \\
\text { the composite assembly under } \\
\text { expected service conditions } \\
\text { including high strain rates } \\
\text { utilizing physics-based model }\end{array}$ & $\leq 15 \%$ accuracy & $\begin{array}{l}\text { Model to test correlation } \\
\text { studies on coupons }\end{array}$ & $\begin{array}{l}\leq 15 \% \text { score, dynamic } \\
\text { behavior has higher error }\end{array}$ \\
\hline & $\begin{array}{l}\text { Part properties } \\
\text { during and after } \\
\text { molding }\end{array}$ & $\begin{array}{l}\text { Microstructure morphology, } \\
\text { optimized cycle time, and local } \\
\text { thickness, fiber length, and } \\
\text { orientation of the final part }\end{array}$ & $\leq 15 \%$ accuracy & $\begin{array}{l}\text { Model correlation to hat } \\
\text { section testing }\end{array}$ & $\leq 15 \%$ agreement \\
\hline & $\begin{array}{l}\text { Assembly } \\
\text { properties after } \\
\text { joining and } \\
\text { assembly }\end{array}$ & $\begin{array}{l}\text { Load to failure, failure location } \\
\text { and mode, stiffness/deflection, } \\
\text { dynamic performance, energy } \\
\text { absorption/crashworthiness }\end{array}$ & $\leq 15 \%$ accuracy & $\begin{array}{l}\text { Model correlation to hat } \\
\text { section testing }\end{array}$ & $\begin{array}{l}\leq 15 \% \text {, some crash modes } \\
\text { have higher error }\end{array}$ \\
\hline 3 & $\begin{array}{l}\text { ICME-based } \\
\text { multi- } \\
\text { disciplinary } \\
\text { optimization } \\
\text { workflow }\end{array}$ & $\begin{array}{l}\text { Construct an ICME-based } \\
\text { multi-disciplinary optimization } \\
\text { workflow connecting multiple } \\
\text { computer-aided design and } \\
\text { computer-aided engineering } \\
\text { tools to optimize an initial } \\
\text { design of a CFRP composite } \\
\text { component }\end{array}$ & $\begin{array}{l}\text { Batch process on } \\
\text { Ford High } \\
\text { Performance } \\
\text { Computing platform }\end{array}$ & $\begin{array}{l}\text { Establish an integrated } \\
\text { workflow to automate the } \\
\text { entire ICME analysis } \\
\text { process, composite } \\
\text { material processing, } \\
\text { microstructure } \\
\text { description, material } \\
\text { properties, and } \\
\text { component performance } \\
\text { with optimization and } \\
\text { results analysis on the } \\
\text { Ford High Performance } \\
\text { Computing platform }\end{array}$ & $\begin{array}{l}\text { Approximately } 100 \text { design } \\
\text { iterations per day }\end{array}$ \\
\hline \multirow[t]{3}{*}{4} & \multirow[t]{3}{*}{$\begin{array}{l}\text { CFRP composite } \\
\text { front subframe } \\
\text { initial design }\end{array}$} & $\begin{array}{l}\text { Develop an initial design of } \\
\text { CFRP composite front subframe } \\
\text { that meets all package, } \\
\text { stiffness, strength, durability, } \\
\text { and safety metrics at the } \\
\text { weight and cost targets }\end{array}$ & - & $\begin{array}{l}\text { Steel Intensive Design: } \\
79 \text { wt.\% steel } \\
16 \text { wt.\% CF-SMC } \\
5 \text { wt.\% CF-unidirectional }\end{array}$ & $\begin{array}{l}\text { CF-SMC Intensive Design: } \\
12 \text { wt.\% steel } \\
83 \text { wt.\% CF-SMC } \\
5 \text { wt.\% CF-unidirectional }\end{array}$ \\
\hline & & Weight saved target & $>25 \%$ weight saved & $30 \%$ weight saved & $41 \%$ weight saved \\
\hline & & $\begin{array}{l}\text { Additional variable cost per } \\
\text { pound of weight saved target }\end{array}$ & $\begin{array}{l}\leq \$ 4.27 \text { per pound } \\
\text { saved }\end{array}$ & $\begin{array}{l}\$ 4.01 \text { additional cost per } \\
\text { pound of weight saved }\end{array}$ & $\begin{array}{l}\$ 8.90 \text { additional cost per } \\
\text { pound of weight saved }\end{array}$ \\
\hline
\end{tabular}


The ICME models were all accurate within $15 \%$ of experimental values, except in the case of specific dynamic, high loading-rate behaviors that require ongoing investigation to refine predictive capabilities. These models are linked together in the multidisciplinary optimization scheme. A variable cost estimate is also included into the multidisciplinary optimization which adds tremendous value for users in the automotive industry by expanding the objectives in the optimization scheme to include cost as well as weight while adding little computational cost or complexity to the optimization procedures. This combination of ICME-based models and tools showcases the advantage of producing an initial design of a complex automotive part with a methodology that concurrently considers materials, performance and costs.

The ICME tools produced an initial design of the front subframe that met the critical engineering performance metrics at the weight-savings and additional cost per pound of weight saved targets. The proposed initial subframe design saves $30 \%$ weight (> $25 \%$ weight save target) and costs an additional $\$ 4.01$ per pound saved ( $<4.27$ per pound weight saved target) compared to a stamped steel baseline subframe. The multi-material design utilizes steel, $79 \mathrm{wt} \%$, with reinforcements of chopped CF SMC, $16 \mathrm{wt} \%$, and selected patches of unidirectional $\mathrm{CF}$ non-crimped fabric composite, $5 \mathrm{wt} \%$. This initial design meets critical stiffness, strength, durability and one safety metric. Figure II.2.6.1 shows the material distribution in the proposed initial subframe design.

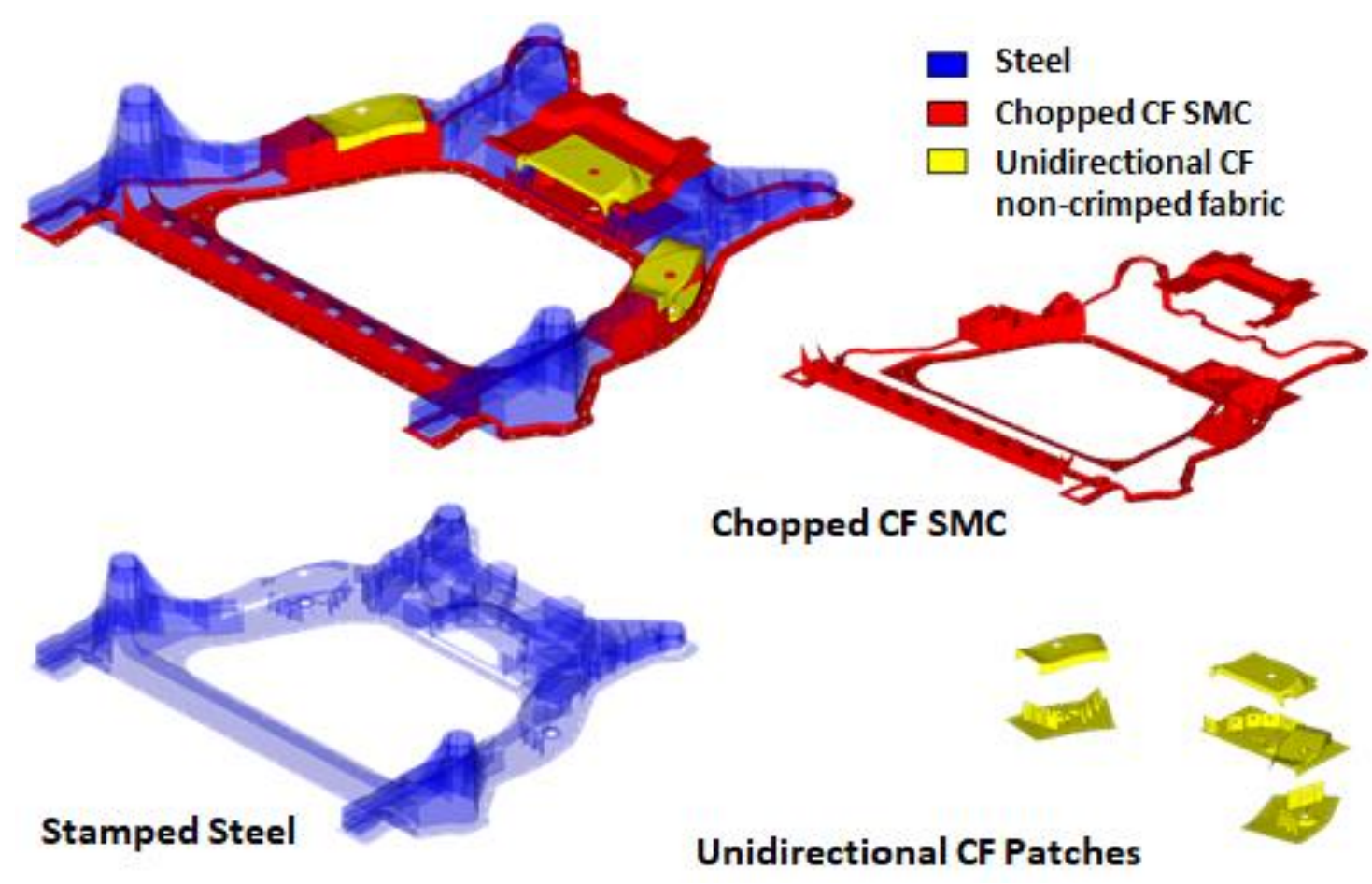

Figure II.2.6.1. Proposed initial subframe design at 30\% weight save and additional cost of $\$ 4.01$ per pound saved material distribution. Source: Ford Motor Company.

Through all the project exercises, certain gaps appeared and are captured as areas for future improvements and future research. In the CFRP composite materials testing, fatigue characterization has been developed and improved but further efforts and refinements are certainly needed. The modeling efforts can be further enhanced by improvements in the multiscale modeling protocols.

The modeling efforts identify gaps in nanoscale simulations of highly crosslinked epoxy resins that offer a promising way for the development of new continuum theories and models. The modeling of chopped CF sheet molding compound (SMC) depends strongly on the processing method to formulate the SMC. In unidirectional and woven CFRP composite, the delamination during crushing that often limits ultimate load 
capacity depends on the processing and formulation. The fatigue modeling future improvements include further understanding of multiaxial fatigue properties of CFRP composites and development of a multiaxial fatigue failure criterion. The modeling tasks made good progress on these models, but further work remains.

One major challenge of implementing the ICME-based multidisciplinary optimization approach for composite design is that the design space is notably enlarged by including design variables from multiple domains. Second, the composite design problem is often mixed-variable type, i.e., the design variables are a mixture of continuous (e.g., part thickness and height, fiber volume fraction), discrete (e.g., fiber rotation angle, levels of speed and force in preforming), or categorical variables (e.g., type of materials), posing challenges to the optimization search due to the combinatorial nature. Therefore, future work needs to refine the design variables and/or improve the computational efficiency of all the analytical methods from preforming, through molding to fatigue and safety simulations.

The subframe design optimization task highlighted the need for improvements in the geometry and architecture morphing to produce high quality, CAE models for manufacturing and engineering performance simulations. The different mesh requirements for the preforming, molding, stiffness, durability, strength and safety simulations presented the most difficult challenge in using these tools in an automated batch optimization process. Particularly, the Moldflow requirement for tetrahedral mesh in a continuous closed volume is so different from the shell mesh that is most appropriate for the durability and safety simulations that an automated process to produce both these meshes proved elusive within this project.

\section{Conclusions}

After four years of efforts, the project extends and complements the knowledge of CFRP epoxy materials with woven carbon fiber, unidirectional carbon fiber and chopped carbon fiber SMC used in compression molding of structural parts. The project creates an industry-first, ICME-based, multidisciplinary optimization modeFRONTIER workflow that combines part geometry, material, and CF composite layup design variables with compression molding simulation to generate local material properties feeding into engineering performance simulations for stiffness, overload strength, HCF durability and crash safety while tracking component weight and estimating variable cost. The workflow demonstrates the full ICME process on a small part, such as a closed top hat section. A similar workflow investigated the design of a large, complex automotive-structural component, the front subframe. This optimization workflow finds an initial design that exceeds the project targets for $>25 \%$ weight save at a variable cost $<\$ 4.27$ additional variable cost per pound of weight saved. The initial subframe design includes 79 wt.\% steel, $16 \mathrm{wt} . \%$ chopped CF SMC and $5 \mathrm{wt} . \%$ unidirectional CF epoxy composites. This initial design saves $30 \%$ weight at an additional variable cost of $\$ 4.01$ per pound of weight saved compared to the production stamped steel subframe.

The material characterization task performed over 700 specimen material characterization tests for mechanical properties. All the test conditions and results are now included in the NIST CFRP composite material database ${ }^{10}$ for the benefit of future researchers and engineers. The link to this website is:. The largest gap in this testing arena is the definition of a robust fatigue testing protocol for the variety of CFRP composites that are under development. This project makes substantial strides forward on fatigue testing, but much more work remains to be done.

The material modeling task developed and improved local material models and engineering performance material models. The largest gap appears to be the fatigue modeling. The fatigue modeling future improvements include further understanding of multiaxial fatigue properties of CFRP composites and development of a multiaxial fatigue failure criterion. The modeling task made good progress on these models, but further work remains.

${ }^{10} \mathrm{https://materialsdata.nist.gov.}$ 
The ICME modeling integration efforts successfully integrated numerous models into a full multidisciplinary workflow. The largest gaps in this area are still the special scripts that must be developed to pass information between the various simulation codes. Also, the multitude of potential design variables make the full ICMEbased optimization problem challenging even for Ford's HPC platform.

The industry-first ICME-based, multidisciplinary optimization scheme highlights and includes all the successes from this large CF composite project. The accomplishments of the project teams advance the stateof-the-art and the knowledge-base for epoxy thermoset CF composites in three forms, unidirectional noncrimped fabric, woven fabric and random chopped SMC.

\section{Key Publications}

1. Bostanabad, R., B. Liang, J. Gao, W. K. Liu, J. Cao, D. Zeng, X. Su, H. Xu, Y. Li, and W. Chen, 2018, "Uncertainty quantification in multiscale simulation of woven fiber composites," Comput. Method Appl. M., Vol. 338, pp. 506-532.

2. Chen, Z., T. Huang, Y. Shao, Y. Li, H. Xu, K. Avery, D. Zeng, W. Chen, and X. Su, 2018, "Multiscale FE modeling of SMC composite structure based on stochastic mesostructure reconstruction," Compos. Struct., Vol. 188, pp. 25-38.

3. Fenner, J. S., and I. M. Daniel, 2018, "Testing the 2-3 shear strength of unidirectional composite," 2018 Society for Experimental Mechanics Annual Conference, 4-7 June 2018, Greenville, SC, USA.

4. Li, Y., Z. Chen, L. Su, W. Chen, X. Jin, and H. Xu, 2018, "Stochastic reconstruction and microstructure modeling of SMC chopped fiber composites," Compos. Struct., Vol. 200, pp. 153-164.

5. Tang, H., Z. Chen, G. Zhou, Y. Li, K. Avery, H. Guo, H. Kang, D. Zeng, and X. Su, 2019, "Correlation between failure and local material property in chopped CF chip-reinforced SMC composites under tensile load," Polym. Composite, Vol. 40, No. S2, pp. E962-E974.

6. Zhang, W., X. Ma, J. Lu, Z. Zhang, Q. J. Wang, X. Su, D. Zeng, M. Mirdamadi, and J. Cao, 2018, "Experimental characterization and numerical modeling of the interaction between CF composite prepregs during a preforming process," J. Manuf. Sci. E., Vol. 140, No. 8, Art. \#081003.

7. Hansoge, N. K., T. Huang, R. Sinko, W. Xia, W. Chen, and S. Keten, 2018, "Materials by design for stiff and tough hairy nanoparticle assemblies," ACS Nano, Vol. 12, No. 8, pp. 7946-7958.

8. Sun, Q., Z. Meng, G. Zhou, S. P. Lin, H. Kang, S. Keten, H. Guo, and X. Su, 2018, "Multiscale computational analysis of unidirectional CFRP composites under various loading conditions," Compos. Struct., Vol. 196, pp. 30-43.

9. Sun, Q., H. Guo, G. Zhou, Z. Meng, Z. Chen, H. Kang, S. Keten, and X. Su, 2018, "Experimental and computational analysis of failure mechanisms in unidirectional CFRP laminates under longitudinal compression loading," Compos. Struct., Vol. 203, pp. 335-348.

10. Zhang, W., R. Bostanabad, B. Liang, X. Su, D. Zeng, M. A. Bessa, Y. Wang, W. Chen, and J. Cao, 2019, "A numerical Bayesian-calibrated characterization method for multiscale prepreg preforming simulations with tension-shear coupling," Compos. Sci. Technol., Vol. 170, pp. 15-24.

11. Liang, B., W. Zhang, J. S. Fenner, J. Gao, Y. Shi, D. Zeng, X. Su, W. K. Liu, and J. Cao, 2019, "Multiscale modeling of mechanical behavior of cured woven textile composites accounting for the influence of yarn angle variation," Compos. Part A-Appl. S., Vol. 124, Art. \#105460.

12. Tang, H., Z. Chen, G. Zhou, X. Sun, Y. Li, L. Huang, H. Guo, H. Kang, D. Zeng, C. Engler-Pinto, and $\mathrm{X}$. Su, 2019, "Effect of fiber orientation distribution on constant fatigue life diagram of chopped CF chipreinforced SMC composite," Int. J. Fatigue, Vol. 125, pp. 394-405. 
13. Chen, Z., H. Tang, Y. Shao, Q. Sun, G. Zhou, Y. Li, H. Xu, D. Zeng, and X. Su, 2019, "Failure of chopped CF SMC composites under UT loading: Computational prediction and experimental analysis," Compos. Part A-Appl. S., Vol. 118, pp. 117-130.

14. Sun, Q., G. Zhou, Z. Meng, H. Guo, Z. Chen, H. Liu, H. Kang, S. Keten, and X. Su, 2019, "Failure criteria of unidirectional CFRP composites informed by a computational micromechanics model," Compos. Sci. Technol., Vol. 172, pp. 81-95.

15. Sun, Q., G. Zhou, H. Guo, Z. Meng, Z. Chen, H. Liu, H. Kang, and X. Su, 2019, "Failure mechanisms of cross-ply CFRP laminates under longitudinal compression with experimental and computational analyses," Compos. Part B-Engineering, Vol. 167, pp. 147-160.

16. Liu, H., A. Ojha, Z. Li, C. C. Engler-Pinto Jr., X. Su, Q. Sun, H. Kang, W. Wen, and H. Cui, 2019, "Fatigue modeling for carbon/epoxy unidirectional composites under various stress ratios considering size effects," Int. J. Fatigue, Vol. 120, pp. 184-200.

17. Gao, J., M. Shakoor, G. Domel, M. Merzkirch, G. Zhou, D. Zeng, X. Su, and W. K. Liu, 2020, "Predictive multiscale modeling for unidirectional CFRP," Compos. Sci. Technol., Vol. 186, Art. \#107922.

\section{Acknowledgements}

The authors thank and gratefully acknowledge our colleagues in Ford Research \& Advanced Engineering, Dow Chemical Company, Northwestern University, NIST, the University of Maryland, and DOE-VTO for their efforts and support of this research project. Researchers and developers from LSTC, HBM Prenscia, Autodesk, and ESTECO have also contributed to this exciting project. Over 100 research scientists and engineers have contributed to this project. 


\title{
II.2.7 Continuous Fiber Malleable Thermoset Composites with Sub-1-minute Dwell Times; Validation of Impact Performance and Evaluation of the Efficacy of the Compression-Forming Process (Mallinda LLC)
}

\author{
Dr. Philip Taynton, Principal Investigator \\ Mallinda LLC \\ 7270 Gilpin Way Ste. 180 \\ Denver, CO, 80229 \\ E-mail: philip@mallinda.com
}

\author{
Dr. Leonard S. Fifield, Co-Principal Investigator \\ Pacific Northwest National Laboratory \\ 902 Battelle Blvd. \\ Richland, WA, 99354 \\ E-mail: leo.fifield@pnnl.gov
}

\section{Dr. Bo Song, Co-Principal Investigator}

Sandia National Laboratories

1515 Eubank Blvd. SE

Albuquerque, NM, 87123

E-mail: bsong@sandia.gov

\section{Dr. Robert Norris Jr., Co-Principal Investigator}

Oak Ridge National Laboratory

1 Bethel Valley Rd.

Oak Ridge, TN, 37830

E-mail: norrisrejr@ornl.gov

\section{Dr. H. Felix Wu, DOE Technology Manager}

U.S. Department of Energy

E-mail: felix.wu@ee.doe.gov

Start Date: October 1, 2018

Project Funding (FY19): \$1,000,000
End Date: September 30, 2020

DOE share: $\$ 500,000 \quad$ Non-DOE share: $\$ 500,000$

\section{Project Introduction}

Despite the significant impact that CF composite materials have on lightweighting, performance, and efficiency in the aerospace industry, adoption of these materials has been slow in high-volume industries, such as automotive. Two major barriers to adoption are cost and cycle time. Current approaches to thermoset composite-part production are dependent on in-mold curing of thermosets, which is not optimal for highvolume, high-throughput production due to limitations and inefficiencies associated with the in-mold cure itself. In addition, ancillary activities - such as transport and the storage of shelf-life-limited uncured or Bstaged prepreg materials - typically necessitate refrigerated transport and storage, which significantly impacts the cost and energy requirements associated with manufacturing. Furthermore, limited out-life means that trimmings cannot typically be used as they partially cure during initial production leading to high scrap rates (more than $20 \%$ is common). Finally, the need to cure in-mold drives cycle times to multiple minutes in the best cases, and from tens of minutes to hours in most cases. 
Mallinda is developing polyimine vitrimer prepreg composite materials, which have excellent mechanical properties (e.g., $100 \mathrm{GPa}$ tensile modulus, $2 \mathrm{GPa}$ tensile strength, 2.4\% elongation at break) and high operating temperatures (e.g., $\mathrm{T}_{\mathrm{g}}>200^{\circ} \mathrm{C}$ ). At scale, polyimine resins are commensurate in price with commodity epoxy resins. What distinguishes malleable thermoset prepreg from traditional thermoset prepreg materials is that they are fully cured during Mallinda's roll-to-roll production of prepreg laminate. This results in five key value-differentiating benefits. First, it simplifies manufacturing logistics by enabling ambient transportation and storage and by significantly extending out-life and shelf-life almost indefinitely. Second, elimination of autoclave curing reduces the cost and energy requirements to the customer. Third, scrap rates can be reduced as malleable thermoset prepreg materials are directly reusable. Fourth, the manufacturing consolidation step can be roughly $10 \times$ faster than that of traditional thermosets because the resin is already cured. Parts can be made via compression-forming by the application of heat and pressure to quickly vitrify and consolidate a multilayer part, easily leading to less than 3-minute cycle times. At a laboratory-scale, we have demonstrated a 20 -second dwell time with room for further optimization. Finally, the closed-loop cradle-to-cradle solutionbased recyclability of malleable thermoset composites can also contribute significantly to the future of sustainable lightweight materials.

Technical background on vitrimers (also known as malleable thermosets) includes:

- Recent major advances in polymer chemistry enable a paradigm shift in the area of processing and the re-processing/recycling of advanced composite materials. Inexpensive compression molding with short cycle times, traditionally available for thermoplastic composites only, are now possible for the thermoset resins based on polyimine chemistry. One key feature of the proposed technology is the malleability of the thermoset polyimines, which allows for thermoplastic-like flow at elevated processing temperatures, but thermoset-like stability and strength at operating temperatures. The re-processability of these malleable thermoset materials (i.e., vitrimers) is attributable to the active exchange of covalent chemical bonds at elevated temperatures. Stress-relaxation studies have shown that below the glass transition temperature $\left(\mathrm{T}_{\mathrm{g}}\right)$, malleable polyimine materials behave as traditional thermosets while, above the $\mathrm{T}_{\mathrm{g}}$, malleable behavior enables molding, welding, and repair [1,2]. These novel and unique malleable properties allow for the facile forming and reforming of fabricated polyimine composite structures in a process analogous to metal compression-forming. Another key feature of the polyimine system is intrinsic recyclability due to dynamic covalent chemistry that enables closed-loop recycling of both resin and filler material (e.g., fibers, ceramic) from the composite. The environmental and economic impact of recoverable resin from advanced composite scrap materials and end-of-life products is significant.

- Despite the promise of malleable thermoset composites, large industry players (who are investing heavily in further optimization of incumbent thermoset-curing techniques) are reluctant to engage until the technology is fully validated. While Mallinda has validated the manufacturability of its resins by conducting full-scale prepreg production trials with a manufacturing partner, a few critical areas remain, and these require experimental validation beyond the capabilities of Mallinda and existing third-party commercial-testing facilities. Fortunately, these capabilities can be found within the LightMAT network at PNNL, Sandia National Laboratories (SNL) and ORNL. Specifically, the ability to nondestructively characterize microscopic structural defects (such as voids and resin-rich areas) and to characterize highspeed impact performance are critical to the validation and adoption of these materials.

\section{Objectives}

The focus of this project is the development, optimization, and validation of malleable thermoset composite materials that exhibit manufacturing cycle times of three minutes or less, high-speed impact performance on par with incumbent technologies, and defect-free consolidation of three-dimensional (3D) parts. 


\section{Approach}

In order to accomplish these objectives, the following LightMAT resources will be included in this project:

1. Test Machine for Automotive-Crashworthiness. PI: Bob Norris, ORNL.

2. Ultrasonic analysis of composite defects in 3D parts. PI: Michael Larche, PNNL.

3. High-speed impact material characterization. PI: Bo Song, SNL.

The following tasks will be accomplished at the industry partner (Mallinda) sites and will be funded by the industry partner (Mallinda) in fulfillment of the in-kind cost-match requirement:

1. Lab-scale development and optimization of malleable thermoset materials for high-throughput compression-forming environment.

2. Analysis of thermal-mechanical properties and performance of composite materials for development and validation purposes (including third-party validation of mechanical properties).

3. Automotive-crashworthiness trials (including material synthesis and sample preparation).

4. High-speed-impact material characterization (including material synthesis and sample preparation).

5. Ultrasonic analysis of composite defects (including material synthesis and sample preparation).

6. Project management and reporting requirements.

The following tasks will be carried out at LightMAT sites and will be funded by the DOE LightMAT consortium:

1. Automotive-crashworthiness trials.

2. Ultrasonic analysis of composite defects.

3. High-speed-impact material characterization.

\section{Results}

In the first year of the project, the Mallinda team successfully demonstrated a wide range of resin formulations to cover a broad spectrum of potential impact performances from low-modulus relatively high-elongation formulations to high-modulus lower-elongation materials. In addition, the Mallinda team has developed custom resin solutions for integration with various fibers to enable the hybrid-fiber approach, including CF, glass fiber, ultrahigh-molecular-weight polyethylene (UHMWPE) fiber, steel fiber, and aramid fibers. The Mallinda team has further scaled the resin and composite preparations and worked with a partner to produce sample automotive parts via compression-forming. In addition, the Mallinda team provided samples to support method-development for both ultrasonic imaging (at PNNL) and split-Hopkinson bar testing (at SNL). The initial or baseline results of both tests were also completed in the first year of this project.

The Mallinda team produced and tested over 100 unique formulations in order to develop custom vitrimer resins to support this project's hybrid-fiber approach. In Figure II.2.7.1 (a), the successful impregnation of $\mathrm{CF} / \mathrm{UHMWPE}$ is demonstrated. This is by far the most challenging hybrid-fiber combination for a thermoset resin to accommodate. In addition to developing resin solutions for the hybrid-fiber approach, the Mallinda team worked with a private partner to develop a production technique for forming complex 3D shapes via compression-forming of precured vitrimer-matrix composite sheets. A finished part resulting from this development is shown in Figure II.2.7.1 (b). 


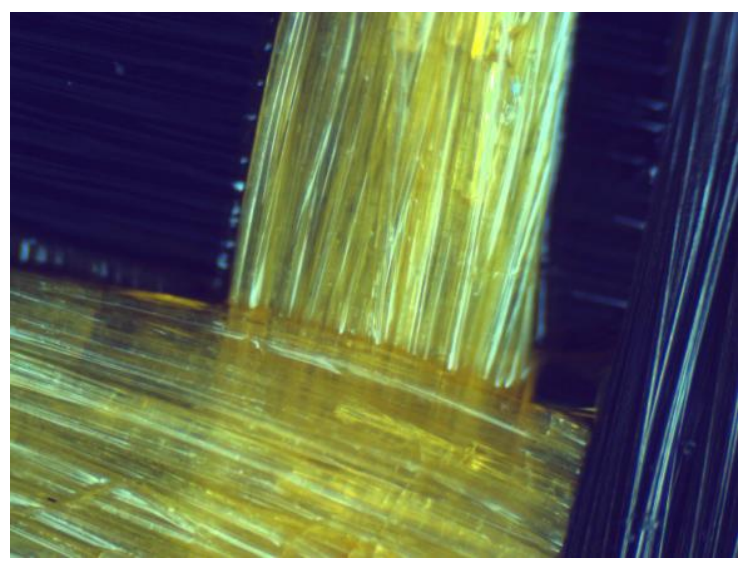

(a)

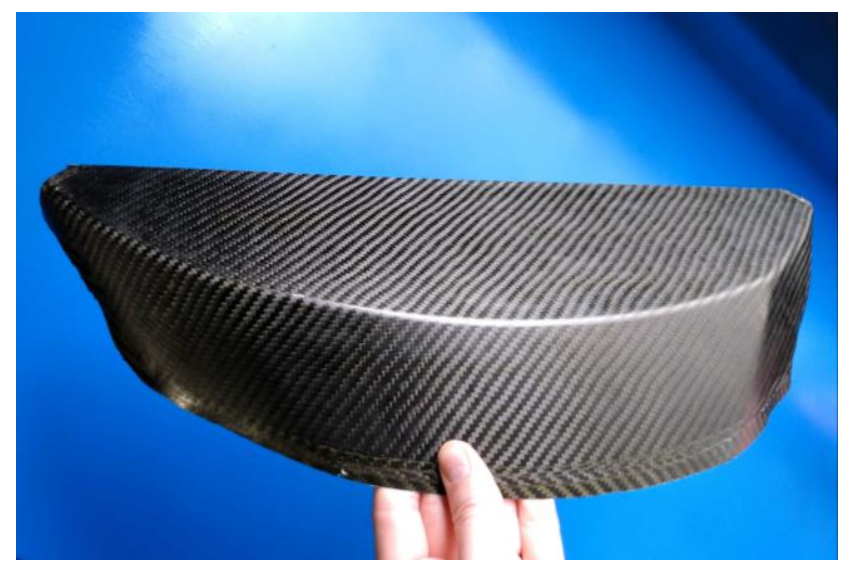

(b)

Figure II.2.7.1. (a) Successful impregnation of CF/UHMWPE hybrid-fiber. (b) Successful demonstration of compressionforming precured vitrimer-matrix parts with complex curvature. Source: Mallinda.

This new compression-forming process will be used to produce parts for this study moving forward including parts for ultrasonic testing, split-Hopkinson bar testing, and automotive-crashworthiness testing (using the Test Machine for Automotive-Crashworthiness). Samples were supplied by the Mallinda team to support initial baseline testing and method-development for both ultrasonic analysis and split-Hopkinson bar testing. Figure II.2.7.2 (a) shows the preliminary results of ultrasonic analysis on the sample materials.

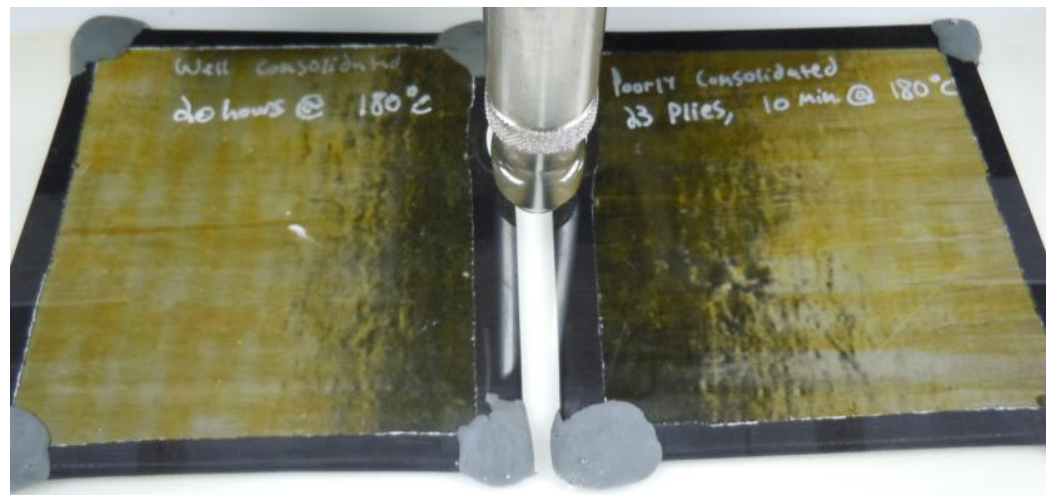

(a)

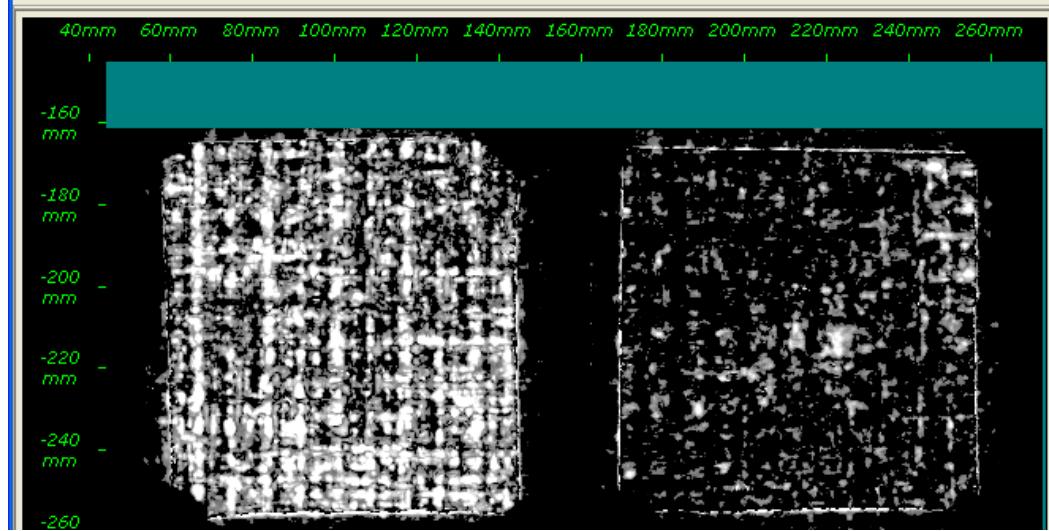

(b)

Figure II.2.7.2. Application of C-scan ultrasonic analysis to vitrimer-matrix composites. (a) Though visibly similar, the difference in consolidation conditions can be clearly seen in (b) the C-scan images. Source: PNNL. 
A well-consolidated sample, as shown on the left of Figure II.2.7.2 (b), shows good reflection of sound off the back surface. This is indicated by the high-amplitude reflections (white pixels) observed in the gated window. By contrast, the poorly consolidated sample on the right of Figure II.2.7.2 (b) shows scattering of the sound through the depth of the sample which is observed by the lack of reflection (black pixels) off the back surface.

Split-Hopkinson bar testing, conceptually illustrated in Figure II.2.7.3, is critical for obtaining a profile of material performance over a range of strain rates. The initial results of split-Hopkinson bar dynamic tensile testing are shown below in Figure II.2.7.4 for a series of strain rates. The data show material strength increasing with increasing strain-rate, a typical strain-rate effect, with the best performance at a rate of $530 \mathrm{~s}^{-1}$.

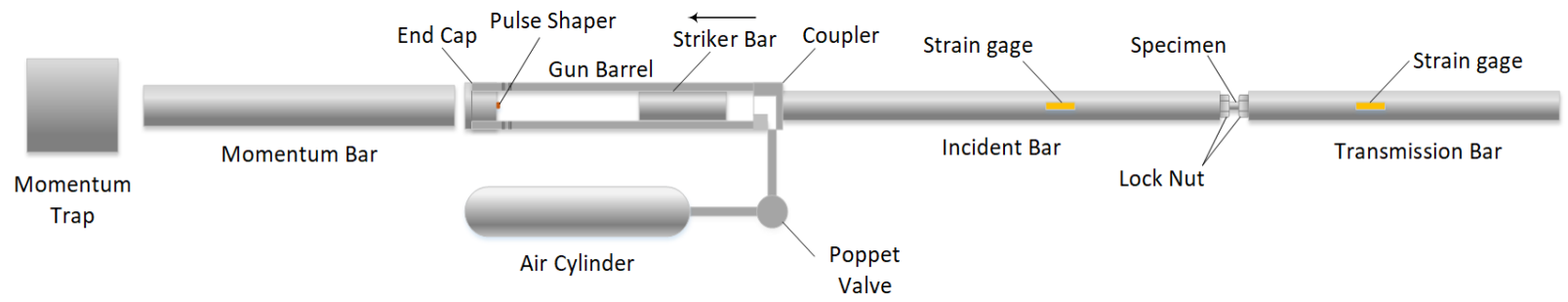

(a)

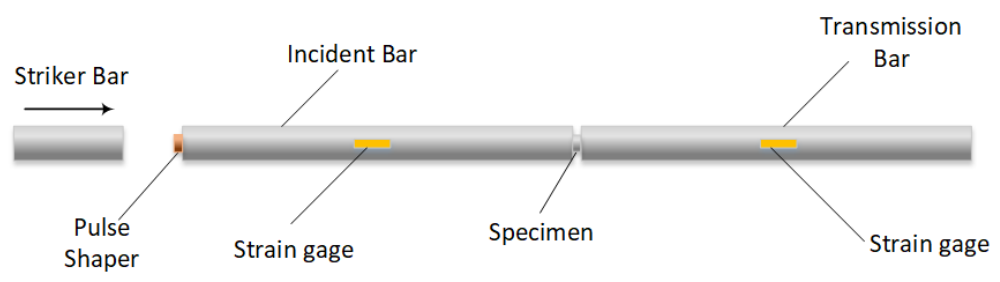

(b)

Figure II.2.7.3. Application of split-Hopkinson bar (Kolsky bar) testing to vitrimer-matrix composites for (a) tension configuration and (b) compression configuration. Source: SNL. 


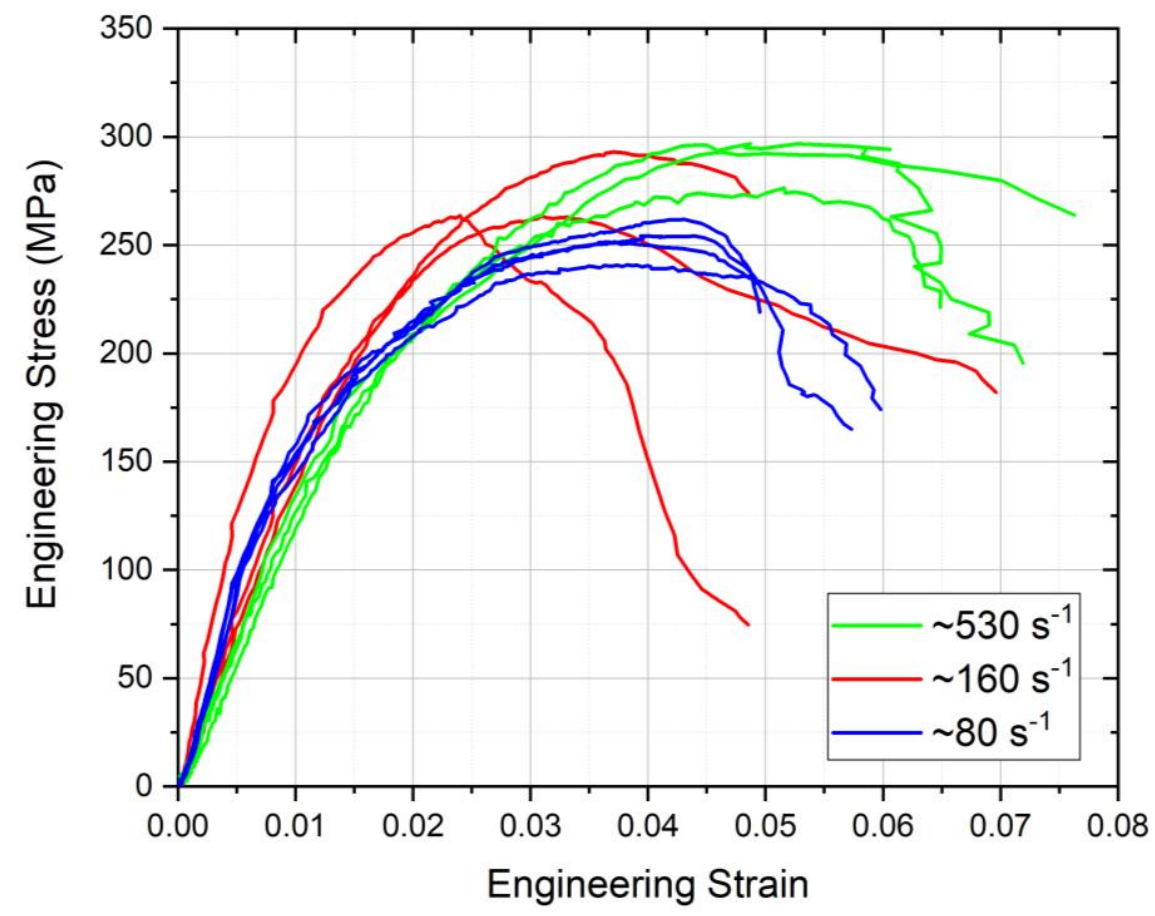

Figure II.2.7.4. Dynamic tensile stress-strain curves at three test rates. Source: SNL.

Initial dynamic compressive testing results are shown below in Figure II.2.7.5 (a) for out-of-plane loading and Figure II.2.7.5 (b) for in-plane loading. The out-of-plane image is for a loading direction perpendicular to the plate while the in-plane image is for a loading direction parallel to the plate ( $\sim 45$ degrees/135 degrees to the fibers). For the out-of-plane data, the stress-strain curves are more linear, the modulus (slope) along this direction is nearly constant and strain-rate effect is insignificant. For the in-plane direction, the slope of the stress-strain curves increases with increasing strain-rate, exhibiting a more significant strain-rate effect than the out-of-plane data.

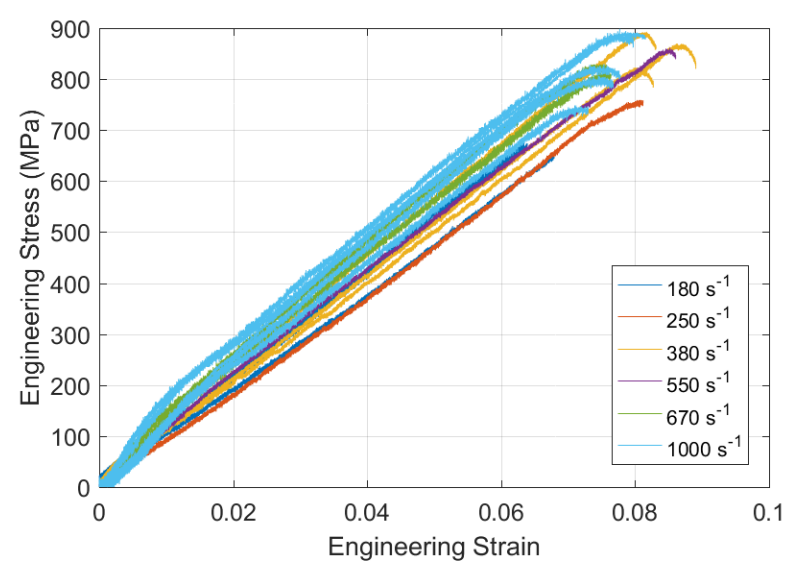

(a)

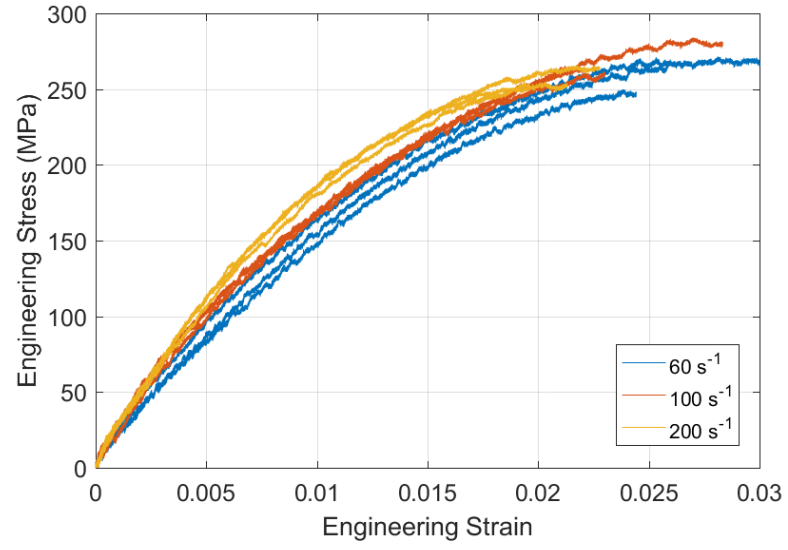

(b)

Figure II.2.7.5. Dynamic compressive stress-strain curves in (a) the out-of-plane and (b) the in-plane directions. Source: SNL. 
The development of the split-Hopkinson method and apparatus for this application will provide the primary test bed for impact performance of hybrid composites for this study. These results will be used to down-select materials for automotive-crashworthiness studies.

\section{Conclusions}

In conclusion, the work carried out in the first year of this project is on track with the initial statement of work, and the second year of the project should see a successful completion of the tasks. While the impact performance of hybrid-fiber vitrimer-matrix composites is still to be determined, the materials and methoddevelopment are now in place to enable demonstration of the key results of this study. Consolidation of cured plies was completed, but further optimization and ultrasonic validation are needed to get this process within the sub-three-minute target time desired by DOE.

\section{References}

1. Taynton, P., K. Yu, R. Shoemaker, Y. Jin, H. J. Qi, and W. Zhang, 2014, "Heat- or water-driven malleability in a highly recyclable covalent network polymer," Adv. Mater., Vol. 26, pp. 3938-3942.

2. Taynton, P., H. Ni, C. Zhu, K. Yu, S. Loob, Y. Jin, H. J. Qi, and W. Zhang, 2016, "Repairable woven CF composites with full recyclability enabled by malleable polyimine networks," Adv. Mater., Vol. 28, pp. 2904-2909. 


\title{
II.2.8 Ultra-Lightweight, Ductile Carbon Fiber Reinforced Polymers (Oak Ridge National Laboratory)
}

\author{
Vlastimil Kunc, Principal Investigator \\ Oak Ridge National Laboratory \\ 1 Bethel Valley Road \\ Oak Ridge, TN 37831 \\ E-mail: kuncv@ornl.gov
}

\section{Dr. H. Felix Wu, DOE Technology Development Manager}

U.S. Department of Energy

E-mail: felix.wu@ee.doe.gov

Start Date: January 1, 2018

Project Funding (FY 19): \$460,000

End Date: September 30, 2020

DOE share: $\$ 460,000 \quad$ Non-DOE share: $\$ 0$

\section{Project Introduction}

CFRP composites are known for their high-stiffness-to-weight ratio and, hence, are of great interest in diverse modern engineering fields, such as aerospace, automotive, and defense, etc. Despite their lightweight and highstiffness, such composites are not suitable for dissipating energy because failure occurs with very little or no plastic deformation. In this work, we present a scalable multi-material stereolithography process for the fabrication of multiphase materials, which enables us to develop a novel design of a three-dimensional lightweight cellular CFRP microlattice to reach its high-stiffness and damping pairs. We measured the damping performance of the microlattice at small- and large-strain regimes via dynamic mechanical analysis (DMA) and quasi-static cyclic compression tests, respectively. Using these measurements, we characterize the intrinsic and structural damping performance in terms of a damping figure of merit, defined as $E^{1 / 3} \tan \delta / \rho$. Finally, we compare the microlattice with other existing materials in a material damping performance map $\left(\tan \delta\right.$ in $\mathrm{x}$-axis and $\mathrm{E}^{1 / 3} / \rho$ in $\mathrm{y}$-axis).

\section{Objectives}

In this project, we proposed the design to address the limitation in the brittleness in additively manufactured CFRP composite and harness the benefit of hierarchical architecture to achieve high-stiffness - damping pairs. To achieve this, the following proposed tasks need to be accomplished:

1. Develop an ultraviolet (UV)-curable CFRP composite feedstock and a light-based AM platform.

2. Design and fabricate a lightweight cellular CFRP microlattice to reach its high-stiffness-damping pairs.

3. Evaluate of the design through experimental test and analytical calculations.

\section{Approach}

Our approach, detailed below, is based on developing a new AM method to produce CFRP composites with a rationally designed dissipative phase. The hybrid material system allows attainment of the upper bound of stiffness and damping pairs at low density. 


\section{Material Preparation and Development of Multi-Material 3D Printing System}

To fabricate the lightweight cellular CFRP microlattice, a UV-curable CFRP composite was first developed. The composite was made of a UV-sensitive resin reinforced with $\sim 70 \mu \mathrm{m} \mathrm{CF}$. A high-energy ball mill was used to mix the monomer, photo-initiator, and CF. This resin mixture method ensured the uniform spatial distribution of the fibers. Incorporating the dissipative inclusion into prescribed sites within the CFRP struts was achieved via a multi-material AM system we developed, as shown in Figure II.2.8.1 (a). The multimaterial AM process involves a resin recoating process and a material switching system. Figure II.2.8.1 (b) is a schematic of the recoating process, depicting the behavior of the viscous resin during the process. To print a structure, a computer-aided design model was sliced into different groups of closely spaced images such that each group represented one material. These $2 \mathrm{D}$ slices were subsequently digitized and sent to a digital micromirror device chip, which projects an image at $405 \mathrm{~nm}$ wavelength through a series of lens onto the corresponding photosensitive resin. This process initiates polymerization of the resin, converting it into a solid single layer part, which has the same shape as the projected images. When the other materials needed to be fabricated in the same layer or the next layer, the transition stage moved the platform to the washing vat, and the previously printed part is cleaned by a cleaning dispenser. After cleaning, the platform moved to the other resin vat to print the next layer. Figure II.2.8.1 (c)-(g) shows pictures of samples with multiple different 3D micro-architectures fabricated with the multi-material AM system. Although we focus on an octet-truss structure in this study, the AM system developed here can be used for any design of micro-architecture. The minimum feature size the system can fabricate is $150 \mu \mathrm{m}$ as shown in the close-up view of Figure II.2.8.1 (h). 

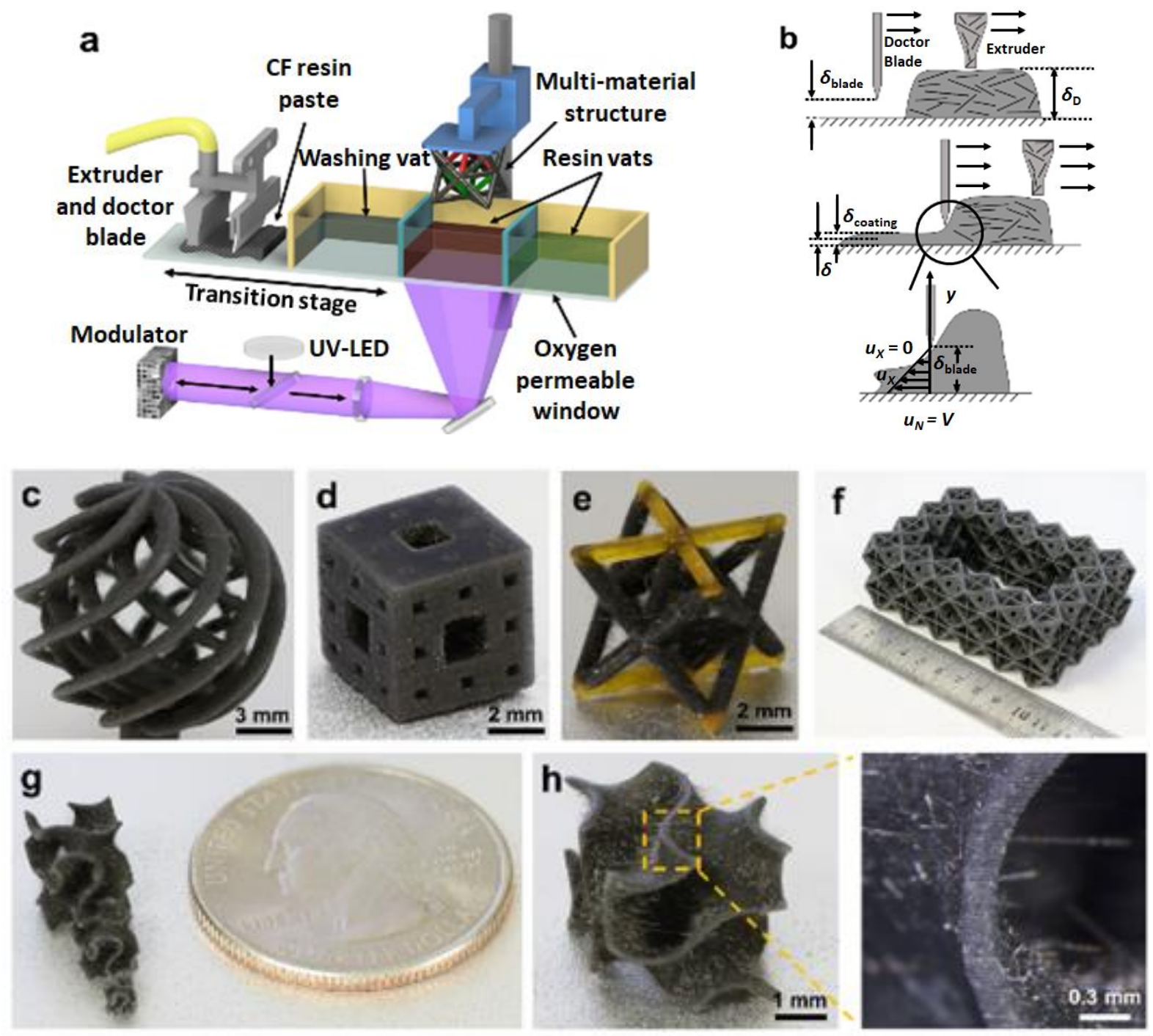

Figure II.2.8.1. Customized multi-material AM system and fabricated samples. (a) Schematic of multi-material projection micro-stereolithography process integrated with the tape-casting method. (b) Schematic of the recoating process, depicting

the behavior of the viscous resin during the process. (c)-(d) Complex 3D structures fabricated by the system. (e) Multimaterial octet-truss unit cell complying with CFRP and polyethylene glycol diacrylate resin. (f) Closed cell made of CFRP in a large-scale. (g-h) Arbitrary gyroid 3D structure with a minimum feature of $150 \mu \mathrm{m}$. Source: Virginia Tech.

\section{Testing Methods Including Small-strain DMA Test and Large-strain Cyclic Test}

Energy dissipation mechanisms can be generally categorized into intrinsic and structural damping $[1,2]$. To capture intrinsic damping properties of the lightweight cellular CFRP microlattice, we performed small-strain DMA tests using the TA Instruments DMA 850. All tests were performed with a maximum strain of $0.05 \%$ to ensure that all fabricated samples were excited elastically at small-strains. The results measured at $0.1 \mathrm{~Hz}$ were adopted here for a direct comparison of damping property (e.g., $\tan \delta$ ) obtained from small-strain DMA and quasi-static large-strain experiments, since the frequency of the quasi-static compression tests is usually considered as $<0.1 \mathrm{~Hz}[1]$. Structural damping properties were investigated by performing large-strain quasistatic cyclic compression tests using an INSTRON 5944. A strain-rate of $10^{-3} / \mathrm{s}$ was adopted to ensure that all tests were performed in a quasi-static regime to suppress the mass inertia effect. From the measured stressstrain hysteresis loop, we computed the effective modulus from a slope of a loading curve in the linear region, dissipated energy $(\Delta U)$ from the area within the stress-strain hysteresis loop, and stored energy from the area under the loading curve $(U)$. The last two measures were used to evaluate the damping performance of the 
microlattice via the loss coefficient $(\Psi)$ defined as $\Psi=\Delta U / U[1]$. This quantity was then converted to $\tan \delta$ via $\Psi=(\pi / 2) \tan \delta[2,3]$, for a direct comparison between the structural damping and intrinsic damping. Note that this conversion represents a quarter of a full cycle compressive loading-unloading response [2].

\section{Results}

\section{Characterization of the Fabrication Process of CFRP}

Our AM technique was designed to achieve a minimum printable 3D feature size of $\sim 50 \mu \mathrm{m}$ in the projection plane. This was determined by the pixel resolution of the digital micromirror device array and the optical components. The resolution in the vertical direction was determined by a driving motor, which was $5 \mu \mathrm{m}$, and we set the layer thickness as $40 \mu \mathrm{m}$. To demonstrate the capabilities of our technique, we fabricated some complex 3D samples from micro- to macro-scales, as shown previously in Figure II.2.8.1 (c)-(h).

\section{Design of a Lightweight, High Stiff and High-Damping CFRP Cellular Microlattice}

It is promising to utilize a two-phase composite layout to achieve a high-stiffness-loss pair [2]. Figure II.2.8.2 (a) illustrates a stiffness-loss tangent relationship of the two-phase material layouts; the percentage in this figure refers to the volume fraction of Phase 1. As depicted in Figure II.2.8.2 (b), the Reuss layout having the volume fraction of Phase 2 equal to approximately $0.9 \%$ was found to exhibit a maximal figure of merit (FOM) for bulk materials. The FOM, defined as $\mathrm{E}^{1 / 3} \tan \delta / \rho$, represents the damping performance of a structure. With this, to design a lightweight structure, we chose an octet-truss cell as our blueprint topology since it is one of the stretch-dominated topologies capable of maintaining a constant specific stiffness at lower-density compared to bending-dominated foams $[4,5]$. This led to a design of a multi-material architecture consisting of multiple octet-truss unit cells made of CFRP and designed soft inclusion in selected out-of-plane struts, as shown in Figure II.2.8.3 (a). The soft inclusion ratio in a single Reuss strut, $V_{\text {soft }}$, defined as the ratio of $h$ to $H$. The relative density of the microlattice $(\bar{\rho})$ is defined approximately as $\bar{\rho}=6 \pi \sqrt{2}(r / l)^{2}$, where $r$ is the strut radius and $l$ is the strut length $[5,6]$. As shown in Figure II.2.8.3 (c), a SEM image was taken at the boundary showing a desired separation between the two phases; in this figure, the top is CFRP, whereas the soft phase is shown at the bottom.

a

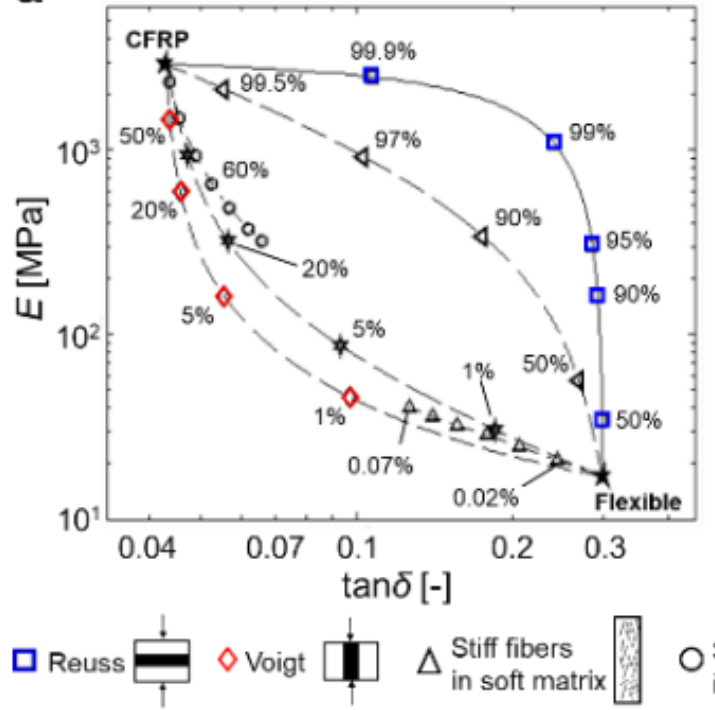

b

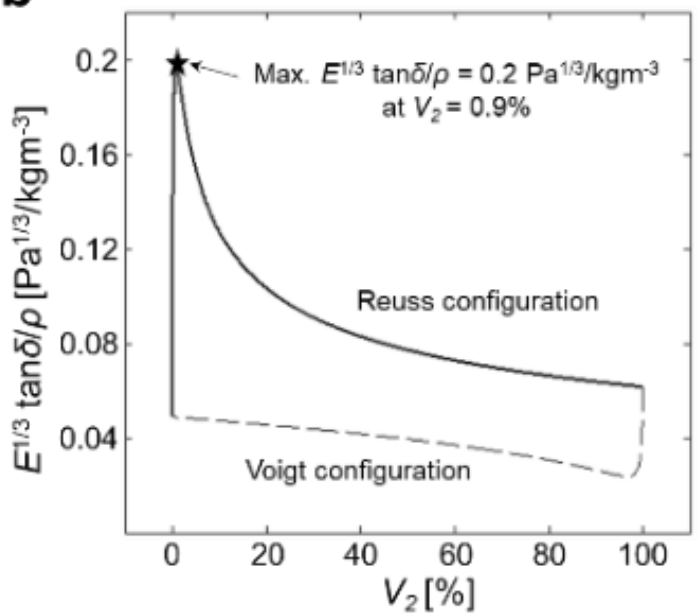

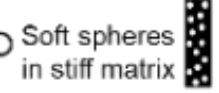

$\triangleleft$ Hashin-Shtrikman lower

Hashin-Shtrikman upper

Figure II.2.8.2. The effect of soft phase on the bulk CFRP stiffness-damping properties. (a) Stiffness ( $E$ ) versus loss tangent (tan $\delta$ ) for various two-phase configurations incorporating CFRP and soft phase of bulk material. The percentage shown here corresponds to the volume fraction of the stiff phase. Graphical legends are provided on the bottom of this figure.

(b) $E^{1 / 3} \tan \delta / \rho$ versus the volume fraction of the soft phase $\left(V_{2}\right)$. This index reaches its maximum at $V_{2}=0.9 \%$.

Source: Virginia Tech. 
a

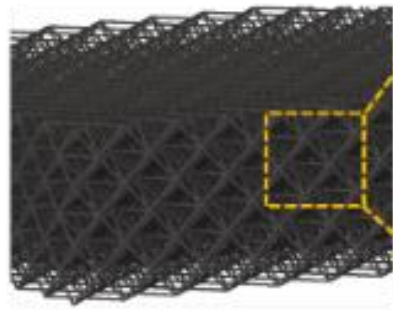

b

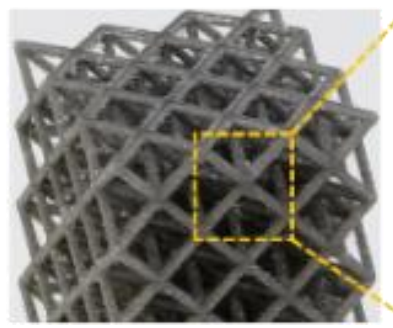

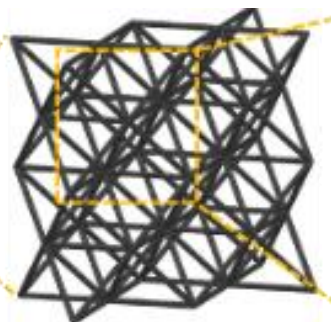

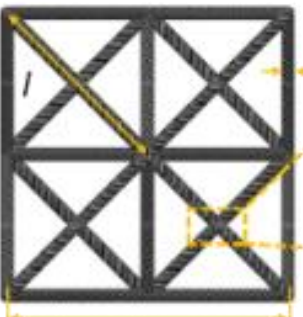

H

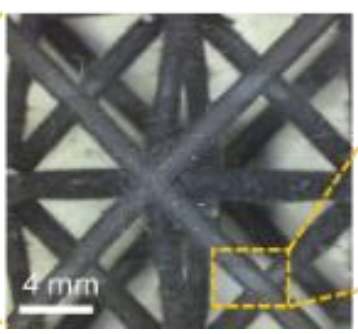

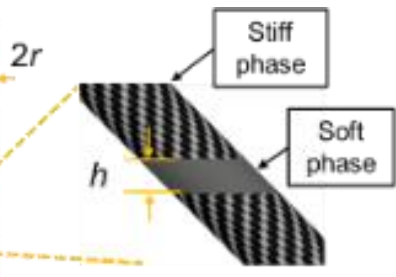

C

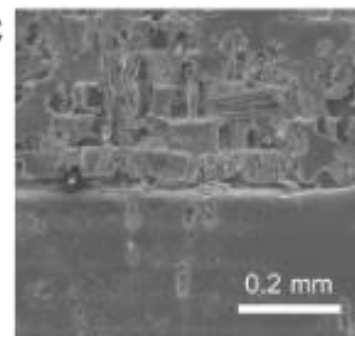

Figure II.2.8.3. Multi-material architecture. (a) Design of lightweight, stiff, high-damping microlattice with two-phase materials incorporating CFRP and soft phase. (b) Fabricated lightweight cellular CFRP microlattice having $\bar{\rho}=7 \%$ with $V_{\text {soft }}=9 \%$. (c) SEM image showing the interface between the two phases. Source: Virginia Tech.

\section{Experimental Investigation of the Energy Dissipation Mechanism}

Figure II.2.8.4 depicted the intrinsic damping property obtained from DMA tests of the samples having $\bar{\rho}=7 \%$ with various soft phase ratios. We observed that the effective modulus (i.e., storage modulus) of the samples was inversely proportional to $V_{\text {soft }}$ and showed an exponential relationship as a function of $V_{\text {soft }}$ via $E=$ $a^{*} \exp \left(b V_{\text {soft }}\right)+c^{*} \exp \left(d V_{\text {soft }}\right)$. Conversely, $\tan \delta$, representing intrinsic damping, is nonlinearly proportional to $V_{\text {soft }}$ via $\tan \delta=a^{*} \exp \left(b V_{\text {soft }}\right)+c$. The computed FOM reaches its peak when $V_{\text {soft }}$ is equal to approximately $10 \%$. In this study, we only considered a single relative density since the intrinsic damping is invariant with respect to the relative density [2].
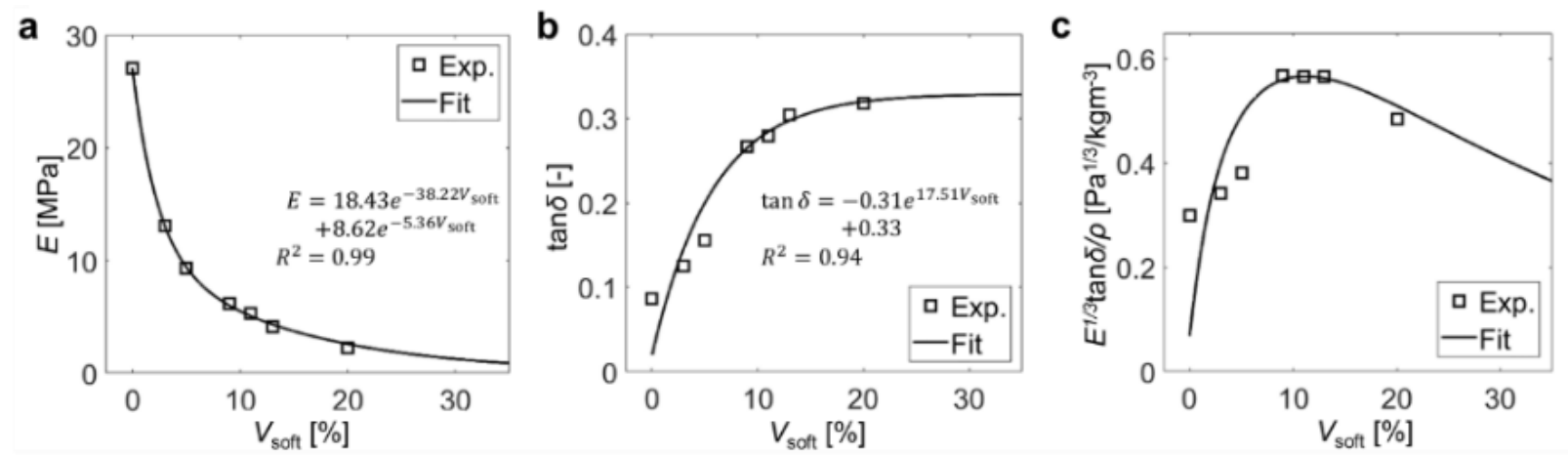

Figure II.2.8.4. Intrinsic damping properties of the lightweight cellular CFRP microlattice having a relative density of $7 \%$ with different soft phase ratios. (a) The effective modulus $(E)$ as a function of the soft phase ratio $V_{\text {soft. }}$ (b) Loss tangent (tan $\delta$ ) as

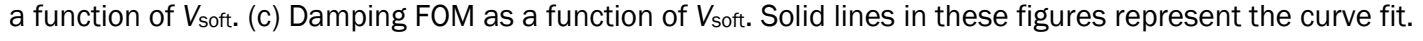

Source: Virginia Tech.

For the structural damping property, stress-strain hysteresis loops of samples with $\bar{\rho}=4 \%$ and $\bar{\rho}=12 \%$ having $V_{\text {soft }}=20 \%$ in response to multicyclic compression (30 cycles) are shown in Figure II.2.8.5 (a) and Figure II.2.8.5 (b), respectively. In Figure II.2.8.5 (c), we investigated the evolution of the stress-strain hysteresis loops over different relative densities while holding $V_{\text {soft }}$ constant. Dominant deformation mechanism at a low relative density $(\bar{\rho}=4 \%)$ was elastic buckling of the constituent struts. However, this buckling response diminished with an increase in the relative density $(\bar{\rho}=7 \%)$, and plastic yielding 
mechanism started to dominate at a higher relative density $(\bar{\rho}=20 \%)$. We also observed that for uniform $V_{\text {soft }}$, the effective modulus of the microlattice was proportional to an increase in the relative density, as shown in Figure II.2.8.5 (d). In addition, all samples exhibited the maximum FOM at specific $V_{\text {soft }}$ as shown in Figure II.2.8.5 (f).
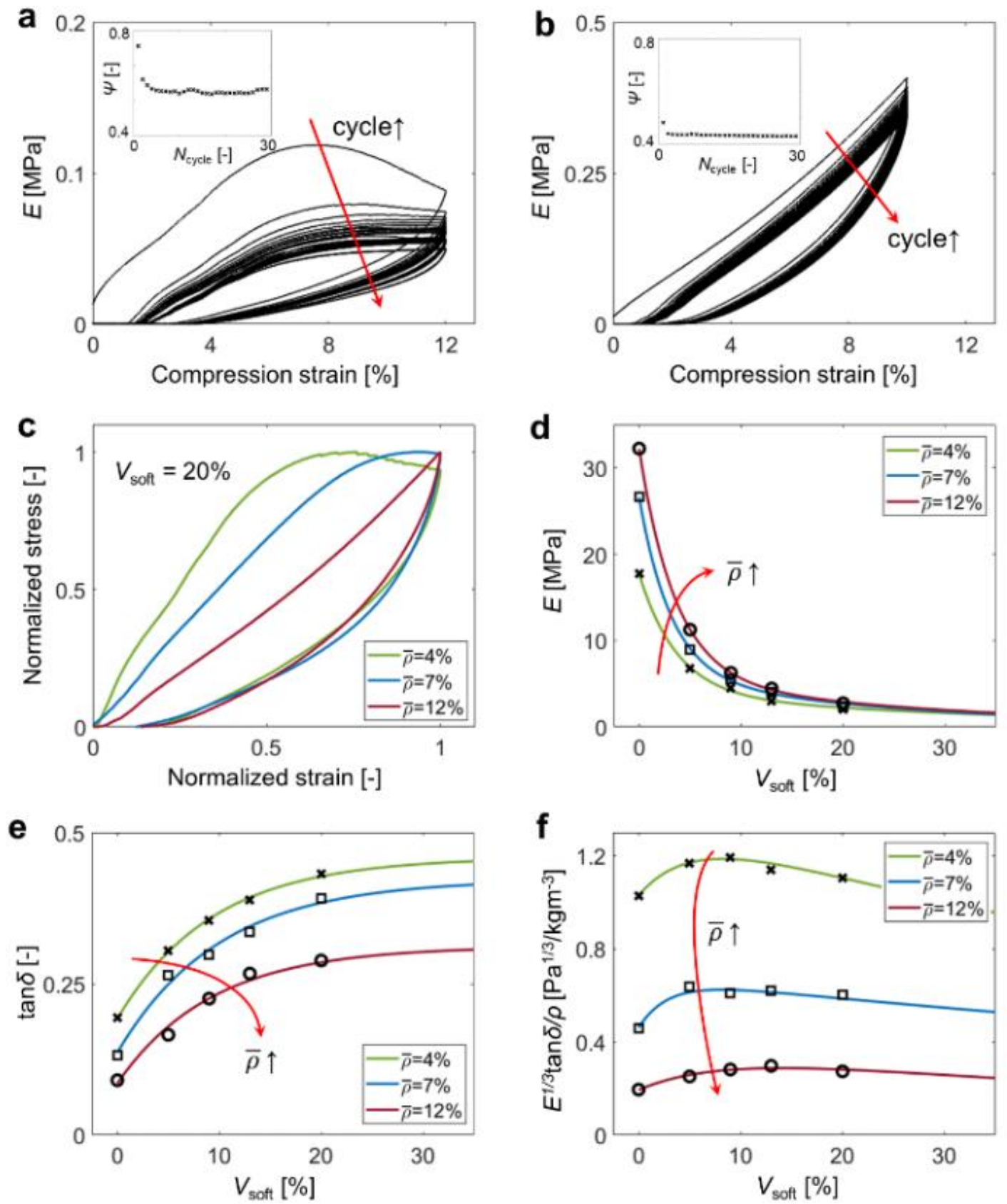

Figure II.2.8.5. Structural damping properties of the lightweight cellular CFRP microlattice obtained from large-strain quasistatic cyclic compression tests. (a)-(b) Hysteresis loops from multicyclic compression tests for 30 cycles and evolution of the loss coefficient with the cycle number. (a) Sample with $\bar{\rho}=4 \%$ having $V_{\text {soft }}=20 \%$. (b) Sample with $\bar{\rho}=12 \%$ having $V_{\text {soft }}=20 \%$. (c) Normalized stress-strain hysteresis loops of samples having $V_{\text {soft }}=20 \%$ with various $\bar{\rho}$. Elastic buckling behavior was observed for $\bar{\rho}=4 \%$ and diminished with an increase in $\bar{\rho}$. (d) The effective modulus $(E)$ as a function of $V_{\text {soft. }}$

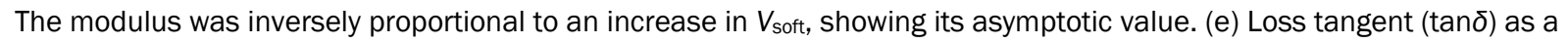
function of $V_{\text {soft. }}$ An increase in $V_{\text {soft }}$ led to an improvement in loss tangent. (f) Damping FOM as a function of $V_{\text {soft. }}$ FOM can be tuned to have a peak when $V_{\text {soft }}$ is approximately $10 \%$. Source: Virginia Tech. 
Tunability maps of the intrinsic and structural damping performance, as shown in Figure II.2.8.6, were obtained by using the curve fit of experimentally obtained FOM. The FOM for both intrinsic and structural damping has its maximum at low relative densities and at the soft inclusion ratio of approximately 5 10\%. Moreover, as illustrated in Figure II.2.8.7, a $E^{1 / 3} / \rho$ - $\tan \delta$ map was created to quantitatively assess the performance of the stiffness-damping pair of the present microlattice alongside other existing materials. We observed that the CFRP microlattice is superior to any CFRPs available by almost two orders of magnitude with respect to $\tan \delta$. Furthermore, the microlattice exhibits a relatively high specific stiffness per density as that of commercial CFRPs and technical ceramics and composites and high-damping comparable to that of elastomers.
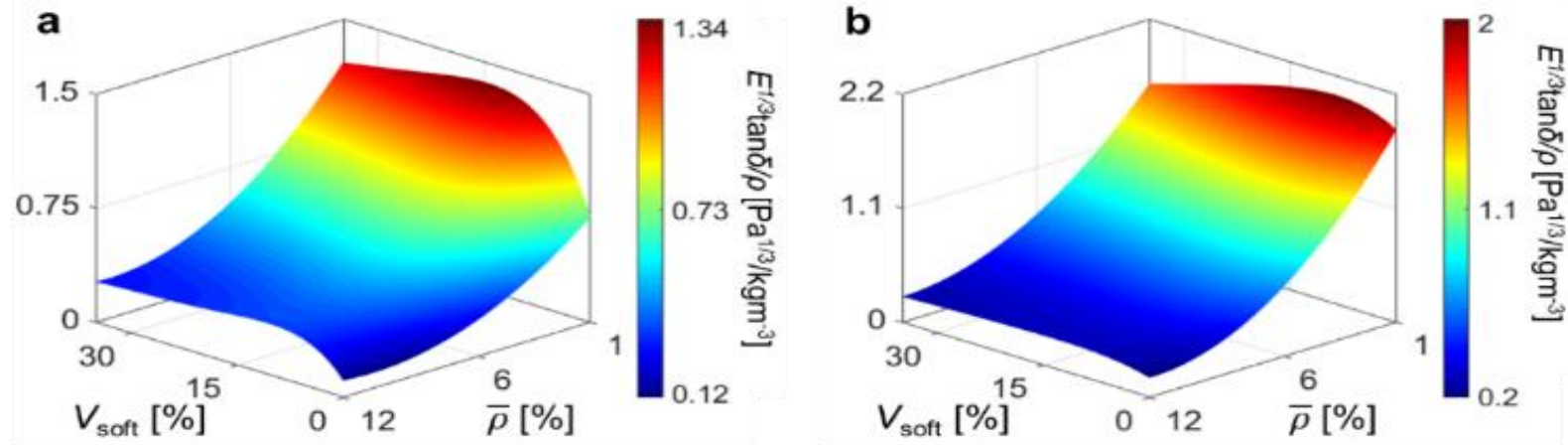

Figure II.2.8.6. Tunability maps for: (a) intrinsic; and (b) structural damping performance in terms of the damping FOM obtained from the experimental measurements. Source: Virginia Tech.

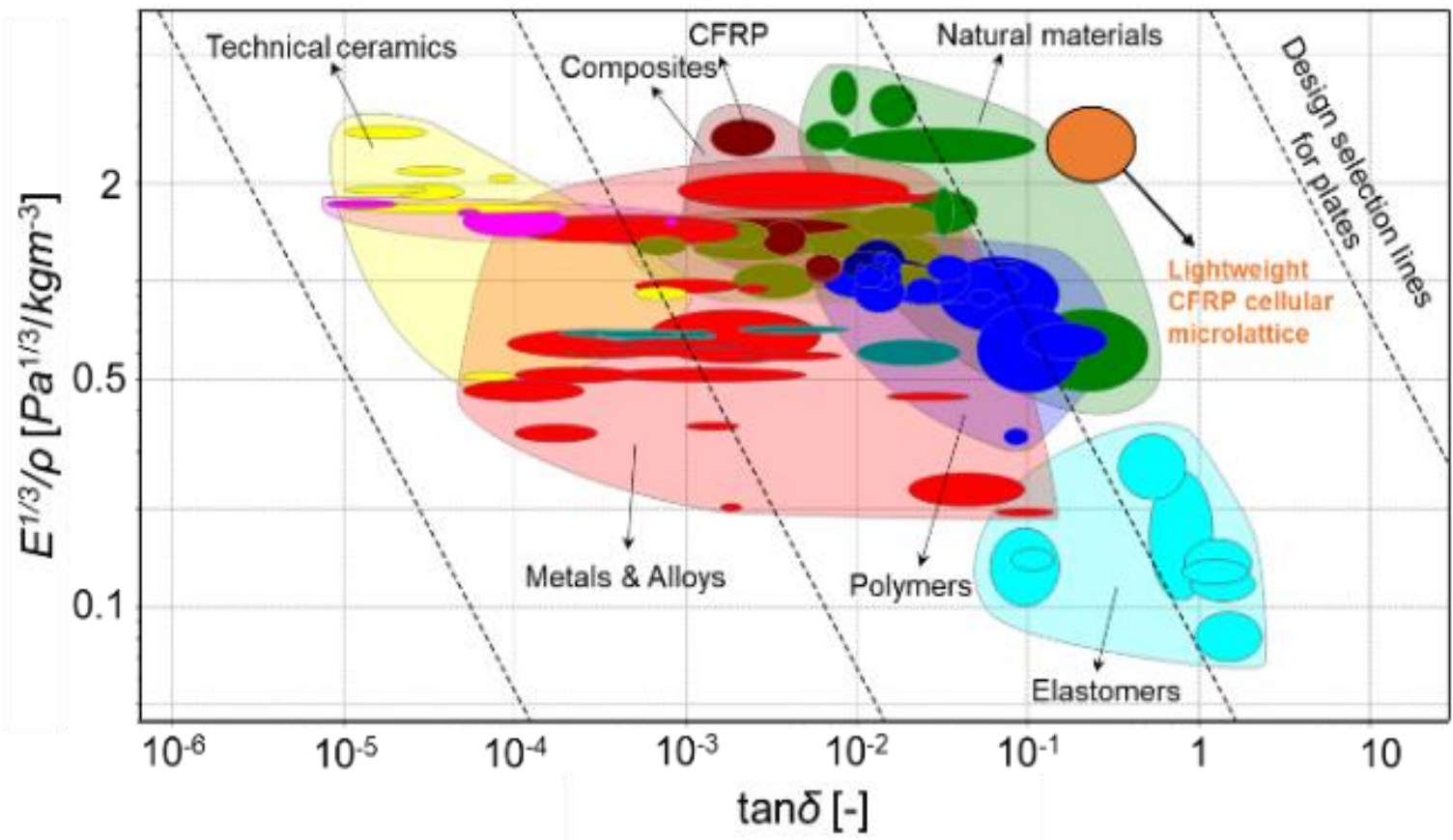

Figure II.2.8.7. Assessment of the lightweight cellular CFRP microlattice developed in this work. The orange ellipse represents the envelope of experimental results for an overall damping property. The CFRP microlattice exhibits similar stiffness as commercially available CFRP composites while providing high-damping as like that of elastomers. (Image is created using CES Granta [7]) The property of the lightweight microlattice (in orange color) is from our experimental results. All the other material properties are from CES Granta library. Source: Virginia Tech. 


\section{Analytical Model}

We developed analytical models valid at small-strains and large-strains separately to compare with the experimental results. By applying the force-moment equilibrium, the Timoshenko beam theory, and the correspondence principle in the context of viscoelasticity together, the effective modulus of the structure under the UC can be written as:

$$
E_{z}^{*}=\frac{2 \sqrt{2}}{9} \pi\left(\frac{r^{2}}{L_{\text {strut }} L_{\text {eff }}}\right)\left[E_{\mathrm{CF}}+2 E_{\text {Reuss }}^{*}\left(1+12 C_{1} \frac{r^{2}}{L_{\text {eff }}^{2}}\right)\right]
$$

where $E_{\text {Reuss }}^{*}=\left[\frac{V_{\text {soft }}}{E_{\text {soft }}^{*}}+\frac{1-V_{\text {soft }}}{E_{\mathrm{CF}}}\right]^{-1}, C_{1}=\left[\frac{12 E_{S} I}{\kappa A G_{\text {Reuss }} L_{\text {eff }}^{2}}\right]^{-1}, G_{\text {Reuss }}=\frac{E_{\text {Reuss }}}{2\left(1+v_{\text {strut }}\right)}$, and $v_{\text {strut }}=\left[\frac{V_{\text {soft }}}{v_{\text {soft }}}+\frac{1-V_{\text {soft }}}{v_{\mathrm{CF}}}\right]^{-1}$, $V_{\text {soft }}$ is the volume fraction of the soft phase, $V_{\mathrm{CF}}$ is the volume fraction of the CFRP phase in the single strut, and $L_{\text {eff }}$ refers to the effective length, defined as $L_{\text {eff }}=L_{\text {strut }}-2 r$ where $L_{\text {strut }}$ is the length of the strut and $r$ is the radius of a circular cross-section of the strut. When the deformation is small, the intrinsic damping can be obtained via $\tan \delta=E^{\prime \prime} / E^{\prime}$ using Eq. (1), where $E_{z}^{\prime}$ is the storage modulus (real part of $E_{z}^{*}$ ) and $E_{z}^{\prime \prime}$ is the loss modulus (imaginary part of $E_{z}^{*}$ ). On the other hand, structural damping is caused by large-strain deformations, and the microlattice results in energy dissipation through either elastic buckling or brittle-like deformation. Here, we assume that in-plane CFRP struts would not dissipate any mechanical energy because CFRP has negligible damping as compared to that of a soft phase $\left(\tan \delta_{\mathrm{CF}} \sim 0.07\right.$ and $\left.\tan \delta_{\mathrm{soft}} \sim 0.3\right)$. In the case of a dominant failure due to elastic buckling, the out-of-plane strut would buckle when the maximum stress reaches to the critical buckling strength of the strut. According to Euler buckling theory and a simple energy balance, the energy dissipation of the microlattice due to buckling can be written as:

$$
\begin{gathered}
E_{\text {strut,buckling }}^{\text {out-of-plane }}=\frac{1}{2} \frac{F_{\text {buckling }}^{\text {cr,strut }}{ }^{2}}{K_{\text {strut }}^{\text {out-strut }}}=\frac{72 \mathrm{E}_{\text {Reuss }}^{*} \pi^{5} r^{2}\left(\frac{r}{L_{\text {eff }}}\right)^{2}}{\left[1+2\left(\frac{L_{\text {eff }}}{r}\right)\right]^{2}\left[\frac{1}{L_{\text {eff }}}+12 C_{1} \frac{r^{2}}{L_{\text {eff }}^{3}}\right]} \\
\Delta U_{\text {dissipated }}^{\text {buckling }}=\frac{f N_{b} \Delta E_{\text {strut,buckling }}^{\text {out-of-plane }}}{W L H}=\frac{24 f \Delta E_{\text {strut,buckling }}^{\text {out-of-plane }}}{L^{3} \sin ^{2} \theta \cos \theta}
\end{gathered}
$$

where $f$ is the fraction of the struts that need to buckling to accommodate the global displacement, $N_{b}$ is the number of struts that need to buckling, $W, L$, and $H$ are the dimensions of the microlattice, and $\Delta E_{\text {strut buckling }}^{\text {out-olane }}$ is the elastic strain energy of the strut due to buckling. The fraction of the struts was assumed as $f=\varepsilon_{\max } /\left(1-2 \sqrt{ } 2^{*} r / L_{\text {eff }}\right)$ [1]. Another main collapse mechanism is brittle-like deformation, which is mainly due to yielding of the base material. This mechanism may occur either globally or locally. With a similar procedure described above, the energy dissipation of the microlattice due to brittle-like deformation can be expressed as:

$$
\Delta E_{\text {strut,yielding }}^{\text {out-of-plane }}=\frac{1}{2} \frac{F_{\text {yielding }}^{\text {cr,strut }}{ }^{2}}{K_{\text {strut }}^{\text {out-strut }}}=\frac{72 \pi r^{2} \sigma_{\mathrm{y}}^{2}}{E_{\text {Reuss }}^{*}\left[1+2\left(\frac{L_{e f f}}{r}\right)\right]^{2}\left[\frac{1}{L_{\text {eff }}}+12 C_{1} \frac{r^{2}}{L_{\text {eff }}^{3}}\right]}
$$

The energy dissipation due to yielding $\Delta U_{\text {dissipated }}^{\text {yielding }}$ can be obtained similarly with Eq. (2b) by replacing $\Delta E$.

Overall, the trends of the effective modulus and damping were reasonably predicted by the analytical model. However, we observed several discrepancies because the model assumes linear viscoelasticity, whereas the samples experienced a localized nodal fracture, finite size effects, possible plastic deformation, and some degree of nonlinearity present in the stress-strain hysteresis loops. Moreover, we approximated a relationship between $\Psi$ and $\tan \delta$ as a linear relation, since there was no reported literature. Nonetheless, the model was intended to provide an estimate of the damping properties with an order-of-magnitude margin. 


\section{Conclusions}

In this work, we presented a scalable tape-casting-integrated multi-material stereolithography process capable of fabricating dissipative soft inclusions embedded in a viscous, highly loaded CF-reinforced resin. Our process was achieved by recoating the highly viscous resin with the uniformly thin film via the doctor blade and by multiple vats allowing two or more material selection. This process is not limited to fabrication of the CFRP composites and can also be applied to other types of viscous resins. To demonstrate our technique, a lightweight cellular CFRP microlattice for high-stiffness-damping pairs was designed, fabricated, and investigated analytically and experimentally. Through DMA tests and quasi-static cyclic compression tests at small- and large-strains, respectively, we observed that a small volume fraction of the soft phase was adequate to improve both intrinsic and structural damping performance with some compensation in the effective stiffness. Tunability maps for the intrinsic and structural damping were also developed by using the experimental measurements for design usefulness in terms of FOM $\left(E^{1 / 3} \tan \delta / \rho\right)$. The present microlattice was compared with the universe of existing materials, indicating that the CFRP microlattice exhibited a comparable stiffness per density as commercial CFRPs and technical ceramics and composites and high-damping as elastomers. Finally, the analytical models were developed and compared with the experimental measurements, which displayed a reasonable agreement.

\section{Key Publications}

1. Xu, Z., C. Ha, R. Kadam, J. Lindahl, S. Kim, Felix Wu, V. Kunc, and X. Zheng, 2019, "AM of two-phase lightweight, stiff, and high-damping CFRP microlattice," Addit. Manuf., under review.

\section{References}

1. Salari-Sharif, L., T. A. Schaedler, and L. Valdevit, 2014, "Energy dissipation mechanisms in hollow metallic microlattices," J. Mater. Res., Vol. 29, No. 16, pp. 1755-1770.

2. Lakes, R., 2009, Viscoelastic Materials, Cambridge University Press: Cambridge, UK.

3. Graesser, E. J., and C. R. Wong, 1992, in: Kinra, V. K., and A. Wolfenden (Eds.), The Relationship of Traditional Damping Measures for Materials with High-Damping Capacity: A Review, pp. 316-343, ASTM International: West Conshohocken, PA, USA.

4. Zheng, X., W. Smith, J. Jackson, B. Moran, H. Cui, D. Chen, J. Ye, N. Fang, N. Rodriguez, T. Weisgraber, and C. M. Spadaccini, 2016, "Multiscale metallic metamaterials," Nat. Mater., Vol. 15, pp. 1100-1106.

5. Deshpande, V. S., N. A. Fleck, and M. F. Ashby, 2001, "Effective properties of the octet-truss lattice material," J. Mech. Phys. Solids, Vol. 49, No. 8, pp. 1747-1769.

6. Dong, L., V. Deshpande, and H. Wadley, 2015, "Mechanical response of Ti-6Al-4V octet-truss lattice structures," Int. J. Solids Struct., Vol. 60-61, pp. 107-124.

7. Granta webpage, Available at: https://grantadesign.com/education/ces-edupack/, last accessed 2 January 2020 .

\section{Acknowledgements}

This research was conducted in collaboration with Dr. Xiaoyu "Rayne" Zheng at the Virginia Polytechnic Institute and State University. 


\title{
II.2.9 Spider Silk as Carbon Fiber Precursor (Oak Ridge National Laboratory)
}

\author{
Dr. Felix L. Paulauskas, Co-Principal Investigator \\ Chemical Science Division, Carbon and Composites \\ Oak Ridge National Laboratory \\ 1 Bethel Valley Road \\ Oak Ridge, TN 37831 \\ E-mail: paulauskasfl@ornl.gov
}

\author{
Prof. Randy Lewis, Co-Principal Investigator \\ Utah State University \\ 9820 Old Main Hill \\ Logan, UT 84341 \\ E-mail: randy.lewis@usu.edu
}

\section{Dr. H. Felix Wu, DOE Technology Development Manager}

\section{U.S. Department of Energy}

E-mail: felix.wu@ee.doe.gov

Start Date: January 1, 2018

Project Funding (FY 19): \$420,000

End Date: December 31, 2018

DOE share: $\$ 420,000 \quad$ Non-DOE share: $\$ 0$

\section{Project Introduction}

Spider silk fibers have a unique combination of tensile strength and elasticity that can be used directly to create composite materials with application to lightweight vehicles. For the past several years, Utah State University (USU) has developed methods to produce spider silk proteins, spin fibers from them, and conduct preliminary studies on the use of those fibers in epoxy-based composite materials. The fibers show very strong adherence to the composite matrix, while the spider silk proteins themselves can serve as impressive adhesives.

In Phase I of this project (up to 2015) involving the same partners, it was demonstrated that synthetic spider silk can be converted into CF. The limitations of this work were: (1) low material production; and (2) weak final mechanical properties (e.g., $100 \mathrm{ksi}$ ). The aim of this project is to address these two limitations and demonstrate that this type of biosynthetic polymer is suitable for CF precursor application by accelerating the development of genetically modified fiber from silkworm silk and exceeding the mechanical properties obtained in the previous work. Beyond this project, the finality is to propose novel non-petroleum-based precursors for CFs that are suitable for composites used in vehicle technologies.

\section{Objectives}

The objective of this project is to develop an unconventional non-petroleum-based CF precursors that have the potential to be produced in high yield and quantities. Methods will be developed to produce pilot-scale quantities of fibers from spider silk proteins with mechanical properties at least $75 \%$ that of the natural dragline silk fibers in tensile strength and elongations of less than $5 \%$. The precursor fibers will be converted to CFs, with a goal of $>250 \mathrm{ksi}$ strength and $1 \%$ to $2 \%$ elongation. The process will be optimized, and a cost analysis will be performed.

This twelve-month project (i.e., Phase II) is a continuation of previous efforts that already demonstrated the feasibility of converting "synthetic" spider silk fibers into CFs. This current project will determine the best fibers for conversion and develop an optimized conversion process. USU will be developing the protein production and fiber spinning methods, while ORNL will develop the conversion processes. 


\section{Approach}

This project was detailed in six main tasks, which were distributed over four calendar steps, one per quarter, as listed in Table II.2.9.1.

Table II.2.9.1. Detail of the Major Calendar Steps of the Spider Silk Conversion Project.

\begin{tabular}{|c|c|c|c|c|}
\hline Phase & Name & Calendar & $\begin{array}{c}\text { Status } \\
\text { (Sept. 2018) }\end{array}$ & Description \\
\hline 1 & Protein production & FY 2018 Q2 & Completed & $\begin{array}{l}\text { Production of } 25 \mathrm{ft} \text { of } 24 \text { tow fiber from two spider } \\
\text { silk proteins. }\end{array}$ \\
\hline 2 & Filament extrusion & FY 2018 Q3 & Completed & $\begin{array}{l}\text { Production of five } 1000 \mathrm{ft} \text { samples with } \\
24 \text { filaments per tow from the best two proteins } \\
\text { previously developed. }\end{array}$ \\
\hline 3 & $\begin{array}{l}\text { Protein production } \\
\text { and conversion }\end{array}$ & FY 2018 Q4 & $\begin{array}{l}\text { Not } \\
\text { achieved }\end{array}$ & $\begin{array}{l}\text { Develop conversion parameters. Exceed best } \\
\text { results of previous work (100 ksi). }\end{array}$ \\
\hline 4 & $\begin{array}{l}\text { Protein production } \\
\text { and optimization }\end{array}$ & FY 2019 Q1 & $\begin{array}{l}\text { Not } \\
\text { achieved }\end{array}$ & $\begin{array}{l}\text { Increase protein production by } 2 \text {-fold, from } 1 g / \mathrm{l} \text { to } \\
2 \mathrm{~g} / \mathrm{l} \text {. Exceed best results of previous work (e.g., } \\
100 \mathrm{ksi}) \text {. }\end{array}$ \\
\hline
\end{tabular}

The success of this project is based on the expertise of the parties involved. While USU is a world-class expert in the production of biosynthetic spider silk, ORNL has extensive experience with the conversion of polymers into CFs. The two teams worked together previously on the conversion of synthetic spider silk. Together they have the experience to evaluate the tasks detailed here:

1. Four different spider silk proteins will be tested. The first will be the minor ampullate spidroin (MaSP1) protein from the minor ampullate gland, which is like one of the dragline silk proteins, but with much less elongation ability. The next two will be genetically made analogs of a combination of dragline and capture spiral (flagelliform) silk proteins known to have very interesting mechanical properties; one of which has a substantial percentage of amino acids that can be crosslinked via our new method. Finally, we will be testing aciniform silk protein, which has been tested very little in its natural form due to its very fine fiber diameter. It has a very different amino acid composition than most other silks, being low in glycine, which is the predominant amino acid in other silks that could aid in carbonization.

2. A proprietary crosslinking method will be used to determine if that will improve the carbonization process and generate improved CFs. This will involve developing a method of crosslinking in situ (during the fiber spinning process) or as a post-treatment.

3. The effort will be focused on the fiber spinning process, not on the fibers we produce as the starting material. The aim is to assess the effects of various spinning methods on the final CFs. In particular, a new 24 filament spinning head was specifically designed to prevent the fibers from fusing. New rollers with divided sections were implemented to prevent filament interaction. It is expected that several modifications to the current process will be required to create an unfused tow.

4. The production levels of spider silk proteins will be improved by increasing both the protein produced in the fermentation, as well as the purification yield.

5. The preliminary conversion protocol will be developed. Extensive studies will be conducted to determine an appropriate conversion protocol for a precursor without fused filaments. This will result in a fundamental understanding of what is required to convert the engineered polymer into CF. This step is somewhat independent of applied stretch and focuses on chemical changes in the precursor and on the use of single or small filament sample quantities.

6. The conversion process will be optimized. Based on the accomplishments of the previous task, an optimization of the conversion conditions will be conducted to increase the final fiber mechanical properties. 


\section{Results}

This project was initially funded for the full 12-month-period of FY 2018, but its calendar has been shifted by three months. It effectively began on January 1, 2018, and is planned to conclude on December 31, 2018. This report covers the context and activity on the spider silk conversion for the first quarter of FY 2019 only. Our major achievements were related to the production of the material using a novel process (pCold). The production of the protein was doubled, while the yield of full-length protein was increased from $45-80 \%$. Figure II.2.9.1 (a) shows images of spooled spider silk fiber and Figure II.2.9.1 (b) provides a microscopic comparison of natural and transgenic silkworm silk. Additionally, larger tows of 24 filaments were spun using the spinning equipment shown in Figure II.2.9.2 as compared to the tows of eight filaments in the past. Increasing the production method and capacity of the precursor was one of the main goals of this project. This goal was reached by the end of June at USU leaving the rest of the year for the carbonization work at ORNL.

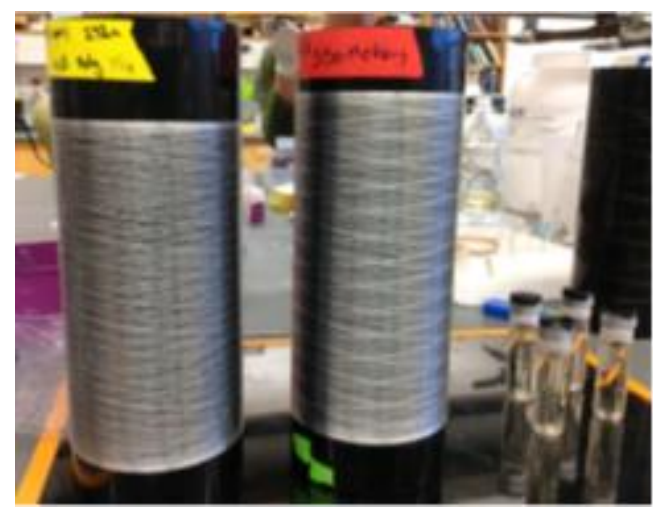

(a)

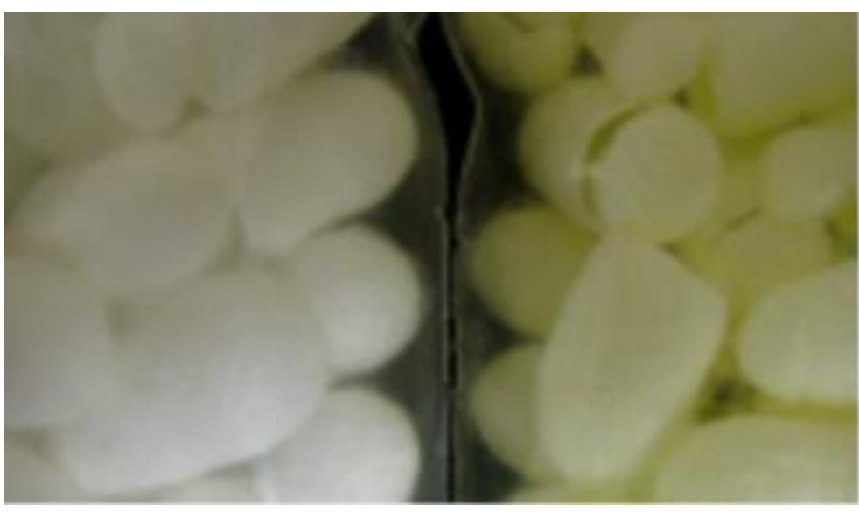

(b)

Figure II.2.9.1. (a) Spooled samples of $100 \%$ bacterially produced spider silk fiber; and (b) comparative photos of the natural (white) and transgenic silkworm silk (green). Source: USU.

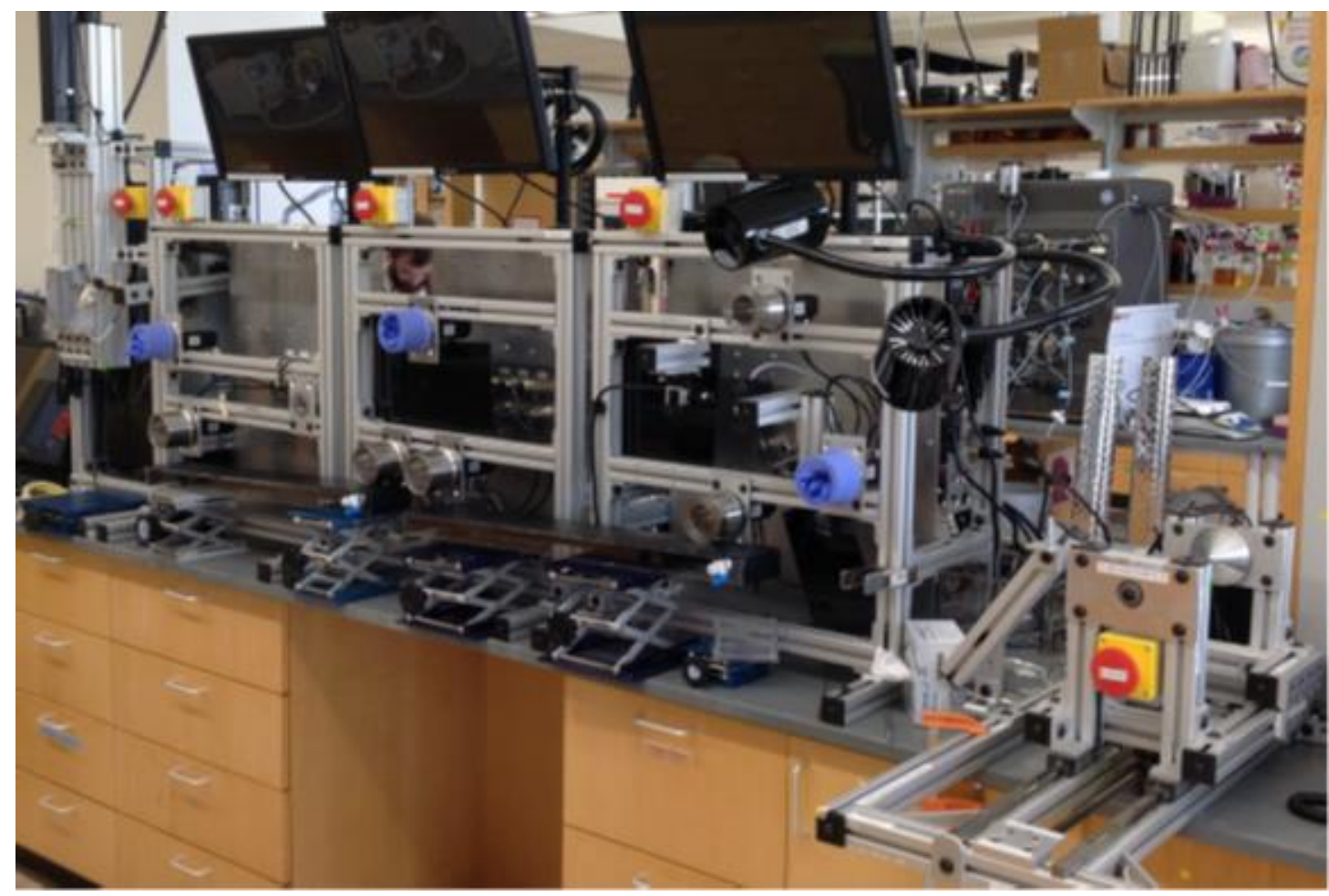

Figure II.2.9.2. Spinning equipment used at USU for the filament extrusion. Source: USU. 
By increasing the production, improvements to the handling and quality of the material were expected. Regarding the handling, the conversion of tiny tow was one of the main challenges of the previous work. ${ }^{11}$ With the new larger tows, the combining step prior to conversion could be skipped, allowing a significant time gain. On the other hand, the quality of the material was not improved - the filaments remained fused with random cross-sections for individual filaments shown in Figure II.2.9.3 (a) and for non-degummed MaSP1 shown in Figure II.2.9.3 (b).

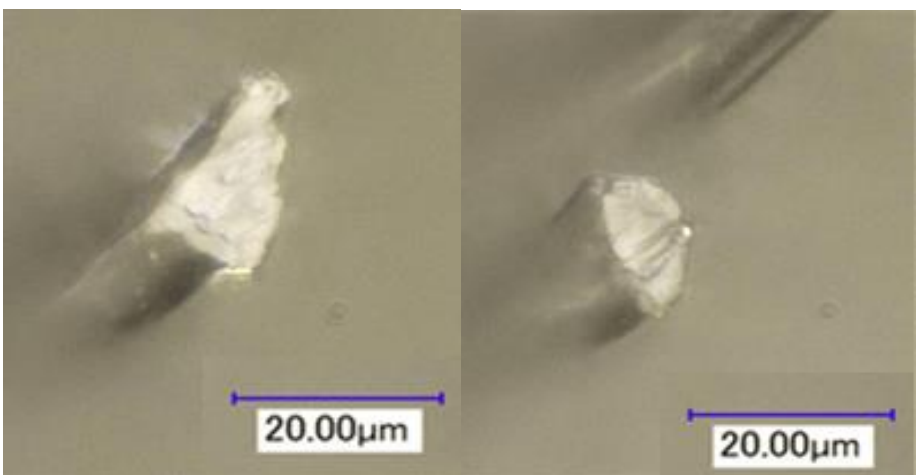

(a)

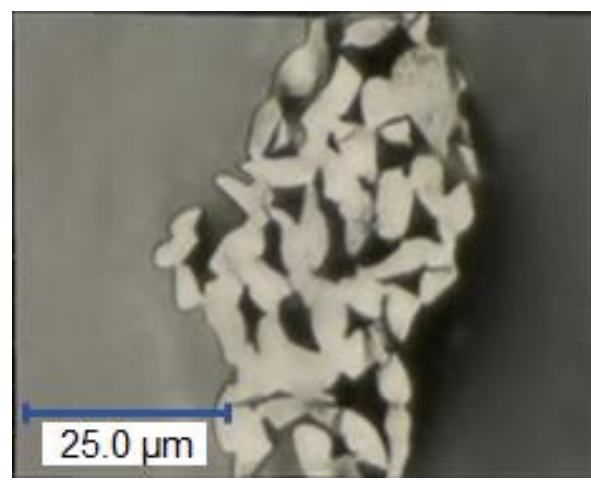

(b)

Figure II.2.9.3. (a) Two examples of isolated MaSp1 filament by optical-microscopy. These two pictures highlight the irregularity of the filament cross-sections. (b) Observation of the cross-section of a MaSp1 non-degummed strand before conversion. The filaments were fused during the spinning phase. Source: ORNL.

This last quarter of FY 2019 was focused on the conversion of the material using the same conventional setup as in FY 2018. The scope of the work was a series of 27 attempts of conversion. Regrettably, none of these attempts could produce a sample fully converted without breaking the fibers during the process of conversion. As a result, no mechanical data could be collected from this series of tests. ${ }^{12}$

The two major issues encountered in the conversion process were (1) a repeated failure at the edge of the material (i.e., static batch) and (2) the lack of reproducibility (some process conditions from FY 2018 that allowed the samples to survive the entire conversion process could not be successfully duplicated).

The failure mode of the batches was initially thought to be correlated to the geometry of the setup used for the conversion. Indeed, most of the failures occurred on both ends of the batches which are the weakest points. Geometrical modifications of the cavity were implemented to reduce the temperature gradient along the samples. This stopped the failure mode of one side leaving the failure to systematically occur on the other side, as observed in Figure II.2.9.4. This type of failure is thought to be related to both the geometry of the setup and the material properties. On the right of the failure is the processed material, which is laying in the hottest part of the cavity of the heating equipment. On the left side lays the carrier material, which is almost unprocessed. If the sample is allowed to shrink while the temperature rises, the unprocessed material slides progressively inside the hottest part of the cavity, which leads to failure. Preventing the shrinkage also leads to failure. While previous successful samples were able to elongate during this process, thus keeping the unprocessed material away from the hot zone, these samples did not. At the conclusion of this project, this issue had not been adequately solved. The remaining failure mode on the reverse side was diagnosed as being related to the shrinkage of the material. ${ }^{13}$ No acceptable solutions were found to limit this shrinkage without breaking the sample.

\footnotetext{
11 In the work performed in 2015-2016, only bundles of eight filaments were available. They had to be combined at least ten times to be used in batch for conversion. The combining step is delicate because of the thinness of the material and the difficulty to reach even tension with each bundle.

12 Rupture of the sample always occurred at the temperature where the material is expected to experience the major morphological change. The loss of the control of the tension at this stage of the process of conversion allows the material to shrink. This usually produces brittle material that disintegrates with any little tension or pressure, which makes the continuation of the process or the testing phase almost impossible.
}

${ }^{13}$ For a detailed description of the sample failure, see Figure II.2.9.4 and its comment. 


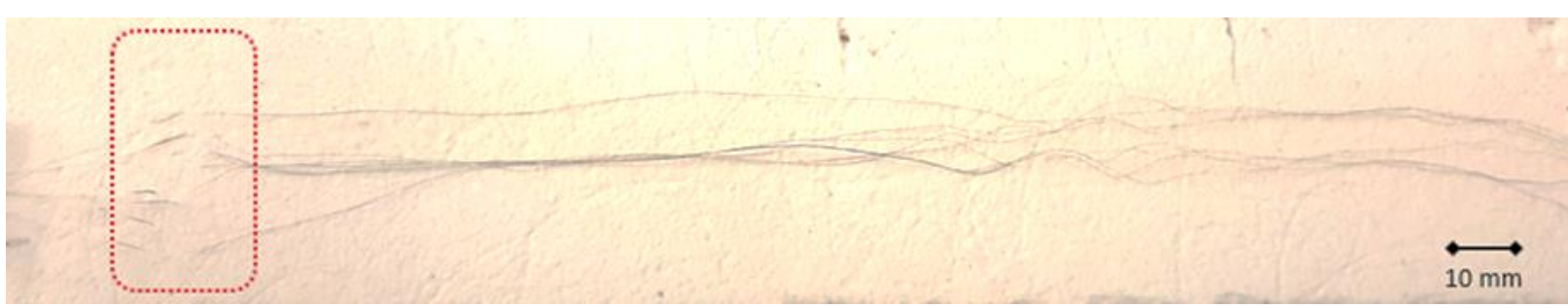

Figure II.2.9.4. The typical failure mode observed during the conversion process is shown in the red dotted frame with the failure in the processed material on the right and the carrier material on the left. Source: ORNL.

Otherwise, the lack of reproducibility of some successful conversions from FY 2018 indicates that the failure could be originated by some inconsistency along the hundreds of meters of materials. However, the fact that the shrinkage was measured as slightly more pronounced than before with all the materials qualifies this judgment and would reinforce the hypotheses of the process conversion and the choice of its parameters being the reason for the shrinkage and the failures. An in-depth characterization of the precursor material would be needed to determine the root cause of the observed lack of reproducibility.

Aside from these process issues, the experimental work was slowed by three technical issues, as listed below:

- Glassware breakage: one flange of the furnace was accidentally broken, stopping any work of conversion with this setup; a custom spare part had to be handcrafted by the local glass shop.

- $\quad$ One relay of the furnace was diagnosed as faulty; this part was ordered and replaced.

- $\quad$ One thermocouple of the furnace failed; this part was ordered and replaced.

Due to these incidents, the experimental work was placed on hold for approximately three weeks. This had limited impact in the progress of the project. By December 31, 2018, a better homogeneity of the process was achieved, but the samples were still not surviving the conversion process. No acceptable solution was found to limit the shrinkage of the material at the project's end.

\section{Conclusions}

This project was a 12-month research program spread over FY 2018 (3/4) and FY 2019 (1/4) from January 1 to December 31, 2018. The largest part of the effort and the resources were allocated during FY 2018 for the development of the protein elaboration process by fermentation (pCold). Most of the results were achieved during this first period-increase of the yield and protein production volume (e.g., the percentage of full protein was increased from $45-80 \%$, and the number of proteins per unit volume by two folds), production and extrusion of the biopolymer, as well as the production of all the samples that could be fully converted and tested for their mechanical properties.

FY 2019 was limited to the continuation of the CF conversion work over the first three months of the fiscal year with the same materials produced in FY 2018. The precursors were long continuous samples of several hundreds of meters of 24 fused filaments offering random cross-section. Between October 1 and December 31, 2018 , a series of 27 conversion attempts were performed. None of these tests provided a fully converted sample without breaking before the end of the process. No material suitable for mechanical testing could be produced. As a result, the weak mechanical properties obtained in FY 2018 could not be improved in FY 2019.

At the conclusion of this project, the production of the protein had been significantly improved in volume, but the quality of the biopolymer and the spun filaments were depleted compared to the previous work. Furthermore, the number of successful conversions, as well as the mechanical properties of the fully carbonized material, remained significantly lower than those obtained in 2016. This led to the conclusion that the current process of filament production is not suitable in its current state to produce a CF precursor. 
With this overall work, a new path to the production of a precursor for CF was explored. It was demonstrated that, like their natural counterparts, synthetic biopolymer can be converted into CF. The biosynthetic material has not yet been able to reach the level of performance achieved by the conversion of natural silk fiber (i.e., a tensile strength of $350 \mathrm{ksi}$ and a modulus of 35Msi) [1]. Nevertheless, it is reasonable to think that it has the potential to outperform these values because, contrary to the natural silk, the synthetic biopolymer offers the advantage a fully controlled process from end-to-end. Compared to the precursors currently used in industry (PAN-based for most of them), each step of the elaboration of the precursor is critical to produce decent CF. The elaboration of a precursor and its associated process of conversion represents years of intense effort in R\&D. In the present case, the research with synthetic biopolymers dedicated to the production of CF is still at its beginning. Many parameters, such as the effects of the composition and the proportion of proteins, or the morphological change during the conversion, remain not completely understood. Additional fundamental work is needed to help this new precursor to reach acceptable performance and usability for composite parts.

\section{References}

1. Cho, S. Y., Y. S. Yun, D. Jang, J. W. Jeon, B. H. Kim, S. Lee, H.-J. Jin, 2017, “Ultra strong pyroprotein fibers with long-range ordering," Nat. Comm., Vol. 8, Art. \#74.

\section{Acknowledgements}

This research was sponsored by DOE-EERE VTO at ORNL, which is managed by UT-Battelle LLC for DOE under contract DEAC05-00OR22725. 


\title{
II.2.10 Carbon Fiber Technology Facility (Oak Ridge National Laboratory)
}

\author{
Merlin Theodore, Co-Principal Investigator \\ Energy and Environmental Sciences \\ Oak Ridge National Laboratory \\ 1 Bethel Valley Road \\ Oak Ridge, TN 37831 \\ E-mail: theodorem@ornl.gov
}

\author{
Amit Naskar, Co-Principal Investigator \\ Energy and Environmental Sciences \\ Oak Ridge National Laboratory \\ 1 Bethel Valley Road \\ Oak Ridge, TN 37831 \\ E-mail: naskarak@ornl.gov \\ Rich Davies, Co-Principal Investigator \\ Energy and Environmental Sciences \\ Oak Ridge National Laboratory \\ 1 Bethel Valley Road \\ Oak Ridge, TN 37831 \\ E-mail: daviesrw@ornl.gov
}

\section{Dr. H. Felix Wu, DOE Technology Development Manager}

U.S. Department of Energy

E-mail: felix.wu@ee.doe.gov

Start Date: March 1, 2011

Project Funding (FY19): \$1,000,000

End Date: Project continuation evaluated annually

DOE share: $\$ 1,000,000 \quad$ Non-DOE share: $\$ 0$

\section{Project Introduction}

In March 2009, DOE-EERE issued a competitive call for proposals to construct and operate a highly flexible, highly instrumented, Carbon Fiber Technology Facility (CFTF) for demonstrating and evaluating low-cost carbon fiber (LCCF) and new low-cost manufacturing technologies at a pilot-scale. Construction began in March 2011 and the CFTF was commissioned for operations in March 2013. The CFTF offers a unique, highly flexible, highly instrumented CF line for demonstrating advanced technology scale-up and for producing market development volumes of prototypical CFs. The CFTF's unique capabilities, including the flexibility to process a range of feed stocks and product forms, are unmatched anywhere in the world. Designed to bridge what has been called the "valley of death" between laboratory research and commercial scale deployment of LCCF technologies, the CFTF fills a critical need for support for industrial competitiveness in the manufacture of CF in this nation. The objective of this project is to accomplish the safe and reliable operation of CFTF and to further DOE's objectives for large-scale LCCF commercialization. The CFTF supports EERE's efforts toward transitioning technologies to industry, specifically with respect to cost-effective CF for composite materials in high-volume energy applications.

The semi-production scale facility is capable of producing up to 25 tons of CF each year (based on processing with the $24 \mathrm{k}$ PAN precursor in the tow format). In addition to a conventional conversion line, the CFTF has a melt-spun precursor fiber production line with a rated capacity of 65 tons per year. The 42,000 -sq. ft. facility has the capacity for future expansion to include the addition of an advanced technology conversion line. 


\section{Objectives}

The objective is to develop LCCF from alternative precursors and accomplish the safe and reliable operation of the CFTF further DOE's objectives for large-scale commercialization of LCCFs. The tasks covered under these agreements are co-funded by the VTO and the Advanced Manufacturing Office (AMO) in support of the efforts by DOE-EERE in transitioning technologies to industry. The CFTF serves as a national resource to assist the industry in overcoming the barriers of CF cost, technology scaling, and product and market development. The CFTF is intended to be the bridge from R\&D to deployment and LCCF commercialization. The CFTF is open for tours so that industry and others can see the technology and the process science being developed to produce LCCF with industry-appropriate mechanical properties from alternative precursor materials.

\section{Approach}

Currently, no commercial manufacturer exists for LCCF, so the CFTF plans to identify cost-effective alternative sources of precursors based on availability, carbon yield, cost, and spinnability. Once the chemistry of the precursor is developed and baseline properties of $250 \mathrm{Ksi}$ tensile strength and 25 Msi modulus are met, a sufficient amount of material will be produced for scaling up to the next level at CFTF. The CFTF staff then develops process conditions for converting the precursor material into LCCF that will exceed baseline properties. Enough CF will be produced for deployment to industry to evaluate, therefore us showcasing the quality of the LCCF on a multitude of end user platforms. This work is intended to produce industry demand for the technology. The CFTF staff continues to hold discussions with industrial partners that are interested in scaling the processes being developed at CFTF. The financial investment for industry to scale the technology is significant and thus not quickly decided upon.

One CFTF mission is to transition this technology to industry for scaling, but numerous technical challenges remain that must be overcome to further reduce the risk for industry to implement the technology. These technical challenges are being addressed in collaboration with industry in order to reduce implementation risks. Some of these challenges are handling the large-tow, increasing throughput, splicing and splitting the largetow, and developing appropriate sizing for the LCCF for specific composite applications. The CFTF continues to be a training ground for any technical staff involved in the production of CF. Key strategies implemented by the project in FY 2019 were under Task 3 of the CFTF Annual Operating Plan with specific milestones. The objectives for the milestones, and their status at the end of FY 2019 as shown in Table II.2.10.1, were to investigate potential alternative CF precursors will allow selection of a candidate precursor not previously studied, and investigation of supplier-to-supplier variation effects in precursor chemistry, physical characteristics on product variability, and performance.

Table II.2.10.1. Task 3 Milestones, Task Descriptions and Status.

\begin{tabular}{|c|c|c|c|c|}
\hline Milestone & Title or Short Description & $\begin{array}{l}\text { Orginal } \\
\text { Plan }\end{array}$ & $\begin{array}{l}\text { Revised } \\
\text { Plan }\end{array}$ & $\begin{array}{l}\text { Percent } \\
\text { Complete }\end{array}$ \\
\hline 3.1 & $\begin{array}{l}\text { Investigate textile precursor modifications to improve, processing, } \\
\text { quality, capacity, and properties. }\end{array}$ & $12 / 31 / 18$ & 03/31/19 & $100 \%$ \\
\hline 3.2 & $\begin{array}{l}\text { CFTF team to deliver mechanical, thermal stability, electrical resistivity, } \\
\text { and carbon content test and analysis on the alternative precursor }\end{array}$ & 03/31/19 & $06 / 30 / 19$ & $100 \%$ \\
\hline 3.3 & $\begin{array}{l}\text { Achieve CF with }>400 \mathrm{ksi} \text { tensile strength, }>35 \mathrm{Msi} \text { tensile modulus, } \\
\text { and }>1 \% \text { elongation which will enable the fiber to be applicable for } \\
\text { more semi-structural and structural applications. }\end{array}$ & 06/30/19 & $06 / 30 / 20$ & $\begin{array}{l}\text { Moved to } \\
\text { CFTF Task } 1 \text { - } \\
\text { Not completed }\end{array}$ \\
\hline 3.4 & $\begin{array}{l}\text { Produce } 500 \mathrm{kgs} \text { of CF material for deployment to industry for joint } \\
\text { industry projects. }\end{array}$ & 09/30/19 & $09 / 30 / 20$ & $\begin{array}{l}\text { Moved to } \\
\text { CFTF Task } 1 \text { - } \\
\text { Not completed }\end{array}$ \\
\hline
\end{tabular}




\section{Results}

\section{Visitors/Tours}

In FY 2019, the CFTF hosted 534 visitors representing 128 organizations.

\section{Precursor and CF Development}

In FY 2019, the CFTF developed process conditions for 13 variations of Montefibre Carbon precursor fiber [1] using 99 process variations, and the mechanical properties are depicted in Figure II.2.10.1 and Figure II.2.10.2. The CFTF achieved mechanical properties values above the VTO mechanical target properties of a tensile strength of $25 \mathrm{Ksi}$, tensile modulus $25 \mathrm{Msi}$ in $77 \%$ of the trials executed.

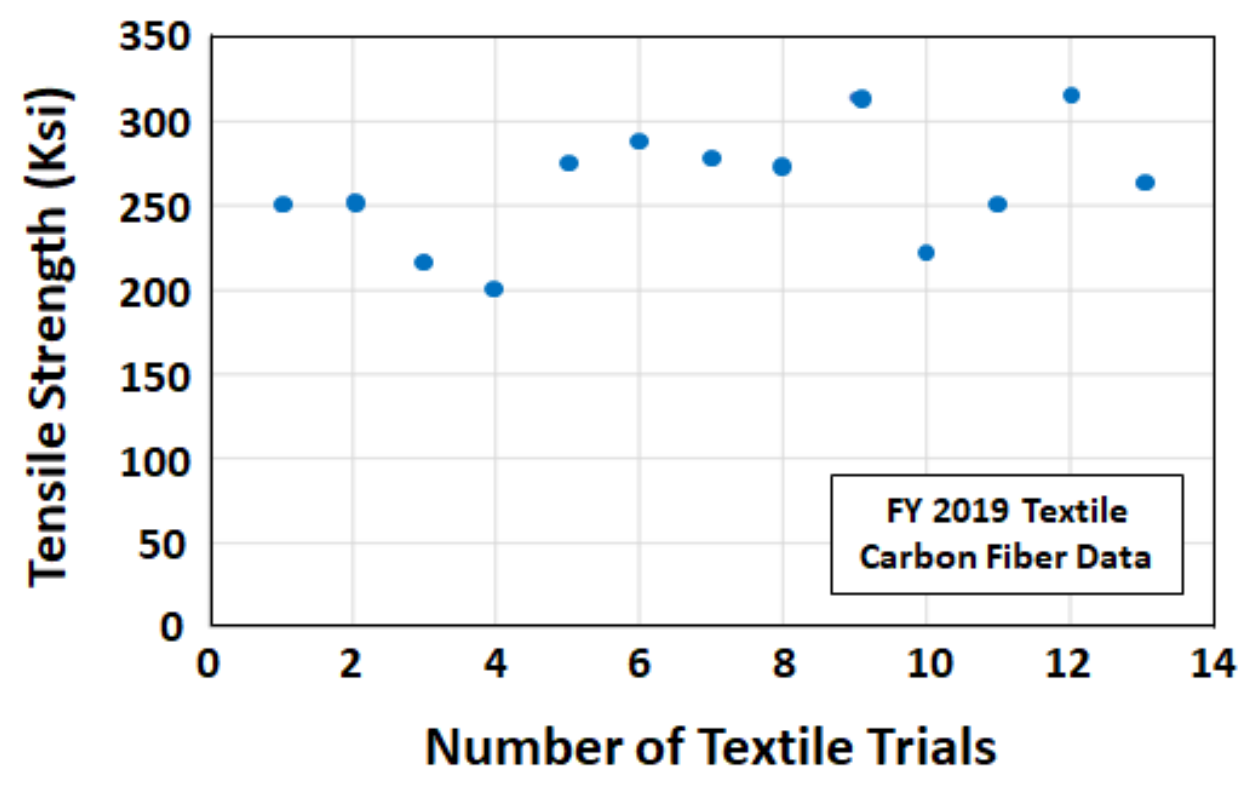

Figure II.2.10.1. Tensile Strength of Textile CF. Source: ORNL.

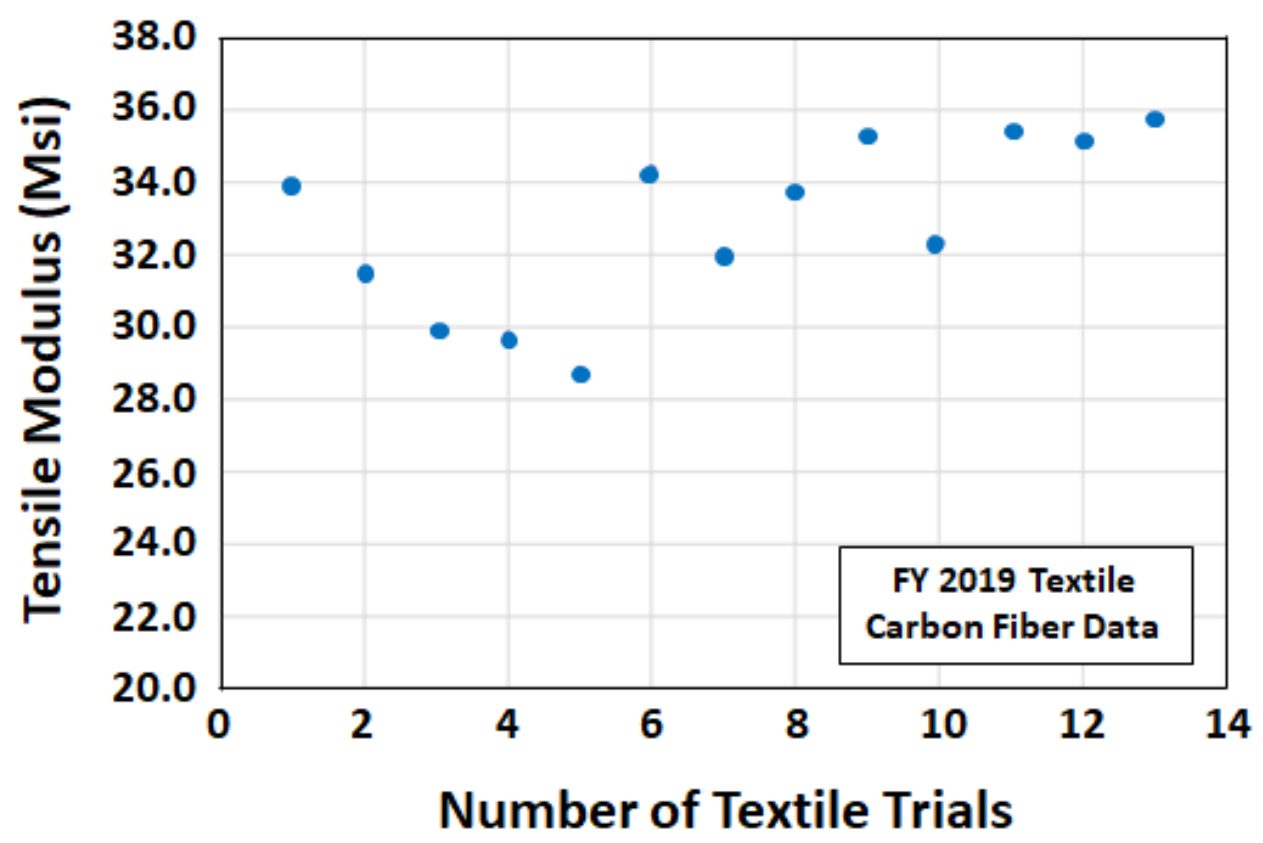

Figure II.2.10.2. Tensile Modulus of Textile CF. Source: ORNL. 
Quality issues are typically the root causes associated with processability issues of precursor fiber, high variations in final properties, and even lower properties. Packaging is one of the top three concerns. The old packaging was not stable, created crossovers of tows, cause tows to fall of the edges, contains knots, easily entangled within itself creating a "birds' nest." Quality issues resulted in frequent shutdowns of the CF manufacturing process, wraps, fiber damage, low mechanical properties, and poor-quality CF. A new packaging system was developed to reduce health and safety risks and processability issues. Figure II.2.10.3 shows the differences between the old packaging method and the new packaging developed in this project. The new packaging was made with cardboard boxes where the precursor fibers where laid differently and not tampered nor compressed. There are no more shutdowns due to packaging issues.

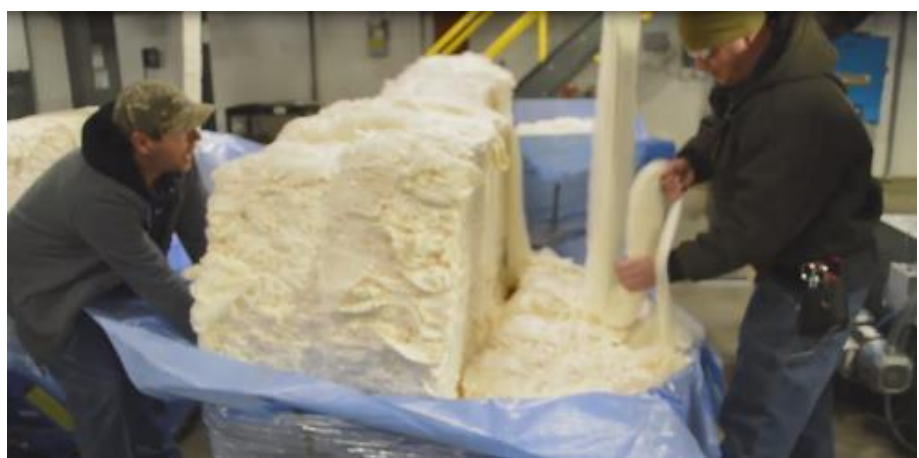

(a)

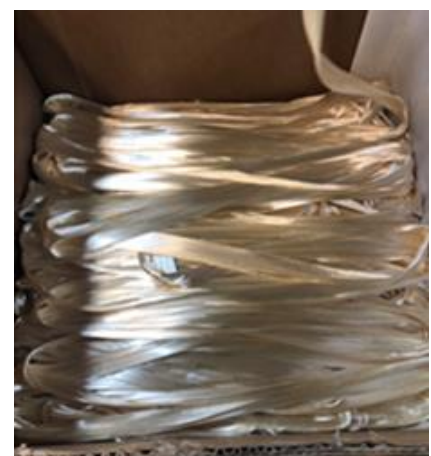

(b)

Figure II.2.10.3. (a) Old packaging method that was bulky and time-consuming; and (b) new simplified packing configuration. Source: ORNL.

The precursor samples were down-selected to the best three lots/trials. Samples from these lots were further characterized and analyzed using thermogravimetric analysis, elemental analysis, and electrical resistivity. Carbon content, decomposition temperature, and electrical resistivity of these fibers were determined and the results are listed in Table II.2.10.2.

Table II.2.10.2. Characterization of the Top Three Montefibre Carbon Materials-Based on Developed Process Conditions.

\begin{tabular}{|c|c|c|c|c|c|c|c|c|}
\hline $\begin{array}{c}\text { Carbon Fiber } \\
\text { Samples }\end{array}$ & $\begin{array}{l}\text { Carbon } \\
\text { Content } \\
(\%)\end{array}$ & $\begin{array}{c}\text { Resistivity } \\
\text { ( } \mu \text {-ohm-cm) }\end{array}$ & $\begin{array}{c}\text { Weight } \\
\text { Loss at } \\
\text { Onset (\%) }\end{array}$ & $\begin{array}{c}\text { Thermogravimetric } \\
\text { Analysis Peak } \\
\text { Temperature }\left({ }^{\circ} \mathrm{C}\right)\end{array}$ & $\begin{array}{l}\text { Weight Loss at } \\
\text { Decomposition } \\
\text { (\%) }\end{array}$ & $\begin{array}{l}\text { Tensile } \\
\text { Strength } \\
\text { (Ksi) }\end{array}$ & $\begin{array}{c}\text { Tensile } \\
\text { Modulus } \\
\text { (Msi) }\end{array}$ & $\begin{array}{l}\text { Carbon Fiber } \\
\text { Density } \\
(\mathrm{g} / \mathrm{cc})\end{array}$ \\
\hline $\begin{array}{c}\text { M180-UA-480K- } \\
\text { TE4801180302- } \\
\text { T003 }\end{array}$ & 99.546 & 1733 & 2.95 & 502.06 & 97.39 & 401.5 & 34.3 & 1.7110 \\
\hline $\begin{array}{c}\text { M180-UA-480K- } \\
\text { TE4801180302- } \\
\text { T010 }\end{array}$ & 99.734 & 1432 & 1.45 & 601.60 & 96.92 & 372.1 & 34.4 & 1.7529 \\
\hline $\begin{array}{c}\text { M180-UA-480K- } \\
\text { TE4801180302- } \\
\text { T012 }\end{array}$ & 100.210 & 1273 & 0.89 & 552.84 & 98.60 & 357.0 & 34.2 & 1.7220 \\
\hline
\end{tabular}

\section{Joint Projects/CF Intermediate Forms}

The CFTF and collaborative industrial efforts in FY 2019 using the Montefibre Carbon materials are discussed in the following sections. Key highlights from these projects were presented at the JEC Group Composites Conference in Paris, France [2]; the Evolution of Composites Workshop in Nashville, TN, USA [3]; the IACMI members meeting in Indianapolis, IN, USA [4], and several others listed in the key publication section. 


\section{Textile CF packaging \& NCF Production - ORNL, McCoy Machinery Co. Inc. (McCoy), Institute for Advanced Composites Manufacturing Innovation (IACMI), Montefibre Carbon}

A major achievement for this task was that over $100 \mathrm{~kg}$ of packaged CF with a tow linear density greater than $10 \mathrm{~g} / \mathrm{m}$ and each continuous tow greater than $500 \mathrm{~m}$ in length was produced and shipped to the Chomarat production facility in South Carolina to produce NCF material. Traditional winder system for CF production can only accommodate tow sizes up to 50K. Results of a joint industry project with McCoy Industries lead to the development a paperless textile winder system, depicted in Figure II.2.10.4, for handling large tows. The textile winder was built with three intentions: (1) develop spools without paper interleave like traditional spools; (2) create spools with more fiber to allow downstream processes to run more efficiently (longer run times and smaller number of spools); and (3) reusability. Figure II.2.10.5 (a) shows old spools with paper interleave on cardboard cores and Figure II.2.10.5 (b) shows the new textile spools on reusable plastic cores ready for shipment. The paper is a nuisance and adds non-value-added steps for downstream process. In addition, it adds weight to the spools which increase shipping costs and require additional overheard to remove the paper in downstream processes.

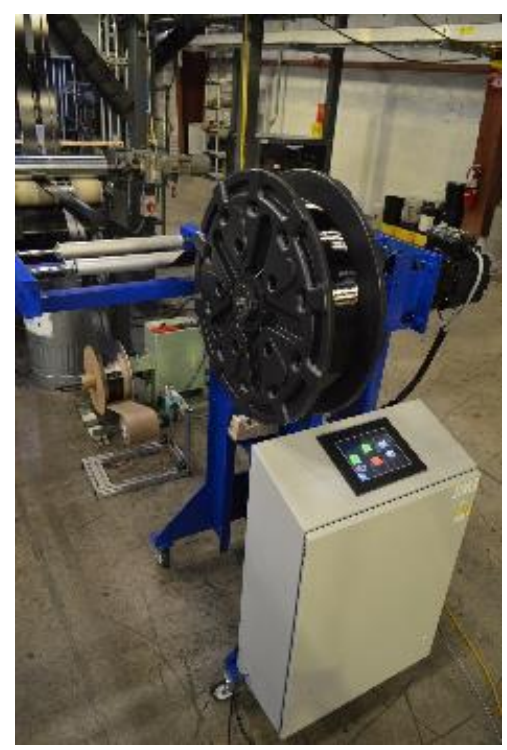

Figure II.2.10.4. Paperless packaging system for downstream processing. Source: ORNL.

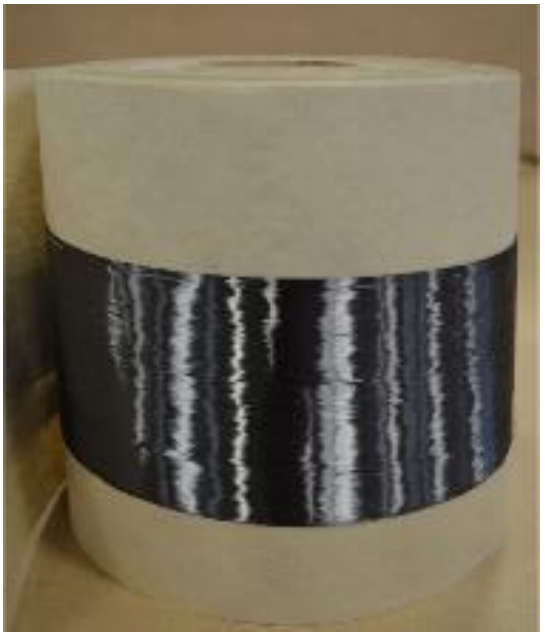

(a)

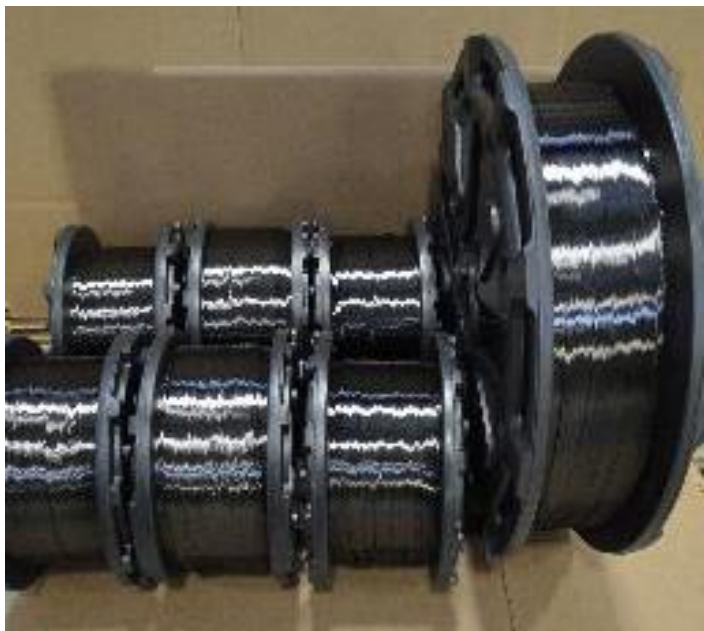

(b)

Figure II.2.10.5. (a) Textile CF on cardboard cores. (b) Textile CF on plastic cores. Source: ORNL. 


\section{Database Generation - ORNL, IACMI, University of Tennessee Knoxville}

A textile $\mathrm{CF}$ and its composites database were generated with representative properties for research partner, industry partners, and the community in general to use in composites projects. IACMI has undertaken a limited study to generate a basic database use in design, modeling, and application development. The data generated is applicable to IACMI core areas, so the materials portfolio is being expanded on continuous basis. CFTF successfully customizes textile fiber with sizing chemistries for use in thermoplastic, thermosets composite systems. Figure II.2.10.6 and Figure II.2.10.7 provide an example of the data generated for the flexural strength and modulus, respectively, of textile $\mathrm{CF}$ in various resin systems testing with various fabrication methods. Results are presented biannually at the IACMI members meeting.

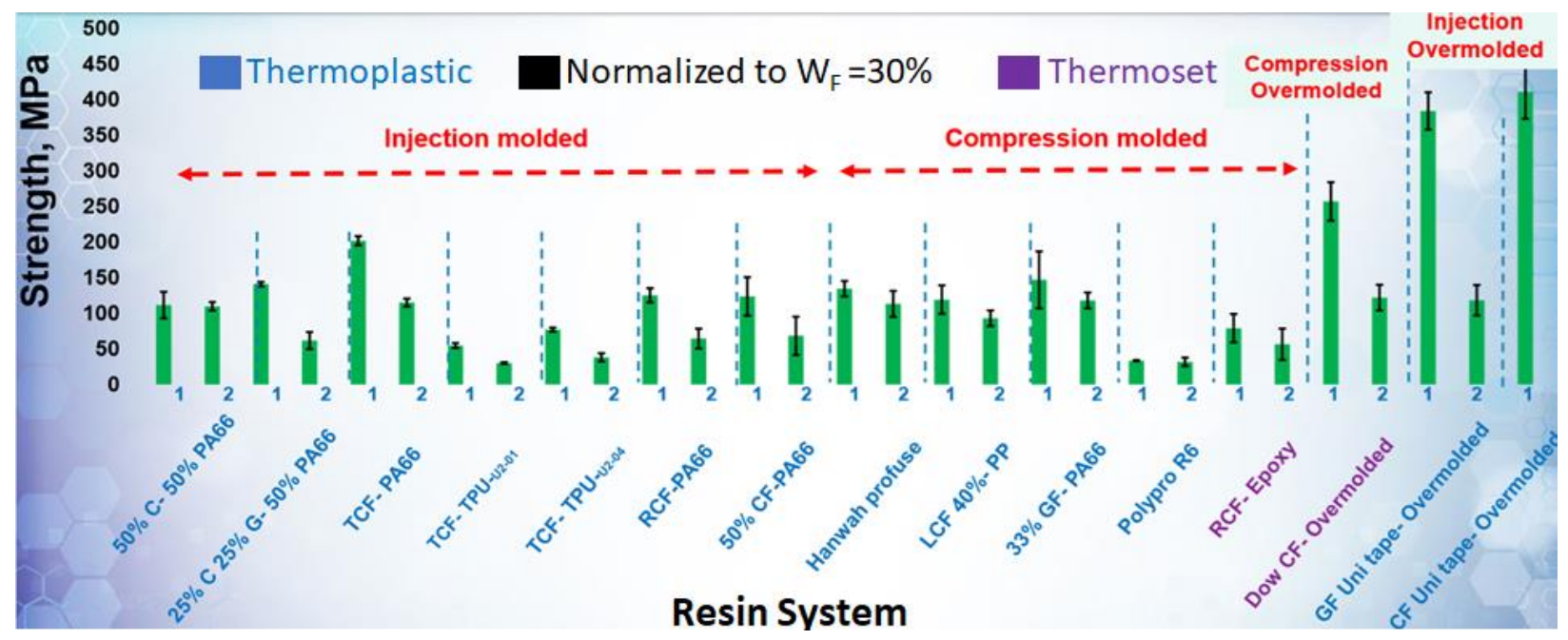

Figure II.2.10.6. Flexural strength of TCF in various resin systems. Source: IACMI.

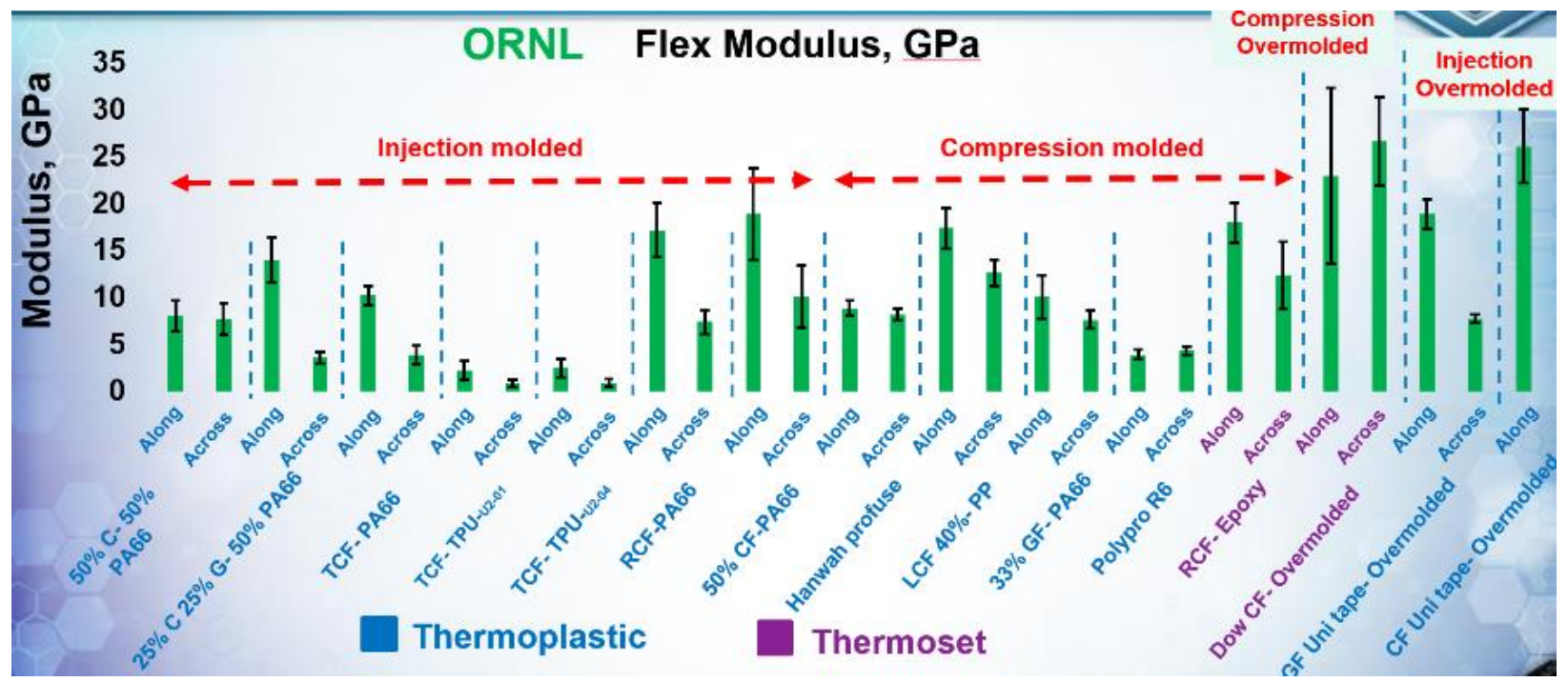

Figure II.2.10.7. Flexural modulus of TCF in various resin systems. Source: IACMI. 


\section{Applications for Developed Textile CF - TPI Composites, Montefibre Carbon, Huntsman, NREL, ORNL, University of Tennessee Knoxville}

The CF production processes developed at the ORNL CFTF in collaboration with industry and academia demonstrated the potential for the use of textile $\mathrm{CF}$ in pultruded composites production. The IACMI/ORNL/UT team, jointly with IACMI partners, made significant progress and commissioned the new pultrusion machine donated to the organization by Strongwell which is pictured in Figure II.2.10.8 at the CFTF. Preliminary trials, shown underway in Figure II.2.10.9, were conducted to determine the use of textile CFs in the pultrusion process. ORNL/IACMI team overcame some challenges and was able to produce a few pultruded samples with textile CF. Some challenges remain to be addressed with the equipment and the material.

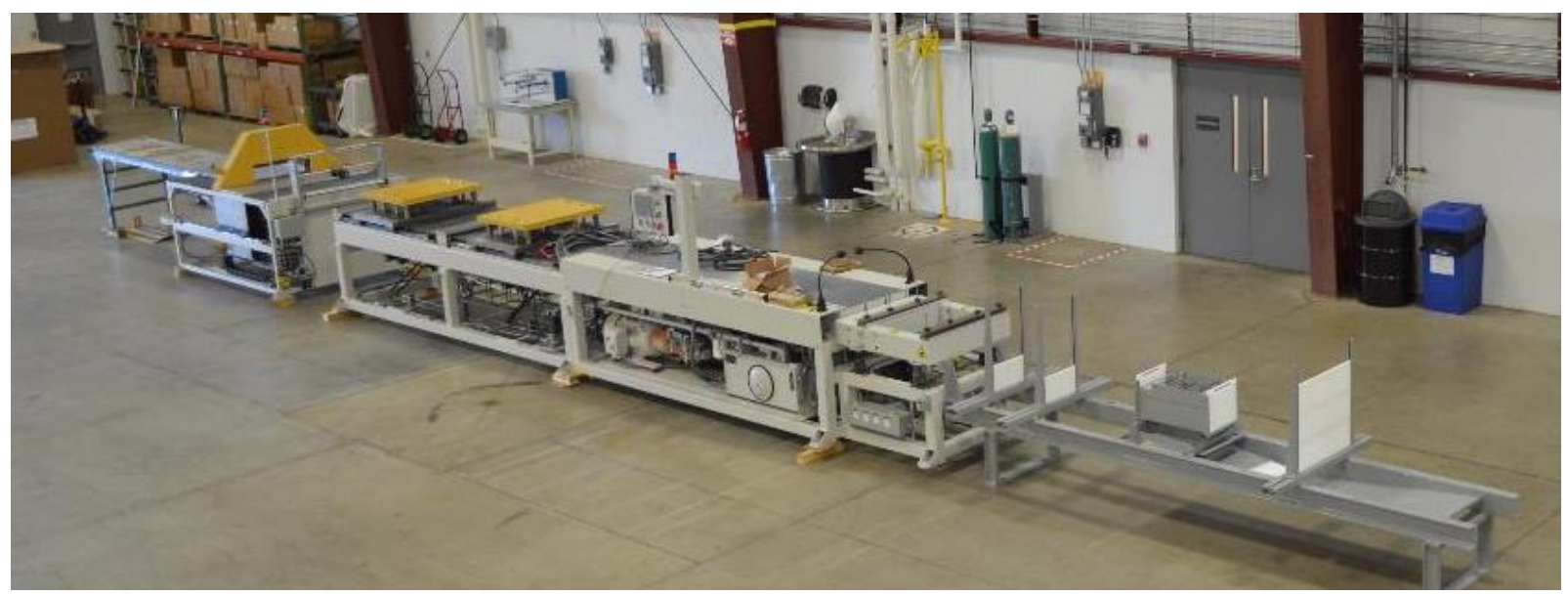

Figure II.2.10.8. Strongwell pultrusion equipment at the CFTF. Source: ORNL.

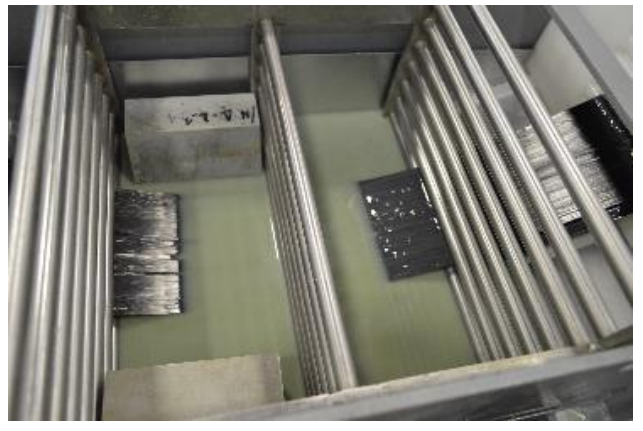

(a)

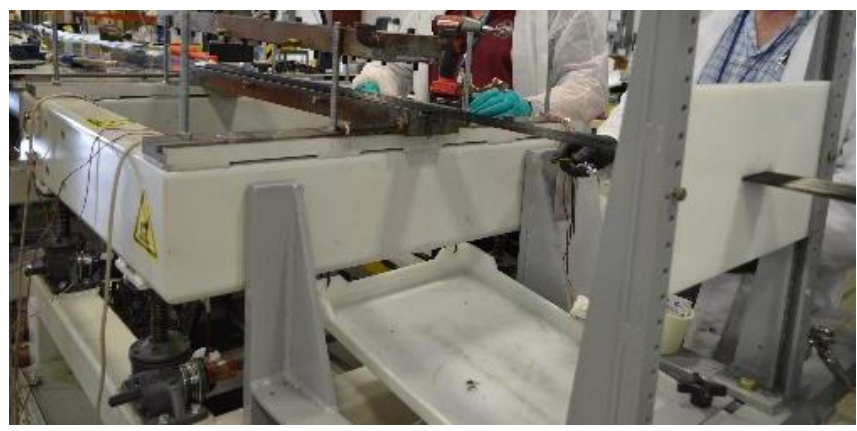

(b)

Figure II.2.10.9. (a) Textile CF wet out resin being pulled through the pultruded process. (b) Pultruded textile CF being pulled through the pultruded process to remove excess resin. Source: ORNL.

\section{Conclusions}

Significant progress was made in developing processes to convert the newest source of textile acrylic fiber to CF with mechanical properties, more specifically the modulus, which is suitable for use in lightweight applications for vehicles and other industrial products. Current compositing equipment is not designed to handle heavy tow textile fiber. CFTF, IACMI, UT, and joint partners are aiding in development of the supply chain for textile CF by developing custom-made equipment and or process for handling the wide tow material in a downstream process was developed. Newly developed spools will enable more efficient downstream processing, lower energy and cost by reducing setup times using a smaller number of spools and longer runtimes. 


\section{Key Publications}

1. Theodore, M., A. Naskar, and R. Davies, 2018, "Low-cost carbon fiber," CAMX 2018, 15-17 October 2018, Dallas, TX, USA.

2. Theodore, M., 2018, Co-chair at Composites World 2018 Conference, 14-16 December 2018, San Diego, CA, USA.

3. Theodore, M., 2019, "Overview of CFTF," Composites Coalition, ORNL, 20 February 2019, Oak Ridge, TN, USA.

4. Theodore, M., 2019, "Overview of CFTF," UTSA/SNL/INL/Texas A\&M Presentation, MDF, ORNL, 6 March 2019, Oak Ridge, TN, USA.

5. Theodore, M., 2019, "Transformative leadership panel," SAMPE, 20-21 May 2019, Charlotte, NC, USA.

6. Theodore, M., 2019, "CFTF overview and activities," Carbon Fibers and Composites Workshop, 11-12 July 2019, New Hope Center, Oak Ridge, TN, USA.

7. Vaidya, U., M. Theodore, D. Penumadu, S. Young, H. Ghossein, and N. Hiremath, 2019, "Textile grade carbon fiber-epoxy composite evaluation for automotive and industrial applications," Submitted to $J$. Comb. Theory B.

\section{References}

1. Mason, H., 2019, "Montefibre Carbon unveils its first CF PAN precursor," Composites World, Available at: https://www.compositesworld.com/news/montefibre-carbon-unveils-its-first-carbon-fiber-panprecursor (accessed 3 December 2019).

2. Theodore, M., 2019, "Cost-effective CF produced from textile grade PAN," JEC World 2019, 11-14 March 2019, Paris, France.

3. Theodore, M., 2019, "Low-cost carbon," Evolution of Composites Workshop, 20-21 March 2019, Nashville, TN, USA.

4. IACMI Members Meeting, 2019, “ORNL Textile CF C-Ply ${ }^{\mathrm{TM}}$," 29-30 January 2019, Indianapolis, IN, USA.

\section{Acknowledgements}

The CFTF Team would like to acknowledge H. Felix Wu, DOE Technology Manager; Craig Blue and Mohammad Khaleel, IACMI Institute; and James Parks II, Raynella Connatser, Vincent Paquit, James Humphries, Yarom Polsky, Roger Kisner, Dr. Mongi Abidi, Dr. Vaidya Uday, Cliff Eberle, Michael Lance, Edward Western, William Peter, Lonnie Love, Soydan Ozcan, Vlastimil Kunc, Edgar Lara-Curzio, Nidia Gallejo, James Klett, Nadya Ally, Jennifer Burke, Jennifer Palmer, Rita Ayers, Karen Triplett, Samuel Lewis, Daljeet Singh, Vinoy Thomas, Amol Vaidya, Catherine Schuman, Angie Blankenship, Debra Garrett, Teddie Reagan, Alan Liby, Hiram Rogers, Randale Strong, Claus Daniel, and Dayakar Penumadu for their sponsorship, contributions, and/or support of the ORNL CFTF program. 


\title{
II.2.11 Proximity Electromagnetic Carbonization (Oak Ridge National Laboratory)
}

\author{
Dr. Felix L. Paulauskas, Principal Investigator \\ Materials Science and Technology Division \\ Oak Ridge National Laboratory \\ 1 Bethel Valley Road \\ Oak Ridge, TN 37831-6053 \\ E-mail: paulauskasfl@ornl.gov
}

\section{Dr. H. Felix Wu, DOE Technology Manager}

U.S. Department of Energy

E-mail: felix.wu@ee.doe.gov

Start Date: October 1, 2015

Project Funding (FY19): \$930,000

End Date: September 30, 2020

DOE share: $\$ 930,000$

Non-DOE share: $\$ 0$

\section{Project Introduction}

The goal of the proximity electromagnetic carbonization (CPEC) project is to develop a novel carbonization technique to replace existing conventional low temperature carbonization (LTC) equipment in the CF industry. It is a joint development project fully funded by DOE between ORNL and 4XTechnologies, LLC (formerly RMX Technologies). Preliminary work on electromagnetic carbonization was jointly conducted dating back to 2013. This former work is the impetus of the CPEC project. This report outlines the progress of the work on the CPEC project FY 2019.

At the beginning of FY 2018, the former setup, CPEC-3, could produce low temperature CF from PAN precursor, on a continuous single tow, with direct electromagnetic radiation. The output fiber was measured to have a density of $1.5 \mathrm{~g} / \mathrm{cc}$ or greater and, by November 2017, the final CF (after full carbonization) reached the milestone requirements. In the meantime, the modeling of the current iteration of the CPEC technology, CPEC-4, began and was completed in April 2018. For scalability and cost reasons, the design of CPEC-4 was entirely revised. As designed, CPEC-4 is can process up to 8 tows and works at a lower power per unit mass of material than the previous version.

Over FY 2019, the effort focus was to mitigate the negative impact of the delays with the procurement of components for CPEC-4. Indeed, several suppliers were unable to honor the lead time they had promised. Among the late parts, the generator, a critical element for commissioning the setup, came in two separate deliveries: the first in March 2019 (delayed 8.5 months) and the second in June 2019 (delayed 11.5 months). This important delay reduced the time available to six months to complete the installation, test the performance of the equipment, commission the setup, and perform the experimental work.

At the end of FY 2019, an intense effort was dedicated to fix the technical issues discovered during the commissioning of the overall LTC processing system. Few experimental tests were conducted at this time. Finally, the conversion of oxidized PAN fiber (OPF) was observed on a limited area of a static batch, but the antenna system failed. The design of the antenna arrangement needs to be revisited. Negotiations for a one year extension of this project began in September 2019. This extension was approved on October 31, 2019. 


\section{Objectives}

The objective of the project is to develop a faster and more efficient carbonization process than the actual conventional process. The concept is to elevate the temperature of the material only and to increase the throughput by approximately $30 \%$, while producing the same (or better) quality CF. Also, this technology is expected to reduce unit energy consumption of the LTC stage in $\mathrm{kWh} / \mathrm{kg}$ by approximately $50 \%$ (which represents about a 5\% of the cost-reduction on the CF overall manufacturing process). Physically, the CPEC technology could be scaled later to a nameplate capacity of one annual metric ton and will demonstrate this capability by the end of the project.

\section{Approach}

The CPEC project is split into four major design and construction phases with each phase related to a separate experimental setup. These phases are reported in Table II.2.11.1. This chart shows the four phases of the project, from its preliminary work in FY 2013 to its completion. All the tasks accomplished in FY 2019 are encompassed in Phase 4. The two main tasks accomplished in FY 2019 were the construction and commission of the fourth iteration of CPEC-4. This technology is co-developed by ORNL and 4XTechnologies.

Table II.2.11.1. Phase Approach for CPEC-4.

\begin{tabular}{|c|c|c|c|c|}
\hline Phase & Name & Date & $\begin{array}{l}\text { Status } \\
\text { (Sept. 2016) }\end{array}$ & Description \\
\hline 1 & CPEC-1 & FY 2013 & Completed & Demonstration of feasibility (static mode). \\
\hline \multirow[t]{2}{*}{2} & CPEC-2 & FY 2016 & Aborted & $\begin{array}{l}\text { Upgrade of CPEC-1 (with automatic impedance matching); } \\
\text { abandoned in favor of CPEC- } 2 \mathrm{~V} \text {. }\end{array}$ \\
\hline & CPEC-2V & FY 2016 & Completed & Optimization of the CPEC concept using a computational model. \\
\hline 3 & CPEC-3 & FY 2016 & Completed & $\begin{array}{l}\text { Physical construction of CPEC- } 2 \mathrm{~V} \text { (continuous process at lab- } \\
\text { scale). }\end{array}$ \\
\hline 4 & CPEC-4 & $\begin{array}{l}\text { FY } 2017 \\
\text { FY } 2019\end{array}$ & In progress & Scale-up of CPEC-3 to a 1 ton/year furnace. \\
\hline
\end{tabular}

\section{Results}

\section{CPEC-4 Construction}

CPEC-3 was a benchtop reactor designed as a proof-of-concept, whereas CPEC-4 is a part of a pilot line with a nameplate capacity of one metric ton per year of $\mathrm{CF} .{ }^{14}$ As a result, it is a larger and more complex setup, using more advanced technologies. CPEC-4 mainly differs from CPEC-3 in the following aspects: ${ }^{15}$

- the type of generator used

- the transmission line design

- the antenna system design

- $\quad$ the vessel design/geometry, size (enlarged), and configuration

- $\quad$ the integrated fiber handling system

- $\quad$ the control system (more complex).

14 Both prototypes (CPEC-3 and CPEC-4) are designed for continuous process, with a larger capacity for CPEC-4.

${ }^{15}$ All CPEC designs are proprietary and cannot be disclosed in this report. 
CPEC-4 was partially operational in early April 2019, shortly after the first lot of the generator system was delivered with 8.5 months of delay. This significant delay has been a limiting factor in the achievement of the CPEC project for FY 2019. The first six months of the FY were used to install and commission the other subsystems that were delayed. Among them, the most noticeable were:

- $\quad$ The power: The installation of suitable power in the facility (e.g., $480 \mathrm{~V}, 3$ phases), along with the installation of an outdoor transformer and a new connection to the grid. There was also a delay with this installation of three months, due to legal reasons and weather conditions.

- $\quad$ The fiber handling system: A custom-made system had to be ordered for the CPEC project. This is the second largest subsystem of the setup, but also the largest delay in the procurement. Once received, this subcomponent had to be installed and connected to the control system.

- $\quad$ The transmission line: This system was also delayed by approximately four weeks. It was assembled and tested when received in October 2019.

- $\quad$ Other: Several non-critical parts, such as the safety probes, came with several weeks and up to several months of delay (this is the case for the radiation safety probes), but had no impact on the project other than delaying the adjustments of the control system.

It must be mentioned that based on the lead times claimed by the suppliers, each subsystem - such as the cooling units, pretreatment unit, parts of the vessel, etc.- - was ordered on time in FY 2018. Because of these delays, however, work on this project had to be lower than average over the first half of FY 2019. ${ }^{16}$

Nonetheless, some efforts related to the construction was still required in FY 2019. For example, the control system could not be totally completed until the hardware to be controlled was delivered.

\section{CPEC-4 Commissioning}

The commissioning of the separate subsystems began after their installation. The most important element that could be checked before the generator system arrived was the cavity. The cavity has been probed over the frequency range of interest using a virtual network analyzer. Figure II.2.11.1 shows an example of these measurements. In this example, we can see two preferable bands of operation. The position and the intensity of these bands are in accordance with the prediction of the computational electromagnetic modeling (CEM). This measurement was performed on each port. All the measurements showed consistent results.

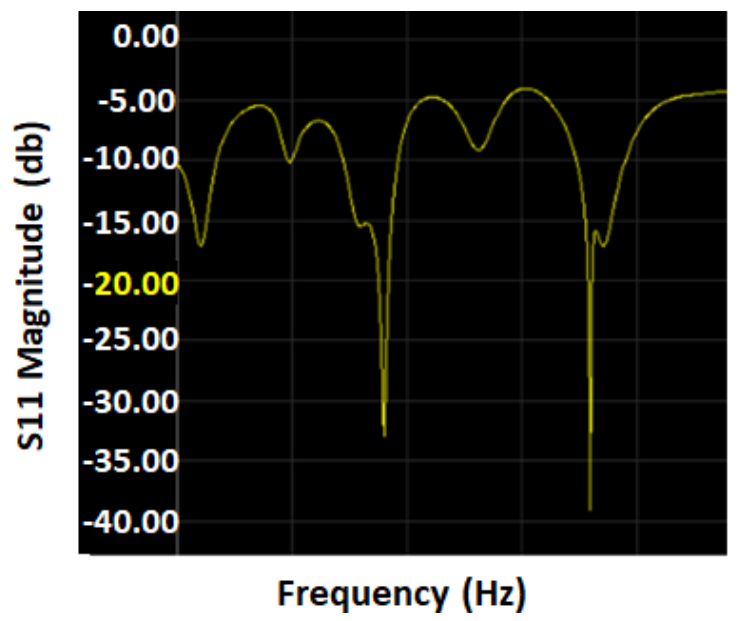

Figure II.2.11.1. An example of a virtual network analyzer measurement of one port of the vessel. Two bands are showing a low reflection $(<30 \mathrm{~dB})$, ensuring an effective power transfer at these frequencies. Data generated at 4XTechnologies.

16 During this period, several options were investigated as a substitute to the generator system. Unfortunately, none of them satisfied both the technical and financial requirements. 
As soon as the first generator was delivered, more measurements became possible. Among the most critical characteristics of the radiative system is the isolation of the antennas vs. their relative position. ${ }^{17}$ Indeed, crosstalk $^{18}$ between the lines and their respective antennas is undesirable. As of March 28, based on the model, the two configurations that were expected to be the extremes were tested at two power levels - the "favorable configuration" and the "unfavorable configuration." These tests were performed without load, which is the most stressful configuration for the setup. As observed in Table II.2.11.2, the results match the model by confirming that a relatively good isolation was obtained without load with the favorable configuration and a high crosstalk was obtained with the unfavorable configuration.

\section{Table II.2.11.2. Measurements of the Crosstalk between the Antennas and Their Lines with Two Configurations at Two Power Levels.}

\begin{tabular}{|c|c|c|}
\hline Power Level & \multicolumn{2}{|c|}{$\begin{array}{c}\text { Crosstalk between } \\
\text { Antennas (\% of power) }\end{array}$} \\
\cline { 2 - 3 } & $\begin{array}{c}\text { Favorable } \\
\text { configuration }\end{array}$ & $\begin{array}{c}\text { Unfavorable } \\
\text { configuration }\end{array}$ \\
\hline A & 11.1 & $>25 *$ \\
\hline B = A x 10 & 8.6 & $>25 *$ \\
\hline
\end{tabular}

* Values could not be measured, but are known to be over the $25 \%$ threshold, after which the control system is designed to stop the generator for safety reasons. Data generated at 4 XTechnologies.

Additionally, the entire generator system was tested. Its effective power delivered was measured using a specific benchtop setup. ${ }^{19}$ One measurement was smaller than expected, but the others agreed with the specification. As is, the performance of the generator system is enough to perform its task. On the other hand, despite being operational, the software of this system is a second edition and still contains some glitches. It is expected that it will soon receive updates.

The safety tests were first conducted at the same time as the performance tests of the generator system, and then on the entire system. The major risk was related to electromagnetic (EM) radiation leakage through the joints between the assembled parts and through the main openings of the system. A three-axis probe was used to measure the electric field at multiple points close to the surface of the equipment $(\sim 50 \mathrm{~mm})$, as well as in the vicinity of the equipment (e.g., in the entire lab space and outdoor). At full power, all measured levels were below the general population exposure limits. ${ }^{20}$

The generator system was tested and connected to the processing system. A safety procedure was conducted on the overall system. Then, the system was ready to be energized. Finally, the EM field distribution inside the vessel could be evaluated. Some media was used to experimentally measure the EM field density distribution inside the vessel. This test showed media exposure closely matched with the predictive CEM. This validated the CEM effort dedicated to designing CPEC-4.

\footnotetext{
17 The antenna system of CPEC-4 is made of multiple radiative elements and several ports. These elements have some degree of freedom to allow tunability and field distribution arrangements. By changing the position of the elements, the mutual exposure of one element to another (e.g., "crosstalk") is also changed.

18 The crosstalk from port to port is seen as reflected power by the source (e.g., the generator). Crosstalk is highly undesirable.

19 Because of their export controlled and proprietary nature, these measurements and the characteristics of the benchtop system cannot be disclosed in this document.

20 Standard used: "ICNIRP guidelines for limiting exposure to time-varying electric, magnetic and electromagnetic fields (up to $300 \mathrm{GHz}$ )," Health Physics, 1998, Vol. 74, No. 4, pp. 494-522.
} 
Experimental work with fiber in the cavity began in early May, prior to receiving the second generator system. For these first tests, a load of four static tows of OPF (50k filaments each) was positioned and several tests were performed. The fiber did not convert. Eventually, this series of tests was interrupted by a second failure, identical to the first, which occurred during the evaluation of the EM field distribution. This failure happened under similar conditions (e.g., at maximum power). This led to two conclusions:

- A single subsystem was discovered to not produce the expected effects on the entire system (despite matching the specification). It was suspected to be the main reason why the OPF did not convert. Subsequently, several important hardware modifications were undertaken to modify the vessel and make the process possible.

- The hardware failures were due to the usage of materials that do not match the specification. A potential substitute material was found and tested. This replacement had negative effects on the distribution of the EM field. Tests showed that this substitute material could not be used without a long design effort. No other suitable configuration could be found in a reasonable time. The project had to continue with another batch of the same material.

Both hardware modifications and material failure required CEM efforts to inform appropriate corrective actions.

On August 26, an acceptable technical solution to this issue was conceived and tested at a small-scale. This concept was the result of a several-months-long effort and a multitude of attempts at corrective action. Implementation of this concept at a larger scale (for 8 tows of 50k each) required several additional days for hardware adjustments. On September 10, the modification was completed, and the setup became ready for testing with a batch of 8 static tows.

On September 11, a region of undamaged filaments about 8 in. long was found among the tows exhibiting a color change from the deep shiny black of the OPF to a dark grey with some brown reflections. This color change indicates possible conversion, as observed in Figure II.2.11.2. Conversion was confirmed by additional characterization. The bundle became conductive, as seen in Figure II.2.11.3, and its chemistry changed, as seen in Figure II.2.11.4. Unfortunately, the previously experienced hardware failures again occurred during this test.

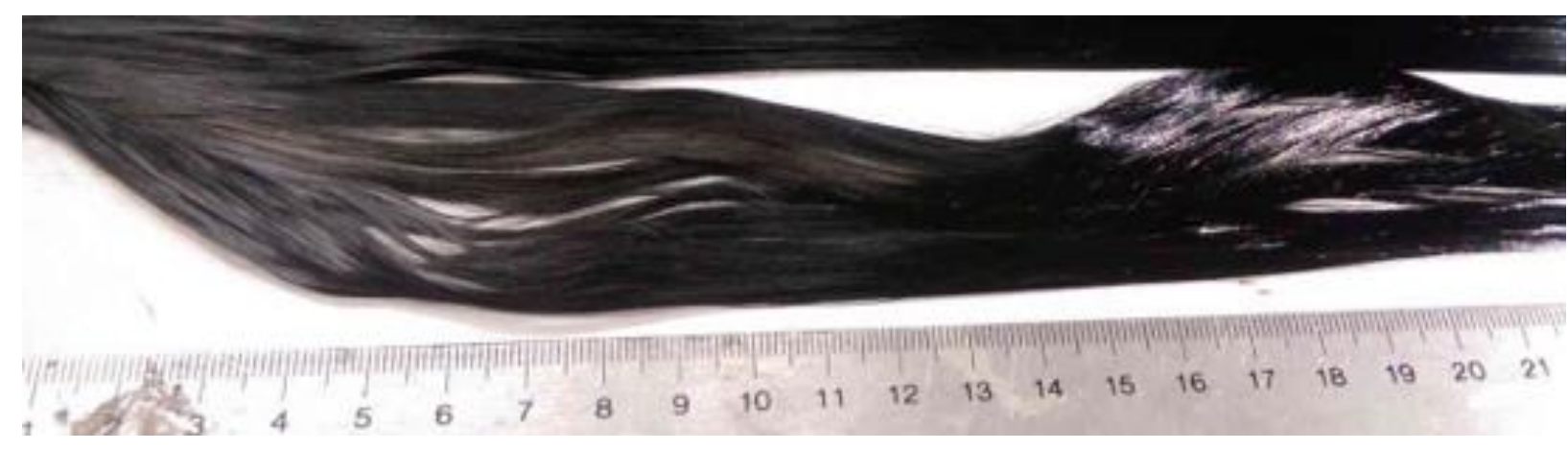

Figure II.2.11.2. A bundle of several hundred of grey filaments between $10 \mathrm{~mm}$ and $120 \mathrm{~mm}$ was found among the 8 tows of the static batch. Its total length was measured to ca. 8 in. Source: 4XTechnologies. 


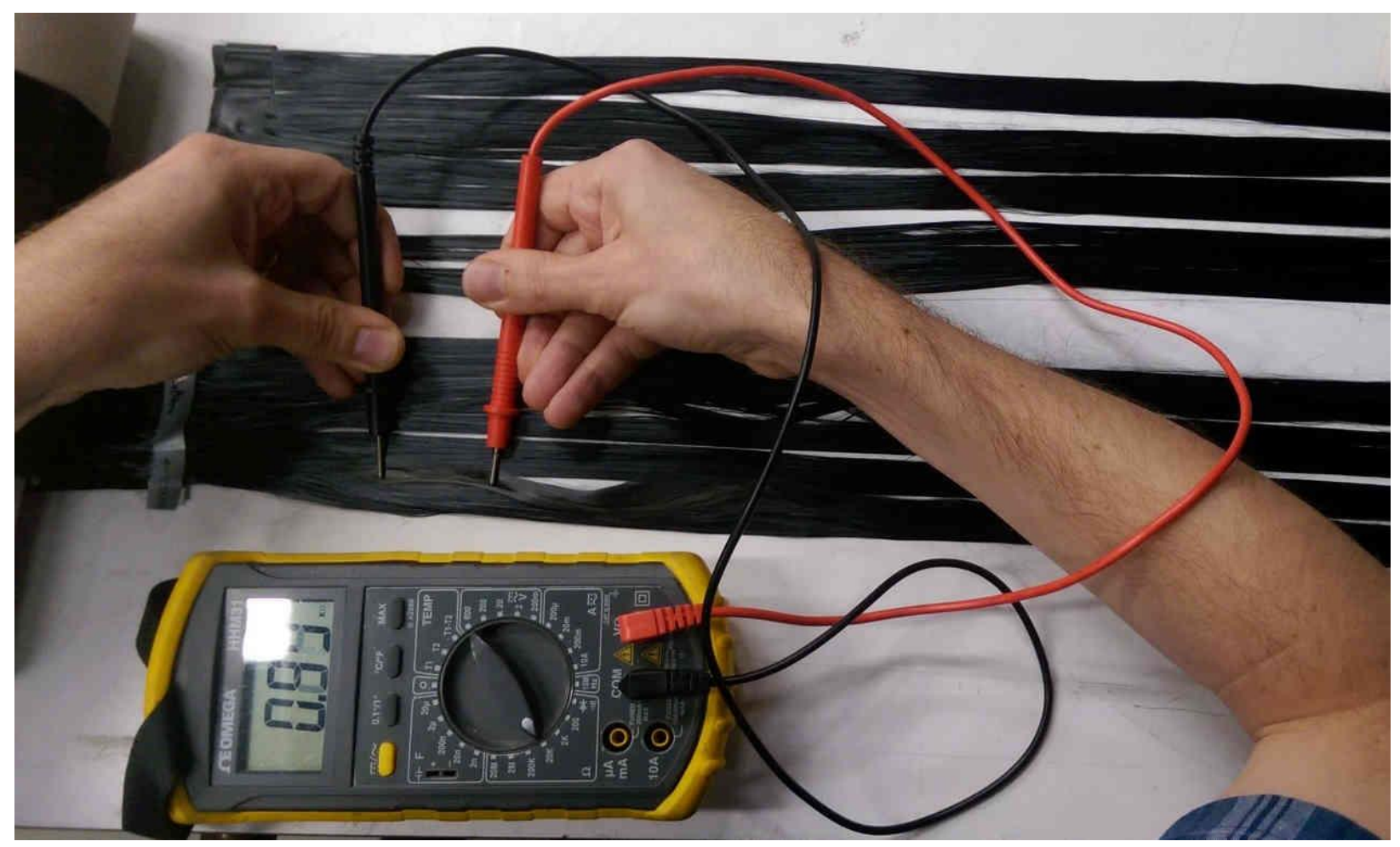

Figure II.2.11.3. By using a regular multimeter, the fiber can be measured to be conductive in the area of interest, which confirms that the material has been carbonized. Source: 4XTechnologies.

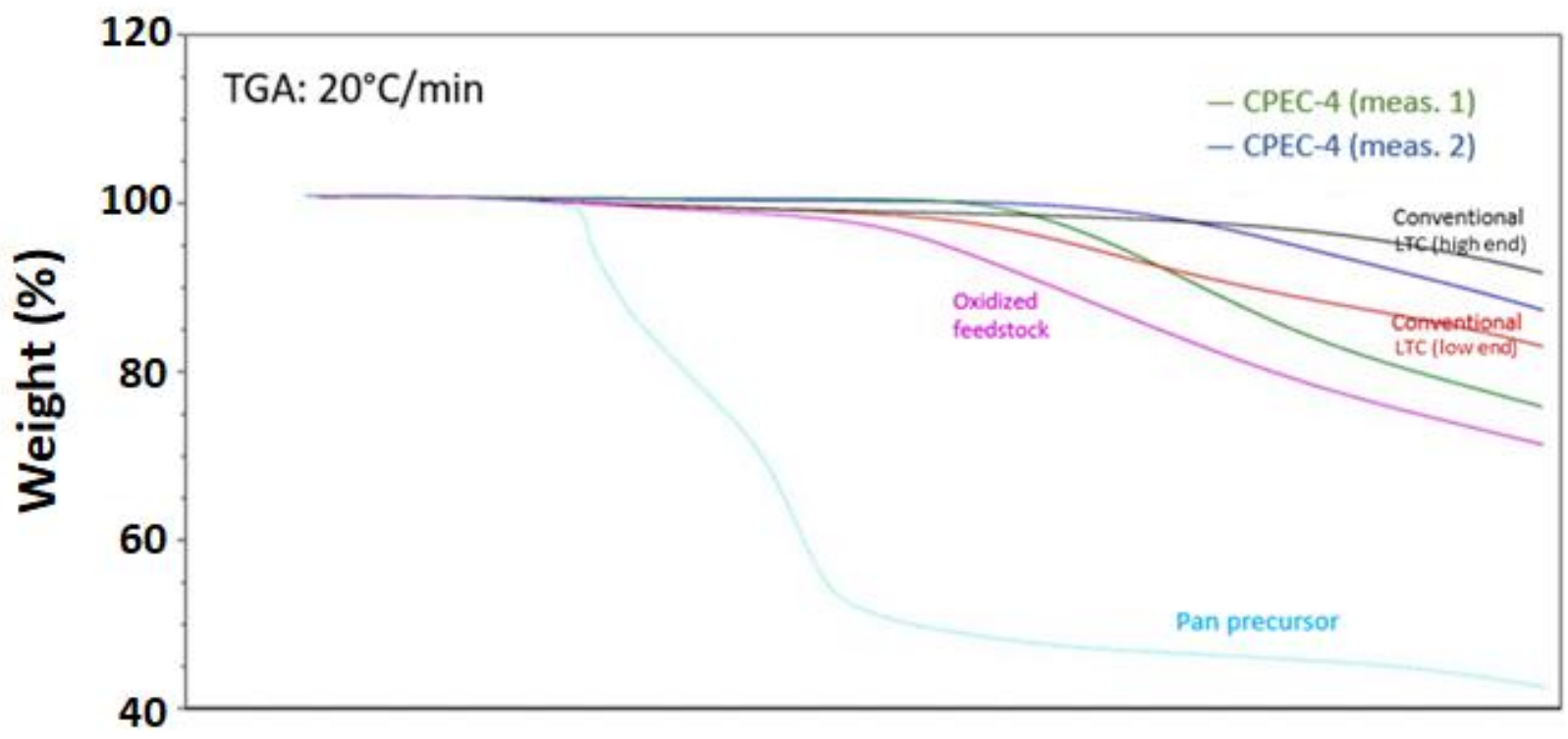

Figure II.2.11.4. TGA characterization for the OPF conversion. Sample analyzed at ORNL.

For the thermogravimetric analysis, two measurements were performed with the bundle of interest. These are the green and dark blue curves in Figure II.2.11.4. Other curves shown for reference are turquoise = PAN; purple $=$ OPF; red $=$ OPF processed conventionally at the lower limit; and black $=$ OPF processed conventionally at the higher limit of the temperature range of LTC. The onset of the weight reduction shown by the green and the blue curves occurs between the red and the black curves. This indicates that the bundle of 
interest has been treated in the temperature range defined by the two references (red and black curves). Furthermore, the value of the blue curve remains higher than the red curve over the entire test, but lower than the black curve at the end of the characterization. This confirms that the bundle of interest was processed at a higher temperature than the sample "conventional LTC (low end)" [red] but less than the "conventional (high end)" [black]. On the other side, compare to measurement 2 [blue], the sharpest onset and the lower weight of measurement 1 [green] at the end of the characterization $\left(\sim 77^{\circ} \%\right)$ indicates that both samples were exposed to similar temperature, but different time of exposure (shorter for measurement 1 [green]).

The results of these tests led to two major observations: (1) carbonization did occur in CPEC-4, but it was localized, suggesting the EM field distribution is not as expected; and (2) repeated failure indicates the failing part is not made with the appropriate material. The correction of those two issues is expected to be timeintensive. Part of the design will have to be revisited using the CEM, then the system will require further modification. These tasks are estimated to take at least five months, which cannot be completed prior to the scheduled conclusion of the project in December 2019. Therefore, the final activities in FY 2019 were to continue the CEM activity to determine what type of modifications will be required to the system and engage DOE in negotiating revised milestones and budget for this project to make it successful.

\section{Conclusions}

The LTC project using CPEC made limited progress due to major delays in the procurement of a critical subsystem, as well as technical issues associated with the novelty of the design. These delays made the completion of the programmatic milestone schedule impossible. At the end of FY 2019, a test with a static batch showed an area with several hundred filaments carbonized in CPEC-4, where the fiber of interest was characterized by multiple methods as LTC fibers. In the meantime, one part of the system failed. This experimental work indicates that CPEC-4 can accomplish what it is designed for, but some parts are not stable and need to be revisited. Already several options are identified, some parts must be redesigned and then fabricated. This effort is mandatory prior any further experimental work and is estimated to be an additional five months effort.

The repartition of the effort on this project was dictated by the availability of the equipment during the first half of the FY, as the effort was reduced prior to the reception of the generator system in March 2019. More resources were allocated afterwards, when it became possible to install, test, and commission the equipment. The second half of the year was mostly dedicated to the commissioning of the equipment, the implementation of corrective actions, and eventually some experimental work in September 2019 (e.g., lab work and CEM work). While maintaining the effort on the CEM, the final efforts of the year were dedicated to the preparation of an extension with a new milestone schedule for this project.

\section{Acknowledgements}

This research was sponsored by the DOE-EERE VTO, performed at ORNL, and managed by UT-Battelle, LLC, for DOE under contract DEAC05-00OR22725. 


\section{II.3 Multi-Material Joining}

\section{II.3.1 A Multiscale Computational Platform for Predictive Modeling of Corrosion in Aluminum-Steel Joints (University of Michigan)}

\section{Mihaela Banu, Principal Investigator (2019-2021)}

University of Michigan

2350 Hayward Street

Ann Arbor, MI, 48109

E-mail: mbanu@umich.edu

\section{S. Jack Hu, Principal Investigator (2018-2019)}

University of Michigan

2350 Hayward Street

Ann Arbor, MI, 48109

E-mail: jackhu@umich.edu

\section{Sarah Kleinbaum, DOE Technology Development Manager}

U.S. Department of Energy

E-mail: sarah.kleinbaum@ee.doe.gov

Start Date: January 24, 2019

End Date: December 31, 2021

Project Funding: \$1,978,423

DOE share: $\$ 1,499,992$

Non-DOE share: $\$ 478,431$

\section{Project Introduction}

The goal of this project is to develop multiscale models for predicting the location and extent of corrosion and the impact of such corrosion on the performance of dissimilar materials joints. The selected joining technologies are RSW, self-piercing riveting (SPR), and rivet-welding (R-W) and are applied to coupons made of Al alloy AA 6022, $2.0 \mathrm{~mm}$ thickness, as well as hot-dipped galvanized high-strength steel low alloy (HDG HSLA) 340 steel, $1.2 \mathrm{~mm}$ thickness. The performance models will include joint strength and fatigue life predictions. These models will be validated by experiments with the goal of achieving strength and fatigue prediction accuracy within $10 \%$ of experiments.

\section{Objectives}

To achieve the project goal, three objectives are considered: (1) to develop models at the microscale level starting from DFT and simulating the initiation of the corrosion; (2) to develop corrosion mesoscopic models by bridging the scales from atom to grain structure, with the mesoscale material properties being homogenized using uncertainty quantification; (3) to develop FE performance and fatigue life predictions by integrating models at different scales. These models will be validated as they are developed.

\section{Approach}

The multiscale models will integrate: (1) the atomic phase level modeling with new high-throughput DFT and calculation of phase diagram (CALPHAD) simulations; (2) the mesoscopic level modeling of corrosion evolution with a FE model, material property homogenization, and uncertainty quantification; (3) the macrolevel modeling of joint performance with experimental validations; and (4) the integrations of these models into a computational platform. The models will be validated by experiments with the goal of achieving an accuracy in strength and fatigue life prediction within $10 \%$ of experiments. 


\section{Results}

\section{Joint Fabrication, Corrosion Experiments, and Characterization}

RSW and SPR processes were used to fabricate joints for accelerated corrosion tests and characterization before and after corrosion. The optimization of the joining processes was performed at three locations. Testing for RSW was done at GM, Henrob completed the SPR, and Optimal Process Technologies, LLC did the R-W to identify a robust process window producing the highest quality of joints. A fractional factorial experiment was used to determine the factor combinations and number of replicates for corrosion tests and performance tests. Each group of replicates was considered as a sample set for subsequent experiments. Figure II.3.1.1 shows an example of the RSW and SPR samples, both uncoated and E-coated. E-coat material is based on 0-5 wt.\% titanium oxide and applied after the samples are joined.
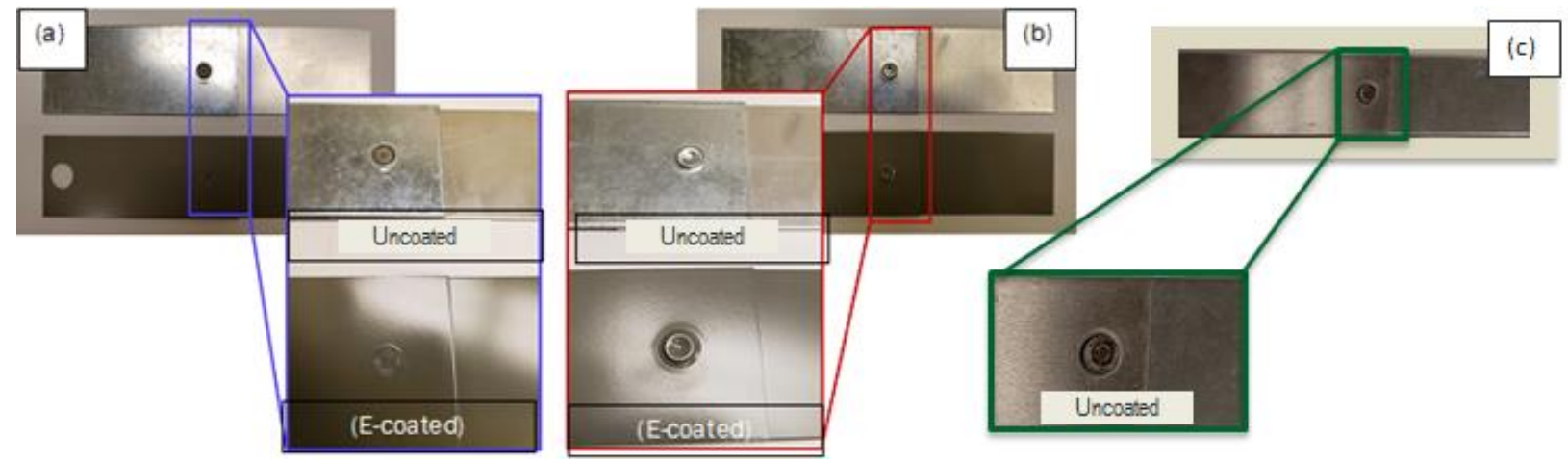

Figure II.3.1.1. Joint samples: (a) RSW, uncoated samples and E-coated samples; (b) SPR; and (c) R-W.

Source: University of Michigan.

Electrochemical measurements of all three materials were performed using a three-electrode cell, in which a saturated calomel reference electrode, a pure Pt-mesh counter electrode, and a working electrode (sample) were used. A $3.5 \mathrm{wt} . \% \mathrm{NaCl}$ solution was used as the electrolyte. To determine the corrosion tendencies and corrosion rate of the materials, open-circuit potential measurements and polarization scanning were performed. The results of the open-circuit potential measurements for the HDG zinc coating, steel substrate (HSLA 340), and $\mathrm{Al}$ alloy were $-1.03,-0.7$, and -0.74 voltages of the saturated calomel electrode, respectively. This indicates that HDG zinc coating is the most active material, and that AA 6022 and HSLA 340 are the noblest materials. Upon exposure to an electrolyte, it is expected that the zinc coating will corrode first, followed by AA 6022, then HSLA 340. According to the polarization curve, AA 6022 has the slowest corrosion rate and HSLA 340 has the fastest corrosion rate.

For characterization purposes in the first year, two configurations of the uncoated RSW and SPR joints were probed. A backscattered diffraction technique was used, along with EDS using a Thermo Scientific Apreo SEM, to ascertain the microstructural features and existing phases. Figure II.3.1.2 shows a cross-sectional overview of the non-coated RSW and SPR joints, for which three different interface regions (i.e., regions 1, 2, and 3) are examined. 

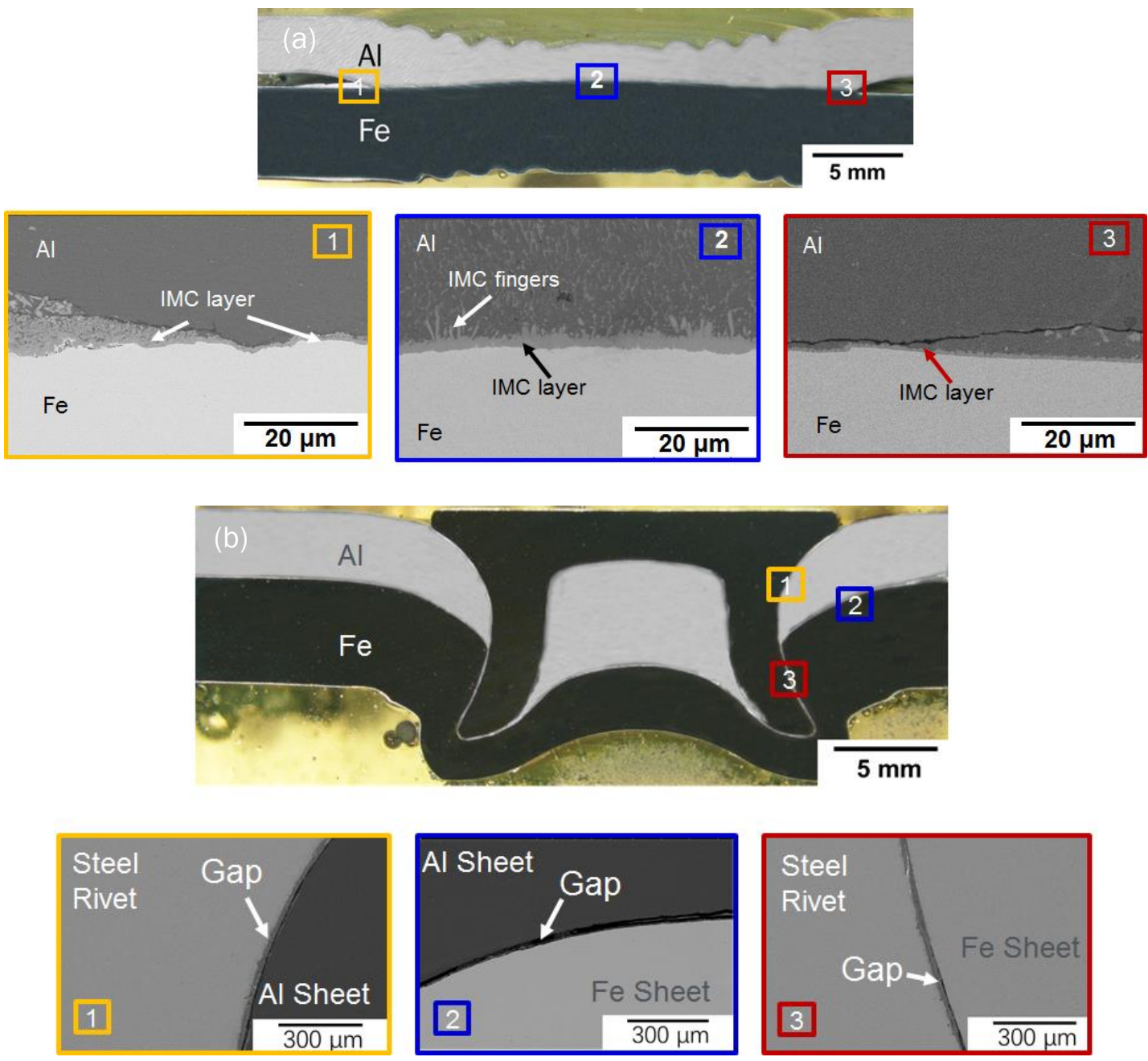

Figure II.3.1.2. Cross-sections of an: (a) uncoated RSW joint; and (b) uncoated SPR joint. Source: Penn State University.

For RSW, as shown in Figure II.3.1.2 (a), region 1 is an intermetallic compound (IMC) layer of approximately $8 \mu \mathrm{m}$. Further, microcracks are visible on both sides of the IMC. Region 2 is the middle part of the joint, where an IMC layer approximately $2 \mu \mathrm{m}$ thick is evident, with finger-like structures protruding into the $\mathrm{Al}$ alloy sheet. However, no defects (i.e., microcracks or holes) are visible along this interface. In addition, small IMC fringes are also discernible in the Al. Region 3 is a thin IMC layer with a continuous microcrack towards the $\mathrm{Al}$ side. A point spectrum analysis-marked with black dots-of the interfacial regions of 1, 2, and 3 suggests the presence of either $\mathrm{Al}_{3} \mathrm{Fe}$ (i.e., $\mathrm{Al}_{13} \mathrm{~F}_{4}$ ) and/or $\mathrm{Al}_{5} \mathrm{Fe}_{2}$. However, the IMC phases will be ascertained more precisely in future analyses using TEM and thermodynamic/kinetic modeling.

In a similar vein, three different regions: (1) steel rivet-Al sheet interface; (2) Al sheet-steel sheet interface; and (3) steel rivet-steel sheet interface were all analyzed for each SPR joint configuration (i.e., uncoated and E-coated), as seen in Figure II.3.1.2 (b). This figure presents the morphology of the steel rivet-Al sheet interface and steel rivet-steel sheet interface, where a thick coating of $\mathrm{Zn}$ towards the rivet side is evident for both interfaces. Moreover, for both interfaces, a gap is evident between the rivet and workpiece materials in regions 1 and 3, suggesting that no chemical bonding has occurred during the riveting process. Region 2 is the $\mathrm{Al}$ sheet-steel sheet interface where a small gap between the two worksheets is present. 
Accelerated corrosion tests were performed for uncoated RSW and SPR joints in customized cabinets. These cabinets simulate field environments by controlling $\mathrm{pH}$, humidity, and temperature. The test parameters were set as follows: $0.90 \% \mathrm{NaCl}, 0.10 \% \mathrm{CaCl}_{2}, 0.075 \% \mathrm{NaHCO}_{3}$, and $98.925 \%$ water (content by weight) for a 60-gal. batch. Corrosion tests were repeated in 1 cycle, 7 cycles, 14 cycles, 26 cycles, 48 cycles, and 72 cycles $\left(1 \mathrm{cycle}=24 \mathrm{~h}: 8 \mathrm{~h}\right.$ salt spray exposure $+8 \mathrm{~h}$ humidity exposure at $49{ }^{\circ} \mathrm{C}+8 \mathrm{~h}$ drying at $\left.60{ }^{\circ} \mathrm{C}\right)$. The corroded samples are presented in Figure II.3.1.3 (a) and Figure II.3.1.3 (b). Further, the samples were lap-sheared, and the results are presented in Figure II.3.1.3 (c). The performance of RSW of SPR joints is equally affected by corrosion after 48 cycles when it is noticed a significant drop in the maximum load.
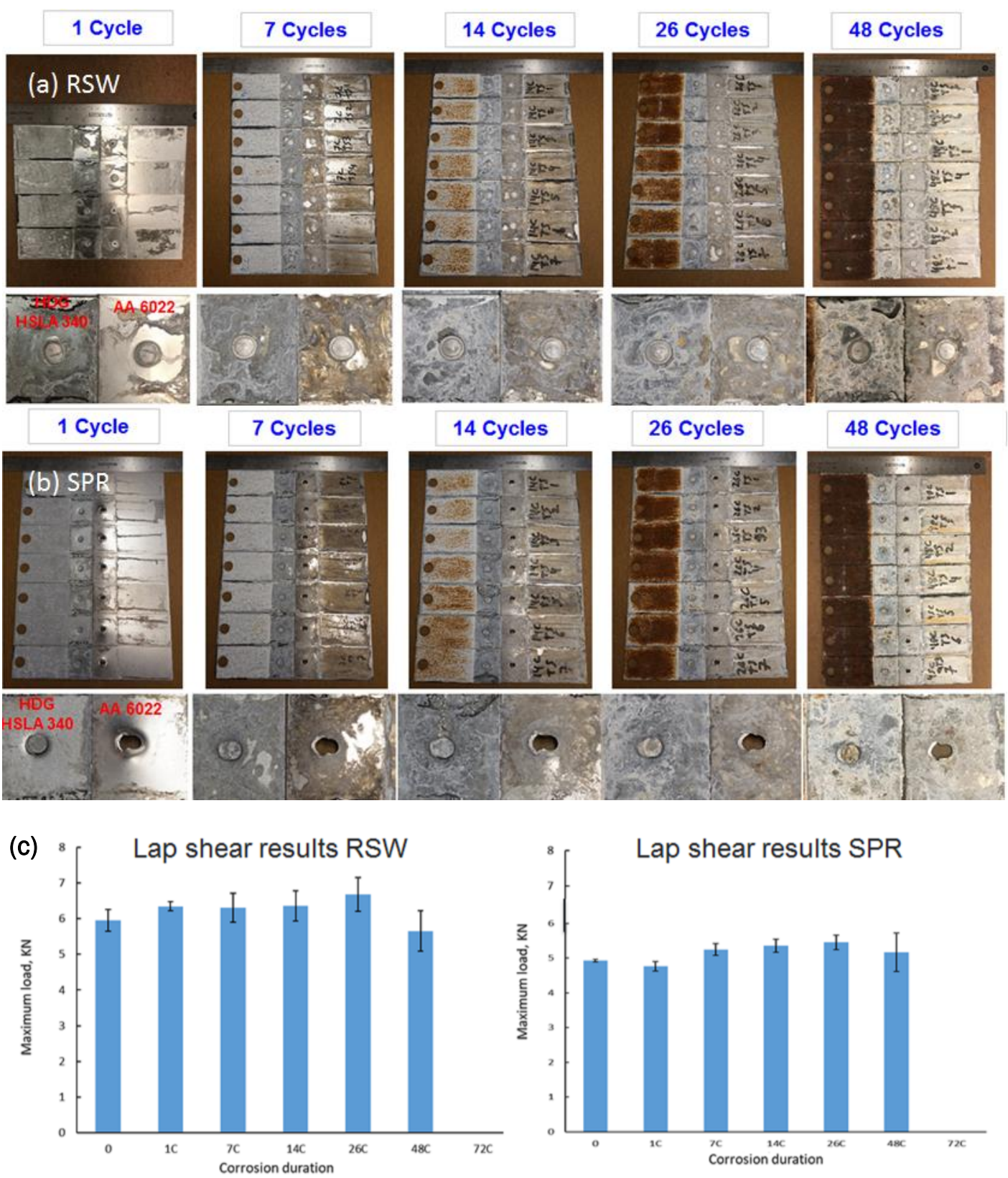

Figure II.3.1.3. Corroded samples (a) RSW; (b) SPR joints uncoated after lap-shear tests; and (c) lap-shear results. Source: University of Michigan. 
Microscopic analysis and lap-shear tests of the uncoated corroded SPR samples showed evidence of corrosion formation starting at 7 cycles. Microscopic analysis revealed the corrosion sites in the joints. For example, Figure II.3.1.4 shows a comparison between corrosion after 1 cycle and 26 cycles. It was noticed that the corrosion starts and grows in the crevice formed by the rivet and the $\mathrm{Al}$ sheet corresponding to region 1 in Figure II.3.1.2 (b). Then, the electrolyte penetrates this gap and generates a second site of corrosion situated at the intersection between the Al-steel and the rivet as illustrated by the red boxes of Figure II.3.1.4 (a), which corresponds to the area between regions 1 and 3 in Figure II.3.1.2 (b). An additional three corrosion sites were noticed at the bottom of the rivet. In total, seven corrosion sites were identified on the SPR joints, as indicated in Figure II.3.1.4 (b). Further analyses and comparisons will be performed for the other cycles, with the purpose of creating a full map of the corrosion nucleation sites and their evolution, as well as to quantify the mass loss. This information will be used to validate the multiscale models.
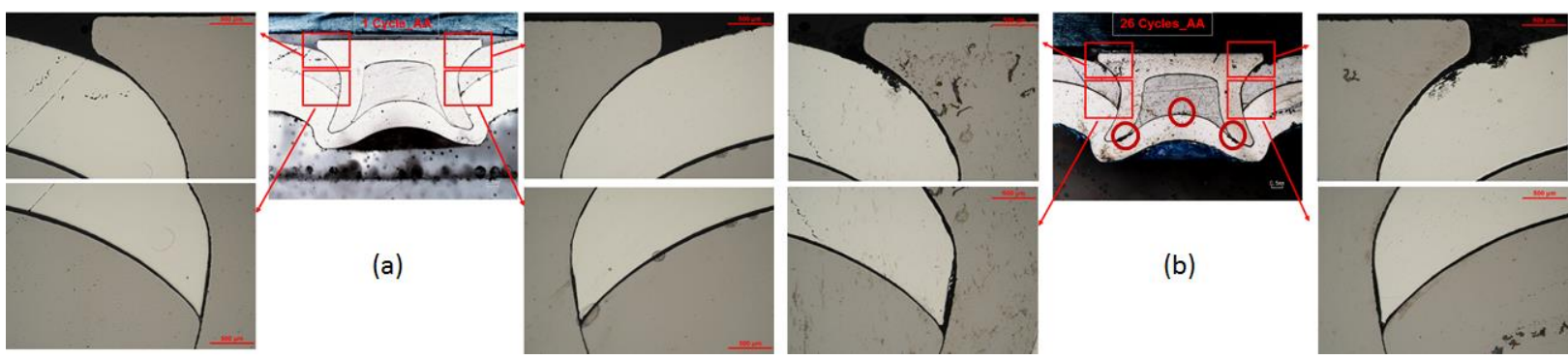

Figure II.3.1.4. SPR joints uncoated with corrosion after (a) 1 cycle and (b) 26 cycles. Source: University of Michigan.

\section{Prediction of Intermetallic Phases through CALPHAD Modeling and DFT Calculations}

To analyze the formation of IMCs in RSW and R-W welded Al-Fe joints, the phase stability of Al-Fe IMCs was studied as a function of composition, temperature, and pressure in terms of: (i) DFT-based first principles and phonon calculations and (ii) thermodynamic calculations using CALPHAD modeling approach. In addition, the Pourbaix corrosion diagram was studied to develop an open-source code DFT tool kit for highthroughput first-principle calculations, while PyCalphad/ extensible self-optimizing phase equilibria infrastructure (ESPEI) was used to determine the high-throughput CALPHAD modeling [1].

Figure II.3.1.5 shows the preliminary DFT-predicted enthalpy of formation of Al-Fe IMCs at $0 \mathrm{~K}$, indicating that the stable IMCs are $\mathrm{Al}_{13} \mathrm{Fe}_{4}, \mathrm{Al}_{2} \mathrm{Fe}$, and $\mathrm{Al}_{3} \mathrm{Fe}$, which are located on the convex hull. The IMCs, which are close to the convex hull, include $\mathrm{Al}_{6} \mathrm{Fe}, \mathrm{Al}_{5} \mathrm{Fe}_{2}$ and $\mathrm{Al}_{12} \mathrm{Fe}_{7}$. By considering the effects of pressure and temperature due to entropy and thermal electrons (i.e., using a quasi-harmonic approach) [2], it was found that $\mathrm{Al}_{6} \mathrm{Fe}$ is a pressure-favorable phase-stabilized IMC at high-pressure and temperature. Note that $\mathrm{Al}_{6} \mathrm{Fe}$ is a metastable phase observed in direct-chill cast $\mathrm{Al}$ sheet [3]. $\mathrm{Al}_{5} \mathrm{Fe}_{2}$ is a temperature-favorable phase-stabilized IMC at high temperatures, while $\mathrm{Al}_{2} \mathrm{Fe}$ is a pressure-favorable, but temperature-unfavorable, phase. Both $\mathrm{Al}_{12} \mathrm{Fe}_{7}$ and $\mathrm{Al}_{8} \mathrm{Fe}_{5}$ are pressure-unfavorable and are not stable at high temperatures and pressures. Note that $\mathrm{Al}_{5} \mathrm{Fe}_{2}$ becomes more stable if the atomic positions are adjusted, because some of their Wyckoff sites are partially occupied. In addition to DFT predictions, we also studied the formation of non-equilibrium IMCs using the equilibrium Al-Fe phase diagram and the metastable "liquid + bcc" phase region, as seen in Figure II.3.1.6. By assuming a peak temperature of $1000 \mathrm{~K}$ during the welding of Al-Fe joints, the mole fraction of $\mathrm{Fe}(\mathrm{xFe})$ on the metastable dashed line in Figure II.3.1.6 was 0.281, while the IMC phases around this composition (with the possibility from high to low) were $\mathrm{Al}_{13} \mathrm{Fe}_{4}, \mathrm{Al}_{5} \mathrm{Fe}_{2}$, and $\mathrm{Al}_{8} \mathrm{Fe}_{5}$ based on their thermodynamic driving forces [4]. Correspondingly, at $1200 \mathrm{~K}$ and $\mathrm{xFe}=0.35$, the $\mathrm{IMC}$ phases were $\mathrm{Al}_{5} \mathrm{Fe}_{2}$, $\mathrm{Al}_{13} \mathrm{Fe}_{4}$, and $\mathrm{Al}_{2} \mathrm{Fe}$. At $1400 \mathrm{~K}$ and $\mathrm{xFe}=0.438$, the IMC phases were $\mathrm{Al}_{2} \mathrm{Fe}, \mathrm{Al}_{8} \mathrm{Fe}_{5}$, and $\mathrm{Al}_{5} \mathrm{Fe}_{2}$. We assumed a welding temperature above liquidus and the fast cooling of Al-Fe joints, with no solid-phase diffusion, but infinitely fast liquid-phase diffusion (i.e., the Scheil simulation conditions). Figure II.3.1.7 (a) shows the solid fraction variations for two $\mathrm{Al}-\mathrm{Fe}$ alloys $(\mathrm{xFe}=0.3$ and 0.6$)$ during the Scheil simulations. When $\mathrm{xFe}=0.3$, $\mathrm{Al}_{5} \mathrm{Fe}_{2}$ will form from a liquid in a small temperature range (1430-1427 K), and $\mathrm{Al}_{8} \mathrm{Fe}_{5}$ will form at $1427 \mathrm{~K}$. 


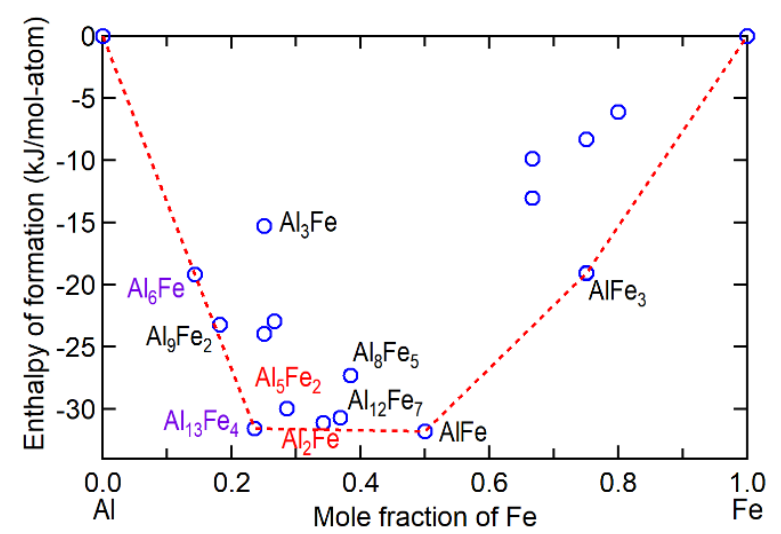

Figure II.3.1.5. DFT calculated phase stability of AI-Fe IMCs at O K. Red lines indicate convex hull. Source: Penn State University.

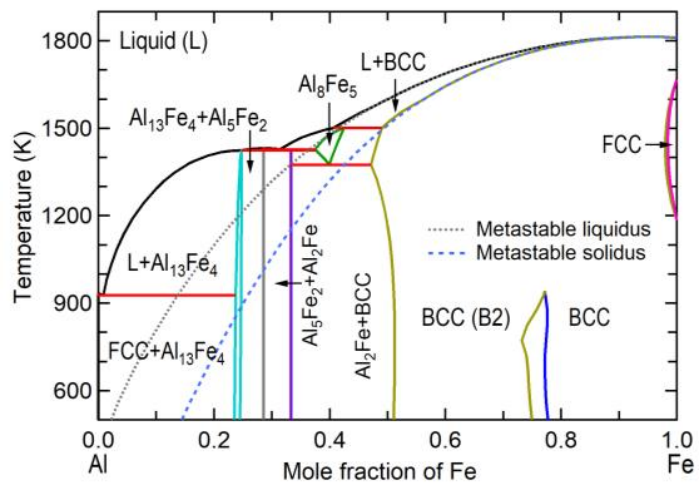

Figure II.3.1.6. Equilibrium Al-Fe phase diagram by CALPHAD modeling and the metastable phase region (Liquid+BCC/B2, the dotted \& dashed lines). Source: Penn State University.
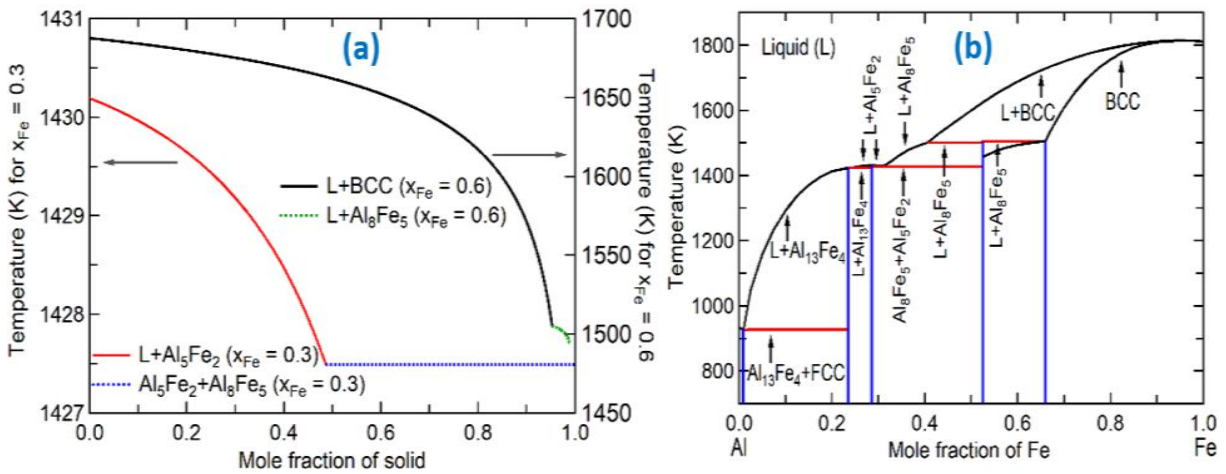

Figure II.3.1.7. Scheil simulations for (a) $\mathrm{X}_{\mathrm{Fe}}=0.3$ and 0.6 and (b) non-equilibrium Al-Fe phase diagram based on Scheil simulations. Source: Penn State University.

When $\mathrm{x}_{\mathrm{Fe}}=0.6, \mathrm{BCC}$ (or the ordered $\mathrm{BCC}$ phase of $\mathrm{B} 2$ ) will form at high temperatures from 1688-1505 K, while $\mathrm{Al}_{8} \mathrm{Fe}_{5}$ will form at $1505 \mathrm{~K}$. Because this reaction needs high temperatures (>1500 K), it may be impossible to form these IMCs during the Al-Fe welding process. Figure II.3.1.7 (b) shows a complete Al-Fe non-equilibrium phase diagram based on Scheil simulations, indicating that $\mathrm{Al}_{13} \mathrm{Fe}_{4}$ and $\mathrm{Al}_{5} \mathrm{Fe}_{2}$ are the most frequently formed phases at lower temperatures (900-1400 K). The experimental tests confirmed that the IMC phases are $\mathrm{Al}_{5} \mathrm{Fe}_{2}$ and $\mathrm{Al}_{13} \mathrm{Fe}_{4}$. In summary, the most likely Al-Fe IMCs to form during welding are $\mathrm{Al}_{13} \mathrm{Fe}_{4}$ and $\mathrm{Al}_{5} \mathrm{Fe}_{2}$. 


\section{FE Based Mesoscopic Modeling for Corrosion Evolution}

A FE simulation method was adopted to investigate the galvanic/crevice corrosion behavior of the proposed joints. During BP1, we studied the effects of spatial and temporal multi-corrosion sites (nucleation) on the crevice corrosion processes of the Al-Fe SPR joints. In the model, the moving of the metal surfaces in the corrosion evolution process can be simulated through the moving boundary trace with the Arbitrary Lagrangian-Eulerian-based moving mesh technique [5]. Specifically, transport of the ionic species in electrolyte obeys Nernst-Planck Equation and the Conservation of Mass, while the anodic and cathodic reaction kinetics at the $\mathrm{Al}$ and $\mathrm{Fe}$ electrode surfaces follow the polarization curves obtained from the experiments [6].

The implemented model consists of a single sealed electrolyte solution domain and several electrolyte-metal interfaces (serving as the cathode or anode surfaces) shown in Figure II.3.1.8 (a), similar to the conditions identified in the experiment. Two corrosion sites (anodes) are selected arbitrarily, with sizes of $2 \mathrm{~mm}$ (site 1) and $1.5 \mathrm{~mm}$ (site 2), as shown in Figure II.3.1.8 (c). A distance of $2 \mathrm{~mm}$ is assigned between the two sites, and the corrosion at site 1 is assumed to occur three days earlier than that at site 2 . The electrolyte potential changes and localized corrosion transformations during the 10-day corrosion evolution process are illustrated in Figure II.3.1.8 (c).

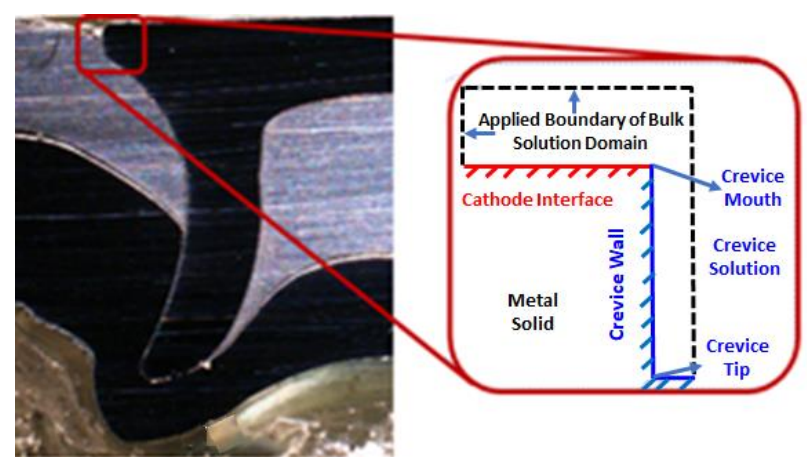

(a)

(b)

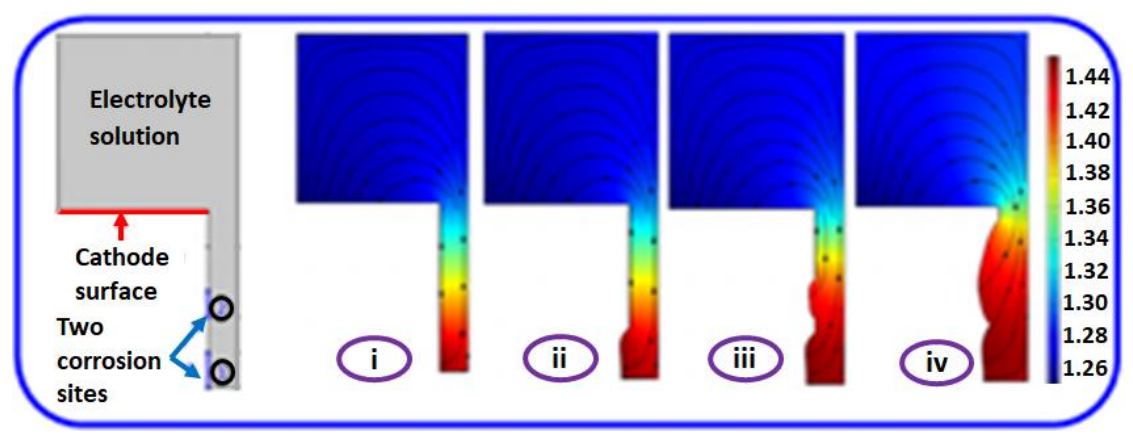

(c)

Figure II.3.1.8. (a) Crevice localized at the interface between the rivet and top sheet in a SPR joint. (b) 2D FE model of galvanic/crevice corrosion. (c) Electrolyte potential change contours and structural deformation during the corrosion evolution process. Source: University of Illinois Urbana-Champaign.

Because of depressed ion diffusivity in the narrow crevice solution in the early stages, the results show that corrosion rates are relatively low; afterwards, with the propagation of corrosion, the localized corrosion transforms to a uniform corrosion, and the sharp corners corrode more rapidly before eventually diminishing. In addition, to consider more complex geometric crevice structures in a realistic scenario, and to better compare the simulated results and experimental data, 3D models are currently in development. 
Figure II.3.1.9 (a) illustrates the 3D structure of the joint, including the two metal bulks, single rivet in the center, and narrow gap domains filled with electrolyte (blue domains). Corrosion is assumed to occur at the interface between the electrolyte and the Al. Figure II.3.1.9 (b) shows a visualization of the electrolyte potential change contours and transformation of the electrolyte domain along the $\mathrm{Al}$ anode surface during three cycles $(72 \mathrm{~h}$ ) of the corrosion process. The results show that uniform corrosion occurs on the $\mathrm{Al}$ anode surface. The corrosion rate near the crevice mouth is higher, and the concentrated electrolyte current density near the cathode surface increases the reaction rate. Additionally, the narrow crevice suppresses the diffusion of ions in the electrolyte, reducing reactions at the crevice tip.
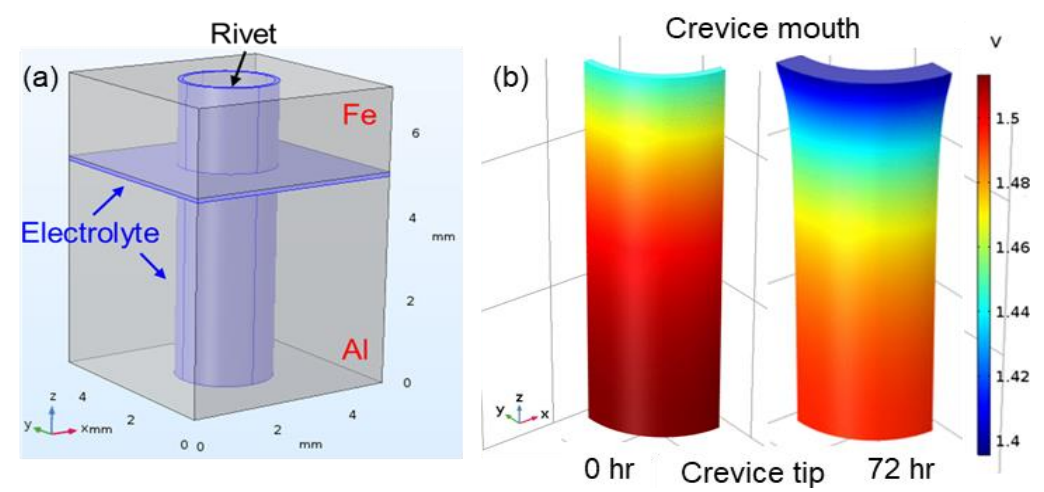

Figure II.3.1.9. (a) The 3D visualization of crevice corrosion schematic in the FE model. (b) Electrolyte potential change contours along the anode (Al) surface. Source: University of Illinois Urbana-Champaign.

\section{Prediction of Joining Performance-Integration of Corrosion Behavior in LS-DYNA}

The algorithm used to model joining performance subjected to corrosion was designed to integrate the results from the micro- and meso-scales presented in the previous sections. The algorithm comprises a new userdefined material model that will account for visco-plastic, thermal, phase transformation, and corrosion effects. To simulate loosening effects on corroded joints, the elastic and strength properties of the new material will also be modified as a function of the predicted level of corrosion. The interface scheme for user-defined materials in LS-DYNA is illustrated in Figure II.3.1.10. The input parameters for a user-defined material can be specified via the keyword *MAT_USER_DEFINED_MATERIAL_MODELS. If this keyword is included in the analysis input file, then the main FE program calls a user-defined subroutine in which stresses, at the current time step, are computed from strains, or deformations, evaluated within each individual element. Following the development of this new user-defined material in LS-DYNA, the behavior and the residual performances predicted by high-fidelity numerical models of multi-metal joints will accurately reflect the damage caused by corrosion.

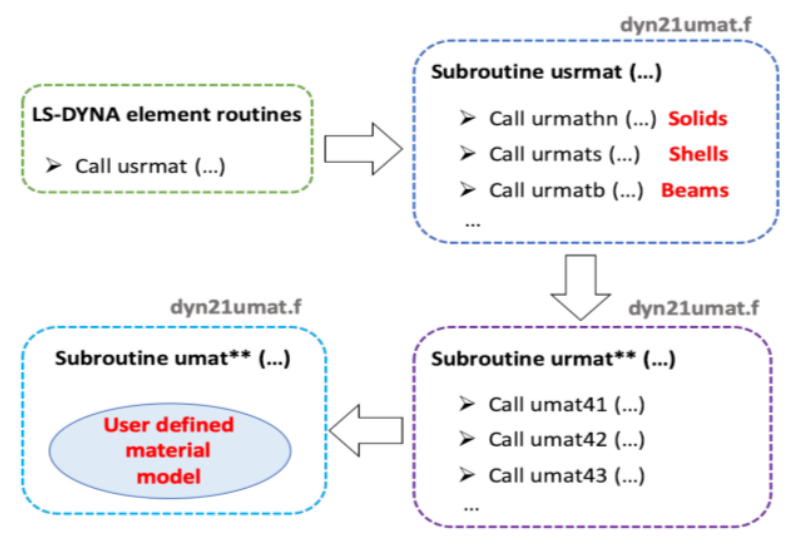

Figure II.3.1.10. Scheme of the user-defined material interface in LS-DYNA. Source: Livermore Software Technology Corporation. 


\section{Conclusions}

In the first year, we performed the extensive fabrication of joints using a robust process window of RSW and SPR processes. These joint samples were subjected to accelerated corrosion tests matching the form and degree of corrosion in field environments. These joint samples were then used in subsequent characterizations to determine their mechanical properties and IMC phases and to map their corrosion nucleation sites and quantify material losses over time. Seven corrosion sites were identified on the SPR joints. In parallel with the experiments, multiscale modeling was initiated. Thus, microscale modeling using DFT calculations and CALPHAD modeling of the thermodynamic properties of an Al-Fe material system predicted two dominant phases in the IMCs of the joints $\left(\mathrm{Al}_{2} \mathrm{Fe}_{5}\right.$ and $\left.\mathrm{Al}_{13} \mathrm{Fe}_{4}\right)$. For mesoscale modeling, a hybrid corrosion pit initiation/growth model with predetermined site conditions and accurate atomic phase scale inputs was built. This model will be further developed and validated using experimental data. For macroscale modeling, a generalized material joint modeling module was initiated and will be further integrated into the LS-DYNA platform.

\section{References}

1. Liu, Z. K., and S. L. Shang, 2019, "Python-based open-source software developed in the PI's group: ESPEI (Http://Espei.Org), Pycalphad (Http://Pycalphad.Org), and DFT Tool Kit. (Http://Github.Com/PhasesResearchLab). Based on Atomate (Http://Atomate.Org).”

2. Shang, S.-L., Y. Wang, D. E. Kim, and Z.-K. Liu, 2010, "First principles thermodynamics from phonon and debye model: Application to Ni and Ni3Al," Comp Mater Sci, Vol. 47, No. 4, pp. 1040-1048.

3. Aliravci, C. A., and M. Ö. Pekgüleryüz, 1998, "Calculation of phase diagrams for the metastable Al-Fe phases forming in direct-chill (DC)-cast $\mathrm{Al}$ alloy ingots," CALPHAD, Vol. 22, No. 2, pp. 147-155.

4. Lee, H. M., S. W. Yoon, and B.-J. Lee, 1998, "Thermodynamic prediction of interface phases at Cu/solder joints," J Electron Mater, Vol. 27, No. 11, pp. 1161-1166.

5. Hu, H. H., N. A. Patankar, and M. Y. Zhu, 2001, "Direct numerical simulations of fluid-solid systems using the arbitrary Lagrangian-Eulerian technique," J Comput Phys, Vol. 169, No. 2, pp. 427-462.

6. Gjelstad, A., K. E. Rasmussen, and S. Pedersen-Bjergaard, 2007, "Simulation of flux during electromembrane extraction based on the Nernst-Planck equation," J Chromatogr A, Vol. 1174, No. 1-2, pp. 104-111.

\section{Acknowledgements}

The authors would like to recognize the following individuals whose contributions to this project have been greatly appreciated:

- Co-PI Blair Carlson, Co-I Joseph Simmer, and Yang Guo, GM

- Co-PI Richard Chinoski, Henrob

- Weiling Wan and Tian Liu, UM

- $\quad$ Co-PIs Jingjing Li and Shunli Shang, Zi-Kui Liu, Yi Wang, and Bo Pan, Pennsylvania State University

- $\quad$ Co-PIs Chenhui Shao, Pingfeng Wang, and Yumeng Li, Post-Doc Zhuoyuan Zheng, and Graduate Student Yuhang Yang, University of Illinois-Urbana-Champaign

- $\quad$ Co-PI Marco S. Pigazzini, Co-I Dandan Lyu, LSTC

- Co-PIs Nenad Eskerica and Boris Bagaric, Optimal Process Technologies. 


\title{
II.3.2 Phase-Field Modeling of Corrosion for Next-Generation Aluminum-Magnesium Vehicle Joints (Worcester Polytechnic Institute)
}

\author{
Adam C. Powell, IV, Principal Investigator \\ Worcester Polytechnic Institute \\ 100 Institute Rd. \\ Worcester, MA 01691 \\ E-mail: acpowell@wpi.edu
}

\section{Sarah Kleinbaum, DOE Technology Development Manager}

U.S. Department of Energy

E-mail: sarah.kleinbaum@ee.doe.gov

Start Date: October 1, 2018

Project Funding (FY19): \$630,299
End Date: December 31, 2021

DOE share: $\$ 499,450$
Non-DOE share: $\$ 130,849$

\section{Project Introduction}

The next frontier in vehicle body lightweighting could make extensive use of Mg alloy sheet for its outstanding stiffness and strength-to-weight, with an Al alloy sheet providing low-cost paintable Class A finish surfaces. Already, the Lincoln MKT and Chrysler Pacifica liftgates have used cast Mg inner and $\mathrm{Al}$ sheet outer components, and the Magna-FCA next-generation ultralight door will likely use similar materials. However, corrosion of Al-Mg fusion welds is a serious problem, limiting widespread use of this material pair in a vehicle's body (e.g., steel self-piercing rivets in $\mathrm{Mg}$ would create more severe corrosion).

FSW leads to significant grain refinement, second-phase refinement, homogenization, and densification; all characteristics known to have beneficial effects on the corrosion resistance of light metals. Al corrosion studies suggest grain refinement via equal-channel angular pressing improves corrosion resistance primarily via impurity breakup and homogenization leading to reduced microgalvanic current. Similarly, FSW in wrought Mg-Yttrium RE and AA5083 Al alloys shows potential for improved corrosion resistance likely due to breakdown and dispersion of intergranular precipitates reducing the mass loss rate.

That said, there is no quantitative link between microstructure and corrosion performance. Closing this missing link in understanding will facilitate design of cost-effective processes for making robust joints whose geometry and microstructure reduces corrosion and joint failure, even when coatings fail.

\section{Objectives}

The objective of this project is to provide a quantitative link between FSW microstructure and corrosion performance. In particular, the team aims to develop and validate a grain-level phase-field model of microgalvanic corrosion, and coupled micromechanics model of mechanical failure, in FSW Al-Mg alloy joints to predict strength and fatigue lifetime of corroded joints within $10 \%$ of measured performance.

This project will develop the models using $6022 \mathrm{Al}$ and ZEK100 Mg alloys, which were selected for the Magna-FCA ultralight door project. Test joints between these sheet materials will use diffusion-bonding and FSW. The team will attempt to validate the model using 7xxx-series Al alloys. 


\section{Approach}

The phase-field corrosion model starts with an expression for free energy as a function of composition in the metal, electrolyte, and oxide and/or hydroxide corrosion product phases. This free energy expression begins with a fit to thermodynamic data on the $\mathrm{Al}$ and $\mathrm{Mg}$ alloy systems, including the base metals and IMCs, with higher free energy in composition ranges between IMCs to tune the interfacial energies. The electrolyte is an aqueous solution with $\mathrm{Al}^{3+}$ and $\mathrm{Mg}^{2+}$ ions and dissolved oxygen. Using a representation of the full $\mathrm{Al}-\mathrm{Mg}$ system free energy function both produces the correct compositions at phase boundaries, and leads to correct chemical potential of both species at metal-electrolyte boundaries, which automatically creates electronically mediated microgalvanic corrosion reactions between the various phases.

Corrosion model validation begins with fabrication of diffusion-bonded and welded joints. Diffusion-bonded sheets of pure $\mathrm{Mg}$ and $\mathrm{Al}$ provide an ideal model system for understanding the fundamentals of galvanic corrosion. Diffusion-bonded alloy sheets deepen this understanding to include additional phases in each alloy. When the phase-field corrosion model is proven for diffusion-bonded couples, we will then try to use it to describe corrosion in a complex FSW microstructure.

Prediction of FSW microstructure is beyond the scope of this study. Instead, advanced characterization techniques, such as SEM with EDS and EBSD, will produce maps of composition and grain orientation across a FSW joint. This will include using in situ FIB and plasma etching to expose and characterize multiple layers in a joint leading to a 3-D microstructure. The microstructure thus characterized will provide the initial condition for the corrosion model.

This project consists of four tasks with an end goal of a validated accurate model of corrosion and mechanical failure:

1. $\quad$ Produce Diffusion-Bonded and Welded Coupons for Testing:

Produce coupons with joints between pure and/or alloyed $\mathrm{Mg}$ and $\mathrm{Al}$ sheets by either diffusion-bonding or friction stir and/or fusion welding.

2. Conduct Corrosion and Mechanical Testing:

Run accelerated corrosion tests and tensile and cyclic loading tests to determine corrosion morphology and its effect on strength and fatigue performance of $\mathrm{Mg}-\mathrm{Al}$ joints.

3. Characterize Welded Joints and Corrosion and Mechanical Test Samples:

Use advanced characterization methods including ESM with EDS and EBSD, as well as small angle neutron scattering, to understand the structure of corrosion products and fracture surfaces and provide input geometry/morphology as an initial condition for models.

4. Develop Corrosion and Mechanics Model:

Use phase-field and CP modeling based on PRISMS tools from the UM to build a grain-level model of corrosion and mechanical deformation of $\mathrm{Mg}-\mathrm{Al}$ joints.

\section{Results}

Produce Diffusion-Bonded and Welded Coupons for Testing

Methods progressed significantly in both diffusion-bonding and FSW Mg sheet to Al sheet as discussed below. 


\section{Diffusion-Bonding}

An argon atmosphere tube furnace shown in Figure II.3.2.1 (a) was utilized to perform diffusion-bonding of well-polished pure $\mathrm{Mg}$ and Al. A simple screw-driven fixture, as observed in Figure II.3.2.1 (c) was fabricated and used to apply adequate pressure at the $\mathrm{Mg} / \mathrm{Al}$ interface. The fixture is equipped with a thermocouple that is in contact with diffusion couple. This allowed us to obtain more accurate data than relying on furnace temperature. After some trial-and-error, a temperature of $400^{\circ} \mathrm{C}$ for $2 \mathrm{~h}$ provided a well-bonded $\mathrm{Mg}$ - $\mathrm{Al}$ sample. Preliminary SEM characterization was performed on a manually de-bonded 1 in. $\times 1$ in. sample shown in Figure II.3.2.1 (b) during the trial-and-error phase and showed the presence of an IMC with $\mathrm{Mg}-(38$ at \%) Al. This condition was used to produce several diffusion-bonded couples, like the one shown in Figure II.3.2.1 (d), though a noticeable gap was observed between the two sheets as well as poor-quality.
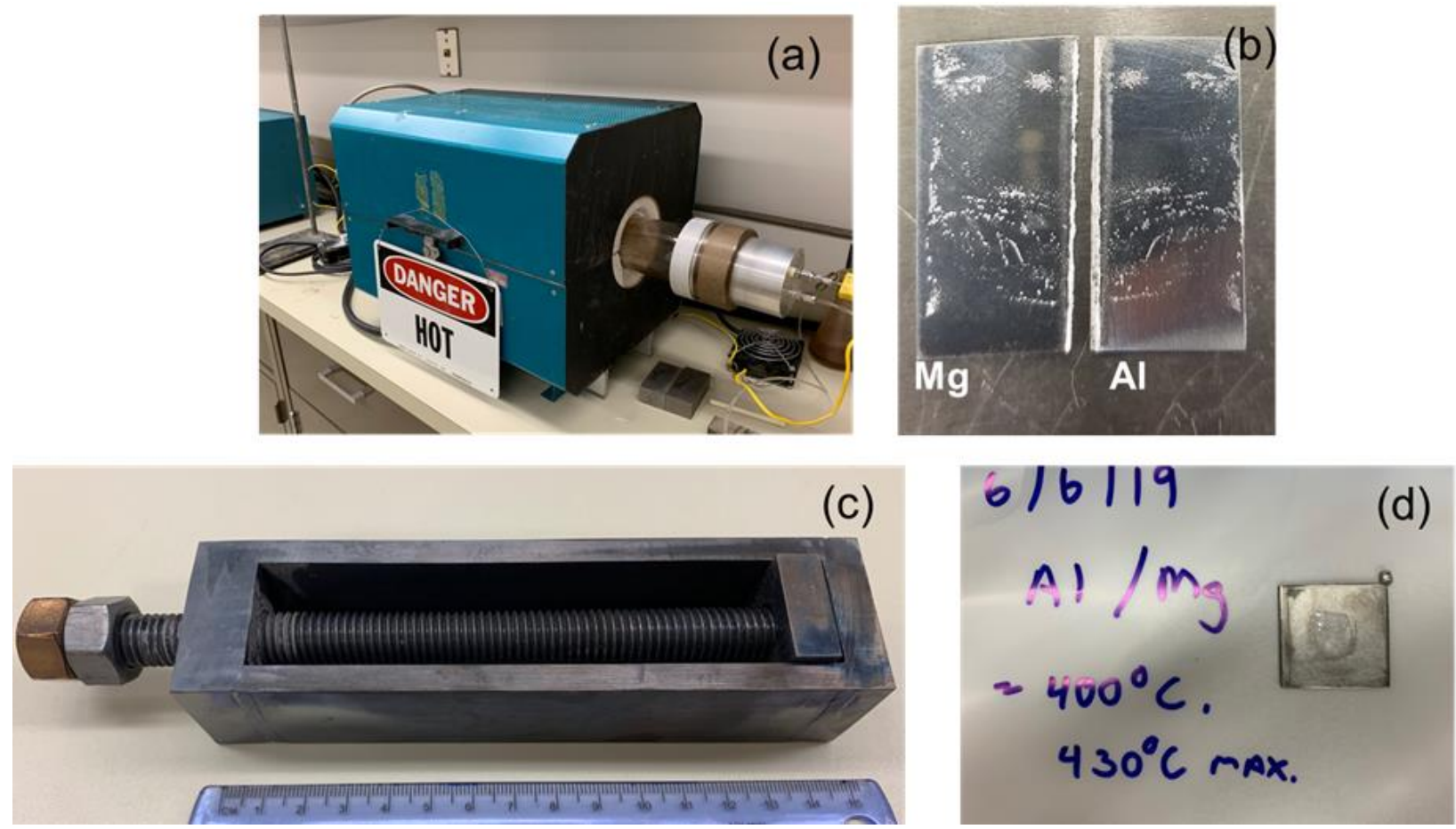

Figure II.3.2.1. Al-Mg sheet diffusion-bonding photos: (a) argon tube furnace; (b) manually de-bonded sample; (c) screw-driven fixture providing compression force; and (d) diffusion-bonded piece. Source: PNNL.

Due to poor bonding quality in these samples, the team moved to a more rigorous procedure of sample preparation and storage prior to diffusion-bonding. The samples were polished to $10 \mu \mathrm{m}$, stored, and transported submerged in mineral oil, as shown in Figure II.3.2.2 (a), to reduce the chance of oxidation on the polished surface. When ready to diffusion bond, the oil was wiped off the sample surfaces. The two surfaces of the bonding pair were then connected and submerged in alcohol. While still wet, the pair was loaded onto the fixture, shown in Figure II.3.2.2 (b), and transferred to the furnace, heated at $390^{\circ} \mathrm{C}$ for $2 \mathrm{~h}$. This method resulted in a bond between 6022 and ZEK100 material pairs that is continuous across the joint surface area. Detailed cross-section analysis including phase identification remains in progress. The team is working on a paper describing the improved diffusion-bonding methodology and an associated phase-field model of diffusion in both the pure metal and alloy sheet samples. 


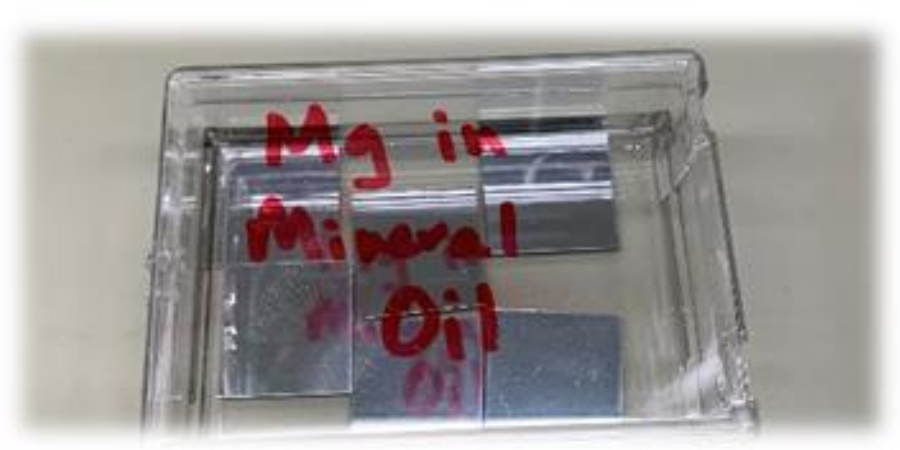

(a)

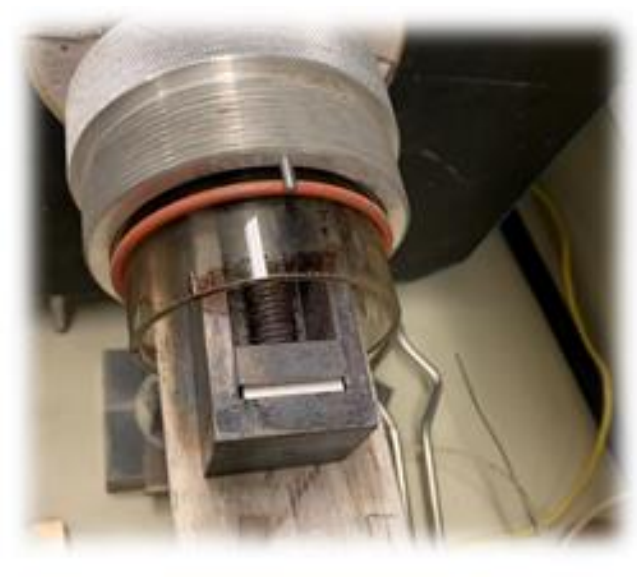

(b)

Figure II.3.2.2. (a) Polished samples submerged in mineral oil ready for diffusion-bonding. (b) Mg and Al samples loaded in the fixture for diffusion-bonding. Source: PNNL.

\section{Friction Stir Welding}

The team began work on FSW parameter development using the candidate materials set - Mg-E717 (1.5 mm) and Al-6022-T43 $(1.27 \mathrm{~mm})$. The initial weld development approach with three pin lengths is summarized schematically in Figure II.3.2.3.

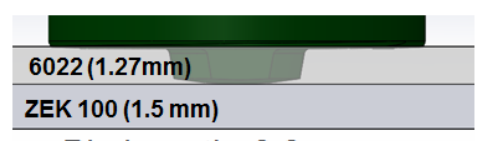

Pin Length: $0.9 \mathrm{~mm}$

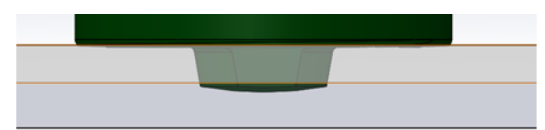

Pin Length: $1.6 \mathrm{~mm}$

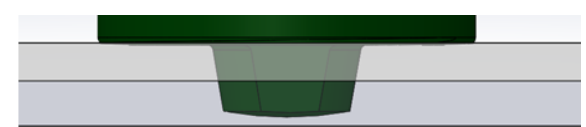

Pin Length: $2.5 \mathrm{~mm}$

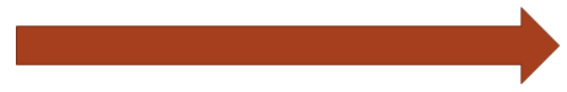

\section{Greater engagement of bottom Mg sheet.}

Figure II.3.2.3. FSW weld development approach. Source: PNNL.

Given the challenge of having to weld a harder material ( $\mathrm{Al}$ ) on top of a softer material $(\mathrm{Mg})$, we evaluated a relatively cold welding temperature at gradually increasing pin length. In addition to the length of the pin we also evaluated the effect of several welding variables, including tool tilt angle, shoulder geometry, welding speed, and revolutions per minute. Previous work in this material system indicate that the primary challenge of joining $\mathrm{Mg} / \mathrm{Al}$ is the management of IMCs that inevitably form at the interface. Proper management of the interface geometry and the thickness of the IMC will be needed to increase the load-bearing capacity of the joint. Figure II.3.2.4 shows preliminary results of weld characterization. A lap-shear strength of $\sim 2.3 \mathrm{kN}$ per one inch of weld length was obtained. 


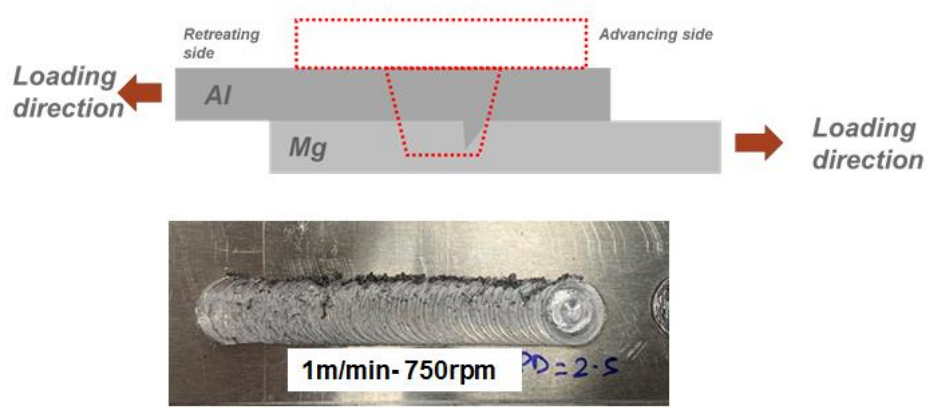

(a)

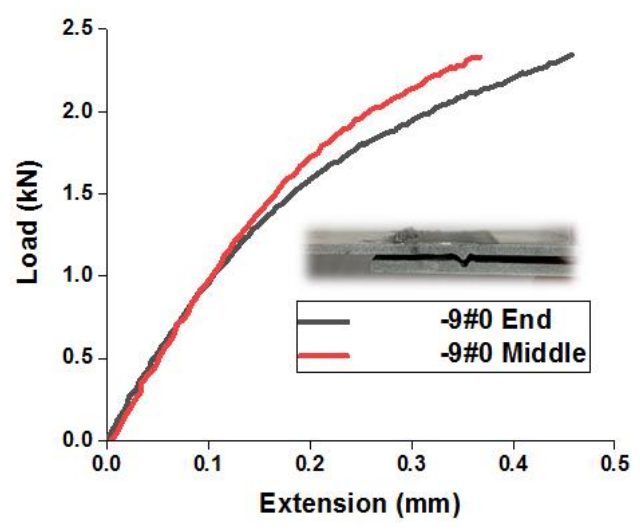

(b)

Figure II.3.2.4. (a) Weld geometry (top) and preliminary optical characterization with the length direction from left to right, and (b) lap-shear test results. Source: PNNL.

The team examined the cross-sections of the welded samples and changed several welding parameters including welding speed, revolutions per minute, and tool tilt angle while keeping the FSW tool geometry constant. Digital image correlation strain mapping during lap-shear testing suggests mechanical hooking may be the primary method of bonding. Typical cross-sections of the joint show a penetrating feature of $\mathrm{Al}$ into the Mg matrix as can be seen in Figure II.3.2.5. Using EDS analysis of the interface in SEM, we observed a fairly thin $(2.5-3 \mu \mathrm{m})$ IMC layer. We also observed some voids and lack of consolidation in the joints. Using a lower welding speed and higher tool tilt angle, we demonstrated higher lap-shear strength in the $\mathrm{Mg} / \mathrm{Al}$ joints — as high as $60 \%$ of the base ZEK100 sheet. Nevertheless, a fair amount of variation in lap-shear strength across the length of the joints was observed from $40 \%$ to $60 \%$ of the base ZEK 100 value as shown by the data in Figure II.3.2.6. This indicates process instability and may be the result of intermittent IMC formation. We are further characterizing the joints to understand this better.

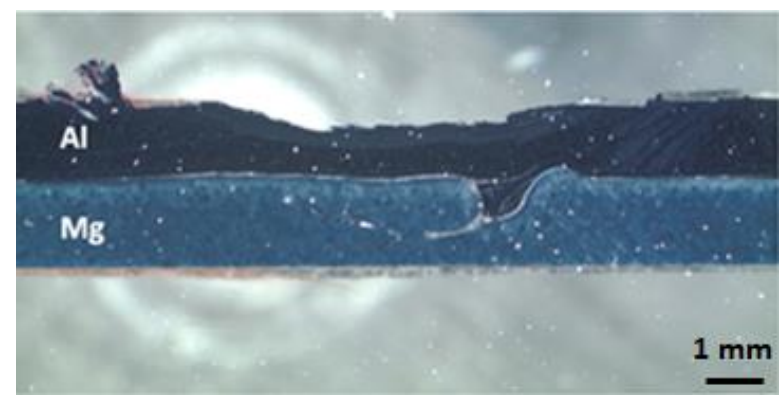

(a)

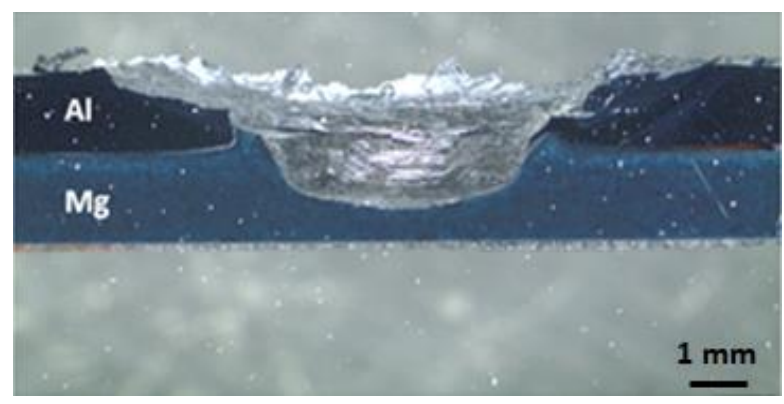

(b)

Figure II.3.2.5. Optical micrograph of Mg/Al joint cross-section: (a) in the middle of the weld line; and (b) at the exit hole. Source: PNNL. 


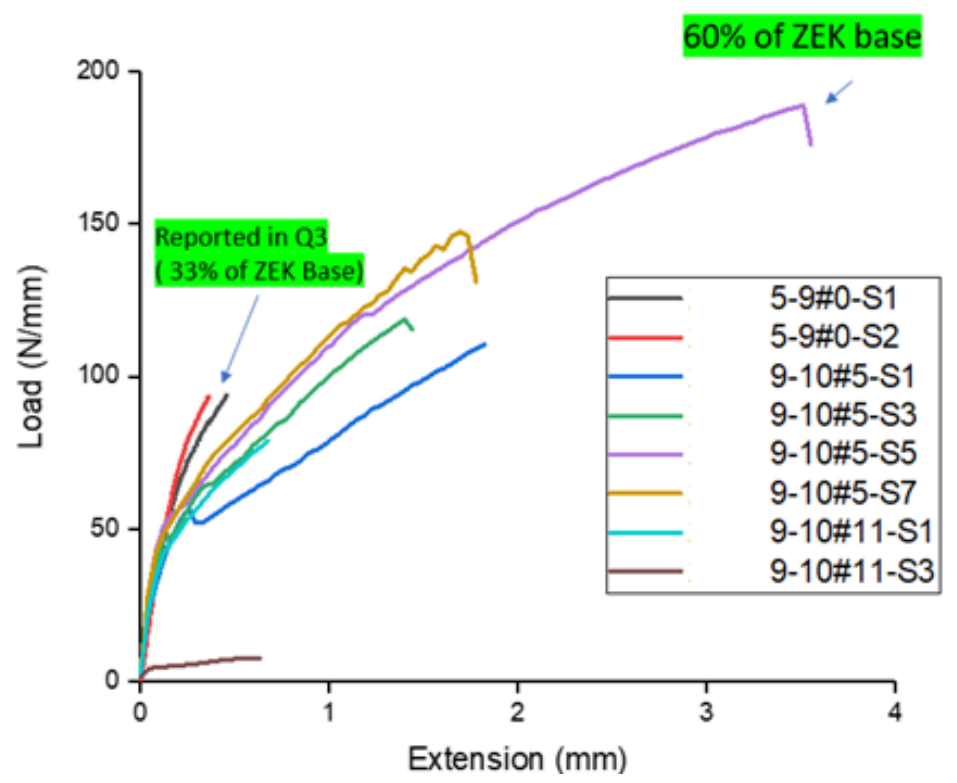

Figure II.3.2.6. Load as a function of extension for Mg-Al joints showing progress in load-bearing capacity. Note the variation in joint strength from 115-180N/mm. Source: PNNL.

\section{Corrosion and Mechanical Testing}

During BP1, this task focused on corrosion testing. Initial corrosion tests on baseline pure $\mathrm{Al}$ and $\mathrm{Mg}$ sheet samples ran in a vessel using two electrolyte solutions: (1) $0.9 \% \mathrm{NaCl}, 0.1 \% \mathrm{CaCl}_{2}$, and $0.25 \% \mathrm{NaHCO}_{3}$ with $\mathrm{pH}=7.4$ and (2) a milder salt solution of the SAE J2334 cyclic corrosion testing (CCT) test standard. The Gamry paracell kit in Figure II.3.2.7 (a) and the Reference 600 potentiostat in Figure II.3.2.7 (b) were used to measure corrosion potential and current (rate) over time.

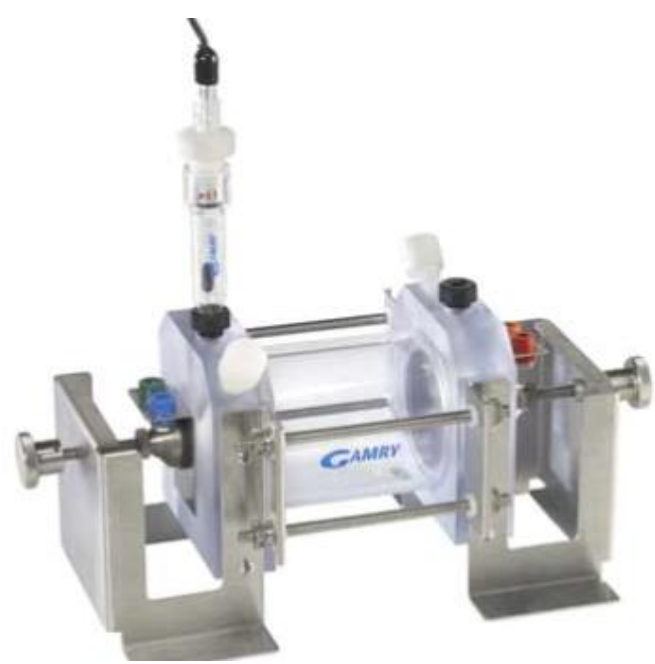

(a)

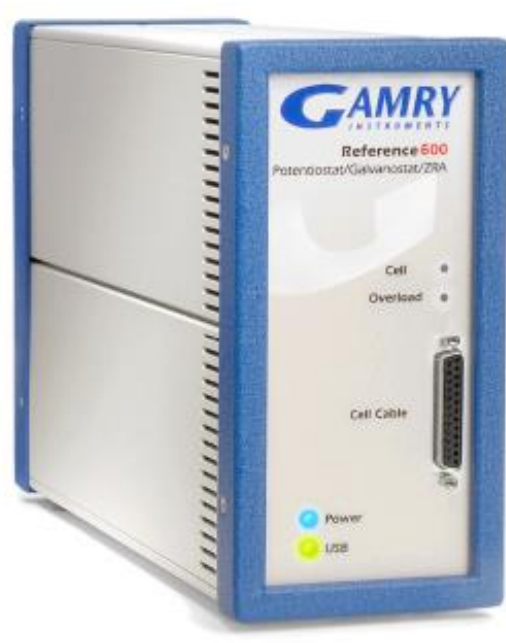

(b)

Figure II.3.2.7. (a) Gamry paracell kit; and (b) Reference 600 potentiostat. Source: Gamry Instruments.

For Solution 1, the measured corrosion potential was $-0.742 \mathrm{~V}$ for pure $\mathrm{Al}$ and $-1.77 \mathrm{~V}$ for pure $\mathrm{Mg}$. The linear polarization test showed a corrosion rate of $0.578 \mathrm{mils} / \mathrm{year}(12.8 \mu \mathrm{m} / \mathrm{year})$ for a pure $\mathrm{Al}$ sheet and $831.7 \mathrm{mils} /$ year $(21.1 \mathrm{~mm} / \mathrm{year})$ for pure $\mathrm{Mg}$. In Solution 2, the corrosion rate was 0.6 mil/year (15.24 $\mu \mathrm{m} /$ year) for pure $\mathrm{Al}$ sheet and 57 mils/year $(1.45 \mathrm{~mm} /$ year) for pure $\mathrm{Mg}$. Representative polarization graphs are presented in Figure II.3.2.8 (a)-(d) for both solutions. 

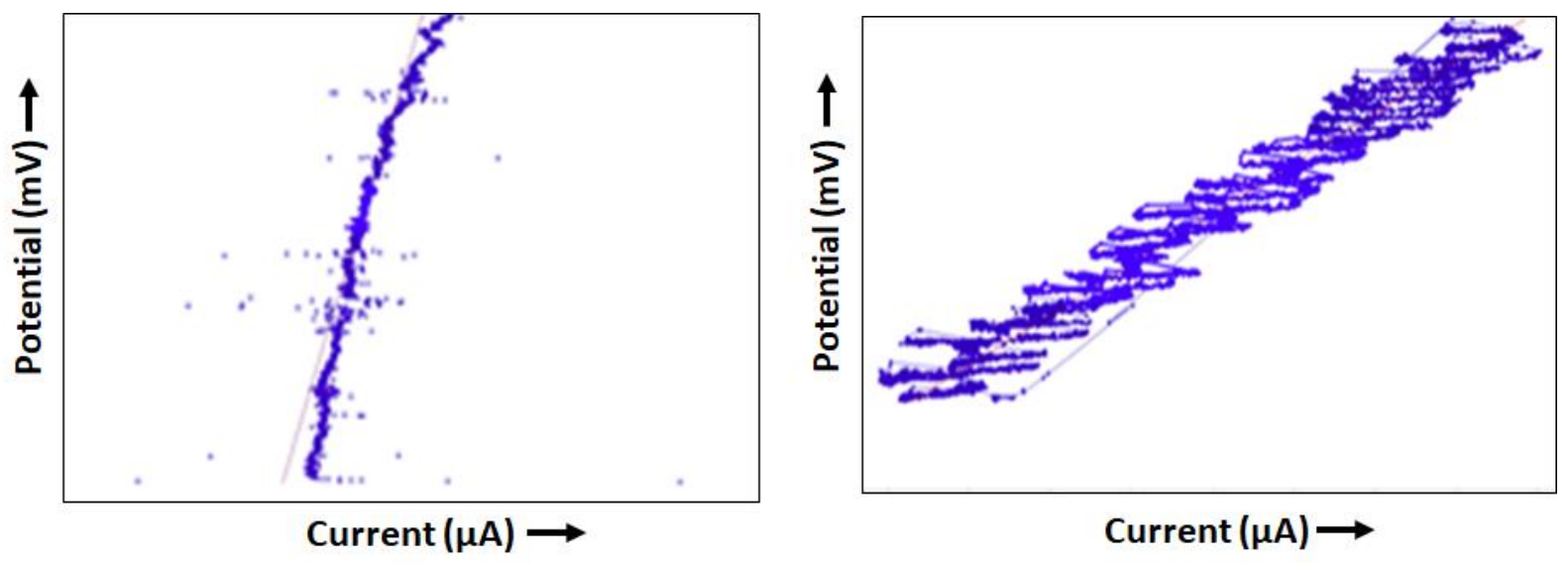

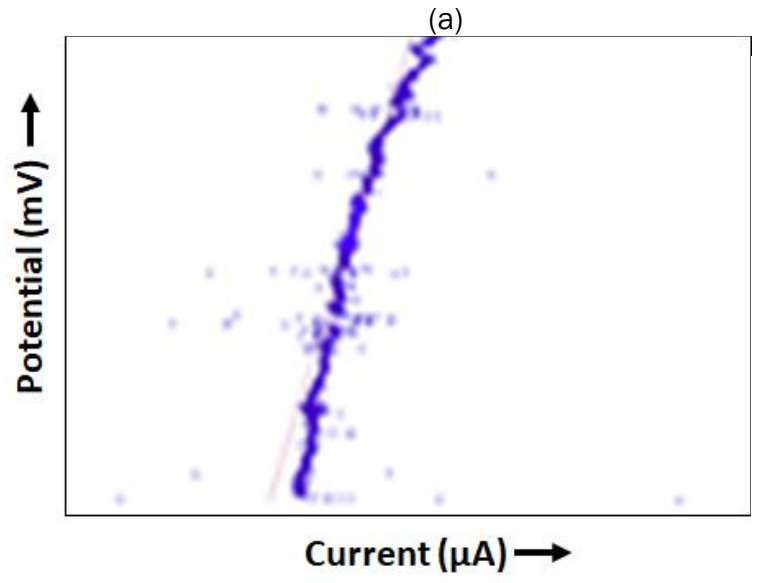

(c)

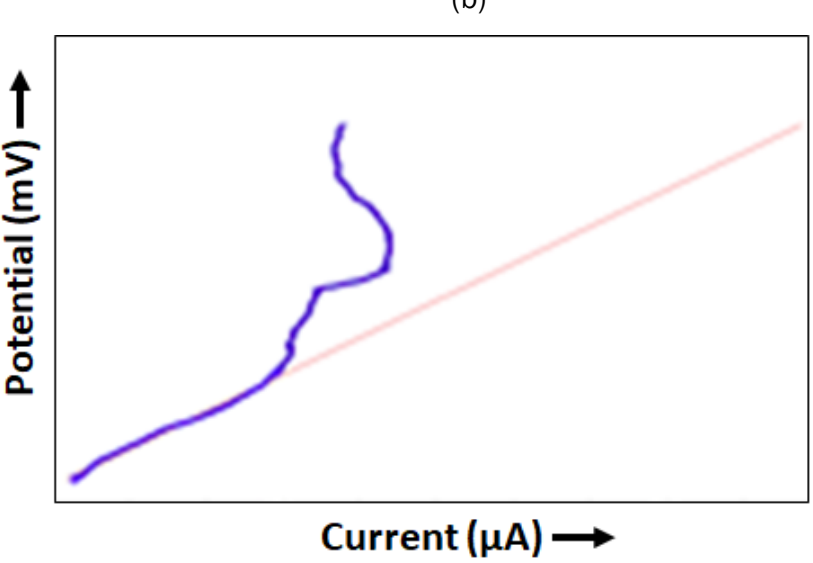

(d)

Figure II.3.2.8. Linear polarization test graphs for Solution 1: (a) Al and (b) Mg; and Solution 2: (c) Al and (d) Mg. Source: WPI.

The WeiCe Testing Instrument Co. Ltd. (WeiCe) CCT chamber and controller shown in Figure II.3.2.9 was installed and leveled, and will be used for salt-fog/spray and CCT corrosion testing for the remainder of the project.

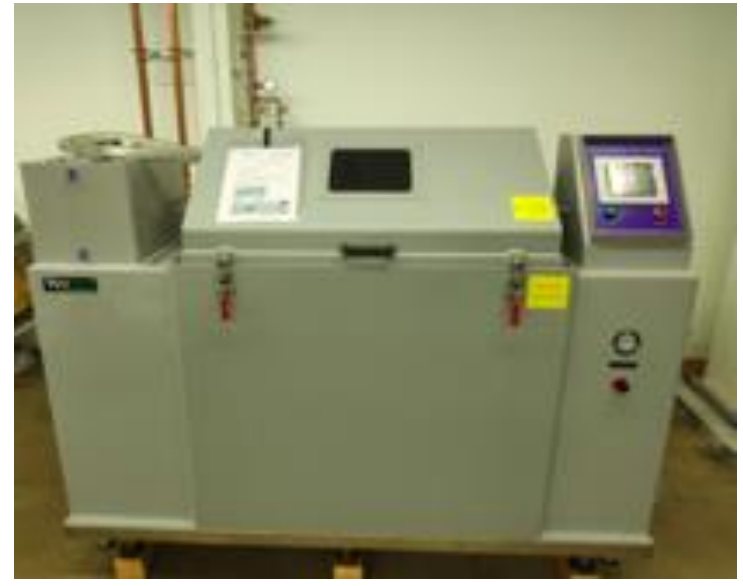

(a)

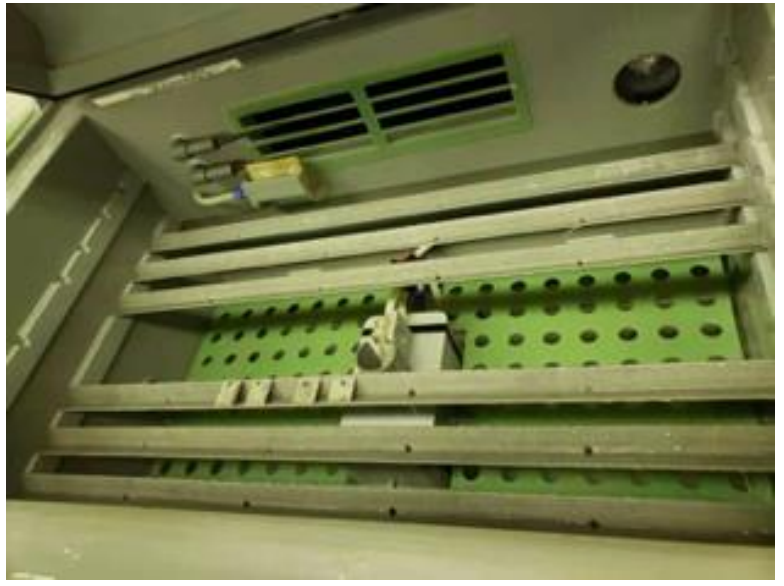

(b)

Figure II.3.2.9. (a) WeiCe CCT equipment; and (b) corrosion chamber with samples. Source: WPI. 


\section{Characterize Welded Joints and Corrosion and Mechanical Test Samples}

\section{Baseline Pure and Alloy Sheet Materials}

Surface roughness measurements of pure Mg sheet and pure Al sheet were acquired using a Keyence VR-3000 series (Keyence, Osaka, Japan) 3D measuring macroscope. The root mean square roughness from equal areas $\left(\sim 1.1 \mathrm{~mm}^{2}\right)$ on as-received surfaces of the two samples was found to be $2.73 \mu \mathrm{m}$ for the $\mathrm{Al}$ and $1.26 \mu \mathrm{m}$ for the $\mathrm{Mg}$. Initial EBSD data from the pure Al sheet shows texturing in the [001] direction and grain sizes in the 125$150 \mu \mathrm{m}$ range for plan view orientation as can be seen in Figure II.3.2.10 (a). Additionally, grain boundary rotation in the $\mathrm{Al}$ sample was sampled and showed that $5-15^{\circ}$ rotations between grains makes up $32.6 \%$ of the grain boundaries as shown in Figure II.3.2.10 (b). Texturing in the Al sample can affect diffusion during joining as well as influence the joint's resulting mechanical properties. EBSD of the Mg sheet was not as successful as the $\mathrm{Al}$ due to the surface preparation of the sample. Mg polishing is done without water and the final colloidal silica step was not performed since it is an aqueous suspension. The EBSD data, although noisy, does show that the $\mathrm{Mg}$ is also textured in the [001] direction in the plan view.

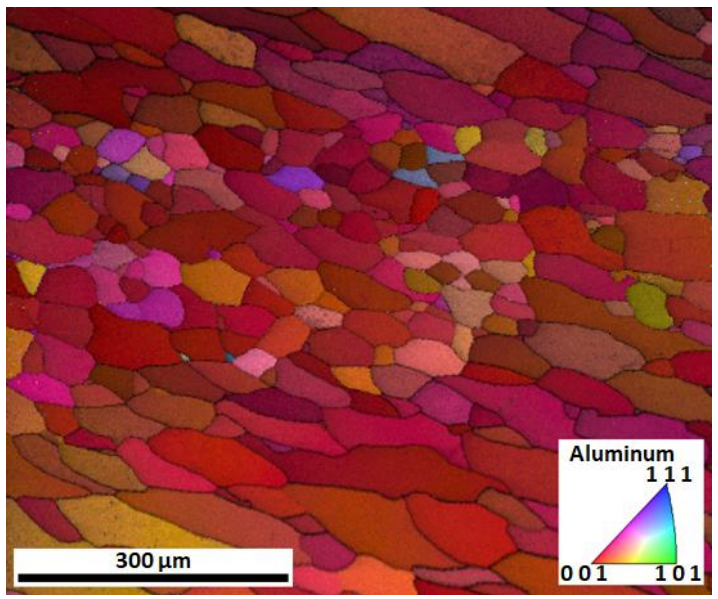

(a)

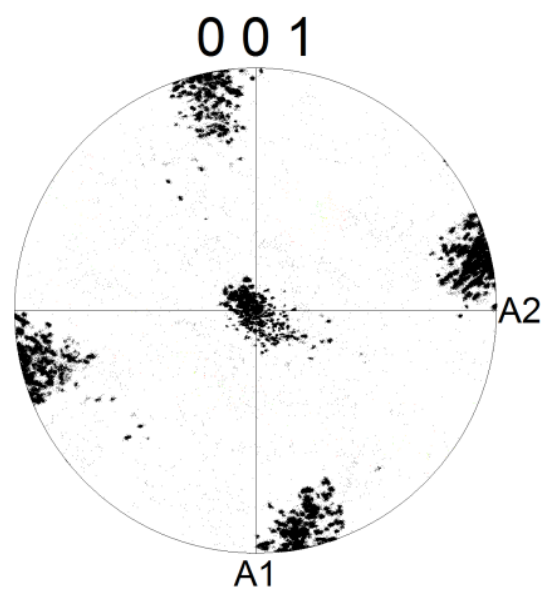

(b)

Figure II.3.2.10. (a) Al sheet EBSD inverse pole figure map showing the [001] texturing. (b) The pole figure of the Al sheet is typical of textured materials. Source: ORNL.

A backscattered electron (BSE) micrograph of the Mg sheet seen in Figure II.3.2.11 showed spherical secondphase particles rich in Si and $\mathrm{Zn}$ measuring $\sim 1 \mu \mathrm{m}$ and smaller. Grain size was not determined due to the lack of channeling contrast in BSE.

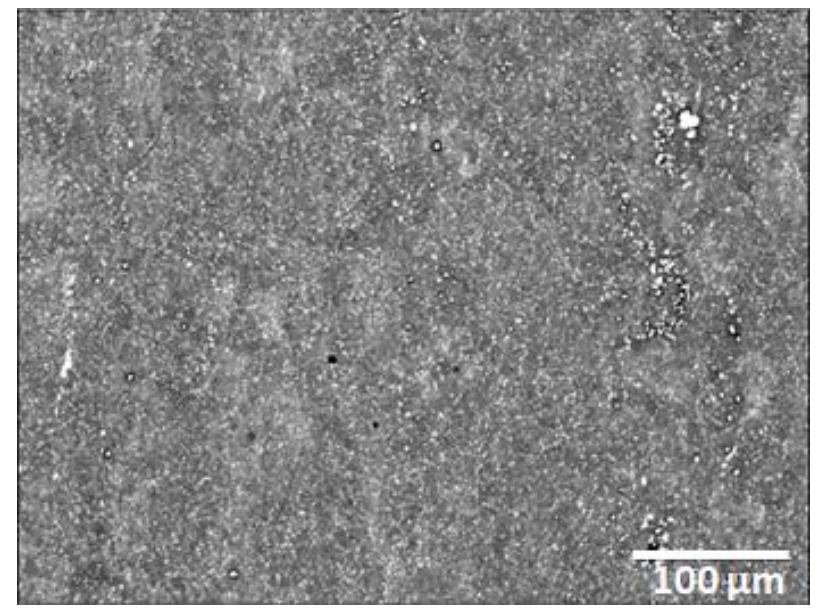

Figure II.3.2.11. SEM-BSE micrograph of as-received Mg sheet. Source: ORNL. 
For this reason, EBSD and STEM with FIB will be used to determine starting grain size range, and STEM/EDS will be used to determine the second-phase composition quantitatively, since electron probe microanalyzer (EPMA) does not have the ability to resolve the second-phase and consequently cannot determine the exact elemental composition due to the larger analytical volume produced in the bulk sample. The Mg sheet phase map did not pick up the spherical second-phase particles, and STEM/EDS will be needed to better understand the morphology and elemental composition of these features in the as-received material. For the as-received Al sheet, channeling contrast in the BSE image, as shown in Figure II.3.2.12 (a), clearly shows grain size on the order of 10's to $\sim 100 \mu \mathrm{m}$ and bright second-phase particles in the 5-10 $\mu \mathrm{m}$ size range. Phase maps of the matrix and second-phase particles indicate the second-phase particles are composed of $\mathrm{Si}$ and Fe, which are the bright spots Figure II.3.2.12 (b).
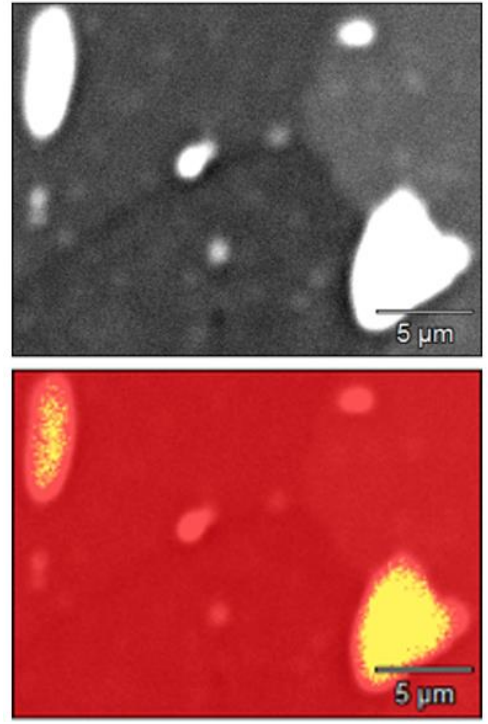

(a)

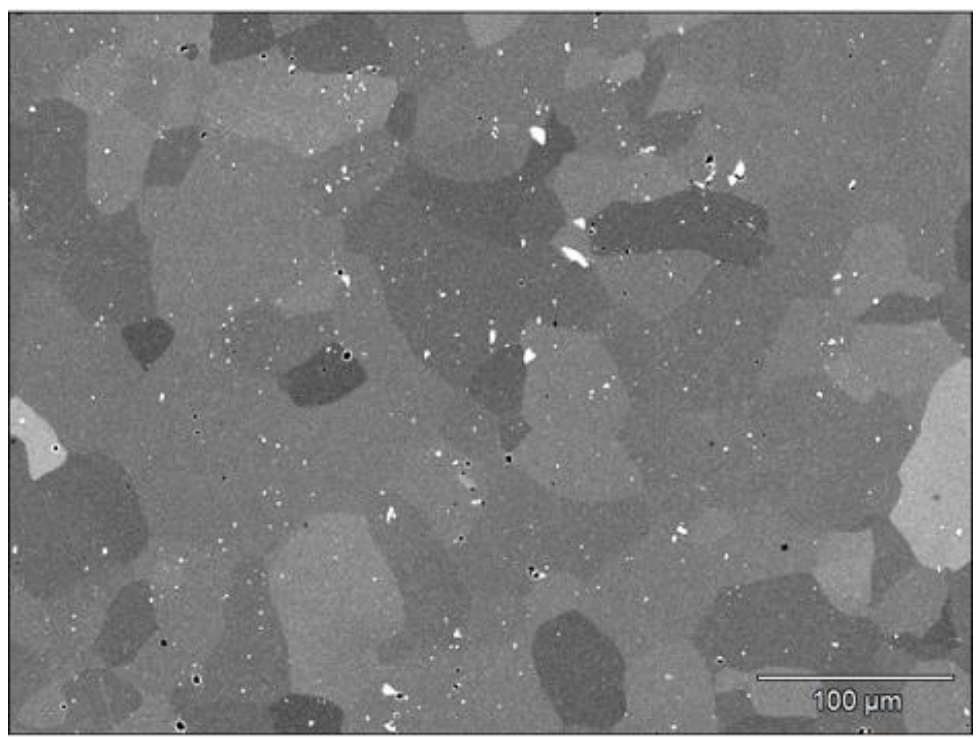

(b)

Figure II.3.2.12. (a) SEM-BSE micrograph of as-received Al sheet; and (b) phase map of as-received Al sheet. Source: ORNL.

\section{Diffusion-Bonded Couples}

Characterization began with four diffusion-bonded pure $\mathrm{Mg}$ and $\mathrm{Al}$ samples that were sliced into four pieces/sample and metallographically prepared so that the cross-section of the samples could be polished. These were then examined as-bonded. Note that these were produced using the earlier diffusion-bonding method described previously, not the new method, and so had a low-quality non-uniform bond. After sectioning, it was found by optical-microscopy that the interface between the $\mathrm{Mg}$ and $\mathrm{Al}$ samples had a large gap between metal films. Further examination at higher magnifications with SEM/EDS showed that on the surfaces of the visible gap, at the bond interface, there were IMCs of $\mathrm{Mg}_{2} \mathrm{Al}_{3}$ and a $\mathrm{Mg}$ rich intermediate layer (possibly $\gamma-\mathrm{Al}_{43} \mathrm{Mg}_{57}$ ) at the $\mathrm{Mg}$ side, as shown in Figure II.3.2.13. X-ray mapping, as observed in Figure II.3.2.14, clearly showed that diffusion did occur during the anneal, but the gap formation and its origin is still being debated. X-ray maps showed a $\sim 110 \mu \mathrm{m}$ diffusion zone between the $\mathrm{Al}$ and $\mathrm{Mg}$ sides. One sample inter-diffused then broke in the intermetallic layer with scalloped morphology. Backscattered imaging channeling contrast was used to determine the $\mathrm{Mg}$ grain size to be $75-100 \mu \mathrm{m}$, with the $\mathrm{Al} \sim 200-400 \mu \mathrm{m}$. The high-temperature sample (i.e., $430^{\circ} \mathrm{C}$ ) showed a $\sim 1 \mathrm{~mm}$ diffusion zone. A eutectic microstructure was clearly observed in the high-temperature samples and served as a fiducial for the post-corrosion analysis. 

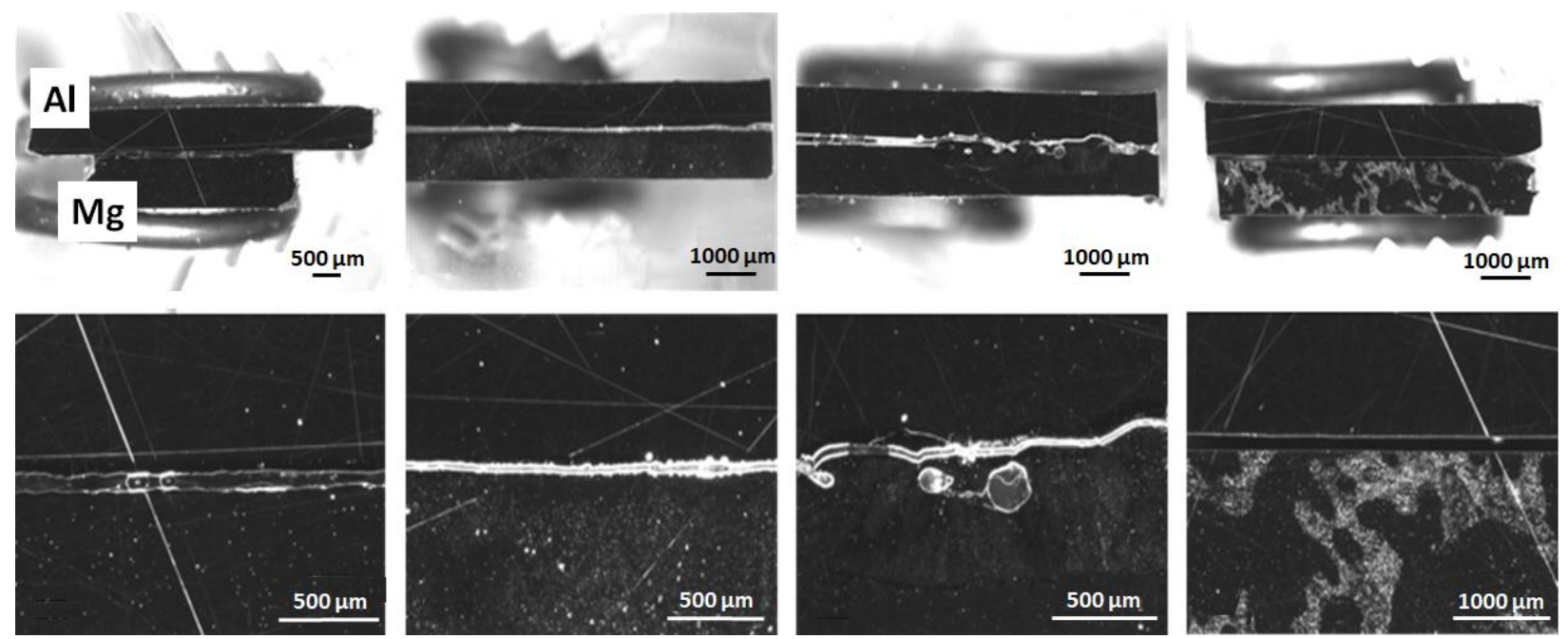

Figure II.3.2.13. SEM images of pure Al and Mg sheet diffusion-bonded samples, from left to right: $430^{\circ} \mathrm{C}$ for two hours; $400-425^{\circ} \mathrm{C}$ for two hours; $400-430^{\circ} \mathrm{C}$ for two hours; and $390-410^{\circ} \mathrm{C}$. Note that none of the interfaces were adherent. Source: ORNL.

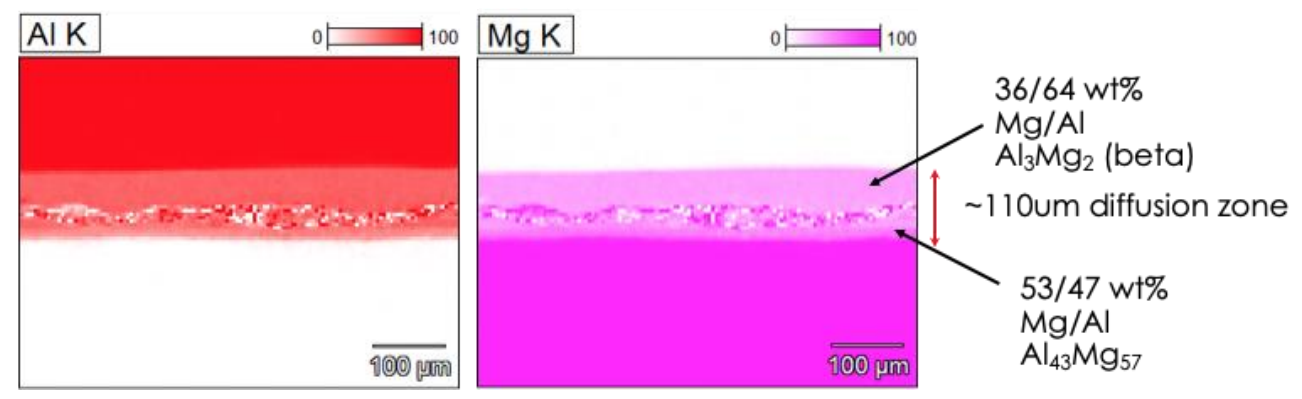

Figure II.3.2.14. X-ray maps of pure Mg and Al sheet diffusion-bonded samples. Source: ORNL/WPI.

\section{Develop Corrosion and Mechanics Model}

\section{Literature Review}

A thorough literature review on phase-field corrosion models was completed. Among all the available models of corrosion, Chadwick et al. [1] and Ansari et al. [2] were judged to be the most applicable candidates for this study, with each having its own advantages and disadvantages. Both studies perform 2D simulation of localized corrosion. The initial domain consists of an electrolyte phase and an anodic metal phase (single grain or polycrystalline). It is assumed that initially a protective coating layer exists, and that a small semicircular pit starts to grow due to the breakdown of the protective coating. The evolution of the metal-electrolyte interface is described.

Figure II.3.2.15 represents the pit morphology evolution into a polycrystalline metal [1]. It is reported that if the metal is a single grain, then the initial semicircular pit retains its semicircular symmetry as it grows, while the formation of mostly flat, sharply defined facets is observed in the case of polycrystalline metal due to the orientation-dependent reaction kinetics. A similar morphology is reported by [2]. 

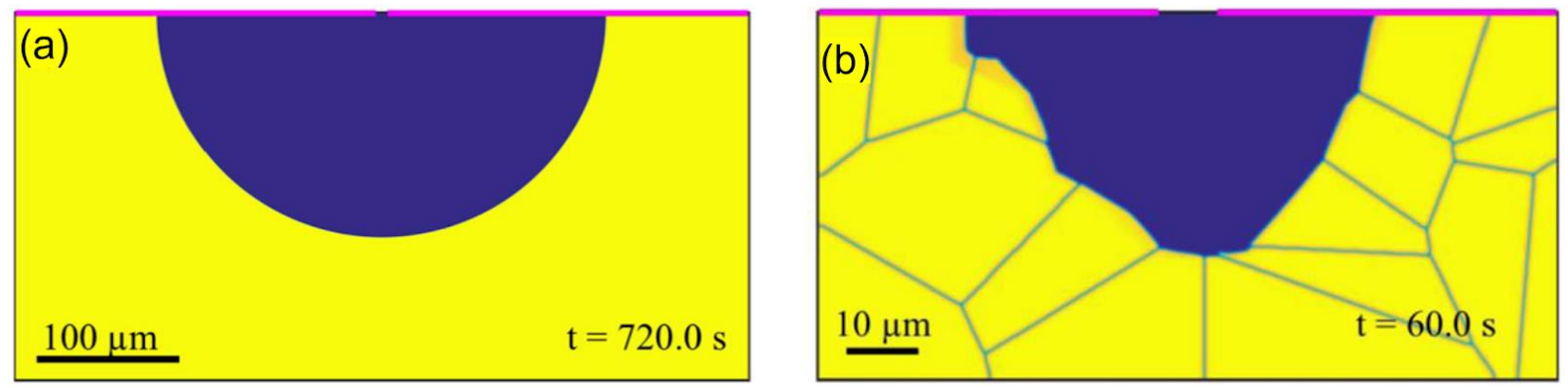

Figure II.3.2.15. The evolution of pit morphology is shown for: (a) a single grain metal; and (b) for a polycrystalline metal. Source: [1].

Both models account for ionic transport and variation of electrostatic potential within the electrolyte, as well as nonlinear reaction kinetics at the electrolyte-metal interface through the Butler-Volmer or Tafel equation. Chadwick's model considers only anodic reactions at the metal-electrolyte interface, while both anodic and cathodic reactions are accounted for in Ansari's model. The main difference in both studies is in the employed phase-field ( $\mathrm{PF}$ ) models. Chadwick uses multiple Cahn-Hillard $(\mathrm{CH})$ parameters to describe the electrolyte and metal grains, which makes the model computationally expensive. In addition, it is based on the Smoothed Boundary Method (SBM), which is utilized to restrict the equations to be solved inside a given boundary (e.g., inside the electrolyte phase). The use of SBM in the model also adds an extra difficulty in implementation and increases the computational burden. Ansari's model, on the other hand, is based on Kim, Kim, and Suzuki [3], which uses coupled Allen-Cahn (AC) and $\mathrm{CH}$ parameters to describe the entire system (electrolyte + metal) and reduces the number of equations to be solved. In addition, there is no need to use SBM to solve the equations.

It is worthwhile to note that Chadwick's model is developed by the PRISMS-PF team, and already implemented in the open-source PRISMS-PF Software. However, the WPI modeling team does not prefer to use that model due to its high computational cost associated with the employed PF method and SBM, as discussed above. Compared to Chadwick's model, Ansari's is less computationally intensive. However, due to the PF formulation [3] adopted in Ansari's model, an AC parameter coupled with a $\mathrm{CH}$ parameter should be used to describe each phase, which means solving multiple $\mathrm{AC}$ and $\mathrm{CH}$ equations for a multiphase system. On the contrary, one can use a single or reduced number of order parameters to describe a multiphase,

multicomponent system, which is enabled by constructing a single free energy density for the entire system, as reported in Pongsaksawad, Powell, and Dussault [4]. Reducing the number of order parameters for describing the system is important, especially for 3D simulations with high computational cost in general.

\section{New Phase-Field Corrosion Formulation}

In order to model galvanic and pitting corrosion in welded joints, a new formulation based on [4] was developed. They originally developed their formulation for simulating the transport-limited electrolysis process, in which a high-temperature environment is assumed with negligible charge transfer limitation [4]. Here, we reformulate the original model to account for low temperature conditions and other important phenomena associated with corrosion process as described in Chadwick's and Ansari's models. The new model will consider a multicomponent (e.g., $6022 \mathrm{Al}, \mathrm{ZEK} 100 \mathrm{Mg}, \mathrm{H}_{2} \mathrm{O}, \mathrm{Al}(\mathrm{OH})_{3}, \mathrm{Mg}(\mathrm{OH})_{2}$ ) multiphase system with intermetallic phases $\left(\mathrm{Al}_{3} \mathrm{Mg}_{2}\right.$ and $\left.\mathrm{Al}_{43} \mathrm{Mg}_{57}\right)$ and will account for the following phenomena: nonlinear reaction kinetics at the interface (via Tafel equation), very different anodic and cathodic reactions, and orientation and grain boundary effects in a polycrystalline material. An example of the PF model results for IMC layer growth in diffusion-bonding can be seen in Figure II.3.2.16. 


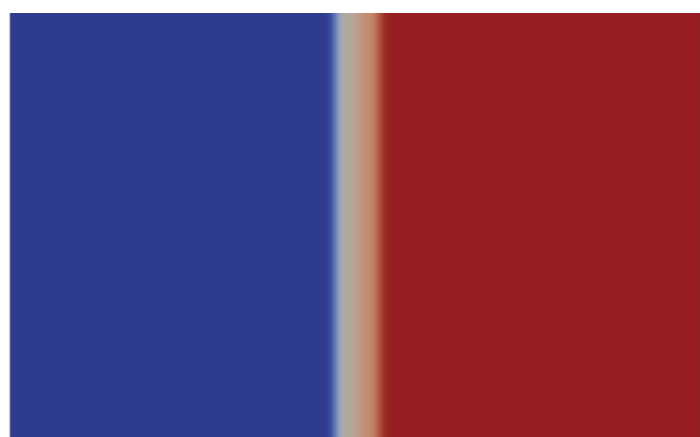

(a)

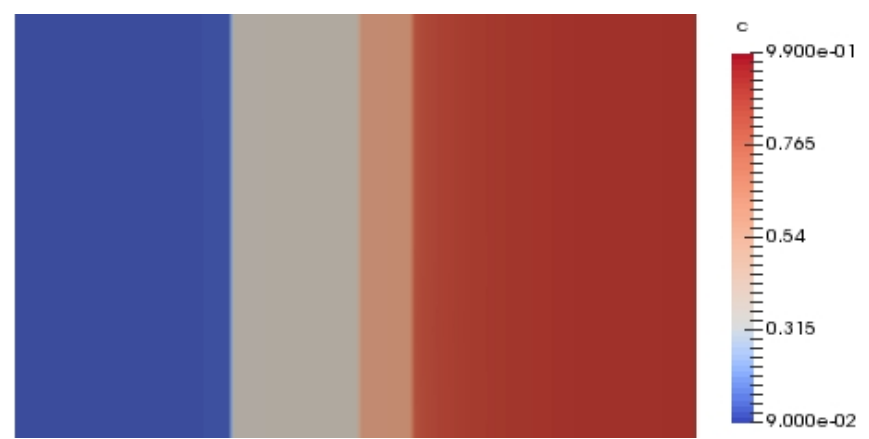

(b)

Figure II.3.2.16. PF model of IMC layer growth in diffusion-bonding for (a) the initial condition and (b) after significant growth. The scale bar on the right shows $\mathrm{Mg}$ mole fraction. Note the faster growth of $\mathrm{Al}_{3} \mathrm{Mg}_{2}$ (grey) than $\mathrm{Al}_{43} \mathrm{Mg}_{57}$ (pink) as seen in the experiments. Source: WPI.

\section{Preliminary Results for Simulations}

For the first simulation case, a diffusion couple of pure Al-Mg with IMC phases was considered. The homogeneous free energy of the multiphase system was described through a single equation, following [4], with a gradient energy as well. Currently, four phases are considered: Al-fcc, Mg-hcp and two IMC phases $\left(\mathrm{Al}_{3} \mathrm{Mg}_{2}\right.$ and $\left.\mathrm{Al}_{43} \mathrm{Mg}_{57}\right)$. Free energy data at $400^{\circ} \mathrm{C}$ from Thermo-Calc Software were fitted to a polynomial function with four minima corresponding to the four phases as shown in Figure II.3.2.17. The complete corrosion model formulation, including other metal components and inclusions and an aqueous electrolyte phase, is under development.

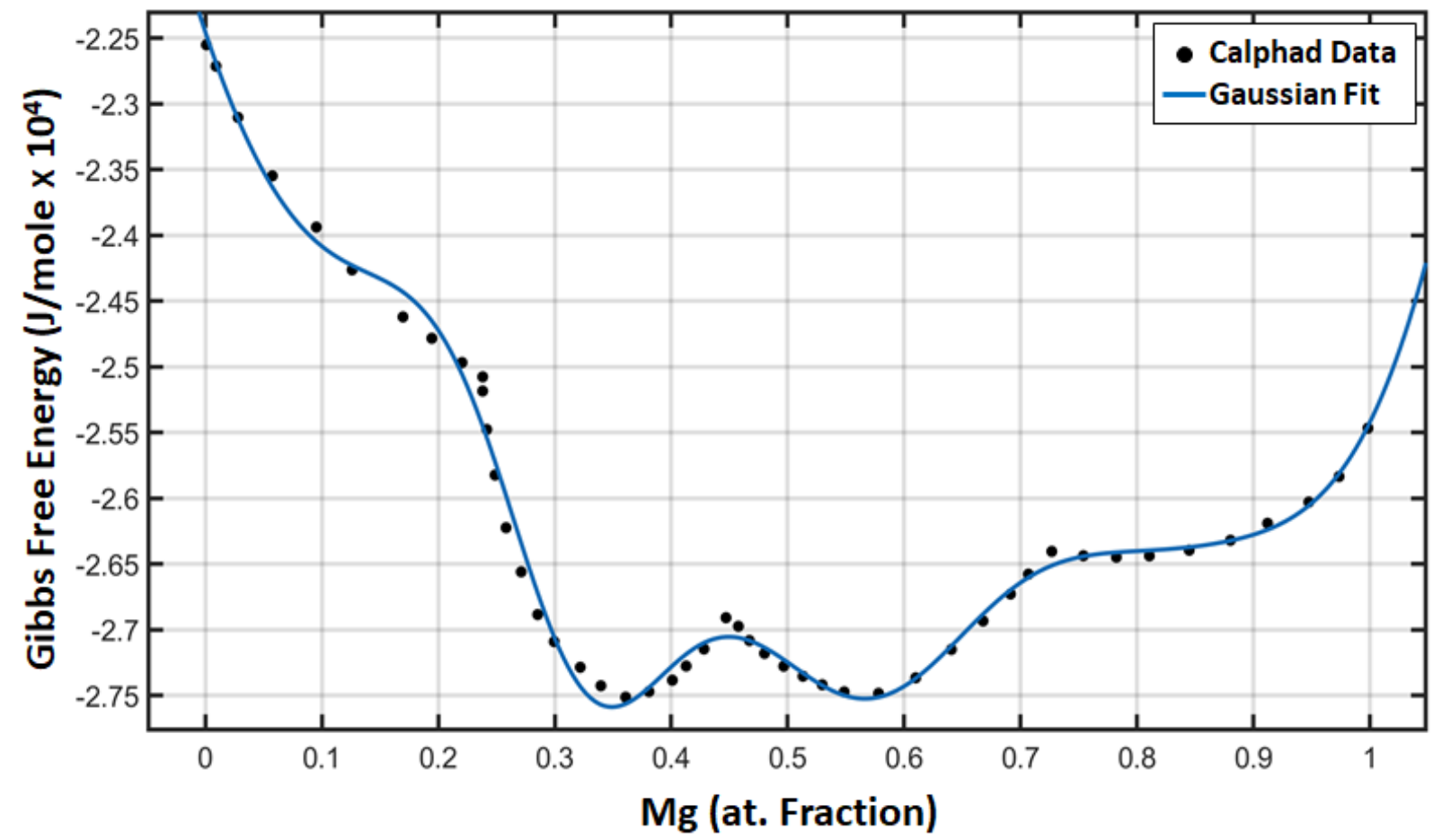

Figure II.3.2.17. Al-Mg binary free energy function used in the PF model of diffusion-bonding. Source: WPI. 


\section{Conclusions}

The project thus far has established the foundation for studying corrosion in Al-Mg welds. Major accomplishments include:

- The development of a method for diffusion-bonding $\mathrm{Al}$ and $\mathrm{Mg}$ sheets, and the production of multiple diffusion-bonded couples.

- The development of FSW capability with the tool penetrating through a harder $6022 \mathrm{Al}$ alloy sheet into a softer ZEK100 Mg alloy sheet.

- The installation of a corrosion chamber capable of salt-fog and CCT.

- The development of a Cahn-Hilliard formulation for modeling galvanic reactions between $\mathrm{Al}$ and $\mathrm{Mg}$, with validation in a diffusion-bonded couple.

The project will proceed to validate the corrosion model, add a CP model of deformation and fracture and refine the models to predict tensile and fatigue strength of corroded FSWs.

\section{References}

1. Chadwick, A. F., J. A. Stewart, R. A. Enrique, S. Du, and K. Thornton, 2018, "Numerical modeling of localized corrosion using PF and smoothed boundary methods," J Electrochem Soc, Vol. 165, No. 10, pp. C633-C646.

2. Ansari, T. Q., Z. Xiao, S. Hu, Y. Li, J.-L. Luo, and S.-Q. Shi, 2018, "PF model of pitting corrosion kinetics in metallic materials," NPJ Computational Materials, Vol. 4, No. 1, pp. 1-9.

3. Kim, S. G., W. T. Kim, and T. Suzuki, 1999, "PF model for binary alloys," Phys. Rev. E, Vol. 60, No. 6, pp. 7186-7197.

4. Pongsaksawad, W., A. C. Powell, and D. Dussault, 2007, "PF modeling of transport-limited electrolysis in solid and liquid states," J Electrochem. Soc., Vol. 154, No. 6, pp. F122-F133.

\section{Acknowledgements}

This work was supported by DOE Contract DE-EE0008454. 


\title{
II.3.3 A Hybrid Physics-based Data-Driven Approach to Model Damage Accumulation in Corrosion of Polymeric Adhesives (Michigan State University)
}

\author{
Michigan State University \\ 428 S. Shaw Ln., Rm. 3567 \\ East Lansing, MI 48824 \\ E-mail: roozbeh@msu.edu
}

Dr. Roozbeh Dargazany, Principal Investigator

\section{Sarah Kleinbaum, DOE Technology Development Manager}

U.S. Department of Energy

E-mail: sarah.kleinbaum@ee.doe.gov

Start Date: October 1, 2018

Project Funding (FY19): \$451,659
End Date: December 31, 2021

DOE share: $\$ 308,662 \quad$ Non-DOE share: $\$ 142,997$

\section{Project Introduction}

Degradation of polymeric adhesives in dissimilar joints is a menace to electrical and structural components of the automotive industry. Often in polymeric adhesives, failure occurs due to damage accumulated from discrete sources such as thermal degradation, oxidation, hydrolysis, radiation, and moisture combined with progressive damage mechanisms like aging and fatigue. Most current efforts are directed towards a singlephenomenon, single model approach where a model covers one damage mechanism only. While this approach is advantageous in exploring single mechanisms, it is inconclusive for parallel damage mechanisms. We propose to take a fresh perspective by designing a hybrid constitutive/data-driven approach that allows models of different damage mechanisms to be integrated into one model and then use it to explore failure of polymeric adhesives. The theoretical-computational part of this work is divided into four research components.

\section{Objectives}

Our objective is to develop and validate a model to predict environmentally induced failure in polymeric adhesives with respect to damage accumulated by different corrosion mechanisms within $10 \%$ error. The adhesives included for study in the project are Dow Corning (DC) silicone adhesive (Dowsil 7091), two polyurethane adhesives (3M-590 and 3M-DP6310), and acrylic (Lord 810/20GB). Models of different damage mechanisms will be integrated into one platform and then used to explore the degradation-induced failure of polymeric joints. Initially, the focus will be on modeling and coupling of visco-elasticity, thermo-oxidation, photo-oxidation, and moisture degradation. The platform output will be given to a neural network engine to calculate the remaining service life. Using the neural network, a fatigue failure model will be developed to consider the effect of degradation.

\section{Approach}

This report will cover our results during BP1 where our focus was on the understanding, characterizing, and modeling of individual degradation mechanisms. The Principal Investigator's involvement in development of models for predicting the effects on elastomers led to the generation of the following four models:

- Vibration-induced damage of adhesives is being developed by coupling previous quasi-static and viscoelastic models into one damage function. The model will describe the contribution of engine vibration into the accumulative damage of adhesives by considering the damage accumulated in each load cycle.

- Thermal-oxidative degradation of adhesives is being micro-mechanically modeled with respect to changes that occurred in the matrix substructure and morphology of the polymer chains. The goal is to predict the performance loss of crosslinked polymers in the course of thermo-oxidative degradation. 
- Hydrolysis is being modeled by coupling two models, a Langmuir diffusion model of mass uptake and water diffusion as a damage precursor and will integrate them into the network evolution theory.

- Photo-oxidation will be modeled by advancing the concept of oxygen diffusion in thermo-oxidative aging. Damage equations will be reformulated based on a new damage precursor; ultraviolet (UV) energy dosage.

\section{Results}

As shown in Figure II.3.3.1, the constitutive behavior of adhesives in quasi-static, UT test can provide significant information over their inelastic properties, such as strain softening, permanent set and hysteresis. The developed quasi-static model can predict the elastic/inelastic behavior of adhesives in the absence of plasticity. The developed framework is built on the same basis as all other aging and degradation models. The total energy of the adhesive matrix can be estimated through statistical mechanics of the polymer chains which gives the strain energy of the matrix as the summation of energy of chain bundles in each direction, namely:

$$
\Psi_{M}=\frac{1}{A_{S}} \int_{S} W_{M}^{\vec{a}} d u^{\vec{d}} \cong \sum_{i=1}^{k} \omega_{i} \int_{D_{A}\left(\lambda_{\max } \vec{a}_{i}\right.} N(n) \psi_{c}(n, \bar{r}) d n
$$

where $\omega_{i}$ and $d_{i}$ are the weight and direction of numerical integration respectively. $N(n)$ is the number of polymer chains with length $n$ and $\psi_{c}(n, \bar{r})$ is the energy of a single chain.

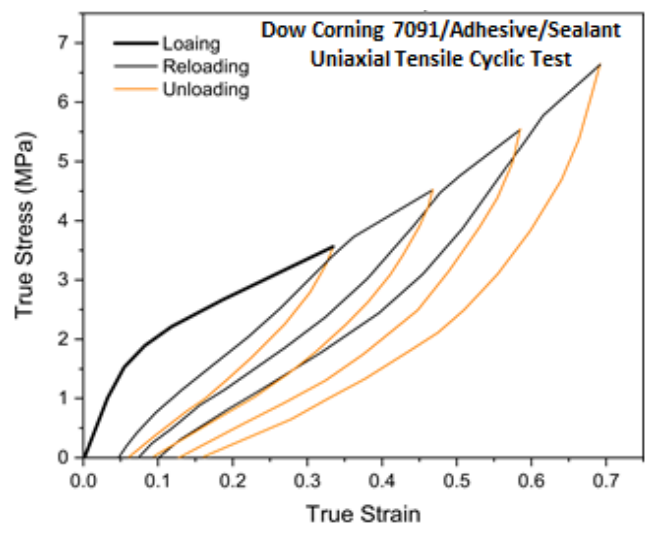

Figure II.3.3.1. Inelastic effects in silicon adhesives. Source: MSU High Performance Materials Group.

\section{Vibration-induced Damage}

Behavior of adhesives subjected to continuous vibration shows significant damage accumulation with three features: (1) stress-softening in the first cycle; (2) increased permanent set in the sample; and (3) stiffness reduction. Energy dissipation is maximum in the first cycle, significantly decreases during the second cycle and will become negligible as the number of cycles increases. We are mainly considering the effect of the number of cycles on the stress-softening of the material and the energy dissipation in the first few cycles are not considered. The evolution of damage accumulation in the crosslinked network is derived based on the effect of force on the rate of chain scission and the dissociation of bonds. To this end, the mechano-chemistry theory of history-dependent damage is used to predict the number of active chains with a constant length. A probability distribution of the chains with different lengths evolve during cyclic deformation, which results in non-uniform stress-softening in the material. The kinetics of irreversible chain scission is represented by $d N(n) / d \tau=-K_{s} N(n)$ where $K_{s}$ is the rate of chain scission. Considering the experimentally established relationship between the material lifetime and its load, the rate of the surviving chains, $\mathrm{N}$, can be written as: 


$$
K_{S}=n K_{S 0} e^{\frac{\alpha(f(\lambda, n)-f(1, n))}{k_{B} T}}
$$

where $K_{s 0}$ is the Arrhenius rate factor, $\alpha$ is the activation length, while $k_{B}$ and $T$ are the Boltzmann constant and the absolute temperature, respectively. Moreover, $f(\lambda, n)$ is the entropic force of the chain with the $n$ segment at deformation $\lambda$. The activation force, $f(1, n)$, is considered to remove the effect of equilibrium chain forces on the damage evolution. The rate is multiplied by chain length, $\mathrm{n}$, to consider the same rupture probability for each bond on the chain, which is enough for chain scission. The number of survived chains with $\mathrm{n}$ segment at time $\tau$ can be calculated by solving the kinetics equation. Thus, the evolution of the probability of the chain length in each direction during cyclic loading yields:

$$
\stackrel{d_{i}}{\stackrel{P}{P}}(n)=P_{0}(n) e^{-C_{S}(n) j}, C_{s}(n)=\int_{c y c l e} \exp \left[\frac{\alpha}{k_{B} T}\left(\mathcal{L}^{-1}\left(\frac{R \lambda^{d_{i}}}{n}\right)-f_{a}\right)\right] d t
$$

To validate the proposed concept of damage accumulation, the model is benchmarked against a set of UT tests after 1, 1000, and 2000 cycles of loading and unloading represented in Figure II.3.3.2 (a). The experimental data for the evaluation of the constitutive model of this work is taken from the work of Zhang et al. [1] focusing on UT loading and damage accumulation over thousands of cycles for a sample. In another test, loss of stress at stretch 1.65 was reported against cycle numbers during uniaxial cyclic loading of samples as shown in Figure II.3.3.2 (b). By means of six material parameters, the model is fitted to the first cycle of the tensile test. In addition, two material parameters of damage accumulation are calculated by only considering maximum stress after 10 and 4000 cycles. The response at 1000 and 2000 cycles is predicted.

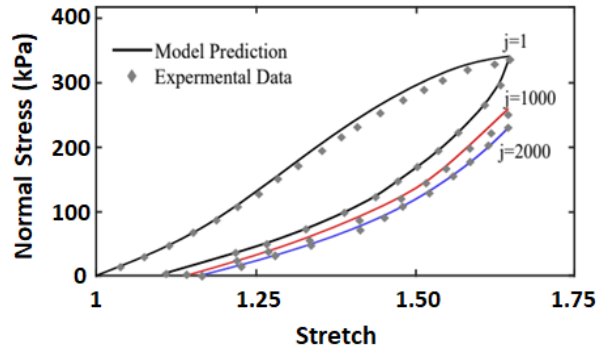

(a)

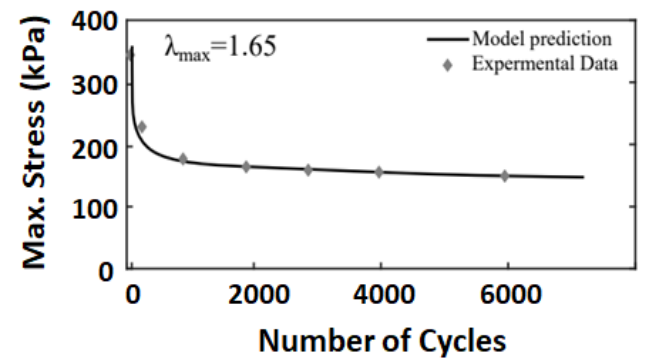

(b)

Figure II.3.3.2. Comparison of model against (a) UT tests after 1, 1000, and 2000 cycles and (b) stress-relaxation over the number of loading cycles [1]. Source: MSU High Performance Materials Group.

\section{Thermo-oxidative}

In the automotive industry, adhesive joints and anti-vibration systems can be located under the hood. For under the hood applications, the temperature is usually high due to radiative heat from the engine. These conditions make these materials prone to thermo-oxidation. Moreover, adhesive joints are often subjected to continuous loads and harsh environmental conditions for a long-time. To predict the changes in the properties of those adhesives in the course of degradation, we chose micromechanical approaches over phenomenological ones to describe damage evolution behavior and later couple it with other damage mechanisms. To characterize thermo-oxidative aging of adhesives, a set of experimental tests based on ASTM D412 were designed where adhesives were exposed to $60^{\circ} \mathrm{C}, 80^{\circ} \mathrm{C}$, or $95^{\circ} \mathrm{C}$ for different times up to 30 days. To prevent humidity and UV radiation from influencing the results, samples were put into sealed buckets with a RH absorbing salt. Styrenebutadiene rubber (SBR) was used as the control sample set. 
In our model, we describe aging as the competition of two types of chemical reactions - chain scission and crosslinking. Chain scission causes dissolution and degradation of the original network. Crosslinking reinforces the matrix by creating a whole new set of bonds. Accordingly, during long thermo-oxidative aging, all the original crosslinks of the matrix are fully replaced by many more new ones which leads to a new stiffer matrix with more crosslinks. After this point, no further aging will occur at that stretch level. This assumption leads to a dual network hypothesis, where aging is considered the transition of the polymer matrix from one state - virgin at time 0 - to another state - aged at time infinity. Any other state in the middle of these two can be defined as a transition state. Accordingly, the strain energy of the polymer matrix, $\Psi_{M}$, at each stage of aging can be simply represented with respect to the energies of the network at the two states, $\Psi_{0}$ and $\Psi_{\infty}$ as:

$$
\begin{gathered}
\Psi_{M}(t, T, \boldsymbol{F})=\rho(t, T) \Psi_{0}(\boldsymbol{F})+(1-\rho(t, T)) \Psi_{\infty}(\boldsymbol{F}), \\
\text { where } \rho(t, T)=A_{1} \exp \left(-\tau_{1} \exp \left(-\frac{E_{a 1}}{R T}\right) t\right)+A_{2} \exp \left(-\tau_{2} \exp \left(-\frac{E_{a 2}}{R T}\right) t\right)
\end{gathered}
$$

where $\rho(t, T)$ represents the weight of each state. To validate the proposed model, its predictions were benchmarked against our experimental data specifically designed to capture constitutive behavior for the effects of thermo-oxidative aging illustrated by the examples shown Figure II.3.3.3 (a) and (b) for silicone adhesive and Figure II.3.3.3 (c) and (d) for black polyurethane.

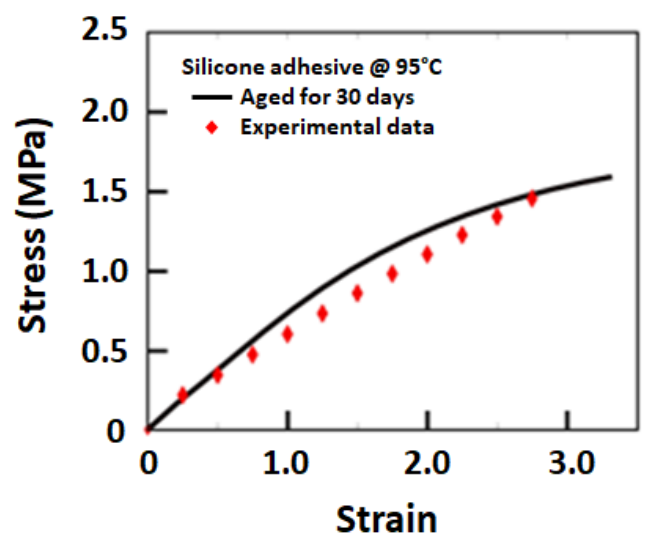

(a)

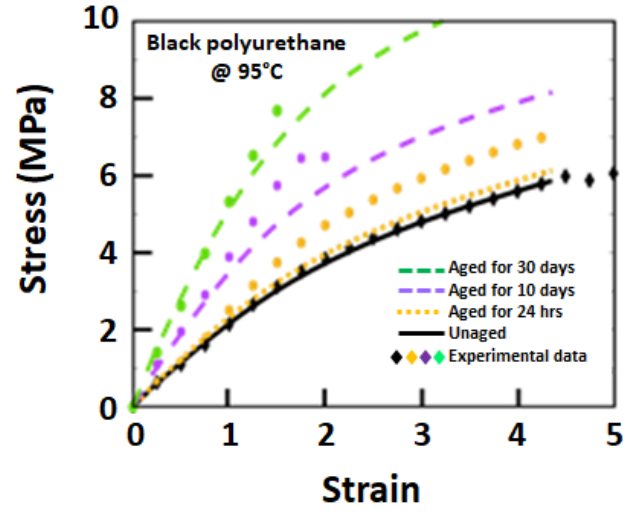

(c)

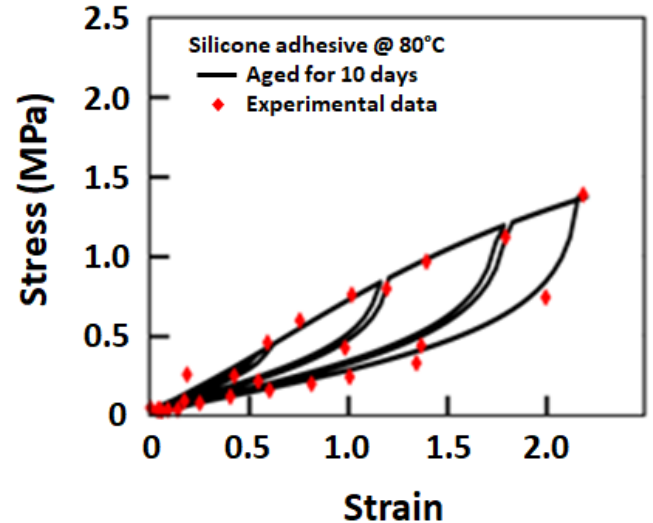

(b)

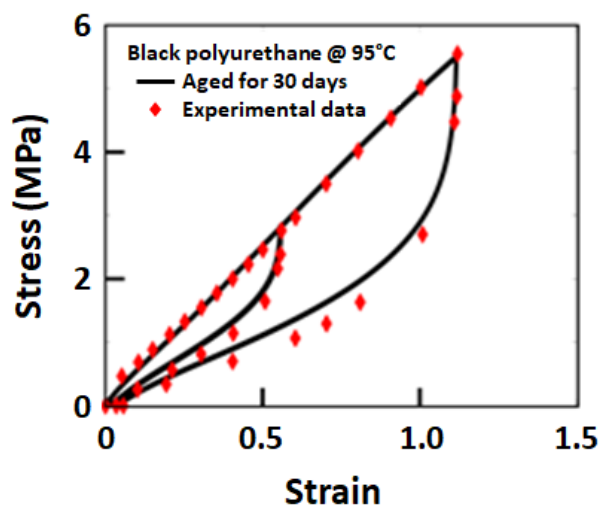

(d)

Figure II.3.3.3. Comparison of model to measured constitutive behavior for: (a) failure of DC Dowsil 7091 silicone adhesive aged for 30 days at $95^{\circ} \mathrm{C}$; (b) cyclic behavior of DC Dowsil 7091 silicone adhesive aged for 10 days at $80^{\circ} \mathrm{C}$; (c) failure for $3 \mathrm{M}-590$ polyurethane aged for various times at $95^{\circ} \mathrm{C}$; and (d) cyclic behavior of $3 \mathrm{M}-590$ polyurethane aged for 30 days at $95^{\circ} \mathrm{C}$. Source: MSU High Performance Materials Group. 


\section{Photo-oxidation}

While most adhesives used in automotive would not be exposed to sunlight, the adhesive used for windshields and some interior components could be an exception. These polymeric materials are prone to photo-oxidative reactions when they are under UV radiation. UV radiation can significantly expedite the oxidation process. While radiation affects matrix oxidation similar to thermal loads, radiation should be considered more as a catalyst rather than an individual driving force since we cannot de-couple the thermal loads. Even at extremely low temperature, heat generated by radiation prevents us from isolating thermo- and photo-oxidation. As such, for photo-oxidation, the goal is to find a damage function complementary to that of the thermo-oxidative model which can take into account the effects of radiation, temperature, static strain and loading history.

To characterize the performance loss of adhesives during photo-oxidative aging, a series of tests based on ASTM D4329 were completed using Q-Panel UV machines. The samples were subjected to radiation at $80^{\circ} \mathrm{C}$ for different times up to 30 days and then their behavior was characterized as shown in Figure II.3.3.4 (a)-(d) for silicone, black polyurethane, acrylic and green polyurethane adhesives, respectively.

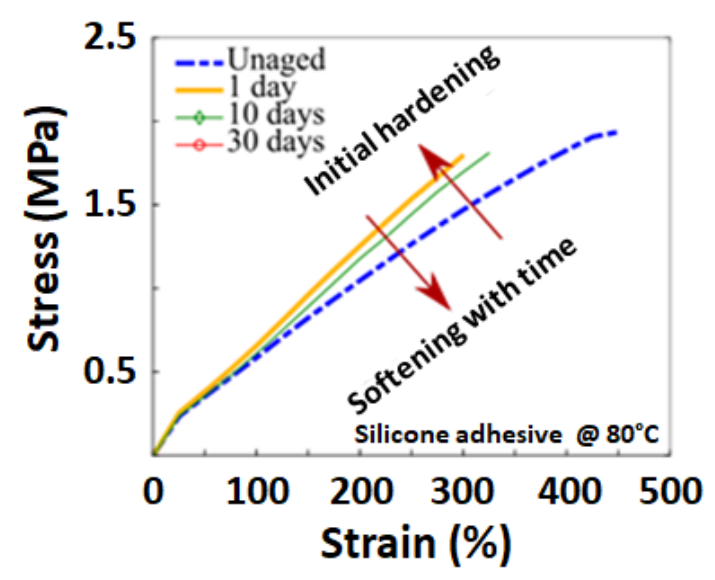

(a)

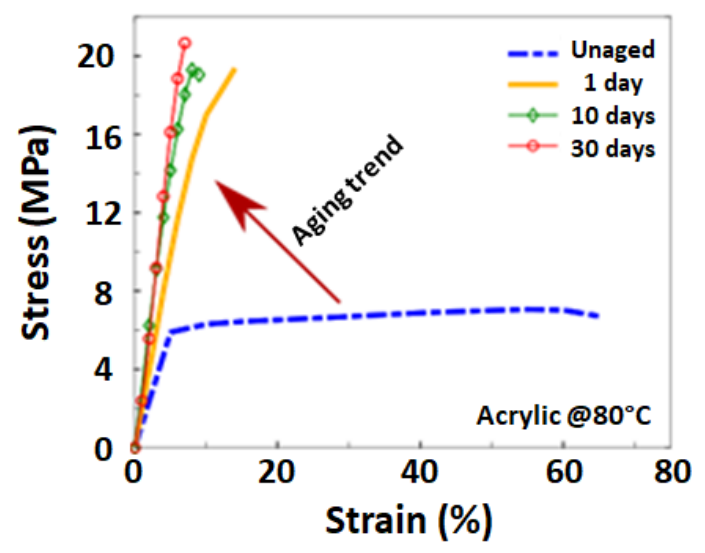

(c)

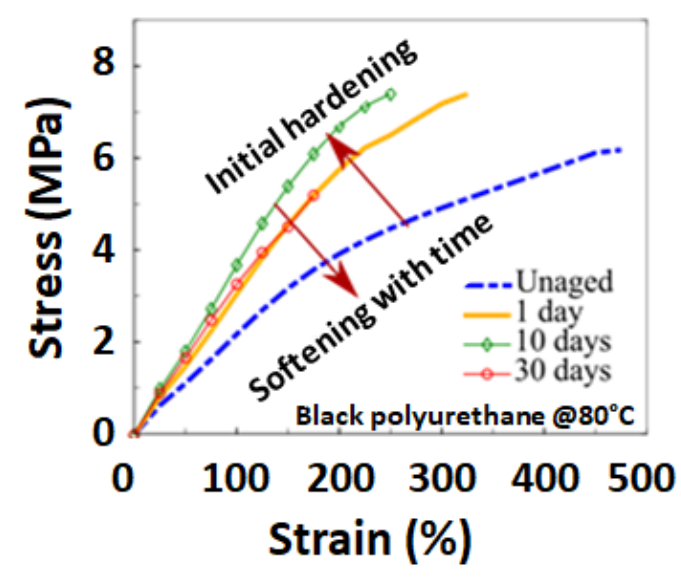

(b)

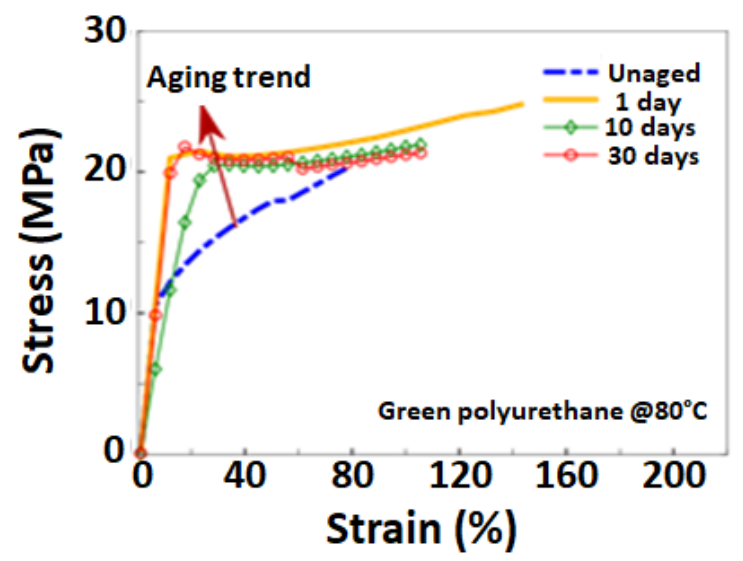

(d)

Figure II.3.3.4. Constitutive behavior of UV test samples for (a) Dow Corning adhesive, (b) black polyurethane adhesive, (c) acrylic, and green polyurethane. Source: MSU High Performance Materials Group. 
UV exposure can be considered a catalyst in both chain scission and crosslinking that occurs during thermooxidative aging. Accordingly, using the dual network hypothesis, the strain energy of a sample exposed to a certain UV dosage rate, $\gamma$, can be written through a decay function such as:

$$
\begin{gathered}
\Psi_{M}(t, T, \gamma, \boldsymbol{F})=\rho(t, T, \gamma) \Psi_{0}(\boldsymbol{F})+(1-\rho(t, T, \gamma)) \Psi_{\infty}(\boldsymbol{F}), \\
\text { where } \Psi_{M}(t, T, \gamma, \boldsymbol{F}) \cong \sum_{i=1}^{k} \omega_{i} W_{M}^{d_{i}} \text { and } W_{M}^{d}=\rho_{0}(t, T, \gamma) W_{0}^{d}+(1-\rho(t, T, \gamma)) W_{\infty}^{d} \\
\rho(t, T)=A_{1} \exp \left(-\tau_{1} \exp \left(-\frac{E_{a 1}+E_{\text {photo }} \gamma}{R T}\right) t\right)+A_{2} \exp \left(-\tau_{2} \exp \left(-\frac{E_{a 2}+E_{\text {photo }} \gamma}{R T}\right) t\right)
\end{gathered}
$$

where $\Psi_{M}$ is the strain energy of the whole matrix and $W^{d}$ are the strain energies of each subnetwork in direction $d$. All the other parameters are as defined in Equation 4.

\section{Hydrolysis}

In the automotive industry, adhesive joints may be located near the engine where the temperature is high while also being exposed to saltwater splashes in the winter. These conditions could induce hygro-thermal aging. Additionally, since adhesive joints can be exposed to atmospheric humidity over a long-time, a model for hydrolytic aging and its effect on the mechanical properties of crosslinked adhesives is of major relevance to the automotive industry and original equipment manufacturers. Only a few phenomenological models can consider the effect of hydrolytic aging on behavior of polymeric materials and no micromechanical model with those capabilities currently exists.

To investigate hydrolysis, the control samples of SBR were first characterized in pilot experiments to determine their constitutive behavior at various aging times as shown in Figure II.3.3.5 (a) and Figure II.3.3.5 (b). Aging experiments of more relevant adhesive materials including silicone adhesive (Dowsil 7091), two polyurethane adhesives (3M-590 and 3M-DP6310), and acrylic (Lord 810/20GB) are in progress. Samples were fully immersed in several sealed containers filled with distilled and saline water at temperatures of $60^{\circ} \mathrm{C}, 80^{\circ} \mathrm{C}$, and $95^{\circ} \mathrm{C}$ under constant pressure for different times up to 30 days. After aging, the samples were removed and dried at RT (i.e., $23^{\circ} \mathrm{C}$ ) for one day before characterization.

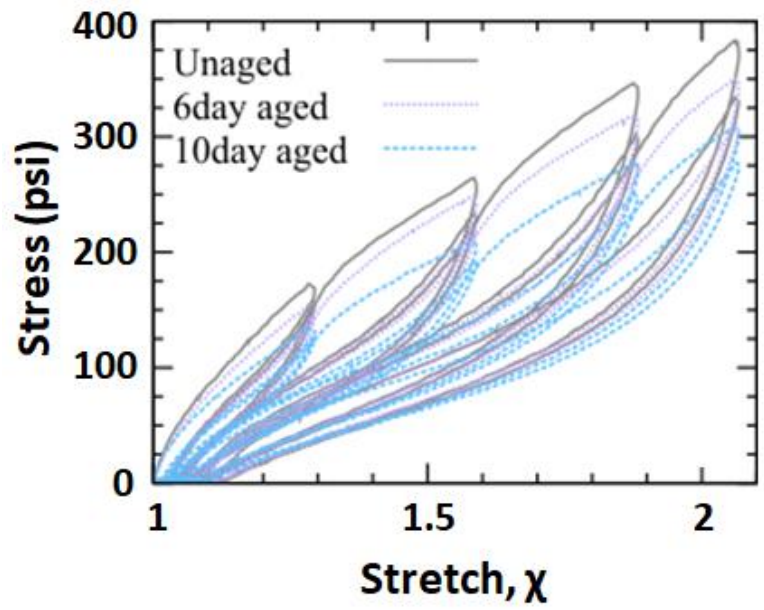

(a)

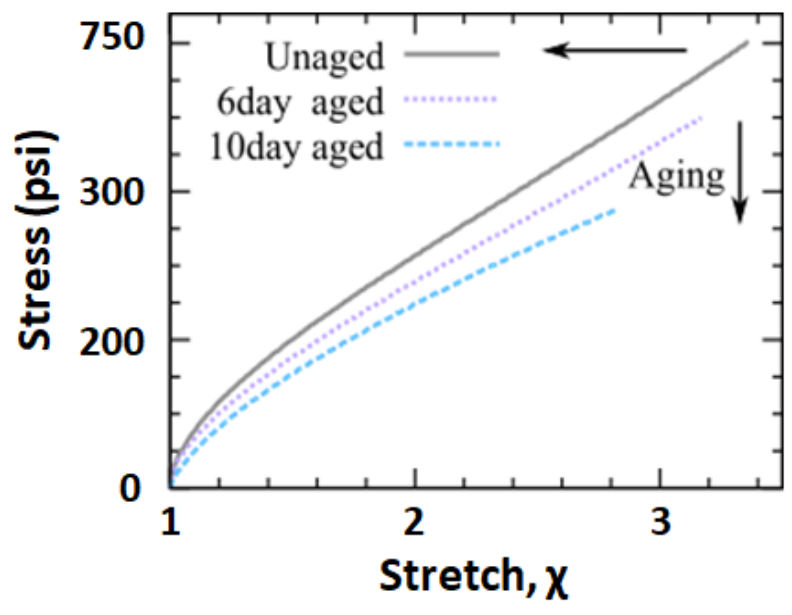

(b)

Figure II.3.3.5. SBR sample constitutive behavior at various aging times shown in: (a) cyclic tests performed at $60^{\circ} \mathrm{C}$ and (b) at failure of the adhesive. Source: MSU High Performance Materials Group. 
The hydrolytic damage function should be derived with respect to three independent variables - temperature, $\mathrm{T}$, time, $\mathrm{t}$, and deformation given by the deformation gradient tensor, $\boldsymbol{F}$. To describe the state of damage evolution with respect to time, one can use the end status of the matrix at time zero and $\infty$ as the reference points, and then define a shape function to interpolate the damage status at time, t. Representing the energy of the matrix in the virgin state by $\Psi_{0}$ and following the indefinite aging time by $\Psi_{\infty}$, one can approximate the strain energy of the matrix $\Psi_{M}$ at time, t, as:

$$
\Psi_{M}(t, T, \boldsymbol{F})=N(t, T) \Psi_{0}(\boldsymbol{F})+N^{\prime}(t, T) \Psi_{\infty}(\boldsymbol{F}), \text { where } N(t, T)=\exp \left(-\gamma \exp \left(-\frac{E_{a}}{R T}\right) t\right)
$$

where $\mathrm{N}(\mathrm{t}, \mathrm{T})$ and $\mathrm{N}^{\prime}(\mathrm{t}, \mathrm{T})=1-\mathrm{N}(\mathrm{t}, \mathrm{T})$ represent the weight of each state. To define the hydrolytic aging of the matrix, we used experiments to define the shape function $\mathrm{N}$ and the energy states $\Psi_{0}$, and $\Psi_{\infty}$, as seen in Figure II.3.3.6 (a) and Figure II.3.3.6 (b).

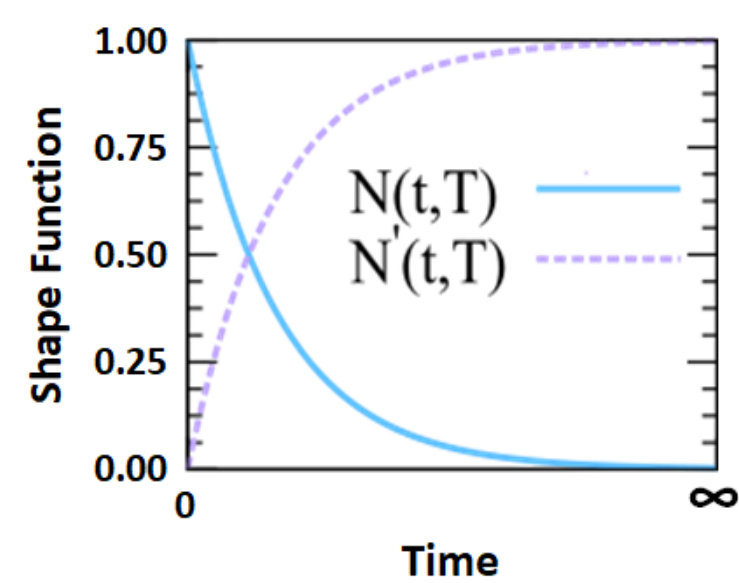

(a)

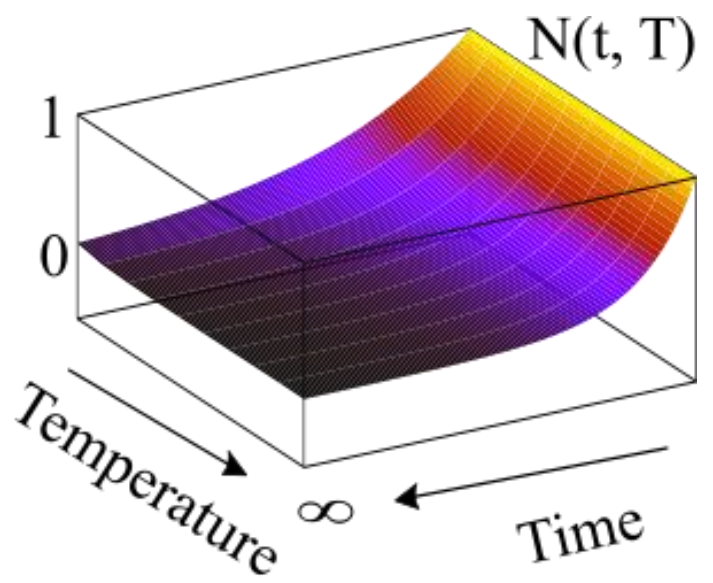

(b)

Figure II.3.3.6. (a) Shape functions $(\mathrm{N})$ as a function of time and temperature; and (b) the reconstructed surface of $N(t, T)$. Source: MSU High Performance Materials Group.

Once water attacks the matrix, two parallel phenomena occur-reduction of the crosslinks, which increases average chain length in the matrix, and energy dissipation due to the reduction of polymer active agents. To this end, a fully attacked matrix $\Psi_{\infty}$, can be decomposed into two independent networks, namely a newly morphed network $\Psi_{m}$ and a deactivated network $\Psi_{d}$, which can be defined as $\Psi_{\infty}=\alpha \Psi_{m}+(1-\alpha) \Psi_{d}$ where $\Psi_{m}$ and $\Psi_{d}$ represent the energies of the morphed and deactivated networks, respectively. The morphed network, $\Psi_{m}$, results from the reduction of the un-attacked network crosslinks, which consequently has longer chains than the virgin matrix. The deactivated network, $\Psi_{d}$, then results from the water-attacking polymer chains. For validation, model predictions were benchmarked against our experimental data specifically designed to capture the effects of hydrolysis with respect to $t, T$, and $F(t)$. Examples are shown in Figure II.3.3.7 (a)-(d) for SBR aged for six days at $60^{\circ} \mathrm{C}$ and $80^{\circ} \mathrm{C}$ and 10 days at the same temperatures. 


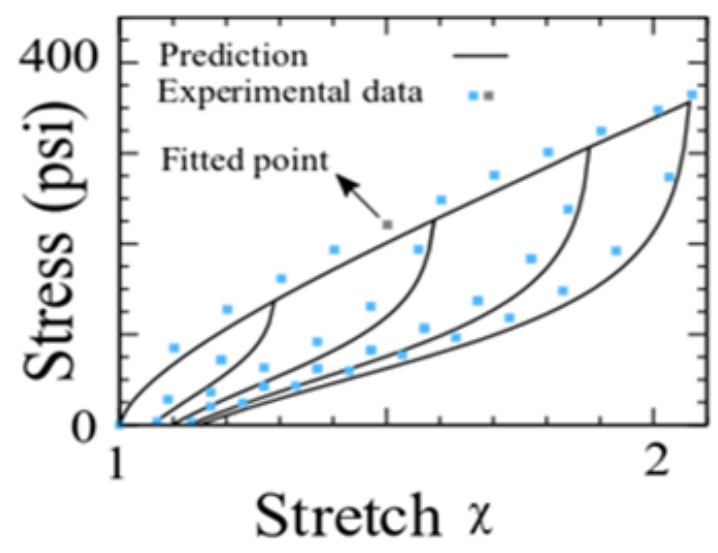

(a)

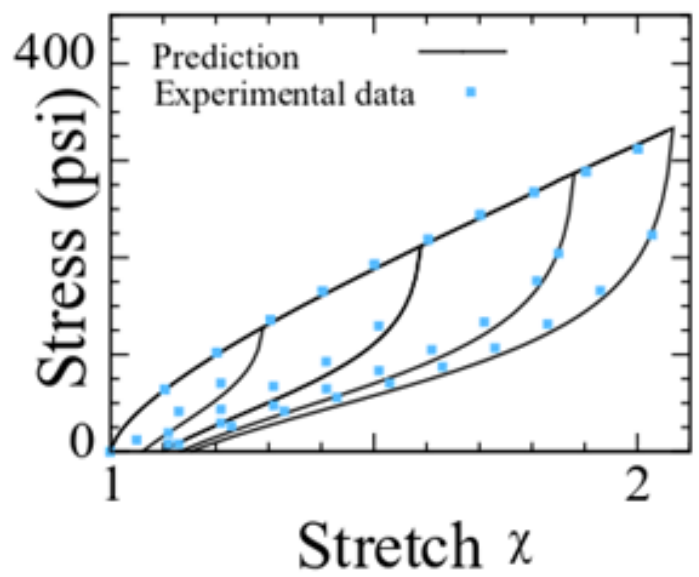

(c)

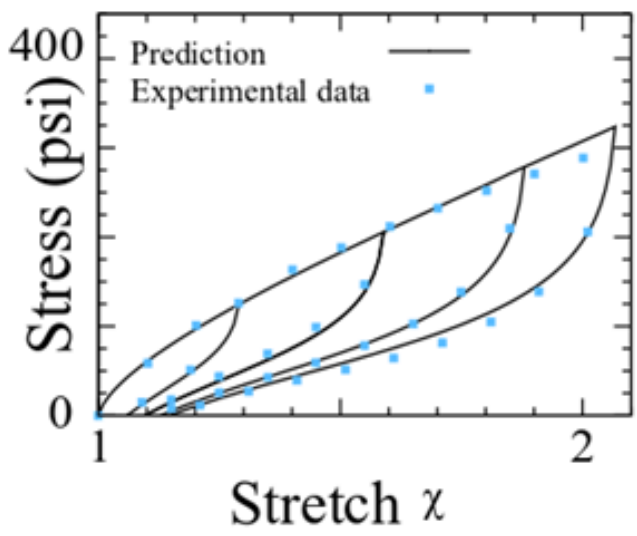

(b)

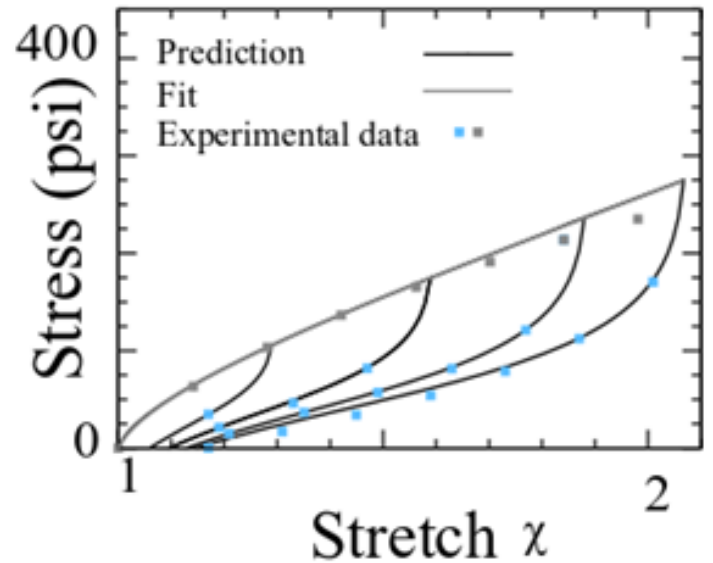

(d)

Figure II.3.3.7. Model validation for aging of control compound SBR in multiple conditions. Constitutive behavior for samples aged for 6 days at: (a) $60^{\circ} \mathrm{C}$ and (b) $80^{\circ} \mathrm{C}$ and those aged for 10 days at (c) $60^{\circ} \mathrm{C}$ and (d) $80^{\circ} \mathrm{C}$. Source: MSU High Performance Materials Group.

\section{Conclusions}

An integrated experimental, computational, and analytical approach was developed to understand and model the mechanisms of degradation in polymeric adhesives through a set of constitutive models supported by an extensive experimental program. This approach should provide predictive capabilities on the state of damage accumulation for cases when corrosion is induced by multiple parallel mechanical and environmental loads. The proposed platform is expected to provide guidance for future durability research of compounds. The micromechanical concepts and modeling strategies employed in this project are evolving to reflect best practices to provide the most confidence in predicted results. In view of the experimental tests provided on different adhesives, most adhesives with a crosslinked network are highly susceptible to damage when environmental loads are combined with mechanical loads. The project focus is currently on modeling individual and dual-coupled damage mechanisms. Ongoing activities should include the following:

- $\quad$ Evaluate environmental and mechanical damages in the selected set of adhesives during accelerated aging.

- Identify damage resolution needed for candidate adhesives to be suitable for validation of theoretical models. 
- Continue integration of constitutive models of the following phenomena to assist in adhesive durability analysis:

- quasi-static deformation

○ vibration

○ thermo-oxidative degradation

$\circ$ photo-oxidative degradation

○ hydrolysis degradation

$\circ$ hygro-thermal degradation.

\section{Key Publications}

1. Morovati, V., and R. Dargazany, 2019, "Improved approximations of non-Gaussian probability, force, and energy of a single polymer chain," Phys. Rev. E, Vol. 99, Art. \#052502.

2. Morovati, V., and R. Dargazany, 2019, "NET v1.0: A framework to simulate permanent damage in elastomers under quasi-static deformations," Softwarex, Vol. 10, Art. \#100229.

3. Mohammadi, H., A. Bahrololoumi, Y. Chen, and R. Dargazany, 2019, "A micromechanical model for constitutive behavior of elastomers during thermo-oxidative aging," In: Huneau, B., J.-B. Le Cam, Y. Marco, and E. Verron, Constitutive Models for Rubber XI: Proceedings of the 11th European Conference on Constitutive Models for Rubber (ECCMR 2019), Nantes, France, 25-27 June 2019, pp. 542-547, CRC Press: New York.

4. Morovati, V., and R. Dargazany, 2019, "Modeling damage accumulation during cyclic loading in elastomeric gels with interpenetrating networks," ASME 2019 International Mechanical Engineering Congress and Exposition, Salt Lake City, UT, USA, 8-14 November 2019.

5. Bahrololoumi, A., and R. Dargazany, 2019, "Hydrolytic aging in rubber-like materials: A micromechanical approach to modeling," ASME 2019 International Mechanical Engineering Congress and Exposition, Salt Lake City, UT, USA, 8-14 November 2019.

\section{References}

1. Zhang, W., X. Liu, J. Wang, J. Tang, J. Hu, T. Lu, and Z. Suo, 2018, "Fatigue of double-network hydrogels," Eng. Fract. Mech., Vol. 187, pp. 74-93.

\section{Acknowledgements}

The authors would like to thank Sarah Kleinbaum, DOE Technology Development Manager, for her assistance and guidance on the experimentation procedure and continuous feedback and Mr. John Terneus for managing the progress of the project. 


\section{II.3.4 High-Strength Steel-Aluminum Components by Vaporizing Foil Actuator Welding (The Ohio State University)}

\section{Glenn Daehn, Co-Principal Investigator}

Department of Materials Science and Engineering

The Ohio State University

295 Watts Hall, 2041 College Rd. N

Columbus, $\mathrm{OH} 43210$

E-mail: daehn.1@osu.edu

\section{Anupam Vivek, Co-Principal Investigator}

Department of Materials Science and Engineering

The Ohio State University

348 MacQuigg Labs, 105 W. Woodruff Ave.

Columbus, OH 43210

E-mail: vivek.4@osu.edu

\section{Sarah Kleinbaum, DOE Technology Development Manager}

U.S. Department of Energy

E-mail: sarah.kleinbaum@ee.doe.gov

Start Date: October 1, 2016

End Date: September 30, 2020

Project Funding (FY19): \$1,071,665

DOE share: $\$ 986,165$

Non-DOE share: $\$ 85,500$

\section{Project Introduction}

This project aims to address the challenge of effectively welding dissimilar materials - high-strength steel and high-strength $\mathrm{Al}$ for creating lightweight, multi-material automotive components. Traditional fusion-based welding between dissimilar combinations of $\mathrm{Al}$ and steel is difficult due to starkly disparate melting points and usually leads to the formation of brittle IMCs at the weld interface. Such joints are weak and cannot be implemented in crash-sensitive components. Structural adhesives and mechanical fasteners of various types including self-piercing rivets, flow-drilled screws, friction element welds, and RIVTAC ${ }^{\circledR}$ are currently being utilized to address this issue. Solid-state welding techniques, like friction stir and impact welding, offer an elegant alternative solution for joining dissimilar metals without the use of a third body, such as rivets. This project utilizes Vaporizing Foil Actuator Welding (VFAW), an impact welding method developed at OSU [1]. Details on this process can be found in the Materials 2017 and 2018 Annual Progress Reports [2, 3], as well as several journal articles published on this technology.

VFAW has demonstrated much success in welding a wide range of alloys, including $\mathrm{Mg}-\mathrm{Al}, \mathrm{Al}-\mathrm{Cu}, \mathrm{Ti}-\mathrm{Cu}$, bulk metallic glass-Cu, and $\mathrm{Ni}$-Ti. In this project, the focus is on welding specific grades of steel and $\mathrm{Al}$ that are relevant to automotive sub-frames and developing the technology to a level where it can be transitioned to an automotive supplier or an OEM. Besides the research on weldability of different material combinations and determining the ideal parameters through experiment and simulation, the project also includes design, production, and testing of a prototype subframe component, as well as the production equipment. Robustness of the process for application in a serial production setting is demonstrated via robotic implementation. 
The work in FY 2019 was focused on validating and optimizing the welding process and developing the prototype welding head towards the final prototype production. The prototype design was upgraded with a final weight reduction of $12 \%$ compared with the $8 \%$ estimated in last year's report. The two-shot VFAW method was created, which achieved greater repeatability and robustness. Several new high-strength Al alloys were introduced, and lap-shear tested with coupon scale welded samples. Through collaboration with Magna and Coldwater Machine, the prototype welding head was designed, and a pre-prototype welding head was machined and tested. Meanwhile at OSU, a fully automated pedestal welder was developed and is nearing completion.

\section{Objectives}

This project accelerated and focused the development of VFAW to produce an automotive component. At project completion, the technology will be ready for adoption within the $\mathrm{R} \& \mathrm{D}$ groups of Tier 1 and original equipment manufacturers for assembly of any mixed/advanced material bodies.

The overall objectives of the project are:

- A 20\% weight reduction of the current all steel automotive component from a 2017 mid-size sedan at a cost premium of $\$ 3 / \mathrm{lb}$-saved by developing a mixed-material joining technology capable of high-volume production.

- The produced component should meet or exceed the strength and durability of the incumbent component.

- To develop a predictive modeling capability for relating process, structure, and property of VFAW joints.

The specific objectives for FY 2019 were:

- $\quad$ To optimize the VFAW process parameters and system for a specific material pair within geometric constraints of the prototype component assembly for maximum reproducibility, using coupon testing.

- To design a prototype Al-steel engine cradle including the location and required properties of individual welds to meet automotive performance objectives of strength, durability in corrosion, durability in fatigue, and stiffness.

- To design, construct, and machine a welding head to produce the prototype component.

- To design and construct an automation system achieving fully automated coupon feeding, foil feeding, VFA spot welding, and cleaning to show the potential for automation capability of the VFAW process.

\section{Approach}

The overall approach for this project is to perform coupon scale testing at OSU, develop welding systems and part fixtures at OSU and Coldwater, prototype-scale manufacture and testing at Magna, and process simulation at PNNL. Ashland, Arconic, Hydro, Novelis, and Magna are providing material. The pre-prototype welding heads and final welding head will be fabricated at Coldwater and tested at OSU. The final welding head, along with a capacitor bank, will be installed at Magna. Coupon data will be imported into the design of the prototype component, while simulated testing and design iterations will lead to the final part. Once the prototype design is released, Magna will produce subcomponents and perform the assembly at its prototype facility. The assembled prototypes will be tested for durability and corrosion performance.

\section{Coupon Testing}

The Al-steel combination was finalized as hot-rolled $4.2 \mathrm{~mm}$-thick AA5182-O and $2.2 \mathrm{~mm}$-thick high-strength low-alloy (HSLA) 340, based on the screening results and actual material inventory at Magna's material supplier, Novelis. This was a change from the $4 \mathrm{~mm}$-thick AA5754-O that was selected as the flyer and tested in Year 2. In addition, an $\mathrm{Al}$ flyer of $4 \mathrm{~mm}$ and $5.1 \mathrm{~mm}$-thick AA5052-O and $4.5 \mathrm{~mm}$-thick AA5182-O were 
also examined as the test for the pre-prototype welding head went on. For the HSLA 340 target, a concern about cracking on the pre-strained HSLA 340 from deformation arose from forming simulations at Magna. To imitate the actual condition of the prototype, welding to HSLA 340 with pre-strain from $10 \%$ to $30 \%$ was also tested.

For this project, VFAW requires a 1 mm-thick AA3003-H14 intermediate layer (interlayer). Standoff gaps between the interlayer and target were provided by dimpling the target sheet. Geometry of the preformed dimples was finalized based on the trials conducted to generate the strength and durability data used in the prototype design simulation. For the single-shot method, standoff gaps between the flyer, interlayer, and target were provided by dimpling the target and interlayer sheets. In a single-shot, the flyer impacts the interlayer, which in turn impacts the target, and the three layers are then welded to each other. This stack-up is shown in Figure II.3.4.1. For the two-shot method, the first shot drives the interlayer to impact the pre-deformed target, and then the second shot joins the flyer to the target at the pre-welded interlayer. A schematic of this process is shown in Figure II.3.4.2. Comparing processing needed for each method, the two-shot method requires one more shot, but with no pre-deformation of the interlayer, less-strict alignments of the stack-up, and reduced input-energy for each shot. Welds were created using the two-shot method and lap-shear tests were performed afterwards. Welds in the prototype design were created with the weld's length parallel to that of the coupon (regular), as well as perpendicular to the long dimension of the coupon, as shown in Figure II.3.4.3.

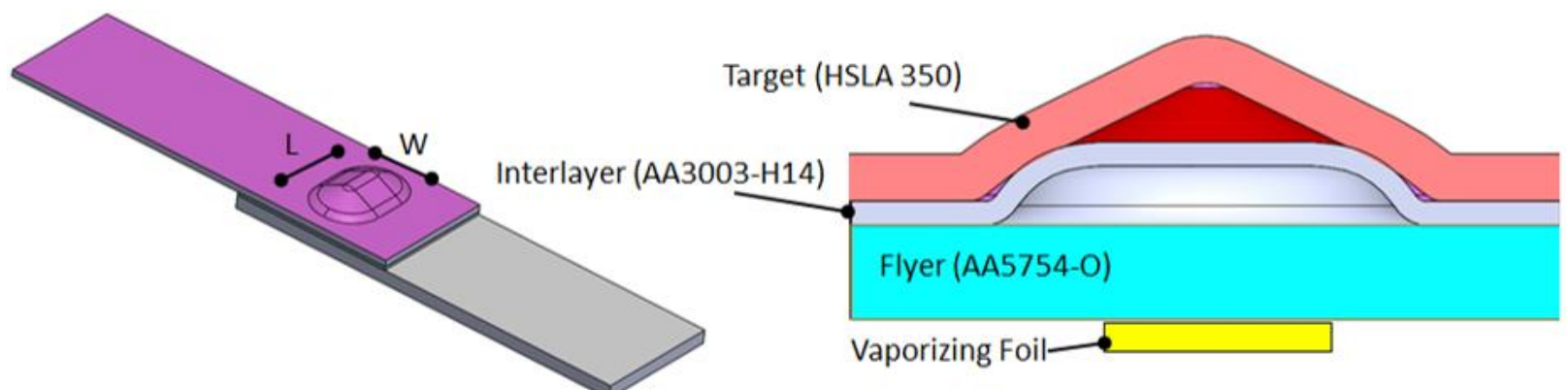

(a)

(b)

Figure II.3.4.1. Illustrations of: (a) the lap-joint showing the dimple length (L) and width (W), which varied; and (b) the VFAW layup with the interlayer. Source: OSU.

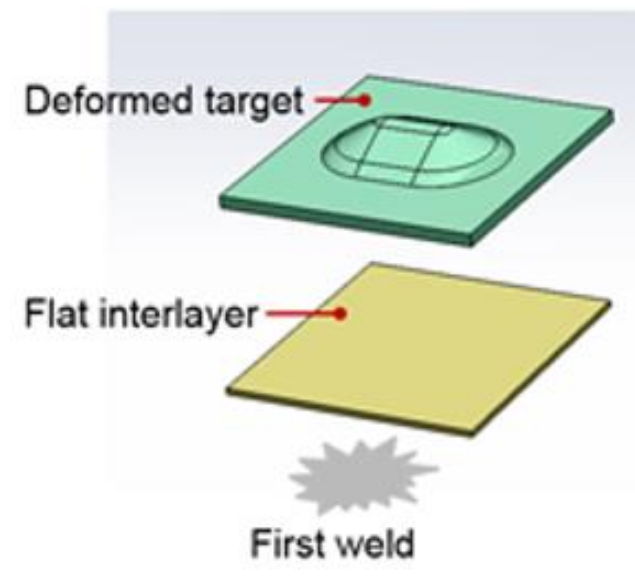

(a)

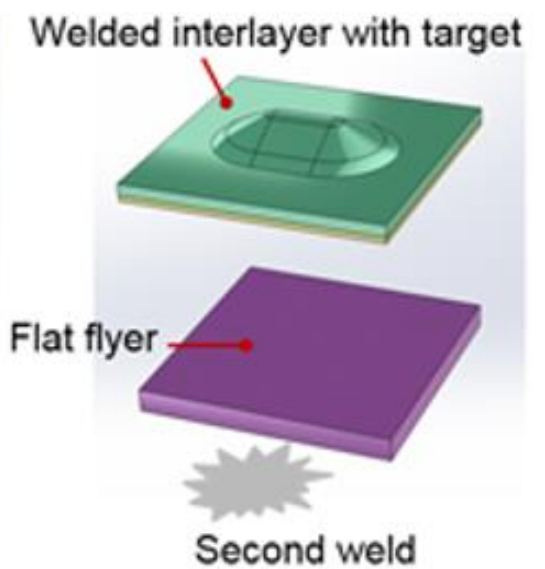

(b)

Figure II.3.4.2. Illustrations of the two-shot VFAW: (a) the first shot welds the flat interlayer to the deformed target; and (b) the second shot welds the flyer to the welded flyer-target stack-up. Source: OSU. 


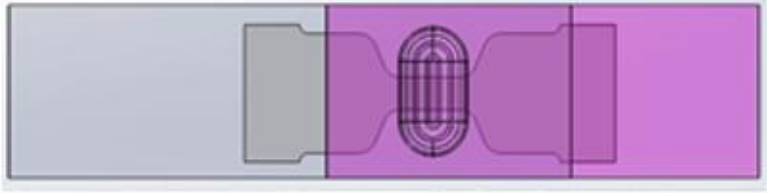

(a)

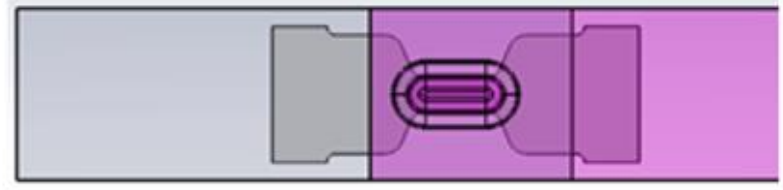

(b)

Figure II.3.4.3. Welds of two directions: (a) the length of the weld is perpendicular to the length of the sample; and (b) the length of the weld is parallel to the length of the sample. Source: OSU.

Several samples of the AA5754-O (4 mm-thick flyer) and the HSLA 340 (2.2 mm-thick target) created with the single-shot method were prepared with an adhesive between the interlayer and the target. The adhesive was applied around the dimple in order to avoid having to weld through the adhesive. Ashland's two-part, room temperature-curing adhesive attaches the interlayer to the target, as well as providing a seal around the final weld between the two pieces. There is no adhesive between the flyer and the interlayer as they are both made of $\mathrm{Al}$ and are not susceptible to galvanic corrosion. Tension-tension fatigue tests to identify low-(5000 cycles), medium-(100,000 cycles) and high-(1,000,000 cycles) cycle failure loads at a frequency of $10 \mathrm{~Hz}$ were performed.

\section{Development of Weld Head}

Previously, the ideas of a fixed weld head to which the part is to be brought or a weld head hanging from a tool balancer were discussed and presented under this project. However, for better maneuverability and to accelerate the technology, a robot-mountable prototyping weld head has been designed and is now in production. The size of the component, various approach angles and space constraints were taken into consideration during the weld head design. Several design review meetings and a few iterations on the physical tooling ("pre"-prototyping weld heads) were conducted to test key aspects of the prototyping weld head, such as foil clamping and removal, welding on flanges, and force due to foil vaporization.

\section{Results}

\section{Coupon Testing}

The deformation of the pre-strained HSLA 340 target coupons are shown in the images of Figure II.3.4.4 (a). The images show that after deformation all the pre-strain samples have small orange-peel patterns at the very top center of the outer side, but no cracking is observed at the corners as can be seen in Figure II.3.4.4 (b). Additionally, a weld was made using $20 \%$ pre-strained HSLA 340, which is assumed to be a reasonable upper limit for prototype production, and nothing abnormal is observed compared with a $0 \%$ pre-strained target. 

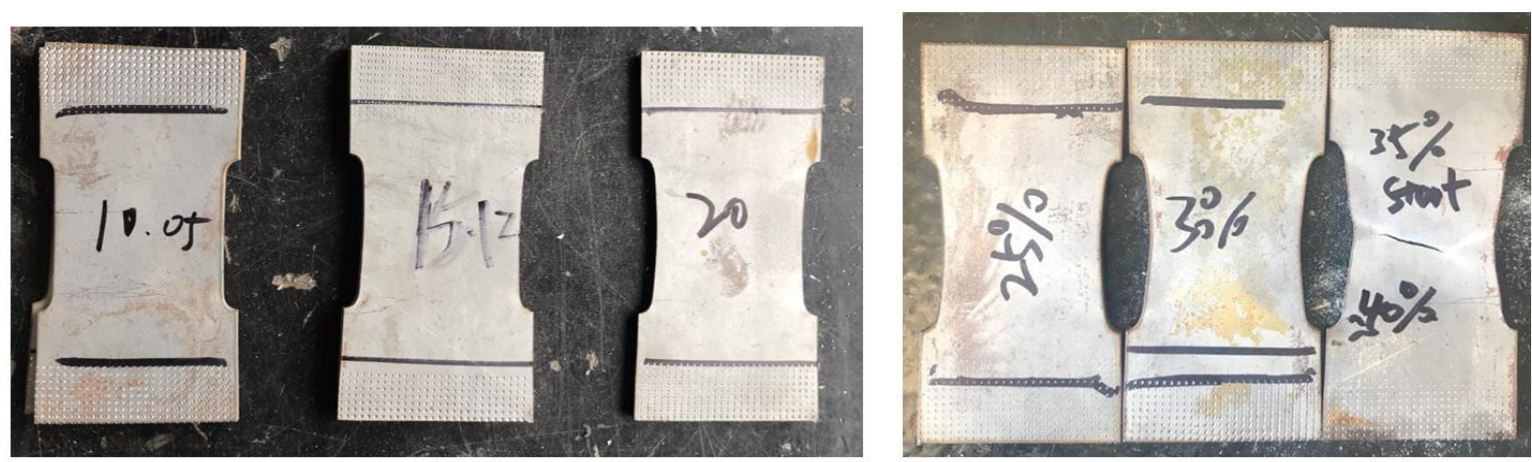

(a)
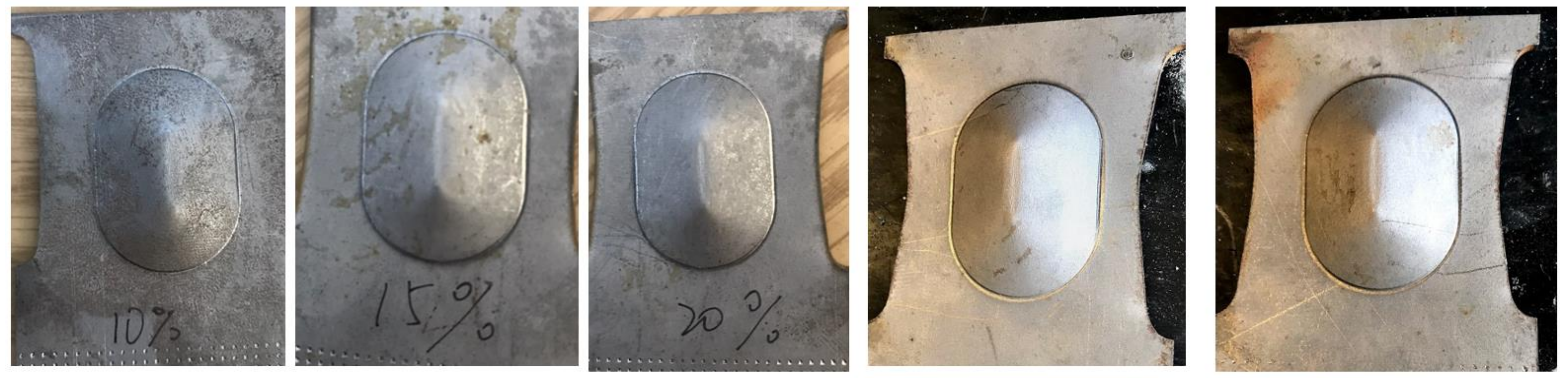

(b)

Figure II.3.4.4. (a) Pre-strained samples and (b) pre-strained and deformedsamples. The pre-strain increases from left to right as labeled. Source: OSU.

The results of fatigue tests for welds of 4-mm-thick AA5754-O to 2.2-mm-thick HSLA 340 with the singleshot method are shown in Figure II.3.4.5. Peak tensile load is $16.55 \mathrm{kN}$ for the lap-shear tests. HCF failure occurred around $10^{6}$ cycles (i.e., for 4 out of 5 samples) at $40 \%$ of peak load, but some inconsistency in failure mode was observed. Samples tested at $45 \%$ peak load also showed large variability with cycles to failure ranging from $7.4 \times 10^{3}$ to $6.9 \times 10^{4}$ (error bars left off of figure). Better repeatability was observed for samples cycled at $50 \%$ and $60 \%$ of peak lead which resulted in $8.0 \times 10^{3}$ and $2.0 \times 10^{3}$ cycles to failure respectively. The manual weld setup creates variation which is evident in static results and in cyclic loading the inconsistency is exacerbated.

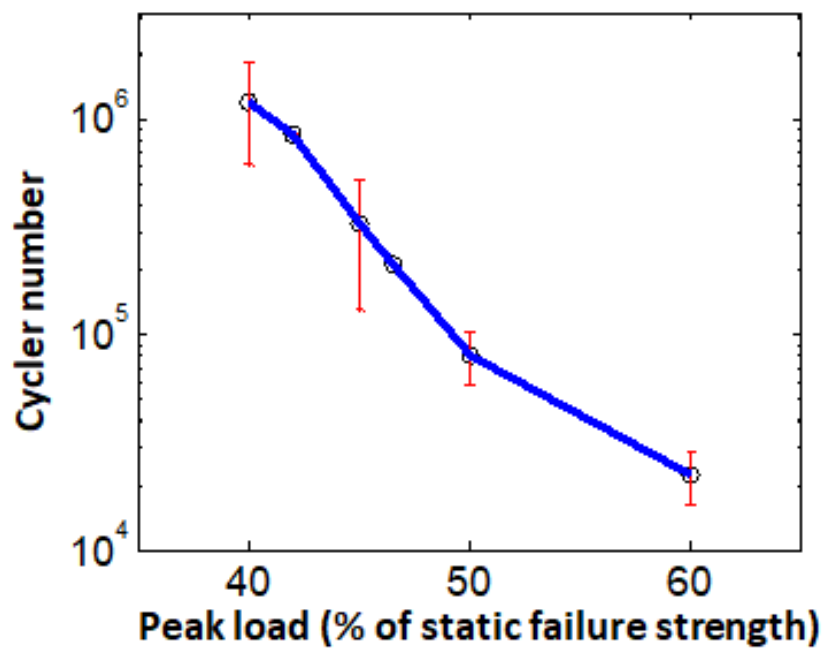

Figure II.3.4.5. Fatigue matrix of 4-mm-thick AA5754-0 to 2.2-mm-thick HSLA 340 with the single-shot method. Source: OSU. 
Welds of 4 mm AA5052-HSLA 340 were created and tested using the two-shot method with pre-prototype welding head \#1. The results are shown in Figure II.3.4.6. The input-energy of first and second welding process is $2.0 \mathrm{~kJ}$ and $3.0 \mathrm{~kJ}$, respectively. They show good repeatability with a peak load around $22 \mathrm{kN}-$ despite no strict alignment-and the entire process was easily conducted.
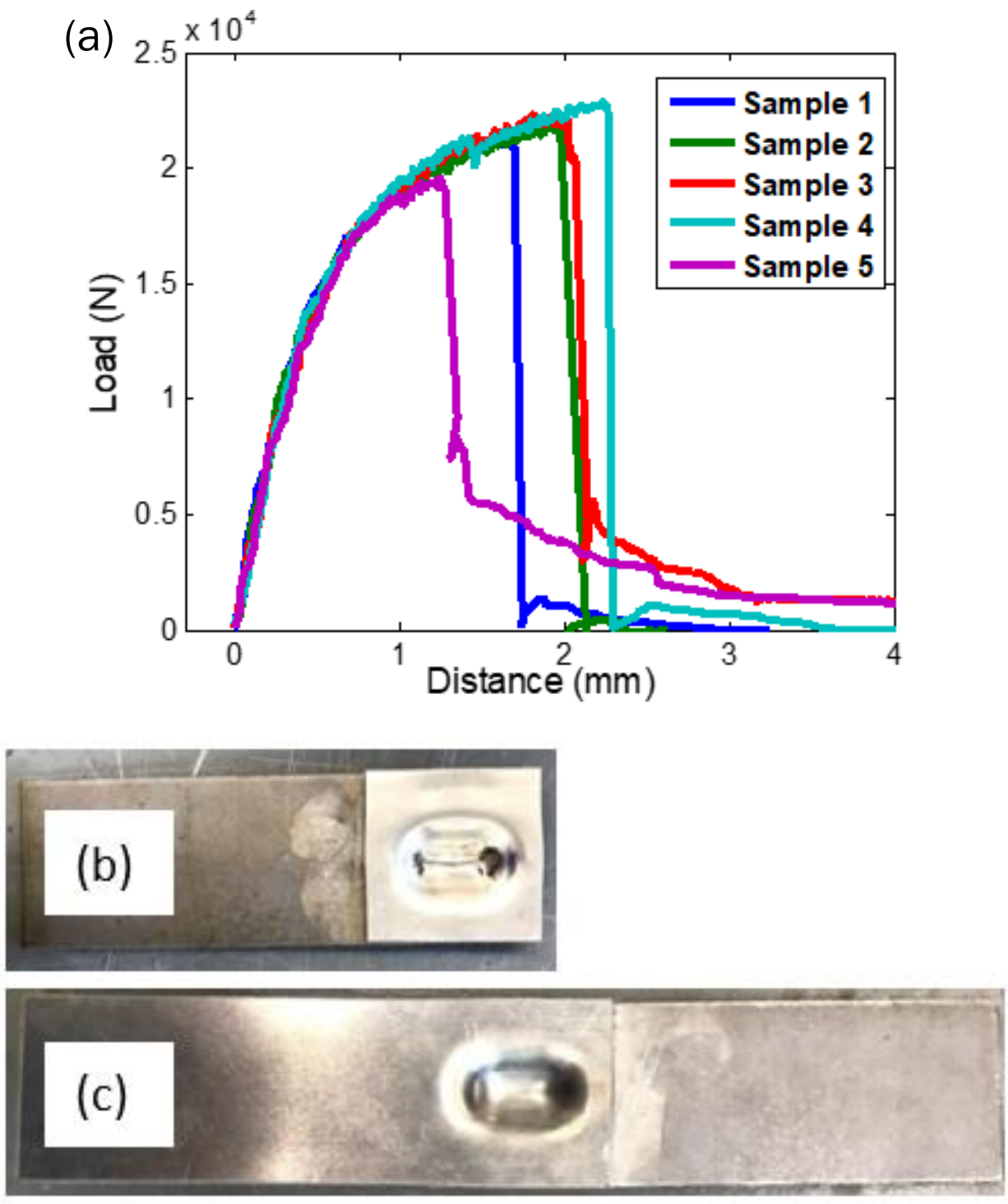

Figure II.3.4.6. Welds of $4 \mathrm{~mm}$ AA5052-HSLA 340 using the two-shot method created with the pre-prototype welding head \#1: (a) results of lap-shear tests; (b) a weld of interlayer to target after first shot; and (c) a weld created using the two-shot method. Source: OSU.

A direct comparison between the two-shot method and the single-shot method was performed with preprototype welding head \#2. The results are shown in Figure II.3.4.7. The single-shot welds were produced at the input-energy of $3.5 \mathrm{~kJ}$. With the same failure mode of interfacial failure between the interlayer and the target, the two-shot method produces a higher peak load and fracture toughness. Single-shot welds yielded a brittle failure. Despite various issues with the pre-prototype welding head \#2, which will be discussed in next section, the welds show good repeatability. 


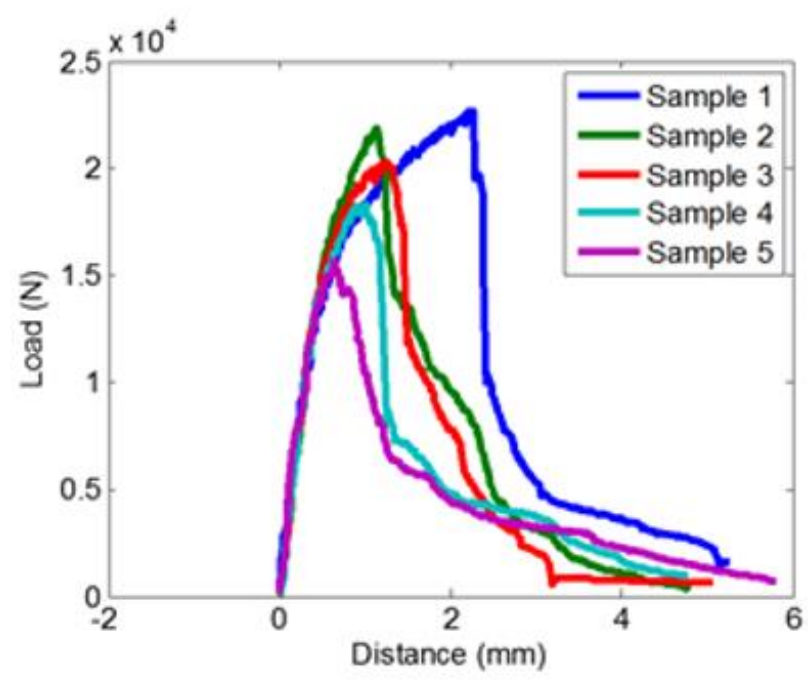

(a)

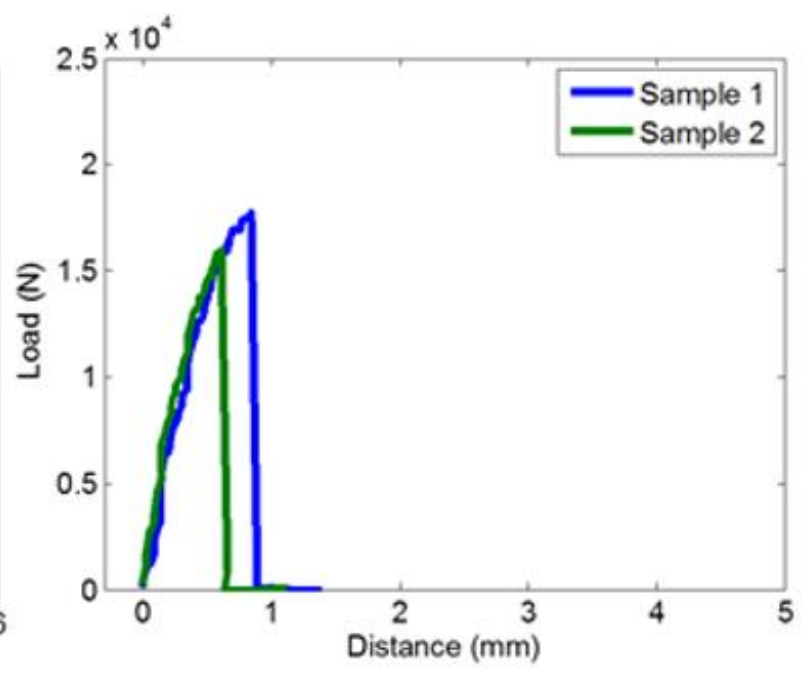

(b)

Figure II.3.4.7. Lap-shear tests of welds of 4 mm AA5052-HSLA 340 using: (a) the two-shot method; and (b) the single-shot method created with pre-prototype welding head \#2. Source: OSU.

Additionally, welds of different orientations with reference to the sample length were created and tested with a $4.5 \mathrm{~mm}$ AA5182, and a $5.1 \mathrm{~mm}$ hot-rolled AA5052 as the flyer, using the two-shot method with pre-prototype welding head \#2. The results as seen in Figure II.3.4.8 show that VFAW creates welds with a similar strength in both orientations and the two-shot method worked even for material as thick as $5.1 \mathrm{~mm}$ hot-rolled AA5052 with a peak load around $16 \mathrm{kN}$.

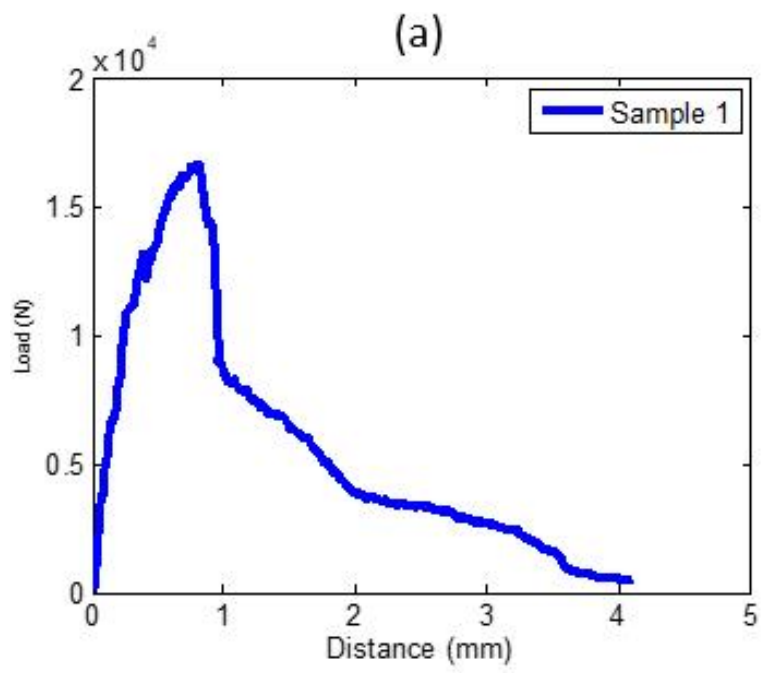

(b)
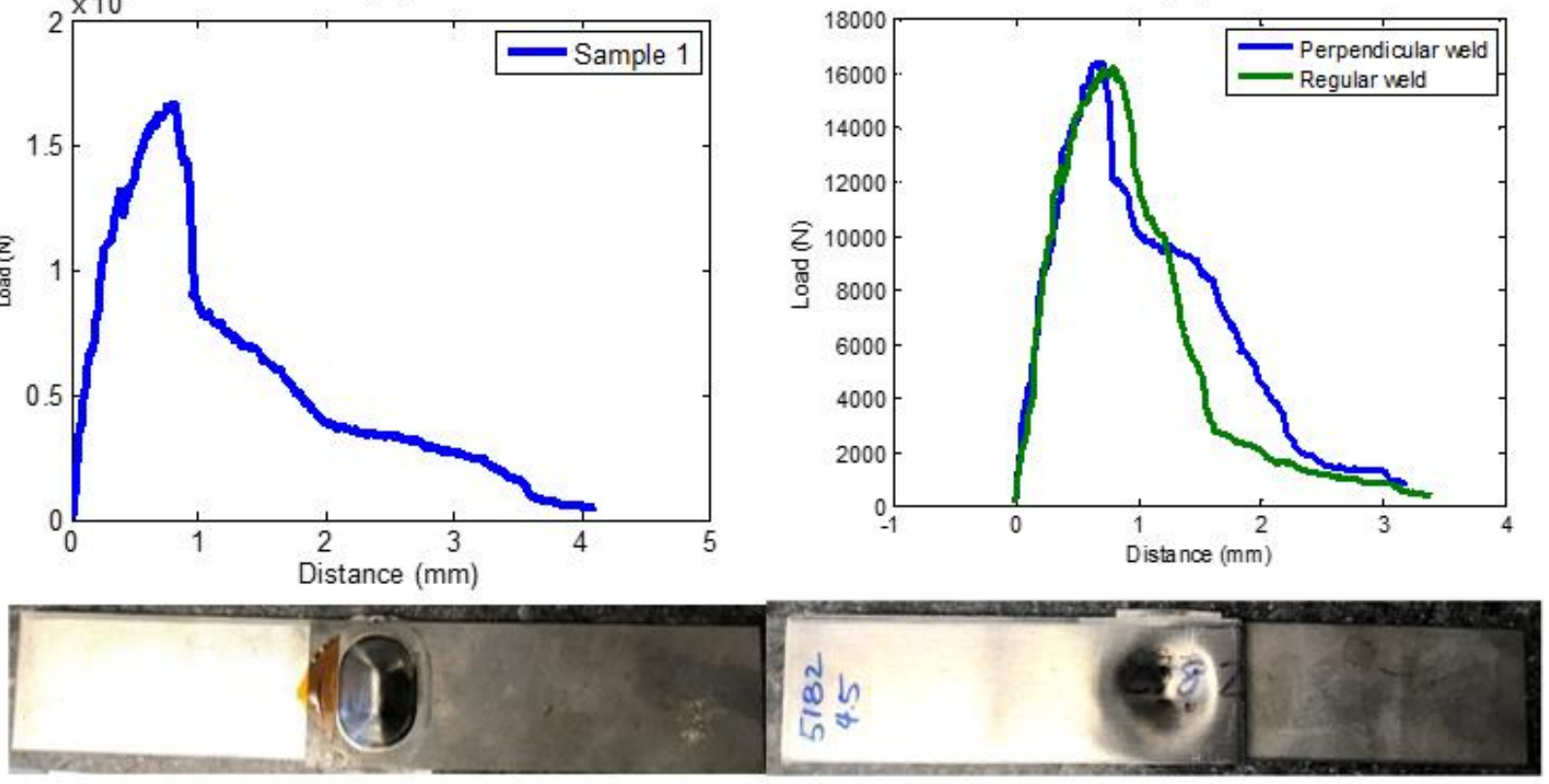

(C)

Figure II.3.4.8. Lap-shear tests of (a) a weld of with $4.5 \mathrm{~mm}$ AA5182-HSLA 340 in the perpendicular direction; (b) welds of $5.1 \mathrm{~mm}$ hot-rolled AA5052-HSLA 340 in both directions; and (c) a photo of the front and back sides of an AA5182-HSLA 340 weld in the perpendicular direction. Source: OSU. 


\section{Development of Prototype Component}

Magna has finalized a multi-material VFAW front subframe design, as shown in Figure II.3.4.9. Assuming an adequate weld performance, the design currently meets most performance targets. The original mass objective was to achieve a $20 \%$ weight reduction from the current all steel production design. This weight-savings was achievable with the original concept of an entirely $\mathrm{Al}$ rear that would be welded to the steel front rails using VFAW. The final design provides a $12 \%$ mass reduction based on adherence to stiffness targets, while making several more welds than initially planned. Based on the prototype design, the abuse loading condition was modeled with NASTRAN and ABAQUS, showing that the max force on a weld is around $2.5 \mathrm{kN}$ with extreme loading at the Front Lower Control Arm (FLCA) location, as shown in Figure II.3.4.10.

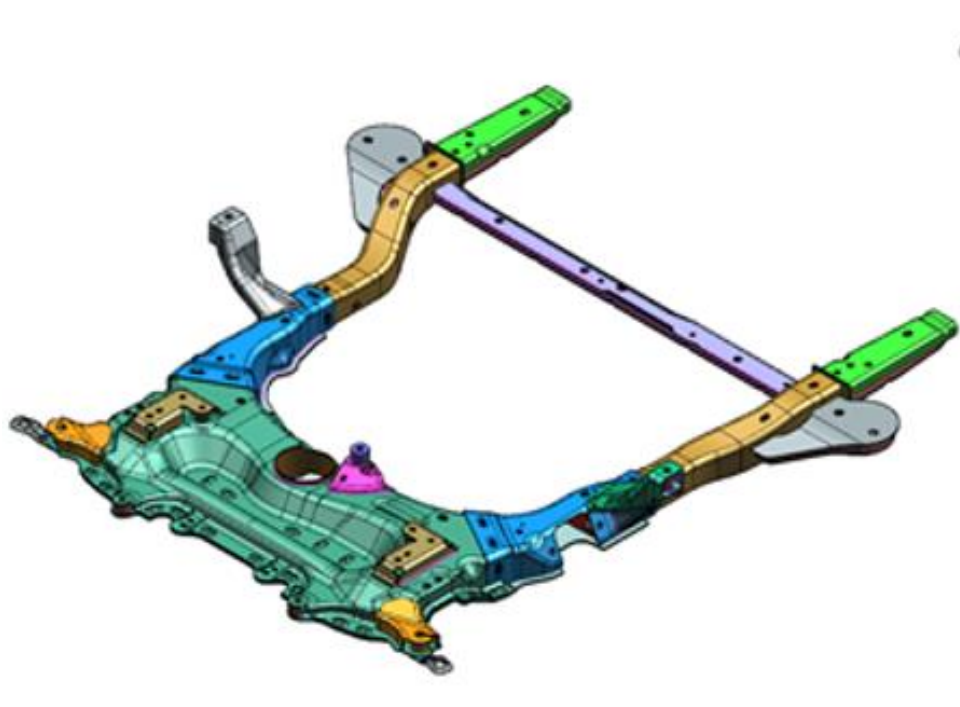

(a)

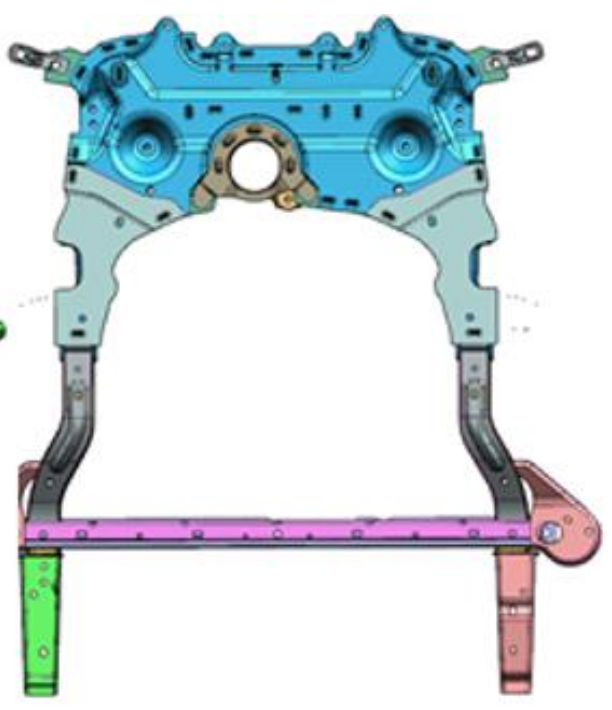

(b)

Figure II.3.4.9. Upgrade design of subframe: (a) frontside; and (b) backside. Source: Magna. 


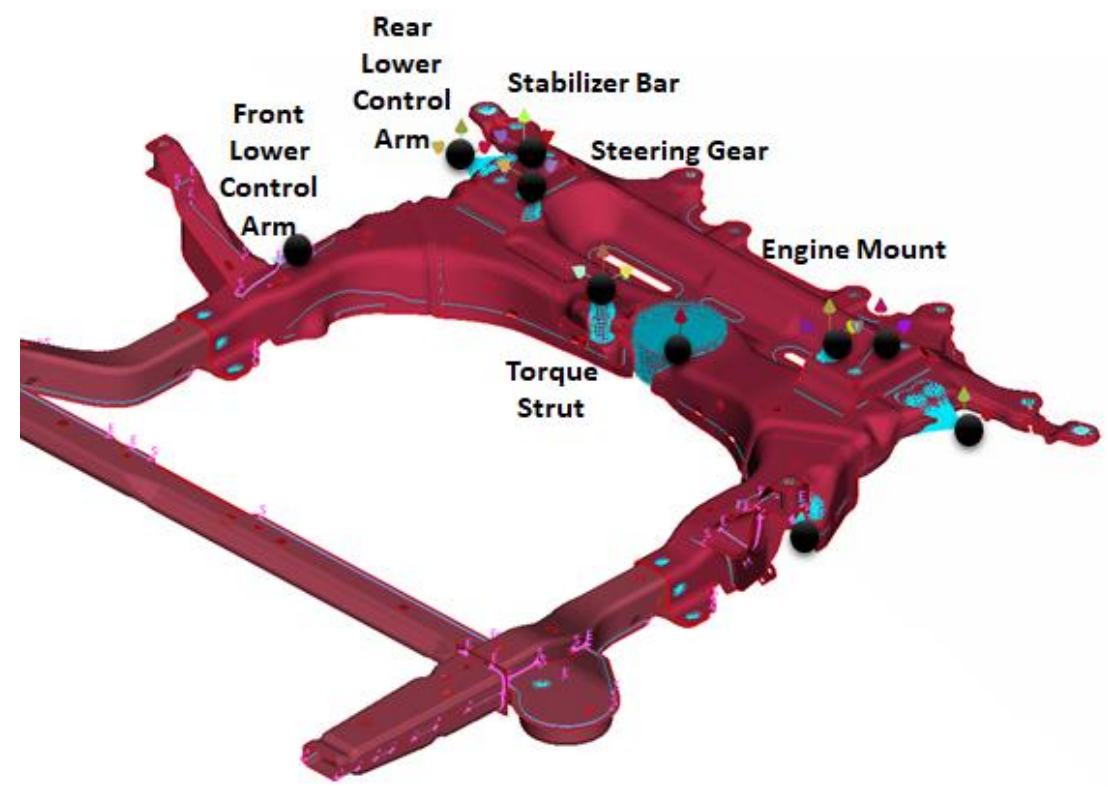

(a)

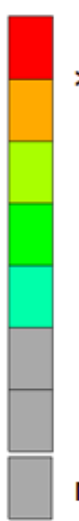

$>2479.24$
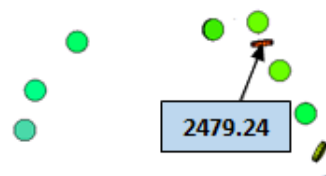

2001.27

1523.30

1045.33

567.35

$<89.385$
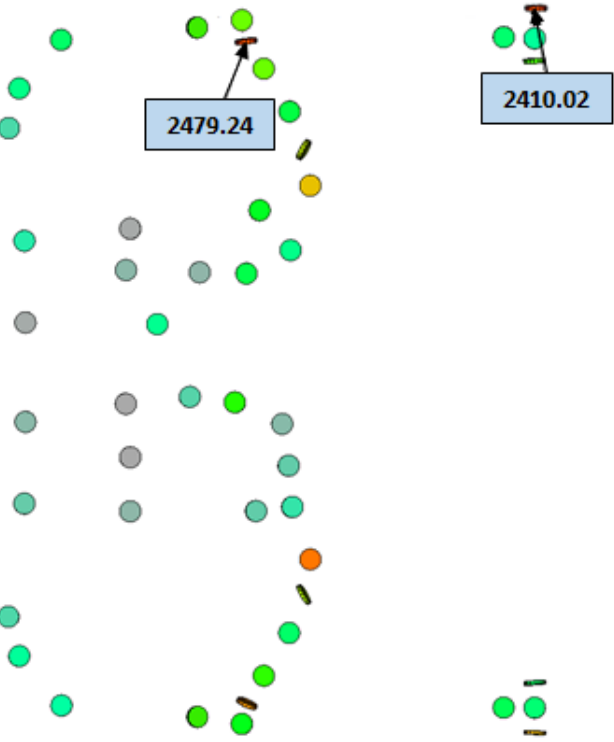

No Value

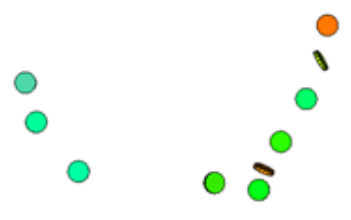

(b)

Figure II.3.4.10. (a) FLCA locations and (b) results of modeling of extreme loading condition. Source: Magna.

\section{Development of Weld Head}

As a transition from the welding fixture used in the lab environment and the prototype welding fixture, a simplified pre-prototype system was built and tested. The system has a welding head (e.g., pre-prototype welding head \#1), which is a greatly simplified version of the prototype welding fixture, a safety enclosure, and a custom-built capacitor bank, which will be used for the prototype production. This system is shown in Figure II.3.4.11. Processing parameters including input-energy in the range of 3.6-4.2 kJ and fastening torque in the range of 5-30 ft.lbf were tested. A combination of $3.8 \mathrm{~kJ}$ with a fastening torque of $10 \mathrm{ft} \cdot \mathrm{lbf}$ for the single-shot method and $2 \mathrm{~kJ}+3 \mathrm{~kJ}$ at the same fastening torque for the two-shot method works for making welds on most of the material combinations chosen for the prototype component. A set of coupon tests using the preprototype welding head \#1 was shown previously in Figure II.3.4.6. 


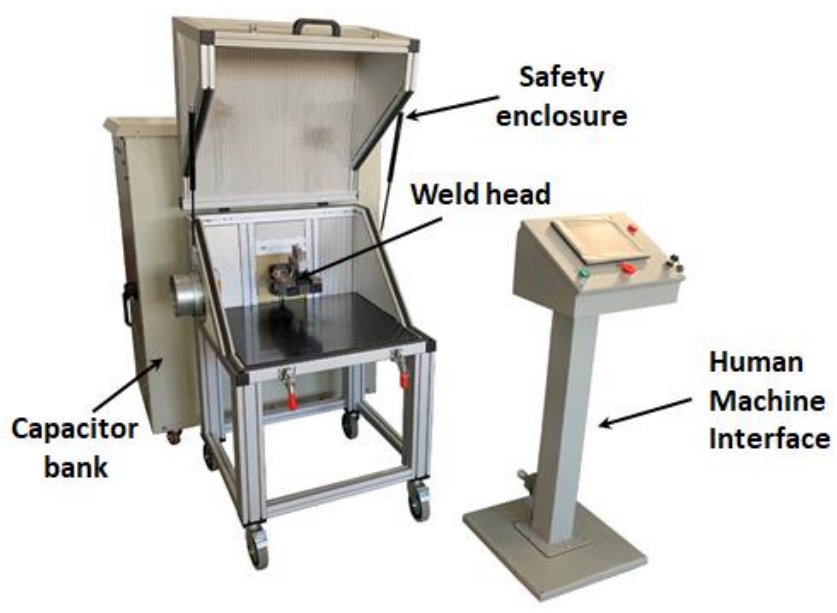

(a)

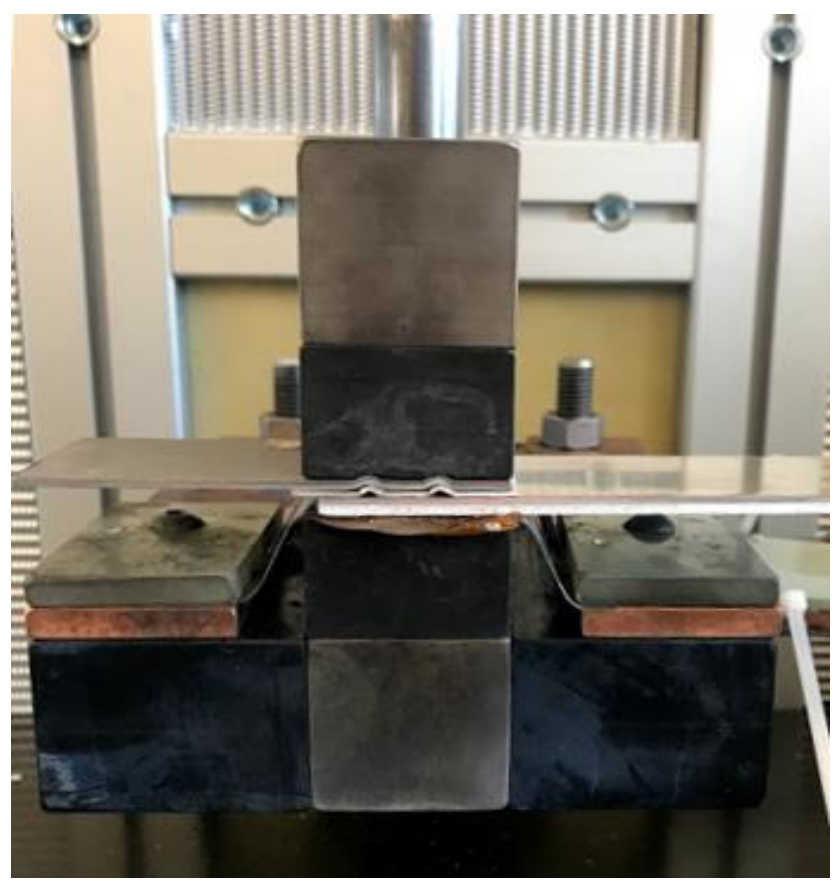

(b)

Figure II.3.4.11. (a) A pre-prototype system and (b) a closer view of the pre-prototype welding head \#1. Source: OSU.

Another pre-prototype welding fixture - pre-prototype welding head \#2-was used to estimate foil vaporization loads and test a novel foil placement and clamping mechanism. This welding fixture shown in Figure II.3.4.12 is designed to be capable of quick change of anvil and breech. The impact force - estimated to be $3000 \mathrm{lbs}$. using the shearing pin - was used as the input parameter for FE model simulation of the state-ofthe-fixture during welding. The simulation result shows the pre-prototype welding fixture operates properly at less than $3.5 \mathrm{~kJ}$ with only minor elastic deformation on the main structural components. Both the single-shot method at $3.5 \mathrm{~kJ}$ and the two-shot method were tested using the pre-prototype welding fixture. The mechanical test results were shown previously in Figure II.3.4.7. During the tests, issues such as arcing from the electrodes, cracking on the weaker components, debris accumulation, and difficulty in reaching and cleaning the electrodes were found and addressed. Once these issues were corrected, the welding process with this fixture showed good repeatability. 
The final review of the prototype welding head is complete and is in production at Coldwater Machine. It will achieve a quick change of anvil and breech in order to reach the welds in the lower portion of the stampings, accurate foil placement, and easy cleaning action. Figure II.3.4.13 shows the configuration when welding on the flange and the schematic of mounting on an articulated robot arm. The welding head will be equipped with hydraulic cylinders to apply a preload, as well as backing force during welding. Flexible coaxial cables will connect the weld head electrodes to the capacitor bank terminals for the desired freedom of movement.

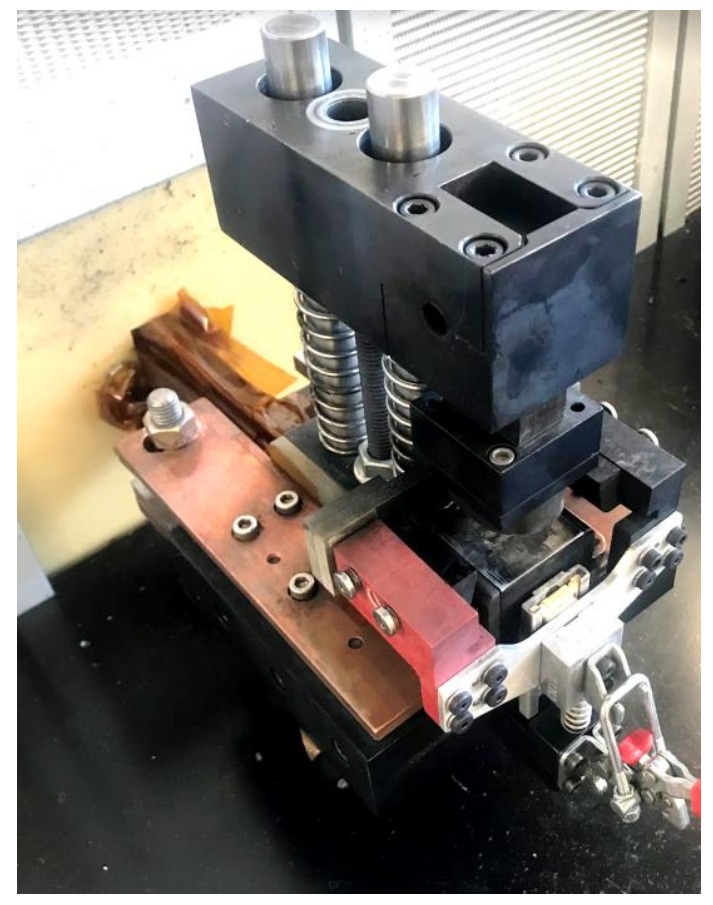

(a)

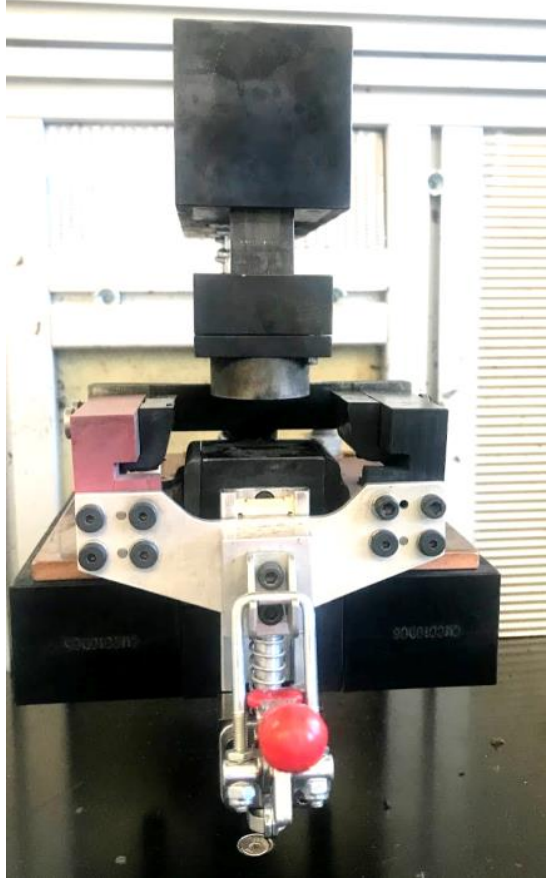

(b)

Figure II.3.4.12. Pre-prototype welding head \#2 (a) top and (b) front. Source: OSU.

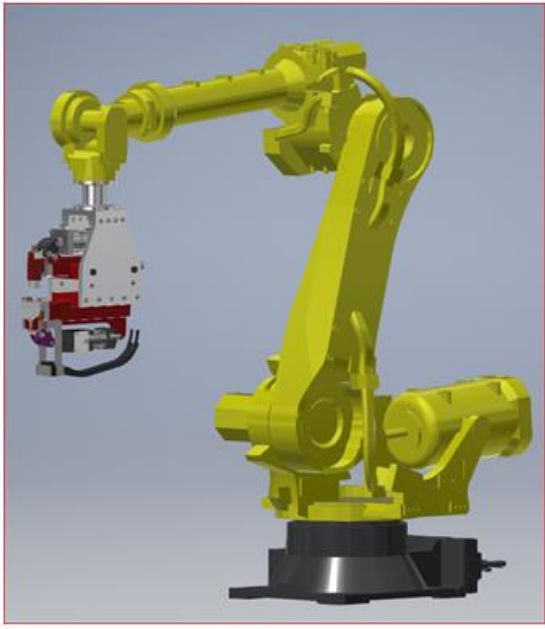

(a)

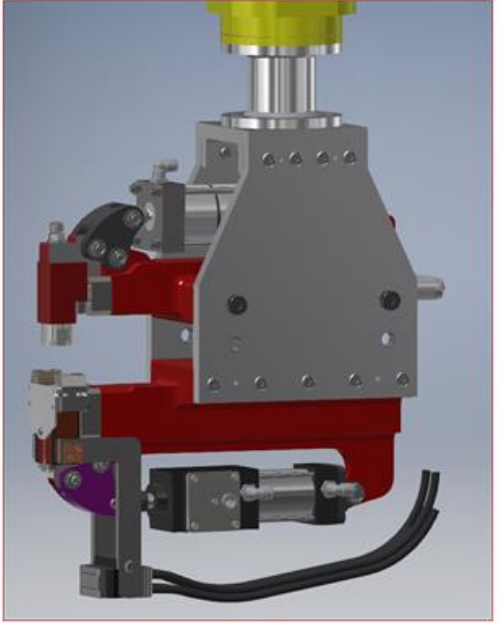

(b)

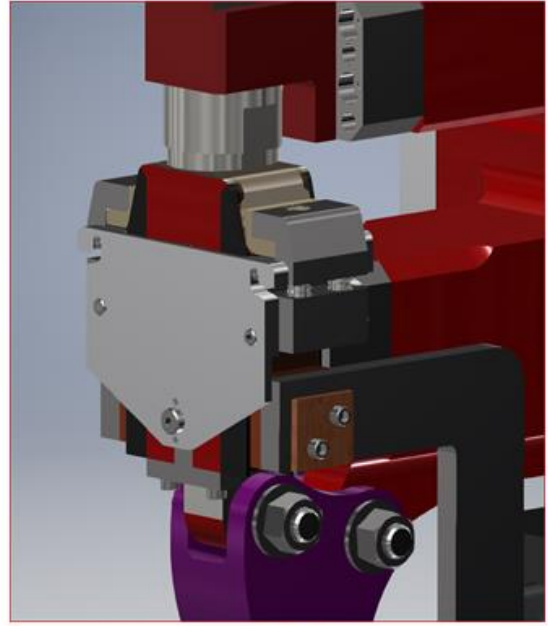

(c)

Figure II.3.4.13. (a) Weld head mounted on a 6-axis robot. (b) VFAW head in configuration with lower initial gap between anvil and breech for welding on the outside flange of the prototype component. (c) Close-up view of the weld head shown in closed position with the foil in place. Source: Coldwater Machine. 


\section{Development of Automated Work Cell}

An automated work cell is being constructed to test the robustness and stability of the process. The cell is being designed for a lights-out, closed-door level of automation, which will include coupon dispensing, adhesive dispensing, part conveyance, welding, and cleaning of the weld area. An image of this system is shown in Figure II.3.4.14. All the automation equipment has been installed on an existing pedestal welder system, tested, aligned, and is fully functional. Some work still needs to be done on the foil feeder and the communication with the capacitor bank in order to get the equipment fully operational. The system is designed around a mix of pneumatic and electric equipment to achieve high repeatability at a low-cost. This cell will build confidence in the performance of the technology without human input as well as allow for process inefficiencies to be identified.

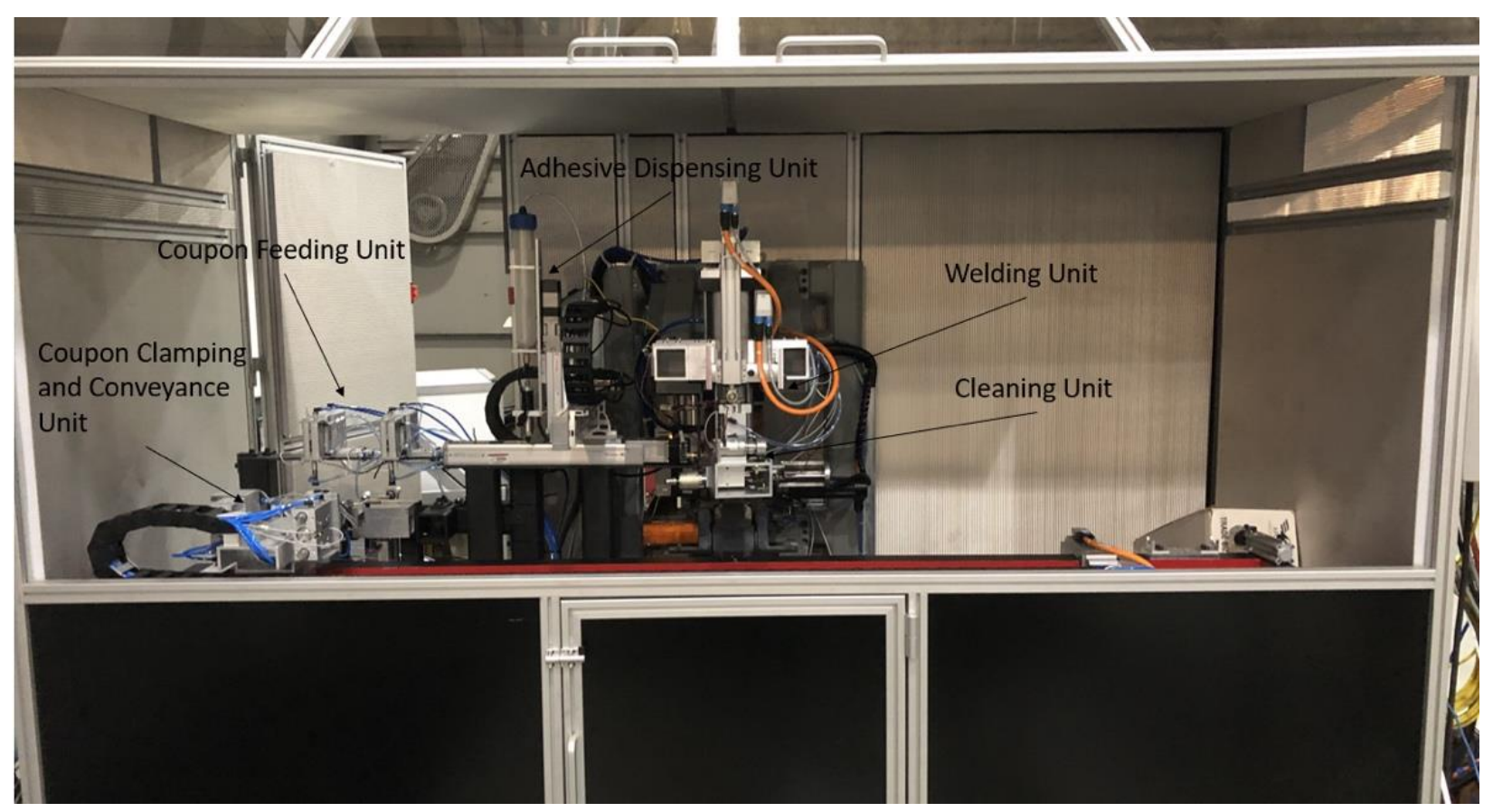

Figure II.3.4.14. Fully automated work cell with all equipment installed. Source: OSU.

\section{Conclusions}

VFAW has been used for welding HSLA 340 steel and 5000-series Al in various stack-up thicknesses appearing in the prototype component design. Both in static and cyclic testing, the welds performed better than the requirement. With the development of new fixtures, repeatability of weld strength was improved. The dissimilar welds in the prototype component will be made with a robotic VFAW welding head, which is in production. Process automation, including foil, part, and adhesive feed, is also nearing completion.

\section{Key Publications}

1. Sridharan, N., J. Poplawsky, A. Vivek, A. Bhattacharya, W. Guo, H. Meyer, Y. Mao, T. Lee, G. Daehn, 2019, "Cascading microstructures in Al-steel interfaces created by impact welding." Mater. Charact., Vol. 151, pp. 119-128.

2. Gupta, V., T. Lee, A. Vivek, K. S. Choi, Y. Mao, and X. Sun, 2019, “A robust process-structure model for predicting the joint interface structure in impact welding," J. Mater. Process. Tech., Vol. 264, pp. 107-118. 
3. Mao, Y., V. Gupta, B. Ufferman, A. Vivek, K. S. Choi, X. Sun, and G. S. Daehn, 2018, "On process, structure, property relationships in impact welding of Al 6061 and Steel 4130," Eighth International Conference on High-Speed Forming, May 14-15, 2018, Columbus, OH, USA.

4. Gupta, V., X. Sun, K. Choi, A. Vivek, Y. Mao, and G. Daehn. 2017, “A computational modeling approach to predict the interfacial characteristics obtained in high velocity impact welding of metals," Materials Science \& Technology 2017, October 8-12, 2017, Pittsburgh, PA, USA.

5. Gupta, V., K. Choi, A. Vivek, Y. Mao, X. Sun, and G. Daehn, 2018, "Prediction of joint properties obtained in the high velocity impact welding of dissimilar metals," TMS 2018 Annual Meeting \& Exhibition, March 11-15, 2018, Phoenix, AZ, USA.

\section{References}

1. Vivek, A., S. R. Hansen, B. C. Liu, and G. S. Daehn, 2013, "Vaporizing foil actuator: A tool for collision welding," J. Mater. Process. Tech., Vol. 213, No. 12, pp. 2304-2311.

2. DOE Vehicle Technologies Office, 2018, "Materials 2017 Annual Progress Report," DOE-EERE VTO Report No. DOE/EE-1711, May 2018.

3. DOE Vehicle Technologies Office, 2019, "Materials 2018 Annual Progress Report," DOE-EERE VTO Report No. DOE/EE-1827, April 2019.

4. Gupta, V., T. Lee, A. Vivek, K. S. Choi, Y. Mao, and X. Sun, 2019, "A robust process-structure model for predicting the joint interface structure in impact welding," J. Mater. Process. Tech., Vol. 264, pp. 107-118. 


\section{II.3.5 Corrosion Control in Carbon Fiber Reinforced Polymer Composite-Aluminum Closure Panel Hem Joints (PPG Industries, Inc.)}

\section{Brian Okerberg, Principal Investigator}

PPG Industries, Inc.

Coatings Innovation Center

4325 Rosanna Dr.

Alison Park, PA 15101

E-mail: okerberg@ppg.com

\section{Sarah Kleinbaum, DOE Technology Development Manager}

U.S. Department of Energy

E-mail: sarah.kleinbaum@ee.doe.gov

Start Date: October 1, 2016

Project Funding (FY19): \$685,578
End Date: March 31, 2020

DOE share: $\$ 476,353$
Non-DOE share: $\$ 209,225$

\section{Project Introduction}

This project seeks to develop new coatings, adhesives, and new joint geometries, and establish and validate corrosion characterization techniques that enable CFRP/Al hem flange joints. The CFRP/Al closure panels will save over $40 \mathrm{~kg}$ per vehicle compared to a vehicle with all $\mathrm{Al}$ closure panels. The CFRP/Al hem will meet current specifications for mechanical performance, corrosion resistance, and appearance when cured between $150-180^{\circ} \mathrm{C}$. Critical success factors to achieve this goal include the development of novel coatings, adhesives, and/or joining geometries to fully isolate the inner panel from the outer panel in the joint while maintaining the ability to electrostatically paint the inner panel in the paint line. These materials must cure at a lower temperature and be designed to mitigate the CTE mismatch while enabling processing through a standard paint shop. Extensive electrochemical and stress corrosion cracking (SCC) testing will be conducted to evaluate the corrosion performance of the new solutions and determine the appropriate accelerated test methods. Outdoor on-vehicle exposure testing will be conducted to verify the proper laboratory accelerated corrosion test method for CFRP/Al hem joints.

\section{Objectives}

The objective of this project is to develop new coatings, adhesives, and new joint geometries, and establish and validate corrosion characterization techniques that enable Ford Motor Company to implement lightweight closure panels based on a CFRP inner/Al outer joined by a hem flange.

\section{Approach}

The project has been divided into three project/BPs. The focus of the first budget period (BP1) was to understand the nature and extent of the corrosion problem resulting from the CFRP/Al joint. Testing to evaluate the susceptibility to galvanic corrosion and SCC was begun. An investigation began into the level of conductivity needed to promote electrostatic painting, but not galvanic coupling. Development of adhesives and coatings to improve the required cure and corrosion-preventive characteristics in the required temperature range was initiated.

During BP2, additional formulation of prototype conductive primers, adhesives, and E-coats was done. The decision was made to use a conventional hem flange for the upcoming demonstration of the prototype technologies developed under the program. Work continued to study galvanic corrosion and SCC, and to develop an accelerated corrosion test procedure. 
During this report period (comprising the remainder of BP2 and continuing into BP3), prototype conductive primers, adhesives, and E-coat formulations were finalized. Five liftgate assemblies incorporating CRFP/Al hem joints were produced using the prototype materials from PPG. Galvanic corrosion, SCC, and accelerated corrosion test procedure development work was continued.

Finally, during the remainder of BP3, the five demonstration liftgate assemblies will be tested by Ford for structural integrity and corrosion performance and compared to laboratory test results and the accelerated corrosion test results from OSU.

\section{Results}

\section{Subtasks 1.1.2 and 2.3: Galvanic Corrosion Susceptibility Assessment (OSU)}

Initial electrochemical corrosion analysis of primed and unprimed CFRP was completed in May 2018 on newly primed surfaces by carrying out potentiodynamic polarization in $5 \mathrm{wt} . \% \mathrm{NaCl}$ solution in open-air conditions. The testing was done in $5 \mathrm{wt} . \% \mathrm{NaCl}$ to match the same concentration used in the B117accelerated chamber testing. For the studies in FY 2019, the standard $3.5 \mathrm{wt} . \% \mathrm{NaCl}$ solution was selected for the electrochemical experiments to match the salt concentration of Ford's standard CETP 00.00-L-467 cyclic testing, which have been shown to correlate very well with real-world performance on vehicles. The polarization curves in $3.5 \mathrm{wt}$ \% $\mathrm{NaCl}$ were repeated for primed CFRP after approximately one year in storage in the laboratory environments where significant differences in electrochemical activity were observed. Figure II.3.5.1 shows the polarization curves of unprimed and 100, 50, and 0 primed (i.e., high, medium, and no/low conductivity primers) on CFRP-Random and Twill, as well as uncoated AA6022 and AA6111 in $5 \mathrm{wt} \% \mathrm{NaCl}$ solution from 2018. Oxygen reduction and hydrogen reduction regions can be clearly distinguished in the 100-primed CFRP. Polarization was carried out on 0 conductive primed CFRP, but the conductivity was so low that no current was recorded and the data are not presented.

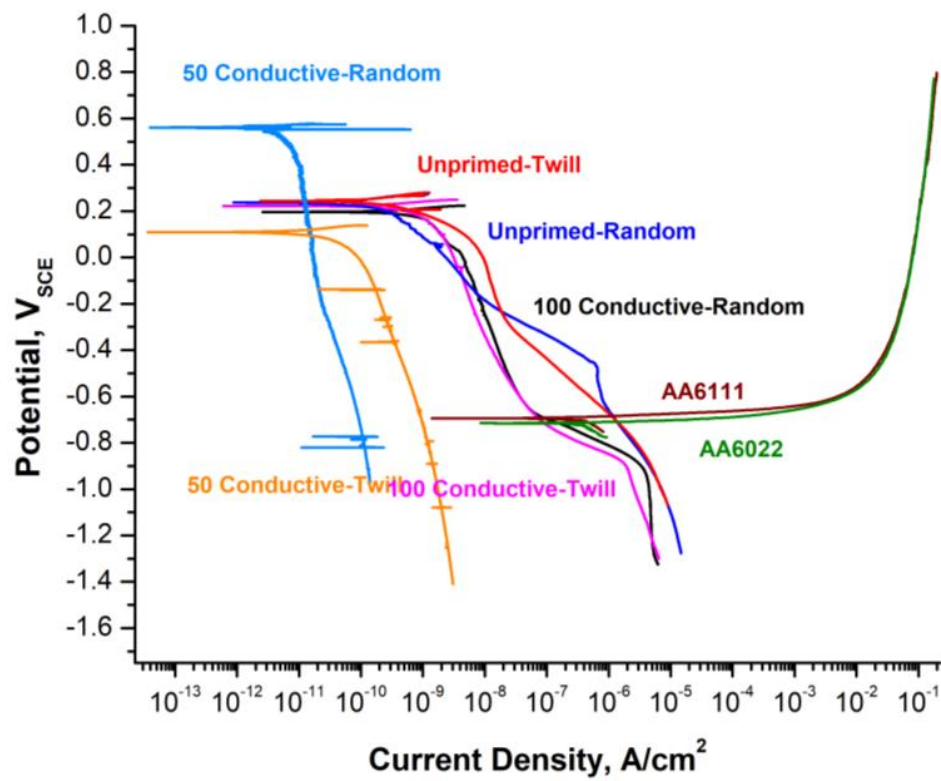

Figure II.3.5.1. Potentiodynamic polarization of primed (100, 50), unprimed CFRP (Twill, Random), and AA (6111, 6022) in 5 wt.\% aerated $\mathrm{NaCl}$ at $27^{\circ} \mathrm{C}$ (May 2018 results for comparison). Source: The Ohio State University.

Based on these initial results, it was then suggested that galvanic corrosion between CFRP and AA could be controlled by using 50 conductive primed CFRP as it showed low cathodic kinetics, which minimizes the interaction. Furthermore, it has enough conductivity to enable subsequent E-coating. Figure II.3.5.2 shows the potentiodynamic polarization plots in $3.5 \mathrm{wt} . \% \mathrm{NaCl}$ for primed CFRP-Twill and primed CFRP-random, 
respectively, after storage for one year. The 0 conductive primed sample exhibited cathodic kinetics close to those of unprimed CFRP. Based on these findings, the predicted galvanic current, $i_{\text {galv }}$, between AA and unprimed 100, 50, 0-conductive primed CFRP-Twill, and random are almost equal. These results indicate that the protective nature of 0,50 , and 100 conductive primed CFRP degraded over time to the extent that their electrochemical behavior was like that of unprimed CFRP. Possible reasons for changes in electrochemical behavior of primed CFRP with time are: (a) moisture absorption by the primer on CFRP enhanced the electrochemical activity; (b) segregation of carbon black in the primer matrix led to non-uniform dispersion and increased electrochemical activity; and (c) changes in CFRP-substrate and primer interaction such as disbondment. Each of these hypotheses will be investigated in future work.

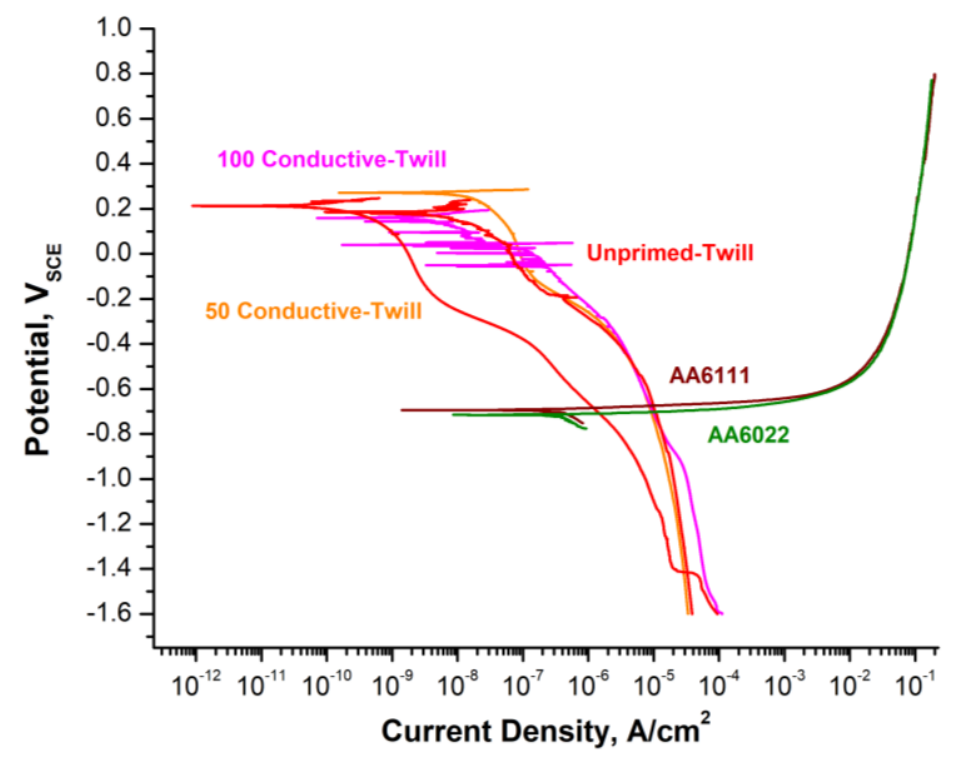

(a)

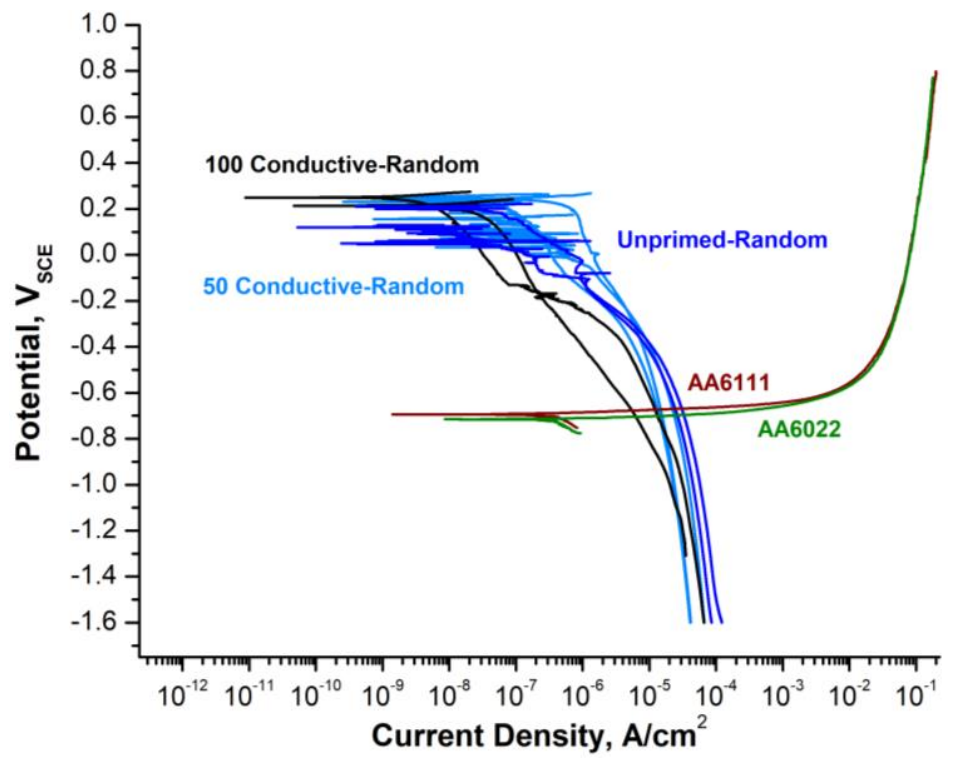

(b)

Figure II.3.5.2. Potentiodynamic polarization of (a) unprimed, primed $(0,50,100)$ CFRP-Twill and (b) CFRP- Random and AA $(6111,6022)$ in 3.5 wt.\% aerated $\mathrm{NaCl}$ at $27^{\circ} \mathrm{C}$. Source: The Ohio State University. 
Uncoated and E-coated AA6111 and AA6022 coupled with CFRP-Twill and CFRP-random coupons were tested for corrosion in the Ford-accelerated L-467 chamber. Galvanic current is being measured on each coupon during a 24-hour cycle, once every 2 weeks. The coupons were exposed for 10 weeks. Optical profilometry will be performed to determine the material loss and area under blisters. Galvanic current measurements, volume loss measurements, and analysis along with pictures of corrosion attack will be obtained. Alternate immersion testing to determine SCC behavior of smooth AA6111-T8-like specimens with no pre-crack began in FY 2019. These tests will assist in determining the role of SCC initiation versus crack propagation.

\section{Subtask 1.1.3: Effect of Galvanic Coupling on SCC-Susceptibility (OSU)}

AA6111-T8-like extended compact ESE(T) coupons were tested under full immersion in $3.5 \mathrm{wt} \% \mathrm{NaCl}$ at a crosshead displacement rate of $1.05 \times 10^{-7} \mathrm{in} / \mathrm{sec}$, and the threshold stress intensity $\left(\mathrm{K}_{\mathrm{TH}}\right)$ was determined using the $95 \%$ secant method as per ASTM E1820 and E561/B646. The following polarization conditions were tested and compared with air $\mathrm{K}_{\mathrm{TH}}$ values: $-756 \mathrm{mV}_{\mathrm{SCE}}$ (iso-potential baseline) and $-656 \mathrm{mV}_{\mathrm{SCE}}(+100 \mathrm{mV}$ anodically polarized with respect to the iso-potential baseline).

To confirm strain-rate is not an influencing factor in the results. Testing was conducted at a second and slower displacement rate of $4.50 \times 10^{-8} \mathrm{in} / \mathrm{sec}$. To compare full immersion tests to cracking in an environment where no SCC will occur, testing was also conducted in air at the same displacement rate. This $\mathrm{K}_{\mathrm{TH}}$ is equivalent to the upper limit of SCC behavior, or fracture toughness, and was determined to be $19.3-21.5 \mathrm{MPa} \sqrt{\mathrm{m}}$ as per both ASTM analysis methods. Full immersion testing was performed at the following polarization conditions and compared with air $\mathrm{K}_{\mathrm{TH}}$ values: $-756 \mathrm{mV}_{\mathrm{SCE}}$ (iso-potential baseline), $-706 \mathrm{mV}_{\mathrm{SCE}}(+50 \mathrm{mV}$ anodically polarized with respect to the iso-potential baseline), $-656 \mathrm{mV}_{\mathrm{SCE}}(+100 \mathrm{mV}$ anodically polarized). Results can be found in Figure II.3.5.3 as a plot of effective stress intensity as a function of effective crack extension, and in Figure II.3.5.4 (b) as a plot of $\mathrm{K}_{\mathrm{TH}}$ as a function of applied polarization potential. The measured values of $\mathrm{K}_{\mathrm{TH}}$ for $-756 \mathrm{mV}_{\mathrm{SCE}}$ and $-706 \mathrm{mV}_{\mathrm{SCE}}$ are close to the air $\mathrm{K}_{\mathrm{TH}}$ values, indicating only mild SCC is occurring at these levels of polarization; however, since only one air test was conducted, normal scatter in experimental data may compensate for the small difference in $\mathrm{K}_{\mathrm{TH}}$. Anodic polarization greatly decreases SCC resistance, causing $\mathrm{K}_{\mathrm{TH}}$ to fall to lower than an experimentally measurable value at $-656 \mathrm{mV}_{\mathrm{SCE}}$.

Comparing results at displacement rates of $4.50 \times 10^{-8} \mathrm{in} / \mathrm{sec}$ and $1.05 \times 10^{-7} \mathrm{in} / \mathrm{sec}$ for ASTM E1820 and ASTM E561, as shown in Figure II.3.5.4 (a), show that the strain rates utilized are sufficiently slow for valid SCC testing. Convergence of values indicates testing is being conducted at a sufficiently slow rate. Conducting SCC testing at overly rapid displacement rates can cause falsely high $\mathrm{K}_{\mathrm{TH}}$ values to be measured. For both displacement rates at $-756 \mathrm{mV}_{\mathrm{SCE}}$, measured $\mathrm{K}_{\mathrm{TH}}$ was $16-18 \mathrm{MPa} \sqrt{\mathrm{m}}$; and at $-656 \mathrm{mV}_{\mathrm{SCE}}, \mathrm{K}_{\mathrm{TH}}$ was less than 8.3-6.0 MPa $\sqrt{\mathrm{m}}$. The converging values can be found in Figure II.3.5.4 (a). The dashed lines represent the inair $\mathrm{K}_{\mathrm{TH}}$ determined by both methods for one test. The decrease in $\mathrm{K}_{\mathrm{TH}}$ indicates an increase in SCCsusceptibility with anodic polarization. Figure II.3.5.4 (b) provides additional data for the two test methods using the same two test methods at a single displacement rate. 


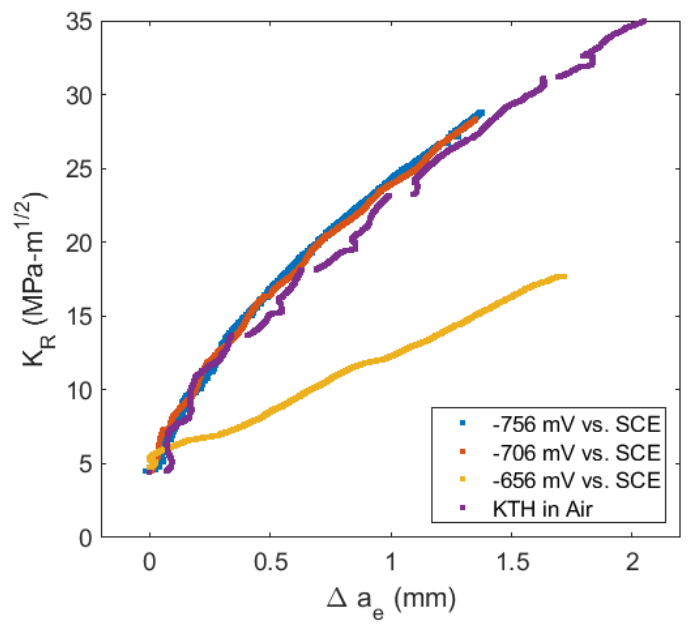

Figure II.3.5.3. Effective stress intensity as a function of effective crack extension for slow strain-rate testing at crosshead displacement rate of $4.50 \times 10-8 \mathrm{in} / \mathrm{sec}$. A PS plastic zone size correction was applied to calculate effective values, and full immersion SCC tests are plotted with an in-air test for comparison to "no-environment" cracking behavior.

Source: The Ohio State University.

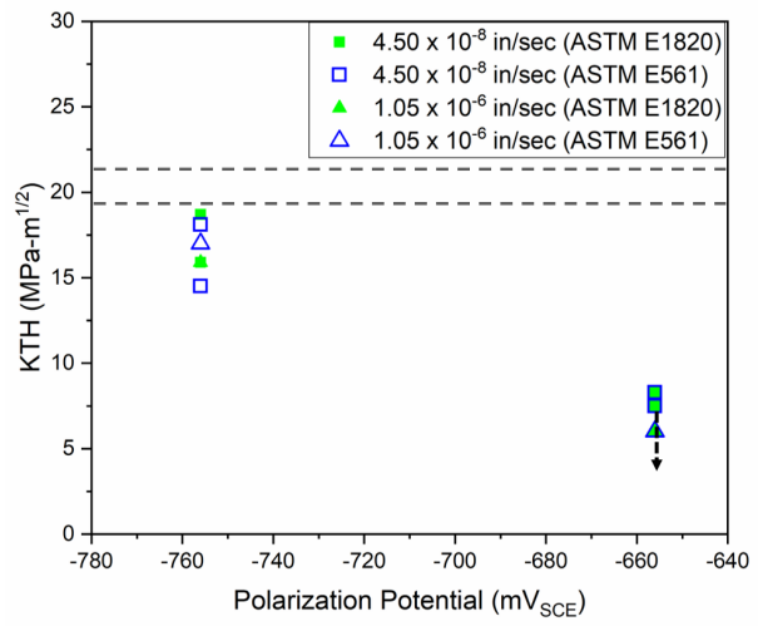

(a)

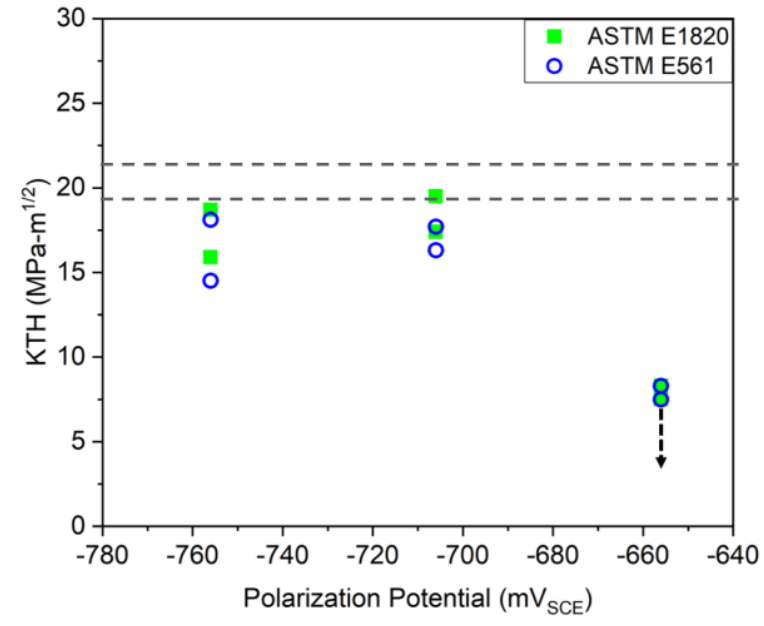

(b)

Figure II.3.5.4. Plot of threshold stress intensity $\left(\mathrm{K}_{\mathrm{TH}}\right)$ as a function of applied polarization potential for rising displacement SCC testing of AA6111-T8 in 3.5 wt.\% NaCl using ASTM E1820 and ASTM \#561. (a) KTH results at displacement rates of $4.50 \times 10^{-8} \mathrm{in} / \mathrm{sec}$ and $1.05 \times 10^{-6} \mathrm{in} / \mathrm{sec}$ as a function of polarization potential. (b) $\mathrm{K}_{T H}$ results at displacement rate of $4.50 \times 10^{-8} \mathrm{in} / \mathrm{sec}$ as a function of polarization potential. Source: The Ohio State University.

\section{Subtasks 1.1.4 and 2.4: Accelerated Corrosion Test Procedure Analysis/Development (OSU)}

On-bus on-road exposure testing of uncoated and E-coated AA6111, AA6022 coupled with CFRP-Twill, and random coupons began during the second week of December 2018. Pictures of the samples have been taken once every three months. The combination of AA6111 (uncoated and E-coated) coupled with random CFRP exhibited the maximum extent of corrosion in the form surface attack (uncoated) and blistering (E-coated), whereas AA6022 (uncoated and E-coated) coupled with Twill exhibited the maximum corrosion resistance. Significant differences can be seen in E-coated AA6111-Random and E-coated AA6022-Twill in the form of blistering. However, in uncoated coupons, major corrosion damage might be confined within the overlap region, resulting in little difference upon visual inspection. Hence, it has been proven again that the combination of AA6111-random and AA6022-Twill exhibit the highest and the lowest extent of corrosion attack, respectively. AA6022 contains 0.2 wt.\% $\mathrm{Cu}$, whereas AA6111 contains $0.5-0.8$ wt. \% Cu. The higher 
content of $\mathrm{Cu}$ in the AA enhances the overall corrosion activity. It is possible that there is stronger galvanic coupling between the CFRP and the $\mathrm{Cu}$-depleted zone around $\mathrm{Cu}$ containing intermetallics, leading to more preferential dissolution of the $\mathrm{Cu}$-depleted zone (Al matrix) in AA6111. However, this needs further investigation to establish microstructural evidence for more corrosion in AA6111 containing higher $\mathrm{Cu}$ content than AA6022 and the role of $\mathrm{Cu}$ during anodic polarization of AA in an AA-CFRP galvanic couple.

\section{Subtask 2.1.1: Development of Low temperature Cure E-coat (PPG)}

The two previously identified E-coat formulations meeting the key properties of cure response, appearance, and corrosion resistance were further examined for throwpower and corrosion behavior on various metal substrates. The throwpower was determined by a Nagoya box test, which measures the ratio of the interior panel (the $\mathrm{G}$ face) to exterior panel (the A face) coating film thickness. Compared to the control value of 55\%, the P11 variable had a slightly lower G/A value of 51\% and the P1B variable had a higher (improved) value of $69 \%$, as observed in Table II.3.5.1. The results were highly reproducible and plasticizer level was identified as the main formulation component influencing throwpower performance.

Table II.3.5.1. Formulation Details, Key Properties, and Throwpower for the Selected Prototype and Control Electrocoats.

\begin{tabular}{|c|c|c|c|c|c|c|c|}
\hline \multirow{4}{*}{ ID } & \multicolumn{4}{|c|}{ Formulation Variables } & Cure & Appearance & Throwpower \\
\hline & Resin & XL & Additive & Catalyst & $\begin{array}{c}150^{\circ} \mathbf{C} \text { for } \\
10 \text { min }\end{array}$ & Wb $(\mu \mathrm{m})$ & G/A $(\%)$ \\
\hline P1B & A & XL1 & C & E & Pass & 0.12 & 69 \\
\hline P11 & B & XL1 & G & F & Pass & 0.13 & 51 \\
\hline Control & C & XL3 & C & E & N/A & 0.11 & 55 \\
\hline
\end{tabular}

The two selected prototype low cure E-coats were further evaluated for their corrosion resistance over various metal substrates, including electrogalvanized steel, CRS, and two bake conditions (i.e., $150^{\circ} \mathrm{C}$ for 13 min and $175^{\circ} \mathrm{C}$ for $25 \mathrm{~min}$ ). The samples were submitted for six weeks of L-467 testing, including a standard bake $\left(175^{\circ} \mathrm{C}\right.$ for $\left.25 \mathrm{~min}\right)$ control. The results clearly indicated that the corrosion resistance of the two selected prototype coatings were comparable with those of the control.

The key properties of two low cure prototype E-coats and those for the control are summarized in Table II.3.5.1. The cure at the target bake condition of $150^{\circ} \mathrm{C}$ for $10 \mathrm{~min}$ (metal), appearance, and corrosion resistance were comparable with those of the control, satisfying the key target properties for the project. In addition, the prototype E-coats showed comparable or better corrosion resistance for various metal substrates in L-467 corrosion testing for six weeks. The P1B formulation further showed improved throwpower relative to the other prototype E-coat and the control. In addition, the P1B formulation was determined to exceed the robustness requirements for the scale-up. Thus, the P1B formulation was selected as the candidate for BP3 technical activities and was successfully scaled-up June 2019.

\section{Subtask 2.1.2: Development of Low temperature Cure Adhesives (PPG)}

A low temperature adhesive for hem flanges was developed to meet project targets and specifications. Changing formulation components such as resin and filler types/chemistries led to a system having the required cure response, lap-shear strength, fracture toughness, and rheological behavior. The formulation parameters were used to control the glass transition temperature ( $\mathrm{Tg}$ ) and modulus; both of which are crucial in mitigating the effect of the CTE mismatch on the integrity of the bonded joints. The relationship of the storage modulus, as an indicator of $\mathrm{Tg}$, and $\tan \delta$ as a function of temperature for two developmental adhesives is shown in Figure II.3.5.5. 

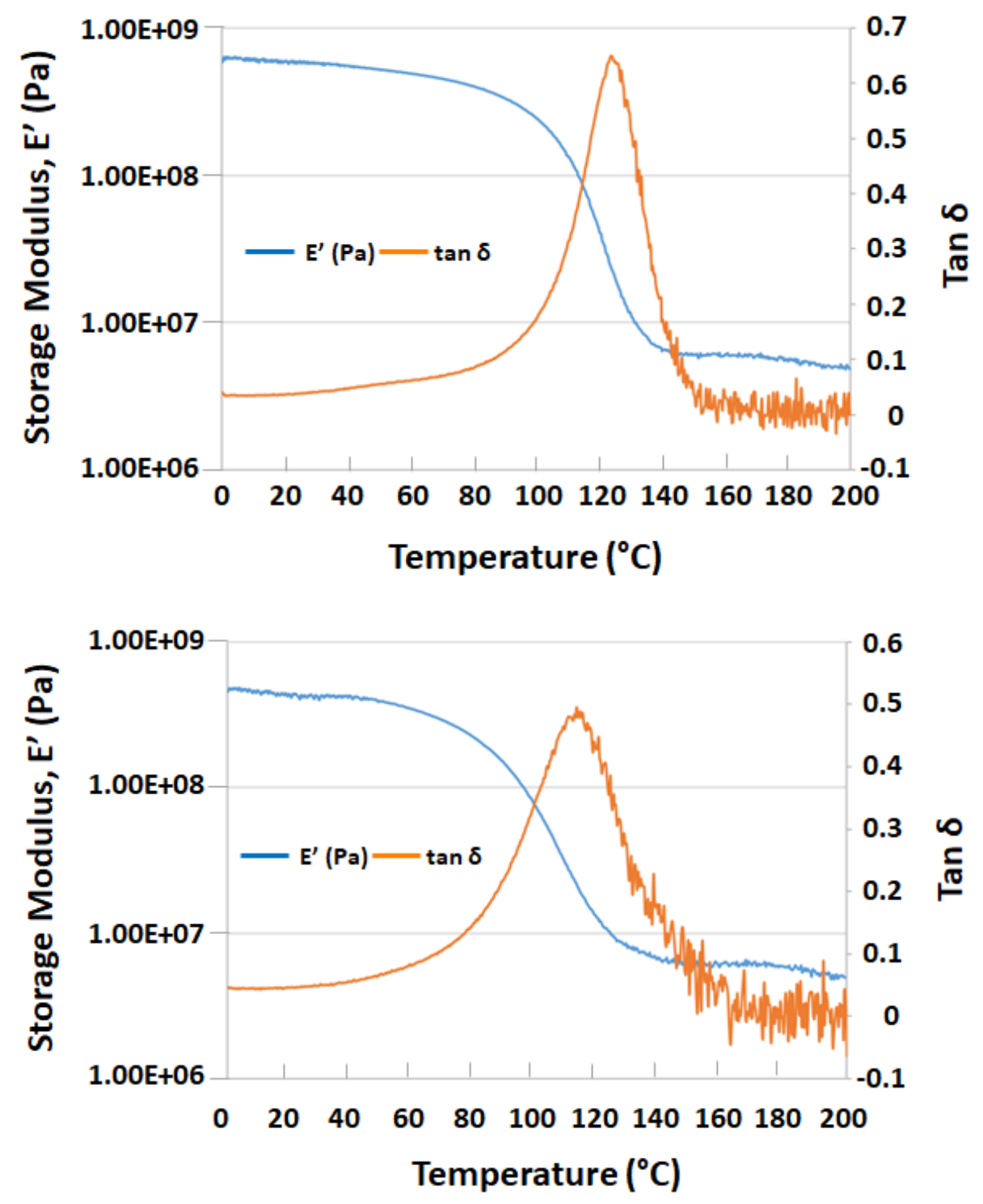

Figure II.3.5.5. Storage modulus (representative of $\mathrm{Tg}$ ) and tan $\delta$ as a function of temperature for two developmental adhesives. Source: PPG Industries, Inc.

After observing degradation of adhesion performance for the low cure adhesives in humid environments, additional reformulation worked focusing on modification of the catalyst was completed and an additional soak test was added to the evaluation protocol to understand the impact of moisture on performance. The reformulated adhesive candidates and a control high-bake formulation were tested in lap-shear and t-peel configuration before and a 2-day soak in a $50^{\circ} \mathrm{C}, 5 \%$ salt solution. The results for these tests are shown in Figure II.3.5.6. 


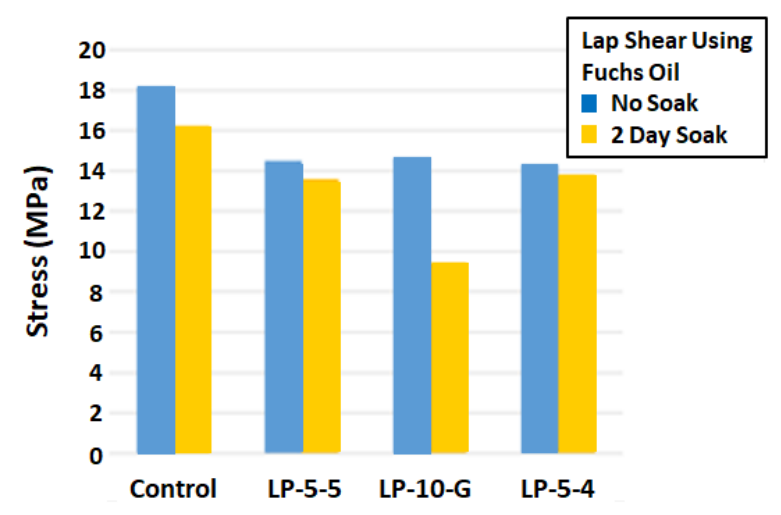

(a)

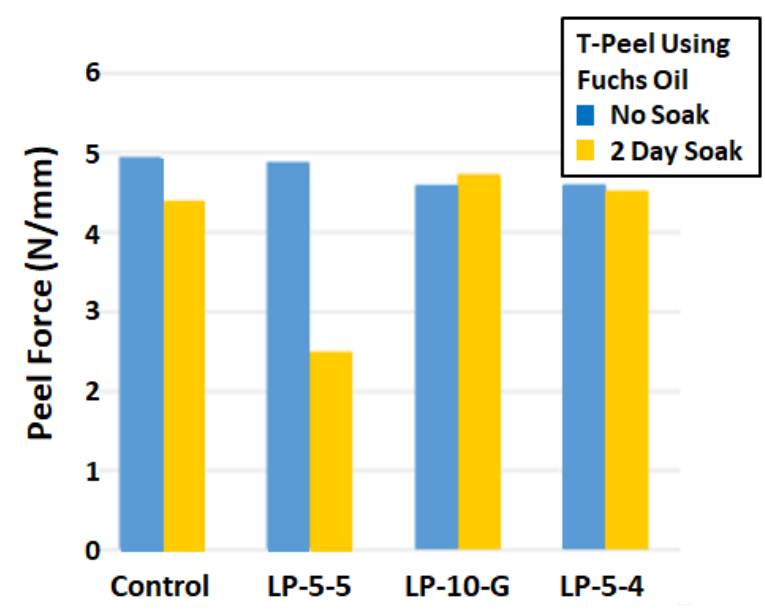

(b)

Figure II.3.5.6. (a) Lap-shear (b) and t-peel results comparing the control formula baked at $175^{\circ} \mathrm{C}$ with a few prototype formulations baked at $150^{\circ} \mathrm{C}$ and showing progress in optimizing anticorrosion properties for low cure adhesive systems. Source: PPG Industries, Inc.

The developmental formula, LP-5-4, maintained both lap-shear and T-peel strengths better after the solution soak test, while maintaining perfect cohesive failure. Long CFRP/Al beams were then prepared to assess the residual deformation and adhesive robustness of the new prototype. The curvature of the specimens after simulated E-coat bakes are shown in Figure II.3.5.7. The prototype of the low temperature cure adhesive specimen displayed less deformation than the control high-bake adhesive specimen as detailed in Figure II.3.5.7. 


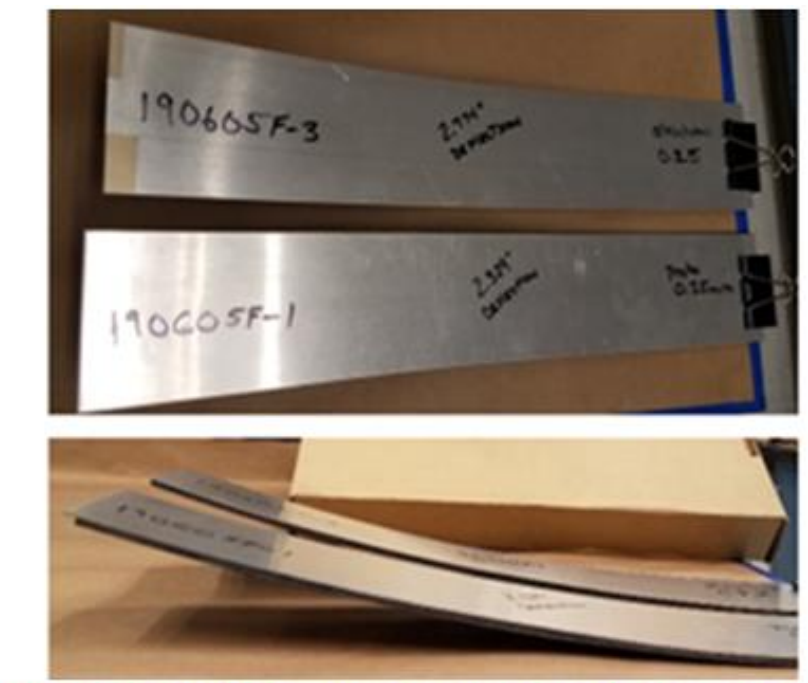

Note: Photos are for illustration only! Ends are loosely clamped.

End Deflection Measured (inches)

with a $0.25 \mathrm{~mm}$ Bond Gap:

Structural Adhesive: 2.774

Prototype Adhesive: 2.329

Figure II.3.5.7. Long-beam CFRP-Al adhesive specimens made from high (top sample) and low temperature (bottom sample) bake adhesives with a comparison of the deflection for structural vs. prototype adhesives.

Source: Ford Motor Company.

Teardown of the newly formulated low temperature bake adhesive showed excellent adhesion and toughness gave confidence that the newly formulated adhesive would work well for the prototype build.

The final adhesive formulation was scaled-up (differing slightly from the LP-54), loaded into cartridges, and used in the assembly of the prototype liftgates as described in Subtasks 3.1.2 and 3.1.3.

\section{Subtask 2.1.3: Reformulation of Conductive Primers (PPG)}

Two primers with high and low conductivity were selected based on conductivity level, appearance, ability to be sprayed, and adhesion to CFRP and $\mathrm{Al}$ which are target dissimilar joint components. The selected conductive primer formulations were successfully scaled-up in support of the BP3 liftgate demonstration.

\section{Task 2.3: Continued Galvanic Characterization and Assessment (Ford)}

The results obtained in BP1 clearly showed that the presence of CFRP near Al drives extensive galvanic corrosion of the Al. To more clearly quantify this behavior, a second set of coupon testing was conducted in which the joining methods were more representative of those that would be used in an automotive closure panel. These coupons were joined with adhesives in one of two configurations, as shown in Figure II.3.5.8. The two configurations were meant to simulate possible outcomes on the vehicle where water could flow through a crevice or be trapped in a crevice between the Al and CFRP panels. Coupons were constructed of the random SMC CFRP and AA6022. In addition, as corrosion mitigation strategies are at the core of the project, various material combinations were assessed. These included high and low conductivity primer for the CFRP, flexible and rigid adhesives, and high and low temperature bake E-coat. The full test matrix is shown in Table II.3.5.2. After assembly and coating, the coupons were tested in either L-467 accelerated corrosion testing, Ford's accelerated chamber test, or R-343 which is Ford's full vehicle corrosion test. In the latter case, the coupons were affixed to the lower body side of an F-150 truck that was subjected to 12 weeks of R-343 testing. 


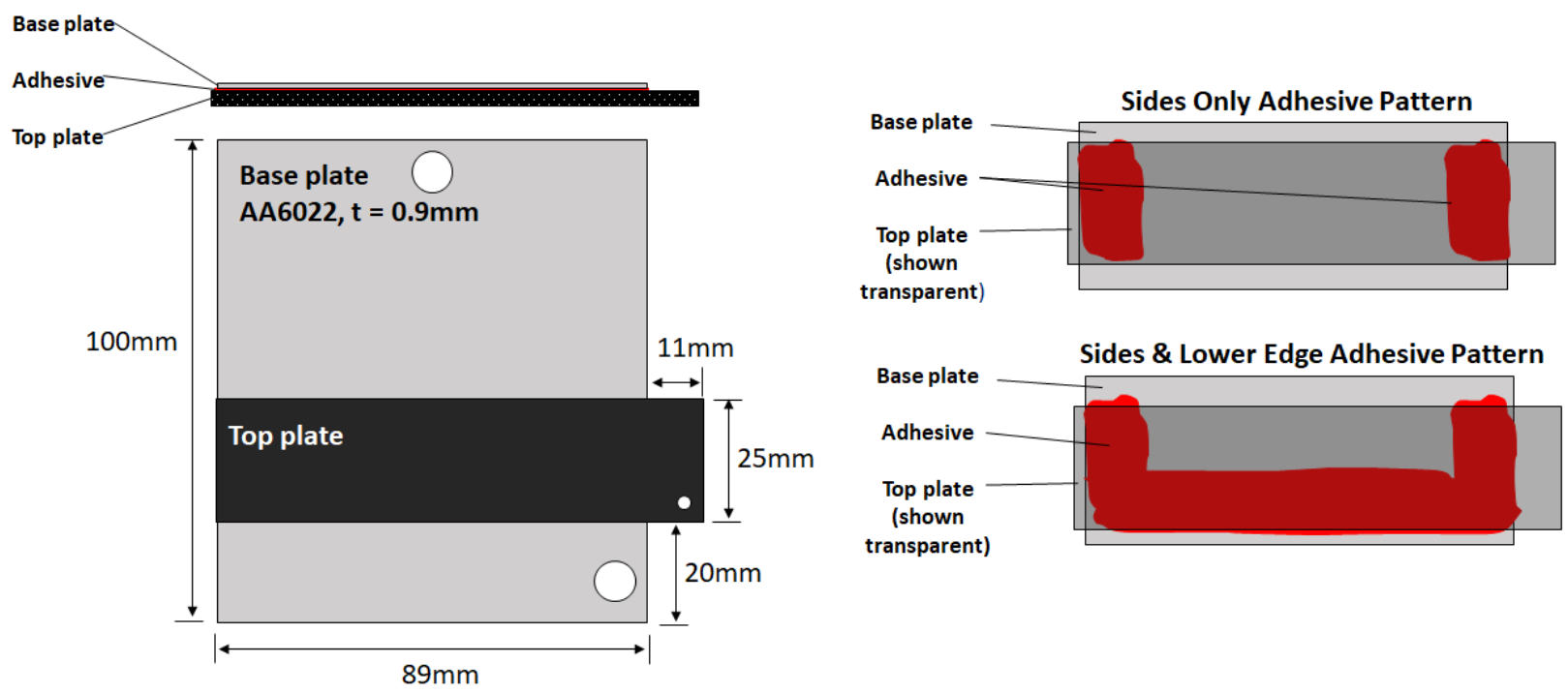

Figure II.3.5.8. Accelerated corrosion coupon geometry and adhesive placement conditions. Source: Ford Motor Company.

Table II.3.5.2. Full Test Matrix for Coupons Used in Accelerated Corrosion Testing.

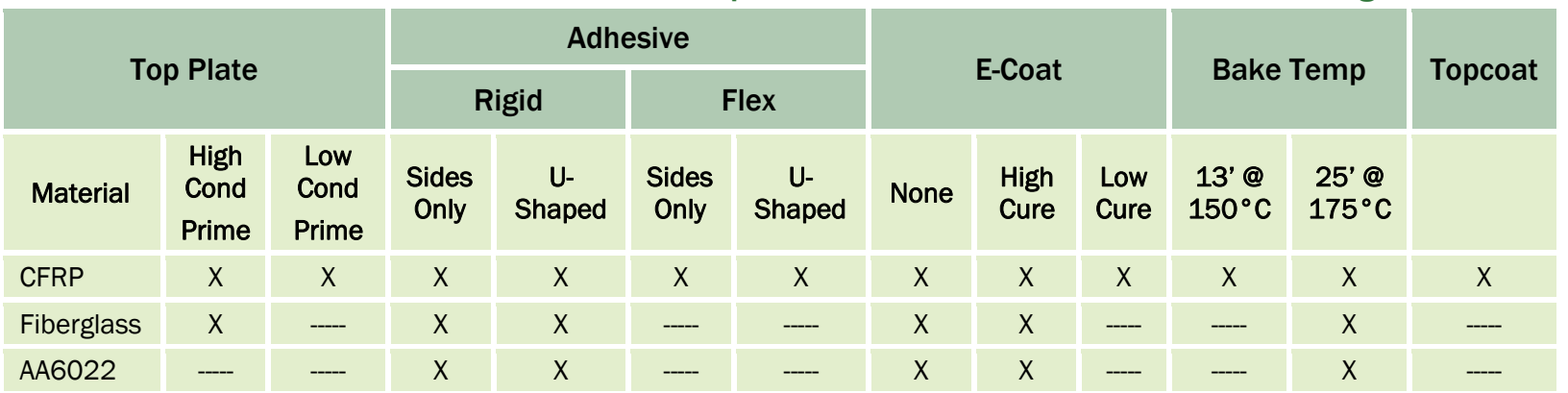

Unfortunately, the prototype flexible adhesive supplied by PPG displayed some anomalous moisture sensitivity, which led to premature failure of some of the coupons and an incomplete assessment of the variables tested. However, some conclusions could be drawn from the experiments. In general, the coupons exposed to R-343 (on-vehicle) testing showed more corrosion, as measured by undercutting and gauge loss. Those coupons with the U-shaped adhesive application showed more corrosion that the coupons with side-only adhesive. The different adhesive geometries were chosen specifically to stimulate corrosion and mimic manufacturing noise that can occur during vehicle assembly. A coupon with perfect perimeter adhesive application would likely have shown no corrosion.

During FY 2019, PPG reformulated their flexible, prototype adhesive and preliminary testing showed excellent compatibility with the Al and CFRP substrates, in Figure II.3.5.9 as compared to the control structural adhesive PPG provided (not showing in the figure). New coupons were manufactured with completion and analysis scheduled before the end of the project. 


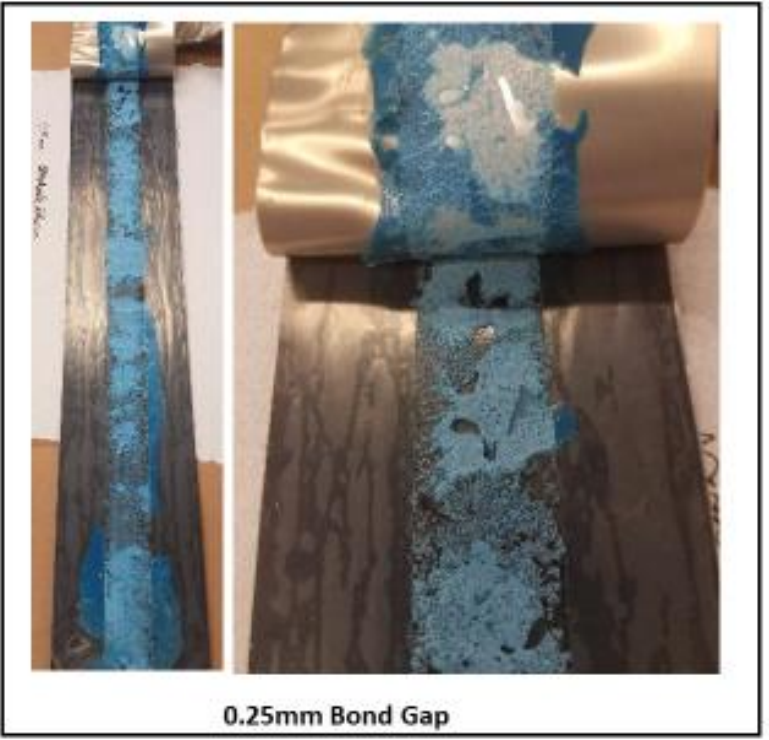

(a)

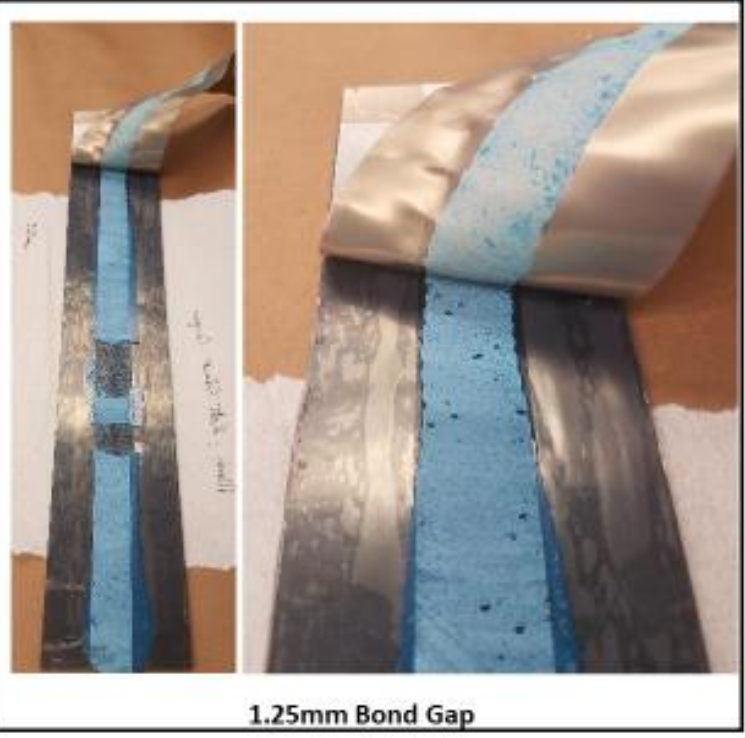

(b)

Figure II.3.5.9. (a) \& (b) Prototype PPG adhesive showing excellent adhesion and toughness between Al and CFRP at (a) a bond gap of $0.25 \mathrm{~mm}$ and (b) a bond gap of $1.25 \mathrm{~mm}$. Source: Ford Motor Company.

\section{Subtask 3.1.1: Preparing for the Prototype Build}

As a proof of corrosion mitigation strategies and manufacturing processes, prototype closure panels were manufactured to assess their dimensional stability and corrosion performance. The closure panel chosen was based on the Mondeo liftgate, currently produced by Ford in Europe. The inner panel was chosen to be manufactured from the SMC CFRP and the outer panel was the production Al component. The component was chosen because a tool had previously been made for the inner part, which could be re-utilized for this research project.

A test matrix was designed to demonstrate the effects of conductive primer conductivity, conductive primer placement (one side or two sides of CFRP panel), adhesive stiffness, and electrocoat bake temperature on the dimensional stability and corrosion performance of the component. The matrix of materials and process parameters are shown in Table II.3.5.3.

Table II.3.5.3. Test Matrix of Liftgates Manufactured to Prove Out Dimensional Stability and Corrosion Performance.

\begin{tabular}{|l|c|c|c|c|c|}
\hline Material & $\begin{array}{c}\text { Standard Component } \\
\text { (Control) }\end{array}$ & $\begin{array}{c}\text { Component } \\
\text { High }\end{array}$ & $\begin{array}{c}\text { Component } \\
\mathbf{2}\end{array}$ & $\begin{array}{c}\text { Component } \\
\mathbf{3}\end{array}$ & Component \\
\hline Conductivity of Primer & Howh & High & Low \\
\hline $\begin{array}{l}\text { Conductive Primer } \\
\text { Application }\end{array}$ & Outside only & Both sides & Outside only & Both sides & Both sides \\
\hline Adhesive Type & High T, & Low T, & Low T, & High T, & Low T, \\
\hline rigid & flexible & flexible & flexible \\
\hline E-Coat Type & High T Cure & Low T Cure & High T cure & High T cure & Low T cure \\
\hline
\end{tabular}




\section{Subtask 3.1.2: Prototype Manufacture}

The inner SMC CFRP parts were molded in January 2019 in Detroit. These parts were then transported to PPG's Flint facility where they were conductively primed with one of two different formulations (low or high conductivity). The parts were then shipped back to Ford in Dearborn, MI, where they were joined to the Al outer panels with one of two different adhesives (e.g., standard rigid or prototype flexible). The adhesive was manually dispensed onto the parts and the hem joint was created manually. The parts were then shipped to a PPG facility in Lima, OH, for pretreatment and electrocoat application. Of note, one extra part was made, and was used to test process compatibility at the Lima facility. During this "test" run, it was determined that the part was too buoyant to be properly submerged in the process baths. Extra holes were added to the subsequent parts to prevent this error state. All five parts were later processed at Lima without issue. After application of the appropriate electrocoat (e.g., high or low temperature bakes), the parts were shipped back to PPG's Flint facility where they were top-coated. After shipment back to Ford, the parts were scanned with a laser scanner to compare their dimensions to engineering CAD. The parts were then sent to Ford's proving ground in Romeo, MI, where they were affixed to a sled for R-343 corrosion testing.

\section{Subtask 3.1.3: Prototype Testing and Analysis}

Laser scanning of the prototype parts showed improved dimensional distortion when using the flexible adhesive and low temperature curing E-coat, as shown in Figure II.3.5.10. As seen in Table II.3.5.3, the part distortion was reduced from a maximum of $\sim 10 \mathrm{~mm}$ on the control liftgate (standard [rigid adhesive, high-bake E-coat]) to $\sim 5 \mathrm{~mm}$ on the liftgate with flexible adhesive and low temperature cure E-coat (Component 1), which is now in the range for manufacturing that can be addressed by design changes and/or additional adhesive formulation development. This demonstrates the value in moving to lower temperature processes and flexible adhesives.

Corrosion testing commenced on September 3, 2019 and will continue for 12 weeks. After 12 weeks of exposure, the parts will be disassembled to assess the extent of corrosion in the hem flange area, as well as manufacturing noise effects. 


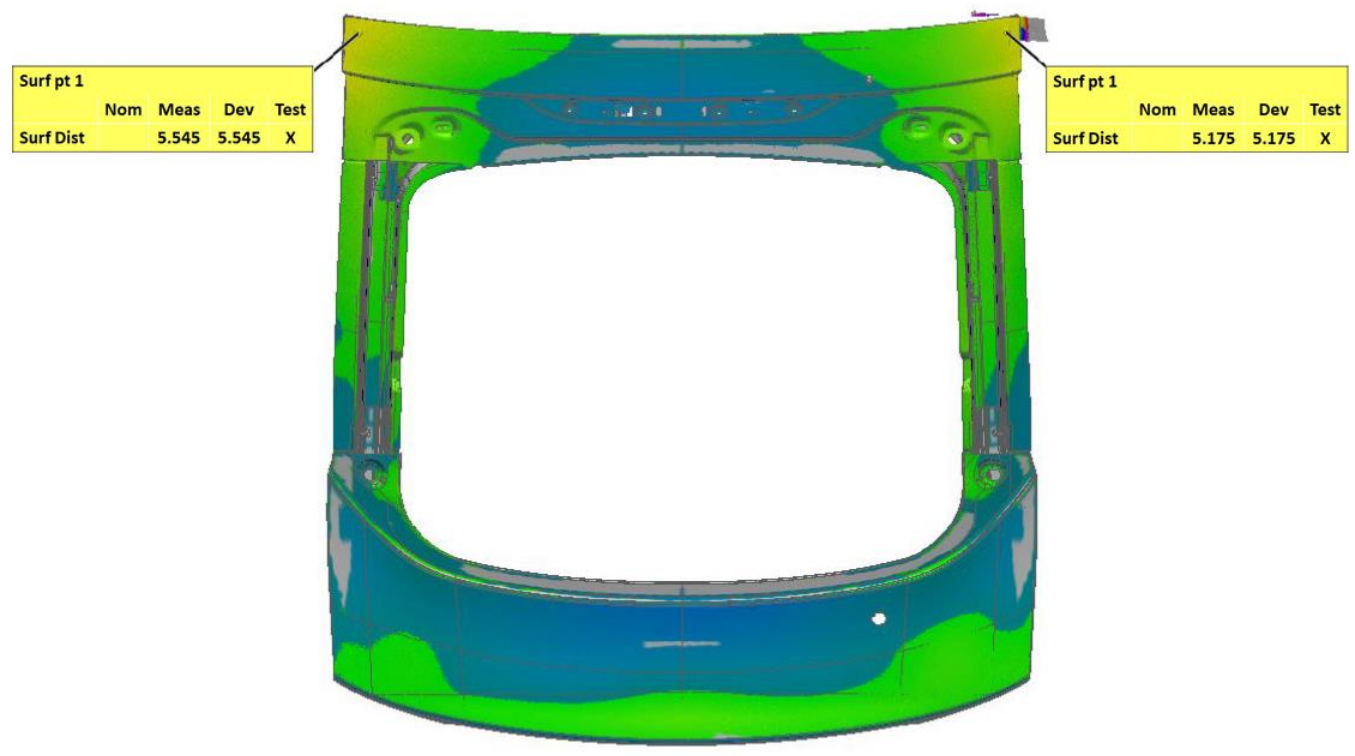

(a)

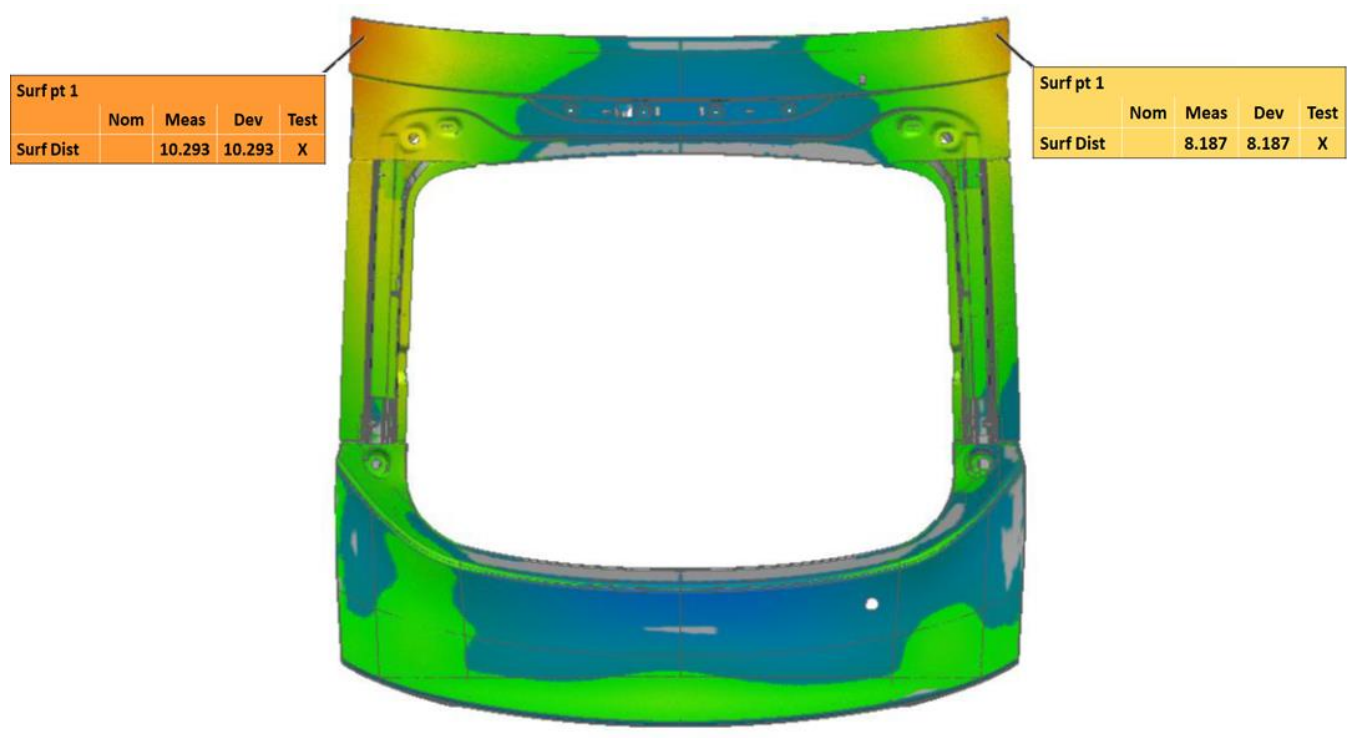

(b)

Figure II.3.5.10. Laser scanning output of liftgates (a) manufactured with a low-temperature cure E-coat and flexible adhesive, and (b) control process and materials. Maximum deflection is approximately $50 \%$ less with a low-temperature process. Source: Ford Motor Company.

\section{Conclusions}

The work performed on this project during FY 2019 further explored the corrosion and SCC issues associated with the CFRP/Al couple and resulted in the development of prototype electrocoat, adhesive, and conductive primer formulations that meet or exceed performance and application requirements while curing at the lower temperatures required by the presence of the CFRP material. Additional development took place on an electrochemical technique to further understand the conductive features of the CFRP surfaces and an accelerated test protocol that can accurately predict in-service corrosion performance. Five liftgate assemblies were produced for structural integrity and corrosion testing during BP3. 


\title{
II.3.6 Assembly of Dissimilar Aluminum Alloys for Automotive Applications (Pacific Northwest National Laboratory)
}

\author{
Piyush Upadhyay, Principal Investigator \\ Pacific Northwest National Laboratory \\ 902 Battelle Boulevard \\ Richland, WA 99352 \\ E-mail: piyush.upadhyay@pnnl.gov
}

\section{Sarah Kleinbaum, DOE Technology Development Manager}

\author{
U.S. Department of Energy \\ E-mail: sarah.kleinbaum@ee.doe.gov
}

Start Date: July 1, 2017

Project Funding: $\$ 1,000,000$
End Date: December 31, 2019

DOE share: $\$ 500,000$
Non-DOE share: $\$ 500,000$

\section{Project Introduction}

While the FSW process has been implemented in butt-joint configurations over the years, it is mostly lap joints that enable the assembly of the modular components in the automotive industry. Assembly techniques include hemming, clinching, fasteners, and adhesives; however, each has specific challenges when applied to join $\mathrm{Al}$ alloy components. High-speed friction stir lap welding (FSLW) has the potential to enable greater versatility and cost-effectiveness in the integration of $\mathrm{Al}$ sheet components. In FSLW, two or more sheets of different thicknesses, alloying elements, surface treatments, and/or strengths are stacked in the desired order with required overlap. A rotating FSW tool plunges into the material stack-up extending through the bottom of the top sheet and into the bottom sheet creating a continuous linear bond between the faying surfaces. Unlike the butt-joint, the lap interface is oriented perpendicularly to the tool rotation axis. Because of this, FSLW is more prone to inadequate mixing resulting in undesirable material upturn on either side of the nugget, often referred to as "hooks." These hooks are formed adjacent to the tool and are detrimental to joint performance. In addition, the FSLW bonded area is exclusively dependent on a smaller pin diameter rather than the much larger shoulder diameter, as is the case in butt-joining; thereby making material flow around the pin more critical for FSLW.

Lap geometry intrinsically has crack-like regions containing hook features that can form on both edges of the overlap. This feature can either deviate towards the top or bottom of the joint, reducing the effective sheet thickness and concurrently acting as a site for crack initiation and propagation, thus decreasing static and dynamic joint property. At higher welding speeds, defects can form either at the crown or at the root of the weld, resulting in premature joint fracture. Certain combinations of welding control parameters and tool geometries have been shown to perform better [1-3]; however, these parameters are highly specific to a chosen material stack-up. The reported strength values have large scatter and can range anywhere between $20 \%$ and $60 \%$ of the BM. In addition to the reported variation in weld strength, welding speed is another important issue that persists as a primary obstacle to commercialization. The welding speeds reported are relatively low (i.e., most of the welds are performed at around $0.3-0.6 \mathrm{~m} / \mathrm{min}$ while only a few show capabilities up to $1 \mathrm{~m} / \mathrm{min}$ ) and do not justify commercial investment nor a switch to FSLW technology unless a significant improvement in welding speed can be demonstrated. A comprehensive series of weld trials will be conducted on material combinations that are most relevant to industry using a wide variety of welding parameters and tool designs. These trials are designed to help build functional relationships between several identified critical factors, like effective sheet thickness, interface morphology, tool geometry, control parameters, and resulting lap-shear strength. Having developed these fundamental relationships, the industry will be able to more effectively move towards commercialization by understanding characteristics, such as the effect of material stack-up order 
(placement of the advancing or retreating side, minimum trim edge requirement per alloy type, minimum clamping requirements, etc.).

\section{Objectives}

The purpose of this project is to develop the joining technology needed to enable broader use of lightweight Al sheets in automotive assemblies. The project plan is to accomplish this by maturing FSLW technology towards high-volume applications. In collaboration with an automotive OEM and a material supplier, this project will develop FSLW technology in relevant alloys, thicknesses, and geometries. Specifically, the work will develop process techniques, tools, and control parameters needed to advance the FSLW method using experimental trials, testing, and validation at the laboratory-scale and transition this work for integration into a productionlike environment. The resulting body of work and knowledge-base will enable automotive original equipment manufacturers to effectively move towards commercialization of the joining technology for lightweight $\mathrm{Al}$ sheets.

\section{Approach}

The project is divided into three main task areas to ensure risk mitigation and successful completion. Task 1 focused on initial FSLW development and characterization. This task concluded with a performance milestone that enabled continued development and process improvements. Task 2 expanded upon the work to be accomplished in Task 1 by optimizing the tool design and demonstrating applicability across alloys, thicknesses, and material combinations. This task ultimately led to a decision gate for the use of the process in the prototypical demonstrations associated with Task 3 by providing the information necessary to justify capital investments and product design for technology commercialization.

Task 3 is divided into two subtasks that will prepare the process for commercialization and establish the process in a demonstration hat section for near prototype testing. The target is to evaluate the joining method for its viability in integrating stamped $\mathrm{Al}$ alloys within the existing body construction so that a functionspecific Al assembly can be tailored based on specific property needs. This is illustrated in Figure II.3.6.1, which shows a representative future sedan structure with an $\mathrm{Al}$ cabin on a steel platform. The front part may be made using two thicker structural 7055 sheets with thinner 6022 as an outer thin section. Similarly, the back of the cabin, which is stiffness critical, can be made using more ductile, lower strength Al like a 6111 or 5754 series alloy. The three-material set layup including alloy types and the thickness (e.g., $1.0 \mathrm{t}$ ) that is used in this work is also shown in Figure II.3.6.1.

Technology transfer will be made directly through the project industry participants (i.e., Honda R\&D Americas, Inc. [HRA] and Arconic). PNNL will support technology transfer of the developed FSLW technique in the selected dissimilar Al assembly for demonstration at PNNL and functional validation at HRA's facility in a specific production concept. This will include the integration of FSLW tooling, parameters, and fixtures integrated with HRA's anticipated product development equipment. 

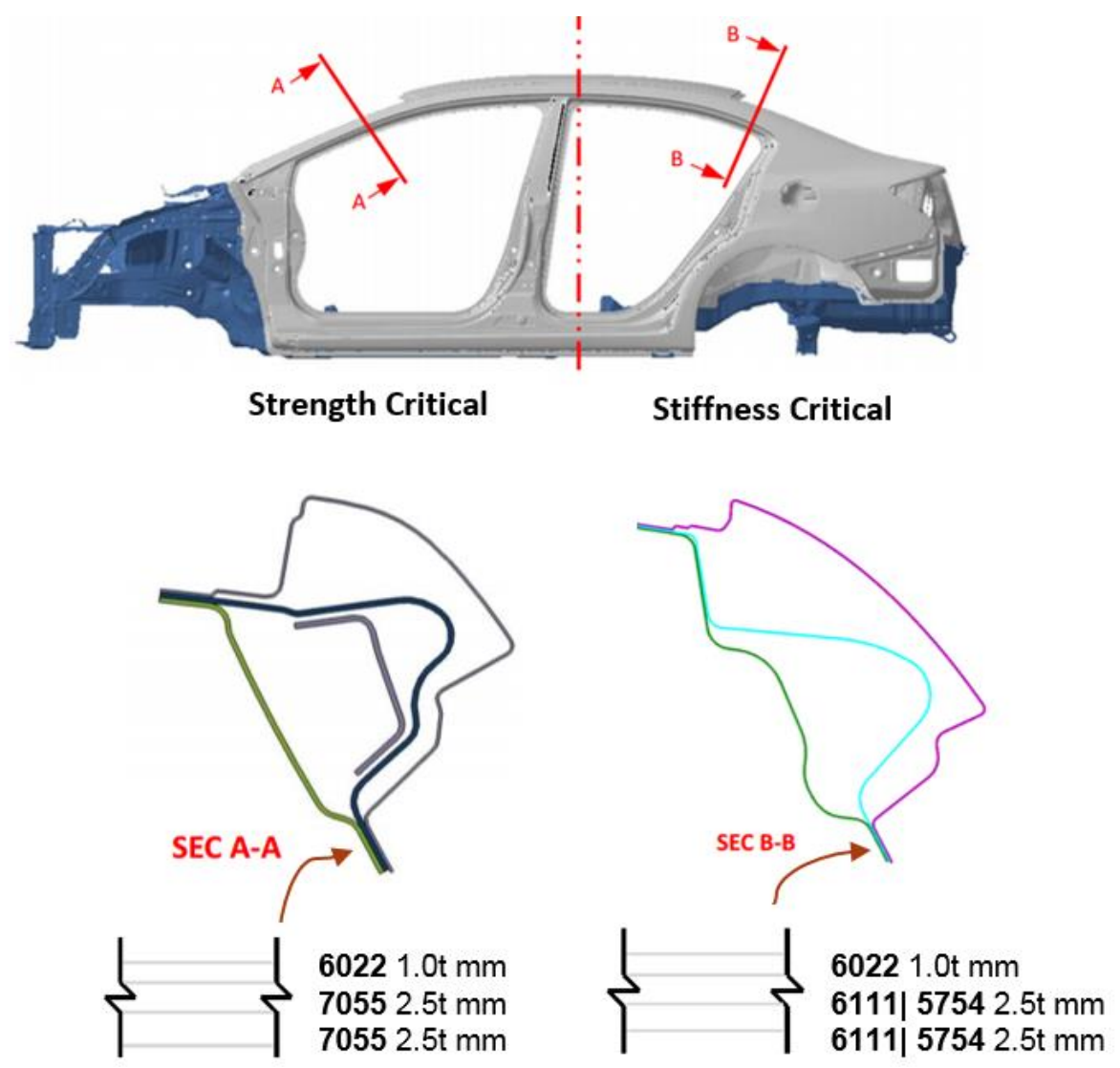

Figure II.3.6.1. Examples of sample placement used for exposure orientation assessment. Source: OSU.

\section{Results}

Load per unit weld width versus measured extension curves for lap-shear FSLW joints are shown in Figure II.3.6.2 (a). At a welding speed of $1 \mathrm{~m} / \mathrm{min}$, we demonstrated around $60 \%$ and $50 \%$ of load-bearing capacity (compared to the BM) for 3- and 2-sheet configurations, respectively. The full-field hardness distribution of the FSLW joint discussed above is shown in Figure II.3.6.2 (b). The fracture location is coincident with the location of the HAZ minimum hardness location on the advancing and retreating side depending on the final fracture location. This indicates that fracture propagation can be limited by increasing the strength around the interface region. While it is difficult to make any direct comparison to alternative joining methods without considering economic aspects like cost, speed, and other material considerations, a simple load-bearing comparison was attempted with RSW standard data available in the open literature. The lap-shear strengths measured exceed that of the dotted line in Figure II.3.6.2 (a), which represents the minimum load-bearing requirements for RSWs of 7xxx series alloy with a 15-mm spot spacing per AWS D17.2. Similar results of exceeding the minimum requirements were also obtained for the other two material sets as well.

The load-carrying capacity per $40 \mathrm{~mm}$ weld length in lap-shear is shown in Figure II.3.6.3. In all three stackups, we exceeded the target (proprietary) set by HRA in Task 1 . Note that for the 5754 and 6111 sets, the demonstrated welding speed is $2 \mathrm{~m} / \mathrm{min}$. Owing to its high hardness and consequent high-temperature flow stress, 7055 presented a unique set of challenges in FSLW. As is well-known compared to the 5754 and 6111 set, the 7055 set required significantly greater power input. The welding parameters and tool design that produced "good" welds in 6xxx and 5xxx series resulted in various types of defects including advancing side worm-hole defect either near the root region or near the crown. Additionally, higher planer forces at welding speed $(<1 \mathrm{~m} / \mathrm{min})$ often resulted in tool failures. After some process development and tool geometry changes, we found a reasonable material flow regime in the 7055 material set at a lower welding speed. 


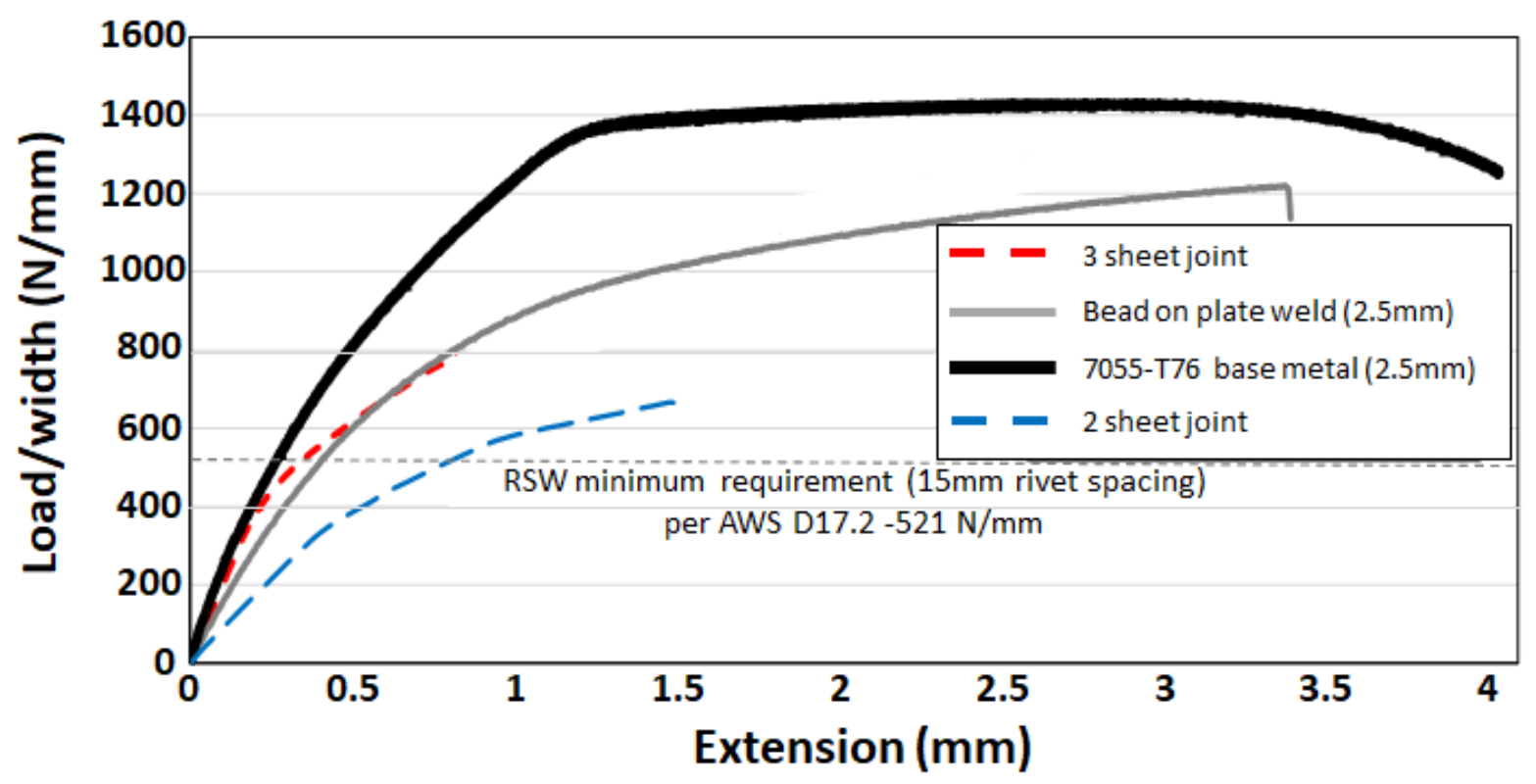

(a)

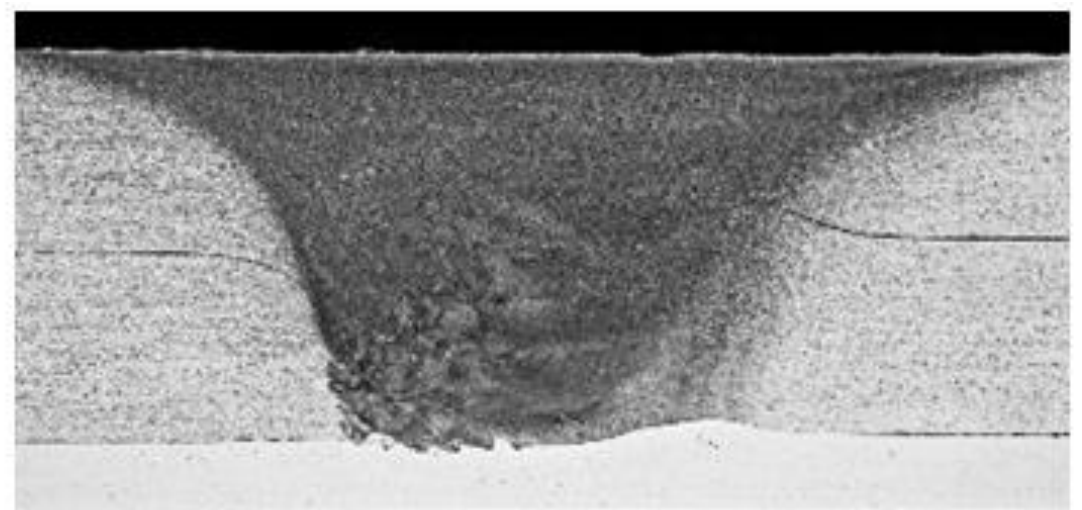

\section{$1 \mathrm{~mm}$}

\section{Joint cross-section}

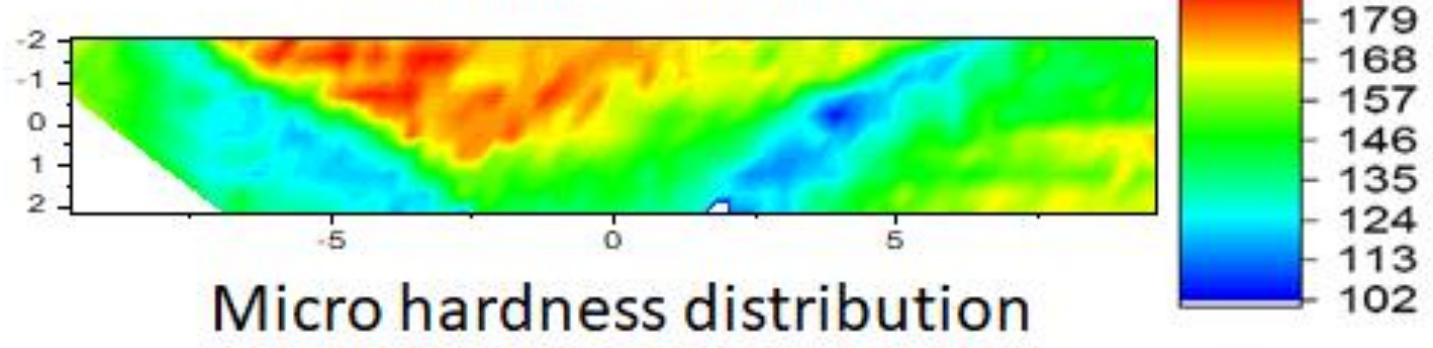

(b)

Figure II.3.6.2. 7055 Load-bearing capacity comparison. (a) Load per unit weld width plotted against measured extension during lap-shear testing of two [7055 (2 mm)-7055 (2 mm] and three sheets [7055 (2 mm)-7055 (2 mm)-6022 (1 mm)]

FSLW joints. Tensile test results for base 7055 sheet and bead-on-plate welds are also shown for reference. The dotted line indicates the minimum load-bearing capacity required for RSW for $15 \mathrm{~mm}$ spacing as per AWS D17.2. (b) A representative joint cross-section for a 3 sheet joint. Full-field hardness color map showing HAZ minimum hardness regions around the weld nugget. Source: PNNL. 


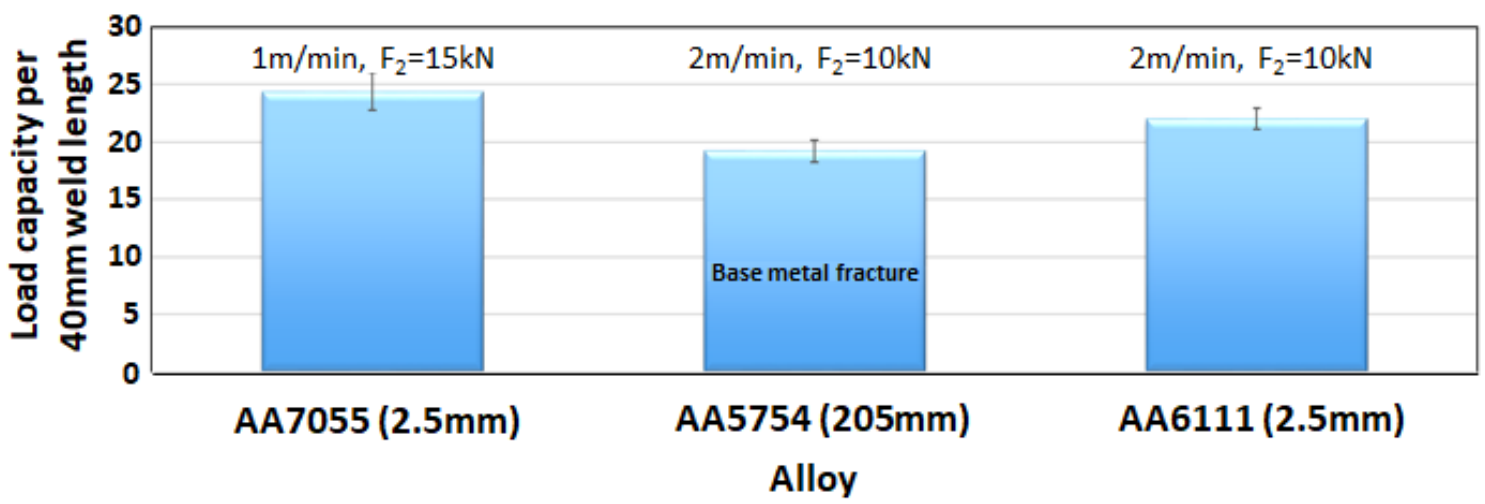

Figure II.3.6.3. Bar chart representing the average load-bearing capacity observed in three sheet FSLW for three types of alloys considered. The corresponding welding speeds and Z forces are also shown. Standard deviations with three samples in each case are also shown. In all cases, the target set by HRA was met. Source: PNNL.

After reaching the target for lap-shear strengths, we evaluated and developed welding parameters for T-peel performance. The effects of advancing side defect and upturn hook on the retreating side were more pronounced in T-peel testing as opposed to the lap-shear tests. Several fracture modes were observed, as shown in Figure II.3.6.4. The interfacial fracture on the left side of Figure II.3.6.4 is indicative of inadequate vertical material flow, which resulted in the lowest load-bearing capacity. The HAZ fracture either on the top or bottom sheet yielded the highest load-bearing capacity. In the case of the 6111 set, where the nugget region is softer, a ductile nugget fracture was observed. The right most fracture mode occurred when a crack line initiated at the retreating side hook feature was met with the worm-hole defect on the advancing side. This shows how defects/features on either side of the nugget can significantly affect the load-bearing capacity. In general, the fracture on the bottom sheet (HAZ/bottom sheet edge) of the nugget yielded the highest loadbearing capacity, as observed in the middle fracture of Figure II.3.6.4.

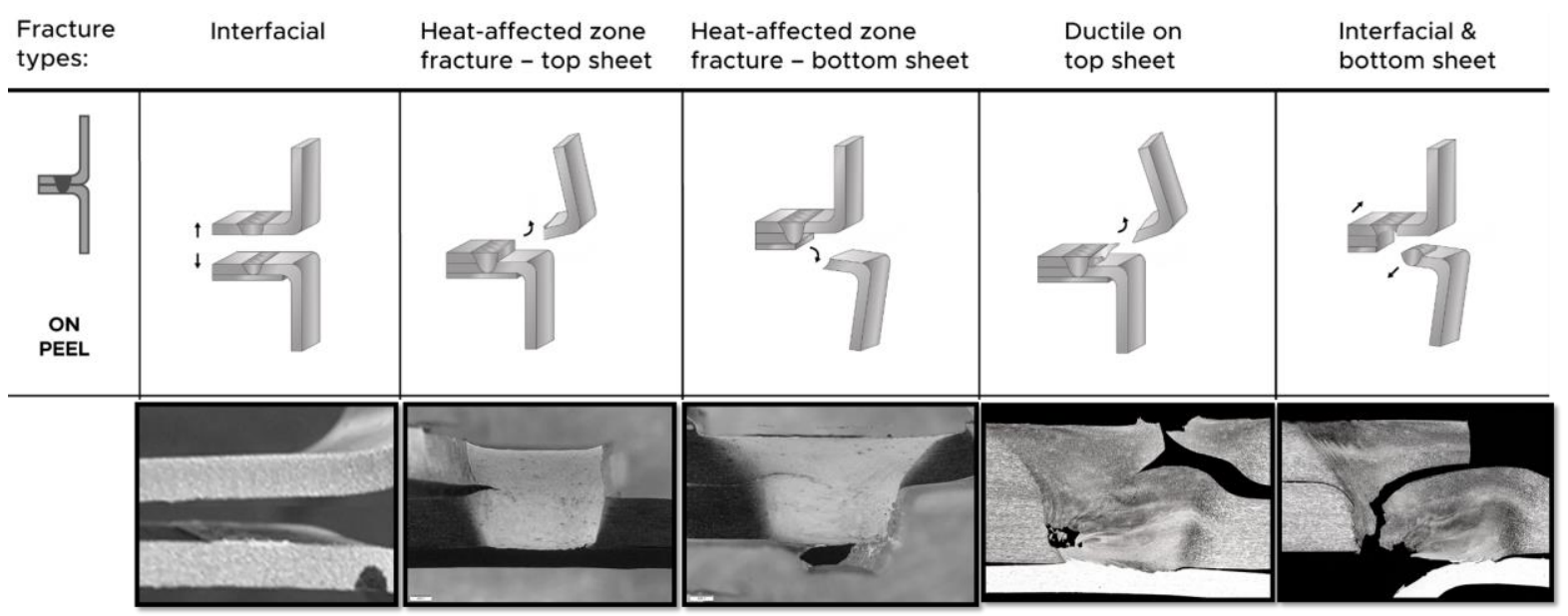

Figure II.3.6.4. Several types of fracture modes observed in three sheet FSLW. Results are shown for whole welds samples for each case with a 30-mm weld length. Source: PNNL. 
In the course of obtaining the T-peel samples, which met and exceeded the requirement set by HRA, we investigated the effects of cut versus whole samples. Here cut samples refer to samples obtained by the waterjet/ band saw cutting of the weld section resulting in an open cross-section on either side of the sample. In contrast, the whole weld has both the start and stop on the sample, as seen in the inset of Figure II.3.6.4. For the 7055 set, a 50\% increase in T-peel and lap-shear performance was observed when the whole weld was tested in place of the cut welds. For the 6111 and 5754 sets, the increase in performance with whole weld was only modest. The peel performance of the "whole" FSLW in the three-material sets is summarized in Figure II.3.6.5. This observation led us to further investigate the effects of start/stop and stitch configurations in peel and lap-shear setup and U-peel configuration. The fracture mode in all the cases was the HAZ fracture of the bottom sheet, as shown in the third column of Figure II.3.6.4 above. The load-carrying capacity and stiffness behavior is the highest with the 7055 material set, albeit the strength is not proportional to the base material strength. This indicates that there may be additional room for improvement in peel performance for the 7055 set. Nevertheless, by utilizing the whole weld testing samples, we were able to exceed the T-peel load-carrying capacity set by HRA.

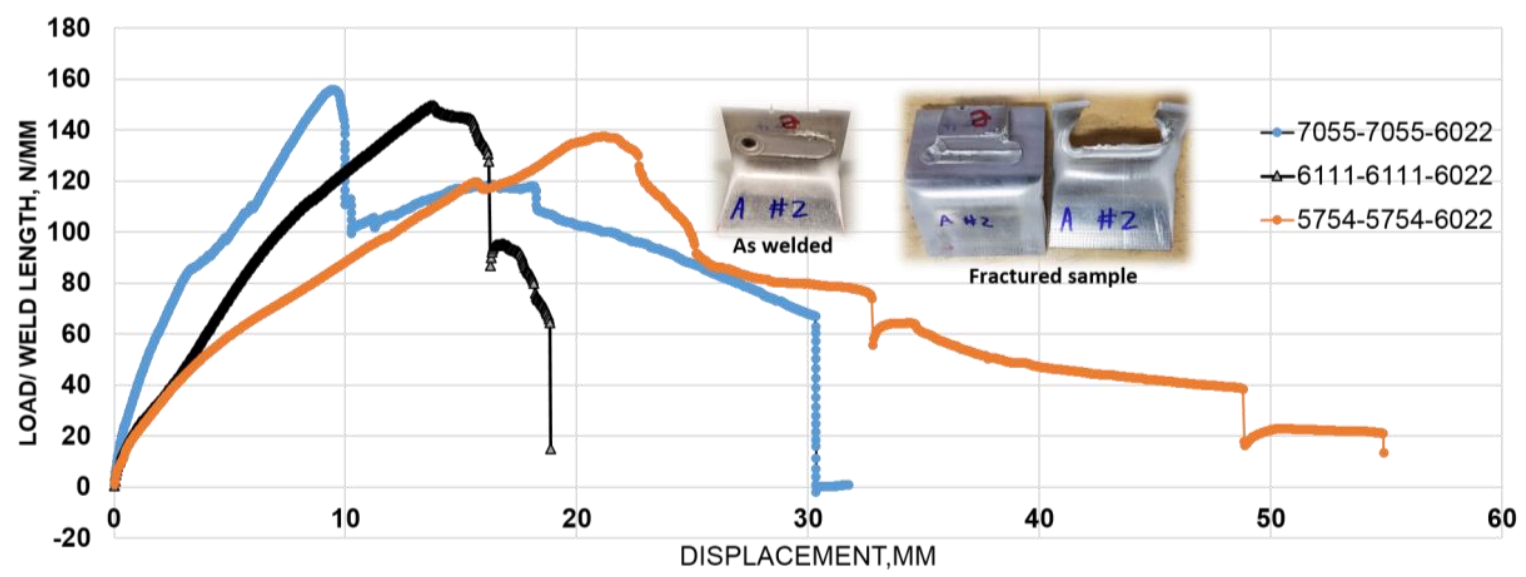

Figure II.3.6.5. Load per unit weld length plotted against displacement for T-peel FSLW samples for the three alloys layup. An example of a fractured sample is shown for each fracture mode for the 6111-6111-6022 set. Note that the start and stop of the weld is within the tested joint. The total weld length in each case was $30 \mathrm{~mm}$. Source: PNNL.

While coupon-level work provides welding parameters necessary for reaching the target set by HRA, near prototype-scale demonstration joints are necessary to make a case for the commercial viability of FSLW. To that end, it is necessary to evaluate FSLW in loading conditions like what is currently being used in the automotive industry. Since most of the joining methods in the industry are "point joints" rather than linear joints, direct comparison to existing testing method is problematic since linear joints are asymmetrically loaded upon mechanical testing (e.g., lap-shear, T-peel, and cross-tension tests). To address this gap (at least partially), we devised and implemented a KS2 fixture configuration to test joints in U-peel conditions shown in Figure II.3.6.6 (a). Two FSLWs are made on two sides of U-shaped sheets with end clamping, as seen in Figure II.3.6.6 (b). Welds were made such that loading sides were identical in two cases as seen in Figure II.3.6.6 (c). This weld was then tested on the KS2 testing fixture. This KS2 welding setup enabled the project team to evaluate different welding conditions including start, stop, multiple starts, and stiches. Unlike a lap-shear or T-peel case, the U-peel test shows at least two loading peaks, as seen in Figure II.3.6.6 (f). The fracture typically began at the root on the loading side and traveled towards the weld start. The mode of fracture was similar to the HAZ bottom sheet fracture. A max load of $370 \mathrm{~N} / \mathrm{mm}$ and $266 \mathrm{~N} / \mathrm{mm}$ was obtained for the 7055 and 6111 set, respectively. Several different configurations of short stitches have been tested recently to ascertain the effects of multiple stats and stop. We are currently compiling this information which can serve as important input in the FE model at the HRA facility. We intend to use this capability to produce an ideal demonstration hat section welding. 


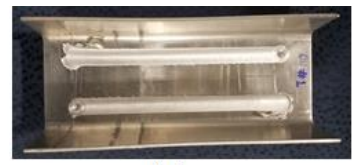

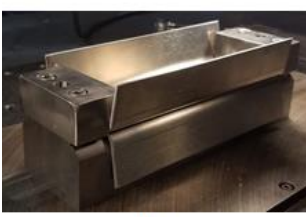

(b)

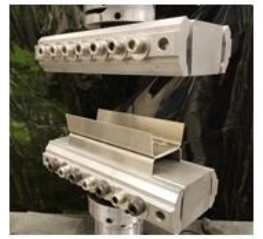

(a)

(c)

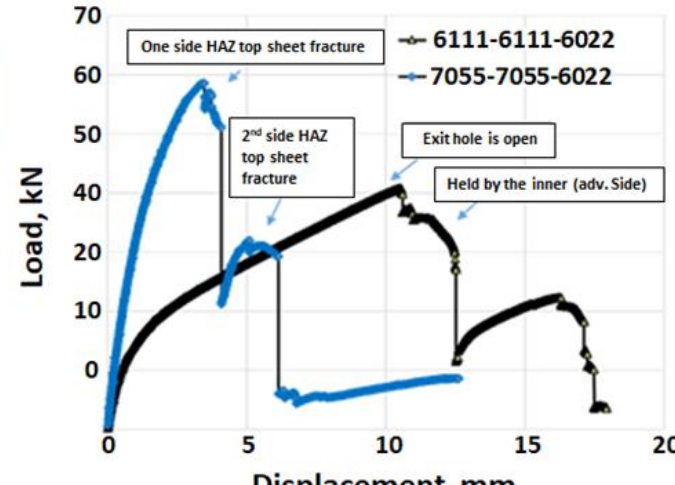

(f)

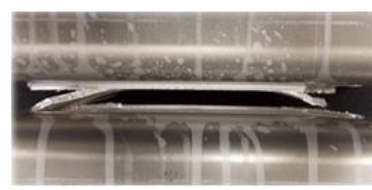

(d)

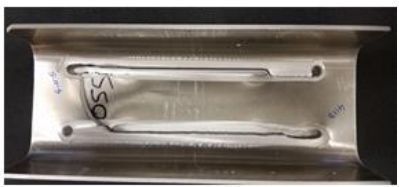

(e)

Figure II.3.6.6. Clockwise from bottom left: (a) KS2 sample testing fixture holding a KS2 sample; (b) KS2 welding fixture with KS2 sample clamped; (c) a top view of KS2 sample; (d) a side view of tested fractured KS2 (7055-7055-6022);

(e) a top view of fractured KS2 (7055-7055-6022). Source: PNNL.

Figure II.3.6.7 (a) shows the fixturing and clamping arrangement for the demonstration hat section joints designed by HRA. We have implemented this design and fabricated fixturing, as seen in Figure II.3.6.7 (b) and Figure II.3.6.7 (c), to be used at the PNNL FSW facility. Having received a sufficient shipment of hat section demonstration samples, we are preparing to make the hat section for the axial crush shown in Figure II.3.6.7 (d) and the 3-point bend sample shown in Figure II.3.6.7 (f) for testing at the HRA facility. An example completed hat section weld is shown in Figure II.3.6.7 (e).

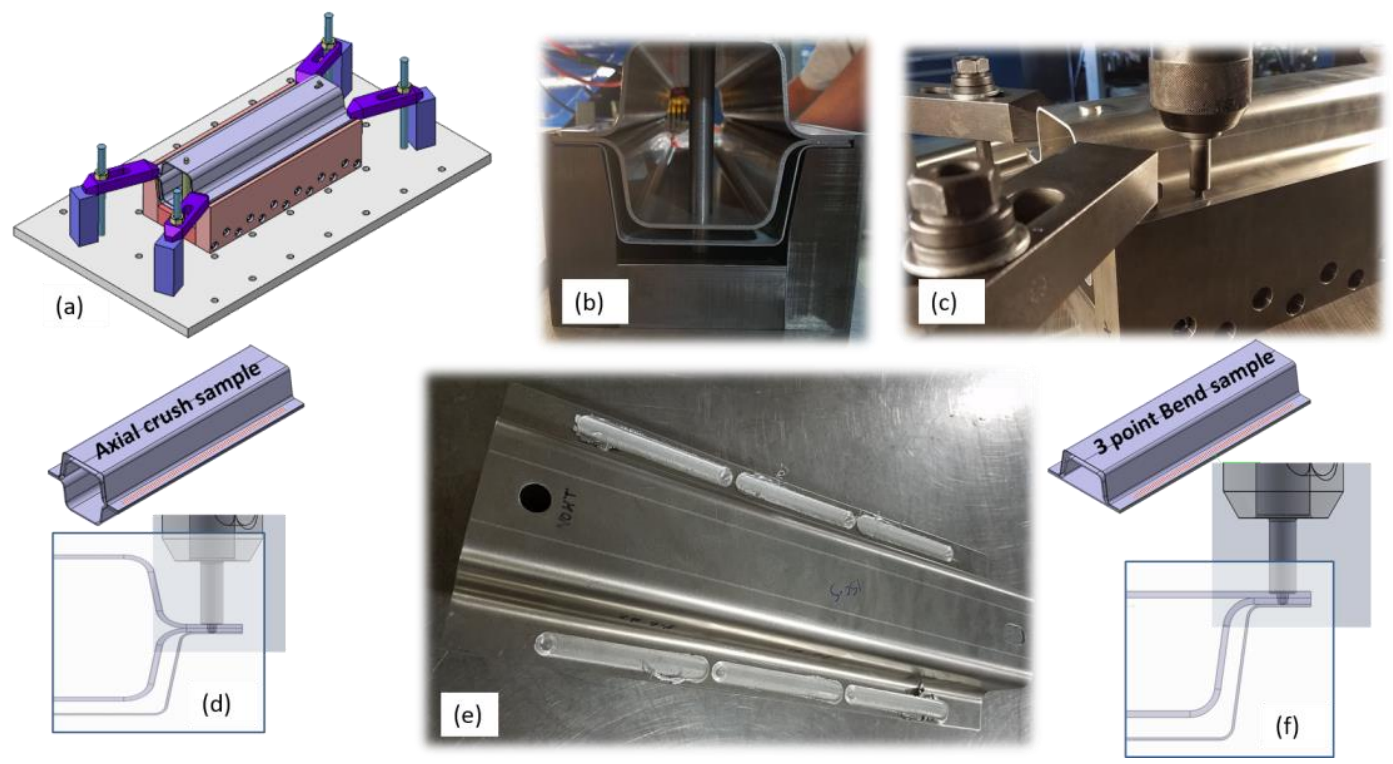

Figure II.3.6.7. (a) A CAD of demonstration hat section atop of fixture with desired clamping scheme. Source: HRA.

(b) A side view of axial crush sample loaded on the hat section fixture. Source: PNNL.

(c) Hat sections clamped down on fixture ready to be welded. Source: PNNL.

(d) FSLW setup sketch illustrating welding configuration for axial crush sample. (e) An example of a welded axial crush sample with three weld lines on each side. Source: PNNL.

(f) FSLW setup sketch illustrating welding configuration for the 3-point bend sample. Source: HRA. 


\section{Conclusions}

This project aims to develop a FSLW technique in automotive $\mathrm{Al}$ sheets such that effective joints can be made at industrially viable welding speed for commercialization. Building upon the work done in FY 2018, this year we made progress on several fronts towards achieving the objectives outlined in Task 2 and Task 3 above, specifically in three areas. (1) For all the three-material sets identified for this project, we demonstrated mechanical performance in lap-shear and T-peel configuration that met/ exceeded target set by the automotive partner, HRA, in Task 1 at commercially viable welding speeds. (2) In concert with HRA, we developed and implemented U-peel welding and testing fixturing that enabled evaluation of FSLW in KS2 configuration. This is the first time that linear FSLW joints have been characterized in cross-tension configuration. (3) We designed demonstration hat section welds in for 3-point bend and crush testing at HRA facility. We are near ready to fabricate hat section welds in three selected welding configurations. We anticipate that final project deliverable will be completed by the end of CY 2019.

\section{Key Publications}

1. Upadhyay, P., L. Xiao, and T. Roosendaal, 2019, "High-speed FSLW of automotive Al alloys," MS\&T 2019, Portland, OR, October 2019.

\section{References}

1. Cederqvist, L., and A. P. Reynolds, 2001, "Factors affecting the properties of friction stir welded Al lap joints," Welding Journal-New York, Vol. 80, No. 12, pp. 281-s.

2. Song, Y., X. Yang, L. Cui, X. Hou, Z. Shen, and Y. Xu, 2014, "Defect features and mechanical properties of friction stir lap welded dissimilar AA2024-AA7075 Al alloy sheets," Mater. Design, Vol. 55, pp. 9-18.

3. Soundararajan, V., E. Yarrapareddy, and R. Kovacevic, 2007, "Investigation of the FSLW of Al alloys AA 5182 and AA 6022," J. Mater. Eng. Perform., Vol. 16, No. 4, pp. 477-484.

4. Upadhyay, P., and A. P. Reynolds. "Effects of thermal boundary conditions in friction stir welded AA7050-T7 sheets." Mater. Sci. Eng. A-Struct., Vol. 527, No. 6, pp. 1537-1543.

\section{Acknowledgements}

The authors recognize the regular engagement and support from Eric Boettcher's team at Honda R\&D Americas and Russell Long's team at Arconic, Inc. At PNNL, welding and data analysis activities were supported by Daniel Graff, Li Xiao, and Angel Ortiz. Testing and characterization of joints were supported by Tim Roosendaal, Mark Rhodes, and Anthony Guzman. 


\title{
II.3.7 Corrosion Protection of Dissimilar Material and Joining for Next-Generation Lightweight Vehicles (Arconic, Inc.)
}

\section{Donald J. Spinella, Principal Investigator}

Arconic Technology Center

100 Technical Dr.

New Kensington, PA 15069

E-mail: DJ.Spinella@arconic.com

\section{Sarah Kleinbaum, DOE Technology Development Manager}

\author{
U.S. Department of Energy \\ E-mail: sarah.kleinbaum@ee.doe.gov
}

Start Date: October 1, 2019

Project Funding (FY19): \$1,996,071
End Date: June 30, 2020

DOE share: $\$ 1,514,293$
Non-DOE share: $\$ 481,778$

\section{Project Introduction}

The goal of the project is to demonstrate the use of RSRs to join steel to $\mathrm{Al}$ and $\mathrm{Al}$ to CFRP on a prototypescale. This new technology is being developed by Arconic to leverage the existing automotive RSW infrastructure for high-performance joining of steel to $\mathrm{Al}$ and $\mathrm{Al}$ to CFRP.

When using this process to join an $\mathrm{Al}$ sheet to a steel sheet, a steel rivet is fed to a spot weld gun. The initial spot weld cycle heats the steel rivet, which is then forced through the $\mathrm{Al}$ sheet. Upon contact with the steel sheet, a resistance spot weld is made between the steel sheet and the rivet. An alternative is to use a pilot hole in the $\mathrm{Al}$ sheet and directly weld the rivet to the steel sheet while simultaneously mechanically locking the $\mathrm{Al}$ sheet. The process can be reversed by employing an Al RSR, if joining through steel, or CFRP and welding to an $\mathrm{Al}$ sheet.

\section{Objectives}

In this project, the joint strength and corrosion performance will be documented with and without adhesives between steel and the $\mathrm{Al}$ sheet and $\mathrm{Al}$ and the CFRP sheet. The weld quality will be evaluated under different process conditions. The production viability will be evaluated with the feed system and integration of the feed system onto a robotic system.

\section{Approach}

The third year of this project focused on completing the corrosion studies initiated at both OSU and Honda. Experimental studies were completed that established production boundaries for the RSR process including part angularity, flange widths, joint gaps, and rivet-to-hole misalignments. Additionally, a fully robotic rivet delivery system (RDS) was installed and commissioned at an Arconic Fastening System (AFS) integrator who was selected based on the ability to produce, integrate, and provide field support in North America. The RDS successfully produced robotic RSR joints under a variety of feed angles and completed extensive cycle testing. The demonstrator assembly design was finalized with parts to be fabricated and assembled in late 2019. Due to delays in completing the production condition testing and commissioning of the RDS pilot line, the program was extended 6 months with BP-3 activities initiated in July 2019 and estimated to close by the end of June 2020. 


\section{Results}

OSU initiated cyclic corrosion testing (CCT-1) in addition to the ASTM B117 and ASTM G85-A2 procedures reported previously. Bare samples (without e-coat and joint adhesives) made with different alloys of $\mathrm{Al}$ and steel were subjected to each exposure test to compare the corrosion of different metal couplings up to 32.4 days for all three test methods. The ASTM B117 test method employs a constant spray of $5 \% \mathrm{NaCl}$ solution at $35^{\circ} \mathrm{C}$. The ASTM G85-A2 test consists of a spray of $5 \% \mathrm{NaCl}$ solution, adjusted to $\mathrm{pH}$ of 3.0 with the addition of acetic acid for 45 minutes, followed by a dry cycle of 120 minutes at $7 \%$ to $40 \% \mathrm{RH}$, followed by a humidified cycle for 195 minutes at $95 \% \mathrm{RH}$ and a temperature of $45^{\circ} \mathrm{C}$. Finally, the CCT-1 test employs a $5 \% \mathrm{NaCl}$ salt spray for 240 minutes at $35^{\circ} \mathrm{C}$ followed by a drying time of 120 minutes at $60^{\circ} \mathrm{C}$ and a humidifying time for 240 minutes at $50^{\circ} \mathrm{C}$ and $95 \% \mathrm{RH}$.

After reviewing the corrosion attack depth data between the RSR, SPR, and FDS joints, the team determined a breakout study was necessary to understand if the sample orientation in the test chambers could influence the results. Figure II.3.7.1 shows the various orientations of the samples that are available in the test chambers. As a result, a new definition shown schematically in Figure II.3.7.2 was developed to identify the location of the corrosion in reference to its chamber exposure orientation.

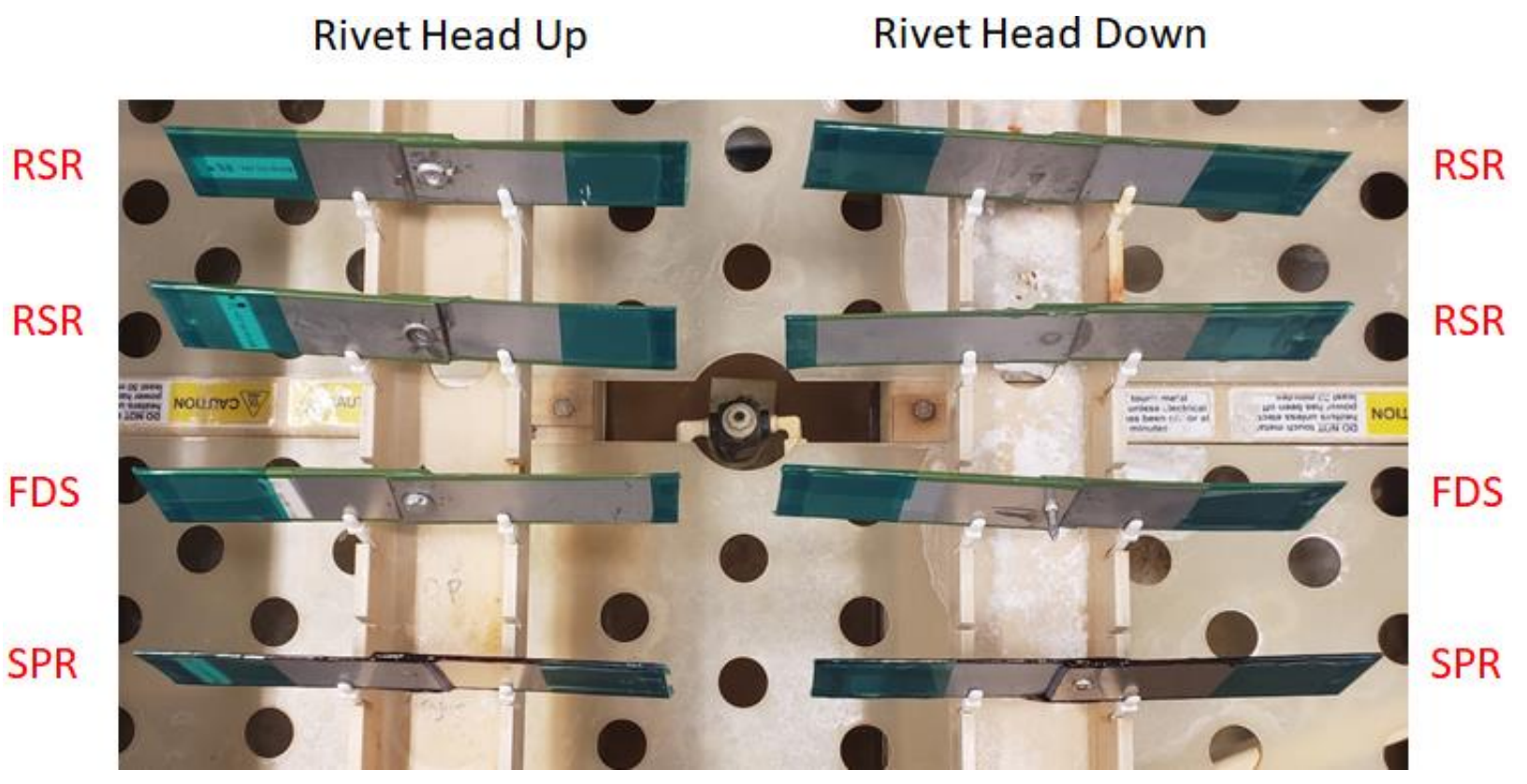

Figure II.3.7.1. Examples of sample placement used for exposure orientation assessment. Source: OSU.

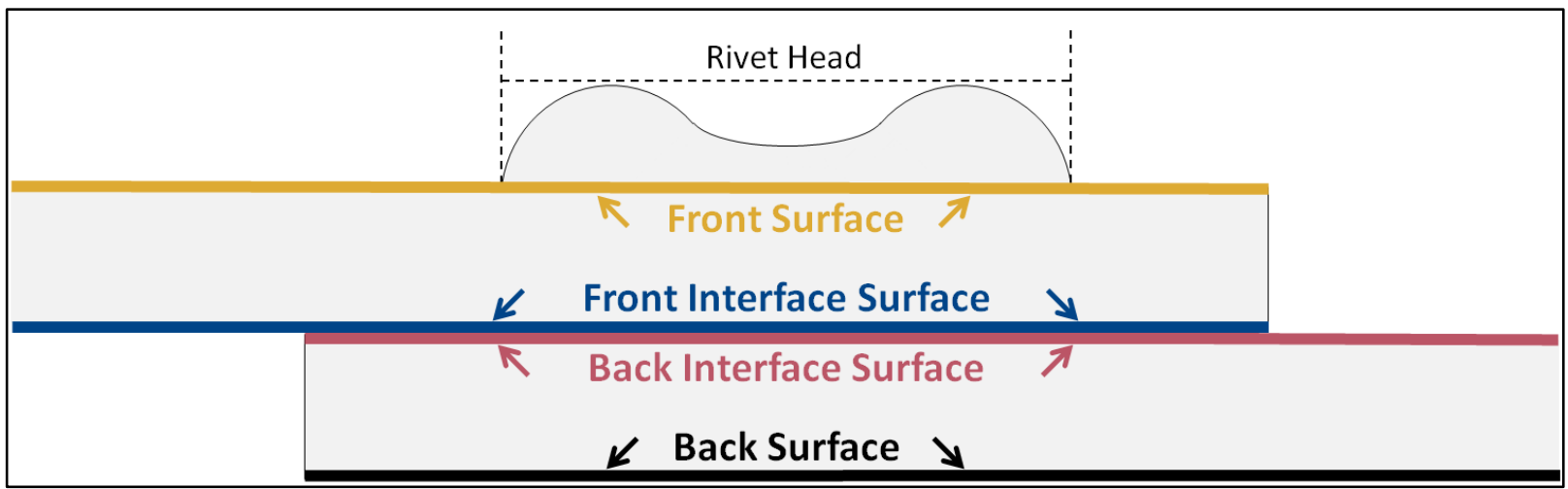

Figure II.3.7.2. Surface corrosion location definitions. Source: OSU. 
Figure II.3.7.3 shows the mechanical performance of samples exposed to ASTM G85-A2 for RSR, SPR and FDS joining technologies. As expected, the samples with adhesive (i.e., SPR and FDS joints only) sustained the highest loads across all exposure times. It was anticipated that RSR joints would have similar increased maximum loads with the addition of adhesive. When comparing the maximum sustained loads of joints without adhesive, RSR joints have higher maximum loads than FDS and SPR joints at all exposure times. The strength comparisons were based on bare specimens (without a joint adhesive). SPR with adhesive does not displace as much adhesive, so it is possible for the SPR joint to have a higher strength in the cured condition. The exposure orientation difference between the RSR (steel down) and the SPR and FDS (steel up) could provide a reason as to why the maximum load is increased but will be confirmed with additional mechanical testing in the steel down orientation.

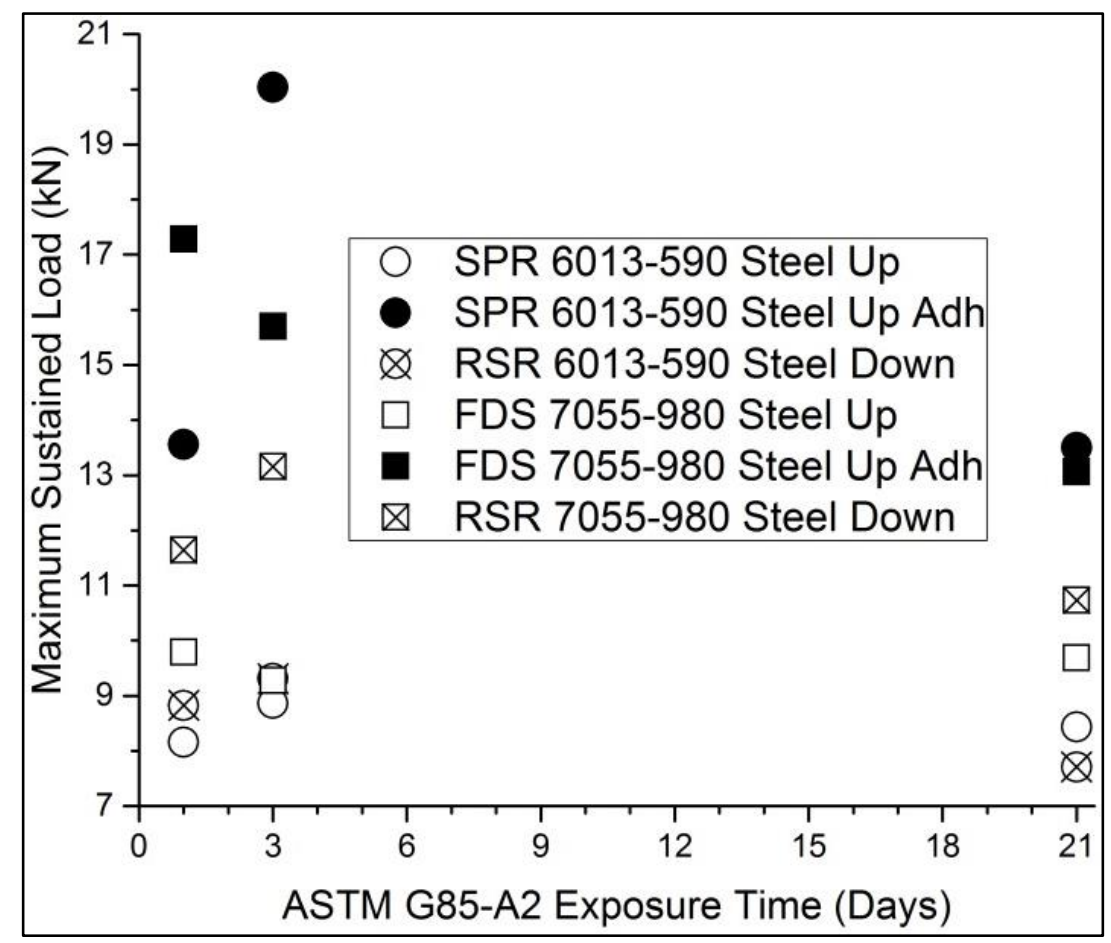

Figure II.3.7.3. Maximum sustained load of samples joined by SPR, RSR, or FDS after ASTM G85-A2 exposure. Source: OSU.

Figure II.3.7.4 and Figure II.3.7.5 show test chamber orientation differences between RSR and FDS using ASTM G85-A2 exposure. Localized corrosion was most severe on a 7055 sheet just outside the crevice mouth on the front interface surface, independent of orientation as shown in Figure II.3.7.4. More severe corrosion was observed when the steel sheet was oriented up, allowing the salt solution to accumulate on the $\mathrm{Al}$ surface and increasing the galvanic contact with the steel sheet.

Figure II.3.7.6 and Figure II.3.7.7 show test chamber orientation differences between RSR and SPR for 6013 Al joined to 590 steel using ASTM G85-A2 exposure. The impact of sample orientation was similar to 7055. The corrosion attack level is highly dependent on the specimen orientation in the test chamber and care must be taken when comparing the technologies. 

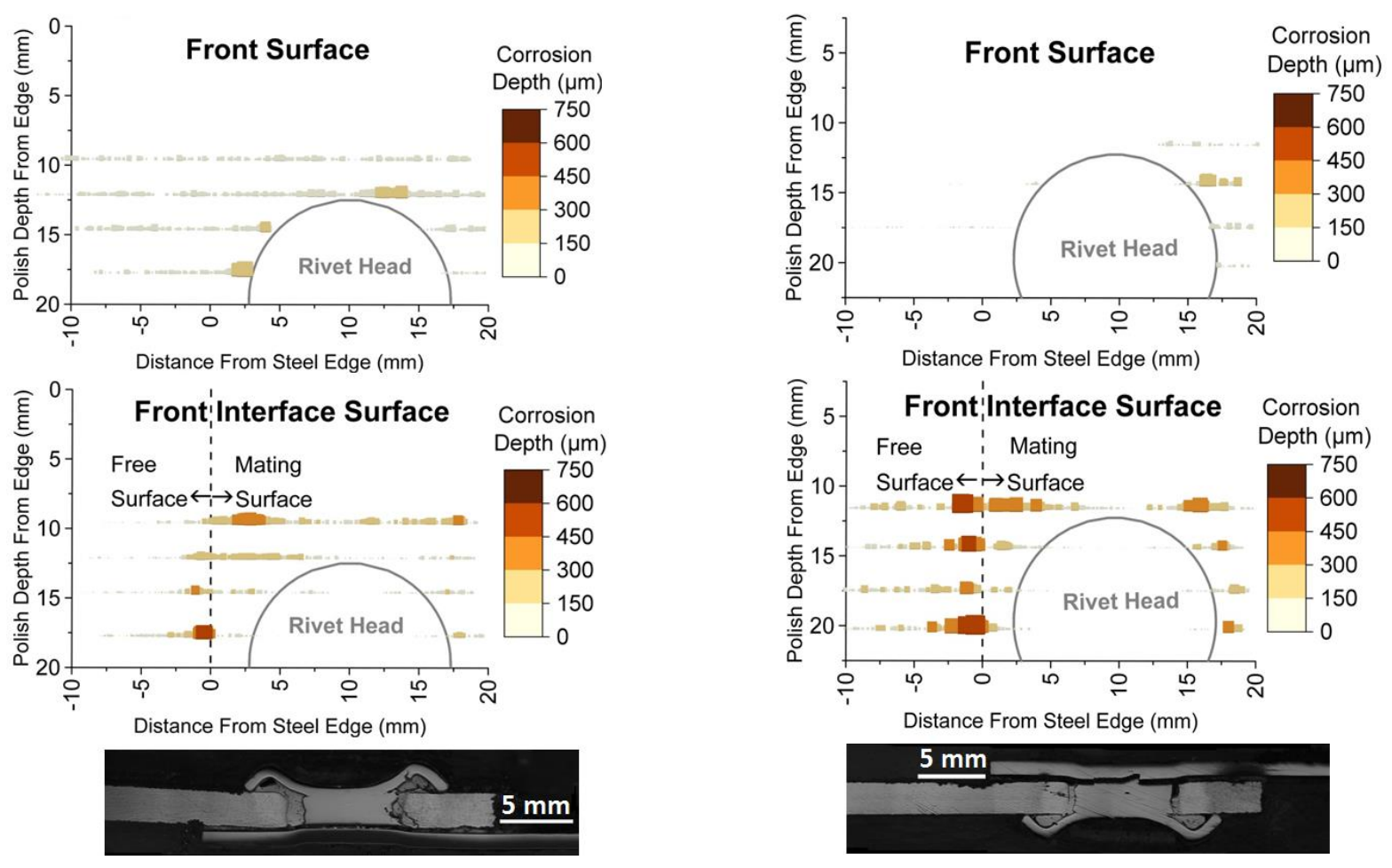

(a)

(b)

Figure II.3.7.4. Cross-section data of AA7055-T76 joined to 980 steel via RSR orientation after 21 days of ASTM G85-A2 exposure: (a) RSR exposed up and (b) RSR exposed down. Source: OSU.
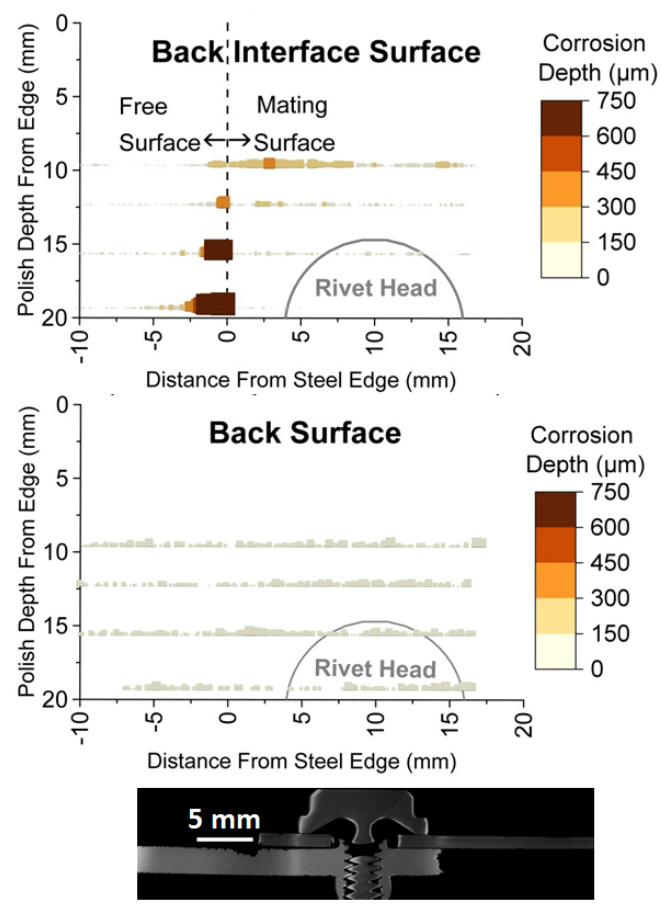

(a)
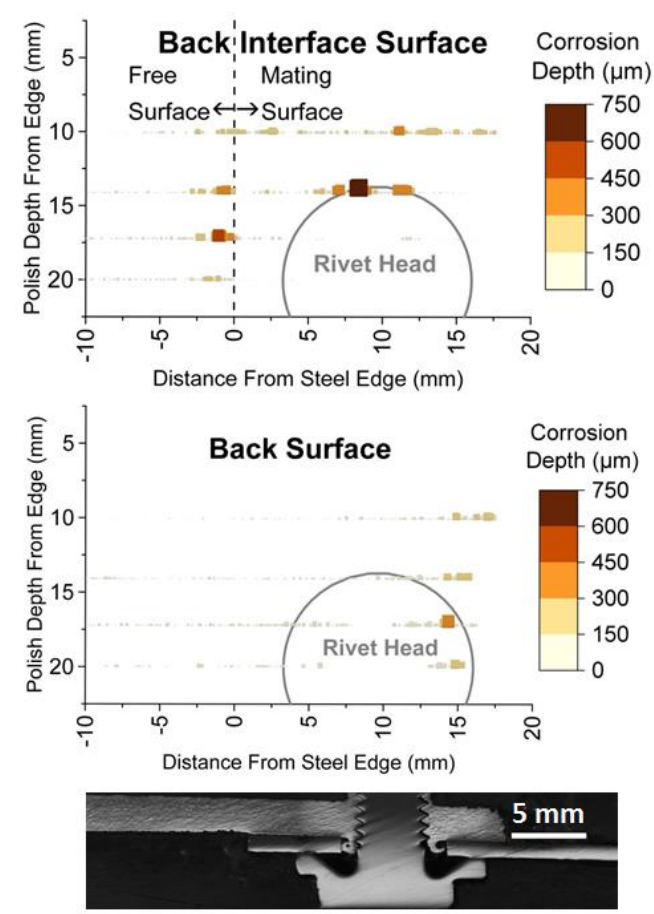

(b)

Figure II.3.7.5. Cross-section data of AA7055-T76 joined to 980 steel via FDS orientation after 21 days of ASTM G85-A2 exposure: (a) FDS exposed up and (b) FDS exposed down. Source: OSU. 

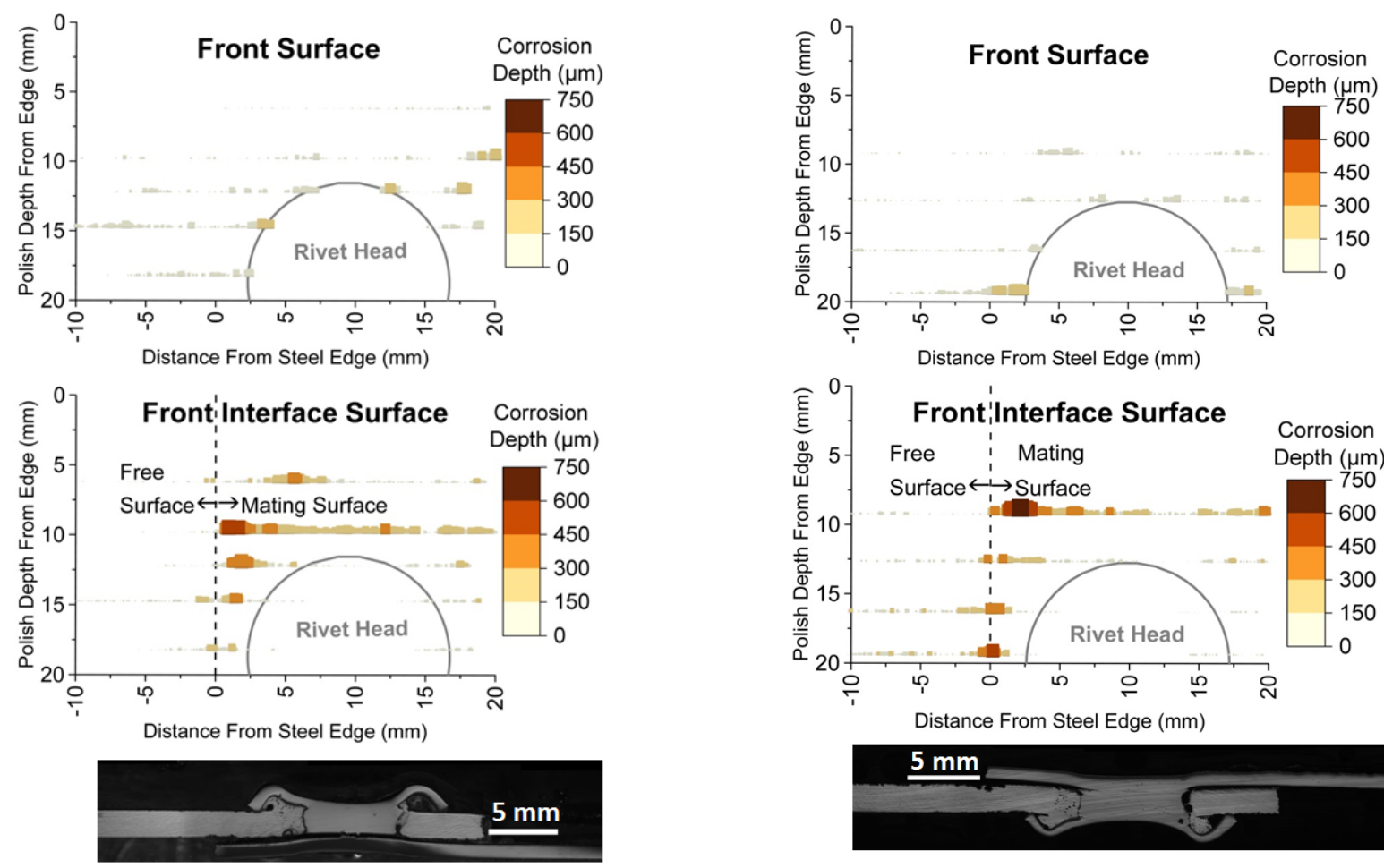

(a)

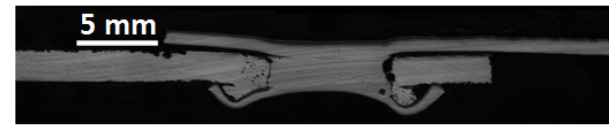

(b)

Figure II.3.7.6. Cross-section data of AA6013-T4 joined to 590 steel via RSR orientation after 21 days of ASTM G85-A2 exposure: (a) RSR exposed up and (b) RSR exposed down. Source: OSU.
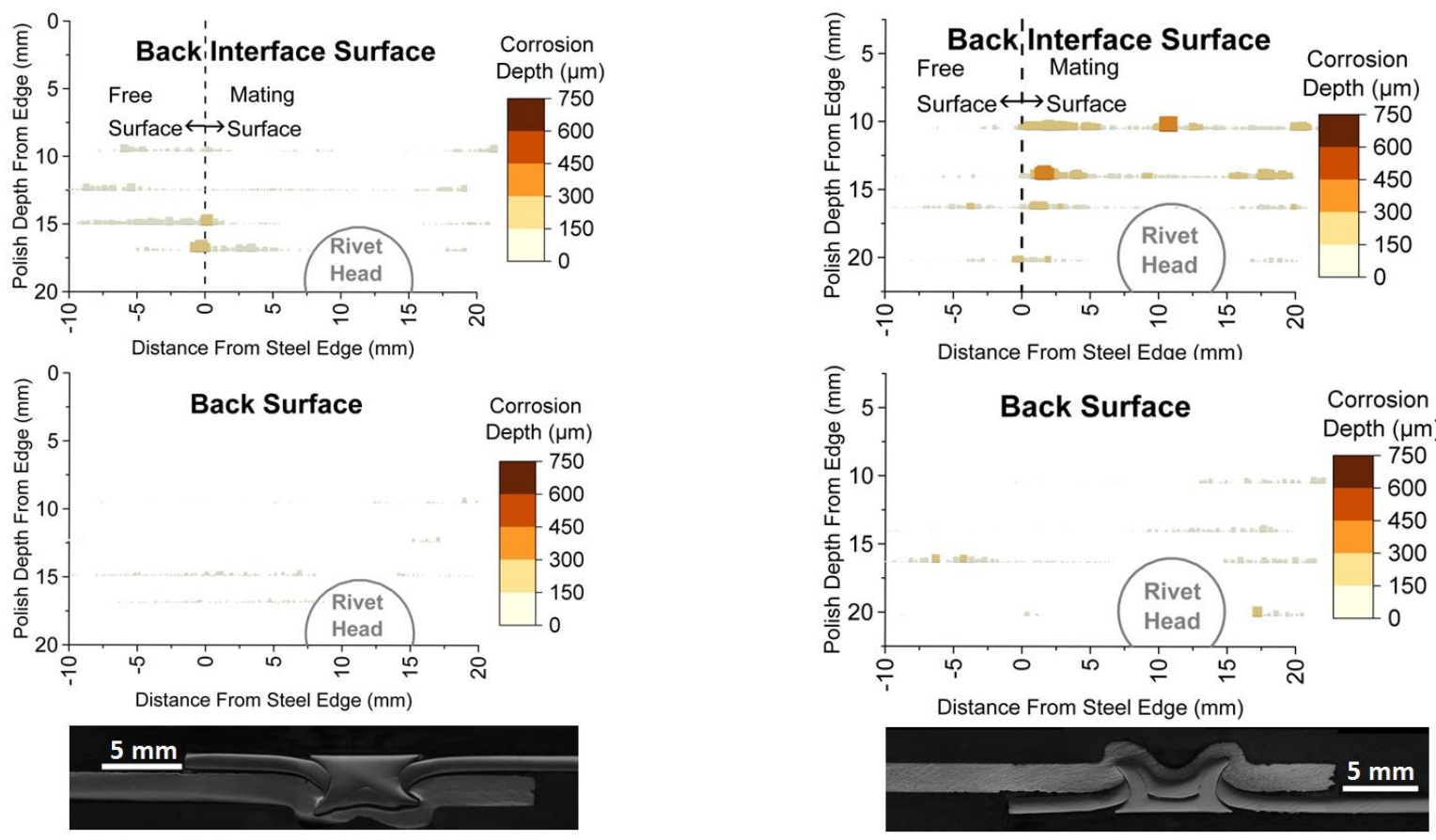

(a)

(b)

Figure II.3.7.7. Cross-section data of AA6013-T4 joined to 590 steel via SPR orientation after 21 days of ASTM G85-A2 exposure: (a) SPR exposed up and (b) SPR exposed down. Source: OSU. 
Figure II.3.7.8 shows the cumulative probability of finding a pit at a specific depth for RSR and FDS, after 21 days ASTM G85-A2 exposure. While the FDS samples experienced greater corrosion depths, the RSR samples have a greater probability of having deeper corrosion sites, as denoted by the slight shift in the RSR curves. Additional statistical analysis is planned to determine if the cumulative probability of all samples is statistically similar or different. This is important given the low sample number $(n=1)$ for each stack-up type currently investigated.

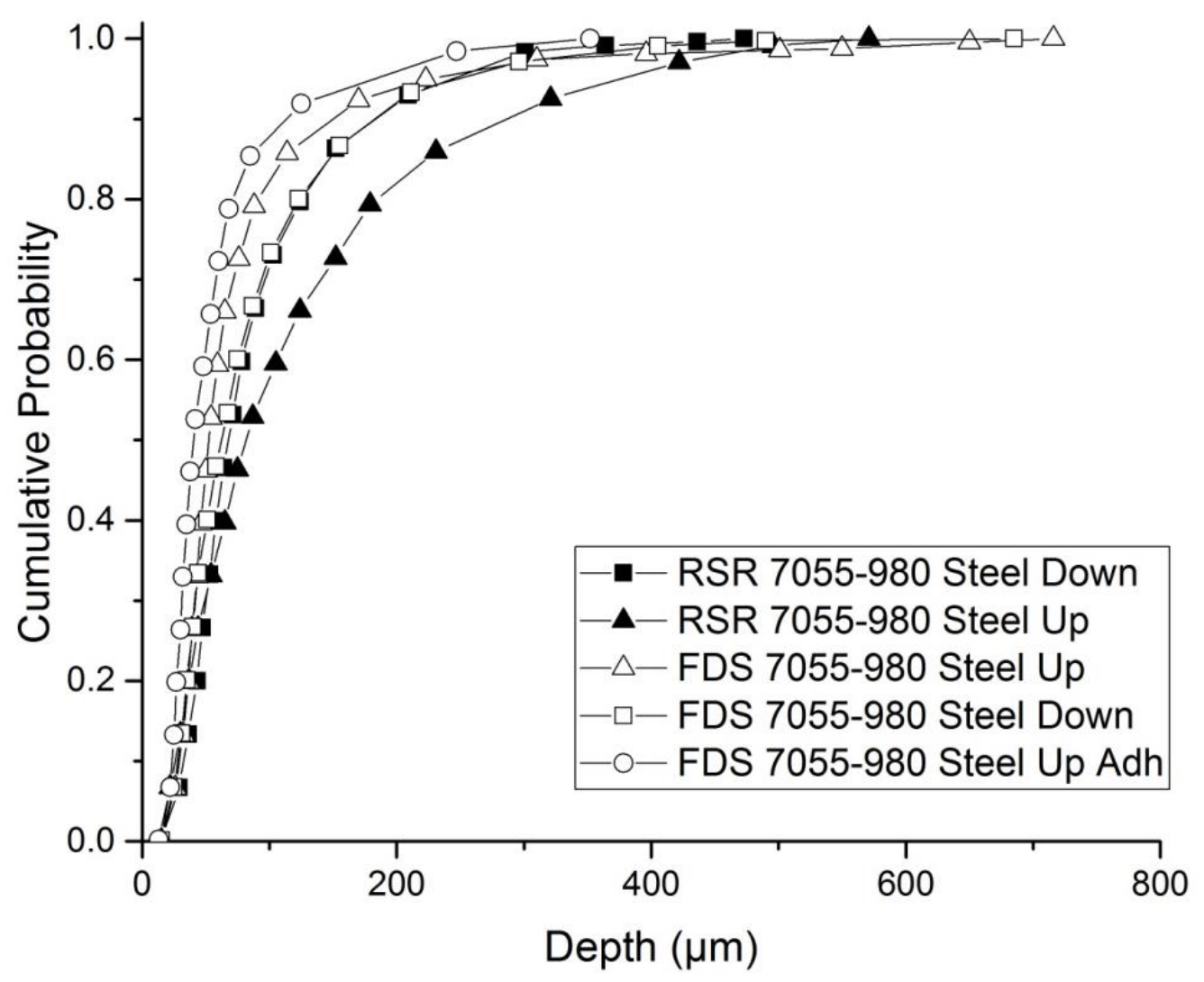

Figure II.3.7.8. Cumulative probability of corrosion depths of AA7055 joined to 980 steel via RSR or FDS after 21 days ASTM G85-A2 exposure. Note: the data was fit to a line and symbols were added for legibility. The symbols do not represent a single data point collected. Source: OSU.

Figure II.3.7.9, Figure II.3.7.10, and Figure II.3.7.11 show the difference in corrosion of $\mathrm{Al}$ to steel stacks joined by RSR as a result of the three exposure methods (i.e., ASTM B117, ASTM G85-A2, and CCT-1) at 32.4 days exposure. Samples comprised of AA5754 joined to steel 980 by RSR and exposed for 32.4 days showed that the ASTM B117 test method was the least aggressive, with a maximum corrosion depth of 195 $\mu \mathrm{m}$, as shown in Figure II.3.7.9. The CCT-1 and ASTM G85-A2 showed similar results, with the deepest pit depths of $510 \mu \mathrm{m}$ and $595 \mu \mathrm{m}$, respectively. When the samples were made with AA6013 joined to 980 steel, the results showed that the B117 test again gave the shallowest pit depth of $265 \mu \mathrm{m}$ when comparing all three tests, as shown in Figure II.3.7.10. G85-A2 showed a maximum pit depth of $519 \mu \mathrm{m}$, while CCT-1 showed $668 \mu \mathrm{m}$. Lastly, the RSR samples joining Aural2 to 980 steel yielded different results in terms of maximum pit depth using different exposure conditions as shown in Figure II.3.7.11. The least corrosive test was the G85A2 test, having a maximum pit depth of $694 \mu \mathrm{m}$, closely followed by the B117 test, which had a maximum corrosion depth of $732 \mu \mathrm{m}$. The CCT-1 test revealed that the greatest pit depth was $977 \mu \mathrm{m}$. In all three comparable sets, the severity of corrosion between the three tests changed, depending on the stack-up type. More data will be collected between samples made via RSR and joining different combinations of material stack-ups to determine the effect different exposure conditions have on corrosion. 

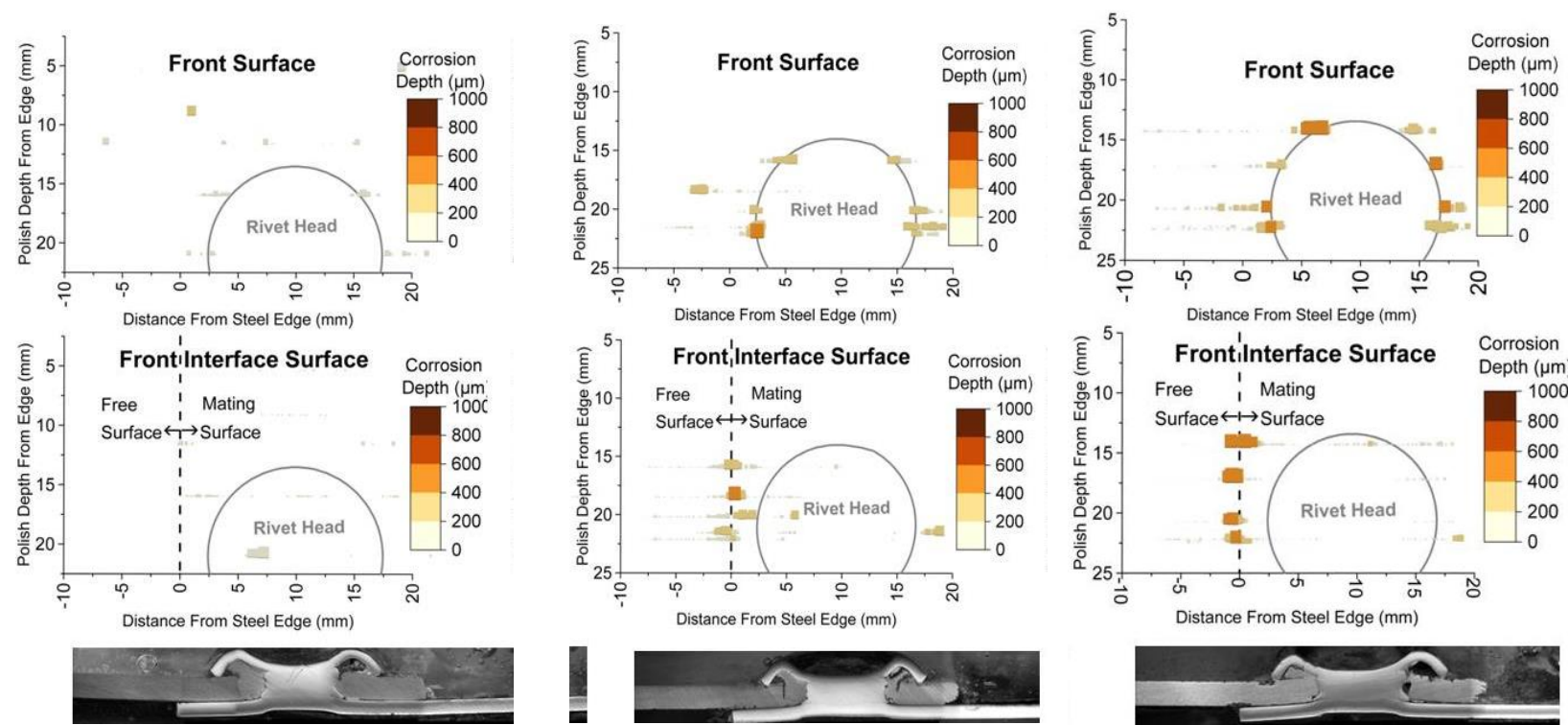

(a)

(b)

(c)

Figure II.3.7.9. Cross-section data of AA5754-0 joined to 590 steel via RSR after 32.4 days exposure: (a) ASTM B117; (b) ASTM G85-A2; and (c) CCT-1. Source: OSU.

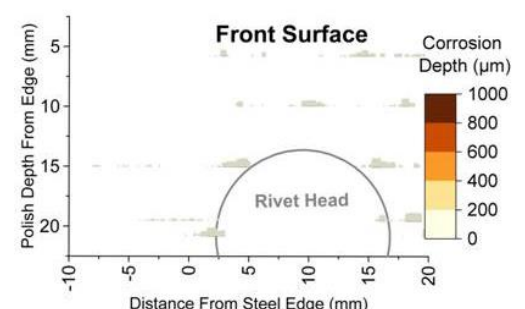

Distance From Steel Edge (mm)

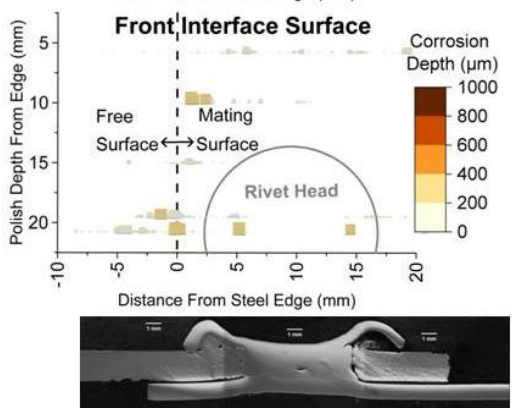

(a)
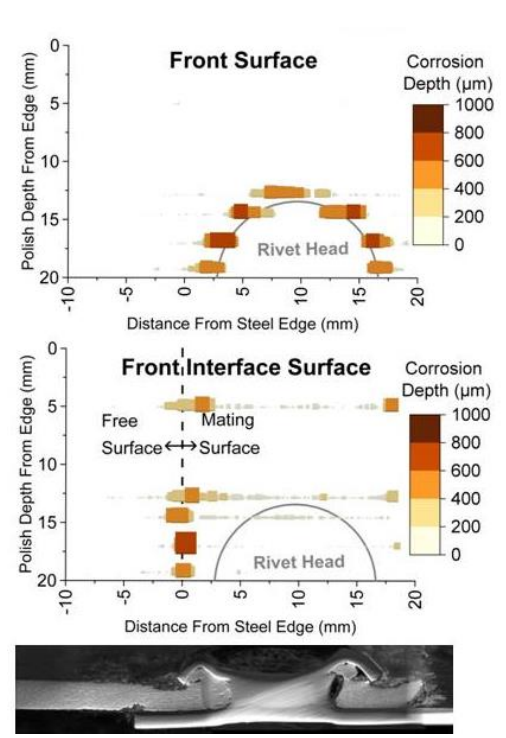

(b)
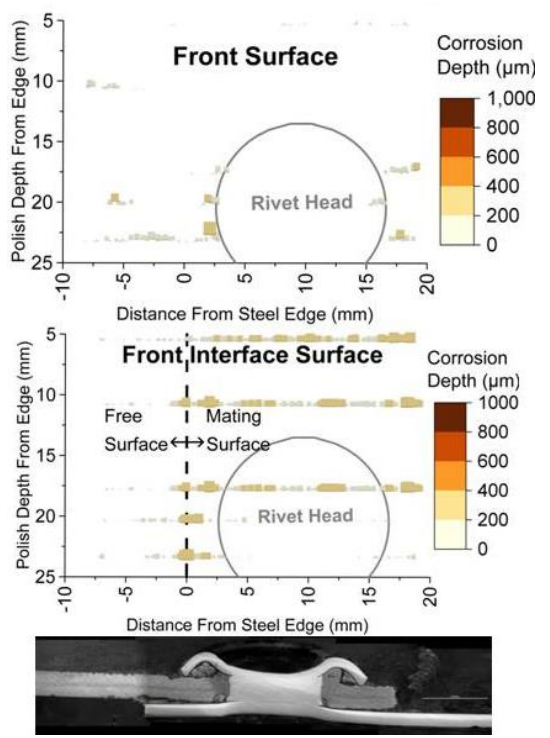

(c)

Figure II.3.7.10. Cross-section data of AA6013-T4 joined to 980 steel via RSR after 32.4 days exposure: (a) ASTM B117; (b) ASTM G85-A2; and (c) CCT-1. Source: OSU. 


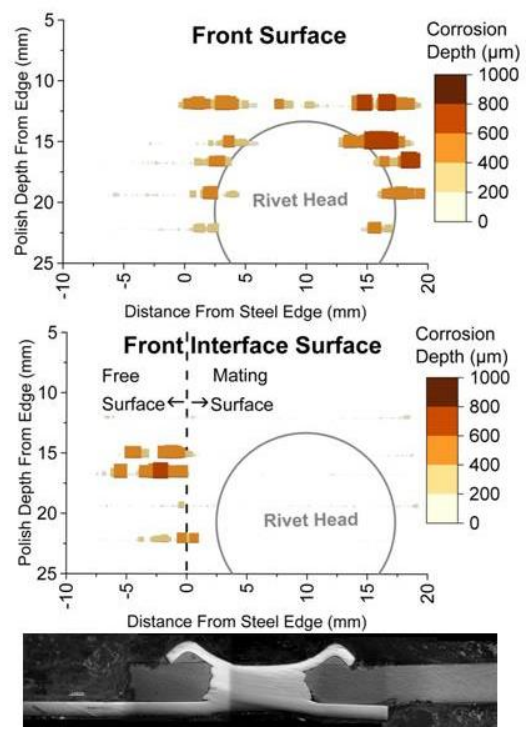

(a)
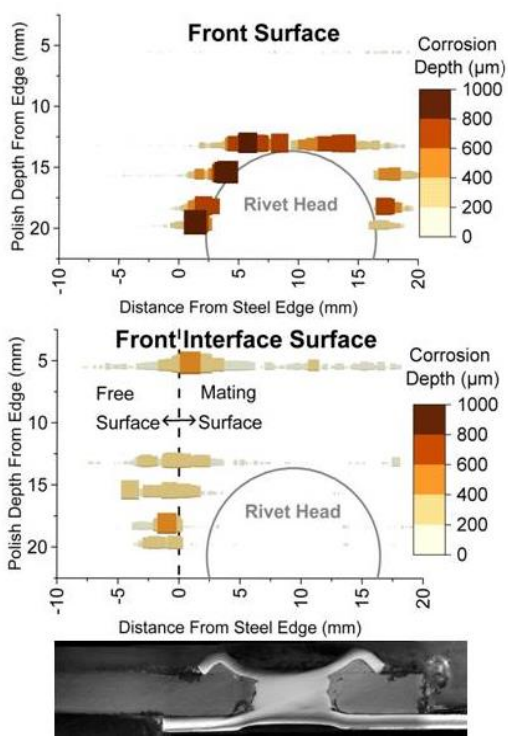

(b)
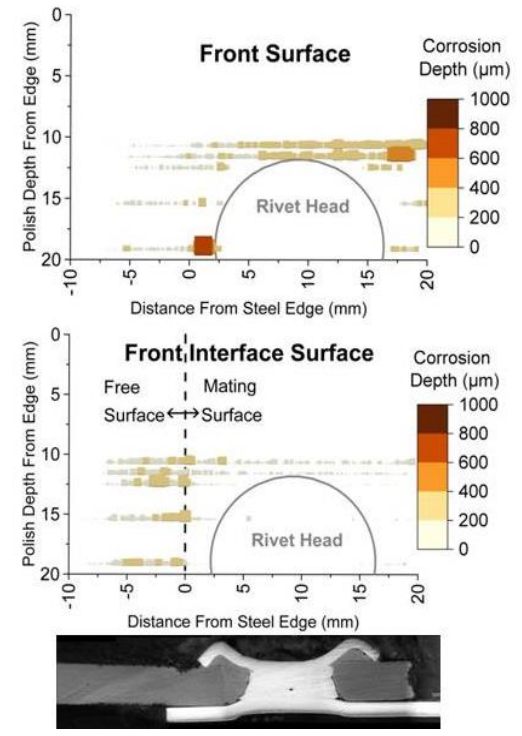

(c)

Figure II.3.7.11. Cross-section data of Aural2 joined to 980 steel via RSR after 32.4 days exposure: (a) ASTM B117;

(b) ASTM G85-A2; and (c) CCT-1. Source: OSU.

Mechanical loading results from Honda's proprietary cyclic corrosion standard tests, designated as CCT, are shown in Figure II.3.7.12 and Figure II.3.7.13 for three different piloted (rivet head side of the joint has a predrilled hole) conditions. A joint adhesive was present during welding between the $\mathrm{Al}$ and steel interfaces. Corrosion testing for both tensile shear strength (TSS) and cross-tension strength (CTS) samples manufactured during electrode life trials was then conducted. All samples were e-coated prior to exposure and various levels of corrosion mitigation were assessed including:

- $\quad$ E-coat only (baseline)

- $\quad$ Edge paint

- $\quad$ No edge paint, dust sealer applied to joint edges

- $\quad$ No edge paint, dust sealer applied to both edges and rivet head.

Figure II.3.7.12 shows the TSS results of Al and steel RSR joints exposed to CCT as compared to the baseline. In both rivet materials, samples without head or edge protection displayed an approximate $50 \%$ reduction in functionality and had clear signs of galvanic corrosion. Samples with edge protection only at the multi-material interface still displayed galvanic corrosion around the rivet head. Only samples with both head and edge protection had no immediate signs of galvanic corrosion but displayed similar strength performance to the controls. It was determined that, with the standard geometry TSS coupon employed, sufficient edge protection and general coverage of the fastener head were able to prevent immediate galvanic corrosion. 


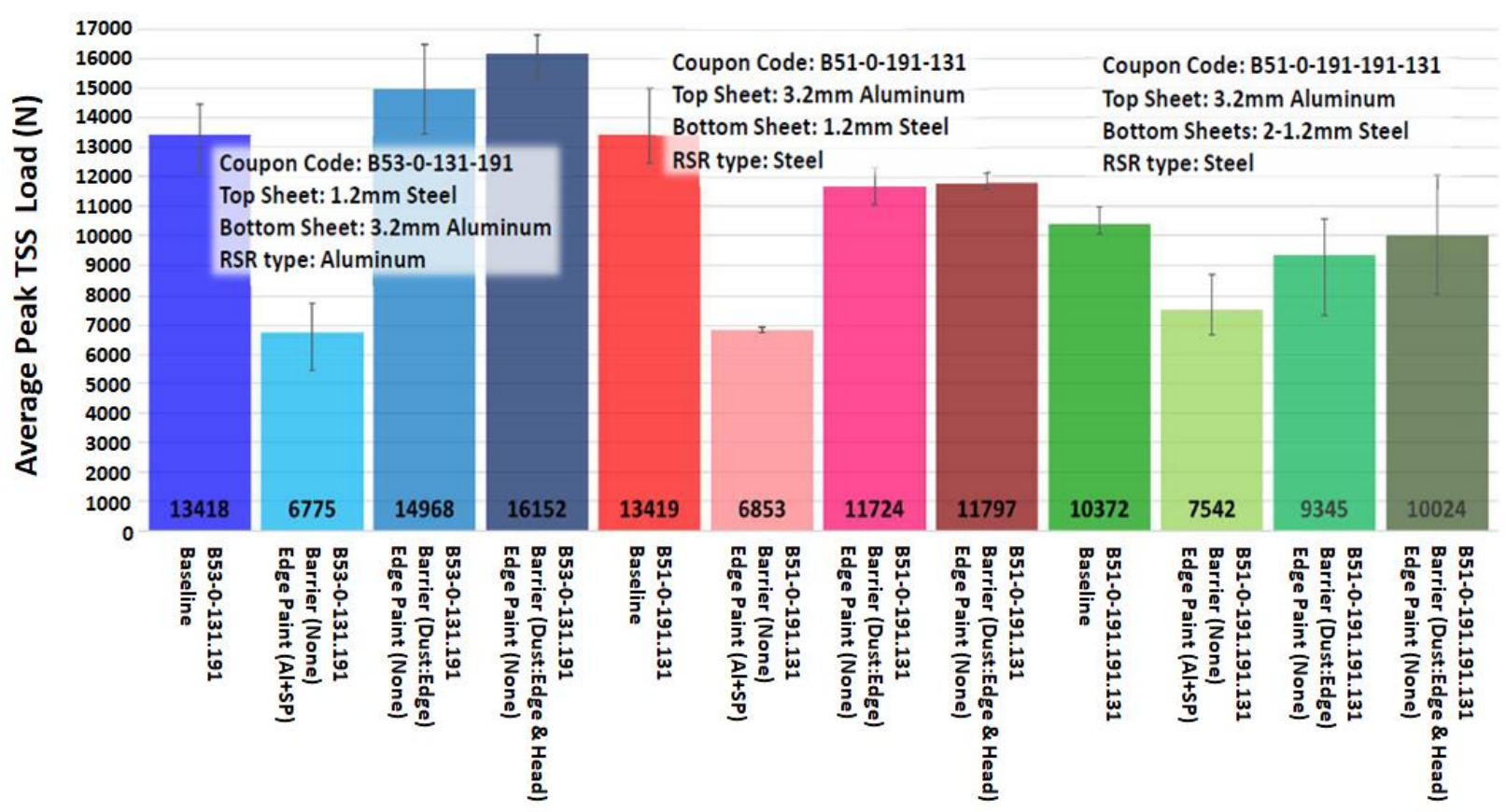

Figure II.3.7.12. Honda CCT results - TSS coupons (with Pilot Holes). Source: Honda.

Figure II.3.7.13 shows the CTS results for the same conditions in the previous figure. In terms of the Al RSR, samples without head or edge protection showed a larger range in functionality, attributed to ranges in galvanic corrosion. Also, samples with only edge protection at the multi-material interface had significant galvanic corrosion of and around the Al head. Samples with both head and edge protection had no immediate signs on galvanic corrosion in the joint area, but the resulting reaction force range was larger than the baseline, possibly due to surface corrosion variations affecting strength.

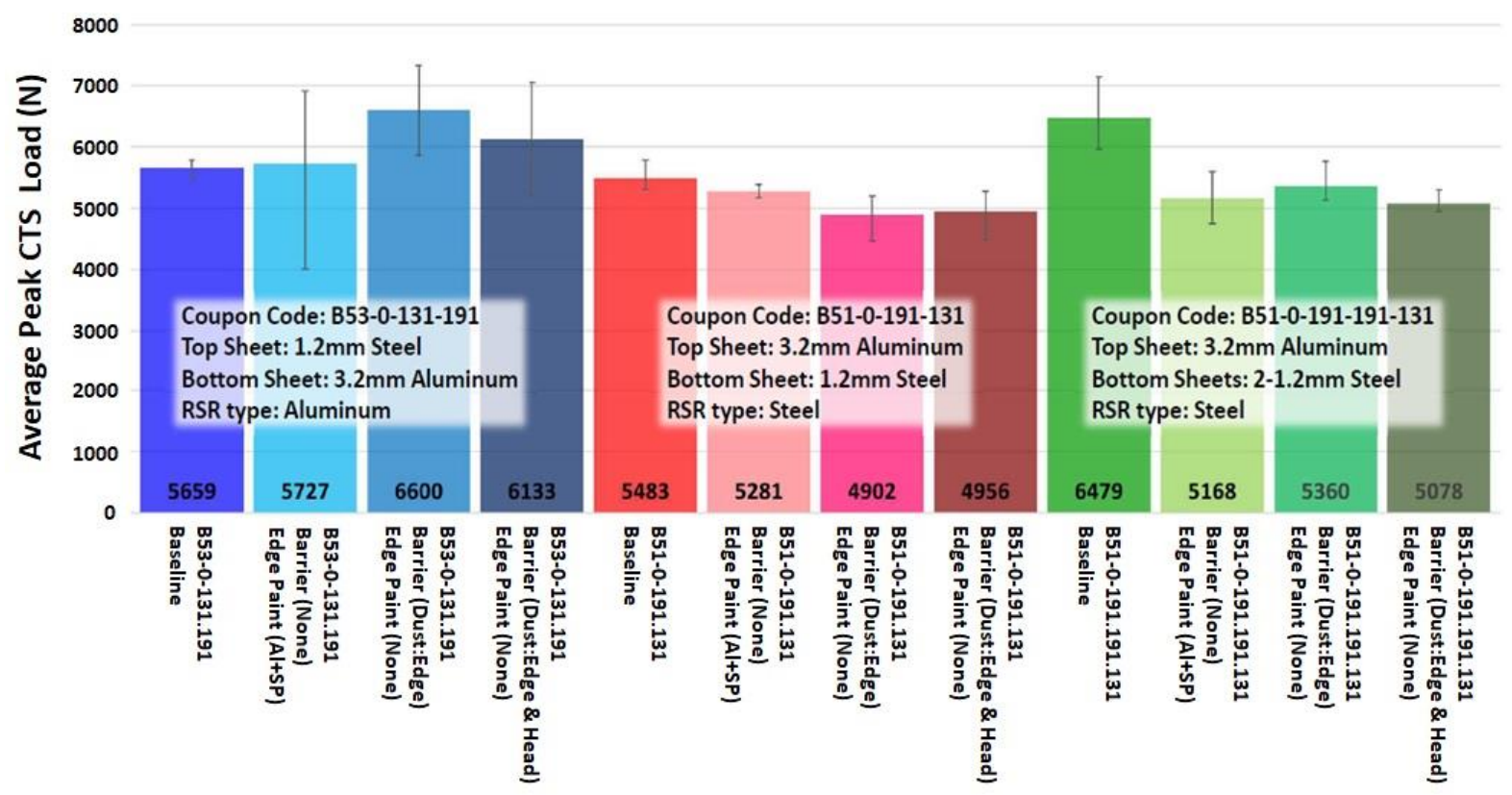

Figure II.3.7.13. Honda CCT test results - CTS coupons (with Pilot Holes). Source: Honda. 
A picture of the TSS samples showing the head and edge protection can be seen in Figure II.3.7.14 for the dust seal applied to both edges and rivet head for two steel RSR conditions. The steel RSR fasteners employed venting features to displace adhesive during the installation process. A visual (subjective) finding indicated that expulsion from head vents could create e-coat deposition flaws which can defeat the head coverage leading to galvanic corrosion and degraded corrosion toughness. Self-piloted (no predrilled holes) samples have not been fully evaluated but generally do not display the head venting expulsion. Overall the samples assessment showed the prospect to manage corrosion as well as the concerns of surface corrosion affecting toughness.

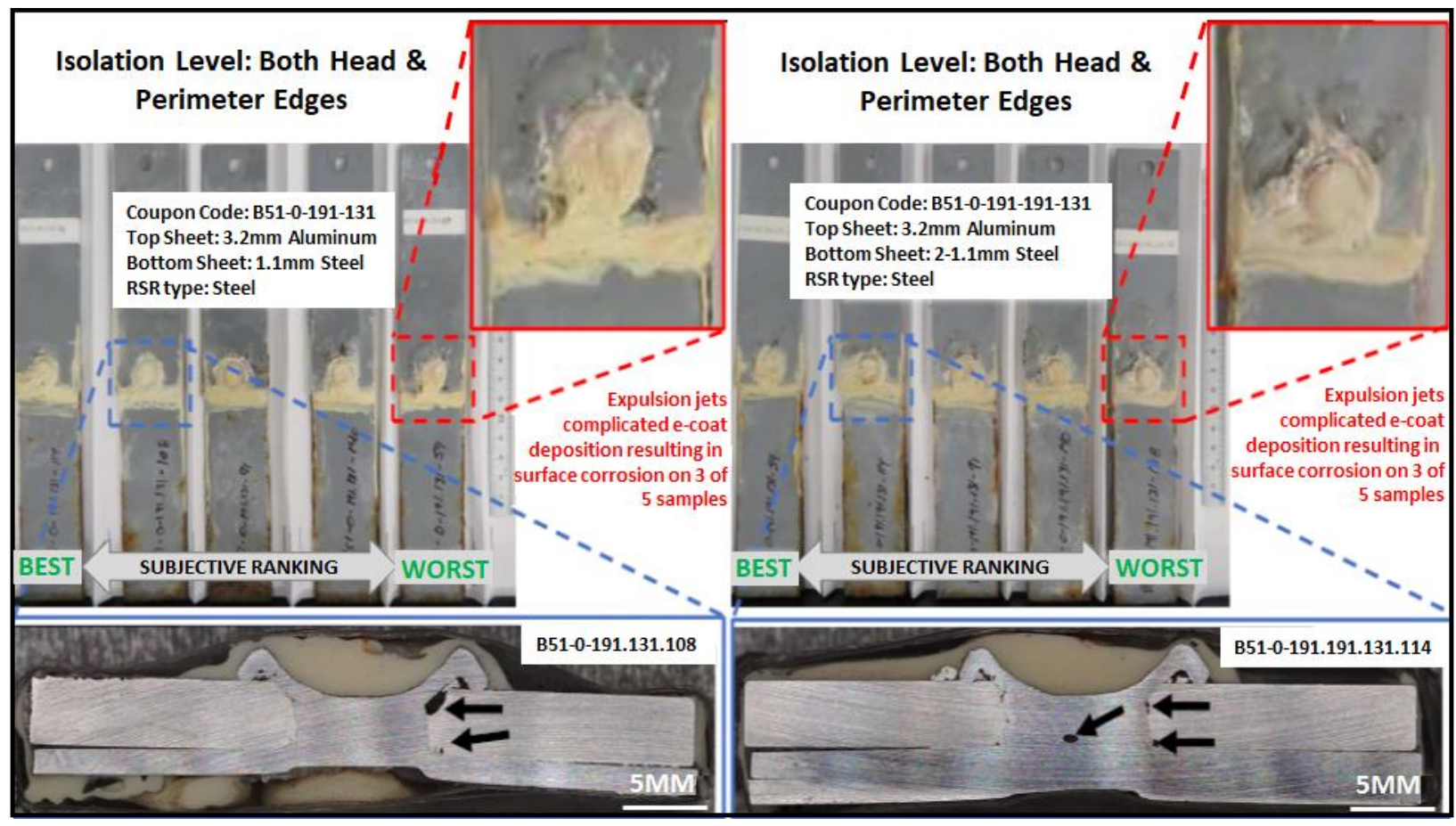

Figure II.3.7.14. Honda CCT TSS Coupons (with pilot holes). Source: Honda.

Table II.3.7.1 and Figure II.3.7.15 provide a description of the production conditions and range of variations evaluated for both piloted and self-piloted applications. The types of production conditions included part angularity, part gaps (between the $\mathrm{Al}$ and steel interfaces), rivet offset along and across the flange, flange overlap, and pilot hole diameter. These tests were meant to simulate issues associated with real-world occurrences in robotic welding and part fit up. Lap-shear tensile strength was evaluated for all production conditions with peel/pry checks (to understand cross-tension type loading) and weld sectioning. Honda provided guidance on the type of tests with the purpose of establishing the performance tradeoffs for each variable independently as a guide for production recommendations. 
Table II.3.7.1. Production Conditions.

\begin{tabular}{|c|c|c|}
\hline & $\begin{array}{c}\text { Piloted }(2 \mathrm{~mm}, 3 \mathrm{~mm} \mathrm{Al})-3 \text { Pilot Hole } \\
\text { Diameters }\end{array}$ & Self-Piloted (1, 3mm Al) \\
\hline Rivet Offset - Width & $\frac{1}{2}($ Pilot - Pin Diameter $) \pm 0.5 \mathrm{~mm}$ & Not Applicable \\
\hline Rivet Offset - Length & $\pm\left(\frac{1}{2}(\right.$ Pilot - Pin Diameter $\left.)+0.5 \mathrm{~mm}\right)$ & $\pm 1.0 \mathrm{~mm}, \pm 2.0 \mathrm{~mm}$ \\
\hline Angularity - Width & \multicolumn{2}{|c|}{$3^{\circ}, 5^{\circ}, 7^{\circ}$ Across TSS width } \\
\hline Angularity - Length & \multicolumn{2}{|c|}{$3^{\circ}, 5^{\circ}, 7^{\circ}$ Across TSS width } \\
\hline Gap & \multicolumn{2}{|c|}{$0.5,1.0,1.5,20 . \mathrm{mm}$} \\
\hline Flange Width & \multicolumn{2}{|c|}{$\begin{array}{c}20 \mathrm{~mm} \text { for Piloted } 2 \mathrm{~mm} / 3 \mathrm{~mm} \mathrm{AL} \text {, Self-Piloted } 1 \mathrm{~mm} \mathrm{AL} \\
22,24,26 \mathrm{~mm} \text { for Self Piloting }>3 \mathrm{~mm} \mathrm{AL}\end{array}$} \\
\hline Pilot Hold Diameter & $6,7,8 \mathrm{~mm}$ & Not Applicable \\
\hline
\end{tabular}

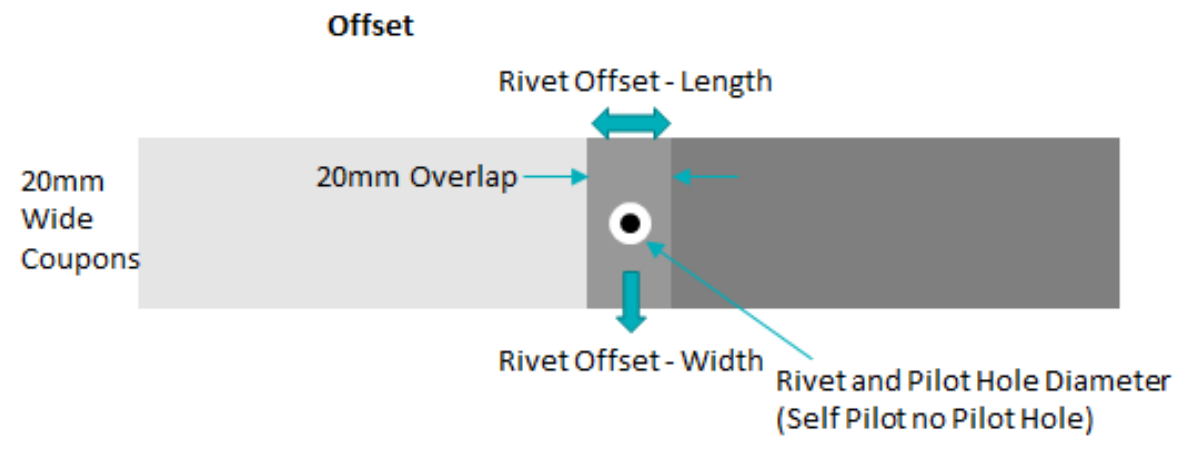

Angularity - Length

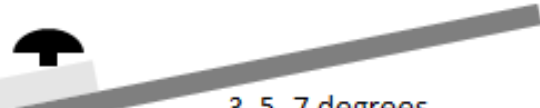

3, 5, 7 degrees

Angularity - Width

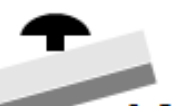

$3,5,7$ degrees

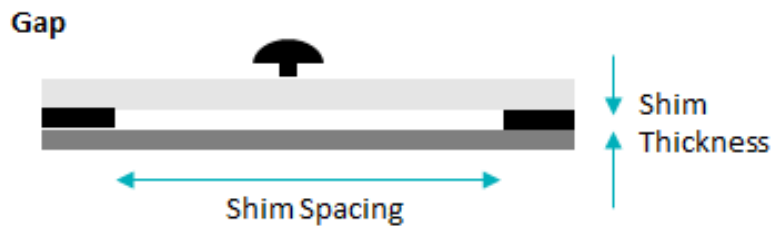

Figure II.3.7.15. Production condition specimen examples (e.g., rivet offset, angularity, gap). Source: Arconic.

Figure II.3.7.16 and Figure II.3.7.17 show the TSS changes measured as the angle was increased 3, 5, and 7 degrees from normal for the self-piloted (i.e., no hole condition). Figure II.3.7.16 shows the thin gauge Al while Figure II.3.7.17 compares the strength for the 3-mm Aural 2 casting. In all cases, acceptable strengths were achieved even under aggressive (5 and 7 degree) angles. 


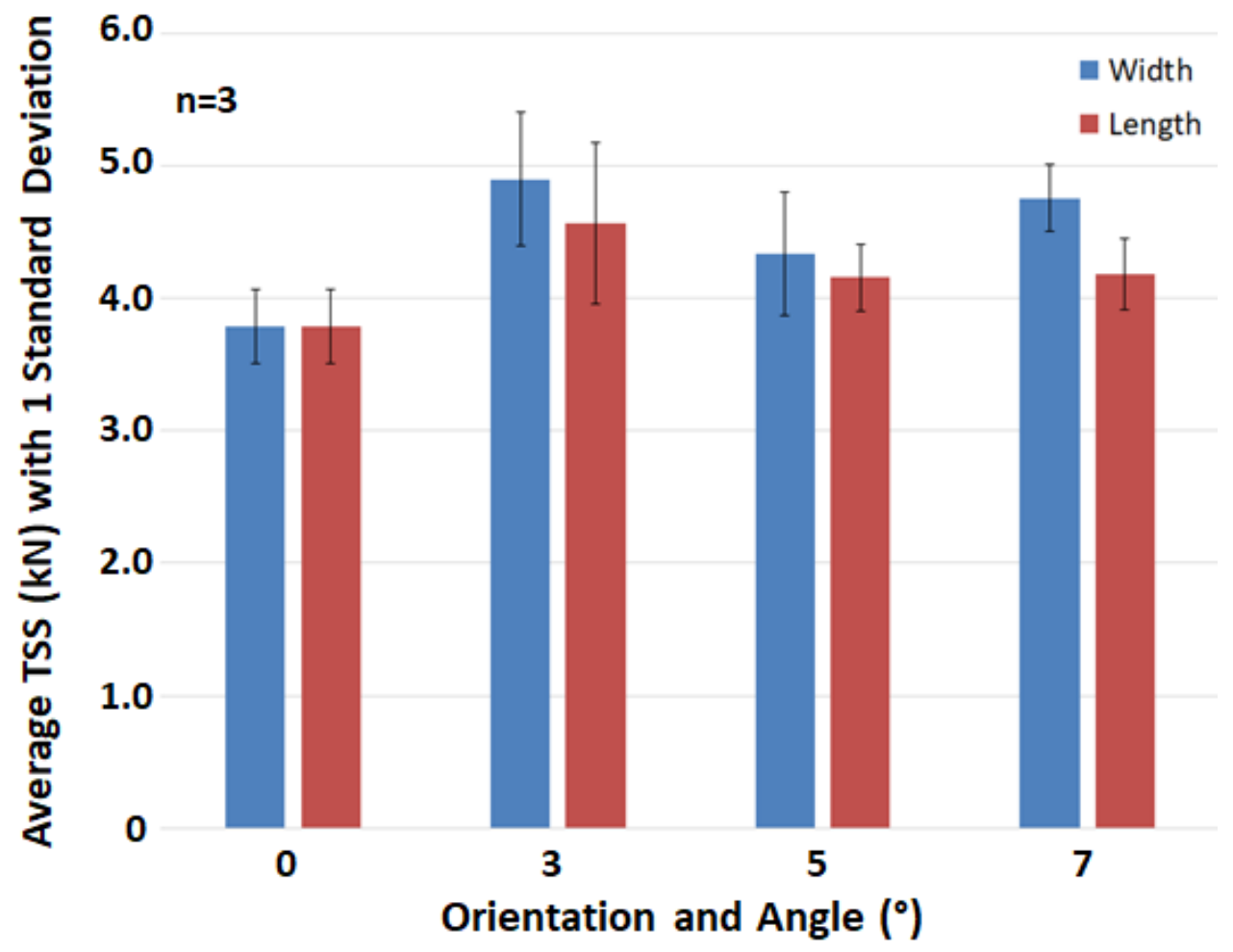

Figure II.3.7.16. Effect of angularity for self-piloted (no hole) joints between 1.0-mm MMHF-T4 Al and two sheets of 1.2-mm 980-MPa steel. Source: Arconic.

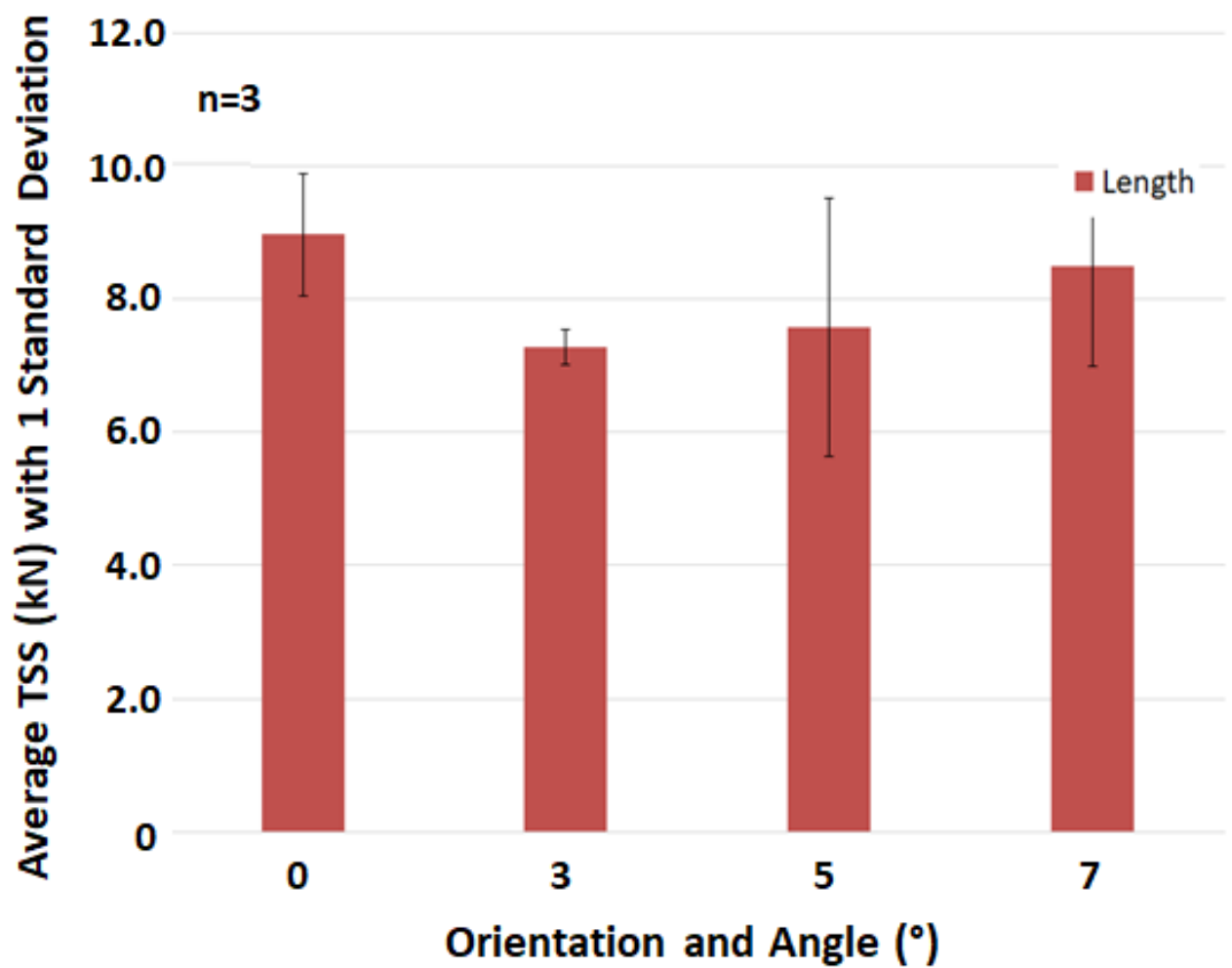

Figure II.3.7.17. Effect of angularity for self-piloted (no hole) joints between 3-mm Aural2-T7 Al and two sheets of 1.2-mm 980-MPa steel. Source: Arconic. 
Figure II.3.7.18 and Figure II.3.7.19 show the same combinations except for varying gaps between the $\mathrm{Al}$ and steel sheets. In both stack-ups, excellent lap-shear strengths were achieved for all gap conditions. It should be noted that three samples were created for each production condition and error bars represent one standard deviation.

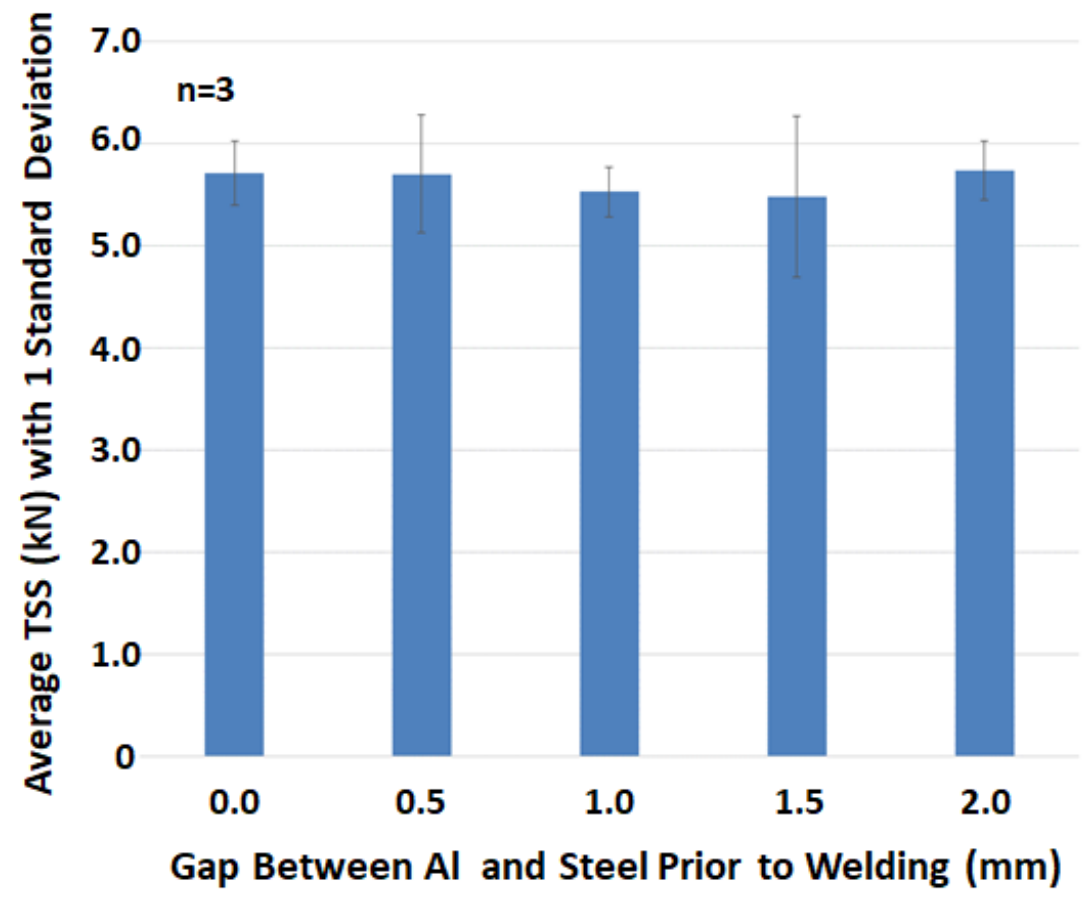

Figure II.3.7.18. Effect of gap testing for self-piloted (no hole) joints between 1.0-mm MMHF-T4 Al and two sheets of 1.2mm 980-MPa steel. Source: Arconic.

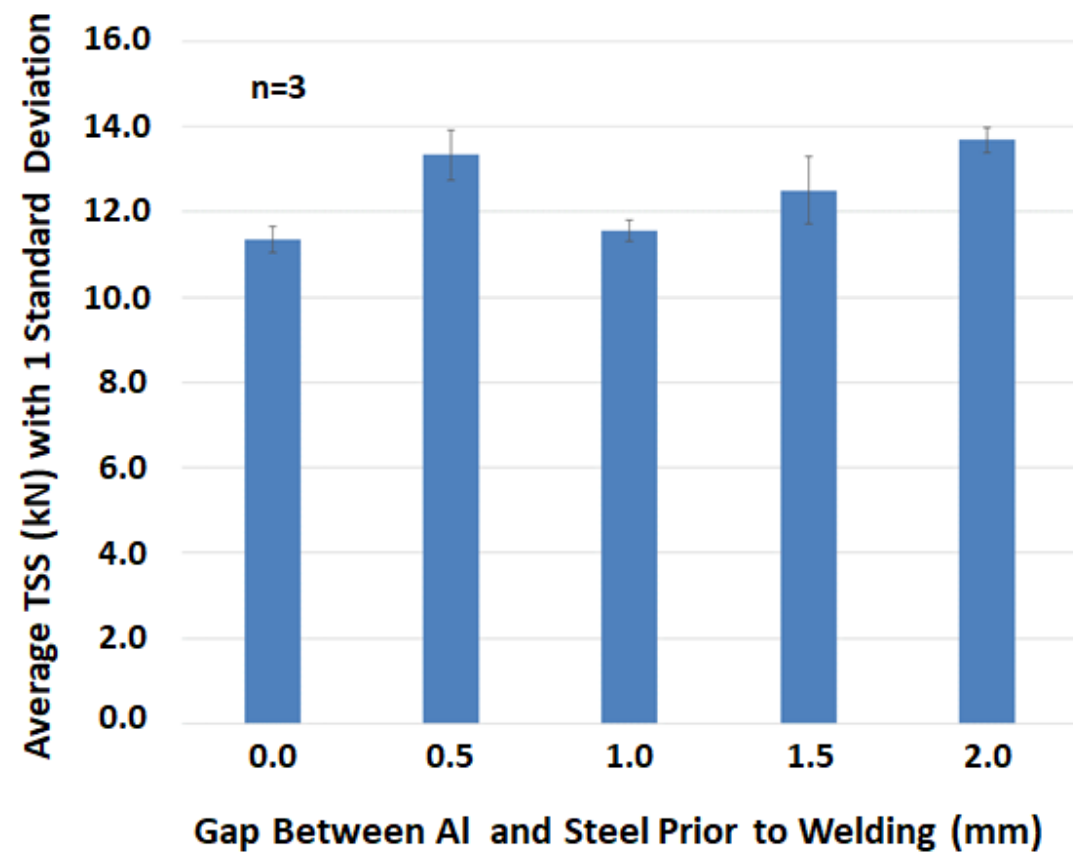

Figure II.3.7.19. Effect of gap testing for self-piloted (no hole) joints between 3-mm Aural2-T7 Al and two sheets of 1.2-mm 980-MPa steel. Source: Arconic. 
The final integration of the RSR RDS into an automated robotic cell was completed by AFS and its integrator CenterLine, Ltd. in the second quarter of FY 2019. The system was evaluated for feed reliability and achieved several thousand cycles without faulting. RSR welding trials were initiated on a double hat section, as shown in Figure II.3.7.20, in both a flat and vertical position, to demonstrate the ability to successfully feed rivets and weld in multiple positions. A meeting was held July 16, 2019 at CenterLine, Ltd. between Arconic, Honda, OSU, and DOE representatives. A full demonstration of the robotic RDS was provided during the meeting. The demonstration included all aspects of the RDS including bowl feeder, refill station, rivet magazine, and collet. In addition to the tour of the RDS, a status update of the BP3 demonstration articles was provided by Honda.

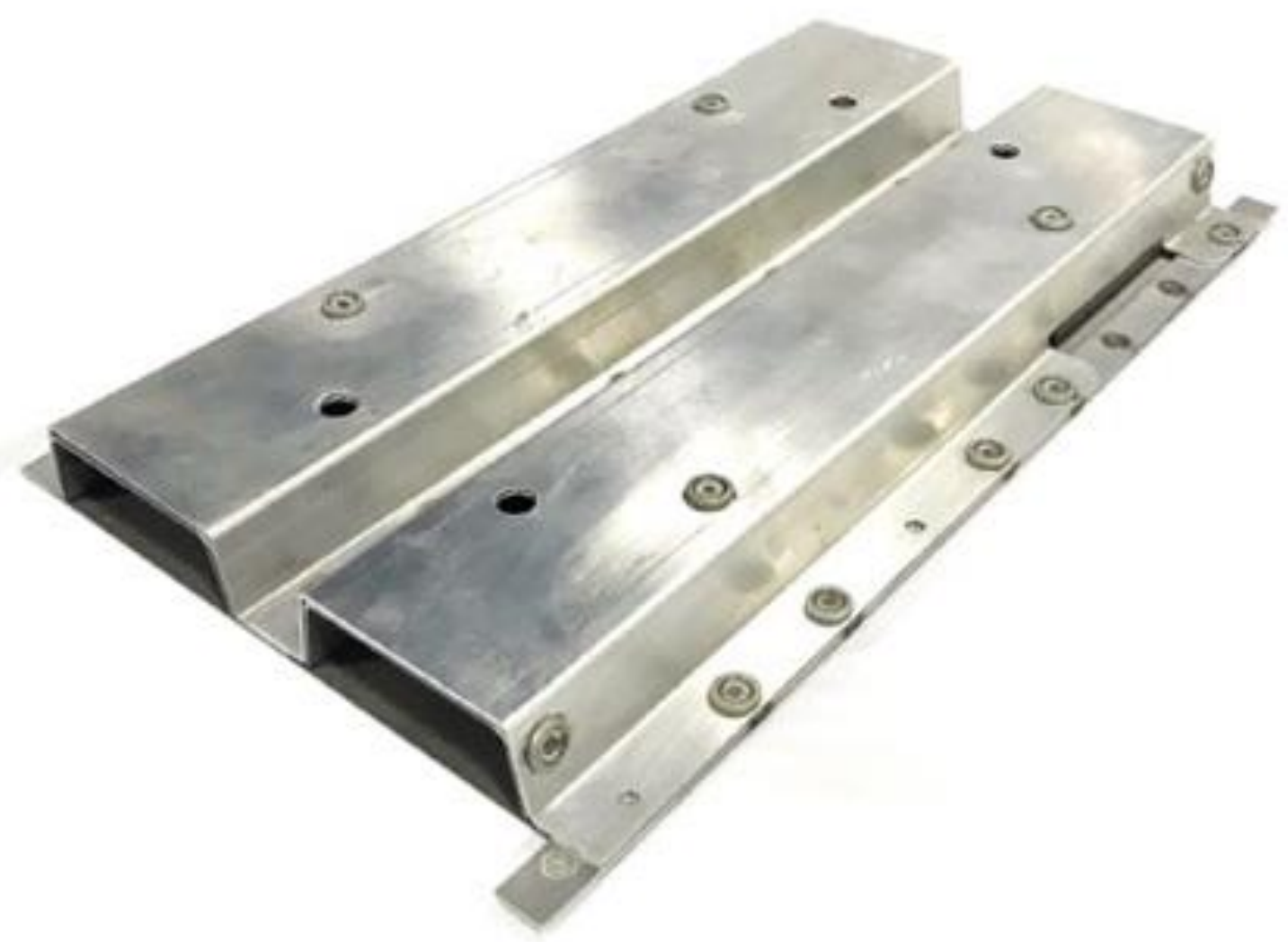

Figure II.3.7.20. Double hat assembly used to demonstrate multi-axis RSR robotic capability. Source: Arconic.

Honda completed the initial design of the demonstrator and assembly tools/sequence as shown in Figure II.3.7.21 and Figure II.3.7.22. FEA assessments of the demonstrator, tool design, manufacturing sequencing, part fabrication, and component assembly were scheduled for completion. 
Initial Design Concept (Mar 2019

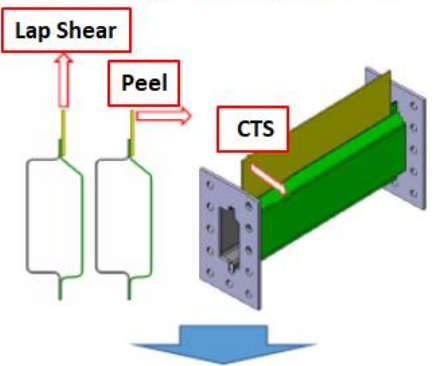

Finalized Design (Sep 2019)

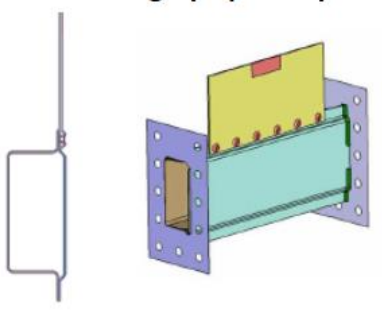

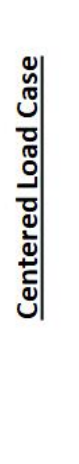
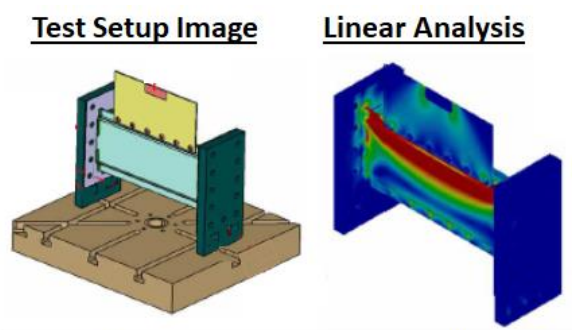

Basic test frame setup used to conduct preliminary linear analysis, affirming stress flow patterns to validate fastener load trends. Non-linear analysis used to predict functional response levels and potential failure patterns.
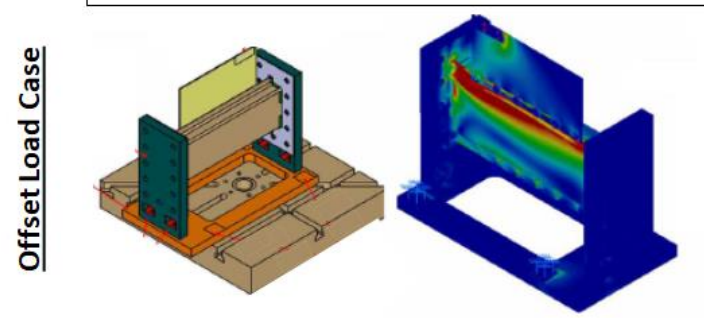

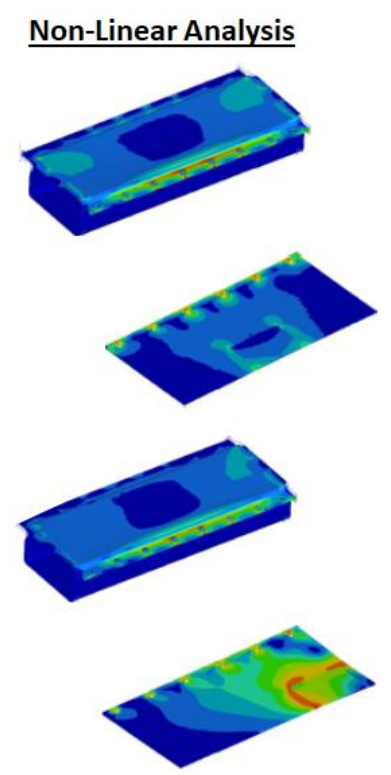

Figure II.3.7.21. Demonstration assembly process with the first step to design maturation. Source: Honda.

\section{Assembly sequence for steel sample preparation}
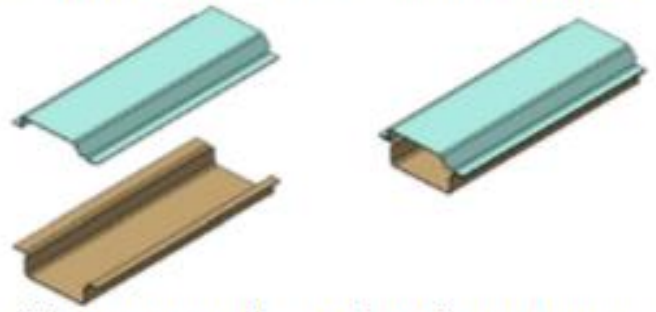
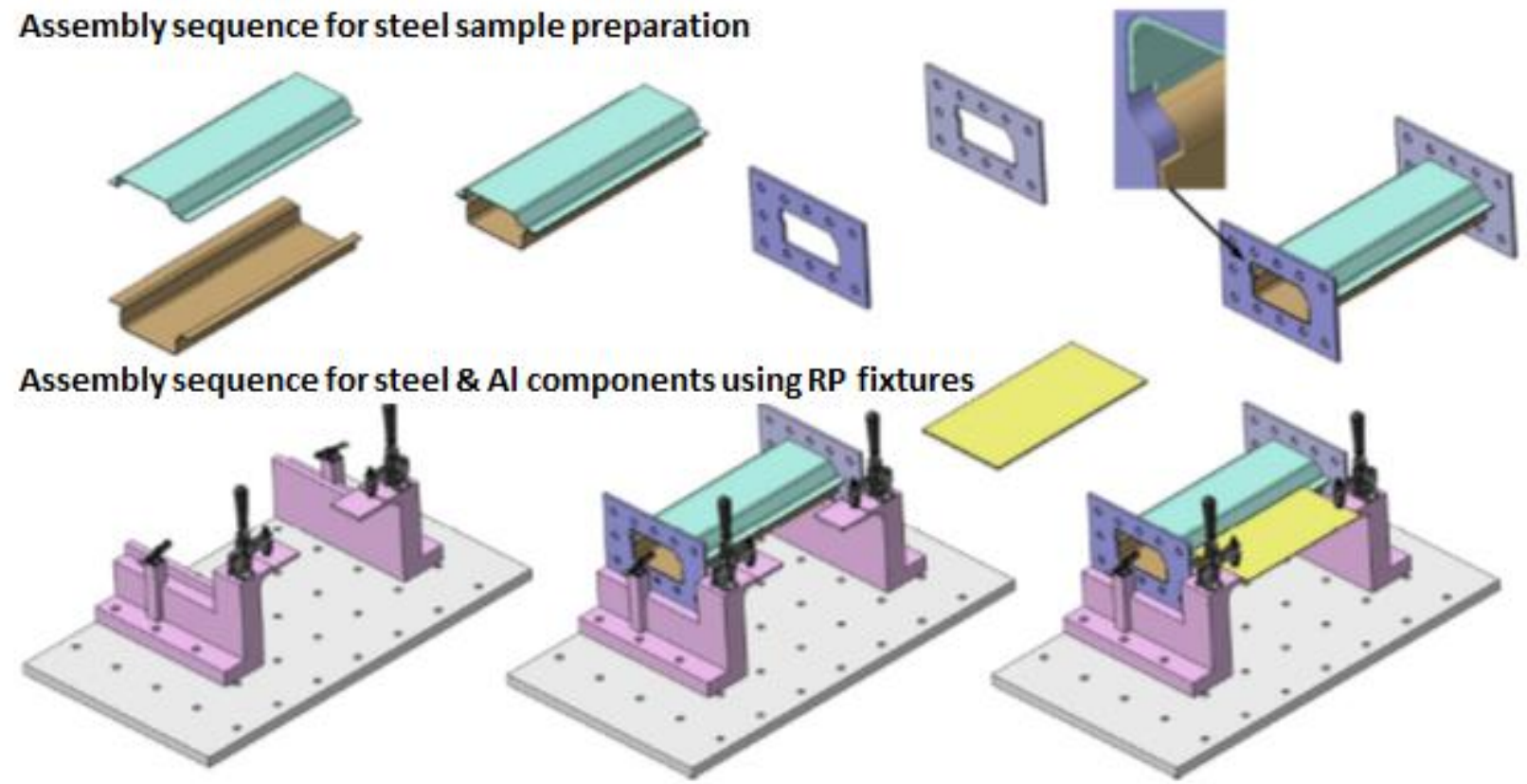

Figure II.3.7.22. Demonstration assembly manufacturing sequences. Source: Honda.

\section{Conclusions}

Corrosion work under a variety of test protocols neared completion at both OSU and Honda including joints between $\mathrm{Al}$ to steel and $\mathrm{Al}$ to CFRP. OSU observed that the orientation of the samples in the corrosion chamber can influence the results, and additional testing was commissioned to model the impact for RSR and competing technologies for a variety of joint combinations. While the overall trends remained similar between the various joining technologies, the orientation can influence the aggressiveness of the galvanic corrosion pitting so special care must be noted when comparing technologies. Additionally, OSU has completed testing under the ASTM B117, ASTM G85-A2, and CCT-1 methods, and observed the severity of corrosion varied among different material combinations. 
Honda has completed the majority of the CCT testing under various production scenarios, including the influence of e-coat and paint seal (along the joint and rivet head) for the piloted Al to steel combinations. In both rivet materials, samples without head or edge protection displayed a wider range in functionality, which included both objective and subjective categories (most of the functionality was based on strength) and had clear signs of galvanic corrosion. Samples with edge protection only at the multi-material interface still displayed galvanic corrosion around the rivet head. Only samples with both head and edge protection had no immediate signs of galvanic corrosion and displayed similar strength performance to the controls. It was determined that, with the standard geometry for the TSS and CTS coupons employed, sufficient edge protection and general coverage of the fastener head was able to prevent immediate galvanic corrosion.

Production conditions including part angularity, gaps (between the A, and steel interfaces), rivet offset, flange overlap, and pilot hole diameter were evaluated for both piloted and self-piloted applications. These tests were meant to simulate issues associated with real-world occurrences in robotic welding and part fit up. Lap-shear tensile strength was established for a wide range of production conditions broader than typical automation.

The final integration of the RSR RDS into an automated robotic cell was completed by AFS and its integrator CenterLine, Ltd. The system was evaluated for feed reliability, achieving several thousand cycles without faulting. RSR welding trials were initiated on a double hat section in both a flat and vertical position, which demonstrated the ability to successfully feed rivets and weld in multiple positions. Honda completed the initial design of the demonstrator and assembly tools/sequence. The tool design, manufacturing sequencing, part fabrication, and component assembly will initiate in Q4.

\section{References}

1. ASTM International, 2018, Standard Practice for Operating Salt Spray (Fog) Apparatus, ASTM B117-18, West Conshohocken, PA, USA. https://doi.org/10.1520/B0117-18.

2. ASTM International, 2018, Standard Practice for Modified Salt Spray (Fog) Testing, ASTM G85-11, West Conshohocken, PA, USA. https://doi.org/10.1520/G0085-11. 


\title{
II.3.8 Adhesive-Bonding of CF Reinforced Polymer to Advanced High-Strength Steels (Oak Ridge National Laboratory)
}

\section{Zhili Feng, Co-Principal Investigator}

Oak Ridge National Laboratory

1 Bethel Valley Rd.

Oak Ridge, TN 37831

E-mail: fengz@ornl.gov

\author{
Kevin Simmons, Co-Principal Investigator \\ Pacific Northwest National Laboratory \\ 902 Battelle Blvd. \\ Richland, WA 99354 \\ E-mail: kl.simmons@pnnl.gov
}

\section{Sarah Kleinbaum, DOE Technology Development Manager}

U.S. Department of Energy

E-mail: sarah.kleinbaum@ee.doe.gov

Start Date: October 1, 2017

Project Funding (FY19): \$750,000

End Date: September 30, 2020

DOE share: $\$ 750,000 \quad$ Non-DOE share: $\$ 0$

\section{Project Introduction}

Adhesive-bonding is widely used in the automotive industry, primarily for structurally non-critical components. For structurally critical components, weld-bonding that combines adhesive-bonding and spot welds is more common for joining lightweight materials to meet performance requirements in body stiffness, crash and safety performance, and enhanced noise-vibration-harshness characteristics. These lightweight materials include joining AHSS to AHSS, or Al alloys to other $\mathrm{Al}$ alloys, and fiber-reinforced polymers. Innovations in adhesive-bonding would allow for the development of new and innovative designs of mixed materials - high-strength steels, non-ferrous metals, plastics, and composites - that provide more efficient assembly and weight reductions.

Purely adhesive-bonded joints historically suffer from limitations in peel strength, brittle cleavage failure, relatively low toughness, long-term aging degradation, and poor environmental performance. They also have low crack-arrest resistance in dynamic crash events under compressive load. For dissimilar material bonding, a number of unique challenges must be addressed. They include tailoring adhesives to provide strong bonding for two different materials, compatibility with mismatches in coefficients of thermal expansion, and galvanic corrosion effect.

This multiyear early stage R\&D project plans to address several key aspects of adhesive-bonding, including the effects of surface conditions of substrates, adhesive chemistry and additives, long-term performance and degradations, inhibition of galvanic corrosion, compatibility with the CTE mismatch, health monitoring of curing and manufacturing processes, and structural soundness in service. Overcoming each of these factors or their combination could lead to significant advancements in multi-material joining. 


\section{Objectives}

This project focuses on the fundamentals of adhesive-bonding of CFRP to AHSS. In concert with a parallel interface by design simulation task, innovative adhesive-bonding concepts are identified and explored, and predictive tools are developed for adhesive-bonding performance, joint design, and lifetime prediction. The goal is to significantly improve the performance and productivity of adhesive-bonding in high-volume autobody production to enable increased use of CFRP in multi-material body structures for weight reduction. ORNL and PNNL are working together on this research to effectively utilize the complementary research capabilities, facilities, and technical knowledge of two National Laboratories to achieve the project goals.

\section{Approach}

Figure II.3.8.1 outlines the overall research approach and plan. It is organized into four major categories: key governing factors, methods to improve and innovations, interface/adhesive characterization, and joint properties. In recognizing the nature and risks associated with early stage research, a phased approach will be taken in proposed R\&D. The first phase is a 12-18-month effort to explore the feasibility of concept and ideas identified as promising. Depending on the outcome from the first phase effort, promising alternatives will be down-selected for more-comprehensive R\&D in later phases of the project. Major R\&D activities in FY 2019 were marked with red-outlined boxes in Figure II.3.8.1.

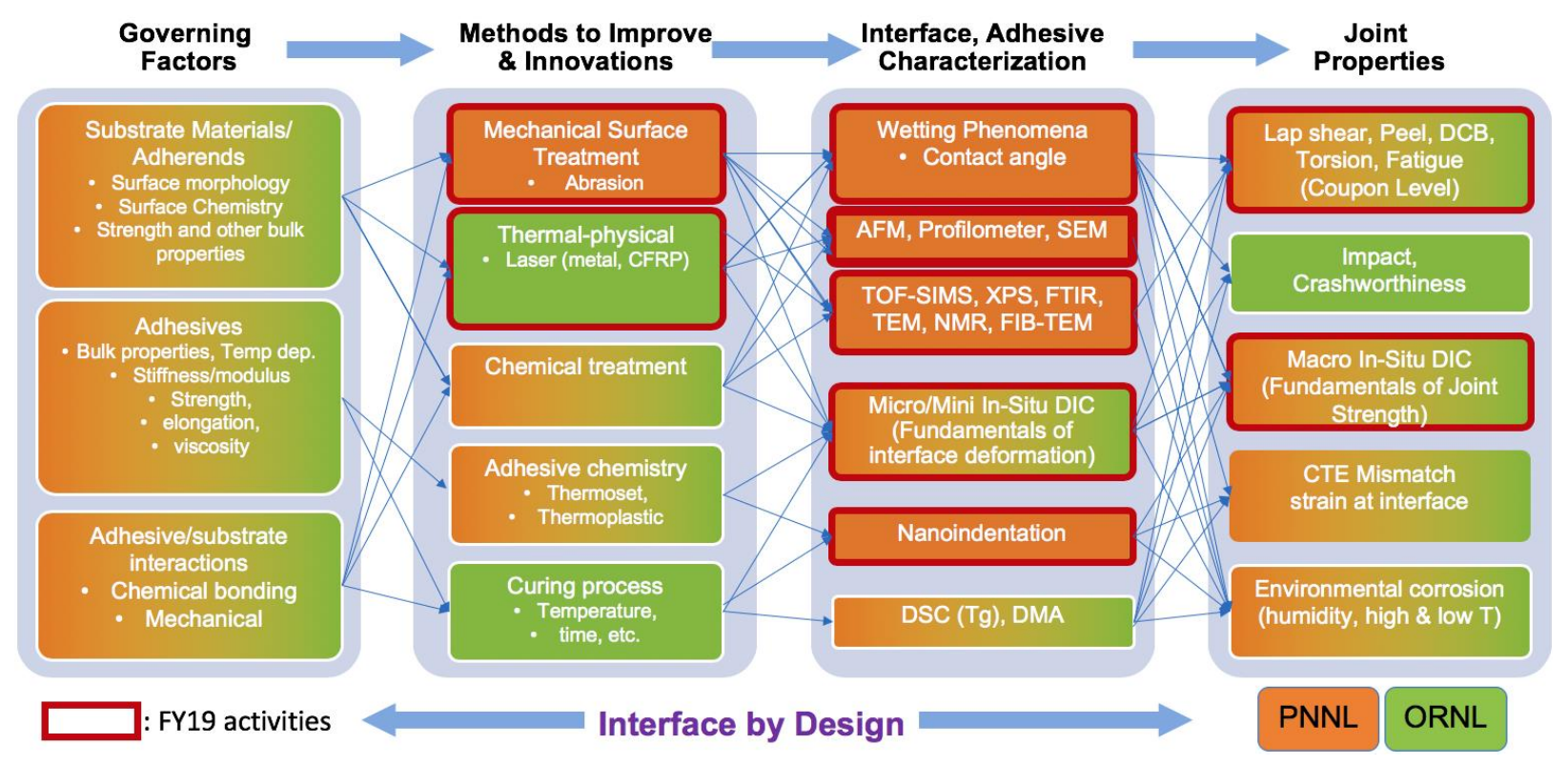

Figure II.3.8.1. Overall research plan. Source: ORNL.

The proposed research will closely interact with a parallel interface by design simulation task. Design concepts from the simulation task directs the experimental research in this work. At the same time, data from experimental work in this project assists the simulation effort and provides the necessary experimental validation of the simulations.

Chemical bonding of adhesive to the interface of the substrate material requires characterization by chemical analysis of surface and surface roughness. The dissimilar materials of interest have varied chemistry. The steel surface chemistry has a variety of metal oxides based on the alloying elements of $\mathrm{Si}, \mathrm{Mn}$, and $\mathrm{Cr}$ in $\mathrm{Fe}$. Galvanized-coated metal has a different set of surface chemistries and is more difficult to adhesively bond. Traditional adhesive-bonding typically has special surface preparation instructions to achieve the best bond line performance to metals. 
Polymer composite systems have a completely different set of chemistries that can be even more complex. These materials are also surface-modified to maximize adhesive performance. The chemistry of these surfaces influences the interphase region, which is a transition zone from the surface into the bulk adhesive. Characterization of the interface and its chemical influence into the transition zone will be important to optimize bonding and understand how it changes over time in extreme environments.

It is well-known that surface conditions (e.g., chemistry, roughness, morphology) significantly influence adhesive-bonding strength. Techniques that alter surface conditions (e.g., laser surfacing, mechanical abrasion) have demonstrated benefits; however, there is a lack of understanding of the surface condition and morphology most appropriate to improve bonding. With interface by design modeling tools, it is possible to proactively design surface conditions for bonding. This task will take the designs and concepts from interface by design simulations and identify surface-modification processes and methodologies to implement such interface-design concepts to achieve improved bonding strength. Mechanical testing capabilities will provide full characterization of substrate and adhesive materials. The testing will also provide joint efficiency performance. With new real-time imaging techniques (such as digital image correlation (DIC)) for analysis, we will focus on understanding how fracture and failure are occurring and how that changes with environmental stresses over time. The data from this task will also be provided as inputs to the lifetime-performance predictions for model correlation and validation.

\section{Results}

\section{Interface and Bondline Characterization and Property Testing}

After evaluating surface characteristics of the different selected adherends (e.g., thermoplastic CFRP [polyphthalamide] [PPA] with $40 \%$ short carbon fiber, thermoset laminated carbon fiber/epoxy [Toray G83C_7-11], and dual-phase 980 steel, we focused on evaluating the adhesive-bonding strength of as-received (AR) surfaces of thermoplastic carbon fiber reinforced polymer polyphthalamide (TP-CFRP-PPA) and thermoset carbon fiber reinforced polymer (TS-CFRP) to DP 980 without zinc coating. Adhesive joint strength is a function of bond area, surface chemistry, and varies with adhesive bondline thickness. Adherend test geometries were cut by a waterjet into 25.4- $\mathrm{mm} \times 101.6-\mathrm{mm}$ plaques and shear-machined for DP980. As summarized in Table II.3.8.1, different modulus epoxy-based adhesives from Dow, L\&L Products, and 3M were applied and thermally cured according to the manufacturer's instructions. To protect business-sensitive information for each adhesive company, name and modulus of each adhesive is labeled as a numeric number and high, mid, or low, based on the modulus category. Orientation of adherend pieces during bonding was maintained using engineered jigs. Bondline thickness was initially controlled using glass spheres of a known diameter (i.e., $250 \mu \mathrm{m}$ ) in the bondline. A 25.4-mm overlap was used for the lap-shear coupon. Different material combinations were used to evaluate the adhesive bond strength with various epoxy-based adhesives.

Table II.3.8.1. Summary of Various Adhesives with Different Modulus.

\begin{tabular}{|c|c|c|c|c|c|c|}
\hline Adhesive & Adhesive 1 & Adhesive 2 & Adhesive 5 & Adhesive 6 & Adhesive 7 & Adhesive 8 \\
\hline Modulus & $\begin{array}{c}>2000 \\
\mathrm{MPa}\end{array}$ & $\begin{array}{l}\text { Between } \\
1000 \text { and } \\
2000 \mathrm{MPa}\end{array}$ & $\begin{array}{l}\text { Between } \\
1000 \text { and } \\
2000 \mathrm{MPa}\end{array}$ & $\begin{array}{c}<1000 \\
\mathrm{MPa}\end{array}$ & $\begin{array}{c}<1000 \\
\mathrm{MPa}\end{array}$ & $\begin{array}{c}<1000 \\
\mathrm{MPa}\end{array}$ \\
\hline $\begin{array}{c}\text { Modulus } \\
\text { Label }\end{array}$ & High & Mid & Mid & Low & Low & Low \\
\hline
\end{tabular}

Figure II.3.8.2 summarizes lap-shear failure load for steel/steel and steel/TS-CFRP cases. No surface treatment on adherends was applied. In general, lap-shear peak-failure load is increased with increased modulus of adhesive. This result can be used as a guidance for automotive industries where different desired application (e.g., structural, semi-structural, and sealing/elastic bonding) properties are required. Also, fracture load for steel-steel is higher than for the steel-TS-CFRP case. All lap-shear samples showed a cohesive failure mode where the bulk layer of the adhesive fails. It is usually the desired mode of failure for adhesive-bonding. 


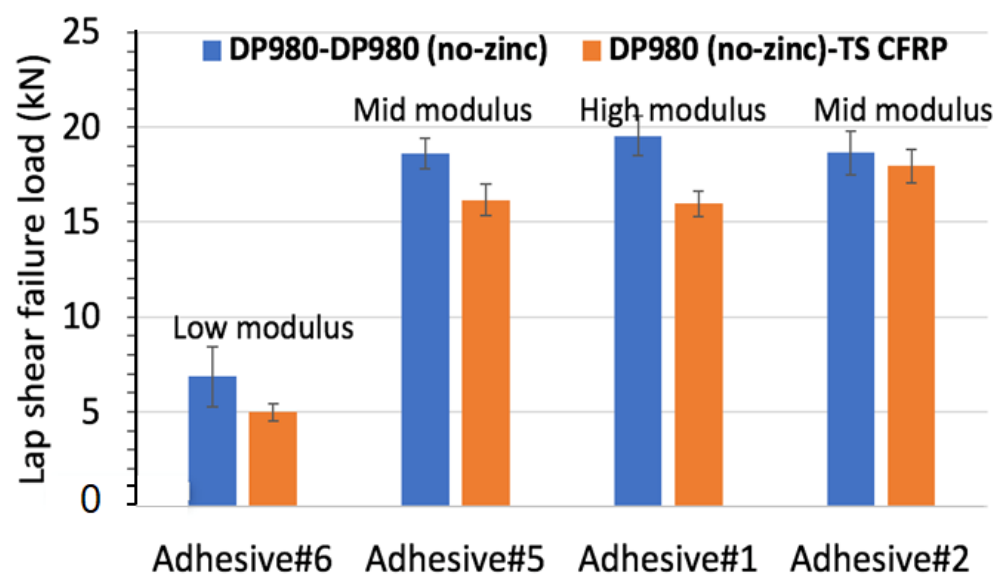

Figure II.3.8.2. Summary of adhesive-bonding strength for DP980-DP980 (no-zinc) and DP980 (no-zinc)-TS-CFRP with different modulus of adhesives. Source: ORNL.

Next, we evaluated adhesive-bonding strength of TP-CFRP-PPA to DP980 with the same lap-joint configuration and dimensions used as TS-CFRP to DP980. Table II.3.8.2 provides a quick summary of lapshear tensile testing results with different modulus of adhesives. Adhesive 1 shows the highest peak-failure load, compared to the other two adhesives, which is potentially due to its higher modulus. For Adhesives \#7 and \#8, average peak fracture loads were 6.89 and 5.97, respectively, and a failure mode was the adhesive failure at the interface between the adhesive and the AR TP-CFRP-PPA surface. This may be due to the low surface energy of polymer composite which results in lower adhesion between adhesive and AR polymer surface. Therefore, an engineered surface-modification on the adherend is required to further enhance the adhesive-bonding strength.

Table II.3.8.2. Summary of Various Adhesives.

\begin{tabular}{|l|c|c|c|}
\hline Adhesive & $\begin{array}{c}\text { Adhesive } \\
\text { Modulus }\end{array}$ & $\begin{array}{c}\text { Average Lap- } \\
\text { Shear Failure } \\
\text { Load }(\mathbf{k N})\end{array}$ & Failue Location \\
\hline Adhesive 1* & High & $8.70 \pm 0.80$ & Base TP-CFRP-PPA \\
\hline Adhesive $7^{* *}$ & Low & $6.89 \pm 0.49$ & Adhesive failure between adhesive and TP-CFRP-PPA \\
\hline Adhesive 8 $^{* *}$ & Low & $5.97 \pm 0.91$ & Adhesive failure between adhesive and TP-CFRP-PPA \\
\hline
\end{tabular}

*DP980: 1.2-mm thickness, * ${ }^{*}$ DP980: 1.0-mm thickness.

To further study the localized deformation of adhesive layers and adjacent adherends with high-resolution and high magnification images, a dedicated microscale DIC system with customized tensile machine was designed and developed in FY 2019. Because higher magnification is required to more accurately monitor and capture interface phenomena between two adherends, field of view is limited. That is, the smaller the field of view, the higher the magnification. For this reason, a small miniature tensile coupon (25.4-mm-long and 5-mm wide) was designed and fabricated which resulted in a field of view in the strain field map of approximately $2.0 \mathrm{~mm} \times 1.3 \mathrm{~mm}$. A $2.0-\mathrm{mm}$ overlap was used for miniature lap-shear coupons. In addition, it is difficult to use a conventional tensile machine for so small a miniature coupon, so a customized tensile machine was developed. Figure II.3.8.3 shows shear-strain maps in an adhesive layer at the interface between DP980 and TP-CFRP-PPA during tensile shear testing and the fractography after final failure. Relatively large shear-strain was developed at the interface between the adhesive and the adherends. At that point, it failed at the interface between adhesive and TP-CFRP-PPA side. This can be due to relatively low surface energy of TP-CFRP-PPA, compared with DP980 surface. For this reason, adhesion on the TP-CFRP-PPA side is lower than the adhesion on the DP980 side. This advanced optical measurement technique can be further used to study adhesive deformation and interface characteristic for adhesive-bonded samples after environmental testing. 


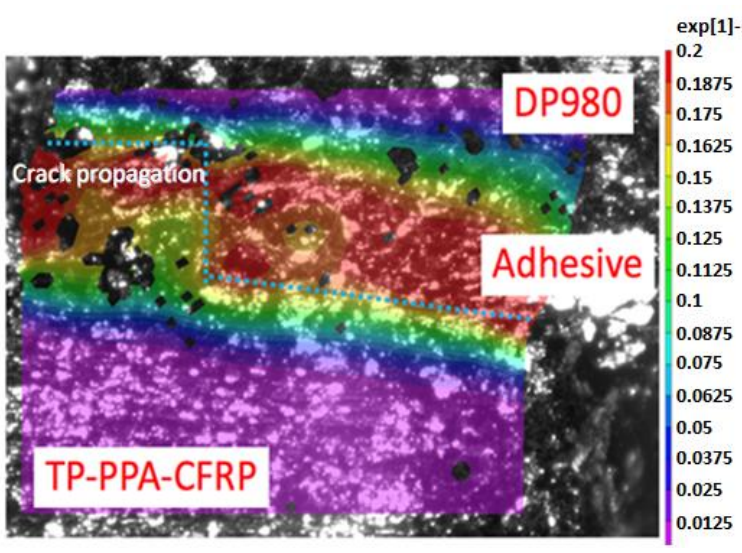

(a)

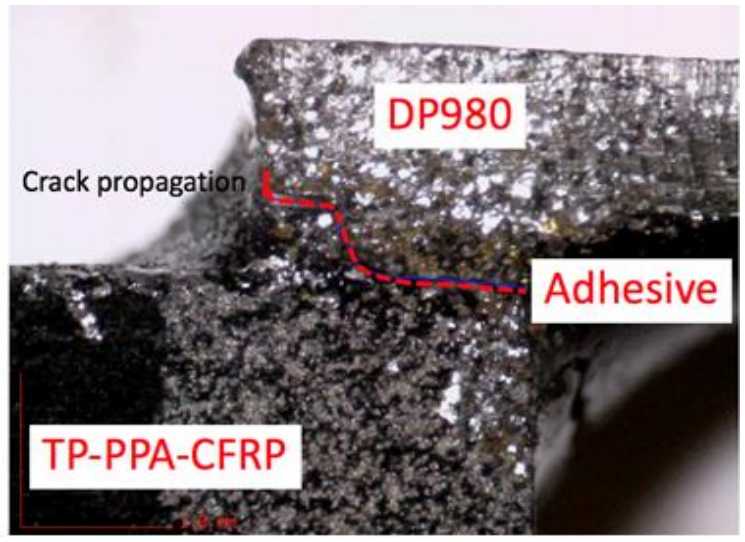

(b)

Figure II.3.8.3. (a) Shear-strain map from the micro-DIC image with the strain field during lap-shear tensile testing and (b) the fractograph of the sample. Source: ORNL.

\section{Surface Energy and Related Surface Characterization}

Adhesive-bonding strengths of TP-CFRP-PPA and DP980 show a relatively lower strength compared with TS-CFRP and DP980. In addition, adhesive fails at the interface between TP-CFRP-PPA and the adhesive, while cohesive failure mode was found for the TS-CFRP and DP980 case. For this reason, adherend (i.e., TP-CFRP-PPA) surface-modification was applied. Various surface-modification methods - such as laser, plasma, chemical, and mechanical abrasion — can be used to enhance adhesive-bonding strength. Laser ablation uses a focused high-energy density beam to modify surface profiles in a non-contact and precise way. During laser ablation, localized plasma can form, so additional chemical surface treatment is possible with different shield gas. In this task, pulsed-laser surface texturing was studied using a technique developed at ORNL to increase adherend surface roughness for improved adhesive-bonding. The profilometry profiles for a lasersurface-textured TP-CFRP-PAA and the AR TP-CFRP-PAA are compared in Figure II.3.8.4 as an example of the surface roughness obtained with laser treatment.

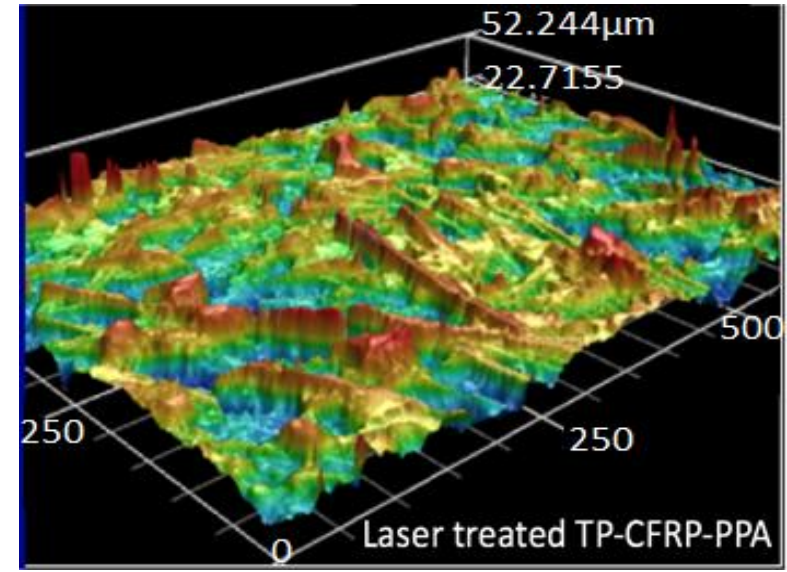

(a)

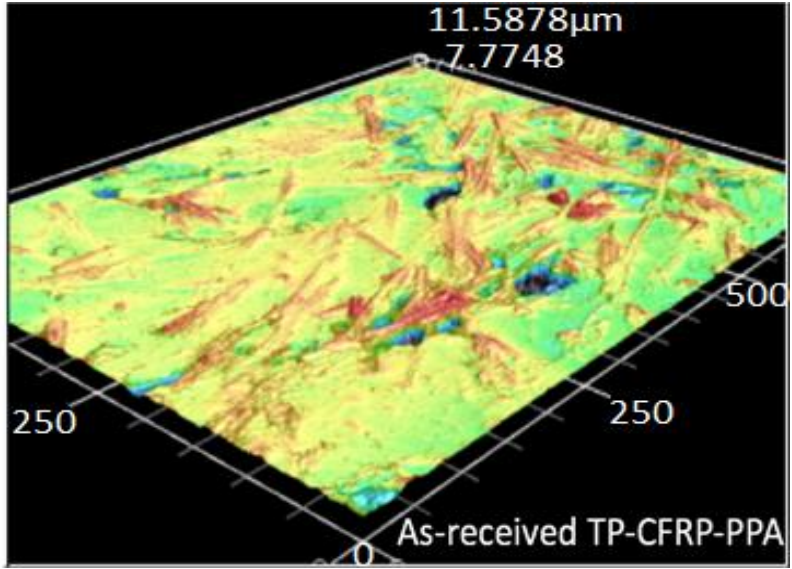

(b)

Figure II.3.8.4. Laser profilomerty in micrometers for (a) laser-surface-textured TP-CFRP-PPA and (b) AR TP-CFRP-PPA. Source: ORNL. 
Figure II.3.8.5 summarizes wetting envelopes plotted from surface energies of mechanically abraded and lasertreated surfaces. In general, a wetting envelope describes the area under of the curve of the polar and dispersive components of surface energy of an adherend. When the surface energy of an adhesive is inside the envelope of the substrate, good wetting and adhesion occurs. When the surface energy of the adhesive is outside the envelope, poor wetting and adhesion occurs. The adhesives we used were hydrophobic as represented by the open circles at the bottom of each graph labeled "adhesive" with values of $\gamma^{\mathrm{d}}=44.63, \gamma^{\mathrm{p}}=$ $1.37 \mathrm{~mJ} / \mathrm{m}^{2}$; and $\gamma^{\mathrm{d}}=43.74, \gamma^{\mathrm{p}}=1.34 \mathrm{~mJ} / \mathrm{m}^{2}$. In our work, both surface treatments result in a significant increase in the wetting area from AR surfaces. The TP-CFRP-PPA samples showed an increase in dispersive components $\left(\gamma^{\mathrm{d}}\right)$ after mechanical abrasion to values equal to those of the adhesive. AR PPA without CF showed dispersive components similar to those of the adhesive and laser treatment primarily affected the polar components. Laser treatment of TS-CFRP and TP-CFRP-PPA significantly increased wetting in both polar and dispersive components beyond that of the adhesives which indicates adhesion would be greatly enhanced. However, laser-textured DP980 and DP980 - without zinc did not show enough increase to match that of the adhesives.
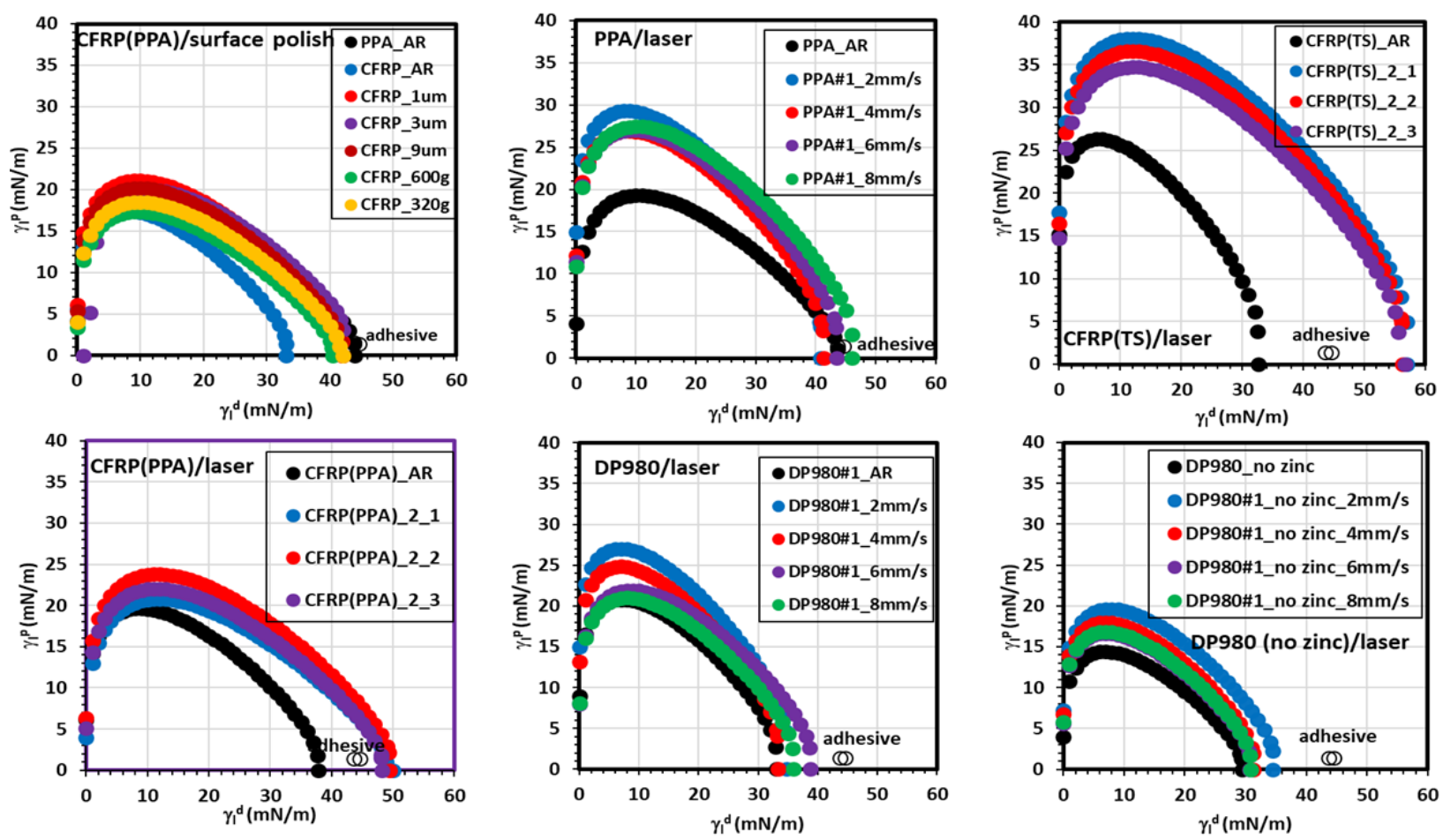

Figure II.3.8.5. Wetting envelopes for surface polished and laser-surface-textured PPA resin only, TP-CFRP-PPA, TS-CFRP, and DP980. Source: PNNL.

PNNL also studied surface roughness effects for improved adhesive-bonding using a tensile pull test. The tensile pull results are normalized to the bond line thickness. Figure II.3.8.6 shows three representative CFRPPPA surfaces: CFRP-PPA-AR, CFRP-PPA-1 $\mu \mathrm{m}$ (polished with $1 \mu \mathrm{m}$ alumina), and CFRP-PPA-320 grit (roughened with 36 micron $\mathrm{SiC}$ abrasive paper). Area factor, $\mathrm{S}_{\mathrm{dr}}$, measured by optical profilometer, was $0.747 \%, 0.0093 \%$, and $71.695 \%$ for CFRP-PPA-AR, CFRP-PPA-1 $\mu \mathrm{m}$, and CFRP-PPA-320 grit, respectively. Unfilled PPA samples were also mechanically abraded with 600 and 320 grit resulting in a $14 \%$ increase in normalized tensile pull. The modified surfaces were extensively characterized by contact angle, Fourier Transform Infrared spectroscopy, X-ray photoelectron spectroscopy, and Time-of-Flight secondary ion mass spectrometry. The tensile pull strength of modified surfaces is also summarized in the graph on Figure II.3.8.6 and shows the effect of surface roughness and the $\mathrm{CF}$ influence on strength. The results demonstrate that increasing the surface roughness increases tensile pull strength. 


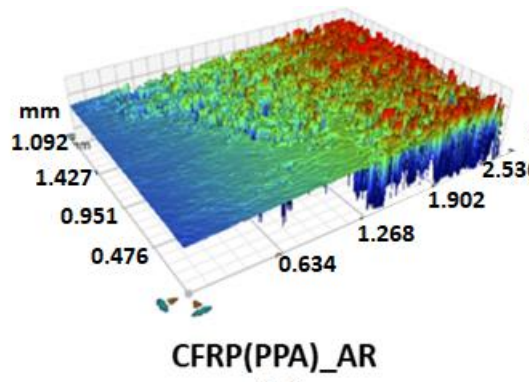

(a)
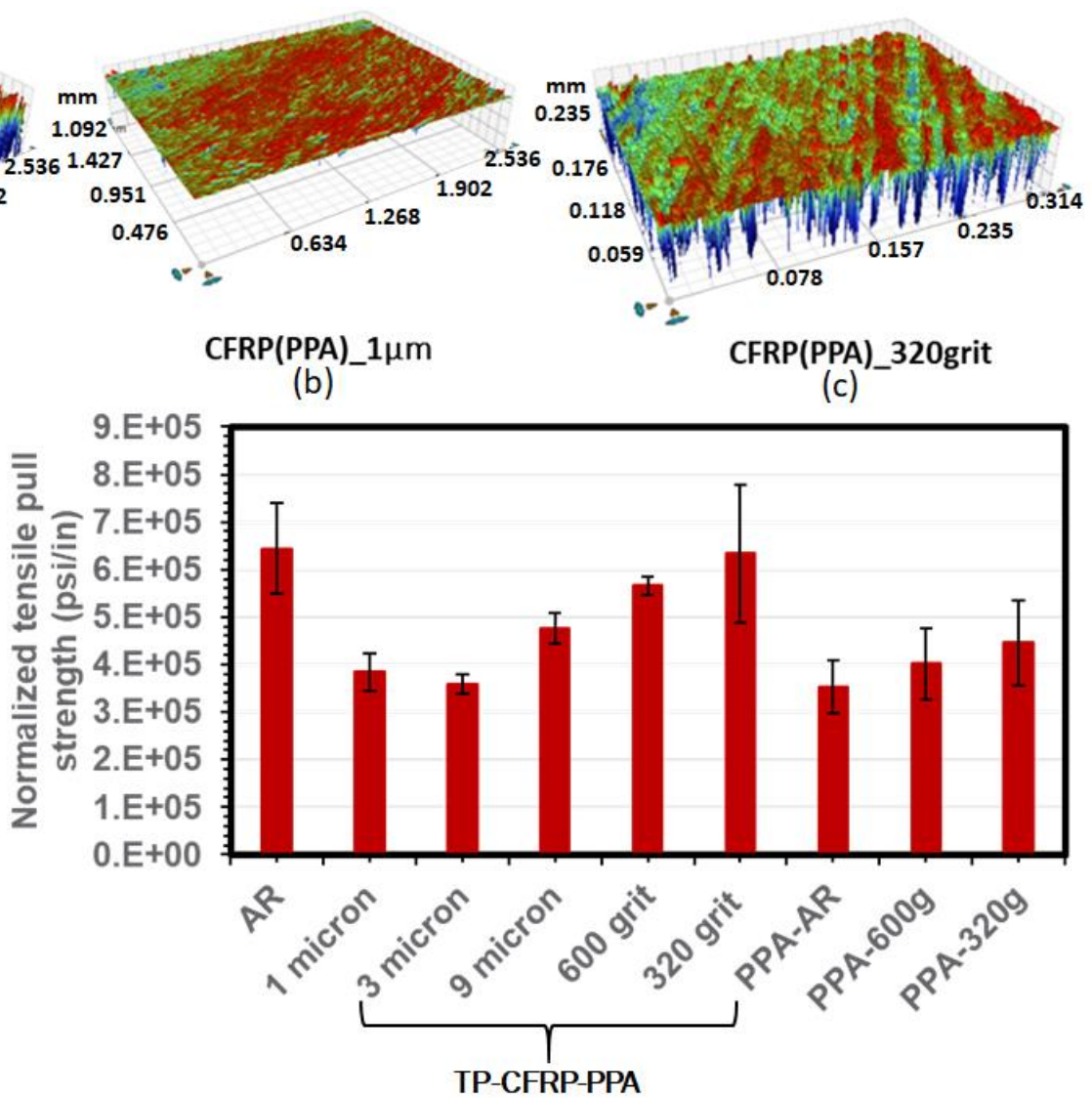

(e)

Figure II.3.8.6. Optical profilometry for: (a) AR-CFRP-PPA; (b) mechnically polished with $1 \mu \mathrm{m}$ alumina; (c) mechanically abraded with 320 grit; (d) example of tensile pull sample; and (e) tensile pull strength test values for surface roughened PPA resin only and TP-CFRP-PPA. Source: PNNL.

Figure II.3.8.7 summarizes adhesive-bonding strength for DP980 to TP-CFRP-PPA, with and without laser surface treatment, with different modulus adhesives. As can be seen, bond strength was increased approximately $25 \%$ and $72.6 \%$ for Adhesives \#7 and \#8 respectively, when direct laser surface texture was applied on TP-CFRP-PPA. This laser surface treatment on the polymer composite increased surface roughness at the microscopic level and, also exposed randomly distributed CFs on the surface, resulting in an anchor (or interlocking) effect between the adhesive and the laser-treated composite surface. For this reason, adhesivebonding strength was greatly improved, and failure location changed to partial adhesive failure between adhesive and DP980 surface for Adhesive \#7 and to the base polymer failure for Adhesive \#8. This was distinct from the adhesive failure at interface between adhesive and AR TP-CFRP-PPA surface seen for the same adhesives when surfaces did not receive the treatment. 


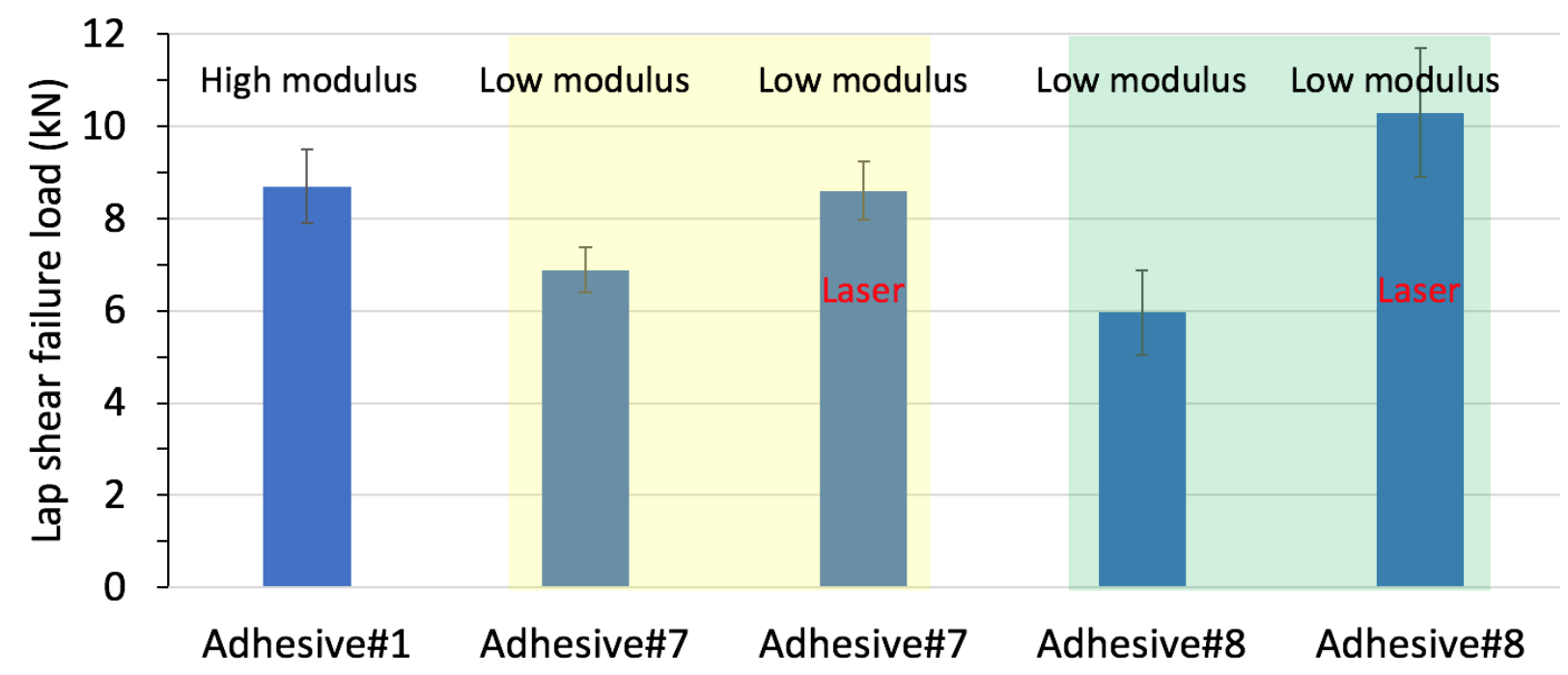

Figure II.3.8.7. Adhesive-bonding strength for DP980 (no-zinc)-TP-CFRP-PPA before and after laser-surface-textured on TP-CFRP-PPA. Source: PNNL.

\section{Conclusions}

Adhesive-bonding of thermoplastic CFRP (PPA) and thermoset CFRP to DP980 was investigated. Engineered surface modifications by laser and mechanical abrasion changed surface morphology and roughness, leading to improved surface energy and bonding strength. A micro-DIC system was developed and applied to capture adhesive deformation and failure propagation using a miniature lap-shear coupon.

\section{Key Publications}

1. Lim, Y. C., J. Chen, H. Huang, N. Nguyen, D. Warren, Z. Feng, K. Simmons, and L. Fifield, 2020, "Adhesive-bonding of AHSSs to CFRPs (polyphthalamide)," accepted to The Adhesion Society $43^{\text {rd }}$ Annual Meeting, 23-26 February 2020, Charleston, SC, USA.

2. Lim, Y. C., J. Chen, N. Nguyen, D. Warren, A. Naskar, Z. Feng, K. Simmons, and L. Fifield, 2019, "Adhesive-bonding of CFRPs to AHSSs," The Adhesion Society $42^{\text {nd }}$ Annual Meeting, 17-20 February 2019, Hilton Head, SC, USA.

\section{Acknowledgements}

The principal investigators would like to recognize A. Naskar, Y. C. Lim, J. Chen, M. Goswami, N. Nguyen, D. Warren, and X. Sun of ORNL; K. Simmons, L. Fifield, D. Graff, A. Ortiz, and Y. Shin of PNNL; and our industry partners-DOW, L\&L, 3M and General Motors-for their support and assistance with this project. 


\title{
II.3.9 Solid-Phase Joining of Magnesium Sheet to High-Strength Steel (Pacific Northwest National Laboratory)
}

\author{
Piyush Upadhyay, Co-Principal Investigator \\ Pacific Northwest National Laboratory \\ 902 Battelle Blvd. \\ Richland, WA 99352 \\ E-mail: piyush.upadhyay@pnnl.gov \\ Zhili Feng, Co-Principal Investigator \\ Oak Ridge National Laboratory \\ 1 Bethel Valley Rd. \\ Oak Ridge, TN 37831 \\ E-mail: fengz@ornl.gov
}

\section{Sarah Kleinbaum, DOE Technology Development Manager}

U.S. Department of Energy

E-mail: sarah.kleinbaum@ee.doe.gov

Start Date: October 1, 2017

Project Funding (FY19): \$525,000

End Date: September 30, 2020

DOE share: $\$ 525,000 \quad$ Non-DOE share: $\$ 0$

\section{Project Introduction}

In a modern multi-material vehicle, lightweight materials such as $\mathrm{Al}$ and $\mathrm{Mg}$ alloys can be a challenge to join and attach to the underlying substructure, usually composed of steel. Even in Al- and Mg-intensive designs where entire substructures may be constructed of lightweight metals, there remains a need to join the substructure with other parts of the vehicle, such as the predominantly steel passenger safety cage. Joining methodologies available in the cost range relevant to automotive manufacturing include RSW, adhesives, linear fusion welding, hemming, clinching, bolting, and riveting. However, because of the highly dissimilar natures of the materials, $\mathrm{Mg}$ to steel joints are extremely problematic. $\mathrm{Mg}$ to steel joints simply cannot be fusion-welded due to the extreme differences in their melt temperatures. Additionally, no intermediate phases exist between a $\mathrm{Mg}$ and $\mathrm{Fe}$ system. Joining methods that require a large amount of plastic strain in the $\mathrm{Mg}$ component suffer from Mg's poor ductility at RT.

FSW and USW methods provide joining options that may be able to overcome some of the technical barriers preventing more robust and reliable joining of $\mathrm{Mg}$ to steel [1-3]. These methods involve creating a large degree of plastic deformation at the interface while at the same time delivering heat from frictional and plastic work. Both methods are solid-state, warm deformation technologies, and take advantage of the enhanced ductility of $\mathrm{Mg}$ and steel at elevated temperatures. There are several identified technical barriers:

1. Lack of understanding of the methods and processing conditions required to achieve robust joints.

2. Lack of comprehensive performance information on joints fabricated by FSW/USW methods (e.g., strength, fatigue, durability, crash performance).

3. Insufficient understanding of the feasible joint geometries.

4. For USW, lack of fundamental understanding of the response of materials and joint geometry to process variables (i.e., frequency, etc.).

5. Lack of understanding of the corrosion protection and mitigation strategies that will be necessary to implement for these joining methods. 


\section{Objectives}

The overall goal of this project is to mature two types of solid-phase joining techniques-FSW and USW-by developing an understanding of the methods and processing conditions required to achieve robust joints between $\mathrm{Mg}$ and steel, thus integrating lightweight materials for multi-material vehicles.

\section{Approach}

This project is designed to overcome many of these technical barriers by performing three primary tasks to ensure risk mitigation and successful completion. These tasks and associated deliverables are intended to enable a broader application of solid-phase joining technologies, while further facilitating the joining of $\mathrm{Mg}$ to steel. The three main tasks are as follows:

- $\quad$ Task 1: The development and assessment of FSW and USW techniques with and without geometric joint line features.

- $\quad$ Task 2: The fundamental study of bond formation and associated mechanical properties.

- $\quad$ Task 3: Interface control and tailoring for improved corrosion and strength.

By utilizing several advanced characterization techniques, we intend to correlate process parameters and variables to resulting interface chemistry and properties to tailor the joint interface that maximizes strength, ductility, and corrosion resistance. This would enable us to develop an applied understanding of the following:

1. The localized metal-forming and potential metallurgical bonding that develops during FSW techniques and USW methods.

2. The intermetallic formation at the interface strategies to tailor the joint interface that maximizes strength, ductility, and corrosion resistance.

3. How the process parameters influence the joint strength and performance of the joints and assemblies produced.

4. How both processes interact with existing corrosion protection methods (coatings) and how they affect the overall corrosion performance of hybrid $\mathrm{Mg} /$ steel assemblies.

\section{Results}

\section{Friction Stir Welding}

In FY 2019, the project team built upon the work performed in previous year to better understand the joint line interface. This included interfacial property measurements, fracture modes, and interfacial chemistry assessments and joint line study. The summary of this work is presented below.

PNNL investigated the IMC layer that forms between $\mathrm{Mg}$ /steel joints made using friction stir scribe technique when $\mathrm{Zn}$-coating is present. As we work closely with the modeling team to enable better prediction of joint fracture behavior and load-bearing capacity, there is a need to assign accurate mechanical properties in the model that reflect the material property changes as a result of welding conditions. This necessitated measurement of the mechanical property of the interfacial components. A viable way to obtain the properties are via nano indentation which provides load versus displacement behavior at a submicron scale as shown in Figure II.3.9.1 (a), which can be used to calculate both elastic and plastic properties of a given substrate. 


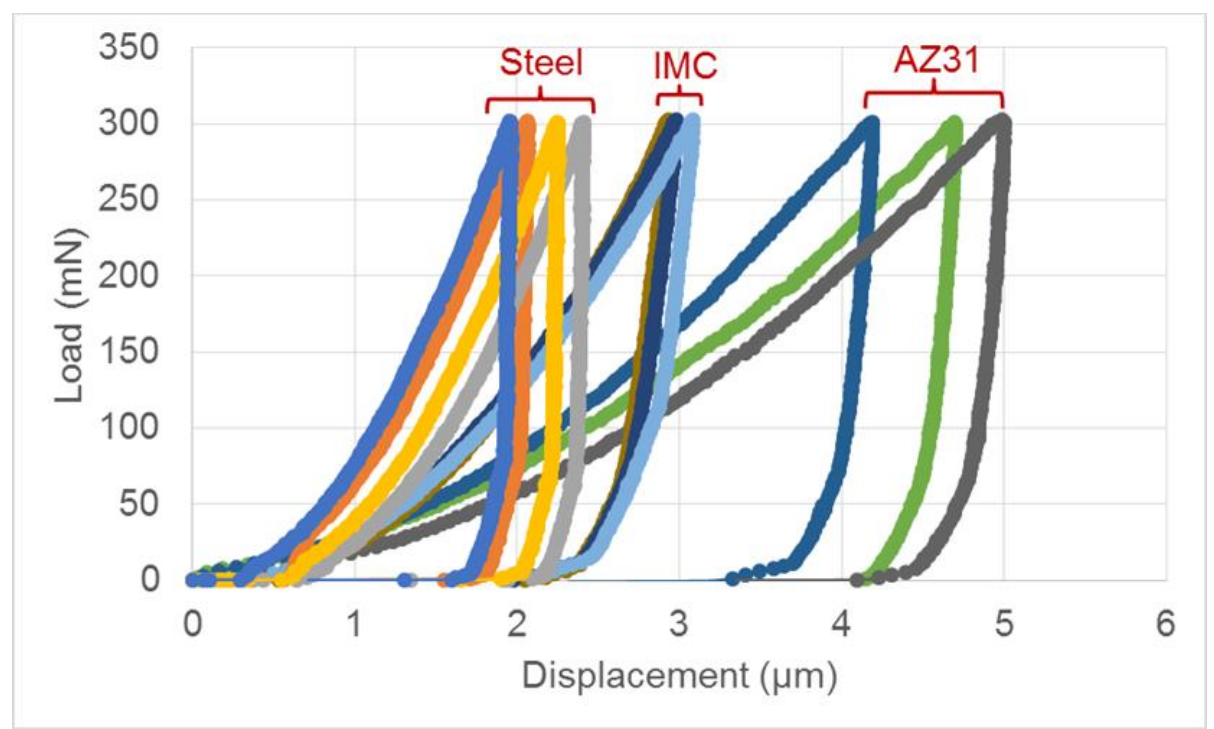

(a)

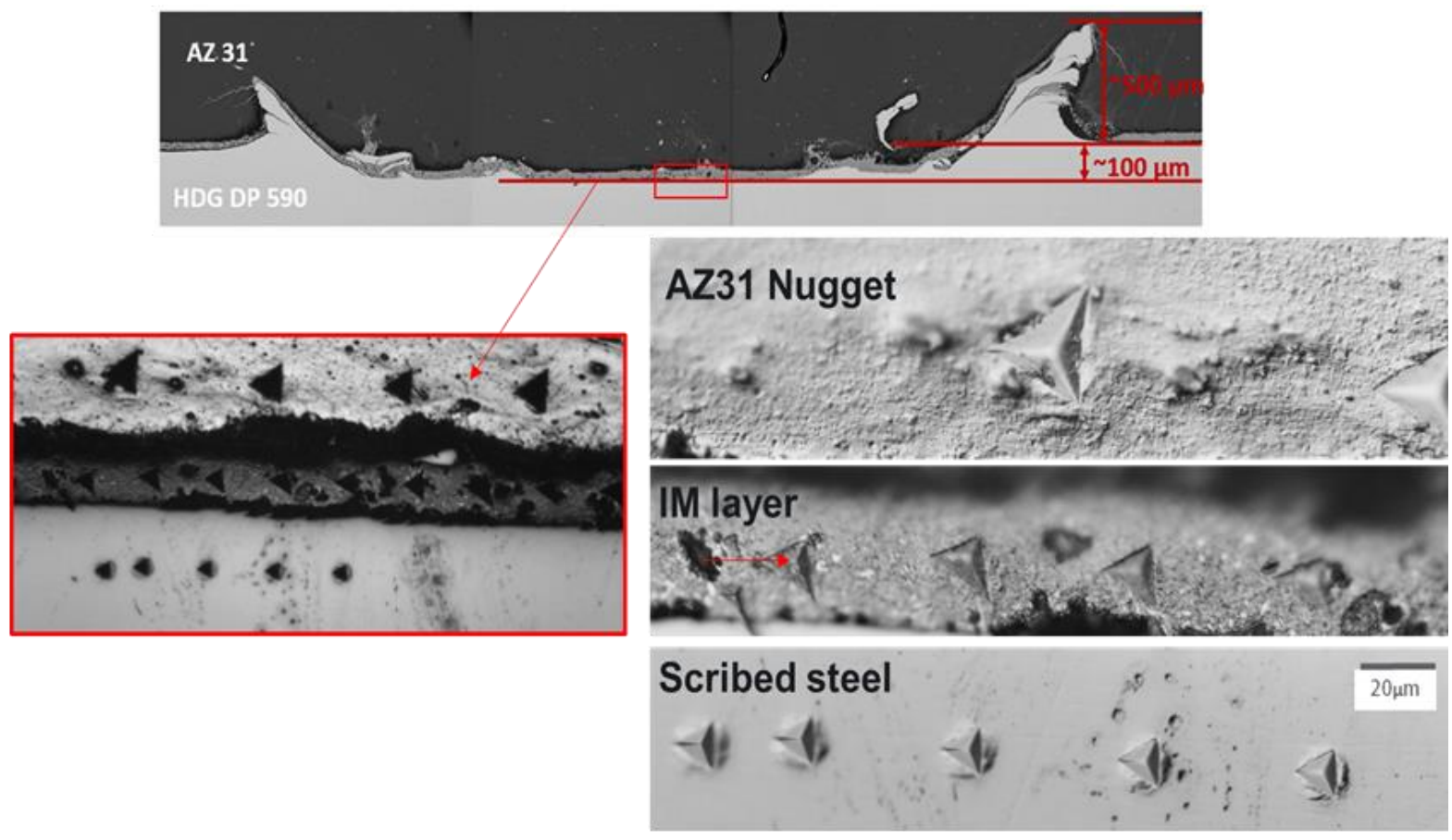

(b)

Figure II.3.9.1. (a) Representative load versus displacement curves obtained during nano indentation of the weld interface. (b) Snapshot of joint cross-section at various magnification shows nano indents at AZ31 weld nugget near the interface, IM layer at the interface, and steel near the interface. Source: PNNL.

Figure II.3.9.1 (b) provides a snapshot of nano indents at various locations on and around the interface. To validate the nano indentation technique, we obtained nano indents on both the AZ31 nugget and scribed steel areas near the interface. The hardness values for both these materials were found to be in good agreement with more established microhardness methods (microhardness values not shown). Figure II.3.9.2 summarizes the results of the nano indentation measurements. The weld region hardness and elastic modulus (orange) is compared to the corresponding base values (blue). For the intermetallic (IM) layer, the base value refers to 
measurements obtained from the IMC layer away from the weld region (outside the hook area). The IM layer found in between the hook region is significantly harder ( $\sim 3$ times) and more ductile ( 2 times) than the IM layer away from the hook region. While both layers in the IM (inside and outside of the hook) are composed of $\mathrm{Zn} / \mathrm{Mg}$ eutectic, the nano indentation results indicate that the phases present in both cases are different. Greater ductility of the IM layer at the bonded interface can provide a favorable joint performance. This dataset was provided to the Interface by Design team for input into the model, which may allow for greater predictive capability towards specific joint characteristics, including IM thickness layer, hook geometry, etc.

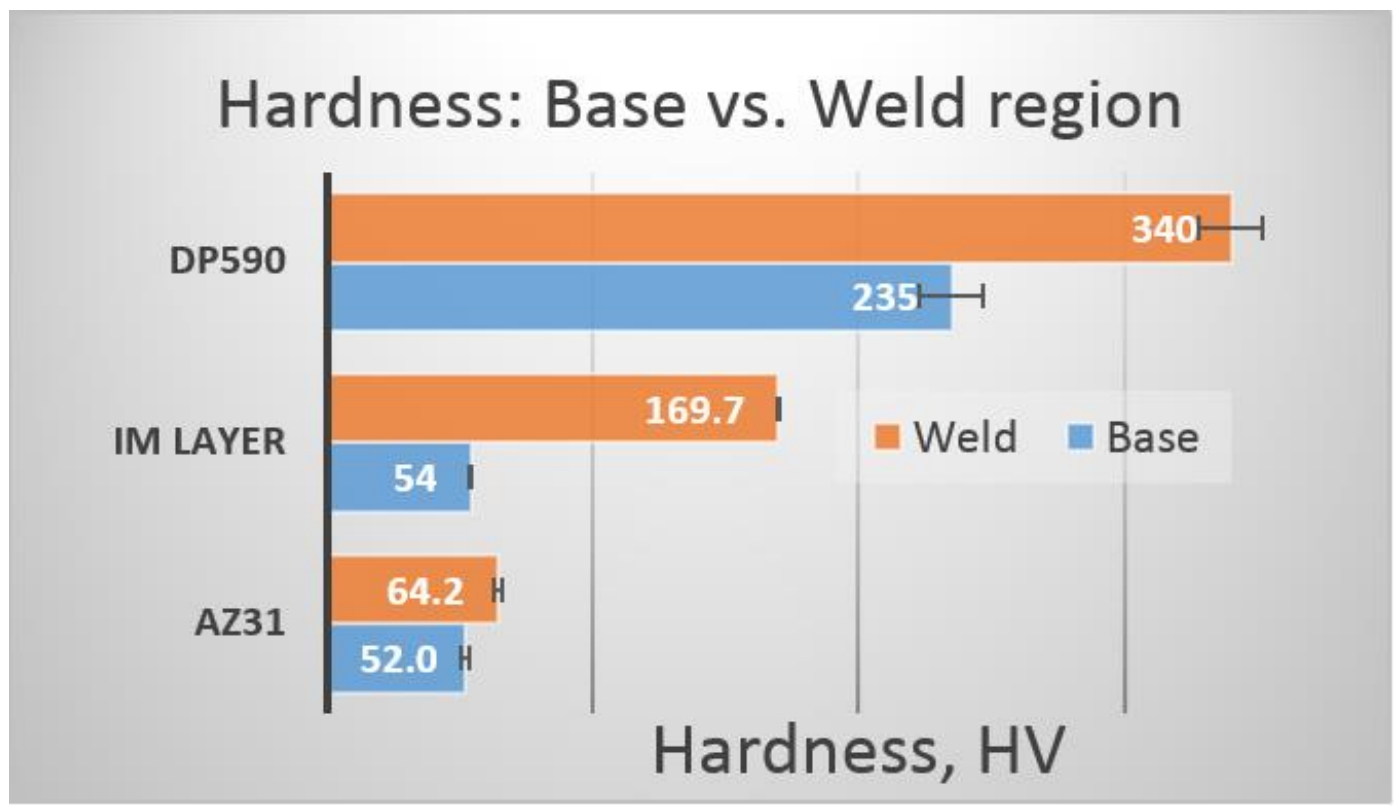

(a)

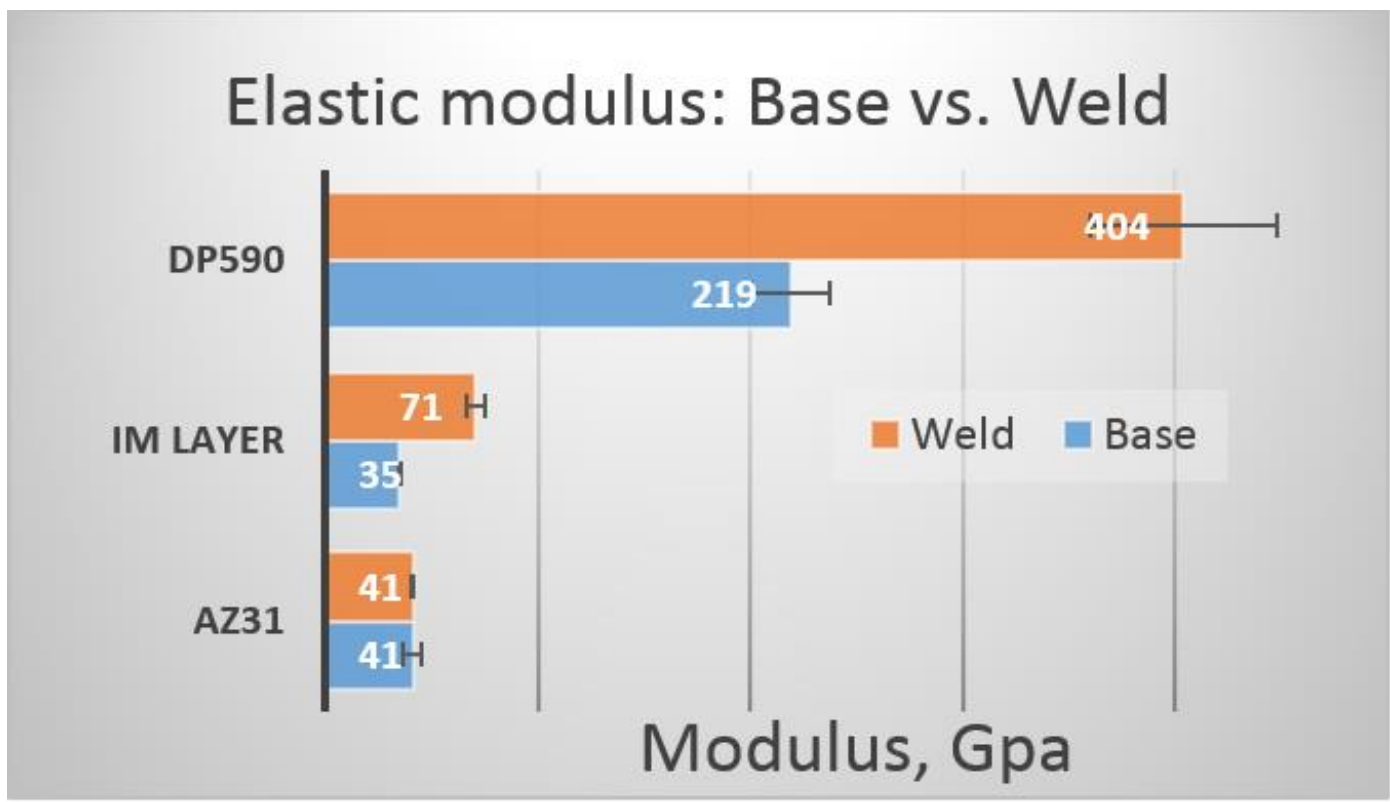

(b)

Figure II.3.9.2. Comparison of (a) hardness and (b) elastic modulus calculated from nano indentation tests near the weld interface and base material. For IMC, the base represents the IMC layer found away from the weld region. Source: PNNL. 
SEM micrographs of several fractured samples tested (some reported in last year's report) were investigated. Representative fractography for two of these cases are shown in Figure II.3.9.3 (e.g., DP590 with Zn-coating) and Figure II.3.9.4 (Bare DP590). In cases of interfacial fracture with Zn-coated steel joints, fracture occurred at $\mathrm{Zn} / \mathrm{Mg}$ eutectic and steel interface. While some stray steel was observed on the $\mathrm{Mg}$ side, and some $\mathrm{Zn} / \mathrm{Mg}$ eutectic observed in the steel side, most of the $\mathrm{Zn} / \mathrm{Mg}$ eutectic was attached to the $\mathrm{Mg}$ side. Additionally, interpenetrating/interlocking features are observed near the hook region, as seen in Figure II.3.9.4 (b3). It is important that this longitudinal feature is accurately captured in addition to the cross-sectional hook feature structure-property modeling efforts.
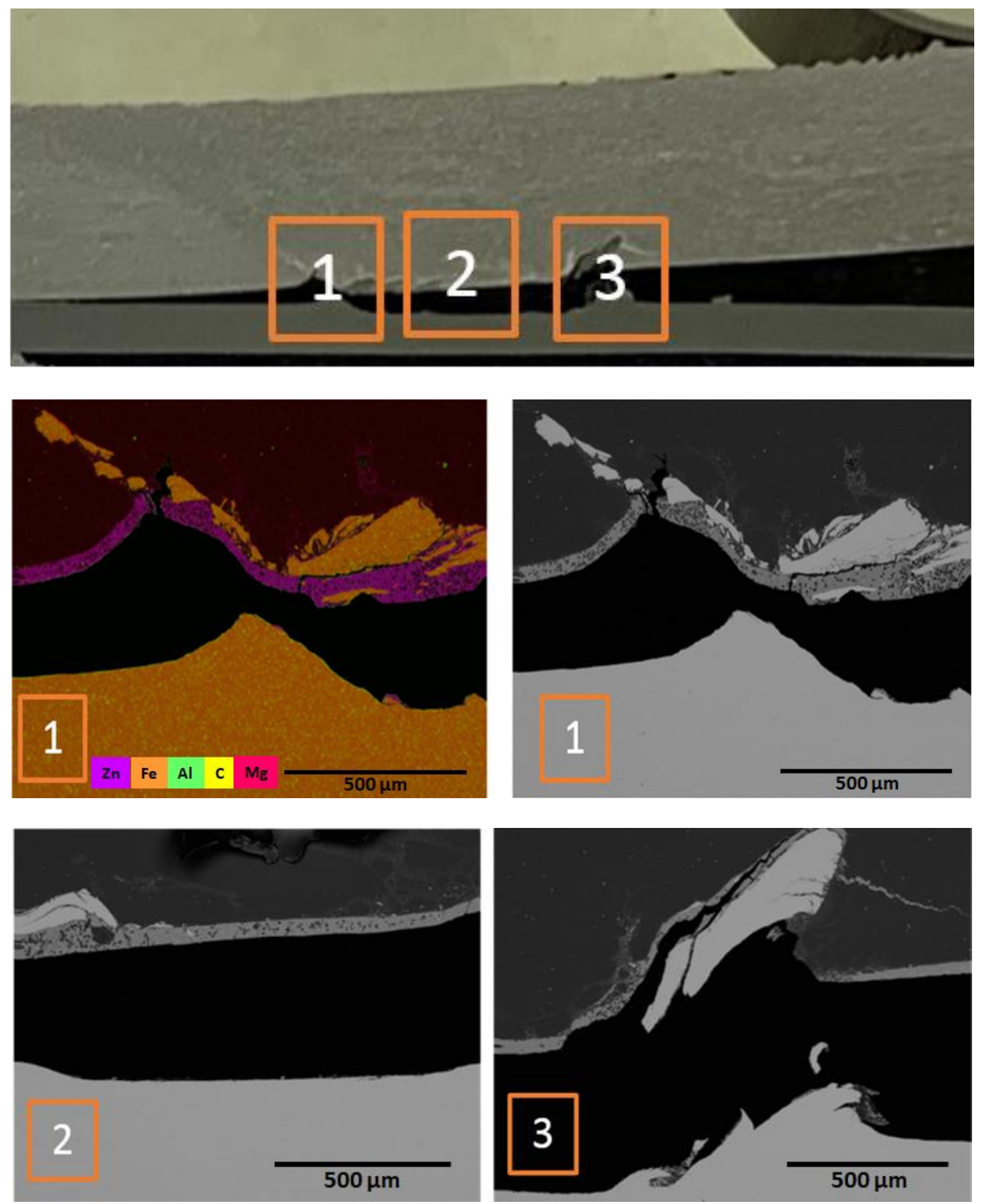

Figure II.3.9.3. SEM micrographs of a fractured Mg/steel joing. Images 1 and 3 are the two hook locations while 2 is the area in between the hooks. The $\mathrm{Zn} / \mathrm{Mg}$ eutectic is distinctly observed in elemental color mapping in the upper left micrograph. Source: PNNL. 


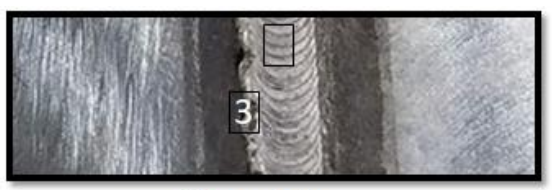

Mg side
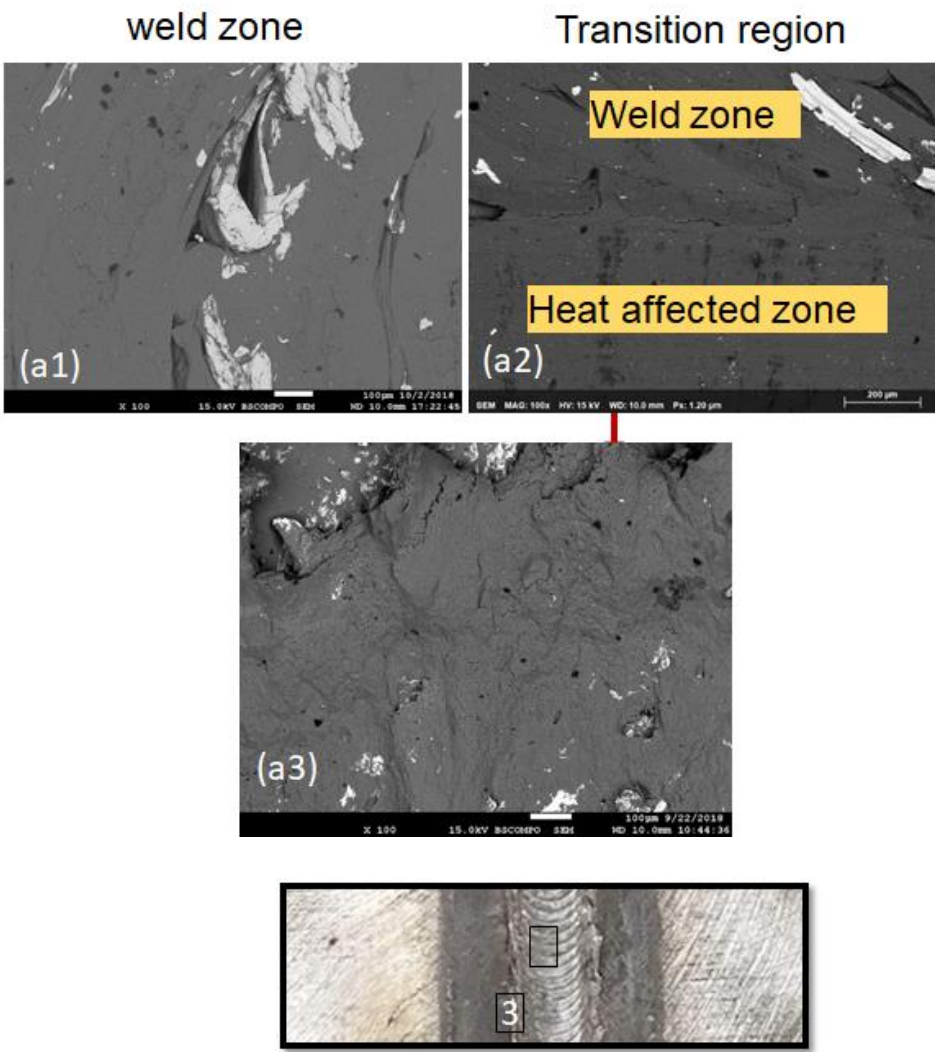

Steel side
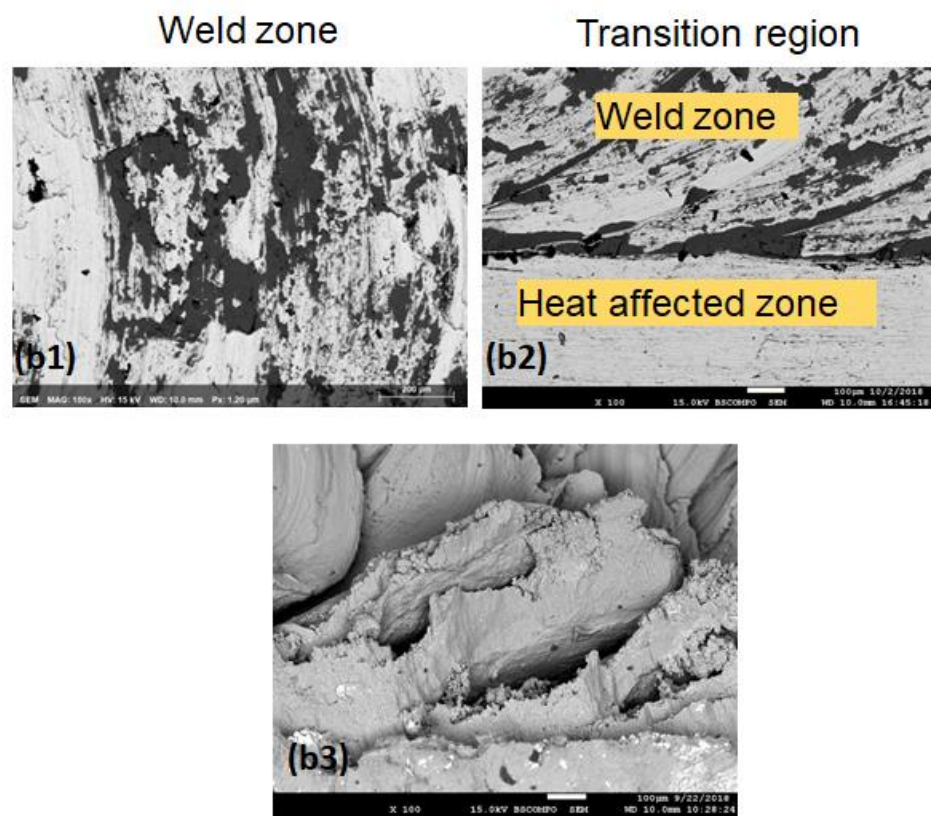

Figure II.3.9.4. SEM fractography of the Mg and steel side on scribe joints made with bare steel. Source: PNNL. 
To decouple the mechanical and metallurgical contribution to the bond strength in the dissimilar joints, we evaluated scribe joints with gradually reduced hook features, as observed in Figure II.3.9.5. This was attained by progressively decreasing the engagement of the scribe on the steel sheet. The joint strength expressed in terms of $\mathrm{N} / \mathrm{mm}$ is plotted against measured extension for several joints in Figure II.3.9.6. There are a couple of interesting observations. The joint lap strength is not directly proportional to the size of the hook. The loadbearing capacity remained very similar for welds with different hook sizes $(150-60 \mu \mathrm{m})$ and all other things being equal. This can be observed in the grouping of square data points between 180 and $210 \mathrm{~N} / \mathrm{mm}$. The orange square data point had hook size of $\sim 10 \mu \mathrm{m}$. Perhaps this represents the smallest scribe engagement necessary for viable joint in the scribe process. This does not mean that the hook size has no effect on mechanical properties; a large increase in joint ductility was observed with larger hook size while load-bearing capacity was not significantly affected in $2.5-\mathrm{mm} \mathrm{Mg}$-steel joints [4]. A significant increase in load before fracture is observed when the joint line (distance between the hooks in transverse cross-section) is increased (see 2 triangles nearing $340 \mathrm{~N} / \mathrm{mm}$ ). This observation also suggests the role of the region in between the hooks for an increase in load-bearing capacity. While these observations are helpful to understand relationships between microstructural features, the mechanical versus metallurgical contribution to the bond strength appears still elusive. Thus far, the advanced SEM characterization has not shown any distinct layer of IMC or interfacial diffusion that can account for metallurgical bonding other than the presence of $\mathrm{Zn} / \mathrm{Mg}$ eutectic in place of $\mathrm{Zn}$-coated steel. We continue to characterize joints in different configurations including mini tensile tests of different locations of the joints.

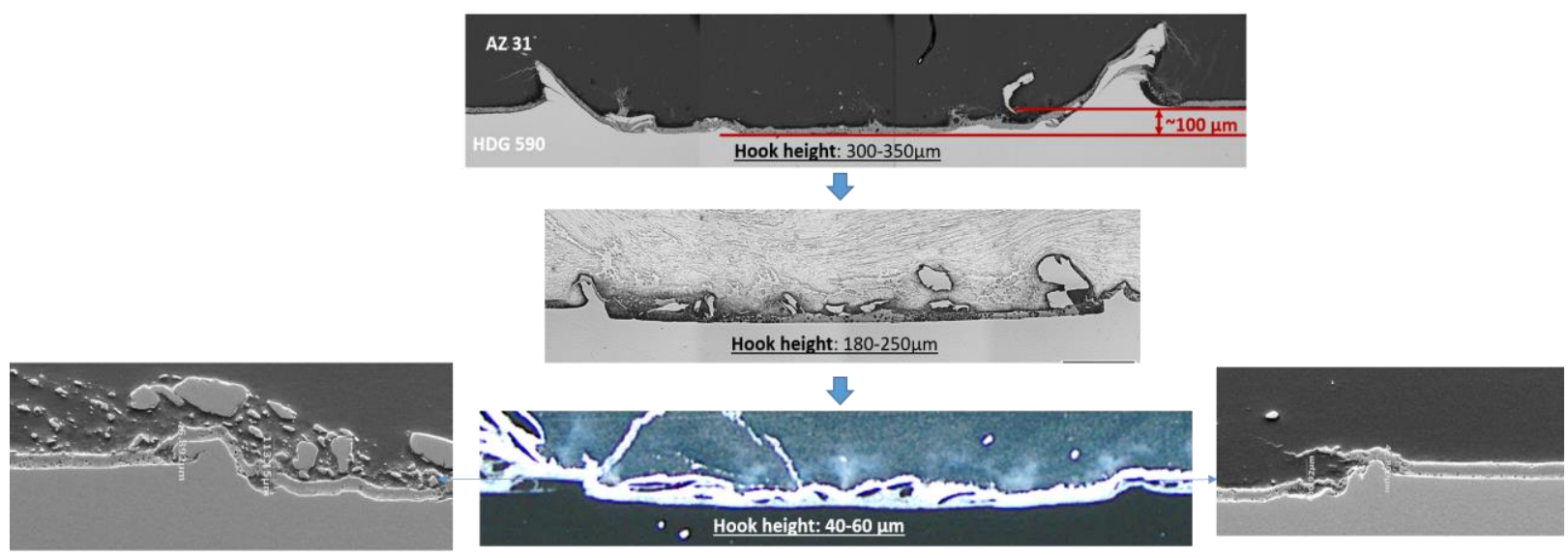

Figure II.3.9.5. Progressively decreasing hook features $(350 \mu \mathrm{m}-60 \mu \mathrm{m})$ at the interface of Mg/steel joints. Source: PNNL. 


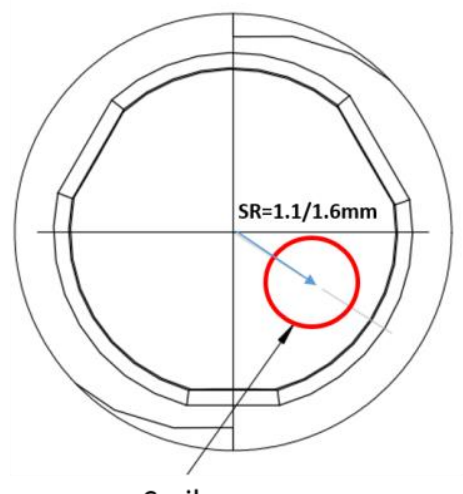

Scribe
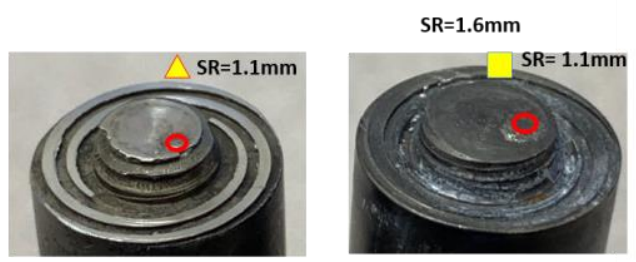

Figure II.3.9.6. Fracture load plotted against fracture extension for various Mg/steel joints made at 2 scribe swept radius. Pictures and sketch on the left shown two swept radius scribe tooling. Source: PNNL.

In addition to the above efforts, in FY 2019 we completed the three tests (fixture, sample design, and implication) for cross-tension, T-peel, and U-peel configuration. The completed test data was supplied to the Interface by Design team for inclusion into their structure-property model.

\section{Ultrasonic Welding}

Joining $\mathrm{Mg}$ or $\mathrm{Mg}$ alloy to steel is challenging because of their different physical and chemical properties and the absence of a Fe-Mg intermetallic or Fe-Mg solid-solution in an $\mathrm{Mg}$-Fe system. In the past, most of the research is focused on joining these two materials with a presence of an interlayer or coating such as $\mathrm{Zn}, \mathrm{Sn}$, and Ni coating. This is because these elements could form IMCs or solid solutions with Mg or Fe. Joining Mg to steel without the presence of interlayer elements is considered virtually impossible.

ORNL's team has demonstrated the feasibility to use USW to join (1) 2-mm-thick AZ31B Mg alloy to 1-mmthick Zn-coated DP590 steel, as well as (2) 2-mm-thick AZ31B Mg to 1-mm-thick bare DP590 steel, and (3) 2-mm-thick pure $\mathrm{Mg}$ (Mg 99.95wt\%, Fe 0.04wt\%, $\mathrm{Al} \mathrm{0.01wt. \%} \mathrm{and} \mathrm{Zn}$ less than 0.01wt.\%) to 1-mm-thick bare DP590.

Figure II.3.9.7 shows the SEM image and the energy dispersive x-ray (EDX) spectroscopy chemical mapping at the joint interface of a USW sample made of AZ31B Mg alloy to Zn-coated DP590 steel. The AZ31B sheet is on the right side. At the interface between the AZ31B and steel sheet is a Mg- $\mathrm{Zn}$ reaction layer. It is generally considered that such a reaction layer would facilitate the formation of the metallurgical bond through brazing. 

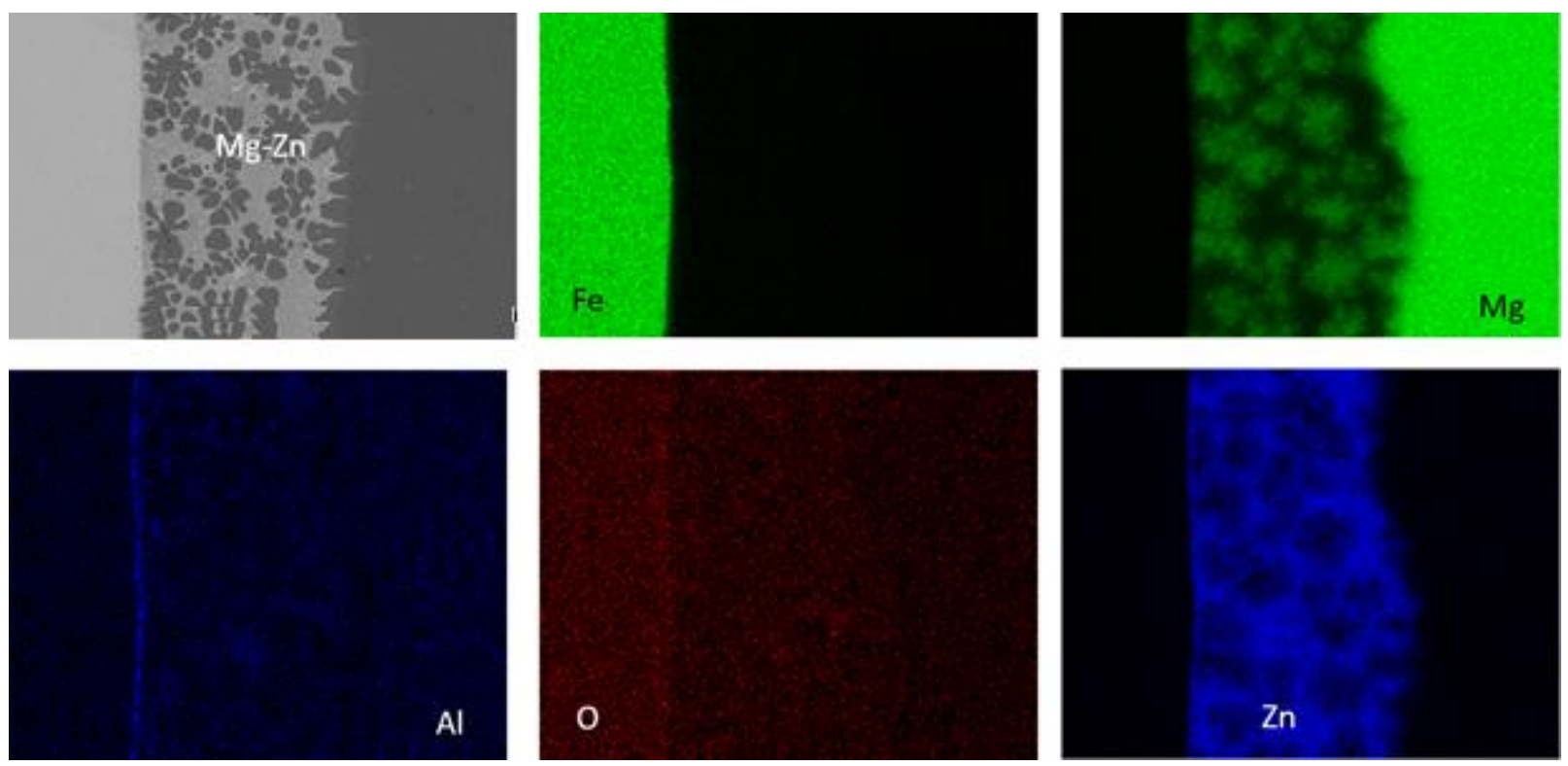

Figure II.3.9.7. SEM and EDX mapping of a USW sample made of AZ31B Mg alloy to Zn-coated DP590 steel. Source: ORNL.

Joining $\mathrm{Mg}$ or $\mathrm{Mg}$ alloy to steels without $\mathrm{Zn}$ surface-coating is also successfully demonstrated. As shown in Figure II.3.9.8 (a), lap-shear tensile specimens were made with both AZ31B to bare DP950 and pure Mg to bare DP590. As shown in Figure II.3.9.8 (b), the average lap-shear tensile strength of the AZ31B-bare DP590 USW samples is $63 \mathrm{MPa}$, and the average lap-shear tensile strength of the pure Mg-bare DP590 USW samples is $28 \mathrm{MPa}$. The difference could be due to the different tensile strength of AZ31B and pure $\mathrm{Mg}$ (260 MPa and $83 \mathrm{MPa}$, respectively).

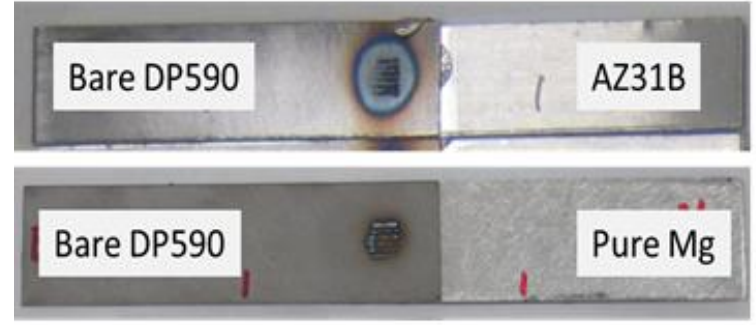

(a)

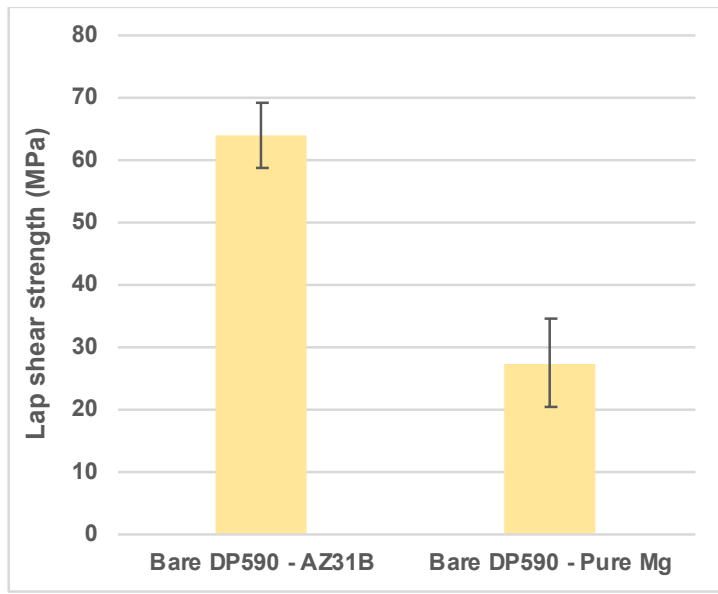

(b)

Figure II.3.9.8. (a) Lap-shear USW samples of AZ31B and bare DP590 (top) and pure Mg and bare DP590 (bottom) and (b) results for the lap-shear strength. Source: ORNL

Figure II.3.9.9 shows the TEM image and the EDX chemical mapping at the joint interface of a USW sample made of AZ31B Mg alloy to bare DP590 steel. A separate chemical analysis on the bare DP590 and AZ31B was performed. In bare DP590, $\mathrm{Al}$ content is $0.04 \mathrm{wt} \%$, and $\mathrm{Zn}$ content is less than $0.01 \mathrm{wt} \%$. In $\mathrm{AZ31B}, \mathrm{Al}$ is approximately $2.99 \mathrm{wt} . \%$ and $\mathrm{Zn}$ is $0.91 \mathrm{wt} . \%$. Thus, $\mathrm{Zn}$ and the $\mathrm{Al}$ composition adjacent to the joint interface shown in the EDX mapping in Figure II.3.9.9 is mainly from the AZ31B BM. 

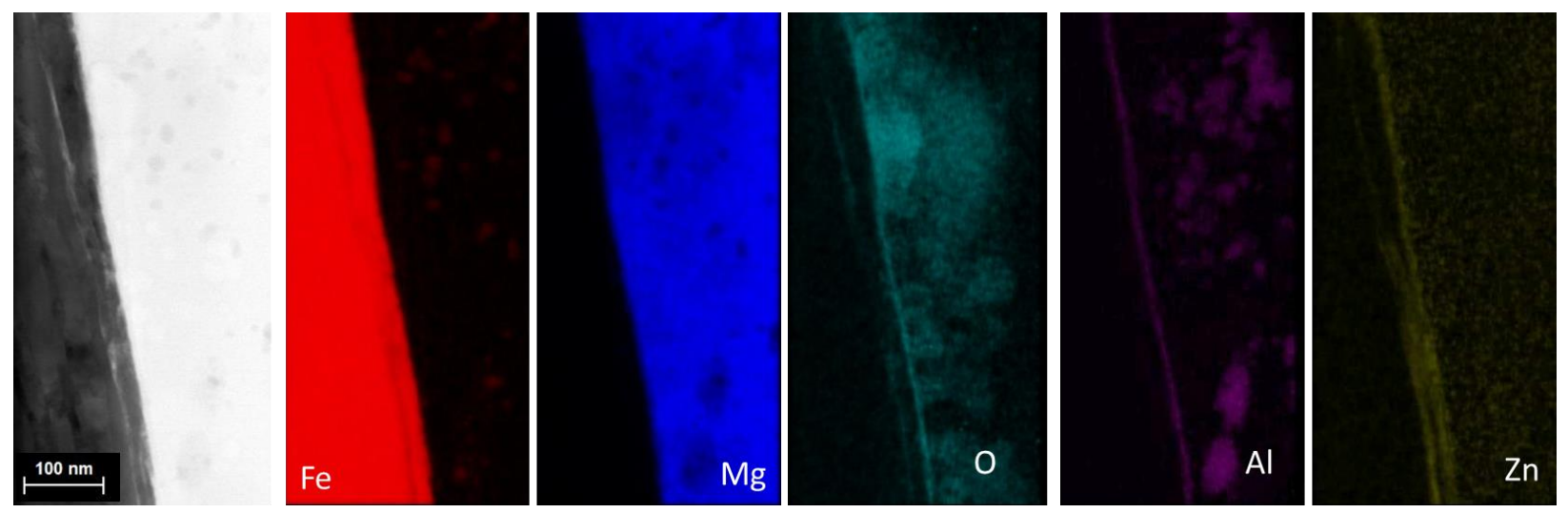

Figure II.3.9.9. SEM and EDX mapping of a USW sample made of AZ31B Mg alloy to bare DP590 steel. Source: ORNL.

The SEM, STEM, and EDX mapping at the joint interface of the pure Mg and bare DP590 USW sample are shown in Figure II.3.9.10 and Figure II.3.9.11. The overall joint line is straight. Isolated unbonded region is present in the STEM image. No macro- or micro-scale mechanical interlocking is observed. So, the joint strength is mainly contributed to metallurgical bonding between $\mathrm{Mg}$ and $\mathrm{Fe}$. The EDX mapping reveals a clear separation of $\mathrm{Mg}$ and $\mathrm{Fe}$ across the joint interface and concentrated $\mathrm{O}$-rich layer at the joint interface.

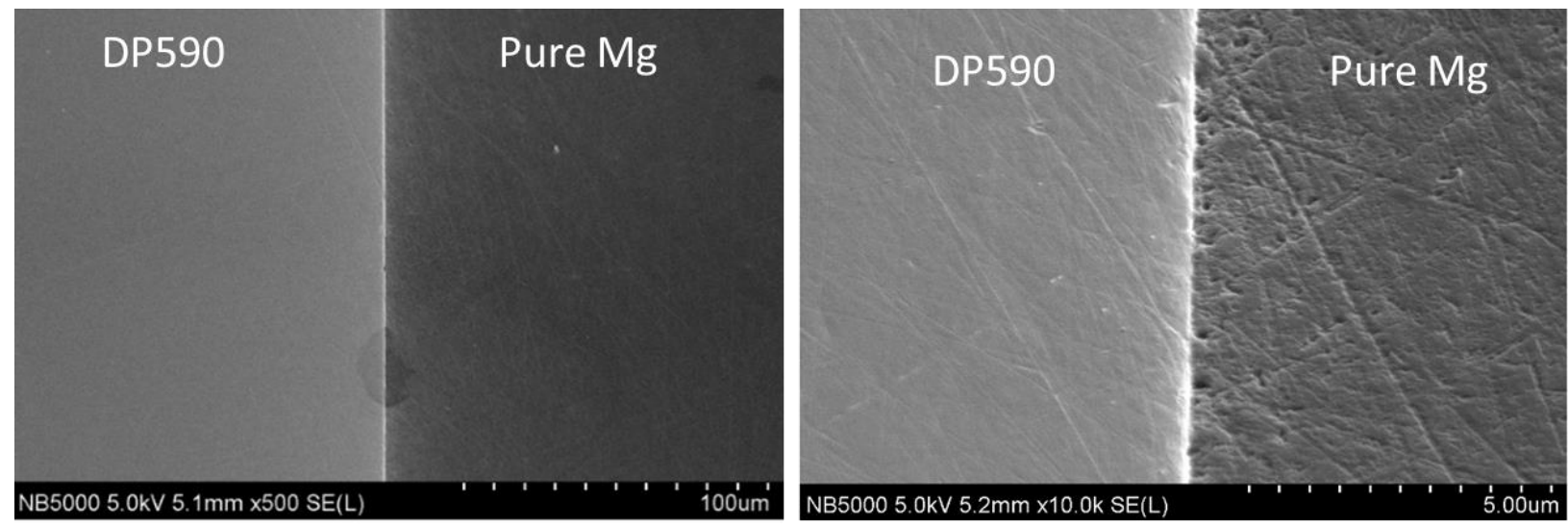

Figure II.3.9.10. SEM images of the joint interface of pure Mg-bare DP590 USW samples. Source: ORNL.
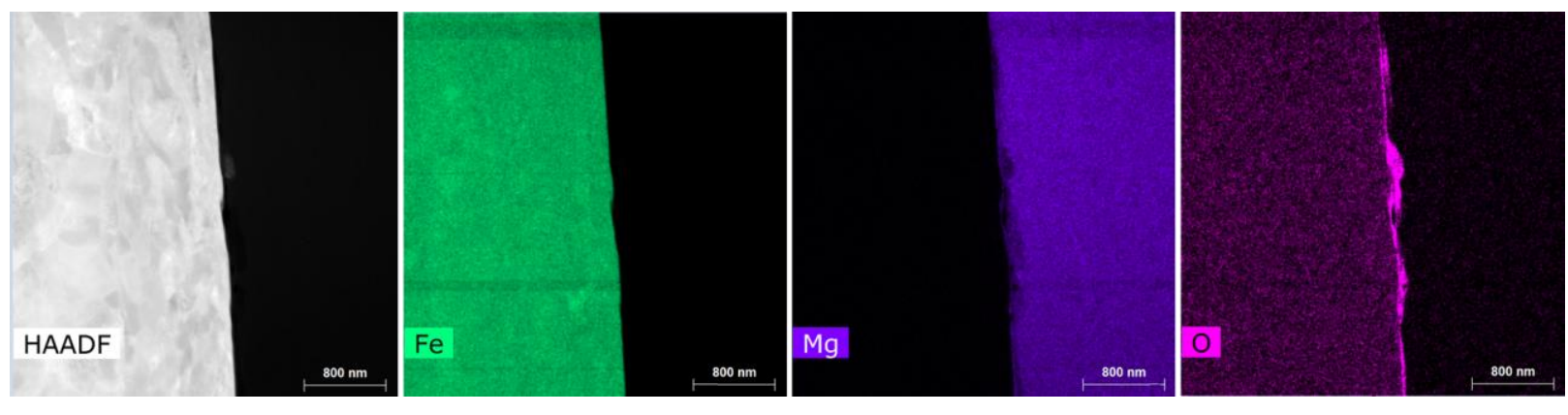

Figure II.3.9.11. STEM image (left) and EDX mapping (right) of the joint interface of pure Mg-bare DP590 USW samples. Source: ORNL. 


\section{Conclusions}

This project aims to develop an applied understanding of two solid-state joining techniques to overcome barriers in joining $\mathrm{Mg}$ to steel such that more reliable and robust multi-material joints can be realized.

In FY 2019, the project team at PNNL (evaluating FSW) and ORNL (evaluating USW) focused on characterization of joint interface to understand interface chemistry and investigate bonding mechanism. For friction stir scribe joints, the hook feature size greater than $\sim 60 \mu \mathrm{m}$ did not result in higher load-bearing capacity all other things being equal (ductility improves with larger hook sizes). Successful joints that result in the fracture of $\mathrm{Mg}$ sheets can be obtained by using a larger joint. For USW, the results reveal that the bonding between AZ31B and Zn-coated DP590 was mainly due to the reaction between Mg and the Zn-coating. Joining $\mathrm{Mg}$ or $\mathrm{Mg}$ alloy to steels without interlayer is much more challenging because $\mathrm{Mg}$ and $\mathrm{Fe}$ are immiscible. However, we successfully demonstrated that metallurgical bonds with reasonable strength could also be formed using USW. We will be utilizing advanced microscopy to further determine interface chemistry and properties so that the precise mechanism of $\mathrm{Mg} /$ steel bonding can be established. This information coupled with interface by design modeling activity will enable the project team to create interface microstructure to tailor the joints for optimized joint strength, ductility, and corrosion performance.

\section{Key Publications}

1. Upadhyay, P., 2019, "Solid-state joining of Mg sheet to high-strength steel," Annual Merit Review Meeting, 10-13 June 2019, Arlington, VA, USA.

2. Chen, J., Lim, Y., Huang, H., Feng, Z., \& Sun, X., 2019, “Ultrasonic welding of AZ31B Mg alloy,” MRS Bulletin, Vol. 44, No. 8, pp. 630-636.

3. Chen, J., Lim, Y., Huang, H., Feng, Z., \& Sun, X., 2019, “Ultrasonic Joining of Mg to Steel,” AWS Professional Program, Nov 2018, Atlanta, GA, USA.

4. Wang, T., et al, "Effect of interfacial characteristics on the mechanical performance of $\mathrm{Mg}$ to steel joint obtained using FAST" submitted to Materials and Design Journal.

\section{References}

1. Jana, S., Y. Hovanski, and G. J. Grant, 2010, "FSLW of Mg alloy to steel: A preliminary investigation," Metall. Mater. Trans. A, Vol. 41, No. 12, pp. 3173-3182.

2. Patel, V. K., S. D. Bhole, and D. L. Chen, 2013, "Formation of zinc interlayer texture during dissimilar ultrasonic spot welding of Mg and high-strength low alloy steel," Mater. Des., Vol. 45, pp. 236-40.

3. Wang, T., H. Sidhar, R. S. Mishra, Y. Hovanski, P. Upadhyay, and B. Carlson, 2017, "Friction stir scribe welding technique for dissimilar joining of $\mathrm{Al}$ and galvanized steel," Sci. Technol. Weld. Joi., Vol. 23, No. 3, pp. 249-255.

4. Wang, T., H. Sidhar, R. S. Mishra, Y. Hovanski, P. Upadhyay, and B. Carlson, 2019, "Evaluation of IMC layer at Al/steel interface joined by friction stir scribe technology," Mater. Design, Vol. 174, Art. 107795.

\section{Acknowledgements}

At PNNL, welding and data analysis activities were supported by R. E. Rabby and L. Xiao. Testing and characterization of joints were performed by T. Roosendaal, H. Jiang, and A. Guzman. Supervisory roles by D. Herling and G. Grant are also acknowledged. J. Chen and Y.C. Lim performed and characterized the USW at ORNL. 


\section{II.3.10 Joining Magnesium Alloys to Carbon Fiber Reinforced Polymers (Pacific Northwest National Laboratory)}

\section{Keerti Kappagantula, Co-Principal Investigator}

Pacific Northwest National Laboratory

902 Battelle Blvd.

Richland, WA 99354

E-mail: keertisahithi.kappagantula@pnnl.gov

\section{Yong Chae Lim, Co-Principal Investigator}

Oak Ridge National Laboratory

1 Bethel Valley Road

Oak Ridge, TN 37831

E-mail: limy@ornl.gov

\section{David Warren, Co-Principal Investigator}

Oak Ridge National Laboratory

1 Bethel Valley Road

Oak Ridge, TN 37831

E-mail: warrencd@ornl.gov

\section{Sarah Kleinbaum, DOE Technology Development Manager}

U.S. Department of Energy

E-mail: sarah.kleinbaum@ee.doe.gov

Start Date: October 1, 2017

Project Funding (FY19): \$650,000
End Date: September 30, 2020

DOE share: $\$ 650,000 \quad$ Non-DOE share: $\$ 0$

\section{Project Introduction}

Existing methods for joining Mg alloys to CFRCs are challenged in varying degrees by corrosion, strength, surface treatment requirements, compatibility with high-volume production or being too application specific. As part of the Joining Core Program, a task on joining Mg to CFRC has been established to address these challenges. PNNL and ORNL have combined forces in the areas of process development, materials characterization, and materials testing in an effort to identify new and promising technologies for joining $\mathrm{Mg}$ to CFRC.

Down-selection was performed by the DOE technology manager, based on the evaluation criteria submitted by the team at the end-of-year project-review meeting held at ORNL on October 10, 2018. The projects selected for continued development in FY 2019 were friction stir interlocking (FSI), conventional bolting, and friction self-piercing riveting (FSPR). Techniques investigated within this project are now centered on new methods for creating robust mechanical interlocking between $\mathrm{Mg}$ and CFRC.

\section{Objectives}

The objective of the FSI task was to manufacture Mg-CFRC joints without any defects on the surface or in the weld zone. The FSPR and bolting tasks focused on evaluating the corrosion phenomena at the Mg-CFRC joint interface and developing optimal corrosion mitigation strategies. 


\section{Approach}

To achieve defect-free Mg-CFRC joints using FSI, a design of experiments approach was used to understand the influence of tool features and process parameters on preliminary bead-on-plate configuration.

Microstructural analysis was used to ensure optimal mixing of the interlock and the $\mathrm{Mg}$ plate, as well as the absence of any defects. Lap-shear testing was performed to determine the load-carrying capacity of the $\mathrm{Mg}$ CFRC FSI joints.

In the FSPR and bolting tasks, corrosion of Mg-CFRC joints was studied to develop corrosion mitigation approaches. It was challenging to study corrosion behavior focused at the dissimilar joint due to massive general corrosion of Mg from ASTM B 117 corrosion testing. Therefore, a systematic and new approach to study corrosion of Mg-CFRC joint was developed.

\section{Results}

\section{Method 1: FSI (PNNL Lead)}

This task demonstrated proof-of-concept for joining an AZ31 Mg alloy sheet to thermoplastic (TP)-CFRC panels by FSI, where Mg interlocks are inserted through CFRC panels to which a Mg sheet is joined by FSW in a lap configuration, as shown in Figure II.3.10.1. In FY 2018, when tools with 5-mm-long pins were used to make joints at a tilt angle of 0-2 degrees, CFRC was ejected onto the joint surface, causing defects. Simultaneously, the CFRC plate deflected, and the plasticized $\mathrm{Mg}$ flowed into the distended CFRC, causing internal defects as well. In FY 2019, friction stir tools with shorter pin length and a correspondingly lower plunge depth were used. Initially, bead-on-plate $(\mathrm{BoP})$ tests were performed using a design of experiments approach - with varying tool-traverse speed, rotation rate, plunge depth, and tilt angle - to identify process parameters resulting in defect-free surfaces and minimal CFRC deflection. Microstructural images of the joint cross-sections obtained perpendicular to tool-traverse direction showed the deflection in CFRC was minimal, and there were no defects observed either on the joint surface or the interior after the BoP process parameter optimization experiments.

(a)

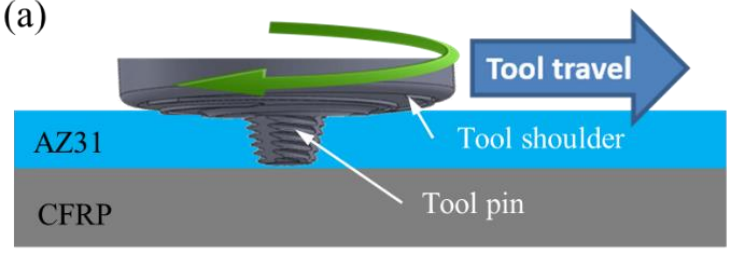

(b)

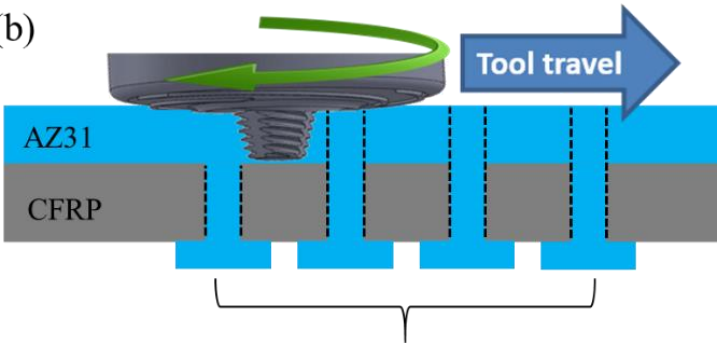

AZ31 inserts

(c)

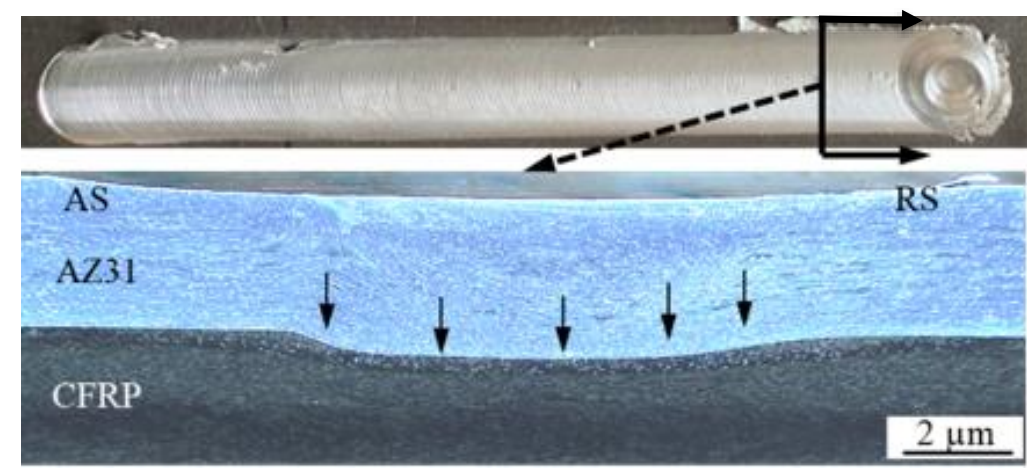

Figure II.3.10.1. Schematic of Mg (AZ31) and CFRC plates joined via (a) BoP configurations, and (b) FSI. (c) Top view of the Mg-CFRC BoP joint showing a defect-free surface. (d) Microstructure of the Mg-CFRC BoP joint showing minimal CFRC deflection and no defects. Source: PNNL. 
Subsequently, these optimal process parameters were applied to join Mg and CFRC plates in the FSI configuration. Results showed that the FSI joints manufactured using shorter pin tools demonstrated no defects on the surface or internally, as shown in Figure II.3.10.2, when the tool tilt angle was increased to 4 degrees. Microstructural images still showed that the CFRC deflected in defect-free joints manufactured in FY 2019. However, defect formation observed in FY 2018 was mitigated when the short pin tool was used to make FSI joints. SEM images of the Mg-CFRC interface showed that the CFRC polymer matrix demonstrated characteristic bubbles that were indicative of the polymer melting and re-solidifying during the process. This served to adhere the polymer to the Mg plate, effectively sealing the interface.

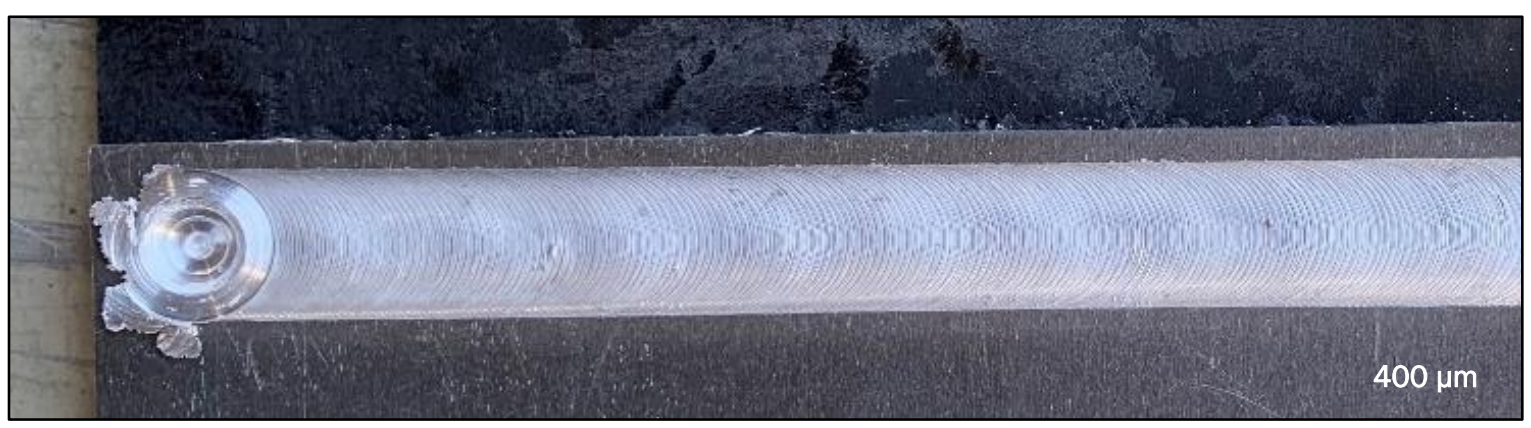

(a)

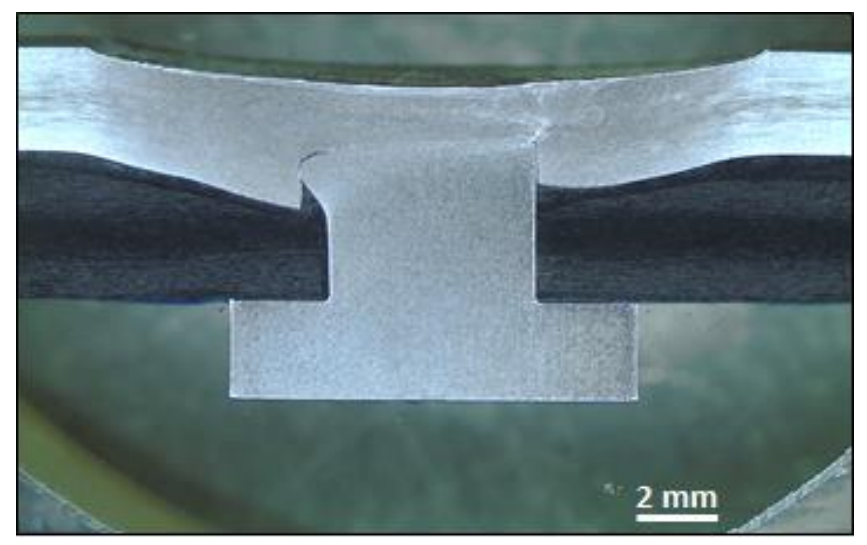

(b)

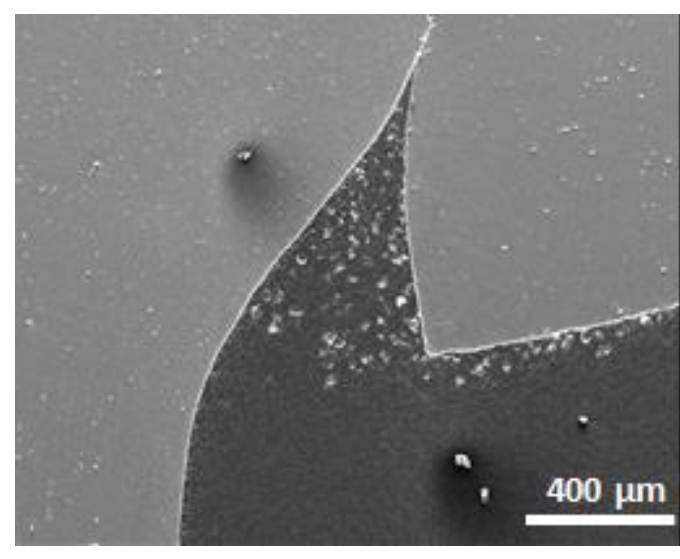

(c)

Figure II.3.10.2. Top view (a) and cross-section view (b) of Mg-CFRC joint made using FSI; SEM image (c) of the Mg-CFRC interface. Source: PNNL.

Lap-shear testing was performed on samples using an MTS load frame, the representative images of which are shown in Figure II.3.10.3 (a)-(c) in various configurations. These identify which parts of the joint are the loadbearing members. Mg-CFRC FSI joints with a width of $15 \mathrm{~mm}$ demonstrated a load capacity of $0.1006 \mathrm{kN} / \mathrm{mm}$ with an elongation of about $0.5 \mathrm{~mm}$ prior to failure, as shown in Figure II.3.10.3 (d). All samples failed in the CFRC with cracks initiating along the Mg-CFRC interface at the hole in the CFRC plates. 


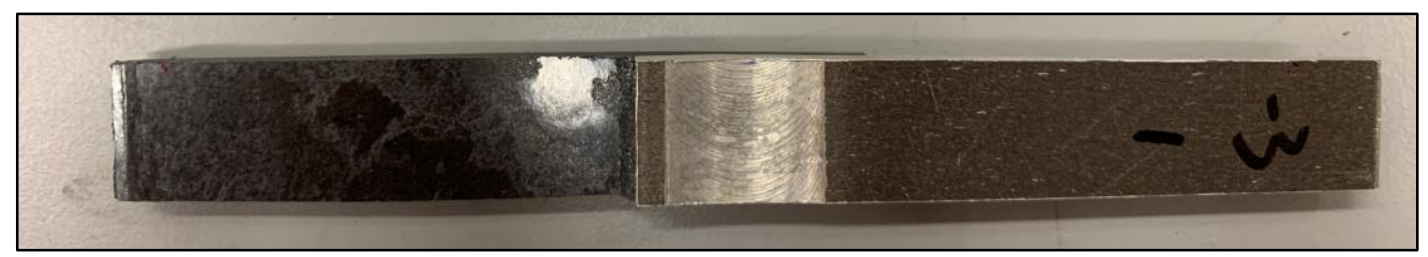

(a)

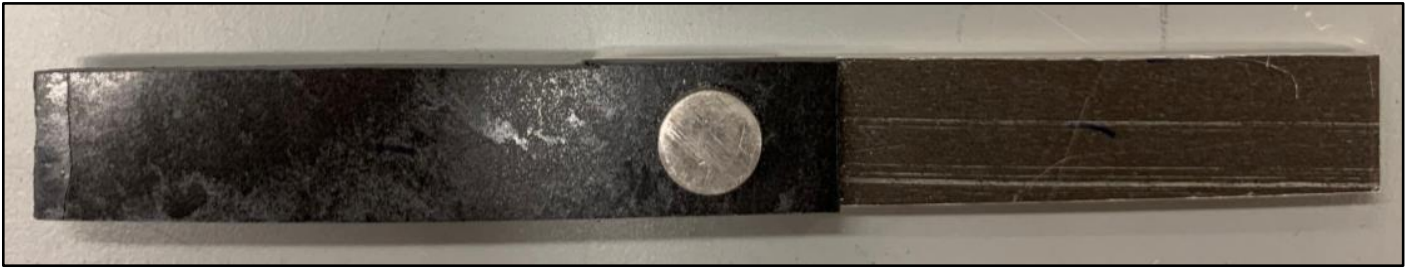

(b)

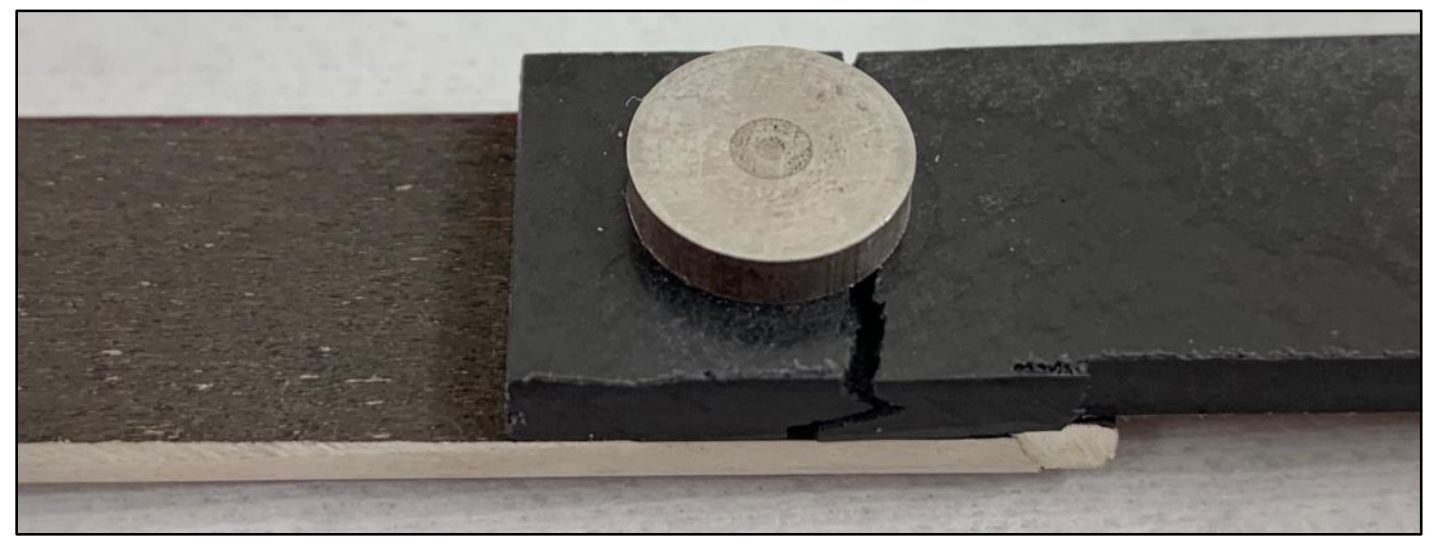

(c)

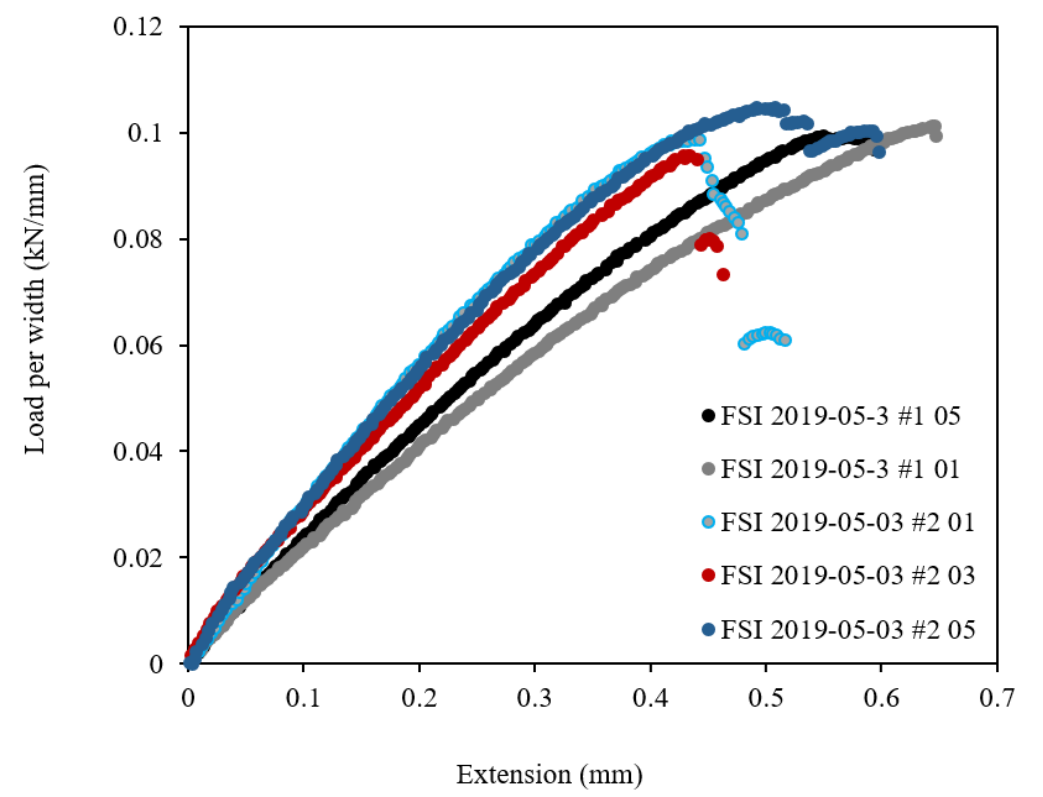

(d)

Figure II.3.10.3. (a) Top and (b) bottom view of Mg-CFRC joint made using FSI; (c) fractures Mg-CFRC sample post lap-shear testing and (d) load per sample width as a function of sample extension depicting the lap-shear behavior of the multiple Mg-CFRC FSI joints manufactured with 4 degrees tool tilt angle (legend labels indicate sample names). Source: PNNL. 


\section{Method 2: Conventional Bolting (ORNL Lead)}

This task demonstrated proof-of-concept for mitigating the damage created by drilling holes in the composite, and a proof-of-concept for electrochemical isolation of the joint components to inhibit or reduce galvanic corrosion of $\mathrm{Mg}$ in contact with steel fasteners and CFRC. To mitigate the damage in the CFRC, the inside of the hole was coated with a very low-viscosity resin that is compatible with the CFRC polymer matrix and has a surface affinity to be drawn into the microcracks of the composite. Working with $3 \mathrm{M}$, a newly designed polymer was identified for this purpose. A three-step method of isolating the components was also developed. In the first step, the polymer infiltrates the microcracks, blunting the crack tip and reducing microcrack propagation. The crack-filling polymer also provides electrochemical isolation of the CFRC. A separate epoxy designed to adhere to $\mathrm{Mg}$ was "painted" onto the surface of the $\mathrm{Mg}$ as the second step, but only at the places where either the steel washers or the composite will be in contact with the substrate. For the third step, polytetrafluoroethylene (PTFE) insulating tape with a high service temperature $\left(>326^{\circ} \mathrm{C}\right)$ was applied to the steel bolt for electrical isolation and corrosion protection. Based on feedback from original equipment manufacturers, thicker thermoset CFRC (4-mm-thick, 20 ply, $+45^{\circ} /-45^{\circ}$ stacking sequence) and AZ31B (3.29mm-thick) plates were used to evaluate corrosion performance of bolted Mg-CFRC joints. It is noted that the standard lap-shear specimen was modified due to the larger bolt size $(\Phi 12.7 \mathrm{~mm})$ and the length was increased to still allow for a square overlap, leading to a $38.1 \mathrm{~mm} \times 114.3 \mathrm{~mm}$ coupon size. For the corrosion study, $0.1 \mathrm{M} \mathrm{NaCl}$ immersion testing, with selective exposure area on AZ31B, was used to investigate corrosion attack on the joint area without causing severe general corrosion of AZ31B observed in ASTM B117 testing. This procedure is illustrated in Figure II.3.10.4 and a summary of immersion corrosion testing conditions is provided in Table II.3.10.1.
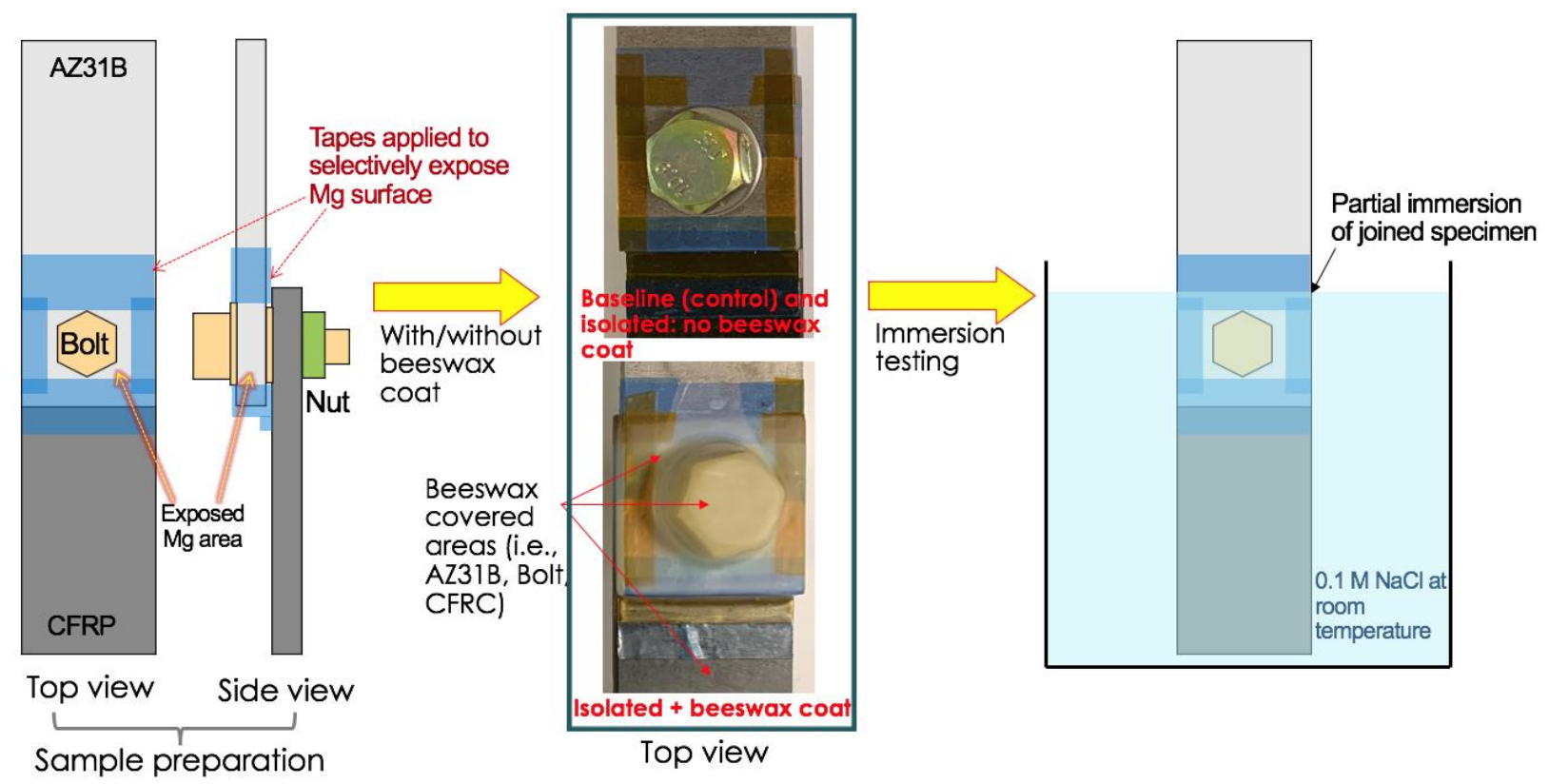

Figure II.3.10.4. Schematic of immersion corrosion testing of bolted thermoset CFRC-AZ31B joint. Source: ORNL.

Table II.3.10.1. Summary of Immersion Corrosion Testing Condition.

\begin{tabular}{|c|c|c|c|c|}
\hline Condition & Baseline (control) & Isolated (three-step process) & $\begin{array}{c}\text { Isolated (three-step process) and } \\
\text { Additional Coating with Beeswax }\end{array}$ \\
\hline Description & $\begin{array}{c}\text { No gavanic } \\
\text { isolation between } \\
\text { bolt and AZ31B }\end{array}$ & $\begin{array}{c}\text { Low-viscosity resin was applied on } \\
\text { CFRC. Epoxy coated at joint } \\
\text { interface AZ31B and CFRP. PTFE } \\
\text { tape was applied on the bolt }\end{array}$ & $\begin{array}{c}\text { Isolated condition + beeswax coating on } \\
\text { the bolt \& CFRP and partial coating on } \\
\text { Mg-CFRP joint area (see Figure II.3.10.4) }\end{array}$ \\
\hline
\end{tabular}


Figure II.3.10.5 shows optical images of post-corroded samples with different conditions after 24-hour immersion testing. For the baseline (control) case in Figure II.3.10.5 (a), corrosion was observed on AZ31B (front and side exposure area) due to the formation of galvanic cell between the bolt and AZ31B. For the isolated case shown in Figure II.3.10.5 (b), much less corrosion was found on AZ31B compared with the baseline case. No corrosion was observed on the joint coated in beeswax in Figure II.3.10.5 (c), indicating total protection of the materials from the electrolyte.

(a) Baseline (Control)

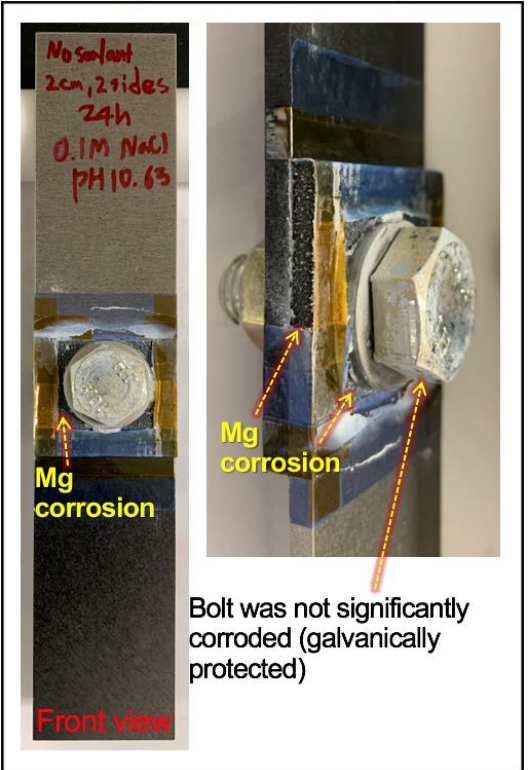

(b) Isolated (three-step process)

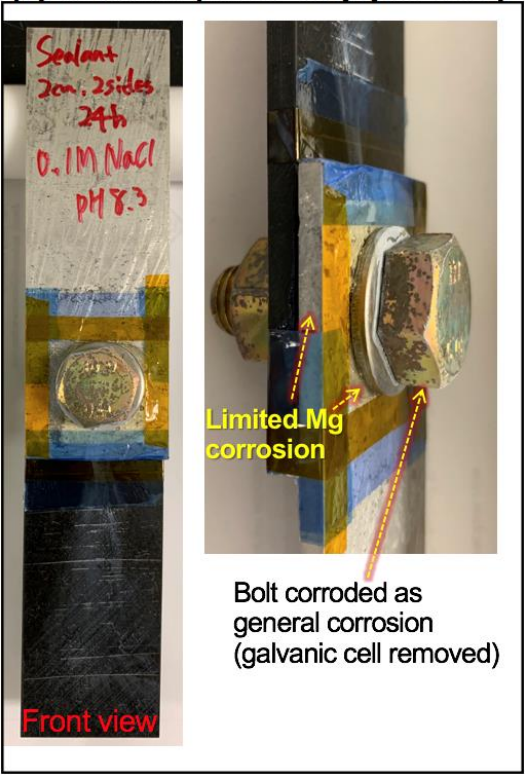

(c) Isolated + coating with beeswax

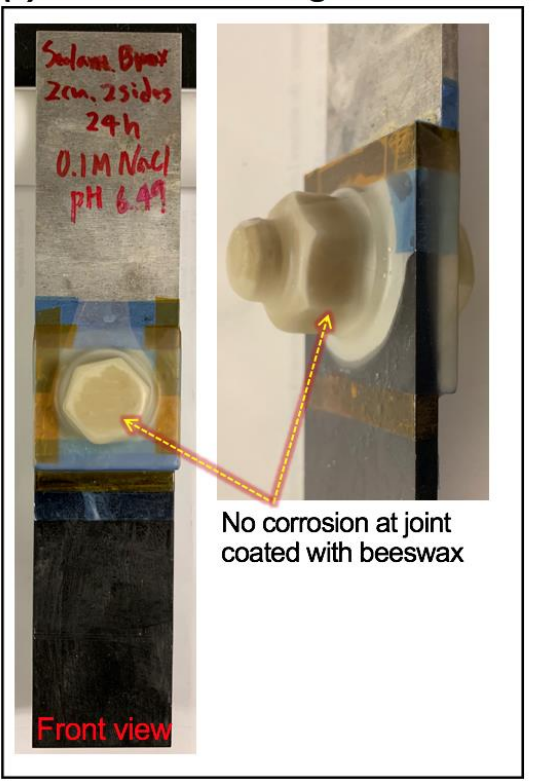

Figure II.3.10.5. Optical images of post-corroded lap-shear samples for: (a) the baseline control; (b) isolated for the threestep process; and (c) isolated plus the coating with beeswax. Source: ORNL.

Electrochemical isolation approaches, including coating at the joint interface and PTFE tape on the bolt, significantly reduced galvanic corrosion effect. Finally, when bolted samples were additionally covered by beeswax on the joint area (bolt + CFRP) and Mg-CFRP area, no corrosion at the joint was seen. Note that beeswax was used for the current study simply to simulate fully protected surfaces at the bolted joint, but future collaborative efforts with the coating industry will be necessary to identify a suitable coating technology. The values of $\mathrm{NaCl}$ solution $\mathrm{pH}$ after 24 hours corrosion testing were measured as $10.63,8.3$ and 6.49 for baseline, isolated and isolated with additional beeswax coating cases, where the initial solution $\mathrm{pH}$ for $0.1 \mathrm{M} \mathrm{NaCl}$ was approximately 6.2. This solution $\mathrm{pH}$ measurement also shows an indirect indication of $\mathrm{Mg}$ corrosion because the $\mathrm{pH}$ would rise with increasing $\mathrm{Mg}$ corrosion. The current study quantitatively indicates that isolation of the galvanic circuit for a dissimilar joint is effective to prevent galvanic corrosion.

\section{Method 3: FSPR (ORNL Lead)}

The FSPR process uses a rotating piercing rivet to generate localized frictional heating during the process. The frictional heating softens the CFRC to facilitate piercing. The frictional heating further increases the local ductility of Mg to avoid cracking issues commonly associated with SPR of Mg alloy. In FY 2018, FSPR was used successfully to join both thermoplastic and thermoset CFRC to Mg alloy AZ31B. Initial evaluation of the corrosion of the dissimilar material joint was also completed. In FY 2019, the focus was on quantitative corrosion study of dissimilar joint and mitigation approaches. For FSPR joining, three corrosion mechanisms - i.e., general corrosion of each material, crevice (i.e., a confined gap) corrosion and galvanic corrosion (e.g., steel rivet and Mg alloy, CFRP and Mg alloy) - will be expected, and these corrosion mechanisms must be addressed for future application of dissimilar joints. General concepts to address the corrosion mechanisms are stated here. General corrosion of each material can be addressed by applying 
coating to isolate a direct-exposure surface (i.e., through a physical barrier and/or sacrificial-anode protection) from the corrosive environment. Crevice corrosion can be mitigated by applying sealant or adhesive to prevent corrosion medium infiltration at the joint interface. This approach is generally called a hybrid or weld-bonding process, and it shows effective mitigation for dissimilar joint $[1,2]$. Electrochemical isolation should be applied on the dissimilar joint to prevent galvanic circuit formation.

To prevent general corrosion of bare steel rivets, a zinc-nickel ( $\mathrm{Zn}-\mathrm{Ni}$ ) coating (i.e., one of common coatings in automotive industry) with a thickness ranging from $8-10 \mu \mathrm{m}$ was applied on steel rivets. Then the corrosion potential of the $\mathrm{Zn}-\mathrm{Ni}$-coated steel rivet was measured and compared with other materials, as summarized in Table II.3.10.2.

Table II.3.10.2. Summary of Corrosion Potential Measurement for Individual Material.

\begin{tabular}{|l|c|}
\hline \multicolumn{1}{|c|}{ Material } & Corrosion Potential $\left(E_{\text {corr }}\right)$ \\
\hline AZ31B & $-1.52 \mathrm{~V}_{\mathrm{SCE}}$ \\
\hline Zn-Ni Coated Rivet & $-0.75 \mathrm{~V}_{\mathrm{SCE}}$ \\
\hline Bare Steel Rivet & $-0.55 \mathrm{~V}_{\mathrm{SCE}}$ \\
\hline Thermoset CFRC & $-0.22 \mathrm{~V}_{\mathrm{SCE}}$ \\
\hline
\end{tabular}

The corrosion potential of the $\mathrm{Zn}-\mathrm{Ni}$ coated rivet was approximately $200 \mathrm{mV}$ lower than the value of a bare steel rivet resulting in a sacrificial-anode effect. That is, $\mathrm{Zn}-\mathrm{Ni}$ coating can galvanically protect the steel rivet. Advanced electron microscopy analysis of FSPR joints made with the Zn-Ni coated steel rivet showed most of the $\mathrm{Zn}$-Ni coating remained on the steel rivet after the joining process, but the coating was removed at localized areas due to interaction with the tool holder and materials. For this reason, wear resistance as well as a corrosion-resistant coating should be further explored.

To quantitatively study corrosion of FSPR joints, immersion testing in a $0.1 \mathrm{M} \mathrm{NaCl}$ solution was performed with selective corrosion exposure in AZ31B (e.g., on two sides and half exposure at the joint), similar to the approach used for the bolting task described previously. This study provided measurable corrosion depth caused by galvanic effect in the dissimilar joint. Figure II.3.10.6 shows optical images of post-corroded FSPR joints with different corrosion exposure times. Figure II.3.10.6 (a) depicts a side view of selective exposure areas at the FSPR joint after 25 hours of immersion corrosion testing. Figure II.3.10.6 (b) and (c) show a backside view of the FSPR joint displaying progressive corrosion of AZ31B at increased corrosion exposure time. Maximum corrosion depth (indicated by red dotted circles) on AZ31B for 25 and 50 hours was measured to be 1.44 and $2.6 \mathrm{~mm}$, respectively. From lap-shear tensile testing for post-corroded samples, retained mechanical joint strength for 25 and 50 hours was 4.67 and $4.33 \mathrm{kN}$, respectively. Approximately, 90\% of the original strength remained after 50 hours of exposure time. Longer corrosion testing will be required to further study quantitative corrosion depth measurement and correlation with mechanical joint strength. 


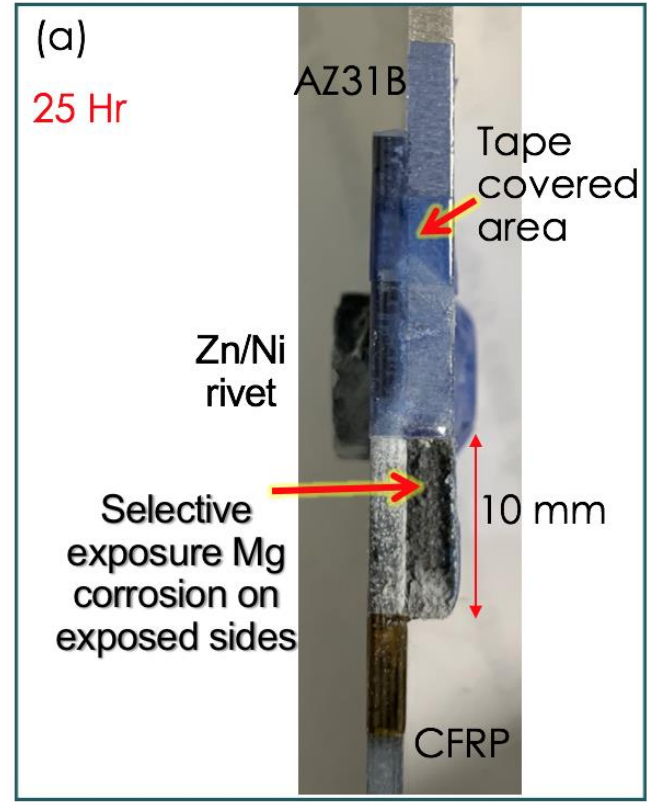

Side view of F-SPR
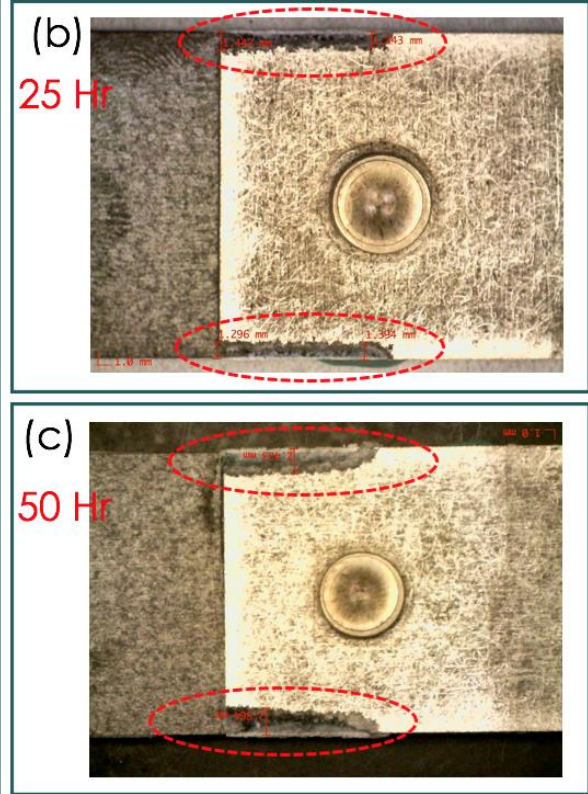

Backside view of F-SPR joint

Figure II.3.10.6. Optical images of post-corroded lap-shear samples with different conditions. (a) Side view of selective exposure areas at the FSPR joint, and (b) and (c) backside view of the FSPR joint displaying progressive corrosion. Source: ORNL.

To address crevice corrosion, the FSPR process was combined with structural adhesive to isolate corrosion medium infiltration at the joint area. Figure II.3.10.7 depicts load and displacement curves from lap-shear tensile testing for weld-bonded samples with a representative fractography image shown in the inset. From load and displacement curves, two distinct peak-failure loads were observed. The first peak load corresponded to the strength of the adhesive bond and the second peak was from the FSPR joint. One of the advantages of the weld-bonding process is to further increase mechanical joint strength while providing a barrier layer from corrosion-solution penetration at the joint. The fractography image of a weld-bonded sample also shows the two failure modes. The first one is a cohesive failure mode from adhesive-bonding where the adhesive (aquacolored) layer is fractured. The second failure was button pullout from AZ31B, which is the same as is seen in an FSPR joint without adhesive. For future work, corrosion behavior of adhesive-bonded only and weldbonded samples should be evaluated.

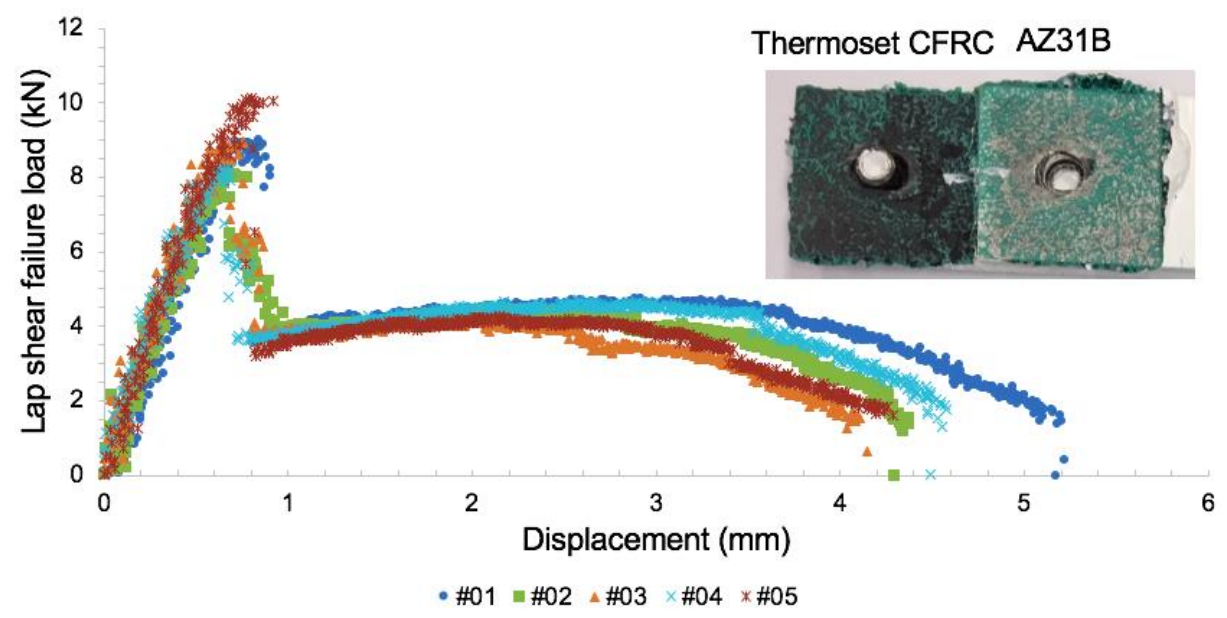

Figure II.3.10.7. Load versus displacement curves from lap-shear testing for five weld-bonded samples. Source: ORNL. 


\section{Conclusions}

At the end of FY 2019, all joining techniques used for Mg-CFRC joining have demonstrated proof-of-concept. The FSI task demonstrated mechanically sound Mg-CFRC joints by developing an understanding of the effects of tool and process parameters on the heat generated during the joining process and effectively managing it. The bolting and FSPR tasks studied the corrosion behavior of the Mg-CFRC joint by demonstrating methods of isolating the joint during corrosion testing along with demonstrating corrosion mitigation strategies using relevant coatings.

\section{Key Publications}

1. Upadhyay, P., M. Reza, E. Rabby, and S. A. Whalen, 2019, "Joining of Mg to reinforced polymers using FSI," TMS Friction Stir Joining and Welding X, 10-14 March 2019, San Antonio, TX, USA.

2. Wang, T., P. Upadhyay, S. A. Whalen, and K. S. Kappagantula, 2019. "Joining of Mg to CFRPs using FSI technique," MS\&T 2019, 30 September -3 October 2019, Portland, OR, USA.

3. Wang, T., S. A. Whalen, P. Upadhyay, and K. S. Kappagantula, 2019, "Joining dissimilar materials via rotational hammer riveting technique," Magnesium Technology 2020. Accepted for publication.

4. Whalen, S. A., T. Wang, P. Upadhyay, and K. S. Kappagantula, 2020, "Joining Mg and polymer by friction stir technologies," TMS 2020, 23-27 February 2020, San Diego, CA, USA. Accepted for presentation.

5. Lim, Y., J. Jun, J. Chen, M. Brady, D. Leonard, C. Warren, J. Chen, and Z. Feng, 2020, “Corrosion study on CFRP-AZ31B spot joined by friction self-pierce rivet," TMS Coatings and Surface Engineering for Environmental Protection II, 23-27 February 2020, San Diego, CA, USA. Accepted for presentation.

6. Lim, Y., C. Warren, J. Chen, J. Jun, and Z. Feng, 2019, "Joining of CFRPs to AZ31B using FSPR," $M S \& T$ 2019, 30 September - 3 October 2019, Portland, OR, USA.

7. Lim, Y. C., C. D. Warren, J. Chen, and Z. Feng, 2019, "Joining of lightweight dissimilar materials by FSPR” In: Hovanski, Y., R. Mishra, Y. Sato, P. Upadhyay, and D. Yan (eds), Friction Stir Welding and Processing $X$. The Minerals, Metals \& Materials Series. Springer, Cham.

8. Lim, Y., C. Warren, J. Chen, and Z. Feng, 2019, "Joining of lightweight dissimilar materials by friction self-pierce riveting," TMS Friction Stir Joining and Welding X, 10-14 March 2019, San Antonio, TX, USA.

\section{References}

1. Lim, Y. C., L. Squires, T.-Y. Pan, M. Miles, G.-L. Song, Y. Wang, and Z. Feng, 2015, "Study of mechanical joint strength of Al alloy 7075-T6 and dual-phase steel 980 welded by friction bit joining and weld-bonding under corrosion medium," Mater. Des., Vol. 69, pp. 37-43.

2. Lim, Y. C., L. Squires, T.-Y. Pan, M. Miles, J. K. Kuem, G.-L. Song, Y. Wang, and Z. Feng, 2017, "Corrosion behavior of friction-bit-joined and weld-bonded AA7075-T6/galvannealed DP980,"

Sci. Technol. Weld. Join., Vol. 22, pp. 455-464. 


\title{
II.3.11 High-Performance Computing and High-throughput Characterizations towards Interfaces-by-Design for Dissimilar Materials Joining (Oak Ridge National Laboratory)
}

\author{
Xin Sun, Co-Principal Investigator \\ Oak Ridge National Laboratory \\ 1 Bethel Valley Rd. \\ Oak Ridge, TN 37831 \\ E-mail: $\underline{\text { unx1@ornl.gov }}$
}

\section{Erin Barker, Co-Principal Investigator}

Pacific Northwest National Laboratory

902 Battelle Blvd.

Richland, WA 99354

E-mail: erin.barker@pnnl.gov

\section{Sarah Kleinbaum, DOE Technology Development Manager}

U.S. Department of Energy

E-mail: $\underline{\text { sarah.kleinbaum@ee.doe.gov }}$

Start Date: October 1, 2017

End Date: September 30, 2020

Project Funding (FY19): \$850,000

DOE share: $\$ 850,000$

Non-DOE share: $\$ 0$

\section{Project Introduction}

Further weight reduction of automotive body structures depends on the industry's ability to reliably and costeffectively assemble multiple lightweight materials, including CFRPs, Mg alloys, Al alloys, and AHSSs. Because of their extreme disparities in physical properties, such as melting temperature, thermal conductivity, and thermal expansion coefficient, dissimilar materials pose a serious challenge for traditional joining process development. There is a lack of fundamental understanding on the interfacial bond formation mechanisms for the different dissimilar materials pairs to enable process development.

\section{Objectives}

With the recent advancements in HPC and high-throughput experiments at various materials length scales, this project aims to establish a scientific framework for designing the hierarchical interfacial features and the associated chemical and thermomechanical kinetic pathways to achieve them.

\section{Approach}

For each set of materials interfaces, the interfacial characteristics at different length scales are simulated with multiscale materials processing models and the results will be experimentally validated and calibrated with associated experiments at the corresponding scales. The calibrated modeling parameters at the lower length scale will be hierarchically passed to the higher length scale to predict the robust processing parameter windows with quantitative confidence. Once the hierarchical multiscale interfacial modeling framework is established, it can be used to inversely design the dissimilar materials interface and the associated welding process and parameters, thereby accelerating the development and deployment of dissimilar materials joining technologies. 
In FY 2019, the interface by design model development focused on joining Mg to uncoated steel. The intention of selecting these two materials is to develop and demonstrate the interface by design framework with the most challenging material pair, $\mathrm{Mg}$ and iron (Fe), which are typically considered un-weldable due to their high physical-property disparities, low mutual solubility, and lack of chemical reactions. The success in joining the immiscible $\mathrm{Mg}$ to uncoated steel will enable the generalization of the design framework to other dissimilar materials. Two different joining methods, ultrasonic welding (USW) and friction stir scribe welding (FSSW), are investigated in parallel, and the technical approaches for interface by design were carried out in two parallel subtasks corresponding to each joining method. Macroscale coupled thermomechanical finite element model (FEM) of USW was established to quantify the heat generation and plastic deformation for joining $\mathrm{Mg}$ sheet and uncoated steel. Modeling results were validated with infrared measurements and lap-shear test. Using the validated model, the surface roughness effect on heat generation was predicted and an optimized surface engineering method was identified. In micro- and lower-length scales, the chemical components and structure of $\mathrm{Mg} / \mathrm{steel}$ interface was investigated under SEM and HRTEM. MD model was used to study the underlying mechanism and the intrinsic interface structure and strength. The macro- and micro- simulation results are then connected to identify the interface-formation condition to guide the welding process parameter development. For FSSW, a FEM is developed to link underlying structure of the friction stir scribe (FSS) joint to its properties. After validation of the model, $\mathrm{Mg}$-steel lap-joint performance simulations are conducted to identify interfacial characteristics leading to desired joint mechanical performance. In addition, an approach is developed by combining both experimental output and FE modeling to determine the cohesive law parameters to mechanically characterize the interface obtained in dissimilar FSW joints.

\section{Results}

\section{Task 1. Interface by Design for Ultrasonic Welding of AZ31/DP590}

A coupled thermomechanical FEA model was developed to predict the heat generation in the UW of AZ31 Mg sheet and bare DP590 steel sheet, as shown in Figure II.3.11.1 (a). The thickness of the DP590 sheet is 1.08 $\mathrm{mm}$, while the AZ31 sheet measures $2.34 \mathrm{~mm}$. The zinc coating was removed from the DP590 surface by acid with energy dispersive spectroscopy confirmation. An UW machine with two sonotrodes was employed to weld the 1-in. $\times 1$-in. coupon for the fundamental understanding of interface temperature evolution. An infrared camera was used to measure temperature at the edge of sheets. The welding power was preset as 1000 $\mathrm{W}$, vibrating frequency was set at $20 \mathrm{kHz}$, and there was a nominal clamping pressure of $50 \mathrm{psi}$. In the simulation, the Johnson-Cook model was used to model the plasticity of the AZ31 and DP590, respectively. The friction coefficient at the interface was a function of temperature $(T)$ and pressure $(p)$. The modeled temperature features an oval distribution due to the frictional heat at faying interface. The indentation on the $\mathrm{Mg}$ sheet is large under the combined effect of thermal softening and acoustic softening. Figure II.3.11.1 (b) plots the comparison between infrared measurements of temperature history versus simulation results, as well as the effects of vibration amplitude and clamping load. Achieved peak temperature is higher in the cases with larger clamp load and especially with higher vibration amplitude. Even though no bonding is found between the two sheets under all conditions, the reasonable temperature comparison between the prediction and the measurement serves as model validation. 


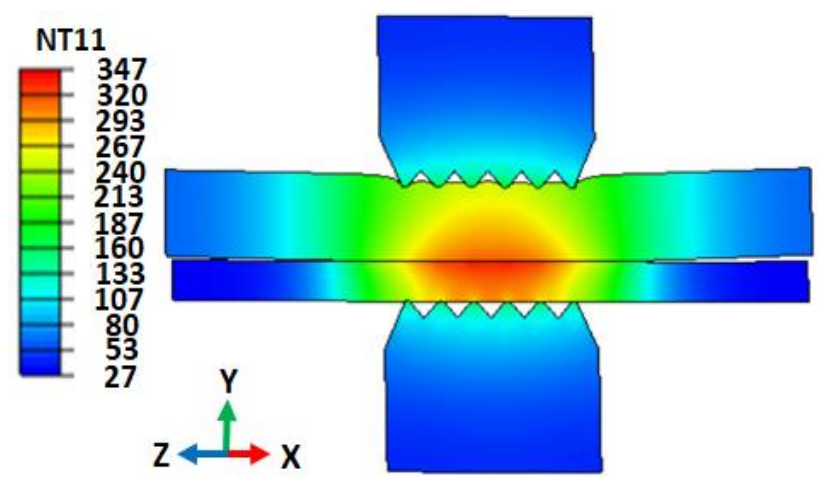

(a)

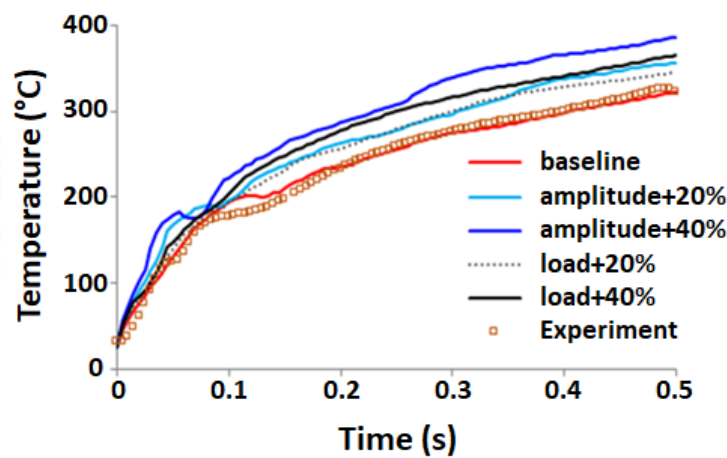

(b)

Figure II.3.11.1. Prediction of heat generation for UW: (a) predicted temperature distribution and (b) comparison of predicted and measured temperature histories under different welding conditions. Source: ORNL.

We hypothesized that the absence of welded interface is because of the low temperature and the very low solubility of $\mathrm{Mg}$ in $\mathrm{Fe}$ at the interface. To validate the above hypothesis and further improve the $\mathrm{Mg}-\mathrm{Fe}$ interface, we conducted a study on the Mg-steel interface formed under extreme heat generation and rapid cooling achieved by VFAW, where a 1.8-mm-thick AZ31 sheet flyer is shot to a 0.8-mm-thick DP590 (uncoated) target at an impact velocity of $730 \mathrm{~m} / \mathrm{s}$. The details of the experiment can be found in Reference [1]. The very high impact velocity causes a significant temperature increase at the interface through the rapid conversion from kinetic energy to local heat generation. The two sheets are found bonded after the impact. SEM micrograph is used to characterize the resulting AZ31-DP590 interface microstructure, and the results are shown in Figure II.3.11.2 (a). An "interlayer" is found at the interface between AZ31 and DP590, which acts as a bridge to bond the two materials, as shown in the high magnification SEM image in Figure II.3.11.2 (b). The interlayer is characterized by having abundant particles with varying sizes from a few nanometers to a few micrometers. The thickness of the interlayer is around $\sim 200 \mu \mathrm{m}$ in the corner of the weld and gradually "vanishes" toward the center of the impact sample. The HRTEM micrograph of the interlayer-DP590 interface is shown in Figure II.3.11.2 (c), which shows the atomic-level conforming interface between the interlayer and DP590 and indicates a strong joint. In Figure II.3.11.2 (c), nano-size particles are revealed close to the interlayer-DP590 interface and short-range order in the lattice structure. This is in contrast with the amorphous interface nanostructure reported in the impact welded interfaces of other dissimilar materials. The chemical composition of the interlayer is revealed in Figure II.3.11.2 (d) using EDS. The matrix of the interlayer is found to be $\mathrm{Mg}$, and the chemical composition of the particles is dominated by $\mathrm{Fe}$, with a slight amount of Mn. At the interlayer-DP590 interface, the Fe-Mg transition zone is about 20-nm thick. Interestingly, no $\mathrm{Al}$ or $\mathrm{Zn}$ intermetallic phase or concentration is observed in the interlayer, and they do not appear as a constitutive component of the Fe-based particles. No concentration of $\mathrm{Al}$ or $\mathrm{Zn}$ is observed at the interface either, due to the fast cooling rate and limited time for $\mathrm{Al}$ or $\mathrm{Zn}$ to diffuse to the interface This is different from some previous reports on welded Mg-Fe samples where Al- or Zn-based IMC layers are critical for bonding. 


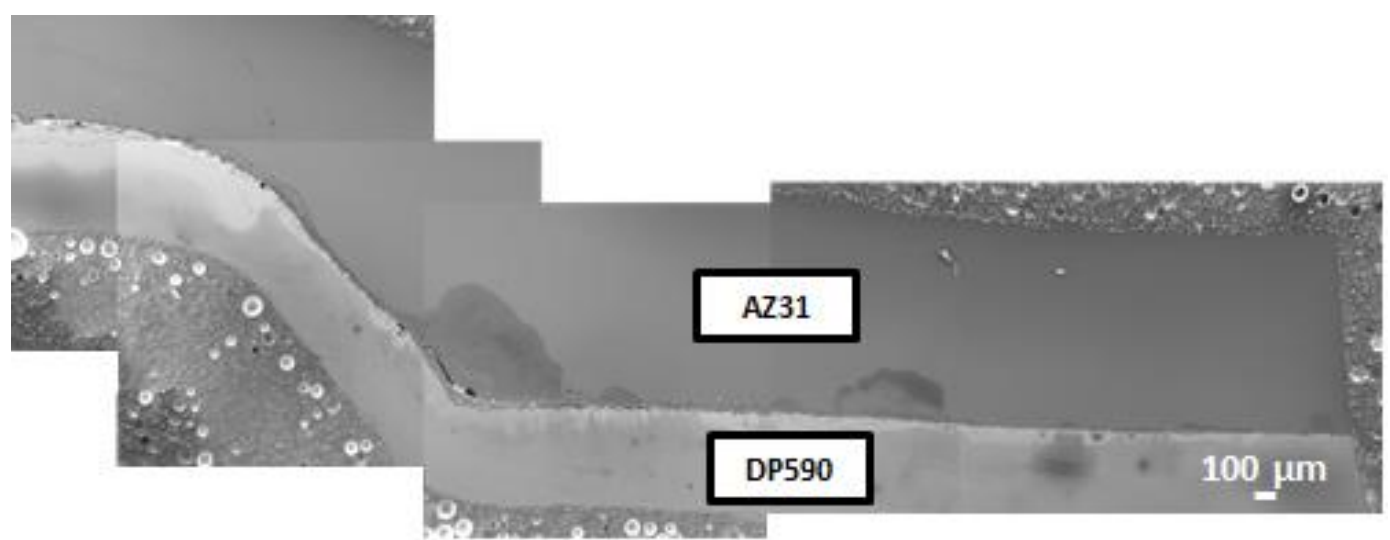

(a)

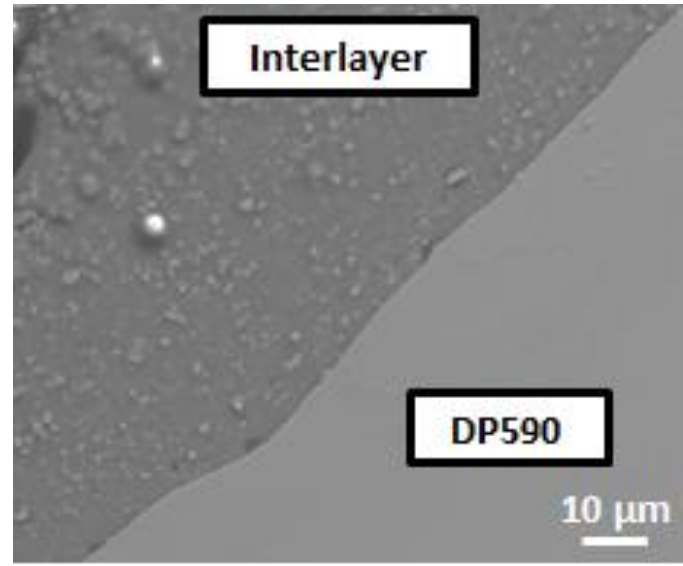

(b)

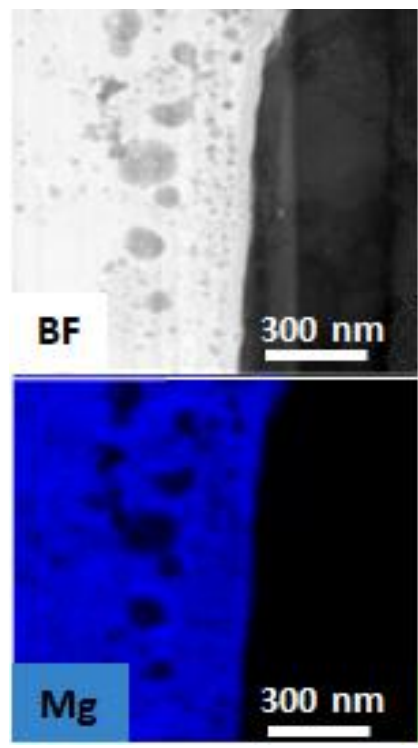

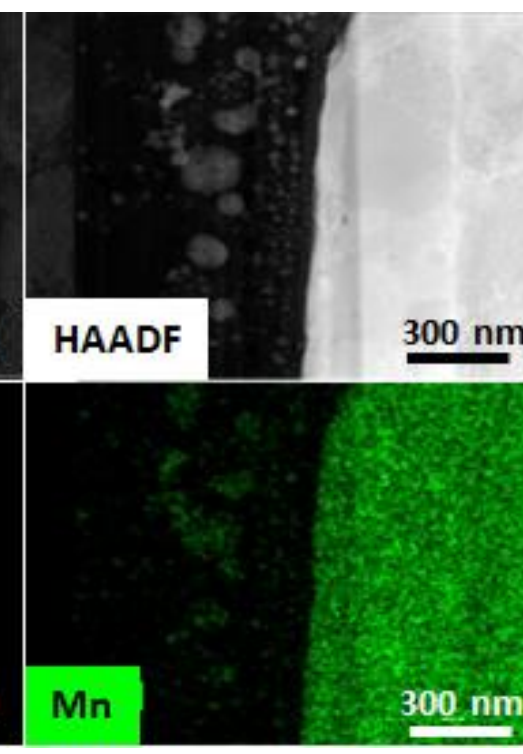

(d)

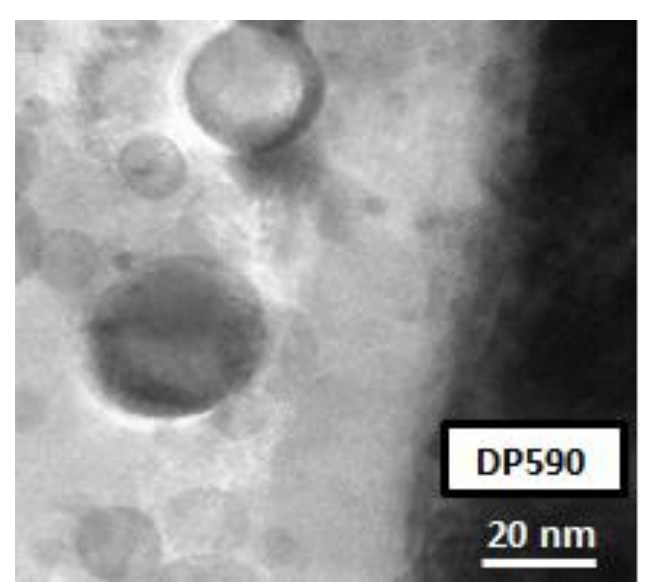

(c)

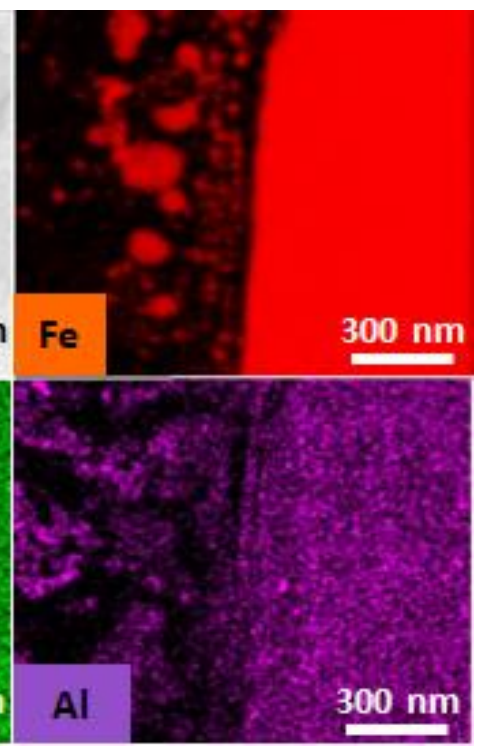

$00 \mathrm{~nm}$

Figure II.3.11.2. SEM and TEM micrograph showing the AZ31/DP590 impact weld result by VFAW. (a) SEM micrograph of the AZ31/DP590 impact weld sample cross-section. (b) Higher magnification SEM image of the corner region, which shows an interlayer between AZ31 and DP590. (c) HRTEM images of the interlayer and the interface between interlayer and DP590, abundant Fe particles are observed. (d) STEM-EDS image of chemical components of the interlayer. Source: ORNL. 
To understand the bonding mechanism at the interface, it is critical to know the local conditions (e.g., local velocity and temperature at impact interface), which is different from the macroscopic applied condition. This is analyzed using an Eulerian-based coupled thermal-mechanical FE model within the commercial FE software ABAQUS/Explicit.

The model setup is shown in Figure II.3.11.3 (a) and the details about mesh, boundary conditions, and material properties are described in Reference [1]. The simulation results are shown in Figure II.3.11.3 (b)-(e). The Mg flyer impacts the tilted edge of steel target first. A thin layer of ejected Mg flows ahead of the collision front, known as impact jetting, and is then observed during the impact of the Mg flyer on the steel target edge, as shown in Figure II.3.11.3 (b). The jetting is a function of impact angle and impact velocity. The velocity transverse to the impact surface and temperature at the jet front increases sharply during the propagation of the impact and jetting, as shown in Figure II.3.11.3 (c) and (d). The jet layer reaches a high-temperature of $1200 \mathrm{~K}$ when it reaches the corner of steel target, which indicates the jet layer is already molten (melting point of $\mathrm{Mg}$ is $923 \mathrm{~K}$ ). The jet velocity is very high (i.e., $V_{j e t}>2000 \mathrm{~m} / \mathrm{s}$ ) at the end of the tilted surface. The high velocity allows the jet layer to reach the flat steel surface earlier than the rest of the $\mathrm{Mg}$ flyer, giving it a short

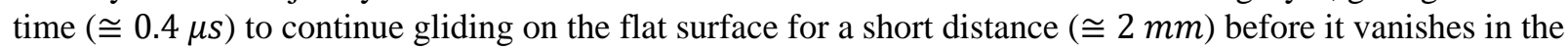
collision between the Mg flyer and the steel target. Figure II.3.11.3 (e) illustrates the transverse component of velocity $\left(V_{x}\right)$ at the moment of impact between flyer, jet layer, and target. The FE simulation results help to identify the local impact conditions at the interface - a Mg intermediate jetting layer exists between the AZ31 flyer plate and the DP590 target, which has high-temperature and high transverse velocity. The impact indeed occurred like a "sandwich" structure - the Mg flyer impacted the jet layer and then pushed/squeezed it to the steel target.

Next, a MD simulation was conducted to study the interfacial characteristics by considering the FE-predicted results as the initial and boundary conditions for the local interface layer. The setup of the MD model is shown in Figure II.3.11.4 (a). The jetting velocity and temperature $\left(V_{\text {normal }}=750 \mathrm{~m} \cdot \mathrm{s}^{-1}, V_{\text {transverse }}=\right.$ $3000 \mathrm{~m} \cdot \mathrm{s}^{-1}$ and $T=900 \mathrm{~K}$ ) are adopted as the initial condition of the $\mathrm{Mg}$ atoms. The details for simulation of this process are described in Reference [1]. Figure II.3.11.4 (b)-(e) plots the MD predictions of the interface at different times. Figure II.3.11.4 (b) shows the atoms mixing at the beginning of impact. The very high transverse velocity of the Mg flyer "stirred" the atoms at the impact interface, which caused a significant amount of $\mathrm{Fe}$ atoms to gradually become mixed into the $\mathrm{Mg}$. Meanwhile, the Fe target did not preserve the lattice structure locally at the interface. The temperature in this nanoscale interfacial region increased sharply due to the conversion of the high kinetic energy from the bulk of jetting layer into the internal energy. The very high-shear velocity provides a mechanical "mixing" force that locally blends the atoms of the two materials, which are otherwise immiscible at RT, as shown in Figure II.3.11.4 (c). Next, cooling is introduced into the MD simulation by reducing the temperature under canonical (number of particles, volume and temperature) ensembles. Figure II.3.11.4 (d) and (e) shows the system cooled to 300K within 1.0 nanosecond at constant rate. When the temperature drops, the Fe and $\mathrm{Mg}$ segregation occurs owing to the decrease in solubility. The $\mathrm{Fe}$ atoms within the $\mathrm{Mg}$ start to assemble into particles, and the Fe-Mg interface gradually becomes sharp, as shown in Figure II.3.11.4 (d). The Fe atoms nucleate crystallographic lattice structure from the interface and within the particles to minimize the free energy of the system. When the temperature further decreases, the $\mathrm{Mg}$ lattice structure tends to nucleate from the existing Fe lattice at the interface with coherency, as observed in Figure II.3.11.4 (e). The MD simulation results closely resemble the HRTEM observations of the weld interface observed previously in Figure II.3.11.2 (c), which explains the formation of the experimentally observed features: (1) the formation of the Fe particles is from the jetting induced mechanical mixing of $\mathrm{Mg}$ and $\mathrm{Fe}$, followed by cooling induced segregation; (2) the sharp $\mathrm{Mg}$-Fe joining interface is the result of low solubility at low temperature; and (3) during the cooling induced crystallization, the $\mathrm{Mg}$ and Fe lattices forms low-mismatching structure, leading to the bonding between the two materials. 


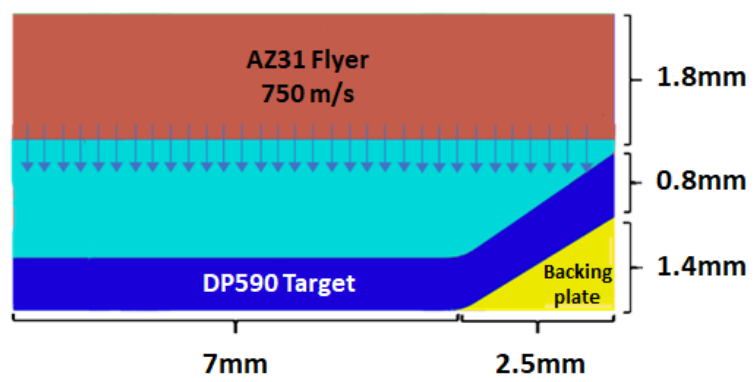

(a)
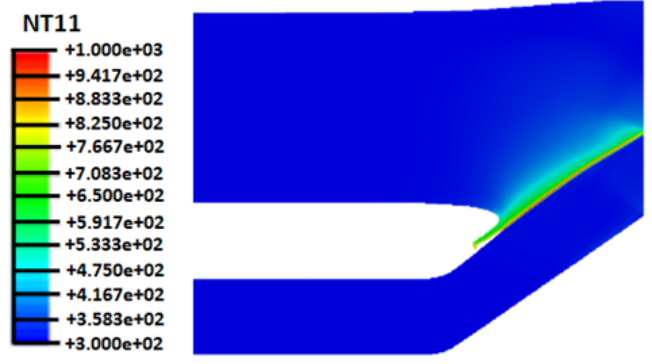

(c)

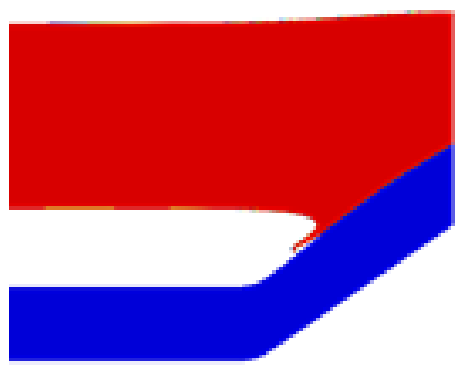

(b)

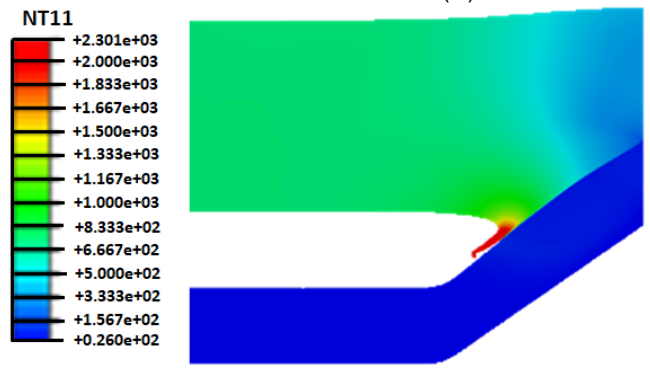

(d)
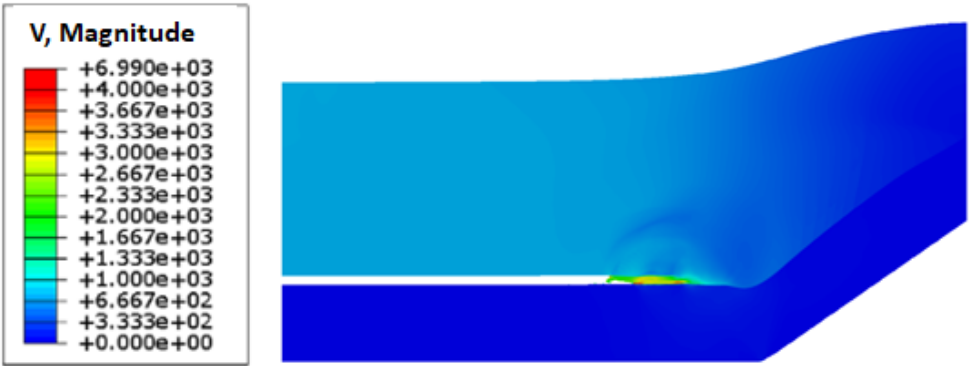

(e)

Figure II.3.11.3. Eulerian FEM simulation results of the AZ31-DP590 impact welding process. (a) Model setup with applied initial boundary conditions. (b) AZ31 material flow ejected from the impact front, known as jetting, is formed as a result of the impact angle. (c, d) A simulation showing the corresponding temperature and velocity distributions, respectively. (e) the velocity distribution after the jet flow propagates to the flat region of impact interface. Source: ORNL.

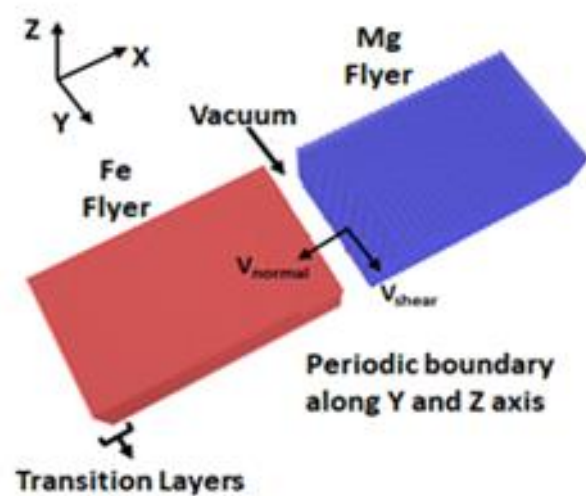

(a)

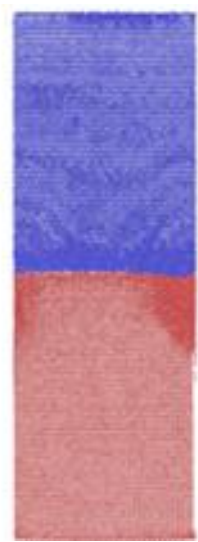

(b)

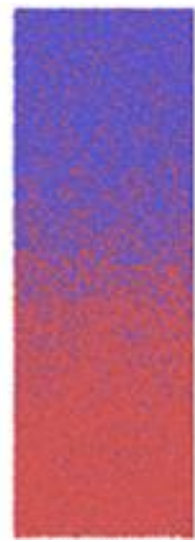

(c)

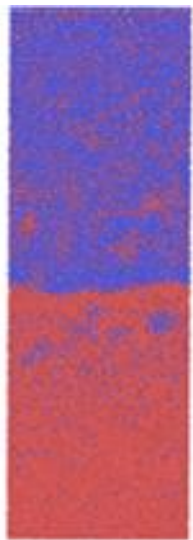

(d)

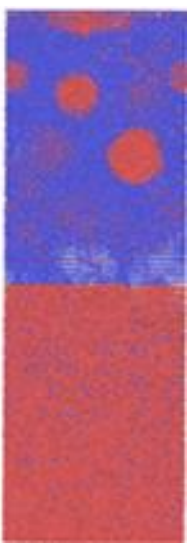

(e)

Figure II.3.11.4. MD simulation prediction of impact interface nanostructure evolution: (a) setup of MD simulation with applied initial boundary conditions; (b) at the begining (10 ps after) of the impact; (c) before the unloading; (d) after cooling

for $0.6 \mathrm{~ns}$ to $2500 \mathrm{~K}$, segregation of $\mathrm{Mg} / \mathrm{Fe}$ atoms at the interface is observed; and (e) after cooling to RT, Fe atoms segreagated into particles in Mg matrix and lattice structure formed at interface. Source: ORNL. 
We further studied the possible methods of enhancing the bonding strength between Mg alloy and uncoated steel via USW. First, a different friction coefficient at the interface was considered. The reference case used the temperature and pressure dependent friction coefficient established during research in FY 2018. Three cases were investigated by assuming the friction coefficient at faying surfaces offset +0.1 and -0.1 , as well as a +0.2 increase at all surfaces (e.g., $\mathrm{Mg}$ sheet-horn, $\mathrm{Mg}$ sheet-Fe sheet, and Fe sheet-horn). The modeling results indicate that heat generation cannot be improved by solely increasing or decreasing the friction at the faying surface but requires the modification to the top and interfaces between the sonotrode and the substrates. To achieve the desired surface condition, the sheet surfaces were engineered by polishing and filing. The surface roughness for each condition was measured using an optical profiler at three locations and the averaged values are summarized in Table II.3.11.1. For DP590, the surface sanded with sanding paper with different grit sizes showed a lower roughness ( $\mathrm{Ra}$ ) value than the filed case. For AZ31, a similar level of surface roughness is achieved by sanding with P120 and filing. The surface data for filed samples are shown in Figure II.3.11.5 (a) for AZ31 and in Figure I.1.1.5 (b) for DP590. Similar level of surface roughness for Mg and Fe sheets can be observed. The texture pattern created through filing will promote the mechanical interlocking as well as the friction coefficient by increased roughness. From Reference [2], the friction coefficient was increased with surface roughness, Ra, in a range between $0.75 \mu \mathrm{m}$ and $1.5 \mu \mathrm{m}$. Further, a meso-scale model was developed to correlate the surface roughness with friction coefficient for the $\mathrm{Mg}$-Fe dissimilar material pair, proving a monotonic increase of friction coefficient with respect to Ra in a range of $1.2 \sim 3.6 \mu \mathrm{m}$. In this study, the concept of surface engineering to enhance heat generation is validated by experimental temperature measurement.

Table Il.3.11.1. Surface Roughness (Ra) of Specimen under Different Engineering Conditions.

\begin{tabular}{|c|c|c|c|c|c|}
\hline Processing & As-received & $\begin{array}{c}\text { Sanded by } \\
\text { P120 }\end{array}$ & $\begin{array}{c}\text { Sanded by } \\
\text { P320 }\end{array}$ & $\begin{array}{c}\text { Sanded by } \\
\text { P1000 }\end{array}$ & Filed \\
\hline AZ31 sheet & $0.19 \mu \mathrm{m}$ & $2.64 \mu \mathrm{m}$ & $0.92 \mu \mathrm{m}$ & $0.31 \mu \mathrm{m}$ & $2.39 \mu \mathrm{m}$ \\
\hline DP590 sheet & $1.57 \mu \mathrm{m}$ & $1.03 \mu \mathrm{m}$ & $0.56 \mu \mathrm{m}$ & $0.29 \mu \mathrm{m}$ & $1.85 \mu \mathrm{m}$ \\
\hline
\end{tabular}

Compared with engineered surface using sandpaper, heat generation was substantially enhanced in the filed cases as indicated by the infrared camera measurement in Figure II.3.11.6 (a). The difference in peak temperature is more than $70^{\circ} \mathrm{C}$, which is consistent with numerical prediction. Figure II.3.11.6 (b) compares the measured temperature profile at the center of the faying interface between AZ31 and uncoated DP590. The experiment with a specimen sanded at the faying surface by P120 shows the lowest heat generation rate. The specimen prepared by intermediate grit size P320 sanding has a higher heating rate at the early stage of welding than other sanded specimens. Reasonable agreement between an experiment and the model can be confirmed for the studied cases. Again, no bond between the two sheets is formed since the temperature at the faying interface is still low.

The microscale study indicates the high local shear velocity at $\mathrm{Mg}$-Fe interface induces mechanical mixing between the $\mathrm{Mg}$ and $\mathrm{Fe}$ and creates an interlayer, which is critical for bond formation. With this information, the macroscopic UW test was revised to increase the vibration amplitude. A bond is formed under a welding power of $3500 \mathrm{~W}$ and a clamping pressure of $60 \mathrm{psi}$, with the horn-anvil setup, as seen in Figure II.3.11.7 (a). 

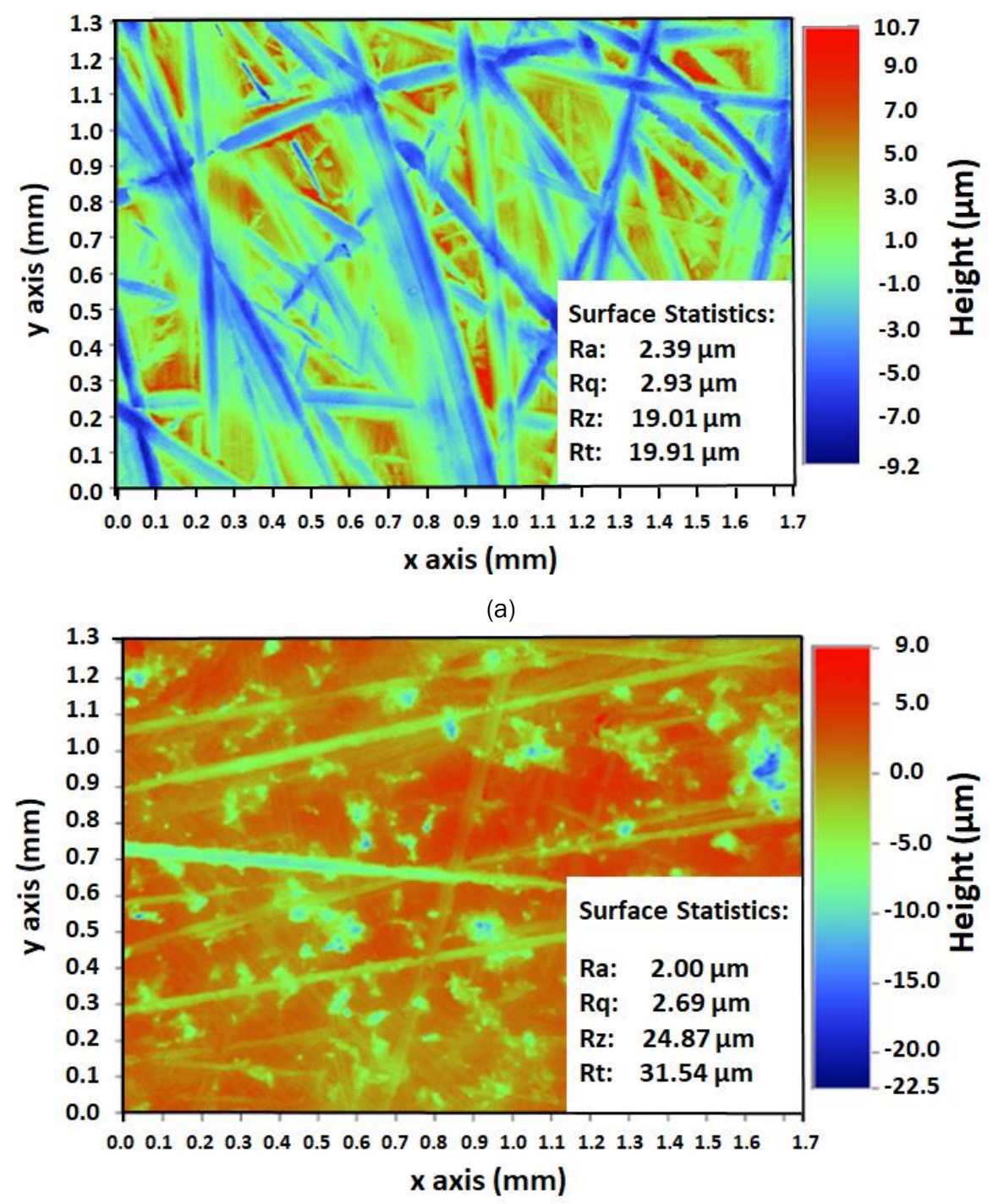

(b)

Figure II.3.11.5. Roughness measurements of filed surfaces. (a) AZ31 and (b) DP590. Source: ORNL.
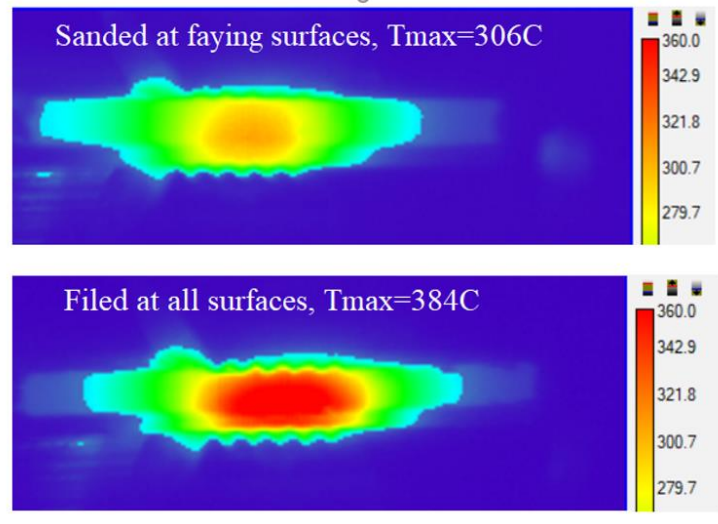

(a)

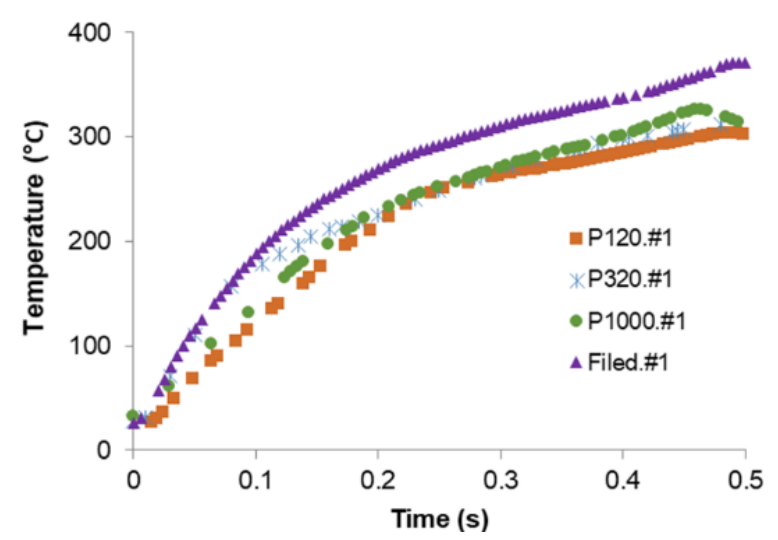

(b)

Figure II.3.11.6. Temperature by infrared measurement for USW with different surface conditions: (a) Peak temperature and (b) at center. Source: ORNL. 
For the horn-anvil setup, a numerical study was performed using the same concept of surface engineering. A bonded interface is formed under welding power of $3500 \mathrm{~W}$ and welding time longer than 2 secs. For the 4sec. welding, the interfacial temperature on $\mathrm{Mg}$ side was increased to $662^{\circ} \mathrm{C}$ with the sheet surfaces filed before welding, as shown in Figure II.3.11.7 (b). Figure II.3.11.7 (c) shows that the temperature rings are obviously larger than the baseline case. The resulting lap-shear strength for the filed samples was also improved by about $10 \%$ as summarized in Table II.3.11.2. The increase of joint strength can also be attributed to the increased bond area and mechanical interlocking. With a larger sonotrode diameter, pressure can be distributed in a wider region to enable larger bond area. We plan to further increase the bond strength by combined surface engineering and process optimization (e.g., tool diameter, clamping load).

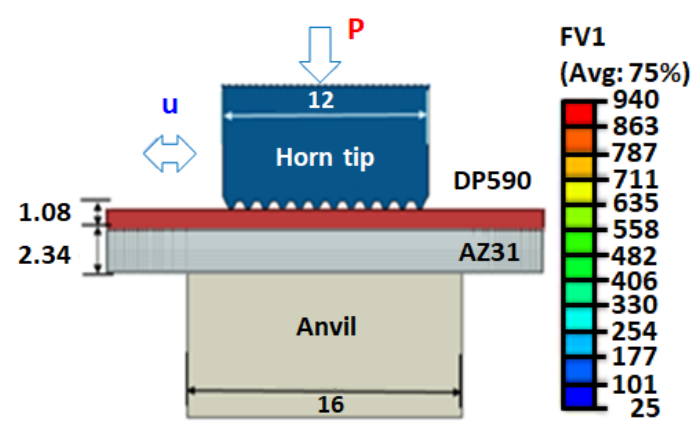

(a)

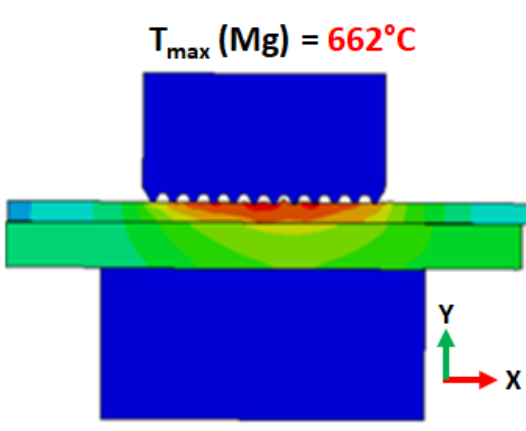

(b)

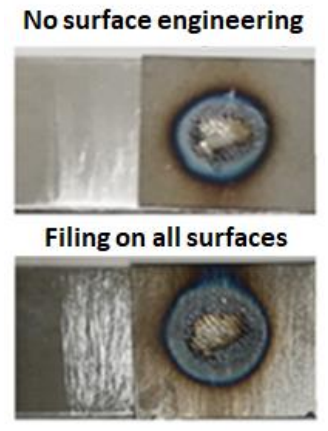

(c)

Figure II.3.11.7. Horn-anvil welding setup and results of Mg-Steel (uncoated) USW. (a) Sheet stack-up and dimension;

(b) predicted temperature distribution in two-sided filed sheets; and (c) the effect of surface engineering on the temperature mark on the steel surface. Source: ORNL.

Table II.3.11.2. Bonding Strength of Mg-Steel USW Joint under Different Process Conditions.

\begin{tabular}{|c|c|c|c|c|c|}
\hline Case & Surface Condition & Faying Surface & Other Surfaces & $\begin{array}{l}\text { Welding Power } \\
\text { and Time }\end{array}$ & Tensile Load \\
\hline & 0 & Sanded P80 (Mg) & As-received & $2500 \mathrm{~W}, 2 \mathrm{~s}$ & Fail Drop Test \\
\hline & 1 & Sanded P80 (Mg) & As-received & $3500 \mathrm{~W}, 2 \mathrm{~s}$ & Pass Drop Test \\
\hline & 2 & Sanded P80 (Mg) & As-received & $3500 \mathrm{~W}, 4 \mathrm{~s}$ & $3.9 \pm 0.5 \mathrm{kN}$ \\
\hline & 3 & Filed & Filed & $3500 \mathrm{~W}, 4 \mathrm{~s}$ & $4.2 \pm 0.4 \mathrm{kN}$ \\
\hline
\end{tabular}

Tool diameter also plays a critical role in weld quality. Figure II.3.11.8 shows the numerical results for different tool diameters, while keeping the tooth shape the same. With a reduced tool size, the heat generation becomes higher, especially at the regions near the sonotrode tip. An additional investigation on the temperature distribution along the faying surfaces indicates that the temperature increase is more than $150^{\circ} \mathrm{C}$ for reduced tool diameters, as shown in Figure II.3.11.9 (a). On the other hand, the pressure distribution, as shown in Figure II.3.11.9 (b), becomes more localized for smaller horns, which can reduce the bond area due to insufficient pressure and shear deformation. Higher stress concentration will also accelerate the wear rate of the sonotrode tip, which can reduce the tool life and affect the weld quality unexpectedly. Moreover, the steel sheet tends to develop large plastic deformation due to increased temperature and stress. Just by increasing the pressure from $60 \mathrm{psi}$ to $100 \mathrm{psi}$, the numerical model with an 8-mm-diameter sonotrode collapsed at 3.5 secs with the steel plate fully penetrated. 

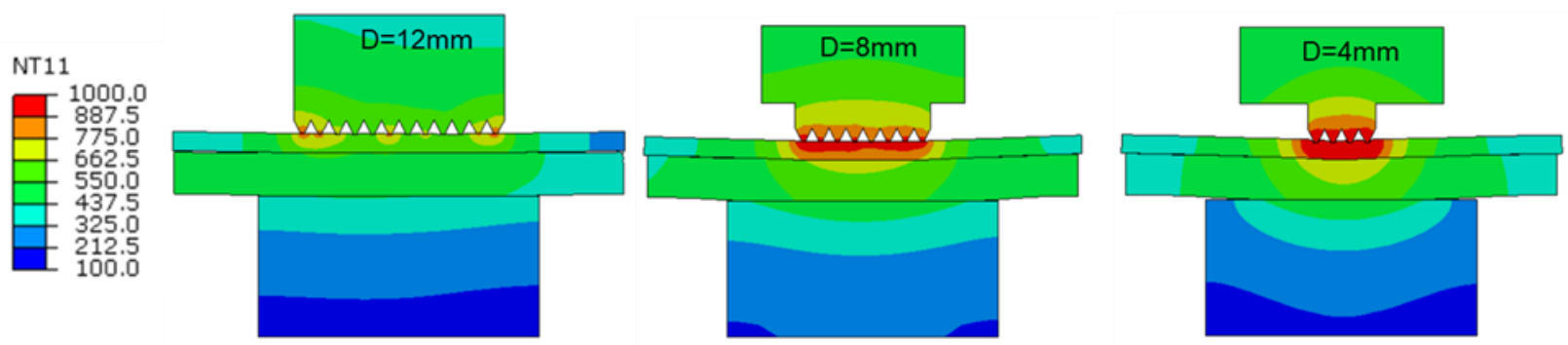

Figure II.3.11.8. Influence of horn tip diameter on the heat generation for three different diameters. Source: ORNL.

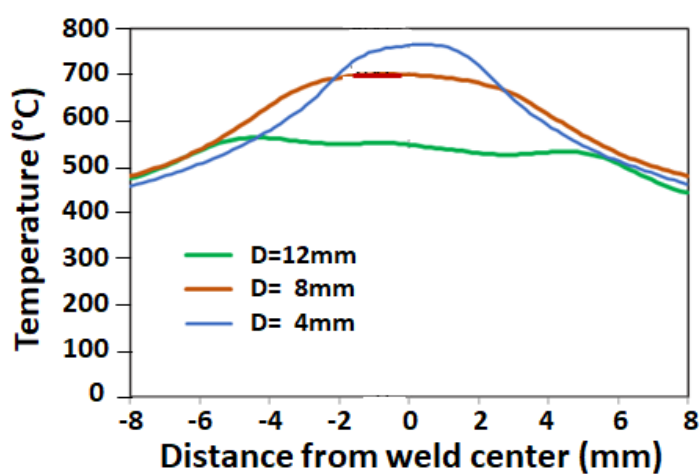

(a)

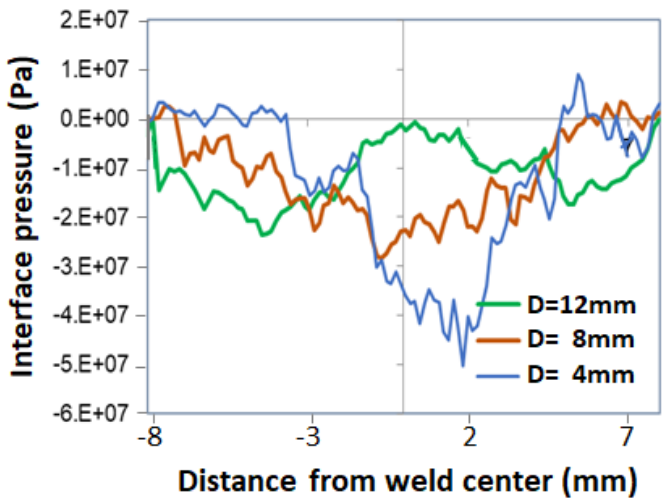

(b)

Figure II.3.11.9. Numerical simulation of horn diameter effect with: (a) temperature distribution; and (b) pressure distribution. Source: ORNL.

For high-strength steel USW, tool wear is an emerging issue due to the aggressive welding conditions. Once the tool is worn to a certain extent, the contact area will be reduced and similar phenomena in reduced tool diameter can occur. A sensitivity study was done with the teeth on the right-hand side of the sonotrode worn off by $30 \mu \mathrm{m}$. The temperature distribution is found to be asymmetric with localized low temperature regions, which can result in lower bond strength. One approach to reduce the wear rate is to use a sonotrode with blunter (flatter) teeth to alleviate stress concentration, as shown in Figure II.3.11.10 (a). A numerical model was used to simulate the heat generation and stress distribution. From the temperature profile, seen in Figure II.3.11.10 (a), the heat generation was minimally influenced, and the temperature is actually higher on the shoulder. The pressure distribution, seen in Figure II.3.11.10 (b), becomes more uniform, which is important to mitigate any voids at the faying surfaces and ensure a sound bond. We plan to customize the sonotrode tool based on this concept to validate the numerical model in FY 2020. 


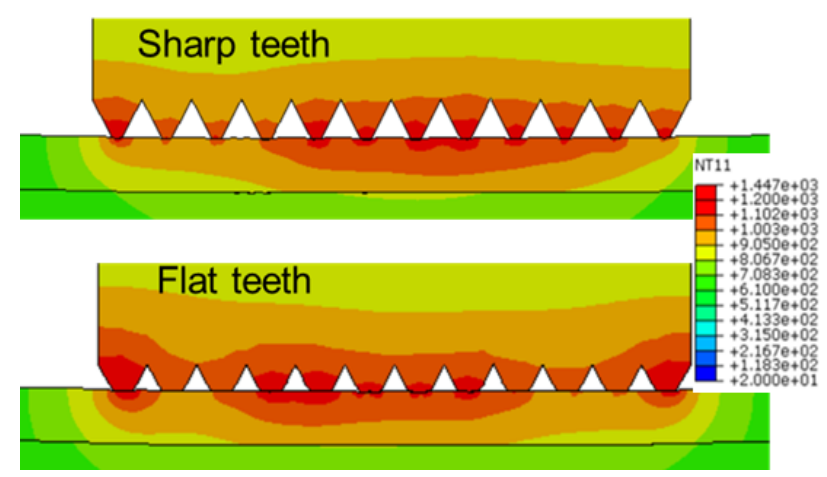

(a)

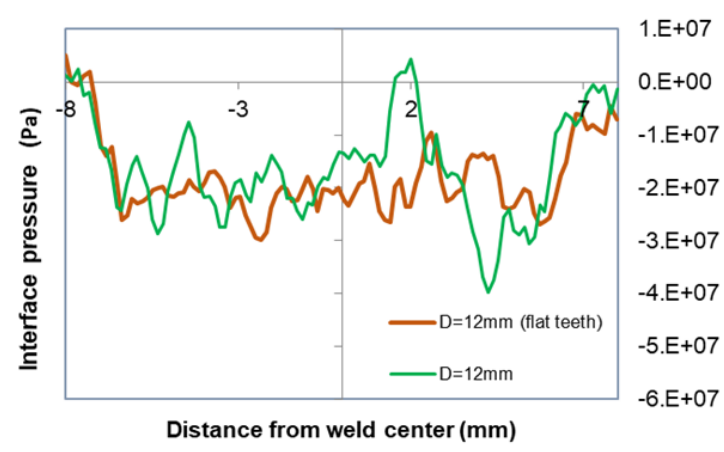

(b)

Figure II.3.11.10. Comparison of USW using sharp-tooth sonotrode and flat-tooth sonotrode: (a) temperature; and (b) contact pressure. Source: ORNL.

\section{Task 2. Interface by Design for FSSW of Dissimilar Materials}

Within this task, the research efforts are focused on (1) a sensitivity study to predict and advise on the Mg-steel interfacial design to maximize strength and ductility using FSS technique to produce a lap-joint and (2) determination of cohesive parameters characterizing the interfacial response for the Mg-steel FSS joint.

In the first subtask, a finite element-based modeling framework was developed that was used to study the impact of different interfacial morphologies and properties on the mechanical behavior of the Mg-steel FSS joint. Figure II.3.11.11 (a) shows an SEM image of a cross-section obtained from a representative FSS joint between Mg-steel. It also shows an overlay of the interfacial geometry features that were used to create the initial baseline FEM. In the modeling framework, the mechanical interlocks or hooks were generated using cubic splines and the process was automated to generate several such hook geometries by defining a few points along the curve. The processing of material in FSW can lead to distinct material regions in the welded plates. This was ascertained using hardness mapping performed by indentation tests. These different material regions have been considered in the FEM as shown in Figure II.3.11.11 (b) and (c). The material properties for these different regions were obtained by scaling the base material properties based on the hardness values obtained from the indentation tests. The weld interface is modeled as a cohesive zone defined by traction-separation law, the reasonable parameters for which are used from previous references for now. In practice, this interfacial property can be altered by local pressure and temperature during the welding, for example. 


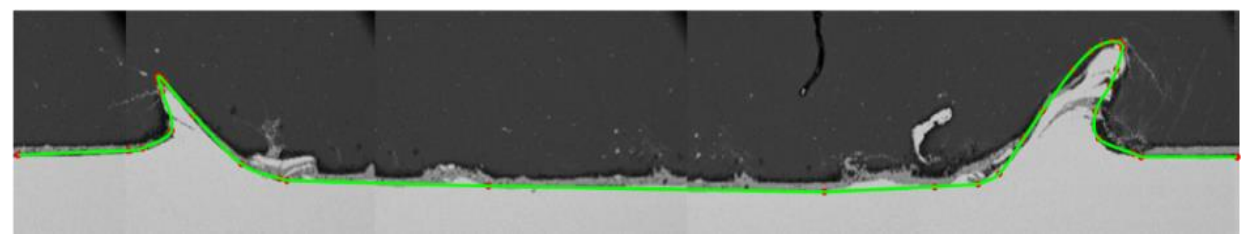

(a)

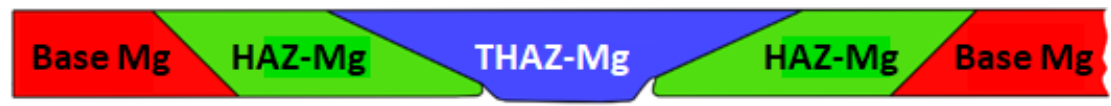

(b)

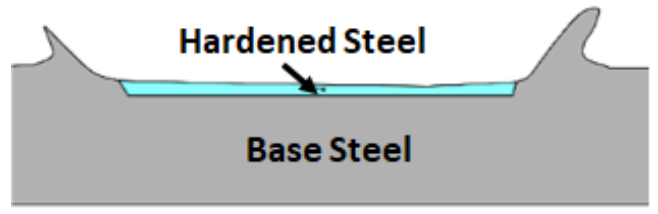

(c)

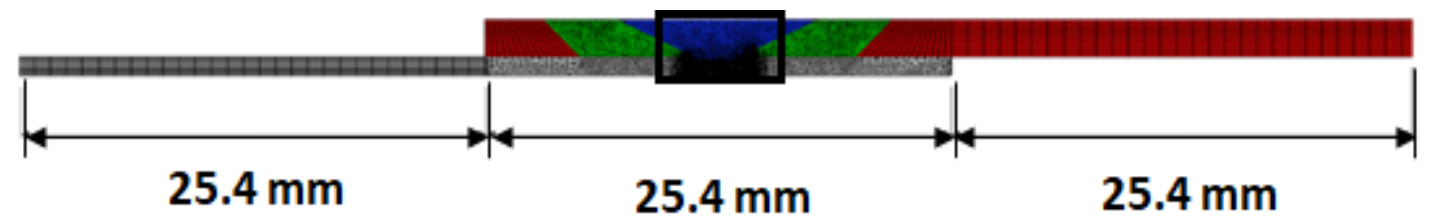

(d)

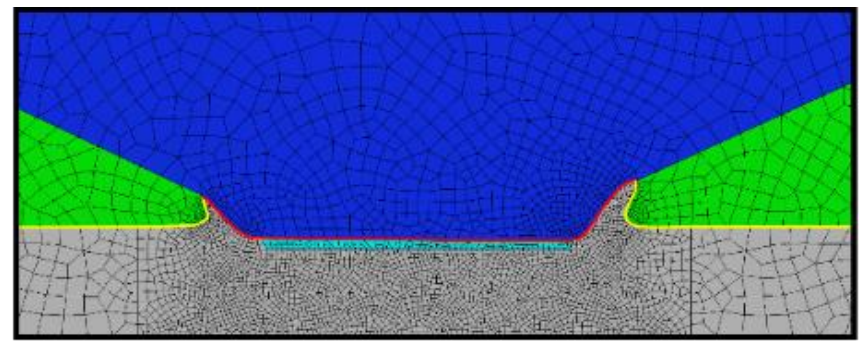

(e)

Figure II.3.11.11. (a) SEM image of a representative Mg-steel FSS joint cross-section with an overlay of interfacial geometry. FEM for the Mg-Steel lap-joint: (b) and (c) different material regions considered in the Mg and steel plates respectively. (d) Dimensions and FE mesh for the model. (e) Close-up of the FEM close to the interface where red line indicates region with cohesive interaction and yellow line indicates penalty contact region to avoid any penetration. Source: PNNL.

The determination of these parameters through a combined experimental and modeling approach is discussed below. Figure II.3.11.12 (a) shows the deformed configuration of the joint in lap-shear testing, where the circled regions show two most likely locations of failure: (1) interfacial (cohesive zone) failure; and (2) material failure due to plastic strain accumulation. The model can predict the failure mode and capture the competition between the two modes of failure, depending on the geometry and properties of the interface. The load-displacement behavior along with the failure mode obtained for several different representative hook geometries are provided in Figure II.3.11.12 (b). The cartoons on the top left show the different left and right hook shapes respectively that were used to simulate the joint performance. Color of each load-displacement curve corresponds to the color of the hook shapes as depicted in the cartoon illustration. Plots also indicate the damage initiation and final failure points for cohesive zone and material damage, respectively. The graph also indicates the damage initiation and final failure points along each curve. When the left hook is sufficiently inclined to the left, a mechanical locking is created and the stresses at the interface are alleviated. Then, if the 
right hook is large enough and inclined to the right, it induces a stress concentration in the Mg plate resulting in failure of the material. In addition to providing a quantitative prediction of joint peak load and ductility, the model captures the failure mode for any given interfacial geometry and properties. Since the simulation framework is automated, several different cases of geometries, material and cohesive zone properties, boundary conditions, etc., were considered in the simulations, but only representative cases are presented here. These analyses will provide feedback to the experimental team on the desired interfacial characteristics that can be targeted during the FSS processing to achieve robust Mg-steel FSW joints.

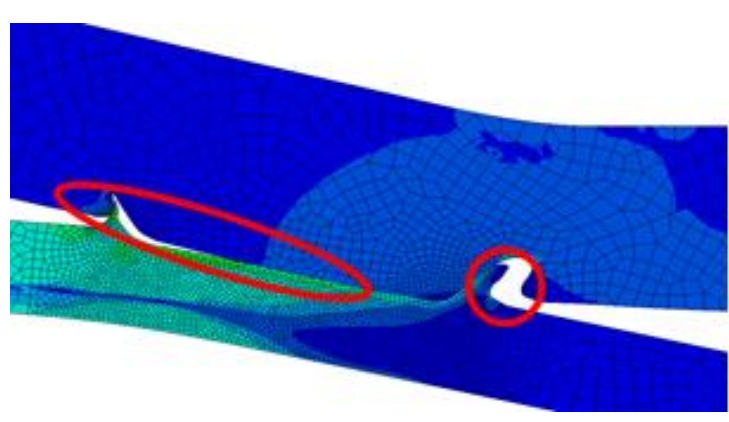

(a)

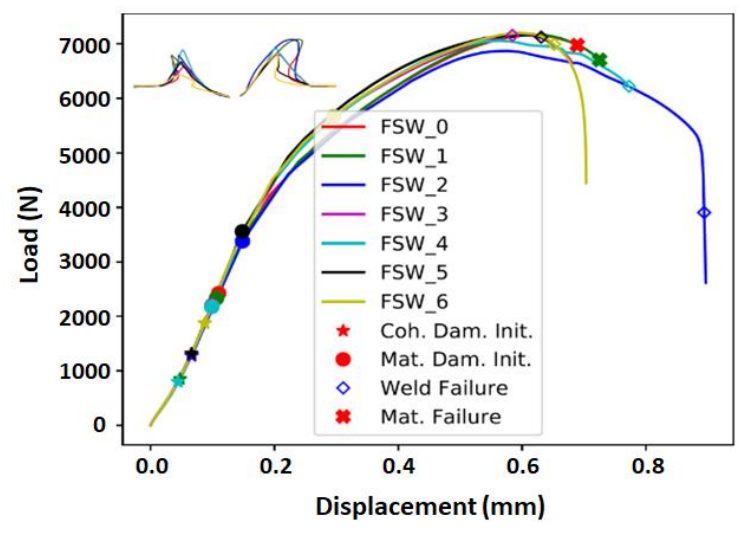

(b)

Figure II.3.11.12. (a) Deformed configuration of the Mg-steel joint tested in lap configuration showing the contour plot for von Mises stress. Highlighted regions indicate the most likely locations for failure. (b) Load-displacement curves obtained for different hook shapes. Source: PNNL.

The determination of cohesive parameters for the Mg-steel interface in FSS joints was conducted next. Typical fracture testing configurations to obtain the parameters for a cohesive law involve assumptions including large weld areas and undisturbed substrate, making those techniques unsuitable for FSW joints. In FY 2019, we developed an approach that involves combining experimental measurements and FE modeling to determine the cohesive law parameters to mechanically characterize the interface obtained in dissimilar FSW joints. Figure II.3.11.13 shows the schematics of the three mechanical tests developed to determine and validate the two cohesive parameters for Mode-I failure of the interface; i.e., strength and fracture toughness. The crosstension test utilizes adhesively bonded $\mathrm{Al}$ backing plates to ensure a uniform normal stress distribution on the weld interface. The interfacial strength is then uniquely determined by the peak load observed in the test divided by the area of the weld and is independent of the fracture toughness. Once the interfacial strength is determined, the results of the T-peel test are used to calibrate the fracture toughness value for the joint interface. To avoid any asymmetric bending and resulting shear at the interface during the T-peel test, the flexural stiffness on the two sides is balanced by adhesively attaching the weld base plates on the opposite sides (i.e., steel plate on $\mathrm{Mg}$ and $\mathrm{Mg}$ plate on the steel side). The results of the U-peel test, being sensitive to both strength and toughness, are used to validate the cohesive parameters obtained from the other two tests. 


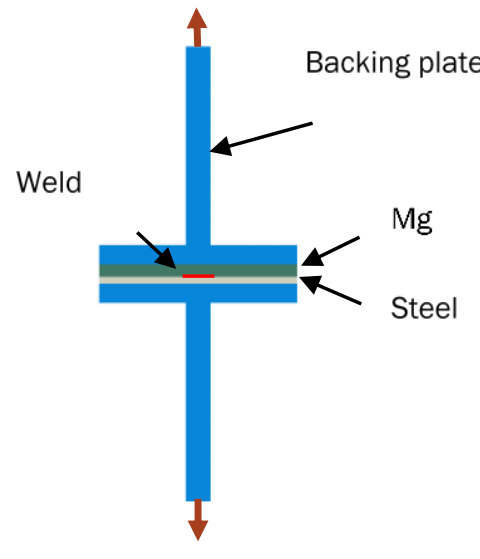

(a)

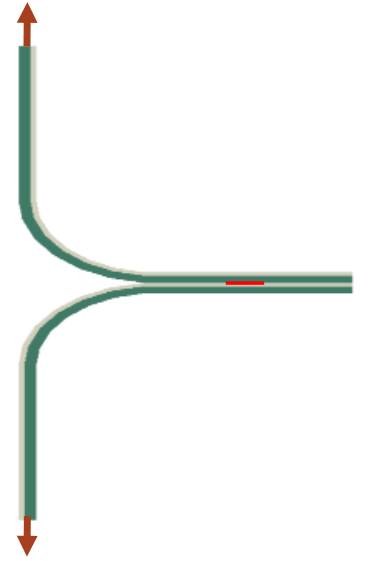

(b)

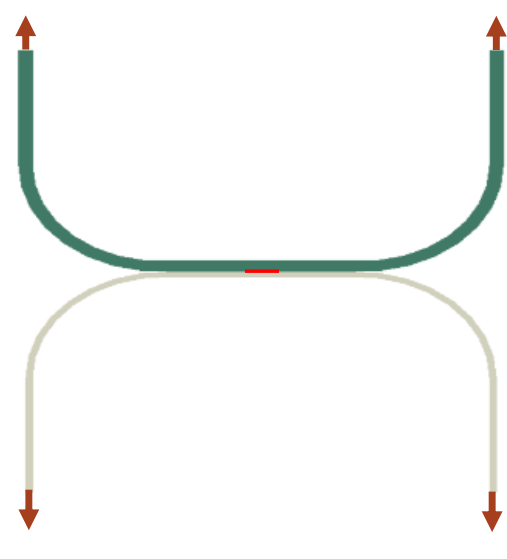

(c)

Figure II.3.11.13. Schematics of the mechanical tests in: (a) cross-tension; (b) T-peel; and (c) U-peel configurations to determine the interfacial parameters for a dissimilar friction stir welded joint. Source: PNNL.

Several different samples were created in the three mechanical test configurations from the welds performed under the same (as much as possible) process conditions. The average peak load from the cross-tension test data yielded a value of $32.98 \mathrm{~N} / \mathrm{mm}^{2}$ for the interfacial strength. Using this value for the interfacial strength, the fracture toughness for the cohesive law was calibrated using simulations from a FE model of the T-peel test. The calibration was performed such that the peak load from the simulation matched the average of the peak loads obtained from different samples of T-peel configuration. This led to a fracture toughness value of $0.44 \mathrm{~kJ} / \mathrm{m}^{2}$. It is noted that the T-peel samples were prepared such that there were equal number of samples loaded from both advancing and retreating sides of the weld. Figure II.3.11.14 (a) shows the deformed configuration and contour plot for the maximum principal stress obtained from an intermediate step of the FE simulation of the T-peel test. The load-displacement curves obtained from the experimental tests and the FE model with calibrated cohesive parameters are shown in Figure II.3.11.14 (b). The model and experiments seem to be in good agreement. The FE model shows a slightly stiffer response compared to the experimental load-displacement curves, which might be resulting from the geometric approximations and other assumptions made in the model. After obtaining strength and toughness from the above two tests, they are used as input cohesive law parameters for the validation case of U-peel test. Figure II.3.11.14 (c) shows the loaddisplacement behavior obtained from the FE simulation of U-peel test alongside the experimental curves. The experimental data show a significant spread in the peak load, but otherwise the overall load-displacement behavior obtained from the FE simulation is in good agreement with the experiments; hence, it validates the developed approach. More experimental testing for the U-peel geometry is underway to establish statistical significance in the data and the results are awaited at the time of writing this report. 


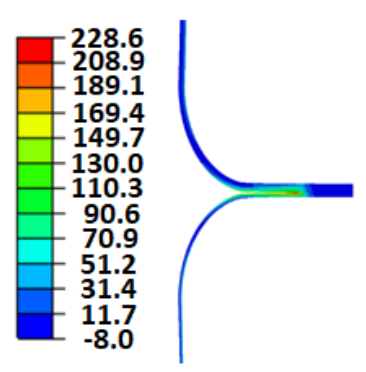

(a)

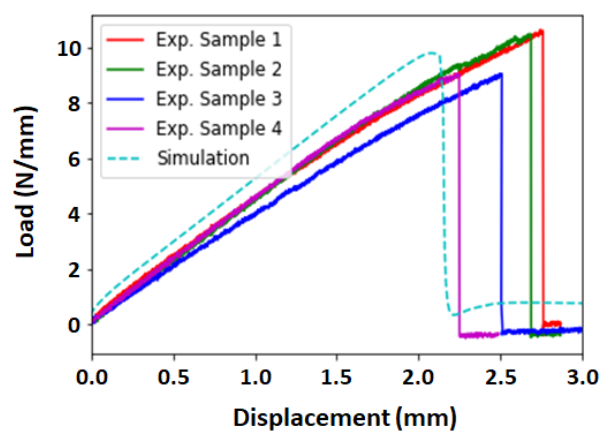

(b)

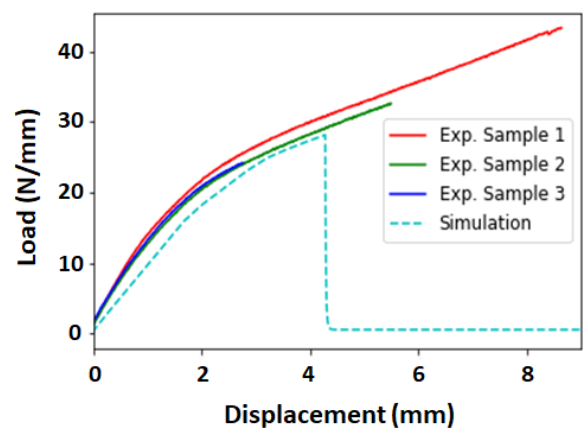

(c)

Figure II.3.11.14. (a) Contour plot showing the maximum principal stress (MPa) in the welded plates loaded in the T-peel configuration. (b) Load-displacement behavior obtained through the experiments and FE simulation of the T-peel test.

(c) Load-displacement behavior obtained through the experiments and FE simulation of the U-peel test. Source: PNNL.

\section{Conclusions}

This work aims to develop a multiscale hierarchical modeling framework to design the joint interface between lightweight $\mathrm{Mg}$ alloy and advanced high-strength steel-the pair that is conventionally believed to be not weldable. In micro- and lower-scale evaluation of impact welded samples, an interlayer formation of $\mathrm{Fe}$ and $\mathrm{Mg}$ is found. The SEM and HRTEM characterization showed the interlayer bonds the steel substrate to $\mathrm{Mg}$ without the formation of Al- or Zn-based IMC. The coupled thermal-mechanical FE-based process simulation indicates the formation of the interlayer is a result of the impact jetting, and the MD analyses showed the high transverse velocity of the $\mathrm{Mg}$ jet causes mechanical mixing of Fe atoms into Mg. During subsequent cooling, the Mg-Fe forms a low-lattice match interface, relaxes the strain energy, and leads to considerable bonding strength.

Guided by the microscopic analysis, the macroscopic thermomechanical model established in FY 2018 was extended to identify the USW process control parameter that optimize the transverse velocity and heat generation between the AZ31 and DP590 sheets. Surface roughness created by filing at both sides of specimen was found to be helpful to enhance heat generation and joint strength. The temperature was increased by $20 \%$ in the dual-sonotrode UW setup and the lap-shear strength was increased by $10 \%$ in the horn-anvil welding setup. The parametric study on sonotrode diameter indicates that local temperature is high in UW with smaller tools while the pressure is more concentrated, which can reduce the bonding area. The sonotrode tip with blunt (flat) teeth will have comparable heat generation rate relative to the case with sharp teeth. Due to less stress concentration, the wear rate of sonotrode tip will be reduced, and the pressure distribution across the interface will be more uniform, which is favorable to a reliable bond.

For the FSS welding approach, a finite element-based model to evaluate the mechanical performance of MgSteel FSS joints was developed. The model was used to study the impact of different interfacial morphologies and properties on the mechanical (load-displacement) behavior of the Mg-Steel FSS joint. Based on the sensitivity analysis conducted, it is demonstrated that a combination of parameters can affect whether the lapjoint fails at the weld interface or in the material. The results from the simulations indicate that there exists a fine balance between the hook morphology and interfacial parameters that can result in different failure modes. This study can provide feedback regarding desirable interfacial and microstructural characteristics that can be targeted during the FSW process. 
In addition, a calibration procedure involving both modeling and experiment was developed to obtain the parameters defining the interfacial response of the dissimilar friction stir welded joint. The approach is agnostic to the joining technique used and can be applied to, for example, ultrasonic welds. The results from this approach are presented for one of the representative Mg-steel FSSW process conditions. The interfacial strength is obtained from the cross-tension test, which is then utilized to obtain the interface toughness from the results of T-peel test. The results are validated with the U-peel test, which is the third, independent test geometry. Since strength and toughness are the fundamental quantities defining the mechanical response of a joint interface, they are independent of the test and loading geometry, unlike the global load-displacement behavior obtained in a mechanical test. Therefore, with these quantities it is possible to compare different joining techniques, including USW, FSW and adhesive-bonding that are underway in the joining core program on an equal footing.

\section{Key Publications and Presentations}

1. Huang, H., J. Chen, Y. C. Lim, Z. L. Feng, X. H. Hu, J. H. Cheng, and X. Sun, 2019, "Heat generation and deformation in UW of Mg alloy AZ31," J. Mater. Process. Tech., Vol. 272, pp. 125-136.

2. Huang, H., J. Chen, Y. C. Lim, Z. L. Feng, and X. Sun, 2018, "A validated computational study on heat generation mechanisms in UW joint," American Welding Society, Professional Program, Atlanta, GA, USA, November 2018.

3. Cheng, J., X. Hu, X Sun, V. Anupam, D. Glenn, and D. Cullen, 2019, "Multiscale Characterization and Simulation of Impact Welding Between Immiscible Mg/Steel Alloys," Acta Materialia, submitted.

4. Gupta, V., E. Barker, P. Upadhyay, and D. Herling, 2019, "Mechanical characterization of the interface obtained in friction stir welded joints using cohesive zone modeling," TMS, San Antonio, TX, USA, March 2019.

5. Wang, T., D. Ramirez, P. Kitsopoulos, X. Jiang, N. Canfield, W. Kuang, V. Gupta, E. Barker, and P. Upadhyay, 2019, "Effect of interfacial characteristics on mechanical performance of welded $\mathrm{Mg}$ and steel obtained via FSS technique: Utilizing a combined experimental and computational approach," Mater. Design, submitted 2019.

\section{References}

1. Cheng, J., X. Hu, X. Sun, V. Anupam, D. Glenn, and D. Cullen, 2019, "Multiscale characterization and simulation of impact welding between immiscible $\mathrm{mg} / \mathrm{steel}$ alloys," submitted to Mat. Sci. Eng. A-Struct.

2. Lee, B. H., Y. T. Keum, and R. H. Wagoner, 2002, "Modeling of the friction caused by lubrication and surface roughness in sheet metal-forming," J. Mater. Process. Tech., Vol. 130, pp. 60-63.

\section{Acknowledgements}

Computing support by ORNL's Compute and Data Environment for Science (CADES) is gratefully acknowledged. 


\title{
II.3.12 Solid-State BIW Spot Joining of Al to AHSS at a Prototype-Scale (Oak Ridge National Laboratory)
}

\section{Zhili Feng, Co-Principal Investigator}

Oak Ridge National Laboratory

1 Bethel Valley Rd.

Oak Ridge, TN 37831

E-mail: fengz@ornl.gov

\section{Eric Boettcher, Co-Principal Investigator}

Honda R\&D Americas, Inc.

21001 State Rte. 739

Raymond, OH 43067

E-mail: eboettvher@oh.hra.com

\section{Sarah Kleinbaum, DOE Technology Development Manager}

\author{
U.S. Department of Energy
}

E-mail: $\underline{\text { sarah.kleinbaum@ee.doe.gov }}$

Start Date: November 1, 2014

Project Funding (FY19): \$450,000
End Date: June 30, 2020

DOE share: $\$ 200,000$
Non-DOE share: $\$ 250,000$

\section{Project Introduction}

This project focuses on developing and demonstrating two emerging solid-state, friction-heating-based spot joining processes - friction bit joining (FBJ) and FSSW - to join AHSS to high-strength Al alloys (HSA alloys) from the coupon scale to the prototype part level. Application of these joining processes to HSA and AHSS in the BIW production environment requires further R\&D to address a number of technical obstacles (i.e., joint performance, productivity, maintaining consistency of joint quality under expected dimensional variations of stamped and/or formed parts, and cost-effectiveness).

The project consists of two phases. During Phase 1, both FBJ and FSSW methods were developed and evaluated at a coupon scale. Furthermore, because corrosion management is a primary concern for the joining of dissimilar materials, both FBJ and FSSW were combined with adhesive-bonding as an isolation approach to protect the spot joint from galvanic corrosion. Finally, an integrated computational welding engineering modeling framework, developed by the project team over the years, is being adopted, refined, and applied. The purpose of the integrated computational welding engineering model is to: (1) refine and optimize the solidstate joining process; (2) understand microstructure changes in the weld region and their effects on the strength/properties of an individual joint; and (3) optimize joinability and joint performance at the prototype assembly level.

During Phase 2, FBJ was selected as the "winning" process for further development using prototype-scale BIW assembly level joining. Hardware of the winning joining process is being integrated with an assembly line welding robot. Prototype BIW subsystems, selected by the OEM, will be welded and assembled with the robotic joining system to evaluate and validate the production readiness of the joining technology for highvolume mass production BIW assembly. Al-steel joints, at both the coupon scale and the prototype-scale, will be tested and characterized to determine performance of the joints in accordance with the requirements in the funding opportunity announcement and a set of process and performance criteria from the OEM, Tier 1 supplier, and industry partners. The project assembled an excellent project team composed of automotive manufacturers and autopart suppliers to carry out the proposed process R\&D: engineering testing, joining system integration, and eventual commercialization and implementation. 
The project is led by ORNL, with participation from Honda R\&D Americas, Alcoa, DOW Chemical, L\&L Products, Cosma Engineering, G-NAC, Mega-Stir Technologies, Brigham Young University, and OSU.

\section{Objectives}

This project aims to develop, mature, and validate near-production readiness of a solid-state spot joining technology to join prototype-scale automobile BIW subsystems made of AHSS and 7000/6000 series HSA alloys to meet the dissimilar metal joining challenges in high-volume mass production. The project focuses on spot joints - the most common form of joints in BIW structures of high-volume production vehicles. Thus, it enables the broadest insertion of lightweight materials in BIW and has the highest potential as a joining technology to support the reduction of petroleum consumption, environmental and economic impacts in the transportation sector.

\section{Approach}

FBJ is a newly invented solid-state joining process particularly suitable for dissimilar materials. FBJ creates a metallurgical-bonded spot joint in two or more sheets of dissimilar metals through a combination of frictional drilling and bonding action of a high-strength consumable joining bit. FSSW is a derivative of the FSW process. A rotating fixed-pin tool is plunged into the upper sheet with normal force to generate frictional heat. The heated and softened material adjacent to the tool deforms plastically and a solid-state bond is made between two dissimilar metals, such as $\mathrm{Al}$ and steel. After critical review of both processes by project teams, FBJ was down-selected for Phase 2.

The development of joining technology must be coupled with corrosion management, which is critical for automotive applications, especially for any joint between dissimilar materials. An adhesive was applied at all proposed contact locations between HSA and AHSS components to serve as an insulator. The solid-state joining techniques, adhesive-bonding, and a combination of the two were initially assessed using laboratoryscale coupons. The in situ distortion measurement at the component level is to gain a comprehensive understanding and provide a potential solution for part distortion caused by the mismatch of the thermal expansion of $\mathrm{Al}$ and steel during paint-baking and curing of adhesives during the automotive body assembly process. The experiment will guide, improve, and verify the distortion model developed in this project.

Modeling the joining process provides insights on physical phenomena (e.g., thermal and deformation histories) during joining. This model is a prerequisite for subsequent weld microstructure and performance modeling and understanding the effect of temperature rise due to joining on the thermal expansion coefficient mismatch of two different metals. In addition, component level modeling provides an analytical modeling tool for assisting in design optimization to reduce welding distortion and stresses to a tolerable level. The integrated computational welding engineering approach has been adapted to refine the joining process and to predict joint performance under manufacturing conditions (e.g., paint-baking).

During Phase 2, FBJ was selected for further development using prototype-scale BIW assembly level joining. The main objective in Phase 2 is to demonstrate the FBJ process in a controlled production environment. A new FBJ welding head will be designed, manufactured, and integrated with a robot system along with fixtures for coupons and demonstration parts. Coupons and demonstration parts produced by the prototype FBJ welding cell will be further tested and evaluated for mechanical and corrosion performance. Prototype BIW subsystems, selected by the OEM, will be welded and assembled with the robotic joining system to evaluate and validate production readiness of the joining technology for high-volume mass production BIW assembly. Al-steel joints, at both the coupon and prototype scales, will be tested and characterized to determine the performance of the joints in accordance with the requirements in the funding opportunity announcement and a set of process and performance criteria from the OEM, Tier 1 supplier, and industry partners. 


\section{Results}

\section{Development of Component Level Thermal Distortion Model}

The primary objective of component level modeling is to understand the part distortion of the bimetallic structure during the paint-bake process. The distortion mode was treated as a thermal elasto-plastic deformation problem. ABAQUS was used to simulate the thermal distortion. The simulation model has two channels - one for $\mathrm{Al}$ and another for steel — that were bolted together. Figure II.3.12.1 shows a comparison of the gap at the center as measured by DIC and predicted by a numerical model for a full-length panel, halfflange height $(22.55 \mathrm{~mm})$, and AA6111 case. Deflection of the $\mathrm{Al}$ and steel component was plotted along the longitudinal distance to the center for DIC measurement and numerical model prediction. The measured gap at center by DIC was $9.1 \mathrm{~mm}$ at peak temperature, while the predicted gap at the center by numerical model was $10.0 \mathrm{~mm}$. The model prediction shows a good agreement with experimental result.

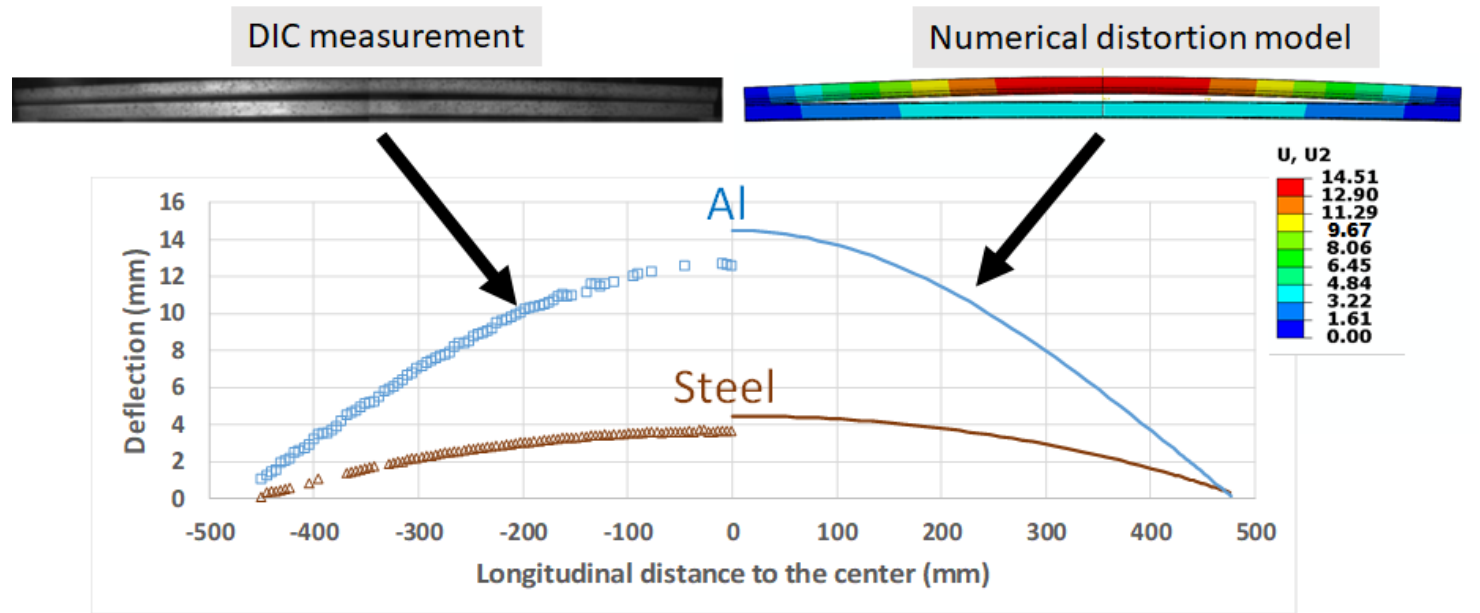

Figure II.3.12.1. Comparison of gap measured by DIC and predicted numerical model at peak-bake temperature. Source: ORNL.

In FY 2019, the validated thermal distortion model was applied to explore options to minimize thermally induced distortion of $\mathrm{Al}$ and steel subassembly due to CTE mismatch. Figure II.3.12.2 depicts predicted distortion with different weld pitches from $900 \mathrm{~mm}$ to $55 \mathrm{~mm}$ at the peak paint-baking temperature. Figure II.3.12.3 plots predicted gap from the center to edge of coupon panel at the peak paint-baking temperature (left) and residual gap after cooling down to RT (right). A maximum gap was found to be approximately $10 \mathrm{~mm}$ at the peak paint-baking temperature when the weld pitch was $900 \mathrm{~mm}$. Alternatively, $0.4 \mathrm{~mm}$ was predicted as a maximum gap when the weld pitch was $55 \mathrm{~mm}$. Similarly, a maximum residual gap was predicted $0.25 \mathrm{~mm}$ and $0.03 \mathrm{~mm}$ for a weld pitch of $900 \mathrm{~mm}$ and $55 \mathrm{~mm}$ at RT. Therefore, controlling weld pitch distance is critical to greatly reduce thermal distortion for an Al-steel bimetallic structure. In addition, this numerical modeling result can be used to further guide required effective weld pitch for a different length of coupons. 

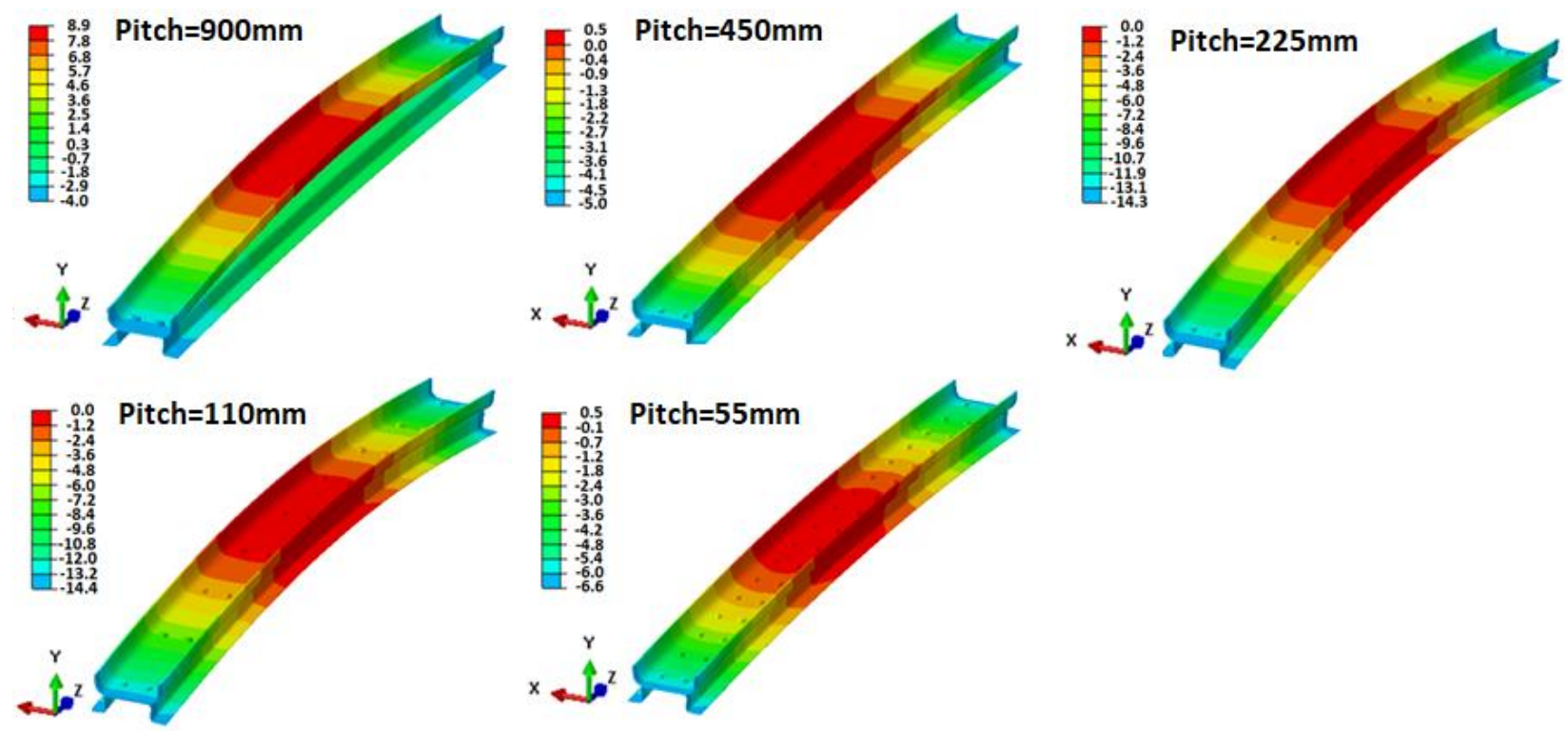

Figure II.3.12.2. Predicted distortion with different weld pitch from $900 \mathrm{~mm}$ to $55 \mathrm{~mm}$ at peak paint-bake tempeature. Source: ORNL.

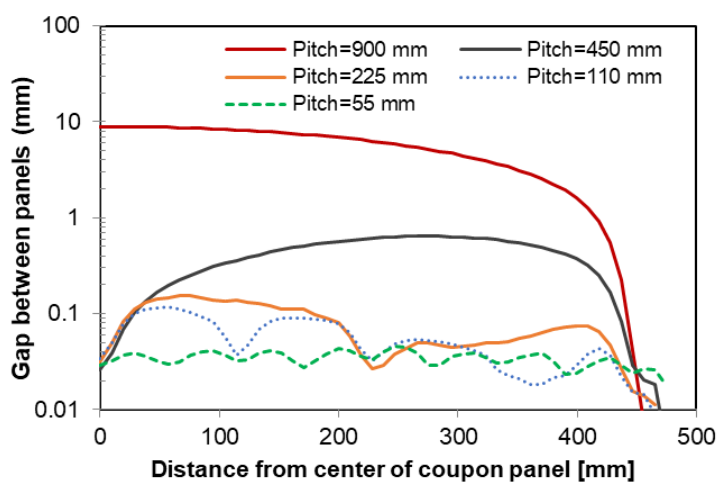

(a)

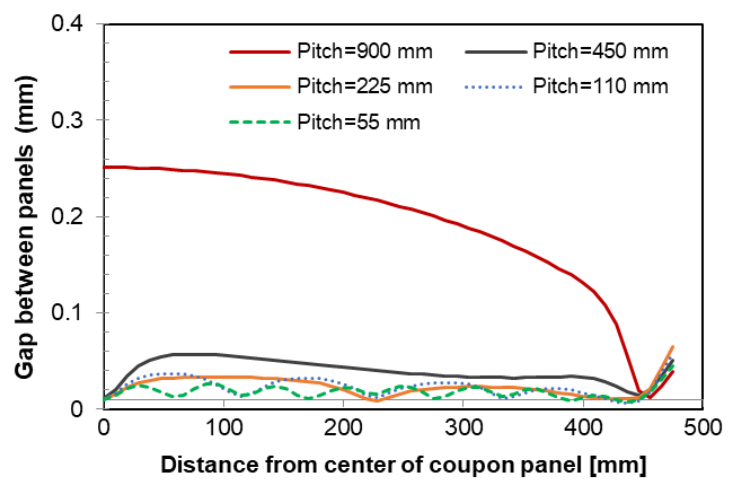

(b)

Figure II.3.12.3. (a) Predicted gap between Al and steel panel with different weld pitch from $900 \mathrm{~mm}$ to $55 \mathrm{~mm}$ at peak paint-bake tempeature. (b) After cool down to RT. Source: ORNL.

\section{Prototype FBJ Work Cell for Demonstration Part Joining}

The FBJ process was selected for further development at a prototype-scale BIW assembly level in Phase 2. The goal of Phase 2 is a successful demonstration of the FBJ process in a controlled and fully automated welding cell to assemble the prototype-scale BIW component selected by project OEM by FBJ and adhesivebonding of 7xxx Al and steel parts. In FY 2019, Mega-Stir, Brigham Young University, Honda R\&D America, and ORNL worked as a team to develop and execute the R\&D plan in Phase 2. The main accomplishment is the design of an improved new FBJ welding head based on the learnings of the FBJ technology in Phase I, the requirements of automated robotic assembly cell typical in a high-volume autobody assembly production line, and the prototype-scale BIW component selected for the project demonstration. The new FBJ welder was optimized for functionality and for meeting the weight requirement for attachment to the robot welding system. Figure II.3.12.4 illustrates the new FBJ welder attached to a Fanuc Robotic arm for fully automated robotic FBJ joining/assembly cell. 


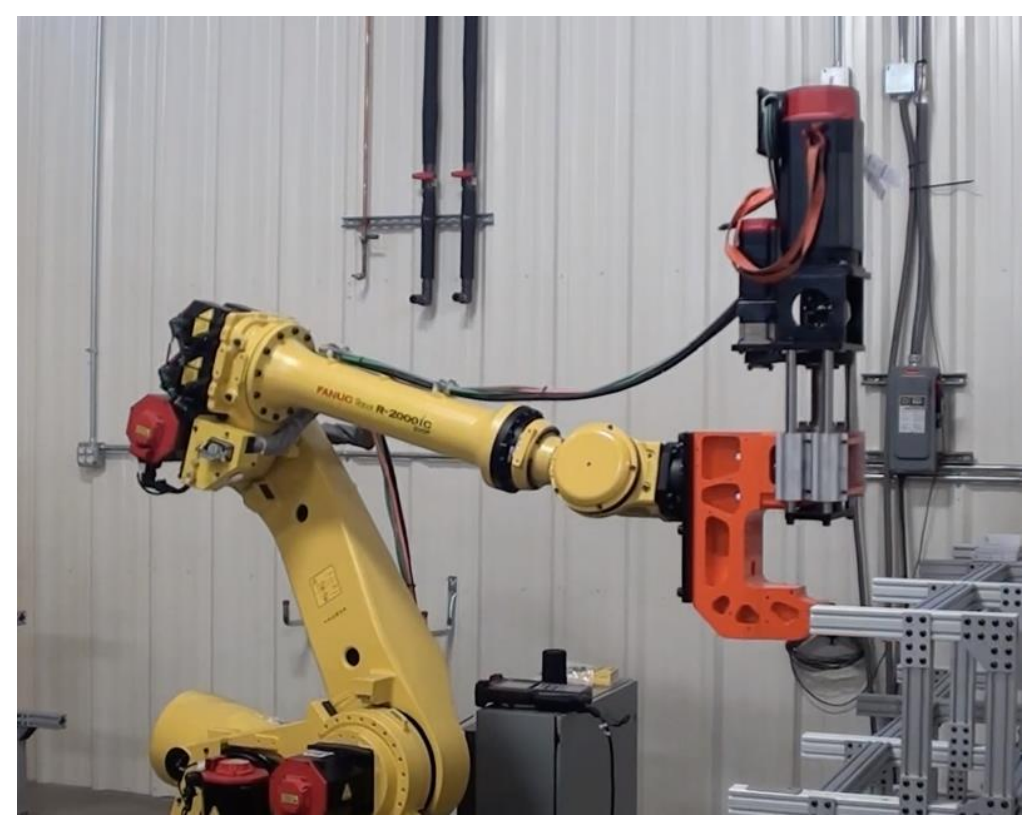

Figure II.3.12.4. Finalized new FBJ machine design. Source: ORNL.

The robotic FBJ system has been tested successfully for functionality. Coupon-level FBJ trials yielded satisfactory results that were consistent with the Phase I development results in terms of joint strength. Table II.3.12.1 summarizes the lap-shear tensile testing results for FBJ coupons spot-welded by the integrated FBJ robotic welding system. Average peak-failure load was found to be $13.1 \mathrm{kN}$, which is 2.6 times the target strength for FBJ $(5 \mathrm{kN})$. Also, lap-shear tensile testing resulted in good repeatability with the new robotic FBJ system. More extensive system test, adjustment, and refinement will continue for the prototype-scale BIW component assembly planned through December 2019. A go/no go review meeting is scheduled for the project to move forward to the prototype-scale BIW assembly study and demonstration.

Table II.3.12.1. Summary of Lap-Shear Tensile Testing for FBJ Samples by the New FBJ Machine.

\begin{tabular}{|c|c|}
\hline FBJ Sample Number & $\begin{array}{c}\text { Peak Lap-Shear } \\
\text { Failure Load }(\mathbf{k N})\end{array}$ \\
\hline $\mathbf{1}$ & 12.99 \\
\hline $\mathbf{2}$ & 12.74 \\
\hline $\mathbf{3}$ & 13.26 \\
\hline $\mathbf{4}$ & 14.27 \\
\hline $\mathbf{5}$ & 12.55 \\
\hline $\mathbf{6}$ & 12.06 \\
\hline $\mathbf{7}$ & 12.76 \\
\hline $\mathbf{8}$ & 14.80 \\
\hline $\mathbf{9}$ & 12.70 \\
\hline $\mathbf{1 0}$ & 12.89 \\
\hline Average \pm STDEV & $13.10 \pm 0.83$ \\
\hline
\end{tabular}

In addition to the robotic FBJ system, the joining/assembly cell will also be comprised of fixtures necessary for joining and assembly of the prototype-scale BIW components. The assembly cell that is being constructed is depicted schematically in Figure II.3.12.5. 


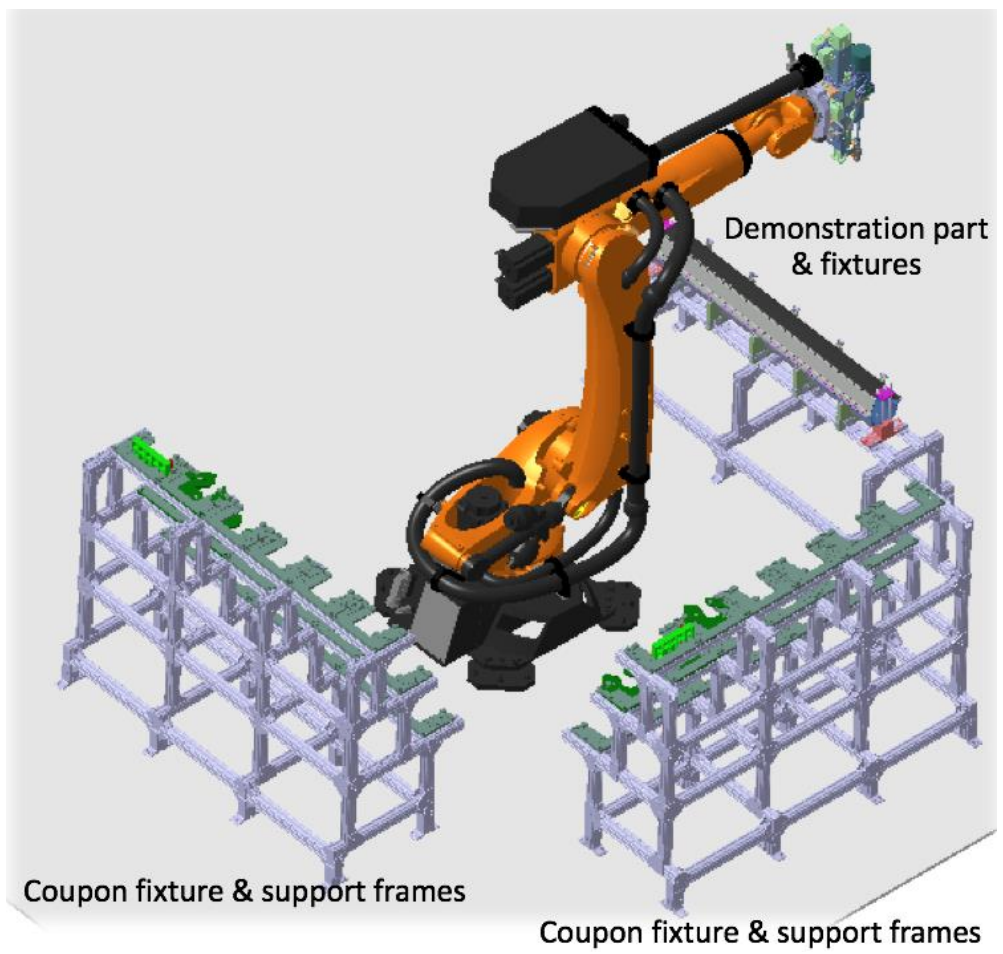

Figure II.3.12.5. Finalized new FBJ machine and joining/assembly cell design. Source: ORNL.

\section{Conclusions}

In this report period, the project transitioned from Phase 1 to Phase 2. A validated FE model was used to evaluate various factors and scenarios that would influence the thermally induced distortion of the Al-steel body components. For the simple channel assembly panels, the thermal distortion modeling revealed the significant influence of the weld pitch. A 55-mm weld pitch distance predicted a maximum gap of $0.4 \mathrm{~mm}$ at peak paint-baking temperature and $0.03 \mathrm{~mm}$ at RT. A new FBJ welder was designed, manufactured, and integrated with robot system. Lap-shear FBJ coupons were produced by newly integrated FBJ machine, and the TSS exceeded the OEM requirement. Design of FBJ weld cell was finalized and integrated with the robot mounted new FBJ machine and weld fixtures for final demonstration.

\section{Acknowledgements}

The principal investigators would like to thank the following individuals for their contributions to this work:

- $\quad$ Oak Ridge National Laboratory: Yong Chae Lim, Yanli Wang, Lianshan Lin, Ward Manneschmidt

- Honda R\&D Americas: Eric Boettcher, Alan Seid

- $\quad$ Brigham Young University: Michael Miles, Kevin Shirley, Shane Wood

- Mega-Stir: Dale Fleck, Bill Johnson, Russel Steel

- $\quad$ OSU: Wei Zhang

- DOW: Mirdamadi Mansour, Gary Hayes

- L\&L Products: David Kosal, Stuart Crump

- Cosma Engineering: Arvind Srilatha, Changji Shi, Erryn Ashmore

- G-NAC: Eric Yousey, Susumu Wakameda, Nick Weisenberger, Robert Clark

- $\quad$ Alcoa: Russel Long, Donald Spinella, Daniel Bergstrom. 


\title{
II.3.13 Nondestructive Inspection of Aluminum-Steel Weld Bond (Oak Ridge National Laboratory)
}

\author{
Zhili Feng, Principal Investigator \\ Oak Ridge National Laboratory \\ 1 Bethel Valley Rd. \\ Oak Ridge, TN 37831 \\ E-mail: fengz@ornl.gov
}

\section{Sarah Kleinbaum, DOE Technology Development Manager}

U.S. Department of Energy

E-mail: $\underline{\text { sarah.kleinbaum@ee.doe.gov }}$

Start Date: October 1, 2017

Project Funding (FY19): \$200,000
End Date: March 31, 2019

DOE share: $\$ 200,000$
Non-DOE share: $\$ 0$

\section{Project Introduction}

Material joining or welding is the primary assembly method in the automotive industry. The quality of the welds is critical to the crash resistance and performance of vehicles. The auto industry demands effective and reliable nondestructive examination (NDE) methods to inspect each critical joint. It is even more important for dissimilar material joining. The lack of effective and reliable NDE tools suitable to the mass production environment is a barrier to the adoption of lightweight materials and suitable dissimilar material joining technologies by the automotive industry.

As the auto industry increasingly relies on multi-material strategy to balance the performance, fuel efficiency, and cost of auto body structures, joining of dissimilar materials by means of spot welds and adhesives has become an integral part of dissimilar materials joining solution as exemplified by the introduction of RSW of $\mathrm{Al}$ to steel process joints in the seatback of the Cadillac CT6. There is a critical need for reliable and costeffective NDE technologies that can be used in a high-volume auto structure-manufacturing environment to inspect adhesively bonded joints or joints with rough and complex surface patterns. Conventional NDE tools, such as ultrasound, have been difficult to use for such applications. Ultrasound typically requires physical contact between the transducer and the material surface through use of a gel or fluid couplant at the interface. The patterned surface topography of the resistance spot weld causes difficulty for obtaining good couplant conditions for ultrasonic transmission. Therefore, it is the goal of this project to introduce and develop an NDE method that is insensitive to the complex surface topography.

Sponsored by DOE VTO and with close collaboration with automotive industry original equipment manufacturers and Tier One suppliers, ORNL has successfully developed a non-contact online weld NDE technology for inspecting RSWs based on the infrared thermography-based NDE technique (IR NDE) in the past few years. This research was recognized with an R\&D 100 Award in 2015 and has received considerable interest for licensing and technology commercialization. However, ORNL's IR NDE development was primarily limited to AHSSs, although limited testing trials suggested feasibility for Al RSW.

In this work, ORNL, in partnership with GM, proposes to develop an IR NDE method that can effectively and reliably inspect dissimilar material joints with complex surface patterns in a highly automated manufacturing environment. This method will be built upon our extensive experience with IR NDE. We plan to evaluate options to address critical bottlenecks hindering the use of IR NDE for RSW and adhesive bond inspection. Based on such evaluation, the technique will be refined and optimized. A prototype system will be built and tested in a simulative manufacturing environment as a demonstration of this technology. 


\section{Objectives}

The objective of this research is to extend ORNL's award-winning IR NDE technique to weld-bonding of Al to AHSSs to address an immediate and critical NDE need identified by automotive original equipment manufacturers. To achieve this goal, new or improved NDE measurement techniques will be developed to solve unique challenges in dissimilar materials NDE and new thermal signature recognition algorithms specific for Al-steel welds will be investigated. For the latter, artificial intelligence-based ML tools that have become much more powerful in recent years will be explored as a new way to solve the very complicated relationship between joint quality and NDE measurement results. This will lay the groundwork for adoption and application of artificial intelligence-based ML tools for future R\&D of NDE and in situ monitoring of dissimilar material joining.

\section{Approach}

\section{Resistance Spot Welding}

RSW was performed using a medium frequency direct current welding machine designed for spot welding $\mathrm{Al}$ alloys. The system used an inverter weld control from Welding Technology Corp, Farmington Hills, MI with medium frequency direct current transformers from RoMan Manufacturing, Grand Rapids, MI. Pneumatic actuators were used to apply weld force. Distilled water at ambient temperature was used to cool the welding electrodes at a flow rate of 1.5-2.0 gpm. Weld currents were adjusted to produce the desired weld size in a hand peel sample.

Seven weld variations were evaluated in this study that include low carbon steel (LCS) welded to itself, Al welded to itself, and stack-ups with dissimilar metals, thicknesses, and welding electrodes shown in Table II.3.13.1. All steel materials were hot-dip galvanize (HDG) coated and the Al alloys were uncoated. No cleaning or other surface preparation was used on either material prior to welding the sheet stack-ups.

\begin{tabular}{|c|c|}
\hline Stack-Up & Electrodes \\
\hline $1.0 \mathrm{~mm} \mathrm{HDG} \mathrm{LCS} \mathrm{-} 1.0 \mathrm{~mm}$ HDG LCS & 6006 Ballnose \\
\hline $1.0 \mathrm{~mm}$ HDG LCS - $1.0 \mathrm{~mm}$ HDG LCS & $6066 \mathrm{MRD}$ \\
\hline $2.0 \mathrm{~mm}$ HDG LCS - $2.0 \mathrm{~mm}$ HDG LCS & $6148 \mathrm{MRD}$ \\
\hline $0.8 \mathrm{~mm} \mathrm{Al}-0.8 \mathrm{~mm} \mathrm{Al}$ & $6066 \mathrm{MRD}$ \\
\hline $1.2 \mathrm{~mm} \mathrm{Al}-1.2 \mathrm{~mm} \mathrm{Al}$ & $6148 \mathrm{MRD}$ \\
\hline $0.8 \mathrm{~mm} \mathrm{Al}-1.0$ mm HDG LCS & 6006 Hybrid (MRD/Ballnose) \\
\hline $1.2 \mathrm{~mm} \mathrm{Al}-2.0 \mathrm{~mm}$ HDG LCS & 6148 MRD \\
\hline
\end{tabular}

Material stack-ups were welded with either 6006 (16-mm diameter) or 6148 (19-mm diameter) CuZr C15000 copper alloy electrodes. Ballnose electrodes were machined to include a $\sim 5$-mm face. MRD electrode geometry is per GM's patented MRD electrode [1]. All welding tests were performed with the Al alloy sheet contacting the positive welding electrode. Electrodes were chosen for each stack-up based on the size and type of electrode that would be used in a production body shop environment.

\section{IR NDE Methods}

Three IR NDE methods were used: (1) inline imaging, as seen in Figure II.3.13.1 (a); (2) post-weld off-line imaging using flash lamp pulsed auxiliary heating, as seen in Figure II.3.13.1 (b); and (3) post-weld imaging using auxiliary induction heating, also as seen in Figure II.3.13.1 (c). 


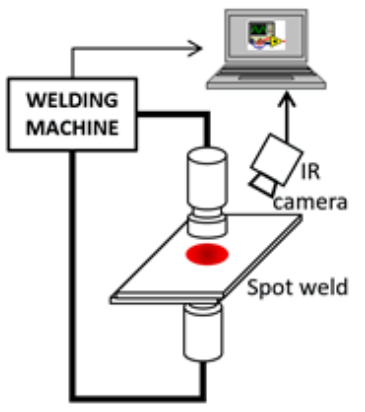

(a)

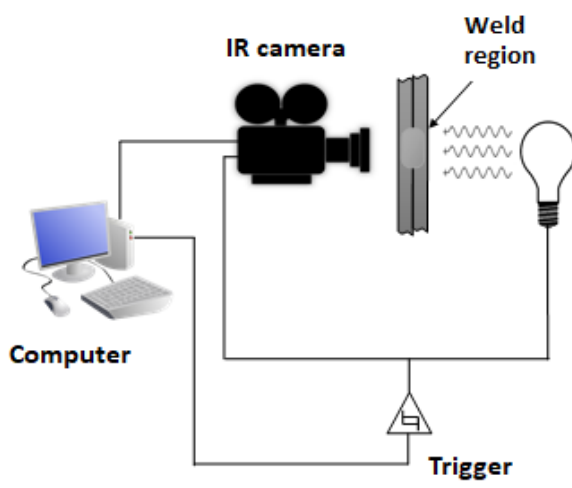

(b)

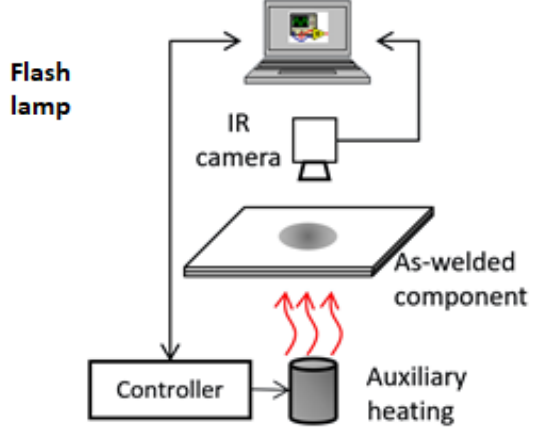

(c)

Figure II.3.13.1. IR NDE methods used were (a) inline imaging , (b) off-line imaging using flash lamp pulsed auxiliary heating and (c) post-weld imaging using auxiliary induction heating. Source: ORNL.

1. Inline imaging: Thermal images were taken during and immediately after welding. For this setup, a FLIR Systems A655sc was positioned at an angle with a view of the spot weld that was unobstructed after the weld electrode raised. The camera was automatically triggered to record as the welder started and captured at least three seconds of data at a rate of $200 \mathrm{~Hz}$.

2. $\quad$ Post-weld imaging (flash lamp): Pulsed thermography was employed, using a flash lamp (Elinchrom 1200RX) to provide a single pulsation of heat at $1200 \mathrm{Ws}$ with a duration of $1 / 1450$ seconds. The samples were mounted to a garolite fixture on the end of the lamp to ensure consistency in placement relative to the camera and light source. Care was taken to isolate the strobe light from the camera to avoid overexposure and contamination of the data. The camera was triggered to record slightly before the pulsed light was triggered. A FLIR Systems SC8300 thermal camera was used with a frame rate of 187 frames/sec.

3. $\quad$ Post-weld imaging (induction heating): Additionally, post-weld through-transmission thermography imaging was conducted using induction for excitation. An induction heater (Ambrell EasyHeat 0112) of 1200 watts was used to provide pulse heating. During heating, the sample was positioned on a ring stand so that the sample was at a distance less than $1 \mathrm{~mm}$ from the induction heater. The camera was set to record for 3.5 seconds beginning at the time of the heater excitation. The entire sample set was evaluated using the induction heating method.

\section{Sample Analysis}

Metallographic specimens were sectioned and polished to a surface finish of $0.04 \mu \mathrm{m}$ using silica polishing media. Samples were examined in the unetched state to evaluate the weld nugget size. ImageJ image analysis software (U.S. National Institutes of Health, Bethesda, MD) was used to measure nugget diameter at the faying interface (straight line tool). Three welds per weld condition were measured using ImageJ.

\section{Results}

\section{Metallographic Results}

The structure of Al-steel RSWs is very distinct compared to similar metal spot welds as shown in Figure II.3.13.2. In Al-Al and steel-steel spot welds, for instance, the weld structure consists of a single-weld nugget comprised of a mixture of material from both metal sheets. In contrast, Al-steel spot weld joints feature a planar interface between the $\mathrm{Al}$ and steel with a weld nugget contained only within the $\mathrm{Al}$ alloy sheet. The $\mathrm{Al}$ weld nugget is separated from the steel substrate by a layer of IMCs at the faying interface. These IMCs are primarily Fe2Al5 adjacent to the steel substrate and FeAl3 adjacent to the $\mathrm{Al}$ alloy weld nugget. In some instances, a very small steel nugget can form in the center of the steel sheet surrounded by a large HAZ. 


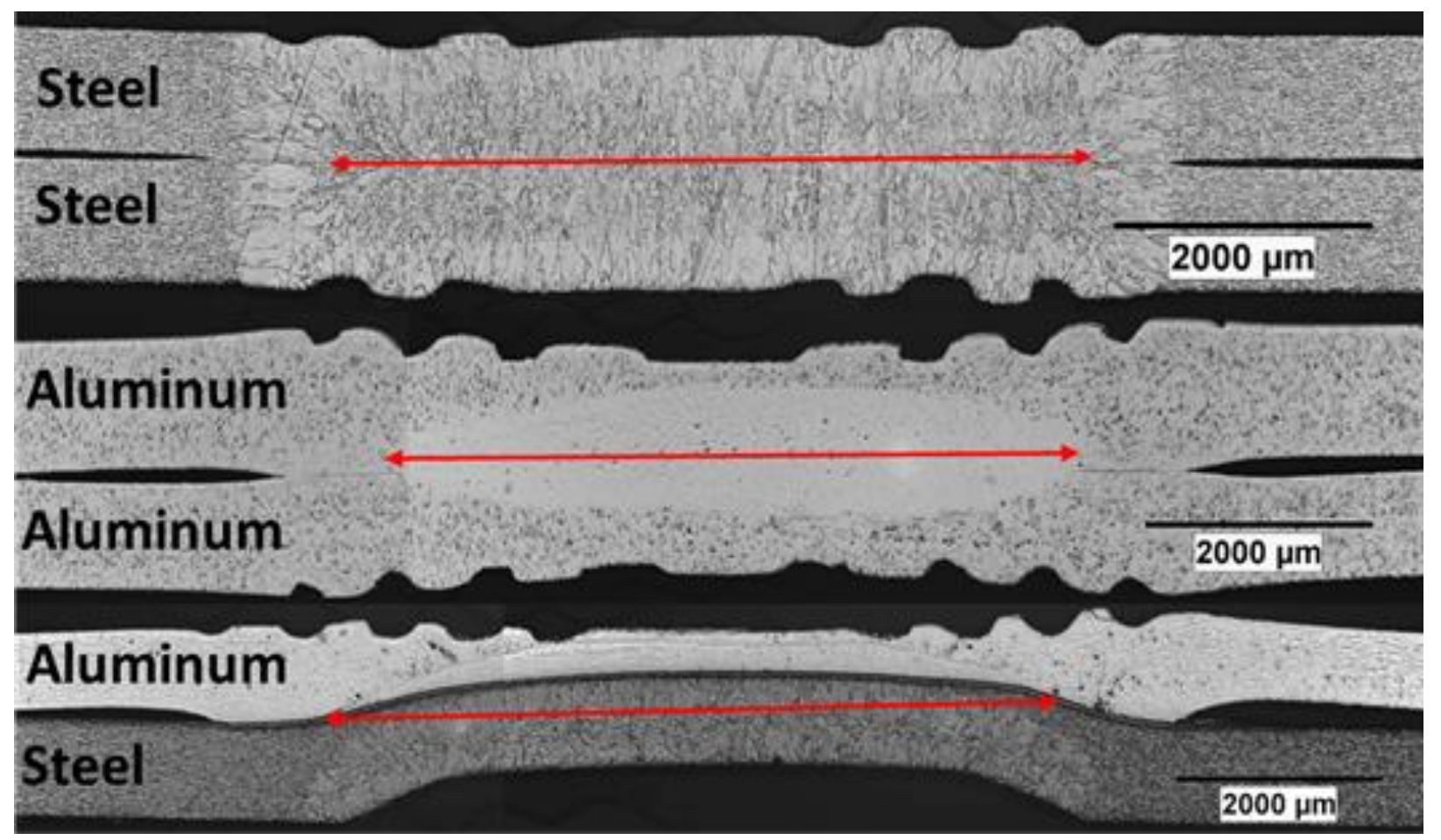

Figure II.3.13.2. Examples of steel, Al, and Al-steel weld cross-sections with nugget diameters highlighted by red arrows. Source: [1].

Three nugget diameters for each stack-up and weld size have been measured. This data is used as a comparison to the nugget diameters predicted by each of the nondestructive evaluation methods.

\section{IR NDE Results}

Figure II.3.13.3 (a)-(c) shows the results for the predicted weld nugget diameters for the entire sample set analyzed by (a) inline IR NDE, (b) off-line pulsed induction heating and (c) off-line pulsed flash lamp heating methods, respectively. The comparison to the cross-sectionally measured diameters is also plotted. The individual measurements are denoted by the black dots and the expected 1:1 ratio is denoted by the diagonal line. A proportional trend is observed between the predicted versus real diameters. Overall, inline and off-line induction heating methods yielded better prediction, while the off-line flash lamp heating method has a relatively scattered prediction. This is due to the low signal-to-noise ratio resulting from the very small temperature changes (typically on the order of $0.1^{\circ} \mathrm{C}$ ) induced by the flash lamp method. 


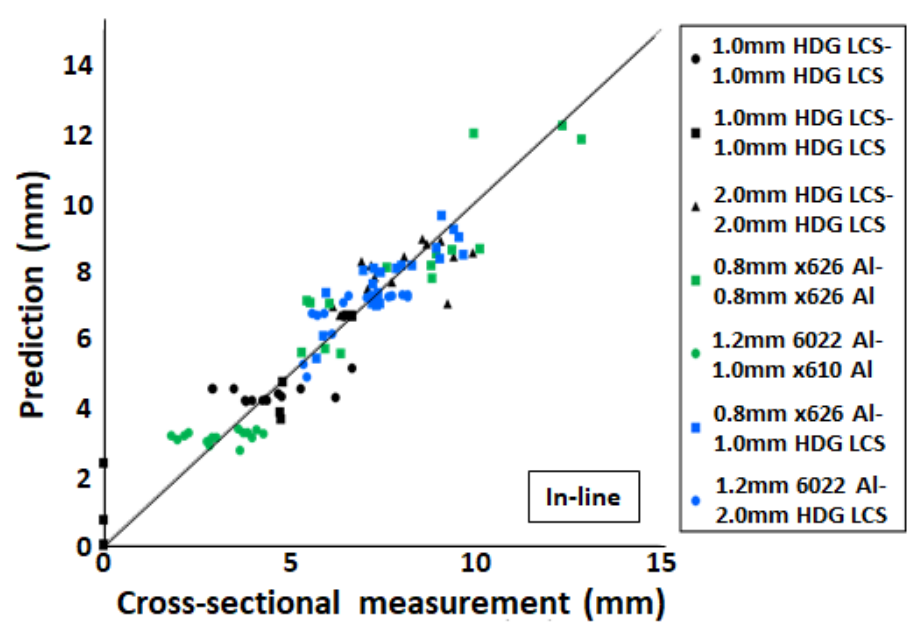

(a)

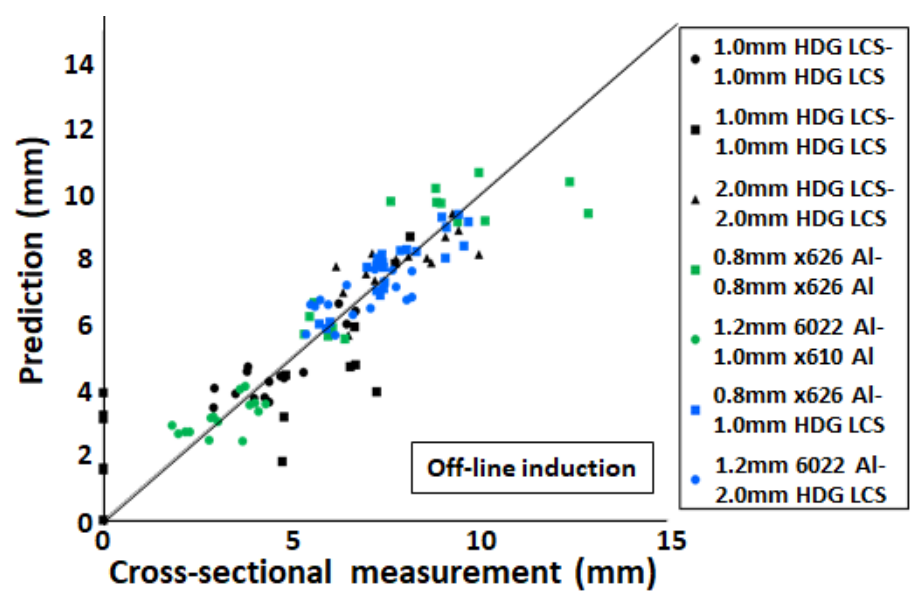

(b)

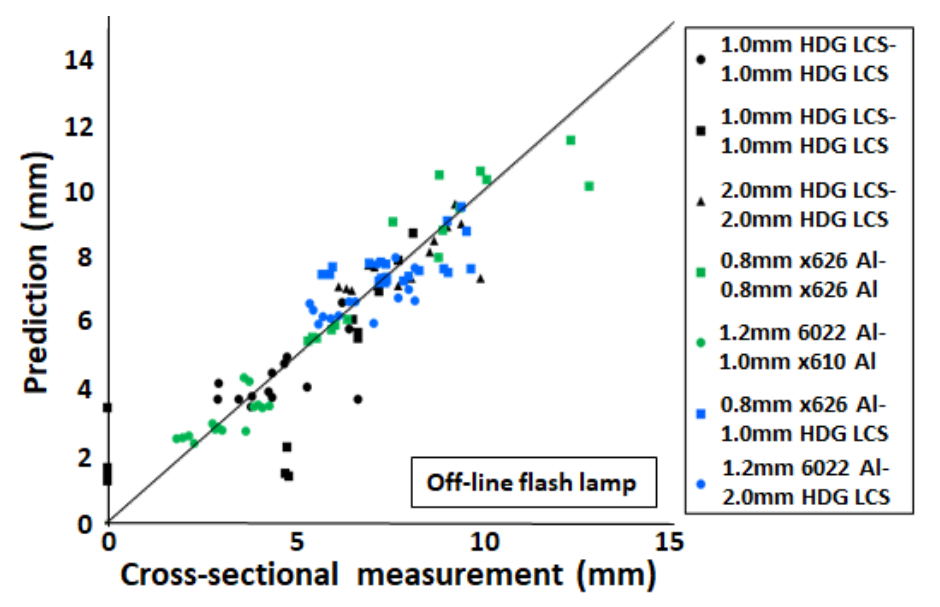

(c)

Figure II.3.13.3. Predicted diameters versus real measured diameters for the entire sample set, where measurements are denoted by the black dots and the expected trend is represented by the diagonal grey line, for (a) inline IR NDE, (b) off-line pulsed induction method and (c) off-line pulsed flash lamp. Source: ORNL. 


\section{Conclusions}

Steel-steel, $\mathrm{Al}-\mathrm{Al}$ and $\mathrm{Al}$-steel spots welds produced at different welding conditions were measured using inline and off-line (pulsed induction and pulsed flashlamp heating) methods. Data obtained from inline measurements and off-line pulsed induction heating methods exhibited a relatively good proportional relationship, while the off-line flashlamp heating method yielded a scattered prediction due to the low signalto-noise ratio resulting from the very small temperature changes induced by the flash lamp method.

\section{Key Publications}

1. Carlson, B. E., A. S. Haselhuhn, J. Chen, and Z. Feng, 2019, "Nondestructive evaluation of resistance spot-welded Al-steel joints," MRS Bulletin, Vol. 44, No. 8, pp. 619-624.

\section{References}

1. Carlson, B. E., A. S. Haselhuhn, J. Chen, and Z. Feng, 2019, "Nondestructive evaluation of resistance spot-welded Al-steel joints," MRS Bulletin, Vol. 44, No. 8, pp. 619-624.

\section{Acknowledgements}

The principal investigator would like to recognize Jian Chen of ORNL and Blair Carlson, Megan McGovern, Teresa Rinker, and Amberlee Haselhuhn of GM for their support and assistance in this project. 


\title{
II.3.14 Corrosion Mechanisms in Magnesium-Steel Dissimilar Joints (Pacific Northwest National Laboratory and Oak Ridge National Laboratory)
}

\author{
Vineet Joshi, Co-Principal Investigator \\ Pacific Northwest National Laboratory \\ 902 Battelle Blvd. \\ Richland, WA 99354 \\ E-mail: vineet.joshi@pnnl.gov
}

\section{Donovan N. Leonard, Co-Principal Investigator}

Oak Ridge National Laboratory

One Bethel Valley Road

Oak Ridge, TN 37831

E-mail: leonarddn@ornl.gov

\section{Sarah Kleinbaum, DOE Technology Development Manager}

\author{
U.S. Department of Energy
}

E-mail: $\underline{\text { sarah.kleinbaum@ee.doe.gov }}$

Start Date: October 1, 2018

Project Funding (FY19): \$300,000
End Date: September 30, 2020

DOE share: $\$ 300,000$
Non-DOE share: $\$ 0$

\section{Project Introduction}

The Joining Core Program is a collaborative research effort initiated jointly between ORNL and PNNL, leveraging existing expertise and past efforts in joining technology development and lightweight materials R\&D capabilities. Collaborative, early stage research conducted through this program is being used to address multi-material joining and associated compatibility challenges that currently limit the integration of lightweight materials for vehicle structures. One of the initial four research thrusts focused on the joining of $\mathrm{Mg}$ to steel with an objective to deliver relevant joining technologies that enable increased used of lightweight materials in vehicles.

Currently, FSSW and USW methods are being investigated as viable techniques to join $\mathrm{Mg}$ to steel. Both involve creating a large degree of plastic deformation at an interface while delivering heat from frictional and plastic work dissipation mechanisms. This results in creating unique microstructural and mechanical features at the joint interfaces which enhances the joint strength. Despite the potential success of these processes, their application is limited because of $\mathrm{Mg}$ 's poor corrosion resistance. $\mathrm{Mg}$ inherently corrodes at a faster rate than steel and, due to it being the most anodic of the structural materials, is susceptible to galvanic corrosion and corrodes severely when interfaced with steel. To mitigate/decrease the corrosion in these joints, we not only need to understand the design of the joints but also the impact of the microstructural features at the interface on the corrosion rate. Such considerations will also provide baseline information for understanding and optimizing protective coatings for $\mathrm{Mg}$-Steel joints, which will be needed in automotive service.

Technical barriers/challenges associated with joining $\mathrm{Mg}$ to steel with respect to corrosion include:

1. Corrosion Mechanism and Products:

- Identifying the phases and microstructural features at the interface and their respective galvanic potentials.

- Determining the corrosion mechanism and distinguishing between corroded $\mathrm{Mg}$ due to galvanic coupling or inherent corrosion.

- Identifying corrosion products and their potential impact. 
2. Design and Methods:

- Minimizing the current density by tailoring the thickness of the Mg to steel.

- Providing baseline understanding and guidance for the development and optimization of protective coating schemes.

In this work, we will determine the critical thickness ratios of $\mathrm{Mg}$ to steel needed to minimize the corrosion in FSSW and USW joints. The COMSOL Multiphysics modeling tool will be used to predict the corrosion rate. This tool will be important to not only understand the thickness ratios and permissible overlap (i.e., lap-joint), but it will also be useful in determining the effect of zinc ( $\mathrm{Zn})$ coated steel on the $\mathrm{Mg}$-Steel joint corrosion. This task will also help validate the experimental aqueous corrosion observations made at ORNL and provide baseline understanding to enable possible solutions to mitigate corrosion in these systems. Scanning electrochemical cell microscopy (SECCM) is a versatile technique for microscopic electrochemistry study [1, 2], and will be used to complement the advanced microstructural characterization efforts at ORNL devoted to micro- and nano- structure features in the corroded joints. The technique can be used successfully for microscopic corrosion studies, such as the effects of crystallographic orientation and second-phase particles in electrochemical response in a polycrystalline material. In addition, this technique can be used for studying corrosion behavior at an interface for two dissimilar metals or alloys joined by the friction stir method. Understanding the electrochemical response at joined interfaces will help in designing improved joint properties.

\section{Objectives}

In modeling focused approach, there are two main objectives for this task. First, we will determine the critical thickness ratios of $\mathrm{Mg}$ to steel needed to minimize the corrosion in FSSW and USW joints. The COMSOL Multiphysics ${ }^{\circledR}$ modeling tool will be used to predict the corrosion rate. This tool will be important to not only understand the thickness ratios and permissible overlap (i.e., lap-joint), but it will also be useful for determining the effect of $\mathrm{Zn}$-coated steel on the corrosion. This task will also help validate the experimental observations made at ORNL and provide solutions to mitigate corrosion in these systems. Second, this task will help understand the role of crystal orientation, second-phase particles, and galvanically graded potential at an interface in corrosion behavior in two dissimilar metals joined by FSSW. This will be followed by gathering the microscopic corrosion information, such as corrosion potential $\left(\mathrm{E}_{\mathrm{Corr}}\right)$, corrosion current, and electrochemical impedance spectroscopy (EIS) for developing a COMSOL-based model.

In experimental and characterization focused approaches, the objectives are to quantify galvanic corrosion of $\mathrm{Mg}$ influenced by weld method as well as $\mathrm{Zn}$ coatings on the steel side of the joints and to investigate the microstructures of the bonded area in FSSW and USW joints. The initial joints to be studied are Mg alloy, AZ31B, joined to steel alloy, DP590, with and without a Zn-coating. For simplicity, throughout this report the AZ31B Mg alloy is referred to as "Mg" and the steel alloy as "steel"

\section{Approach}

\section{Modeling Approach}

The corrosion rate for an $\mathrm{Mg}$ bar electrode in contact with a steel bar electrode was determined using two configurations:

1. Mg on top.

2. Steel on top. 
A $0.1-\mathrm{M} \mathrm{NaCl}$ electrolyte $10 \mathrm{~cm}$ deep was assumed to be present above the electrodes. During the run, the electrolyte concentration was assumed to be constant. The anodic reaction was oxidation of $\mathrm{Mg}$ to $\mathrm{Mg}^{+2}$ while the cathodic reaction depends on $\mathrm{pH}$. The polarization curves for the anodic and cathodic reactions were taken from literature [3-5]. The experimental setup consisted of two overlapping bars with a given length, thickness, and overlap that is shown schematically in Figure II.3.14.1 (a). The geometry can be uniquely defined as shown in Figure II.3.14.1 (b) which describes the height of the electrolyte as $h_{\text {electrolyte, }}$ a top bar with a length of $l_{\text {top }}$ and a thickness of $l_{\text {thickness, }}$, and the overlap between the top and bottom bar with a length of $l_{\text {bottom. }}$.

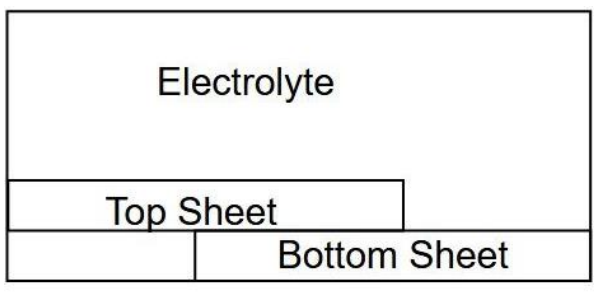

(a)

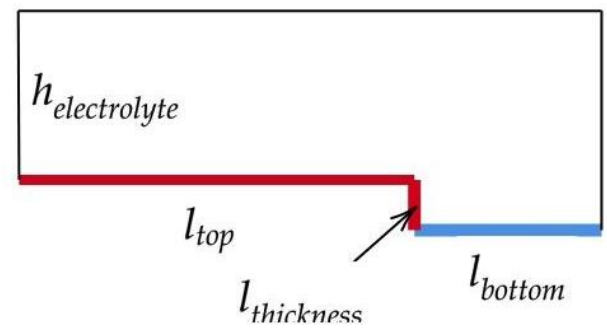

(b)

Figure II.3.14.1. (a) Simplification of the geometry to produce (b) one domain for COMSOL. Source: PNNL.

Protocol was optimized for SECCM measurements during the microscopic corrosion study. A micron-sized double-barreled theta pipette $(\sim 1-50 \mu \mathrm{m})$ filled with electrolyte solution is being used as a probe. The two barrels are connected by reference and counter electrodes. A very thin droplet or meniscus formed in front of the theta probe and was used for the microscopic corrosion study and electrochemical imaging. The theta capillary with $\sim 6.6 \mu \mathrm{m}$ was used for measurement. Corrosion was studied by measuring Tafel and EIS measurements from multiple locations in a microstructure. For demonstration and measurement protocol development purposes, primary measurement and analyses were carried out on a LCS sample. The optimized measurement protocol will be used for the microscopic corrosion study across the interface in a FSSW sample.

\section{Experimental and Characterization Approach}

Galvanic corrosion of Mg in FSSW and USW joints was assessed experimentally using a novel method called 'selective joint masking and immersion' where an insulation tape was applied over the joint specimen but not on the lower sides of the joints as shown in the plan and side views of Figure II.3.14.2 (a) and Figure II.3.14.2 (b). When the selectively masked joint specimens were immersed in $0.1 \mathrm{M} \mathrm{NaCl}$ solution, the corrosion of $\mathrm{Mg}$ under galvanic influence progressed toward the center, creating a measurable corrosion depth on each side as described schematically in Figure II.3.14.2 (c). During $0.1 \mathrm{M} \mathrm{NaCl}$ immersion, the corrosion potentials of the selectively masked joints were also monitored to assess the degree of galvanic polarization with respect to the single $\mathrm{Mg}$ electrode. All corrosion experiments were conducted at RT with $0.1 \mathrm{M} \mathrm{NaCl}$ solution open to air.

Materials characterization of the as-joined and post-corrosion samples was accomplished using correlative multi-length scale microscopy \& microanalysis. Low magnification $(25 \mathrm{X}-160 \mathrm{X}), 3 \mathrm{D}$ OEM was employed to determine morphology differences of various corrosion products formed during aqueous immersion and the resulting galvanic corrosion. Higher magnification SEM, in the same regions of interest interrogated with OM, allowed for both morphology and elemental composition of corrosion products to be determined on the micron to nanoscale and at much higher spatial resolutions than OM. When merited, the FIB prep of specific features and areas of interest was performed followed by STEM and EDS to determine nanoscale second phases, crystalline or amorphous corrosion products and elemental composition of these. 


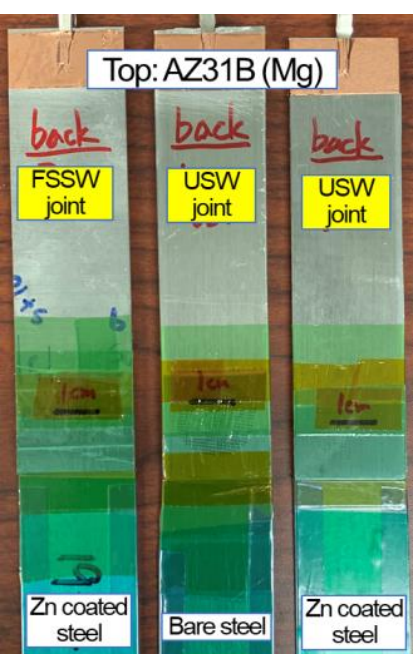

(a)

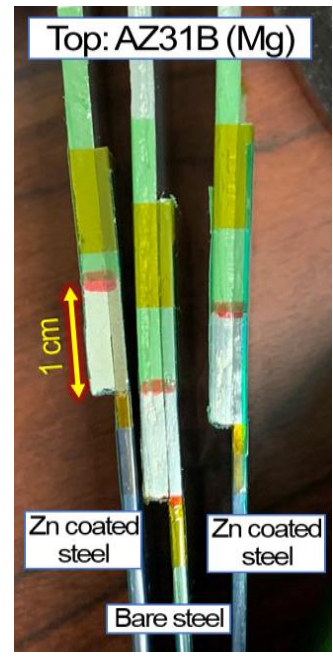

(b)
Selective corrosion exposure in $0.1 \mathrm{M} \mathrm{NaCl}$ to produce corrosion depth
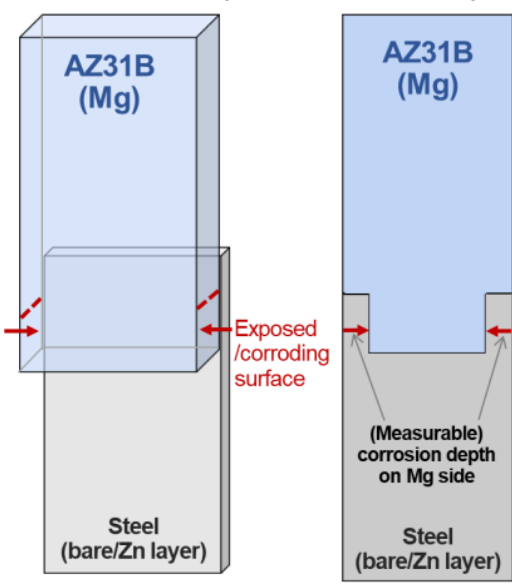

(c)

Figure II.3.14.2. Selectively masked FSSW and USW joint specimens: (a) plan and (b) side view. The lower side of joints were remained unmasked with $1 \mathrm{~cm}$ length as shown in (b). (c) The schematic of exposed and corroding surfaces in a selectively masked joint and the resulting corrosion depth on Mg. Source: ORNL.

\section{Results}

Due to the simplicity of the corrosion modeling approach selected, a run can be conducted in a matter of seconds; meaning it is straightforward to perform a parametric analysis. All three dimensions of $1_{\text {top }}, 1_{\text {thickness }}$, and $l_{\text {bottom }}$ were varied along with their combinations resulting in about 1300 runs. An example of one selected COMSOL Multiphysics ${ }^{\circledR}$ run is provided in Figure II.3.14.3 and shows that most of the current is concentrated inside the corner between the top and bottom bar, rapidly falling off outside this area. This also demonstrates that the electrolyte height is large enough to not have much of an effect on the amount of corrosion. As expected, peak currents occur close to the joint with higher peaks near the corner where the bars meet. This could be problematic; hence, it is useful to determine the effects of bar thickness on peak currents for both configurations as shown in Figure II.3.14.4 and Figure II.3.14.5. There are some edge effects shown in the top corner to the left in each figure where the current rapidly falls off.

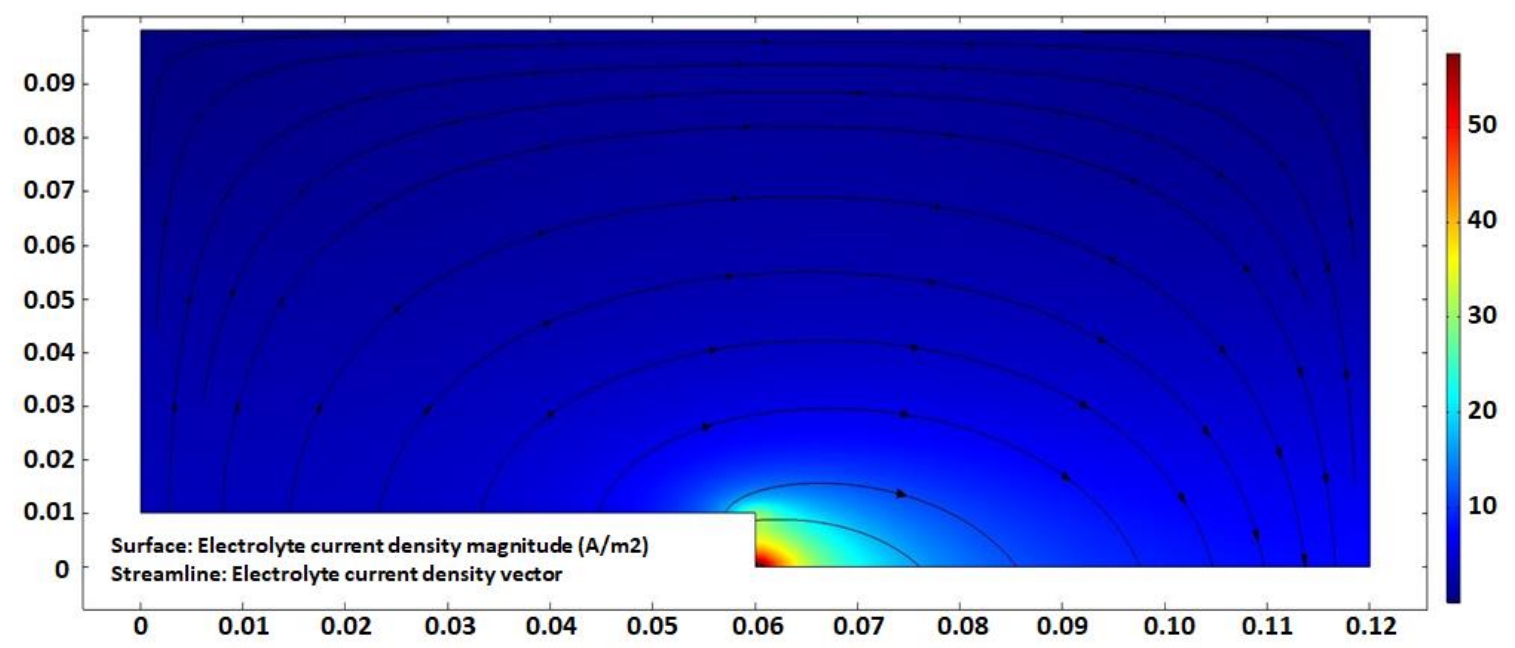

Figure II.3.14.3. Electrolyte potential profile (color surface) and current density distribution (arrows) using COMSOL Multiphysics ${ }^{\circledR}$ software for $\mathrm{Mg}$ on top, $1 \mathrm{~cm}$ sheet thickness, and $6 \mathrm{~cm}$ top and bottom lengths. Source: PNNL. 


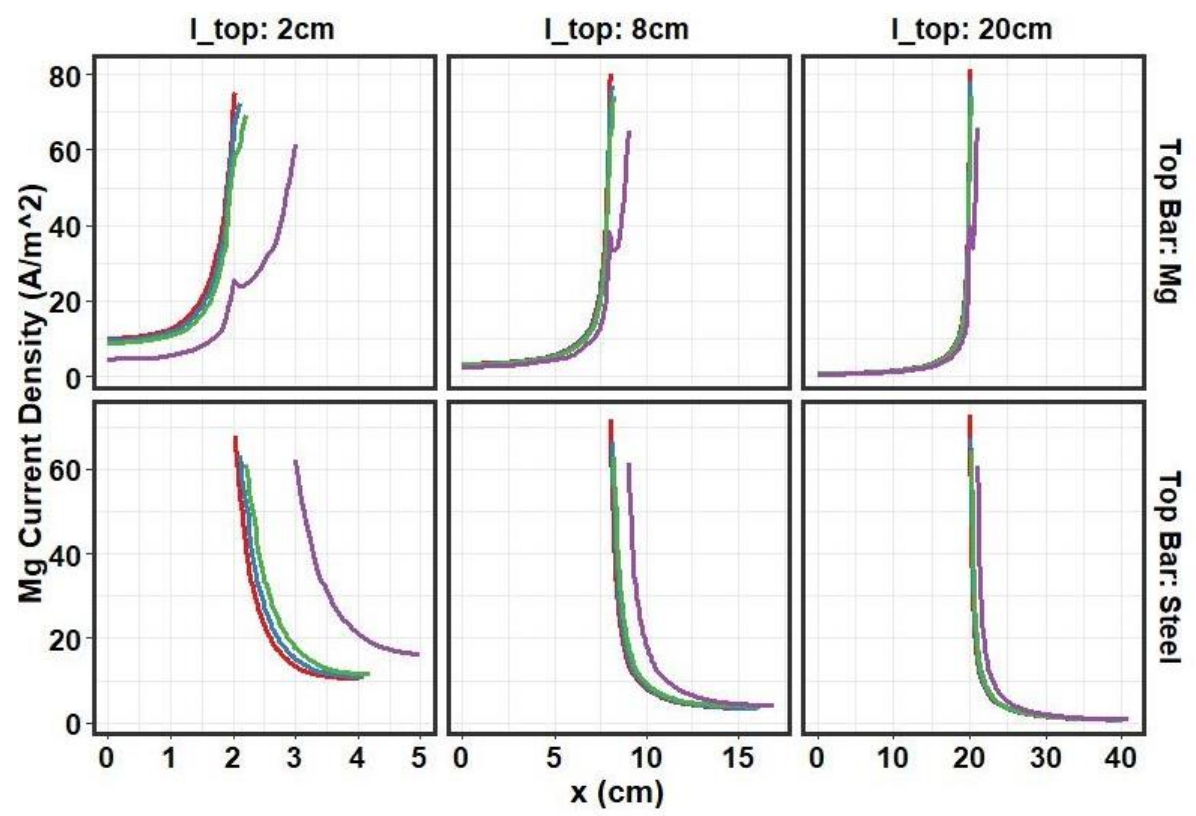

I_thickness(mm) $-0.25-1-2-10$

Figure II.3.14.4. Mg current distribution for selected cases where I $_{\text {top }}=$ l $_{\text {bottom. }}$ Source: PNNL.

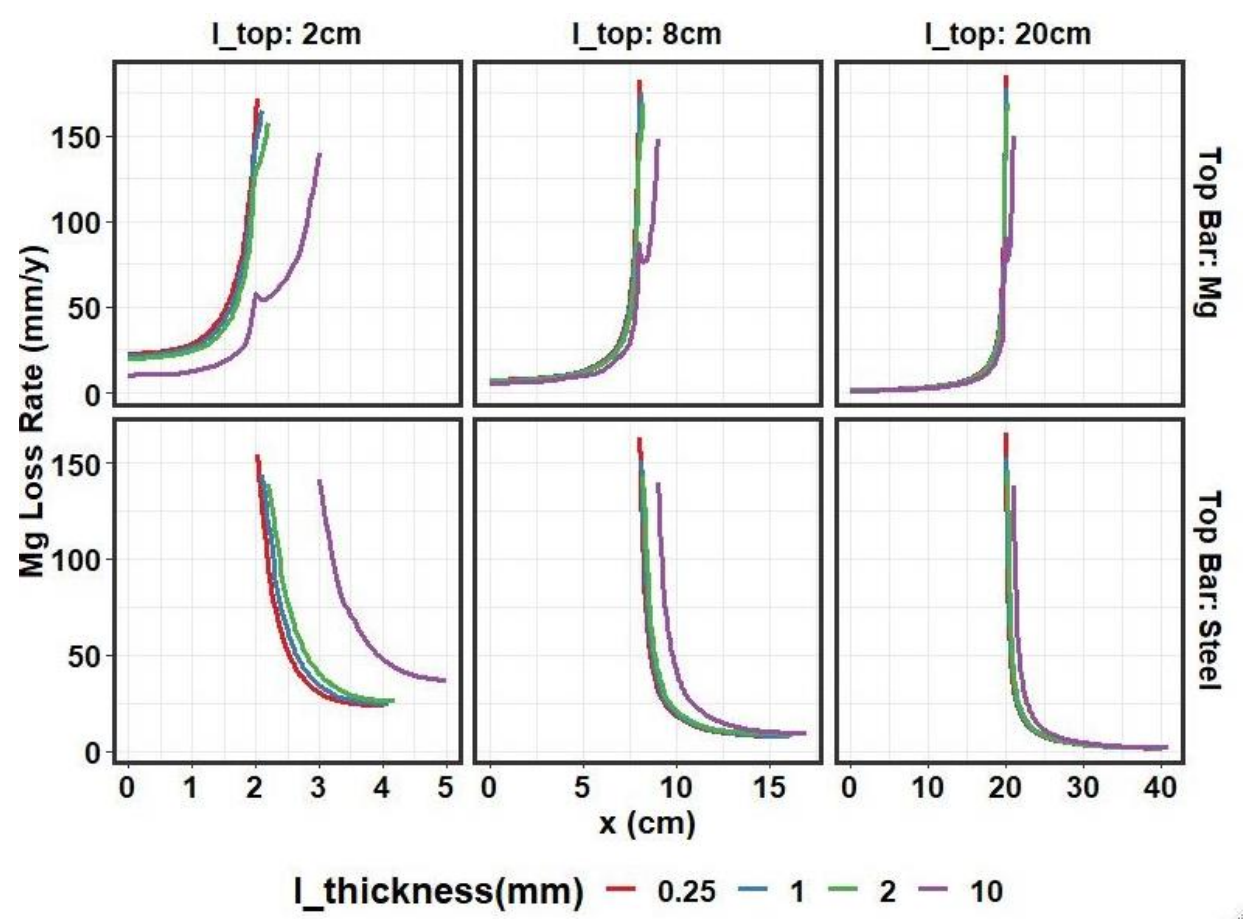

Figure II.3.14.5. Mg loss rate for selected cases where $I_{\text {top }}=I_{\text {bottom. }}$ Source: PNNL.

The distribution of current along the bars is given in Figure II.3.14.6 and Figure II.3.14.7, with the former being the actual current density and the latter being the $\mathrm{Mg}$ loss rate. For each $\mathrm{A} / \mathrm{m}^{2}$ of $\mathrm{Mg}$ current, $2.28 \mathrm{~mm} / \mathrm{yr}$ is lost. The plots start with $\mathrm{x}=0$ referring to the far left of the top bar. Local maxima can be observed at the corner on the top bar. Current is maximized at the inside of the bottom corner. It is not clear why the maximum anodic current density increases when length of the $\mathrm{Mg}$ top bar increases, whereas the increase of maximum anode current density with increasing length of the top steel bar is consistent. 


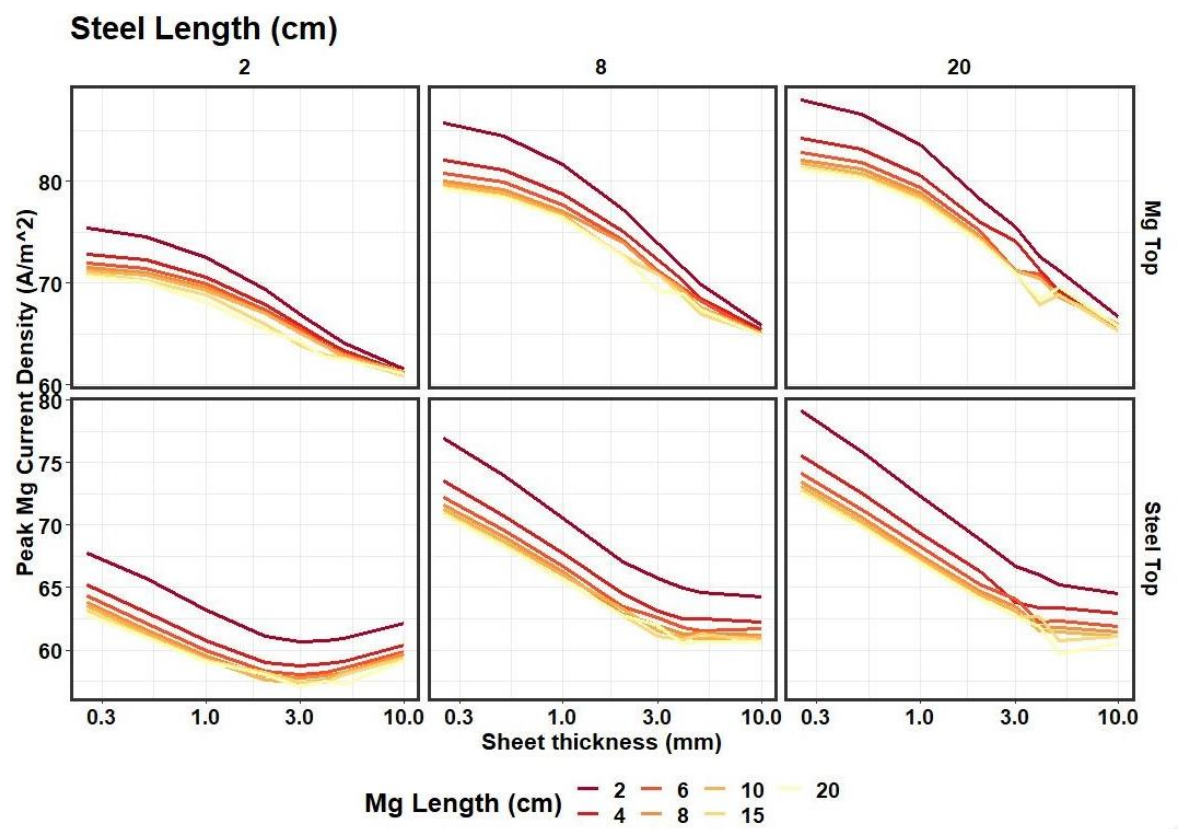

Figure II.3.14.6. Peak Mg current density at selected steel lengths. Source: PNNL.

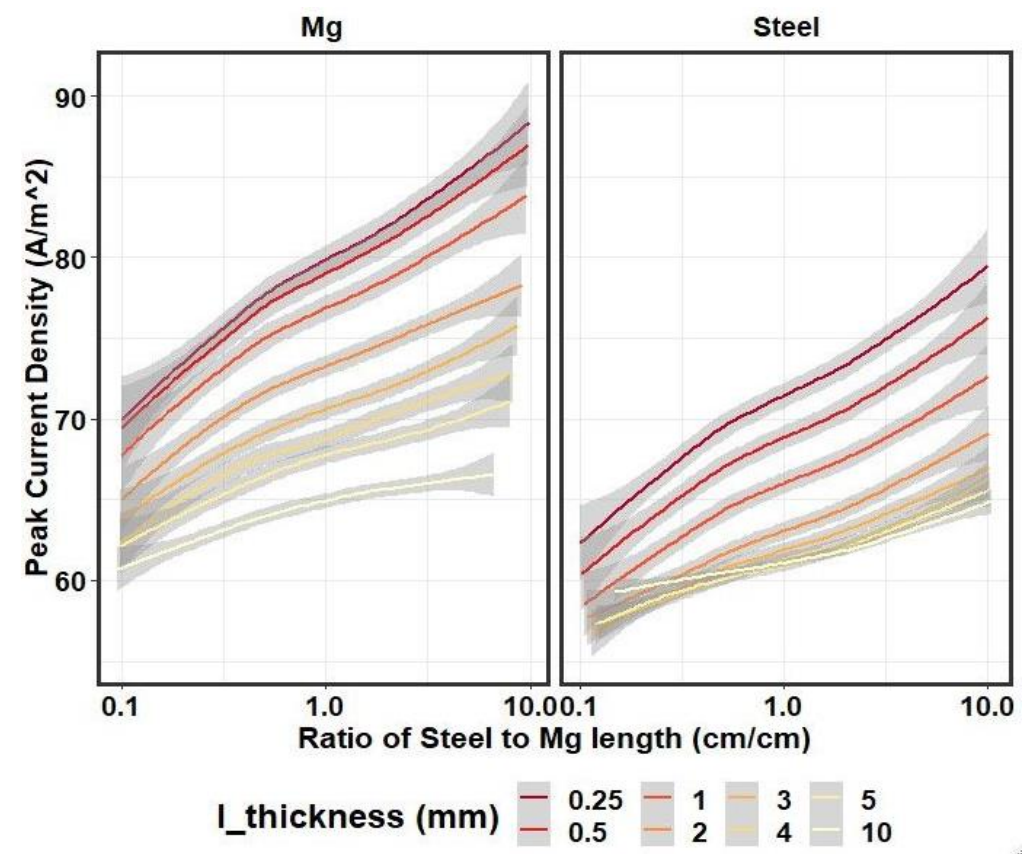

Figure II.3.14.7. Peak Mg current density for Mg on top and Steel on top at various thicknesses as a function of the ratio of bar lengths. Source: PNNL.

The peak current is an important part of the distribution, especially as this peak occurs right at the joint thereby weakening it. To get a better idea of how the lengths effect the peak current, the peak current is plotted as a function of the various lengths in Figure II.3.14.7. In general, as expected, increasing the steel length results in a larger peak current for $\mathrm{Mg}$. In addition, increasing the thickness of the top bar results in a lower peak current. Having the $\mathrm{Mg}$ on top generally results in a higher peak current. Increasing the length of the Mg bar results in a lower peak current. 
To consolidate these trends further, the peak $\mathrm{Mg}$ current density was plotted as a function of a ratio of steel-to$\mathrm{Mg}$ lengths in Figure II.3.14.7, showing you can mostly find the peak $\mathrm{Mg}$ current just from knowing the ratio of the bar lengths and the thickness and which is on top. Again, $\mathrm{Mg}$ on top increases the peak current, having a larger top bar thickness decreases the peak current, and having a high ratio of steel-length-to-Mg-length increases the peak current. Note that larger thickness is a proxy for the angle between bars approaching $90^{\circ}$.

Figure II.3.14.8 (a) shows the microstructure and Figure II.3.14.8 (b) and Figure II.3.14.8 (c) show the electrochemical results for a model steel sample used to establish the SECCM characterization protocols. The optical microstructure shows the footprint residue of SECCM measurement locations. Locations are marked by yellow and red circles. The starting spot was very large due to initial leakage of the electrolyte. The yellow and red circles represent the grain interior and grain boundary locations. The Tafel plot shows the variation of $E_{\text {Corr }}$ value among the grains. The $E_{\text {Corr }}$ values corresponding to Point 6 and Point 7 towards the more negative sides which indicate that the grain boundaries are more susceptible to corrosion. For demonstration purposes, the EIS was measured at a 0 volt potential. The Nyquist plot shown in Figure II.3.14.8 (c) corresponding to all the point shows very similar results. This indicates that the instrument is reliable, and data is reproducible. Instrument reliability was further verified by calculating the resistance from current-voltage (I-V) and EIS measurements. The resistance calculated from two different and independent techniques is shown in Table II.3.14.1 and is very similar, which demonstrates the instrument's reliability.

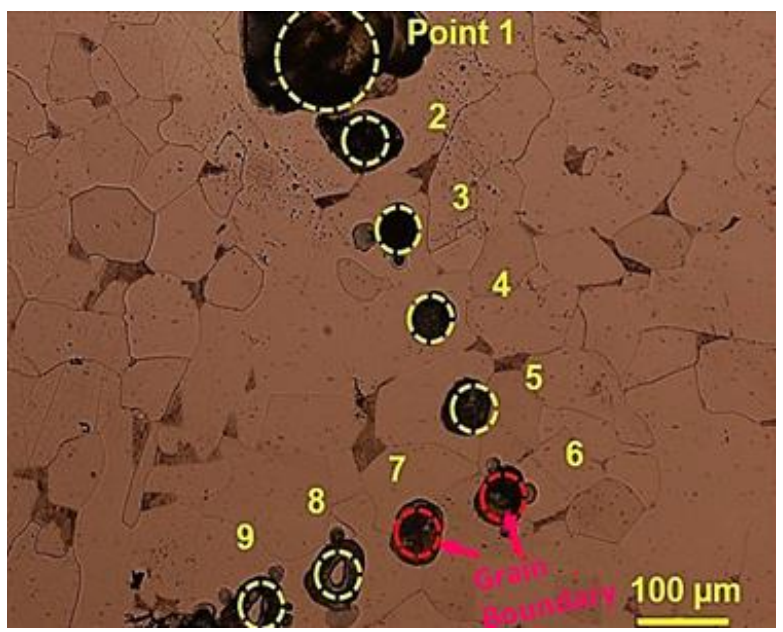

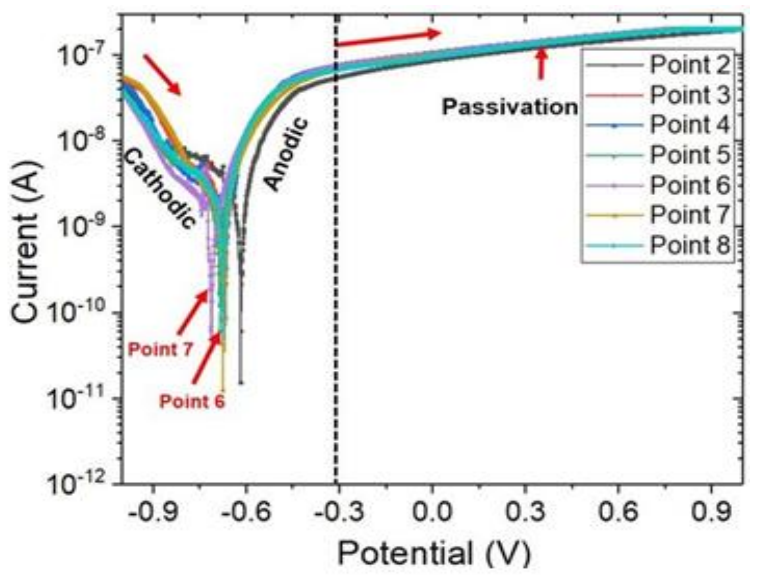

(b)

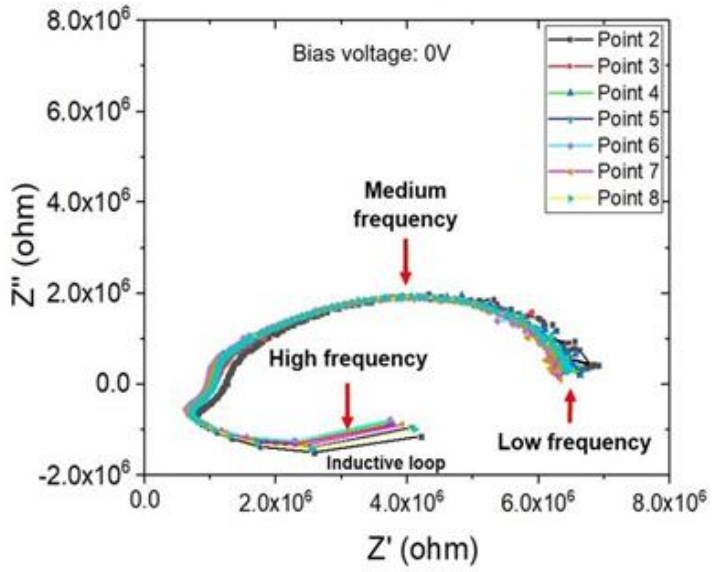

(c)

Figure II.3.14.8. (a) Optical microstructure taken after measurements showing the different locations where data was generated to produce the (b) Tafel and (c) Nyquist plots for the corresponding points marked. The starting measurement point was relatively large. Yellow circles represent individual grains and red circles are grain boundaries. Source: PNNL. 
Table II.3.14.1. Resistance Calculated From DC and AC Methods.

\begin{tabular}{|c|c|c|}
\hline Location & $\begin{array}{c}\text { DC method/ I-V plot } \\
(\text { ohm })\end{array}$ & $\begin{array}{c}\text { AC method/ } \\
\text { Nyquist plot (ohm) }\end{array}$ \\
\hline Point 2 & $9.1 \times 10^{6}$ & $\sim 6.7 \times 10^{6}$ \\
\hline Point 3 & $8.0 \times 10^{6}$ & $\sim 6.5 \times 10^{6}$ \\
\hline Point 4 & $8.4 \times 10^{6}$ & $\sim 6.4 \times 10^{6}$ \\
\hline Point 5 & $8.3 \times 10^{6}$ & $\sim 6.3 \times 10^{6}$ \\
\hline Point 6 & $8.2 \times 10^{6}$ & $\sim 6.3 \times 10^{6}$ \\
\hline Point 7 & $8.2 \times 10^{6}$ & $\sim 6.4 \times 10^{6}$ \\
\hline Point 8 & $8.2 \times 10^{6}$ & $\sim 6.5 \times 10^{6}$ \\
\hline
\end{tabular}

Selective joint masking and immersion in $0.1 \mathrm{M} \mathrm{NaCl}$ was conducted for 208 hours for three types of joints: (1) USW Mg/bare steel; (2) USW Mg/Zn-coated steel; and (3) FSSW Mg/Zn-coated steel. The corrosion of $\mathrm{Mg}$ in the three tested joints are shown in Figure II.3.14.9 (a)-(c) along with the reconstructed corrosion morphologies also shown in Figure II.3.14.9 (d)-(f). The most and least severe corrosion of Mg were observed in the USW joints with bare and Zn-coated steels, respectively, while the FSSW joint with Zn-coated steel resulted in intermediate corrosion of $\mathrm{Mg}$. The bare steel substrate exposed after $\mathrm{Mg}$ corrosion did not produce any visible corrosion product and maintained a flat profile as seen in Figure II.3.14.9 (a) and (d), indicating that the bare steel surface did not corrode. This is because the bare steel surface functioned as a cathode and was galvanically protected by the Mg acting as an anode in the joint. Meanwhile, the $\mathrm{Zn}$-coated steel substrates exposed after Mg corrosion were covered with white corrosion products with uneven surface profiles for both USW and FSSW joints that can be seen in Figure II.3.14.9 (b) and (e). This indicates that Zn-coating on steels corroded simultaneously with $\mathrm{Mg}$ and did not function as an effective cathode like bare steel. These results highlight a beneficial effect of $\mathrm{Zn}$-coating on mitigating the galvanic corrosion of $\mathrm{Mg}$ in welded joints.

The maximum corrosion depths of Mg, shown in Figure II.3.14.10 (a), and corrosion potentials, shown in Figure II.3.14.10 (b) were measured during the immersion test of the joints presented above and plotted as a function of time. The corrosion depths of $\mathrm{Mg}$ were higher in the joint with bare steel than the joint with $\mathrm{Zn}$ coated steel for the entire immersion test. For Mg joined with Zn-coated steels, the USW joint resulted in lower corrosion depth than the FSSW joint. For all three joints, the growth of corrosion depth decreased notably after approximately $112 \mathrm{~h}$.

In the meantime, the measured corrosion potentials appeared higher in the following order: $\mathrm{Mg} / \mathrm{bare}$ steel

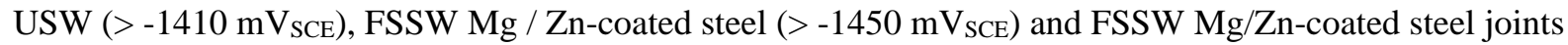
$\left(>-1460 \mathrm{mV}_{\mathrm{SCE}}\right)$. All measured corrosion potentials of the joints were higher than the corrosion potential of single $\mathrm{Mg}$ electrode, i.e. lower than $-1540 \mathrm{mV}_{\mathrm{SCE}}$ in $0.1 \mathrm{M} \mathrm{NaCl}$ under the same condition. As the degree of galvanic influence can be approximated by the corrosion potential difference of single $\mathrm{Mg}$ and the joints, the galvanic corrosion of $\mathrm{Mg}$ would have been greater in $\mathrm{Mg}$ joined with bare steel, and this agreed well with the corrosion depths measured in the joints. It is also noticed that the rise of corrosion potentials slowed down after approximately $120 \mathrm{~h}$, which somewhat coincided with the time when the growth of corrosion depths decreased. This contradicts the idea that corrosion of $\mathrm{Mg}$ would accelerate under a higher galvanic potential. The most probable reason for this behavior is the increase of ohmic drop, caused by diffusion and corrosion product layers, for the corroding surface of $\mathrm{Mg}$, which will need further investigation. 


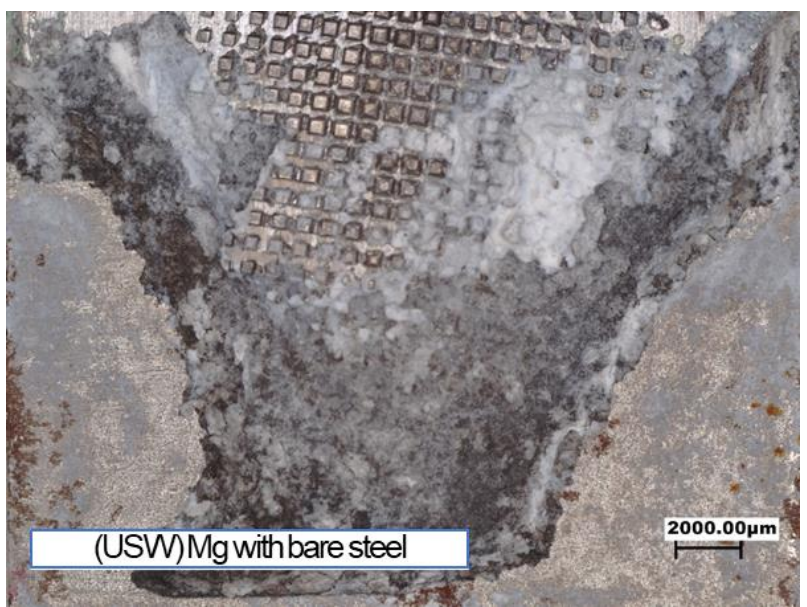

(a)

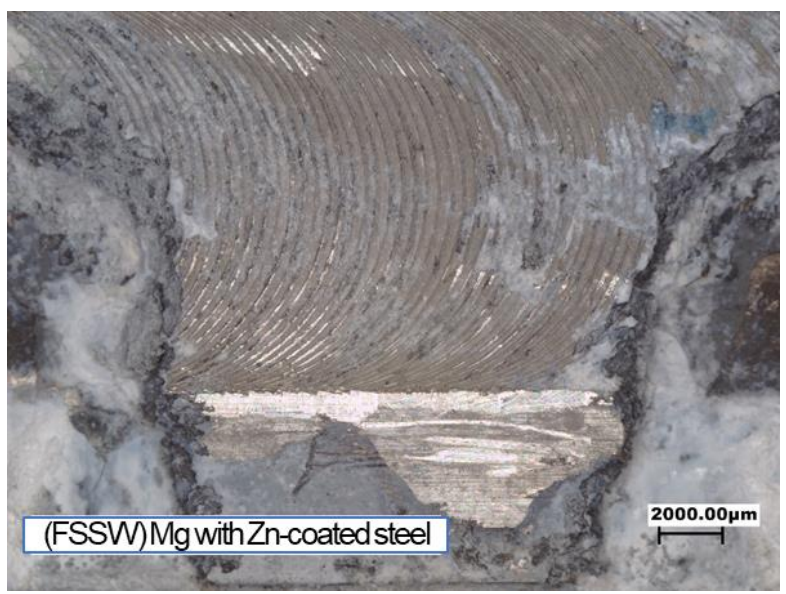

(c)

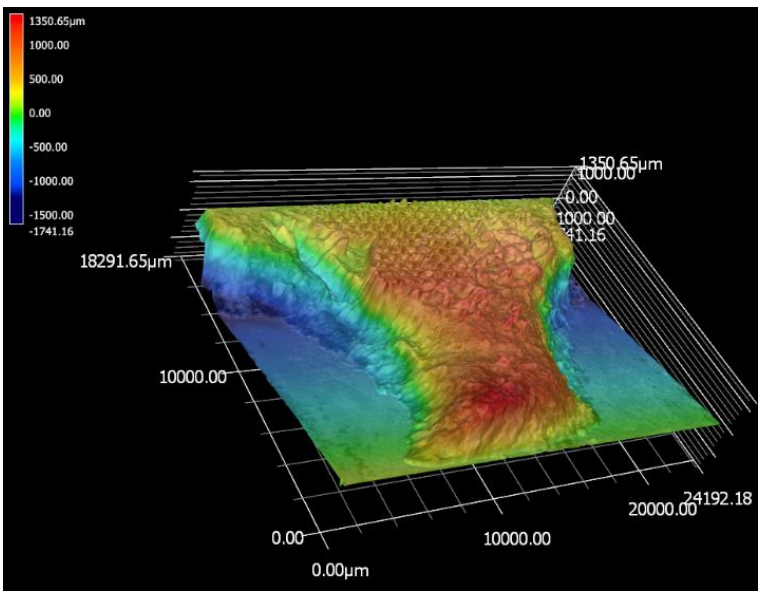

(e)

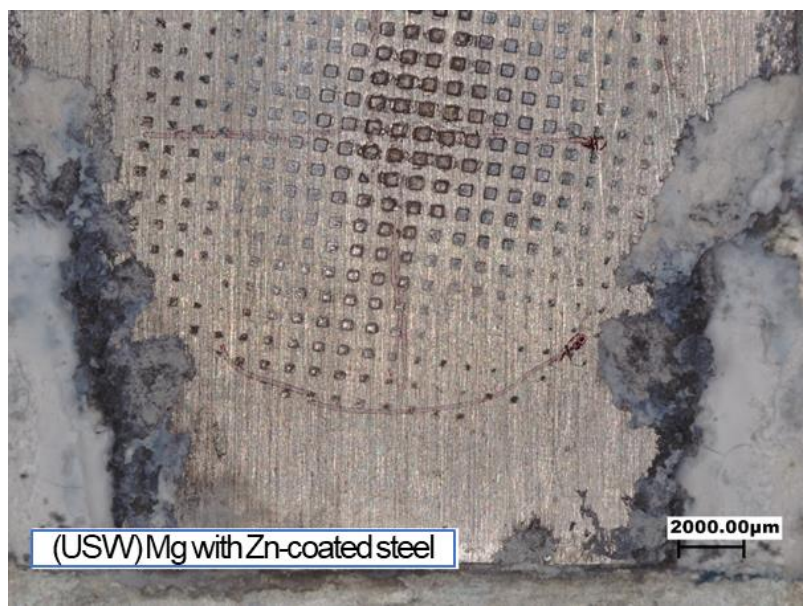

(b)

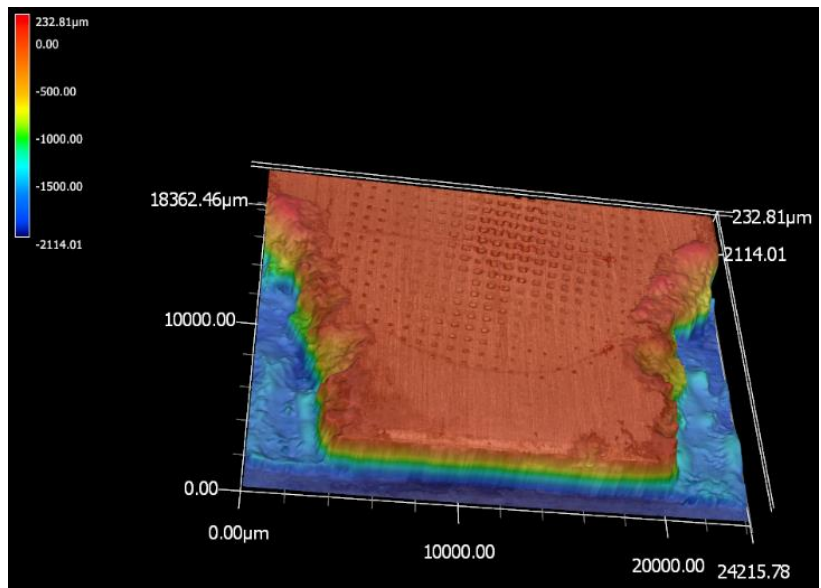

(d)

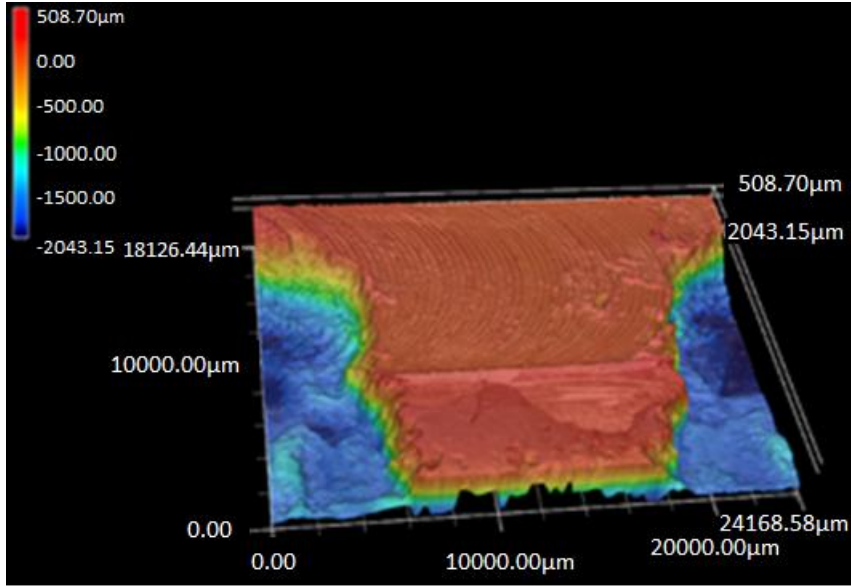

(f)

Figure II.3.14.9. Plan view of corroded (a) USW joint of Mg and bare steel; (b) USW joint of Mg and Zn-coated steel; and

(c) FSSW joint of $\mathrm{Mg}$ and $\mathrm{Zn}$-coated steel after $208 \mathrm{~h}$ immersion in $0.1 \mathrm{M} \mathrm{NaCl}$. The related corrosion morphologies reconstructed by an image analysis software are shown in (d)-(f), respectively. Notable difference of Mg corrosion volume can be observed between the joints. Source: ORNL. 


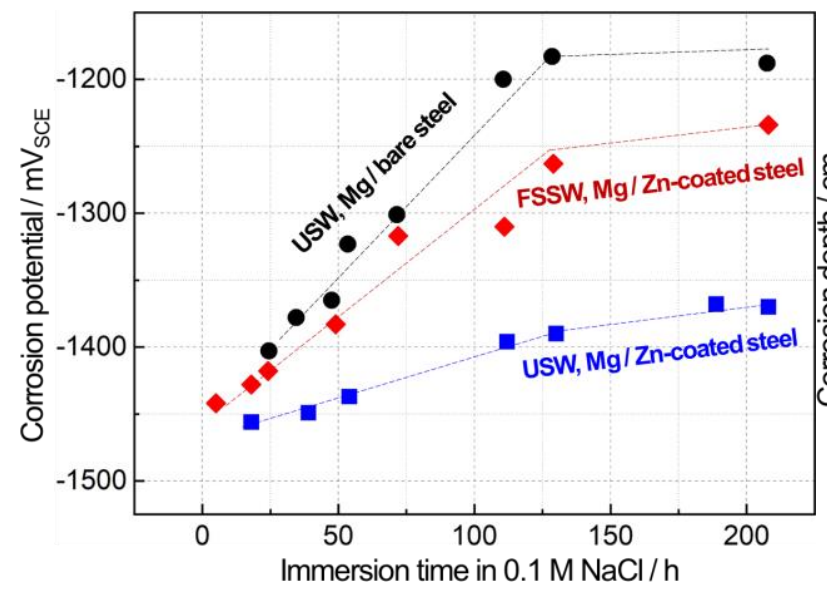

(a)

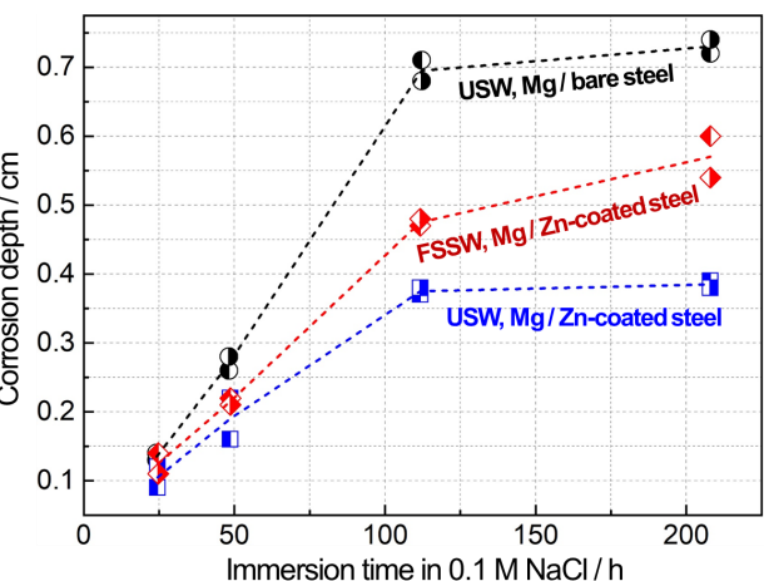

(b)

Figure II.3.14.10. (a) The maximum corrosion depths measured for the two sides of Mg (left- and right-half filled symbols for the left and right sides, respectively) and their average trends (dotted lines) as a function of time; and (b) the corrosion potentials of the joints measured during $0.1 \mathrm{M} \mathrm{NaCl}$ immersion using saturated calomel reference electrode also as a function of time. Arbitrary trend lines are added for corrosion potential data in (b). Source: ORNL.

Multi-length scale microscopy and microanalysis of the USW and FSSW post-corrosion joints helped to understand the extent of corrosion and the types of corrosion products which evolved during the selective joint masking and immersion. To illustrate the new knowledge gained by the materials characterization Figure II.3.14.11 shows images of galvanic corrosion produced on the millimeter and micron length scales of the $\mathrm{Zn}$-coated steel and Mg sheet used in the joint. The OM of Figure II.3.14.11 was acquired after the corroded joint was fractured during a tensile test. The shiny round area at the center of the OM shows a top down view of the USW joint. One empirical observation from this micrograph is that the USW joint did not corrode during the immersion. Near the outer edge of this joint, there was evidence that corrosion had taken place because of the changes in contrast in the field of view indicating that the corrosion products scatter the light differently. To better understand corrosion product morphology, a 3D optical profilometer was used to capture height based images from the same sample region observed with OM. A thicker type corrosion product was observed with this method, but the elemental composition was still unknown. Using the SEM and EDS, the exact same feature identified in the 3D images was found again in the SEM and an X-ray map was acquired. X-ray elemental analysis determined that the corrosion products with different morphologies were in fact different in composition too. From the SEM/EDS, it is clearly shown that the more abundant porous corrosion product was $\mathrm{Mg}$ and O-rich, yet the taller, denser appearing corrosion product contained $\mathrm{Zn}, \mathrm{Mg}$, and $\mathrm{O}$.

The analysis described above was done first on the $\mathrm{Zn}$-coated steel side of the fractured joined specimen. To determine if the corrosion products and morphology were similar on the $\mathrm{Mg}$ side of the fractured joint, the same multi-length scale materials characterization was performed. The SEM micrograph in Figure II.3.14.12 (a) shows the surface of the weld, corrosion product, and un-corroded $\mathrm{Mg}$ in the weld region. These regions were better visualized using the $\mathrm{x}$-ray mapping and the maps for $\mathrm{Mg}, \mathrm{Zn}, \mathrm{O}$ and $\mathrm{Cl}$ are shown in Figure II.3.14.12 (b). Lightly to un-corroded Mg was a surprise finding at first, but it is presumed that the formation of Zn-rich corrosion products along with trapped hydrogen bubbles in the narrow weld gap may have blocked the access of $\mathrm{NaCl}$ solution for this local spot so that $\mathrm{Mg}$ did not appreciably corrode.

With experimental evidence indicating that the welds themselves in FSSW and USW joints were not extensively corroding, rather only the materials around them were extensively corroded, we attempted to observe corrosion of a FSSW joint cross-section. Applying the selective masking and immersion approach to a metallographically polished FSSW joint cross-section allowed the first look at how the TMAZ, weld region (nugget), Zn-coated steel, and Mg corrosion proceeds in the as-joined FSSW configuration. 

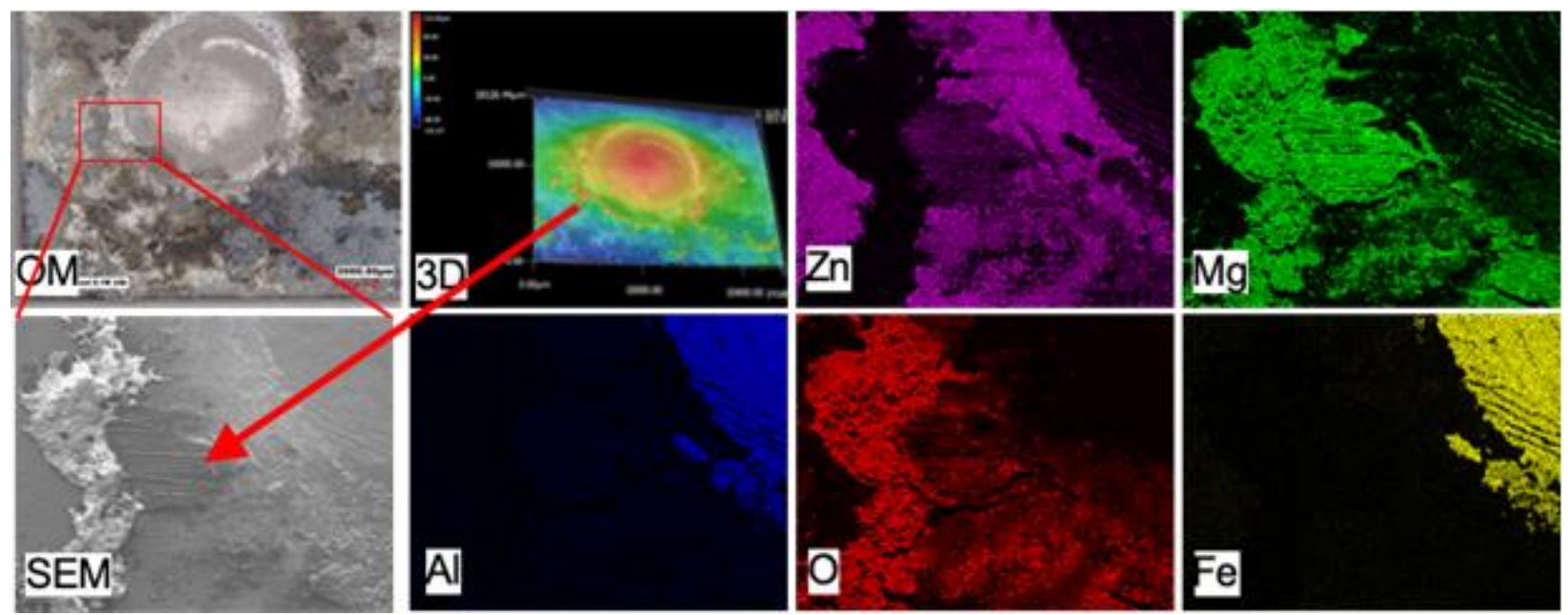

Figure II.3.14.11. Multi-length scale imaging of post-corrosion Mg to Zn-coated steel USW joint. OM and 3D imaging were used to survey the fracture surface of the USW weld. SEM and X-ray maps determined two types of corrosion product present post-corrosion. Mg and O-rich corrosion product and a Mg, ,Zn and O-rich feature were present on the Zn side of the fractured USW joint. Source: ORNL.

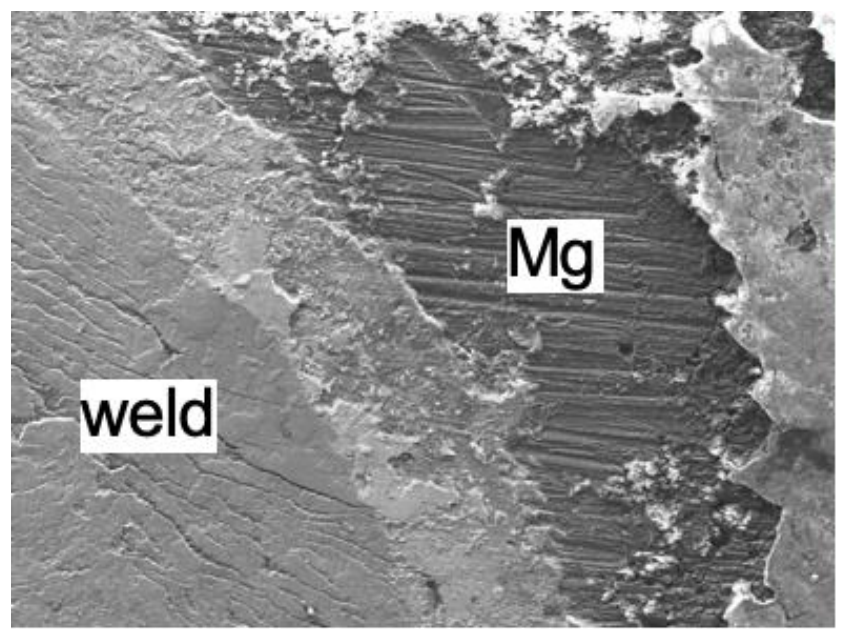

(a)
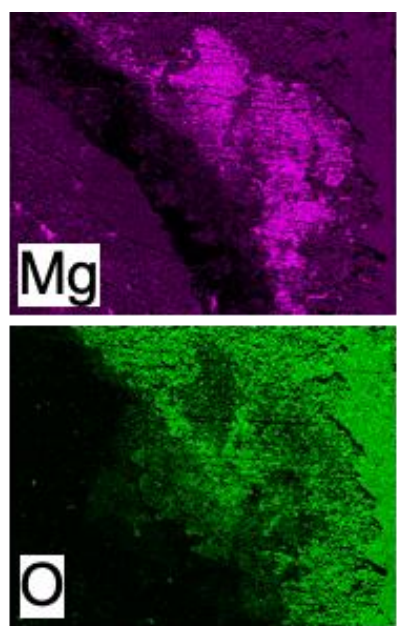
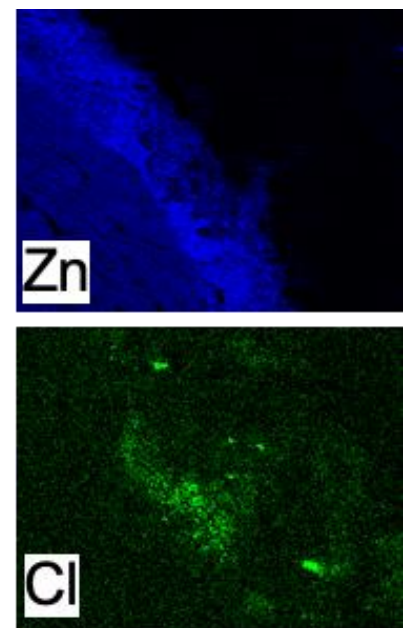

(b)

Figure II.3.14.12. Post-corrosion USW sample, Mg side of sample in Figure II.3.14.9: (a) The SEM micrograph shows that un-corroded Mg existed near the USW joint, in addition to the Mg oxide corrosion product; (b) X-ray maps show the distribution of $\mathrm{Mg}$ oxide scale near the joint, the $\mathrm{Zn}$ which diffused from the steel coating and $\mathrm{Cl}$ ions left over from the immersion. Source: ORNL.

At the top of Figure II.3.14.13, a low magnification OM image of the entire post-corrosion FSSW joint crosssection shows evidence that different regions of the joint corroded at different rates and produced different morphologies of corrosion products after a two hour immersion in $0.1 \mathrm{M} \mathrm{NaCl}$ at $\mathrm{RT}$. Dotted red line delineates different corrosive attack in the TMAZ and nugget regions of the joint. The TMAZ zone appeared to corrode much less and with a filamentous morphology while the Mg below this TMAZ region corroded much more aggressively. Further examination at higher magnifications with SEM/EDS showed the $\mathrm{Zn} / \mathrm{Mg}$ eutectic at the steel/Mg interface did not undergo significant corrosion when compared to the $\mathrm{Mg}$ right above this interfacial layer (see bottom left of Figure II.3.14.13). A low density corrosion product in the Mg below the TMAZ was in stark contrast to the filamentous corrosion observed in the TMAZ region of the joint, and severe corrosion of the $\mathrm{Mg}$ above the $\mathrm{Zn}$-coated steel was revealed. The nugget of the joint also appeared to corrode more heavily when compared to the TMAZ region. The EDS x-ray mapping, shown right-center of 
Figure II.3.14.13, confirmed $\mathrm{Cl}, \mathrm{Al}$ and $\mathrm{O}$ present in the filamentous corrosion regions. Further investigations, on the nanoscale with STEM/EDS is planned to understand more about the microstructure and elemental composition in this distinctly different region of the post-corrosion cross-section. The filamentous corrosion morphology was somewhat unexpected for these Mg-steel joint samples, and additional studies will be pursued to ensure these features are not related to some type of artifact of the test or characterization procedure.
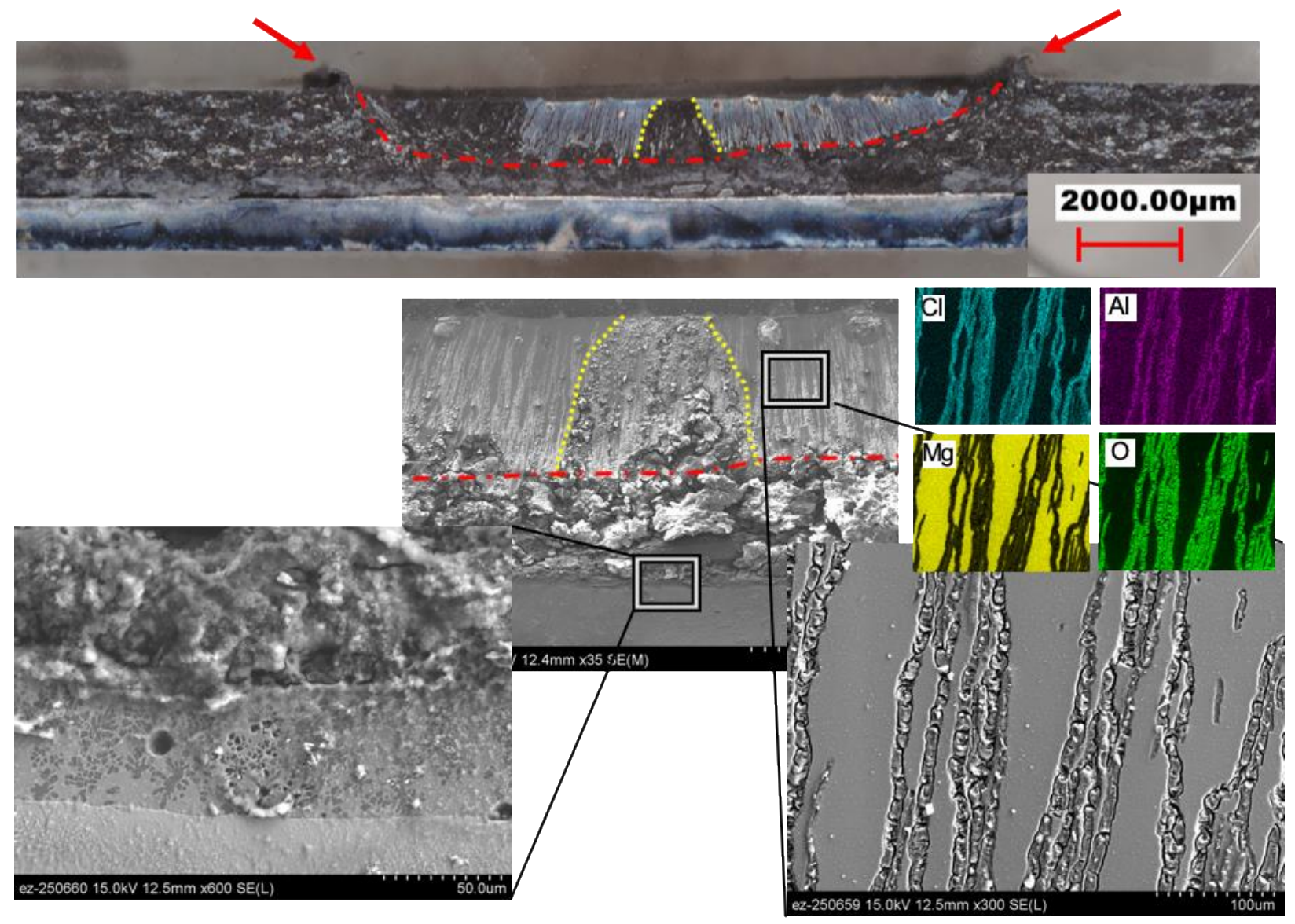

Figure II.3.14.13. Post-corrosion analysis of PNNL FSSW Mg/Zn-coated steel joint cross-section. Source: ORNL.

\section{Conclusions}

1. The maximum current density is computationally observed when the ratio of the $\mathrm{Mg}$ (anode)-to-steel (cathode)-overlap is high. When $\mathrm{Mg}$ is placed on top of the steel, the peak current density is approximately two times more than when steel is placed on top of the $\mathrm{Mg}$.

2. The effect of hermeticity and sheared edge was also computationally investigated, and it was observed that the average current density is highest when the seal is not hermetic. As the angle of the sheared edge between $\mathrm{Mg}$ and steel is reduced, the average current density decreases by approximately three times.

3. The effect of a $\mathrm{Zn}$-coating on the steel was investigated and it was determined that the $\mathrm{Zn}$ interlayer helps in reducing the anodic current density.

4. The effect of steel/Mg thickness ratios on the corrosion density was computationally investigated. A ratio of 0.25 helps to minimize corrosion in this system. It is desirable to have $\mathrm{Mg}$, which is at least four times the thickness of the steel sample, to reduce the average galvanic current density to near zero. 
5. In the SECCM study, a testing protocol for microscopic corrosion study was developed. Two independent electrochemical techniques, DC and AC, showed very similar resistance values that demonstrate the instrumental reliability. Measurement spots (or grains) are identified after measurement. Orientationdependent corrosion properties and the role of the second-phase particles in the overall corrosion behavior can be established now. This can help in designing corrosion resistance structural materials.

6. Selective joint masking and immersion tests were conducted to quantify the galvanic corrosion of $\mathrm{Mg}$ in different weld joints in $0.1 \mathrm{M} \mathrm{NaCl}$ at RT. The measured $\mathrm{Mg}$ corrosion depths can be summarized: $\mathrm{Mg}$ / bare steel USW > Mg / Zn-coated steel FSSW > Mg / Zn-coated steel USW.

7. The Mg / bare steel USW joint with the greatest corrosion depth exhibited the highest corrosion potential while the $\mathrm{Mg} / \mathrm{Zn}$-coated steel with the lowest corrosion depth showed the lowest corrosion potential. This result agreed well with the idea that the Mg galvanic corrosion would increase at a higher corrosion potential of a galvanic couple.

8. Experimental evidence indicated that the welds of FSSW and USW joints were only minimally corroding, only the materials around them were susceptible to corrosion.

9. Post-corrosion examination of FSSW joint cross-section showed distinct morphological differences in corrosive attack due to microstructure and/or elemental composition.

\section{Key Publications}

1. Spies, K. A., V. V. Viswanathan, A. Soulami, Y. Hovanski, and V. V. Joshi, 2019, "Galvanically graded interface: A computational model for mitigating galvanic corrosion between $\mathrm{Mg}$ and mild steel," Magnesium Technology, 2019, pp. 135-144.

\section{References}

1. Yule, L. C., C. L. Bentley, G. West, B. A. Shollock, and P. R. Unwin, 2019, "SECCM: A versatile method for highly localized corrosion-related measurements on metal surfaces," Electrochim. Acta, Vol. 298, pp. 80-88.

2. Ebejer, N., A. G. Güell, S. Lai, K. McKelvey, M. E. Snowden, and P. R. Unwin, 2013, "SECCM: A versatile technique for nanoscale electrochemistry and functional imaging," Annu. Rev. Anal. Chem., Vol. 6, pp. 329-351.

3. Shimamura, R., A. Sugimoto, T. Fujiwara, and O. Seri, 2011, "Polarization behavior and its analysis of $1050 \mathrm{Al}$ in solution containing chloride ions," J. Jpn. I. Light Met., Vol. 61, 2011, pp. 303-309.

4. Südholz, A. D., N. T. Kirkland, R. G. Buchheit, and N. Birbilis, 2011, "Electrochemical properties of intermetallic phases and common impurity elements in Mg alloys," Electrochem. Solid St., Vol. 14, No. 2, pp. C5-C7.

5. Sukiman, N. L., X. Zhou, N. Birbilis, A. E. Hughes, J. M. C. Mol, S. J. Garcia, and G. E. Thompson, 2012, "Durability and corrosion of $\mathrm{Al}$ and its alloys: Overview, property space, techniques, and developments," in Ahmad, Z. (ed.), Aluminum Alloys - New Trends in Fabrication and Applications, pp. 47-97. 


\section{Acknowledgements}

At PNNL, the authors wish to recognize the exceptional contributions from Alasdair Crawford and Vilayanur V. Viswanathan, who performed the COMSOL modeling work. The authors also recognize Rajib Kalsar, Venkateshkumar Prabhakaran, and Olga A. Marina, who performed the SECCM work. At ORNL, the authors wish to recognize Jay Jun, who led the aqueous corrosion studies and designed the corrosion assessment protocols, and Mike Brady, who provided guidance on $\mathrm{Mg}$ corrosion testing and interpretation. We also acknowledge the joining expertise, knowledge and samples supplied by Piyush Upadhyay (PNNL, FSW) and Jian Chen (ORNL, UW). 
(This page intentionally left blank) 


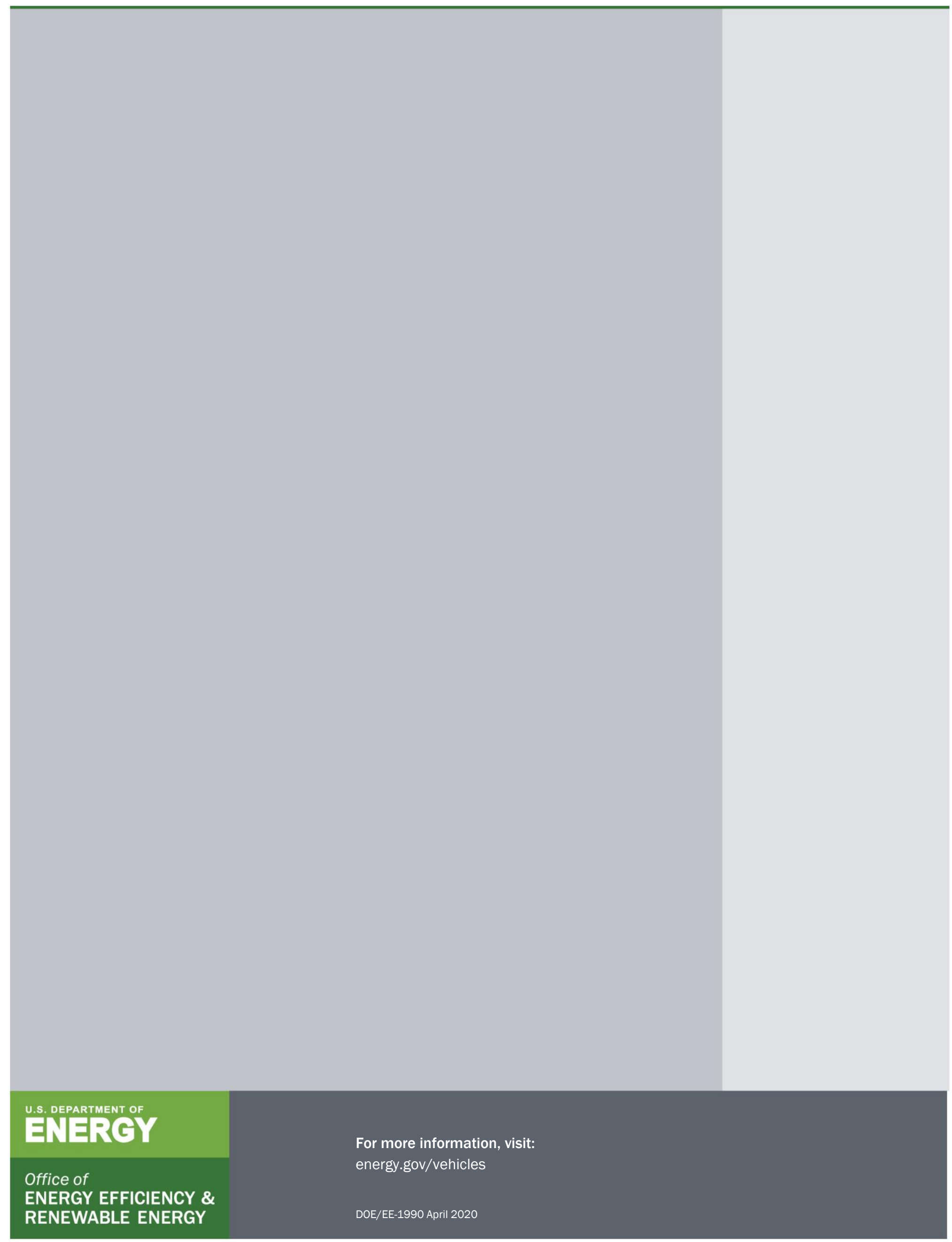

